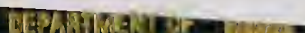

GOMAMERCE

PUDMLARTION

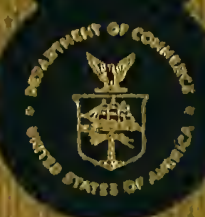

NBS SPECIAL PUBLICATION

\title{
VOLUME "
}

\author{
Precision \\ Measurement \\ and \\ Calibration
}

\section{Radiometry and Photometry}

U.S. DEPARTMENT of COMMERGE

National Bureau of Standards 


\section{Precision Measurement and Calibration}

Volumes available or to be published in the NBS Special Publication 300 series

SP300, Vol. 1, Statistical Concepts and Procedures, H. H. Ku, Editor, Feb. 1969, 436 pages, (SD Catalog No. C 13.10:300/v.1)* Price $\$ 5.50$

SP300, Vol. 2, Temperature, J. F. Swindells, Editor, Aug. 1968, 520 pages, (SD Catalog No. C 13.10:300/v.2)*

Price $\$ 4.75$

SP300, Vol. 3, Electricity-Low Frequency, F. L. Hermach and R. F. Dziuba, Editors, Dec. 1968, 498 pages, (SD Catalog No. C 13.10:300/v.3)*

Price $\$ 4.50$

SP300, Vol. 4, Electricity-Radio Frequency, A. J. Estin, Editor, June 1970, 450 pages, (SD Catalog No. C 13.10:300/v.4)*

Price $\$ 5.50$

SP300, Vol. 5, Frequency and Time, B. E. Blair and A. H. Morgan, Editors, May 1971, 565 pages, (C 13.10:300/v.5)

In Press

SP300, Vol. 6, Heat, D. C. Ginnings, Editor, Feb. 1970, 400 pages, (SD Catalog No. C $13.10: 300 / v .6)$

Price $\$ 5.00$

SP300, Vol. 7, Radiometry and Photometry, H. K. Hammond, III, and H. L. Mason, Editors, Nov • 1971, 690 pages, (SD Catalog No. C 13.10:300/v.7)*

Price $\$ 7.00$

\section{Volumes in Press**}

SP300, Vol. 8, Mechanics, R. L. Bloss and Mary J. Orloski, Editors.

SP300, Vol. 9, Colorimetry, I. Nimeroff, Editor.

SP300, Vol. 10, Spectrochemical Analysis, B. F. Scribner, Editor.

SP300, Vol. 11, Ionizing Radiation, Elmer H. Eisenhower, Editor.

SP300, Vol. 12, Image Optics, Calvin S. McCamy, Editor.

* Available by purchase from the Superintendent of Documents, Government Printing Office, Washington, D. C. 20402. Order by the Supt. of Documents Catalog number as given for each volume.

** For announcements of new volumes as issued, complete the form (Notification key N-353) in the back of this volume and mail it to the Superintendent of Documents office. 



$$
\text { . }
$$




\title{
NATIONAL
}

United STATES DEPaRTMEnT OF COMmerce - Maurice H. Stans, Secretary $\checkmark$ S. NATIONAL BUREAU OF STANDARDS - Lewis M. Branscomb, Director $\cdots$

\section{Precision Measurement and Calibration}

\author{
Selected NBS Papers on
}

\section{Radiometry and Photometry}

H. K. Hammond III, and H. L. Mason, Editors

Institute for Applied Technology

National Bureau of Standards

Washington, D.C. 20234

A compilation of previously published papers by the staff of the National Bureau of Standards, including selected abstracts by NBS and non-NBS authors. Issued in several volumes, see page Iv.

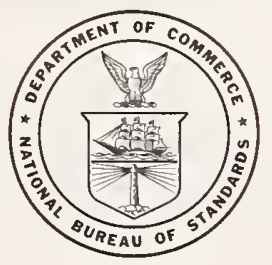

NBS Special Publication 300 - Volume 7

Coden Nat. Bur. Stand. (U.S.), Spec. Publ. 300, V. 7, 685 pages, (Nov. 1971) CODEN: XNBSA

Issued November 1971 


\section{A bstract}

This volume is one of an extended series which brings together some of the previously published papers, monographs, abstracts and bibliographies by NBS authors dealing with the precision measurement of specific physical quantities and the calibration of the related metrology equipment. The contents have been selected as being useful to the standards laboratories of the United States in tracing to NBS standards the accuracies of measurement needed for research work, factory production, or field evaluation.

Volume 7 contains reprints of more than 60 items on radiometry and photometry published between 1957 and 1970. The material has been placed in groups according to the following subjects: general radiometry, emissivity standards, emissivity measurements and techniques, material properties, irradiance standards, radiometric measurement techniques, radiance standards, flux measurement, reflectometry, general photometry, projectors, flashing lights, and chronological bibliography of publications by NBS staff.

Key words: Emissivity, flashing light, flux measurement, irradiance, material properties, photometry, projectors, radiance, .radiometry, reflectometry.

Library of Congress Catalog Card Number: 68-60042 


\section{For ew ord}

In the 1950's the tremendous increase in industrial activity, particularly in the missile and satellite fields, led to an unprecedented demand for precision measurement, which, in turn, brought about the establishment of hundreds of new standards laboratories. To aid these laboratories in transmitting the accuracies of the national standards to the shops of industry, NBS in 1959 gathered together and reprinted a number of technical papers by members of its staff describing methods of precision measurement and the design and calibration of standards and instruments. These reprints, representing papers written over a period of several decades, were published as NBS Handbook 77, Precision Measurement and Calibration, in three volumes: Electricity and Electronics; Heat and Mechanics; Optics, Metrology, and Radiation.

Some of the papers in Handbook 77 are still useful, but new theoretical knowledge, improved materials, and increasingly complex experimental techniques have so advanced the art and science of measurement that a new compilation has become necessary. The present volume is part of a new reprint collection, designated NBS Special Publication 300, which has been planned to fill this need. Besides previously published papers by the NBS staff, the collection includes selected abstracts by both NBS and non-NBS authors. It is hoped that SP 300 will serve both as a textbook and as a reference source for the many scientists and engineers who fill responsible positions in standards laboratories.

Lewis M. Branscomb, Director. 


\section{Preface}

The general plan for this compilation has been reviewed by the Information Committee of the National Conference of Standards Laboratories. The plan calls for Special Publication 300 to be published in 12 volumes as presented on the inside of the front cover.

The division of subject matter has been chosen to assure knowledgeable selection of context rather than to attain uniform size. It is believed, however, that the larger volumes, of approximately 500 pages, will still be small enough for convenient handling in the laboratory.

The compilation consists primarily of original papers by NBS authors which have been reprinted by photoreproduction, with occasional updating of graphs or numerical data when this has appeared desirable. In addition, some important publications by nonNBS authors that are too long to be included, are represented by abstracts or references; the abstracts are signed by the individuals who wrote them, unless written by the author.

Each volume has a subject index and author index, and within each volume, contents are grouped by subtopics to facilitate browsing. Many entries follow the recent Bureau practice of assigning several key words or phrases to each document; these may be collated with titles in the index. Pagination is continuous within the volume, the page numbers in the original publications also being retained and combined with the volume page numbers, for example 100-10. The index notation 7-134 refers to volume 7, page 134 of this volume. A convenient list of SI (Système International) physical units and a conversion table are to be found inside the back cover.

The publications listed herein for which a price is indicated are available from the Superintendent of Documents, U.S. Government Printing Office, Washington, D.C. 20402 (foreign postage, one-fourth additional). Many documents in the various NBS nonperiodical series are also available from the NBS National Technical Information Service, Springfield, Va. 22151. Reprints from the NBS Journal of Research or from non-NBS journals may sometimes be obtained directly from an author.

Suggestions as to the selection of papers which should be included in future editions will be welcome. Current developments in measurement technology at NBS are covered in annual seminars held at either the Gaithersburg (Maryland) or the Boulder (Colorado) laboratories. These developments are summarized, along with a running list of publications by NBS authors, in the monthly NBS Technical News Bulletin.

HENRY L. MASON,

Office of Measurement Services, NBS Institute for Basic Standards. 


\section{Editor's Note}

The papers reprinted in this volume are those that seemed to the editors and their colleagues to merit inclusion in this kind of compendium. The radiometry papers are grouped under related headings; they include papers on emissivity, emittance, radiance, and irradiance standards as well as related measurement techniques, including reflectometry and the increasingly important topic of laser radiometry.

Photometry is actually a specialized branch of radiometry where radiant flux is weighted spectrally in accord with the spectral sensitivity of the average human eye. For convenience of photometrists, the papers on this subject have been grouped separately.

Of the more than ninety items included in this volume, all but sixteen, which have historical value, bear publication dates of 1960 or later. Papers selected are for the most part by NBS authors. Space limitation permits inclusion of only a few important publications by others, such as the Optical Resource Letter on Radiometry, (Paper 11.6), and the paper (11.7), which covers the effect of the "International Practical Temperature Scale of 1968" on the designations of light sources.

HARRY K. HAMMOND III, and

HENRY L. MASON, Editors. 
Abstract_-_-_-_-_-

Foreword

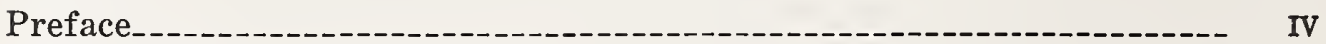

Editor's Note

\section{General Radiometry}

\section{Papers}

1.1. Standards, Sources, and Detectors in Radiation Measurements. Ralph Stair and William E. Schneider, Symposium on Thermal Radiation of Solids, San Francisco, Calif., Mar. 4-6, 1964, pp. 217-231 (NASA SP-55, Nat. Aeron. and Space Admin., Washington, D.C., 1965).

Key words: Cavity detectors; filter spectroradiometer; radiation measurement; radiometric standards; sensitivesurface uniformity; thermopiles

1.2. On the Use of Thermopiles for Absolute Radiometry in the Far Ultraviolet.

R. G. Johnston and R. P. Madden, Appl. Opt. 4, No. 12, 15741580 (Dec. 1965).

Key words: Absolute radiometry; electron cooling; goldblack radiometry; reflective scattering; surface sensitivity maps; thermopiles; ultraviolet radiometry----------------

1.3. A New Radiometric Equation and its Application.

H. J. Kostkowski, Appl. Opt. 5, No. 12, p. 1959 (Dec. 1966). Key words: Non-blackbody radiation; Planck equation; radiometric equation; radiometry; spectral radiance; tungsten-strip lamp; Wien equation

1.4. Comparison of an Ionization Chamber and a Thermopile as Absolute Detectors in the Extreme Ultraviolet.

L. R. Canfield, R. G. Johnston, K. Codling, and R. P. Madden, Appl. Opt. 6, No 11, 1886-1888 (Nov. 1967).

Key words: Absolute radiometry; ionization chamber; radiometry; thermopile; ultraviolet radiometry 


\section{Emissivity (Emittance) Standards}

2.1. On Kirchhoff's Law and Its Generalized Application to Absorption and Emission by Cavities.

Francis J. Kelly, J. Res. Nat. Bur. Stand. (U.S.) 69B, No. 3, 165-171 (July-Sept. 1965).

Key words: Cavity absorption; cavity emission; Kirchhoff's

Law

2.2. A Note on the Numerical Evaluation of Thermal Radiation Characteristics of Diffuse Cylindrical and Conical Cavities. B.A. Peavy, J. Res. Nat. Bur. Stand. (U.S.) 70C, No. 2, 139148 (Apr.-June 1966).

Key words : Cavities ; conical ; cylindrical ; emissivity ; nonisothermal ; isothermal; radiation.

2.3. An Equation for the Local Thermal Emissivity at the Vertex of A Diffuse Conical or V-Groove Cavity. Francis J. Kelly, Appl. Opt., 5, No. 6, 925-927 (June 1966).

Key words: Angle factor; blackbody ; conical cavity ; emissivity; radiant heat transfer; V-groove cavity

2.4. The Apparent Thermal Radiation Properties of an Isothermal V-Groove with Specularly Reflecting Walls.

Richard B. Zipin, J. Res. Nat. Bur. Stand. (U.S.) 70C, No. 4, 275-280 (Oct.-Dec. 1966).

Key words: Cavity; conical cavity; emissivity; radiation properties; V-groove cavity

2.5. An Approach to Thermal Emittance Standards.

J. C. Richmond, W. N. Harrison, and F. J. Shorten, Symposium on Measurement of Thermal Radiation Properties, Dayton, Ohio, September 5-7, 1962, pp. 403-423 (NASA SP-31, Nat. Aeron. and Space Admin., Washington, D. C., 1963)

Key words: Spectral emittance standard; spectral emittance measurement; total emittance computation

2.6. A Test of Analytical Expressions for the Thermal Emissivity of Shallow Cylindrical Cavities.

F. J. Kelly, and Dwight G. Moore, Appl. Opt. 4, No. 1, 31-40 (Jan. 1965).

Key words: Analysis; emittance; paper lined cavity; shallow cavity reflectance; specular component.

2.7. Investigation of Shallow Reference Cavities for High-Temperature Emittance Measurements.

Dwight G. Moore, Symposium on Measurement of Thermal Radiation Properties of Solids, Dayton, Ohio, September 5-7, 1962, pp. 515-526 (NASA SP-31, Nat. Aeron. and Space Admin., Washington, D. C., 1963).

Key words: Alumina ; cavity ; emittance of oxidized Inconel ; graphite; high-temperature; emittance measurement; oxidized nickel; shallow cavity 


\section{Emissivity (Emittance) Standards - Continued}

Papers

2.8. Instructions for Handling Emittance Standards.

Office of Standard Reference Materials, Nat. Bu. Stds., 1 p. (1965).

Key words: Emittance; standards \& handling

2.9. Certificate of Normal Spectral Emittance, Standard Reference

Materials 1402 to 1409, Platinum-13 Percent Rhodium Alloy.

Office of Standard Reference Materials, 7 pp. (1966).

Key words: Emittance; platinum; platinum-rhodium alloy; standard

2.10. Certificate of Normal Spectral Emittance, Standard Reference Materials 1420-1428, Oxidized Kanthal.

Office of Standard Reference Materials, 4 pp. (1965).

Key words: Emittance; Kanthal; standard

2.11. Certificate of Normal Spectral Emittance, Standard Reference Materials 1440 to 1447, Oxidized Inconel.

Office of Standard Reference Materials, 4 pp. (1965).

Key words: Emittance; Inconel; standard

3. Emissivity and Emittance Measurements and Techniques

3.1. Standardization of Thermal Emittance Measurements Part IV: Normal Spectral Emittance $800-1400^{\circ} \mathrm{K}$.

William N. Harrison, Joseph C. Richmond, Frederick J. Shorten and Horace M. Joseph, abstracted from Tech. Rept. WADCTR-59-510, Part IV, Wright Patterson Air Force Base, 90 pages, (1963).

Key words: Emittance measurement; normal spectral emittance; standardization

3.2. Equipment and Procedures for Evaluation of Total Hemispherical Emittance.

Joseph C. Richmond and William N. Harrison, Am. Ceram. Soc. Bull. 39, No. 11, 668-673 (Nov. 15, 1960).

Key words: Emittance change on vacuum heating; hemispherical emittance measurement.

3.3. Method of Measuring Emissivities of Metals in the Infrared. Arthur G. Maki and Earle K. Plyler, J. Res. Nat. Bur. Stand. (U.S.) 66C, No. 3, 283-287 (Sept. 1962).

Key words: Emissivity measurement; gold; metals ; infrared

3.4. Comments on the Surface Characterization of Real Metals.

David P. DeWitt, Symposium on Measurement of Thermal Radiation of Solids, San Francisco, Cal., Mar. 4-6, 1964, pp. 141-145 (NASA SP-55, 1965). Nat. Aeron. and Space Admin., Washington, D.C.

Key words: Emittance of metals; roughness effect; surface characterization; thermal history 
3. Emissivity and Emittance Measurements and Techniques - Continued

Papers

3.5. Importance of Surface Films.

Joseph C. Richmond, Symposium on Thermal Radiation of Solids, San Francisco, Cal., Mar. 4-6, 1964, pp. 157-158 (NASA SP-55, Nat. Aeron. and Space Admin., Washington, D.C., 1965).

Key words: Hemispherical emittance; Inconel; surface films

3.6. A Rotating Cylinder Method for Measuring Normal Spectral Emittance of Ceramic Oxide Specimens from $1200^{\circ}$ to $1600^{\circ} \mathrm{K}$.

Howard E. Clark and Dwight G. Moore, J. Res. Nat. Bur. Stand. (U.S.) 70A, No. 5, 393-415 (Sept.-Oct. 1966).

Key words: Alumina; ceramic oxides; emittance; error sources; magnesia; measurement uncertainties; rotating cylinder; spectral emittance; thermal radiation; thoria; zirconia

3.7. Apparatus for the Measurement of the Normal Spectral Emissivity in the Infrared.

A. G. Maki, Ralph Stair, and R. G. Johnston, J. Res. Nat. Bur. Stand. (U.S.) 64C, No. 2, 99-102 (Apr.-June 1960).

Key words: Emissivity of platinum; oxidized Inconel ; spectral emissivity measurement

3.8. Approximate Normal Emissivity in the Infrared at Elevated Temperatures of Single-Crystal and Polycrystalline Calcium Fluoride.

W. Fussell and J. Geist, Appl. Opt. 6, No. 1, 119-124 (Jan. 1967).

Key words: Calcium fluoride ; infrared absorption coefficient; elevated-temperature emissivity ; infrared emissivity ; polycrystalline calcium fluoride; single-crystal calcium fluoride

3.9. Periodic Heat Flow in a Hollow Cylinder Rotating in a Furnace with a Viewing Port.

B. A. Peavy and A. G. Eubanks, Symposium on Measurement of Thermal Radiation Properties of Solids, Dayton, Ohio, Sept. 5-7, 1962, pp. 553-563, (NASA SP-31, Nat. Aeron. and Space Admin., Washington, D.C., 1963).

Key words: Rotating-specimen furnace; spectral emittance; total emittance

3.10. Survey of Infrared Measurement Techniques and Computational Methods of Radiant Heat Transfer.

S. Thomas Dunn, Joseph C. Richmond, and Jerome F. Parmer, J. Spacecraft and Rockets, Vol. 3, No. 7, 967-975 (July 1966). Key words: Computational methods; emittance; heat transfer; infrared; measurement techniques ; reflectance. 
3. Emissivity and Emittance Measurements and Techniques - Continud Abstracts

3.11. Thermal Radiation Property Measurement Techniques.

S. T. Dunn, J. C. Geist, D. G. Moore, H. E. Clark and J. C. Richmond, Nat. Bur. Stand. (U.S.) Tech. Note 415, 77 pp., (Apr. 27, 1967).

Key words: Averaging spheres; diffuse reflectance; emissivity; emittance; flux averaging devices; infrared reflectance; spectral reflectance; spectral emittance; specular

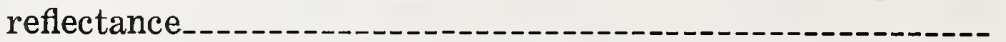

3.12. Procedures for Precise Determination of Thermal Radiation Properties, November 1962 to October 1963.

J. C. Richmond, D. P. DeWitt, and W. D. Hayes, Jr., Nat. Bur. Stand. (U.S.) Tech. Note 252, 55 pp. (Nov. 20, 1964). (Table 3 included in this volume.)

Key words: Emissivity of metals ; radiation property determination; standards calibration; total emittance computa-

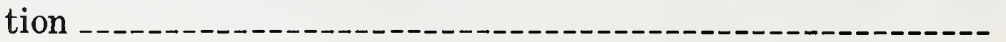

3.13. Procedures for Precise Determination of Thermal Radiation Properties, November 1963 to October 1964.

J. C. Richmond, S. T. Dunn, D. P. DeWitt, and W. D. Hayes, Jr., Nat. Bur. Stand. (U.S.) Tech. Note 267, 62 pp., (Dec. 17, 1965).

Key words: Emissivity ; emittance; high temperature reflectance; infrared reflectance; radiation properties; reflectance; spectral emittance; spectral reflectance; thermal radiation

3.14. Procedures for Precise Determination of Thermal Radiation Properties, November 1964 to October 1965.

J. C. Richmond, G. J. Kneissel, D. L. Kelley and F. J. Kelly, Nat. Bur. Stand. (U.S.), Tech. Note 292, 80 pp. (Feb. 10, 1967).

Key words: Emittance error sources ; emittance from shallow cavity; emittance measurement; radiation property determination; standards calibration

\section{Material Properties}

Papers

4.1. Effect of Surface Roughness on Emittance of Nonmetals.

J. C. Richmond, J. Opt. Soc. Am. 56, No. 2, 253-254 (Feb. 1966).

Key words: Emittance; nonmetals; surface roughness

4.2. Thermal Radiation Properties of Ceramic Materials.

J. C. Richmond, Proc. Symposium on Mechanical and Thermal Properties of Ceramics, Gaithersburg, Md., Apr. 1-2, 1968, Nat. Bur. Stand. (U.S.) Spec. Publ. 303, pages 125-137 (May 1969).

Key words: Alumina; ceramics; emittance; magnesia ; reflectance; temperature coefficient of emittance; thermal radiation properties; thoria; zirconia_.....-...-...- 


\section{Material Properties - Continued}

Papers

4.3. Relation of Emittance to Other Optical Properties.

J. C. Richmond, J. Res. Nat. Bur. Stand. (U.S.) 67C, No. 3, 217-226 (Sept. 1963).

Key words: Emittance; layer properties; mathematical relations

4.4. Total Hemispherical Emittance of Coated and Uncoated Inconel and Types 321 and 430 Stainless Steel.

J. C. Richmond and W. N. Harrison, J. Res. Nat. Bur. Stand. (U.S.) 66C, No. 3, 261-269 (Sept. 1962).

Key words: Alloys; ceramic coatings; emittance; Inconel; stainless steel; surface treatment

4.5. Spectral Emittance of Ceramic-Coated and Uncoated Specimens of Inconel and Stainless Steel.

J. C. Richmond, and J. E. Stewart, J. Am. Ceram. Soc. 42, No. 12, 633-640 (Dec. 1959).

Key words: Ceramic-coated materials; emittance; Inconel ; spectral emittance; stainless steel

Abstract

4.6. Theory and Measurement of the Thermal Radiative Properties of Metals.

D. P. DeWitt and J. C. Richmond, Techniques of Metals Research, Vol. VI, (John Wiley \& Sons, New York, N.Y., 1970).

Key words: Absorptance; electromagnetic theory; emittance; measurement techniques; metals; reflectance; surface effects; thermal radiation properties

\section{Irradiance Standards}

Papers

5.1. Memorandum on a Procedure for Obtaining Spectral Radiant Intensities of Tungsten-Filament Lamps, 400-700 $\mathrm{m} \mu$.

L. E. Barbrow, J. Opt. Soc. Am. 49, No. 11, p. 1122, (Nov. 1959).

Key words: Irradiance; spectral irradiance; standard_-----

5.2. The New Tungsten-Filament Lamp Standards of Total Irradiance.

Ralph Stair, William E. Schneider and William B. Fussell, Appl. Opt. 6, No. 1, 101-106 (Jan. 1967).

Key words: Carbon-filament lamp; irradiance; standard ; total irradiance; tungsten-filament lamp

5.3. Instructions for Using the NBS Tungsten-Filament Lamp Standards of Total Irradiance, (March 1966).

Key words: Irradiance; standard; total irradiance; tung-

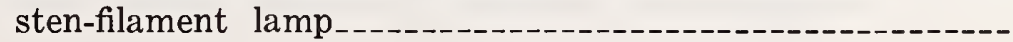




\section{Irradiance Standards - Continued}

Papers

5.4. A New Standard of Spectral Irradiance.

Ralph Stair, W. E. Schneider, and John K. Jackson, Appl. Opt. 2, No. 11, 1151-1154 (Nov. 1963).

Key words: Irradiance; quartz-iodine lamp; spectral irradiance; standard

5.5. Instructions for Using the NBS 1000-Watt Quartz-Iodine Lamp Standards of Spectral Irradiance, (Nov. 1964).

Key words: Irradiance; quartz-iodine lamp; spectral irradiance; standard

5.6. A Standard for Extremely Low Values of Spectral Irradiance. Ralph Stair, William B. Fussell and William E. Schneider, Appl. Opt. 4, No. 1, 85-89 (Jan. 1965).

Key words: Irradiance; spectral irradiance; standard

5.7. A One-Solar-Constant Irradiance Standard.

William E. Schneider, Appl. Opt. 9, No. 6, 1410-1418 (June 1970).

Key words: Calibration techniques; radiometric standards; spectral irradiance; solar constant; total irradiance..-...--.

\section{Radiometric Measurement Techniques}

6.1. Spectral Irradiance as Determined Through the Use of Prism and Filter Spectroradiometric Techniques.

William E. Schneider, Ralph Stair, and John K. Jackson, Appl. Opt. 6, No. 9, 1479-1486 (Sept. 1967).

Key words: Filter spectroradiometry; measurement techniques; prism spectroradiometry; radiometry; sources; spectral irradiance

6.2. The Measurement of Solar Radiation with Principal Emphasis on the Ultraviolet Component.

Ralph Stair, Air and Wat. Pollut. Int. J. 10, pp. 665-688 (1966).

Key words: Solar radiation measurement; ultraviolet radiation

6.3. Some Factors Affecting the Sensitivity and Spectral Response of Thermoelectric (Radiometric) Detectors.

Ralph Stair, William E. Schneider, William R. Waters, and John K. Jackson, Appl. Opt. 4, No. 6, 703-710 (June 1965).

Key words: Area sensitivity; detector surface uniformity; spectral response; thermal detectors; thermopiles

6.4. Radiometric Methods for Measuring Laser Output.

D. A. MeSparron, C. A. Douglas, and H. L. Badger, Nat. Bur. Stand. (U.S.) Tech. Note 418, 16 pages (Nov. 1967).

Key words: Laser; radiometry ; laser energy measurement; radiometric calibration; photoelectric photometry; optical attenuator 


\section{Radiometric Measurement Techniques - Continued}

Papers

6.5. Effects Upon Radiant Intensity Measurements due to Scattering Page by Optical Elements.

William H. Venable, Jr., Appl. Opt. 9, No. 3, pp. 609-615 (March 1970).

Key words: Instrument function; radiance; scattering

\section{Radiance Standards}

\section{Papers}

7.1. Standard of Spectral Radiance for the Region of 0.25 to 2.6 Microns.

Ralph Stair, Russell G. Johnston, and E. W. Halback, J. Res. Nat. Bur. Stand. (U.S.) 64A, No. 4, 291-296 (July-Aug. 1960). Key words: Blackbody; spectral radiance; standard; tung-

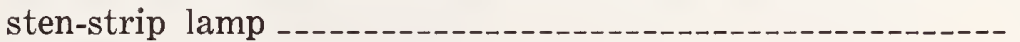

7.2. Spectral Radiance of a Low Current Graphite Arc.

Albert T. Hattenberg, Appl. Opt. 6, No. 1, 95-100 (Jan. 1967). Key words: Arc; graphite; spectral radiance; spectroradiometer; temperature blackbody

7.3. High-Accuracy Spectral Radiance Calibration of Tungsten-Strip Lamps.

H. J. Kostkowski, D. E. Erminy, A. T. Hattenburg, Ch. 4. Advances in Geophysics, Vol. 14, Academic Press, N.Y. (1970).

Key words: Blackbody; high temperature measurement; radiometry; spectral irradiance; tungsten-strip lamp _.---

7.4. The NBS Photoelectric Pyrometer and its Use in Realizing the International Practical Temperature Scale above $1063^{\circ} \mathrm{C}$. R. D. Lee, Metrologia, 2, No. 4, 150-162 (Oct. 1966).

Key words: Blackbody; freeze; gold point; temperature scale

Abstracts

7.5. Construction and Operation of a Simple High-Precision CopperPoint Blackbody and Furnace.

R. D. Lee, Nat. Bur. Stand. (U.S.) Tech. Note 483, 15 pages (May 1969).

Key words: Blackbody; copper-point temperature; optical pyrometry; radiometry

7.6. Radiance Temperature at $6550 \AA$ of the Graphite Arc.

R. D. Lee and E. Lewis, Appl. Opt. 5, No. 11, p. 1858 (Nov. 1966).

Key words: Arc; graphite; spectral radiance; radiance temperature 


\section{Radiance Standards - Continued}

Abstract

7.7. Theory and Methods of Optical Pyrometry.

H. J. Kostkowski and R. D. Lee, Nat. Bur. Stand. (U.S.)

NBS Monogr. 41, 28 pages, (Reprinted Sept. 1962).

Key words: Blackbodies; gold point; optical pyrometry; tungsten-strip lamps

\section{Flux Measurement}

Papers

8.1. Theory, Construction, and Use of the Photometric Integrating Sphere.

E. B. Rosa and A. H. Taylor, Bul. BS 18, 281-323, (1922-23) S447.

Key words: Integrating sphere; measurements of luminous flux; spherical candlepower

8.2. Application of Sulfur Coatings to Integrating Spheres.

S. Thomas Dunn, Appl. Opt. 4, No. 7, 877 (July 1965).

Key words: Integrating sphere; sulphur coating

Abstracts

8.3. Flux Averaging Devices for the Infrared.

S. Thomas Dunn, Nat. Bur. Stand. (U.S.) Tech. Note 279, 14 pages (Dec. 1965).

Key words: Angular sensitivity; flux averaging; infrared detector; integrating sphere; mixing mechanism; spatial sensitivity

8.4. A 5-Meter Integrating Sphere.

Mamoru Nonaka, Tokeo Kashima, and Yatoro Knodo, Appl. Opt. 6, No. 4, 757-771 (April 1967).

Key words: Integrating sphere; measurement of luminous flux

\section{Reflectometry}

Papers

9.1. Terms, Definitions and Symbols in Reflectometry.

Deane B. Judd, J. Opt. Soc. Am. 57, No. 4, 445-452, (Apr. 1967).

Key words: Nomenclature; photometry; radiometry; re-

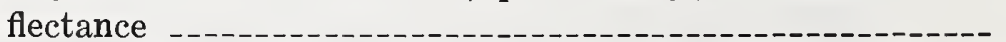

9.2. Use of an Auxiliary Sphere with a Spectroreflectometer to Obtain Absolute Reflectance.

David G. Goebel, B. Patrick Caldwell and Harry K. Hammond III, J. Opt. Soc. Am. 56, No. 6, 783-788, (June 1966).

Key words: Absolute reflectance; auxiliary sphere; re-

flectance; spectrophotometry 


\section{Reflectometry - Continued}

9.3. Generalized Integrating-Sphere Theory.

David G. Goebel, Appl. Opt. 6, No. 1, 125-128, (Jan. 1967). Key words: Absolute reflectance; integrating sphere; reflectance; sphere efficiency

9.4. Effect of Surface Texture on Diffuse Spectral Reflectance: A. Diffuse Spectral Reflectance of Metal Surfaces; H. J. Keegan, J. C. Schleter, and V. R. Weidner; B. Surface Texture Measurement of Metal Surfaces; D. B. Spangenberg, A. G. Strang, and J. L. Chamberlain, Symposium on Thermal Radiation of Solids, San Francisco, Cal. Mar. 4-6, 1964, pp. 165-177 (NASA SP-55, Nat. Aeron. and Space Admin., Washington, D.C. 1965).

Key words : Aluminum ; beryllium ; diffuse reflectance ; interferograms; platinum; steel surface finish; surface texture; stylus technique

9.5. Ellipsoidal Mirror Reflectometer.

S. T. Dunn, J. C. Richmond, and J. A. Wiebelt, J. Res. Nat. Bur. Stand. (U.S.) 70C, No. 2, 75-88, (Apr.-June 1966). Key words: Averaging spheres; bidirectional reflectance; diffuse reflectance; ellipsoidal reflectometer; infrared; infrared detectors; reflectance; reflectometer; spatial sensitivity; spectral reflectance; specular reflectance

9.6. High Purity Powdered CsI as a High Reflectance Infrared Diffuser.

Jon Geist, Gerhart J. Kneissl, and V. R. Weidner, Appl. Opt. 6, No. 7, 1280 (July 1967).

Key words: Cesium iodine; diffusing reflector; hemispherical reflectance factor

9.7. Preparation and Colorimetric Properties of a Magnesium Oxide Reflectance Standard.

Nat. Bur. Stand. (U.S.) Letter Circ. LC1050, 3 pp. (Revised June 1970).

Key words: Diffuse reflectance; CIE illuminants; luminous reflectance; magnesium oxide; chromaticity coordinates

Abstract

9.8. A Laser-Source Integrating Sphere Reflectometer. G. J. Kneissl and J. C. Richmond, Nat. Bur. Stand. (U.S.) Tech. Note 439, 69 pp., (Feb. 1968).

Key words: Diffuse reflectance; graphite; high temperature reflectance; infrared reflectance; integrating sphere reflectometer; laser; reflectance; sodium chloride coating; synchronous amplification; thoria; tungsten 


\section{Laser Radiometry}

Papers

10.1. Calorimetric Measurement of Pulsed Laser Output Energy.

D. A. Jennings, IEEE Trans. Instr. \& Meas. IM-15, No. 4, 161-164 (Dec. 1966).

Key words: Absorption cell ; calorimeter; energy measurement; pulsed ruby laser; substitution calibration

10.2. Radiometric Methods for Measuring Laser Output.

D. A. MeSparron, C. A. Douglas, and H. L. Badger, Nat. Bu. Stand. (U.S.) Tech. Note 418, 12 pp. (Nov. 1967).

Key words: Laser; laser energy measurement; optical attenuator; photoelectric photometry; radiometric calibra-

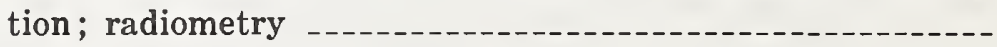

10.3. Laser Power Meter for Large Beams.

D. A. Jennings and E. D. West, Rev. Sci. Instrum. 41, No. 4, 565-567 (April 1970).

Key words: C. W. laser power; diverging laser beam; large diameter laser beam; pulsed laser power

10.4. Laser Power and Energy Measurements.

D. A. Jennings, E. D. West, K. M. Evenson, A. L. Rasmussen, and W. R. Simmons, Nat. Bur. Stand. (U.S.) Tech. Note 382, 64 pp. (Oct. 1969).

Key words: Absorption cell calorimeters ; laser energy measurement; laser power measurement; metal plate calorimeters; pulsed ruby laser

\section{General Photometry}

\section{Papers}

11.1. The Waidner-Burgess Standard of Light.

H. T. Wensel, Wm. F. Roeser, L. E. Barbrow, and F. R. Caldwell, J. Res. Nat. Bur. Stand. (U.S.) 6, 1103-1117 (RP325, June 1931).

Key words: Platinum blackbody ; primary standard of light; SI unit of luminous intensity

11.2. Announcement of Changes in Electrical and Photometric Units. Sect. III Photometric Units, p. 5-7 (May 1947).

Key words: International photometric units of 1948 ; platinum blackbody radiator

11.3. Vertical Distribution of Light from Gas Filled Candlepower Standards.

L. E. Barbrow, S. W. Wilson, Illum. Eng. 53, No. 12, 645-648, (Dec. 1958).

Key words: Lamp intensity ; standard lamps; variation with angle; vertical distribution 


\section{General Photometry - Continued}

Papers

11.4. Concepts, Terminology, and Notation for Optical Modulation. C. S. McCamy, Photog. Sci. \& Engrg. 10, No. 6, 314-325 \& Note (Nov.-Dec. 1966).

Key words: Absorptance; contrast; modulation reflectance; notation; propagance; optical density; reflectance factor; symbols; transmittance factor

11.5. The Metric System in Illuminating Engineering.

L. E. Barbrow, Illum. Eng. 62, No. 11, 638-640, (Nov. 1967). Key words: Illuminating engineering; metric system; nomenclature; photometric units; International System of Units

11.6. Optical Resource Letter on Radiometry.

Fred E. Nicodemus, J. Opt. Soc. Amer. 59, No. 3, 243-248 (Mar. 1969).

Key words: Bibliography; illumination; infrared radiometry ; light; ultraviolet radiometry -_-_-_-_-_-_-_._-_-_-_

11.7. Introduction of the International Practical Temperature Scale 1968: some effects in relation to light sources, colour temperature, and colorimetry.

J. S. Preston, Lighting Res. \& Tech., 1, No. 4, 248-250 (196.9). Key words: Color temperature; nomenclature; SI unit; spectral distribution; luminous efficacy; tungsten-filament lamps ; platinum blackbody

11.8. Photometric Unit Chart.

Francis Clark and L. E. Barbrow, Illum. Engrg. 64, No. 9, 603 (Sept. 1969).

Key words: Illumination; luminance; luminous energy ; luminous exitance; luminous flux; luminous intensity; units_-

11.9. Filters for the Reproduction of Sunlight and Daylight and the Determination of Color Temperature.

Raymond Davis and K. S. Gibson, Misc. Pub. 114, Nat. Bur. Stand. (U.S.), 165 pp. (Jan. 21, 1931); reprinted 1970 as (PB192894).

Key words: Sunlight; daylight; color temperature, 1,600 to $20,000 \mathrm{~K}$; color filters ; colorimetry ; spectrophotometry _-

11.10. A Physical Photometer.

Ray P. Teele, J. Res. Nat. Bur. Stand. 27, RP1415, 217-228 (Sept. 1941).

Key words ; CIE luminous efficiency function ; luminous efficiency filter; luminous intensity; standard observer 


\section{General Photometry - Continued}

Abstracts

11.11. New Principle of Absolute Photometry.

Calvin S. McCamy, Abstract MC16, 1963 Spring Meeting, Jacksonville, Florida. J. Opt. Soc. Am. 53, No. 4, p. 511 (Apr. 1963).

Key words: Adjustable light source; photometric comparator; quantitative attenuation; Riemann integration; sliding shutter; optical modulation

11.12. Changes in the U.S. Unit of Luminous Flux.

Tech. News Bull. 54, No. 3, p. 55 (March 1970).

Key words: Color temperature $2800 \mathrm{~K}$; gas-filled tungstenfilament lamps; total luminous flux; U.S. standard

\section{Applied Photometry}

Papers

12.1. The Use of Zonal Constants in the Calculation of Beam Flux. Theodore H. Projector, Illum. Eng. 48, No. 4, 189-191 (April 1953).

Key words: Luminous flux; searchlights; floodlights ; zonal constants ; candlepower distribution

12.2. Versatile Goniometer for Projection Photometry.

Theodore H. Projector, Illum. Eng. 48, No. 4, 192-196 (April 1953).

Key words; Goniometry; projection photometry ; 100-foot photometric range; searchlights

12.3. Photometry of Projectors at the National Bureau of Standards. L. Chernoff, Nat. Bur. Stand. (U.S.) Tech. Note 198, 28 pages, (Dec. 1963).

Key words: Projectors; searchlights

12.4. Review of Elementary Theory of the Photometry of Projection Apparatus.

C. A. Douglas, Illum. Eng. 62, No. 4, 246-253. (Apr. 1967). Key words: Critical distance; illumination; light projectors; photometric distance; signal lighting

12.5. Photometer for Measurement of Effective Intensity of Condenser-Discharge Lights.

C. A. Douglas, Illum. Eng. 53, No. 4, 205-208, (Apr. 1958).

Key words: Flashed light intensity ; photometer

12.6. Effective Intensity of Flashing Lights.

T. H. Projector, Illum. Eng. 52, No. 12, 630-640, (Dec. 1957).

Key words: Flashing light; effective intensity 


\section{Applied Photometry - Continued}

Papers

12.7. Computation of the Effective Intensity of Flashing Lights.

C. A. Douglas, Illum. Eng. 52, No. 12, 641-646 (Dec. 1957).

Key words; Flashing light; effective intensity computation 640

12.8. Photometer for Luminescent Materials.

Ray P. Teele, J. Opt. Soc. Amer. 35, No. 6, 373-378 (June 1945).

Key words: Dark adapted eye; low luminance photometry ; luminescent materials

12.9. On the Standard Source for Low Level Photometry.

J. Terrien, J. Opt. Soc. Amer. 39, No. 10, Letter to Editor,

p. 888 (Oct. 1949).

Key words: Color temperature $2042 \mathrm{~K}$; dark adapted observer; low luminance; platinum blackbody ; Purkinje effect

651

12.10. The Photometry of Colored Light.

A. C. Wall, Illum. Eng. 62, No. 4, 239-242 (Apr. 1967).

Key words: Filters; photocells; photometers; phototubes spectral correction

Abstracts

12.11. Absolute Light-Scattering Photometer: I, Design and Operation.

Donald McIntyre and G. C. Doderer, J. Res. Nat. Bur. Stand. (U.S.) 62, No. 4, 153-159 (April 1959).

Key words: Absolute photometry ; angular scattering ; photometer design; polymer solutions; ratio scattered to incident light; research photometer

12.12. Absolute Light-Scattering Photometer: II, Direct Determination of Scattered Light from Solutions.

Donald McIntrye, J. Res. Nat. Bur. Stand. (U.S.) 68A, No. 1, 87-96 (Jan.-Feb. 1964).

Key words : Absolute photometry ; polymer solutions ; transmission by solutions; transverse measurements of light scattering

Author index

SI physical units (inside back cover) 



\section{General Radiometry}

Papers

1.1. Standards, Sources, and Detectors in Radiation Measurements.

Ralph Stair and William E. Schneider, Symposium on Thermal Radiation of Solids, San Francisco, Calif., Mar. 4-6, 1964, pp. 217-231 (NASA SP-55, Nat. Aeron. and Space Admin., Washington, D.C., 1965).

Key words: Cavity detectors; filter spectroradiometer; radiation measurement; radiometric standards ; sensitive-surface uniformity; thermopiles

1.2. On the Use of Thermopiles for Absolute Radiometry in the Far Ultraviolet.

R. G. Johnston and R. P. Madden, Appl. Opt., 4, No. 12, 15741580 (Dec. 1965).

Key words: Absolute radiometry; electron cooling; goldblack; radiometry; reflective scattering; surface sensitivity maps; thermopiles; ultraviolet radiometry

1.3. A New Radiometric Equation and its Application.

H. J. Kostkowski, Appl. Opt. 5, No. 12, p. 1959 (Dec. 1966).

Key words: Non-blackbody radiation; Planck equation; radiometric equation; radiometry ; spectral radiance; tungsten strip lamp; Wien equation

1.4. Comparison of an Ionization Chamber and a Thermopile as Absolute Detectors in the Extreme Ultraviolet.

L. R. Canfield, R. G. Johnston, K. Codling, and R. P. Madden, Appl. Opt., 6, No. 11, 1886-1888 (Nov. 1967).

Key words: Absolute radiometry; ionization chamber; radiometry ; thermopile; ultraviolet radiometry 



\title{
24. Standards, Sources, and Detectors in Radiation Measurements
}

\author{
RALPH STAIR AND WILLIAM E. SGHNEIDER
}

NATIONAL BUREAU OF STANDARDS, WASHINGTON, D.C.

\begin{abstract}
The NBS standards of total and of spectral irradiance and of spectral radiance are based upon the Stefan-Boltzmann and Planck laws of radiation and, as set up in terms of lamp sources, are in close agreement with each other and with the NBS standard of luminous intensity. Their use in the calibration of various sources requires great skill in the operation of spectroradiometric equipment employing detectors having sensitivities that vary over the detector surface or that vary with wavelength. Examples are given illustrating the wide ranges in sensitivity which may exist over the surface of certain detectors and also the large variation of sensitivity with wavelength of supposedly neutral thermal detectors. General suggestions are made regarding methods for obtaining accurate results in the different types of measurement.
\end{abstract}

The precise measurement of the radiation from a source requires the use of either a calibrated detector or a calibrated source with which comparison may be made by a transfer operation. At the NBS, the standards of total and of spectral irradiance and of spectral radiance are based upon the radiance from a blackbody as defined by the Stefan-Boltzmann and the Planck laws of radiation.

The Stefan-Boltzmann law is the fundamental law of the blackbody. It relates the total radiant flux $W$ from a unit area of a blackbody to the absolute temperature by the relationship

$$
W=\sigma T^{4}
$$

The precise value of the Stefan-Boltzmann constant $\sigma$ can be derived from other physical constants by the relationship

where

$$
\sigma=\frac{2 \pi^{5} k^{4}}{15 c^{2} h^{3}}
$$

$k$ Boltzmann constant

$c$ speed of light

$h$ Planck constant of action
When the NBS standard of total irradiance was set up in 1913 (ref. 1), a value for $\sigma$ of $5.70 \times 10^{-12}$ watt $/ \mathrm{cm}^{2}-\mathrm{deg}^{4} \mathrm{~K}$ was accepted as best representing the extensive experimental radiometric work previously performed with a blackbody. The precision possible in radiometry cannot furnish a hope of improving this value greatly. However, the extensive researches on the physical constants of many investigators through the years have furnished data (ref. 2) which establish the value of $\sigma$ to possibly five significant figures as follows:

$$
\begin{aligned}
& c=2.997925 \times 10^{10} \mathrm{~cm} / \mathrm{sec} \\
& h=6.6256 \times 10^{-27} \mathrm{erg}-\mathrm{sec} \\
& k=1.38054 \times 10^{-16} \mathrm{erg} / \mathrm{deg} \mathrm{K}
\end{aligned}
$$

hence,

$$
\sigma=5.6697 \times 10^{-12} \mathrm{watt} / \mathrm{cm}^{2}-\mathrm{deg}^{4} \mathrm{~K}
$$

differing but little from the value employed in 1913. Further refinement of this value cannot be expected to affect the results of future radiometric work. 
The Planck law of radiation relates the radiance $N_{\lambda}$ at a particular wavelength to the absolute temperature $T$ on the International Practical Temperature Scale (IPTS) by the relationship

$$
N_{\lambda}=\frac{c_{1} \lambda^{-5}}{\mathrm{e}^{c_{2} / \lambda T-1}}
$$

where $c_{1}$ and $c_{2}$ are the first and second radiation constants and have the dimensions watt- $\mathrm{cm}^{2}$ and $\mathrm{cm}-\operatorname{deg} \mathrm{K}$, respectively. With

$c_{1}=2 c^{2} h=1.19088 \times 10^{-12}$ watt-cm ${ }^{2} / \mathrm{ster}$

$c_{2}=1.4380$ cm-deg $\mathrm{K}$ by definition (IPTS, ref. 3 )

and wavelength $\lambda$ in centimeters, $N_{\lambda}$ is in watts/ $\mathrm{cm}^{2}$-ster-cm wavelength interval.

\section{THE NBS RADIOMETRIC STANDARDS}

\section{The Standard of Total Irradiance}

The NBS standard of total irradiance as presently issued consists of a carbon-filament lamp operated at a temperature around $1600^{\circ}$ to $2200^{\circ} \mathrm{K}$. At these temperatures most of the irradiance falls between wavelengths of about 1 and 3 microns. As previously indicated, this standard was set up through comparisons of the irradiances from a group of lamps with the irradiance from a blackbody. For this work the blackbody temperature was usually set at approximately $1400^{\circ} \mathrm{K}$ and a thermopile heavily coated with lampblack was employed as detector. Such a heavily coated thermopile has been found to be nearly uniform in sensitivity with wavelength between the visible and about 3 microns in the infrared, hence, it will give an acceptably accurate evaluation of an $1800^{\circ} \mathrm{K}$ lamp filament in terms of a $1400^{\circ} \mathrm{K}$ blackbody.

Our recent reviews of detectors substantiate the validity of the earlier measurements in this area, so that the carbon-filament lamp standard of total irradiance is an adequate standard for use in the calibration of properly blackened thermal detectors over the range from a few microwatts to several hundred microwatts per $\mathrm{cm}^{2}$. To cover higher ranges of irradiance, work is in progress toward setting up a secondary standard yielding an irradiance approximating 100 to $150 \mathrm{mw} / \mathrm{cm}^{2}$. There appears to be little interest in a total irradiance standard having an irradiance lower than a few microwatts per $\mathrm{cm}^{2}$. A low intensity standard of spectral irradiance recently set up (ref. 4) and discussed below should fill any existing needs in this area.

\section{Standards of Spectral Radiance}

Three standards of spectral radiance have been set up covering the regions of 0.25 to 0.75 micron, 0.5 to 2.6 microns, and 0.25 to 2.6 microns (fig. 1). The first two were set up independently through the use of two blackbodies having temperatures of about $2200^{\circ}$ to $2600^{\circ} \mathrm{K}$ and $1200^{\circ}$ to $1400^{\circ} \mathrm{K}$. This work has been described in detail elsewhere (ref. 5). One of the setups employed is shown in figure 2.

The experimental work consisted of alternately allowing the radiation from the blackbody (set at a specific temperature) and that from a strip lamp (set at a fixed current) to enter a spectroradiometer after being focused on the entrance slit by the same optical system, and measuring the relative radiances of the two sources at selected wavelengths. For the system illustrated, the wavelength range was 0.25 to 0.75 micron, and a $1 \mathrm{P}-28$ photomultiplier was used as the detector.

A supplementary setup consisting of similar optics and electronics and a $1400^{\circ} \mathrm{K}$ blackbody covered the spectral range of 0.5 to 2.6 microns. In this case, an Eastman lead sulfide ( $\mathrm{PbS}$ ) cell, supplemented by an RCA 7102 photomultiplier, was employed as detector. Thus, the spectral radiances of two groups of strip lamps were evaluated in terms of those of the two blackbodies at specific temperatures as defined by the Planck law of radiation. Although set up independently, the two standards are in close agreement over the common spectral range of 0.5 to 0.75 micron.

The third standard of spectral radiance simply combines the two spectral ranges by means of a single strip lamp operated at a single current with calibration covering the full range from 0.25 to 2.6 microns.

\section{Standard of Spectral Irradiance}

The experimental work connected with setting up the standard of spectral irradiance 


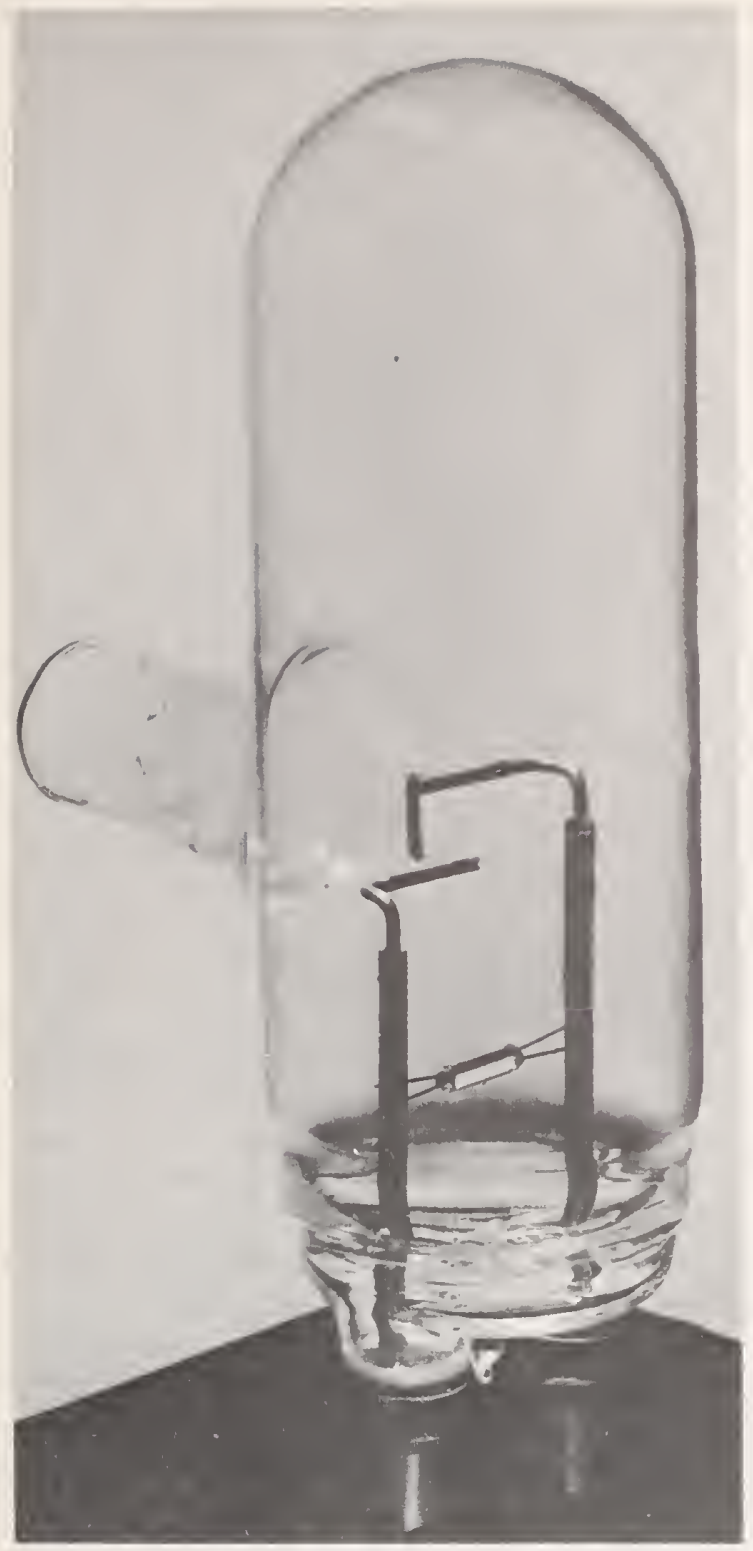

Figure 1.-Tungsten-ribbon-strip lamp standard of spectral radiance.

(fig. 3) offered an opportunity to check the agreement between the existing standards of spectral radiance, total irradiance, and luminous intensity. Although consideration was given to setting up this new standard directly against a blackbody, a number of difficulties involved in that procedure led us to set it up through comparisons with the standards of spectral radiance supplemented by measure-

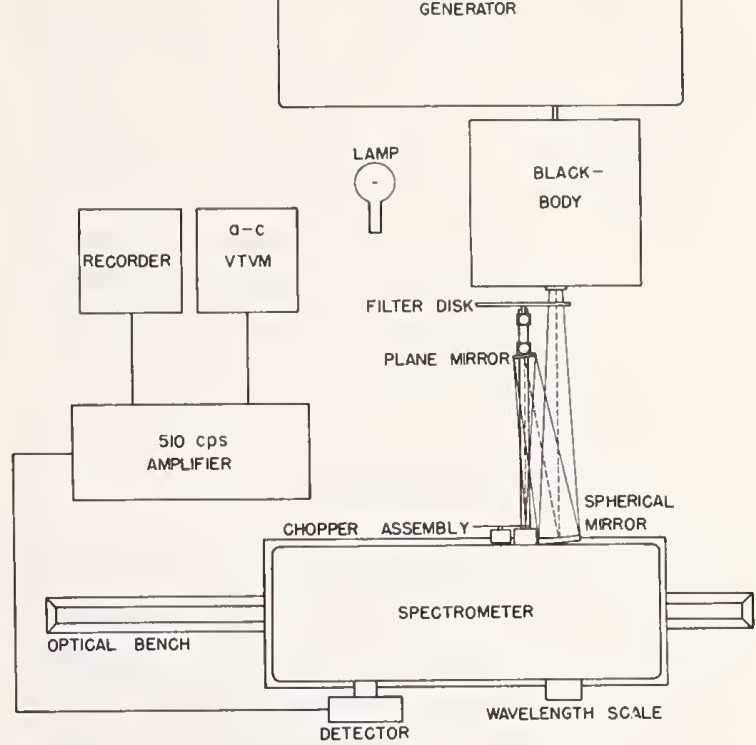

Figure 2.-Instrumental setup of blackbody, monochromator, lamp, and associated equipment for the wavelength region of 0.25 to 0.75 micron.

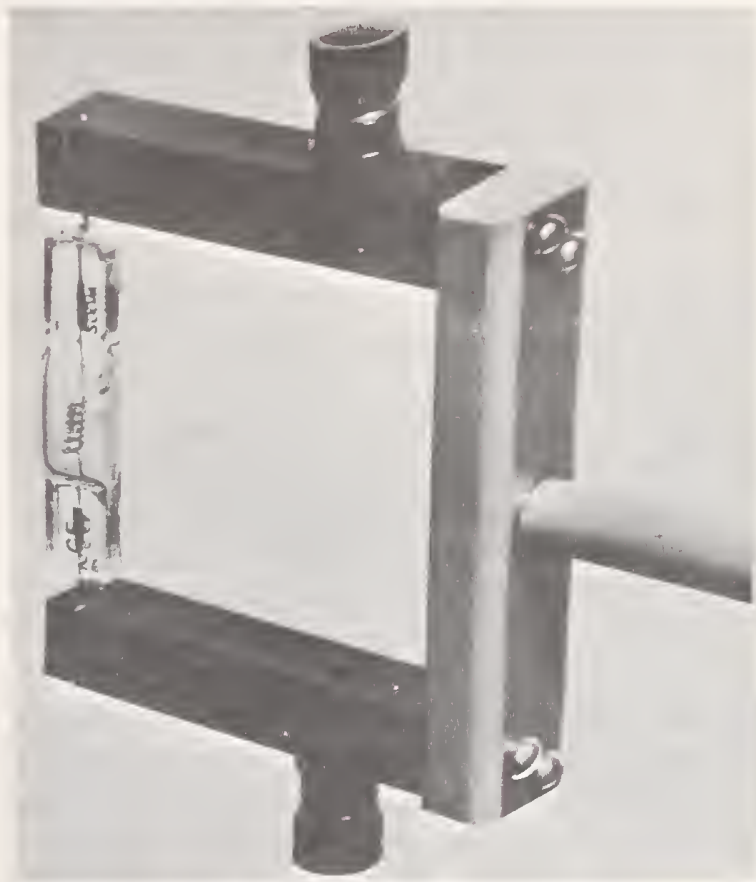

Figure 3.-Quartz-iodine lamp standard of spectral irradiance. 
ments against the standards of total irradiance and of luminous intensity, all three of which had been established through direct comparisons with the radiances from blackbodies at specific temperatures. This work is described in detail elsewhere (ref. 6). The results indicated close agreement between these standards, in some cases to 1 percent and certainly in all cases to within a few percent. Hence, all the NBS standards in this area are based upon the radiance of the blackbody as defined by the Planck and the Stefan-Boltzmann laws of radiation.

\section{Low Intensity Standard of Spectral Irradiance}

To serve the interests of workers in certain fields of extremely low irradiance such as is present in the air glow, and in phosphorescent, fluorescent, biochemical, and associated areas, an extremely low intensity standard of spectral irradiance has been set up (ref. 4). This standara, in effect, combines the NBS standard of spectral radiance with a small aperture which serves as a point source of radiant flux of known spectral intensity. This in combination with one or more convex mirrors set up after the method described by Engstrom (ref. 7) may be readily arranged to supply an irradiance as low as $10^{-16}$ watt $/ \mathrm{cm}^{2} /$ nanometer wavelength interval.

\section{SELECTED APPLICATIONS OF SOURCES AND DETECTORS}

Sources and detectors will be considered together since they are so closely related for most purposes of this symposium. Furthermore, their consideration is closely linked with the geometrical characteristics of the standards employed. Little difficulty arises from the use of uhermal detectors when they are employed with a standard of thermal irradiance or any source of irradiance as long as the flux from the source irradiates the detector uniformly and the detector has a flat response (with wavelength) throughout the spectral interval under investigation. Most sources (including the standard) emit radiant energy over a wide spectral range, and most thermal detectors available fall far short of having an equally wide spectral range of constant sensitivity. Usually, the thermal detector will have a window cover of selective transmission characteristics. Also, the black coating may vary widely in effective blackness as a function of wavelength. Some information can be deduced from the character, thickness, or measured transmittance or reflectance of similar coatings. But the final answer comes only through a direct measurement of the spectral response of the detector (with its window in place) since the response is affected also by such things as the conductive properties of the coating and receiver elements, the emissivity of the receiver, the reflectance of the receiver metal, and the chopping rate (if the signal is chopped). Hence, the true spectral sensitivity of a specific detector can be obtained only through its individual calibration, and this has not been previously possible since no standard in this area has been available. Work is progressing, however, in a number of laboratories (refs. 6 and 8).

The use of detectors, be they thermal or photoelectric, in conjunction with a spectroradiometer or spectrophotometer of some type, in the measurement of spectral radiance or irradiance, or in comparison of the intensities of two sources, may require a knowledge of the geometrical variations in their sensitivity and relative spectral _sensitivity. A spectrometer produces a spectrum, but this spectrum is distorted in a number of ways, especially in a double monochromator. Not only are certain regions of the spectrum more compressed than others, but we have polarization effects, selective absorption, mechanical defects of spectral drive between the two sections of the instrument, imperfect optics, imperfect achromatism, scattered radiation, etc., so that the beam at the exit slit emerges in an uneven pattern covering an area much larger than the first and second defining slits of the instrument. The result may not be too bad if the detector has a uniformly sensitive surface with wavelength and position on its surface. But there is no known commercial detector meeting these specifications. 


\section{The New Cavity Detector}

The nearest approach to a spectrally "flat" detector is the new cavity or "blackbody" detector recently constructed (ref. 6 and 8), one of which is shown diagrammatically in figure 4 . This is constructed in the form of a cone of small angle and coated with carbon or other black on the inside surface. To reduce heat capacity and thereby increase response rate the cone is made of the thinnest gold foil having sufficient strength to insure adequate support under laboratory conditions. Along the outside fold of the foil of the unit illustrated, several wires are connected to serve the double duty of supporting members and thermojunction connectors. Thus, two or more thermojunctions are wired in parallel, resulting in reduced electrical circuit resistance and more rapid thermoelectric response. Increased symmetry and uniformity of response over the conical surface may be obtained by constructing the cone with two or more elements, thereby permitting the attachment of thermal elements at two or more positions around the circumference. However, such construction increases the receiver mass, thereby slowing its response.

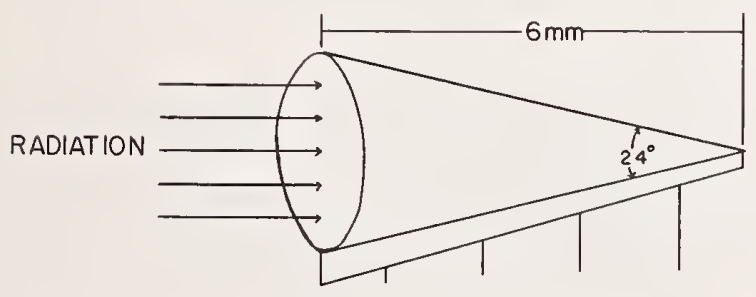

FIGURE 4.-Gold-foil conical cavity detector.

The maximum in flatness of response to be expected from this detector may be approximately calculated (ref. 9 and 10) from a knowledge of its geometry and surface reflectance. Since the cavity shape is not the ideal as a blackbody detector, any spectral variations in reflectance become important. Hence, to insure the greatest degree of spectral flatness, a coating having the most nearly uniform spectral reflectance (or absorptance) should be employed.

To date, several cavity detectors have been constructed and coated with various blacks. Figure 5 shows a comparison between two of these as a function of wavelength. There are good reasons to believe these are neutral, or uniform in response, from the ultraviolet to about 2 microns. At longer wavelengths, detector 2 is suspected of having a reduced sensitivity because of a higher reflectance of carbon black over camphor black, at least for the respective thicknesses employed on these two detectors. Detector 3 is much better than detector 2 at these longer wavelengths, but there is no assurance from the limited data in this illustration that it, too, does not drop somewhat in sensitivity at the longer wavelengths. However, preliminary comparisons between detector 3 and a surface thermopile heavily coated with Parsons' black indicated equal relative sensitivities between the ultraviolet and about 20 microns. Only additional comparisons with "blacker" surfaces (or with receivers having smaller conical angles or thicker coatings) can decide this point. These investigations are in progress. Meanwhile, detector 3 has the most uniform sensitivity of all detectors (conical or flat) examined in the course of this investigation to date.

\section{Some Commercial and Laboratory Thermal Detectors}

Let us now return our attention to the readily available detectors. The usual thermopile or thermocouple consists of a flat surface (usually gold foil) coated with one of the common blacks, such as gold black, carbon black, camphor black, or graphite, in a layer whose thickness is inversely proportional to the "speed" required. The end result is usually an element having selective spectral sensitivity and varying in sensitivity over its surface. The following figures show some of the geometrical variations present in the elements examined. In all cases the thermopile was scanned by a fine line of incandescent lamp flux (set at right angles to the direction of movement) and moved slowly lengthwise with the thermopile or transversely across the element. Figure 6 shows the results 


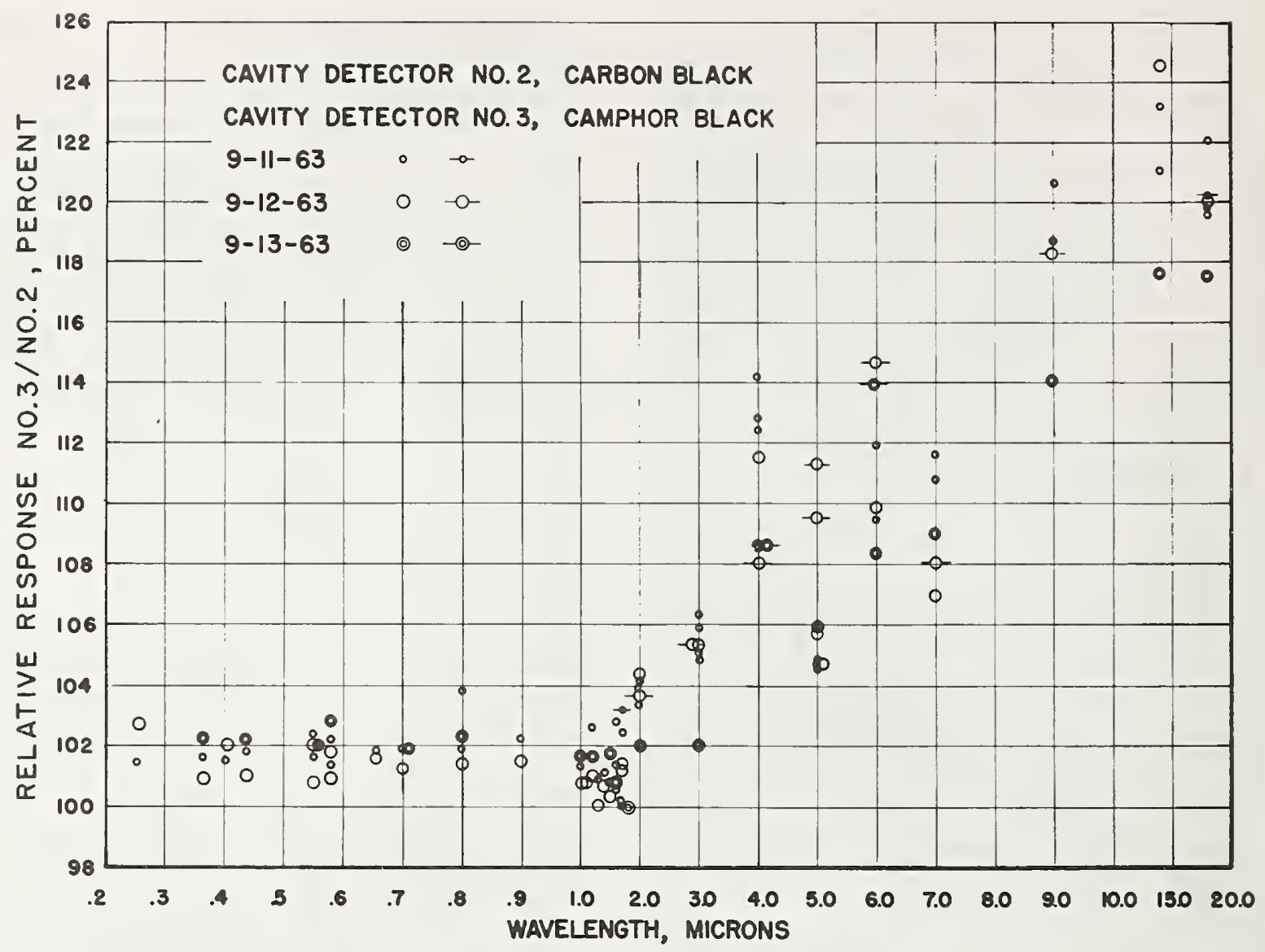

FIGURE 5.-Ratio of spectral sensitivity of cavity detector 3 (camphor black) to that of cavity detector 2 (carbon black).

when a Coblentz 12-junction thermopile was scanned lengthwise. In this illustration, the light beam was chopped at $13 \mathrm{cps}$ and amplified by a Perkin-Elmer 107 amplifier. Eleven peaks of varying sensitivity are noted. The 12th receiver element may have partially separated thermally from the thermoelement. Variations in the degree of thermal contact probably are in part responsible for some of the other variations.

Figure 7 shows the results when the same thermopile is scanned lengthwise, but by measuring the d-c output with a Keithley millimicrovoltmeter. In this case all twelve junctions are represented with corresponding peak responses. Figure 8 shows the results when the same thermopile is scanned crosswise, and with the use of the 13-cycle chopper amplifier. It is noted that somewhere near the two edges, areas of high sensitivity exist. It would be extremely interesting to study detectors of this type with a point light source if the required sensitivity of amplifying equipment were available. Then each element could be plotted showing its true "valleys and hills" of sensitivity. Such a plot could be expected to show greater contrasts than any pictures available at this time. Figure 9 illustrates the results when this thermopile is scanned crosswise and the $\mathrm{d}-\mathrm{c}$ output measured. In summary, the results in these four figures show that this thermopile varies significantly in sensitivity over its area, but that the variations are much smaller when it is used as a d-c detector.

Similarly, in figure 10 are shown the variations in lengthwise sensitivity of a Reeder Company thermopile when the signal is chopped at $13 \mathrm{cps}$. Only noise is produced near one end of this unit. Then follow several peaks and troughs in 


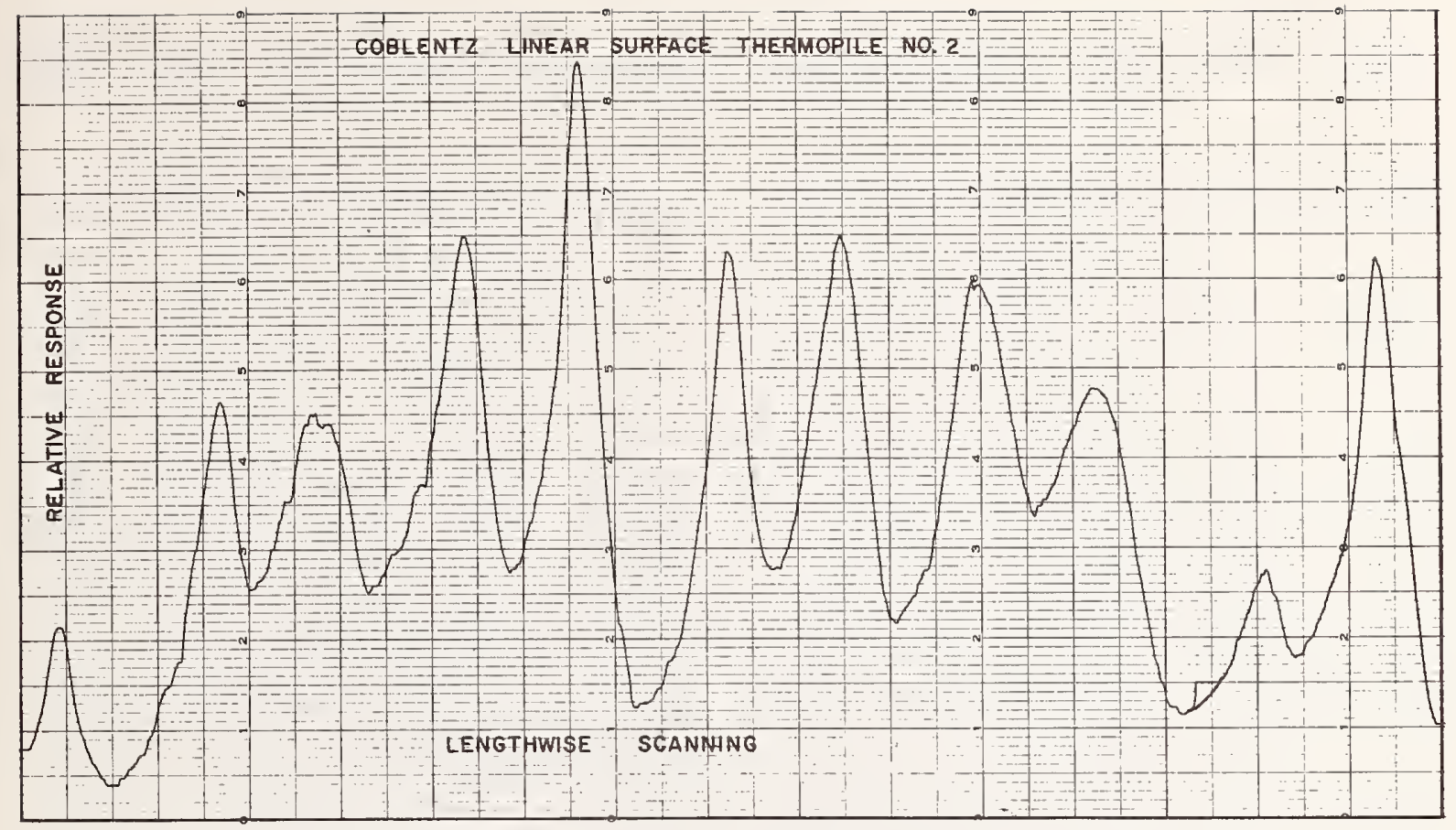

FIGURE 6.-Linear sensitivity of Coblentz thermopile 2 scanned lengthwise (at $19 \mathrm{cps}$ ).

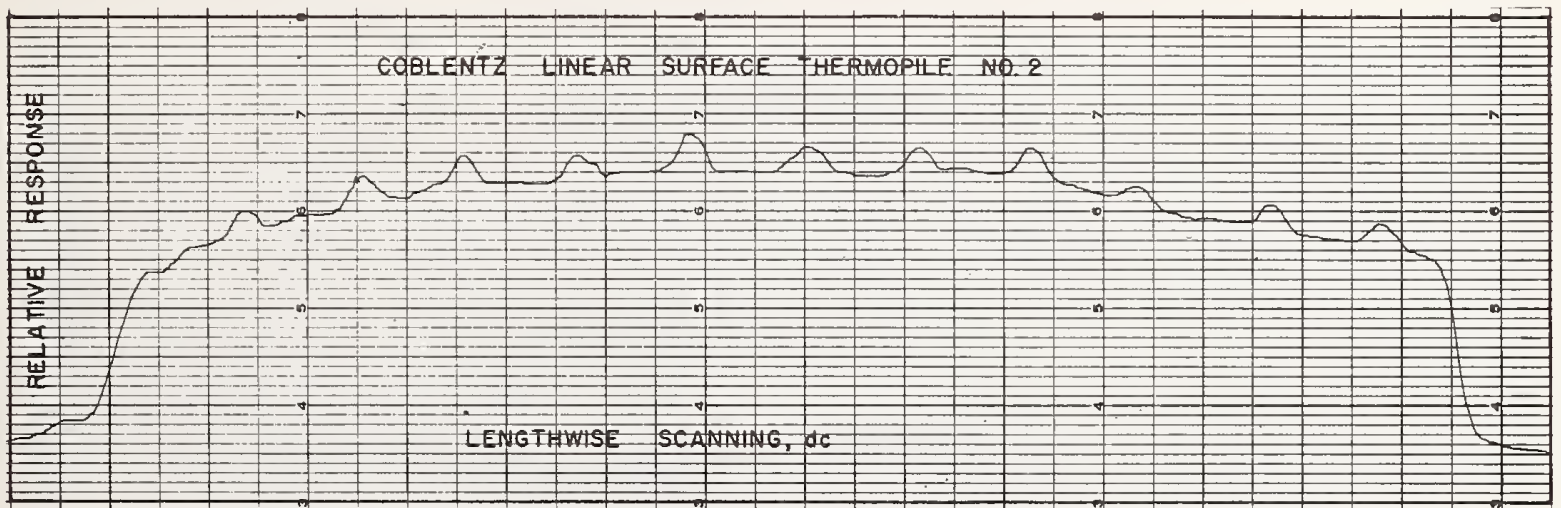

FIGORE 7.-Linear sensitivity of Coblentz thermopile 2 scanned lengthwise (measured by $d$-c method).

sensitivity. Several of these are negative (below zero). Two are very large, such that the recorder trace is off the scale. The complete explanation for these large negative values is unknown to the authors, but an analysis of similar measurements on other detectors indicates that there is a phase change in the signal resulting from the time lag between the absorption of the light and the resulting build-up of an electric potential in the thermal junction.
Thus, the Perkin-Elmer 107 amplifier picks up a response out of phase with the signal itself. If near $180^{\circ}$ out of phase, the signal would be negative, if near $90^{\circ}$, zero, etc. Incidentally, the placing of a narrow shield over the thermopile indicated that this response came solely from the blackened receiver. The number of receivers in this thermopile cannot be determined from this chart. In figure 11 is shown the record obtained when this thermopile is 
again scanned lengthwise, but with the d-c output recorded. The resulting tracing is

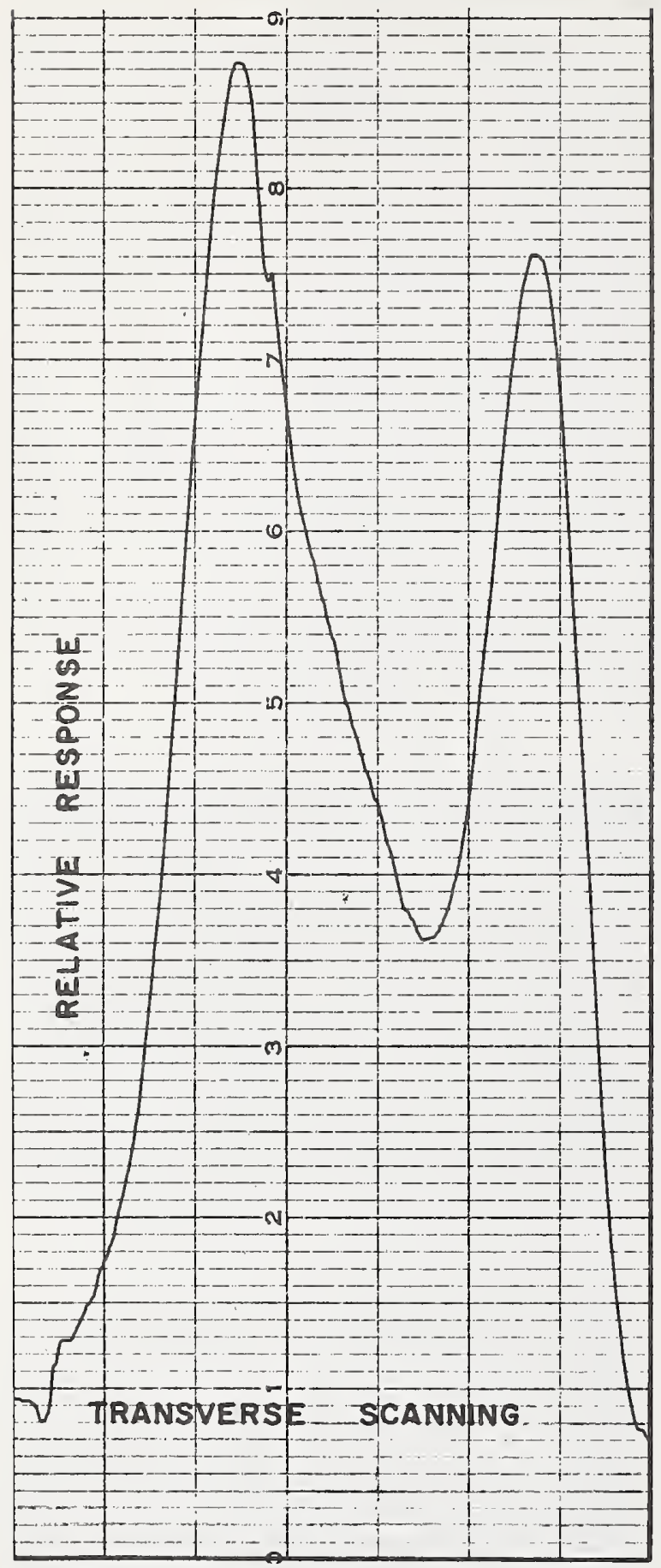

Figure 8.-Linear sensitivity of Coblentz thermopile * scanned crosswise (at $13 \mathrm{cps}$ ). notably different, there being no recognizable similarity between the two tracings. When the scanning was crosswise, tracings similar to those of figures 8 and 9 were obtained.

Figure 12 shows the recorded response of an Eppley thermopile scanned lengthwise with the signal chopped at 13 cps. Each receiver element of this unit stands out in terms of a definite response since the six elements are physically separated by small distances-not uniform, but definite. A zone of high sensitivity is noted near one end of each receiver element. When scanned in the opposite direction (fig. 13) the pattern is reversed but not exactly since the reverse scanning employs the d-c method with a Keithley millimicrovoltmeter. These differences are small, especially in view of the fact that this is not a fast type of thermopile, the receivers being relatively massive with a response rating near 1 sec. On crosswise scanning (d-c) this thermopile gave a response similar to that shown in figure 9 .

The data presented in the previous figures are not specially picked but represent what may be expected as to the geometrical variations in the sensitivity over the surface of represent-

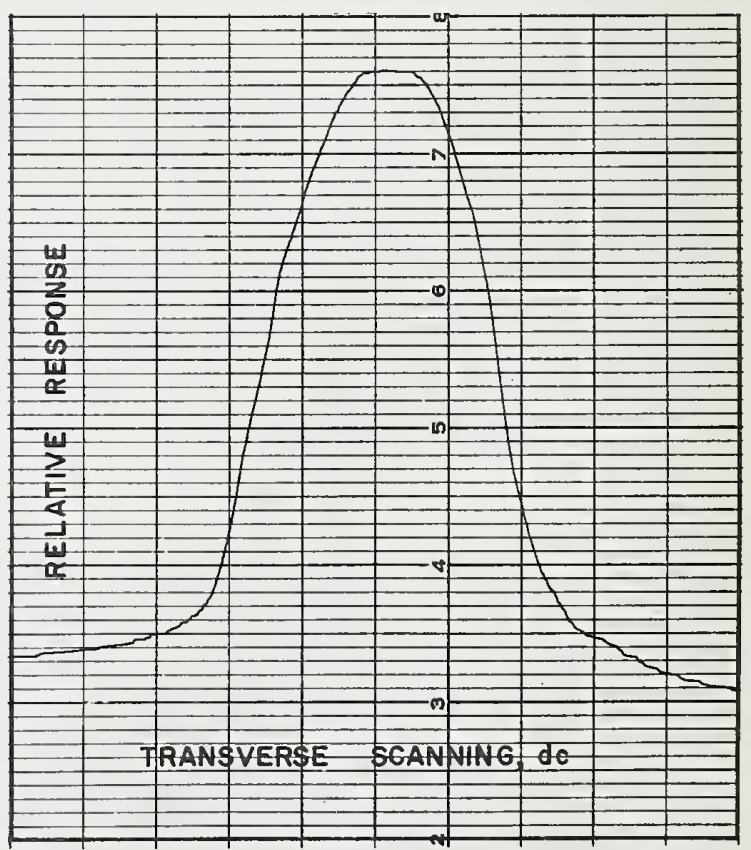

Figure 9.-Linear sensitivity of Coblentz thermopile 2 scanned crosswise (measured by d-c method). 


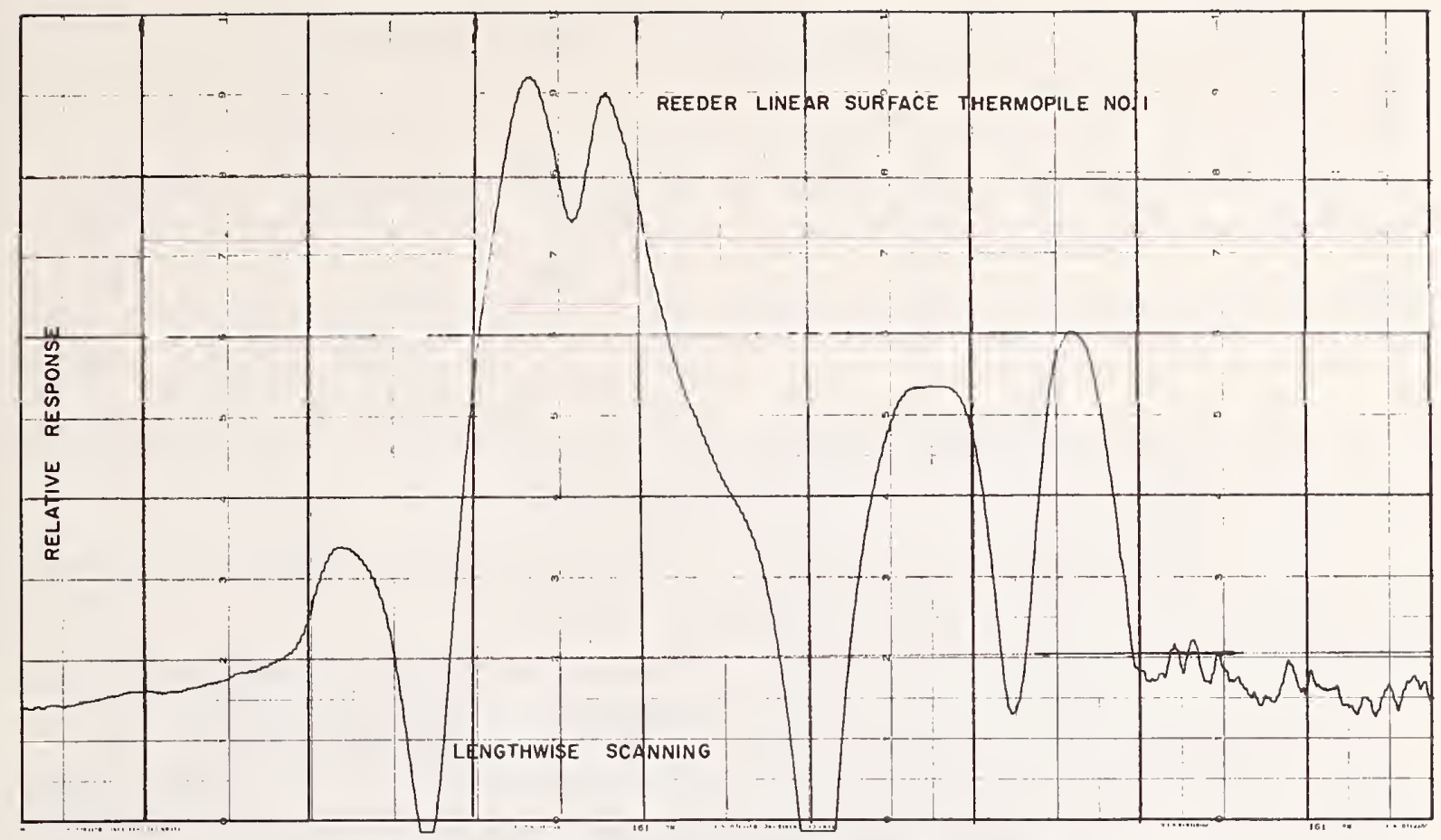

FIGORE 10.-Linear sensitivity of Reeder thermopile 1 scanned lengthwise (at $19 \mathrm{cps}$ ).

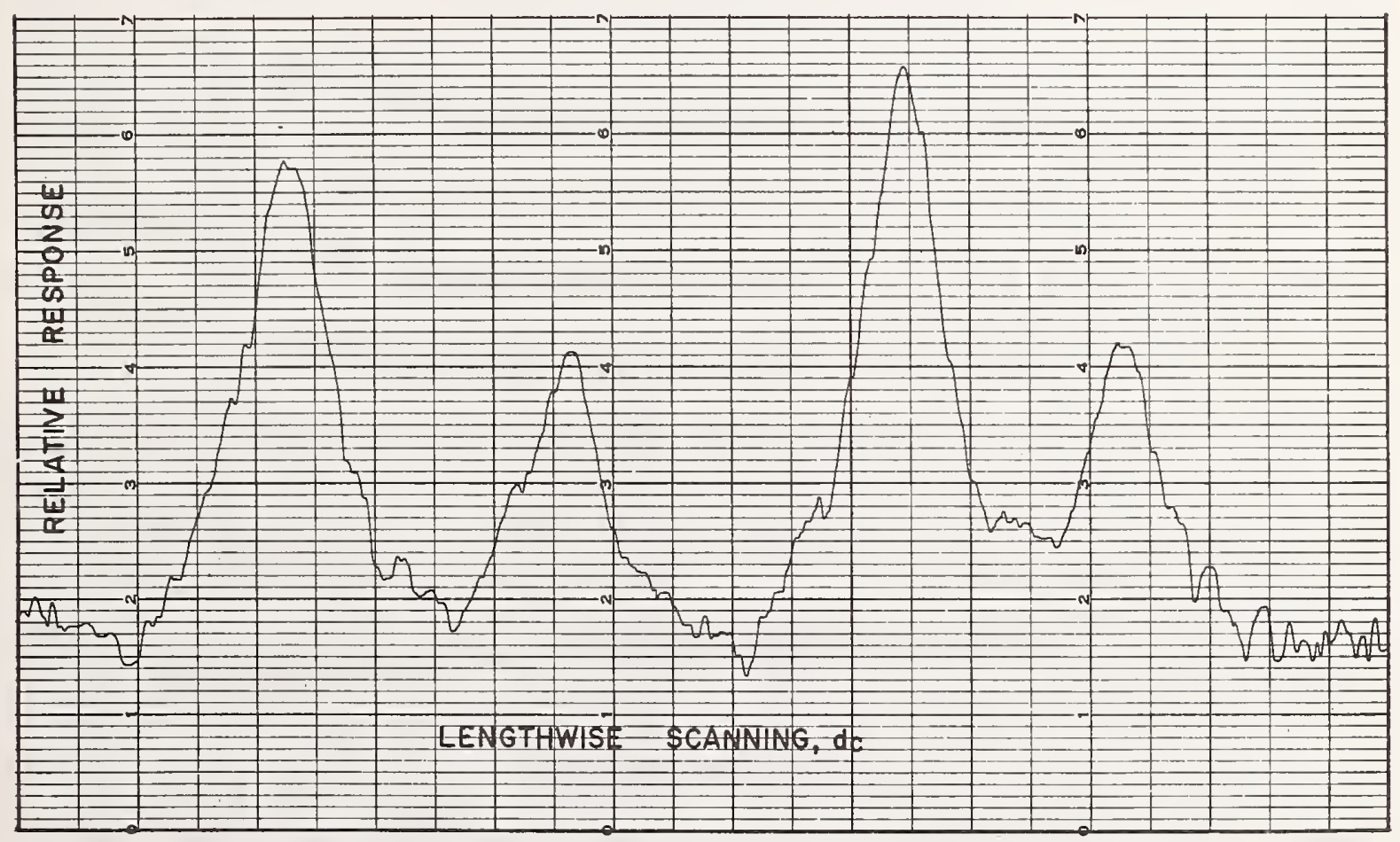

FIGURE 11.-Linear sensitivity of Reeder thermopile 1 scanned lengthwise (measured by $d-c$ method). 


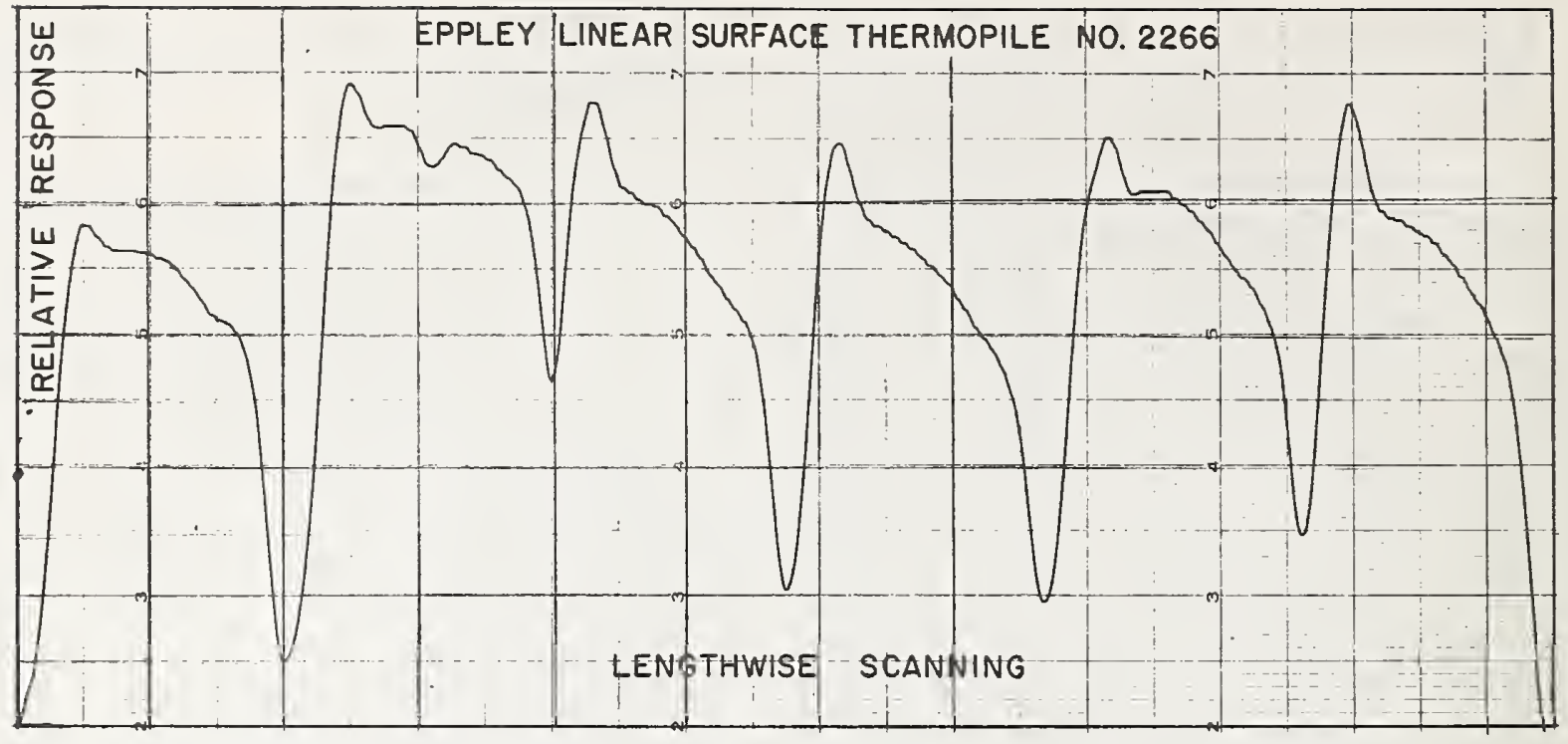

FIGURE 12.- Linear sensitivity of Eppley thermopile 2266 scanned lengthwise (at $13 \mathrm{cps}$ ).

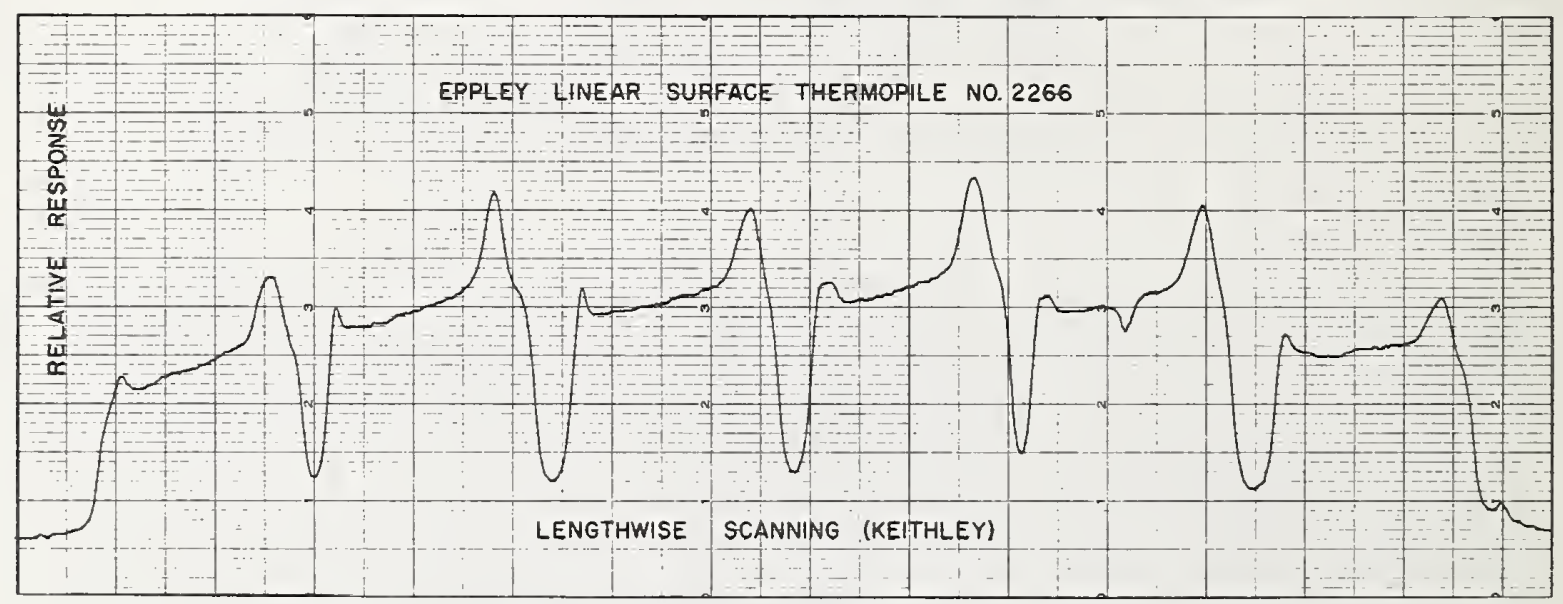

FIGURE 13.-Linear sensitivity of Eppley thermopile 2266 scanned lengthwise (measured by d-c method).

ative thermal detectors of this type. The Golay cell and bolometer may be regarded as having sensitivities more nearly uniform over their surfaces-but most observers are using thermocouples or thermopiles.

A few figures follow which show variations in the spectral sensitivity of available thermal detectors. Bolometers would be expected to have similar responses. Golay cells are yet to be investigated in this respect. These data, and also those illustrated in figure 5, were obtained with a newly developed filter spectroradiometer shown by block diagram in figure 14 .
By means of selected sources, such as low- and high-pressure mercury arcs, incandescent lamps, and glowers, used in conjunction with glass color filters and special multiple unit interference plates, approximately 30 narrow spectral bands of radiant energy are isolated. Data may be taken at a chopping rate of 13 cycles or for d-c output of the thermopiles. The spectral transmittance of a typical filter unit is shown in figure 15.

Figure 16 records a comparison between the spectral sensitivities of the Coblentz thermopile No. 1 and cavity detector No. 2. Since this 


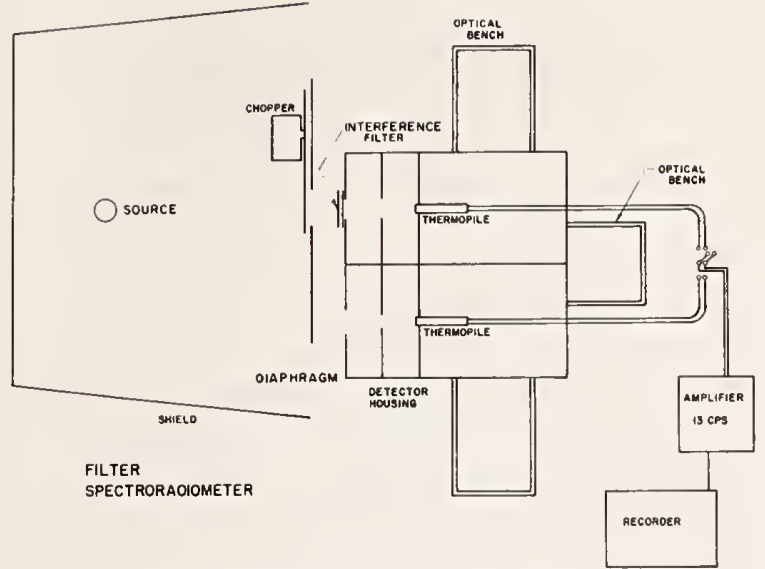

FigURE 14.-Block diagram of filter spectroradiometer.

curve is flat between 0.6 micron and 3 microns and since that for cavity unit No. 3 vs. cavity unit No. 2 was also flat within this spectral range to 2 microns, it may be assumed that all three receivers are relatively flat between 0.6 and 2.0 microns. At longer wavelengths the Coblentz thermopile and cavity unit No. 2

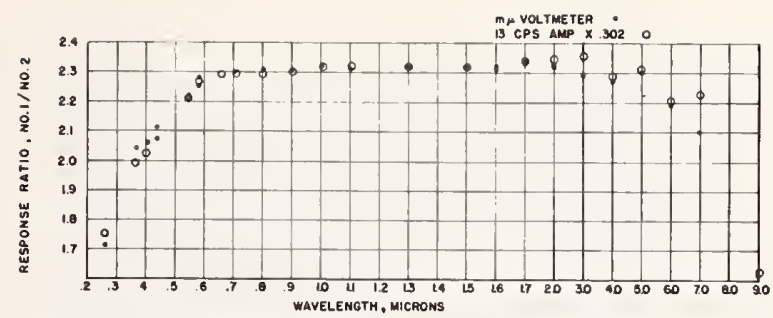

FIgURE 16.-Ratio of spectral sensitivity of Coblentz thermopile 1 to that of cavity detector 2.

appear about equally sensitive, which is reasonable because both are of carbon black. The drop at 7 to 9 microns results from window absorption. The reason for the drop at short wavelengths is undetermined. It is interesting that the data obtained by the two methods (Keithley d-c and Perkin-Elmer chopped at 13 cycles) are in close agreement. Other measurements, especially on thermopiles having thicker coatings, show spectral differences between the two methods, possibly resulting from the fact that the coatings absorb radiation of different wavelengths at different depths, thereby

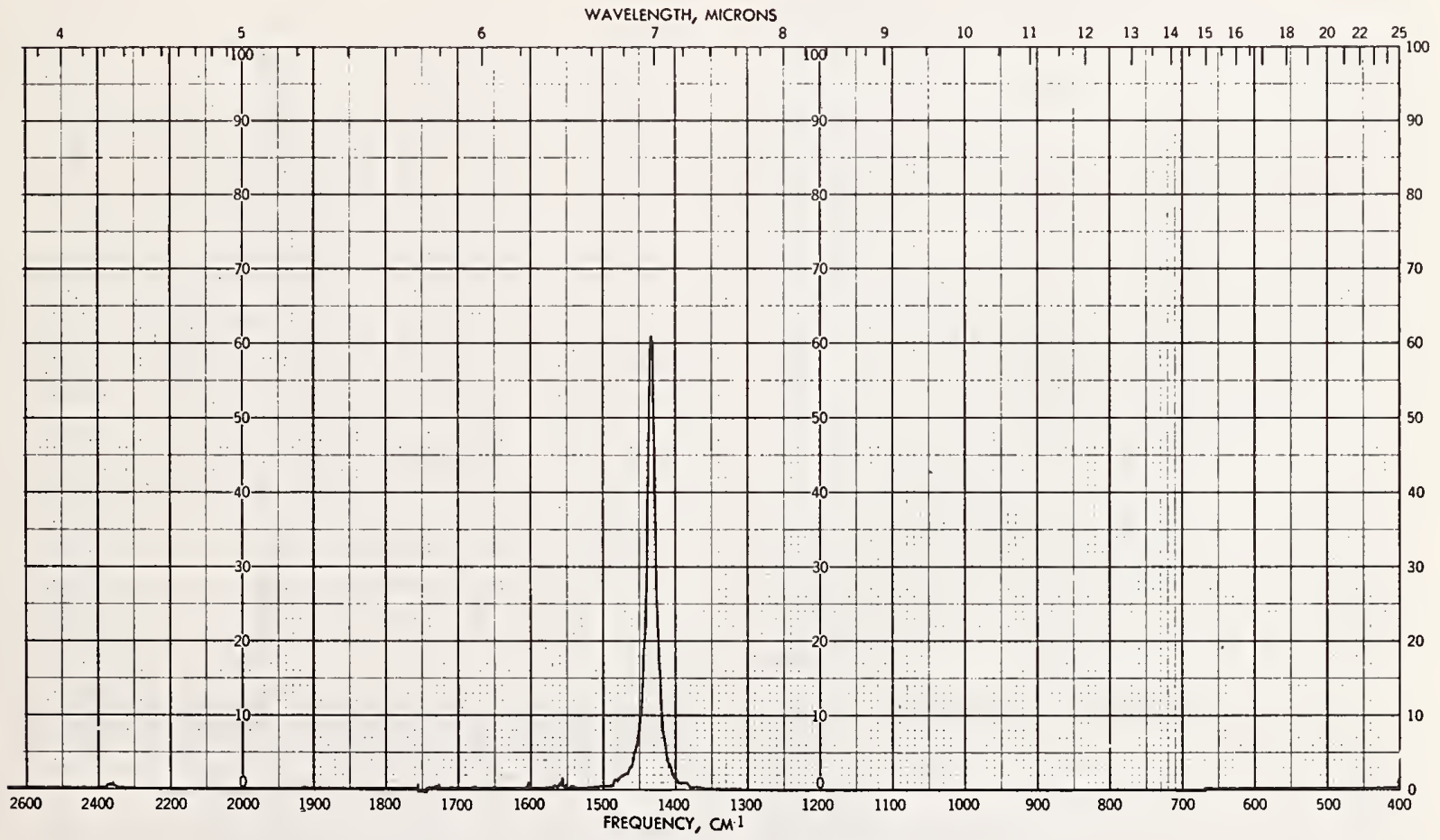

FIGURE 15.- Spectral transmittance of a representative filter. 


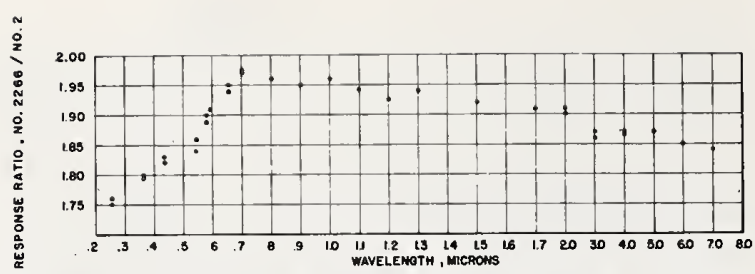

FIgURe 17.- Ratio of spectral sensitivity of Eppley thermopile 2266 to that of cavity detector 2 (13-cps amplifier).

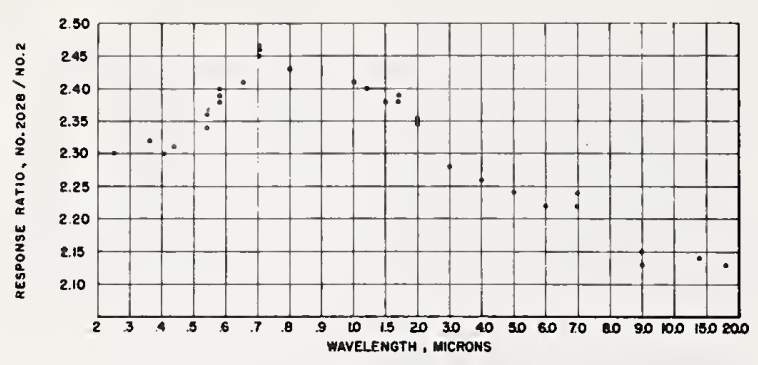

Figure 18.-Ratio of spectral sensitivity of Eppley thermopile 2028 to that of cavity detector 2 (13-cps amplifier).

producing phase angle variations with wavelength for the chopped signal.

Figure 17 shows a similar comparison between Eppley thermopile 2266 and cavity detector 2. The Eppley thermopile has a gold black surface and shows a drop in sensitivity at wavelengths both longer and shorter than about 0.7 micron. For wavelengths above about 2 microns the drop is even greater than that recorded for cavity detector 2 . Similar data were obtained for Eppley thermopile 2028 (replacement element 2650) having a lampblack surface and no window (fig. 18). Although this thermopile has a coating similar to that of the Coblentz 1 unit, its response drops off at longer wavelengths apparently because the coating is thinner and also because the substrate is gold. For the longer wavelengths, the Coblentz thermopile also has receivers consisting of thin sheets of tin having a lower reflectance than gold.

\section{Photosensitive Detectors}

Two types of photosensitive detectors will be considered as representative of what one may encounter in the laboratory-a photomultiplier and a $\mathrm{PbS}$ cell. It is realized that others offer different problems and may be worse or better in certain respects. The two detectors chosen have wide use. For the multiplier type, data are given on an RCA type 1P-28. (Fig. 19). This illustration shows the variations in sensitivity over the cathode surface of a particular tube-one chosen at random from a group of about a dozen having similar characteristics. From this figure it becomes evident that in order to measure radiant flux accurately, the light beam must always fall exactly upon the same position of the cathode in all cases. A movement of the light beam by even a few thousandths of an inch may result in a response change of many percent. A crosswise scanning of the cathode of the same tube in figure 20 shows changes in response across the center of the cathode for blue light and for red light. An appreciable color sensitivity exists. In other tubes much larger color variations have been noted. The third curve (the solid circles)

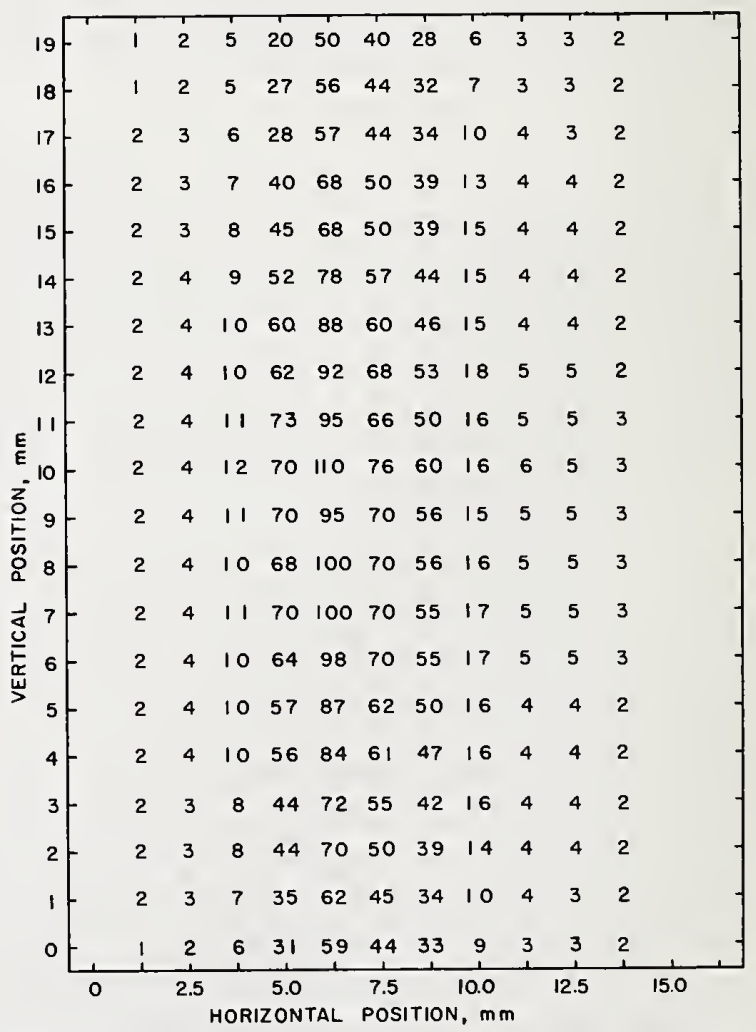

FIgURE 19.-Variation in sensitivity over the surface of a 1P-28 photomultiplier. 
shows what may be accomplished with incandescent lamp flux by covering the multiplier

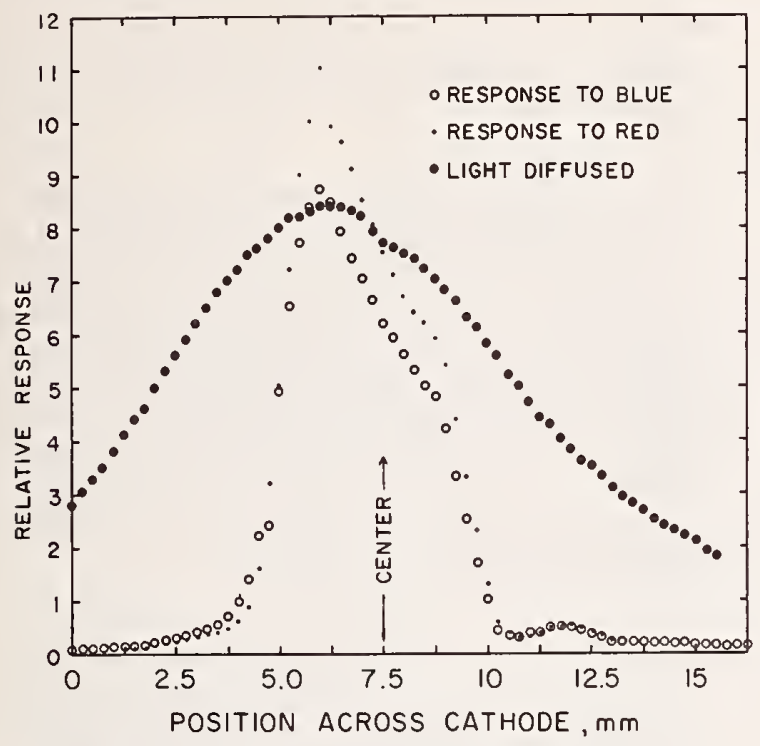

FIgURE 20.-Variation in sensitivity for blue and red light across the cathode of the 1P-28 photomultiplier of figure 19.

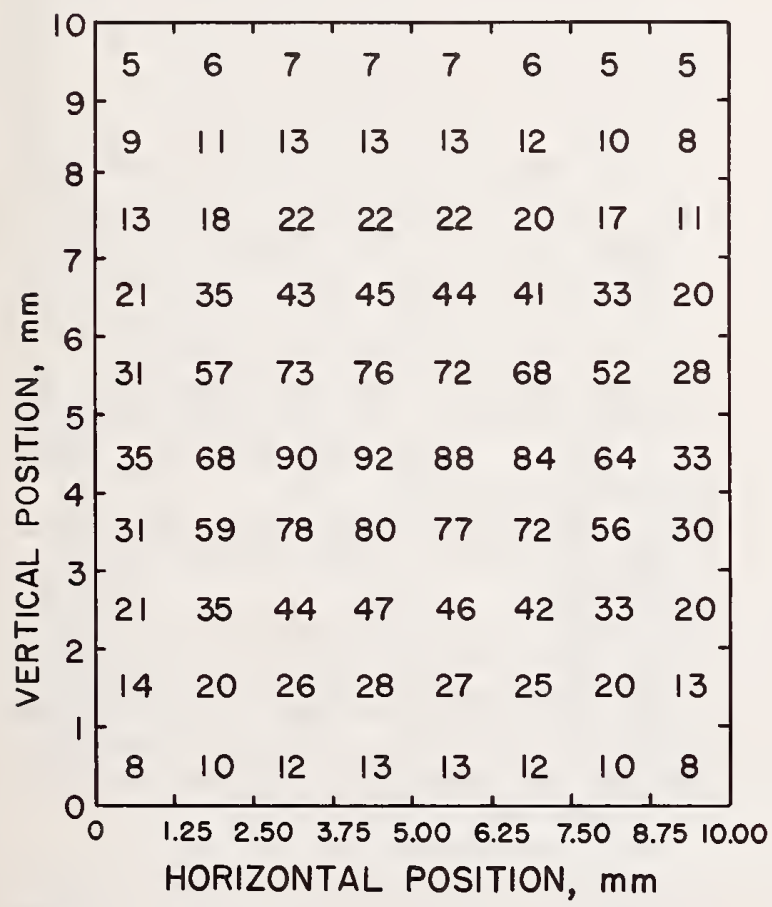

FIgURE 21.-Variation in sensitivity over the surface of an Eastman lead sulfide cell. with a doubly diffusing fine ground quartz glass plate. A lesser effect may be obtained through roughening (sandblasting) the tube envelope before or after manufacture.

In figure 21 are shown the variations in sensitivity over the surface of a $10 \mathrm{~mm} \times 10 \mathrm{~mm}$ Eastman $\mathrm{PbS}$ cell when scanned with a small spot of incandescent lamp flux. Highest sensitivity occurs across the center of the element between the electrodes (located at 0 - and 10$\mathrm{mm}$ positions). Lower sensitivities exist near the upper and lower edges of the element. A detector of this type should be set up with the spectroradiometer slit along the ridge of highest sensitivity for highest efficiency and highest signal-to-noise ratio. However, for certain purposes a more nearly constant output may be obtained with the cell rotated $90^{\circ}$.

\section{CONCLUDING REMARKS}

When consideration is given to the large variations that may exist over the surfaces of detectors, coupled with the nonuniformity of the emergent beam of the spectroradiometer, the direct comparisons of sources spectroradiometrically become very difficult. For example, with one detector, source A may appear to have several times the intensity of source B at the set wavelength, but with a change of detector (or even with a resetting of the original detector) a new measurement may indicate just the opposite-that source B has several times the intensity of source A at the set wavelength. Even with very careful optical adjustments using two similar sources (two lamps of the same type and size) errors of 50 to 100 percent may occur. With sources of unlike size and shape accurate comparisons are impossible by direct radiometric comparisons without auxiliary equipment.

Similarly, when a single source is used, the comparison of two detectors spectroradiometrically poses a like problem. After much effort in this direction, the authors concluded that for thermal detectors having relatively flat responses with wavelength the problem could best be solved through the use of a filter method. Accordingly, the filter spectroradiometer shown in figure 14 employing twenty odd narrow band interference and other type filters was set up 
and employed in the comparisons of the thermal detectors described herein. This instrument will be described in greater detail in a coming report.

Accurate comparisons between like sources (two lamps of the same type for example) may be made spectroradiometrically through the use of detectors having surfaces of uniform sensitivity provided each lamp is set to irradiate the spectroradiometer in exactly the same way. This may be simply accomplished through the use of identical auxiliary optics as shown in figure 2 in radiance measurements, but in irradiance work the difficulty is greater. Even the small differences in filament shapes between two lamps of the same type may be sufficient to upset the measurements by many percent. However, in practice the two lamps may be individually set at the optimum position by obsurving the radiometric deflection and satting each of the lamps (individually) at the position for maximum reading after the detector has been mounted with its position of maximum sensitivity centered on the slit and the wavelength drum of the spectroradiometer has been set for peak response for the particular lamp and detector. With these precautions two like sources may be compared accurately, the same results being obtainable with different detectors.

But when the sources are different-as will usually be the case with an unknown source being measured in terms of a standard-the results will depend greatly upon the experimental setup. With radiance measurements, the results will simply be relative for a particular area of the unknown-as, for example, a limited section of the arc between the electrodes of a xenon arc. Such a result is of little value for most purposes. A more meaningful measurement must include the entire source and will usually require that it be made in terms of irradiance. Since the two sources, the standard and unknown, are of different geometrical shape and area as viewed from the spectroradiometer slit, some optical method must be included to produce like sources as seen from the spectrometer. This can best be accomplished through the use of a diffusing sphere or spheres which are alternately illumi- nated by the two sources. This is not a new idea, but one which is often by-passed if sufficiently useful information can be had without resorting to its use. For a sphere coating, magnesium oxide offers good reflectance from about 0.25 to 2 microns (ref. 11, 12, and 13) and may be usable to about 11 microns. At wavelengths longer than 2 microns metallic surfaces are probably best. Some of the ceramics appear promising if methods of coating or casting can be worked out. In lieu of spheres for approximate measurements, good diffusing surfaces may be employed and set such that the diffusing surface receives and reflects or transmits the radiant energy in the same manner in the two cases, if sufficient energy is available.

\section{REFERENCES}

1. Coblentz, W. W.: Measurements on Standards of Radiation in Absolute Value. Bull. Bur. Standards, vol. 11, no. 1, Nov. 15, 1914, pp. 87-96.

2. Anon.: NBS Technical New Bulletin, vol. 47, Oct. 1963, p. 10.

3. Strmson, H. F.: International Practical Temperature Scale of 1948-Text Revision of 1960 . Res. Paper 65A3-96, Jour. Res. Nat. Bur. Standards, vol. 65A, no. 3, May-June 1961, pp. 139-145.

4. Stajr, Ralph, Fussell, William B., and Schneider, William E.: A Standard for Extremely Low Values of Spectral Irradiance. Appl. Optics, vol. 4, no. 1, Jan. 1965, pp. 85-89.

5. Stajr, Ralph, Johnston, Russell G., and HalBACH, E. W.: Standard of Spectral Radiance for the Region of $\mathbf{0 . 2 5}$ to 2.6 Microns. Res. Paper 65A-5P, Jour. Res. Nat. Bur. Standards, vol. 64A, no. 4, July-Aug. 1960, pp. 291-296.

6. Stair, Ralph, Schneider, William E., and JACKson, JoHN K.: A New Standard of Spectral Irradiance. Appl. Optics, vol. 2, no. 11, Nov. 1963, pp. 1151-1154.

7. Engstrom, R. W.: Luminous Microflux Standard. Rev. Sci. Instr., vol. 26, no. 6, June 1955, pp. 622-623.

8. Eisenman, W. L., Bates, R. L., and Merriam, J. D.: Black Radiation Detector. Jour. Optical Soc. of America, vol. 53, no. 6, June 1963, pp. 729-734.

9. Goufft, ANdre: Aperture Corrections for Artificial Blackbodies. Revue d'Optique, vol. 24, nos. 1-3, Jan.-Mar. 1945, pp. 1-10.

10. Sparrow, E. M., and Jonsson, V. K.: Radiant Emission Characteristics of Diffuse Conical Cavities. Jour. Optical Soc. of America, vol. 53, no. 7, July 1963 , pp. 816-821. 
11. Middleton, W. E. K., and Sanders, C. L.: An Improved Sphere Paint. Illuminating Eng., vol. 48, no. 5, May 1953, pp. 254-256.

12. McAloren, J. T.: A Reproducible Magnesium Oxide Standard for Reflectance Measurement
From 0.3 to $2.6 \mu$. Nature, vol. 195, no. 4843 , Aug. 25, 1962, pp. 797-798.

13. Gier, J. T., Dunkle, R. V., and Bevans, J. T.: Measurement of Absolute Spectral Reflectivity From 1.0 to 15 Microns. Jour. Optical Soc. of America, vol. 44, no. 7, July 1954, pp. 558-562.

\section{DISCUSSION}

Thomas Limperis, University of Michigan: Perhaps the change in responsivity with wavelength for the a-c and d-c modes might be a thickness effect; for example, at longer wavelengths $I$ would think that the radiation might be absorbed further down into the material and that the recovery time of the detector might be a little longer. Have you reduced the chopping frequencies somewhat to determine whether recovery is toward the d-c level?

SCHNEIDER: No. We are now working on that; we plan to use a variable-frequency-type amplifier and then examine this effect further. That was one of our considerations.

LIMPERIs: What was the band pass of your electronics, operating at $13 \mathrm{cps}$ ?

SchNeIDER: I do not know the exact band pass.

DWIGHT Moore, National Bureau of Standards: I would like to make a comment on a statement by Clayton, earlier, that the thermal emittance standards were not easy to get from the National Bureau of Standards. This may have been true 3 or 4 years ago, but for the past year, it has been quite easy to obtain these standards from our laboratory. I would guess that some 20 or 25 sets have been sent out over this period. Of course, he might have been referring to the cost-we do charge for them. For a complete set (low-, medium-, and high-emittance standards calibrated from 1 to 15 microns at temperatures of $800^{\circ}$, $1100^{\circ}$, and $1400^{\circ} \mathrm{K}$ ) the price is about $\$ 600$, with the exact amount depending on the particular size that is required. Possibly, Mr. Schneider would like to comment on how easy it is to obtain the standards that he referred to in the early part of his talk.

SCHNEIDER: The standards of total irradiance and spectral radiance (the tungsten strip lamps) can be purchased from The Eppley Laboratory, Inc., Newport, Rhode Island. The standard of spectral irradiance can be purchased directly from the National Bureau of Standards. However, there is a delay of about a month.

Michael T. Surh, University of Michigan: In the instructions for the use of standards of spectral radiance or irradiance, it is indicated that the uncertainty of the spectral values that are furnished in the certificate of calibration varies from approximately 8 percent at the shortest wavelength to 3 percent at the longest wavelength. I wonder if in the future a more detailed statement relating to the accuracy of the standard can be given. I have seen many papers in which the authors claim that they have made radiation measurements with an uncertainty of 1 or 2 percent using a standard which is 3 to 8 percent uncertain. I believe that some of this confusion could be eliminated if the NBS or Eppley would supply data on precision or statistical spread of the values they measure. You mentioned that, in scanning the thermopile lengthwise or vertically, you found some large negative values; I wonder if this is due to the irradiation of the cold junction rather than the hot junction.

SCHNEIDER: I do not think the negative deflections are due to the cold junctions heating up, since we do not observe these deflections with the d-c setup. For one thermopile there was some negative deflection near the edges, which was probably due to the cold junction heating up; but the negative deflections occurring along the length of one of the thermopiles spaced somewhere in the middle of the element are probably not due to cold junctions heating up. However, it has been found that the apparent sensitivity pattern observed changes radically both with change in the chopping frequency and with angular adjustment of the light beam interceptor blade. These results indicate that there is a phase change in the signal resulting from the time lag between the absorption of the light and the resulting buildup of an electric potential in the thermal junctions. Thus, the Perkin-Elmer 107 amplifier picks up a response out of phase with the signal itself.

Richard M. Jansson, Honeywell, Aeronautical Division, Boston: In the past, The Eppley Laboratory has calibrated thermopiles for solar simulation use by comparison with a set of pyrheliometers. Do you know when your high-intensity standard might be aviilable for more accurate calibrations and what its characteristics will be?

Schneider: This standard will be a 1000-watt tungsten lamp calibrated for total irradiance. Work on this standard is just beginning. As I mentioned, this standard will have an irradiance of about 100 to $150 \mathrm{mw} / \mathrm{cm}^{2}$. 


\title{
On the Use of Thermopiles for Absolute Radiometry in the Far Ultraviolet
}

\author{
R. G. Johnston and R. P. Madden
}

\begin{abstract}
Thermopiles used for absolute radiometry in the far ultraviolet spectral region are generally calibrated with visible and near infrared radiation. Three possibilities for the failure of the sensitivity of thermopiles to be independent of wavelength have been investigated. It was found that gold-black typical of that used on thermopiles for far ultraviolet applications reflectively scattered $2-4 \%$ of the incident far ultraviolet radiation. Since the same gold-black scattered a similar fraction of incident visible and near infrared radiation, the correction for this effect was small. An analysis of surface sensitivity maps indicated that a thermopile of $0.04-\mathrm{sec}$ time constant showed no wavelength variation of sensitivity when either de detection or ac synchronous detection with $13 \mathrm{cps}$ chopping was used. The most significant wavelength-dependent phenomenon was found to be the photoelectric effect. Ejected electrons carry a significant amount of energy away from gold-blacked thermopiles in the spectral region below $1600 \AA$. The maximum correction determined for a particular thermopile was $5.2 \%$ at $\lambda 735 \AA$.
\end{abstract}

\section{Introduction}

Thermopiles have often been used in the vacuum ultraviolet spectral region as absolute detectors. Much of the data which exists on photoionization yields in gases $^{1-3}$ and photoelectric yields for solids ${ }^{1,4-7}$ in the vacuum ultraviolet depends on thermal detection for the absolute measurement of radiant flux. Furthermore, the wavelength dependence of the efficiency of far ultraviolet radiation detectors is often determined by comparison to the fluorescent yield of a sodium salicylate phosphor, the relative response of which was determined by comparison with that of a thermopile. ${ }^{8-10}$

A thermopile destined for use as a standard in the vacuum ultraviolet is first calibrated in the visible region with sources of known blackness and temperature. It is then assumed that the thermopile will develop a given voltage for a specific wattage of incident radiant energy, independent of the wavelength of the radiation. This paper reports on the investigation of three possibilities for the failure of this assumption. First, the fraction of incident radiation reflectively scattered by the thermopile black has been measured in the visible and the far ultraviolet. Second, the energy carried away from the thermopile by photoejected electrons has been determined. Finally, the thermopile surface sensitivity has been mapped at several wave-

The authors are with the National Bureau of Standards, Washington, D.C.

Received 17 May 1965. lengths using both ac and dc methods to reveal any wavelength dependence of the thermopile time constant. ${ }^{11}$ Such a dependence would, of course, influence the calibration of the thermopile if ac methods were employed.

The first possibility mentioned above, namely, the failure of the blackness to be independent of wavelength, has been partially checked in a few instances. The specularly reflected component has been found small by several investigators, ${ }^{12,13}$ but the determination of the total scattered radiation from such blacks has not been previously undertaken in the vacuum ultraviolet. The energy loss due to photoejected electrons had apparently been overlooked previous to the initiation of the present study, although it is well known that most surfaces exhibit a marked increase in photoelectric yield when the photon energy exceeds $9-10 \mathrm{eV}, 1,6,7$

The present studies were conducted using a particular type of thermopile and black; however, the ones chosen are typical of those which have been used in the far ultraviolet as absolute detectors. Also, these results bear on the general problem of the applicability of thermal detectors for absolute radiometry in the far ultraviolet.

\section{Reflective Scattering from Gold-Black}

A. Experimental Arrangement

1. Source and Monochromator

A normal-incidence vacuum ultraviolet monochromator was employed for these measurements which utilized a $1-\mathrm{m}$ radius, 600 lines $/ \mathrm{mm}$, concave grating blazed at 


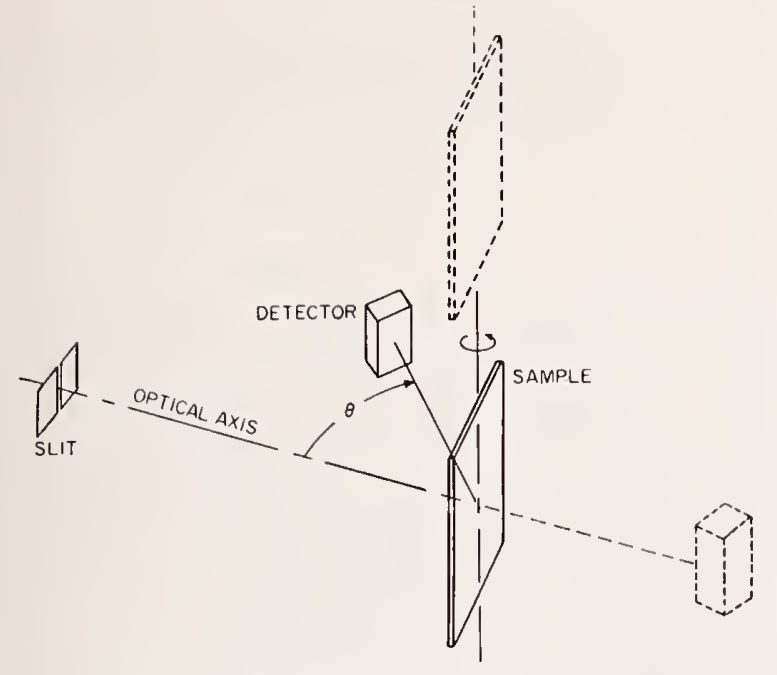

Fig. 1. Diagram showing the experimental arrangement used for the measurement of the reflective scattering from gold-black in the far ultraviolet spectral region. The dashed portion of the diagram indicates the position of the sample and detector when the direct beam is measured.

$1500 \AA$ A. This mounting has fixed entrance and exit slits at an angular separation of $15^{\circ}$. The grating was overcoated with fresh aluminum and $250 \AA$ of $\mathrm{MgF}_{2}$ to enhance its efficiency down to at least $\lambda 1000 \AA$. The light source, a windowless dc capillary discharge tube, was used to obtain the resonance lines of the rare gases, the Lyman series in hydrogen, and the molecular hydrogen spectrum.

\section{Experimental Chamber}

A 50-cm diam vacuum chamber was attached to the exit arm of the monochromator. This chamber and pumping system could be isolated from the monochromator. All of the measurements were made with a chamber pressure on the order of $5 \times 10^{-5} \mathrm{~mm} \mathrm{Hg}$.

The detector was mounted in the experimental chamber on a table which could be rotated from outside the vacuum system. The sample to be studied was mounted in a holder which could be moved vertically in and out of the exit beam and rotated about a vertical axis intersecting the beam. These relationships are indicated in Fig. 1. The sample holder was mounted so that the sample surface was located at the center of rotation of the detector table.

The signal from the detector, a magnetic multiplier with a tungsten photocathode (insensitive above $\lambda 1600$ $\AA)$, was fed into a dc amplifier and recorded.

\section{B. Procedure}

Let us assume a beam of intensity $I_{0}$ to be incident on a scattering surface, and a detector, of area $A$, scanning the scattered radiation at a constant radial separation $r$ from that surface. Let $\theta$ be the angle of observation relative to the incident beam, and $I_{s}(\theta)$ the intensity of the scattered radiation observed by the detector at that angle. Then, assuming $I_{s}(\theta)$ is properly averaged over any directional anisotropies in the scattering distribution, the ratio of the total scattered intensity to the incident intensity is given by

$$
\frac{2 \pi r^{2}}{A I_{0}} \int_{0}^{\pi / 2} I_{\varepsilon}(\theta) \sin \theta d \theta .
$$

To obtain a detector having a uniform response, the surface sensitivity of the photocathode was mapped using various dynode voltages. The voltages yielding the most uniform response were used, and the detector was ultimately masked to a selected $9-\mathrm{mm} \times 9-\mathrm{mm}$ area.

The grating was masked to limit the exit beam divergence to $1.5^{\circ}$ vertically and $1.5^{\circ}$ horizontally. The height of the exit slit was reduced to give a beam $3 \mathrm{~mm}$ high at the scattering sample. These adjustments resulted in an exit beam cross section of $3 \mathrm{~mm} \times 3 \mathrm{~mm}$ on the sample.

The scattering sample was set normal to the beam, and measurements were made every $5^{\circ}$ from $10^{\circ}$ to $80^{\circ}$ on each side of the optical axis. The scattering sample was then set at an angle of $10^{\circ}$ and the specular peak was scanned. The sample was then rotated through $90^{\circ}$ about an axis perpendicular to its face and the above procedure repeated so as to detect any anisotropies in the scattering distribution.

The intensity of the incident beam was measured by moving the scattering sample vertically out of the beam; however, the direct beam signal was higher than the scatter signal by a factor of $10^{2}$ to $10^{5}$. To reduce the demands on the linearity of the detector, an attenuating screen was used in the measurement of the incident beam. The screen transmission $(2.1 \%$ ) was measured using the same geometry and wavelength as used in the scatter measurement.

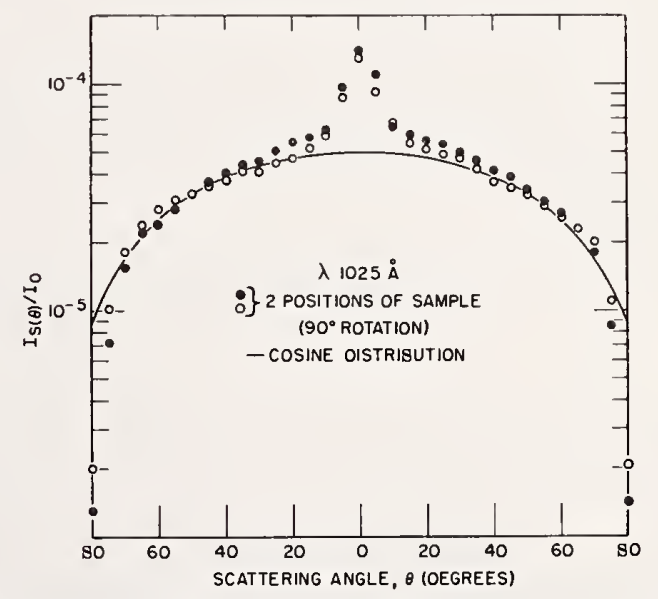

Fig. 2. Angular profile of the beam reflectively scattered from a sample of gold-black on a gold substrate. The ordinate is the ratio of the scattered intensity at angle $\theta$ to the incident beam intensity. A cosine distribution (perfect scatterer) is shown for comparison. Note the specular peak which is apparent for this thickness of black. When used on a thermopile, this thickness resulted in an over-all time constant of $0.04 \sec (\lambda=1025 \AA)$. 


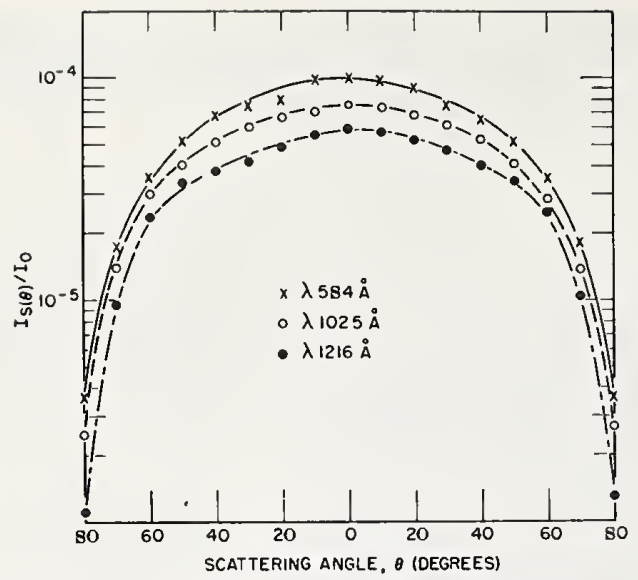

Fig. 3. Angular profile of the beam reflectively scattered from a second sample of gold-black (thicker than that used for Fig. 2) on a gold substrate at three wavelengths in the far ultraviolet. The ordinate is the ratio of the scattered intensity at angle $\theta$ to the incident beam intensity.

\section{Results}

The samples of gold-black measured in these experiments were prepared by a thermopile manufacturer (C. Reeder) and are typical of the blacks used on thermopiles for the far ultraviolet. Two thicknesses of black on gold-leaf substrates were studied. The thinner of the two blacks, and the black deposited on the thermopile discussed in Secs. III and IV of this paper, were manufactured in the same evaporation. This thermopile has a time constant of roughly 0.04 sec. The thicker gold-black, if applied to the same thermopile, would yield a longer time constant, which the manufacturer suggests would be on the order of 0.09 sec.

As a preliminary check of the scatter-measuring apparatus, a highly polished sample of silica was studied. The angular distribution of the reflected beam from the sample closely followed the distribution of the direct beam, thus indicating that the highly polished flat did not scatter appreciably at $\lambda 584 \AA$, and that the measuring apparatus was not recording unwanted scatteredlight signals.

Figure 2 indicates the ratio $I_{s}(\theta) / I_{0}$ as a function of $\theta$ obtained at $\lambda 1025 \AA$ when the scattering surface was the thinner of the two gold-black samples (presumably identical to that used on the thermopile tested in Secs. III and IV). In Fig. 2, two scans are shown, the difference between them being a $90^{\circ}$ rotation of the scattering sample about the incident beam axis. The difference between the two curves indicates some anisotropy in the angular scattering by the gold-black. Also shown in Fig. 2 is a curve representing the distribution of a perfect scattering surface, i.e., where $I_{s}(\theta) / I_{0} \sim$ $\cos \theta$. It is apparent that the angular distribution of the scattering by the gold-black-on-gold resembles the distribution of a perfect scattering surface, with a specular reflection component added on. The specular component is due to the gold substrate which is not completely obscured by the gold-black deposit. The peak of this specular component is less than $0.014 \%$ of $I_{0}$. The total scattered intensity, obtained from this distribution by the integration indicated in Sec. II.B, is $3.1 \%$. A similar distribution was obtained for this sample of gold-black at $\lambda 584 \AA$ and at $\lambda 1216 \AA$. The integrated scattered radiation at $\lambda 584 \AA$ was $3.6 \%$ and at $\lambda 1216 \AA$ was $3.1 \%$.

The specular component of the reflectively scattered radiation was absent in the heavier gold-black-on-gold sample tested, as can be seen in Fig. 3, where the distributions are plotted for the same three wavelengths in the far ultraviolet. It can be seen that the scattering loss increases slightly with decreasing wavelength which may reflect the changing ratio of wavelength-toparticle size of the gold-black. The integrated total scattering for this heavier black was $2.1 \%$ at $\lambda 584 \AA$, $2.0 \%$ at $\lambda 1025 \AA$, and $1.9 \%$ at $\lambda 1216 \AA$.

These same two samples of gold-black-on-gold were tested for total scattering in the visible and near infrared spectral regions using an integrating sphere and an appropriate spectrophotometer. The thin sample showed a total scatter of $1.6-3.1( \pm 0.5) \%$ and the thicker sample 1.5-2.9 $( \pm 0.5) \%$ over the region $0.4 \mu$ to $2.2 \mu$. Thus, while reflective scattering is a nonnegligible loss for thermopile blacks, it would seem that the similarity of this loss in the visible and the far ultraviolet indicates that the uncertainty in the use of the thermopile at the short wavelengths after calibrating in the visible is less than $1 \%$.

\section{Thermopile Cooling due to Ejected Photoelectrons}

Two independent methods have been used to determine the loss of thermopile signal due to the cooling caused by the loss of photoejected electrons. In each method, the basic idea is to eliminate the energy loss mechanism due to the photoelectric effect by returning the electrons to the thermopile with the same energy which they carried away. The first method is to surround the thermopile with an electrostatic field sufficiently strong to prevent a loss of photoelectrons. Care must be taken to prevent driving stray electrons

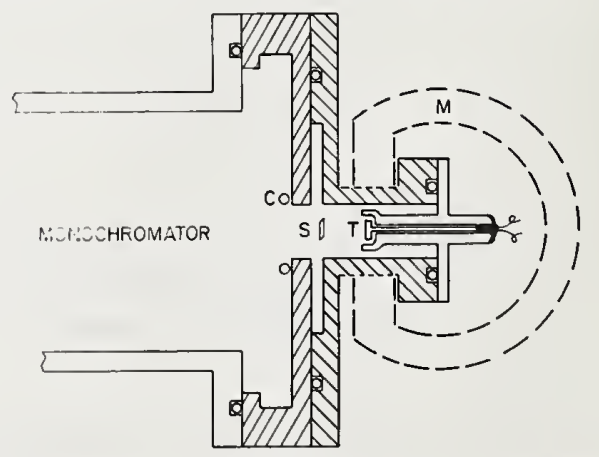

Fig. 4. A sectional drawing of the exit slit and thermopile housing, showing the relative locations of the exit slit (S) and thermopile ( $\mathrm{T}$ ). The collector ring $(\mathrm{C})$ is used in the electrostatic method of returning photoejected electrons to the thermopile and the magnet (M) is used in the magnetic method. 
and/or negative ions into the thermopile. The second is to immerse the thermopile in a de magnetic field so that ejected electrons will be turned back to the surface. This method has the advantage that stray electrons or ions are repelled from the thermopile.

\section{A. Experimental Arrangement}

The monochromator and light source described in Sec. II were used for these measurements. However, for this part of the work the radiation from the source was chopped between the source and the monochromator entrance slit with a frequency of $13 \mathrm{cps}$. The chopping motion, the mechanical design of which has been described, ${ }^{14}$ was fed into the vacuum system through a bellows seal. A substantial gain in signal-toscattered light ratio can be achieved by the proper choice of chopper blade materials. An additional advantage to the use of chopped source radiation applies when a thermal detector is used, namely, the de voltage generated at the detector due to thermal radiation within the monochromator itself is not accepted by the ac amplifier.

\section{Thermopile}

The thermopile used for this experiment is a series connection of four elements which are composed of bismuth-tellurium and bismuth-antimony alloys. ${ }^{*}$ The thermal junctions are extended using gold flakes of $1-\mathrm{mm} \times 2-\mathrm{mm}$ area. Together they form a sensitive area $1 \mathrm{~mm} \times 8 \mathrm{~mm}$. The thermopile is compensated, designed for a $13 \mathrm{cps}$ chopping frequency, and constructed entirely of nonmagnetic materials. The thin gold-black coating on the gold flakes is similar, if not identical, to the thin black tested for scattering in Sec. II. The time constant of the thermopile is on the order of $0.04 \mathrm{sec}$, and when calibrated in the visible, the evacuated thermopile had a sensitivity of $4 \mu \mathrm{V} / \mu \mathrm{W}$ and an ENI power of approximately $10^{-9} \mathrm{~W}$.

The thermopile was located approximately $15 \mathrm{~mm}$ behind the exit slit of the monochromator. This slit was adjusted to $0.6-\mathrm{mm}$ width and $7-\mathrm{mm}$ height, assuring that all the flux which passed through the exit slit of the monochromator would be intercepted by the sensitive area of the thermopile. The physical housing of the thermopile is indicated in Fig. 4. It was designed so that a dc magnet could be placed outside the vacuum housing but in close proximity with the thermopile, which was centered between the poles with the magnetic field lines parallel to the long dimensions of the sensitive surface.

\section{Electronics}

An impedance matching transformer was utilized between the thermopile and a preamplifier. The thermopile could either be grounded to its surroundings, or made electropositive with a variety of potentials available from battery sources.

\footnotetext{
* This thermopile was manufactured and mounted in a special
} housing by the C. Reeder Co., Detroit, Michigan.
The signal from the preamplifier was fed to a 13-eps broadly tuned amplifier. The output of this amplifier was rectified synchronously with the chopping frequency by a mechanically coupled rectifier. The signal was then filtered, further amplified, and fed into a voltageto-frequency converter. The converter signal was fed to a $10^{5}$ count scaler. By proper adjustment of the voltage to the converter, the signal could be integrated for any desired period.* In addition to the counter readout, a recorder continuously displayed the rectified and filtered output of the 13-cps amplifier. Thus the source stability during the integration time could be monitored.

Background due to local interference was reduced to a negligible level by placing the electronics and the experimental apparatus inside a double Faraday cage.

\section{B. Electrostatic Method}

\section{Procedure}

The thermopile signal was first recorded without biasing the element with respect to its surroundings. Then, a de bias was applied and the signal again recorded. A comparison of these signals should indicate the loss caused by the photoejected electrons carrying away a portion of the incident radiant energy, assuming the bias voltage was sufficiently high and that no stray electrons or ions were being driven erroneously into the thermopile. Early results indicated some difficulty with the latter effect, probably due primarily to photoelectrons from the exit slit jaws. A positively biased collector electrode was installed on the monochromator side of the exit slit see (C in Fig. 4) in an attempt to collect these stray electrons before they were influenced by the retarding field of the thermopile.

The change in thermopile signal resulting from an increase in the collector potential is indicated in Fig. 5, for three thermopile bias potentials, at $\lambda 584 \AA$. It can be seen that a collector potential of $+45 \mathrm{~V}$ with respect to the grounded monochromator was sufficient to eliminate the effects of the unwanted electrons and ions. Furthermore, it is apparent that a thermopile bias voltage of $+45 \mathrm{~V}$ with respect to the grounded surroundings is sufficient to retard essentially all of the ejected electrons. For the purpose of obtaining the data below, the thermopile retarding potential and the collector ring potential were set at $+67 \mathrm{~V}$.

\section{Results}

The change in the thermopile signal caused by switching on and off the electrostatic retarding field is shown in Fig. 6 for a number of wavelengths. The data were obtained by allowing the scaler to integrate the signal for $10 \mathrm{sec}$ without a retarding field, then for $10 \mathrm{sec}$ with a retarding field. The procedure was continued for 10 or 12 cycles allowing a good check on zero drifts and light source fluctuations.

* The use of the voltage-to-frequency converter and scaler system was inspired by a visit to the laboratory of $\mathrm{H}$. E. Bennett who has been using this method for infrared signal detection. 


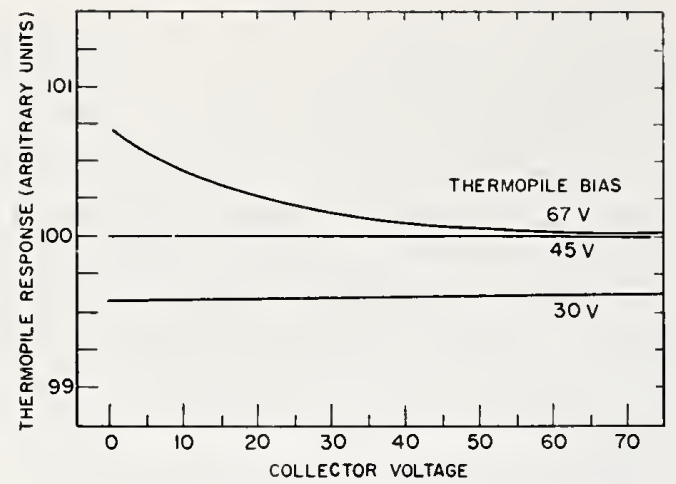

Fig. 5. Thermopile response vs collector potential for several thermopile bias voltages. The bias voltage provides the electrostatic field to return photoejected electrons to the thermopile. The collector ring potential (see Fig. 4) is required to prevent stray electrons or ions from reaching the thermopile.

\section{Magnetic Method}

The maximum orbital radius of electrons photoejected from the thermopile surface in the presence of a magnetic field is readily calculated from the following equation:

$$
r=\frac{\sqrt{2 m E}}{B e},
$$

where $m, e$ are the mass and charge of the electron, $E$ is the electron energy, and $B$ the magnetic field strength. For illustration let us consider electrons leaving with $40-\mathrm{eV}$ energy, remembering, however, that most photoejected electrons are expected to have energies well below that of the incident photons due to scattering effects in the solid. In the case of $40-\mathrm{eV}$ electrons a magnetic field strength of $1000 \mathrm{G}$ yields a maximum orbital radius of $0.2 \mathrm{~mm}$.

The maximum radius orbit is achieved when the velocity of the ejected electron is normal to the magnetic field. If the thermopile surface is parallel to the field, and one assumes an electron leaving the surface with a velocity oriented $1^{\circ}$ from the direction of the field of strength $1000 \mathrm{G}$ with an energy of $40 \mathrm{eV}$ then the electron will cycloid back to the surface in approximately a $1.3-\mathrm{mm}$ vertical displacement. One can conclude from these considerations that a negligible number of photoejected electrons would be expected to escape from a $1-\mathrm{mm} \times 8-\mathrm{mm}$ thermopile immersed in a 1000-G magnetic field for photon energies up to $100 \mathrm{eV}$. Furthermore, electrons and ions from the monochromator would have little chance of striking the thermopile.

In the present experiment, a permanent magnet with a gap field of $1600 \mathrm{G}$ was used. The housing and exit slit (as well as the thermopile) were constructed of nonmagnetic materials. The thermopile signal was recorded with and without the magnet positioned as shown in Fig. 4. The data taking procedure was the same as that described for the electrostatic field method in Sec. III.A,B. The change in the thermopile signal expressed in percent is shown for various wavelengths in Fig. 6, where the data compare favorably with the results of the electrostatic field method. The solid curve shown in Fig. 6 is merely a guide through the experimental points.

The enhancement of the thermopile signal in the presence of the magnetic field was also checked in the visible spectral region as a safeguard against extraneous effects. Application of the magnetic field caused no change in signal when visible light was incident upon the thermopile.

\section{Surface Sensitivity Maps and Time-Constant Considerations}

If the time constant of the thermopile is not short compared with the chopping period, a drop in over-all ac sensitivity will occur. A change of the time constant with wavelength would, in this case, cause variations in ac sensitivity such as have been previously noted."1 A thermopile calibrated in the visible would, therefore, give erroneous results if used in the far ultraviolet. A dc method would circumvent this difficulty, but the ac technique has a great advantage in discriminating against unwanted signals.

If the experimental arrangement used for the calibration in the visible is identical to that of the far ultraviolet application, it would seem that a wavelength dependence of the time constant could only be effected by a change in the absorption process, such as a variation in the penetration of the radiation in the absorbing black. Without dwelling on the possible mechanism for such a time-constant variation, it is of practical importance to determine the extent to which it exists in a typical far ultraviolet thermopile. In this section we report on measurements which yield a map of the sensitivity variation over the surface of the thermopile, using both visible and far ultraviolet incident radiation, and both ac and de methods. Any difference between the ac and the dc maps would indicate the extent to which the time constant of the thermopile was not negligibly short with respect to the chopping period, and any variation of the ac maps with wavelength

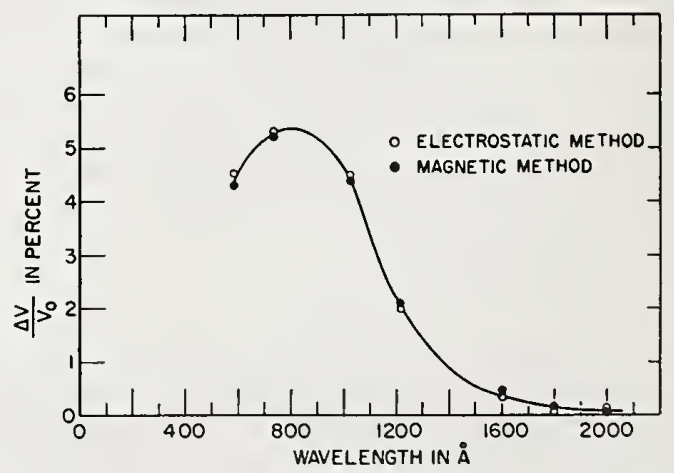

Fig. 6. The percent increase in thermopile signal achieved by returning the photoejected electrons to the detector, as a function of wavelength in the far ultraviolet. The results of the electrostatic method and the magnetic method are compared. The solid line merely connects the experimental points. 


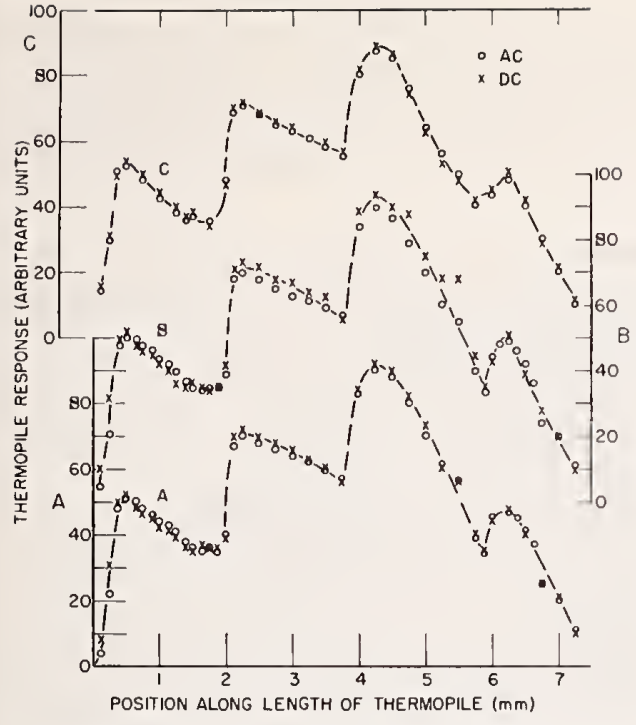

Fig. 7. One-dimensional sensitivity maps showing the variation of the thermopile response as a function of displacement along the long dimension of the thermopile. The data obtained at three wavelengths are shown, and the results using de detection are compared with those using ac synchronous detection and 13-cps chopping. The data obtained at the three wavelengths ( $\mathrm{A}-$ $584 \AA ; B-1606 \AA ; C-5461 \AA$ ) have been given arbitrary vertical displacements. The results of the ac method are arbitrarily normalized to the results of the dc method for each wavelength.

would be a danger sign in the ac use of the thermopile outside the wavelength range of the calibration.

\section{A. Experimental Procedure}

The monochromator and light source were described in Sec. II, and the thermopile in Sec. III. For these measurements the thermopile was mounted $8 \mathrm{~mm}$ from the exit slit on a platform which could be translated perpendicular to the exit beam. The exit beam was limited so that its cross section at the thermopile was $0.3 \mathrm{~mm} \times 8.0 \mathrm{~mm}$, with the long dimension vertical. The thermopile was oriented in the beam with its long dimension horizontal, parallel to the translational direction of the table on which it was mounted. The thermopile was then scanned slowly through the beam and a one-dimensional map of the surface sensitivity was determined at different wavelengths.

The output of the thermopile was measured by two methods. In the first, an unchopped light source was used, and the thermopile signal was amplified with a dc amplifier and displayed on a recorder. In the second, the light source was chopped at $13 \mathrm{cps}$ and the signal amplified using a broad-band 13-cps amplifier. The output of the amplifier was rectified synchronously with the chopping frequency, filtered, and displayed on a recorder.

\section{B. Results}

The one-dimensional sensitivity maps of the thermopile which were obtained at $\lambda$ 's $5461 \AA, 1606 \AA$, and 584
$\AA$ are shown in Fig. 7. Data obtained by both the ac and de methods are indicated. The ac and de data obtained at each wavelength are arbitrarily normalized since only the variation in sensitivity along the thermopile is of interest. The curves for three wavelengths have been given an arbitrary vertical displacement in Fig. 7. It can be seen that the ac and dc sensitivity maps for each wavelength are essentially identical, the deviations being within the combined reproducibility of these measurements. The curves show, incidentally, that the thermopile deviates considerably from uniform sensitivity along its length. The four segments of the thermopile can be easily recognized, and it is apparent that they are not all equally effective. Furthermore, it is obvious (and expected) that the thermal emf generated at the two ends of each extended junction are not equal in magnitude, thus the sensitivity of each segment is a ramp function. Since the ac and de sensitivity maps are essentially identical at all wavelengths studied (several intermediate wavelengths were examined), it would appear that the time constant for this thermopile is, effectively, short compared to the chopping frequency.

In Fig. 8 the ac sensitivity map for the three wavelengths $5461 \AA, 1606 \AA$, and $584 \AA$ are plotted on a single coordinate system. Again, an arbitrary normalization has been used. It is apparent from the figure that each of these incident wavelengths produces a nearly identical sensitivity map. From this result we conclude that any variation in the time constant of this thermopile with wavelength is of negligible practical importance.

Sensitivity maps were also obtained with the thermopile rotated through $90^{\circ}$ so that its long dimension was parallel to the exit slit. These relatively lower resolution scans produced rather rounded rectangular distribution functions which showed no differences under ac and de operation and no wavelength dependence.

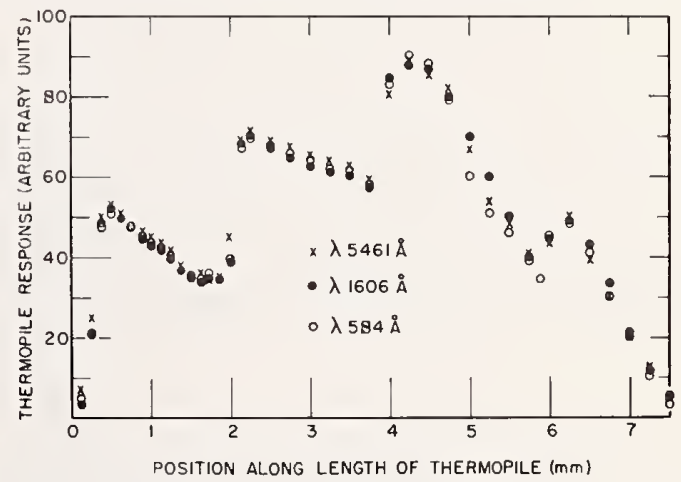

Fig. 8. One-dimensional sensitivity maps showing the variation of the thermopile response as a function of displacement along the long dimension of the thermopile for three different wavelengths. Only the results from the ac detection method (13-cps chopping) are shown. The scans for the different wavelengths were arbitrarily normalized at one point along the thermopile. 


\section{Conclusions}

The foregoing sections have discussed three aspects of the performance of a thermopile which are of importance for radiometric applications in the far ultraviolet. It has been shown that the reflective scattering from thin gold-black-on-gold foil, while not insignificant, is similar in the visible and far ultraviolet. Therefore, if the thermopile is calibrated in the visible and used in the far ultraviolet, this phenomena should not require a correction, if radiometric accuracy in the $1 \%$ range is sufficient. It was further determined that this thermopile has a surface sensitivity variation and a time constant which is independent of wavelength in the regions of interest. It would appear, in fact, that the only significant correction which would be required for this thermopile is due to the photoelectric effect, which causes a negligible loss in the visible region, but as high as a $5 \%$ loss in the far ultraviolet.

These results apply only to the thermopile which was used in this study, and a generalization of them is not necessarily justified. However, these results increase our confidence in the validity of this method of radiometry for the far ultraviolet. We feel that if these corrections are carefully established for an optimum thermopile, a radiometric detector standard will have been developed for the far ultraviolet which will be reliable in the few percent range. If this detector compared favorably with the rare gas ionization chamber detectors $^{15}$ in their regions of effectiveness, and with the source standards being developed at $\mathrm{NBS}^{16}$ in the region above $\lambda 2000 \AA$, we feel it would be justifiable to use such a thermopile to calibrate more sensitive detectors throughout the far ultraviolet.

The work reported in this paper was supported by the National Aeronautics and Space Administration.

\section{References}

1. N. Wainfan, W. C. Walker, and G. L. Weissler, J. Appl. Phys. 24, 1318 (1953).

2. K. Watanabe, F. F. Marmo, and E. C. Y. Inn, Phys. Rev. 91, 1155 (1953).

3. N. Wainfan, E. C. Walker, and G. L. Weissler, Phys. Rev. 99, 542 (1955).

4. C. Kenty, Phys. Rev. 44, 891 (1933).

5. R. Baker, J. Opt. Soc. Am. 28, 55 (1938).

6. H. E. Hinteregger and K. Watanabe, J. Opt. Soc. Am. 43, 604 (1953).

7. W. C. Walker, N. Wainfan, and G. L. Weissler, J. Appl. Phys. 26, 1366 (1955).

8. D. M. Packer and C. Lock, J. Opt. Soc. Am. 41, 699 (1951).

9. F. S. Johnson, K. Watanabe, and R. Tousey, J. Opt. Soc. Am. 41, 702 (1951).

10. K. Watanabe and E. C. Y. Inn, J. Opt. Soc. Am. 43, 32 (1953).

11. R. Stair, W. Schneider, W. Waters, and J. Jackson, Appl. Opt. 4, 703 (1965).

12. A. Smith, thesis, University of Rochester, 1961.

13. L. R. Canfield, private communication.

14. R. P. Madden and L. R. Canfield, J. Opt. Soc. Am. 51, 838 (1961).

15. J. A. R. Samson, J. Opt. Soc. Am. 54, 6 (1964).

16. H. J. Kostkowski, D. E. Erminy, and A. T. Hattenberg, J. Opt. Soc. Am. 54, 1386 (1964).

Reprinted from:

APPLIED OPTICS

Vol. 4, No. 12, December 1965 


\section{A New Radiometric Equation and Its Application}

\section{Henry J. Kostkowski}

National Bureau of Standards, Washington, D. C. 20234.

Received 4 August 1966.

A simple equation has been found which gives, at any wavelength, the ratio of the spectral radiances of two arbitrary sources when their spectral radiances at some particular wavelength are equal. The new equation does not provide any information not available from computations using the Planck equation and emissivity factors. Moreover its derivation requires the use of Wien's rather than Planck's radiation equation. Nevertheless, because of its simplicity and because it does not involve the temperature explicitly, the equation has been useful in various radiometric and thermometric problems. This letter presents the derivation and various applications of this equation.

Let the temperature and spectral emissivity of the radiating surface and the spectral transmittance $r$ ay window surrounding it be denoted by $T_{1}, \epsilon_{1}(\lambda), \tau_{1}(\lambda)$ and $1_{2}, \epsilon_{2}(\lambda), \tau_{2}(\lambda)$, respectively for the two sources. Using Wien's radiation equation, the equality of the spectral radiances at $\lambda_{0}$ and their ratio at $\lambda$ are

$$
\begin{gathered}
\epsilon_{1}\left(\lambda_{0}\right) \tau_{1}\left(\lambda_{0}\right) O_{1} C_{0}^{-5} e^{-C_{2} / \lambda_{0} T_{1}}=\epsilon_{2}\left(\lambda_{0}\right) \tau_{2}\left(\lambda_{0}\right) C_{1} \lambda_{0}{ }^{-5} e^{-C_{2} / \lambda_{0} T_{2}} ; \\
\frac{L_{2}(\lambda)}{L_{1}(\lambda)}=\frac{\epsilon_{2}(\lambda) \tau_{2}(\lambda) C_{1} \lambda^{-5} e^{-C_{2} / \lambda T_{2}}}{\epsilon_{1}(\lambda) \tau_{1}(\lambda) C_{1} \lambda^{-5} e^{-C_{2} / \lambda T_{1}}}
\end{gathered}
$$

Equation (1) can be reduced to

$$
C_{2} / T_{1}=\left(C_{2} / T_{2}\right)+\lambda_{0} \ln \left[\epsilon_{1}\left(\lambda_{0}\right) \tau_{1}\left(\lambda_{0}\right) / \epsilon_{2}\left(\lambda_{0}\right) \tau_{2}\left(\lambda_{0}\right)\right]
$$

Substituting Eq. (3) into Eq. (2) and rearranging terms results in the desired equation:

$$
\frac{L_{2}(\lambda)}{L_{1}(\lambda)}=\frac{\left[\epsilon_{1}\left(\lambda_{0}\right) \tau_{1}\left(\lambda_{0}\right) / \epsilon_{2}\left(\lambda_{0}\right) \tau_{2}\left(\lambda_{0}\right)\right]^{\lambda_{0} / \lambda}}{\left[\epsilon_{1}(\lambda) \tau_{1}(\lambda) / \epsilon_{2}(\lambda) \tau_{2}(\lambda)\right]}
$$

The usefulness of Eq. (4a) results from the fact that the temperature does not occur explicitly in the equation and the implicit dependence is usually not great. In addition, when $\lambda$ is near $\lambda_{0}$, the ratio of the spectral radiances is essentially a function of the ratio of the spectral emissivities and transmittances at the two wavelengths for each of the sources. Such ratios are generally more accurately known than the emissivities or transmittances themselves.

Equation (4a) has been used in a number of applications, a few of which the author believes are worth describing here. In these applications, Eq. (4a) has been simplified further as a result of using a specialized source or sources. These simplified equations and their sources are listed below:

source 2 is a blackbody without a window

$$
L_{b}(\lambda) / L_{1}(\lambda)=\left[\left(\epsilon_{1}\left(\lambda_{0}\right) \tau_{1}\left(\lambda_{0}\right)\right]^{\lambda_{0} / \lambda} / \epsilon_{1}(\lambda) \tau_{1}(\lambda) ;\right.
$$

source 2 is a blackbody without a window and source 1 is a graybody with a window having a constant spectral transmittance:

$$
L_{b}(\lambda) / L_{\theta}(\lambda)=\left(\epsilon_{g} \tau_{\theta}\right)^{\left(\lambda_{0}-\lambda\right) / \lambda}
$$

sources 1 and 2 are graybodies without windows:

$$
L_{\theta_{2}}(\lambda) / L_{\theta_{1}}(\lambda)=\left(\epsilon_{1} / \epsilon_{2}\right)^{\left(\lambda_{0}-\lambda\right) / \lambda} .
$$

Equation (4b) has been used to calculate the spectral radiance of a tungsten strip lamp relative to a blackbody of temperature $T$ when the brightness temperature of the lamp at $6500 \AA$ has been determined to be $T$ with an optical pyrometer. Differences in the spectral radiance ratios when using DeVos'1 and Larrabee's ${ }^{2}$ emissivities increase monotonically from $6500 \AA$ to $3100 \AA$ and to $8000 \AA$ where the differences are about $5 \%$ and $2 \%$, respectively.

Equation (4b) has been particularly useful in extrapolating the spectral radiance of a tungsten strip lamp a few hundred angstroms from a spectral calibration point. The difference between the radiances using DeVos' and Larrabee's emissivities anywhere in the visible and near uv region is about $1 / 4 \%, 1 / 2 \%$, and $1 \%$ for a $100 \AA, 200 \AA$, and $400 \AA$ extrapolation, respectively.

Equation (4b) can also be used to check the quality of a cavity in a wavelength range where one suspects the emissivity to depart from unity in a range of $50 \AA$ or $100 \AA$ owing to some continuum absorption. This is accomplished by adjusting the spectral radiance of the strip lamp and the cavity to be equal just outside this region, where one is confident of an emissivity of one, and experimentally determining the ratio at a wavelength in question and comparing it to a calculated ratio using Eq. (4b).

The obvious application of Eq. (4c) is in calculating the correction to the spectral radiance of a blackbody having a constant spectral transmittance window when its brightness temperature $T_{B}$ is determined with an optical pyrometer at $6500 \AA$. Such a source can be considered a graybody and

$$
L_{o}(\lambda)=L_{b}\left(\lambda, T_{B}\right) /\left(\tau_{g}\right)^{\left(\lambda_{0}-\lambda\right) / \lambda} .
$$

If the spectral transmittance of the window is not constant, Eq. (4b) can be used.

Eq. (4d) has been used for determining the partial reflectivity of the wall opposite a cavity opening and therefore the emissivity of the cavity. To a first approximation, the spectral emissivity of a cavity having only one opening and having walls of uniform temperature is $\epsilon_{\lambda}=1-r_{\lambda} \Omega$ (Ref. 3), where $\Omega$ is the solid angle originating at the wall target and determined by the cavity opening, and $r_{\lambda}$ is the spectral partial reflectivity of the wall target associated with the angle between the wall normal and line of sight of the cavity. In many cases, to a useful approximation, such a cavity can be considered to be a graybody so that the emissivity and partial reflectivities are independent of wavelength. By adjusting two cavities, identical except for the solid angle $\Omega$, so that $L g_{1}\left(\lambda_{0}\right)=L g_{2}\left(\lambda_{0}\right)$ and then determining $L g_{2}(\lambda) / L g_{1}(\lambda)$, the partial reflectivity $r$ can be obtained from Eq. (4d), which becomes

$$
L g_{2}(\lambda) / L g_{1}(\lambda)=\left[\left(1-r \Omega_{1}\right) /\left(1-r \Omega_{2}\right)^{\left(\lambda_{0}-\lambda\right) / \lambda} .\right.
$$

One cavity can be used if it is possible to modify the size of its opening and therefore solid angle $\Omega$. In this case, it is convenient to use a tungsten strip lamp as a reference source to compare the two cavities.

The various applications of Eq. (4a) described in this letter have been useful in precise radiometry and, in addition, have given a better qualitative understanding of the radiation from nonblackbody sources.

\section{References}

1. J. C. DeVos, Physica 20, 690 (1954).

2. R. D. Larrabee, J. Opt. Soc. Am. 49, 619 (1959).

3. J. C. DeVos, Physica 20,669 (1954). 


\title{
Comparison of an lonization Chamber and a Thermopile as Absolute Detectors in the Extreme Ultraviolet
}

\author{
L. R. Canfield, R. G. Johnson, K. Codling, and R. P. Madden
}

\begin{abstract}
A comparison has been made between a calibrated thermopile and an argon ionization chamber as absolute detectors of radiant flux of wavelengths $584 \AA$ and $735 \AA$. Corrections were applied to the data in order to account for the absorption due to gases in the monochromator, the energy carried away from the thermopile by photoejected electrons, nonuniformity of response across the surface of the thermopile, and uneven illumination of the thermopile by the flux from the monochromator. The two detectors were found to agree within the $3 \%$ estimated probable error in the measurements.
\end{abstract}

\section{Introduction}

Samson ${ }^{1}$ has proposed the use of the photoionization of rare gases for absolute detection of radiant flux in the wavelength region below $1022 \AA$. He demonstrated that, under prescribed conditions, the photoionization yields of $\mathrm{Ne}, \mathrm{Ar}, \mathrm{Kr}$, and $\mathrm{Xe}$ are the same to $\pm 5 \%$. If this common value is unity, which is obviously the most probable, the ion current is a direct measure of photon flux. Matsunaga et al. ${ }^{2}$ have recently shown this to be the case for Xe from $860 \AA$ to $1022 \AA$. Such an absolute detector is particularly attractive in a spectral region where the energy typically available approaches the noise level of the best thermopiles.

The problems inherent in the use of a thermopile for absolute intensity measurements in the vacuum uv have been discussed in a previous paper, ${ }^{3}$ where three possible sources of error were considered. The first was the assumption that the thermopile absorbs the same percentage of incident photons in the vacuum uv as in the region where it was calibrated. The second was the possibility that energy is lost via ejected photoelectrons at short wavelengths. And the third was the possible variation of ac sensitivity that may occur if the time constant of the thermopile has a wavelength dependence.

It was shown that of these possible problems, only the photoelectric energy loss need be accounted for when using a thermopile with a properly selected response time and absorbing coating. Thus, it has become possible to conduct a careful experimental comparison of two entirely independent methods of measuring absolute intensity in the extreme uv. Such a comparison,

All authors were with the National Bureau of Standards, Washington, D.C. 20234, when this work was done; K. Codling is now with the University of Reading, Berkshire, U.K.

Received 7 July 1967. using the relatively intense and stable resonance lines of neutral $\mathrm{Ne}$ and $\mathrm{He}$, is the subject of this work.

\section{Experimental Arrangement}

Vacuum uv energy of $584-\AA$ and $735-\AA$ wavelength was obtained from a 1-m normal incidence, grating monochromator, equipped with a windowless de cold cathode light source. A 13-Hz chopper, located between the light source and the entrance slit, was used during thermopile measurements to interrupt the light entering the monochromator. Attached to the exit arm of the monochromator was a vacuum chamber which housed the ion chamber and thermopile. The arrangement is represented schematically in Fig. 1.

The Reeder thermopile used was of the extended junction variety, employing four such junctions to give a sensitive area $1 \mathrm{~mm} \times 8 \mathrm{~mm}$. It was essentially identical to the one used in the earlier study of thermopiles. ${ }^{3}$ To ensure the use of a specific portion of the thermopile and of the $f / 25$ light beam emerging from the monochromator, a rectangular mask with an opening about $\frac{1}{2}$ the area of the thermopile was fabricated. A three point mounting, designed kinematically for accurate relocation, positioned the mask relative to the light beam from the monochromator and a second similar arrangement located it relative to the surface of the thermopile, about $0.5 \mathrm{~mm}$ from its surface. The locations could be effected individually or simultaneously. The thermopile signal was amplified by a $13-\mathrm{Hz}$ amplifier, rectified synchronously with the chopping frequency and recorded on a potentiometric recorder. A carbon filament total irradiance standard lamp* was used to calibrate the thermopile. For this calibration, the rectangular mask was fastened in its location relative to the

* This lamp, calibrated at NBS, has a stated accuracy of $2 \%$. 


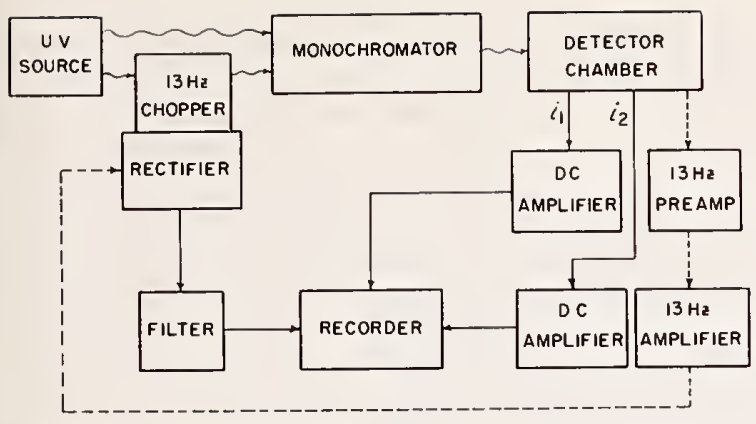

Fig. 1. Schematic diagram of the apparatus used to compare the ionization chamber and thermopile as absolute detectors.
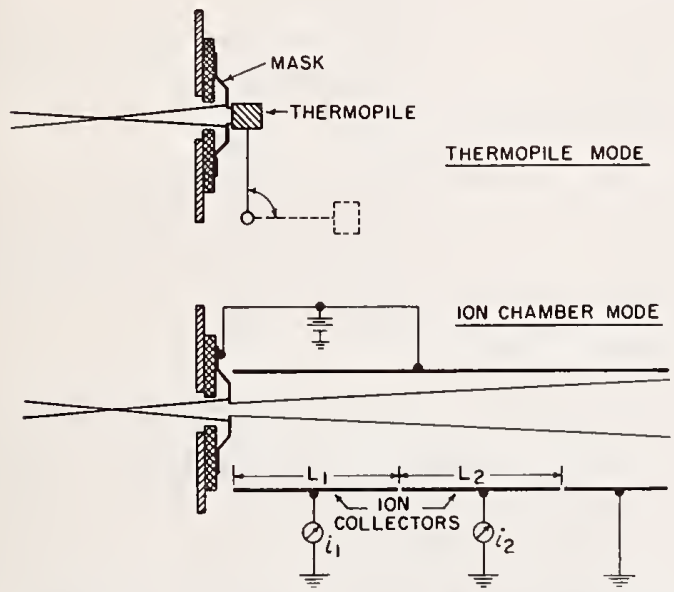

Fig .2. Schematic of detector chamber, showing thermopile and ion chamber modes of operation.

thermopile surface and the assembly oriented so as to face the standard lamp through a calibrated silica window on the rear of the vaccum chamber. The standard lamp was chopped in a manner carefully matched to that used on the monochromator, and the entire detection system was calibrated as a unit.

Measurements of the flux from the monochromator were taken with the mask located in the exit beam and the thermopile housing engaged with the mask. The thermopile housing could be disengaged from the mask following a measurement, allowing the masked beam to pass unobstructed into the ion chamber for a comparative measurement (see Fig. 2).

The ion chamber used was a double ion chamber similar to that described by Samson ${ }^{1}$; this is also represented in Fig. 2. The axis of the light beam was 0.8 $\mathrm{cm}$ from the positive plate which, with the mask, was $18 \mathrm{~V}$ positive with respect to the collector plates. The length $L$ of each collector plate was $10.2 \mathrm{~cm}$, and the forward edge of the \#1 plate was positioned in the plane of the mask. Argon was used as the absorbing gas, with the pressure appropriate to give a factor of two to three between the currents from the collector plates.

\section{Procedure}

Data to enable a comparison between thermopile and ion chamber to be made were taken in the following manner. The thermopile housing was engaged with the mask and the resulting signal recorded by the 13$\mathrm{Hz}$ detection system (with the source chopper operating). Then the thermopile was removed from the mask (by an external motion) and lowered clear of the ion chamber plates. Argon was allowed to flow into the chamber to an appropriate pressure, the chopper was stopped, and measurements of the collector currents were recorded. A check for possible light source drift was then made by returning to the thermopile mode.

Measurements of this nature were made at $584 \AA$ and $735 \AA$ with argon as the ion chamber gas. Several argon pressures (within the range giving a factor of two to three in collector currents) and light source currents were used for each set of measurements.

The following corrections were then applied to the recorded data.

(a) The measured sensitivity of the thermopile was corrected for the error caused by the nonuniformity of illumination from the monochromator and the variations in sensitivity across the surface of the thermopile. ${ }^{3}$ The thermopile used was scanned (with the mask in place) behind a narrow slit mounted in the beam perpendicular to the long dimension of the thermopile. The resulting sensitivity profile over the length of the thermopile, shown in Fig. 3, exhibited irregularities similar to those previously observed. ${ }^{3}$ The slit was then rotated through $90^{\circ}$ and the thermopile surface scanned across its smaller dimension. The sensitivity was found to be essentially uniform.

The intensity distribution of the beam from the monochromator was also studied, in this case by scanning the auxiliary slit vertically through the beam using the ion chamber as the detector. The distribution shown in Fig. 4, for $584 \AA$, was obtained after careful adjustment of the instrument and light source.

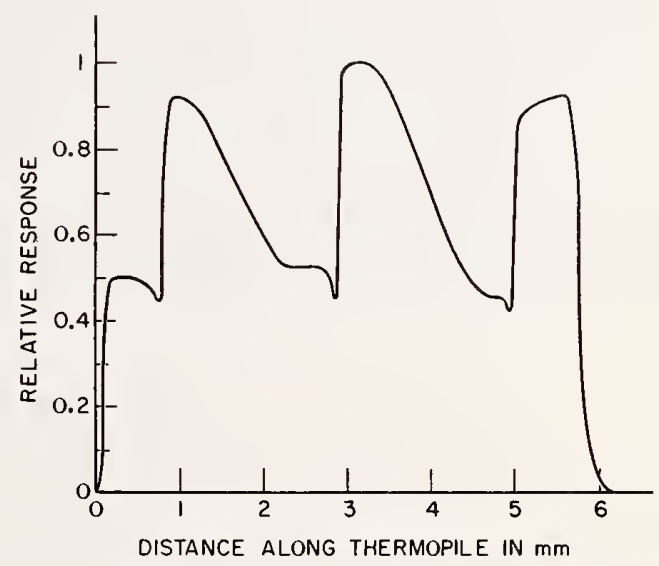

Fig. 3. Sensitivity profile of the thermopile when kinematically mounted to the mask and scanned lengthwise across a narrow light beam. 


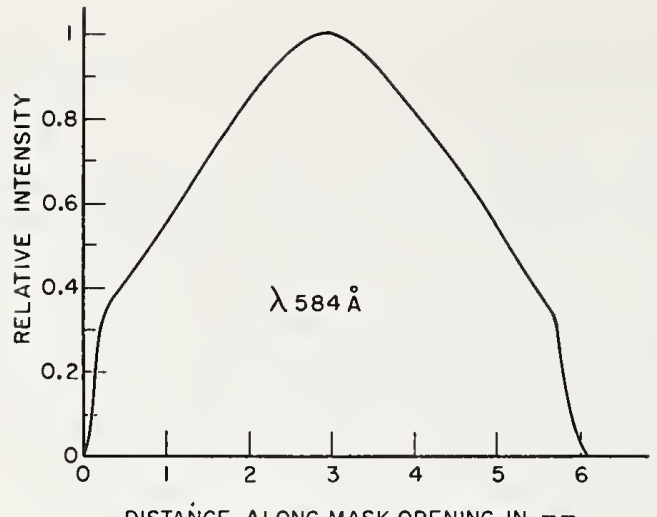

Fig. 4. Vertical intensity distribution of the 584- $\AA$ line. The ionization chamber was used as the detector and a horizontal slit scanned at the mask.

Table 1. Comparison of Absolute Flux Levels Measured by Ion Chamber and Thermopile

\begin{tabular}{cc}
\hline & $2\left(\mathrm{~N}_{1}-N_{2}\right) /\left(N_{1}+N_{2}\right)$ \\
\hline $584 \AA$ & $-0.0052 \pm 0.0214^{a}$ \\
$735 \AA$ & $0.0044 \pm 0.0132^{a}$ \\
\hline
\end{tabular}

- This error represents the standard deviation for a set of ten independent comparisons.

The thermopile sensitivity obtained from the standard lamp calibration represented an average over the entire illuminated area. This value served to normalize the relative sensitivity curve of Fig. 3 , which was then folded with the relative intensity curve of Fig. 4 to yield the average sensitivity of the thermopile when illuminated with flux distributed according to Fig. 4. The sensitivity, obtained in this way, differed by about $3 \%$ from that which would have been obtained if either distribution had been assumed uniform.

(b) A correction was made to the indicated thermopile signal for the energy lost through photoemission.

It has been shown ${ }^{3}$ that the energy carried away by photoejected electrons from the gold black absorbing surface of this type of thermopile is significant in the far uv. A correction for this loss was made according to the results previously obtained. The correction amounted to $4.4 \%$ at $584 \AA$ and $5.2 \%$ at $735 \AA$.

(c) A further correction was included for the reduction of flux entering the ion chamber due to absorption by argon in the monochromator.
Although the leak at the windowless connection between experimental chamber and monochromator was restricted to the aperture of the optical mask, a sufficient quantity of argon from the ion chamber was present in the monochromator to reduce noticeably the flux reaching the mask from the level seen by the thermopile. The amount of this self-absorption was measured as a function of monochromator pressure, using a phosphored photomultiplier in back of the mask. Thus, by monitoring the pressure during an ion chamber measurement, the appropriate correction for this effect could be applied. The magnitude of this correction was approximately $4 \%$.

\section{Results}

The reduction of data from the ion chamber involves only the use of the measured currents $i_{1}$ and $i_{2}$ (since $L_{1}=L_{2}$ and the photoelectric yield was assumed unity). In particular, the number of photons per second entering the ion chamber is given by $i_{1}{ }^{2} / e\left(i_{1}-i_{2}\right)$.

This rate can be compared with that predicted by the thermopile after the above mentioned corrections have been applied. Let the corrected rates predicted by the thermopile and the ion chamber be $N_{1}$ and $N_{2}$, respectively. The experimental agreement between the two is indicated in Table $\mathrm{I}$.

The table indicates that the agreement between the absolute radiant energy determined by the thermopile and the ionization chamber is well within the bounds of the experimental repeatability. However, systematic errors must also be evaluated, such as the meter and geometrical accuracies in the calibration procedure for the thermopile, the probable error in the photoelectric and sensitivity corrections, the geometrical accuracy of the ion chamber, and the repeatability of the pressure meter on the monochromator. A consideration of these possible sources or error yields a most probable systematic error in the above comparison of $3 \%$. Thus, these results show that within this uncertainty, the ionization chamber and the thermopile, with proper corrections, determine the same absolute value of radiant energy at $584 \AA$ and $735 \AA$.

This work was supported by the National Aeronautics and Space Administration.

\section{References}

1. J. A. R. Samson, J. Opt. Soc. Am. 54, 6 (1964).

2. F. J. Matsunaga, R. S. Jackson, and K. Watanabe, J. Quant. Spectry. Radiative Transfer 5, 329 (1965).

3. R. G. Johnston and R. P. Madden, Appl. Opt. 4, 1574 (1965). 


\section{Emissivity Standards}

Papers

2.1. On Kirchhoff's Law and Its Generalized Application to Absorption and Emission by Cavities.

Francis J. Kelly, J. Res. Nat. Bur. Stand. (U.S.) 69B, No. 3, 165-171 (July-Sept. 1965).

Key words: Cavity absorption; cavity emission; Kirchhoff's Law --------------------------_-------------------

2.2. A Note on the Numerical Evaluation of Thermal Radiation.

Characteristics of Diffuse Cylindrical and Conical Cavities. B. A. Peavy, J. Res. Nat. Bur. Stand. (U.S.) 70C, No. 2, 139148 (Apr.-June 1966).

Key words: Cavities ; conical ; cylindrical ; emissivity ; nonisothermal; isothermal; radiation

2.3. An Equation for the Local Thermal Emissivity at the Vertex of A Diffuse Conical or V-Groove Cavity.

Francis J. Kelly, Appl. Opt. 5, No. 6, 925-927 (June 1966).

Key words: Angle factor; blackbody radiation; conical cavity ; emissivity ; radiant heat transfer ; V-groove cavity -.---.

2.4. The Apparent Thermal Radiation Properties of an Isothermal V-Groove with Specularly Reflecting Walls.

Richard B. Zipin, J. Res. Nat. Bur. Stand. (U.S.) 70C, No. 4, 275-280 (Oct.-Dec. 1966).

Key words: Cavity; conical cavity; emissivity; radiation

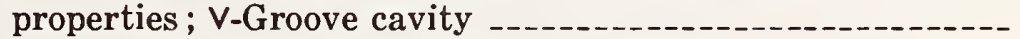

2.5. An Approach to Thermal Emittance Standards.

J. C. Richmond, W. N. Harrison, and F. J. Shorten, Symposium on Measurement of Thermal Radiation Properties, Dayton, Ohio, September 5-7, 1962, pp. 403-423 (NASA SP-31, Nat. Aeron. and Space Admin., Washington, D.C., 1963).

Key words: Spectral emittance standards; spectral emittance measurement; total emittance computation

2.6. A Test of Analytical Expressions for the Thermal Emissivity of Shallow Cylindrical Cavities.

F. J. Kelly, and Dwight G. Moore, Appl. Opt. 4, No. 1, 31-40 (Jan. 1965).

Key words: Analysis; emittance; paper lined cavity ; shallow cavity reflectance; specular component 


\section{Emissivity Standards - Continued}

Papers

2.7. Investigation of Shallow Reference Cavities for High-Temperature Emittance Measurements.

Dwight G. Moore, Symposium on Measurement of Thermal Radiation Properties of Solids, Dayton, Ohio, September 5-7, 1962, pp. 515-526 (NASA SP-31, Nat. Aeron. and Space Admin., Washington, D.C., 1963).

Key words: Alumina ; emittance of oxidized Inconel ; graphite; high-temperature emittance measurement; oxidized

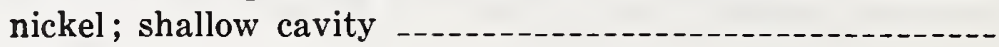

2.8. Instructions for Handling Emittance Standards.

Office of Standard Reference Materials, 1 p. (1965).

Key words: Emittance; standards \& handling

2.9. Certificate of Normal Spectral Emittance, Standard Reference Materials 1402 to 1409, Platinum-13 Percent Rhodium Alloy. Office of Standard Reference Materials, $7 \mathrm{pp}$. (1966).

Key words: Emittance; Kanthal; standard

2.10. Certificate of Normal Spectral Emittance, Standard Reference Materials 1420-1428, Oxidized Kanthal.

Office of Standard Reference Materials, 4 pp. (1965).

Key words: Emittance; Kanthal; standard

2.11. Certificate of Normal Spectral Emittance, Standard Reference Materials 1440 to 1447, Oxidized Inconel.

Office of Standard Reference Materials, 4 pp. (1965).

Key words: Emittance; Inconel; standard 


\title{
On Kirchhoff's Law and Its Generalized Application to Absorption and Emission by Cavities*
}

\author{
Francis J. Kelly
}

(March 15, 1965)

\begin{abstract}
Several authors have made the assumption that Kirchhoff's Law holds for the apparent local spectral emittance and apparent local spectral absorptance of any point on the interior surface of a cavity. The correctness of this assumption is demonstrated under certain general conditions, and it practical application to the calculation of the total flux absorbed by a cavity or spacecraft is discussed. A further application to the case of a nonisothermal cavity or spacecraft is derived. By this derivation an easy method for determining the total flux emitted from such a nonisothermal cavity is found when the distribution of the apparent local spectral emittance of the isothermal cavity is known. The economy and versatility of this method is shown by the calculation of the total flux emitted from a nonisothermal cylindrical cavity for several arbitrary cases of temperature distribution on the interior surface of the cavity. Finally, the integral equation for a diffuse cavity whose wall emittance varies with position on the wall is transformed to an equation having a symmetric kernel.
\end{abstract}

\section{Introduction}

Kirchhoff's Law states that at a point on the surface of a thermal radiator at any temperature and wavelength, the spectral directional emittance is equal to the spectral absorptance for radiation incident from the same direction. ${ }^{1}$ This law, coupled with the law of conservation of energy, permits us to relate the emittance, absorptance, and reflectance at a point on the surface of an opaque thermal radiator by eq (1).

$$
\epsilon(\lambda)=\alpha(\lambda)=1-\rho(\lambda)
$$

This relation has been used by Wien and Lummer, Ribaud, Gouffé, Michaud, and Page (refs. $\left.[1-5]^{2}\right)$, to derive expressions for the emittance of a cavity or enclosure with a finite opening. In these derivations the implicit assumption seems to be made (without explicit proof) that the apparent local spectral emittance at a point within an isothermal cavity is equal to the apparent local spectral absorptance at that same point. ${ }^{3}$ Although it is not immediately obvious that Kirchhoff's Law can be thus extended to these "apparent local" quantities, the analysis presented in section 2 based on the assumptions and techniques of diffuse interreflection theory shows that it does hold. The proof is purposely made for the general case, so as to be applicable to opaque isothermal cavities of any shape with one or more openings. It thus applies to any spacecraft configuration as a special case of a cavity. The emittance of the wall material is not assumed to be uniform over the cavity walls, but the emittance instead is permitted to vary with position within the cavity. The proof, however, is limited to a cavity with diffusely reflecting and emitting walls. Although a proof for the more general case of a a cavity with nondiffusely reflecting and emitting walls is not presented here, such a proof might possibly be developed by an approach similar to that of C. von Fragstein [6].

\footnotetext{
*This paper was presented at the AlAA Second Aerospace Sciences Meeting, New York, N.Y., on January $27,1965 . \quad$ This work was done under the sponsorship of the Marshall Space Flight Center of NASA and of the Air Force Materials Laboratory, Research and Technology Divisions, Air Force Systems Command.

1 The spectral directional emittance is defined as the ratio of the flux per unit area, unit solid angle, and unit wavelength emitted at wavelength $\lambda$ in a particular direction $(\varphi, \theta)$ from a surface element to the radiant flux per unit area, unit solid angle, and unit wavelength emitted at wavelength $\lambda$ in the same direction $(\varphi, \theta)$ from the surface of a blackbody radiator at the same temperature.

${ }^{2}$ Figures in brackets indicate the literature references at the end of this paper.

"For the definitions of these "apparent local" quantities, see section 2.
} 
In section 3 the equations developed in section 2 are discussed, and they are shown to have a practical application to the problem of determining the radiant flux absorbed by a cavity. In section 4 a novel method is derived for determining the total flux emitted from a nonisothermal cavity, while in section 5 the method derived in section 4 is applied to some sample calculations. This is done by determining the heat flux from a cylindrical cavity for several different cases of temperature distribution within the cavity. Finally in the appendix the integral equation charac. teristic of a cavity with nonuniform wall emittance is transformed into an integral equation with a symmetric kernel.

\section{Proof of Kirchhoff's Law for a Diffuse Cavity}

First the, general reflecting geometry of a cavity will be established. An enclosure $C$ of arbitrary shape which has one or more openings is considered throughout. Each point on the interior surface of the enclosure is identified with a coordinate vector, $\mathbf{x}$ in a convenient frame of reference. The coordinate vector, $\mathbf{x}$ is used here because some quantities will be thought of as functions of position on the interior surface, or equivalently, as functions of $\mathbf{x}$. It is assumed that the enclosure's walls are opaque and that they reflect and emit diffusely. It is further assumed that the material and temperature of the interior wall may vary as a function of position on the wall surface (that is as a function of $\mathbf{x}$ ), but that the emittances of the various wall materials are independent of temperature. The spectral emittance, absorptance, and reflectance of the wall material at the point $\mathbf{x}$ are denoted by $\epsilon(\lambda, \mathbf{x}), \alpha(\lambda, \mathbf{x})$ and $\rho(\lambda, \mathbf{x})$ respectively. In accordance with Kirchhoff's Law the wall material's emittance, absorptance, and reflectance at each point $\mathbf{x}$ are related to one another according to (2)

$$
\boldsymbol{\epsilon}(\lambda, \mathbf{x})=\alpha(\lambda, \mathbf{x})=1-\rho(\lambda, \mathbf{x})
$$

It is assumed that (2) is valid whateyer the wavelength or angular distribution of flux falling on the surface. Thus all fluorescence and stimulated emission effects that might invalidate (2) are neglected.

Next, the presence of radiant flux within the cavity is considered. The symbol $B_{\lambda}(\mathbf{x})$ stands for the spectral radiosity or the radiant flux per unit area and unit wavelength leaving an element $d A(\mathbf{x})$ on the cavity interior. The symbol $E_{\lambda}(\mathbf{x})$ stands for the spectral irradiance or the radiant flux per unit area and unit wavelength incident on $d A(\mathbf{x})$. The radiosity is given by

$$
B_{\lambda}(\mathbf{x})=\epsilon(\lambda, \mathbf{x}) B_{\lambda, b}[T(\mathbf{x})]+[1-\epsilon(\lambda, \mathbf{x})] E_{\lambda}(\mathbf{x})
$$

where $B_{\lambda, b}[T(\mathbf{x})]$ stands for the flux per unit area and unit wavelength emitted from a blackbody radiator at temperature $T(\mathbf{x})$ and wavelength $\lambda$. It is convenient for the purposes of this paper to divide $E_{\lambda}(\mathbf{x})$ into two parts: $E_{\lambda, e}(\mathbf{x})$, the external spectral irradiance is the flux per unit area and unit wavelength incident on $\mathbf{x}$ directly from a source outside the cavity; $E_{\lambda, c}(\mathbf{x})$, the cavity spectral irradiance is the spectral flux per unit area and unit wavelength incident on $\mathbf{x}$ whose source is the radiosity distribution $B_{\lambda}\left(\mathbf{x}_{0}\right)$ at all points $\mathbf{x}_{0}$ within the cavity. $E_{\lambda}(\mathbf{x})$ is given by

$$
E_{\lambda}(\mathbf{x})=E_{\lambda, e}(\mathbf{x})+E_{\lambda, c}(\mathbf{x})
$$

The cavity irradiance at a point $\mathbf{x}, E_{\lambda, c}(\mathbf{x})$, is related to the radiosity, $B_{\lambda}\left(\mathbf{x}_{0}\right)$, of every point $\mathbf{x}_{\mathbf{0}}$ according to

$$
E_{\lambda, c}(\mathbf{x}) \doteq \int_{c} B_{\lambda}\left(\mathbf{x}_{\mathbf{0}}\right) K\left(\mathbf{x}_{\mathbf{0}}, \mathbf{x}\right) d A\left(\mathbf{x}_{\mathbf{0}}\right)
$$

where $K\left(\mathbf{x}_{0}, \mathbf{x}\right)$ is the diffuse angle factor or configuration factor which gives the irradiance at $\mathbf{x}$ 
caused by a unit area of a diffuse source located at $\mathbf{x}_{0}$. The following important relation is obtained by combining (3), (4), and (5).

$$
B_{\lambda}(\mathbf{x})=\epsilon(\lambda, \mathbf{x}) B_{\lambda, b}[T(\mathbf{x})]+[1-\epsilon(\lambda, \mathbf{x})] E_{\lambda, e}(\mathbf{x})+[1-\epsilon(\lambda, \mathbf{x})] \int_{c} B_{\lambda}\left(\mathbf{x}_{0}\right) K\left(\mathbf{x}_{0}, \mathbf{x}\right) d A\left(\mathbf{x}_{0}\right) .
$$

There is only one function $B_{\lambda}(\mathbf{x})$ which will "satisfy" this equation when the functions $T(\mathbf{x})$, $E_{\lambda, e}(\mathbf{x}), \epsilon(\lambda, \mathbf{x})$, and $K\left(\mathbf{x}_{0}, \mathbf{x}\right)$ are given. Equation (6) in which $B_{\lambda}(\mathbf{x})$ is the only unknown function, thus completely determines $B_{\lambda}(\mathbf{x})$. Many well-known methods exist for finding the solution, $B_{\lambda}(\mathbf{x})$, to such an integral equation, although in some cases the actual evaluation of $B_{\lambda}(\mathbf{x})$ may be somewhat tedious. The transformation of eq (6) into the form of a Fredholm integral equation of the second kind with a symmetric kernel, is shown in the appendix.

Next the apparent local spectral emittance inside an isothermal emitting cavity which is not irradiated by an outside source will be defined. For this circumstance $T(\mathbf{x})=T$ (a constant) and $E_{\lambda, e}(\mathbf{x})=0$ everywhere over the interior surface of the cavity. Then the apparent local spectral emittance $\epsilon_{a}(\lambda, \mathbf{x})$ is defined as

$$
\epsilon_{a}(\lambda, \mathbf{x}) \equiv \frac{B_{\lambda}(\mathbf{x})}{B_{\lambda, b}[T]}
$$

where $B_{\lambda}(\mathbf{x})$ is the radiosity distribution within the cavity. The integral equation which governs $\epsilon_{a}(\lambda, \mathbf{x})$ is obtained by substituting the above conditions in (6) and dividing both sides by $B_{\lambda, b}[T]$.

$$
\epsilon_{a}(\lambda, \mathbf{x})=\epsilon(\lambda, \mathbf{x})+[1-\epsilon(\lambda, \mathbf{x})] \int_{c} \epsilon_{a}\left(\lambda, \mathbf{x}_{0}\right) K\left(\mathbf{x}_{0}, \mathbf{x}\right) d A\left(\mathbf{x}_{0}\right) .
$$

Equation (8) completely determines the function $\epsilon_{a}(\lambda, \mathbf{x})$ as truly characteristic of the cavity because $\epsilon_{a}(\lambda, \mathbf{x})$ depends only on the cavity geometry and the emittance of the wall material.

Next, the apparent local spectral absorptance $\alpha_{a}(\lambda, \mathbf{x})$ is defined as a dimensionless function of position $\mathbf{x}$ such that if $Q_{\lambda, \alpha}$ is the total monochromatic flux absorbed within the cavity from any monochromatic external irradiance distribution $E_{\lambda, e}(\mathbf{x})$, then $Q_{\lambda, \alpha}$ may be calculated from

$$
Q_{\lambda, \alpha}=\int_{c} \alpha_{a}(\lambda, \mathbf{x}) E_{\lambda, e}(\mathbf{x}) d A(\mathbf{x})
$$

An equivalent definition of $\alpha_{a}(\lambda, \mathbf{x})$ is: $\alpha_{a}(\lambda, \mathbf{x})$ represents the dimensionless fraction of the monochromatic flux from outside the cavity incident on an element of area, $d A(\mathbf{x})$, that is absorbed within the cavity. This is illustrated in figure $l$ for a focused incident beam, where a finite flux $Q_{\lambda, e}$ is shown striking the infinitesimal area $d A(\mathbf{x})$. For this focused beam, an amount $Q_{\lambda, e} \alpha(\lambda, \mathbf{x})$ is absorbed on the first reflection. The remainder is diffusely reflected both to the other parts of the cavity and to the cavity opening. The irradiance on the other points $\mathbf{x}_{0}$ within the cavity after this first reflection but before the second is given by (10).

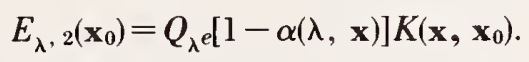

FIGURE 1. Focused beam incident at a point inside a cavity.

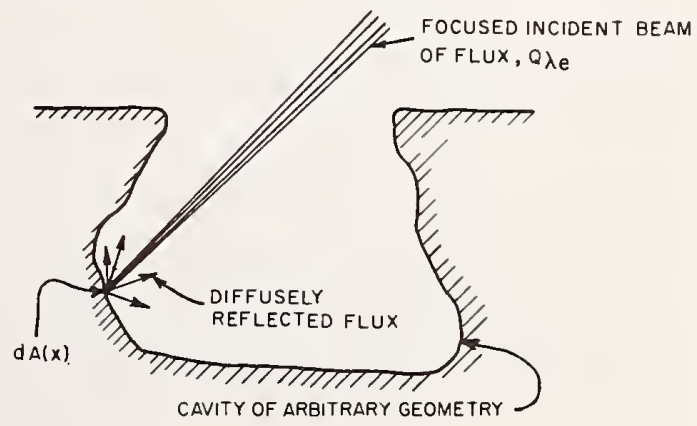


Since $E_{\lambda, 2}\left(\mathbf{x}_{0}\right)$ could be exactly simulated in principle by an irradiance from outside the cavity, the total flux absorbed by the cavity from $E_{\lambda, 2}\left(\mathbf{x}_{0}\right)$ may be calculated from (9). Thus we obtain (11) for the total flux absorbed.

$$
Q_{\lambda, \alpha}=Q_{\lambda, e} \alpha(\lambda, \mathbf{x})+Q_{\lambda, e}[1-\alpha(\lambda, \mathbf{x})] \int_{c} \alpha_{a}\left(\lambda, \mathbf{x}_{0}\right) K\left(\mathbf{x}, \mathbf{x}_{0}\right) d A\left(\mathbf{x}_{0}\right)
$$

Because $\alpha(\lambda, \mathbf{x})=\epsilon(\lambda, \mathbf{x})$ from Kirchhoff's law, $K\left(\mathbf{x}, \mathbf{x}_{\mathbf{0}}\right)=K\left(\mathbf{x}_{\mathbf{0}}, \mathbf{x}\right)$ from angle factor algebra, and for the focused radiation considered, $\alpha_{a}(\lambda, \mathbf{x})=Q_{\lambda}, \alpha Q_{\lambda}, e$ by definition, we obtain (12) for $\alpha_{a}(\lambda, \mathbf{x})$, which is equivalent to (8) for $\epsilon_{a}(\lambda, \mathbf{x})$.

$$
\alpha_{a}(\lambda, \mathbf{x})=\epsilon(\lambda, \mathbf{x})+[1-\epsilon(\lambda, \mathbf{x})] \int_{c} \alpha_{a}\left(\lambda, \mathbf{x}_{0}\right) K\left(\mathbf{x}, \mathbf{x}_{\mathbf{0}}\right) d A\left(\mathbf{x}_{0}\right)
$$

Thus, it follows that

$$
\epsilon_{a}(\lambda, \mathbf{x})=\alpha_{a}(\lambda, \mathbf{x})
$$

i.e., the apparent local spectral emittance and apparent local spectral absorptance are equal, and Kirchhoff's law holds.

\section{Application to Cavity Absorption}

A direct consequence of (9) and (11) is that the solution of the integral equation governing the apparent local spectral emittance within a cavity also gives the information necessary for determining the total flux absorbed by the cavity for any given external irradiance. It is obvious that any conceivable spacecraft configuration is just a special case of the general geometric cavity configuration discussed in the first paragraph of section 2. If the spacecraft has diffusely reflecting surfaces, then $\epsilon_{a}(\lambda, \mathbf{x})$ for its particular configuration may be calculated by (8). The total flux absorbed by the spacecraft for any given external irradiance distribution (which would correspond to any given orientation of the spacecraft with respect to the sun, earth, moon or any other celestial object in the neighborhood) can be calculated easily by simply evaluating the integral in (9) based on the solution of (8). The different values of flux absorbed which correspond to the many different possible source positions with respect to the spacecraft can be evaluated quickly to any desired degree of accuracy by using modern computer techniques. This calculational procedure has actually been employed for certain cases of illumination in shallow cylindrical cavities and good agreement with experimental values was found [7].

\section{Application to Nonisothermal Cavity Emission}

Another application of the equation derived in section 2 is to the calculation of the total flux emitted through the opening of a nonisothermal heated cavity (or equivalently from the surface of a nonisothermal spacecraft). It is evident from (6) that both $\epsilon(\lambda, \mathbf{x}) B_{\lambda, b}[T(\mathbf{x})]$ and $[1-\epsilon(\lambda, \mathbf{x})]$ $E_{\lambda, e}(\mathbf{x})$ play the same mathematical role in determining $B_{\lambda}(\mathbf{x})$. As a consequence, both of the following cases will give rise to identical radiosity distributions within the cavity and fluxes leaving the cavity.

Case 1. Arbitrary temperature distribution $=T(\mathbf{x})$; external irradiance distribution $=E_{\lambda,}(\mathbf{x})=0$, everywhere.

Case 2. Temperature distribution $=T^{\prime}(\mathbf{x})=0$, everywhere; external irradiance distribution $=E_{\lambda, e}^{\prime}(\mathbf{x})=\epsilon(\lambda, \mathbf{x})[1-\epsilon(\lambda, \mathbf{x})]^{-1} B_{\lambda, b}[T(\mathbf{x})]$.

This can easily be seen by substituting both Case 1 and 2 into (6) and noting that exactly the same equation results in both cases. In order to find the total flux emitted by the cavity in Case 1, 
it is necessary to find in Case 2 the difference between the total incident flux and the total absorbed flux. The total incident flux $Q_{\lambda, e}$, is simply given by (14).

$$
Q_{\lambda, e}=\int_{c} E_{\lambda, e}^{\prime}(\mathbf{x}) d A(\mathbf{x})
$$

The total absorbed flux $Q_{\lambda, \alpha}$ is given by (9). Hence the total flux emitted, $Q_{\lambda, \epsilon}$ is

$$
Q_{\lambda, \epsilon}=\int_{c}\left[1-\alpha_{a}(\lambda, \mathbf{x})\right] E_{\lambda, e}^{\prime}(\mathbf{x}) d A(\mathbf{x})
$$

or in terms of the arbitrary temperature distribution $T(\mathbf{x})$ and the apparent local spectral emittance $\epsilon_{a}(\lambda, \mathbf{x})$

$$
Q_{\lambda, \epsilon}=\int_{c}\left[1-\epsilon_{a}(\lambda, \mathbf{x})\right] \epsilon(\lambda, \mathbf{x})[1-\epsilon(\lambda, \mathbf{x})]^{-1} B_{\lambda, b}[T(\mathbf{x})] d A(\mathbf{x}) .
$$

Because $T(\mathbf{x})$ is completely arbitrary, and because no restrictions have been made on the possible geometry of the cavity (or spacecraft), eq (15b) applies to all situations.

\section{The Flux From a Cylindrical Cavity}

To illustrate the usefulness of (15b) the total heat flux from a cylindrical cavity has been calculated for several different temperature distributions (see fig. 2).

Figure 2. Arbitrary temperature distributions within a cylindrical cavity.

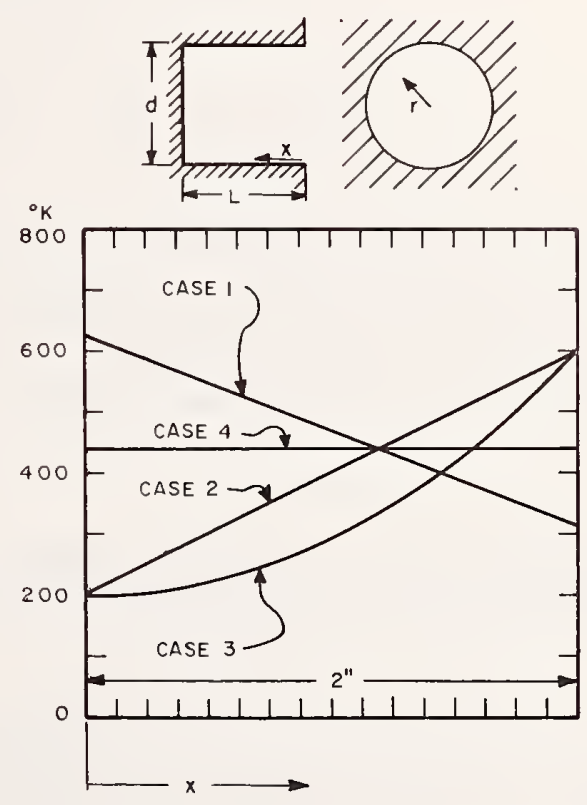

The apparent local spectral emittance $\epsilon_{a}(\lambda, \mathbf{x})$ has been published for a diffuse cylindrical cavity of uniform wall emitiance, $\epsilon(\lambda)$ of 0.5 [8]. In the following calculations, these results are used to determine the total flux from an identical nonisothermal cavity. The cavity considered had a 1.0 in. radius and a 2.0 in. depth. Four arbitrary temperature distributions shown in figure 2 were considered. These temperature distributions are assumed to be angularly symmetrical about the central axis of the cylinder. The base of the cavity is assumed to be isothermal with its temperature equal to the temperature at $x=2 \mathrm{in}$. in all four cases. These four temperature distributions were chosen to show that wide variations of temperature are easily handled by this method. Here $x$ is the distance from the mouth of the cavity along the walls; $r$ is the radial distance from the center of the cavity base. The apparent local spectral emittance distribution 
$\epsilon_{a}(\lambda, x)$ and $\epsilon_{a}(\lambda, r)$ were taken from reference [7], figure 4. For convenience, it is assumed that the cavity wall material is gray (its spectral emittance does not vary with wavelength). For this case the Stefan-Boltzmann law is applicable and $Q_{\lambda, \epsilon}, B_{\lambda}, b[T(\mathbf{x})], \epsilon(\lambda)$ and $\epsilon_{a}(\lambda, x)$ can be replaced by $Q_{\epsilon}, \sigma[T(\mathbf{x})]^{4}, \epsilon$, and $\epsilon_{a}(\mathbf{x})$ respectively. The total flux emitted from the mouth is given by (15c).

$$
\left.Q_{\epsilon}=2 \pi \epsilon \sigma[1-\epsilon]^{-1}\left\{\frac{d}{2} \int_{0}^{L}\left[1-\epsilon_{a}(x)\right][T(x)]^{4} d x+\int_{0}^{d / 2}\left[1-\epsilon_{d} r\right)\right][T(r)]^{4} r d r\right\} .
$$

The integrals in (15c) were approximated by Simpson's rule summations. Table 1 gives the total heat flux for each of the four cases. The first line of table 1 gives the results calculated with $\Delta x$ and $\Delta r$ equal to $0.1 \mathrm{in}$. The second line gives the results when $\Delta x$ and $\Delta r$ are taken to be $0.05 \mathrm{in}$. The results of the calculation based on the larger $\Delta x$ and $\Delta r$ agree to within 0.6 percent with those using the smaller $\Delta x$ and $\Delta r$. These results were obtained with relatively little expenditure of time and effort. For the three cases which have the same average temperature $(1,2$, and 4$)$, the isothermal case emits the least flux, and the cavity whose temperature decreased from a maximum at the front emits the greatest flux. The values obtained for the (isothermal) case 4 can be directly compared to data given in [8]. Using $Q_{\epsilon} / \sigma T^{4}\left(\pi d^{2} / 4\right)=0.8084$ from table 2 of [8], one calculates $Q_{\epsilon}=3.484 \mathrm{~W}$, whereas the result from the integration in the present paper gives $Q_{\epsilon}=3.49370 \mathrm{~W}$. When $T(x)=T$ (a constant) everywhere within the cavity, eq (15c) of the present paper reduces to eq (11a) of [8].

TABLE 1. Q $\mathrm{Q}_{\mathrm{f}}$ for cases 1 to 4

\begin{tabular}{r|r|r|r|r}
\hline \hline & \multicolumn{1}{c|}{ Case 1 } & \multicolumn{1}{c|}{ Case 2 } & \multicolumn{1}{c|}{ Case 3 } & \multicolumn{1}{c}{ Case 4 } \\
\cline { 2 - 5 }$\Delta x=0.1$ in .................. & 5.94 & 4.269 & 3.348 & 3.49373 \\
$\Delta x=0.05$ in ................... & 5.97 & 4.265 & 3.346 & 3.49370 \\
\hline
\end{tabular}

The main limitation on the application of (15b) is the need for the function $\epsilon_{a}(\lambda, \mathbf{x})$ for various cavity (or spacecraft) geometries. The function $\epsilon_{a}(\lambda, \mathbf{x})$ has been determined for only a limited number of simple geometries like the sphere, the cone, and the cylinder. [9-11]. It is to be hoped that more $\epsilon_{a}(\lambda, \mathbf{x})$ data will appear in the future, because its knowledge permits the determination of the flux to and from a cavity under a great number of conditions.

The author thanks D. G. Moore, J. C. Richmond, and A. C. Kelly for their valuable assistance in the preparation of this paper; and Dr. E. M. Sparrow for a helpful conversation.

\section{Appendix}

Equation (8) may be transformed into the form of a Fredholm Integral equation of the second kind with a symmetric kernel, $K^{*}\left(\mathbf{x}_{0}, \mathbf{x}\right)$, by the following transformation:

$$
\begin{gathered}
B_{\lambda}^{*}(\mathbf{x})=B_{\lambda}(\mathbf{x})[1-\epsilon(\lambda, \mathbf{x})]^{1 / 2} \\
K^{*}\left(\mathbf{x}_{0}, \mathbf{x}\right)=[1-\epsilon(\lambda, \mathbf{x})]^{1 / 2}\left[1-\epsilon\left(\lambda, \mathbf{x}_{0}\right)\right]^{1 / 2} K\left(\mathbf{x}_{0}, \mathbf{x}\right) .
\end{gathered}
$$

The resulting equation is given below:

$$
\begin{aligned}
B_{\lambda}^{*}(\mathbf{x})=\epsilon(\lambda, \mathbf{x})[1-\epsilon(\lambda, \mathbf{x})]^{-1} B_{b, \lambda}[T(\mathbf{x})]+[1-\epsilon(\lambda, \mathbf{x})]^{1 / 2} E_{\lambda_{,}} e(\mathbf{x}) & \\
& +\int_{c} B_{\lambda}^{*}\left(\mathbf{x}_{0}\right) K^{*}\left(\mathbf{x}_{0}, \mathbf{x}\right) d A\left(\mathbf{x}_{0}\right) .
\end{aligned}
$$


This transformation is interesting because it permits the use of certain theorems and methods introduced by Moon [12] even when $\epsilon(\lambda, \mathbf{x})$ is not constant.

\section{References}

[1] W. Wein and O. Lummer, Methode zur Prufung des Strahlungsgesetzes absolut schwarzer Korper, Annalen der Physik 56, 451-456 (1895).

[2] G. Ribaud, Traité' de Pryométrie (Edition de la Revue d'Optique Theroique et Instrumentale), Paris, 1931, p. 231.

[3] A. Gouffé, Corrections d'ouverture des corps-noirs artificiels compte tenu des diffusions multiples internes, Rev. d'Optique, 24, No. 1-3, 1-10 (Jan.-Mar., 1945).

[4] M. Michaud, Facteur d'Emission des Cavités de Formes Geometriques Simples, Comptes Rendus 226, 999 (1948).

[5] C. H. Page, private communication.

[6] C. von Fragstein, On the formulation of Kirchhoff's Law and its use for a suitable definition of diffuse reflection factors, Translation from Optik, 12, No. 2,60-70(1955), S.L.A. Translation Center.

[7] F. J. Kelly and D. G. Moore, A test of analytical expressions for the thermal emissivity of shallow cylindrical cavities, Applied Optics 4, 31 (1965).

[8] E. M. Sparrow, L. U. Albers and E. R. G. Eckert, Thermal radiation characteristics of cylindrical enclosures, J. Heat Transfer, Trans. ASME, Series C, pp. 73-81 (Feb. 1962).

[9] E. M. Sparrow and L. U. Albers, Apparent emissivity and heat transfer in a long cylindrical hole, J. Heat Transfer, Trans. ASME, Series C, 82, 253-255 (1960).

[10] E. M. Sparrow and V. K. Jonsson, Absorption and emission characteristics of diffuse spherical enclosures, J. Heat Transfer, Trans. ASME, Series C, 84, No. 2, 188-189 (1962).

[11] E. M. Sparrow and V. K. Jonsson, Radiant emission characteristics of diffuse conical cavities, J. Opt. Soc. Am. 53, No. 7, 816-821 (July 1963).

[12] P. Moon, On interreflections, J. Opt. Soc. Am. pp. 195-205 (30 May 1940).

(Paper 69B3-148) 


\title{
A Note on the Numerical Evaluation of Thermal Radiation Characteristics of Diffuse Cylindrical and Conical Cavities
}

\author{
B. A. Peavy \\ Institute for Applied Technology, National Bureau of Standards, Washington, D.C. \\ (January 14, 1966)

\begin{abstract}
Presented are methods that avoid the need to employ an extrapolation technique in the region of the critical points for evaluation of the apparent emmissivity of diffuse cylindrical and conical cavities. The methods involve appropriate substitutions in the integrands of integral equations that are used in analytical solutions for determining the thermal radiation characteristics of diffuse and conical cavities. Equations for either isothermal or nonisothermal surface temperature conditions are provided in a direct form for computations. Numerical results are presented for a general linear temperature distribution along the length of a cylindrical cavity. The method is equally applicable for the solution of other problems in integral equations where discontinuities are encountered.
\end{abstract}

Key Words: Conical, cylindrical, emissivity, nonisothermal.

\section{Introduction}

Analytical formulations have been derived $\left[1,2,3 l^{1}\right.$ for determining the radiant interchange in finite length cylindrical cavities, and conical cavities whose bounding surfaces radiate in a gray diffuse manner. These solutions are in the form of integral equations where, unfortunately, most of the integrands exhibit discontinuities with finite limits at critical points, such as the corner of the cylindrical cavity and the apex of the conical cavity. Numerical solutions of these equations in the region of the discontinuities have been previously evaluated by extrapolation techniques. Also, to a lesser degree of difficulty, some of the integrands exhibit slope discontinuities, which for accurate numerical results may involve intricate numerical integration procedures.

The purpose of this paper is to present methods for numerical evaluation of the apparent emissivity in these cavities that avoid use of an extrapolation technique in the region of the critical points. For the main part, the methods involve appropriate substitutions which make the integrands go to zero at the critical points and at the points of slope discontinuity. Impetus for this investigation has been the numerical evaluation of thermal radiation characteristics for parameters not included in the scope of references 1 and 2. A particular need has been the determination of the thermal characteristics for low values of the surface emissivity of shallow cylindrical cavities investigated by Kelly and Moore [4].

${ }^{1}$ Figures in brackets indicate the literature references at the end of this paper.
Also presented is a system of integral equations for determining the thermal characteristics of diffuse cylindrical and conical cavities whose surfaces are nonisothermal. A few selected numerical results are included for the cylindrical cavity with an arbitrary linear temperature variation over its length.

\section{Cylindrical Cavity}

Analytical formulations for the apparent emissivity of the surfaces of cylindrical enclosures have been derived by Sparrow, Albers, and Eckert [1]. Using the same nomenclature, their eqs (8) and (9) are presented here in a different form.

$$
\begin{aligned}
\epsilon_{a}\left(x_{0}\right)=\epsilon+(1-\epsilon) & \int_{0}^{L / d} \epsilon_{a}(x) K_{1}\left(x_{0}, x\right) d x \\
& +\frac{1-\epsilon}{4}\left(\frac{L}{d}-x_{0}\right) \int_{0}^{1} \epsilon_{a}(r) K_{2}\left(x_{0}, r\right) r d r \\
\epsilon_{a}(r)=\epsilon+ & \frac{1-\epsilon}{2} \int_{0}^{L / d} \epsilon_{a}(x) K_{2}(x, r)\left(\frac{L}{d}-x\right) d x
\end{aligned}
$$

where $\quad K_{1}\left(x_{0}, x\right)=1-\frac{\left|x_{0}-x\right|}{2} \cdot \frac{2\left(x_{0}-x\right)^{2}+3}{\left\{\left(x_{0}-x\right)^{2}+1\right\}^{3 / 2}}$

$$
\begin{aligned}
K_{2}(x, r) & =\frac{(z+w)}{\left\{z^{2}+(1-2 w) z+w^{2}\right\}^{3 / 2}} \\
z & =\left(\frac{L}{d}-x\right)^{2} \quad \text { and } \quad w=\frac{1-r^{2}}{4}
\end{aligned}
$$


and $\epsilon_{a}$ is the apparent emissivity of a surface location; $\epsilon_{a}\left(x_{0}\right)$ and $\epsilon_{a}(r)$ represent values on the curved surface and flat surface, respectively, of the cylindrical cavity. The physical system is described in [1]. $L$ is the length of the cavity, $d$ is the diameter of the cavity, $x$ is a ratio of the distance measured along the cavity wall from the opening to the diameter, and $r$ is the ratio of the radius of a position on the bottom of the cavity to the cavity radius, $R$.

Initial attempts by the author at obtaining numerical solutions of (1) and (2) were frustrated by the erratic behavior of the kernels $K_{2}\left(x_{0}, r\right)$ and $K_{2}(x, r)$ as $r$ approaches unity and $x$ and $x_{0}$ approach $L / d$ (the corner of the system). Visual inspection of these kernels and integrals involved indicates an indeterminate form at these limits. In correspondence with L. U. Albers (coauthor of [1]), it was disclosed that an extrapolation technique was employed in the region of the corner, and that the results were quite insensitive to wide variations in types of extrapolation. Attempts at extrapolation by the author proved to be inconclusive. Indications were that the second differences were quite large in this region, showing that linear extrapolation may be inaccurate.

The suggested method for obtaining solutions is to make the integrand zero at the corner and make contributions to the results negligibly small in the region of the corner. This may be performed by making the substitutions

$$
\begin{aligned}
& \epsilon_{a}(x)=A_{0}+A_{1} z+\phi(x) \\
& \epsilon_{a}(r)=B_{0}+B_{1} w+\psi(r)
\end{aligned}
$$

in the integrals of (1) and (2). $A_{0}, A_{1}, B_{0}$, and $B_{1}$ are chosen such that $\phi(L / d)=\phi(0)=0$ and $\psi(1)=\psi(0)=0$. Substitution of the first two terms of (3) and (4) in the appropriate integrals of (2) and (1), and by $r d r=-2 d w$, and $\left(\frac{L}{d}-x\right) d x=-d z / 2$ gives

$$
\begin{aligned}
& I_{1}=2 z_{0}^{1 / 2} \int_{0}^{1 / 4} \frac{\left(B_{0}+B_{1} w\right)\left(z_{0}+w\right) d w}{\left\{w^{2}-2 z_{0} w+z_{0}+z_{0}^{2}\right\}^{3 / 2}} \\
& I_{2}=\frac{1}{2} \int_{0}^{(L / d)^{2}} \frac{\left(A_{0}+A_{1} z\right)(z+w) d z}{\left\{z^{2}+(1-2 w) z+w^{2}\right\}^{3 / 2}} .
\end{aligned}
$$

From a table of integrals

$$
\begin{gathered}
I_{1}=2\left[\frac{\left(2 z_{0}+1\right) B_{0}+2 z_{0}\left(z_{0}+1\right) B_{1}}{\left(z_{0}+1\right)^{1 / 2}}\right. \\
-z_{0}^{1 / 2}\left\{2 B_{0}+\left(1+2 z_{0}\right) B_{1}\right\} \\
\left.\quad-z_{0}^{1 / 2} B_{1} \ln \left[2\left\{\left(z_{0}+1\right)^{1 / 2}-z_{0}\right\}\right]\right]
\end{gathered}
$$

$$
\begin{aligned}
& I_{2}=A_{0}+A_{1} w+\frac{\left[(L / d)^{2}-w\right]\left[A_{0}+A_{1}+A_{1} w\right]-A_{1}(L / d)^{2}}{\left[\left\{(L / d)^{2}-w\right\}^{2}+(L / d)^{2}\right]^{1 / 2}} \\
& +A_{1} / 2 \ln \left[2\left\{\left[(L / d)^{2}-w\right]^{2}+(L / d)^{2}\right\}^{1 / 2}+2(L / d)^{2}+1-2 w\right]
\end{aligned}
$$

The kernel $K_{1}\left(x_{0}, x\right)$ has a discontinuous derivative at $x=x_{0}$. This did not seem to have an appreciable effect on the accuracy of numerical integration by Simpson's rule if the subdivision of $L / d$ was sufficiently small. The smaller the increment, the longer becomes the time needed to compute apparent emissivities, and it is therefore expedient to use as large an increment as possible. This can be done by making the integrand equal to zero at $x=x_{0}$, and quite small in the vicinity of $x_{0}$, or by the substitution

$$
\epsilon_{a}(x)=\epsilon_{a}\left(x_{0}\right)+\lambda(x)
$$

in the first integral of eq (1). Letting

$$
I_{3}=\epsilon_{a}\left(x_{0}\right)\left\{\int_{0}^{x_{0}} K_{1}\left(x_{0}, x\right) d x+\int_{x_{0}}^{L / d} K_{1}\left(x_{0}, x\right) d x\right\}
$$

and performing the indicated integration, we obtain

$$
I_{3}=\epsilon_{a}\left(x_{0}\right)\left[\frac{L}{d}-1-\frac{2 x_{0}^{2}+1}{2\left(x_{0}^{2}+1\right)^{1 / 2}}-\frac{2\left(\frac{L}{d}-x_{0}\right)^{2}+1}{2\left\{\left(\frac{L}{d}-x_{0}\right)+1\right\}^{1 / 2}}\right] .
$$

With the substitution of (3), (4), and (7), eqs (1) and (2) become

$$
\begin{aligned}
\epsilon_{a}\left(x_{0}\right) & =\epsilon+(1-\epsilon)\left[I_{3}+\int_{0}^{L / d} \lambda(x) K_{1}\left(x_{0}, x\right) d x\right] \\
+ & \frac{(1-\epsilon)}{4}\left\{I_{1}+\left(\frac{L}{d}-x_{0}\right) \int_{0}^{1} \psi(r) K_{2}\left(x_{0}, r\right) r d r\right\} \\
\epsilon_{a}(r) & =\epsilon+\frac{(1-\epsilon)}{2}\left\{I_{2}+\int_{0}^{L / d} \varphi(x) K_{2}(x, r)\left(\frac{L}{d}-x\right) d x\right\}
\end{aligned}
$$

Numerical solutions were obtained from (la) and (2a) by the process of iteration, where all numerical integrations were performed by Simpson's rule and employed an increment equal to $1 / 64$ of the difference of the limits of integration. This increment was proved adequate by comparison with results obtained using an increment of $1 / 128$. For a fixed value of $L / d$, the kernel functions were computed only one time for all variations in the surface emissivity, $\epsilon . K_{1}\left(x_{0}, x\right)$ was computed in a one-dimensional array where proper indexing gave translation about a given value of $x_{0}$. $K_{2}(x, r)$ was computed in two-dimensional array.

Initial values of $\epsilon_{a}(x)$ were determined from rather crude polynomial approximations. Placing the values 


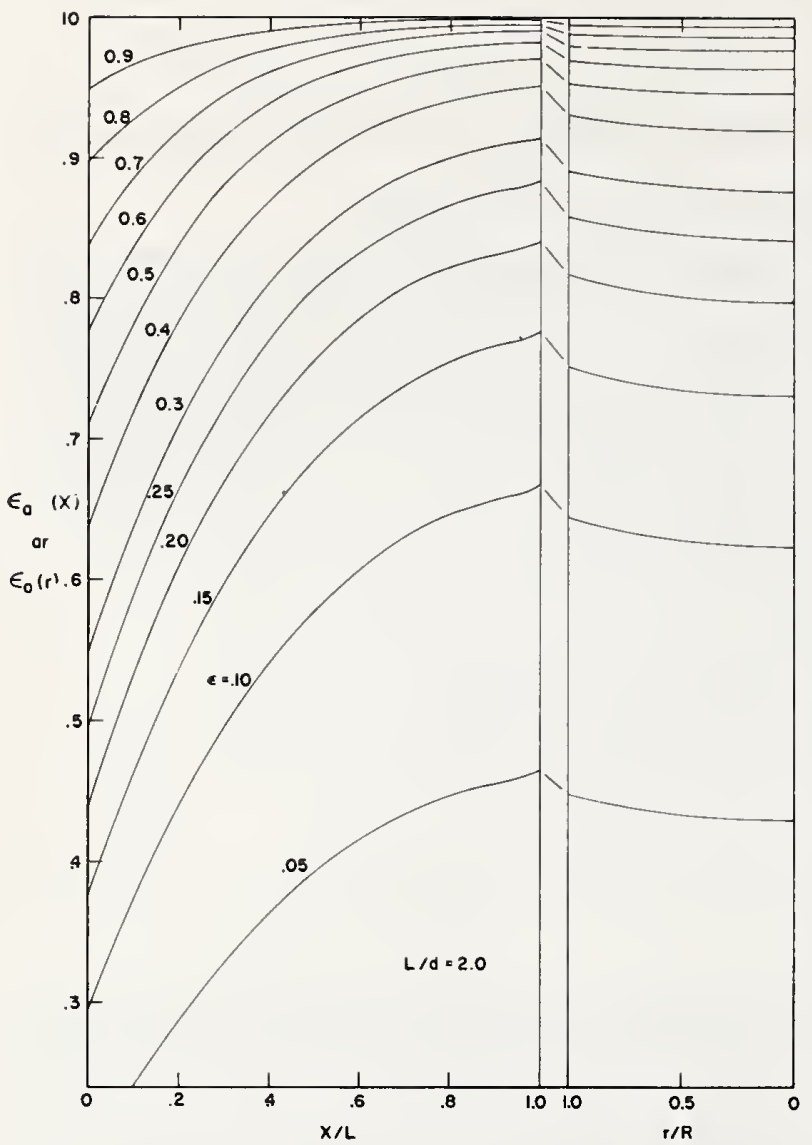

Figure 1. Apparent emissivity in a cylindrical cavity, $\mathrm{L} / \mathrm{d}=2$.

for $\epsilon_{a}(x)$ in (2a), gave values for $\epsilon_{a}(r)$, which with the values of $\epsilon_{a}(x)$ placed in (la) gave new values for $\epsilon_{a}(x)$. This process was repeated using the new values in (2a). The number of iterations $(n)$ necessary for convergence was set by the criterion

$$
\left|\frac{\epsilon_{a}(x=0)_{n}-\epsilon_{a}(x=0)_{n-1}}{\epsilon_{a}(x=0)_{n}}\right| \leqslant 0.0005
$$

which was usually satisfied for $n=4$ or less. A typical time to compute 16 cases involving various values of $L / d$ and $\epsilon$ was about 2 min for an IBM 7094 digital computer.

Figures 1, 2, 3, and 4 give values for apparent emissivity versus dimension ratios for $L / d=2,1,0.5$, and 0.25 , respectively, and for various values of the surface emissivity, $\epsilon$. Where the same parameters occur, there is agreement with values given in table 1 of reference [1] to within 0.1 percent. Figure 5 is a plot of $\epsilon_{a}(r=0)$ versus $\epsilon$ for $L / d=2,1,0.5$, and 0.25 . Figure 6 is a plot of the ratio of the overall emissivity of the cavity to the surface emissivity $\left(Q / \sigma \in \pi R^{2} T^{4}\right)$ versus $\epsilon$ for $L / d=2,1,0.5$, and 0.25 . The ratio was computed by numerical integration of eq (11b) of [1].

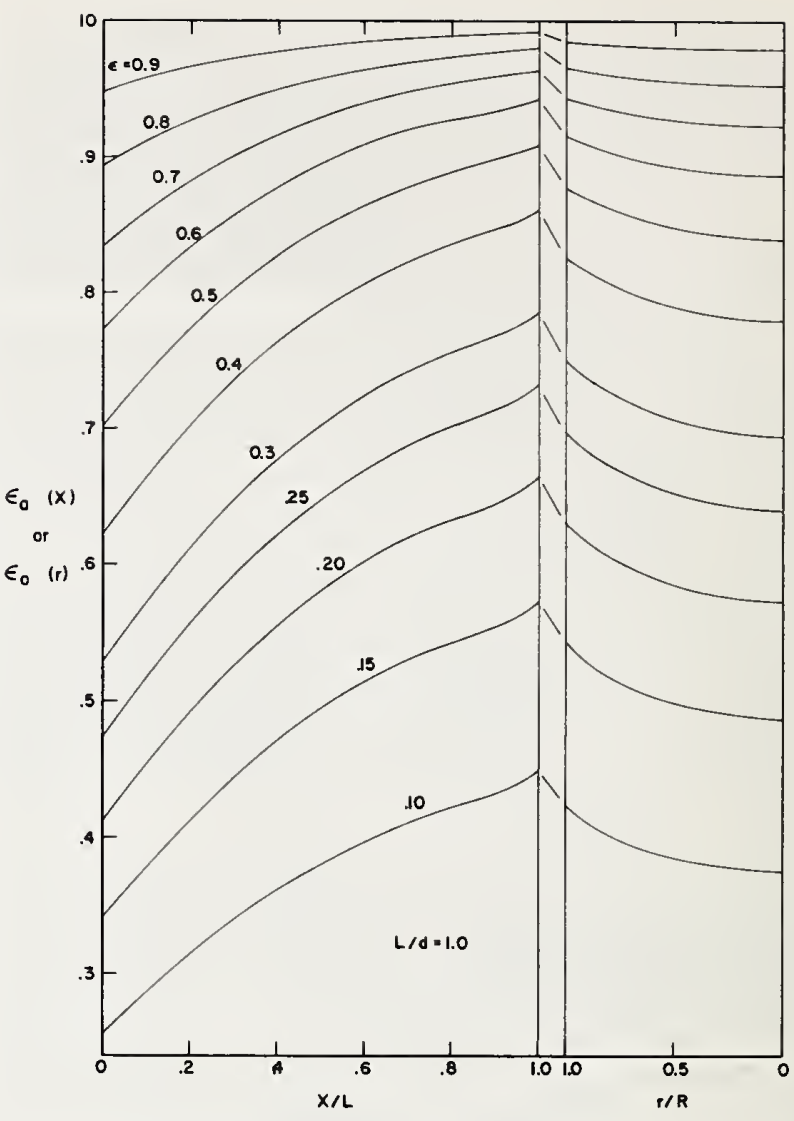

FiguRe 2. Apparent emissivity in a cylindrical cavity, $\mathrm{L} / \mathrm{d}=1$.

TABLE 1

\begin{tabular}{|c|c|c|c|c|c|c|c|c|}
\hline \multicolumn{9}{|c|}{ Values of $\epsilon_{a}(r=0)$ and $\epsilon_{a}(r=1)$} \\
\hline$\epsilon$ & \multicolumn{2}{|c|}{$L / d=2$} & \multicolumn{2}{|c|}{1.0} & \multicolumn{2}{|c|}{0.5} & \multicolumn{2}{|c|}{0.25} \\
\hline 0.9 & 0.9936 & 0.9944 & 0.9785 & 0.9843 & 0.9482 & 0.9707 & 0.9191 & 0.9600 \\
\hline .8 & .9860 & .9877 & .9532 & .9659 & .8924 & .9371 & .8361 & .9149 \\
\hline .7 & .9764 & .9795 & .9231 & .9435 & .8314 & .8977 & .7506 & .8633 \\
\hline .6 & .9637 & .9686 & .8862 & .9153 & .7640 & .8504 & .6619 & .8030 \\
\hline .5 & .9460 & .9534 & .8396 & .8778 & .6879 & .7916 & .5694 & .7316 \\
\hline .4 & .9196 & .9302 & .7789 & 8264 & .6005 & .7168 & .4719 & .6452 \\
\hline .3 & .8766 & .8913 & .6951 & .7504 & .4976 & .6185 & .3684 & .5388 \\
\hline .25 & $\begin{array}{l}.8000 \\
.8442\end{array}$ & .8612 & $\begin{array}{l}.0901 \\
.6401\end{array}$ & .6980 & .4383 & .5568 & .3138 & .4758 \\
\hline .2 & .7986 & .8179 & .5728 & .63 & .3724 & .4841 & .2570 & .4046 \\
\hline .15 & .7313 & .7526 & .4874 & .54 & .2982 & .39 & .1977 & .3237 \\
\hline .1 & .6238 & .6457 & .3756 & 42 & .213 & .29 & .1354 & 2311 \\
\hline .05 & .4305 & .4488 & .2228 & .2554 & .1156 & .1624 & .0697 & .1242 \\
\hline
\end{tabular}

\begin{tabular}{c|c|c|c|c}
\multicolumn{5}{c|}{ Values of $Q /\left(\sigma \epsilon \pi R^{2} T^{\Psi}\right)$} \\
\hline 0.9 & 1.0829 & 1.0801 & 1.0688 & 1.0483 \\
.8 & 1.1829 & 1.1755 & 1.1482 & 1.1025 \\
.7 & 1.3060 & 1.2908 & 1.2410 & 1.1637 \\
.6 & 1.4620 & 1.4330 & 1.3507 & 1.2337 \\
.5 & 1.6662 & 1.6156 & 1.4844 & 1.3138 \\
.4 & 1.9482 & 1.8540 & 1.4480 & 1.4070 \\
.3 & 2.3670 & 2.1877 & 1.8543 & 1.5163 \\
.25 & 2.6609 & 2.4072 & 1.9788 & 1.5784 \\
.2 & 3.0550 & 2.6770 & 2.1820 & 1.6465 \\
.15 & 3.6080 & 3.0207 & 2.2880 & 1.7213 \\
.1 & 4.4500 & 3.4720 & 2.4840 & 1.8040 \\
.05 & 5.8980 & 4.0920 & 2.7160 & 1.8960 \\
\hline
\end{tabular}




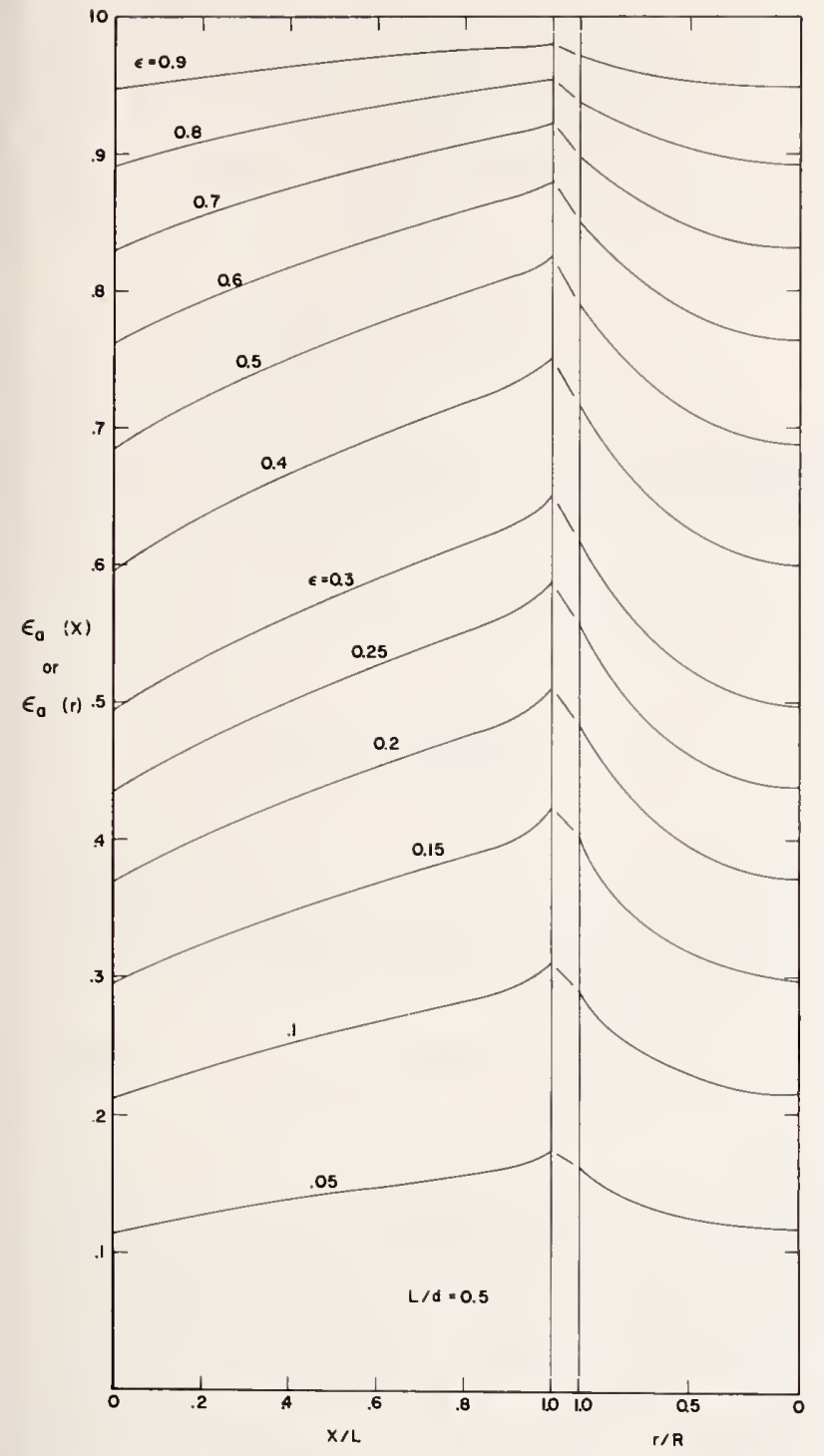

FigURE 3. Apparent emissivity in a cylindrical cavity, $\mathrm{L} / \mathrm{d}=0.5$.

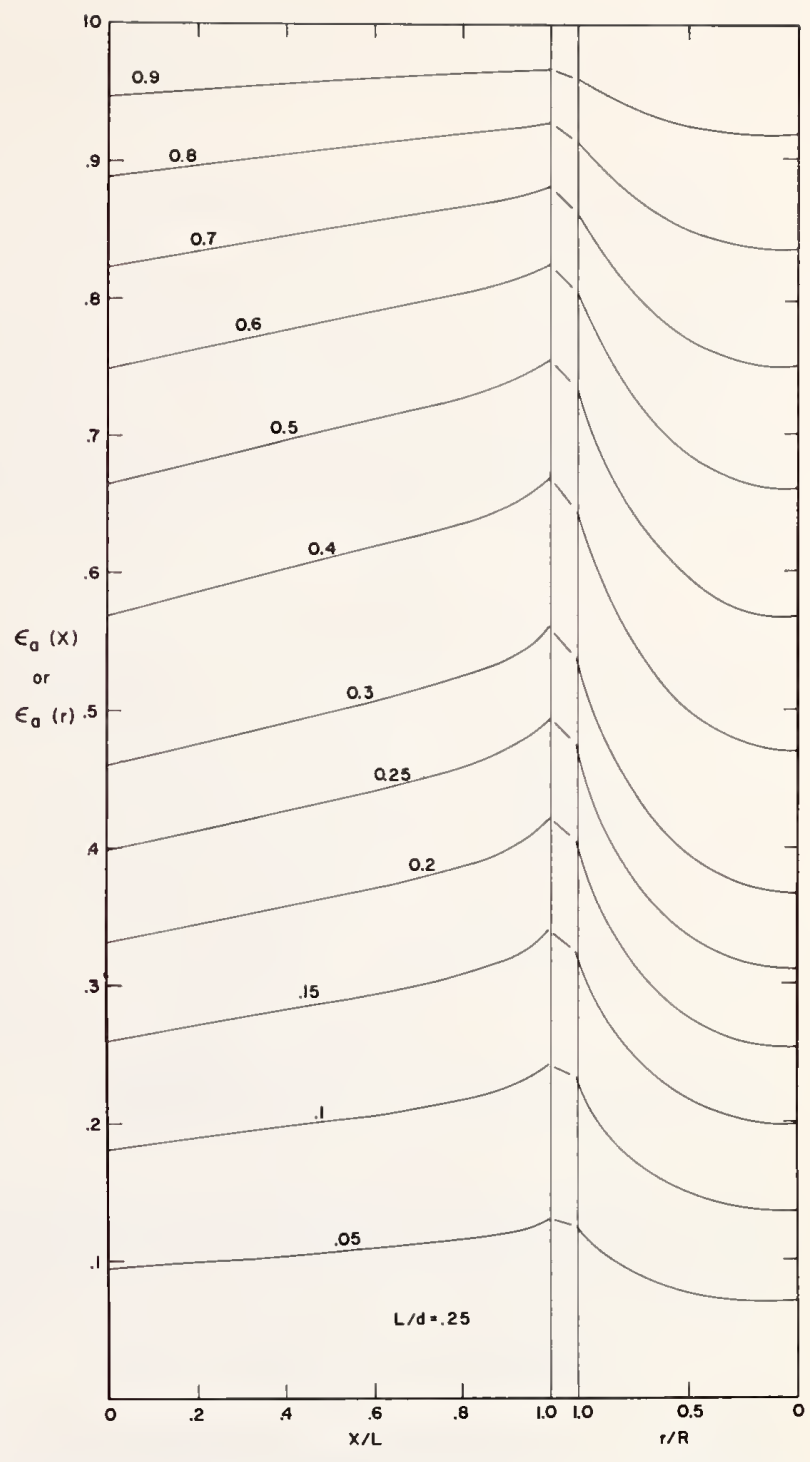

Figure 4. Apparent emissivity in a cylindrical cavity, $\mathrm{L} / \mathrm{d}=0.25$.

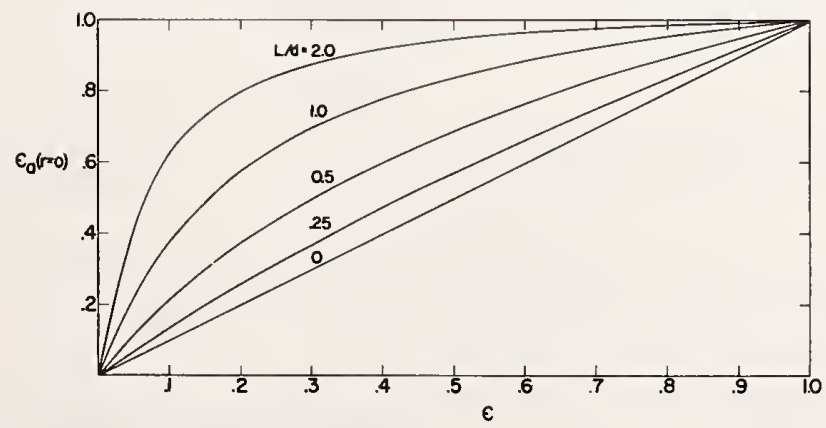

Figure 5. Apparent emissivity versus surface emissivity at the center of the bottom of cylindrical cavity. 


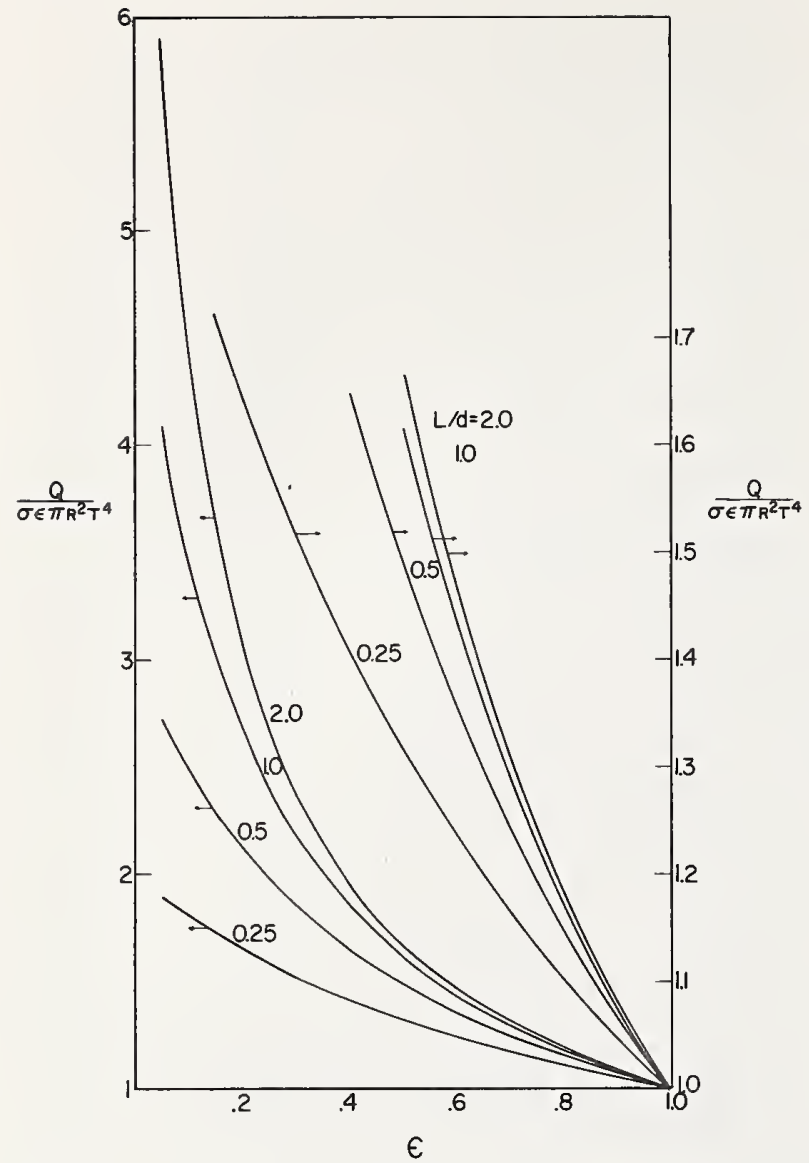

FIGURE 6. Overall heat transfer rate from cylindrical cavities versus surface emissivity.

\section{Conical Cavity}

Analytical solutions for the apparent emissivity of the surfaces of diffuse conical cavities were derived by Sparrow and Jonsson [2]. Here, the problem is to evaluate an integral of the form

$$
\frac{\cos ^{2}(\theta / 2)}{2 y \sin (\theta / 2)} \int_{0}^{1} \epsilon_{t(t)}(t) K_{3}(y, t) d t
$$

where

$$
K_{3}(y, t)=1-|t-y| \frac{\left[(t-y)^{2}+6 t y \sin ^{2}(\theta / 2)\right]}{\left[(t-y)^{2}+4 t y \sin ^{2}(\theta / 2)\right]^{3 / 2}}
$$

and $y$ is the ratio of a surface position measured from the apex to the total length of a cone. The kernel function $K_{3}(y, t)$ has a discontinuous derivative at $y=t$ and a problem arises for the case $y=0$, which by visual inspection gives an indeterminant form. This suggests a substitution of the form

$$
\epsilon_{a l}(t)=\epsilon_{\| \prime}(y)+\gamma(t) .
$$

Letting

$$
I_{4}(y)=\frac{\cos ^{2}(\theta / 2)}{2 y \sin (\theta / 2)}\left[\int_{0}^{y} K_{3}(y, t) d t+\int_{y}^{1} K_{3}(y, t) d t\right]
$$

and performing the indicated integration, we obtain

$$
\begin{aligned}
I_{4}(y)=\frac{2 \sin (\theta / 2)-1}{2 \sin (\theta / 2)}+\frac{\cos ^{2}(\theta / 2)}{2 y \sin (\theta / 2)} \\
\quad\left[1-y-\frac{(1-y)^{2}+y(1+y) \tan ^{2}(\theta / 2)}{\left\{(1-y)^{2}+4 y \sin ^{2}(\theta / 2)\right\}^{1 / 2}}\right] .
\end{aligned}
$$

For the case $y=0$, the second term is an indeter. minant, but can be evaluated by L'Hospital's rule. This yields

$$
I_{4}(0)=1-\sin ^{3}(\theta / 2)
$$

The int egral (9) then becomes

$$
\epsilon_{q}(y) I_{4}(y)+\frac{\cos ^{2}(\theta / 2)}{2 y \sin (\theta / 2)} \int_{0}^{1} \gamma(t) K_{3}(y, t) d t
$$

where the second term still contains the indeterminant form for $y=0$. By letting $t>0$, and applying L'Hospital's rule, we find that the integrand goes to zero. Also, $\gamma(0)=0$, so that the second term goes to zero for $y=0$, and the limit becomes

$$
\operatorname{Lim}_{y \rightarrow 0} \frac{\cos ^{2}(\theta / 2)}{2 y \sin (\theta / 2)} \int_{0}^{1} \epsilon_{a}(t) K_{3}(y, t) d t=\epsilon_{a}(0)\left[1-\sin ^{3}(\theta / 2)\right] .
$$

This is the same result derived by C. H. Page in an internal NBS Report (1952), where it was intuitively assumed that the apparent emissivity at the apex of the cone would be independent of the length of the cone, or that the cone appears infinite. The upper limit of (9) was changed to infinity: this yields $\epsilon_{a}(t)$ constant, hence equal to $\epsilon_{q}(0)$. Another way to arrive at this limit for the case $y=0$ is to assume $\gamma(t)=A_{1} t$ $+A_{2} t^{2}+\ldots$ in eq (13) and perform the indicated integration and limiting process which will give the same result as eq (14).

By making the substitution $y=x / L$ in eq (5) of [2], the equation for the apparent emissivity of a cavity maintained at uniform temperature is

$$
\begin{aligned}
\epsilon_{t \prime}(y)=\epsilon+(1-\epsilon) & \left\{\epsilon_{a}(y) I_{4}(y)\right. \\
+ & \left.+\frac{\cos ^{2}(\theta / 2)}{2 y \sin (\theta / 2)} \int_{0}^{1} \gamma(t) K_{3}(y, t) d t\right\}
\end{aligned}
$$

which has a unique solution at $y=0$,

$$
\epsilon_{\prime \prime}(0)=\frac{\epsilon}{\epsilon+(1-\epsilon) \sin ^{3}(\theta / 2)} .
$$

This is the expression for conical cavities shown by F. J. Kelly, in a paper soon to be published, as derivable from the expression for cavities used by Gouffé. It 
is also the expression derived by C. H. Page by another method (see discussion following eq (14)). Comparison with graphical quantities given in [2] for $\epsilon\left[1-\epsilon_{a}(0)\right] /(1-\epsilon)$ show almost identical agreement.

As in [2], numerical solutions were obtained by the process of iteration, where initial values of $\epsilon_{11}(y)$ were computed from

$$
\epsilon_{\text {II }}(y)_{1}=\frac{\epsilon}{1-(1-\epsilon) I_{4}(y)} .
$$

Because graphical presentations have appeared in [2], numerical values are not shown in this paper.

Equation (17) can be considered a good approximation to eq (15) for all cavities with surface emissivities equal to or greater than 0.7 and for all cavities with apex angles greater than $120^{\circ}$ and emissivity greater than 0.3. For emissivities and apex angles below the values cited above, the deviation between the two equations is not acceptable. An example of the percentage deviation

$$
\gamma(y)=\frac{100\left[\epsilon_{a}(y)-\epsilon_{a}(y)_{1}\right]}{\epsilon_{a}(y)}
$$

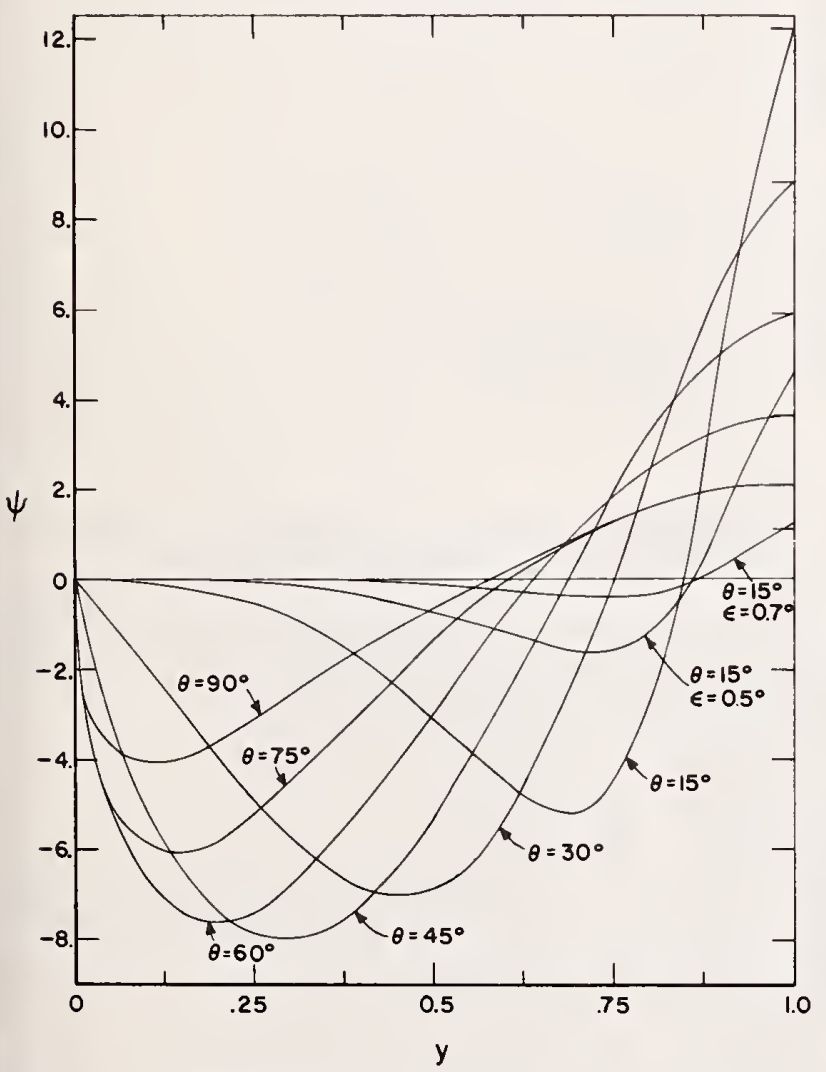

Fic:ure 7. Percentage deviation of eq (/7) from eq (1.5) versus position from apex of a conical cavity.

Lunless wherwine nosted. surtace emisivity. $\epsilon=0,3$ for various apex angles is given in figure 7 , where the average deviation over the length is -2.3 percent for $\epsilon=0.3$ and $\theta=45^{\circ}$.

\section{Cavities With Nonisothermal Surfaces}

Equations 1, 2, and 15, are concerned with cavities for which the surfaces were assumed isothermal. In the succeeding paragraphs, there are presented systems for determining the apparent emissivities of cylindrical and conical cavities with arbitrary variations of surface temperature in respect to dimensions of length and radius, assuming the surface emissivity is constant and not a function of temperature.

\subsection{Cylindrical Cavity}

For the cylindrical cavity, the integral equations take the form

$$
\begin{gathered}
\epsilon_{a}\left(x_{0}\right)=\frac{B\left(x_{0}\right)}{\sigma T_{0}^{4}}=\epsilon f\left(x_{0}\right)+(1-\epsilon) G_{1}\left[\epsilon_{a}(x), \epsilon_{a}(r)\right] \\
\epsilon_{a}(r)=\frac{B(r)}{\sigma T_{0}^{4}}=\epsilon g(r)+(1-\epsilon) G_{2}\left[\epsilon_{a}(x)\right]
\end{gathered}
$$

where

$$
\begin{aligned}
T^{4}\left(x_{0}\right) & =T_{f} f\left(x_{0}\right): f\left(x_{0}\right)=C_{0}+C_{1} y+\ldots+C_{n} y^{m} \\
T^{4}(r) & =T_{0}^{4} g(r) ; g(r)=1+D_{1} r^{2}+\ldots+D_{k} r^{2 k},
\end{aligned}
$$

$y=x_{0} /(L / d), T_{0}$ is an arbitrary temperature which is taken to be the temperature at $r=0$, and $G_{1}$ and $G_{2}$ are the coefficients of $(l-\epsilon)$ in $(l a)$ and $(2 a)$, respectively. Two sets of integral equations are defined by

$$
\begin{aligned}
E_{n}\left(x_{0}\right) & =\epsilon y^{n}+(1-\epsilon) G_{1}\left[E_{n}(x), E_{n}(r)\right] \\
E_{n}(r) & =\epsilon+(1-\epsilon) G_{2}\left[E_{n}(x)\right] \\
n & =0,1,2, \ldots m
\end{aligned}
$$

and

$$
\begin{aligned}
M_{j}\left(x_{0}\right) & =\epsilon+(1-\epsilon) G_{1}\left[M_{j}(x), M_{j}(r)\right] \\
M_{j}(r) & =\epsilon \gamma^{2 j}+(1-\epsilon) G_{2}\left[M_{j}(x)\right] \\
j & =1,2, \ldots . k
\end{aligned}
$$

The apparent emissivity is then found from the following:

$$
\begin{array}{r}
\epsilon_{a}\left(x_{0}\right)=C_{0}^{\prime} E_{0}\left(x_{0}\right)+C_{1} E_{1}\left(x_{0}\right)+\ldots+C_{m} E_{m}\left(x_{0}\right) \\
+D_{1} M_{1}\left(x_{0}\right)+\ldots+D_{h^{\prime}} M_{k}\left(x_{0}\right) \\
\epsilon_{a l}(r)=C_{0}^{\prime} E_{0}(r)+C_{1} E_{1}(r)+\ldots+C_{m} E_{m}(r) \\
+D_{1} M_{1}(r)+\ldots+D_{k} M_{k \cdot(r)}
\end{array}
$$




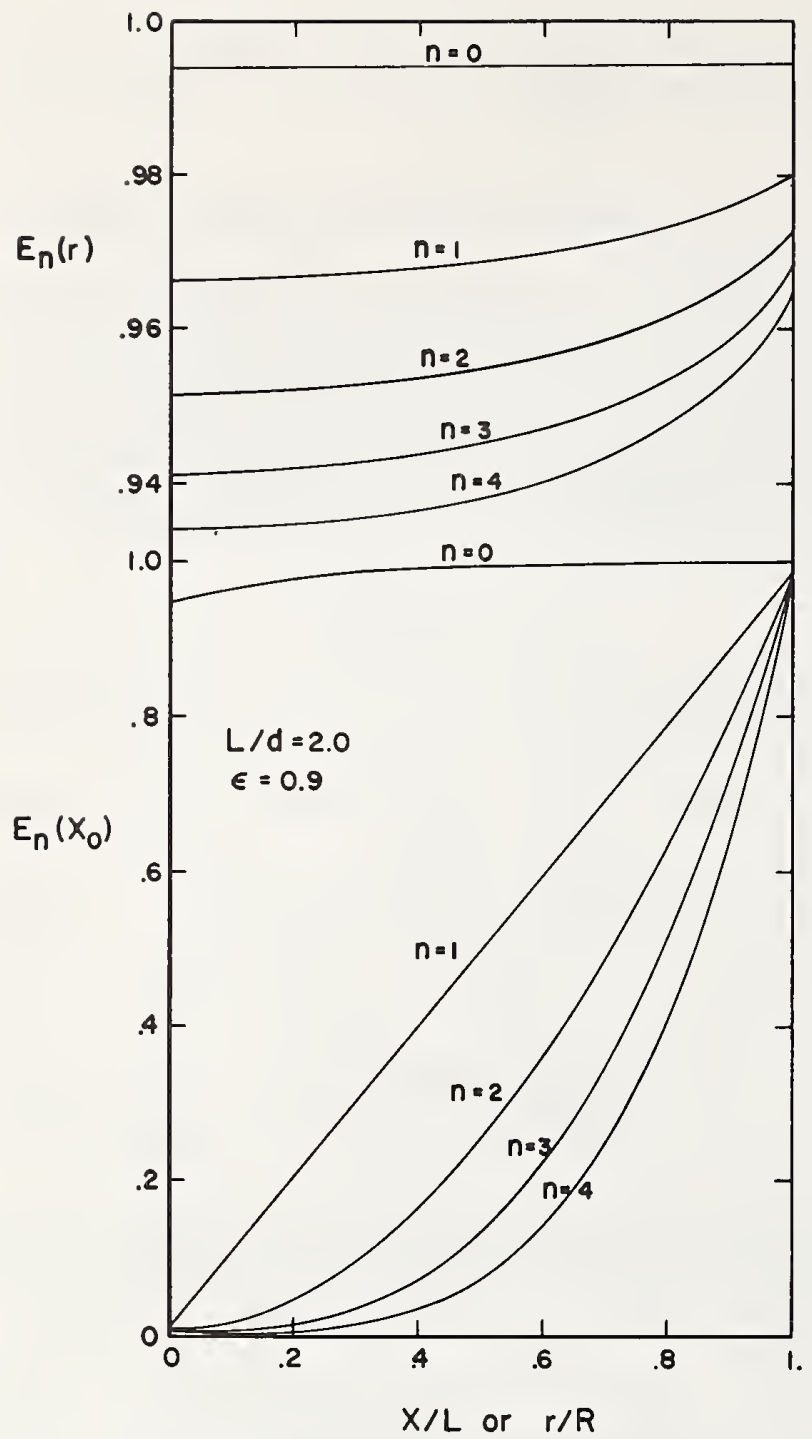

FIGURE 8. Component apparent emissivity values versus position in cylindrical cavity $(\mathrm{L} / \mathrm{d}=2.0)$ for a surface emissivity $=0.9$.

which satisfies (19) and (20) for $C_{0}^{\prime}=C_{0}-D_{1}-D_{22}-\ldots$ $D_{k}$, which gives continuity to the temperature at $x_{0}=L / d$ and $r=1$. Figures 8 and 9 show solutions of (23) and (24) for $\epsilon=0.9$ and 0.5 , respectively, with $L / d=2.0$, and $n=0,1,2,3$, and 4 .

One simplifying procedure is to assume that the bottom of the cavity is isothermal at temperature $T_{0}$. This eliminates the numerical solutions of (25) and (26); and (27) and (28) become

$$
\begin{gathered}
\epsilon_{11}\left(x_{0}\right)=\sum_{n=0}^{m} C_{n} E_{n}\left(x_{0}\right) \\
\epsilon_{11}(r)=\sum_{n=0}^{m} C_{n} E_{n}(r) .
\end{gathered}
$$

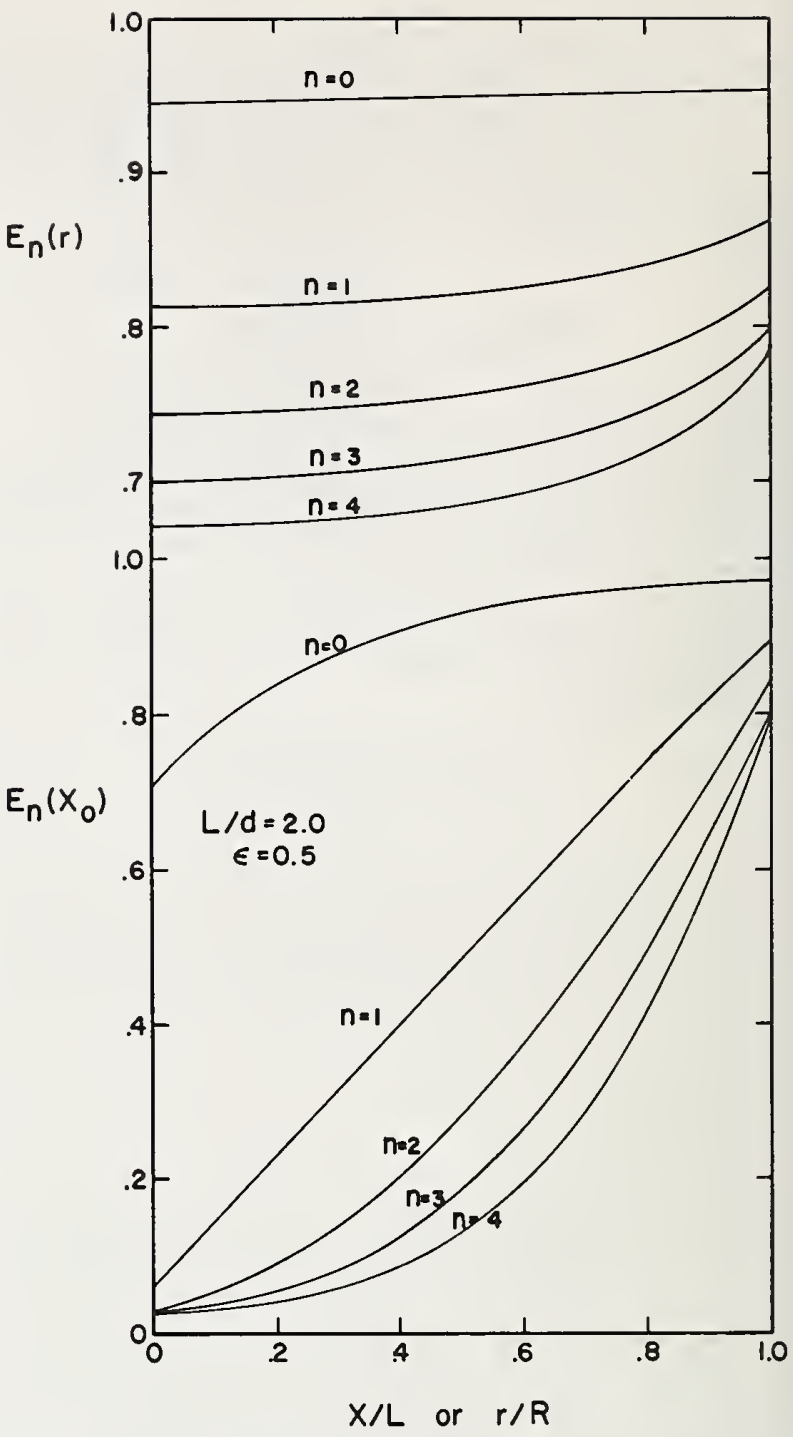

Figune 9. Component apparent emissivity values versus position in cylindrical cavity $(\mathrm{L} / \mathrm{d}=2.0)$ for a surface emissivity $=0.5$.

The ratio of the cavity radiant heat exchange $Q$ with the environment through the opening to that of a black disk of equal area at temperature $T_{0}$ is

$$
\begin{aligned}
\frac{Q}{\sigma T_{0}^{4} \pi R^{2}}=\frac{\epsilon}{(1-\epsilon)} & {[4} \\
& \frac{L}{d} \int_{0}^{1}\left\{f\left(x_{0}\right)-\epsilon_{n}\left(x_{0}\right)\right\} d x \\
& \left.+2 \int_{0}^{1}\left\{1-\epsilon_{a}(r)\right\} r d r\right]=\sum_{n=0}^{m} C_{n} Q_{n}
\end{aligned}
$$

where

$Q_{n}=\frac{\epsilon}{1-\epsilon}\left[4 \frac{L}{d} \int_{0}^{1}\left\{x_{0}^{n}-E_{n}\left(x_{0}\right)\right\} d x+2 \int_{0}^{01}\left\{1-E_{n}(r)\right\} r d r\right]$. 
TABLE 2. Component thermal characteristics for a cylindrical cavity, $\mathrm{L} / \mathrm{d}=0.5$, with a nonisothermal wall

\begin{tabular}{|c|c|c|c|c|c|c|}
\hline & $\epsilon=$ & 0.5 & 0.6 & 0.7 & 0.8 & 0.9 \\
\hline$E_{n}(r=0)$ & $\begin{aligned} n=0 \\
1 \\
2 \\
3 \\
4\end{aligned}$ & $\begin{array}{r}0.68786 \\
.59722 \\
.56747 \\
.55334 \\
.54539\end{array}$ & $\begin{array}{r}0.76398 \\
.68197 \\
.65522 \\
.64260 \\
.63554\end{array}$ & $\begin{array}{r}0.83139 \\
.76344 \\
.74133 \\
.73095 \\
.72516\end{array}$ & $\begin{array}{r}0.89236 \\
.84298 \\
.82695 \\
.81946 \\
.81530\end{array}$ & $\begin{array}{r}0.94823 \\
.92158 \\
.91295 \\
.90893 \\
.90670\end{array}$ \\
\hline$E_{\|}(r=1)$ & $\begin{array}{r}n=0 \\
1 \\
2 \\
3 \\
4\end{array}$ & $\begin{array}{l}.79159 \\
.74185 \\
.72380 \\
.71433 \\
.70850\end{array}$ & $\begin{array}{l}.85038 \\
.80906 \\
.79416 \\
.78637 \\
.78158\end{array}$ & $\begin{array}{r}.89769 \\
.86620 \\
.85485 \\
.84894 \\
.84530\end{array}$ & $\begin{array}{l}.93707 \\
.91592 \\
.90831 \\
.90436 \\
.90193\end{array}$ & $\begin{array}{l}.97066 \\
.96006 \\
.95627 \\
.95421 \\
.95310\end{array}$ \\
\hline$E_{n t}\left(x_{n}=0\right)$ & $\begin{aligned} & n= 0 \\
& 1 \\
& 2 \\
& 3 \\
& 4\end{aligned}$ & $\begin{array}{l}.68571 \\
.11781 \\
.09700 \\
.08713 \\
.08143\end{array}$ & $\begin{array}{l}.76237 \\
.10232 \\
.08423 \\
.07574 \\
.07087\end{array}$ & $\begin{array}{l}.83139 \\
.08175 \\
.06729 \\
.06057 \\
.05674\end{array}$ & $\begin{array}{l}.89188 \\
.05731 \\
.04718 \\
.04252 \\
.03988\end{array}$ & $\begin{array}{l}.94810 \\
.02986 \\
.02459 \\
.02219 \\
.02084\end{array}$ \\
\hline$Q_{*}$ & $\begin{aligned} & n= 0 \\
& 1 \\
& 2 \\
& 3 \\
& 4\end{aligned}$ & $\begin{array}{l}.7422 \\
.4802 \\
.4006 \\
.3630 \\
.3412\end{array}$ & $\begin{array}{l}.8104 \\
.5223 \\
.4362 \\
.3959 \\
.3728\end{array}$ & $\begin{array}{l}.8687 \\
.5576 \\
.4662 \\
.4240 \\
.3999\end{array}$ & $\begin{array}{l}.9186 \\
.5876 \\
.4921 \\
.4484 \\
.4238\end{array}$ & $\begin{array}{l}.9619 \\
.6136 \\
.5149 \\
.4703 \\
.4453\end{array}$ \\
\hline
\end{tabular}

TABLE 3. Component thermal characteristics for a cylindrical cavity, $\mathrm{L} / \mathrm{d}=1$, with a nonisothermal wall

\begin{tabular}{|c|c|c|c|c|c|c|}
\hline & $\epsilon=$ & 0.5 & 0.6 & 0.7 & 0.8 & 0.9 \\
\hline$E_{N}(r=0)$ & $n=0 \begin{array}{r}0 \\
1 \\
2 \\
3 \\
4\end{array}$ & $\begin{array}{r}0.83957 \\
.70223 \\
.64592 \\
.61520 \\
.59658\end{array}$ & $\begin{array}{r}0.88625 \\
.76827 \\
.71951 \\
.69291 \\
.67656\end{array}$ & $\begin{array}{r}0.92309 \\
.82950 \\
.79050 \\
.76914 \\
.75593\end{array}$ & $\begin{array}{r}0.95324 \\
.88782 \\
.86032 \\
.84521 \\
.83583\end{array}$ & $\begin{array}{r}0.97847 \\
.94438 \\
.92993 \\
.92196 \\
.91700\end{array}$ \\
\hline$E_{w}(r=1)$ & $\begin{array}{r}n=0 \\
1 \\
2 \\
3 \\
4\end{array}$ & $\begin{array}{l}.87784 \\
.79987 \\
.76831 \\
.75077 \\
.73989\end{array}$ & $\begin{array}{l}.91531 \\
.85305 \\
.82782 \\
.81384 \\
.80500\end{array}$ & $\begin{array}{l}.94353 \\
.89741 \\
.87869 \\
.86831 \\
.86170\end{array}$ & $\begin{array}{l}.96591 \\
.93563 \\
.92333 \\
.91651 \\
.91216\end{array}$ & $\begin{array}{l}.98431 \\
.96942 \\
.96338 \\
.96003 \\
.95789\end{array}$ \\
\hline$E_{11}\left(x_{11}=0\right)$ & $\begin{array}{r}n=0 \\
1 \\
2 \\
3 \\
4\end{array}$ & $\begin{array}{l}.70148 \\
.09719 \\
.06924 \\
.05704 \\
.05059\end{array}$ & $\begin{array}{l}.77174 \\
.08009 \\
.05627 \\
.04613 \\
.04075\end{array}$ & $\begin{array}{l}.83533 \\
.06114 \\
.04237 \\
.03455 \\
.03043\end{array}$ & $\begin{array}{l}.89394 \\
.04117 \\
.02816 \\
.02284 \\
.02007\end{array}$ & $\begin{array}{l}.94858 \\
.02068 \\
.01396 \\
.01127 \\
.00988\end{array}$ \\
\hline$Q_{n}$ & $\begin{array}{r}n=0 \\
1 \\
2 \\
3 \\
4\end{array}$ & $\begin{array}{l}.8078 \\
.4088 \\
.3018 \\
.2552 \\
.2281\end{array}$ & $\begin{array}{l}.8598 \\
.4235 \\
.3100 \\
.2614 \\
.2343\end{array}$ & $\begin{array}{l}.9036 \\
.4334 \\
.3149 \\
.2651 \\
.2382\end{array}$ & $\begin{array}{l}.9404 \\
.4401 \\
.3176 \\
.2671 \\
.2405\end{array}$ & $\begin{array}{l}.9721 \\
.4447 \\
.3189 \\
.2682 \\
.2419\end{array}$ \\
\hline
\end{tabular}

TABLE 4. Component thermal characteristics for a cylindrical cavity, $\mathrm{L} / \mathrm{d}=2$, with a nonisothermal wall

\begin{tabular}{|c|c|c|c|c|c|c|}
\hline & $\epsilon=$ & 0.5 & 0.6 & 0.7 & 0.8 & 0.9 \\
\hline$E_{\| l}(r=0)$ & $n=\begin{array}{r}0 \\
1 \\
2 \\
3 \\
4\end{array}$ & $\begin{array}{r}0.94601 \\
.81188 \\
.74333 \\
.70026 \\
.67076\end{array}$ & $\begin{array}{r}0.96371 \\
.85575 \\
.79923 \\
.76313 \\
.73806\end{array}$ & $\begin{array}{r}0.97639 \\
.89514 \\
.85163 \\
.82354 \\
.80386\end{array}$ & $\begin{array}{r}0.98599 \\
.93171 \\
.90204 \\
.88269 \\
.86902\end{array}$ & $\begin{array}{r}0.99364 \\
.96644 \\
.95130 \\
.94132 \\
.93423\end{array}$ \\
\hline$E_{n t} t r=11$ & $\begin{array}{r}n=0 \\
1 \\
2 \\
3 \\
4\end{array}$ & $\begin{array}{l}.95338 \\
.86738 \\
.82589 \\
.80054 \\
.78341\end{array}$ & $\begin{array}{l}.96864 \\
.90331 \\
.87139 \\
.85166 \\
.83819\end{array}$ & $\begin{array}{l}.97949 \\
.93281 \\
.90974 \\
.89543 \\
.88559\end{array}$ & $\begin{array}{l}.98773 \\
.95796 \\
.94312 \\
.93388 \\
.92750\end{array}$ & $\begin{array}{l}.99436 \\
.98006 \\
.97288 \\
.96840 \\
.96530\end{array}$ \\
\hline$E_{u t}\left(x_{1}=0\right)$ & $n=\begin{array}{r}0 \\
1 \\
2 \\
3 \\
4\end{array}$ & $\begin{array}{l}.70647 \\
.06475 \\
.03595 \\
.02561 \\
.02066\end{array}$ & $\begin{array}{l}.77428 \\
.05108 \\
.02713 \\
.01890 \\
.01500\end{array}$ & $\begin{array}{l}.83653 \\
.03755 \\
.01920 \\
.01309 \\
.01034\end{array}$ & $\begin{array}{l}.89437 \\
.02455 \\
.01209 \\
.00809 \\
.00633\end{array}$ & $\begin{array}{l}.94868 \\
.01204 \\
.00573 \\
.00376 \\
.00292\end{array}$ \\
\hline$Q_{u}$ & $\begin{array}{r}n=0 \\
1 \\
2 \\
3 \\
4\end{array}$ & $\begin{array}{l}.8331 \\
.2884 \\
.1737 \\
.1309 \\
.1087\end{array}$ & $\begin{array}{l}.8772 \\
.2863 \\
.1679 \\
.1258 \\
.1051\end{array}$ & $\begin{array}{l}.9142 \\
.2835 \\
.1628 \\
.1210 \\
.1012\end{array}$ & $\begin{array}{l}.9463 \\
.2801 \\
.1580 \\
.1167 \\
.0977\end{array}$ & $\begin{array}{l}.9746 \\
.2766 \\
.1534 \\
.1130 \\
.0948\end{array}$ \\
\hline
\end{tabular}

Tables 2, 3, and 4 give values of the component thermal radiation characteristics $E_{n}(r=0), E_{n}(r=1)$, $E_{n}\left(x_{0}=0\right)$ and $Q_{n}$ for $L / d=2,1$, and $0.5, \epsilon=0.5,0.6$, $0.7,0.8$, and 0.9 , and $n=0,1,2,3$, and 4 . For the sake of a numerical example, assume a cylindrical cavity, $\epsilon=0.7$, where the temperature decreases linearly from a temperature $T_{0}$ at its base to a value $0.9 T_{0}$ at the open end, or, from $(21), f(x)=0.6561+$ $0.2916 y+0.0486 y^{2}+0.0036 y^{3}+0.0001 y^{4}$. For $L / d=2$. from eq (31) and table 4 , we find

$$
\begin{aligned}
\frac{Q}{\sigma T^{4} \pi R^{2}}= & 0.6561 \times 0.9142+ \\
+ & 0.2916 \times 0.2835 \\
+0.048 \times 0.1628+ & 0.0036 \times 0.1210 \\
& +0.0001 \times 0.1012=0.6908
\end{aligned}
$$

which is compared to a value of 0.9142 for the isothermal cavity at $T_{0}$.

\subsection{Conical Cavity}

For the conical cavity, the integral equation takes the form

$$
\epsilon_{a}(y)=\frac{B(y)}{\sigma T_{0}}=\epsilon f(y)+(1-\epsilon) G_{3}\left[\epsilon_{a}(t)\right]
$$

where

$$
T^{4}(y)=T_{0}^{4} f(y)=1+C_{1} y+C_{2} y^{2}+\ldots+C_{m} y^{m}
$$

and $T_{0}$ is the temperature at the apex of the cone, $G_{3}\left[\epsilon_{a}(t)\right]$ is the coefficient of $(1-\epsilon)$ in (15) and $B(y)$ is the radiant emission.

A set of $m$ integral equations is defined by

$$
\begin{gathered}
E_{n}(y)=\epsilon y^{n}+(1-\epsilon) G_{3}\left[\epsilon_{a}(t)\right] \\
n=0,1,2, \ldots, m .
\end{gathered}
$$

Then the apparent emissivity is

$$
\epsilon_{a}(y)=E_{0}(y)+C_{1} E_{1}(y)+\ldots+C_{m} E_{m}(y) .
$$

Evaluation of (34) for substitution in (35) gives an exact solution to (32). An approximation from eq (17) useful within the limits cited in section 3 is

$$
\epsilon_{a}(y)=\frac{\epsilon f(y)}{1-(1-\epsilon) I_{4}(y)} .
$$

\section{Discussion}

Sections 2 and 3 present an analytical treatment by appropriate substitution in integrals which exhibit slope discontinuities or apparent discontinuities or both at critical points, such as the corner of a cylin- 
drical cavity and the apex of a conical cavity. The substitutions (3), (4), (7), and (10) make the appropriate integrands go to zero when evaluated at the critical points and points of slope discontinuity, whereby the awkward behavior of the integrand is practically eliminated. With the transformed problem, as given by (1a), (2a), and (15), accurate numerical results are readily obtained by use of a sufficiently small integration step and the fulfillment of the convergence criterion for the successive iterations.

It may be remarked that the very good agreement found between the numerical results arrived at by extrapolation in references 1,2 , and 3 , and the rigorous analytical formulation given here shows that any error introduced by linear extrapolation was negligible. However, to avoid the uncertainties involved in extrapolation, this paper does provide the equations (isothermal and nonisothermal) in a direct form for computation. The suggested method is equally applicable in other cases of integral equations in which similar discontinuities are encountered.

The nonisothermal cylindrical cavity has been treated by Sparrow [5]. For the same values of the parameters, there is generally good agreement between the numerical results presented in this paper and reference 5. Two assumptions were made in the treatment [5]; namely, (1) the temperature over the base of the cavity was isothermal, and (2) the apparent emissivity over the base was constant. For relatively deep cavities, these assumptions can be considered reasonable, but for shallow cavities there is some question as to the validity of these assumptions. Figures 2,3 , and 4 , for example, show a considerable variation of apparent emissivity $\epsilon_{l l}(r)$ over the base of the cavity.

Although numerical results are not presented in this paper for temperature variations over the base of the cavity, this can be accomplished by evaluation of eqs (25) and (26). Also, numerical results are presented for general linear temperature distributions, but may be evaluated for all temperature distributions that may be represented in a polynomial or transcendental form.

\section{References}

[1] E. M. Sparrow. L. U. Albers, and E. R. G. Eckert. Thermal radiation characteristics of cylindrical enclosures. J. Heat Transfer. Trans. ASME, Series C 84, 73-81 (1962).

[2] E. M. Sparrow and V. K. Jonsson. Radiant emission characteristics of diffuse conical cavities. J. Opt. Soc. Am. 53, 816-821 (1963).

[3] S. H. Lin, Radiant Interchange in Cavities and Passages with Specularly and Diffusely Reflecting Surfaces, Ph. D. Thesis, Department of Mechanical Engineering, University of Minnesota, Minneapolis, Minn., March 1964.

[4] F. J. Kelly and D. G. Moore, A test of analytical expressions for the thermal emissivity of shallow cylindrical cavities, Appl. Opt. 4, 31 (1965).

[5] E. M. Sparrow, Radiant emission charact eristics of nonisothermal cylindrical cavities, Appl. Opt. 4, 41-43 (1965).

(Paper 70C2-224) 


\title{
An Equation for the Local Thermal Emissivity at the Vertex of a Diffuse Conical or V-Groove Cavity
}

\author{
Francis J. Kelly
}

\begin{abstract}
An exact, closed-form expression is derived for the apparent local emissivity of a point at the vertex of a diffuse conical or V-groove cavity. This expression is obtained by a substitution into an approximate equation for the apparent local emissivity of a point within a cavity of any shape. The exact expression depends only on the emissivity of the cavity wall material and the angle factor between the point at the cavity vertex and the cavity opening. The values predicted by this expression agree well with those obtained from a numerical solution of the integral equation characteristic of a diffuse conical cavity.
\end{abstract}

\section{Introduction}

In the past, the radiative transport characteristics of many configurations of diffuse surfaces have been calculated. Usually, these calculations involved the numerical solution of an integral equation or an equiv alent system of linear algebraic equations. In addition to these exact methods, there exist a number of approximate formulas that are useful for determining either the local apparent emissivity of an area within a cavity or the hemispherical apparent emissivity of the cavity opening. ${ }^{1-4}$ Some of these equations were deduced by approximating the kernel of the integral equation mentioned above. ${ }^{1.2}$ Others were obtained by finding approximate expressions for the apparent reflectance of a cavity and then applying Kirchhoff's law to obtain the apparent emissivity.

The author intends to present a closed-form expression for the apparent local emissivity at a point on the wall of a diffuse isothermal conical or V-groove cavity in the limit as the point approaches the vertex of the cavity. This equation shares the exactness of the numerical solutions of the integral equations and the ease of computation of the above mentioned approximations. Incidental to this aim, an approximate equation for the apparent local emissivity of any point on the wall of a diffuse isothermal cavity of any shape will be presented, because the expression for the exact

The author is with the U.S. Naval Ordnance Laboratory, Silver Spring, Maryland; the work described in this paper was done while he was with the National Bureau of Standards.

Received 2 February 1966.

This work was done under the sponsorship of the Marshall Space Flight Center of NASA and of the Air Force Material Laboratory, Research and Technology Divisions, Air Force Systems Command. value of the apparent local emissivity at the vertex of a conical cavity is obtained from the approximate equation by a mere substitution.

\section{The Approximate Equation}

Consider a parallel incident beam of radiation that enters a cavity through its opening and falls on a small area $d A(x)$ centered about a point $x$ on the cavity wall. For a perfect reflector, a fraction $F_{x \rightarrow o}$ of the incident radiation will escape through the opening on the first reflection. $\quad F_{x \rightarrow 0}$ is called an angle factor, geometric factor, or configuration factor. If $\rho$ is the reflectance of the walls, the fraction $\rho F_{x \rightarrow o}$ of the incident beam emerges through the cavity opening after the first reflection, and $\rho\left(1-F_{x-o}\right)$ remains inside as radiant flux to undergo a second reflection. One assumes that after the second and all higher order reflections the radiant flux striking the walls after each reflection is uniformly distributed over the interior of the cavity. Then, since $s / S$ is the ratio of the plane area of the opening of the cavity to the total area of the cavity, opening included, a fraction $\rho^{2}(s / S)\left(1-F_{x \rightarrow 0}\right)$ of the original beam emerges after the second reflection. Likewise, after the third reflection, the fraction $\rho^{2}(s / S)\left(1-F_{x-0}\right) \rho(1-s / S)$ emerges, and after the $n$th reflection, $\rho^{2}(s / S)\left(1-F_{x-0}\right)$ $[\rho(1-s / S)]^{n-2}$ of the original flux emerges. The total fraction of the incident radiation that leaves the cavity is $\rho_{a}(x)$, the apparent local reflectance of $d A(x)$, and is given by the sum of a geometric series,

$$
\begin{aligned}
\rho_{a}(x) & =\rho F_{x-0}+\rho^{2}(s / S)\left(1-F_{x-o}\right)[1+\rho(1-s / S) \\
& \left.+\rho^{2}(1-s / S)^{2}+\ldots\right] \\
& =\rho F_{x-o}+\left\{\rho^{2}(s / S)\left(1-F_{x-o}\right) /[1-\rho(1-s / S)]\right\} .
\end{aligned}
$$

From Kirchhoff's law we write

$$
\epsilon=\alpha=1-\rho,
$$


where $\epsilon$ is the emissivity of the opaque wall material, and $\alpha$ is its absorptance. Also, it has been shown ${ }^{5}$ that

$$
\epsilon_{a}(x)=\alpha_{a}(x)=1-\rho_{a}(x) .
$$

These quantities are defined as follows: the apparent local emissivity $\epsilon_{a}(x)$ is the ratio of the flux per unit area leaving an infinitesimal area $d A(x)$ centered about the point $x$ on the wall of the cavity when the cavity is maintained at a uniform temperature and there is no radiation entering the cavity from any outside source, to the flux per unit area leaving a blackbody radiator maintained at the same temperature. The apparent local reflectance $\rho_{a}(x)$ is the ratio of the flux that escapes from the cavity opening to the total flux entering the cavity in the form of a focused beam incident on an element of area $d A(x)$. The apparent local absorptance $\alpha_{a}(x)$ is just one minus the apparent local reflectance and corresponds to the fraction of the flux that is absorbed by the cavity under the same conditions of irradiation described above.

Substituting Eqs. (2) and (3) into Eq. (1) gives

$$
\epsilon_{a}(x)=\frac{\epsilon\left[1+(1-\epsilon)\left(s / S-F_{x-o}\right)\right]}{\epsilon(1-s / S)+s / S} .
$$

Equation (4) is general and applies to a cavity of any shape.

In an approximation first used by Gouffe, ${ }^{3}$ an expression is given that differs from the above only in that Gouffe's equation has a term involving a ratio of spherical areas rather than the slightly more general $F_{x \rightarrow 0}$ term.

\section{Derivation of the Exact Equation}

The exact expression for the apparent local emissivity at a point on the surface of a conical or $V$-groove in the limit as the point approaches the vertex $t$ of the cavity follows straightforwardly from Eq. (4). One simply notes that the assumption, made in deriving Eq. (4), that the radiation becomes uniformly distributed after the second and higher order reflections does not apply when the first reflection takes place in the neighborhood of the vertex of a conical or V-groove cavity. On the contrary, radiation reflected from an area infinitesimally close to the vertex of the cavity must either pass out through the opening of the cavity or remain in a region infinitesimally close to the vertex. This is because a straight line joining any point on the wall of a cavity with the vertex of the cavity will be perpendicular to a line normal to the cavity wall. Thus, no point on the wall can be irradiated by a point source at the vertex. The infinitesimal area at the vertex can be considered as a point source, because conceptually it can be made arbitrarily small. However, this point source can irradiate a point in a region infinitesimally close to the vertex. It follows, then, that the second and all higher order reflections must take place in a region infinitesimally close to the vertex. After each reflection, a fraction $F_{t \rightarrow o}$ of the reflected radiation escapes from the mouth of the cone, where $F_{t \rightarrow 0}$ is the angle factor between an infinitesimal annular ring at

\begin{tabular}{|c|c|c|c|}
\hline$\epsilon$ & $\begin{array}{c}\text { Cone } \\
\text { angle, } \theta\end{array}$ & $\begin{array}{c}\text { Sparrow } \\
\text { and } \\
\text { Jonsson }\end{array}$ & Eq. (5) \\
\hline \multirow[t]{4}{*}{0.9} & $30^{\circ}$ & 0.998 & 0.998 \\
\hline & $60^{\circ}$ & 0.986 & 0.986 \\
\hline & $90^{\circ}$ & 0.962 & 0.962 \\
\hline & $120^{\circ}$ & 0.932 & 0.932 \\
\hline \multirow[t]{4}{*}{0.7} & $30^{\circ}$ & 0.991 & 0.993 \\
\hline & $60^{\circ}$ & 0.949 & 0.949 \\
\hline & $90^{\circ}$ & 0.867 & 0.868 \\
\hline & $120^{\circ}$ & 0.782 & 0.782 \\
\hline \multirow[t]{4}{*}{0.5} & $30^{\circ}$ & 0.980 & 0.983 \\
\hline & $60^{\circ}$ & 0.895 & 0.889 \\
\hline & $90^{\circ}$ & 0.740 & 0.739 \\
\hline & $120^{\circ}$ & 0.610 & 0.606 \\
\hline \multirow[t]{4}{*}{0.3} & $30^{\circ}$ & 0.953 & 0.961 \\
\hline & $60^{\circ}$ & 0.767 & 0.774 \\
\hline & $90^{\circ}$ & 0.557 & 0.548 \\
\hline & $120^{\circ}$ & 0.394 & 3.397 \\
\hline
\end{tabular}

Table I. Computed Apparent Emissivities of Cone Tip

the cavity vertex and disk stretched across the cavity opening It follows that $s / S$ and $F_{x \rightarrow 0}$ should be replaced by $F_{t-o}$ in Eq. (4) to give an exact expression for the local apparent emissivity at the vertex of a conical or V-groove cavity

$$
\epsilon_{a}(t)=\epsilon /\left[\epsilon+(1-\epsilon) F_{t-o}\right]
$$

Equation (5) is identical with an expression derived by a somewhat different approach by $\mathrm{C}$. H. Page of the National Bureau of Standards, whose work appeared as an internal report in 1956 but was never published. ${ }^{7}$

The angle factor $F_{t \rightarrow o}$ between the vertex $t$ and the opening of a conical cavity has been found by evaluating the following equation [Eq. (16) of Ref. 6]:

$$
\begin{gathered}
F_{t-o}=\lim _{\substack{x \rightarrow 0 \\
\xi \rightarrow 1}} F_{r-d}(x, \xi)=\lim _{\substack{x \rightarrow 0 \\
\xi \rightarrow 1}}\left[\xi^{2} \sin (\theta / 2) / 2 x\right] \\
\\
\quad \times\left(\partial F_{d-d} / \partial x\right)=\sin ^{3}(\theta / 2),
\end{gathered}
$$

where

$$
\begin{aligned}
& F_{d-d}(\xi, x)= \\
& \frac{x^{2}+\xi^{2}-2 x \xi \cos ^{2}(\theta / 2)-|\xi-x|\left[(x+\xi)^{2}-4 x \xi \cos ^{2}(\theta / 2)\right]^{\frac{1}{2}}}{2 \xi^{2} \sin ^{2}(\theta / 2)} .
\end{aligned}
$$

In the above equations, $x$ and $\xi$ are dimensionless lengths giving the position of points on the conical cavity surface. They are chosen so that $x$ and $\xi$ equal zero at the vertex of the cone and $x$ and $\xi$ equal one at the opening of the cone. The angle $\theta$ is the vertex angle of the cone. The quantity $F_{r-d}(x, \xi)$ is the angle factor between an annular ring on the surface of the cone located at $x$ and a circular disk $d$ stretched across the cone at $\xi$. The quantity $F_{d-d}(\xi, x)$ is the angle factor between a disk stretched across the cone at $\xi$ and one stretched across the cone at $x$. The angle factor $F_{t \rightarrow 0}$ is the product of the angle factor between an element of area on the wall of a spherical cavity that has the same depth and same circular opening as the cone, 
and a factor $\sin (\theta / 2)$ that takes the orientation of the conical cavity's surface element into account. Both methods lead to the value $F_{t \rightarrow 0}=\sin ^{3}(\theta / 2)$. Likewise, one can show that for the $V$-groove cavity, $F_{\imath \rightarrow 0}=$ $\sin ^{2}(\theta / 2)$, where $\theta$ is the vertex angle of the groove.

In Ref. 6, calculated data were presented from which the apparent local emissivity in the neighborhood of the vertex of a conical cavity can be determined. These can be compared with the values predicted by Eq. (5), using $F_{t-o}=\sin ^{3}(\theta / 2)$ (Table I). The agreement between the two sets of values is good.

The author wishes to thank D. G. Moore, J. C. Richmond, D. Gross, W. D. Hayes, H. E. Clark, H. E.
Robinson, and especially A. C. Kelly for helpful conversations.

\section{References}

1. H. Buckley, Phil. Mag. 4, 753 (1927); 6, 447 (1928); 17, 576 (1934).

2. Z. Yamauti, Comité International des Poids et Mesures, Procès-Verbaux 16, 243 (1933).

3. A. Gouffé, Rev. Opt. 24, 1 (1945).

4. E. W. Treuenfels, J. Opt. Soc. Am. 53, 1162 (1963).

5. F. J. Kelly, J. Res. Natl. Bur. Std. 69B, 165 (1965).

6. E. M. Sparrow and V. K. Jonsson, J. Opt. Soc. Am. 53, 816 (1963).

7. C. H. Page, Natl. Bur. Std. Rept. 1673 (15 May 1952), Natl. Bur. Std. Project 1002-43-1030. 


\title{
The Apparent Thermal Radiation Properties of an Isothermal V-Groove With Specularly Reflecting Walls
}

\author{
Richard B. Zipin*
}

(July 26, 1966)

\begin{abstract}
The reflection of a parallel beam of light incident in a $V$-groove is treated for the case when the walls are specularly reflecting. Methods of calculating apparent reflectances and absorptances are given. Experiments have been performed to test the theoretical calculations, and the agreement has been found to be satisfactory.
\end{abstract}

Key Words: Reflectance, specular walls, thermal radiation, $V$-grooves.

\section{Introduction}

Recently there has been a great deal of work reported in the literature on the problem of predicting the apparent thermal radiation properties of cavities and rough surfaces when the properties of ideally smooth surfaces of the same material are known. An interesting problem that has not been previously treated very completely is reflection by an isothermal $\checkmark$-groove, whose walls are specularly reflecting. Sparrow and Lin [3] ${ }^{1}$ have reported some results for the effective absorptance of such a groove. Their method of analysis is followed here using a simpler procedure, extended somewhat, and results are given for effective directional reflectance.

The main limitation on the results reported here is due to the assumption that the $V$-groove has specular walls. This assumption is valid when the wavelength of the incident radiation is much less than the characteristic dimensions of the groove, and at the same time much greater than the characteristic dimensions of the roughness of the groove walls. Experimental results reported by the author [4] have shown this to be true.

\section{Reflection Within a V-Groove With Specularly Reflecting Walls}

The problem to be considered is illustrated in figure 1. The $V$-groove has an opening angle of $2 \theta$, a depth $h$, and is infinite in the direction normal to the plane of incidence. The method of calculation is based on the fact that light reflected from a specular surface appears to come from an image located

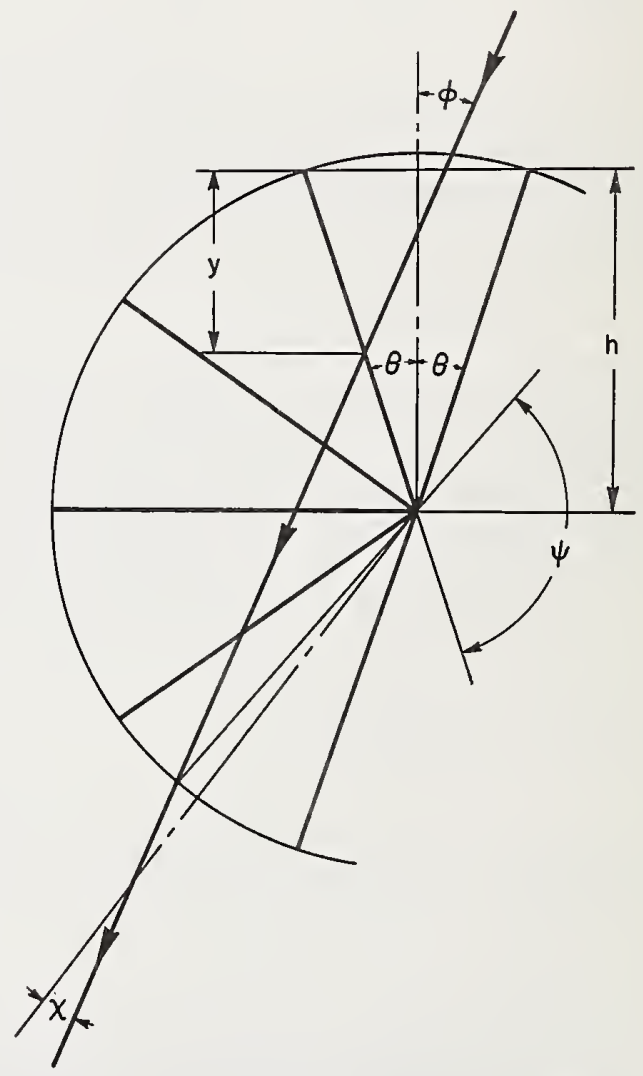

Figure 1. A single incident beam being specularly reflected within a V-groove.

"Present address: The Sheffield Corporation, Dayton, Ohio.

' Figures in brackets indicate the literature references at the end of this paper. 
behind the surface at a distance equal to the distance of the source from the front of the specular surface [2]. The number of reflections, $n$, that an incident beam makes at the walls of the groove before it leaves determines the apparent thermal radiation properties exhibited by the groove, and this may be determined by counting the number of mirror images of the first surface that are crossed by an undeviated beam. A single beam is incident in the groove at an acute angle $\phi$ measured with respect to the normal to the groove opening, and leaves the groove at an acute angle $\chi$ measured with respect to the same normal.

The number of reflections is given by

$$
n=\left[\frac{\psi}{2 \theta}\right]
$$

where $\psi$ is defined in figure 1 , and the square brackets indicate that the quantity enclosed within them should be rounded off to the nearest higher integer. This is because there can never be a fraction of a reflection, and all beams incident in a groove must make at least one reflection.

There are two separate cases to be distinguished here:

(1) If $\phi>\theta$, an incident beam can only strike the far wall on entering the groove, and,

(2) if $\phi<\theta$, an incident beammay strike either wall on entering the groove.

For beams incident on the far wall, the law of sines gives

$$
\frac{h / \cos \theta}{\sin (\theta+\phi)}=\frac{\left(h-y_{f}\right) / \cos \theta}{\sin \left(\pi-\psi_{f}-\theta-\phi\right)}
$$

where $y$ is the vertical distance from the groove opening at which a beam strikes the wall, and the subscript $f$ indicates a quantity associated with a beam incident on the far wall. Now,

$$
\psi_{f}=\pi-\theta-\phi-\sin ^{-1}\left\{\left(1-\frac{y_{f}}{h}\right) \sin (\theta+\phi)\right\}
$$

and by geometry

$$
\chi_{f}=(-1)^{n}\left(\pi-\phi-2 n_{f} \theta\right) .
$$

For beams incident on the near wall, the subscript $n$ is used, and

$$
\frac{h / \cos \theta}{\sin (\theta-\phi)}=\frac{\left(h-y_{n}\right) / \cos \theta}{\sin \left(-\pi+\psi_{n}+\theta+\phi\right)}
$$

so that

$$
\psi_{n}=\pi-\theta-\phi+\sin ^{-1}\left\{\left(1-\frac{y_{n}}{h}\right) \sin (\theta-\phi)\right\}
$$

and in this case

$$
\chi_{n}=(-1)^{n-1}\left(\pi+\phi-2 n_{n} \theta\right) .
$$

A value of $\chi$ obtained from either of eqs (4) and (7) which is equal to or greater than $90^{\circ}$ is obviously forbidden unless $\phi=90^{\circ}$.

By the use of eq (1), with eqs (3) and (4) or eqs (6) and (7), the reflections undergone by any beam incident in the groove may be determined.

If the groove is fully illuminated and $\phi<\theta$ both walls will have energy directly incident on them, but if $\phi>\theta$ only the far wall will receive energy by direct incidence. In the latter case the far wall will be directly illuminated from $y=0$ to $y=y_{\max }$, where $y_{\max }$ is the value of $y$ at which the incident beam that just grazes the lip of the groove on entering strikes the far wall. To calculate $y_{\max }$, the law of sines is used again, and

$$
\frac{h / \cos \theta}{\sin (\pi-\theta-\phi)}=\frac{\left(h-y_{\max }\right) / \cos \theta}{\sin (\phi-\theta)} .
$$

Therefore,

$$
y_{\max }=\frac{2 h \cos \phi \sin \theta}{\sin (\theta+\phi)}
$$

and when $\phi=\theta, y_{\max }=h$ as required.

All beams incident on both the near and the far walls of the groove will undergo the same number of reflections and leave the groove at the same angle as either the $y=0$ or the $y=y_{\max }$ beams. ${ }^{2} \quad$ The subscripts 0 and max denote quantities related to the beams incident at $y=0$ and $y=y_{\max }$ respectively, and $y_{c}$ will denote the critical value of $y$ at which $n$ changes from $n_{0}$ to $n_{\max }$ and $\chi$ changes from $\chi_{0}$ to $\chi_{\max }$. $A$ beam incident at $y_{c}$ will just graze the lip of the groove on leaving. For the far wall, the law of sines is again used, and

$$
\frac{h / \cos \theta}{\sin (\theta+\phi)}=\frac{\left(h-y_{c, f}\right) / \cos \theta}{\sin \left(\pi-2 n_{0, f} \theta-\theta-\phi\right)}
$$

so that

$$
y_{c, f}=h\left\{1-\frac{\sin \left(2 n_{0, f} \theta+\theta+\phi\right)}{\sin (\theta+\phi)}\right\} .
$$

\footnotetext{
${ }^{2}$ For the near wall, $\}_{\max }$ must be $h$.
} 
For the near wall,

$$
\frac{h / \cos \theta}{\sin (\theta-\phi)}=\frac{\left(h-y_{c, n}\right) / \cos \theta}{\sin \left(\pi-2 n_{0, n} \theta-\theta+\phi\right)}
$$

and so

$$
y_{c, n}=h\left\{1-\frac{\sin \left(2 n_{0, n} \theta+\theta-\phi\right)}{\sin (\theta-\phi)}\right\}
$$

If $\phi=0$, there is no distinction between the near and far walls, and eqs (11) and (13) give identical results.

The angle. $\chi$ will be said to be negative if it is measured in the same direction from the normal as $\phi$ is, and positive if it is measured in the opposite direction. When $\phi>\theta, \chi$ is positive if $n$ is even and negative when $n$, is odd.

\section{The Calculation of the Apparent Thermal Radiation Properties}

If the average reflectance of the groove walls is $\rho$, and the walls are opaque, then the average absorptance of the walls is $\alpha=1-\rho$. When a single narrow beam of energy per unit time $I_{i}$ is incident in the groove at an angle $\phi>\theta$, it will strike the far wall, be reflected $n$ times within the groove, and then leave at an angle $\chi$. $\chi$ will have one of the two values $\chi_{0}$ or $\chi_{\max }$, according to the value of $y$ at which the beam is incident.

On the first reflection of the beam, $(1-\rho) I_{i}$ is absorbed and $\rho I_{i}$ is reflected. On the second reflection, $(1-\rho) \rho I_{i}$ is absorbed and $\rho^{2} I_{i}$ is reflected. This continues until the final, $n$th reflection, when $(1-\rho) \rho^{n-1} I_{\text {i }}$ is absorbed and $\rho^{n} I_{i}$ is reflected. The energy-rate of the beam leaving the groove after the $n$ reflections is therefore $I_{r}=\rho^{\prime \prime} I_{i}$.

If the effective reflectance is defined as the ratio of the energy reflected at the angle. $\chi$ to the energy incident at the angle $\phi$, it is given by

$$
\rho_{\mathrm{efr}}(\phi, \chi)=\frac{I_{r}}{I_{i}}=\rho^{\prime \prime}
$$

where the value of $n$ used must correspond to the value $\chi$ considered.

If $\phi<\theta$, a single narrow beam may strike either the near or the far wall of the groove. In this case $\chi$ will have one of the four values, $\chi_{0, f}, \chi_{\max , f}, \chi_{0, n}$. or $\chi_{\max }, \ldots$ Equation (14) may be used to find the effective reflectance.

Now consider the groove to be fully illuminated. If $\phi>\theta$, energy is incident on the far wall from $y=0$ to $y=y_{\max }$. Let the incident beam carry energy per unit time $I_{i}$ per unit width in the plane of incidence and normal to its direction of propagation, or equivalently $I_{i} \sin (\theta+\phi)$ per unit width along the groove wall, so that the total energy incident in the groove per unit time is

$$
I(\phi>\theta)=I_{i} \sin (\theta+\phi) y_{\max } / \cos \theta .
$$

Recalling eq (9), and substituting for $y_{\max }$, this becomes

$$
I(\phi>\theta)=2 I_{\mathrm{i}} h \cos \phi \tan \theta .
$$

The fraction of this energy that leaves after $n_{0}$ reflections at an angle $\chi_{0}$ is $\frac{y_{c}}{y_{\max }} \rho^{n_{0}}$, while the fraction that leaves after $n_{\max }$ reflections at $\chi_{\max }$ is $\left(1-\frac{y_{c}}{y_{\max }}\right) \rho^{\prime \prime} \max$.

As before, the effective reflectance at an angle $\chi$ will be defined as the ratio of the energy leaving the groove in the direction $\chi$ to the total energy incident in the groove. In this case we have two such reflectances,

$$
\rho_{\mathrm{eff}}\left(\phi, \chi_{0}\right)=\frac{y_{c}}{y_{\max }} \rho^{n_{0}}
$$

and

$$
\rho_{\text {eff }}\left(\phi, \chi_{\max }\right)=\left(1-\frac{y_{c}}{y_{\max }}\right) \rho^{\prime \prime \max }
$$

Since the total energy reflected by the groove is the sum of these two reflectances multiplied by the incident energy, the total energy absorbed is

$$
\left\{1-\rho_{\mathrm{eff}}\left(\phi, \chi_{0}\right)-\rho_{\mathrm{eff}}\left(\phi, \chi_{\max }\right)\right\} I(\phi>\theta)
$$

and so the effective absorptance is

$$
\alpha_{\text {eff }}(\phi>\theta)=1-\rho_{\text {eff }}\left(\phi, \chi_{0}\right)-\rho_{\text {eff }}\left(\phi, \chi_{\max }\right) .
$$

This expression is only a function of $\phi$.

If $\phi<\theta$ and the groove is fully illuminated, energy is incident on both walls from $y=0$ to $y=h$. If the incident beam carries energy per unit time $I_{i}$ per unit width in the plane of incidence and normal to its direction of propagation, then it will have $I_{i} \sin (\theta+\phi)$ per unit width along the far wall and $I_{i} \sin (\theta-\phi)$ per unit width along the near wall. The total energy incident on the far wall is $I_{i} \sin (\theta+\phi) h / \cos \theta$ and on the near wall is $I_{i} \sin (\theta-\phi) h / \cos \theta$, so that the total energy incident in the groove is

$$
I(\phi<\theta)=2 I_{i} h \cos \phi \tan \theta=I(\phi>\theta) .
$$

From the above it is easily seen that the fraction of the total encrgy incident in the groove that is directly incident on the near wall is $\frac{\sin (\theta-\phi)}{2 \sin \theta \cos \phi}$ and the fraction of this energy that leaves after $n_{0, n}$ reflections at $\chi_{0, n}$ is $\frac{y_{(, n}}{h} \rho^{n_{0, n}}$ and that leaves after $n_{\text {max }, n}$ reflec- 


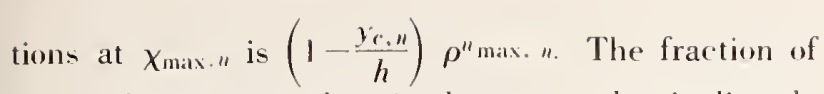
the total energy incident in the groove that is directly incident on the far wall is $\frac{\sin (\theta+\phi)}{2 \sin \theta \cos \phi}$. The effective reflectances are, therefore, given by

$$
\rho_{\mathrm{ent}}\left(\phi, \chi_{1, n}\right)=\frac{\sin (\theta-\phi)}{2 \sin \theta \cos \phi} \frac{y_{c, n}}{h} \rho^{\prime \prime 0, n}
$$

$$
\rho_{\text {eff }}\left(\phi, \chi_{\text {max. }}\right)=\frac{\sin (\theta-\phi)}{2 \sin \theta \cos \phi}\left(1-\frac{y_{c, n}}{h}\right) \rho^{\prime \prime m a x . ~} n
$$

$$
\rho_{\mathrm{eff}}\left(\phi, \chi^{0, f}\right)=\frac{\sin (\theta+\phi)}{2 \sin \theta \cos \phi} \frac{y_{i, f}}{h} \rho^{\prime \prime} 0, f
$$

and

$$
\rho_{\text {exft }}\left(\phi . \chi_{\max . f}\right)=\frac{\sin (\theta+\phi)}{2 \sin \theta \cos \phi}\left(1-\frac{y_{c \cdot f}}{h}\right) \rho^{\prime \prime m a x . f} .
$$

These results depend on the angles only, and are independent of $h$.

The effective absorptance is

$$
\begin{aligned}
& \alpha_{\text {eff }}(\phi<\theta)=1-\rho_{\text {eff }}\left(\phi, \chi_{01}{ }_{n}\right)-\rho_{\text {eff }}\left(\phi, \chi_{\text {max. n }}\right) \\
& -\rho_{\mathrm{etr}}(\phi, \chi \cdot f)-\rho_{\mathrm{eff}}\left(\phi, \chi_{\max . j}\right) .
\end{aligned}
$$

If $\phi=\theta$, eq (26) gives the same result as eq (20) for the $\phi>\theta$ case. This is expected because when $\phi=\theta$. no energy is actually directly incident on the near wall of the groove. If $\phi=0$, there can be no distinction between the two walls of the groove, and they will both have the same quantity of energy incident on them. In this case

$$
\rho_{\mathrm{efr}}\left(\phi, \chi_{0, n}\right)=\rho_{\mathrm{efr}}\left(\phi, \chi_{0, f}\right)
$$

and

$$
\rho_{\text {eff }}\left(\phi, \chi_{\max , n}\right)=\rho_{\text {eff }}\left(\phi, \chi_{\max } . \jmath^{\prime}\right)
$$

\section{Comparison of the Theoretical and Experi- mental Results}

Measurements have been made to test the theoretical results presented above. The angular distribution of energy reflected in the plane of incidence by carefully prepared $V$-grooved surfaces, which were obtained from Bausch and Lomb, Inc., was studied as a function of angle of incidence. Details of the apparatus and the specimens may be found elsewhere [4].

Figure 2 shows the positions of the peaks in the reflected energy distribution as calculated with eqs

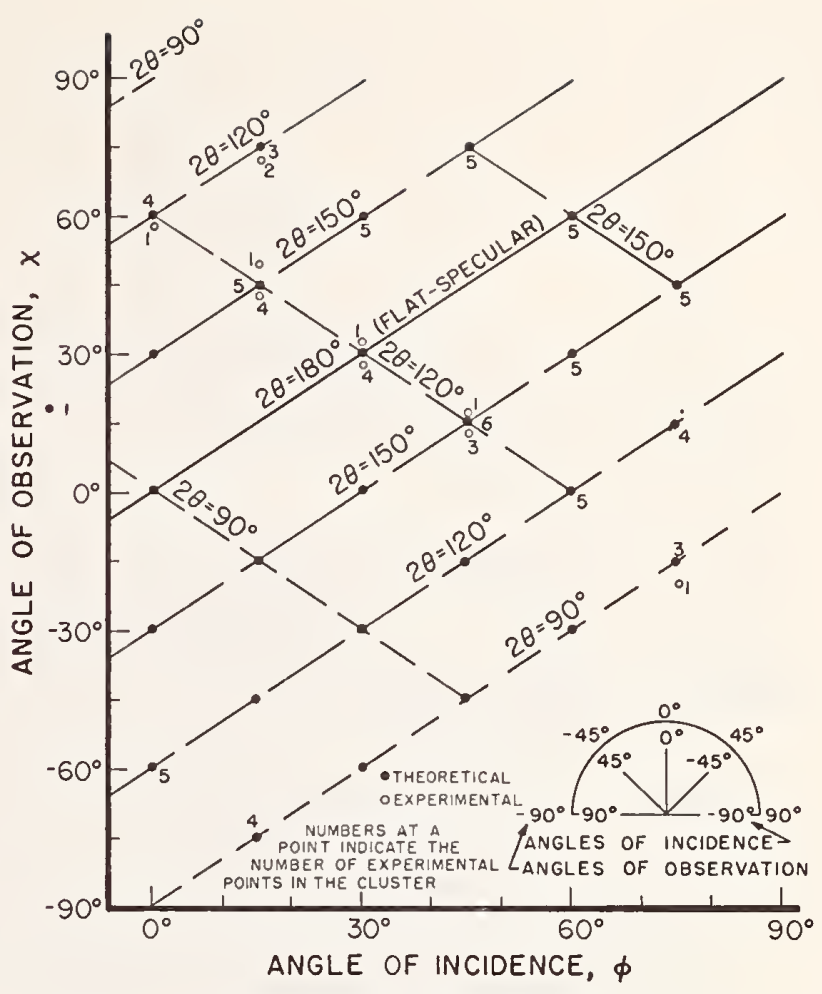

FIgURE 2. The positions of the peaks of energy reflected by the V-grooves with specular walls.

(4) and (7), for $V$-grooves having included angles of $90^{\circ}, 120^{\circ}$, and $150^{\circ}$. The experimentally determined positions are also shown in the figure, and they agree very well with the theoretical results.

In figures 3,4, and 5, the theoretical and experimental values of the reflectance ratio, $R$. at the peaks of the reflected energy distribution are plotted as a function of angle of incidence, for the three values of groove angle. The reflectance ratio is defined as

$$
R=\frac{\rho_{\text {eff }}\left(\phi_{i}, \chi_{i}\right)}{\rho_{\text {eff }}\left(\phi_{j}, \chi_{j}\right)}
$$

In each figure, the point at which the experimental value of $R$ was made unity by choosing the measured value of $\rho_{\text {efr }}\left(\phi_{j}, \chi_{j}\right)$ as the reference is $;$-idicated. At these points the theoretical values of $\rho_{\text {eff }}\left(\phi_{j}, \chi_{j}\right)$ are unity.

Due to the nature of the apparatus, many of the reflected peaks could not be observed. The boxes in the figures are used to indicate which theoretical point the experimental points are to be compared with, wherever there is a possibility of confusion. The theoretical points at which the reflectance ratio vanishes at either grazing observation $\left(\chi=90^{\circ}\right)$ for nongrazing incidence, or at nongrazing observation for grazing incidence $\left(\phi=90^{\circ}\right)$ are the limiting cases obtained from eqs (4) and (7), but peaks cannot exist exactly at these angles, as is proven by Beckmann [1]. 


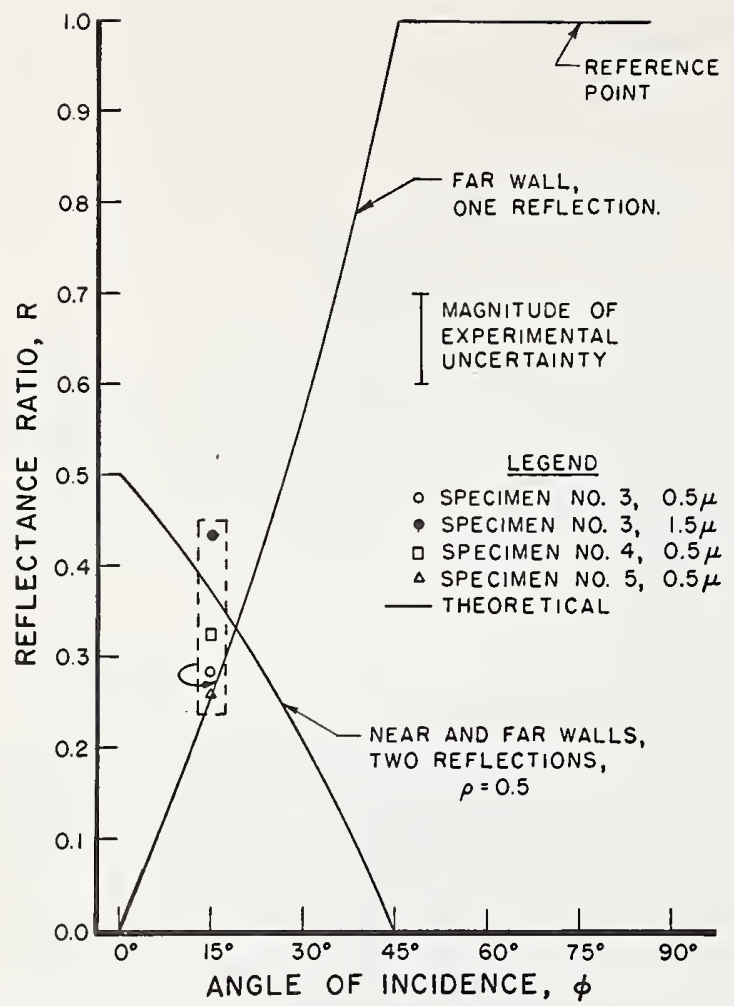

Figure 3. Comparison of theory and experiment for V-grooves with specular walls, $2 \theta=90^{\circ}$.

Except for a few cases of large deviations between the experimental and the theoretical values of $R$. figures 3,4 , and 5 show that reasonable agreement exists between them. The agreement is much better at $0.5 \mu$ than at $1.5 \mu$, since the assumption that the walls of the grooves reflect specularly becomes better as the wavelength is decreased. Theoretical curves for cases of more than one reflection were calculated using $\rho=0.5$.

\section{Discussion}

The assumption that the average reflectance of the groove wall surfaces is known is only rigorous when the wall reflectance is not a function of the angle of incidence on the wall. If the reflectance is a function of the angle of incidence on the wall, the calculations would have to be repeated, and terms such as $\rho^{n}$ would be changed to a product of $n$ different values of $\rho$, each one the reflectance of the groove wall at the angle of incidence of one of the $n$ reflections. Except for this the calculations are valid for monochromatic radiation of any wavelength, or for total radiation if the properties of the groove wall are independent of wavelength. ${ }^{3}$

"It should be noted that the only wavelength dependence in the analysis is due to the value of $\rho$ used.

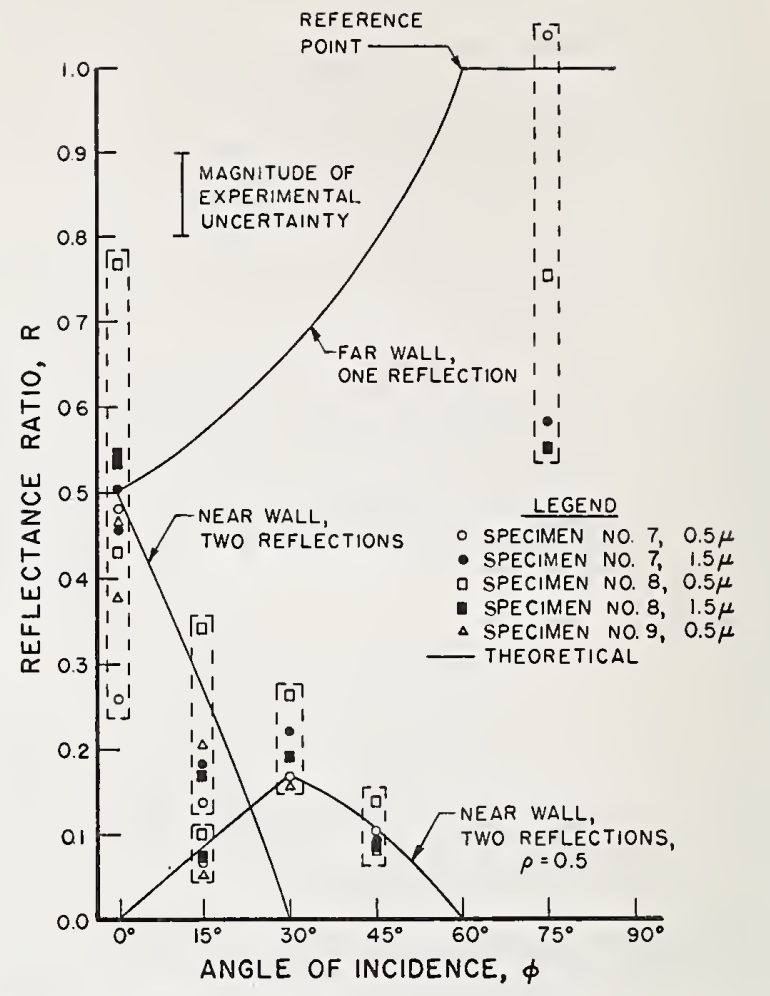

Figure 4. Comparison of theory and experiment for V-grooves with specular walls, $2 \theta=120^{\circ}$.

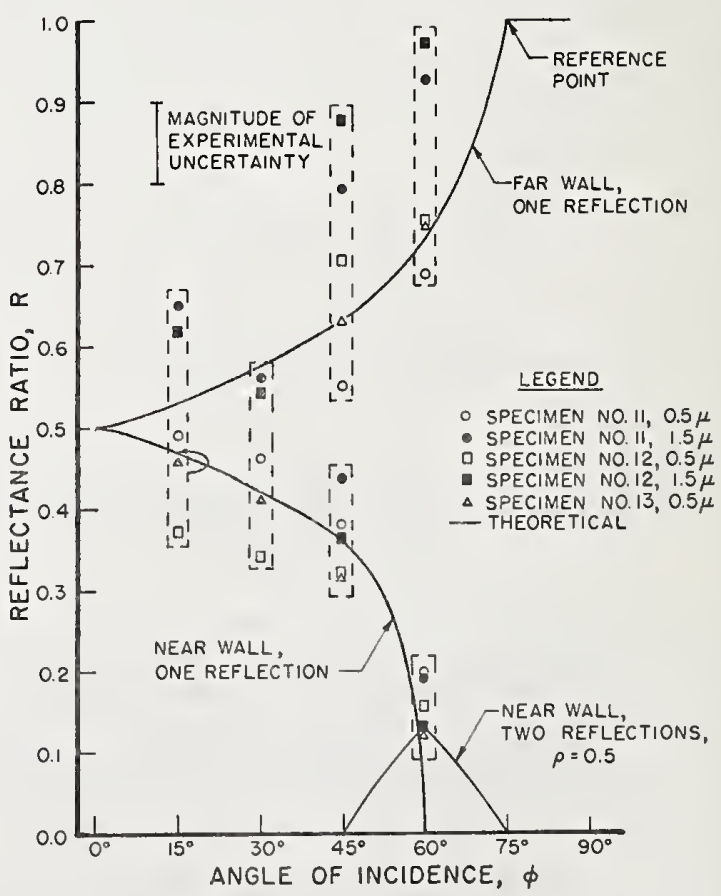

FlGURE 5. Comparison of theory and experiment for V-grooves with specular walls, $2 \theta=150^{\circ}$. 
If the groove walls have properties that are not independent of wavelength and calculations are desired for total radiation, another complication enters the situation. Let $\rho(\lambda)$ be the wall reflectance as a function of wavelength, and $I(\lambda)$ be the spectral distribution of the source, then any function $G(\rho(\lambda))$ can be calculated for total radiation by the use of the following expression:

$$
\{G(\rho(\lambda))\}_{\text {total }}=\frac{\int G(\rho(\lambda)) I(\lambda) d \lambda}{\int I(\lambda) d \lambda}
$$

where the integrations are performed over all wavelengths emitted by the source.

Any desired calculations can now be made for the apparent absorptance or reflectance when the walls are specular. It should be noted that sometimes an equation will yield absurd results which have no physical significance. Should this occur, the appropriateness of the parameters under consideration should be reexamined to ascertain their validity for the case under consideration.

A conclusion which may be drawn from the results is that the effective absorptance increases and the effective reflectances decrease as $\phi$ decreases. In the case $\phi>\theta$, there will always be a region at the vertex of the groove which will be in shadow, i.e., it will not be illuminated either directly or due to internal reflections. In the case $\phi<\theta$ the whole internal surface of the groove will be illuminated.

The author expresses his thanks to his major professor, Dr. Y. S. Touloukian for his advice and encouragement during the course of this work, and to the Purdue Research Foundation for support in the form of a David Ross Grant. Thanks are also due Dr. D. P. DeWitt for suggestions and stimulating discussions.

\section{Nomenclature}

$G=$ Any radiation function, as used in eq (30).

$h=$ The depth of a $\mathrm{V}$-groove.

$I=$ Energy per unit time carried by a beam of radiation. $n=$ The number of reflections within a specular $\checkmark$-groove.

$R=$ Reflectance ratio.

$y=$ A distance measured vertically from the lip of a $\checkmark$-groove.

$\alpha=$ Absorptance.

$\theta=$ The half-angle of a $\mathrm{V}$-groove.

$\lambda=$ Wavelength.

$\rho=$ Reflectance.

$\phi=$ The angle of incidence.

$\chi=$ An angle of reflection.

$\psi=$ An angle defined in figure 1 .

[.] = Brackets indicating a quantity to be rounded off to the nearest higher integer.

\section{Subscripts}

$c=$ A critical value.

eff = An effective or apparent value of some quantity. $f, n=\mathrm{A}$ quantity associated with the far or near wall of a $V$-groove, respectively.

$i, j=$ Indexes.

$i, r=$ A quantity associated with an incident or reflected beam, respectively.

$\max =\mathrm{A}$ quantity associated with the maximum value of $y$ on the far wall of a $V$-groove.

$0=$ A quantity associated with $y=0$.

\section{References}

[1] Beckmann, P.. and Spizzichino, A., The Scattering of Electromagnetic Waves from Rough Surfaces, p. 33 (Perganion and Macmillan, 1963).

[2] Sparrow, E. M., Eckert, E. R. G., and Jonsson, V. K., An enclosure theory for radiative exchange between specularly and diffusely reflecting surfaces, Trans. ASME, J. Heat Transfer 84C, No. 4, 294-300 (1962).

[3] Sparrow E. M., and Lin, S. H., Absorption of thermal radiation in a V.(iroove cavity. Int. J. Heat Mass Transfer 5, 1111-1115 (1962).

[4] Zipin, R. B., The Directional Spectral Reflectance of WellCharacterized Symmetric V-Grooved Surfaces, Ph. D. Thesis, Purdue University, 1965.

(Paper 70C4-238) 


\title{
41-AN APPROACH TO THERMAL EMITTANCE STANDARDS
}

\author{
BY JOSEPH C. RICHMOND, WLLIAM N. HARRISON, AND FREDERICK J. SHORTEN
}

NATIONAL BUREAU OF STANDARDS, WASHINGTON, D.C.

\begin{abstract}
A double-beam ratio-recording infrared spectrometer was modified to record directly the normal spectral emittance of strip specimens that are heated by passing a current through them. A laboratory blackbody furnace and a hot specimen at the same temperature serve as sources for the respective beams. Temperature equalization is achieved by means of a differential thermocouple. Automatic data-processing equipment corrects for "zero-line" and " $100 \%$-line" errors on the basis of previously-recorded calibrations, and also computes from the spectral data, as the measurement progresses, total emittance or absorptance for radiant energy having any known spectral distribution of flux.
\end{abstract}

This paper describes the development of equipment and procedures ${ }^{1}$ for the measurement of normal spectral emittance of metals and other materials that can be heated by passing a current through them, at temperatures in the range of $800^{\circ}$ to $1400^{\circ} \mathrm{K}$, and over the wavelength range of approximately 1 to $15 \mu$. The equipment includes a data-processing attachment to automatically correct for calibration errors of the spectroradiometer, to record the corrected spectral emittance on punched paper tape in a form suitable for direct entry into an electronic digital computer, and to compute a single value for total emittance from the recorded spectral emittance.

In only a limited sense does the report cover the development of thermal emittance standards as such, although the selection and calibration of working standards of normal spectral emittance was included among the objectives. In a broader sense, it points out the need for standardization in the entire field of thermal emittance measurements, and in the field of terminology it makes several definite recommendations.

This paper is intended to be an elementary treatise covering the many elements involved in the production of thermal emittance standards.

1 The work was done under the sponsorship and with the financial assistance of the Aeronautical Systems Division, U.S. Air Force.

\section{DEFINITIONS}

Several important items of nomenclature in the field of electromagnetic radiation have not been firmly established. Different meanings are ascribed to the same words by different individuals, and some of the conflicting definitions have been adopted by different technical and scientific organizations of national scope. The terminology used in this investigation follows that of Worthing (ref. 1) in that the word-ending "ivity" is reserved for the properties of materials, and "ance" for the properties of specimens. The word-ending "ion," as in reflection, absorption, and transmission, is applied to acts or processes, rather than properties.

All bodies are constantly emitting electromagnetic radiation as a result of the thermal vibration of the particles, atoms and molecules comprising the body; this process is known as thermal emission, and the rate of such emission per unit area is often referred to as thermal emissive power.

Radiant flux is the rate of flow of radiant energy.

Emittance is a property of a specimen; it is the ratio of the emitted radiant flux per unit area to that of a blackbody radiator at the same temperature and under the same conditions.

A blackbody absorbs all radiation incident upon it and at any given temperature has the maximum possible rate of thermal emission. 
An ideal blackbody is a complete enclosure with opaque walls at a uniform temperature. The amount, and also the geometric and spectral (or wavelength) distribution of radiant flux emitted, per unit area, by a blackbody at a given temperature can be computed from the Stefan-Boltzmann relationship, Lambert's cosine law and the Planck radiation law, respectively. Laboratory blackbody furnaces which radiate energy are approximate blackbodies, because the opening through which the radiant energy is emitted destroys the ideal character. The extent to which they deviate from the ideal depends upon such factors as the relative size of the opening, the emittance of the inside surface, and the thermal gradients that may be present in the cavity. The rate of thermal emission of any real specimen is less than that of the blackbody at the same temperature.

Emissivity is a special case of emittance; it is a fundamental property of a material, and is measured as the emittance of a specimen of the material which has an optically smooth surface, ${ }^{2}$ and is sufficiently thick to be opaque.

Reflectance is a property of a specimen; it is the ratio of reflected radiant flux to incident radiant flux.

Reflectivity is a special case of reflectance; it is a fundamental property of a material, and is measured as the reflectance of a specimen of the material that has an optically smooth surface, and is sufficiently thick to be opaque.

Transmittance is a property of a specimen; it is the ratio of transmitted radiant flux to incident radiant flux.

Absorptance is a property of a specimen; it is the ratio of absorbed radiant flux to incident radiant flux.

Absorptivity is a special case of absorptance; it is a fundamental property of a material and is measured as the absorptance of a specimen of the material that has an optically smooth surface and is sufficiently thick to be opaque.

A specimen is opaque when none of the radiant energy incident upon it is transmitted through it. All materials theoretically require infinite thickness to become opaque. In prac-

\footnotetext{
2 Any surface contamination may alter the thermal radiation properties, hence a contamination-free surface is also essentisl.
}

tice, a specimen is considered to be opaque when the transmitted radiant flux is too small to be detected, a condition which occurs at a very small thickness for some materials, and at great thicknesses for others. The thickness required for a specimen to be opaque also varies with the wavelength of the incident flux, for any one material.

All of the properties that are defined above vary with the spectral (or wavelength) distribution of the radiant flux. All are temperature dependent, some strongly so, and others only moderately. All of the properties are influenced by the angular distribution of flux in the radiant energy. All of the properties that pertain to specimens are influenced by the surface texture of the specirnen, and by the thickness, unless the specimen is opaque.

Since the emission, reflection and absorption characteristics of a specimen are influenced by the direction of propagation, relative to the surface, of the emitted, incident, or reflected energy, and by the spectral distribution of radiant flux, most of the terms defined above must be qualified in order to convey precise meanings. For reflection, the direction of propagation of both the incident and reflected radiation must be specified. Specular, referring to reflection, means in the direction of mirror reflection. Diffuse, referring to reflection, means in all possible directions. Emission in all possible directions is referred to as hemispherical. When limited directions of propagation are involved, the word directional may be used. Normal is a special case of directional, and means in a direction normal to the surface. For other directions, the angle of deviation from the normal is stated. The terms normal and directional apply strictly to beams of parallel radiation, but are also used for beams contained within a small solid angle, in which case the direction of propagation is taken as that of the axial ray. Since all laboratory sources, specimens and receptors are of finite size, significant deviations from parallelism exist in all laboratory instruments. Hence the source and field apertures should be specified to define the extent of such deviations from parallelism.

Radiant energy having a stated wavelength, or more precisely, that is within a narrow 
wavelength interval centered on a specified wavelength, is referred to as spectral. Spectral emittance at a stated temperature is frequently plotted as a function of wavelength to produce a spectral emittance curve. The word total as used to modify terms describing thermal emission characteristics, means that the modified term pertains to the integral of rates of spectral emission at all wavelengths.

Some examples of the qualified expressions that are required to convey precise meanings are: "total hemispherical emittance at $500^{\circ} \mathrm{K}$," "normal spectral emittance at $1400^{\circ} \mathrm{K}$ in the wavelength interval 1 to $15 \mu$," and "spectral diffuse reflectance from normal illumination over the wave-length interval 0.3 to $3.0 \mu$."

\section{CONSIDERATIONS IN SELECTION OF EQUIP- MENT AND PROCEDURE}

In general, accurate absolute measurcments of radiant energy are not easy to make. Comparative measurements of radiant energy can be made more readily. For this reason it appears logical to base emittance determinations on direct comparison of the radiant flux from a specimen at a given temperature to that from a comparison standard.

Since any comparison standard other than a blackbody furnace must be calibrated against a blackbody furnace at each temperature of test, and even then may change with continued use, it is desirable to use a blackbody furnace as the comparison standard.

The total radiant flux density of a blackbody varies as the fourth power of its absolute temperature. Thus, even a small error in temperature measurement could lead to a large error in emittance. Emittance, however, and particularly spectral emittance, varies only slightly with small changes in temperature. The critical requirements with respect to temperature control in the determination of emittance are (a) that the thermal gradients over the surface of the specimen and comparison standard be reduced as far as praticable, and (b) that the standard and specimen be kept at the same temperature within acceptable tolerances. A thermocouple produces an EMF that is a function of the temperature difference between the hot and cold junctions. Thus, small temperature differences can be measured more accurately by a single direct measurement by means of dif- ferent thermocouple systems. Thus, it is desirable to use a differential thermocouple with suitable instrumentation to maintain the specimen and the comparison standard at the same temperature.

The spectral emittance curve of a solid material heated to a temperature below its melting point does not show the sharp peaks and ralleys that are characteristics of the emission and absorption curves of solutions and gaseous materials. Hence, prism monochromators with relatively wide slits are suitable for use in spectral emittance determinations, in spite of their rather poor wavelength resolution, because they pass relatively large amounts of radiant flux for measurement over a side spectral range. The use of a variable-width slit is desirable so that in wavelength regions of low radiant flux the amount passed can be increased by broadening the wavelength band accepted for measurement. Thus, the available radiant flux in the comparison beam can be maintained at a level commensurate with the sensitivity of the detector.

\section{DEVELOPMENT OF EQUIPMENT FOR NORMAL SPECTRAL EMITTANCE MEASUREMENTS}

The general plan that was followed in developing equipment for determination of normal spectral emittance was to use a doublebeam ratio-recording infrared spetroncter with a laboratory blackbody furnace and a heated specimen as the respective sources for the two beams. With this arrangement the instrument will record directly the normal sprectral emittance of the specimen, if the following conditions are met within acceptable tolerances: (1) the temperature of the specimen must be the same as that of the blackbody furnace; (2) the optical path length in the two beams must be equal or, preferably, the instrument must operate in a nonabsorbing atmosphere or a vacuum, in order to minimize the effects of differential atmosphcric absorption in the two beams; (3) front-surface mirror optics must be used throughout, exrept for the prism, and equivalent optical elements must be used in the two beams in order to minimize attenuation of the beams by absorption in the optical elements; and (4) the source and field apertures of the two beams must be equal in order to ensure that radiant flux in the two beams compared by the apparatus will pertain to 
equal areas of the sources and equal solid angles of emission.

It soon became apparent that the conditions specified under 2,3 , and 4 were not met within sufficiently close tolerances to permit direct recording of normal spectral emittance with the equipment described. It was necessary to use a second, or reference, laboratory blackbody furnace, identical to the first, or comparison, blackbody furnace, in order to correct for these errors.

\section{Description of Spectrometer}

The Perkin-Elmer model 13 spectrometer used for the measurements is equipped with a wavelength drive that provides automatic scanning of the spectrum of radiant flux, and a slit servomechanism that automatically opens and closes the slits to minimize the variations of radiant flux in the comparison beam. Three prisms were available: (1) fused silica, to cover the spectral range of 0.25 to $3.5 \mu$, (2) sodium chloride to cover the spectral range 0.7 to $15 \mu$, and (3) cesium bromide to cover the spectral range of 15 to $38 \mu$. Several photomultiplier detectors were available for use in the spectral range of 0.25 to $1.0 \mu$, a lead sulfide detector for use in the spectral range 1.0 to $2.0 \mu$, a vacuum thermocouple with sodium chloride window for use in the spectral range 1 to $15 \mu$, and a vacuum thermocouple with cesium bromide window for use in the spectral range 1 to $40 \mu$.

The source optics of the instrument were modified by incorporation of an external optical system, which is the standard Perkin-Elmer transfer optical system, used with the hohlraum reflectrometer. Two $90^{\circ}$ folds in a vertical plane were introduced in the specimen beam, to raise the axial ray of the beam 9 inches above the optical plane of the spectrometer. The comparison blackbody furnace was mounted in a fixed position to act as the source of the comparison beam, and the specimen furnace and reference blackbody furnace were mounted side by side on a movable plate attached to a slide, in positions such that they could be brought alternately into position to serve as source for the specimen beam, by sliding the plate against fixed stops on the lathe bed. A sketch of the complete optical path is shown in figure 41-1, and a photograph of the external optical system with furnaces in place and cover removed is shown in figure 41-2.

It was found by experience that when the

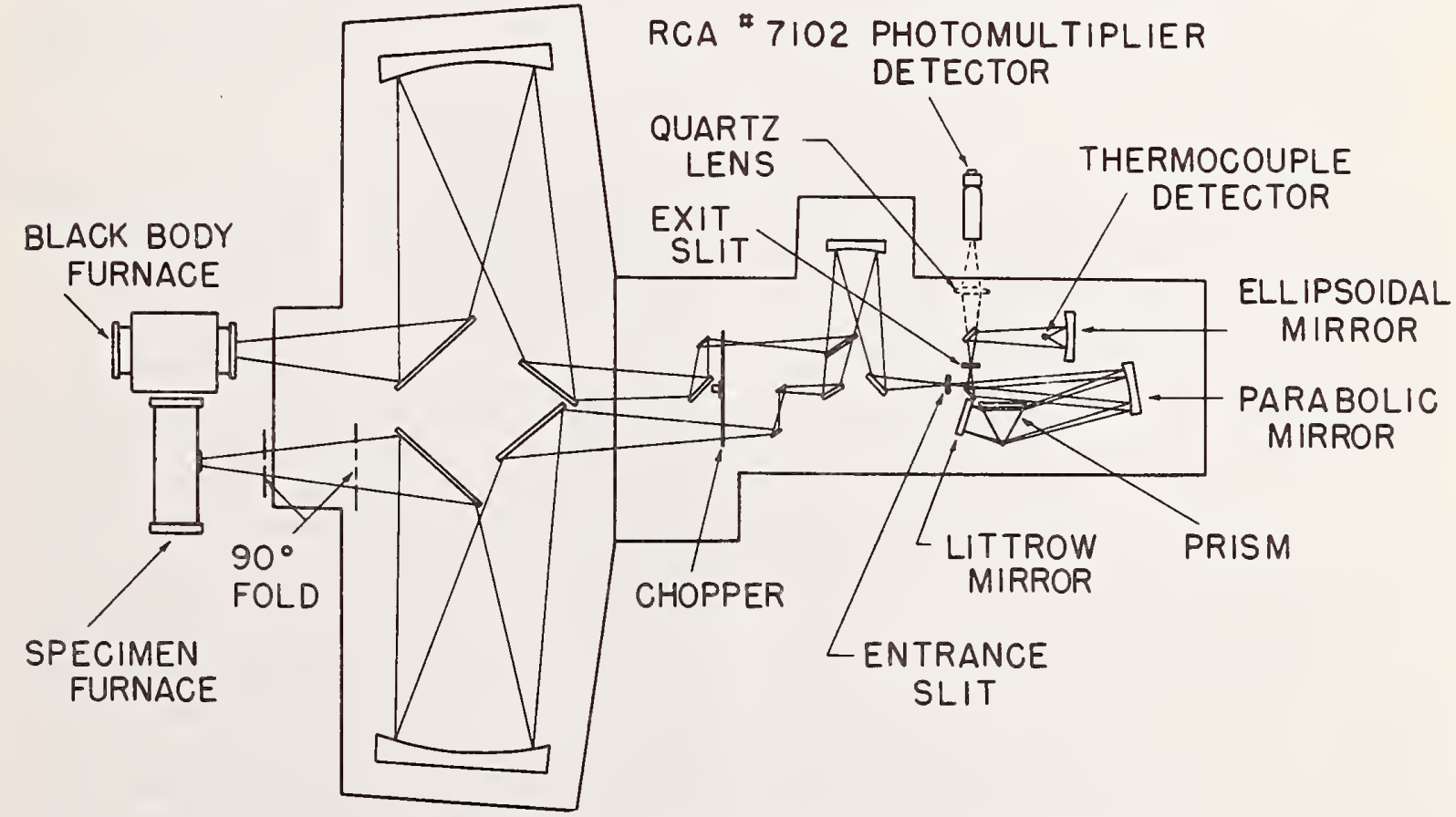

Figure 41-1.-Optical paths of the spectrophotometer. 


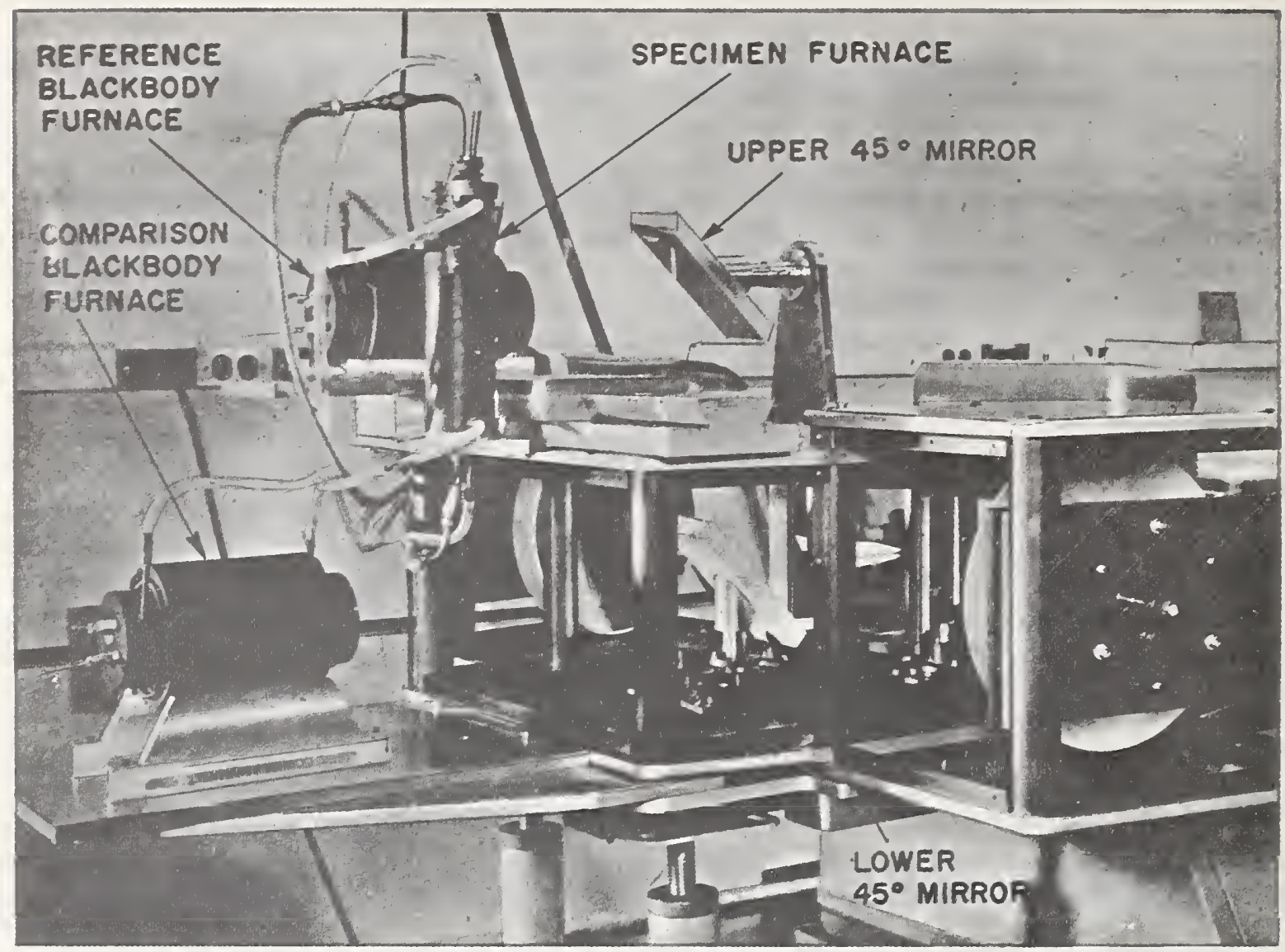

FIGURE 41-2.-External optical system of the spectroradiometer. The comparison blackbody furnace remains in position to act as the source for thn comparison beam. The specimen is in position to act as source for the specimen beam, but can be replaced by the reference blackbody furnace by shifting the movable table on the slide.

two blackbody furnaces were used as sources for the respective beams of the spectrometer, deviations of the recorded " $100 \%$ curve" occurred at the wavelength intervals within which there is significant absorption of radiant energy by water vapor and carbon dioxide in the atmosphere. Such absorption should be identical in both beams if the spectral distribution of flux and path lengths are identical. However, the two beams are separated in time, passing through the monochromator alternately at a frequency of $13 \mathrm{cps}$. Since the spectrum is being traversed continuously, one energy pulse is displaced relative to the other on the wavelength scale, by a very small amount. The effect of this displacement is negligible over most of the range, where the slope of the energy-wavelength curve is not large. But at the wa velengths near those of maximum absorp- tion, the energy-wavelength curves become very steep, and the slight spectral displacement of the successive energy pulses can produce significant deviations in the ratios which are continuously recorded on the instrument.

In order to reduce the effects of absorption by atmospheric water vapor and carbon dioxide, the entire external optical system of the normal spectral emittance equipment, including the blackbody furnaces and specimen furnace, was enclosed in a Lucite box. Gas-tight connecting passages were designed to join (1) the Lucite box enclosing the external optics, (2) the metal cover for the chopper assembly, (3) the metal cover for the combining optics, and (4) the metal cover for the monochromator. Thus, the entire length of both optical paths in the instrument was enclosed in a single system of interconnecting housings that was nearly 
gas-tight. Humidity in the enclosure was monitored and recorded by means of a humidigraph which records temperature and humidity. Immediately before a test the enclosure was purged with "super-dry" nitrogen, free from carbon dioxide, which was fed into the enclosure through several inlets, at a slow, constant rate, so that the enclosure was maintained at a slight positive pressure relative to the surrounding atmosphere. Tests were not started until the recorded humidity was less than $5 \%$.

\section{Blackbody and Specimen Furnaces}

Two small laboratory blackbody furnaces were designed and built. Figure $41-3$ is a sectional drawing of a furnace. The core was a Nichrome $\mathrm{V}$ casting, $1 \frac{3 / 16}{10}$ inches in diameter and 4 inches long. The cavity was $3 / 4$ inch in diameter and $3 \frac{3 \%}{8}$ inches deep, threaded with 13 threads to the inch. The threads were cast in place. The Nichrome $\mathrm{V}$ oxidizes to produce a high-emittance surface in the cavity. The furnace was heated by means of a platinum-20\% rhodium winding. There were two taps on the heating coil approximately one inch from each end. Shunts across the taps and leads were used to reduce thermal grudients in the cavity.
The aperture in the cavity was reduced to a slit approximately $1 / 4$ by $3 / 4$ inches in size by means of a Nichrome cover plate.

The temperature of the blackbody furnace was measured by means of a platinum, platinum-10\% rhodium thermocouple, the bare bead of which extended about $1 / 4$ inch into the cavity from the rear. The thermocouple leads were insulated from the core by high-alumina refractory tubing, which was surrounded by a platinum tube that was grounded, in order to prevent pickup by the thermocouple of spurious signals due to electrical leakage from the winding.

The efficiency of the blackbody furnace, computed from the Gouffé (ref. 2) equation, and assuming that the interior of the cavity is at uniform temperature, and is a completely diffuse reflector, was better than 0.999. Both of these assumptions are known to be only approximations, so that the actual efficiency was somewhat less than the computed value. The thermocouple bead in the back of the cavity cannot be seen, even with an optical pyrometer, when the furnace is in operation, which indicates that the efficiency is very high.

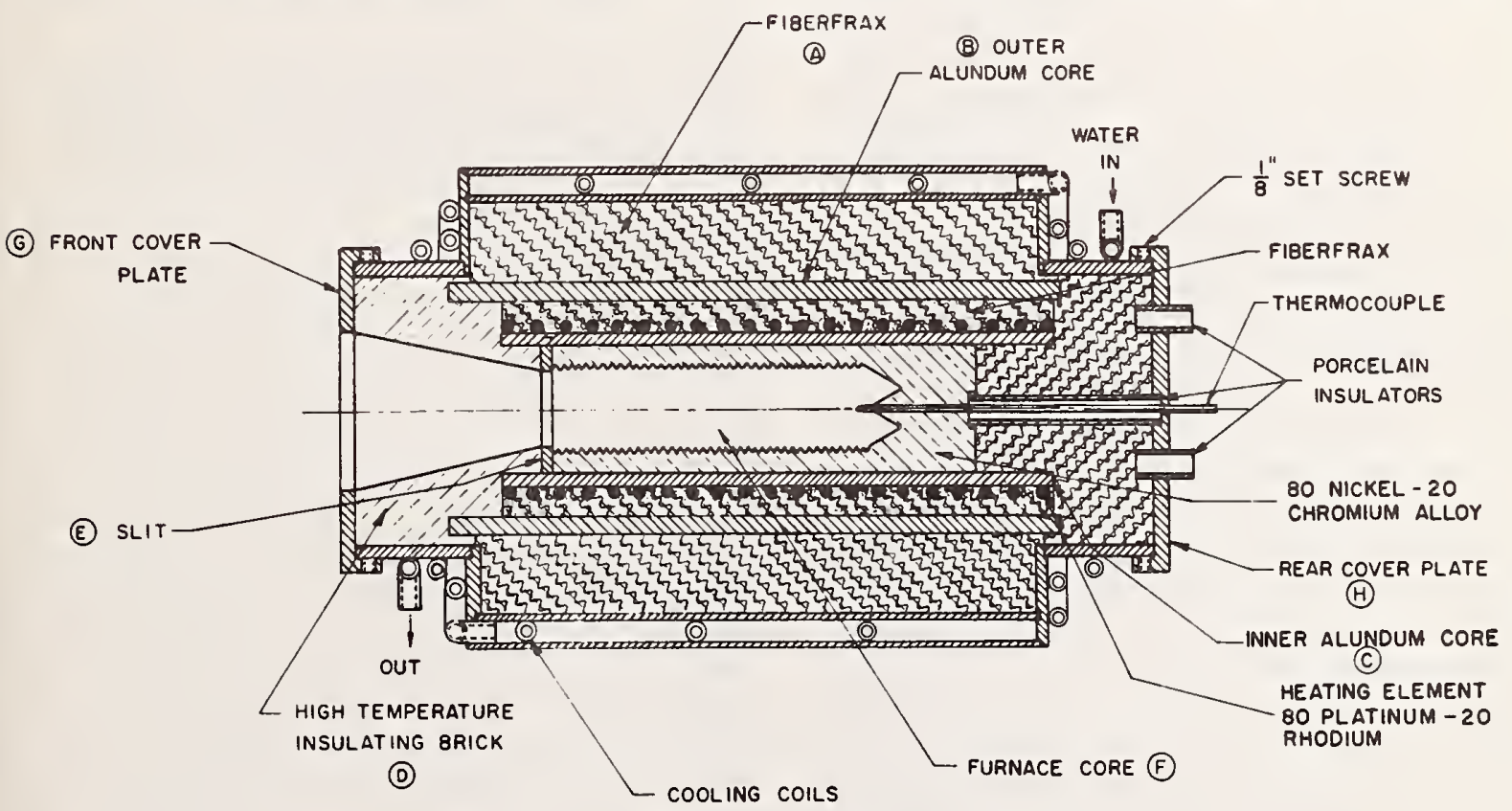

Frgore 41-3.-Sectional drawing of blackbody furnace. 
The specimens were strips of metal, $1 / 4$ inch wide by 8 inches long, of any convenient thickness in the range of approximately 0.010 to 0.060 inch. A sketch of the specimen furnace is shown in figure 41-4. The outer shell was water cooled, and its inner surface was of Inconel that had been threaded with a No. 80 thread and then oxidized in air at a temperature above $1350^{\circ} \mathrm{K}$ for 6 hours to produce a surface having a reflectance of less than 0.05 at the operating temperature of the water-cooled walls. The specimen was positioned off-center in the cylindrical enclosure, so that any radiant flux reflected from the walls would be reflected twice before hitting the specimen.

The electrodes were of water-cooled copper and were insulated from the brass end caps of the furnace by means of bakelite sleeves. The lower electrode fitted loosely in the hole through the end cap to permit the specimen to expand without buckling when heated. The specimen was viewed through a window about $3 / 4$ by 1 inch in size. A viewing window insert reduced the opening to about $1 / 4$ by $3 / 4$ inch.

Adjustable baffles above and below the viewing window were used to reduce convection and the resulting temperature fluctuations and thermal gradients. Adjustable telescoping cylindrical platinum reflectors surround the specimen at each end. These were used to reduce heat loss at the ends of the specimen, and hence the thermal gradient along the specimen.

\section{Tomperature Control Equipment}

The power input to the comparison blackbody furnace was adjusted manually by means of an autotransformer. The heater winding of the furnace had two taps, located approximately one inch from each end of the winding. Variable resistors were connected as shunts across the taps and from the taps to the input leads, as indicated in the wiring diagram, figure 41-5. Adjustment of these resistors pernitted variation of the power distribution along the cavity of the furnace, by ineans of which thermal gradients in the cavity could be reduced as far as practicable. It was found by experience that the heat capacity of the blackbody furnace was large enough to practically eliminate temperature fluctuations due to momentary voltage fluctuations in the power supply. A cold furnace could be brought to $1400^{\circ} \mathrm{K}$ and stabilized

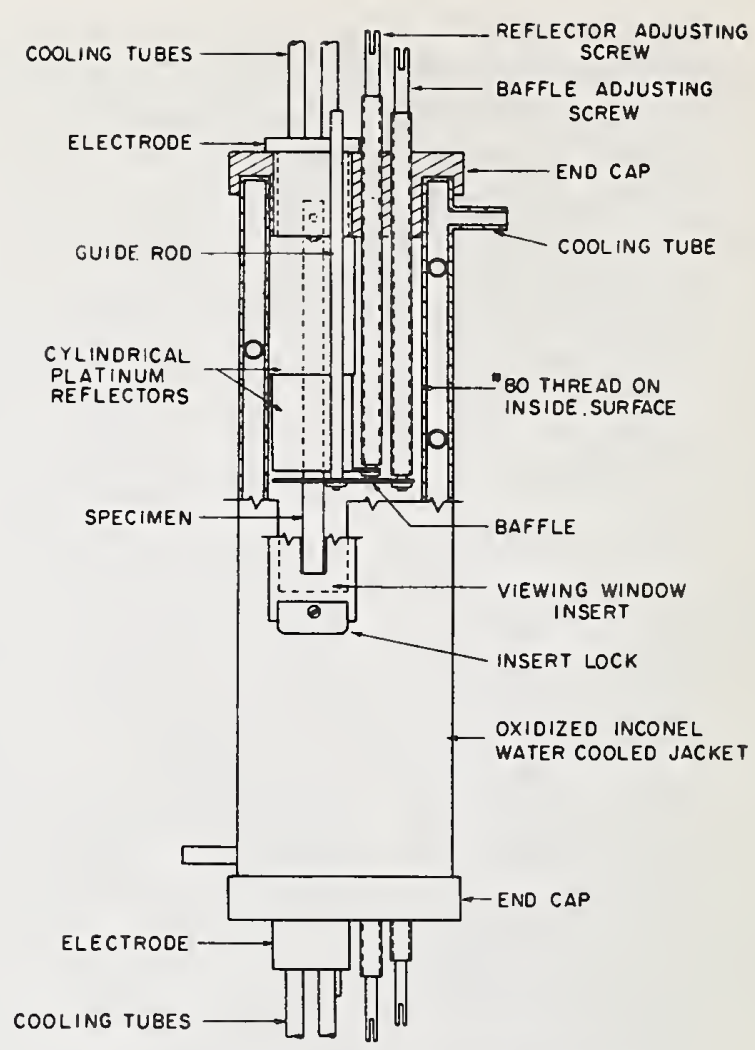

Figure 41-4,--Specimen enclosure.

at that temperature in about 6 hours, and only about $30 \mathrm{~min}$ was required to achieve stability after a temperature change of up to about $30^{\circ} \mathrm{K}$. Normally the blackbody furnaces were heated overnight before use.

The power supply for the reference blackbody furnace was identical to that shown in figure 41-5, except that when the temperature was to be controlled a saturable core reactor was substituted for the autotransformer, as is described below.

The temperature of the specimen (or reference blackbody furnace) was controlled to that of the comparison blackbody furnace, within narrow limits, by means of a differential thermocouple. One bead of the differential thermocouple was in the cavity of the comparison blackbody furnace, and the other was attached to the back of the specimen, in the center of the area being viewed, as described later in this report. The signal from the differential therınocouple was amplified by a $d-c$ amplifier and fed to a center-zero recordercontroller. The output of the recorder- 


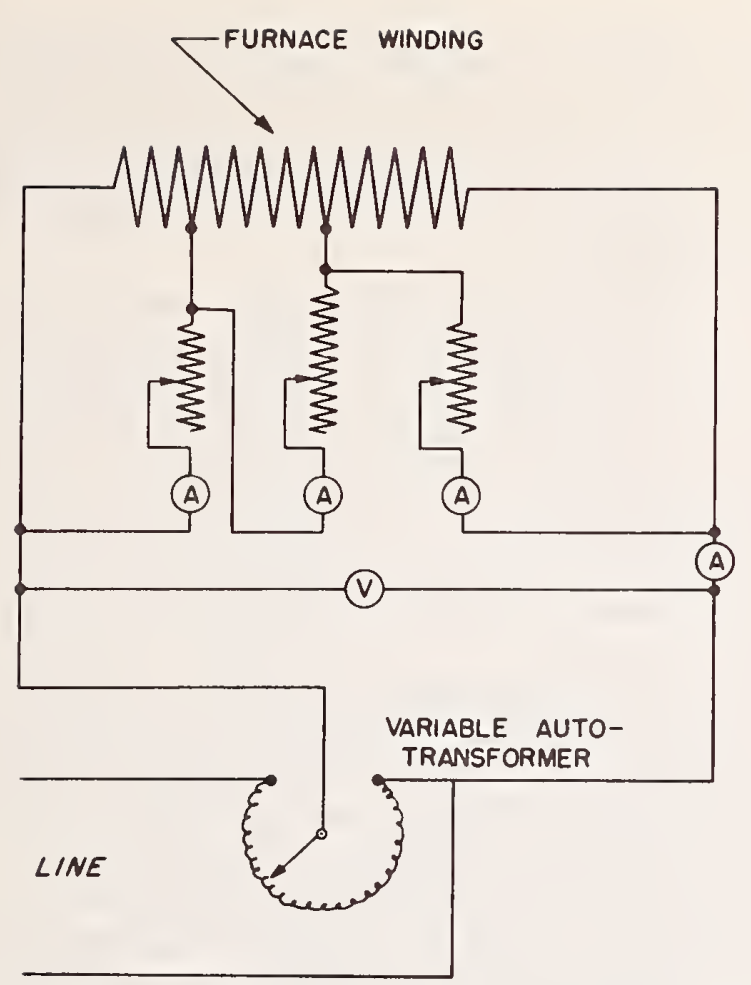

Frgure 41-5.-Wiring diagram for power supply to comparison blackbody furnace.

controller is proportional to the distance between the control pointer and the recorder pointer on the scale, which is in turn proportional to the temperature difference between blackbody furnace and specimen. The output of the recorder-controller was fed to a currentactuating-type controller, the output of which was separated into three portions, each of which could be varied as desired. One portion was proportional to the input signal, one was proportional to the first time derivative of the input signal, and one was proportional to the second time derivative of the input signal. The output of the current-actuating-type control was fed to the coil of a saturable core reactor which varied the power input to the specimen. It was possible to reduce undershoot and overshoot of the controlled temperature by proper adjustment of the three components in the output of the currentactuating-type control. A block diagram of the temperature control equipment is shown in figure $41-6$.

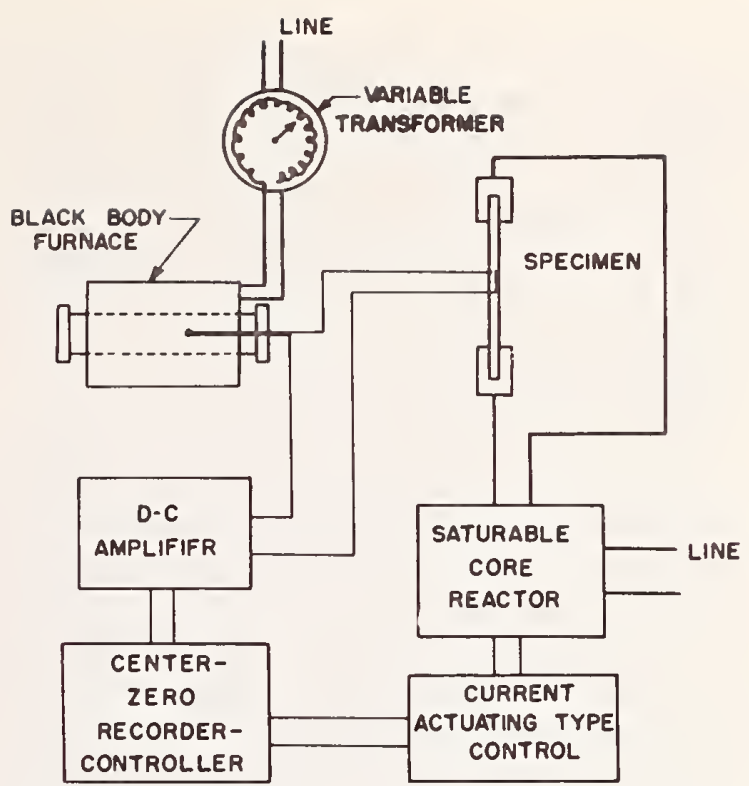

Figure 41-6.-Block diagram of temperature control equipment. The controller operates from a signal produced from a differential thermocouple.

With the described control equipment it has been possible to hold the temperature of a specimen as indicated by the welded thermocouple to well within $\pm 1^{\circ} \mathrm{K}$ of that of the comparison blackbody furnace, and the temperature of the reference blackbody furnace to within $\pm 0.5^{\circ} \mathrm{K}$ of that of the comparison blackbody furnace.

\section{CALIBRATION OF EMITTANCE EQUIPMENT Wavelength Calibration of Monochromator}

A complete wavelength calibration was made of the spectrometer with the fused silica and sodium chloride prisms, respectively, mounted in the monochromator. In calibrating the equipment with the fused silica prism, emission spectra of a helium arc and a mercury arc, and absorption spectra of a didymium glass and a polystyrene film were recorded. The emission and absorption peaks having known wavelengths were identified in the respective curves, and for each peak the observed wavelength drum position at which the peak occurred was plotted as 8, function of the known wavelength of the peak (ref. 3). A total of 66 such points, at wavelengths in the range 0.24 to $2.2 \mu$, was plotted, and a smooth curve was drawn between the points to produce the calibration curve. 
A similar procedure was followed in calibrating the equipment with the sodium chloride prism mounted in the monochromator. In this case, the atmospheric absorption curve replaced the absorption curve for didymium glass. A total of 52 points, at wavelengths from 0.4 to $15 \mu$, was plotted to produce the calibration curve.

\section{Callbration for Linearity of Response}

All of the work done with the spectrometer had been based on the assumption that the response of the instrument (the height of the recorded emittance curve above the recorded "zero curve") was linear with (1) the radiant flux (within the varying wavelength interval encompassed by the exit slit) passing through the monochromator, when the instrument is operated in single-beam mode, or (2) the ratio of the fluxes (within the varying wavelength interval encompassed by the exit slit) in the respective beams that pass through the monochromator when the instrument is operated double-beam in ratio mode. This assumption had not been checked, and a confirmation of (1) and (2) above was considered desirable.

\section{Sitit Linearity Calibration}

The entrance and exit slits of the monochromator open and close simultaneously, and both are controlled at the same width by the slit micrometer. Because of this construction, when a true image of a source is formed at the entrance slit of the monochromator, somewhat larger than the slit at its maximum width, the radiant flux reaching the detector from the monochromator varies as the square of the slit width. This relationship was used to check the linearity of detector response when the instrument is used single-beam in direct mode (item (1) above). A single blackbody furnace, at $1400^{\circ} \mathrm{K}$, was used as a source, and each series of measurements was made at a number of central wavelengths throughout the sensitive range of the thermocouple detector. Experimental results were in conformity with the theoretical relationship. In every case, when the height of the recorder curve was plotted against the square of the slit width, the points obtained with the sodium chloride prism and thermocouple detector fell on a straight line intersecting the origin, within the error of measurement.
Similar measurements were also made at several wavelengths when the spectrometer was operated with the fused silica prism and the lead sulfide detector. Again, experimental results were in conformity with the theoretical relationship.

When the spectrometer was used with the fused silica prism and 1P21 detector, appreciable deviations from the theoretical relationship were found at first, but after careful realignment of the monochromator optics, all of the points again fell on a straight line, within experimental error.

\section{Calibrations With Sector-Disk Attenu- ATOR}

A sector-disk attenuator for use in calibrating the normal spectral emittance equipment was designed and built. The attenuator consists of a variable speed motor, 0 to $4000 \mathrm{rpm}$, with an attenuator disk mounted on its shaft.

Five interchangeable attenuator disks were prepared, having nominal transmissions of $75,50,25,12.5$ and $5.0 \%$, respectively. The disks were machined from sheet aluminum, 0.065 inch thick. The $5 \%$ disk is 10 inches in diameter, and has four equally spaced radial notches, each $4.5^{\circ}$ wide, extending inward from the rim for 3 inches. The other disks are 9 inches in diameter, and have eight notches each, extending inward from the rim for $2 \frac{1}{6}$ inches. The notches are $33 \%^{3}{ }^{\circ}$ wide in the $75 \%$ disk $22 \%^{\circ}$ wide for the $50 \%$ disk, $11 y_{4}^{\circ}$ wide for the $25 \%$ disk, and $5 \%^{\circ}$ wide for the $12.5 \%$ disk.

The attenuator is normally operated at about $1300 \mathrm{rpm}$, and the direction of rotation is opposite to that of the chopper of the spectrometer. At this speed the beam is interrupted at a frequency of more than 85 cps by the $5 \%$ disk, and more than $170 \mathrm{cps}$ by the other disks. The chopper in the spectrometer interrupts the beam at a frequency of $13 \mathrm{cps}$. No coupling has been observed between the attenuator and chopper frequencies.

The attenuator disks were calibrated in the Engineering Meteorology Section of NBS by measuring the angular width of the notches and blades on each disk, at two positions corresponding to the radial positions at which the top and bottom, respectively, of the interrupted beam strike the disks when in use. The unobstructed area of the disk between the two circles was 
computed as a percentage of the total area between the circles, with the results shown below:

$\begin{gathered}\text { Nominal } \\ \text { Transmiltance, } \\ \%\end{gathered}$
75 $\ldots \ldots \begin{gathered}\text { Measured } \\ \text { Unobstrucled Area, } \\ \%\end{gathered}$

The measured values were assigned to the respective sector-disk attenuators.

In the next group of experiments, made for additional checking of item (1) above, the sector-disk attenuator was introduced in to the beam near the blackbody furnace. Each series of measurements was made at a single wavelength, a single slit width, and a single gain setting. In each case the gain was adjusted to give a reading of slightly less than 100 units on the strip chart without the attenuator. The attenuator was then introduced into the beam, and operated with each of the disks in turn. The height of the recorder curve was plotted against the previously measured fraction of the beam passed by the attenuator. In every case the points fell on a straight line passing through the origin, indicating lineur detector response for every combination of slit width and wavelength tested, with both the sodium chloride and fused silica prisms, and with the thermocouple, as well as the lead sulfide and photomultiplier detectors.

In the preceding experiments for which the instrument was being used in direct mode, with single beam, the height of the recorder curve varied linenrly with the signal produced by the detector. This signal was proportional to the radiant flux passing through the monochromator. Normally, however, the instrument is used in ratio mode, with double beam. In this case, the height of the recorder curve should vary linearly with the ratio of the two signals produced by the detcetor wien it is receiving flux alternately from the respective beains.

In order to check item (2) above, the two blackbody furnaces, controlled very closely to the same temperature (about $1400^{\circ} \mathrm{K}$ ), were used as sourses for the two beams. The instrument was adjusted so that the " $100 \%$ curve" would actually fall between 90 and 100 on the chart, after which such a curve was obtained over the wavelength range of interest. The sector-disk attenuator was then introduced into the specimen beam near the blackbody furnace, and operated witn each of the disks in turn, to obtain " $75 \%, 50 \%, 25 \%, 12.5 \%$ and $5 \%$ curves", each over the wavelength range of interest. The heights of the respective curves were then measured at 100 selected wavelengths, and the height of each curve above the experimentally obtained zero for the pertinent wavelength was plotted against the percentage of the flux in the specimen beam that was passed by the attenuator. In every case the points fell on a straight line intersecting the origin, within the error of measurement. No significant departures from linearity of response were detected for any combination of prism and detector, at any point within the wavelength range at which they could be used.

The data for the thermocouple detector and and sodium chloride prism are shown in figure 41-7. In this case, the "apparent emittance" of the combination of the reference blackbody furnace and each attenuator disk is plotted as a function of wavelength. Every value of apparent emittance was within \pm 0.01 of the measured transmittance of the respective disk.

\section{EXPERIMENTAL PROCEDURE}

It was found by experience that the $100 \%$ curve, obtained when the two blackbody fur-

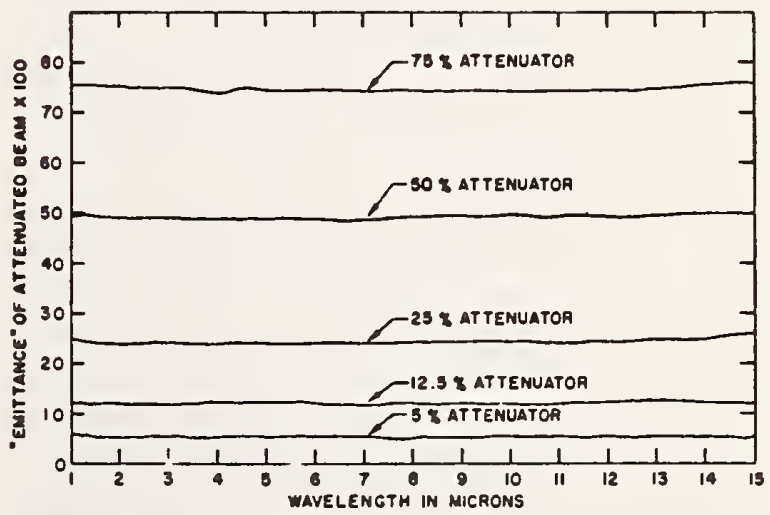

Figdre 41-7.-Spectral "emittance" of the combination of the reference blackbody furnace and sector-disk attenuator when using the $75 \%, 50 \%, 25 \%, 12.5 \%$ and $5 \%$ attenuator disks. Data are for the spectrometer with the sodium chloride prism and thermocouple detector. 
naces at the same temperature served as sources for the respective beams of the spectrometer, deviated appreciably from flatness. These deviations may be due to either (1) variations in spectral absorption or other losses along the respective optical paths from source to detector, or (2) variations in spectral sensitivity of the detector to radiant flux from the two beams. There are two more mirrors in the specimen beam than in the comparison beam, as is shown in figure 41-1. The two beams follow different paths from the sources to the spherical mirror in the combining optics, and are reflected by different mirrors in this portion of the path. From the sphcrical mirror in the combining optics to the detector, the two beams follow the same general path and are reflected by the same mirrors. However, the paths arc not identical, and the beams are reflected by slightly different areas of each mirror, and are transmitted by different areas of the prism and the detector window. These differences could account for spectral differences in losses in the two beams, due to absorption, and to scattering, for instance, by dust particles on the mirrors. The two beams are also focused on slightly different areas of the sensitive surface of the detector. No detector is completely black, and variations in spectral absorptance over the sensitive surface of the detector could be responsible for part of the observed effect.

It was also found by experience that the zero curve, obtained when the specimen beam was blocked near the specimen furnace, deviated from flatness. These deviations are undoubtedly due to stray radiation in the monochromator, which produces a spurious signal when there is in fact no radiant energy being supplied by the specimen.

A test procedure was developed to correct for the errors which result in the deviations of the " $100 \%$ line" and zero line referred to above. The procedure is as follows:

(1) The two blackbody furnaces are placed in position to act as sources for the respective beams of the spectrometer, and are brought to the same temperature. The "Full Scale" control of the spectrometer is adjusted to bring the $100 \%$ line to a position between 90 and 100 on the chart. The specimen beam is blocked, and the "Ratio Zero" control on the spectrometer is adjusted to bring the zero line to a position between 1 and 3 on the chart. The specimen beam is unblocked, and the position of the $100 \%$ line is checked. These instrument settings then remain fixed throughout the emittance determinations on the specimen being tested.

(2) The $100 \%$ line, over the wavelength range of interest, is obtained by automatic recording on the strip chart, after which the chart paper is rerolled.

(3) The specimen beam is blocked near the source, and the zero line is similarly obtained over the wavelength range of interest, after which the chart paper is again rerolled.

(4) The specimen enclosure is next substituted for the reference blackbody furnace, in position to act as source for the specimen beam of the spectrometer. The temperature of the specimen is brought to and held at the temperature of the comparison blackbody furnace. The specimen beam is unblocked, and the "specimen line" is obtained over the wavelength range of interest.

The heights of the respective curves are measured at preselected positions of the wavelength drum (corresponding to known wavelengths), as indicated in figure $41-8$, and the normal spectral emittance is computed for each such wavelength. If $Z_{\lambda}$ is the height of the zero line, $S_{\lambda}$ the height of the specimen line, and $H_{\lambda}$ the height of the $100 \%$ line, at some wavelength $\lambda$, the normal spectral emittance, $E_{\lambda}$ is given by

$$
E_{\lambda}=\frac{S_{\lambda}-Z_{\lambda}}{H_{\lambda}-Z_{\lambda}}
$$

Values of $E_{\lambda}$ are computed for each of 100 wavelengths in the range of 1 to $15 \mu$, and $E_{\lambda}$ is plotted as a function of wavelength. A curve is drawn through the plotted points to represent the spectral emittance curve of the specimen.

\section{SELECTION AND CALIBRATION OF WORKING STANDARDS}

The selection and calibration of suitable working standards of normal spectral emittance, to be used for the comparison of results in several different laboratories, was one of the objectives of this program. 


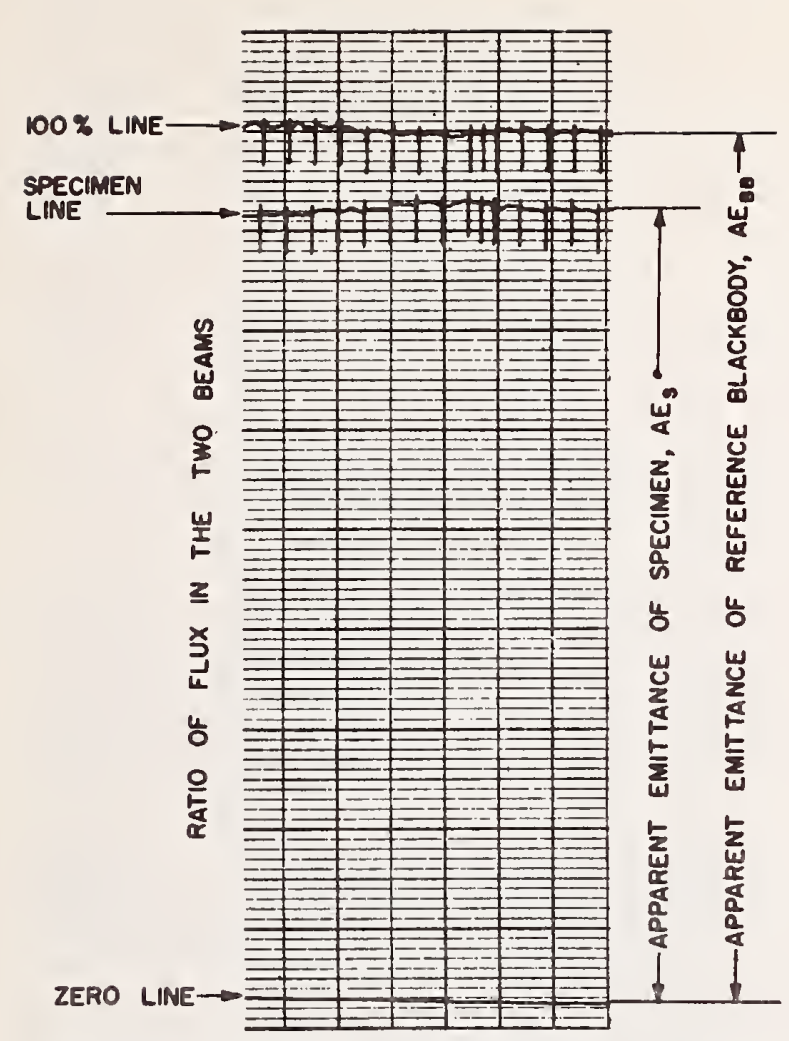

Figdre 41-8.-Section of recorder chart, showing sections of a $100 \%$ line, specimen line, and zero line. The normal spectral emittance is the ratio $A E_{0} / A E_{B B}$.

Working standards having high, intermediate, and low emittance were desired. In addition to having the desired normal spectral emittances, the working standards, to be useful, should be stable on heating in air at temperatures up to the maximum temperature at which they can be used, for times of several hundred hours. They should also be of a material that is not easily damaged in use. In order to be suitable for measurement with the equipment described in this report, the specimens are required to have electrical properties that will permit them to be heated by passing a current through them.

Specimens machined from sheet material of uniform thickness, three of each material in each of several different sizes and shapes were desired, suitable for measurement in the equipment used by different laboratories, as follows:

strips, $1 / 4 \times 8,8 / 4 \times 10$ and $1 \times 10$ inches in size

squares, $2 \times 2$ inches in size

disks, $7 / 8,1,1 \frac{1}{8}$, and $1 \frac{1}{4}$ inches in diameter.
Because it was not possible to measure the normal spectral emittance of specimens of all of the above shapes and sizes with the equipment described in this report, it was decided that the measurements would be confined to six 1/4-x-8-inch strip specimens of each material. All of the specimens of a single material were cut from a single sheet of metal, and all were treated as nearly alike as possible during surface preparation.

After an extensive series of tests, the following materials were selected on the basis of the above criteria, for use in preparing the working standards:

1. for standards of low normal spectral emittance, 0.035 -inch platinum sheet that had been polished and then annealed

2. for standards of intermediate normal spectral emittance, 0.043-inch Kanthal sheet that had been sandblasted and then oxidized

3. for standards of high normal spectral emittance, 0.053-inch Inconel sheet that had been sandblasted and then oxidized.

The tests indicated that specimens of these three materials, when prepared as outlined below; were stable in total hemispherical emittance to better than 0.02 on heating in air at the maximum temperature at which they were calibrated for periods of 200 hours.

\section{Platinum Worklng Standards}

The platinum specimens were received from the fabricator as 0.035 -inch sheet, six $1 / 4-x-8-$ inch sirips and three each in the following shapes and sizes: $7 / 8-, 1-, 1 \frac{1}{8}-$ and 1/4/inch diameter disks, $2-\mathrm{x}$-2-inch squares, and 1- $\mathrm{x}$-10and $8 / 4-x-10$-inch strips. It had been specified that the specimens were to be supplied with highly polished surfaces. The finish actually supplied was not as smooth as had been desired, but it was decided to use the finish supplied, rather than send the specimens back for reworking. A $1 / 8$-inch hole was drilled in one end of each strip specimen, to facilitate hanging during annealing.

Each specimen was washed in hot tap water to which a commercial detergent had been added, rinsed in running hot tap water, then in distilled water, and finally in ethyl alcohol. Rubber surgical gloves were worn at all times while handling the specimens, and the central 
portion, observed for emittance determinations, was not touched after cleaning. The specimens were dried in air and placed in a closed container, supported by the ends or edges only, for storage prior to annealing.

All specimens were annealed in an electricallyheated, silicon carbide element furnace. The strip specimeus were hung by means of platinum hooks suspended from aluminum oxide rods in the furnace; the square and disk specimens were supported by the edges only on cerrnic forms resting. on a flat ceramic slab. All of the specimens were then enclosed in a ceramic muffle: Starting with a cold furnace the temperature of the furnace was raised to $1523^{\circ} \mathrm{K}\left(1250^{\circ} \mathrm{C}\right)$ over a period of 6 hours, and held at that temperature for 1 hour. The power was then turned off, and the specimens were allowed to cool in the furnace, which required 2 days.

The specimens were removed from the furnace by means of cleaned platinum-tipped tongs and were placed in individual plastic holders, in which they were supported only by the ends or edges. Each plastic holder, containing a specimen, was then placed in an individual cardboard box, to protect it from contamination.

The six $1 / 4-x-8$-inch strips were prepared for measurement by welding a platinum-platinum, $10 \%$ rhodium thermocouple to each specimen. A shallow groove was scratched in each specimen, normal to its axis and located at the midlength. The 10-mil thermocouple wires were separately welded to the specimen by means of a condenser-discharge type of electronic spot welder. Each wire was laid in the shallow groove to position it for welding, and the welding operation was observed through a low-power microscope.

Precautions were taken at all times to aroid contamination of the specimens. They were handled as little as possible, and when handling was unavoidable the use of rubber gloves was continued, and even then the center portion of the specimen was not touched.

Three sets of curves were made for each strip specimen at each of three temperatures: $800^{\circ} \mathrm{K}$, $1100^{\circ} \mathrm{K}$, and $1400^{\circ} \mathrm{K}$. Each set of curves consisted of (1) a $100 \%$ curve, obtained when the two blackbody furnaces at the test tem- perature were the sources for the respective beams; (2) a zero curve, obtained when the specimen beam was blocked near the specimen furnace; and (3) a specimen curve, obtained with the comparison blackbody at the test temperature as one source and the specimen at the same tempcrature, as the other. Each curve was recorded over the range of wavelength drum settings corresponding to a wavelength range of approximately 1.0 to $15.0 \mu$.

The rormal spectral emittance was computed at a total of 100 wavelengths, approximately uniformly spaced from 1 to $15 \mu$.

The 18 values ( 3 each on 6 specimens) at each temperature and wavelength drum setting were tabulated, and the following values were computed: (1) $E$, the arithmetic average of the 18 measured values, (2) $\sigma_{t}$, the total standard deviation ${ }^{3}$ of the 18 values about the average of the 18 values, (3) $e$, the $95 \%$ confidence error of $E$, (4) $\sigma_{m}$, the average of six standard deviations, each computed from the three measured values on one of the six specimens, and (5) $\sigma_{s}$, the standard deviation of the six average values, one for each of the six specimens, about the grand average for all six.

The average normal spectral emittance, $E$, of the six platinum specimens at $1100^{\circ} \mathrm{K}$ is plotted as a function of wavelength in figure 41-9. The $95 \%$ confidence errors ${ }^{4}$ associated with the plotted average emittance values are also plotted in the same figure.

The value $\sigma_{m}$ is a measure of the overall reproducibility of the test procedure or the precision of measurement. The average value of $\sigma_{m}$ for all of the 100 wavelengths, is 0.45 at $1100^{\circ} \mathrm{K}$. Thus the overall precision of measurement on platinum is better than 0.005 ,

${ }^{3}$ All standard deviations computed in this study are precisely defined as "estimates of the standard deviation of the parent population from which the measurements were drawn." This quantity is assigned the symbol $\sigma$, to distinguish it from the root-mean-square standard deviation, S. D., of individual values from the mean of a given sample.

4 The $95 \%$ confidence error has the following statistical significance. If the measurements were repeated a large number of times, say 1000 times, and the average and $95 \%$ confidence error was computed for each group of 18 measurements, then the limits of the group average \pm the $95 \%$ confidence error would bracket the overall average of the 1000 groups of measurements about 19 times out of 20 . 


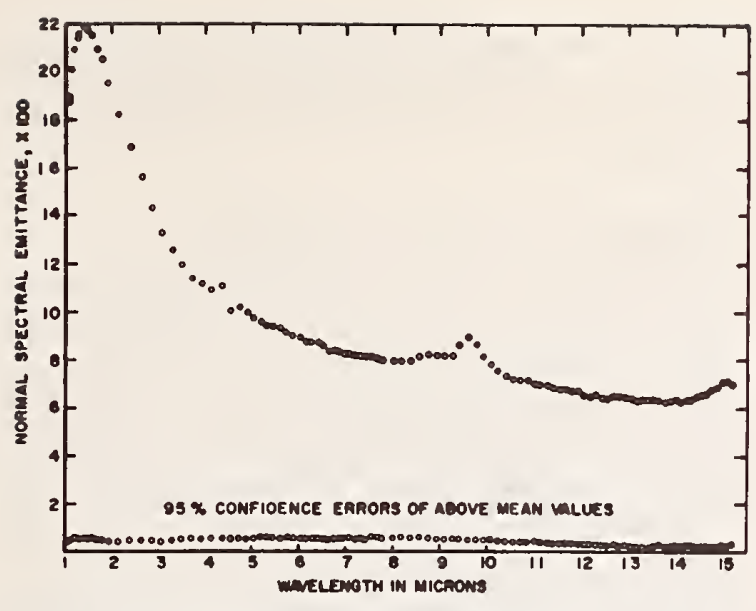

Figure 41-9.-Normal spectral emittance at $1100^{\circ} \mathrm{K}$ of platinum working standards. The points in the upper curve represent averages of 18 measurements, 3 each on 6 specimens. The points in the lower curve represent the $95 \%$ confidence errors of the a verage values in the upper curve.

expressed as a standard deviation in units of emittance. In order to show any trend of $\sigma_{m}$ with wavelength, the moving average of 5 values at adjacent wavelengths was computed, and plotted as a function of wavelength as the lower curve in figure 41-10. The scatter due to errors of measurement, $\sigma_{m}$, shows significantly less variation than $\sigma_{8}$.

The value $\sigma_{2}$ indicates actual differences in emittance of the specimens that were measured. The average value of $\sigma_{8}$ for all of the 100 wavelengths was 0.74 . In order to show any trend of $\sigma_{s}$ with wavelength, the moving average of five values at adjacent wavelengths was computed and plotted as a function of wavelength to form the upper curve in figure 41-10. There appears to be a significant variation in $\sigma_{\text {s }}$ with wavelength.

The values of $\sigma$, are larger than the corresponding values of $\sigma_{m}$ by an amount sufficient to demonstrate statistically that the observed differences between specimens are real, and could not occur due to chance fluctuations of the error of measurement.

Curves similar to those shown in figures 41-9 and 41-10 were prepared from the data obtained at $800^{\circ} \mathrm{K}$ and $1400^{\circ} \mathrm{K}$. In general the curves of data obtained at $800^{\circ} \mathrm{K}$ and $1400^{\circ} \mathrm{K}$ were similar in shape to the corresponding curves at $1100^{\circ} \mathrm{K}$. The normal

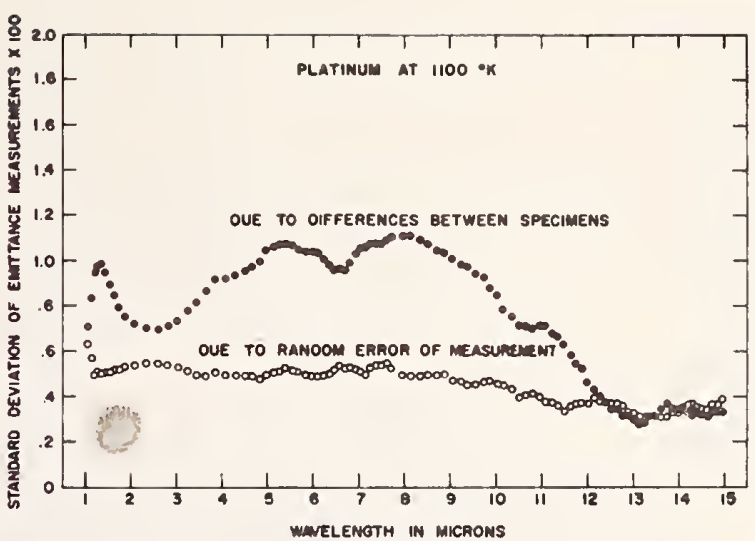

Figure 41-10.-Spectral distribution of two categories of standard deviations, each computed from 18 measured emittance values obtained at $1100^{\circ} \mathrm{K}$, 3 each on 6 platinum working standards. The upper curve represents standard deviations due to real differences in emittance between specimens, identified as $\sigma_{\text {, }}$ in the text. The lower curve represents standard deviations due to random error, identified as $\sigma_{m}$ in the text. In both curves each point represents the moving average of five adjacent values.

spectral emittence at each wavelength increased with an increase in temperature, as would be predicted by the Hagen-Rubens equation. No significant change in $\sigma_{m}$ or $\sigma$, with temperature was noted.

\section{Oxldized Kanthal Worklng Standards}

Specimens of the same sizes and shapes as those referred to above were machined from 0.043-inch Kanthal sheet and were cleaned with acetone to remove any oil or grease from the machine operation. They were then marked for identification, and sandblasted with 60-mesh fused aluminum grit at an air pressure of approximately 70 psi. The sandblasted specimens were cleaned ultrasonically in acetone, passivated for one minute in $10 \%$ nitric acid at $316^{\circ} \mathrm{K}\left(43^{\circ} \mathrm{C}\right)$, rinsed in distilled water and then acetone. They were placed in a cold furnace, which was brought to $1340^{\circ} \mathrm{K}$ and held for 400 hours, after which they were allowed to cool in the furnace.

The normal spertral emittance of the six 1/1-x-8-inch oxidized Kanthal specimens was measured, following the procedure outlined above for platinum specimens, except that the measurements were made at temperatures of $800^{\circ}, 1100^{\circ}$ and $1300^{\circ} \mathrm{K}$. The normal spectrsl 


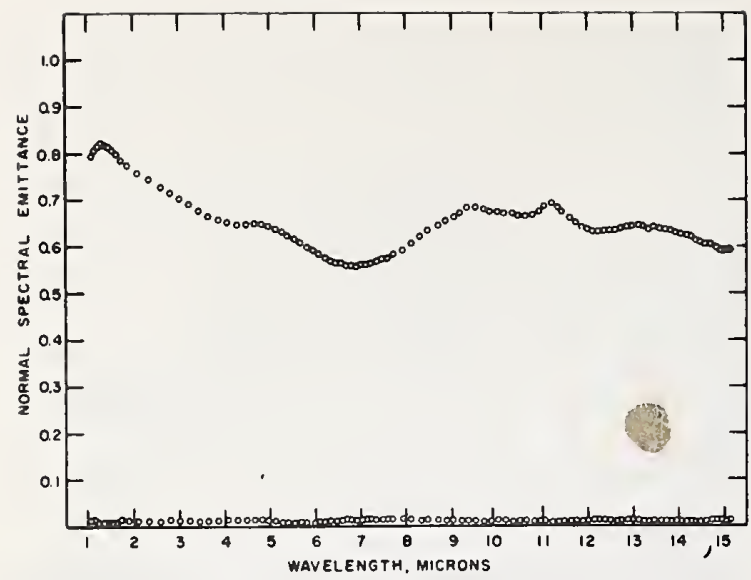

Figure 41-11.- Normal spectral emittance at $1100^{\circ} \mathrm{K}$ of oxidized Kanthal working standards. The points in the upper curve represent averages of 18 measurements, 3 each on 6 specimens. The points in the lower curve represent the $95 \%$ confidence errors of the average values in the upper curve.

emittances and 95\% confidence errors were computed as before and plotted to produce curves of the type shown in figure 41-11 for measurements at $1100^{\circ} \mathrm{K}$ and the standard deviations due to differences in specimens and

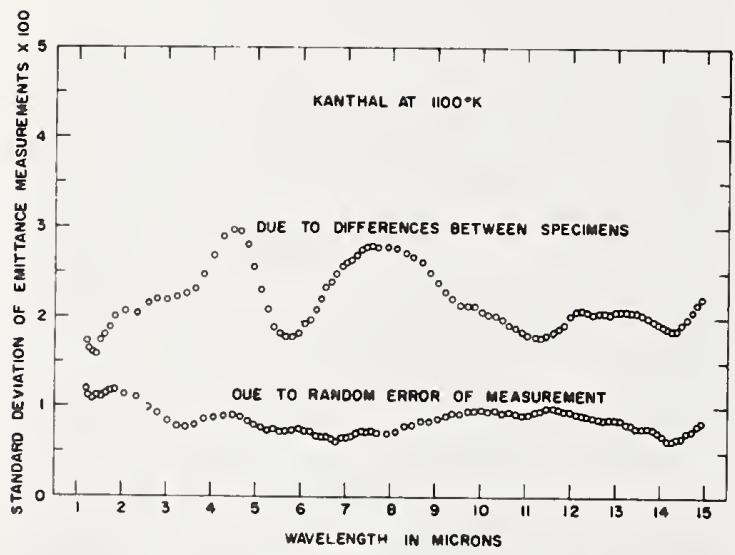

Figore 41-12.-Spectral distribution of 2 categories of standard deviations, each computed from 18 measured emittance values obtained at $1100^{\circ} \mathrm{K}$, 3 each on 6 oxidized Kanthal working standards. The upper curve represents standard deviations due to real differences in emittance between specimens, identified as $\sigma_{0}$ in the text. The lower curve represents standard deviations due to random error of measurement, identified as $\sigma_{m}$ in the text. In both curves each point represents the moving average of 5 adjaoent values. random error of measurement were computed as before and plotted to produce curves of the type shown in figure $41-12$ for measurements at $1100^{\circ} \mathrm{K}$.

\section{Oxidlzed Inconel Working Standards}

Specimens of the same sizes and shapes referred to above were machined from 0.053 inch Inconel sheet, and were cleaned and sandblasted as outlined above for the Kanthal specimens. The cleaned specimens were placed in a cold furnace, which was brought to $1340^{\circ}$ $\mathrm{K}\left(1067^{\circ} \mathrm{C}\right)$ and held for 24 hours; the temperature was then reduced to $1100^{\circ} \mathrm{K}$ and held for an additional 24 hours, after which the specimens were allowed to cool in the furnace.

The normal spectral emittance of the six $1 / 4$ $\mathrm{x}-8$-inch oxidized Inconel specimens was measured, following the procedure outlined above for platinum specimens, except that the measurements were made at temperatures of $800^{\circ}$, $1100^{\circ}$, and $1300^{\circ} \mathrm{K}$. The normal spectral emittances and $95 \%$ confidence errors were computed as before, and plotted to produce curves of the type shown in figure 41-13 for measurements at $1100^{\circ} \mathrm{K}$. The standard deviations due to differences in specimens and to random error of measurement were also computed as before, and plotted to produce curves of the type shown in figure 41-14 for measurements at $1100^{\circ} \mathrm{K}$.

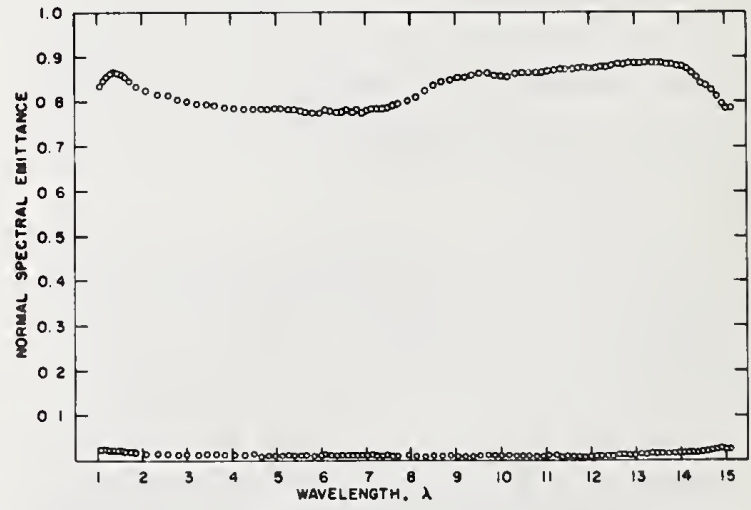

FIGURE 41-13.- Normal spectral emittance at $1100^{\circ} \mathrm{K}$ of oxidized Inconel working standards. The points in the upper curve represent averages of 18 measurements, 3 each on 6 specimens. The points in the lower curve represent the $95 \%$ confidence errors of the average values in the upper curve. 


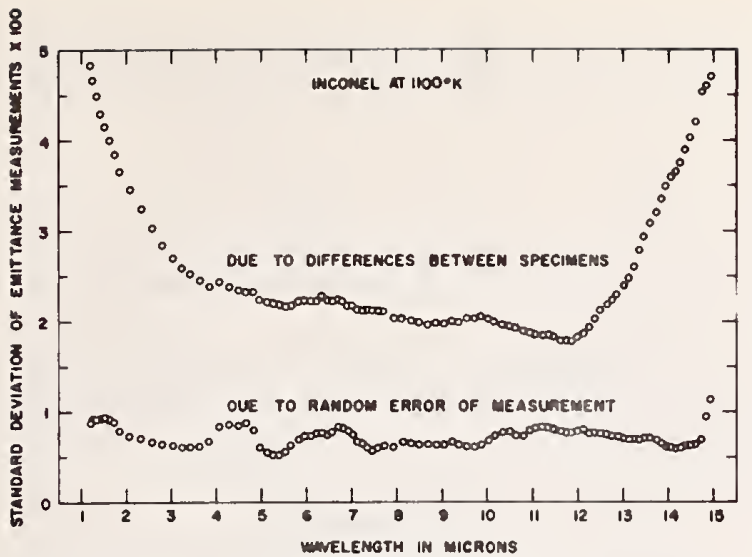

Figore 41-14.-Spectral distribution of 2 categories of standard deviations, each computed from 18 measured emittance values obtained at $1100^{\circ} \mathrm{K}$, 3 each on 6 oxidized Inconel working standards. The upper curve represents standard deviations due to real differences in emittance between specimens, identified as $\sigma$, in the text. The lower curve represents standard deviations due to random error of measurement, identified as $\sigma_{m}$ in the text. In both curves each point represents the moving average of 5 adjacent values.

\section{PRINCIPLES OF DATA REDUCTION}

\section{Computation by the Welghted-Ordinate Methed}

Two main steps are necessary in order to compute the total normal emittance or absorptance of a specimen for radiant flux having a specified spectral distribution from its spectral emittance curve. These steps are described in relation to figure $41-15$, which represents an actual case.

The first step is to weight a series of ordinates chosen at uniform intervals along the emittancewavelength curve for the specimen (curve A) according to the spectral distribution of radiant flux from the source under consideration, which can be represented graphically by a second curve, B. The weighted ordinates, which are the products of ordinates at identical wavelengths for curves $A$ and $B$, provide points for a third curve, C, which indicates the spectral distribution of the energy that would be absorbed in unit time by the specimen, from the specified source. For the special case in which the designated source of radiation, represented by curve $B$, is a blackbody (as it is in figure 41-15) at the temperature of the specimen (which is in thermal equilibrium), curve $\mathrm{C}$ represents, also, the spectral distribution of the

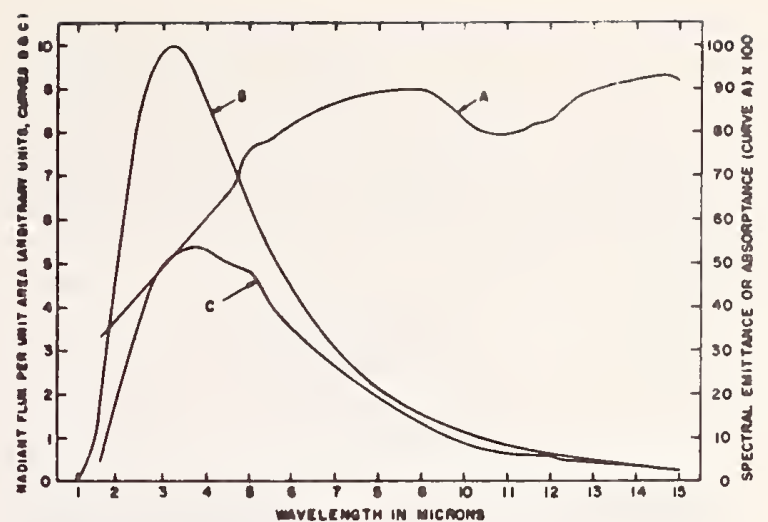

Fradae 41-15.-Grapbical form of data and derived values used in computing from the spectral emittance of a specimen its total emittance, its total absorptance, or its total emissive power. Curve A represents spectral emittance values obtained on a specimen at $650^{\circ} \mathrm{K}$ (about $1200^{\circ} \mathrm{F}$ ). Curve $\mathrm{B}$ represents the spectral distribution of radiant flux from a blackbody at the same temperature. The ordinates of curve $\mathrm{C}$ are in each case the product of the ordinates of curves $A$ and $B$ at the same wavelength. Curve $C$ represents the spectral distribution of flux emitted by the speoimen having the spectral emittance indicated by ourve A.

energy emitted in unit time by the specimen at the temperature under consideration, since. under these specific conditions the rate at which energy is absorbed by the specimen at each wavelength is exactly equal to that at which it is emitted at the same wavelength.

The second step in computing the total emittance or absorptance of the specimen consists of determining the ratio of the area under curve $C$ to that under curve B. A rigorous mathematical expression of the quantity sought is as follows:

$$
E_{u s}=\frac{\int_{0}^{\infty} \epsilon_{b \lambda} E_{3 \lambda} d \lambda}{\int_{0}^{\infty} \epsilon_{b \lambda} d \lambda}
$$

where:

$E_{\imath}=$ total Emittance of specimen

$\epsilon_{b \lambda}=$ rate of energy emission per unit wavelength interval, of a blackbody, for the increment $\lambda$ to $(\lambda+d \lambda)$

$E_{s \lambda}=$ Emittance of the specimen, between $\lambda$ and $(\lambda+d \lambda)$.

In practice, with a sufficient number of uniformly spaced ordinetes (which are taken at 
the same set of wavelengths for all the curves) this ratio of areas is approximated with the required precision by dividing the sum of the ordinates for curve $\mathrm{C}$ by the sum of the ordinates for curve $B$. The mathematical expression of this operation is

$$
E_{t s}=\frac{\sum_{\lambda_{1}}^{\lambda_{2}} \epsilon_{b \lambda} E_{s \lambda} \Delta \lambda}{\sum_{\lambda_{1}}^{\lambda_{2}} \epsilon_{b \lambda} \Delta \lambda}
$$

Since all values of $\Delta \lambda$ are equal, they cancel out numerically as well as dimensionally.

The units in which the ordinates of curve A are expressed are pure numbers, representing spectral-emittance values. The units in which the ordinates for curve $\mathrm{B}$ are expressed occur in both the numerator and denominator of the final ratio; hence it is of no consequence what units are used, provided they are the same for the numerator and denominator, or whether the ordinates for curve $B$ are expressed simply as numbers of the correct relative magnitudes. The final answer in either case is a number signifying the total absorptance of the specimen for radiant flux of the specified spectral distribution.

The procedure is identical for computing either the total emittance of the specimen or its total absorptance for energy from the sun, the earth, or any other source, because the total absorptance for blackbody radiation at the temperature of the specimen is equal to the total emittance of the specimen. Hence to compute total emittance or total solar absorptance as desired, one merely modifies the procedure by selecting for curve B either the spectral distribution of radiant flux for a blackbody at the temperature of the specimen, (which was done for fig. 41-15) or the spectral distribution of radiant flux for solar radiation, or radiation from the earth or any other source.

When it is desired to compute the total amount of radiant energy, for unit time and area, emitted by the specimen at a given temperature, curve B will represent blackbody radiation and its ordinates will be expressed in energy per unit time and area; hence, those of curve $\mathrm{C}$ will also. In this case, the quantity sought is represented by the area under curve C, which can be obtained by integration. The equation that rigorously describes this relationship for all possible wavelengths is:

$$
\epsilon_{\ell \vartheta}=\int_{0}^{\infty} E_{s \lambda} \epsilon_{\partial \lambda} d \lambda
$$

where $\epsilon_{\omega}$ is the total radiant flux density of the specimen.

In practice the value can be computed with the required precision by the following approximation:

$$
\epsilon_{\imath s}=\sum_{\lambda_{1}}^{\lambda_{1}} E_{s \lambda \epsilon_{b \lambda} \Delta \lambda}
$$

Since $E_{s \lambda}$ is a ratio, and $\epsilon_{b \lambda}$ represents radiant flux per unit area and wavelength interval, the $\lambda$ cancels out dimensionally. Numerically, the fraction of the span between $\lambda_{1}$ and $\lambda_{2}$, that is occupied by a single interval, $\Delta \lambda$ is the reciprocal of the number of ordinates, $n$, (each ordinate representing one interval); hence equation (6) may be written:

$$
\frac{\epsilon_{t s}=\sum_{\lambda_{1}}^{\lambda_{3}} E_{s \lambda \epsilon_{b \lambda}}}{n}
$$

This equation states that the total radiant flux per unit area is obtained by simply adding all the ordinates of the points from which curve $C$ was constructed (each of which represents radiant flux per unit area), and dividing by the number of such ordinates. Any required precision of the approximation can be attained by using a sufficient number of ordinates.

As in the case of emittance and absorptance, the procedure is the same whether the object is to compute the total radiant flux emitted by unit area of the specimen or the total radiant flux absorbed upon exposure to a specified source. In the former instance, curve B represents blackbody radiation; in the latter case, it represents radiant flux from the specified source that is incident upon the specimen.

\section{Computation By the Selected Ordinate Method}

Regardless of what method of computation is chosen, the objects remain (1) to obtain the ratio of areas under two curves, $B$ and $C$, for total emittance or total absorptance, and (2) to evaluate the area under one curve, C, for total radiant flux per unit area of the specimen, absorbed from a specified source, or emitted. 
Ordinarily it is preferable to use no more ordinates for the calculations than are needed to give the required precision. The optimum number of ordinates can be chosen by spacing them to represent equal areas under curve $\mathrm{B}$, a device which results in unequal values of $\Delta \lambda$. When this method is used, the first step is to choose ordinates for curve $B$ at increments of $\lambda$ such that the areas between all adjacent pairs of these ordinates correspond to a constant amount, $k$, of radiant flux per unit area. Then the median wavelengths within the respective. intervals between these adjacent pairs of ordinates determine the locations of the selected ordinates. When the ordinates of curve $A$ are read at these specified values of $\lambda$, the rate at which energy is emitted per unit area of the specimen can be obtained with the required accuracy simply by adding up these ordinates and multiplying the sum by the constant, $k$. The basic equation is:

$$
\epsilon_{t s}=\sum_{\lambda_{1}}^{\lambda_{1}} E_{s \lambda}\left(\epsilon_{D \lambda} \Delta \lambda\right)
$$

But since $\left(\epsilon_{b \lambda} \Delta \lambda\right)$ has the constant value $k$, independent of wavelength, the equation becomes :

$$
\epsilon_{\imath s} \cong k \sum_{\lambda_{1}}^{\lambda_{1}} E_{s \lambda}
$$

Often, for convenience, ordinates are selected at intervals such that $k=1$.

The economy in number of ordinates required for a given precision makes this method especially advantageous in the absence of electronic computers. Like the weighted-ordinate method, it is applicable to calculation of total emittance or total absorptance from the spectral data, as well as to calculation of rate of emission or absorption of energy by unit area of the specimen.

\section{DESIGN AND INSTALLATION OF DATA-PROC- ESSING EQUUIPMENT}

A photograph of the spectrophotometer with data-processing equipment installed is shown in figure 41-16. This equipment was designed to perform the following functions: (1) to produce a corrected graphical record of the normal spectral emittance of a specimen as a function of wavelength, (2) to record in digital form on punched paper tape the corrected spectral emittance values, for possible use in a separate electronic digital computer, and (3) to accumulate the digitalized emittances at wavelengths which have been pre-selected to yield specific information as described farther in this report.

The output of the spectrometer is digitized by two shaft encoders, one mounted on the

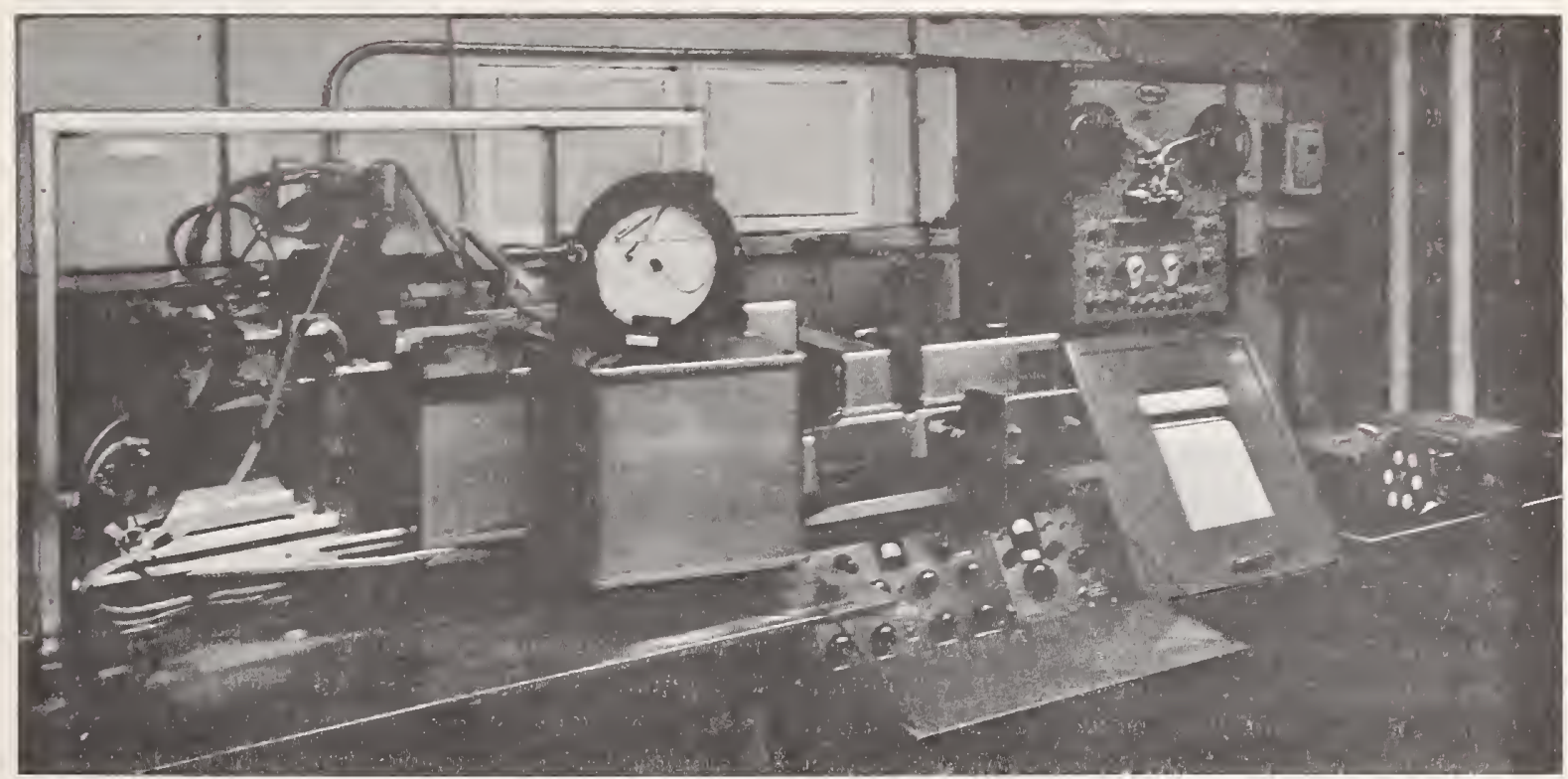

Figdre 41-16.-Datá-processing equipment attached to the spectrometer. The electronic circuits are housed in the chassis above the recorder at the right, and the paper tape punch and punched paper tape reader are on the table at the extreme right. 
wavelength drive shaft, and the other on the recorder pen shaft. Each encoder produces a pulse for each 0.001 revolution of the shaft, and the pulse also contains a signal indicating direction of rotation. These pulses are counted in the data-processing attachment, by reversible counters which increase in count for pulses in the forward direction, and decrease in count for pulses in the backward direction.

During calibration, the pulses from the recorder shaft, encoder are recorded on fourchannel magnetic tape. During measurement of a specimen, the magnetic tape is played back synchronously with the automatic wavelength scanning, and the data-processing attachment automatically corrects for the $100 \%$ line and zero line errors, as is described in more detail in a paper by Horace M. Joseph (to be published). The corrected normal spectral emittance of the specimen appears as the curve drawn by the recording potentiometer. The output of the potentiometer is digitized, as before.

The wavelength drum position and recorder pen position can be automatically recorded on punched paper tape at preselected increments of wavelength drum position. The spectral emittance values can be accumulated at the same or other preselected increments of wavelength drum position.

In order to compute total emittance or absorptance for radiant energy having known spectral distribution of flux, the accumulation must be performed at unequal increments of wavelength drum position, at the selected ordinates which represent equal areas under the spectral energy distribution curve for the blackbody or source as previously described. These unequal increments are prepunched into paper tape, which automatically controls the accumulation process. The sum on the accumulator is then $n$ times the total emittance or absorptance, where $n$ is the number of selected ordinates.

\section{SUMMARY}

Test equipment and procedures were developed to measure normal spectral emittance of strip metal specimens that can be heated by passing a current through them, at temperatures from about $800^{\circ} \mathrm{K}$ to above $1400^{\circ} \mathrm{K}$, and over the wavelength range of 1 to $15 \mu$.

The equipment consisted essentially of a double-beam, ratio-recording infrared spectrometer, in which the source optics were modified to include a blackbody furnace in a fixed position as the source for the comparison beam, and a second blackbody furnace and a specimen furnace mounted on a movable plate attached to a slide so that either could be positioned to serve as the source for the specimen beam. Special control equipment maintained the two sources at the same temperature.

The test procedure corrected for zero line and $100 \%$ line errors in the measured emittance. The zero line error arises as a result of a spurious signal produced in the specimen beam when the beam is blocked near the source. The $100 \%$ line error results from differences in the ratio of the signal produced to radiant flux entering the instrument along the two beams of the spectrometer.

An electronic data-processing attachment was designed and built to (1) automatically correct for "zero line" and " $100 \%$ line" errors, (2) record the corrected data in digital form on punched paper tape, suitable for direct entry into an off-line electronic digital computer and (3) compute, from the spectral data as obtained, a single value of total emittance, or absorptance for radiant energy having any known spectral distribution of flux.

Three types of specimens for use as working standards in other laboratories were prepared and calibrated. These specimens had high, intermediate and low spectral emittance, respectively. Tests of these standards indicated that the overall reproducibility of the method is on the order of 0.005 in emittance, expressed as a standard deviation. 


\section{REFERENCES}

1. Worthing, A. G.: Temperature Radiation Emissivities and Emittances, Temperatureits Measurement and Control in Bcience and Industry. American Institute of Physics. Reinhold Publishing Corp., New York, 1941, pp 1164-1187.

2. Gourft, Andrt: Corrections des Corps Noires Artificiel Compete Teun des Diffusions Multiples Internes, Revue d'Optique 24 [1-3], Jan. to March 1945, pp 1-8.

3. Butrerworth, ED.: Table of Wavenumbers. Int. Union of Pure and Applied Chemistry. 1961.

\section{Discussion}

G. J. Zissis, Institute for Defense Analyses: One thing that worries me a bit is the effect of the corrugations on the walls of the cylinder of the cavity. In the De Vos treatment of a cavity, the first-order correction term gives the equivalent of the Gouffe theory, but the second-order corrections are quite dependent on the amount of radiation coming in through the opening and reflecting off of the walls. I have a feeling that with corrugations a direct return from the sides of the walls through the opening might be larger than it would be if you had, say, a flat wall with a Lambertian distribution. As long as you do not see the walls when you are operating $I$ would imagine this would make only a very small difference, but it might be worth while carrying out the calculation of the emissivity by the De Vos method just to compare.

RIChmond: The walls of the cavity were of 80 nickel-20 chromium alloy which had been oxidized for many hours at temperatures up to $1400{ }^{\circ} \mathrm{K}$ so that there was a rather thick oxide layer which was quite rough. Also, this was a sand casting which had not been smoothed; so the walls were quite rough inside. Under these conditions I believe that the De Vos analysis would not apply. I think the Gouffe analysis would be more nearly correct.

8. SkLARew, The Marquardt Corporation: What is the availability of this type of emittance standard, and are these standards reliable in the visible portion of the spectrum?

Richmond: I am going to ask Bob Winn to answer the first part of that question.

WinN: The standards themselves have been received at ASD; however, the program has been transferred from the Physics Laboratory to the Applications Laboratory, and I would suggest that you contact $\mathrm{Mr}$. Don Stevinson (ASRCEE-2) who will be responsible for making arrangements to lend these standards.

Richmond: The other part of the question was with regard to the reliability of the standards in the visible. So far they have not been calibrated below one micron. We hope to do that eventually. We do not have any information on the reliability in that range. However, we do have some experimental evidence to indicate that these specimens will probably be relatively stable in emittance as long as they are heated in air at temperatures no higher than $1400^{\circ} \mathrm{K}$ for the platinum, $1300^{\circ} \mathrm{K}$ for the other standards, for periods up to at least 200 hours. We have run some of them for periods up to 1,000 hours, and we find probably not over 2 or $3 \%$ change in emittance as a result of this continued heating.
However, they should be handled with care to avoid contamination of the surface because that can really change emittance.

AвbотT, Naval Radiological Defense Laboratory: You mentioned integration of the spectral curve to obtain total normal emittance. Have you done any of this?

Richmond: We have done some of it. The automatic data processing equipment is not yet in operation so what we have done has been done by longhand methods, which is quite tedious as you probably know.

Аввотт: You seem to be making the measurements from 1 to $15 \mu$.

RICHMOND: 1 to 15.

Aввотт: How do you account for the range below and above?

RICHMOND: At the temperatures at which we are working there is only a small percentage of the energy below and above, and we assume that the emittance curve is flat below and above the ranges on which we have actual data.

Аввотт: Do these agree reasonably well with measurements of direct total normal emittance?

Richmond: We do not have any data on the same specimens for direct measurement of normal total, but they agree within 1 or $2 \%$ with direct measurements of total hemispherical when you make a correction for the difference between normal and hemispherical according to the equations given in Jakob.

I. J. Barsy, Armstrong Cork Company: In describing the threaded alloy liner you made the statement that the reflectance is less than 0.05. How did you determine this?

RICHMOND: That was determined first from measured values on flat specimens that had been subjected to the same treatment and then making the correction for the effect of the threading.

I. J. SpIro, The Aerospace Corporation: Mine is not a question but a comment to you on your definitions. In your discussion on emissivity you call attention to the fact that you must have an optically smooth surface. I would like to caution your committee to consider wavelengths when you describe optically smooth surfaces. As you change in wavelengths to 50 and $100 \mu$, what was optically smooth at one place is no longer, and what was an "emittance" may now become an "emissivity".

Richmond: That is very definitely true, but as you go up in wavelength a rough surface tends to appear to be smoother. There has been some data published 
not too long ago (Bennet, I believe was the author), in which it was shown that you can have effects from surface roughness on the order of a hundredth of a wavelength of the light involved. The major effects, however, do not begin to appear until you get up to something on the order of a tenth of the wavelength involved. That is peak-to-valley distance.

Szoulders, General Technology Corporation: When you were showing the curve on platinum you implied that you did not undęrstand why you had a peak at the longer wavelengths around the $15-\mu$ region. Did you mean to imply that you understood why you had the other peaks? I would like to have a little bit of an explanation on that.

Richmond: Suppose I see you after the meeting to talk about that. It gets quite involved. I think briefly that the one around $91 / 2$ or close to $10 \mu$ is probably due to contamination in the specimen, but we are not sure. The one down around $1 \mu$ we believe is due to resonance effects.

Wiluiam Clayton, Boeing Company: I think it might be useful if you were able to state what you believe to be the accuracy of your method, divorced from material changes, of course. Your method is probably two or three times as accurate as anybody else's, at least mine. It might give us all a good target.

RIC RMond: That is a rather difficult question to answer. I think that the precision of our measurements is certainly on the order of 0.01 in emittance. If you wanted it in a percentage, that would depend on the emittance value that you are measuring. The only data we have on the accuracy is what we obtained by use of a sector-disk attenuator; we find that our entire system gives linear response to better than 0.01 . On that basis, assuming that errors are additive, conservatively any value should be good to about 0.02 . I don't have any rigid analytical basis for stating that; that is just my own feeling.

STRED, Lockheed Missiles and Space: Is it possible to clean these standards, and do you have any recommendations in handling them?

RICHMOND: As far as handling them is concerned, the specimens are rounted in plastic holders so that the central area, the area on which you would be making measurements on all specimens, is not touching anything. Our recommendation would be to avoid touching the portion where you would be looking at the specimen. In fact, you would probably be better off if you were to wear surgical gloves at any time when handling the specimen, to avoid fingerprints. Fingerprints can be very very bad, and they are very difficult to remove, particularly if the specimen has been heated before you attempt to remove them. Other than that, I would say avoid letting anything come in contact with that central portion of the specimen. I think probably the specimens could be cleaned if they should become contaminated, but it might be a considerable job. The oxidized Inconel specimens tend to be self-cleaning, becsuse a type of oxide forms on them that gradually spalls away as a very fine powder so that you get a self-renewing surface. I would expect that all you would have to do on those specimens would be to heat them for a few hours, at a high temperature in air. They would probably come back pretty close to what they were originally. That same treatment would not apply to the oxidized Kanthal however, because a very stable oxide film is formed on them.

J. I. WitTEBont, ASD: The problem of thermal etching on the surfaces comes up at high temperatures. Have you noticed much difference in the emittance, at say, $1500^{\circ}$ to $1800^{\circ} \mathrm{F}$ at the beginning of the test and later on after thermal etching has occurred?

Richmond: We did notice that on unannealed platinum specimens. I am not sure that the effect is due to thermal etching, I think it is due to recrystallization, but the specimens had been annealed above the recrystallization temperature before we made any determinations. I think that you have to take the platinum up to temperatures above those at which we have made any measurements in order to get appreciable thermal etching. I am not positive but that is my impression.

KostrowsrI, NBS: I would like to make a comment here that when one begins to get into accuracies of a few percent, one factor that should be taken into account in any type of radiance measurements is the relative size of the two samples one is comparing. For example, in your case, the specimen is of the order of 3/4-inch wide, and the blackbodies, at least the effective size of the blackbody is probably an inch in diameter or greater. Is that right?

RICHMOND: I did not mention this, but the blackbody has an opening approximately 3/-inch wide by 3/4-inch long.

Kostrowskr: But how about the area adjacent to the cavity hole? It is probably still bright, is it not?

RICHMOND: No, it is not. When the blackbody furnace is at a temperature of $1400^{\circ} \mathrm{K}$ that area adjacent to the opening is not incandescent.

Kostrows apply in this cose, but I still think I should mention it. When the relative sizes of the two sources one is comparing are very different, the apparent brightness or radiance of the two can differ by several percent due to diffraction effects alone.

RIChMOND: We are very much aware of that in some of the work Dwight Moore has been doing, which he will describe tomorrow; there we are using an extremely small area of the specimen. 


\title{
A Test of Analytical Expressions for the Thermal Emissivity of Shallow Cylindrical Cavities
}

\author{
Francis J. Kelly and Dwight G. Moore
}

\begin{abstract}
Analytical expressions for the directional thermal emissivity of a shallow cylindrical cavity were tested by means of room temperature reflectance measurements. The measurements were made by placing a paper-lined brass cavity of adjustable depth over the specimen opening of a recording spectrophotometer. Reflectance curves were obtained from $0.4 \mu$ to $0.75 \mu$ with the plunger positioned to give cavity depthto-radius ratios of $0,0.5,1.0,1.5$, and 2.0. The resulting reflectances were then converted to emissivities through use of the relation that, for an opaque material, the emissivity is equal to one minus the reflectance. Two theoretical expressions predicted the emissivity of a cavity with a diffusely reflecting wall to within 0.01. A closed-form expression of Gouffé, which forms the basis of a shallow-hole method for measuring the emissivity of nonmetals at high temperatures, was in as good agreement with the experimental measurements as a more rigorous expression derived by a different approach. Sizable departures of the cavity walls from good diffusers had a negligible effect on the emissivity of the cavity at a depth-to-radius ratio of 0.5 , but the effect of specular components became progressively greater as the cavity depth was increased.
\end{abstract}

\section{Introduction}

The problem of determining the thermal emissivity of a cavity* from a knowledge of the emissivity of the wall material and the geometry of the cavity is insportant both for optical pyrometry and thermal emissivity measurements. Most investigators ${ }^{1-11}$ have been concerned with deep cavities formed in diffusely reflecting materials. Recently, a method of measuring thermal emissivity of polycrystalline ceramics has been proposed. in which cylindrical reference cavities of unprecedentedly shallow depth-to-radius $(L / R)$ ratios are used in order to minimize temperature differences

\footnotetext{
The authors are with the National Bureau of Standards, Washington, D.C.

Received 6 March 1964.

This paper was presented at the Symposium on Thermal Radiation Properties of Solids, 4-6 March 1964, San Francisco, California.

* Emissivity is the ratio of the flux per unit area radiated by a specimen to that radiated by a blackbody at the same temperature and under the same conditions. The conditions refer to the geometric and spectral distribution of measured flux. The directional emissivity of a cavity is a similar ratio for an imaginary plane across the opening of the cavity. The issuing flux, which includes both emitted and reflected components, originates from an area of interior wall opposite the opening. The reflected component is due to radiation from the remainder of the cavity that is incident on the measured area. This reflected component causes the apparent emissivity of a cavity wall, which is called cavity emissivity throughout this paper, to be always greater than its true emissivity.
}

between the reference cavity and the surface. ${ }^{12}$ The reliability of this method depends on the accuracy of analytical expressions for cavity emissivity.

The purpose of the present study was to compare the directional emissivities of cavities predicted by several analytical expressions with those obtained experimentally. The experimental measurements were made by determining the spectral reflectance of an adjustable-depth paper-lined cylindrical brass cavity from $0.4 \mu$ to $0.75 \mu$ and then converting these values to emissivity through use of the relation that, for an opaque material, the emissivity is equal to one minus the reflectance. Because the incident beam of the spectrophotometer was near normal and the viewing was hemispherical, the reflectance measurement was, in effect, the complement of the nornal emissivity, which is the property of interest in the shallow-hole method. ${ }^{12}$

The method used for testing the expressions had three distinct advantages over the more direct approach of measuring emissivity at high temperatures. First, making the measurements at room temperature insured isothermal conditions within the cavity; second, it was possible to obtain a number of different spectral emissivity values from a single spectrophotometer curve by lining the cavity with selectively reflecting materials that would provide a broad range of spectral reflectance values in the range $0.4 \mu$ to 0.75 $\mu$; and, third, the spectrophotometer was capable of measuring reflectances to 1 part in 1000 , which is a precision that could not be achieved easily with available thermal emissivity equipment. 


\section{Expressions for Emissivity of} Shallow Cylindrical Cavities

Three general methods have been employed for deriving equations to express cavity emissivity. ${ }^{13}$ The first, which was used by Ribaud, ${ }^{1,2}$ Gouffé, ${ }^{3}$ and Michaud, ${ }^{4}$ involves the calculation of the reflectance of the cavity, that is, the fraction of the energy incident through the cavity opening that later leaves the cavity after one or more reflections from the walls. According to Kirchhoff's radiation law, the reflectance of the cavity can then be converted to cavity emissivity by subtracting the reflectance from unity.

Of the various expressions derived by this first approach, Gouffe's was of primary interest in the present investigation because of its proposed use in the shallow-hole method. ${ }^{12}$ Its derivation is given in the original paper ${ }^{3}$ and also in ref. 12 and will not be repeated here. Gouffé, however, was not explicit in his published paper about one of the terms used in his expression $s / S_{0}$ (in refs. 3 and 12), and this uncertainty caused some doubt about the validity of his equation for cavity enissivity. However, Appendix I shows that his $s / S_{0}$ term is, in fact, an exact expression for the quantity that is required.

Buckley, ${ }^{5}$ Yamauti, ${ }^{6}$ Rossmann, ${ }^{7}$ Sparrow et al., ${ }^{8}$ and Liebmann ${ }^{9}$ derived their expressions by considering the flux coming from a surface element opposite the opening. This flux includes not only that emitted by the element but also that reflected by the element from other areas of the enclosure. The recent work of Sparrow and co-workers, ${ }^{8}$ who used this approach, is especially noteworthy in that it is possible from their analysis to obtain a rigorous evaluation of the emissivity of any elemental area on the walis of a cylindrical enclosure. This calculation requires the use of a computer, however, because the integral equation expressing the relations is amenable only to a numerical solution.

The third method, used by De Vos, ${ }^{10}$ is based on a calculation of the influence of the hole on the radiation from other surface elcments. The derived cxpression, however, cannot be applied easily to shallow cavities. It should be pointed out that DeVos considers a specularly reflected component in his analysis, whereas the equations derived by ali other investigators apply rigorously only to materials that are perfect diffusers.

The three expressions that seemed best suited for shallow cavities are those of Gouffe, ${ }^{3}$ Buckley, ${ }^{5}$ and Sparrow et al. ${ }^{8}$ These may be written in the following forms:

Gouffé:

$$
\epsilon_{c}=\frac{\epsilon_{w}\left\{1+\left(1-\epsilon_{w}\right)[(a / A)-f]\right\}}{\epsilon_{w}[1-(a / A)+(a / A)]},
$$

where

$$
\begin{aligned}
\boldsymbol{\epsilon}_{c}= & \text { cavity emissivity, } \\
\epsilon_{w}= & \text { emissivity of cavity walls, } \\
f \quad= & 1 /\left[1+(L / R)^{2}\right], \text { where } L \text { is the cavity depth and } R \text { is } \\
& \text { the cavity radins (see } A \text { ppendix I for a derivation of }
\end{aligned}
$$

this $f$ term which is the same as Goufté's $s / S_{0}$ ), $a / A=$ ratio of area of cavity opening to total area of cavity, opening included, $=1 /\{2[1+(L / R)]\}$ for a cylindrical cavity.

Buckley:

$$
\begin{aligned}
\epsilon_{\tau}=1+ & \frac{4 \sqrt{\epsilon_{w}}\left(1-\epsilon_{w}\right) \exp \left(-\sqrt{\epsilon_{w}} \cdot L / R\right)}{\left[\left(1-\epsilon_{w}\right)^{2}-\left(1+\sqrt{\epsilon_{w}}\right)^{2}\right]}, \\
& -\left[\left(1-\epsilon_{w}\right)^{2}-\left(1-\sqrt{\epsilon_{w}}\right)^{2}\right] \exp \left[-2 \sqrt{\left.\epsilon_{w} \cdot L / R\right]}\right.
\end{aligned}
$$

where the symbols are the same as those in Eq. (1)

Sparrow et al.

$$
\begin{aligned}
& \epsilon_{a}\left(x_{0}{ }^{\prime}\right)=\epsilon_{w}+\left(1-\epsilon_{w}\right) \int_{0}^{L / d} \epsilon_{a}\left(x^{\prime}\right) \\
& \left\{1-\left|x_{0}{ }^{\prime}-x^{\prime}\right| \frac{2\left(x^{\prime}-x_{0}\right)^{2}+3}{2\left[\left(x^{\prime}-x_{0}\right)^{2}+1\right]^{3 / 2}}\right\} d x^{\prime} \\
& +4\left(1-\epsilon_{w}\right)\left(\frac{L}{d}-x_{0}{ }^{\prime}\right) \int_{0}^{1} \epsilon_{a}\left(r^{\prime}\right) \\
& \frac{\left\{4\left(\frac{L}{d}-x_{0}^{\prime}\right)^{2}+1-r^{\prime 2}\right\} r^{\prime}}{\left\{\left[4\left(\frac{L}{d}-x_{0}^{\prime}\right)^{2}+1+r^{\prime 2}\right]^{2}-4 r^{\prime 2}\right\}^{3 / 2}} d r^{\prime} \\
& \epsilon_{a}\left(r^{\prime}\right)=\epsilon_{w}+8\left(1-\epsilon_{u}\right) \int_{0}^{L / d} \epsilon_{a}\left(x^{\prime}\right)\left(\frac{L}{d}-x^{\prime}\right) \\
& 4\left(\frac{L}{d}-x^{\prime}\right)^{2}+1-r^{\prime 2} \\
& \left\{\left[4\left(\frac{L}{d}-x^{\prime}\right)^{2}+1+r^{\prime 2}\right]^{2}-4 r^{\prime 2}\right\}^{3 / 2} d x^{\prime},
\end{aligned}
$$

where, in addition to symbols used earlier*

$$
\begin{aligned}
\epsilon_{a}\left(x_{0}{ }^{\prime}\right)= & \text { emissivity of a point } x_{0}{ }^{\prime} \text { on the cylindrical wall of } \\
& \text { the cavity, } \\
\epsilon_{a}\left(r^{\prime}\right)= & \text { emissivity of a point } r^{\prime} \text { on the base of the cavity, } \\
d & \text { diameter of cavity, } \\
x_{0}{ }^{\prime}, x^{\prime}= & \text { dimensionless variable coordinates, } x_{0} / d \text { and } x / d, \\
& \text { where } x_{0} \text { and } x \text { are distances along the cavity wall } \\
& \text { measured from the cavity opening, } \\
= & \text { dimensionless coordinate } r / R \text {, where } r \text { is radial dis- } \\
& \text { tance from center point of cavity bottom, and } R \text { is } \\
& \text { cavity radius. }
\end{aligned}
$$

Equations (3) and (4) must be solved simultaneously. The mathematical scheme used by Sparrow et al. was a numerical solution accomplished with the aid of a digital computer.

Both Michaud ${ }^{4}$ and Vollmer $^{14}$ made experimental neasurements of the emissivity of cylindrical cavities.

* The quantity $\epsilon_{a}\left(x_{0}{ }^{\prime}\right)$ may be defined here precisely as the ratio of the radiant flux diffusely radiating from an infinitesinal area at a point $x_{0}$ ' on the cavity wall to the flux that would radiate from an equal area of a blackbody at the same temperature. In this ratio, the flllx from the wall includes both emitted and reflected radiation. The reflected component originates from radiation from the remainder of the cavify that is incident on $x_{0}$. The definition of $\epsilon_{a}\left(r^{\prime}\right)$ is similar, except that $r^{\prime}$ is a point on the base of the cavity. 


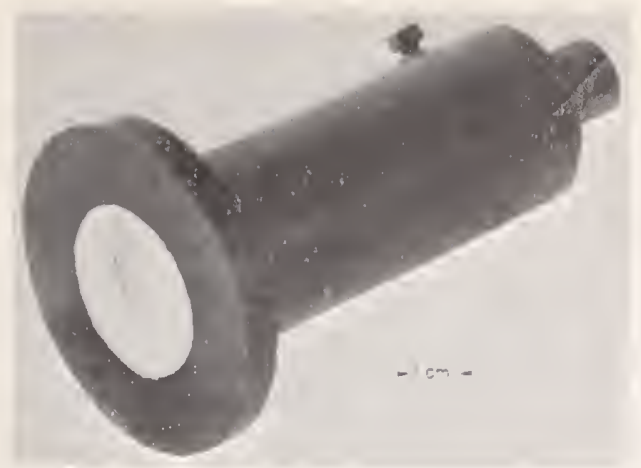

Fig. 1. Paper-lined cylindrical cavity.

Michaud used a variable-depth, water-heated "unpolished brass" cavity with a wall emissivity of 0.6 . His measurements, which were made at $337^{\circ} \mathrm{K}$, agreed with the Gouffé equation [Eq. (1)] to within $2 \%$ over an $L / R$ range that extended from 0.0 to 6.0 . Vollmer used an aluminum oxide cavity at $1100^{\circ} \mathrm{K}$ in conjunction with various optical filters. The filters permitted the cavity to be tested at wall emissivities of $0.09,0.13,0.37$, and 0.40 . Two $L / R$ ratios were used: 2.988 and 3.966. Vollmer altered Buckley's expression to conform with his experimental conditions. His tests showed that the measured radiation intensity agreed with the intensities predicted by this altered expression to within $6 \%$.

\section{Equipment and Procedure}

Figure 1 is a photograph of the adjustable cylindrical cavity used for the reflectance measurements. The cavity was designed so that it could be lined with paper 0.0035 in. $(90 \mu)$ thick without obstructing free movement of the plunger. The clearance between the paper-lined cylinder wall and the plunger was about 0.003 in. $(75 \mu)$.* The paper was attached to the cylinder wall and to the top of the plunger with a thin uniform layer of rubber cement. A scale on the plunger shaft permitted the $L / R$ value of the cavity to be set with a precision of approximately $0.5 \%$.

The cavity opening was the same size as the specimen opening in the General Electric recording spectrophotometer which was used for the reflectance measurements. This instrument has been described previously. ${ }^{15}$ Basically, it consists of: (a) a light source, (b) a double-prism assembly for supplying a monochromatic beam, (c) an integrating sphere lined with smoked magnesium oxide with openings for a specimen and a comparison standard, and (d) a radiation and detection system. The instrument operates in the equivalent of a double-beam mode, so that the reflected energy from the specimen is compared at each wavelength with that from a reference standard which,

* Although the area of clearance between the cavity base and the paper-lined wall is nearly $0.3 \%$ of the total area of the cavity for $L / R=0.5$, it can be shown that the presence of this clearance area will have only a negligible effect on cavity reflectance. in turn, has been calibrated against freshly smoked magnesium oxide. The geometry of the instrument is such that the spectral reflectance is measured under conditions approximating normal illumination and hemispherical viewing, which is the optical equivalent of reflectance for diffusely incident energy and normal viewing; this property, in turn, is the complement of the normal spectral emissivity.

Figure 2 shows that the incident beam of the instrument is at an angle of $6^{\circ}$ from the normal to the plane of the reflectance opening. When the depth of the cavity is zero, the beam, which encompasses approximately $25 \%$ of the area of the specimen opening in the reflectometer, falls on the center of the movable plunger. However, as is evident in Fig. 2, the beam strikes the cavity base more and more off center as the plunger is lowered. Also, since the incident beam is somewhat divergent, the cavity base is illuminated over a slightly larger area as the cavity depth is increased. The effect of these deviations from the conditions assumed in the derivation of the theoretical expressions will be discussed later in the paper.

The reflectance measurements were made after first centering the cavity over the specimen opening of the spectrophotometer. Curves of refiectance vs wavelength were then obtained from $0.40 \mu$ to $0.75 \mu$ for cavity $L / R$ ratios of $0,0.5,1.0,1.5$, and 2.0 . The $L / R$ was changed between determinations except for $L / R=2.0$. Two series of determinations were made: one with $L / R$ increasing in steps of 0.5 to the final value of 2.0 , and the other with $L / R$ decreasing by the same increment to the final value of zero. In all cases, the duplicate measurements agreed within 0.002 . The average reflectance values could be read from the two curves with a precision of \pm 0.001 .

The spectrophotometer curves gave the reflectances of the cavities relative to freshly prepared magnesium oxide. The values taken from these curves were then corrected for the reflectance of the magnesium oxide by using the data of Middleton and Sanders. ${ }^{16}$ The cavity emissivity, $\epsilon_{c} \lambda$, was obtained from the relation

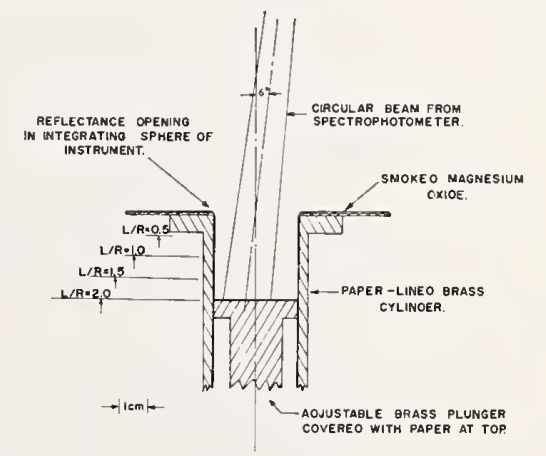

Fig. 2. Positioning of cylindrical cavity with respect to incident beam from spectrophotometer. Positions of plunger for various depth-to-radius $(L / R)$ ratios is indicated at left. 


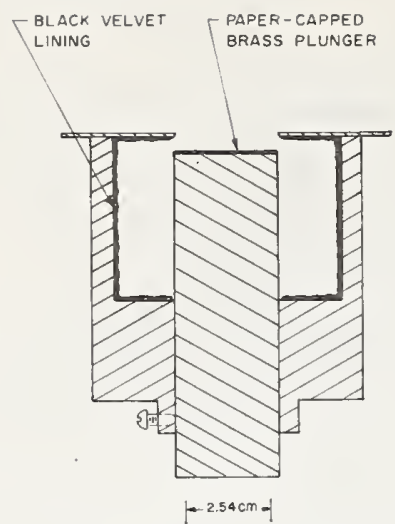

Fig. 3. Felt-lined cavity.

$\epsilon_{c \lambda}=1-\rho_{c \lambda}$, where $\rho_{c \lambda}$ was the corresponding spectral reflectance. The spectral emissivity of the cavity surface, $\epsilon_{w \lambda}$, was measured with $L / R=0$.

After the data reduction, it was possible to plot $\epsilon_{c \lambda}$ against $\epsilon_{w \lambda}$. A broad range of $\epsilon_{w \lambda}$, values was obtained by lining the variable-depth cylinder with different, selectively reflecting papers.

Three different nonglossy papers were used as cavity linings in the initial measurements: one was green, one white, and one black. Goniophotometric measurements were made on each to determine the nature of its deviation from a cosine diffuser. The geometry of the instrument used for the measurements was in accordance with ASTM Standard Practice Recommendation E167-60T. ${ }^{17}$ The light source in these measurements was a collimated beam from a tungsten lamp. All measurements were normalized to $0^{\circ}$ viewing for $75^{\circ}$ incidence and then plotted on polar coordinate paper. The relative intensities of reflected flux for the different angles of incidence for each paper were uncertain, one relative to another, to within $10 \%$.

\section{Corrections for Incident Beam Geometry}

The analytical expression given by Gouffé [Eq. (1)] applies to a normally incident beam of small cross-sectional area striking the center of the cavity bottom. As indicated in Fig. 2, the incident beam in the instrument deviated appreciably from this geometry; hence, it was essential to determine the effect of the deviations. This was done both by experiment and by a theoretical analysis. In both cases, it was assumed that only the first reflection from the cavity would need to be considered.

The experimental measurements were made by using a cavity with $7-\mathrm{cm}$ i.d., lined with black velvet, in which was mounted a movable plunger of $1.6-\mathrm{cm}$ radius, the same size as the specimen opening of the instrument (Fig. 3).* The top surface of this plunger, the only part of the cavity not lined with black velvet, was covered with one of three papers mentioned earlier.

* The design for the cavity was suggested by V. R. Weidner of the Bureau's Photometry and Colorimetry Section.
The velvet was known to be almost completely nonreflecting; hence, the only portion of the incident energy that emerged from the cavity was due to a single reflection from the paper on the top surface of the plunger.

Table I compares the $f$ values obtained from these measurements with values computed by the Gouffe expression for center-point illumination. Computation showed that a significant error would result, especially at low $L / R$, if the $f$ values for center-point illumination were used in Eq. (1) for computing the cavity emissivities.

Theoretical values of $f$ for the geometry of the instrument beam were computed by a graphical method described in Appendix II. As shown in Table I, there is very good agreement between values as computed in this way for the actual incident beam and those measured with the instrument.

In view of the results given in Table I, corrections for incident beam geometry were made by substituting in Eq. (1) the $f$ values obtained graphically for the $f$ values as computed by the Gouffe expression for center-spot illumination. In making these corrections, it was assumed that after the second reflection the radiant energy within the cavity is totally isotropic and homogeneous.

\section{Experimental Results for Cavities Lined with Nonglossy Papers}

Figures 4-6 show the spectral reflectance curves obtained for white, green, and black nonglossy paper linings at different depth-to-radius ratios of the cavity. Goniophotometric curves for the green paper on the same type of brass backing are shown in Fig. 7. The curves for the black and white papers were similar to the green except for somewhat stronger specular components at large angles of incidence.

The cavity emissivities for different wall emissivities are plotted in Fig. 8 for depth-to-radius ratios of 0 , $0.5,1.0,1.5$, and 2.0. The solid curves are those computed from the Gouffe expression with the $f$

\section{Table I. Experimental and Computed $f$ Values for Cylindrical} Cavities

Fraction, $f$, of energy reflected from illuminated area of base that emerges from cavity without a second reflection

\begin{tabular}{cccc}
\cline { 2 - 4 } $\begin{array}{c}\text { Depth-to- } \\
\text { radius ratio } \\
\text { of cavity }\end{array}$ & $\begin{array}{c}\text { Experi- } \\
\text { mental }^{a}\end{array}$ & $\begin{array}{c}\text { Computed } \\
\text { for assumed } \\
\text { center-spot } \\
\text { illumination }^{b}\end{array}$ & $\begin{array}{c}\text { Computed for } \\
\text { actual geometry } \\
\text { of incident beam }^{\text {c }}\end{array}$ \\
\hline 0.25 & 0.919 & 0.941 & 0.916 \\
0.50 & 0.755 & 0.800 & 0.749 \\
1.00 & 0.456 & 0.500 & 0.453 \\
1.50 & 0.289 & 0.305 & 0.285 \\
2.00 & 0.187 & 0.200 & 0.192
\end{tabular}

a Measured with a black velvet-lined cylindrical cavity equipped with an adjustable paper-capped plunger (see text).

${ }^{b}$ Computed from expression for $f$ given in Eq. (1).

${ }^{c}$ See Appendix II 


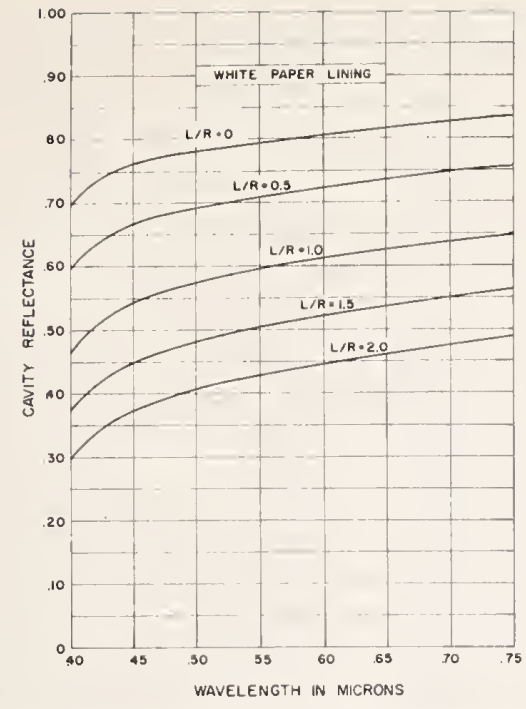

Fig. 4. Spectral reflectances of cavity with white paper lining.

term corrccted for the actual beam geometry. The agreement between the experimental points and the values predicted by the Gouffe equation is exccllent at an $L / R$ of 0.5 , but the agreement becomes progressively poorer as the hole depth increases.

This same type of agreement was also observed when the experimental points were plotted without correcting for the reflectance of smoked magnesium oxide. The principal effect of this correction was to move the points toward lower emissivity for both wall and cavity; the position of curve drawn through the points was changed only slightly by the correction.

\section{Effect of Specular Components}

As shown in Fig. 7, all three papers are good diffusers under the particular condition of the experiment in which the incident beam strikes the paper at $6^{\circ}$ from the normal. This means that the radiant energy is well diffused inside the cavity after the first reflection. The distribution of angles at which this diffused radiation strikes the cylinder walls on the next reflection will determine to a large extent just how well the experimental measurements will agree with a theory for cavity emissivity that assumes that the walls are diffuse reflectors at all angles of incidence. For a cavity of $L / R=0.5$ [Fig. 9(a) $]^{*}$ the energy from the second reflection, because of the small angles of incidence (measured from the normal) will still be almost completely diffuse, and good agreement with theory should occur. However, in a cavity of $L / R=2.0$ [Fig. 9(b)] a significant part of the diffused radiation from the first reflection will strike the wall at fairly large angles of incidence, and because of the specular component of reflected energy that is present at these large angles (see Fig. 7), more of this energy will emerge from the

* Figure 9 is constructed for center-point illumination for ease of illustration. Angles of incidence will, on the average, be greater for the experimental beam.

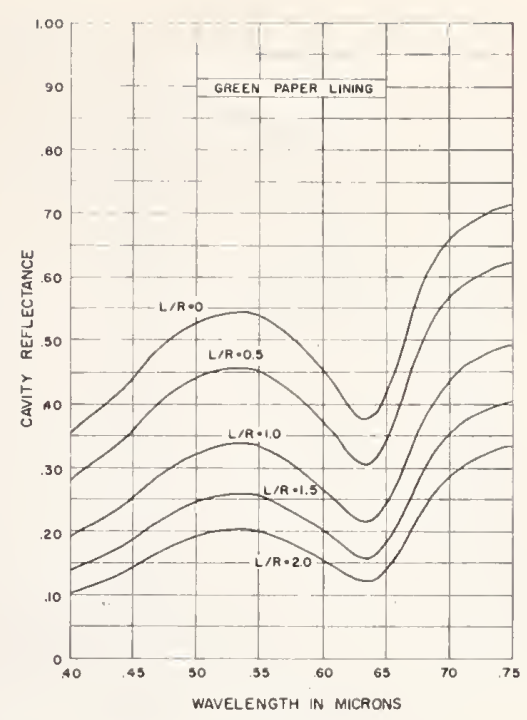

Fig. 5. Spectral reflectances of cavity with green paper lining.

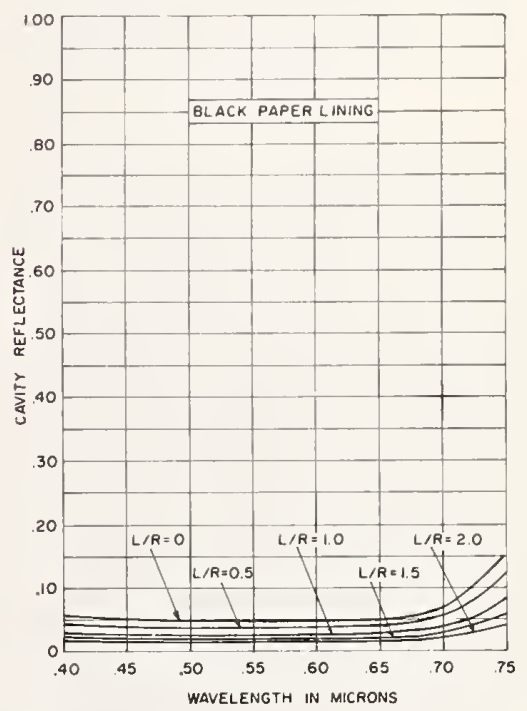

Fig. 6. Spectral reflectances of cavity with black paper lining.

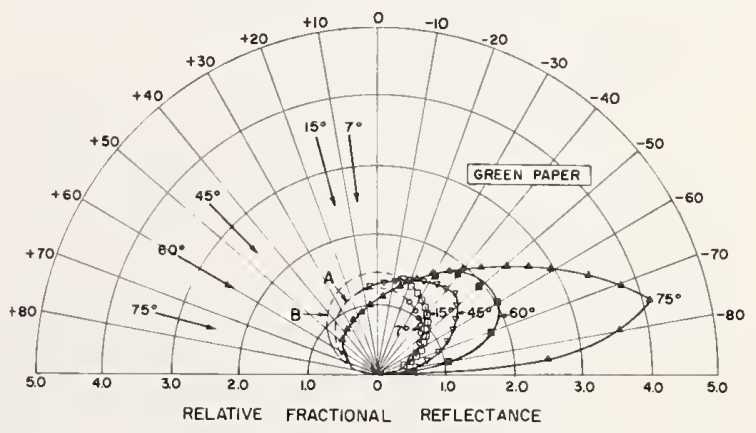

Fig. 7. Goniophotometric curves for the nonglossy green paper on brass backing. Small circle at center represents relative fractional reflectance of a cosine diffuser for $7^{\circ}$ angle of incidence; large circle, for $15^{\circ}$ angle of incidence. 


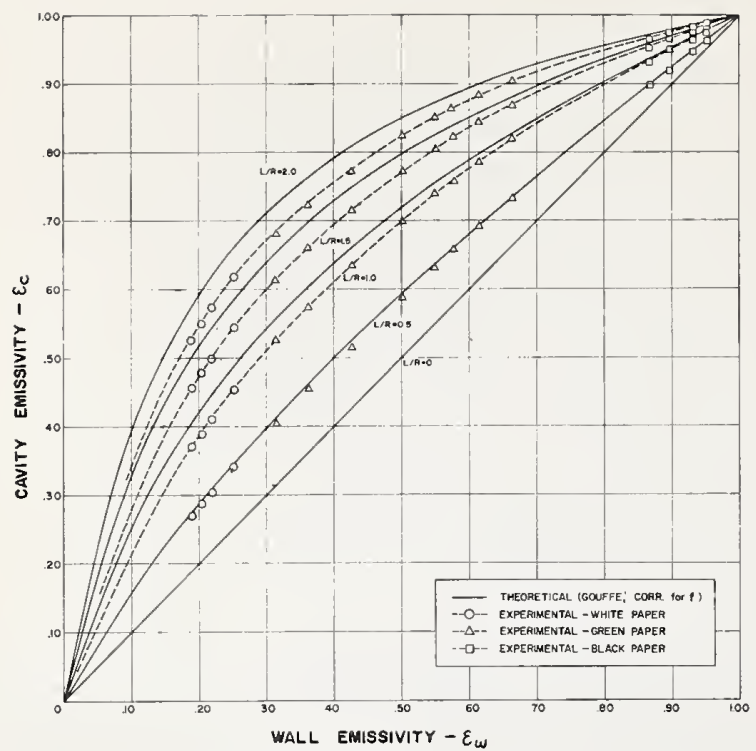

Fig. 8. Emissivities determined by reflectance technique for cavities lined with three nonglossy papers. Solid lines were computed from Gouffé expression with corrections for beam geometry.

opening than would emerge if the wall was a perfect diffuser. Hence, the measured cavity reflectance should be higher (and the cavity emissivity lower) than the values predicted by a theory which is based on a perfectly diffusing wall surface. Figure 8 shows that the cavity emissivities as determined from the reflectance measurements are in good agreement with the Gouffé expression at an $L / R$ of 0.5 . The lack of agreement for deeper cavities might be explained then by the presence of specular components at large angles of incidence.

To determine experimentally whether a better agreement with theory could be achieved if the walls of the cavity were lined with a material that was less specular at large angles of incidence than the papers used for the first measurements, a second test was made in which the brass cavity was lined with a recently developed "glare-free velvet" coating.* A yellow coating was selected for these measurements and applied at uniform thickness to one surface of a white paper. The coated paper was then used to line the cavity.

The goniophotometric curves for the coated paper on a brass backing are shown in Fig. 10. It is apparent from these curves that this particular lining is a much better diffuser than the ordinary matte papers used for the earlier measurements.

The spectral reflectance curves for the cavity with this yellow lining are given in Fig. 11. The wall reflectances were converted to emissivities and plotted against the corresponding cavity emissivities, by the same procedures used earlier. Figure 12 shows the resulting plot. The fact that the experimental points

* Available from 3M Reflective Products Division, Minnesota Mining and Manufacturing Company, 2501 Hudson Road, St. Paul 19, Minnesota. for this "velvet" finish paper are in better agreement with the Gouffé expression than the experimental points obtained from the cavities lined with ordinary matte papers indicates that specular components were affecting the earlier results.

\section{Agreement between Analytical Expressions and Experimental Measurements}

Table II shows the degree of conformity between the emissivity values predicted by each of the three theoretical derivations and the values obtained experimentally. To obtain the eavity emissivities for Sparrow et al., the curves of $\epsilon_{a}\left(r^{\prime}\right)$ vs $r^{\prime}$ in ref. 8 were enlarged photographically by a factor of 5 . Emissivity values were read from these curves with an accuracy of 0.001 , and the $r^{\prime}$ values to an accuracy of $0.01 \mathrm{R}$. Then these values were used to obtain the average emissivity of the area illuminated by the incident beam. The averaging process was very similar to that described in Appendix II.

Values included in the same table show the type of agreement with the Gouffe expression with the $f$ value computed for center-point viewing which is the complement of center-point illumination in the reflectance measurements. This is a condition that could not be duplicated with the spectrophotometer that was used
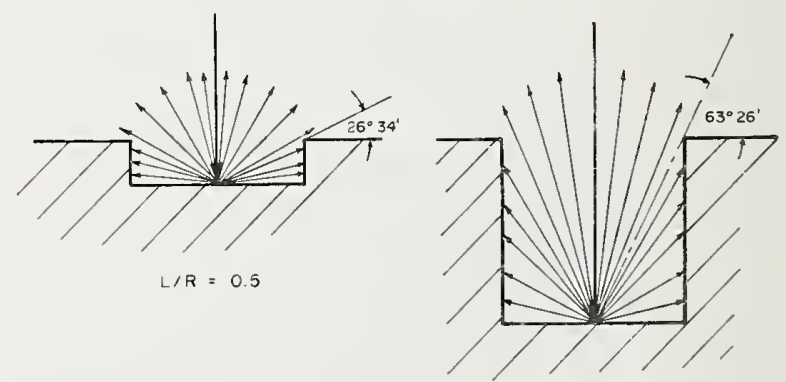

$L / R=2.0$

Fig. 9. Angles of incidence at which radiation diffused by a first reflection (center-spot illumination) strikes the walls on the second reflection.

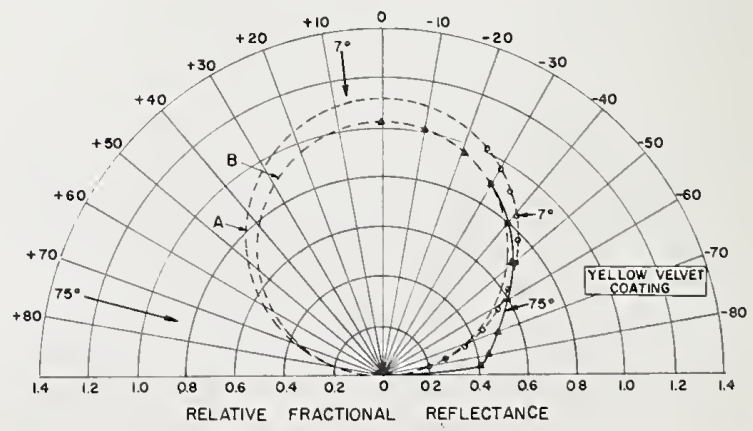

Fig. 10. Goniophotometer curves for the yellow velvet coating on a brass backing. Large circle at center represents relative fractional reflectance of a cosine diffuser for $7^{\circ}$ angle of incidence; small circle, for a $75^{\circ}$ angle of incidence. 
Table II. Comparison of Experimental and Theoretical Cavity Emittances

\begin{tabular}{|c|c|c|c|c|c|c|c|}
\hline \multirow[b]{2}{*}{$L / R$} & \multirow[b]{2}{*}{$\begin{array}{l}\text { Wall } \\
\text { emit., } \epsilon_{w}\end{array}$} & \multicolumn{2}{|c|}{ Experimental $^{a}$} & \multirow[b]{2}{*}{ Gouffe $e^{b}$} & \multirow[b]{2}{*}{$\begin{array}{c}\text { Grouffé } \\
\text { corr. }^{c}\end{array}$} & \multirow[b]{2}{*}{$\begin{array}{l}\text { Sparrow } \\
\text { et al. }{ }^{d}\end{array}$} & \multirow[b]{2}{*}{ Buckley } \\
\hline & & $\begin{array}{l}\text { Maite } \\
\text { papers }\end{array}$ & $\begin{array}{l}\text { Velvet } \\
\text { coating }\end{array}$ & & & & \\
\hline \multirow[t]{5}{*}{0.5} & 0.20 & 0.285 & 0.297 & 0.269 & 0.284 & 一 & 0.314 \\
\hline & 0.40 & 0.492 & 0.507 & 0.481 & 0.498 & - & 0.544 \\
\hline & 0.50 & 0.588 & 0.600 & 0.576 & 0.592 & 0.588 & 0.634 \\
\hline & 0.75 & 0.803 & 0.813 & 0.795 & 0.805 & 0.805 & 0.837 \\
\hline & 0.90 & 0.923 & 0.927 & 0.919 & 0.924 & 0.924 & 0.938 \\
\hline \multirow[t]{5}{*}{1.0} & 0.20 & 0.386 & 0.417 & 0.400 & 0.418 & - & 0.425 \\
\hline & 0.40 & 0.610 & 0.633 & 0.618 & 0.638 & - & 0.659 \\
\hline & 0.50 & 0.698 & 0.716 & 0.700 & 0.718 & 0.706 & 0.741 \\
\hline & 0.75 & 0.871 & 0.875 & 0.865 & 0.876 & 0.872 & 0.894 \\
\hline & 0.90 & 0.951 & 0.951 & 0.949 & 0.953 & 0.951 & 0.961 \\
\hline \multirow[t]{5}{*}{1.5} & 0.20 & 0.472 & 0.514 & 0.508 & 0.516 & - & 0.525 \\
\hline & 0.40 & 0.693 & 0.723 & 0.719 & 0.728 & - & 0.749 \\
\hline & 0.50 & 0.770 & 0.793 & 0.788 & 0.796 & - & 0.817 \\
\hline & 0.75 & 0.908 & 0.917 & 0.911 & 0.917 & - & 0.932 \\
\hline & 0.90 & 0.967 & 0.970 & 0.968 & 0.970 & - & 0.976 \\
\hline \multirow[t]{5}{*}{2.0} & 0.20 & 0.546 & 0.590 & 0.584 & 0.590 & - & 0.613 \\
\hline & 0.40 & 0.755 & 0.788 & 0.784 & 0.790 & - & 0.817 \\
\hline & 0.50 & 0.823 & 0.847 & 0.843 & 0.848 & 0.848 & 0.871 \\
\hline & 0.75 & 0.936 & 0.942 & 0.939 & 0.942 & 0.942 & 0.955 \\
\hline & 0.90 & 0.977 & 0.980 & 0.979 & 0.980 & 0.980 & 0.985 \\
\hline
\end{tabular}

a Interpolated from curves drawn through the data points shown in Figs. 8 and 12.

${ }^{b}$ Computed for center-point viewing; no correction from beam geometry.

${ }^{c}$ Corrected for beam geometry.

${ }^{d}$ Corrected for beam geometry through use of curves given in ref. 9 (see text).

"No correction for beam geometry required.

for the measurements. Poor agreement would be expectcd between experimental values and those computed for center-point viewing, and Table II shows that the agreement was relatively poor. However, when the $f$ term in the Gouffe expression was modified to conform with the off-center, diverging beam of the instrument, the agreement was excellent. It should be emphasized that this modification of the $f$ term was not a fundamental modification of the Gouffé expression; it was necessary only to make the Gouffé expression conform with the beam geometry of the instrument used for the measurements.

When this correction was made for the incident beam, excellent agreement resulted between the experimentally determined cavity emissivities and those predicted by the Gouffé expression. This same type of agreement with the experimental values was also observed for the expression derived by Sparrow et al.* Buckley's expression, however, predicted cavity emissivities that were higher than the experimental values by as much as $7 \%$.

* Total normal emissivities when computed for the cavity center by the two expressions are in excellent agreement in the shallow cavity range. However, for deep holes, the Gouffé emissivities are lower than those of Sparrow et al.; for example, for a wall emissivity of 0.5 and an $L / R$ of 8.0 , the Grouffé expression predicts an emissivity of 0.966 , whereas the Sparrow analysis gives 0.988 .

\section{Application of Results to Shallow-Hole Method}

Gouffé derives his equation for the emissivity of a specimen with a reference cavity of known geometry

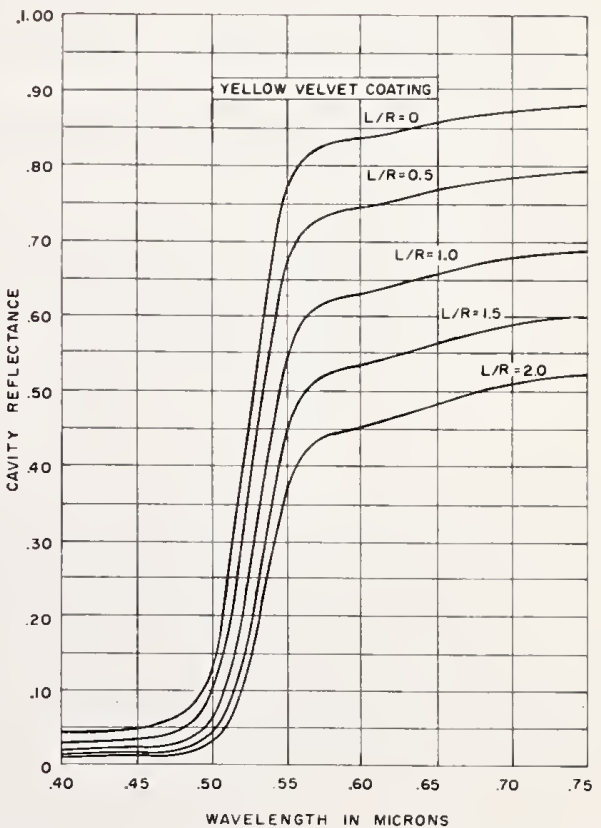

Fig. 11. Spectral reflectances of cavity lined with a yellow glarefree velvet coating. 


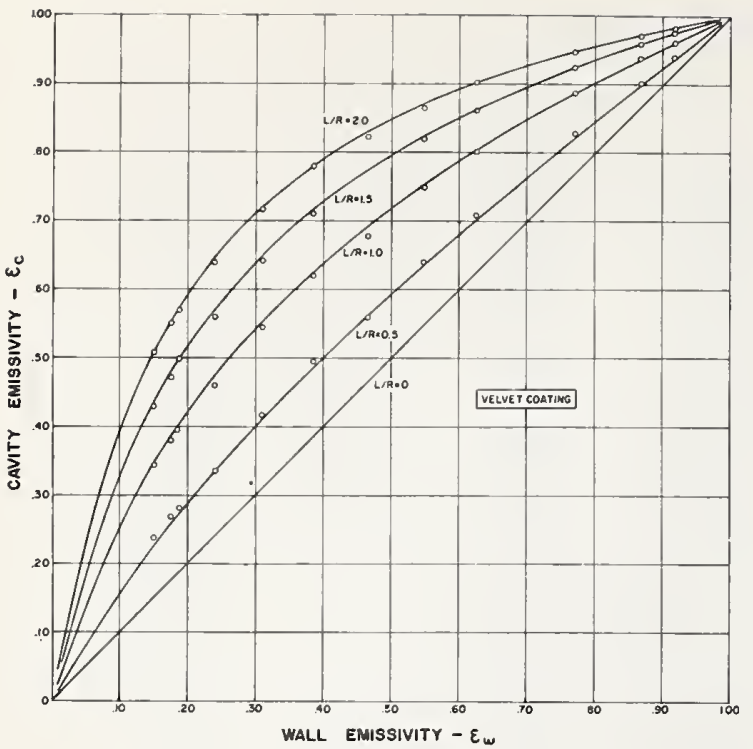

Fig. 12. Emissivities (circled points) determined by reflectance technique for cavities lined with the yellow velvet coating. Solid lines were computed from Gouffé expression with corrections for geometry of incident beam.

by combining Eq. (1) for the emissivity of a cavity with the relation:

$$
E=\frac{I_{s}}{I_{c}}=\frac{I_{s} / I_{B B}}{I_{c} / I_{B B}}=\frac{\epsilon_{s}}{\epsilon_{c}}
$$

where

$E=$ measured ratio of normal radiant flux per unit area from surface and cavity,

$I_{s}=$ normal radiant flux per unit area from a plane (non-cavity) surface of a specimen,

$I_{c}=$ normal radiant flux per unit area from a cavity formed in the specimen material,

$I_{B B}=$ radiant flux per unit area from a blackbody radiator under the same conditions at the same temperature,

$\epsilon_{\mathrm{g}}=$ emissivity of the specimen,

$\epsilon_{s}=$ emissivity of the cavity.

Assuming that the emissivity of the cavity wall, $\epsilon_{w}$, is the same as that of the specimen surface, $\epsilon_{s}$, Gouffé combines Eq. (5) with Eq. (1) to obtain:

$$
\epsilon_{s}=\frac{E[1+(a / A)-f]-(a / A)}{1-(a / A)+[E(a / A)-f]}
$$

Equation (6) is the expression used in the shallow-hole method. ${ }^{12}$ Obviously, it can be valid only if Eq. (1) is valid. Table II shows that the experimental values of cavity emissivity are in excellent agreement with those predicted by Eq. (1), at least for a material that is as good a diffuser as the velvet coating. It would appear then that the shallow-hole method when based on the Gouffé equation can be used with good reliability if the method is restricted to those materials that are diffusely reflecting. Most fine-grained oxide materials fall into this category. However, if the method should be used for materials that have strong specular components at large angles of incidence (relative to the normal), very appreciable errors could be expected. Such errors could be minimized, however, by maintaining the depth-to-radius ratio of the reference cavity at the lowest value consistent with good measurement precision. A second precaution in the use of Eq. (6) with the shallow-hole method is that the $f$ term should be corrected by the method outlined in Appendix II if an appreciable area of the cavity base is viewed by the radiation detector. This correction may be neglected if the viewed area is at the center of the cavity base and is small compared to the total cross-sectional area of the cavity.

\section{Summary}

The results of a study of the emissivities of cylindrical cavities with depth-to-radius ratios of $0.5,1.0,1.5$, and 2.0 have indicated the following:

1. When the wall material was a good diffuse reflector, the theoretically derived expressions of Gouffé and of Sparrow et al. predicted cavity emissivity to within 0.01 . Emissivities predicted by both expressions agreed equally well with the experimentally obtained emissivity.

2 . If the cavity material had relatively large specular components at large angles of incidence, relative to the normal, the experimental and theoretical emissivities still agreed for an $L / R$ ratio of 0.5 . However, the agreement became progressively poorer as the $L / R$ value increased, reaching an emissivity difference of 0.035 at $L / R=2.0$.

3. An expression derived by Buckley predicted cavity emissivities that were always higher than the experimentally determined values; the maximum deviation was 0.037 for $L / R=0.5$.

4. The Gouffé expression can be used with the shallow-hole method of measuring thermal emissivity with good reliability for materials that are good diffuse reflectors.

The assistance of the following members of the National Bureau of Standards staff is gratefully acknowledged by the authors: V. R. Weidner, for measuring the spectral reflectance curves of the cavities and for suggesting the design for the felt-lined cavity; David G. Goebel and Patrick B. Caldwell for obtaining the goniophotometric data; and J. C. Richmond, W. D. Hayes, Jr., and Harry J. Keegan for many helpful criticisms and suggestions during the course of the investigation.

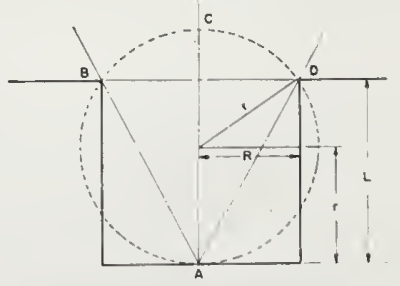

Fig. 13. Section through a cylindrical cavity. 


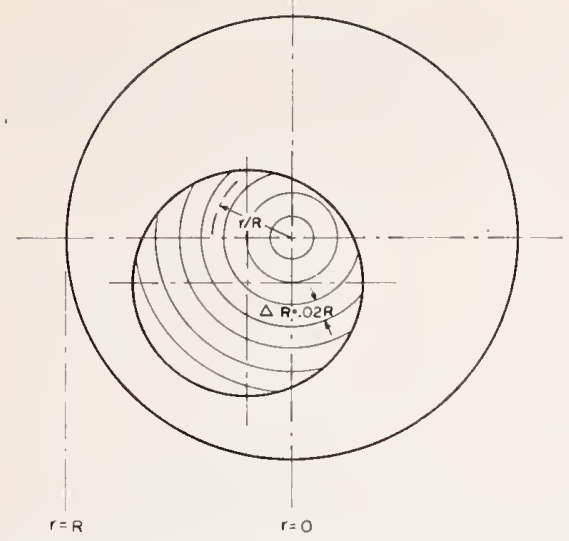

Fig. 14. Base of a cylindrical cavity illuminated by an offcenter incident beam. (Note: For purposes of illustration, circular segments are shown with a larger $\Delta r$ than was used in the graphical analysis.)

\section{Appendix I. Derivation of $f$ in Eq. (1) of Text}

As stated in the text, Gouffé ${ }^{3}$ derived his expression for the emissivity of a cavity through use of a reflectance approach. In his derivation, Gouffé assumes that the radiant flux enters the cavity normally as a beam of imall cross-sectional area and that it strikes the base of the cavity at the center point. He then proceeds to compute the fraction of this incident flux that will later leave the cavity after multiplc reflections from the walls, which are assumed to be diffusely reflecting.

The $f$ term which appears in Eq. (1) is the fraction of the reflected flux that is returned through the opening without a second reflection. This is the same as the $s / S_{0}$ given by Gouffé. The proof that this expression for $f$ is rigorous for a cylindrical cavity follows.

In Fig. 13, consider a narrow beam of radiant flux from an external source striking the cavity bottom at a small area at $A$. This flux will be diffused in accordance with the cosine law so that it will uniformly illuminate the interior of an imaginary sphere drawn through points $A B C D .{ }^{18,19}$ Since the sphere is uniformly illuminated, the fraction, $f$, of the reflected flux that will emerge through the opening $B D$ without a second reflection is given by the ratio of the area, $a_{s}$, of the spherical segment, $B C D$, divided by the total area of the sphere, $A_{0}$, or

where

$$
f=a_{s} / A_{0},
$$

$$
\begin{aligned}
A_{0} & =4 \pi r^{2}, \\
a_{\mathrm{B}} & =4 \pi r^{2}-2 \pi r L, \\
f & =\frac{4 \pi r^{2}-2 \pi r L}{4 \pi r^{2}}=1-\frac{L}{2} .
\end{aligned}
$$

It remains to find $r$ in terms of $L$ and $R$. From Fig. 13,

$$
\begin{aligned}
& r^{2}=R^{2}+(L-r)^{2}, \\
& r=\left(R^{2}+L^{2}\right) / 2 L .
\end{aligned}
$$

The fraction, $f$, then becomes

$$
\begin{aligned}
& f=1-\frac{L}{2\left[\left(R^{2}+L^{2}\right) / 2 L\right]}=1-\frac{L^{2}}{R^{2}+L^{2}}, \text { or } \\
& f=\frac{1}{1+(L / R)^{2}} .
\end{aligned}
$$

\section{Appendix II. Determination of $f$ for an Off- Center Incident Beam}

Consider a cylindrical cavity with diffusely reflecting walls illuminated by an incident beam of finite size that strikes the bottom of the cavity over any given area. The problem is to find $f$, the fraction of the energy reflected from the illuminated area, that emerges from the cavity without a second reflection.

The $f$ value for a beam striking the bottom at any point between the center and the vertical wall is given by Walsh ${ }^{20}$ :

$$
f=\frac{1}{2}\left[1-\frac{c / R}{\left[(c / R)^{2}+1\right]^{1 / 2}}\right]
$$

where:

$$
\frac{c}{R}=\frac{(L / R)^{2}+(r / R)^{2}-1}{2 L / R} .
$$

The average value of $f$ for the illuminated area is needed. Since this average could not be obtained analytically, a graphical solution was performed. Figure 14 illustrates the method. First, the outline of both the incident beam and the cavity base were plotted to scale. Circles concentric with the base wore then inscribed to form circular segments $0.02 \mathrm{R}$ in width. Next the area of each circular segment, $\Delta a$, within the illuminated area was determined and multiplied by the $f$ value computed from Eq. (7) for the average radius, $r / R$, of the segment. The average, $\bar{f}$, for the illuminated area was then computed from $f=\Sigma f \Delta a / \Sigma \Delta a$.

\section{References}

1. G. Ribaud and S. Nikitine, Ann. Phys. (Paris), 10, 451 (1929).

2. G. Ribaud, Traite de pyrométrie optique (Revue d'Optique, Paris, 1931), p. 231.

3. A. Gouffé, Rev. Opt. 24, 1 (1945).

4. M. Michaud, Compt. Rend. 226, 999 (1948).

5. H. Buckley, Phil. Mag. 4, 753 (1927); 6, 447 (1928); 17, 576 (1934).

6. Z. Yamauti, Com. Int. des Poids et Mes. Proc. Verb. 16, 243 (1933).

7. M. G. Rossmann, Brit. J. App. Phys. 6, 262 (1955).

8. E. M. Sparrow, L. U. Albers, and E. R. G. Eckert, J. Heat Transfer 84, 73 (1962).

9. G. Liebmann, Z. tech. Phys. 12, 433 (1931).

10. J. C. De Vos, Physica 20, 669 (1954).

11. C. S. Williams, J. Opt. Soc. Am. 51, 564 (1961)

12. D. G. Moore, Symposium on Measurement of Thermal Radiation Properties of Solids, Dayton, Ohio, NASA SP-31 (U.S. Govt. Printing Office, Washington, D. C., 1963), p. 515.

13. G. A. W. Rutgers, Handbuch der Physik, Bd. 26 (SpringerVerlag, Berlin, 1958), p. 129.

14. J. Vollmer, J. Opt. Soc. Am. 47, 926 (1957). 
15. K. S. Gibson, Spectrophotometry (200 to 1000 millimicrons), NBS Circ. 484 (1949).

16. W. E. K. Middleton and C. L. Sanders, J. Opt. Soc. Am. 41, 419 (1951).

17. Tentative recommended practice for goniophotometry of reflecting objects and materials, ASTM E 167-60T, Part 8, 1961 Book of ASTM Standards, p. 1909.

18. W. E. Sumpner, Proc. Phys. Soc. (London) 12, 10 (1892).

19. W. Wien and O. Lummer, Ann. Phys. 56, 451 (1895).

20. J. W. T. Walsh, Proc. Phys. Soc. (London) 32, 59 (1920).

Reprinted from

\section{APPLIED OPTICS}

Vol. 4, No. 1, January 1965 


\title{
52-INVESTIGATION OF SHALLOW REFER- ENCE CAVITIES FOR HIGH-TEMPERA- TURE EMITTANCE MEASUREMENTS
}

\author{
BY DWIGHT G. MOORE
}

NATIONAL BUREAU OF STANDARDS, WASHINGTON, D.C.

\begin{abstract}
Total normal emittance measurements were made on small specimens with shallow reference holes of circular cross section. The ratio of the radiant flux density from the surface to the flux density from the hole was measured and this value then converted to emittance by a theoretical expression based on hole dimensions. The expression applies only to diffusely reflecting materials.

Measurements were made on four different diffusely reflecting materials. Specimens of oxidized nickel that had reference holes with depth-to-radius ratios of 1.7 to 3.7 gave corrected total normal emittance values at $1350^{\circ} \mathrm{K}$ in the range 0.85 to 0.87 . The emittance of oxidized Inconel, when determined for a specimen with a reference hole having a depth-toradius ratio of 1.7 , was found to be in good agreement with values obtained by a heated strip method. The emittance measurements on four sintered alumina specimens varied from 0.43 to 0.47 when measured in air at $1375^{\circ} \mathrm{K}$.

The determined emittance for a polished specimen of high-purity graphite with a shallow reference hole increased with temperature linearly from 0.59 at $1100^{\circ} \mathrm{K}$ to 0.74 at $2150^{\circ} \mathrm{K}$. These values were in good agreement with those reported for polished graphite when measured by the rotating specimen method.
\end{abstract}

Large errors in emittance measurements may arise when a supposed temperature equality between the specimen surface and the referenceblackbody is not closely approximated. At moderate temperatures close equivalence can be achieved through proper use of differential thermocouples. At temperatures above $1800^{\circ}$ $\mathrm{K}$, however, thermocouples are not always reliable, and some other method is needed to maintain the necessary temperature balance.

Several investigators (ref. 1,2, and 3) have incorporated a reference blackbody cavity into either a tubular or spiral-wound specimen in such a way as to insure that the cavity walls and test surface are at the same temperature when the specimen is heated, usually by internal resistance. This approach is satisfactory for metals than can be fabricated as thin sheets or foils; however, it is obviously unsuited for the brittle nonmetals such as carbides, nitrides and oxides. For materials of this type Blair (ref. 4) and Reithof and coworkers (ref. 5) have used a method in which small cylindrical specimens with deep circular reference cavities were heated to high temperatures by high-frequency induction. The ratio of the radiant-flux density from the surface to that from the reference hole was then taken as the emittance of the specimen.

This deep-hole method when properly used may be suitable for metals and for some of the intermetallics, but for materials of low thermal conductivity, such as most nonmetals, a sizeable temperature difference between the hole bottom and the specimen surface is of ten difficult to avoid. Under most conditions of heating, the magnitude of the difference will increase with hole depth; therefore, if the hole is made shallow, the temperature difference between the hole bottom and test surface will be reduced. While a shallow hole will have an emittance appreciably less than that of a blackbody, it is nevertheless possible to compute its emittance 
from theoretically derived expressions. Further, if the specimen material is a diffuse reflector, as are most nonmetals, it has been shown by Gouffé (ref. 6) that under conditions of temperature equilibrium the specimen emittance can be computed from the following expression:

where:

$$
\epsilon=\frac{E\left(1+R-R_{0}\right)-R}{1-R+E\left(R-R_{0}\right)}
$$

$\epsilon \quad$ emittance of specimen

E ratio of radiant flux density from the surface to the flux density from the hole

R $\frac{1}{2(1+h / r)}$ for a cylindrical cavity and

$\mathrm{R}_{0} \frac{1}{1+(\mathrm{h} / \mathrm{r})^{2}}$

where:

$\mathrm{h}$ hole depth and $\mathrm{r}=$ hole radius.

The purpose of the present investigation' which is still in progress, is first to test the validity of equation (1) for several diffusely reflecting specimens with shallow reference cavities, and second, in the event that an experimental confirmation is achieved, to measure the total normal emittance of a number of refractory nonmetals by the shallow-hole approach at temperatures as high as $3000^{\circ} \mathrm{K}$.

The present paper is a progress report of the investigation. It describes equipment and test procedures, discusses measurement errors that have been encountered and gives the results of preliminary measurements on several different materials. Although additional work is needed, these preliminary results indicate that the shallow-hole approach has considerable promise as a method for the measurement of the emittance of nonmetals.

The author gratefully acknowledges the assistance of Alfred W. Crigler of the NBS staff, who not only constructed much of the equipment but also made many of the measurements.

The investigation is being conducted for the NASA Marshall Space Flight Center, under Contract H-22727.

\section{EQUIPMENT}

Figure $52-1$ is a schematic drawing and figure $52-2$ is a photograph of the furnace equipment which is similar in many respects

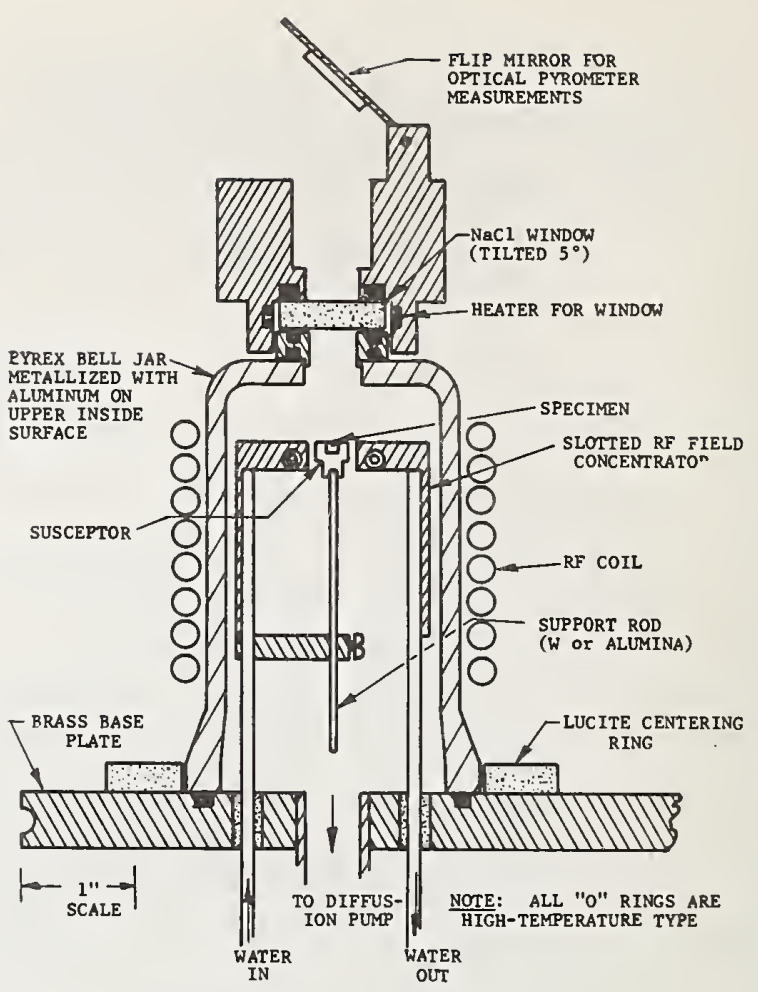

Figdre 52-1.-Schematic of furnace.

to that used by Blair (ref. 4). Two types of specimen are used as indicated in figure 52-3. Susceptors for type 1 specimens have been prepared of platinum, iridium, tungsten, and graphite.

The induced field for heating is supplied by a $10-\mathrm{kw}, 30-\mathrm{mc}$, radio frequency generator with a controlled power output. A water-cooled field concentrator with a longitudinal slot concentrates the energy from the field into the volume occupied by the specimen. The concentrator is fabricated of copper and has a bright plating of silver.

The bell jar consists of a Pyrex glass pipe cap with both ends ground and polished. A $1 / 2$-in.-diameter hole at the top permits viewing of the specimen through a sndium chloride window.

The vacuum system consists of mechanical pump, oil diffusion pump, and liquid-nitrogen cold trap. A needle valve above the cold trap permits argon to be let into the system. A titanium wire filament is positioned inside the bell jar below the concentrator to purify the 


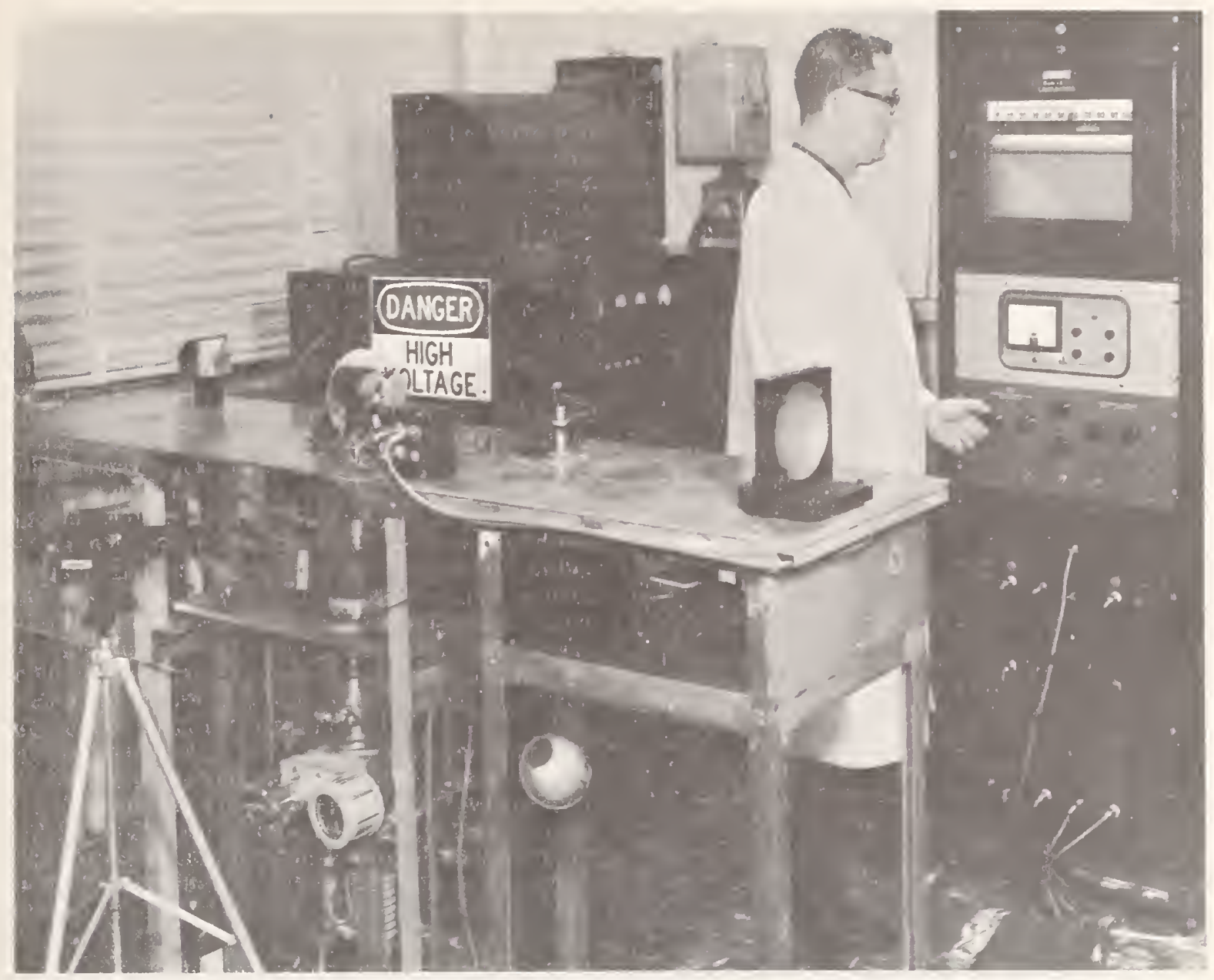

FIGURE 52-2.-Photograph of equipment.

argon. This filament is heated to a bright red heat both before and during test by an auxiliary power supply.

The flip mirror mounted at the top of the bell jar permits the specimen temperature to be measured with a micro optical pyrometer. The pyrometer was calibrated by the Temperature Physics Laboratory of the National Bureau of Standards with both the window and flip mirror in the optical path. The brightness temperature of the test surface was measured at the start and finish of each emittance determination and this temperature was converted to "true" temperature through use of $\epsilon_{0.65}$ values given in the literature. This meant that the specimen temperature was not known with high accuracy; however, because of the relatively minor effect of temperature on thermal emit tance, the error from this source was small.

As shown in figure 52-4 radiant energy from the specimen is directed by a $45^{\circ}$ mirror $\left(90^{\circ}\right.$ fold in fig. 52-4) through a 13-cps chopper, after which it is collected by a 5-in.-diameter spherical mirror and brought to a focus on a thermocouple detector. The path length from the specimen to the mirror is fixed so as to give an image that is twice the size of the specimen.

The detector, which is a commercial vacuumthermocouple type, is mounted on a base plate that can be moved either vertically or horizontally by means of micrometer screws. These screws permit the detector to be centered at any point on the image within $0.1 \mathrm{~mm}$. A small sheet of platinum foil with $0.5-\mathrm{mm}$ - 


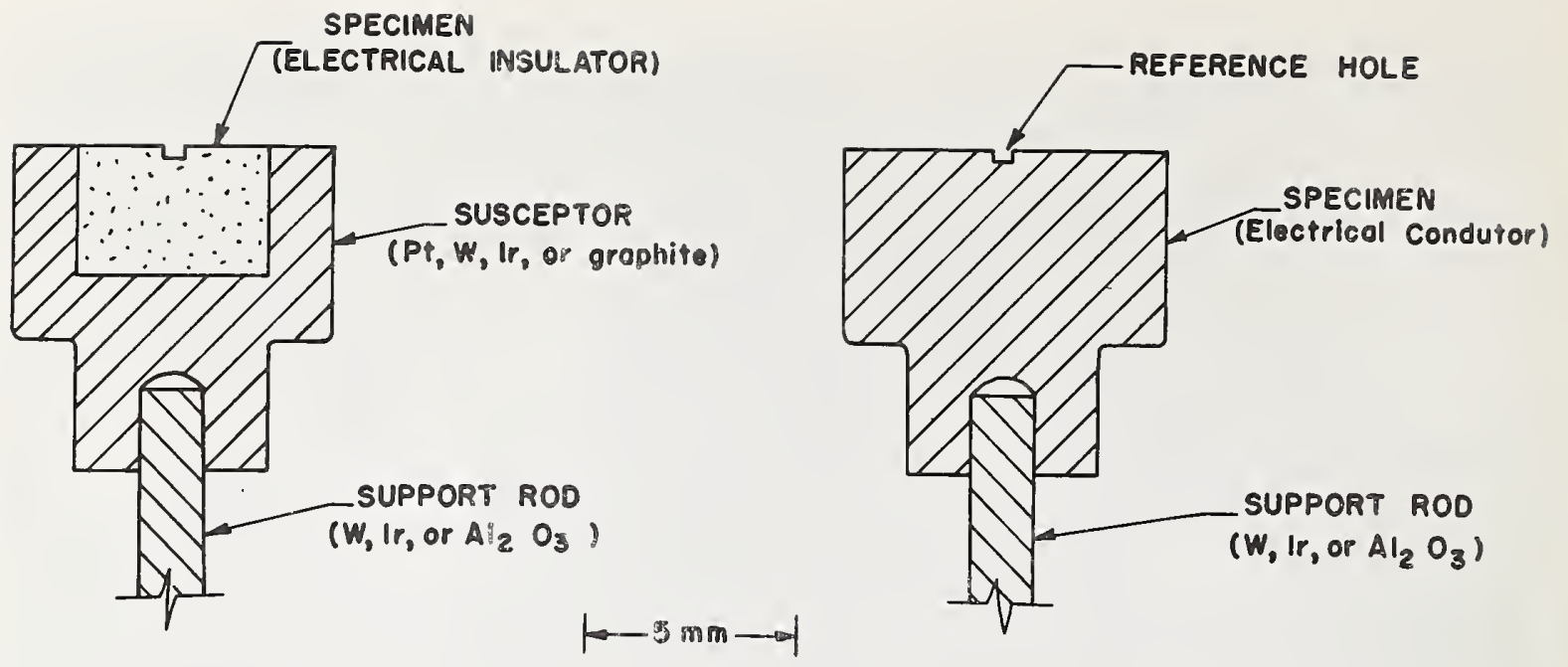

TYPE I SPECIMEN.

\section{TYPE III SPECIMEN.}

Figure 52-3.-Two types of specimens.

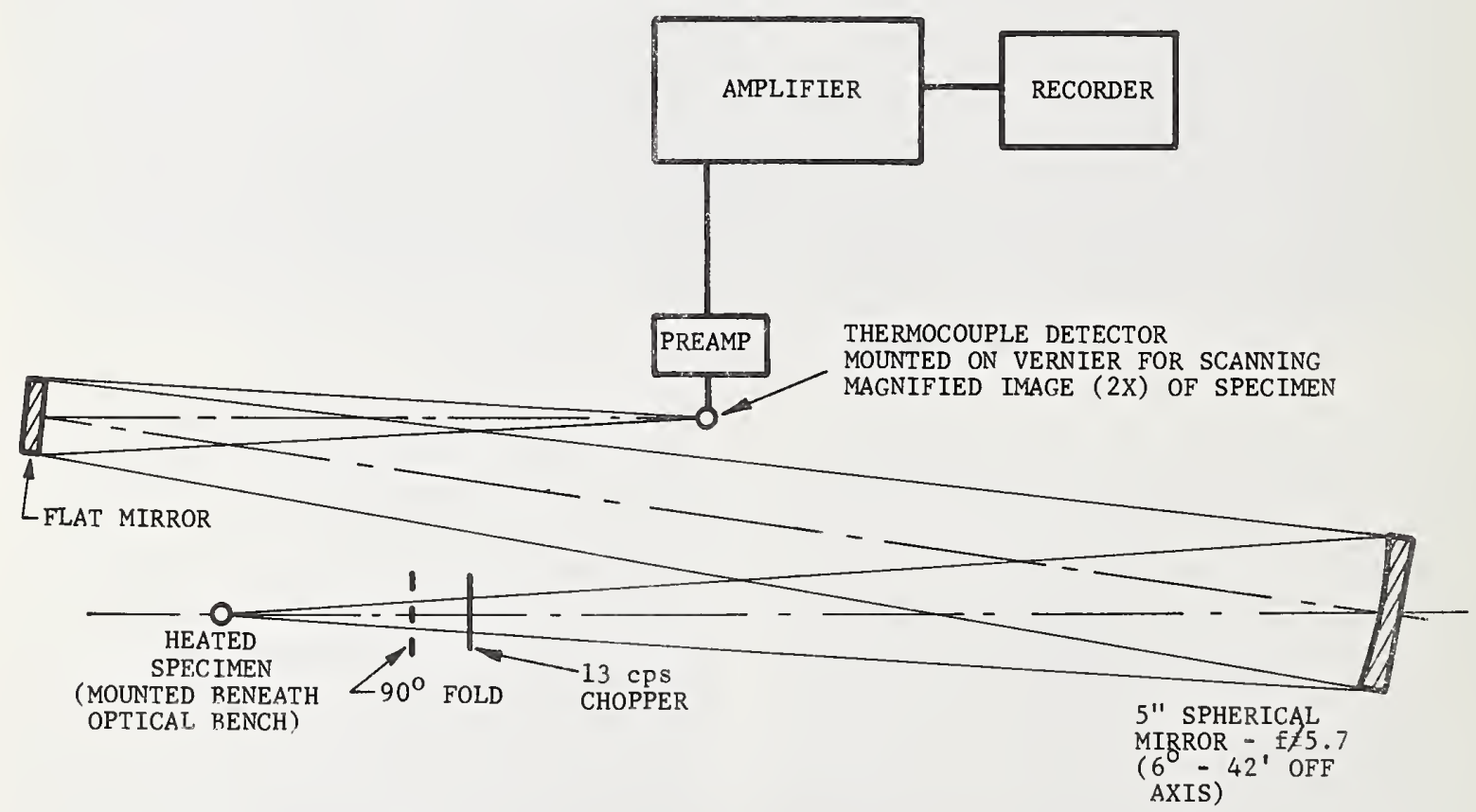

FIGURE 52-4.- Schematic diagram of optics and radiation detection system.

diameter center hole is placed over the front of the detector to serve as a limiting aperture Becsuse of the 2:1 magnification, this means that the detector sees an area of the specimen that is only $0.25 \mathrm{~mm}$ in diameter
The equipment for amplifying the detector signal was obtained commercially, as was the electronic high-speed potentiometer. The linearity of response from the detector-amplifier system was tested by placing high-speed sector- 
disk attenuators in the beam near the chopper. The attenuator blades reduced the radiant flux reaching the detector by $25,50,75,87.5$, and $95 \%$. The response was found to be linear within the limit of error of the measurements.

The optics were aligned visually with a heated specimen as a radiation source. To obtain a satisfactorily uniform detector response from the image of the specimen, it was found that the optical elements had to be accurately aligned so that the radiant flux filled the spherical mirror symmetrically. When this was done, the response from a grit-blasted platinum specimen was found to be uniform to $\pm 1 \%$ over a center area of the image which was $3 \mathrm{~mm}$ in diameter, and to $\pm 3 \%$ over an image area 7-mm diameter.

\section{PREPARATHON OF TEST SPECIMENS}

Type II specimens (fig. 52-3) were prepared by standard machining practices. The ceramic oxide specimens (type I) were formed by grinding bar specimens into $4 . ;$-mm diameter cylindrical rods on a diamond grinder. These rods were then cut into sections about $3.5 \mathrm{~mm}$ long and each section was then ground and polished with No. 14 diamond powder (average particle diameter $14 \mu$ ) to give a length of $3.20 \pm 0.02$ $\mathrm{mm}$. Epoxy resin was then bonded to the face surface and a center hole, approximately $0.5-\mathrm{mm}$ diameter, was formed at the center point with an ultrasonic drill with the No. 14 diamond powder as the cutting medium. The epoxy resin was found to be necessary in order to prevent chipping back of the material from the hole edge. The remaining resin was removed prior to testing by heating the specimen to about $200^{\circ} \mathrm{C}$ which destroyed the bond between the resin and the specimen. Hole dimensions were measured with a microscope. Care was taken with both metals and nonmetals to insure that the bottoms of the holes were flat.

Each specimen was cleaned ultrasonically in benzene prior to testing. In all tests the top of the type I specimens was flush with the top of the susceptor; also, both types of specimen were positioned so as to be flush with the top surface of the field concentrator.

\section{TEST PROCEDURE}

In making a test, the specimen was first centered in the field concentrator and the desired atmosphere achieved. The specimen was then heated to the test temperature as determined with the optical pyrometer sighted on the specimen surface. Once thermal equilibrium was established this temperature was found to remain constant to within about $4^{\circ} \mathrm{K}$. No temperature gradients over the 2-mmdiameter test area of the surface were detected with the micro optical pyrometer for any of the specimens tested. Temperature differences as small as $2^{\circ} \mathrm{K}$ could be detected with the pyrometer.

Prior to making relative flux measurements, the specimen image was scanned with the detector to determine the position of the reference hole. The detector was then positioned over the center of the hole image and the amplifier gain adjusted to give a high reading on the recorder. The detector was next moved to several positions centered on a circle of $1.5-\mathrm{mm}$ radius around the image of the hole center, a reading being obtained at each position. The number of readings varied with specimen uniformity. For most of the porous specimens, eight to twelve readings were required to obtain an average with a satisfactorily low standard deviation, while for polished nonporous specimens, four readings were usually sufficient.

Figure 52-5 is typical of the readings obtained from the two types of specimen. For both types, the ratio, E, in equation (1) was obtained by dividing the average reading from the surface by the reading from the hole center.

\section{RESULTS}

\section{Oxidized Nickel in Air}

Two type II specimens were prepared of high-purity nickel, each of which contained five 1.25-mm-diameter holes of different depths. Four of the holes were equally spaced on a 4-mm-diameter center line; the fifth was drilled at the center. After oxidation for 1 hour at $1400^{\circ} \mathrm{K}$, measurements were made at $1350^{\circ} \mathrm{K}$, in air without a sodium chloride window. The purpose of the test was to determine the effect of hole depth on the emittance of a specimen as computed from the flux ratios by the Gouffé expression; the purpose was not to measure the emittance of a well characterized specimen of oxidized nickel. 


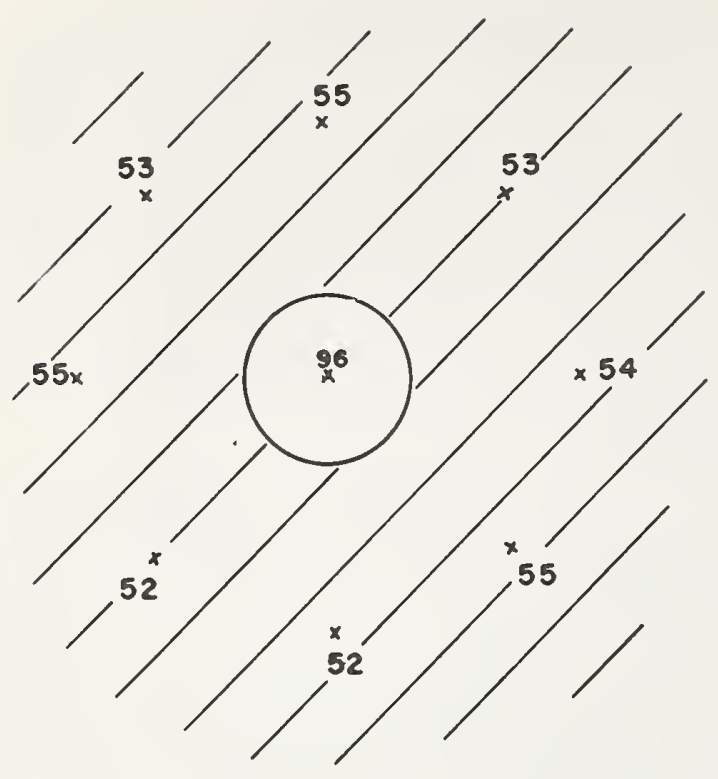

SINTERED

ALUMINA

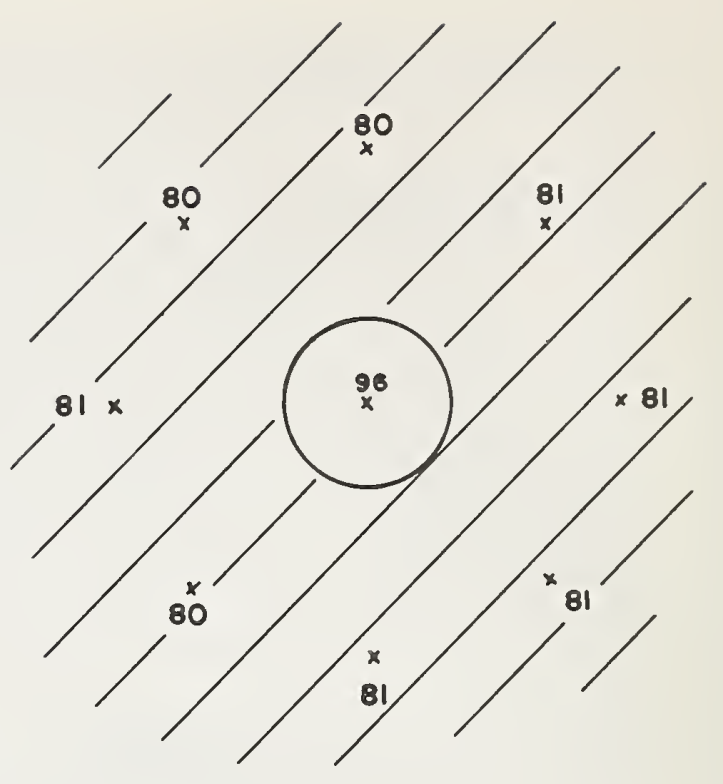

OXIDIZED

INCONEL

FIGURE 52-5.- Relative flux density measurements on a porous specimen (sintered alumina, $h / r=1.5$ ) and on a nonporous specimen (oxidized Inconel, $h / r=1.7$ ). Center circle represents edge of reference hole.

Table $52-1$ lists the results. Agreement between specimens is good; also the emittances as computed from equation (1) from holes with depth-to-radius ratios of 1.7 to 3.7 all fall within the range $0.86 \pm 0.01$. The emittances computed from the two most shallow holes ( $h / r$ of 0.9 and 0.6 ) are significantly lower. This indicates that equation ( 1 ) is not valid at $h / r$ ratios below 1.0 .

\section{Oxidized Inconel in Air}

One type II Inconel specimen was prepared in which a single hole, $0.576 \mathrm{~mm}$ diameter by $0.486 \mathrm{~mm}$ deep, was drilled at the center of the face surface. The specimen was mechanically polished, etched and then oxidized by procedures that were given in detail in a recent report by O'Sullivan and Wade (ref. 7). The radiant flux density from the surface and from the hole was measured at three temperatures and the resulting flux ratios converted to total normal emittance by equation (1). No viewing window was used.

Table 52-II compares the results with those obtained by O'Sullivan and Wade. Agreement is good except for a somewhat lower tempera-
TABLE 52-I.-Total Normal Emittance of Oxidized Nickel at $1350^{\circ} \mathrm{K}$

\begin{tabular}{|c|c|c|c|c|c|}
\hline $\begin{array}{c}\text { (Hole diameter=1.25 mm) } \\
\text { No. }\end{array}$ & $\begin{array}{c}\text { Hole } \\
\text { No. }\end{array}$ & $\begin{array}{c}\text { Hole } \\
\text { Depth, } \\
\text { mm }\end{array}$ & $h / r$ s & $E$ b & $\epsilon_{\ell n^{\circ}}$ \\
\hline & & & & & \\
N-1_- & 1 & 0.54 & 0.9 & 0.913 & 0.81 \\
& 2 & 1.13 & 1.8 & .895 & .86 \\
& 3 & 1.77 & 2.8 & .872 & .86 \\
& 4 & 2.16 & 3.5 & .874 & .86 \\
& 5 & 2.32 & 3.7 & .862 & .85 \\
& 1 & .40 & .6 & .923 & .76 \\
& 2 & 1.06 & 1.7 & .897 & .87 \\
& 3 & 1.71 & 2.7 & .887 & .87 \\
& 4 & 2.32 & 3.7 & .862 & .85 \\
& 5 & 2.25 & 3.6 & .868 & .86 \\
& & & & & \\
\hline
\end{tabular}

- Depth-to-radius ratio.

b Ratio of radiant flux from surface adjacent to reference hole to the flux from the hole.

${ }^{\epsilon_{t n}}=$ tota! normal emittance, computed from equation (1).

ture coefficient for the measurements with the shallow-hole method.

\section{High-Purity Graphite in Vacuum}

A small block of graphite was machined into several type II specimens. Principal impurities 
TABLE 52-II.-Total Normal Emittance of Oxidized Inconel

\begin{tabular}{|c|c|c|c|}
\hline \multicolumn{2}{|c|}{ Temperature } & \multicolumn{2}{c|}{ Total Normal Emittance } \\
\hline${ }^{\circ} \mathrm{K}$ & ${ }^{\circ} \mathrm{F}$ & $\begin{array}{r}\text { o'Sullivan } \\
\text { and Wade }\end{array}$ & $\begin{array}{c}\text { NBS Shal- } \\
\text { low Hole }\end{array}$ \\
\hline & & 0.78 & 0.79 \\
1033 & 1400 & .80 & .80 \\
1144 & 1600 & .82 & .81 \\
1255 & 1800 & .82 \\
\hline
\end{tabular}

- From ref. 7.

b. Depth-to-radius ratio of reference hole, 1.7 .

in the material were, in ppm, 100-200 calcium, $<100$ titanium, $<100$ iron and $<100$ vanadium. The porosity was approximately 25 percent.

The results of measurements made with a highly polished specimen with a depth-toradius ratio of 2.8 are plotted in figure 52-6. The measurements were made in a vacuum of $5 \times 10^{-6} \mathrm{~mm}$ of $\mathrm{Hg}$. As shown in the figure, the values for the polished graphite with filled pores are in good agreement with those reported by Plunkett and Kingery (ref. 8) for a polished commercial-grade graphite as measured by the rotating cylinder method.

The polished graphite used by Plunkett and Kingery contained a small number of pits, caused probably by open pores at the surface. Open pores of this type were filled for the present measurements by first polishing with $3 / 0$ paper that had been wetted with carbon tetrachloride and then heating the specimen in vacuum to $2350^{\circ} \mathrm{K}$ for $15 \mathrm{~min}$ to consolidate the graphite that had entered the pores during the polishing operation. Although the walls of the reference hole were polished, the polish was not equal to that of the specimen surface.

The sizeable increase in emittance caused by presence of a large number of open pores is illustrated in figure 52-6. The specimen used for this test had a depth-to-radius ratio of 2.5. It was dry polished with $3 / 0$ paper and then cleaned ultrasonically to remove the graphite that had entered the pores during polishing.

\section{Sintered Alumina in Air}

Type I specimens of alumina were prepared from bars fabricated from $99.9+$ alumina that had been cold-pressed and then heat treated at $2075^{\circ} \mathrm{K}$ to give a porosity of $15.6 \%$ (Code 15

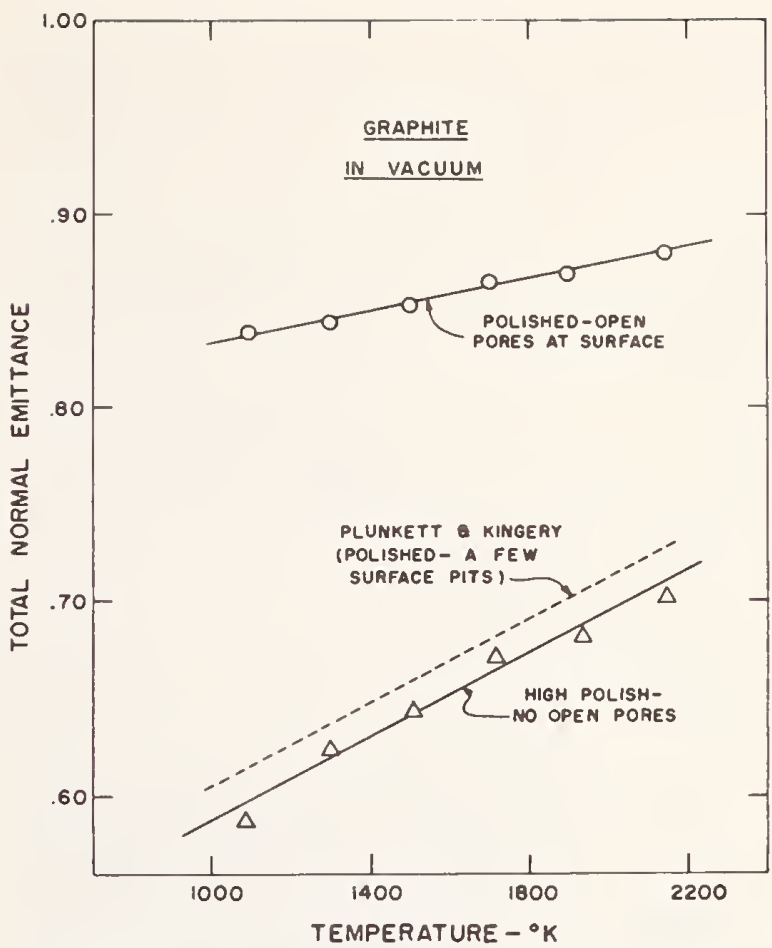

Figdre 52-6. - Total normal emittance of graphite.

in ref. 9). Tests were made in air in a platinum susceptor at temperatures in the range 1200 $1600^{\circ} \mathrm{K}$.

The results at a single temperature $\left(1375^{\circ} \mathrm{K}\right)$ for the four specimens tested are listed in table 52-III. The variation between specimens is fairly high. This could be caused by real differences in emittances.

A specimen of commercially sintered alumina was also tested. This material when examined with a microscope appeared to have a glass phase with corundun crystals in the matrix. There were very few open pores. Figure 52-7 shows the emittance-temperature curves for the two types of alumina.

\section{MEASUREMENT ERRORS}

\section{Precision of Measurement}

Repeated determinations on the same specimen with a fixed hole depth at a single temperature showed that the precision of measurement was within \pm 0.005 emittance unit. This was true even when specimens were removed from the equipment and then repositioned in the field concentrator in a different radial orientation. The same measurement precision was observed for type I and type II specimens. 


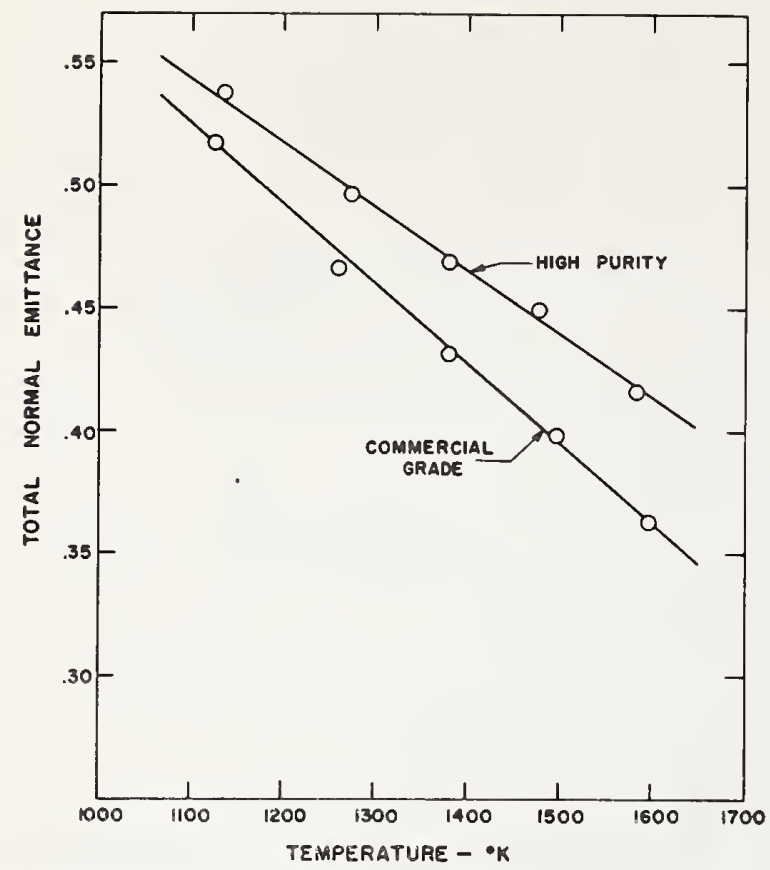

Figure 52-7.-Total normal emittance of two sintered alumina specimens.

'Table 52-III is a report of some typical data.

TABLE 52-III.- Total Normal Emittance of HighPurity Alumina Specimens at $1975^{\circ} \mathrm{K}$.

\begin{tabular}{|c|c|c|c|c|c|}
\hline Spec. No. & $\begin{array}{l}\text { Hole } \\
\text { diam., } \\
\text { mm }\end{array}$ & $\begin{array}{l}\text { Hole } \\
\text { depth, } \\
\text { mm }\end{array}$ & $h / r$ a & $E$ b & $\operatorname{lin}{ }^{\circ}$ \\
\hline$A-1 \ldots$ & 0.580 & 0.433 & 1.5 & 0.575 & 0. 43 \\
\hline $\mathrm{A}-2 \ldots$ & .562 & .510 & 1. 8 & .580 & .47 \\
\hline $\mathrm{A}-3 \ldots$ & .550 & .530 & 1. 9 & .553 & .45 \\
\hline $\mathrm{A}-4 \ldots$ & .554 & .544 & 2.0 & .536 & .43 \\
\hline $\begin{array}{r}\text { Aver- } \\
\text { age }\end{array}$ & & & & & 0. 445 \\
\hline
\end{tabular}

a Depth-to-radius ratio.

b Ratio of radiant fiux from surface adjacent to hole to fiux from hole.

c Total normal emittance as computed from equation (1).

\section{Back Reflection from Bell Bar}

Early in the testing a thin layer of aluminum was vapor deposited on the inside top of the bell jar to serve as a radiation shield and thereby reduce the temperature that the window O-rings would attain during testing. Although no appreciable error from this source was anticipated, recent tests in which measurements were made in air both with and without the bell jar indi- cated that back reflections from the top of the bell jar caused the emittances as herein reported to be high by about $3 \%$. Future measurements will be made without this reflecting film

\section{Error fro:m Viewing Window}

When a sodium chloride window is used in emittance equipment, about $10 \%$ of the radiant flux reaching the window is reflected back toward the source. If the window is parallel with the specimen surface, this back reflected energy will reinforce the flux from the surface to a greater extent than it will that from the reference cavity which, in turn, will cause the emittance readings to be high. Tilting of the window at a small angle to the specimen surface should have prevented this window error in the present design. However, it was found late in the testing that the emittance was from 10 to $20 \%$ higher with either a flat or tilted window in the beam than when no window was present. Numerous tests have indicated that some of the energy that is back reflected from the viewing window eventually reaches the detector because of multiple reflections within the bell jar. This has necessitated a redesign of the furnace chamber with a radiation trap containing an aperture immediately below the window. The data for graphite in vacuum, as shown in figure 52-6, however, were obtained before the design was changed. The data for oxidized nickel, oxidized Inconel, and sintered alumina were obtained with no window in the system; hence, the data for these materials are free of window arror.

\section{CONCLUDING REMARKS}

The Gouffe expression is based on two main assumptions; first, that the specimen is a perfectly diffuse reflector and, second, that no temperature differences exist between hole and surface. Neither of these conditions can be achieved in a real specimen, but they can be approached. It follows that errors will always be present in measurements made by the shallow-hole method based on the Gouffé equation. The measurements completed up to the present time suggest that these errors will not be excessive for oxidized metals. They also suggest that the Gouffe expression is ralid to within a few percent if (a) the specimens are diffusely reflecting and of relatively high thermal conductivity, and (b) the reference hole 
has a depth-to-radius ratio in the range of about 1.5 to 3.7 .

The good agreement obtained between measurements on similarly prepared oxidized Inconel made by the shallow-hole apprcach and those made by O'Sullivan and Wade, who used the heated strip method, can be considered as a confirmation of the Gouffe explession. The agreement observed for graphite with the rotating cylinder measurements of Plunkett and Kingery, however, may be mostly fortuitous, not only because of differences in the two graphites, but also because of the window error. Although this same type of error may have been present from the calcium fluoride window used in the rotating cylinder measurements of Plunkett and Kingery, it seems safe to assume that its magnitude would have been smaller because of their use of a blackened, watercooled viewing port.

It is believed that the shallow-hole method would find its greatest usefulness in measuring the emittance of polycrystalline ceramics. Because of the low thermal conductivity of such materials, sizeable temperature differences between hole and surface would be expected if deep reference cavities were used. In the case of the alumina specimens, holes approximately $0.5-\mathrm{mm}$ diameter by $0.5 \mathrm{~mm}$ deep were employed as reference cavities. While only small temperature differences should be present between holes of this type and the specimen surface, these differences cannot, a priori, be assumed to be negligible. Before the accuracy of the shallow-hole method can be accepted, these differences need to be investigated. Possible approaches include (a) theoretical heattransfer calculations, (b) temperature ineasurements made with microsize thermocouples, and (c) comparison of the emittance of an oxide material as determined by the shallow-hole method with the emittance of the same material determined by the rotating specimen method. Because of the importance of temperature equivalence between hole and surface to the reliability of the measurements, it may be necessary to use all three of these approaches. Until this is done, the results reported for sintered alumina should be considered as preliminary and, hence, of questionable accuracy.

\section{APPENDIX-DERIVATION OF GOUFFE EXPRESSION FOR COMPUTING EMITTANCE}

The original article by Gouffé appeared in Revue d'Optique in 1945. Because it was written in French and also because volumes of this journal may not be readily available, Gouffé's derivation of Equation (1) is given in detail.

\section{CQREECTION FOR EMITTANCE OF A CAVITY}

Consider an enclosure of any form (fig. 52-8) at uniform temperature in whose wall is bored an opening of area $s$, small relative to the total surface area $S$ (opening included) of the wall. This chamber very roughly constitutes a blackbody, whose emittance is, however, not strictly equal to unity.

If $\rho$ denotes the factor of diffused reflection of the material of which the chamber is formed. and $\Omega$, the solid angle in which the radiation of the blackbody is emitted to the outside, the general formula that is often used to express

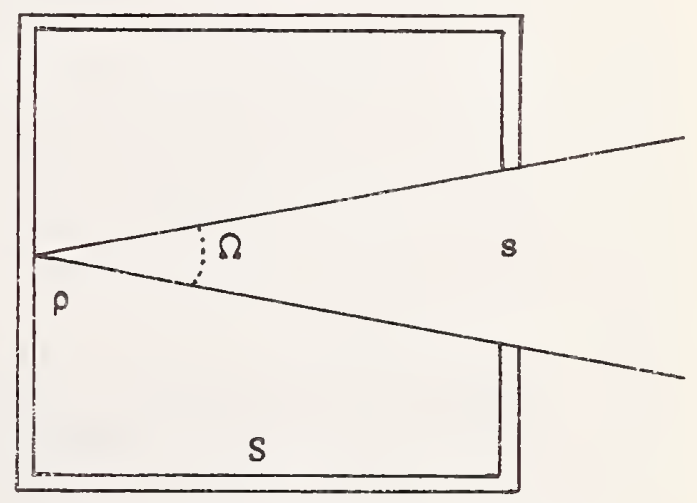

Figure 52-8.-Blackbody enclosure.

the effective emittance, $\epsilon_{0}$, of such a radiator is (ref. 10):

$$
\epsilon_{0}=1-\rho \frac{\Omega}{\pi}
$$

This equation has been derived by supposing 
that a unit incident flux, penetrating into the blackbody through the opening $s$, is diffusely reflected by the portion of the wall opposite this opening according to Lambert's Law and supposing that only the fraction $\rho(\Omega / \pi)$ of this flux goes out again from the chamber diffused into the solid angle $\Omega$ in which the cavity can radiate. One assumes that the portion of incident flux diffusely reflected toward the wall of the chamber is completely absorbed by the latter, which is manifestly inexact, since this wall reflects it diffusely in turn in all directions, particularly toward the opening. The emerging flux therefore must be appreciably larger than $\rho(\Omega / \pi)$.

In order to obtain its exact value, it is necessary to take into account the multiple diffuse reflections which are produced in the interior of the chamber.

If we consider an element $d S$ of the internal wall of the radiator, the total flux which it emits includes:

1. the flux $d F$ due to its own radiation.

2. the flux $\rho d \phi$ diffusely reflected by the element and proceeding from the flux $d \varphi$ emitted by the other parts of the internal surface of the blackbody and incident on $d S$.

Since the element $d S$ can be compared in rough approximation to a perfect blackbody of uniform radiance as defined, the total flux which it emits is proportional to its area $d S$. As the flux, $d F$, due to the natural radiation of the element is also proportional to $d S$, the result is that the flux $d \varphi$ is in turn proportional to $d S$. This means that after multiple reflections in the interior of the chamber, the emitted flux $\varphi$ distributes itself uniformly over the internal surface of the radiator and, for present purposes, it will be assumed that this uniform distribution of emitted flux is brought about as early as the second reflection.

Now return again to the case of a unit incident flux penetrating into the blackbody through the opening, $s$, and consider the fraction of that flux which after an infinite number of diffuse reflections, will be absorbed by the wall. This fraction will be equal to the sought-for emittance of the cavity.

The fraction of the flux leaving through the opening, $s$, after the first reflection has the value $\rho(\Omega / \pi),{ }^{1}$ just as we have already shown at the beginning, and there is left in the chamber, a flux, $1-\rho(\Omega / \pi)$, which undergoes a second reflection after that; conforming to the abovementioned hypothesis we assume that it is uniformly distributed.

Under these conditions, the fraction of the flux twice reflected which leaves through $s$ will have for its value:

$\rho^{2}(1-\Omega / \pi) s / S$ and there will remain in the interior $\rho^{2}(1-\Omega / \pi)(1-s / S)$ which will be reflected again.

The fraction of flux reflected 3 times leaving through the opening will be:

$$
\rho^{3}\left(1-\frac{\Omega}{\pi}\right)\left(1-\frac{s}{S}\right) \frac{s}{S}
$$

and

$$
\rho^{3}\left(1-\frac{\Omega}{\pi}\right)\left(1-\frac{s}{S}\right)^{2}
$$

will remain.

On the whole, a total flux:

$$
\begin{aligned}
\rho \frac{\Omega}{\pi}+\rho^{2} & \left(1-\frac{\Omega}{\pi}\right) \frac{s}{S}\left[1+\rho\left(1-\frac{s}{S}\right)+\rho^{2}\left(1-\frac{s}{S}\right)^{2}\right. \\
+ & \left.\rho^{3}\left(1-\frac{s}{S}\right)^{3}+\cdots\right]=\rho \frac{\Omega}{\pi}+\frac{\rho^{2}\left(1-\frac{\Omega}{\pi}\right) \frac{s}{S}}{1-\rho\left(1-\frac{s}{S}\right)}
\end{aligned}
$$

will be leaving through the opening $s$.

The total emittance of the cavity is then:

$$
\begin{aligned}
\epsilon_{0}=1-\rho \frac{\Omega}{\pi}-\frac{\rho^{2}\left(1-\frac{\Omega}{\pi}\right) \frac{s}{S}}{1-\rho\left(1-\frac{s}{S}\right)} & \\
& =\frac{(1-\rho)\left[1+\rho\left(\frac{s}{S}-\frac{\Omega}{\pi}\right)\right]}{1-\rho\left(1-\frac{s}{S}\right)}
\end{aligned}
$$

But $1-\rho$ is the emittance, $\epsilon$, of the moterial which constitutes the wall (supposedly opaque) of the cavity, hence:

$$
\epsilon_{0}=\frac{\epsilon\left[1+(1-\epsilon)\left(\frac{s}{S}-\frac{\Omega}{\pi}\right)\right]}{\epsilon\left(1-\frac{s}{S}\right)+\frac{s}{S}}
$$

1 This assumes that the material constituting the chamber is a perfectly diffuse reflector, and that $\Omega$ is a small fraction of $\pi$. 
This expression of total emittance of the cavity, although more complex than that which provided equation ( $1 a)$, is much more satisfactory because it includes factors for the shape of the chamber in the form of the ratio $s / S$ in addition to the aperture, $\Omega$, of the radiator and its natural emittance, $\epsilon$.

In the general case of a chamber, of any shape, equation (2) can be written in the form ${ }^{2}$

$$
\epsilon_{0}=\frac{\epsilon\left[1+(1-\epsilon)\left(\frac{s}{S}-\frac{s}{S_{0}}\right)\right]}{\epsilon\left(1-\frac{s}{S}\right)+s / S}
$$

$S_{0}$ representing the surface of a sphere of the same depth as the cavity in the direction normal to the opening, $s$.

In equation (3), $\epsilon_{0}=$ emittance of cavity, $\epsilon=$ emittance of material, $s / S=$ ratio of area of

\footnotetext{
2 Equation (3) is a combined form of Gouffés equations (2b) and (4).
}

opening to area of cavity (opening included) $=$ $1 / 2(1+h / r)$ for a cylindrical cavity of depth, $h$, and radius, $r$, and $s / S_{o}=1 / 1+(h / r)^{2}=\Omega / \pi$ for $(r / h) \rightarrow 0$.

\section{Equation for the Emittance of a Material}

If $E$ is the ratio of the flux density from the surface to that from the bottom of the hole, $\epsilon$ is the emittance of the specimen, and $\epsilon_{0}$ is the emittance of the hole, then

$\epsilon=E \epsilon_{0}$, or when equation (3) is substituted for $\epsilon_{0}$,

$$
\epsilon=\frac{E\left(1+\frac{s}{S}-\frac{s}{S_{0}}\right)-\frac{s}{S}}{1-\frac{s}{S}+E\left(\frac{s}{S}-\frac{s}{S_{0}}\right)}
$$

and, if $s / S=R$ and $s / S_{0}=R_{0}$ as in equation (1) in the main body of the report, then

$$
\epsilon=\frac{E\left(1+R-R_{0}\right)-R}{1-R+E\left(R-R_{0}\right)}
$$

${ }^{3}$ Gouffé equation (5).

\section{REFERENCES}

1. De Vos J. C.: Evaluation of the Quality of a Blackbody. Physica, vol. 20, no. 10, Oct. 1954, pp. 669-689.

2. Ornstein, L. S. (Communicated by): Tables of the Emissivity of Tungsten as a Function of Wavelength From $0.23 \mu-2.0 \mu$ in the Region of Temperature $1600^{\circ}-3000^{\circ} \mathrm{K}$. Physica, vol. 3, no. 6, June 1936, pp. 561-562.

3. Forsythe, W. E.; and Adams, E. Q.: Radiating Characteristics of Tungsten and Tungsten Lamps. Jour. Opt. Soc. Am., vol. 35, no. 2, Feb. 1945, pp. 108-113.

4. Blair, G. R.: Determination of Spectral Emissivity of Ceramic Bodies at Elevated Temperatures. Jour. Am. Cer. Soc., vol. 43, no. 4, Apr. 1960, pp. 197-203.

5. Reithof, T. R.; Achione, B. D.; and Branyad, E. R.: High Temperature Spectral Emissivity Studies on Some Refractory Metals and Carbides. Symp. on Temperature-Its Measurement and Control in Science and Industry. Columbus, March 1961, Preprint no. B. 10. 2.

6. Gouffe, A.: Temperature Corrections of Artificial Black Bodies, Taking Multiple Internal Diffusion into Consideration. NRL Trans. 429, translated from Revue d'Opt. 24, Jan.-Mar. 1945, pp. 1-10.

7. O'Sullivan, William J., Jn.; and Wade, William R.: Theory and Apparatus for Measurement of Emissivity fo, Radiative Cooling of Hypersonic Aircraft with Data for Inconel, Inconel X. Stainless Steel 303, and Titanium Alloy RS-120. NASA TR R-90, 1961.

8. Plunkett, J. D.; and Kingery, W. D.: The Spectral and Integrated Emissivity of Carbon and Graphite. Proc. of the Fourth Conf. on Carbon, Pergaman Press, N.Y., 1959, pp. 457-472.

9. Lang, S. M.: Properties of High-Temperature Ceramics and Cermets; Elasticity and Density at Room Temperature. Nat. Bur. Standards Monograph No. 6, Mar. 1960.

10. Ribaud, G.: Traite de Pyrometric Optique, p. 232. 


\section{DISCUSSION}

Sklarew, The Marquardt Corp.: Have you determined the effect of the hole shape on the accuracy of they system?

Moore: No we haven't. We decided to use a cylindrical hole because holes of this type can be drilled into a ceramic specimen fairly easily with a Cavitron, Also, if we are using metal or some of the non-metals we can drill holes of this type with an ordinary drill. The expression can also be used for holes with a conical bottom, although we haven't tried them. I think you would have more difficulty in forming such holes. The holes that we use are very small; they are only $1 / 2 \mathrm{~mm}$ in diameter.

Scratz, American Machine and Foundry: What would be the calculated emittance of the shallow blackbody cavities having different depth-to-radius ratios, such as 1.5 , and 1.8 .

MOORE: If a material is a diffuse reflector the efficiency can be computed. You have to assume an emittance of your specimen material in order to calculate the emittance of the cavity. I don't know that we have ever done that on a cavity with a depth-toradius ratio of 1.5 . In the case of alumina specimen, I think the emittance of the cavity would be about 0.8 .

Schatz: Isn't that far from what it should be?

Moore: In this method we know that we are not looking at a blackbody cavity, but if the theory is correct, and we think it is, and if we have a diffuse reflector, it all balances out in the Gouffé expression. The nice part of using this expression is that you do not have to know the emittance of the specimen material. The shallow hole method is based on the use of a reference hole of fixed geometry; the emittance of this hole need not be known.

Schatz: I am not sure I understand. Assuming the Gouffé expression for a blackbody with the depth-toradius ratio of 1.5 , would you have a cavity with an emittance approximating 1.0 ?

Moore: No, the blackbody does not approximate 1.0.

Richmond, National Bureau of Standards: I think that I can clear up the misunderstanding. The point is that we do not have a very good blackbody, but since we know how bad it is, we can make corrections for it.

Moore: That is one way of saying it.

LEE, Avco Corporation: You stated, at the beginning of the paper, that you made some assumptions. One was that you should have a perfectly diffuse cavity and that the cavity walls should have the same emittance as the surface. Now, in some of these ceramic materials, sintered materials, or pressed materials, the surface has a very much different character than the substrate material, and the hole is so shallow that you may not be able to realize these assumption. Could you perhaps discuss this a little more?

Moore: Of course, all of these specimens that we prepared were machined specimens. In other words, we were not using the original surface of the ceramic after firing. The test surface was first ground with diamond powder and then we drilled our hole with the same diamond powder using a Cavitron so that the two surfaces in that respect were comparable. Granted there are certain assumptions in use of this shallow hole method. At the moment, we are just interested in seeing what kind of accuraey we can obtain through its use. The method is intended for very high temperature measurements and I know there is much more work needed on it. For one thing, we need a good heattransfer analysis, which is very difficult with the type of specimens that we are using. But we hope to make such an analysis before too long, and again I would like to say that this is a progress report. We are not, as yet, ready to recommend it as an accurate method of emittance measurement.

Zissis, Institute for Defense Analyses: As far as I know the De Vos treatment is more accurate than the Gouffe treatment and is superior to it in every way except in simplicity. If you take the DeVos calculations, assume a perfectly diffuse wall and no temperature gradient, you get the generalized Gouffé expression, $1-\rho(\Omega / \pi)$. Using the De Vos expressions, you have a chance to evaluate the effects of different characteristics of the walls of your shallow cavity and also to consider how big a difference would be introduced by a temperature gradient. I wonder if more theoretical work of this type is being planned, or whether you feel that this is beyond the precision of the experiment.

Moore: Well, we certainly have studied the De Vos expression, as well as some of the others that have been developed. As you say, most of them are much more complex in nature than the Gouffé equation. We plan to investigate some of these other expressions to determine if they will bring the data into better agreement, but right at the moment our problems are not so much in the use of the expressions for calculation of the emittance as they are in the elimination of temperature differences between hole and surface. Large errors will be introduced unless we can reduce this temperature difference to a small value.

R. L. Cox, Chance Vought Astronautics: I would like to compliment Mr. Moore on his very interesting and excellent paper. Some work has been done by Dr. Sparrow at the University of Minnesota on the emission from holes with temperature gradients down the cavity. I believe it might help resolve your temperature gradient problem if such an analysis. were performed.

Moore: I must admit that I had not seen this recent publication by Professor Sparrow, but I have made a note to look that up just as soon as I get back. Of course, one nice thing about the Gouffe expression is that the emittance term is eliminated from the equation that we use. I doubt that this would be possible with some of the other expressions. 


\section{Instructions for Handling Emittance Standards \\ Office of Standard Reference Materials_-December 16, 1965}

1. Read the instructions, including discussion.

2. Leave standards in container except when in use.

3. Wear clean surgical rubber gloves while handling specimens. Handle as little as possible, and then by edges or ends only. Never touch the flat surfaces in the areas to be viewed.

4. Do not lay standards on a desk or bench top. If possible, return to plastic holder-if not, support by edges or ends only on clean glass or stainless steel.

5. In use avoid contamination by oil, grease, dust or condensed volatile materials.

\section{Discussion}

1. INTRODUCTION. The working standards of normal spectral emittance have been carefully prepared and calibrated. However, damage to the surface or contamination can significantly change the emittance.

2. StORAGE Instructions. Each standard is contained in a plastic holder, which supports it by the edges or ends only. The holder containing the specimen is in turn enclosed in a metal tube or plastic box. Each standard should remain in its individual container except during actual use, and the containers should be stored in a clean, dry place at room temperature.

3. Handling of Specimens. Extreme precautions should be taken to prevent contamination or damage to the surface of standards during use. Handling should be kept to the absolute minimum. Wear clean surgical rubber gloves when handling specimens, in order to avoid fingerprints, and touch the ends or edges only. Never touch the flat areas to be viewed, or permit them to come in contact with a bench or desk top. If a specimen must be laid down, return it to its holder, or support it by the ends or edges only on clean glass or stainless steel. Be particularly careful to avoid contamination by oil, grease, dust or condensed volatilized materials.

4. Heating Specimens. The specimens were heated in air during calibration, and should be heated only in a clean air atmosphere at atmospheric pressure. The Kanthal and Inconel specimens have been oxidized in air, and heating in other atmospheres may significantly change the character of the oxide layer, and hence the emittance. While there is no visible oxide layer on the platinum standards, they were calibrated in air, and may change in emittance if heated in other atmospheres. 
U. S. Department of Commerce

John T. Connox - Secretary

National Buiraf Standards

A. V. Astris Director

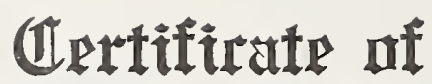

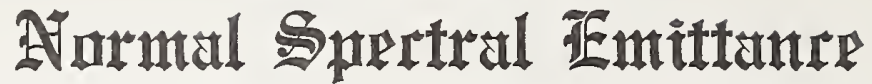

Standard Reference Materials 1402 to 1409

Platinum-13 Percent Rhodium Alloy

\begin{tabular}{|c|c|c|c|c|c|c|c|c|c|}
\hline Wavelength & \multicolumn{3}{|c|}{ At $800^{\circ} \mathbf{K}$} & \multicolumn{3}{|c|}{ At $1100^{\circ} \mathrm{K}$} & \multicolumn{3}{|c|}{ At $1400^{\circ} \mathrm{K}$} \\
\hline $\begin{array}{c}\text { Microns } \\
1.09\end{array}$ & $\underset{0.172}{\epsilon}$ & $\begin{array}{c}\sigma_{m} \\
0.005\end{array}$ & $\begin{array}{c}\sigma_{s} \\
0.038\end{array}$ & $\begin{array}{c}\epsilon \\
0.293\end{array}$ & $\begin{array}{c}\sigma_{m} \\
0.027\end{array}$ & $\begin{array}{c}\sigma_{s} \\
0.042\end{array}$ & $\underset{0.239}{\epsilon}$ & $\sigma_{m}$ & $\sigma_{s}$ \\
\hline $\begin{array}{l}1.09 \\
1.15\end{array}$ & .184 & .005 & $\begin{array}{r}.042 \\
.042\end{array}$ & .296 & .028 & .044 & .233 & $\begin{array}{r}.011 \\
.008\end{array}$ & .013 \\
\hline 1.22 & .191 & .004 & .045 & .297 & .030 & .046 & .227 & .007 & .013 \\
\hline 1.28 & .197 & .004 & .047 & .296 & .032 & .049 & .220 & .007 & .012 \\
\hline 1.36 & .199 & .005 & .048 & .294 & .032 & .055 & .214 & .006 & .011 \\
\hline 1.44 & .198 & .005 & .048 & .289 & .033 & .063 & .207 & .007 & .011 \\
\hline 1.52 & .193 & .005 & .045 & .281 & .030 & .071 & .200 & .007 & .010 \\
\hline 1.63 & .184 & .004 & .040 & .269 & .029 & .074 & .193 & .007 & .010 \\
\hline 1.74 & .172 & .005 & .032 & .250 & .026 & .071 & .184 & .006 & .010 \\
\hline 1.88 & .159 & .004 & .024 & .228 & .023 & .061 & .177 & .006 & .012 \\
\hline 2.10 & .148 & .004 & .018 & .205 & .018 & .046 & .170 & .006 & .013 \\
\hline 2.36 & .138 & .004 & .013 & .185 & .014 & .032 & .162 & .007 & .014 \\
\hline 2.60 & .129 & .005 & .010 & .169 & .011 & .024 & .156 & .006 & .015 \\
\hline 2.81 & .122 & .004 & .008 & .158 & .009 & .019 & .151 & .006 & .017 \\
\hline 3.02 & .117 & .005 & .007 & .149 & .008 & .016 & .147 & .006 & .018 \\
\hline 3.25 & .112 & .005 & .007 & .143 & .007 & .015 & .143 & .006 & .019 \\
\hline 3.45 & .109 & .005 & .008 & .138 & .006 & .014 & .140 & .005 & .021 \\
\hline 3.65 & .106 & .005 & .008 & .134 & .006 & .013 & .138 & .006 & .022 \\
\hline 3.87 & .104 & .004 & .009 & .131 & .006 & .013 & .135 & .006 & .023 \\
\hline 4.09 & .102 & .005 & .009 & .127 & .006 & .013 & .133 & .006 & .024 \\
\hline 4.30 & .098 & .006 & .011 & .124 & .006 & .013 & .129 & .006 & .025 \\
\hline 4.50 & .098 & .004 & .010 & .123 & .006 & .013 & .129 & .006 & .025 \\
\hline 4.67 & .096 & .005 & .010 & .121 & .006 & .013 & .128 & .006 & .026 \\
\hline 4.83 & .095 & .005 & .011 & .119 & .006 & .013 & .126 & .006 & .027 \\
\hline 4.99 & .094 & .005 & .011 & .117 & .005 & .014 & .125 & .006 & .027 \\
\hline 5.13 & .093 & .005 & .011 & .116 & .005 & .014 & .124 & .006 & .028 \\
\hline 5.27 & .093 & .005 & .011 & .115 & .006 & .014 & .123 & .006 & .028 \\
\hline 5.40 & .092 & .005 & .012 & .114 & .006 & .014 & .122 & .006 & .029 \\
\hline 5.54 & .091 & .005 & .012 & .113 & .006 & .014 & .121 & .006 & .029 \\
\hline 5.69 & .090 & .005 & .012 & .111 & .006 & .014 & .120 & .006 & .030 \\
\hline 5.83 & .089 & .005 & .012 & .110 & .006 & .014 & .118 & .006 & .030 \\
\hline 5.97 & .088 & .005 & .012 & .108 & .006 & .014 & .117 & .006 & .030 \\
\hline 6.10 & .087 & .005 & .012 & .107 & .006 & .015 & .116 & .006 & .030 \\
\hline 6.22 & .086 & .004 & .013 & .107 & .006 & .015 & .115 & .006 & .031 \\
\hline 6.35 & .086 & .005 & .013 & .106 & .006 & .015 & .115 & .005 & .031 \\
\hline 6.47 & .085 & .005 & .012 & .104 & .006 & .015 & .113 & .006 & .031 \\
\hline 6.58 & .084 & .005 & .013 & .104 & .006 & .014 & .113 & .005 & .031 \\
\hline 6.70 & .084 & .005 & .013 & .103 & .006 & .015 & .112 & .005 & .031 \\
\hline 6.80 & .083 & .005 & .013 & .103 & .006 & .015 & .112 & .006 & .032 \\
\hline 6.91 & .083 & .005 & .014 & .102 & .006 & .015 & .111 & .005 & .032 \\
\hline
\end{tabular}




\begin{tabular}{|c|c|c|c|c|c|c|c|c|c|}
\hline \multirow{3}{*}{ Wavelength } & \multicolumn{3}{|c|}{ At $800^{\circ} \mathbf{K}$} & \multicolumn{3}{|c|}{ At $1100^{\circ} \mathrm{K}$} & \multicolumn{3}{|c|}{ At $1400^{\circ} \mathrm{K}$} \\
\hline & $\epsilon$ & $\sigma_{m}$ & $\sigma_{s}$ & $\epsilon$ & $\sigma_{m}$ & $\sigma_{s}$ & $\epsilon$ & $\sigma_{m}$ & $\sigma_{s}$ \\
\hline & 0.082 & 0.005 & 0.013 & 0.102 & 0.006 & 0.016 & 0.111 & 0.005 & 0.032 \\
\hline 7.13 & .082 & .005 & .013 & .101 & .006 & .016 & .110 & .005 & .032 \\
\hline 7.25 & .081 & .005 & .013 & .101 & .006 & .016 & .109 & .006 & .032 \\
\hline 7.37 & .081 & .005 & .013 & .100 & .006 & .016 & .109 & .006 & .032 \\
\hline 7.49 & .081 & .005 & .014 & .099 & .006 & .016 & .109 & .006 & .032 \\
\hline 7.60 & .080 & .005 & .014 & .099 & .006 & .016 & .108 & .005 & .032 \\
\hline 7.71 & .080 & .005 & .014 & .099 & .006 & .016 & .108 & .006 & .033 \\
\hline 7.83 & .080 & .005 & .014 & .099 & .006 & .017 & .108 & .005 & .033 \\
\hline 7.94 & .080 & .005 & .014 & .098 & .006 & .016 & .107 & .005 & .033 \\
\hline 8.03 & .080 & .005 & .014 & .098 & .006 & .016 & .107 & .006 & .033 \\
\hline 8.12 & .081 & .006 & .014 & .098 & .006 & .016 & .107 & .005 & .033 \\
\hline 8.22 & .081 & .006 & .014 & .099 & .006 & .016 & .108 & .006 & .033 \\
\hline 8.32 & .083 & .006 & .014 & .099 & .006 & .016 & .108 & .005 & .032 \\
\hline 8.41 & .084 & .005 & .014 & .101 & .006 & .016 & .109 & .006 & .032 \\
\hline 8.50 & .085 & .005 & .014 & .102 & .006 & .016 & .110 & .006 & .032 \\
\hline 8.60 & .086 & .006 & .014 & .103 & .006 & .016 & .111 & .006 & .032 \\
\hline 8.70 & .086 & .006 & .013 & .103 & .006 & .016 & .111 & .006 & .032 \\
\hline 8.79 & .087 & .006 & .013 & .104 & .006 & .016 & .112 & .006 & .032 \\
\hline 8.88 & .087 & .006 & .013 & .104 & .006 & .017 & .113 & .006 & .032 \\
\hline 8.96 & .088 & .005 & .013 & .104 & .006 & .017 & .113 & .006 & .032 \\
\hline 9.05 & .088 & .005 & .013 & .105 & .006 & .016 & .114 & .006 & .032 \\
\hline 9.14 & .088 & .006 & .013 & .105 & .006 & .016 & .114 & .006 & .031 \\
\hline 9.22 & .088 & .005 & .013 & .105 & .005 & .016 & .114 & .006 & .032 \\
\hline 9.30 & .089 & .005 & .013 & .105 & .005 & .016 & .114 & .006 & .032 \\
\hline 9.38 & .090 & .006 & .013 & .106 & .006 & .016 & .114 & .006 & .032 \\
\hline 9.46 & .092 & .006 & .014 & .107 & .005 & .016 & .115 & .006 & .032 \\
\hline 9.55 & .093 & .006 & .014 & .108 & .006 & .017 & .115 & .007 & .032 \\
\hline 9.63 & .095 & .006 & .014 & .110 & .006 & .016 & .116 & .006 & .031 \\
\hline 9.71 & .096 & .006 & .014 & .110 & .006 & .016 & .117 & .006 & .032 \\
\hline 9.79 & .097 & .006 & .014 & .112 & .006 & .015 & .118 & .006 & .031 \\
\hline 9.87 & .098 & .006 & .014 & .112 & .006 & .015 & .118 & .007 & .032 \\
\hline 9.95 & .098 & .006 & .014 & .113 & .006 & .015 & .119 & .006 & .031 \\
\hline 10.03 & .098 & .006 & .014 & .113 & .006 & .015 & .119 & .006 & .031 \\
\hline 10.10 & .098 & .006 & .014 & .113 & .006 & .015 & .119 & .006 & .031 \\
\hline 10.18 & .097 & .006 & .014 & .113 & .006 & .015 & .119 & .006 & .031 \\
\hline 10.26 & .096 & .006 & .014 & .112 & .006 & .015 & .119 & .006 & .031 \\
\hline 10.34 & .095 & .006 & .013 & .111 & .006 & .014 & .118 & .006 & .031 \\
\hline 10.42 & .093 & .006 & .013 & .109 & .006 & .014 & .117 & .006 & .030 \\
\hline 10.50 & .091 & .006 & .013 & .108 & .006 & .014 & .116 & .006 & .030 \\
\hline 10.57 & .090 & .006 & .013 & .106 & .006 & .014 & .115 & .005 & .030 \\
\hline 10.64 & .089 & .006 & .013 & .105 & .005 & .014 & .114 & .006 & .030 \\
\hline 10.72 & .087 & .006 & .013 & .103 & .006 & .013 & .112 & .006 & .030 \\
\hline 10.80 & .085 & .006 & .013 & .102 & .006 & .013 & .111 & .006 & .030 \\
\hline 10.87 & .084 & .006 & .013 & .100 & .005 & .013 & .110 & .006 & .030 \\
\hline 10.94 & .083 & .006 & .013 & .099 & .006 & .013 & .109 & .005 & .030 \\
\hline 11.01 & .081 & .006 & .013 & .097 & .005 & .013 & .108 & .005 & .030 \\
\hline 11.08 & .080 & .006 & .013 & .096 & .006 & .013 & .106 & .005 & .030 \\
\hline 11.15 & .079 & .006 & .013 & .095 & .006 & .012 & .105 & .005 & .030 \\
\hline 11.22 & .078 & .006 & .012 & .093 & .006 & .012 & .104 & .005 & .030 \\
\hline 11.28 & .077 & .006 & .013 & .092 & .006 & .012 & .103 & .005 & .030 \\
\hline
\end{tabular}




\begin{tabular}{|c|c|c|c|c|c|c|c|c|c|}
\hline \multirow{2}{*}{$\begin{array}{l}\text { Wavelength } \\
\text { Microns }\end{array}$} & \multicolumn{3}{|c|}{ At $800^{\circ} \mathbf{K}$} & \multicolumn{3}{|c|}{ At $1100^{\circ} \mathrm{K}$} & \multicolumn{3}{|c|}{ At $1400^{\circ} \mathrm{K}$} \\
\hline & $\epsilon$ & $\sigma_{m}$ & $\sigma_{s}$ & $\epsilon$ & $\sigma_{m}$ & $\sigma_{s}$ & $\epsilon$ & $\sigma_{m}$ & $\sigma_{s}$ \\
\hline 11.35 & 0.076 & 0.006 & 0.013 & 0.091 & 0.006 & 0.012 & 0.102 & 0.005 & 0.030 \\
\hline 11.42 & .075 & .006 & .013 & .090 & .006 & .012 & .101 & .005 & .030 \\
\hline 11.49 & .074 & .006 & .012 & .089 & .006 & .011 & .100 & .005 & .030 \\
\hline 11.55 & .074 & .006 & .012 & .088 & .006 & .011 & .100 & .006 & .030 \\
\hline 11.62 & .073 & .006 & .012 & .088 & .005 & .011 & .099 & .005 & .029 \\
\hline 11.68 & .072 & .006 & .012 & .087 & .005 & .011 & .098 & .005 & .030 \\
\hline 11.74 & .072 & .006 & .012 & .086 & .006 & .011 & .097 & .005 & .029 \\
\hline 11.80 & .072 & .006 & .012 & .085 & .005 & .011 & .097 & .005 & .029 \\
\hline 11.87 & .071 & .006 & .012 & .085 & .005 & .011 & .096 & .005 & .030 \\
\hline 11.94 & .071 & .006 & .012 & .084 & .005 & .010 & .096 & .005 & .029 \\
\hline 12.00 & .071 & .006 & .012 & .084 & .005 & .010 & .095 & .005 & .029 \\
\hline 12.07 & .070 & .006 & .012 & .083 & .005 & .010 & .095 & .005 & .029 \\
\hline 12.13 & .070 & .006 & .012 & .083 & .005 & .010 & .095 & .005 & .029 \\
\hline 12.19 & .070 & .006 & .012 & .083 & .006 & .010 & .095 & .005 & .029 \\
\hline 12.26 & .070 & .006 & .012 & .083 & .006 & .010 & .094 & .005 & .029 \\
\hline 12.32 & .070 & .006 & .012 & .082 & .006 & .009 & .094 & .005 & .029 \\
\hline 12.38 & .070 & .006 & .012 & .082 & .006 & .009 & .094 & .005 & .029 \\
\hline 12.44 & .070 & .006 & .012 & .082 & .005 & .009 & .094 & .005 & .029 \\
\hline 12.50 & .070 & .006 & .012 & .081 & .005 & .009 & .093 & .006 & .029 \\
\hline 12.57 & .069 & .006 & .012 & .081 & .005 & .009 & .093 & .006 & .029 \\
\hline 12.68 & .069 & .006 & .012 & .081 & .006 & .009 & .093 & .006 & .029 \\
\hline 12.69 & .069 & .006 & .012 & .081 & .006 & .009 & .093 & .006 & .029 \\
\hline 12.75 & .069 & .006 & .012 & .080 & .006 & .008 & .092 & .005 & .028 \\
\hline 12.82 & .069 & .006 & .012 & .080 & .006 & .008 & .092 & .005 & .028 \\
\hline 12.88 & .068 & .006 & .011 & .079 & .005 & .008 & .092 & .005 & .028 \\
\hline 12.94 & .068 & .006 & .011 & .079 & .006 & .008 & .091 & .005 & .028 \\
\hline 13.00 & .068 & .006 & .011 & .079 & .006 & .008 & .091 & .005 & .028 \\
\hline 13.06 & .067 & .006 & .011 & .078 & .006 & .008 & .091 & .005 & .028 \\
\hline 13.12 & .067 & .006 & .011 & .078 & .006 & .007 & .091 & .005 & .028 \\
\hline 13.18 & .066 & .006 & .011 & .077 & .006 & .007 & .090 & .005 & .028 \\
\hline 13.24 & .066 & .006 & .011 & .077 & .006 & .007 & .090 & .005 & .028 \\
\hline 13.30 & .066 & .006 & .011 & .076 & .006 & .006 & .089 & .006 & .028 \\
\hline 13.36 & .065 & .006 & .011 & .076 & .006 & .006 & .089 & .006 & .028 \\
\hline 13.42 & .065 & .006 & .011 & .076 & .006 & .006 & .089 & .005 & .027 \\
\hline 13.48 & .065 & .006 & .011 & .075 & .006 & .006 & .088 & .006 & .027 \\
\hline 13.54 & .064 & .006 & .011 & .074 & .006 & .006 & .088 & .006 & .027 \\
\hline 13.60 & .064 & .006 & .011 & .074 & .006 & .005 & .088 & .005 & .027 \\
\hline 13.66 & .064 & .006 & .011 & .074 & .006 & .005 & .088 & .005 & .026 \\
\hline 13.72 & .063 & .006 & .011 & .073 & .006 & .005 & .087 & .005 & .026 \\
\hline 13.78 & .063 & .006 & .011 & .073 & .006 & .005 & .087 & .005 & .026 \\
\hline 13.84 & .063 & .006 & .011 & .072 & .006 & .005 & .086 & .005 & .026 \\
\hline 13.89 & .062 & .006 & .011 & .072 & .006 & .005 & .086 & .006 & .026 \\
\hline 13.95 & .062 & .006 & .010 & .071 & .006 & .005 & .086 & .006 & .025 \\
\hline 14.00 & .062 & .006 & .010 & .071 & .006 & .005 & .085 & .006 & .025 \\
\hline 14.06 & .061 & .006 & .011 & .071 & .006 & .005 & .085 & .006 & .024 \\
\hline 14.11 & .061 & .005 & .010 & .070 & .006 & .004 & .084 & .006 & .024 \\
\hline 14.17 & .060 & .005 & .010 & .070 & .006 & .005 & .084 & .006 & .023 \\
\hline 14.22 & .060 & .005 & .010 & .070 & .006 & .005 & .083 & .006 & .022 \\
\hline 14.28 & .060 & .006 & .010 & .069 & .006 & .005 & .083 & .006 & .021 \\
\hline 14.33 & .059 & .006 & .009 & .069 & .006 & .005 & .082 & .006 & .020 \\
\hline
\end{tabular}




\begin{tabular}{|c|c|c|c|c|c|c|c|c|c|}
\hline Wavelength & \multicolumn{3}{|c|}{ At $800^{\circ} \mathrm{K}$} & \multicolumn{3}{|c|}{ At $1100^{\circ} \mathrm{K}$} & \multicolumn{3}{|c|}{ At $1400^{\circ} \mathrm{K}$} \\
\hline Microns & $\epsilon$ & $\sigma_{m}$ & $\sigma_{s}$ & $\epsilon$ & $\sigma_{m}$ & $\sigma_{s}$ & $\epsilon$ & $\sigma_{m}$ & $\sigma_{s}$ \\
\hline 14.38 & 0.059 & 0.006 & 0.009 & 0.069 & 0.006 & 0.005 & 0.082 & 0.006 & 0.019 \\
\hline 14.44 & .053 & .005 & .008 & .069 & .006 & .005 & .081 & .006 & .018 \\
\hline 14.49 & .057 & .005 & .007 & .068 & .006 & .005 & .080 & .006 & .016 \\
\hline 14.55 & .057 & .005 & .007 & .068 & .006 & .005 & .080 & .006 & .015 \\
\hline 14.60 & .057 & .005 & .007 & .068 & .006 & .005 & .079 & .006 & .014 \\
\hline 14.65 & .056 & .006 & .006 & .068 & .006 & .005 & .079 & .007 & .013 \\
\hline 14.71 & .056 & .005 & .006 & .068 & .006 & .005 & .079 & .007 & .012 \\
\hline 14.76 & .056 & .006 & .006 & .068 & .006 & .005 & .078 & .007 & .012 \\
\hline 14.82 & .055 & .005 & .006 & .067 & .006 & .005 & .077 & .007 & .011 \\
\hline 14.87 & .055 & .005 & .006 & .067 & .006 & .005 & .077 & .006 & .010 \\
\hline 14.92 & .054 & .006 & .006 & .067 & .006 & .005 & .076 & .007 & .009 \\
\hline 14.98 & .054 & .005 & .006 & .067 & .006 & .005 & .076 & .007 & .008 \\
\hline 15.03 & .054 & .006 & .006 & .067 & .006 & .005 & .076 & .007 & .007 \\
\hline 15.08 & .054 & .006 & .006 & .067 & .006 & .005 & .076 & .007 & .007 \\
\hline 15.14 & .053 & .006 & .007 & .067 & .006 & .005 & .076 & .007 & .007 \\
\hline 15.20 & .053 & .006 & .007 & .067 & .006 & .005 & .075 & .007 & .007 \\
\hline
\end{tabular}

From 1 to 15 Microns, at $1600{ }^{\circ} \mathrm{K}$

Wavelength

Wavelength

\begin{tabular}{|c|c|c|c|c|c|c|c|}
\hline Microns & $\epsilon$ & $\sigma_{m}$ & $\sigma_{s}$ & Microns & $\epsilon$ & $\sigma_{m}$ & $\sigma_{s}$ \\
\hline 1.0 & 0.239 & 0.004 & 0.025 & 8.5 & 0.103 & 0.004 & 0.023 \\
\hline 1.5 & .205 & .004 & .020 & 9.0 & .108 & .004 & .024 \\
\hline 2.0 & . 180 & .003 & .020 & 9.5 & .111 & .004 & .024 \\
\hline 2.5 & .163 & .003 & .019 & 10.0 & .118 & .004 & .026 \\
\hline 3.0 & .151 & .003 & .017 & 10.5 & .124 & .004 & .024 \\
\hline 3.5 & .143 & .003 & .020 & 11.0 & .127 & .004 & .024 \\
\hline 4.0 & .137 & .003 & .020 & 11.5 & .120 & .005 & .025 \\
\hline 4.5 & .132 & .003 & .021 & 12.0 & .112 & .004 & .028 \\
\hline 5.0 & .128 & .003 & .020 & 12.5 & .105 & .006 & .026 \\
\hline 5.5 & .123 & .003 & .023 & 13.0 & .101 & .005 & .027 \\
\hline 6.0 & 1119 & .003 & .021 & 13.5 & .099 & .005 & .026 \\
\hline 6.5 & .115 & .003 & .020 & 14.0 & .098 & .005 & .028 \\
\hline 7.0 & .111 & .003 & .022 & 14.5 & .098 & .006 & .031 \\
\hline 7.5 & 107 & .003 & .022 & 15.0 & .100 & .005 & .031 \\
\hline 8.0 & .105 & .004 & .023 & & & & \\
\hline
\end{tabular}


From 14 to 35 Microns, at $1100^{\circ} \mathrm{K}$

Wavelength

Wavelength

\begin{tabular}{|c|c|c|c|c|c|c|c|}
\hline Microns & $\epsilon$ & $\sigma_{m}$ & $\sigma_{s}$ & Microns & $\epsilon$ & $\sigma_{m}$ & $\sigma_{s}$ \\
\hline 14.10 & 0.072 & 0.007 & 0.006 & 23.30 & 0.066 & 0.004 & 0.009 \\
\hline 14.40 & .067 & .005 & .006 & 23.45 & .066 & .003 & .009 \\
\hline 14.70 & .067 & .005 & .006 & 23.55 & .065 & .003 & .009 \\
\hline 15.00 & .065 & .005 & .006 & 23.70 & .065 & .003 & .009 \\
\hline 15.30 & .068 & .005 & .006 & 23.80 & .065 & .003 & .009 \\
\hline 15.60 & .068 & .005 & .007 & 23.95 & .064 & .003 & .009 \\
\hline 15.80 & .068 & .005 & .008 & 24.10 & .063 & .003 & .009 \\
\hline 16.10 & .068 & .005 & .008 & 24.20 & .065 & .003 & .010 \\
\hline 16.30 & .068 & .005 & .009 & 24.30 & .065 & .003 & .010 \\
\hline 16.50 & .067 & .004 & .009 & 24.45 & .064 & .003 & .010 \\
\hline 16.80 & .067 & .005 & .009 & 24.60 & .063 & .003 & .010 \\
\hline 17.00 & .067 & .004 & .009 & 24.70 & .063 & .003 & .010 \\
\hline 17.20 & .067 & .005 & .009 & 24.80 & .064 & .003 & .009 \\
\hline 17.40 & .068 & .005 & .009 & 24.95 & .063 & .003 & .010 \\
\hline 17.60 & .067 & .004 & .010 & 25.10 & .063 & .003 & .010 \\
\hline 17.80 & .067 & .005 & .010 & 25.20 & .061 & .003 & .009 \\
\hline 18.00 & .067 & .005 & .009 & 25.30 & .062 & .004 & .010 \\
\hline 18.20 & .067 & .005 & .009 & 25.45 & .063 & .003 & .010 \\
\hline 18.40 & .067 & .005 & .009 & 25.55 & .062 & .004 & .010 \\
\hline 18.60 & .067 & .005 & .009 & 25.70 & .062 & .004 & .010 \\
\hline 18.75 & .067 & .005 & .010 & 25.80 & .061 & .004 & .010 \\
\hline 18.95 & .067 & .005 & .010 & 25.90 & .061 & .003 & .011 \\
\hline 19.15 & .066 & .005 & .010 & 26.05 & .060 & .003 & .010 \\
\hline 19.30 & .067 & .005 & .010 & 26.15 & .061 & .003 & .010 \\
\hline 19.50 & .067 & .004 & .009 & 26.30 & .061 & .003 & .010 \\
\hline 19.65 & .066 & .004 & .009 & 26.40 & .061 & .004 & .010 \\
\hline 19.80 & .066 & .004 & .009 & 26.50 & .060 & .004 & .010 \\
\hline 20.00 & .066 & .005 & .009 & 26.60 & .060 & .003 & .010 \\
\hline 20.10 & .066 & .005 & .010 & 26.70 & .060 & .003 & .010 \\
\hline 20.30 & .066 & .004 & .010 & 26.80 & .061 & .004 & .011 \\
\hline 20.45 & .066 & .004 & .009 & 26.95 & .060 & .004 & .010 \\
\hline 20.60 & .066 & .004 & .009 & 27.05 & .060 & .003 & .010 \\
\hline 20.80 & .066 & .005 & .010 & 27.20 & .059 & .003 & .010 \\
\hline 20.90 & .066 & .004 & .010 & 27.30 & .060 & .003 & .010 \\
\hline 21.00 & .065 & .005 & .010 & 27.40 & .059 & .004 & .010 \\
\hline 21.15 & .066 & .005 & .010 & 27.50 & .059 & .004 & .010 \\
\hline 21.30 & .066 & .005 & .009 & 27.60 & .059 & .004 & .011 \\
\hline 21.45 & .066 & .005 & .008 & 27.75 & .058 & .004 & .011 \\
\hline 21.60 & .066 & .004 & .008 & 27.90 & .058 & .004 & .011 \\
\hline 21.75 & .067 & .004 & .009 & 28.00 & .058 & .004 & .011 \\
\hline 21.90 & .066 & .005 & .008 & 28.10 & .057 & .004 & .011 \\
\hline 22.05 & .065 & .004 & .008 & 28.20 & .057 & .004 & .010 \\
\hline 22.20 & .065 & .004 & .008 & 28.30 & .057 & .003 & .010 \\
\hline 22.35 & .066 & .005 & .009 & 28.40 & .057 & .004 & .010 \\
\hline 22.45 & .066 & .005 & .008 & 28.50 & .057 & .004 & .011 \\
\hline 22.65 & .066 & .005 & .008 & 28.60 & .057 & .004 & .010 \\
\hline 22.85 & .065 & .005 & .008 & 28.70 & .057 & .004 & .010 \\
\hline 22.90 & .065 & .005 & .008 & 28.80 & .057 & .004 & .010 \\
\hline 23.05 & .066 & .004 & .009 & 28.95 & .056 & .004 & .010 \\
\hline 23.20 & .066 & .004 & .009 & 29.05 & .056 & .004 & .010 \\
\hline
\end{tabular}


From 14 to 35 Microns, at $1100^{\circ} \mathrm{K}$ (Continued)

Wavelength

Wavelength

\begin{tabular}{|c|c|c|c|c|c|c|c|}
\hline Microns & $\epsilon$ & $\begin{array}{c}\sigma_{m} \\
0.004\end{array}$ & $\sigma_{s}$ & Microns & $\epsilon$ & $\sigma_{m}$ & $\sigma_{s}$ \\
\hline 29.15 & 0.056 & 0.004 & 0.011 & 33.30 & 0.050 & 0.006 & 0.011 \\
\hline 29.25 & .056 & .003 & .011 & 33.40 & .050 & .006 & .010 \\
\hline 29.35 & .055 & .003 & .011 & 33.50 & .049 & .006 & .011 \\
\hline 29.50 & .055 & .004 & .011 & 33.60 & .049 & .005 & .011 \\
\hline 29.60 & .054 & .004 & .011 & 33.70 & .048 & .005 & .011 \\
\hline 29.70 & .053 & .004 & .012 & 33.80 & .047 & .005 & .011 \\
\hline 29.80 & .053 & .004 & .011 & 33.90 & .047 & .005 & .011 \\
\hline 29.90 & .054 & .004 & .010 & 34.00 & .047 & .005 & .011 \\
\hline 30.00 & .053 & .004 & .010 & 34.10 & .047 & .005 & .011 \\
\hline 30.10 & .053 & .004 & .010 & 34.20 & .046 & .005 & .011 \\
\hline 30.20 & .052 & .004 & .010 & 34.30 & .046 & .005 & .010 \\
\hline 30.35 & .052 & .004 & .010 & 34.40 & .045 & .005 & .011 \\
\hline 30.45 & .052 & .004 & .010 & 34.50 & .046 & .005 & .011 \\
\hline 30.55 & .052 & .004 & .010 & 34.60 & .046 & .006 & .012 \\
\hline 30.65 & .052 & .004 & .010 & 34.70 & .046 & .006 & .012 \\
\hline 30.75 & .052 & .004 & .011 & 34.85 & .046 & .006 & .011 \\
\hline 30.85 & .053 & .004 & .010 & 34.95 & .046 & .006 & .011 \\
\hline 30.95 & .052 & .004 & .010 & 35.05 & .045 & .006 & .010 \\
\hline 31.05 & .052 & .004 & .011 & 35.15 & .045 & .006 & .011 \\
\hline 31.15 & .052 & .004 & .011 & 35.25 & .044 & .006 & .010 \\
\hline 31.25 & .052 & .004 & .011 & 35.35 & .043 & .006 & .011 \\
\hline 31.35 & .052 & .004 & .011 & 35.45 & .043 & .006 & .010 \\
\hline 31.45 & .051 & .004 & .011 & 35.55 & .043 & .007 & .011 \\
\hline 31.60 & .051 & .004 & .011 & 35.65 & .043 & .008 & .011 \\
\hline 31.70 & .051 & .004 & .010 & 35.75 & .044 & .008 & .011 \\
\hline 31.80 & .051 & .004 & .010 & 35.80 & .044 & .007 & .010 \\
\hline 31.90 & .050 & .004 & .010 & 35.95 & .044 & .007 & .009 \\
\hline 32.00 & .050 & .004 & .011 & 36.05 & .044 & .007 & .011 \\
\hline 32.10 & .050 & .004 & .011 & 36.15 & .043 & .007 & .012 \\
\hline 32.20 & .050 & .003 & .011 & 36.25 & .044 & .007 & .012 \\
\hline 32.30 & .050 & .004 & .011 & 36.35 & .044 & .008 & .011 \\
\hline 32.40 & .050 & .004 & .011 & 36.45 & .042 & .007 & .011 \\
\hline 32.50 & .050 & .005 & .011 & 36.55 & .042 & .007 & .011 \\
\hline 32.60 & .050 & .006 & .011 & 36.65 & .043 & .006 & .013 \\
\hline 32.70 & .049 & .005 & .012 & & & & \\
\hline 32.80 & .049 & .006 & .012 & & & & \\
\hline 32.90 & .049 & .006 & .011 & & & & \\
\hline 33.00 & .049 & .007 & .012 & & & & \\
\hline 33.10 & .049 & .007 & .012 & & & & \\
\hline 33.20 & .049 & .007 & .012 & & & & \\
\hline
\end{tabular}

The standards of normal spectral emittance are intended for use in calibrating equipment used in various laboratories for measuring this property of materials. All of the specimens were prepared from a single sheet of metal at one time, and were subjected as nearly as possible to identical preparation treatments. Because the equipment used for the calibration of these standards from 1 to $15 \mu$ at 800,1100 , and $1400^{\circ} \mathrm{K}$ was suitable only for making measurements on $1 / 4$ in. by 8 in. strips, seven such specimens were prepared from selected locations in the sheet so that the strips measured were statistically representative of the en- 
tire lot of specimens. Three measurements were made on each of the seven samples. The value listed for normal spectral emittance $(\epsilon)$ is the arithmetic average of the 21 measured values. The computed average standard deviation $\left(\sigma_{m}\right)$ of the three measurements on each of the seven specimens about the average value for each specimen-is a measure of the precision of measurement. The standard deviation $\left(\sigma_{s}\right)$ of the average value for each of the seven specimens about the overall average, is indicative of the variation in specimens.

For the measurements from 1 to $15 \mu$ at $1600^{\circ} \mathrm{K}$, and for the measurements from 14 to $35 \mu$ at $1100^{\circ} \mathrm{K}$, crly three specimens were measured. Values for $\epsilon, \sigma_{m}$, and $\sigma_{s}$ were derived, as outlined above, from the three measurements made on each of the three specimens.

Procedures used for the measurements are described in detail in "Standardization of Thermal Emittance Measurements, part 4, Normal Spectral Emittance, 800-1400 'K." Technical Report, No. WADC-TR-59-510, Part IV, by William N. Harrison, Joseph C. Richmond, Frederick J. Shorten, and Horace $\mathbf{M}$. Joseph, available from the Clearinghouse for Federal Scientific and Technical Information, 5285 Port Royal Road, Springfield, Virginia 22171, as publication AD 426846, price $\$ 2.25$.

Because the equipment used for measuring the strip samples has an upper limit of safe operation of $1400^{\circ} \mathrm{K}$, data reported for $1600^{\circ} \mathrm{K}$ were measured by the rotating cylinder procedure described in NBS Monograph "A Rotating Cylinder Method for Measuring Normal Spectral Emittance of Ceramic Dxide Specimens from 1200 to 1.600 'K" by Howard E. Clark and Dwight G. Moore.

Samples are available as $1 / 2$ in. disks, SRM No. 1402; as 7/8 in. disks, SRM No. 1403 ; as 1 in. disks, SRM No. 1404 ; as $11 / 8$ in. disks, SRM No. 1405; as 11/4 in. disks, SRM No. 1406; as 2 in. by 2 in. squares, SRM No. 1407; as 1 in. by 10 in. strips, SRM No. 1408 ; and as $3 / 4$ in. by 10 in. strips, SRin No. 1409.

Washington, D. C. 20234

April 11, 1966.
W. Wayne Meinke, Chief

Office of Standard Reference Materials 
U. S. Department of Commerce

John T. Connor, Secretary

National Burfuluf Standards

Certificate of

A. V. Astin Dirrector

13

Pormal Spectral $\mathbb{E}$ mittance

Standard Reference Materials 1420 to 1428

Oxidized Kanthal

\begin{tabular}{|c|c|c|c|c|c|c|c|c|c|}
\hline \multirow{2}{*}{ Wavelength } & \multicolumn{3}{|c|}{ At $800^{\circ} \mathrm{K}$} & \multicolumn{3}{|c|}{ At $1100^{\circ} \mathrm{K}$} & \multicolumn{3}{|c|}{ At $1300^{\circ} \mathrm{K}$} \\
\hline & $\epsilon$ & $\sigma_{m}$ & $\sigma_{8}$ & $\epsilon$ & $\sigma_{m}$ & $\sigma_{8}$ & $\epsilon$ & $\sigma_{m}$ & $\sigma_{s}$ \\
\hline 1.09 & 0.616 & 0.006 & 0.029 & 0.730 & 0.015 & 0.044 & 0.775 & 0.010 & 0.052 \\
\hline 1.15 & .633 & .008 & .026 & .731 & .012 & .045 & .775 & .010 & .053 \\
\hline 1.22 & .645 & .007 & .025 & .728 & .011 & .046 & .771 & .010 & .051 \\
\hline 1.28 & .653 & .007 & .027 & .728 & .010 & .045 & .764 & .010 & .049 \\
\hline 1.36 & .657 & .006 & .029 & .725 & .010 & .045 & .758 & .009 & .046 \\
\hline 1.44 & .660 & .006 & .029 & .722 & .010 & .043 & .753 & .009 & 044 \\
\hline 1.52 & .660 & .005 & .030 & .718 & .010 & .039 & .747 & .007 & .041 \\
\hline 1.63 & .659 & .005 & .030 & .711 & .010 & .038 & .740 & .007 & .039 \\
\hline 1.74 & .655 & .005 & .030 & .702 & .009 & .035 & .734 & .007 & .037 \\
\hline 1.88 & .650 & .005 & .028 & .693 & .009 & .033 & .725 & .007 & .035 \\
\hline 2.10 & .645 & .005 & .027 & .684 & .008 & .032 & .713 & .007 & .034 \\
\hline 2.36 & .638 & .005 & .026 & .674 & .008 & .030 & .701 & .006 & .033 \\
\hline 2.60 & .631 & .004 & .025 & .664 & .007 & .029 & .690 & .007 & .031 \\
\hline 2.81 & .624 & .005 & .023 & .654 & .007 & .029 & .678 & .007 & .031 \\
\hline 3.02 & .616 & .004 & .023 & .645 & .006 & .028 & .667 & .007 & .029 \\
\hline 3.25 & .610 & .004 & .022 & .636 & .006 & .028 & .658 & .007 & .029 \\
\hline 3.45 & .604 & .004 & .023 & .628 & .006 & .027 & .649 & .007 & .030 \\
\hline 3.65 & .599 & .004 & .022 & .622 & .006 & .027 & .642 & .006 & .029 \\
\hline 3.87 & .594 & .004 & .022 & .616 & .006 & .026 & .634 & .006 & .028 \\
\hline 4.09 & .587 & .003 & .022 & .609 & .005 & .026 & .627 & .006 & .028 \\
\hline 4.30 & .574 & .004 & .024 & .599 & .007 & .030 & .613 & .008 & .028 \\
\hline 4.50 & .578 & .003 & .021 & .597 & .005 & .025 & .614 & .005 & .027 \\
\hline 4.67 & .571 & .003 & .022 & .591 & .005 & .026 & .606 & $.006 ́$ & .028 \\
\hline 4.83 & .568 & .003 & .023 & .587 & .005 & .027 & .602 & .005 & .028 \\
\hline 4.99 & .565 & .003 & .023 & .584 & .005 & .027 & .599 & .005 & .029 \\
\hline 5.13 & .562 & .003 & .023 & .581 & .004 & .027 & .595 & .005 & .029 \\
\hline 5.27 & .559 & .003 & .022 & .577 & .005 & .026 & .591 & .005 & .029 \\
\hline 5.40 & .555 & .003 & .021 & .574 & .004 & .025 & .588 & .005 & .028 \\
\hline 5.54 & .550 & .002 & .020 & .569 & .005 & .024 & .584 & .005 & .027 \\
\hline 5.69 & .545 & .002 & .020 & .565 & .005 & .024 & .579 & .006 & .026 \\
\hline 5.83 & .540 & .002 & .020 & .560 & .004 & .023 & .575 & .006 & .025 \\
\hline 5.97 & .535 & .002 & .020 & .555 & .004 & .023 & .571 & .005 & .026 \\
\hline 6.10 & .532 & .002 & .020 & .551 & .004 & .023 & .568 & .005 & .026 \\
\hline 6.22 & .530 & .002 & .020 & .550 & .004 & .024 & .566 & .005 & .026 \\
\hline 6.35 & .530 & .002 & .022 & .550 & .004 & .025 & .566 & .004 & .027 \\
\hline 6.47 & .530 & .002 & .022 & .551 & .004 & .026 & .567 & .005 & .028 \\
\hline 6.58 & .532 & .002 & .023 & .555 & .004 & .027 & .571 & .005 & .029 \\
\hline 6.70 & .536 & .002 & .024 & .560 & .004 & .027 & .576 & .004 & .030 \\
\hline 6.80 & .540 & .002 & .025 & .564 & .004 & .028 & .581 & .004 & .030 \\
\hline 6.91 & .547 & .002 & .025 & .572 & .004 & .029 & .587 & .004 & .031 \\
\hline
\end{tabular}




\begin{tabular}{|c|c|c|c|c|c|c|c|c|c|}
\hline \multirow{2}{*}{ Wavelength } & \multicolumn{3}{|c|}{ At $800^{\circ} \mathrm{K}$} & \multicolumn{3}{|c|}{ At $1100^{\circ} \mathrm{K}$} & \multicolumn{3}{|c|}{ At $1300^{\circ} \mathrm{K}$} \\
\hline & $\epsilon$ & $\sigma_{m}$ & $\sigma_{\diamond}$ & $\epsilon$ & $\sigma_{m}$ & $\sigma_{s}$ & $\epsilon$ & $\sigma_{m}$ & $\sigma_{s}$ \\
\hline 7.01 & 0.556 & 0.002 & 0.025 & 0.580 & 0.004 & 0.029 & 0.596 & 0.004 & 0.031 \\
\hline 7.13 & .563 & .002 & .025 & .588 & .004 & .030 & .605 & .004 & .032 \\
\hline 7.25 & .569 & .002 & .025 & .596 & .004 & .030 & .613 & .004 & .032 \\
\hline 7.37 & .577 & .003 & .024 & .604 & .004 & .029 & .621 & .004 & .032 \\
\hline 7.49 & .587 & .003 & .024 & .612 & .004 & .029 & .629 & .004 & .032 \\
\hline 7.60 & .595 & .002 & .023 & .621 & .004 & .028 & .637 & .004 & .032 \\
\hline 7.71 & .603 & .002 & .023 & .629 & .004 & .028 & .645 & .004 & .032 \\
\hline 7.83 & .609 & .003 & .022 & .636 & .004 & .027 & .653 & .004 & .032 \\
\hline 7.94 & .615 & .002 & .022 & .642 & .004 & .027 & .659 & .004 & .031 \\
\hline 8.03 & .618 & .003 & .022 & .648 & .005 & .027 & .665 & .004 & .032 \\
\hline 8.12 & .619 & .002 & .022 & .651 & .004 & .027 & .669 & .004 & .032 \\
\hline 8.22 & .620 & .002 & .022 & .653 & .004 & .027 & .672 & .004 & .032 \\
\hline 8.32 & .622 & .002 & .022 & .655 & .004 & .028 & .674 & .004 & .032 \\
\hline 8.41 & .626 & .002 & .023 & .657 & .004 & .027 & .677 & .004 & .032 \\
\hline 8.50 & .631 & .002 & .023 & .661 & .004 & .028 & .680 & .004 & .032 \\
\hline 8.60 & .636 & .002 & .023 & .666 & .004 & .028 & .683 & .004 & .032 \\
\hline 8.70 & .640 & .002 & .024 & .670 & .004 & .029 & .687 & .004 & .033 \\
\hline 8.79 & .644 & .002 & .024 & .674 & .004 & .029 & .692 & .004 & .033 \\
\hline 8.88 & .648 & .002 & .024 & .678 & .004 & .029 & .695 & .004 & .034 \\
\hline 8.96 & .653 & .002 & .024 & .682 & .004 & .030 & .699 & .003 & .034 \\
\hline 9.05 & .659 & .002 & .025 & .687 & .004 & .030 & .704 & .004 & .035 \\
\hline 9.14 & .664 & .002 & .025 & .693 & .004 & .031 & .709 & .003 & .035 \\
\hline 9.22 & .668 & .002 & .025 & .700 & .004 & .031 & .716 & .004 & .035 \\
\hline 9.30 & .671 & .002 & .025 & .705 & .004 & .031 & .722 & .004 & .035 \\
\hline 9.38 & .674 & .002 & .025 & .710 & .004 & .032 & .728 & .004 & .036 \\
\hline 9.46 & .676 & .003 & .026 & .714 & .004 & .032 & .733 & .004 & .036 \\
\hline 9.55 & .680 & .002 & .026 & .717 & .004 & .032 & .738 & .003 & .037 \\
\hline 9.63 & .684 & .002 & .027 & .722 & .004 & .032 & .742 & .004 & .037 \\
\hline 9.71 & .687 & .003 & .027 & .727 & .005 & .033 & .748 & .003 & .037 \\
\hline 9.79 & .691 & .003 & .027 & .731 & .004 & .033 & .753 & .003 & .038 \\
\hline 9.87 & .695 & .003 & .027 & .735 & .005 & .033 & .757 & .003 & .037 \\
\hline 9.95 & .698 & .002 & .027 & .739 & .005 & .033 & .762 & .003 & .037 \\
\hline 10.03 & .702 & .002 & .027 & .744 & .004 & .033 & .767 & .003 & .037 \\
\hline 10.10 & .706 & .003 & .027 & .748 & .004 & .033 & .771 & .003 & .038 \\
\hline 10.18 & .708 & .002 & .027 & .752 & .005 & .033 & .776 & .003 & .038 \\
\hline 10.26 & .709 & .002 & .026 & .756 & .005 & .034 & .779 & .003 & .038 \\
\hline 10.34 & .709 & .003 & .027 & .758 & .004 & .034 & .783 & .004 & .039 \\
\hline 10.42 & .709 & .002 & .026 & .759 & .005 & .033 & .785 & .003 & .039 \\
\hline 10.50 & .710 & .002 & .027 & .759 & .004 & .034 & .787 & .003 & .039 \\
\hline 10.57 & .711 & .003 & .027 & .760 & .004 & .034 & .787 & .004 & .039 \\
\hline 10.64 & .711 & .003 & .027 & .760 & .004 & .034 & .787 & .003 & .040 \\
\hline 10.72 & .713 & .003 & .027 & .760 & .005 & .034 & .787 & .003 & .040 \\
\hline 10.80 & .714 & .003 & .027 & .760 & .004 & .034 & .786 & .003 & .040 \\
\hline 10.87 & .715 & .003 & .028 & .759 & .004 & .034 & .785 & .003 & .040 \\
\hline 10.94 & .715 & .003 & .028 & .758 & .004 & .035 & .783 & .003 & .040 \\
\hline 11.01 & .712 & .004 & .028 & .756 & .005 & .035 & .781 & .003 & .041 \\
\hline 11.08 & .708 & .003 & .028 & .752 & .004 & .035 & .778 & .003 & .041 \\
\hline 11.15 & .703 & .004 & .028 & .748 & .005 & .035 & .774 & .003 & .042 \\
\hline 11.22 & .696 & .004 & .028 & .742 & .004 & .035 & .770 & .003 & .042 \\
\hline 11.28 & .690 & .004 & .028 & .736 & .004 & .035 & .764 & .003 & .041 \\
\hline
\end{tabular}




\begin{tabular}{|c|c|c|c|c|c|c|c|c|c|}
\hline \multirow{2}{*}{$\begin{array}{c}\text { Wavelength } \\
\text { Microns }\end{array}$} & \multicolumn{3}{|c|}{ At $800^{\circ} \mathrm{K}$} & \multicolumn{3}{|c|}{ At $1100^{\circ} \mathrm{K}$} & \multicolumn{3}{|c|}{ At $1300^{\circ} \mathrm{K}$} \\
\hline & $\epsilon$ & $\sigma_{m}$ & $\sigma_{s}$ & $\epsilon$ & $\sigma_{m}$ & $\sigma_{s}$ & $\epsilon$ & $\sigma_{m}$ & $\sigma_{s}$ \\
\hline 11.35 & 0.683 & 0.004 & 0.027 & 0.729 & 0.004 & 0.035 & 0.758 & 0.003 & 0.041 \\
\hline 11.42 & .676 & .004 & .027 & .722 & .004 & .034 & .752 & .003 & .041 \\
\hline 11.49 & .669 & .004 & .026 & .716 & .004 & .034 & .745 & .003 & .041 \\
\hline 11.55 & .663 & .004 & .025 & .710 & .004 & .034 & .740 & .003 & .041 \\
\hline 11.62 & .658 & .005 & .025 & .705 & .003 & .033 & .733 & .003 & .040 \\
\hline 11.68 & .654 & .004 & .025 & .700 & .003 & .033 & .728 & .003 & .040 \\
\hline 11.74 & .651 & .005 & .025 & .695 & .003 & .032 & .723 & .003 & .039 \\
\hline 11.80 & .650 & .004 & .024 & .691 & .003 & .032 & .718 & .003 & .039 \\
\hline 11.87 & .649 & .005 & .024 & .688 & .003 & .032 & .714 & .003 & .039 \\
\hline 11.94 & .648 & .005 & .024 & .686 & .003 & .032 & .711 & .003 & .038 \\
\hline 12.00 & .648 & .004 & .024 & .684 & .003 & .032 & .708 & .003 & .038 \\
\hline 12.07 & .648 & .004 & .024 & .683 & .003 & .032 & .705 & .003 & .038 \\
\hline 12.13 & .648 & .004 & .024 & .682 & .003 & .031 & .703 & .003 & .038 \\
\hline 12.19 & .648 & .004 & .023 & .681 & .003 & .031 & .702 & .003 & .038 \\
\hline 12.26 & .650 & .004 & .023 & .680 & .004 & .031 & .700 & .003 & .037 \\
\hline 12.32 & .651 & .004 & .023 & .680 & .003 & .031 & .699 & .003 & .037 \\
\hline 12.38 & .652 & .004 & .023 & .680 & .003 & .031 & .698 & .003 & 037 \\
\hline 12.44 & .653 & .004 & .023 & .680 & .003 & .030 & .698 & .003 & .037 \\
\hline 12.50 & .653 & .004 & .023 & .681 & .003 & .030 & .697 & .003 & .037 \\
\hline 12.57 & .654 & .004 & .022 & .681 & .002 & .030 & .697 & .003 & .036 \\
\hline 12.63 & .655 & .004 & .022 & .682 & .002 & .029 & .697 & .003 & .036 \\
\hline 12.69 & .656 & .004 & .022 & .682 & .002 & .029 & .697 & .003 & .035 \\
\hline 12.75 & .656 & .004 & .021 & .682 & .002 & .029 & .697 & .002 & .036 \\
\hline 12.82 & .656 & .004 & .021 & .683 & .003 & .028 & .697 & .003 & .035 \\
\hline 12.88 & .656 & .004 & .021 & .683 & .003 & .028 & .697 & .003 & .034 \\
\hline 12.94 & .656 & .004 & .021 & .682 & .002 & .028 & .696 & .002 & .034 \\
\hline 13.00 & .656 & .005 & .020 & .682 & .003 & .028 & .697 & .002 & .034 \\
\hline 13.06 & .656 & .005 & .019 & .682 & .002 & .027 & .696 & .002 & .033 \\
\hline 13.12 & .655 & .005 & .019 & .682 & .003 & .027 & .696 & .002 & .033 \\
\hline 13.18 & .655 & .005 & .018 & .681 & .003 & .026 & .696 & .003 & .032 \\
\hline 13.24 & .654 & .005 & .018 & .680 & .003 & .025 & .695 & .002 & .031 \\
\hline 13.30 & .654 & .004 & .018 & .680 & .003 & .025 & .694 & .002 & .031 \\
\hline 13.36 & .654 & .004 & .017 & .679 & .003 & .024 & .694 & .002 & .030 \\
\hline 13.42 & .652 & .004 & .016 & .678 & .003 & .024 & .693 & .002 & .030 \\
\hline 13.48 & 652 & .004 & .016 & .678 & .003 & .023 & .692 & .003 & .029 \\
\hline 13.54 & .651 & .004 & .016 & .677 & .003 & .023 & .692 & .002 & .029 \\
\hline 13.60 & .650 & .005 & .015 & .676 & .003 & .022 & .691 & .003 & .028 \\
\hline 13.66 & .650 & .004 & .015 & .675 & .003 & .022 & .690 & .003 & .028 \\
\hline 13.72 & .649 & .004 & .014 & .675 & .003 & .021 & .690 & .002 & .026 \\
\hline 13.78 & .649 & .004 & .014 & .674 & .003 & .020 & .689 & .002 & .026 \\
\hline 13.84 & .648 & .004 & .014 & .673 & .003 & .020 & .688 & .002 & .025 \\
\hline 13.89 & .647 & .004 & .013 & .673 & .003 & .020 & .687 & .003 & .024 \\
\hline 13.95 & .647 & .004 & .013 & .672 & .003 & .019 & .687 & .003 & .023 \\
\hline 14.00 & .646 & .004 & .014 & .671 & .003 & .018 & .686 & .002 & .022 \\
\hline 14.06 & .645 & .004 & .014 & .670 & .003 & .018 & .685 & .003 & .022 \\
\hline 14.11 & .645 & .005 & .014 & .669 & .003 & .017 & .684 & .003 & .021 \\
\hline 14.17 & .643 & .005 & .014 & .669 & .003 & .017 & .684 & .003 & .021 \\
\hline 14.22 & .643 & .004 & .015 & .668 & .003 & .017 & .683 & .002 & .020 \\
\hline 14.28 & .641 & .005 & .015 & .667 & .003 & .017 & .682 & .002 & .019 \\
\hline 14.33 & .640 & .005 & .016 & .666 & .003 & .016 & .681 & .002 & .018 \\
\hline
\end{tabular}




\begin{tabular}{|c|c|c|c|c|c|c|c|c|c|}
\hline Wavelength & \multicolumn{3}{|c|}{ At $800^{\circ} \mathrm{K}$} & \multicolumn{3}{|c|}{ At $1100^{\circ} \mathrm{K}$} & \multicolumn{3}{|c|}{ At $1300^{\circ} \mathrm{K}$} \\
\hline Microns & $\epsilon$ & $\sigma_{m}$ & $\sigma_{s}$ & $\epsilon$ & $\sigma_{m}$ & $\sigma_{\theta}$ & $\epsilon$. & $\sigma_{m}$ & $\sigma_{s}$ \\
\hline 14.38 & 0.638 & 0.006 & 0.017 & 0.665 & 0.003 & 0.016 & 0.680 & 0.002 & 0.018 \\
\hline 14.44 & .636 & .007 & .019 & .664 & .003 & .016 & .679 & .002 & .018 \\
\hline 14.49 & .634 & .008 & .021 & .663 & .003 & .017 & .678 & .002 & .017 \\
\hline 14.55 & .631 & .009 & .022 & .662 & .003 & .017 & .676 & .002 & .016 \\
\hline 14.60 & .628 & $: 011$ & .024 & .660 & .003 & .017 & .675 & .002 & .016 \\
\hline 14.65 & .625 & .011 & .026 & .659 & .003 & .018 & .674 & .003 & .016 \\
\hline 14.71 & .623 & .011 & .027 & .658 & .003 & .018 & .673 & .002 & .016 \\
\hline 14.76 & .620 & .010 & .028 & .656 & .002 & .019 & .672 & .002 & .016 \\
\hline 14.82 & .617 & .009 & .029 & .655 & .003 & .020 & .671 & .002 & .016 \\
\hline 14.87 & .612 & .007 & .033 & .653 & .003 & .021 & .670 & .002 & .016 \\
\hline 14.92 & .609 & .004 & .035 & .651 & .003 & .023 & .667 & .002 & .018 \\
\hline 14.98 & .608 & .005 & .032 & .648 & .002 & .024 & .662 & .002 & .018 \\
\hline 15.03 & .609 & .005 & .029 & .647 & .002 & .023 & .662 & .002 & .016 \\
\hline 15.08 & .610 & .004 & .029 & .646 & .003 & .023 & .664 & .003 & .016 \\
\hline 15.14 & .609 & .005 & .032 & .644 & .003 & .024 & .663 & .003 & .019 \\
\hline 15.20 & .607 & .005 & .035 & .642 & .003 & .026 & .662 & .003 & .020 \\
\hline
\end{tabular}

The standards of normal spectral emittance are intended for use in calibrating equipment used in various laboratories for measuring this property of materials. All of the specimens were prepared from a single sheet of metal at one time, and were subjected as nearly as possible to identical preparation treatments. Because the equipment used for the calibration of these standards was suitable only for making measurements on $1 / 4$ inch by 8 inch strips, seven such specimens were prepared from selected locations in the sheet so that the strips measured were statistically representative of the entire lot of specimens.

Three measurements were made on each of the seven samples. The value listed for normal spectral emittance $(\epsilon)$ is the arithmetic average of the 21 measured values. The computed average standard deviation $\left(\sigma_{m}\right)$ of the three measurements on each of the seven specimens about the average value for each specimen is a measure of the precision of measurement. The standard deviation $\left(\sigma_{s}\right)$ of the average value for each of the seven specimens about the overall a verage, is indicative of the variation in specimens.

Procedures used for the measurements are described in detail "Standardization of Thermal Emittance Measurements, part 4, Normal Spectral Emittance, 800-1400 'K." Technical Report No. WADC-TR59-510, Part IV, by William N. Harrison, Joseph C. Richmond, Frederick J. Shorten and Horace M. Joseph, available from the Clearinghouse for Federal Scientific and Technical Information, 5285 Port Royal Road, Springfield, Virginia 22171, as publication AD 426846, price $\$ 2.25$.

Samples are available as $1 / 2$ inch disks, SRM No. 1420; as 7/8 inch disks, SRM No. 1421; as 1 inch disks, SRM No. 1422; as 11/8 inch disks, SRM No. 1423; as 11/4 inch disks, SRM No. 1424; as 2 inch by 2 inch squares, SRM No. 1425; as 1 inch by 10 inch strips, SRM No. 1426; as $3 / 4$ inch by 10 inch strips, SRM No. 1427; and as $1 / 4$ inch by 8 inch strips, SRM No. 1428.

Washington, D. C.

December 16, 1965
W. Wayne Meinke, Chief, Office of Standard Reference Materials. 
U. S. Department of Commerce

John T. Connor, Secretary

National Burwar af Standards

A. V. Asthín D rector

Certificate of

\$2ormal Spectral Emittance

Standard Reference Materials 1440 to 1447

Oxidized Inconel

\begin{tabular}{|c|c|c|c|c|c|c|c|c|c|}
\hline \multirow{2}{*}{$\begin{array}{l}\text { Wavelength } \\
\text { Microns }\end{array}$} & \multicolumn{3}{|c|}{ At $800^{\circ} \mathrm{K}$} & \multicolumn{3}{|c|}{ At $1100^{\circ} \mathrm{K}$} & \multicolumn{3}{|c|}{ At $1300^{\circ} \mathrm{K}$} \\
\hline & $\epsilon$ & $\sigma_{m}$ & $\sigma_{s}$ & $\epsilon$ & $\sigma_{m}$ & $\sigma_{s}$ & $\epsilon$ & $\sigma_{m}$ & $\sigma_{B}$ \\
\hline 1.09 & 0.753 & 0.013 & 0.040 & 0.830 & 0.021 & 0.050 & 0.870 & 0.015 & 0.050 \\
\hline I. 15 & .751 & .009 & .039 & .828 & .020 & .050 & .871 & .016 & .049 \\
\hline I. 22 & .752 & .009 & .039 & .829 & .018 & .050 & .870 & .015 & .049 \\
\hline 1.28 & .755 & .008 & .042 & .828 & .015 & .047 & .869 & .012 & .050 \\
\hline 1.36 & .758 & .009 & .043 & .827 & .014 & .046 & .866 & .012 & .046 \\
\hline 1.44 & .763 & .008 & .044 & .828 & .016 & .045 & .862 & .011 & .044 \\
\hline 1.52 & .768 & .009 & .042 & .829 & .014 & .042 & .859 & .012 & .043 \\
\hline 1.63 & .770 & .008 & .042 & .829 & .015 & .039 & .857 & .013 & .038 \\
\hline 1.74 & .776 & .007 & .040 & .830 & .014 & .039 & .856 & .012 & .037 \\
\hline 1.88 & .781 & .007 & .038 & .831 & .014 & .038 & .855 & .012 & .035 \\
\hline 2.10 & .786 & .007 & .036 & .831 & .014 & .035 & .854 & .012 & .034 \\
\hline 2.36 & .791 & .007 & .034 & .833 & .012 & .033 & .854 & .012 & .033 \\
\hline 2.60 & .794 & .007 & .031 & .834 & .012 & .034 & .854 & .012 & .032 \\
\hline 2.81 & .798 & .007 & .031 & .834 & .013 & .032 & .854 & .012 & .031 \\
\hline 3.02 & .798 & .006 & .029 & .834 & .012 & .033 & .854 & .012 & .031 \\
\hline 3.25 & .802 & .006 & .027 & .835 & .012 & .032 & .855 & .012 & .030 \\
\hline 3.45 & .804 & .006 & .027 & .837 & .013 & .032 & .855 & .012 & .030 \\
\hline 3.65 & .806 & .006 & .027 & .838 & .013 & .032 & .855 & .012 & .030 \\
\hline 3.87 & .808 & .006 & .027 & .839 & .012 & .032 & .856 & .012 & .030 \\
\hline 4.09 & .809 & .005 & .025 & .839 & .012 & .031 & .856 & .012 & .030 \\
\hline 4.30 & .809 & .006 & .026 & .837 & .014 & .030 & .856 & .011 & .029 \\
\hline 4.50 & .812 & .005 & .025 & .840 & .012 & .031 & .857 & .012 & .030 \\
\hline 4.67 & .812 & .005 & .024 & .839 & .012 & .031 & .857 & .012 & .030 \\
\hline 4.83 & .812 & .006 & .024 & .840 & .012 & $.03 \mathrm{I}$ & .856 & .012 & .029 \\
\hline 4.99 & .812 & .006 & .024 & .839 & .012 & .030 & .856 & .012 & .029 \\
\hline 5.13 & 812 & .006 & .024 & .840 & .011 & .030 & .857 & .012 & .030 \\
\hline 5.27 & .812 & .006 & .024 & .839 & .011 & .030 & .856 & .012 & .029 \\
\hline 5.40 & .813 & .005 & .024 & .839 & .011 & .030 & .856 & .012 & .029 \\
\hline 5.45 & .812 & .005 & .024 & .839 & .011 & .030 & .856 & .012 & .030 \\
\hline 5.69 & .812 & .005 & .024 & .838 & .011 & .031 & .855 & .011 & .030 \\
\hline 5.83 & .811 & .005 & .024 & .838 & .012 & .030 & .854 & .012 & .030 \\
\hline 5.97 & .811 & .005 & .024 & .837 & .012 & .031 & .854 & .011 & .029 \\
\hline 6.10 & .810 & .005 & .024 & .837 & .012 & .031 & .854 & .011 & .030 \\
\hline 6.22 & .810 & .005 & .024 & .837 & .012 & .030 & .854 & .012 & .030 \\
\hline 6.35 & .810 & .005 & .024 & .837 & .011 & .030 & .855 & .012 & .030 \\
\hline 6.47 & .809 & .005 & .024 & .837 & .012 & .030 & .854 & .012 & .030 \\
\hline 6.58 & .810 & .005 & .024 & .838 & .011 & .031 & .855 & .012 & .030 \\
\hline 6.70 & .812 & .005 & .024 & .838 & .011 & .031 & .856 & .012 & .030 \\
\hline 6.80 & .813 & .005 & .024 & .839 & .011 & .031 & .856 & .011 & .030 \\
\hline 6.91 & .814 & .005 & .024 & .840 & .010 & .031 & .857 & .011 & .031 \\
\hline
\end{tabular}




\begin{tabular}{|c|c|c|c|c|c|c|c|c|c|}
\hline \multirow{2}{*}{ Wavelength } & \multicolumn{3}{|c|}{ At $800^{\circ} \mathrm{K}$} & \multicolumn{3}{|c|}{ At $1100^{\circ} \mathrm{K}$} & \multicolumn{3}{|c|}{ At $1300^{\circ} \mathrm{K}$} \\
\hline & $\epsilon$ & $\sigma_{m}$ & $\sigma_{s}$ & $\epsilon$ & $\sigma_{m}$ & $\sigma_{\Delta}$ & $\epsilon$ & $\sigma_{m}$ & $\sigma_{s}$ \\
\hline 7.01 & 0.817 & 0.005 & 0.024 & 0.841 & 0.011 & 0.031 & 0.858 & 0.011 & 0.031 \\
\hline 7.13 & .819 & .005 & .024 & .843 & .011 & .031 & .859 & .011 & .031 \\
\hline 7.25 & .822 & .005 & .024 & .845 & .011 & .030 & .861 & .011 & .031 \\
\hline 7.37 & .823 & .005 & .024 & .847 & .011 & .030 & .863 & .011 & .031 \\
\hline 7.49 & .824 & .005 & .024 & .848 & .011 & .030 & .863 & .011 & .031 \\
\hline 7.60 & .826 & .005 & .024 & .849 & .012 & .030 & .864 & .011 & .031 \\
\hline 7.71 & .827 & .005 & .024 & .850 & .011 & .031 & .865 & .011 & .031 \\
\hline 7.83 & .829 & .005 & .024 & .852 & .011 & .031 & .867 & .012 & .032 \\
\hline 7.94 & .833 & .005 & .024 & .854 & .011 & .030 & .868 & .011 & .031 \\
\hline 8.03 & .839 & .005 & .024 & .858 & .012 & .030 & .871 & .012 & .031 \\
\hline 8.12 & .846 & .006 & .024 & .863 & .012 & .030 & .874 & .011 & .031 \\
\hline 8.22 & .852 & .005 & .024 & .867 & .011 & .031 & .878 & .011 & .031 \\
\hline 8.32 & .856 & .005 & .024 & .872 & .012 & .030 & .881 & .011 & .031 \\
\hline 8.41 & .859 & .005 & .025 & .875 & .011 & .031 & .883 & .011 & .032 \\
\hline 8.50 & .862 & .005 & .024 & .878 & .010 & .030 & .886 & .011 & .031 \\
\hline 8.60 & .864 & .005 & .024 & .880 & .011 & .031 & .888 & .011 & .032 \\
\hline 8.70 & .866 & .005 & .024 & .881 & .011 & .030 & .889 & .011 & .032 \\
\hline 8.79 & .868 & .005 & .025 & .882 & .011 & .031 & .890 & .011 & .032 \\
\hline 8.88 & .870 & .005 & .025 & .885 & .011 & .031 & .892 & .011 & .032 \\
\hline 8.96 & .872 & .005 & .025 & .886 & .011 & .031 & .893 & .011 & .031 \\
\hline 9.05 & .874 & .005 & .025 & .888 & .011 & .031 & .895 & .012 & .031 \\
\hline 9.14 & .875 & .005 & .025 & .889 & .011 & .032 & .896 & .011 & .031 \\
\hline 9.22 & .877 & .005 & .026 & .891 & .010 & .032 & .897 & .011 & .031 \\
\hline 9.30 & .876 & .005 & .025 & .891 & .010 & .032 & .898 & .011 & .031 \\
\hline 9.38 & .873 & .005 & .025 & .891 & .010 & .031 & 898 & .012 & .032 \\
\hline 9.46 & .870 & .005 & .025 & .888 & .010 & .031 & .897 & .012 & .031 \\
\hline 9.55 & .868 & .005 & .024 & .887 & .011 & .031 & .896 & .011 & .031 \\
\hline 9.63 & .866 & .005 & .025 & .885 & .010 & .031 & .895 & .011 & .032 \\
\hline 9.71 & .865 & .005 & .025 & .884 & .011 & .031 & .894 & .011 & .032 \\
\hline 9.79 & .863 & .006 & .024 & .884 & .011 & .031 & .894 & .012 & .032 \\
\hline 9.87 & .862 & .005 & .024 & .883 & .011 & .031 & .893 & .011 & .032 \\
\hline 9.95 & .861 & .004 & .024 & .882 & .011 & .031 & .893 & .011 & .032 \\
\hline 10.03 & .861 & .005 & .024 & .882 & .011 & .031 & .893 & .011 & .032 \\
\hline 10.10 & .862 & .005 & .024 & .882 & .011 & .031 & .893 & .011 & .032 \\
\hline 10.18 & .862 & .004 & .024 & .882 & .011 & .031 & .893 & .011 & .032 \\
\hline 10.26 & .863 & .005 & .024 & .882 & .011 & .032 & .893 & .011 & .032 \\
\hline 10.34 & .864 & .005 & .024 & .883 & .011 & .031 & .893 & .011 & .032 \\
\hline 10.42 & .866 & .005 & .024 & .884 & .010 & .031 & .894 & .011 & .032 \\
\hline 10.50 & .868 & .005 & .024 & .885 & .011 & .031 & .895 & .011 & .031 \\
\hline 10.57 & .868 & .005 & .024 & .886 & .011 & .031 & .896 & .011 & .031 \\
\hline 10.64 & .868 & .005 & .024 & .887 & .011 & .031 & .897 & .011 & .031 \\
\hline 10.72 & .868 & .005 & .024 & .888 & .011 & .031 & .898 & .012 & .031 \\
\hline 10.80 & .868 & .005 & .024 & .888 & .010 & .031 & .898 & .011 & .032 \\
\hline 10.87 & .869 & .005 & .025 & .888 & .010 & .032 & .898 & .011 & .032 \\
\hline 10.94 & .870 & .005 & .025 & .888 & .011 & .032 & .898 & .011 & .032 \\
\hline 11.01 & .871 & .005 & .024 & .889 & .011 & .031 & .899 & .011 & .031 \\
\hline 11.08 & .871 & .006 & .024 & .889 & .011 & .032 & .899 & .011 & .032 \\
\hline 11.05 & .872 & .005 & .024 & .890 & .010 & .032 & .899 & .011 & .031 \\
\hline 11.22 & .871 & .006 & .024 & .890 & .011 & .032 & .900 & .011 & .031 \\
\hline 11.28 & .871 & .006 & .024 & .891 & .011 & .031 & .900 & .011 & .031 \\
\hline
\end{tabular}




\begin{tabular}{|c|c|c|c|c|c|c|c|c|c|}
\hline \multirow{2}{*}{$\begin{array}{l}\text { Wavelength } \\
\text { Microns }\end{array}$} & \multicolumn{3}{|c|}{ At $800^{\circ} \mathrm{K}$} & \multicolumn{3}{|c|}{ At $1100^{\circ} \mathrm{K}$} & \multicolumn{3}{|c|}{ At $1300^{\circ} \mathrm{K}$} \\
\hline & $\epsilon$ & $\sigma_{m}$ & $\sigma_{s}$ & $\epsilon$ & $\sigma_{m}$ & $\sigma_{8}$ & $\epsilon$ & $\sigma_{m}$ & $\sigma_{*}$ \\
\hline 11.35 & 0.871 & 0.005 & 0.024 & 0,891 & 0.010 & 0.032 & 0.900 & 0.011 & 0.032 \\
\hline 11.42 & .871 & .005 & .024 & .891 & .011 & .032 & .901 & .011 & .032 \\
\hline 11.49 & .871 & .005 & .024 & .891 & .010 & .032 & .901 & .011 & .031 \\
\hline 11.55 & .871 & .005 & .024 & .892 & .011 & .032 & .901 & .011 & .031 \\
\hline 11.62 & .871 & .005 & .025 & .891 & .011 & .031 & .902 & .011 & .031 \\
\hline 11.68 & .871 & .006 & .024 & .892 & .011 & .032 & .902 & .011 & .032 \\
\hline 11.74 & .871 & .005 & .024 & .892 & .011 & .032 & .902 & .011 & .032 \\
\hline 11.80 & .871 & .006 & .024 & .892 & .011 & .032 & .902 & .011 & .032 \\
\hline 11.87 & .872 & .006 & .024 & .892 & .011 & .032 & .903 & .011 & .032 \\
\hline 11.94 & .872 & .006 & .024 & .893 & .011 & .032 & .903 & .011 & .032 \\
\hline 12.00 & .872 & .005 & .024 & .893 & .010 & .032 & .904 & .012 & .032 \\
\hline 12.07 & .873 & .005 & .024 & .894 & .010 & .031 & .904 & .011 & .032 \\
\hline 12.13 & .874 & .005 & .024 & .894 & .011 & .032 & .904 & .011 & .032 \\
\hline 12.19 & .874 & .005 & .024 & .895 & .011 & .032 & .904 & .011 & .032 \\
\hline 12.26 & .875 & .005 & .024 & .895 & .011 & .032 & .905 & .011 & .033 \\
\hline 12.32 & .877 & .005 & .024 & .895 & .010 & .032 & .906 & .011 & .032 \\
\hline 12.38 & .878 & .005 & .024 & .896 & .011 & .032 & .906 & .011 & .033 \\
\hline 12.44 & .879 & .005 & .024 & .897 & .010 & .032 & .907 & .011 & .033 \\
\hline 12.50 & .880 & .005 & .024 & .898 & .011 & .032 & .908 & .011 & .033 \\
\hline 12.57 & .881 & .005 & .024 & .899 & .010 & .033 & .908 & .011 & .033 \\
\hline 12.63 & .882 & .005 & .024 & .899 & .011 & .032 & .909 & .011 & .033 \\
\hline 12.69 & .883 & .005 & .024 & .900 & .011 & .033 & .910 & .011 & .033 \\
\hline 12.75 & .884 & .005 & .024 & .902 & .011 & .033 & .910 & .011 & .033 \\
\hline 12.82 & .885 & .005 & .024 & .902 & .010 & .033 & .911 & .011 & .034 \\
\hline 12.88 & .886 & .005 & .024 & .903 & .010 & .033 & .912 & .011 & .034 \\
\hline 12.84 & .887 & .005 & .025 & .904 & .011 & .033 & .913 & .011 & .034 \\
\hline 13.00 & .888 & .005 & .025 & .904 & .011 & .033 & .914 & .011 & .034 \\
\hline 13.06 & .889 & .005 & .025 & .905 & .011 & .034 & .915 & .011 & .035 \\
\hline 13.12 & .891 & .005 & .025 & .906 & .011 & .034 & .915 & .011 & .035 \\
\hline 13.18 & .892 & .005 & .025 & .907 & .010 & .035 & .916 & .011 & .035 \\
\hline 13.24 & .893 & .005 & .025 & .908 & .011 & .034 & .916 & .011 & .036 \\
\hline 13.30 & .894 & .005 & .025 & .909 & .011 & .034 & .918 & .011 & .036 \\
\hline 13.36 & .894 & .005 & .026 & .910 & .011 & .035 & .918 & .011 & .036 \\
\hline 13.42 & .895 & .005 & .026 & .910 & .011 & .035 & .918 & .011 & .037 \\
\hline 13.48 & .894 & .004 & .026 & .910 & .011 & .035 & .919 & .011 & .037 \\
\hline 13.54 & .894 & .005 & .027 & .911 & .011 & .035 & .920 & .011 & .038 \\
\hline 13.60 & .893 & .005 & .026 & .911 & .011 & .036 & .920 & .011 & .038 \\
\hline 13.66 & .892 & .005 & .027 & .910 & 011 & .037 & .920 & .011 & .038 \\
\hline 13.72 & .890 & .005 & .027 & .910 & .011 & .037 & 920 & .010 & .039 \\
\hline 13.78 & .887 & .005 & .028 & .909 & .011 & .037 & .920 & .011 & .040 \\
\hline 13.84 & .883 & .005 & .028 & .908 & .011 & .038 & 919 & .011 & .041 \\
\hline 13.89 & .878 & .005 & .029 & .906 & .011 & .038 & .918 & .011 & .041 \\
\hline 13.95 & .873 & .005 & .029 & .904 & .011 & .039 & 918 & .012 & .042 \\
\hline 14.00 & .867 & .005 & .030 & .902 & .011 & .039 & .917 & .012 & .043 \\
\hline 14.06 & .860 & .005 & .030 & .898 & .010 & .039 & .915 & .012 & .044 \\
\hline 14.11 & .853 & .005 & .031 & .895 & .011 & .040 & .913 & .011 & .044 \\
\hline 14.17 & .845 & .005 & .031 & .890 & .011 & .040 & .910 & .011 & .045 \\
\hline 14.22 & .838 & .005 & .031 & .885 & .011 & .040 & .907 & .011 & .046 \\
\hline 14.28 & .831 & .005 & .032 & .879 & 011 & .041 & .904 & .011 & .047 \\
\hline 14.33 & .825 & .005 & .033 & .873 & .011 & .041 & .900 & .011 & .047 \\
\hline
\end{tabular}




\begin{tabular}{|c|c|c|c|c|c|c|c|c|c|}
\hline Wavelength & \multicolumn{3}{|c|}{ At $800^{\circ} \mathrm{K}$} & \multicolumn{3}{|c|}{ At $1100^{\circ} \mathrm{K}$} & \multicolumn{3}{|c|}{ At $1300^{\circ} \mathrm{K}$} \\
\hline Microns & $\epsilon$ & $\boldsymbol{\sigma}_{m}$ & $\sigma_{s}$ & $\epsilon$ & $\sigma_{m}$ & $\sigma_{s}$ & $\epsilon$ & $\sigma_{m}$ & $\sigma_{\theta}$ \\
\hline 14.38 & 0.818 & 0.005 & 0.033 & 0.867 & 0.010 & 0.042 & 0.896 & 0.011 & 0.048 \\
\hline 14.44 & .811 & .005 & .034 & .861 & .010 & .042 & .891 & .011 & .050 \\
\hline 14.49 & .806 & .005 & .034 & .854 & .011 & .043 & .886 & .011 & .051 \\
\hline 14.55 & .800 & .005 & .035 & .848 & .011 & .043 & .881 & .011 & .051 \\
\hline 14.60 & .795 & .005 & .036 & .842 & .011 & .044 & .875 & .011 & .052 \\
\hline 14.65 & .789 & .005 & .037 & .834 & .011 & .045 & .871 & .011 & .053 \\
\hline 14.71 & .784 & .005 & .038 & .831 & .010 & .045 & .865 & .011 & .054 \\
\hline 14.76 & .779 & .005 & .039 & .825 & .012 & .046 & .861 & .011 & .055 \\
\hline 14.82 & .774 & .005 & .040 & .820 & .011 & .047 & .855 & .011 & .056 \\
\hline 14.87 & .768 & .004 & .042 & .813 & .011 & .048 & .850 & .011 & .057 \\
\hline 14.92 & .762 & .004 & .044 & .805 & .012 & .049 & .844 & .011 & .058 \\
\hline 14.98 & .756 & .004 & .044 & .799 & .011 & .048 & .838 & .012 & .058 \\
\hline 15.03 & .753 & .004 & .043 & .796 & .011 & .047 & .834 & .012 & .058 \\
\hline 15.08 & .751 & .004 & .044 & .794 & .011 & .048 & .831 & .012 & .060 \\
\hline 15.14 & .746 & .004 & .046 & .789 & .011 & .050 & .826 & .012 & .061 \\
\hline 15.20 & .740 & .004 & .048 & .783 & .012 & .051 & .822 & .012 & .062 \\
\hline
\end{tabular}

The standards of normal spectral emittance are intended for use in calibrating equipment used in various laboratories for measuring this property of materials. All of the specimens were prepared from a single sheet of metal at one time, and were subjected as nearly as possible to identical preparation treatments. Because the equipment used for the calibration of these standards was suitable only for making measurements on $1 / 4$ inch by 8 inch strips, seven such specimens were prepared from selected locations in the sheet so that the strips measured were statistically representative of the entire lot of specimens.

Three measurements were made on each of the seven samples. The value listed for normal spectral emittance $(\epsilon)$ is the arithmetic average of the 21 measured values. The computed average standard deviation $\left(\sigma_{m}\right)$ of the three measurements on each of the seven specimens about the average value for each specimen is a measure of the precision of measurement. The standard deviation $\left(\sigma_{s}\right)$ of the average value for each of the seven specimens about the overall average, is indicative of the variation in specimens.

Procedures used for the measurements are described in detail "Standardization of Thermal Emittance Measurements, part 4, Normal Spectral Emittance, 800-1400 K." Technical Report No. WADC-TR59-510, Part IV, by William N. Harrison, Joseph C. Richmond, Frederick J. Shorten and Horace M. Joseph, available from the Clearinghouse for Federal Scientific and Technical Information, 5285 Port Royal Road, Springfield, Virginia 22171, as publication AD 426846, price $\$ 2.25$.

Samples are available as $1 / 2$ inch disks, SRM No. 1440; as 7/8 inch disks, SRM No. 1441; as 1 inch disks, SRM No. 1442; as 11/8 inch disks, SRM No. 1443; as $1 \frac{1}{4}$ inch disks, SRM No. 1444; as 2 inch by 2 inch squares, SRM No. 1445; as 1 inch by 10 inch strips, SRM No. 1446; and as $3 / 4$ inch by 10 inch strips, SRM No. 1447.

Washington, D. C.

December 16, 1965
W. Wayne Meinke, Chief

Office of Standard Reference Materials 


\section{Emissivity and Emittance Measurements and Techniques}

Papers

3.1. Standardization of Thermal Emittance Measurements Part IV:

Normal Spectral Emittance $800-1400^{\circ} \mathrm{K}$.

William N. Harrison, Joseph C. Richmond, Frederick J. Shorten, and Horace M. Joseph, abstracted from Tech. Rept. WADC-TR-59-510, Part IV, Wright Patterson Air Force Base, 90 pp. (1963).

Key words: Emittance measurement; normal spectral emittance; standardization

3.2. Equipment and Procedures for Evaluation of Total Hemispherical Emittance.

Joseph C. Richmond and William N. Harrison, Am. Ceram. Soc. Bull. 39, No. 11, 668-673 (Nov. 15, 1960).

Key words: Emittance change on vacuum heating; hemispherical emittance measurement__-_-_-_-_-_-_-_-

3.3. Method of Measuring Emissivities of Metals in the Infrared.

Arthur G. Maki and Earle K. Plyler, J. Res. Nat. Bur. Stand. (U.S.) 66C, No. 3, 283-287 (Sept. 1962).

Key words: Emissivity measurement; gold; metals; infrared

3.4. Comments on the Surface Characterization of Real Metals.

David P. DeWitt, Symposium on Measurement of Thermal Radiation of Solids, San Francisco, Calif., Mar. 4-6, 1964, (NASA SP-55, 1965). Nat. Aeron. and Space Admin., Washington, D.C.

Key words: Emittance of metals; roughness effect; surface characterization; thermal history

3.5. Importance of Surface Films.

Joseph C. Richmond, Symposium on Thermal Radiation of Solids, San Francisco, Calif., Mar. 4-6, 1964, pp. 157-158 (NASA SP-55, Nat. Aeron. and Space Admin., Washington, D.C., 1965).

Key words: Hemispherical emittance; Inconel; surface films

3.6. A Rotating Cylinder Method for Measuring Normal Spectral Emittance of Ceramic Oxide Specimens from $1200^{\circ}$ to $1600^{\circ} \mathrm{K}$.

Howard E. Clark and Dwight G. Moore, J. Res. Nat. Bur. Stand. (U.S.) 70A, No. 5, 393-415 (Sept.-Oct. 1966).

Key words: Alumina; ceramic oxides; emittance; error sources; magnesia; measurement uncertainties; rotating cylinder; spectral emittance; thermal radiation; thoria; zirconia 


\section{Emissivity and Emittance Measurements and Techniques- Continued}

Papers

3.7. Apparatus for the Measurement of the Normal Spectral Emissivity in the Infrared.

A. G. Maki, Ralph Stair, and R. G. Johnston, J. Res. Nat. Bur. Stand. (U.S.) 64C, No. 2, 99-102 (Apr.-June 1960).

Key words: Emissivity of platinum; oxidized Inconel; spectral emissivity measurement.-_-_-_-_-_-_-_-_---.---

3.8. Approximate Normal Emissivity in the Infrared at Elevated

Temperatures of Single-Crystal and Polycrystalline Calcium Fluoride.

W. Fussell and J. Geist, Appl. Opt. 6, No. 1, 119-124 (Jan. 1967).

Key words: Calcium fluoride; infrared absorption coefficient ; elevated-temperature emissivity ; infrared emissivity ; polycrystalline calcium fluoride; single-crystal calcium fluoride

3.9. Periodic Heat Flow in a Hollow Cylinder Rotating in a Furnace with a Viewing Port.

B. A. Peavy and A. G. Eubanks, Symposium on Measurement of Thermal Radiation Properties of Solids, Dayton, Ohio, Sept. 5-7, 1962, pp. 553-563 (NASA SP-31, Nat. Aeron. and Space Admin., Washington, D.C., 1963).

Key words: Rotating-specimen furnace; spectral emittance; total emittance

3.10. Survey of Infrared Measurement Techniques and Computational Methods of Radiant Heat Transfer.

S. Thomas Dunn, Joseph C. Richmond, and Jerome F. Parmer,

J. Spacecraft and Rockets, 961-975 (July 1966).

Key words : Computational methods ; emittance ; heat transfer; infrared ; measurement techniques; reflectance....-.--

Abstracts

3.11. Thermal Radiation Property Measurement Techniques.

S. T. Dunn, J. C. Geist, D. G. Moore, H. E. Clark, and J. C. Richmond, Nat. Bur. Stand. (U.S.) Tech. Note 415, 77 pp., (Apr. 27, 1967).

Key words: Averaging spheres; diffuse reflectance; emissivity; emittance; flux averaging devices; infrared reflectance; spectral reflectance; spectral emittance; specular reflectance 


\section{Emissivity and Emittance Measurements and Techniques- Continued}

Abstracts

3.12. Procedures for Precise Determination of Thermal Radiation Properties, November 1962 to October 1963.

J. C. Richmond, D. P. DeWitt, and W. D. Hayes, Jr., Nat. Bur. Stand. (U.S.) Tech. Note 252, 55 pp. (Nov. 20, 1964). (Table 3 included in this volume).

Key words: Emissivity of metals ; radiation property determination; standards calibration; total emittance computation

3.13. Procedures for Precise Determination of Thermal Radiation Properties, November 1963 to October 1964.

J. C. Richmond, S. T. Dunn, D. P. DeWitt, and W. D. Hayes, Jr., Nat. Bur. Stand. (U.S.) Tech. Note 267, 62 pp. (Dec. 17, 1965).

Key words: Emissivity; emittance; high-temperature reflectance; infrared reflectance; radiation properties; reflectance; spectral emittance; spectral reflectance; thermal radiation

3.14. Procedures for Precise Determination of Thermal Radiation Properties, November 1964 to October 1965.

J. C. Richmond, G. J. Kneissel, D. L. Kelley, and F. J. Kelly, Nat. Bur. Stand. (U.S.) Tech. Note 292, 80 pp. (Feb. 10, 1967). Key words: Emittance error sources; emittance from shallow cavity; emittance measurement; radiation property determination; standards calibration 



\section{STANDARDIZATION OF THERMAL EMITTANCE MEASUREMENTS}

\section{PART IV. NORMAL SPECTRAL EMITTANCE, $800-1400^{\circ} \mathrm{K}$}

TECHNICAL REPORT No. WADC-TR-59-510, PART IV

(Prepared under Contract No. AF 33(616)-61-02 by the

National Bureau of Standards, Washington, D. C.;

William N. Harrison, Joseph C. Richmond, Frederick J. Shorten and Horace M. Joseph, authors.)

\section{ABSTRACT}

Equipment and procedures were developed to measure normal spectral emittance of specimens that can be heated by passing a current through them, at temperatures in the range of 800 to $1400^{\circ} \mathrm{K}$, and over the wavelength range of 1 to 15 microns.

A data-processing attachment for the normal spectral emittance equipment was designed to (1) automatically correct the measured emittance for "100\% line" and "zero line" errors on the basis of previously-recorded calibration tests; (2) record the corrected spectral emittance values and wavelengths at preselected wavelength intervals on punched paper tape in form suitable for direct entry into an electronic digital computer; and (3) to compute during a spectral emittance test on a specimen the total normal emittance, or absorptance for radiant energy of any known spectral distribution of flux, of the specimen.

Working standards of normal spectral emittance having low, intermediate and high emittance values, respectively, were prepared and calibrated for use in other laboratories to check the operation of equipment and procedures used for measuring normal spectral emittance. 
The general plan that was followed in developing equipment for determination of normal spectral emittance was to use a double-beam ratio-recording infrared spectrometer with a laboratory blackbody furnace and a heated specimen mounted normal to the direction of viewing as the respective sources for the two beams. With this arrangement the instrument will record directly the normal spectral emittance of the specimen, if the following conditions are met within acceptable tolerances: 1) The temperature of the specimen must be the same as that of the blackbody furnace; 2) the optical path length in the two beams must be equal or, preferably, the instrument must operate in a non-absorbing atmosphere or a vacuum, in order to eliminate the effects of differential atmospheric absorption in the two beams; 3) front-surface mirror optics must be used throughout, except for the prism, and equivalent optical elements must be used in the two beams in order to reduce and balance attenuation of the beams by absorption in the optical elements; 4) the source and field apertures of the two beams must be equal in order to ensure that radiant $f l u x$ in the two beams compared by the apparatus will pertain to equal areas of the sources and equal solid angles of emission, and 5) the response of the detector-amplifier system must vary linearly with the incident radiant flux.

Early in the investigation it became apparent that the conditions specified under 2, 3 and 4 above were not being met within sufficiently close tolerances to permit direct recording of normal spectral emittance to the desired accuracy. To enable corrections to be made for the errors thus introduced a second, or reference, laboratory blackbody furnace, identical to the first, or comparison blackbody furnace was used.

\section{A. Description of Spectrometer}

The Perkin-Elmer Model 13 spectrometer used for the measurements is equipped with a wavelentth drive that provides automatic scanning of the spectrum of radiant $\mathrm{flux}$, and a slit servomechanism that automatically opens and closes the slits to minimize the variations of radiant flux in the comparison beam. Three prisms were available, 1) fused silica, to cover the spectral range of 0.25 to 3.5 microns, 2) sodium chloride to cover the spectral range 0.7 to 15 microns, and 3) cesium bromide, to cover the spectral range of 15 to 38 microns. Several photomultiplier detectors were available for use in the spectral range of 0.25 to $1.0 \mathrm{micron}$, a 1 ead sulfide detector for use in the spectral range 1.0 to 2.0 microns, a vacuum thermocouple with sodium chloride window for use in the spectral range 1 to 15 microns, and a vacuum thermocouple with cesium bromide window for use in the spectral range 1 to 40 microns. 
The source optics of the instrument were modified by incorporation of an external optical system, which is the standard Perkin-Elmer transfer optical system, used with the hohlraum reflectometer. Two $90^{\circ}$ folds in a vertical plane were introduced in the specimen beam, to raise the axial ray of the beam 9 inches above the optical plane of the spectrometer. The comparison blackbody furnace was mounted in a fixed position to act as the source for the comparison beam, and the specimen furnace and referenze blackbody furnace were mounted side by side on a movable plate attached to a slide in positions such that they could be brought alternately into position to serve as source for the specimen beam, by sliding the plate against fixed stops on the slide. A sketch of the complete optical path is shown in figure 1, and a photograph of the external optical system with furnaces in place and cover removed is shown in figure 2.

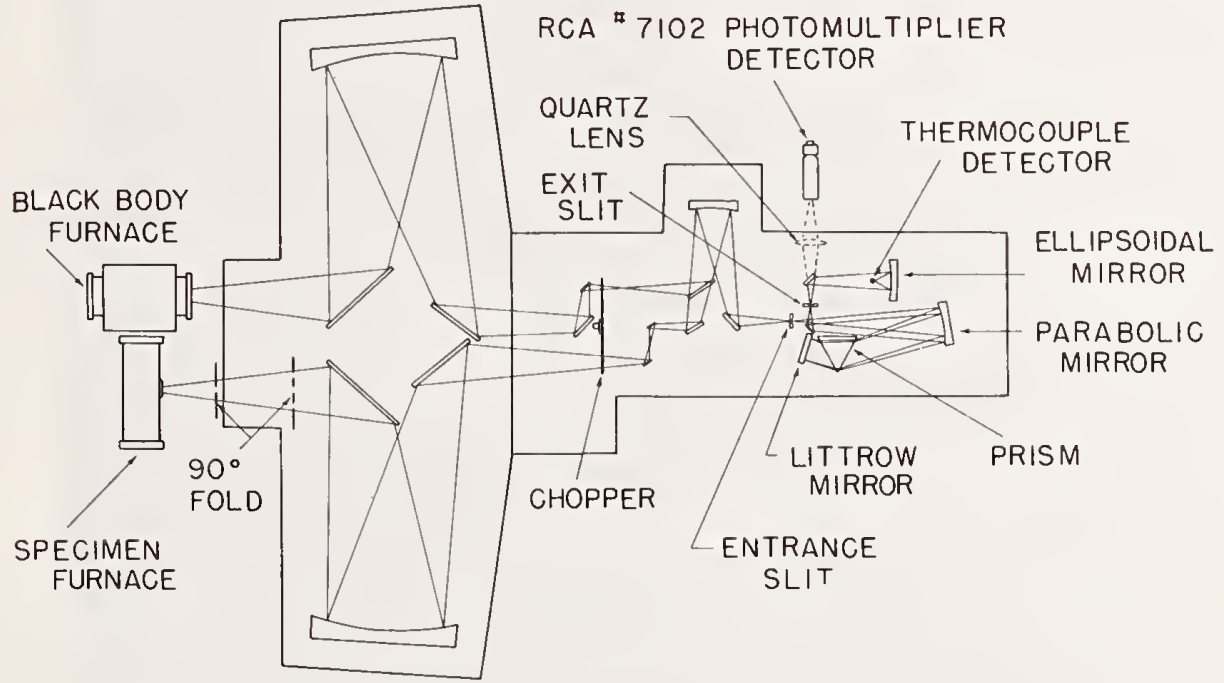

Fig. 1 Sketch of the optical path in the modified spectroradiometer.

It was found by experience that when the two blackbody furnaces were used as sources for the respective beams of the spectrometer, deviations of the recorded "100\% curve" occurred at the wavelength intervals within which there is significant absorption of radiant energy by water vapor and carbon dioxide in the atmosphere. Such absorption should be identical in both beams if the spectral distribution of flux and path lengths are identical. However, the two beams are separated in time, passing through the monochromator alternately at a frequency of 13 cycles per second. Since the spectrum is being traversed continuously, one energy pulse is displaced relative to the other on the wavelength scale, by a very small amount. The effect of this displacement is negligible over most of the range, where the slope of the energy-wavelength curve is not large. But at the wavelengths near those of maximum absorption, the energy-wavelength curves become very steep, and the slight spectral displacement of the successive energy pulses can produce significant deviations in the ratios which are continuously recorded by the instrument. 


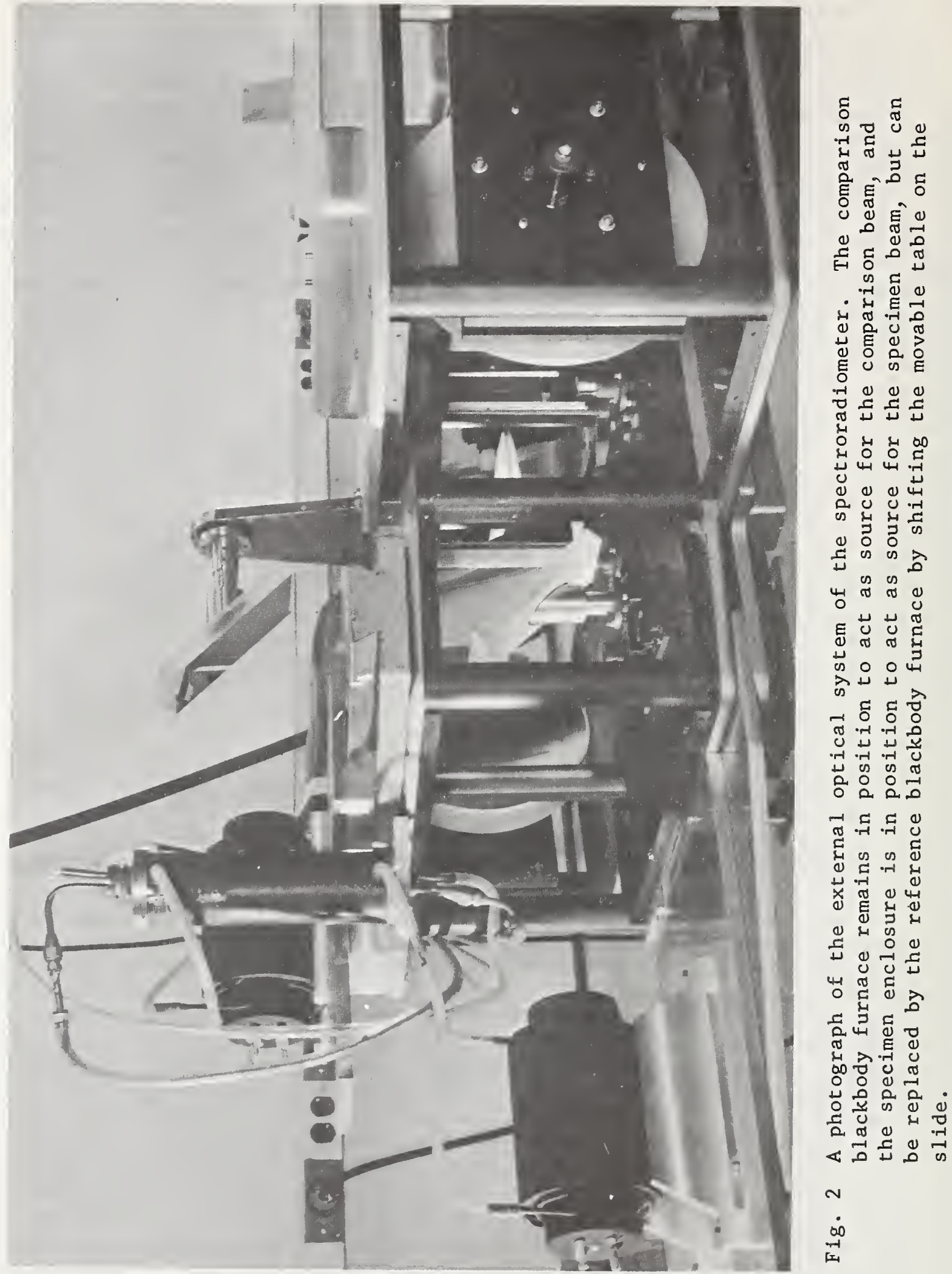


In order to reduce the effects of absorption by atmospheric water vapor and carbon dioxide, the entire external optical system of the normal spectral emittance equipment, Including the blackbody furnaces and specimen furnace, was enclosed in a lucite box. Gas-tight connecting passages were designed to foin 1) the lucite box enclosing the external optics, 2) the metal cover for the chopper assembly, 3) the metal cover for the combining optics, and 4) the metal cover for the monochromator. Thus the entire length of both optical paths in the instrument was enclosed in a single system of interconnecting housings that was nearly gas-tight. Humidity in the enclosure was monitored and recorded by means of a humidigraph which records temperature and humidity. Iminedlately before a test the enclosure was purged with "super-dry" nitrogen, free from carbon dioxide, which was fed into the enclosure through several inlets, at a slow, constant rate, so that the enclosure was malntained at a slight positive pressure relative to the surrounding atmosphere. Tests were not started until the recorded humidity was less than $5 \%$.

\section{B. Blackbody Furnace and Specimen Enclosure}

Two small laboratory blackbody furnaces were designed and built. Figure 3 is a sectional drawing of a furnace. The core was a casting of 80 nickel-20 chromium alloy $13 / 16$ inches in diameter and 4 inches 1 ong. The cavity was $3 / 4$ inch in diameter and $33 / 8$ inches deep, threaded with 13 threads to the inch. The threads were cast in place. The 80 nickel-20 chromium alloy oxidizes to produce a high-emittance surface in the cavity.

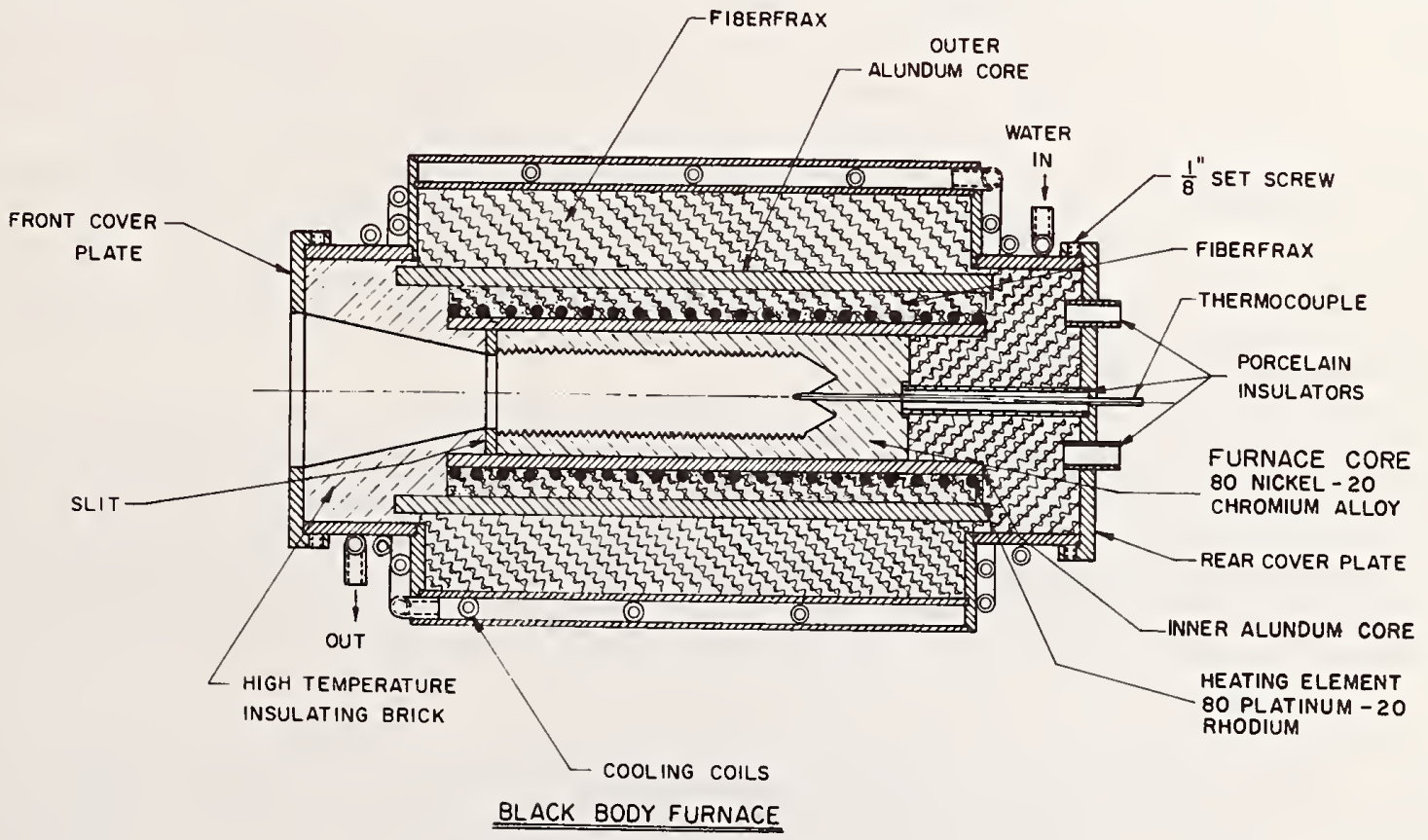

Fig. 3 Sectional drawing of a blackbody furnace. 
The furnace was heated by means of a platinum-20\% rhodium winding. There were two taps on the heating coll approximately one inch from each end. Shunts across the taps and leads were used to reduce thermal gradients in the cavity, as shown in figure 5. The aperture in the cavity was reduced to a slit approximately $1 / 4$ by $3 / 4$ inches in size by means of a Nichrome cover plate.

The temperature of the blackbody furnace was measured by means of a platinum, platinum-10\% rhodium thermocouple, the bare bead of which extended about $1 / 4$ inch into the cavity from the rear. The thermocouple leads were insulated from the core by high-alumina refractory tubing, which was surrounded by a platinum tube that was grounded, in order to prevent pickup by the thermocouple of spurious signals due to electrical leakage from the winding.

The effective emittance of the blackbody furnace, computed from the Gouffe [5] equation, assuming that the interior of the cavity is at uniform temperature, and is a completely diffuse reflector, was better than 0.999. Both of these assumptions are known to be only approximations, so that the actual effective enittance was somewhat less than the computed value. The thermocouple bead in the back of the cavity cannot be seen, even with an optical pyrometer, when the furnace is in operation, which indicates that the effective emittance is very high.

The specimens were strips of metal, $1 / 4$ inch wide by 8 inches long, of any convenient thickness in the range of approximately 0.010 to $0.050 \mathrm{inch}$. They were heated by passing a current through them. A sketch of the specimen enclosure is shown in figure 4. The outer shell was water cooled, and its inner surface was of a nickel - chromium-iron alloy that had been threaded with a No. 80 thread and then oxidized in air at a temperature above $1350^{\circ} \mathrm{K}$ for 6 hours to produce a surface having a reflectance of less than 0.05 at the operating temparature of the water-cooled walls. The specimen was positioned off-center in the cylindrical enclosure, so that any radiant flux specularly reflected from the walls would be reflected twice before hitting the specimen.

The electrodes were of water-cooled copper, and were insulated from the brass end caps of the furnace by means of bakelite sleeves. The lower electrode fitted loosely in the hole through the end cap to permit the specimen to expand without buckling when heated. The specimen was viewed through a window about $3 / 4$ by 1 inch in size. A viewing window insert reduced the opening to about $1 / 4$ by, $3 / 4$ inch.

Adjustable baffles above and below the viewing window were used to reduce convection and the resulting temperature fluctuations and thermal gradients. Adjustable telescoping cylindrical platinum reflectors surrounded the specimen at each end. These were used to reduce heat loss at the ends of the specimen, and hence the thermal gradient along the specimen. 


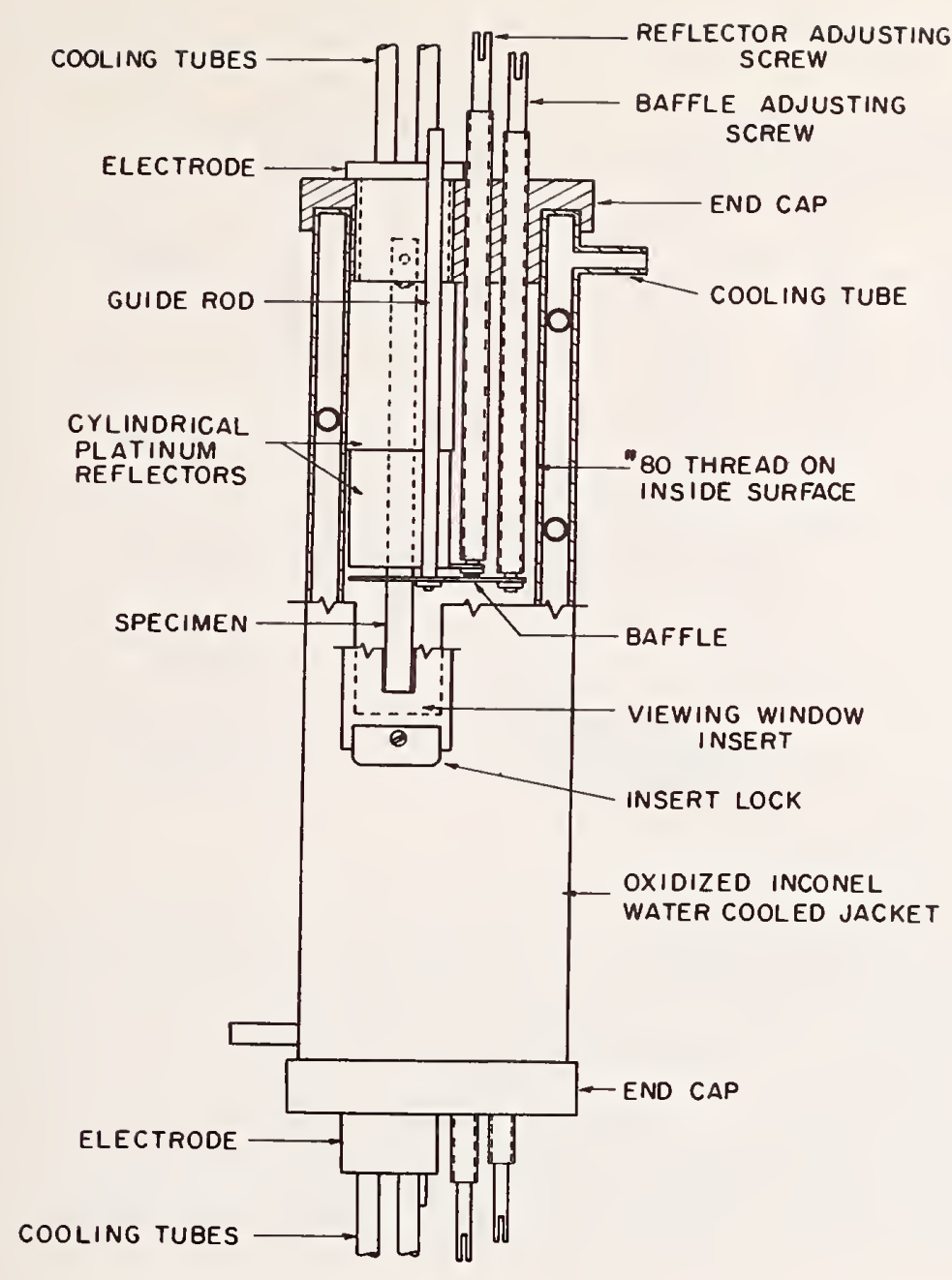

Fig. 4 Sketch of the specimen enclosure. Symmetrica 1 about the mid-length.

\section{Temperature Control Equipment}

The power input to the comparison blackbody furnace was adjusted manually by means of an autotransformer. The heater winding of the furnace had two taps, located approximately one inch from each end of the winding. Variable resistors were connected as shunts across the taps and from the taps to the input leads, as indicated in the wiring diagram, figure 5. Adjustment of these resistors permitted variation of the power distribution along the cavity of the furnace by means of which thermal gradients in the cavity could be minimized. It was found by experience that the heat capacity of the blackbody furnace was large enough to practically eliminate temperature fluctuations due to momentary voltage fluctuations in the power supply. A cold furnace could be brought to $1400^{\circ} \mathrm{K}$ and stabilized at that temperature in about six hours, and only about 30 minutes was required to achieve stability after a temperature change of up to about $30^{\circ} \mathrm{K}$. Normally the blackbody furnaces were heated overnight before use. 


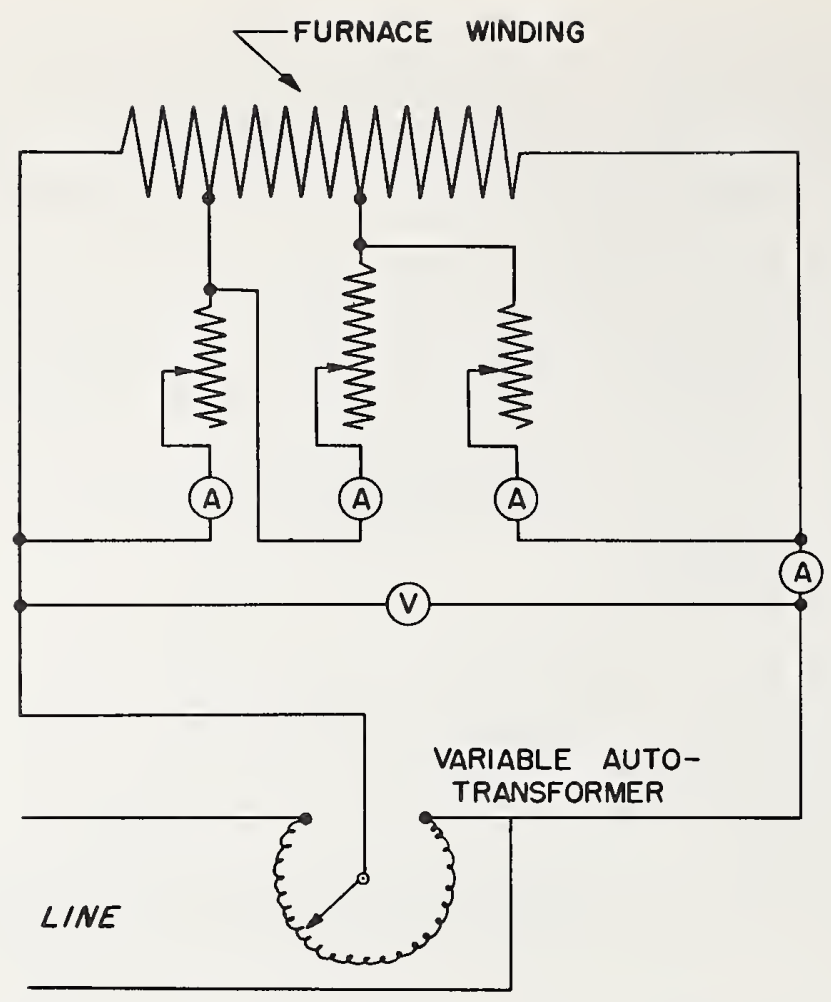

Fig. 5 Wiring diagram of power supply to comparison blackbody furnace.

The power supply for the reference blackbody furnace was identical to that shown in figure 5, except that when the temperature was to be controlled a saturable-core reactor was substituted for the autotransformer, as is described below.

The temperature of the specimen (or reference blackbody furnace) was controlled to that of the comparison blackbody furnace, within narrow 1imits, by means of a differential thermocouple. One bead of the differential thermocouple was in the cavity of the comparison blackbody furnace, and the other was attached to the back of the specimen, in the center of the area being viewed, as described later in this report. The signal from the differential thermocouple was amplified by a d.c. amplifier and fed to a centerzero recorder-controller. The output of the recorder-controller is proportional to the distance between the control pointer and the recorder pointer on the scale, which is in turn proportional to the temperature difference between blackbody furnace and specimen. The output of the recorder-controller was fed to a current-actuating-type controller, the output of which was separated into three portions, each of which could be varied as desired. One portion was proportional to the input signal, one was proportional to the first time derivative of the input signal, and one was proportional to the second time derivative of the input signal. The output of the currentactuating-type control was fed to the coll of a saturable core reactor which varied the power input to the specimen. It was possible to reduce undershoot and overshoot of the controlled temperature by proper adjustment of the three components in the output of the current-actuating-type control. A block diagram of the temperature control equipmeat is shown in figure 6 . 


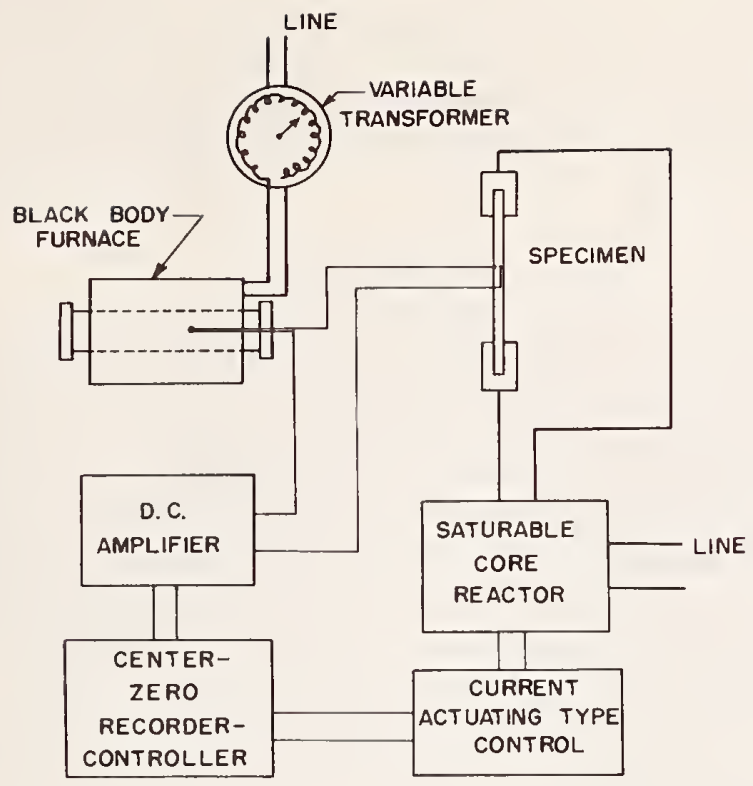

Fig. 6 Block diagram of temperature control equipment. The controller operates from a signal produced fron a differential thermocouple.

With the described control equipment it has been possible to hold the temperature of a specimen as indicated by the welded thermocouple to well within $\pm 1^{\circ} \mathrm{K}$ of that of the comparison blackbody funnace, and the temperature of the reference blackbody furnace to within $\pm 0.5^{\circ} \mathrm{K}$ of that of the comparison blackbody furnace.

\section{CALIBRATION OF EMITTANCE EQUTPMENT}

\section{A. Wavelength Calibration of Monochromator}

A complete wavelength calibration was made of the spectrometer with the fused silica and sodium chloride prisms, respectively, mounted in the monochromator. In calibrating the equipment with the fused silica frism, enission spectra of a helium arc and a mercury arc, and absorption spectra of a didymium glass and a polystyrene film were recorded. The emission and absorption peaks having known wavelengths were identified in the respective curves, and for each peak the observed wavelength drum position at which the peak occurred was plotted as a function of the known wavelength of the paak [6]. A total of 66 such points, at wavelengths in the range 0.24 to 2.2 microns, was plotted, and a smooth curve was drawn between the points to produce a calibration curve. 
A similar procedure was followed in calibrating the equipment with the sodium chloride prism mounted in the monochromator. In this case, the atmospheric absorption curve replaced the absorption curve for didymium glass. A total of 52 points, at wavelengths from 0.4 to 15 microns, was plotted to produce the calibration curve.

\section{B. Calibration for Linearity of Response}

A11 of the work done with the spectrometer had been based on the assumption that the response of the instrument (the height of the recorded emittance curve above the recorded "zero curve") was linear with (1) the radiant flux (within the varying wavelength interval encompassed by the exit slit) passing through the monochromator, when the instrument is operated in single-beam mode, or (2) the ratio of the fluxes (within the varying wavelength interval encompassed by the exit slit) in the respective beams that pass through the monochromator when the instrument is operated double-beam in ratio mode. This assumption had not been checked, and a confirmation of (1) and (2) above was considered desirable.

\section{Linearity Calibration by Varying S1it Width}

The entrance and exit slits of the monochromator open and close simultaneously, and both are controlled at the same width by the slit micrometer. Because of this construction, when a true image of a source is formed at the entrance slit of the monochromator, somewhat larger than the slit at its maximum width, the radiant flux reaching the detector from the monochromator varies as the square of the slit width. This relationship was used to check the linearity of detector response when the instrument is used single-beam in direct mode (item (1) above). A single blackbody furnace, at $1400^{\circ} \mathrm{K}$, was used as a source, and each series of measurements was made at a number of central wavelengths throughout the sensitive range of the thermocouple detector. Experimental results were in conformity with the theoretical relationship. In every case, when the height of the recorder curve was plotted against the square of the slit width, the points obtained with the sodium chloride prism and thermocouple detector fell on a straight line intersecting the origin, within the error of measurement.

Similar measurements were also made at several wavelengths when the spectrometer was operated with the fused silica prism and the lead sulfide detector. Again experimental results were in conformity with the theoretical relationship.

Winen the spectrometer was used with the fused silica prism and 1 P21 detector, appreciable deviations from the theoretical relationship were found at first, but after careful realignment of the monochromator optics, al1 of the points again fell on a straight line, within experimental error. 
A sector-disc attenuator for use in calibrating the normal spectral emittance equipment was designed and built. The attenuator consists of a variable speed motor, 0 to $4000 \mathrm{rpm}$, with an attenuator disc mounted on 1 ts shaft.

Five interchangeable attenuator discs were prepared, having nominal transmissions of $75,50,25,12.5$ and 5.0 percent, respectively. The discs were machined from sheet aluminum 0.065 inch thick. The $5 \%$ disc is 10 inches in diameter, and has four equally spaced radial notches, each $4.5^{\circ}$ wide, extending inward from the rim for 3 inches. The other discs are 9 inches in diameter, and have eight notches each, extending inward from the rim for $21 / 2$ inches. The notches are $333 / 4^{\circ}$ wide in the $75 \%$ disc, $221 / 2^{\circ}$ wide for the $50 \% \mathrm{disc}, 111 / 4^{\circ}$ wide for the $25 \% \mathrm{disc}$, and $55 / 8^{\circ}$ wide for the $12.5 \% \mathrm{disc}$.

The attenuator is normally operated at about $1300 \mathrm{rpm}$, and the direction of rotation is opposite to that of the chopper of the spectrometer. At this speed the beam is interrupted at a frequency of more than 85 cycles per second by the $5 \% \mathrm{disc}$, and more than 170 cycles per second by the other discs. The chopper in the spectrometer interrupts the beam at a frequency of 13 cycles per second. No coupling has been observed between the attenuat or and chopper frequencies.

The attenuator discs were calibrated in the Engineering Metrology Section of NBS by measuring the angular width of the notches and blades on each disc, at two positions corresponding to the radial positions at which the top and bottom, respectively, of the interrupted beam strike the discs when in use. The unobstructed area of the disc between the two circles was computed as a percentage of the total area between the circles, with the results shown below:

Nominal Transmittance
$\%$
75
50
25
12.5
5

Measured Unobstructed Area $\%$

$$
\begin{array}{r}
75.10 \pm 0.02 \\
50.00 \pm 0.02 \\
25.28 \pm 0.14 \\
12.73 \pm 0.14 \\
5.07 \pm 0.03
\end{array}
$$

The measured values were assigned to the respective sector-disc attenuators. 
In the next group of experiments, made for additional check of item (1) above, the sector-disc attenuator was introduced into the beam near the blackbody furnace. Each series of measurements was made at a single wavelength, a single slit width, and a single gain setting. In each case the gain was adjusted to give a reading of slightly less than 100 units on the strip chart without the attenuator. The attenuator was then introduced into the beam, and operated with each of the discs in turn. The height of the recorder curve was plotted against the previously measured fraction of the beam passed by the attenuator. In every case the points fell on a straight line passing through the origin, indicating linear detector response for every combination of slit width and wavelength tested, with both the sodium chloride and fused silica prisms, and with the thermocouple, as well as the lead sulfide and photomultiplier detectors.

In the preceding experiments for which the instrument was being used in direct mode, with single beam, the height of the recorder curve varied linearly with the signal produced by the detector. This signal was proportional to the radiant flux passing through the monochromator. Normally, however, the instrument is used in ratio mode, with double beam. In this case the height of the recorder curve should vary linearly with the ratio of the two signals produced by the detector when it is receiving flux alternately from the respective beams.

In order to check item (2) above, the two blackbody furnaces, controlled very closely to the same temperature (about $1400^{\circ} \mathrm{K}$ ), were used as sources for the two beams. The instrument was adjusted so that the "100\% curve" would actually fall between 90 and 100 on the chart, after which such a curve was obtained over the wavelength range of interest. The sector-disc attenuator was then introduced into the specimen beam near the blackbody furnace, and operated with each of the discs in turn, to obtain $175 \%$, 50\%, 25\%, $12.5 \%$ and 5\% curves", each over the wavelength range of interest. The heights of the respective curves were then measured at 100 selected wavelengths, and the height of each curve above the experimentally obtained zero for the pertinent wavelength was plotted against the percentage of the flux in the specimen beam that was passed by the attenuator. In every case the points fell on a straight line intersecting the origin, within the error of measurement. No significant departures from linearity of response were detected for any combination of prism and detector, at any point within the wavelength range at which they could be used.

The data for the thermocouple detector and sodium chloride prism are shown in figure 7. In this case, the "apparent emittance" of the combination of the reference blackbody furnace and each attenuator disc is plotted as a function of wavelength. Every value of apparent emittance was within \pm 0.01 of the measured transmittance of the respective disc. 


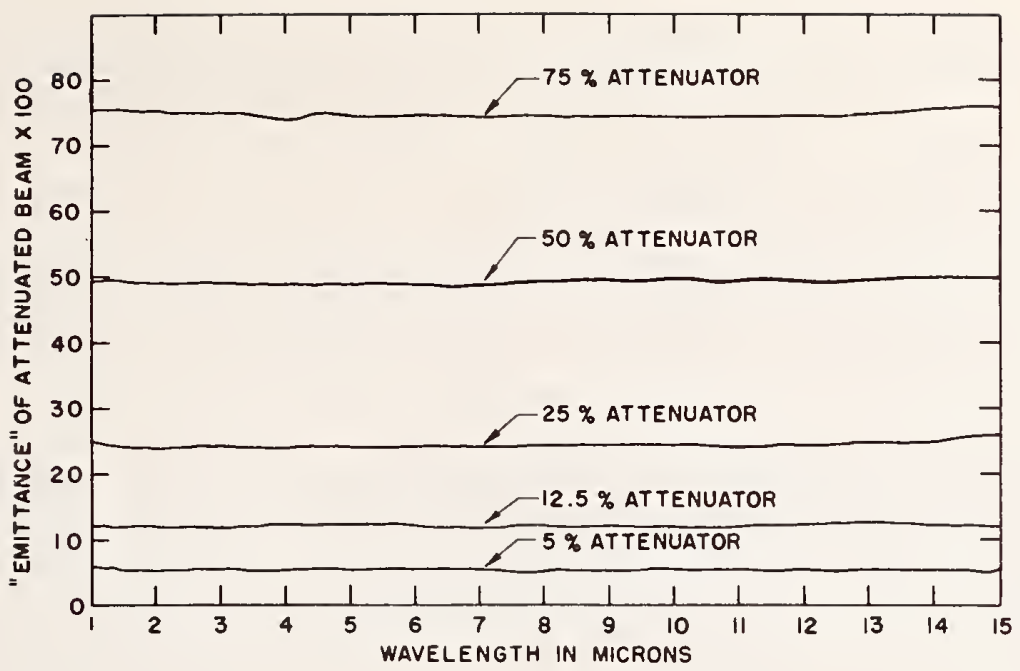

Fig. 7 Spectral "emittance" of the combination of the reference blackbody furnace and sectordisc attenuator when using the $75 \%$, $50 \%$, $25 \%, 12.5 \%$ and $5 \%$ attenuator discs. Diata are for the spectroneter with the sodium chloride prism and thermocouple detector.

\section{EXPERIMENTAL PROCEDURE}

It was found by experience that the "100\% curve", obtained when the two blackbody furnaces at the same temperature served as sources for the respective beams of the spectrometer, deviated appreciably from flatness. These deviations may be due to either (1) variations in spectral absorption or other losses along the respective optical paths from source to detector, or (2) variations in spectral sensitivity of the detector to radiant flux from the two beams. There are two more mirrors in the specimen beam than in the comparison beam, as is shown in figure 1. The two beams follow different paths from the sources to the spherical mirror in the combining optics, and are reflected by different mirrors in this portion of the path. From the spherical mirror in the combining optics to the detector, the two beams follow the same general path and are reflected by the same mirrors. However, the paths are not identical, and the beams are reflected by slightly different areas of each mirror, and are transmitted by different areas of the prism and the detector window. These differences could account for spectral differences in losses in the two beams, due to absorption, and to scattering, for instance by dust particles on the mirrors. The two beams are also focused on slightly different areas of the sensitive surface of the detector. Variations in spectral response over the sensitive surface of the detector could be responsible for part of the observed effect. 
It was also found by experience that the zero curve, obtained when the specimen beam was blocked near the specimen furnace, deviated from flatness. These deviations are undoubtedly due to stray radiation in the monochromator, which produces a spurious signal when there is in fact no radiant energy being supplied by the specimen.

A test procedure was developed to correct for the errors which result in the deviations of the "100\% Line" and "zero Line" referred to above. The procedure is as follows:

(1) The two blackbody furnaces are placed in position to act as sources for the respective beams of the spectrometer, and are brought to the same temperature. The "Full Scale" control of the spectrometer is adjusted to bring the "100\% Line" to a position between 90 and 100 on the chart. The specimen beam is blocked, and the "Ratio Zero" control on the spectrometer is adjusted to bring the "Zero Line" to a position between 1 and 3 on the chart. The specimen beam is unblocked, and the position of the "100\% Line" is checked. These instrument settings then remain fixed throughout the emittance determinations on the specimen being tested.

(2) The $100 \%$ Line", over the wavelength range of interest, is obtained by automatic recording on the strip chart, after which the chart paper is rerolled.

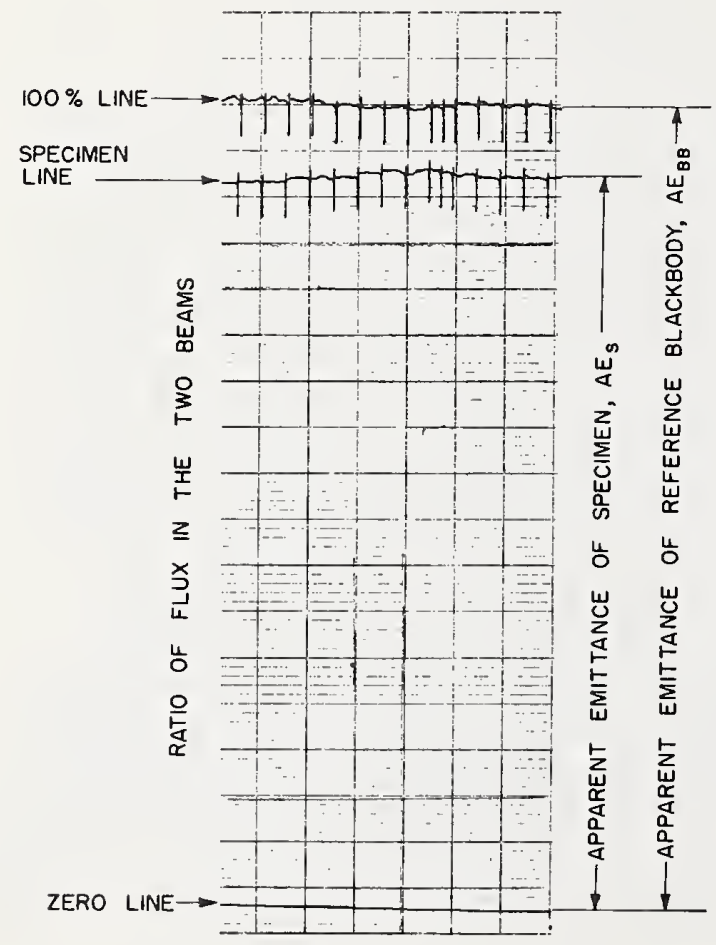

Fig. 8 A section of recorder chart, showing segments of a "100\% Line", "Specimen Line" and "Zero Line." The normal spectral emittance at each wavelength is the ratio $\mathrm{AE}_{\mathrm{s}} / \mathrm{AE}_{\mathrm{BB}}$. 
(3) The specimen beam is blocked near the source, and the "Zero Line" is similarly obtained over the wavelength range of interest, after which the chart paper is again rerolled.

(4) The specimen enclosure is next substituted for the reference blackbody furnace, in position to act as source for the specimen beam of the spectrometer. The temperature of the specimen is brought to and held at the temperature of the comparison blackbody furnace. The specimen beam is unblocked, and the "Specimen Line" is obtained over the wavelength range of interest. A section of the completed recorder chart is shown in fig. 8 .

The heights of the respective curves are measured at preselected positions of the wavelength drum (corresponding to known wavelengths), and the normal spectral emittance is computed for each such wavelength. If $Z_{\lambda}$ is the height of the "Zero Line", $\mathrm{S}_{\lambda}$ the height of the "Specimen Line", and $\mathrm{H}_{\lambda}$ the height of the "100\% Line", at some wavelength $\lambda$, the normal spectral emittance, $E(\lambda)$, is given by

$$
E(\lambda)=\frac{s_{\lambda}-Z_{\lambda}}{H_{\lambda}-Z_{\lambda}}
$$

Values of $E(\lambda)$ are computed for each of 100 wavelengths in the range of 1 to 15 micfons, and $E(\lambda)$ is plotted as a function of wavelength. A variable scale $\mathrm{e}^{1}$ has been found useful for making this computation. Zero on the scale is set at the zero line, 100 on the scale on the $100 \% 1$ ine, and $E(\lambda)$ is read on the scale at the position of the specimen line. A curve is drawn through the plotted points to represent the spectral emittance curve of the specimen. 
Total hemispherical emittance was evaluated by a modification of the hot-filament method. A strip 8 in. long by $1 \%$ in. wide, of any convenient thickness, was heated in vacuum by passing a current through it, under conditions such that essentially all of the heat generated at the mid-length was dissipated by radiation. When thermal equilibrium had been attained, the temperature of and heat input to the specimen (a small known length of the strip at its mid-length) were measured. The temperature of the water-cooled shell surrounding the specimen was also measured. The total hemispherical emittance of the specimen was computed from the measured quantities and the known dimensions of the specimen, by means of an equation derived from the Stefan-Boltzmann relationship. Reasonably good agreement was obtained in cooperative tests made at the General Electric Co. and at the National Bureau of Standards on identical specimens. It was found that the total hemispherical emittance of sevéral chromium-bearing alloys was unstable on heating in vacuum at pressures on the order of $2 \times 10^{-5} \mathrm{~mm}$. of mercury.

\title{
Equipment and Procedures
}

\section{for Evaluation of Total Hemispherical Emittance}

\author{
JOSEPH C. RICHMOND and WILLIAM N. HARRISON \\ National Bureau of Standards, Washington, D. C.
}

The total hemispherical emittance of an object is a measure of its ability to dissipate heat by radiation. It is the amount of radiant energy emitted by a hot body, per unit area and unit time, expressed as a fraction of that emitted by a "blackbody" or complete radiator at the same temperature.

Total hemispherical emittance data are required for accurate computation of heat dissipation by radiation. Significant amounts of heat are dissipated by radiation from objects at temperatures above ambient, and at temperatures above a red heat, radiation becomes the dominant mode of heat transfer. The two other modes of heat transfer, convection and conduction, become less effective as atmospheric pressure decreases with increasing altitude, and become negligibly small at altitudes on the order of 100 kilometers. Hence total hemispherical emittance controls the heat dissipation rate of a space vehicle at any given temperature.

\section{General Description of the Method}

The method herein described for evaluating total hemispherical emittance is a variation of the hot-filament method described by Worthing. ${ }^{1}$ It consists essentially of heating a strip by passing a current through it, under conditions such that all of the heat generated at the mid-length of the strip is dissipated by radiation. If the surface area and temperature of a small segment of the strip at mid-length are known, the total hemispherical emittance can be com-

Presented at the Sixty-First Annual Meeting of The American Ceramic Society in Chicago, Ill., May 18, 1959 (Enamel Division, No. 2-E-59).

1 A. G. Worthing, "Temperature Radiation Emissivities and Emittances," pp. 1164-87 in Temperature, Its Measurement and Control in Science and Industry. American Institute of Physics, Reinhold Publishing Corp., New York, 1941. puted from the power input by use of the Stefan-Boltzmann equation.

In laboratory experiments it is impossible to eliminate completely all heat input to the specimen other than by electrical heating, and to limit the heat output of the specimen solely to radiation. The procedure used in these experiments was as follows: A long, narrow strip was heated in vacuum, in a chamber with black walls maintained at a known temperature. Potential taps were attached by welding, a known small distance apart near the center of the strip, and a fine-wire thermocouple was attached by welding, midway between the potential taps. Under these conditions the total heat input to the specimen (the portion of the strip between potential taps) consisted of (1) that generated by resistance of the specimen to passage of the electric current, designated $W$, and (2) the radiant energy incident upon the specimen that was absorbed, designated $B$. The total heat output was (1) the radiant energy, $C$, emitted by the hot specimen, (2) the.heat lost by conduction and convection through the atmosphere, which was negligibly small at the low pressure used in these experiments, (3) the heat, $G$, lost by conduction through the ends of the specimen, and (4) the heat, $H$, lost by conduction through the thermocouple and potential tap leads.

At equilibrium the heat input equaled the heat output, hence the following equation can be written:

$$
W+B=C+G+H
$$

If the specimen was heated by direct current, $W$ in watts was equal to $I$, the current flowing through the specimen, times $V$, the potential drop across the specimen. This was also true when the specimen was heated by alternating current, since the power factor was 1.00 and the voltage was a true root-mean-square voltage.

$$
W=V \cdot I
$$


The radiant energy incident upon the specimen that is absorbed, $B$, was computed from the absolute temperature of the shell, $T_{2}$, its emittance $E_{2}$, the absorptance, $\alpha$, of the specimen for the radiant energy emitted by the shell, the area of the specimen, $A$, and the Stefan-Boltzmann constant, $\sigma$, by the following equation

$$
B=A E_{2} \alpha \sigma T_{2}
$$

A term could also be added for radiant energy emitted by the specimen that was reflected by the shell back to the surface of the specimen and then absorbed, but this term was negligibly small.

Any complete enclosure with its walls at a uniform temperature is a blackbody radiator. The shell very closely approximated this condition. The hot specimen inside the shell did not appreciably alter the blackbody absorbing characteristics of the shell because (1) the area of the specimen was very small as compared to the area of the shell, and (2) the shell was sandblasted and painted with a mat black paint, hence the small fraction of the radiant energy emitted by the specimen that was not absorbed on striking the shell for the first time was diffusely reflected, and only a negligibly small fraction of it was reflected back to the specimen. Hence $E_{2}=1.00$ with but very slight error. Thus equation (3) may be rewritten as follows:

$$
B=A \alpha \sigma T_{2}^{4}
$$

The radiant energy, $C$, emitted by the hot specimen was computed by means of the Stefan-Boltzmann equation from its emittance, $E_{1}$, and temperature, $T_{1}$ as follows:

$$
C=A E_{1} \sigma T_{1}^{*}
$$

The heat loss, $G$, by conduction through the ends of the specimen could be computed from $k$, the thermal conductivity of the metal, $\Delta T / \Delta X$, the thermal gradient along the specimen, and the cross-sectional area, $a$, by the equation:

$$
G=2 k a \Delta T / \Delta X
$$

In these experiments the thermal conductivity of the metal was on the order of 0.4 watt $\mathrm{cm} .{ }^{-1}{ }^{\circ} \mathrm{C} .{ }^{-1}$, the cross sectional area was about $0.048 \mathrm{~cm}^{2}$, and the measured thermal gradients were on the order of $1^{\circ}$ to $2^{\circ} \mathrm{C}$. $/ \mathrm{cm}$. Substituting these values in equation (6)

$$
G=2 \cdot 0.4 \cdot 0.048 \cdot 2=0.0768 \text { watt }
$$

The power input to the specimen was about 40 watts at $800^{\circ}$ to $1000^{\circ} \mathrm{C}$, hence this heat loss was negligibly small at these temperatures, but it could not be ignored at temperatures of about $100^{\circ} \mathrm{C}$., where the power input is on the order of 0.4 watt.

Equation (6) was also used to compute $H$, the heat loss through the thermocouple and potential leads. There was a total of 4 leads, two from the thermocouple at the center and one from each potential tap. The cross-sectional area of each wire was about $3 \times 10^{-6} \mathrm{~cm} .{ }^{2}$, and the thermal conductivity was again about 0.4 watt $\mathrm{cm}^{-1}{ }^{\circ} \mathrm{C} .{ }^{-1}$, but the thermal gradient was not known with any degree of certainty. A value of $2000^{\circ} \mathrm{C}$. $/ \mathrm{cm}$. was assumed for the gradient, a value that is considered high. The heat loss, $H$, was computed to be

$$
H=4 \cdot 0.4 \cdot 3 \cdot 10^{-6} \cdot 2000=9.6 \times 10^{-3} \text { watt }
$$

which is negligibly small.

Equations (2), (4), and (5) were substituted in equation (1), and $G$ and $H$ were set equal to zero, to obtain

$$
V \cdot I+A \alpha \sigma T_{2}^{4}=A E_{1} \sigma T_{1}^{4}
$$

When $T_{2}=T_{1}, \alpha=E_{1} ; E_{1}$ changes only slightly with temperature, and when $T_{1} \gg T_{2}$, the entire term containing $T_{2}$ becomes negligibly small. Hence only a small error was introduced by substituting $E_{1}$ for $\alpha$ in equation 7 . The equation was then solved for $E_{1}$ to obtain

$$
E_{1}=V \cdot I / A \sigma\left(T_{1}^{4}-T_{2}^{4}\right)
$$

\section{Description of Equipment}

A photograph of the equipment used in this investigation is shown in Fig. 1. It consisted essentially of (1) an oil diffusion pump, associated vacuum gauges, and control equip-

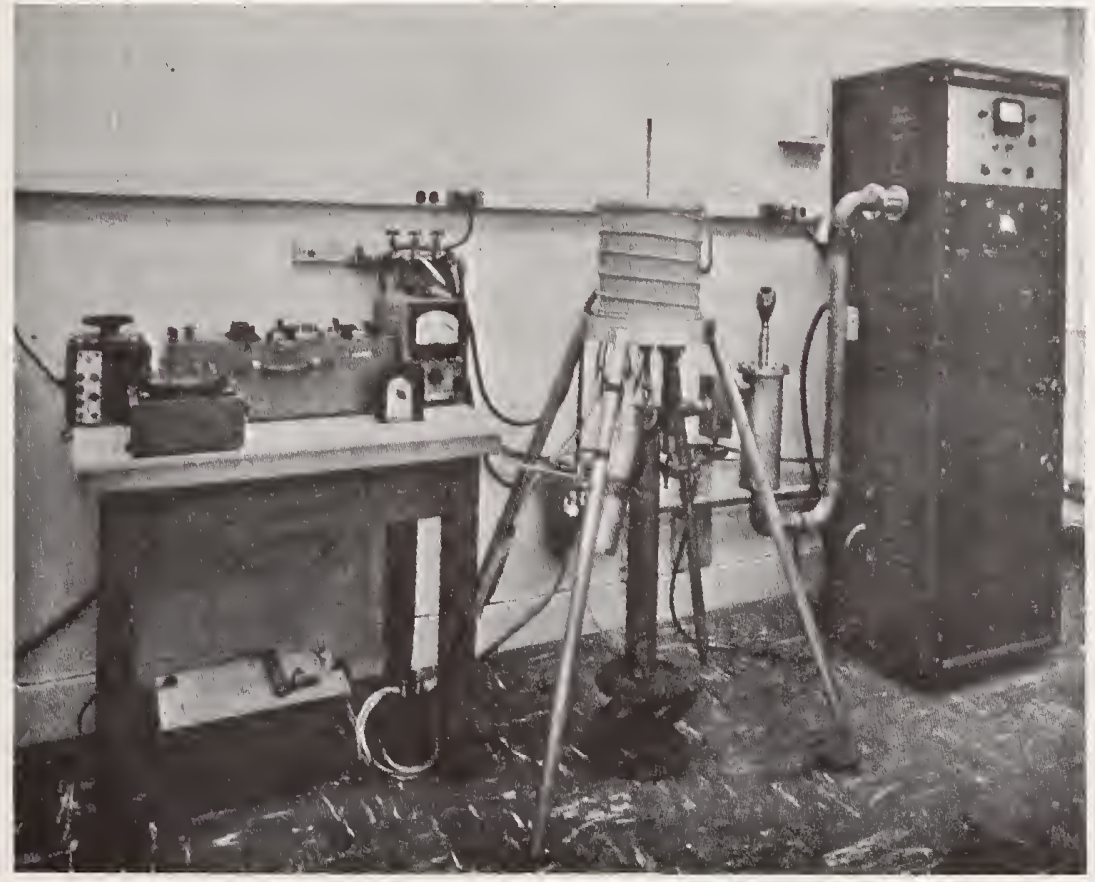

$135-669$
Fig. 1. Equipment for measuring tatal hemispherical emittance. Vacuum pump and associated equipment is at the right, vacuum chamber is in the center, and the electrical meters and transfarmers are at the left. 
Fig. 2. Sectional drawing of the vocuum chomber, with a strip in ploce. $A$ and $A^{\prime}$ are potential tops; $T_{1}, T_{2}$ and $T_{3}$ are welded thermocouples. ment, mounted in the panel at the right, (2) a vacuum chamber in which the strip is mounted, in the center, and (3) various transformers and meters, on the left, used to control and measure the power input to and temperature of the specimen.

Figure 2 is a sectional drawing of the vacuum chamber, with a strip in place. The strip was clamped in two heavy copper water-cooled electrodes. The top electrode was fixed in position, and supported the strip and bottom electrode. The bottom electrode was mounted in a sylphon bellows to permit vertical movement as the strip expanded and contracted during heating and cooling, which prevented the buckling that might have occurred if both ends of the strip were clamped in fixed positions. In order to counteract the effect of air pressure on the sylphon bellows tending to push up the lower electrode when the chamber was evacuated, a 72-pound lead weight was suspended from the lower electrode. The net pull on the specimen when the chamber was evacuated was approximately $1 / 2$ pound, which was sufficient to keep it straight without causing permanent deformation. A scissors-type jack was used to support the weight when the chamber was not evacuated.

Rubber O-ring seals provided ready access to removable parts of the vacuum chamber, and also electrically insulated the upper electrode from the base plate. The low-voltage electrical leads for the thermocouples and potential taps were conducted through the base of the chamber by means of vacuum-tight glass-to-metal seals.

The interior of the vacuum chamber was sandblasted and painted with a mat black paint to give a surface of high absorptance. The walls of the chamber and the electrodes were water cooled to keep them at a uniform low temperature.

Thermocouple leads went directly to cold junctions immersed in oil in a vacuum bottle. Insulated copper leads connected the cold junctions, through a commutator switch, to a precision portable potentiometer. The potential tap leads were connected by a shielded cable to the vacuum-tube voltmeter.

Power was supplied to the strip by means of a low-potential, high-current transformer, the input to which was varied manually by means of an autotransformer. Fluctuations in line voltage were compensated for by means of an electronic voltage regulator. . The water-cooled power leads from the transformer to the strip were connected through a current transformer, which was in turn connected to a precision a.c. ammeter.

The choice of equipment for generating and measuring the power input to the specimen was limited by the requirement that equation (2) should be valid for the measured current and potential. The vacuum-tube voltmeter was selected because it had a very high input impedance, 10
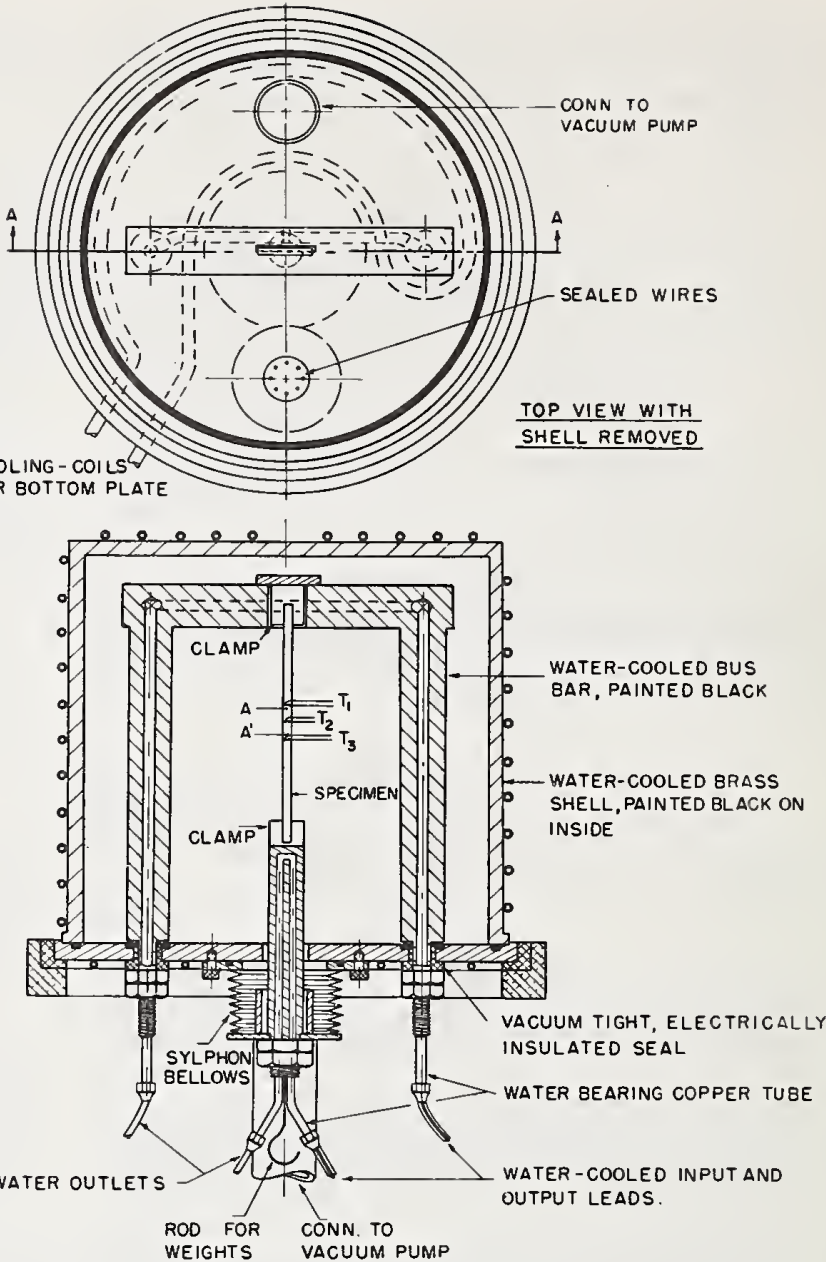

SECTION A-A

megohms, so that the current drain was negligibly small, and it covered the range 0.001 to 300 volts with an accuracy of $\pm 1 \%$. It had the disadvantage, however, that it was somewhat sensitive to the wave form of the applied voltage. The potential indication was proportional to the average absolute value of the applied wave, but the scale was calibrated to read root-mean-square potential of a sine wave. Hence it was necessary to use power transformers that gave an undistorted sine-wave output. The wave form of the power circuit was checked, and was found to be essentially free from distortion.

\section{Test Procedure}

Strips were formed by shearing sheet metal of any convenient thickness in the range 0.01 to $0.2 \mathrm{~cm}$. into strips approximately $20 \mathrm{~cm}$. long by $0.6 \overline{\mathrm{s}} \mathrm{cm}$. wide, after which the edges were machined until the sides were parallel to within $0.001 \mathrm{~cm}$. over the center $10 \mathrm{~cm}$. of each strip.

Three thermocouples and two potential taps were welded to each strip by means of an electronic condenser-discharge type precision spot welder. The thermocouples were spaced $1.88 \mathrm{~cm}$. apart, one at the center and one on either side of the center of the strip. The potential taps were spaced 2.5 


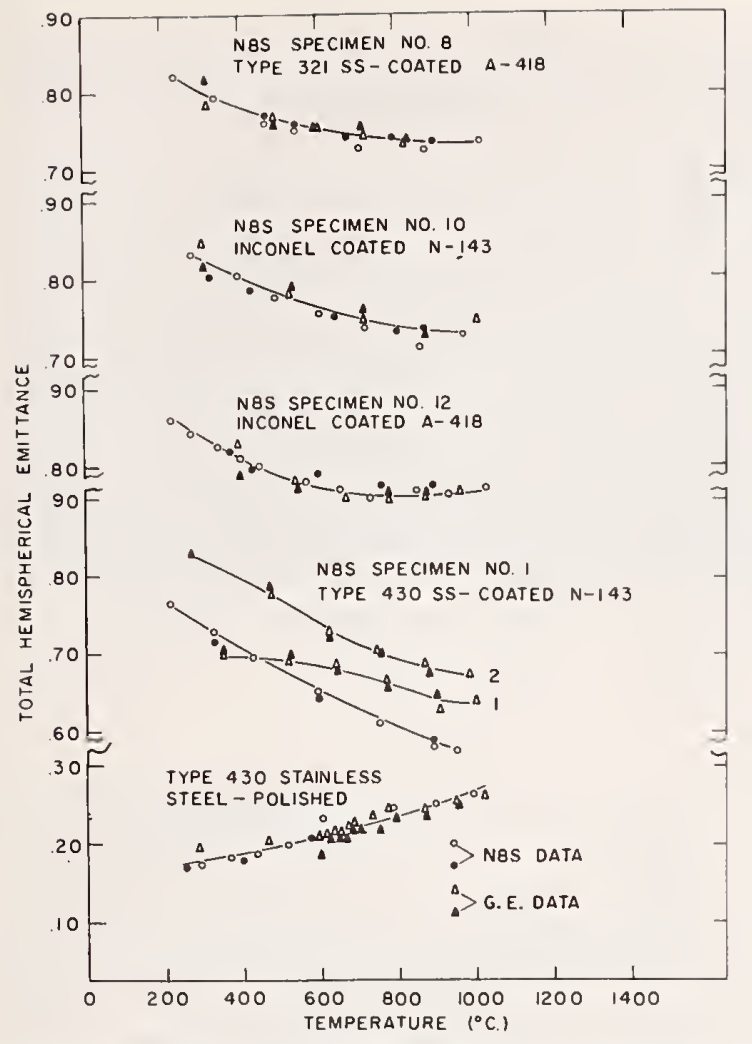

Fig. 3. Results obtained of the Notionol Bureou of Standards and at the Receiving Tube Deparment of Generol Electric Co. on strips supplied by the National Bureau of Standards. Curves 1 and 2 for Specimen No. 1 were determined at different times by General Electric.

cm. apart, $1.25 \mathrm{~cm}$. on either side of the center thermocouple. The thermocouples were formed by separately welding 0.005 -in. Chromel and Alumel wires to the strip. Care was taken to see that the two welds forming each thermocouple were on a line perpendicular to an edge of the strip in order to eliminate 60 -cycle pickup in the thermocouple circuit from the current used to heat the strip.

Before making a test the width and thickness of each strip was measured to the nearest $0.0025 \mathrm{~cm}$., and the distance between potential taps was measured to the nearest 0.01 $\mathrm{cm}$.

In making a test a strip was first mounted in the electrodes in the test chamber, and the chamber was evacuated, care being taken to prevent buckling or stretching of the specimen during the operation.

When a pressure of less than $5 \times 10^{-5} \mathrm{~mm}$. of mercury was attained, the strip was heated to about $100^{\circ} \mathrm{C}$. This usually caused outgassing of the strip, and further pumping was required. When temperature equilibrium had been attained, and when the pressure had dropped to below $4 \times 10^{-5}$ $\mathrm{mm}$. of mercury, the temperature of the specimen and shell, the current, the potential, and the pressure were recorded.

The temperature was increased by increments of approximately $100^{\circ} \mathrm{C}$., and determinations were made at successively higher temperatures until the maximum desired temperature (usually $1000^{\circ} \mathrm{C}$.) had been attained. The temperature was then decreased by decrements of approximately $200^{\circ} \mathrm{C}$., and determinations were made at two or three additional temperatures.

The total hemispherical emittance was computed from equation (8), and plotted as a function of temperature for

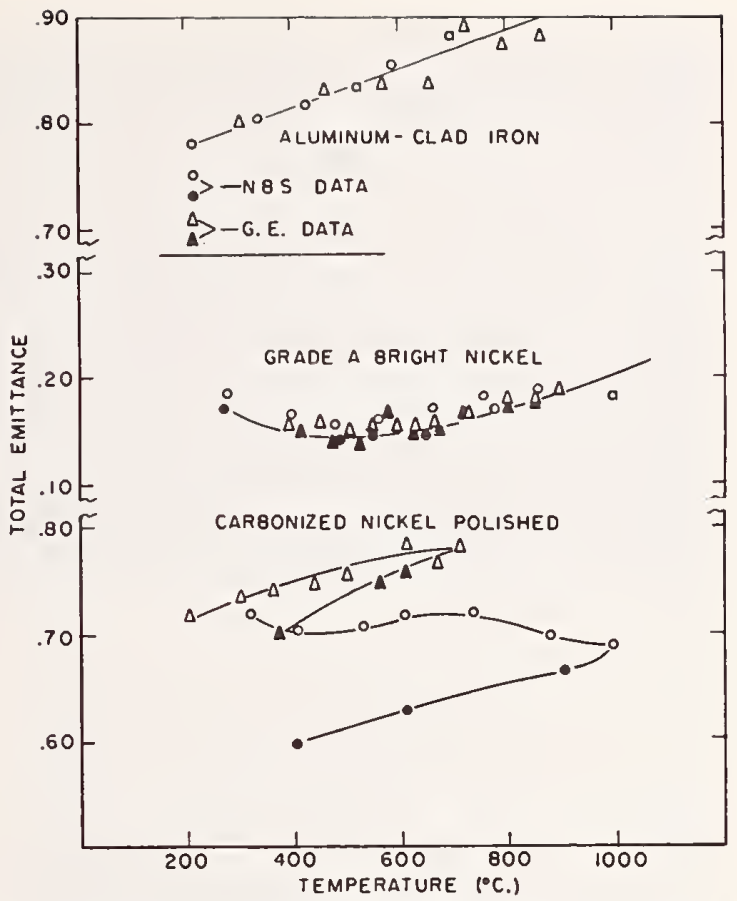

Fig. 4. Results obtained of the Nationol Bureau of Stondards and of the Receiving Tube Department of General Electric Company on sheet metol supplied by General Electric Co.

each specimen. Values at temperatures below $200^{\circ} \mathrm{C}$. were not reported. The value of $\sigma$ was taken as $5.6687 \times 10^{-12}$ watt $\mathrm{cm} .{ }^{-2}{ }^{\circ} \mathrm{K}^{-4}$.

\section{Cooperative Tests}

The Receiving Tube Department of the General Electric Co. at Owensboro, $\mathrm{Ky}$., was also using a modification of the hot filament method to evaluate total hemispherical emittance of sheet metal materials. The principal differences were (1) their vacuum chamber was a glass bell jar, cooled by a fan, (2) their strip was $3 / 4$ by 8 in. in size, and (3) no provision was made to reduce buckling of heated strips.

Arrangements were made to exchange specimens and to compare results obtained in the two laboratories.

Emittance determinations were made in both laboratories on four strips of ceramic-coated alloys and one strip of polished type 430 stainless steel supplied by the National Bureau of Standards. Excellent checks were obtained on four of the five strips, as shown in Fig 3. A less satisfactory check was obtained on the strip of type 430 stainless steel coated with N-143. This discrepancy was later found to be due to a defective thermocouple.

In addition, emittance determinations were made in each laboratory on different strips prepared from aluminumclad iron,* grade A bright nickel, and polished carbonized nickel sheet supplied by the General Electric Co Excellent checks were obtained on two of these three materials, as shown in Fig. 4. The emittance of the polished carbonized nickel was quite unstable on heating in vacuum, as indicated

* The aluminum-clad iron had been coated with aluminum by a process such as flame-spraying, which produced a dark, rough, sandpaper-like coating of partially oxidized aluminum on the iron. These specimens should not be confused with bright aluminized iron. 
by the lower values of emittance obtained during cooling as compared to those obtained during heating. This instability undoubtedly accounts for the discrepancy in results obtained by the two laboratories.

A complete description of the work at the General Electric Co. is contained in one of their reports. ${ }^{2}$

\section{Changes in Emittance on Heating in Vacuum}

The total hemispherical emittance of uncoated and unoxidized specimens of Inconel and types 321 and 430 stainless steel was found to change during heating in vacuum. Results obtained with a specimen of sandblasted Inconel are shown in Fig. 5. The total hemipherical emittance was determined at approximately $200^{\circ}, 300^{\circ}, 400^{\circ}$, and $500^{\circ} \mathrm{C}$, , and again at $200^{\circ} \mathrm{C}$. About 10 minutes was required for each of the determinations. The data show that as a result of this treatment the emittance at $200^{\circ} \mathrm{C}$. dropped from 0.525 to 0.49 . This reduction in emittance is believed to indicate outgassing of the specimen, or removal of gases adsorbed on the surface of the metal.

The specimen was next heated to about $575^{\circ} \mathrm{C}$. and held at constant power input for a period of $11 / 2$ hours, during which frequent emittance determinations were made. During this period the temperature dropped from $575^{\circ}$ to $540^{\circ} \mathrm{C}$., and the emittance increased from 0.535 to 0.615 . On cooling to $200^{\circ} \mathrm{C}$. the emittance at that temperature was found to be 0.545 .

The specimen was then heated to about $620^{\circ} \mathrm{C}$. and held at constant power input for one hour, during which frequent emittance determinations were made. During this period the temperature dropped from $620^{\circ}$ to $598^{\circ} \mathrm{C}$., and the emittance increased from 0.655 to 0.718 . On cooling to $200^{\circ} \mathrm{C}$., the emittance at that temperature was 0.565 .

It is suggested that the increase in emittance on holding at temperatures in the range $500^{\circ}$ to $625^{\circ} \mathrm{C}$. indicates the formation of a thin film of oxide on the surface of the specimen. Even though the specimen was heated at a pressure of less than $5 \times 10^{-5} \mathrm{~mm}$. of mercury, there was enough oxygen present to cause appreciably darkening of the specimen.

The specimen was next heated to about $795^{\circ} \mathrm{C}$. and held at constant power input for $2 \frac{1}{2}$ hours, during which frequent emittance determinations were made. During this period the temperature increased from $795^{\circ}$ to $850^{\circ} \mathrm{C}$., and the emittance decreased from 0.735 to 0.565 . On cooling to $200^{\circ} \mathrm{C}$., the emittance at that temperature was found to be 0.465 .

The decrease in emittance on heating in vacuum at temperatures above $800^{\circ} \mathrm{C}$. is believed to be caused by two different mechanisms. Apparently the oxide formed at lower temperatures dissociated or evaporated on heating in vacuum at temperatures above $800^{\circ} \mathrm{C}$., because specimens that had been held at temperatures above $800^{\circ}$ had a bright oxidefree appearance when removed from the apparatus. Figure 6 shows a light-colored strip that had been tested for total hemispherical emittance at a maximum temperature above $1000^{\circ} \mathrm{C}$. The center portion of the strip had a bright, oxidefree metallic appearance, while a narrow band of oxide could be seen at each end where the strip was at a lower temperature. The dark strip in Fig. 6 was tested for total hemispherical emittance at temperatures up to a maximum of $809^{\circ} \mathrm{C}$. for short periods of time, and was appreciably darkened due to formation of a thin film of oxide.

The data in Fig. 5 and the strips in Fig. 6 indicate that the oxide film does not form at temperatures below about

2 M. W. Russell, "An Accurate Method of Measuring Total Emittance," General Electric Receiving Tube Dept., Tech. Information Ser., No. R 59 E T R J (January 23,1959 ).

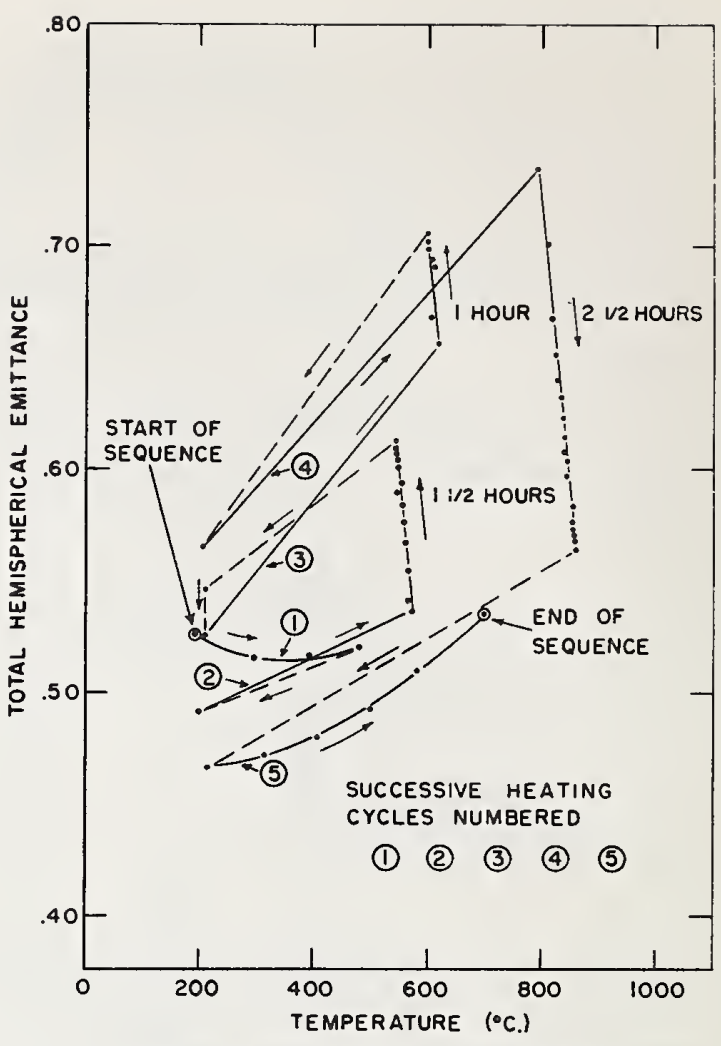

Fig. 5. Total hemispherical emittance of a sandblasted Incanel specimen heated in vacuum, showing changes that occurred during heating.

$500^{\circ} \mathrm{C}$., but that it does form at appreciable rates in the temperature range of about $540^{\circ}$ to $620^{\circ} \mathrm{C}$, and that it dissociates or evaporates on long-time heating above about $790^{\circ} \mathrm{C}$.

The decomposition or evaporation of an oxide film formed at lower temperatures is the first mechanism believed responsible for the observed decrease in emittance at temperatures above $790^{\circ} \mathrm{C}$.

In addition, it was noted that sandblasted strips heated to $1000^{\circ} \mathrm{C}$. had undergone a polishing treatment similar to electropolishing, and a metallic film was deposited on the inside of the vacuum chamber. Spectrochemical analysis showed this film to consist primarily of chromium. Fluorescent X-ray analyses of the strip showed depletion of chromium in the surface layer. It is believed that the observed polishing action is due to volatilization of chromium from the strip. This polishing action is the second mechanism believed responsible for the observed decrease in emittance.

\section{Summary}

An apparatus and procedure have been described for evaluating total hemispherical emittance of coated or uncoated sheet metal specimens. The results appear to be valid at temperatures from about $200^{\circ} \mathrm{C}$. to the maximum that the specimen will withstand.

The method consists essentially of heating a strip in vacuum until equilibrium is attained, then measuring the temperature of and power input to the specimen (a short known length of the strip near its center). It is assumed that all of the heat generated in the specimen is dissipated 


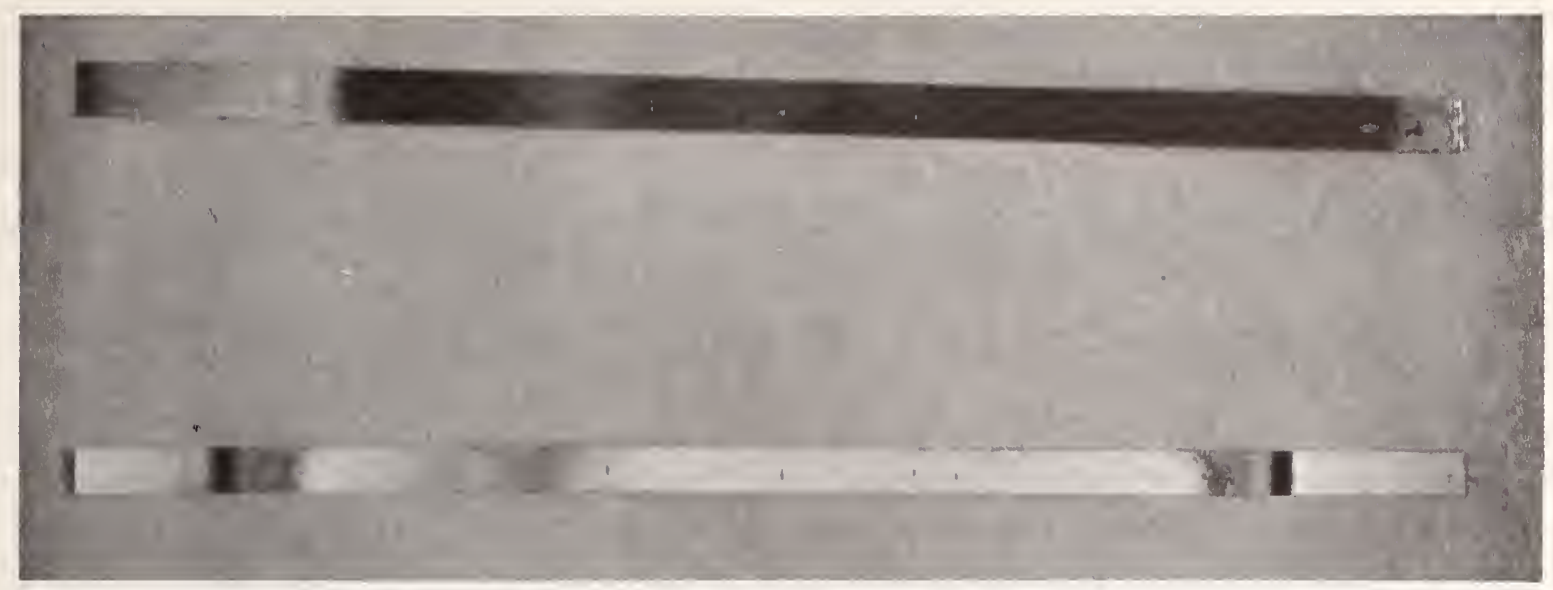

Fig. 6. Strips of Type 430 stoinless steel. The dark strip was tested of a maximum temperoture of $809^{\circ} \mathrm{C}$., ond the bright strip was tested at o moximum temperoture of $1000^{\circ} \mathrm{C}$. The oxide layer that was formed at temperatures in the range of $600-800^{\circ} \mathrm{C}$. opparentiy decomposed or volatilized on heating to $1000^{\circ} \mathrm{C}$.

by radiation, and the total hemispherical emittance is computed by means of an equation derived from the StefanBoltzmann equation.

Reasonably good agreement was obtained in cooperative tests made at the General Electric Co., Receiving Tube Department and at the National Bureau of Standards on identical specimens.

It was found that the total hemispherical emittance of chromium-bearing alloys such as Inconel and types 321 and 430 stainless steel is not stable on heating in vacuum. The changes in emittance of sandblasted Inconel that were observed on heating in vacuum were ascribed to (1) removal of adsorbed films, at temperatures up to about $500^{\circ} \mathrm{C}$.; (2) formation of a thin film of oxide at temperatures in the range of $500^{\circ}$ to $625^{\circ} \mathrm{C}$, even in an air atmosphere at a pressure of less than $4 \times 10^{-5} \mathrm{~mm}$. of mercury, (3) dissociation of previously-formed oxide film at temperatures above about $790^{\circ} \mathrm{C}$., and (4) volatilization of chromium metal, accompanied by a smoothing of the surface at temperatures in the vicinity of $1000^{\circ} \mathrm{C}$.

\title{
ACKNOWLEDGEMENT
}

\author{
This work was done under the sponsorship \\ and with the financial assistance of the \\ Army Ballistic Missile Agency, Redstone \\ Arsenal, Huntsville, Alabama.
}




\title{
Method of Measuring Emissivities of Metals in the Infrared*
}

\author{
Arthur G. Maki and Earle K. Plyler
}

(April 20, 1962)

\begin{abstract}
A method of measuring normal spectral emissivities in the infrared region from 1 to $13 \mu$ is described. It consists of comparing the rate of emission of radiant energy from a blackbody with that from the specimen. The two observed radiances are made equal by adjusting the temperatures. An equation is derived for use in calculating the emissivity for the observed temperatures. The main sources of error arise in the measurement of the temperature of the specimen and the temperature of the blackbody. As an example of the method, the normal spectral emissivity of gold has been measured in the range from 4 to $13 \mu$ at temperatures from 550 to $1,000^{\circ} \mathrm{K}$. The emissivity was found to range from 0.014 at $4 \mu$ and $550^{\circ} \mathrm{K}$ to 0.0256 at $9 \mu$ and $1,000^{\circ} \mathrm{K}$. A table is included which lists the values of emissivity from 4 to $13 \mu$ and from 550 to $1,000{ }^{\circ} \mathrm{K}$ at intervals of $50^{\circ} \mathrm{K}$.
\end{abstract}

\section{Introduction}

The normal spectral emissivity of many metals such as gold, platinum and aluminum is very low in the infrared region from 4 to $13 \mu$. Some measurements have been carried out at high temperatures in the visible spectrum $[1-4],{ }^{1}$ but measurements in the infrared spectrum to $13 \mu$ have not been undertaken. The emissivity of metals in the infrared may be calculated from the reflectivity, $R$, using the Kirchhoff relationship

$$
\epsilon=1-\mathrm{R} \text {. }
$$

For this calculation reflectance measurements made at room temperature are available from several sources, but in most cases the reflectance measurements are only good to about \pm 1 percent thus giving rise to uncertainties of \pm 5 to \pm 50 percent for the calculated emissivities of most metals. A noteworthy exception to this is the recent very accurate reflectance measurements made by Bennett and Koehler [5] on aluminum. In addition Harris and Fowler [6] and Lameris [7] have made some very good ineasurements on gold.

While this paper is primarily concerned with the development of a method for measuring the spectral emissivity of metals, some results are reported on the normal spectral emissivity of gold in the infrared spectrum from 4 to $13 \mu$. A method of measuring the spectral emissivity of metals will be described in the next section.

-This work was supported through a contract with Redstone Arsenal.

2 Figures in brackets indicate the literature references at the end of this paper.

\section{Experimental Procedure}

The experimental arrangement consisted of an infrared spectroradiometer with a sodium chloride prism and thermocouple for measuring the rate of emission of energy in the region from 1 to $13 \mu$. A double pass optical system was employed so that the stray radiation would be greatly reduced. The spectroradiometer was calibrated for wavelength settings with the absorption bands of polystryene, $\mathrm{CO}_{2}$ and $\mathrm{H}_{2} \mathrm{O}$. Rather large spectral slit widths were used in this work varying from $0.18 \mu$ at $2 \mu$ to $0.1 \mu$ at $10 \mu$. However, since neither the blackbody curve nor the emitted-energy curve of the specimen has a very large second derivative with respect to wavelength, the emissivity values which were determined were nearly independent of the slit width used. A blackbody and the specimen to be measured were placed on a platform which could be moved so that the specimen or the blackbody radiated on the entrance slit of the spectrometer. This general type of arrangement has been used in previous work and a more detailed description of the procedure can be found elsewhere [8].

An essentially different method of comparing the radiance from the blackbody and the specimen has been devised. This method consists of adjusting the radiance of both sources (at the same wavelength) to be nearly equal. The effect of nonlinearity of the detector amplifier response is minimized by this procedure. Since the measurements were to be made on metals of low emittance, this required that the blackbody be at a lower temperature than the specimen. In order to reduce the error of ambient change to a minimum, alternate readings were made on the blackbody and specimen during a small time interval. 
'The emissivity calculations were based upon the following equations. If we define

$N_{b}\left(\lambda_{b}\right)=$ rate of emission of radiant energy by a blackbody radiator at temperature $t_{b}$ within the wavelength interval $\lambda \pm d \lambda$ per unit of time, area, and solid angle,

$N_{s}\left(\lambda t_{s}\right)=$ rate of emission of radiant encrgy by the specimen at temperature $t_{s}$ within the wavelength interval $\lambda \pm d \lambda$ per unit of time, area, and solid angle,

$\epsilon_{s}\left(\lambda t_{s}\right)=$ emissivity of the specimen at temperature $t_{s}$ for the warelength interval $\lambda \pm d \lambda$,

$t_{r}=$ rooin temperature,

$t_{s}=$ specimen temperature,

$t_{b}=$ blackbody temperature,

$t_{m}=$ monochromator and detector temperature,

$R_{b}\left(\lambda t_{b}\right)=$ reading of instrument set to view blackbody at temperature $t_{b}$,

$R_{s}\left(\lambda t_{s}\right)=$ reading of instrument set to view specimen at temperature $t_{s}$;

then,

$R_{b}\left(\lambda, t_{b}\right)=k_{1}\left[N_{b}\left(\lambda, t_{b}\right)-N_{b}\left(\lambda, t_{m}\right)\right]$

and

$$
\begin{aligned}
R_{s}\left(\lambda, t_{s}\right)=k_{2}\left\{\left[N_{s}\left(\lambda, t_{s}\right)\right.\right. & \left.-N_{b}\left(\lambda, t_{m}\right)\right] \\
+ & {\left.\left[1-\epsilon_{s}\left(\lambda, t_{s}\right)\right] N_{b}\left(\lambda, t_{r}\right)\right\} . }
\end{aligned}
$$

Here $k_{1}$ and $k_{2}$ are proportionality constants and $k_{1}=k_{2}$ in this method.

Also,

$$
\begin{array}{r}
N_{s}\left(\lambda, t_{s}\right)+\left[1-\epsilon_{s}\left(\lambda, t_{s}\right)\right] N_{b}\left(\lambda, t_{r}\right) \\
=\frac{N_{s}\left(\lambda, t_{s}\right)-N_{b}\left(\lambda, t_{m}\right)+\left[1-\epsilon_{s}\left(\lambda, t_{s}\right)\right] N_{b}\left(\lambda, t_{r}\right)}{N_{b}\left(\lambda, t_{b}\right)-N_{b}\left(\lambda, t_{m}\right)} \\
{\left[N_{b}\left(\lambda, t_{b}\right)-N_{b}\left(\lambda, t_{m}\right)\right]+N_{b}\left(\lambda, t_{m}\right) .}
\end{array}
$$

By substitution,

$$
\begin{aligned}
N_{s}\left(\lambda, t_{s}\right) & =\frac{R_{s}\left(\lambda, t_{s}\right)}{R_{b}\left(\lambda, t_{b}\right)}\left[N_{b}\left(\lambda, t_{b}\right)-N_{b}\left(\lambda, t_{m}\right)\right]+N_{b}\left(\lambda, t_{m}\right) \\
& -\left[1-\epsilon_{s}\left(\lambda, t_{s}\right)\right] N_{b}\left(\lambda, t_{\tau}\right) .
\end{aligned}
$$

Let

Then

$$
\frac{R_{s}\left(\lambda, t_{s}\right)}{R_{b}\left(\lambda, t_{b}\right)}=1 \text {. }
$$

$$
N_{s}\left(\lambda, t_{s}\right)=N_{b}\left(\lambda, t_{b}\right)-\left[1-\epsilon_{s}\left(\lambda, t_{s}\right)\right] N_{b}\left(\lambda, t_{\tau}\right) .
$$

By definition,

From eq (6),

$$
\epsilon_{s}\left(\lambda, t_{s}\right)=\frac{N_{s}\left(\lambda, t_{s}\right)}{N_{b}\left(\lambda, t_{s}\right)}
$$

$$
\epsilon_{s}\left(\lambda, t_{s}\right)=\frac{N_{b}\left(\lambda, t_{b}\right)-\left[1-\epsilon_{s}\left(\lambda, t_{s}\right)\right] N_{b}\left(\lambda, t_{r}\right)}{N_{b}\left(\lambda, t_{s}\right)}
$$

or

$$
\begin{aligned}
\epsilon_{s}\left(\lambda, t_{s}\right) N_{b}\left(\lambda, t_{s}\right)= & N_{b}\left(\lambda, t_{b}\right) \\
& -N_{b}\left(\lambda, t_{r}\right)+\epsilon_{s}\left(\lambda, t_{s}\right) N_{b}\left(\lambda, t_{r}\right) .
\end{aligned}
$$

Combining terms we obtain,

$$
\epsilon_{s}\left(\lambda, t_{s}\right)=\frac{N_{b}\left(\lambda, t_{b}\right)-N_{b}\left(\lambda, t_{r}\right)}{N_{b}\left(\lambda, t_{s}\right)-N_{b}\left(\lambda, t_{r}\right)} .
$$

The values of $N_{b}(\lambda, t)$ in eq 10 can be evaluated from tables of blackbody radiation or calculated from the relation.

$$
N(\lambda, t)=\frac{C_{1}}{\lambda^{5}\left(e^{C_{2} / \lambda T}-1\right)},
$$

where

$$
C_{2}=1.4380 \mathrm{~cm}^{\circ} \mathrm{K} \text {. The constant } C_{1} \text { cancels out. }
$$

\section{Discussion of Possible Errors}

Since the emissivity of a metal is a quantity intrinsic to the substance one may consider sources of error as divided into two catcgories: (1) deviation of the sample from the ideal, and (2) experimental errors.

The first type of error may generally be attributed to impurities in the metal sample and/or nonideal surface effects peculiar to the specimen. It is believed that the purity of our specimen was not a source of error. The gold ribbon which has been measured by this method was part of a sample which had been tested by spectral analysis and found of high purity. The spccimen surface was mirror smooth. It has been shown [9] that small surface irregularities can cause rather large changes in the emittance characteristics. However, at the longer wavelengths of infrared radiation the emissivity is much less sensitive to small surface irregularities than at the shorter wavelengths of visible radiation. The smooth appearance of the surface when vicwed by visible light was taken as an indication that the surface is practically optically smooth in the infrared region and that the errors due to surface roughness are negligible.

Because of the inertness of gold no surface oxide coating is possible. To remove any other possible surface layers the specimen was washed with distilled water and acetone and then heated to $800^{\circ} \mathrm{K}$ for several hours before use. After this initial treatment the samples were maintained at temperatures above $100{ }^{\circ} \mathrm{C}$ until the measurements were completed. As a consequence it is felt that the state of the surface of the specimen was nearly ideal. There is of course some question as to the crystal structure of the optically important region of the metal near the surface, but the manner in which this changes the enittance of metals has not yet been established. Since the radiation observed was within $5 \mathrm{deg}$ of normal to the specimen, polarization effects are negligible and were disregarded.

Errors due to incorrect emissivity values assigned to the specimen are more directly related to the actual experimental measurements. In the emissivity measurements the errors may be divided into three categories: (a) errors in wavelength measurement, (b) errors in energy measurement, and (c) 
errors in temperature measurements. The magnitude of these errors will be discussed separately below.

Wavelength Errors. With modern instrumentation and standard techniques of calibration it is a relatively simple matter to calibrate infrared spectrometers to within $0.02 \mu$ over the entire range used in this work. Inasmuch as both the blackbody curve and the emissivity curve of the specimens are smooth curves with no sharp extremes or discontinuities, errors in wavelength should be entirely negligible in this work. The rather large spectrometer slit used at the shorter wavelengths, however, may introduce a nonnegligible error. The exact value of this error is difficult to determine and is in fact different for each wavelength and temperature. It is estimated to amount to no more than 1 percent.

Errors in Energy Measurements. The measurement of the energy given off by the specimen was accomplished by comparison with a blackbody as described in the section on experimental procedure. In making these measurements the temperatures were so arranged that the energy reaching the detector from the blackbody was nearly equal to that from the specimen. In this way instrumental errors due to amplifier or detector nonlinearity were practically eliminated. Almost all values reported are the average of several determinations with slightly different blackbody temperatures.

The important source of error in energy measurement is due to the signal to noise ratio. For measurements at higher temperatures and at wavelengths near the blackbody radiation maximum the signal to noise ratio is of course very favorable and uncertainties are quite negligible. For lower temperatures and especially for measurements near the long or short wavelength extremes of this work the signal to noise ratio becomes sufficiently small that rather large uncertainties are introduced into the results.

Because of the low energy levels involved in these measurements, especially at long wavelengths, stray radiation might be expected to cause appreciable errors. Use of a polyethylene-black filter to eliminate any short wave stray radiation proved, however, that stray radiation is quite negligible even at the longest wavelengths measured.

Errors in Temperature Measurement. In the determination of the emissivity by the method of equal radiance, the most important measurement is the temperature. Four temperatures must be measured or controlled; the temperature of the detector, the temperature of the room, the temperature of the specimen, and the temperature of the blackbody. The temperature of the room and of the detector can be measured to better than $1 \mathrm{deg} K$ and the errors introduced by these measurements are small compared to the uncertainties in measurement of the temperature of the specimen and the blackbody. The temperature of the specimen is usually measured by an attached thermocouple and when the material is a metal with the form of a thin ribbon, the thermocouple may be welded on the back surface of the specimen. The thermocouple should be made of fine wires but even then there is some conduction of heat from the specimen. When the specimen can be heated to high temperatures the temperature gradients can be evaluated with an optical pyrometer. At lower temperatures the radiation from different areas of the specimen can be focused on the slit of the spectrometer and temperature gradients of the specimen can be detected and evaluated. In the application of this method to measurements on gold it was found that the variation of temperature was never greater than $5 \mathrm{deg} K$ over the area used. The error introduced in the emissivity by a $5 \mathrm{deg}$ error in temperature for a specimen at $800^{\circ} \mathrm{K}$ has been calculated and the results are shown in figure 1 . It will be noticed that the error is between 1 and 2 percent from 6 to $14 \mu$, but at shorter wavelengths the error increases rapidly reaching a value of 6 percent at $2 \mu$. When the specimen is at temperatures above $800^{\circ} \mathrm{K}$, the uncertainty in the emissivity will be smaller than those shown in figure 1 and the error will be greater for temperatures less than $800^{\circ} \mathrm{K}$.

A large source of uncertainty is introduced by errors in the temperature of the blackbody. This temperature should be measured to $\pm 1^{\circ} \mathrm{K}$. When the gold specimen is at a temperature of $800{ }^{\circ} \mathrm{K}$, the blackbody is at $351^{\circ} \mathrm{K}$ for equal energies being recorded at $10 \mu$. In the above example a change of $1^{\circ} \mathrm{K}$ will produce an error of about 4 percent in the emissivity. With an uncertainty of $5{ }^{\circ} \mathrm{K}$ in the temperature measurement of the blackbody the errors become much greater as shown for different wavelengths in figure 2 . It should be noted that large errors will be made in the determination of emissivities by this method unless the temperature of the blackbody is measured with an accuracy of the order of $1^{\circ} \mathrm{K}$.

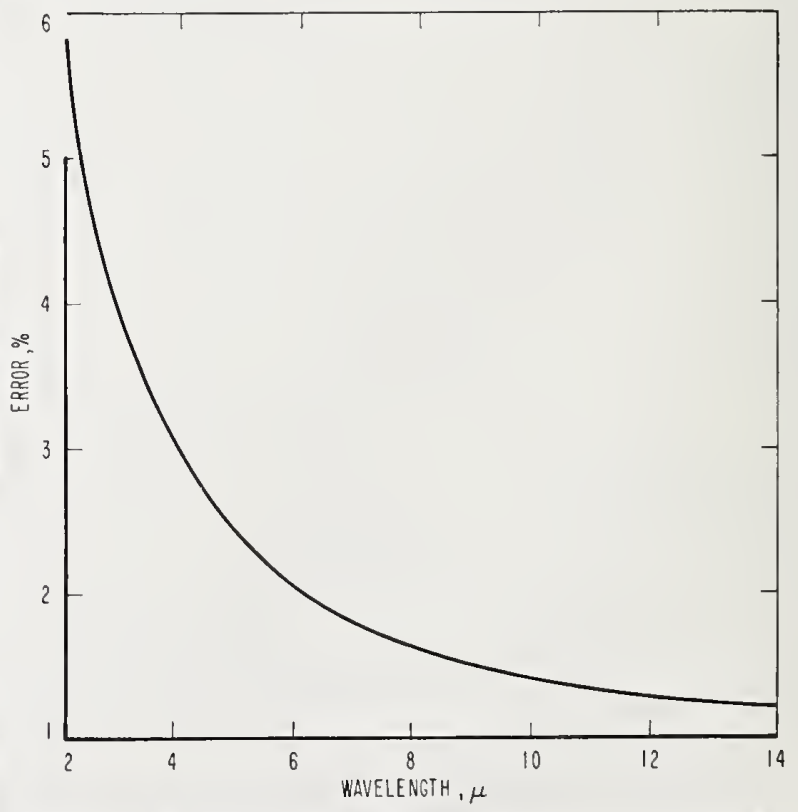

FIGURE 1. Error in emissivity measurement at $800^{\circ} \mathrm{K}$ resulting from an error in specimen temperature measurement of $5^{\circ} \mathrm{K}$. 


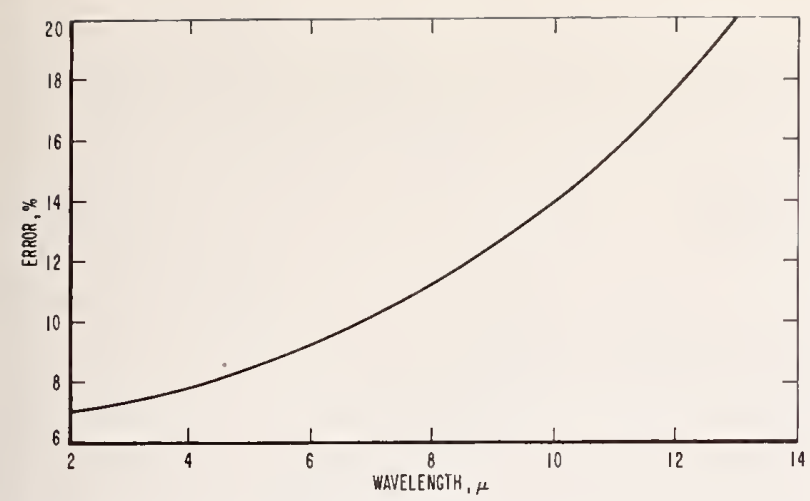

Figure 2, Error in emissivity measurenzent at $1,000^{\circ} \mathrm{K}$ resulting from an error in the blackbody temperature of $5^{\circ} \mathrm{K}$.

\section{Results}

The normal spectral emissivity of gold has been measured over the spectral range from 4 to $13 \mu$. Table 1 lists the values of emissivity determined by this work. In order to make this table, smooth curves of emissivity versus temperature at constant wavelength and emissivity versus wavelength at constant temperature were drawn through the observed experimental values. The table entries were then read from these curves. The results for three temperatures are shown in figure 3. Experimental points which are the average of several determinations have been superimposed on the curves to show how closely the experimental values agree with the smoothed curves. Data were obtained at $50{ }^{\circ} \mathbf{K}$ temperature intervals so that much more data were used in making these curves than is shown in the figure.

The small increase in the emissivity curve at $9 \mu$ is curiously unexpected, but appears consistently. This abnormality may be due to some unknown, consistent error in the data, but all attempts to eliminate it failed. Lameris [7] shows a similar rise in his data at about $10 \mu$ (he made no observations between 7 and $10 \mu$ ).

There seems to be no other measurement of the emissivity or reflectivity of gold in the temperature range reported in this paper. Consequencly, the only independent observations with which our results can be compared are the room temperature measurements for which reflectivity values are given in references 5 and 6 . By extrapolation of the emissivity versus temperature curves it is possible to ise the data of this work to obtain approximate (missivity values at lower temperatures. The extrapolated values are tabulated in table 2 . These values are, of course, considerably less accurate than those given in table 1 since the extrapolation is not very reliable.

TABLE 1. Normal spectral emissivity of gold

\begin{tabular}{|c|c|c|c|c|c|c|c|c|c|c|}
\hline$\lambda_{\mu}$ Temp. & 550 & 600 & 650 & 700 & 750 & 800 & 850 & 900 & 950 & 1000 \\
\hline $\begin{array}{l}4.0 \\
4.5 \\
5.0 \\
5.5 \\
6.0 \\
6.5 \\
7.0 \\
7.5 \\
8.0 \\
8.5 \\
9.0 \\
9.5 \\
10.0 \\
11.0 \\
12.0 \\
13.0\end{array}$ & $\begin{array}{r}0.014 \\
.0142 \\
.0145 \\
.0145 \\
.0142 \\
\\
\end{array}$ & $\begin{array}{c}0.0147 \\
.0151 \\
.0155 \\
.0155 \\
.0152 \\
.0150 \\
.015 \\
.016 \\
\end{array}$ & $\begin{array}{c}0.0158 \\
.0163 \\
.0165 \\
.0165 \\
.0162 \\
.0160 \\
.0162 \\
.0169 \\
.018 \\
.019 \\
\end{array}$ & $\begin{array}{l}0.0169 \\
.0174 \\
.0176 \\
.0176 \\
.0173 \\
.0170 \\
.0172 \\
.0178 \\
.019 \\
.020 \\
\end{array}$ & $\begin{array}{c}0.0181 \\
.0184 \\
.0187 \\
.0187 \\
.0183 \\
.0181 \\
.0183 \\
.0187 \\
.0197 \\
.021 \\
.022 \\
\end{array}$ & $\begin{array}{r}0.0193 \\
.0196 \\
.0198 \\
.0198 \\
.0195 \\
.0193 \\
.0194 \\
.0198 \\
.0206 \\
.0218 \\
.0228 \\
.023 \\
.022 \\
\end{array}$ & $\begin{array}{l}0.0206 \\
.0208 \\
.0210 \\
.0210 \\
.0208 \\
.0206 \\
.0207 \\
.0209 \\
.0215 \\
.0225 \\
.0235 \\
.0234 \\
.023 \\
.023\end{array}$ & $\begin{array}{l}0.0219 \\
.0221 \\
.0223 \\
.0223 \\
.0221 \\
.0220 \\
.0220 \\
.0221 \\
.0225 \\
.0233 \\
.0242 \\
.0241 \\
.0238 \\
.023 \\
.-.-.\end{array}$ & $\begin{array}{c}0.0232 \\
.0234 \\
.0236 \\
.0236 \\
.0235 \\
.0234 \\
.0234 \\
.0234 \\
.0235 \\
.0240 \\
.0249 \\
.0248 \\
.0245 \\
.024 \\
.023\end{array}$ & $\begin{array}{l}0.0246 \\
.0248 \\
.0249 \\
.0249 \\
.0249 \\
.0249 \\
.0248 \\
.0246 \\
.0244 \\
.0247 \\
.0256 \\
.0255 \\
.0252 \\
.024 \\
.024 \\
.023\end{array}$ \\
\hline
\end{tabular}

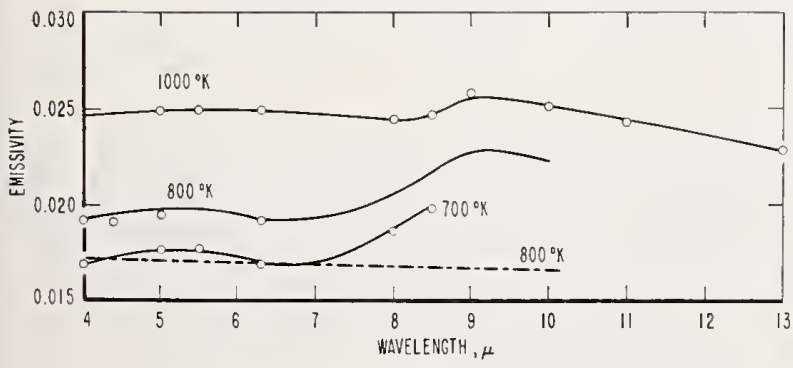

Figure 3. Normal spectral emissivity of gold from 4 to $13 \mu$ at

Dashed line is theoretical emissivity at $800^{\circ} \mathrm{K}$ calculated from Drude equations.]
TABLE 2. Approximate emissivity of gold obtained by extrapolation from higher temperature values

\begin{tabular}{|c|c|c|c|c|}
\hline$\lambda_{\mu}{ }^{\circ} K$ & 200 & 300 & 400 & 500 \\
\hline $4 \ldots$ & 0.0073 & 0.0090 & 0.0108 & 0.0127 \\
\hline $5 \ldots$ & .0083 & .0099 & .0117 & .0135 \\
\hline $6 \ldots$ & .0082 & .0098 & .0115 & . 0133 \\
\hline $7 .$. & .0085 & .0100 & 0117 & .0134 \\
\hline 8... & .0108 & .0123 & .0138 & .0154 \\
\hline
\end{tabular}




\section{Discussion}

It is of considerable interest to compare the observed emissivity values of metals to those predicted by the classical free-electron model proposed by Drude [10]. Roberts has recently shown [11] that Drude's original consideration of several classes of electrons in some cases gives rise to calculated results more compatible with experiment; however, this idea has not been used in this experiment. According to the Drude model the refractive index $(n)$ and the absorption coefficient $(k)$ may be represented by the two equations

and

$$
n^{2}-k^{2}=1-2 \frac{N e^{2}}{2 \pi m^{*}} \frac{1}{\nu^{2}+\gamma^{2}}
$$

where

$$
n k=\frac{\gamma}{\nu} \frac{N e^{2}}{2 \pi m^{*}} \frac{1}{\nu^{2}+\gamma^{2}}
$$

$$
\gamma=\frac{N e^{2}}{2 \pi m^{*}} \rho
$$

$N=$ number of free elcctrons per unit volume,

$e=$ electronic charge,

$m^{*}=$ effective mass of electron,

$\nu=$ frequency, and

$\rho=\mathrm{d}-\mathrm{c}$ electrical resistivity.

A simplification suggested by Hagen and Rubens is often used as a means of making emissivity calculations. This simplification rests upon the assumption that $\nu \ll \gamma$ and conscquently is valid in the long wavelength (low frequency) region. This assumption leads to the result that

$$
n=k=\left(\frac{1}{\rho \nu}\right)^{1 / 2} \text {. }
$$

Substituting this in the equation for emissivity,

$$
\epsilon=1-R=1-\frac{k^{2}+(1-n)^{2}}{k^{2}+(1+n)^{2}},
$$

yields the result

$$
\epsilon=2(\rho \nu)^{1 / 2}-2(\rho \nu)+\ldots .
$$

With appropriate changes of units this becomes the more familiar Hagen-Rubens equation,

$$
\epsilon=0.365\left(\frac{\rho}{\lambda}\right)^{1 / 2}-0.0464 \frac{\rho}{\lambda},
$$

where $\rho=$ resistivity in ohm centimeters and

$$
\lambda=\text { wavelength in centimetcrs. }
$$

Calculations based on the Hagen-Rubens equation give rise to values of the emissivity which are about 100 percent greater than the experimental values. As Schulz [12] has shown, the cause of this discrepancy is evident from an analysis of the assumptions made in the derivation of the Hagen-Rubens equation. Calculation of the value of $\gamma$ indicates that $\nu$ is of the same order of magnitude and in fact is slightly larger than $\gamma$. This means that $n \neq k$. Measurements of $n$ and $k$ by Schulz for the near infrared confirms the fact $n \neq k$ at a wavelength of $1 \mu$. Calculations using Drude's equations indicate that $n$ does not approach the value of $k$ except for rather large wavelengths (about $100 \mu$ ). As a consequence it is not surprising that the HagenRubens equation does not always yield correct values of the emissivity in the ordinary infrared region.

On the other hand, if the Drude equations are used to calculate the emissivity, better agreement with the measured values is obtained. The results of such a calculation for $800^{\circ} \mathrm{K}$ are shown in figure 3 . These calculations assumed one free electron per atom and that the effective electron mass $\left(m^{*}\right)$ is equal to the usual electron rest mass.

The authors express their gratitude to William F. Roeser for his helpful suggestions concerning these measurements. Appreciation is also expressed to Robert 'Thibault who performed the very tangible service of transforming nost of the raw data into values of emissivity.

\section{References}

[1] J. C. DeVos, Physica 20, 690 (1954).

[2] R. D. Larrabee, J. Opt. Soc. Am. 49, 619 (1959).

[3] D. T. F. Marple, J. Opt. Soc. Am. 46, 490 (1956).

[4] D. J. Price, Proc. Phys. Soc. (London) 59, 118 (1947).

[5] H. E. Bennett and W. F. Koehler, J. Opt. Soc. Am. 50, 1 (1960).

[6] Louis Harris and Peter Fowler, J. Opt. Soc. Am. 51, 164 (1961).

[7] P. F. Lameris [R. B. Dingle, Physica 19, 348 (1953)].

[8] A. G. Maki, R. Stair, and R. G. Johnston, J. Research 64C (Eng. and Instr.), 99 (1960).

[9] H. E. Bennett and J. O. Porteus, J. Opt. Soc. Am. 51, 123 (1961).

[10] P. Drude, Ann. Physik 14, 936 (1904).

[11] S. Roberts, Phys. Rev. 118, 1509 (1960).

[12] L. G. Schulz, J. Opt. Soc. Am. 44, 540 (1954).

(Paper 66C3-104) 


\title{
12. Comments on the Surface Characterization of Real Metals
}

\author{
DAVID P. DEWITT \\ NATIONAL BUREAU OF STANDARDS, WASHINGTON, D.C.
}

\begin{abstract}
Radiation properties of metallic specimens have been shown to be very sensitive to methods of preparation, thermal history, and environmental conditions. The magnitude, sensitivity, and complexity are illustrated by an example from the literature. An indication of how a real surface might be characterized is discussed in three general categories: topographical characterization, chemical characterization (species present), and physical (or structural) characterization. Until more systematic methods and techniques for characterization are developed, the experimentalist must assume responsibility for attempting to describe all significant aspects of his physical specimens using conventional laboratory techniques and apparatus.
\end{abstract}

\section{EXAMPLE OF SURFACE EFFECTS ON EMITTANCE}

Radiation properties of metallic specimens have been shown to be very sensitive to methods of preparation, thermal history, and environmental conditions. The magnitude, sensitivity, and complexity of these effects are well illustrated by some recent studies of aluminum surfaces by Reynolds (ref. 1). The measurement technique involved tubular specimens electrically heated in air, with a lateral slit serving as the blackbody cavity opening. Two surfaces were prepared, of roughness $3 \mu$ inch $(0.076 \mu)$ and $115 \mu \operatorname{inch}(2.9 \mu)$, CLA. ${ }^{1}$

Figure 1, which compares the normal spectral emittances ${ }^{2}$ of the two specimens at $326^{\circ} \mathrm{C}$, indicates primarily the effect of surface roughness. It is easily seen that roughening the

1 CLA (centerline average) roughness is defined as the average value of the departure of the profile from its center line, whether above or below it, throughout the prescribed sampling length.

2 The term radiance ratio is used by some workers in the field as equivalent to the term "emittance". surface increases the emittance at all wavelengths. The effect of the natural oxide layer is more apparent on the rougher specimen, where it gives rise to the prominent peak near $11 \mu$. The effects of any structural variations among the specimens and bulk aluminum caused by surface preparation technique cannot be discerned from these data.

Figure 2 shows data for these same two specimens for different temperatures. For the $3-\mu$ inch-roughness specimen, heating from $326^{\circ} \mathrm{C}$ to $532^{\circ} \mathrm{C}$ caused an increase in emittance at all wavelengths and the appearance of a peak near $11 \mu$ due to the oxide layer formed during heating. Subsequent measurements at $326^{\circ} \mathrm{C}$ show that the emittance was increased at all wavelengths, with a very pronounced peak near $11 \mu$. This increase and peak must be attributed to oxidation effects. For the 115$\mu$ inch-roughness specimen the results are similar but more pronounced. Roughening the surface strongly increased the spectral emittance at all wavelengths and accentuated the peak near $11 \mu$ arising from the oxide layer. Subsidiary emittance peaks appeared around $3.5 \mu$ and $6.0 \mu$ as oxidation proceeded. Prolonged heat- 


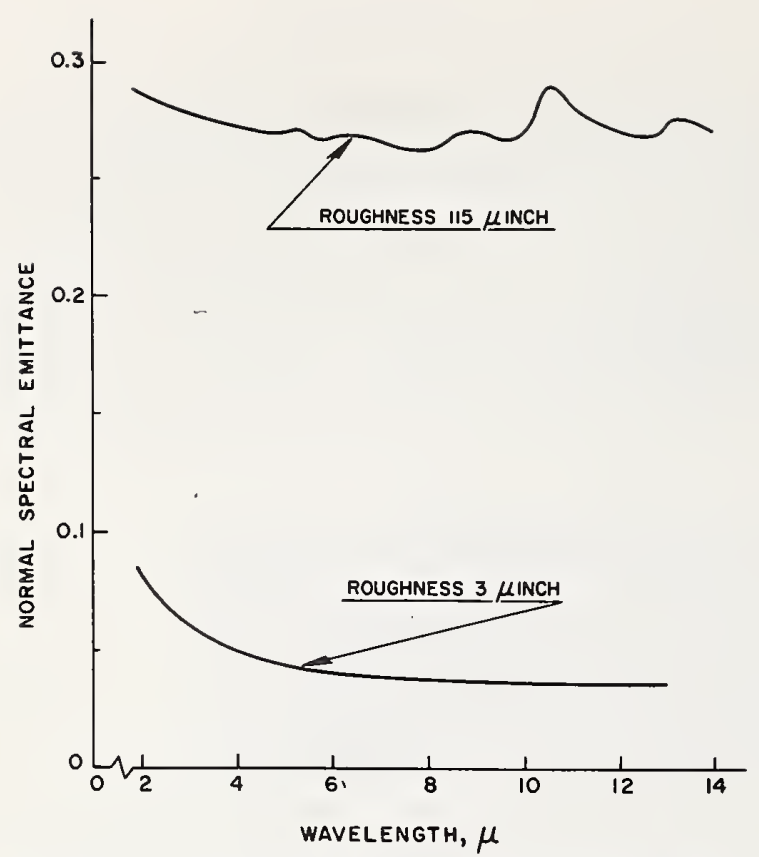

FIGURE 1.-The effect of roughness on normal spectral emittance of aluminum in air at $326^{\circ} \mathrm{C}$. (After Reynolds, ref. 1.)

ing at higher temperatures increased the spectral emittance considerably at wavelengths beyond $10 \mu$.

This example points out the problems involved in understanding the effects of the environment on thermal radiation properties of metals in specific applications. There is further evidence in the literature (ref. 2 and 3) to support this example of extreme dependence of the optical behavior of metals on surface conditions. However, most of this evidence has been collected under unrelated or diversified conditions so that it is not possible to relate observed effects to environmental conditions. It is apparent that the nature of the problemthe identification and control of variablesdemands a highly systematic approach to clarify and categorize conditions which give rise to the effects.

\section{CHARACTERIZATION OF REAL SURFACES}

Even though the nature of surface conditions and their effects on optical properties are not clearly understood, the differences between real

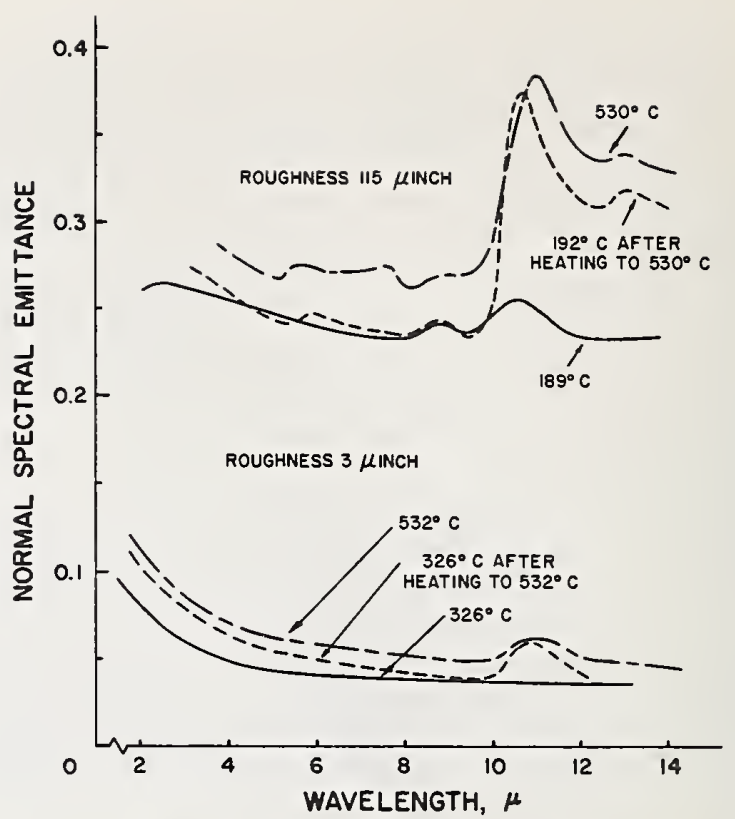

FIGURE 2.-The effects of roughness and oxidation on the normal spectral emittance of aluminum in air. (After Reynolds, ref. 1.)

and ideal surfaces can be distinguished. It seems advantageous to classify these differences under three general headings which give an indication of how a real surface might be characterized; namely, topographical characterization, chemical characterization (species presen $t$ ), and physical (or structural) characterization.

\section{Topographical Characterization}

The profiles of real metal surfaces are always shown as irregular patterns of peaks and valleys. Topographical characterization must provide a parametric description of such surface features as roughness type, roughness distribution, and lay ${ }^{3}$ that affect the optical behavior. Bennett and Porteus (ref. 4, 5, and 6) have shown in a quantitative manner the effect of surface roughness parameters on normal spectral reflectivity. The distinguishing feature of their work was the absence of any effects other than those due to surface topography; consequently

\footnotetext{
${ }^{3}$ Lay refers to the direction of the predominant surface pattern produced by tool marks or grains of the surface ordinarily determined by the production method used.
} 
the results are a significant contribution toward further understanding of topographical characterization. Similar studies that include goniometric measurements need to be made, as it can well be appreciated that the directional characteristics will be very sensitive to surface texture.

\section{Chemical Characterization}

The real metal surface unavoidably has a surface film of one type or another. In engineering applications, these films may be greases or other deposits, but normally they are oxides of the base metal. Even if only the natural oxide layer is considered, characterization is difficult. The layer is a $\cdot$ mixture of several different chemical species (metal atoms, oxygen ions, and one or more metal ions); the interface between oxides and base metal is not smooth; the rate of oxide growth is dependent upon the base metal surface topography, structure, temperature, and atmospheric conditions. The study of oxide layer growth is a problem of current interest. Numerous reviews and studies (ref. 7, 8, and 9) have been reported, but the mechanism of the oxide layer growth is not completely understood for some metals, for there are many conflicting theories and opinions. It is evident that considerable work is necessary to further our understanding of how to chemically characterize a surface.

\section{Physical or Structural Characterization}

The surface characteristics just discussedtopographical and chemical-are easily understood to have important influences on optical behavior. However, the effects caused by physical structure beneath the surface are not so apparent (ref. 10). In the case of a perfectly smooth surface free from surface films, a surface layer several hundred angstroms deep (skin depth of penetration of radiation) is responsible for the optical behavior of the metal. Structural features of this layer, such as adsorbed gas atoms, lattice imperfections, and crystallinity variations, can be expected to have an influence on the optical behavior.

It has been shown that some mechanical polishing processes cause structural changes in layers near the surface (ref. 11). There is evidence of a layer of supercooled fluid metal that fills the scratches caused by the abrasive (ref. 12). This layer (called the Beilby layer) has no clearly defined interface with the underlying metal (ref. 12 and 13). The outermost regions of this layer appear to be completely noncrystalline, and the structure becomes more crystalline as the depth from the outer surface increases. In some cases the layer will contain oxide. It has also been suggested that the polished layer is unstable and will revert, in time, to the ordinary crystalline state (ref. 14).

Heat-treating, cold-working, various surface preparation treatments, and many other processes can give rise to variations in physical and chemical characteristics between the bulk and surface layers. However, concrete evidence is lacking that these variations can in all cases cause significant changes in the optical behavior of metals.

\section{CONCLUSION}

Until systematic methods and techniques for characterization of surfaces are more fully developed, the experimentalist must assume the responsibility for attempting to describe all significant aspects of physical specimens for which data are obtained and reported. Many conventional laboratory techniques and apparatus, such as microphotography, etching techniques, profilometers, and electron microscopy, could be employed to immediate advantage. Certainly for clarity and significance, it is essential that some surface characterization information, no matter how incomplete, accompany numerical values of thermal radiation properties.

\section{REFERENCES}

1. REynolds, P. M.: Spectral Emissivity of $99.7 \%$ Aluminum Between 200 and $540^{\circ}$ C. British Jour. Appl. Phys., vol. 12, no. 3, Mar. 1961, pp. 111-114.

2. Clauss, Francis J., ed.: First SymposiumSurface Effects on Spacecraft Materials. John Wiley \& Sons, Inc., c. 1960.

3. Richmond, J. C.: Discussion of the Third SessionThermal Problems Under Orbiting Conditions. Proc. Fifth Sagamore Ord. Materials Res. Conf--Materials in Space Environment (Contract ROD-30-115-ORD-947), Syracuse Univ. Res. Inst., Sept. 1958, pp. 164-165, 175. 
4. Bennett, H. E., and Porteus, J. O.: Relation Between Surface Roughness and Specular Reflectance at Normal Incidence. Jour. Optical Soc. of America, vol. 51, no. 2, Feb. 1961, pp. 123129.

5. Bennetr, H. E.: Specular Reflectance of Aluminized Ground Glass and the Height Distribution of Surface Irregularities. Jour. Optical Soc. of America, vol. 53, no. 12, Dec. 1963, pp. 13891394.

6. Porteus, J. O.: Relation Between the Height Distribution of a Rough Surface and the Reflectance at Normal Incidence. Jour. Optical Soc. of America, vol. 53, no. 12, Dec. 1963, pp. 13941402.

7. Eubanks, A. G., Moore, D. G., and Pennington, W. A.: Effect of Surface Roughness on the Oxidation Rate of Iron. Jour. Electrochem. Soc., vol. 109, no. 5, May 1962, pp. 382-389.

8. Gulbransen, E. A., and Wrsong, W. S.: Thin Oxide Films on Tungsten. Trans. American
Inst. Mining and Metallurgical Engineers, vol. 175,1948 , pp. 611-627.

9. Kubaschewski, O., and Hopkins, B. E.: Oxidation of Metals and Alloys. Butterworths Scientific Pub. (London), 1953.

10. Donovan, T. M., Ashley, E. J., and Bennett, H. E. : Effect of Surface Damage on the Reflectance of Germanium in the 2650-10,000-A Region. Jour. Optical Soc. of America, vol. 53, no. 12, Dec. 1963, pp. 1403-1409.

11. Samuels, L. E.: Modern Ideas on the Mechanical Polishing of Metals. Res. Appl. in Industry, vol. 13,1960, p. 344 .

12. Beilby, G.: Aggregation and Flow of Solids. MacMillan and Co., Ltd., 1921.

13. Finch, G. Ingle: The Beilby Layer. Sci. Progress, vol. 31 , no. 124, Apr. 1937, pp. 609-625.

14. Lees, C. S.: The Structure of Polished Metal Surfaces. The Structure of Metallic Coatings, Films, and Surfaces. Trans. Faraday Soc. (London), vol. XXXI, 1935, pp. 1102-1106. 


\title{
15. Importance of Surface Films
}

\author{
JOSEPH G. RICHMOND \\ NATIONAL BUREAU OF STANDARDS, WASHINGTON, D.C.
}

\begin{abstract}
The effects of surface conditions, such as roughness and the presence of films, on thermal radiation properties are briefly outlined, and an example is cited where oxide films formed on heating in a good laboratory vacuum (about $10^{-5}$ torr) markedly affected the total hemispherical emittance of a specimen of sandblasted Inconel.
\end{abstract}

Reflection and refraction occur at an interface as a result of the abrupt change in the speed of electromagnetic radiation in passing across the interface. The physical laws relating the fraction of the incident flux that is reflected and the angles of reflection and refraction to the indices of refraction of the materials on the two sides of an optically smooth interface and to the angle of incidence and the amount and direction of polarization of the incident radiation are well known and need not be elaborated here.

In the specific case of a specimen in vacuum (or air), the reflectance at the specimen-vacuum interface determines how much of the externally incident radiation will be refracted into the specimen, where it can be absorbed or transmitted, and how much of the internally incident flux will be refracted through the interface and thus emitted. Hence the reflectance at the vacuum-specimen interface exerts a strong influence on all of the thermal radiation properties of the specimen.

When the interface is not optically smooth, multiple reflection and destructive interference occur, both of which tend to reduce the reflectance. While the general effect of roughness is known in a qualitative way, no rigorous equations have been derived which quantitatively relate roughness to reflectance.

A surface film, of index of refraction less than that of the specimen, will reduce reflectance, and a film of index of refraction higher than that of the specimen will increase reflectance. When films are present, interference effects can also occur, which may greatly increase absorptance. These effects are related to both index of refraction and thickness.

Thus we see that to properly characterize the surface of a specimen we need to describe the departures of its surface from an ideal optically smooth surface, and also the thickness and index of refraction of any surface film that may be present.

Changes in both surface contour and the nature of the surface film can occur as a result of test conditions. As an example, about 5 years ago we showed results (ref. 1) that were obtained on a sandblasted specimen of Inconel, on which the total hemispherical emittance was measured at a pressure of about $10^{-5}$ torr (fig. 1 ). Since the atmosphere in the test chamber was air, the oxygen partial pressure was on the order of $2 \times 10^{-6}$ torr.

Heating at temperatures up to about $500^{\circ} \mathrm{C}$ resulted in a permanent decrease in emittance. 


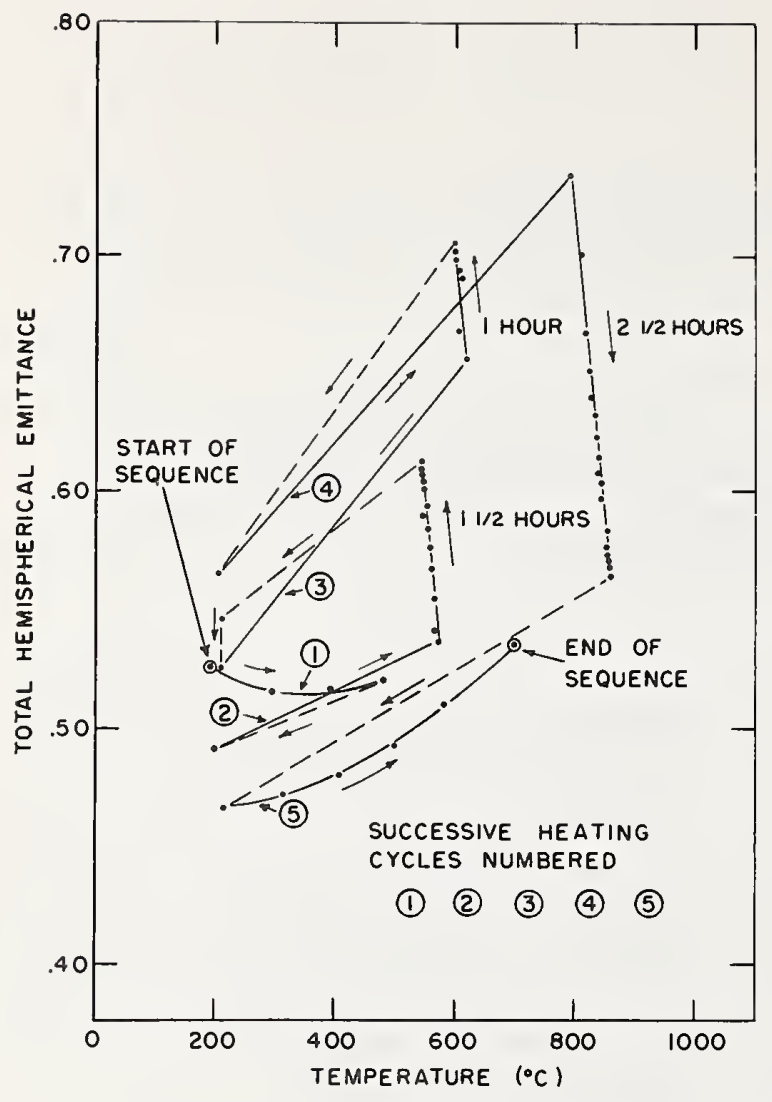

FIGURE 1.-Total hemispherical emittance of a sandblasted Inconel specimen during successive heating cycles in a vacuum with an exygen partial pressure of the order of $2 \times 10^{-6}$ torr.

This was believed to be due to removal of adsorbed films, although the exact nature of the films was not determined.

Heating the specimen at temperatures of about $600^{\circ}$ and $700^{\circ} \mathrm{C}$ resulted in both cases in an appreciable increase in emittance with time. This was due to the formation of an oxide layer which formed on the specimen even at this low oxygen pressure.

At temperatures on the order of $800^{\circ} \mathrm{C}$, the emittance decreased appreciably with timefrom about 0.72 to about 0.55 in $2 \frac{1}{2}$ hours. This effect was caused primarily by removal of the oxide layer. Chromic oxides dissociate in vacuum at temperatures above about $780^{\circ} \mathrm{C}$. At temperatures of $800^{\circ} \mathrm{C}$ and above, chromium volatilizes appreciably and at the same time there is a smoothing effect on the surface of the specimen somewhat similar to that produced by electropolishing. Both peaks and valleys were rounded off, to produce a bright surface that had a much higher reflectance than the initial sandblasted surface. ${ }^{1}$

Our experience with Inconel is presented to emphasize that it is not enough to know the surface roughness and the films present on the specimen before a test is started. We must also know how these change during a test in order to interpret our results properly. But most important of all, if our data are to be really useful, we must be certain that the surface condition of the specimen during test is truly representative of the material as it is used. This is perhaps the most frequently neglected and the most critically important aspect of surface characterization.

\section{REFERENCE}

1. Richmond, Joseph C., and Harrison, William N.: Equipment and Procedures for Evaluation of Total Hemispherical Emittance. American Ceramic Soc. Bull., vol. 39, no. 11, Nov. 15, 1960 , pp. $668-673$.

\footnotetext{
1 See also Paper 19.
} 


\title{
A Rotating Cylinder Method for Measuring Normal Spectral Emittance of Ceramic Oxide Specimens From 1200 to $1600{ }^{\circ} \mathrm{K}$
}

\author{
Howard E. Clark and Dwight G. Moore \\ Institute for Basic Standards, National Bureau of Standards, Washington, D.C. 20234
}

(June 10, 1966)

\begin{abstract}
Equipment was designed and constructed for measuring the spectral emittance of polycrystalline ceramic oxide specimens in the wavelength region 1 to $15 \mu$ and at temperatures of 1200,1400 , and $1600^{\circ} \mathrm{K}$. Specimens consisted of small hollow cylinders that were rotated at $100 \mathrm{rpm}$ in a furnace cavity equipped with a water-cooled viewing port. Emittances were determined by comparing the radiance of the specimen to that of a blackbody at the same temperature.

A series of six measurements (two measurements each on three specimens) was made on commercially pure specimens of alumina, thoria, magnesia, and zirconia. All four materials showed similar behavior in that the emittances were low in the shorter wavelength regions and high at the longer wavelengths. The temperature coefficients of spectral emittance were positive for all four materials, but varied with the material; those for thoria were appreciably higher than the other three. In all cases coefficients were greater at short wavelengths than at long.

Error sources were investigated, and the measurement uncertainties associated with each source were evaluated. It was concluded that at $1200^{\circ} \mathrm{K}$ the true values of emittance at $2 \mu$ for the particular specimens measured might be as much as 0.032 lower or 0.012 higher than the reported values. The data were in good agreement with reflectance measurements of the same material and with recently reported absorption coefficients.
\end{abstract}

Key Words: Alumina, ceramic oxides, emittance, error sources, magnesia, measurement uncertainties, spectral emittance, thermal radiation, thoria, zirconia.

1. Introduction

2. Rotating specimen method.............................

2.1 Selection of method.

2.2 Rotational speed of specimens......................

3. Equipment

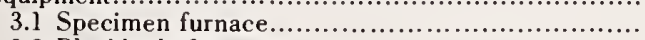

3.2 Blackbody furnaces

a. Details of construction

b. Temperature gradients in blackbody furnaces.

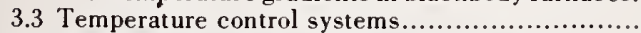

3.4 Spectrophotometer and transfer optics.............

3.5 Calibration for linearity of response.................

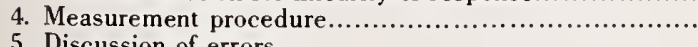

5. Discussion of errors.

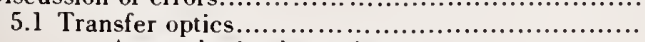

a. Atmospheric absorption..

b. Preferential mirror absorption.

5.2 Extraneous transmitted and reflected radiation...

a. Nonopacity of specimen wall.

\section{Contents}

Page

394

394

394

395

395

395

396

396

397

398

399

400

400

402

402

402

402

402

402 b. Furnace radiation reflected into specimen beam........................................... 5.3 Errors from temperature uncertainties.............. 5.4 Random error of measurement....................... 5.5 Estimated accuracy.

6. Measurements on polycrystalline oxide specimens........ 6.1 Description of specimens........................... 6.2 Results and discussion.

a. Spectral emittance data

b. Statistical scatter of data.

tecimens.........

d. Effect of temperature on emittance.............
e. Comments on use of data for heat transfer computations.

f. Correlation of data for alumina with reported absorption coefficients........................

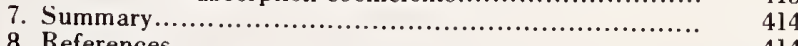

9. Appendix A: Analysis of error caused by nonisothermal blackbody furnace.
Page

402

403

405

405

405

405

406

406

406

409

410 


\section{Introduction}

Knowledge of the spectral emittance ${ }^{1}$ of ceramic oxides at high temperatures is important, not only because of the many potential space applications for materials of this type, but also to obtain an improved understanding of the thermal emission mechanism in solids. Several investigators have made spectral emittance measurements on oxide materials; however, wide variations in the data are evident, especially in the shorter wavelength regions.

The lack of better agreement may be due, at least in part, to certain measurement problems that exist when determining the emittance of ceramic specimens. Tnree of the more important of these problems are

(A) Temperature gradients in specimens. Sizable temperature gradients are generated when a stationary ceramic oxide specimen is heated to a high temperature in such a way that it is free to radiate from an exposed surface. These gradients, which result from the low thermal conductivity of the material, create serious measurement problems. The radiation emitted from a ceramic oxide specimen originates not only from the outermost surface layer, as it does for metals, but also from volume elements at appreciable distances below the surface. Hence, when a gradient is present it becomes virtually impossible to assign a unique temperature to the source of the emitted radiation, and without a knowledge of this temperature, a reliable emittance measurement becomes impossible.

(B) Extraneous reflected radiation. Polycrystal. line ceramic specimens are known to reflect thermal radiation diffusely rather than specularly. This means that any thermal radiation striking the viewed area of the specimen, regardless of the angle of incidence, will be diffusely reflected with some of it entering the specimen beam to give high values of emittance. Thus, unless elaborate precautions are taken to exclude this diffusely reflected radiation, for instance from furnace walls, measurements can be high by sizable amounts. This source of error becomes especially critical with ceramic specimens in the shorter wavelength regions where reflectance often exceeds 0.90 .

(C) Extraneous transmitted radiation. Many single crystal oxides, such as sapphire, have a very high transmittance for radiation in the near infrared. However, when the same oxides are finely ground and then bonded by high temperature sintering, the material becomes less transparent because of internal scattering effects (multiple internal reflections at interfaces) and in this way it is sometimes possible to prepare polycrystalline specimens that will be substantially opaque to radiation in the near infrared at thicknesses as low as $1 / 4$ in. If the specimen to be measured is not opaque, then serious errors will

\footnotetext{
As used in this paper, emittance is the ratio of energy radiated by a specimen per unit time and per unit area to that from a blackbody at the same temperature and under the same conditions. The conditions refer to the geometric and spectral distribution of the radiated energy.
}

result due to radiation from the furnace enclosure passing through the specimen and entering the specimen beam.

A rotating specimen method was chosen for the investigation described herein, since this approach minimizes temperature gradients in specimens. The present report begins by describing the earlier applications of this method. Subsequent sections include a description of the equipment and the measurement procedure, a detailed discussion of errors, and, finally, the results of spectral measurements for polycrystalline specimens of alumina, thoria, magnesia, and zirconia at 1200,1400 , and $1600{ }^{\circ} \mathrm{K}$.

The investigation was conducted at the National Bureau of Standards. However, it was carried forward under the sponsorship and with the financial assistance of the National Aeronautics and Space Administration.

\section{Rotating Specimen Method}

\subsection{Selection of Method}

A review of earlier methods used to measure the emittance of nonmetals indicated that one of the best approaches for reducing temperature gradients in a ceramic specimen to workable levels is to move the heated specimen past a water-cooled viewing port. In this way, a freshly heated surface of the material is continuously arriving at the port for meas. urement. If the movement is sufficiently rapid, a thermal equilibrium is approached in which temperature gradients in the specimen approach minimal values.

The moving specimen concept was first introduced by Féry in 1902 [1]. ${ }^{2}$ In his measurements, and also in those made by Michaud [2] and Pattison [3], cylindrical specimens were rotated while being heated in a gas flame. The ratio of the radiant flux from the surface to that from a hole placed axially at the center of the specimen was then taken as the emittance. No viewing port was used. In other measurements, specimens were rotated in a furnace cavity that was equipped with a water-cooled viewing port. McMahon [4], Slemp and Wade [5], and Olsen and Katz [6], all used a semicircular disk-shaped specimen that was rotated in front of a viewing port so that blackbody radiation from the furnace struck the detector for one-half the cycle, and the detector saw radiation from the specimen for the remaining onehalf cycle. Both McMahon [4] and Olsen and Katz [6] arranged for the periodic insertion of a watercooled shutter behind the specimen so that it was possible, at least in theory, to determine the transmittance of the specimen material. Folweiler [7] described a rotating cylinder apparatus, which incorporated solid cylindrical specimens with a " $V$ "

\footnotetext{
${ }^{2}$ Figures in brackets indicate the literature references on page 414 .
} 
groove positioned around the circumference to serve as a reference blackbody. The specimens were heated in a furnace with silicon carbide heating ele. ments. A water-cooled viewing port was used.

In measurements made by Clayton [8] a horizontal disk-shaped specimen was rotated in a carbon-resistance furnace that was equipped with separate water-cooled viewing ports for detecting radiation from both the specimen and the furnace enclosure. The specimen sighting tube could be moved to two positions. In the first position, the region behind the viewed portion was the hot furnace enclosure, and in the second, it was a water-cooled baffle. Here, again, the aim was to measure both emittance and transmittance.

A careful consideration of the possible errors and of the expected experimental difficulties inherent in each of these methods resulted in the selection for the present measurements of a rotating-cylinder approach with a separate blackbody. Briefly stated, the method consisted of rotating a hollow cylindrical specimen, approximately 1 in in diameter by 1 -in high with $3 / 16$-in thick walls, in a wire-wound furnace equipped with a water-cooled viewing port. Spectral emittance measurements were then made with a double-beam spectrophotometer by comparing the radiance of the rotating specimen to that of a blackbody at the same temperature.

An earlier description of the measurement method has been published [9]. However, only prelim. inary data at one temperature $\left(1200^{\circ} \mathrm{K}\right)$ where given at that time. Also, there was no systematic analysis of errors, since this phase of the investigation had not been completed at the time of the earlier publication.

\subsection{Rotational Speed of Specimens}

A theoretical analysis of periodic heat flow in a specimen rotating before a viewing port, performed as a first phase of the study, has been published by Peavy and Eubanks [10]. The analysis showed that at low rotational speeds the surface temperature of a specimen at the center of the viewing port opening will be substantially lower than the temperature of the thermocouple in the specimen cavity. However, this difference will decrease with increasing speed. The speed at which the difference will become negligibly small will depend on system geometry, thermal properties of the specimen material, and a derived average heat flux for the portion of the cycle during which a point on the specimen is being heated by the furnace.

This analytical treatment may be used to compute the rotational speed required to reduce radial temperature gradients in the specimen to acceptable levels. However, since reliable thermal property data are not presently available for the specimen materials, such computations can be of only limited value. Therefore, in the present work, the minimum permissible speed of rotation was determined experimentally by first rotating a specimen at a very low speed and measuring its emittance at $1.4 \mu{ }^{3}$ As the speed was increased, the indicated emittance at $1.4 \mu$ continued to rise until a point was reached where further increases in speed caused no further increase in the measured emittance. For the materials studied in this report, the maximum value of emittance was reached at about $50 \mathrm{rpm}$. However, as an added insurance against possible errors, actual emittance measurements were made at $100 \mathrm{rpm}$.

\section{Equipment}

\subsection{Specimen Furnace}

Figure 1 is a schematic drawing of the specimen fur. nace. The high-temperature alumina core surrounding the specimen was wound with 0.032 -in-diam platinum -40 percent rhodium wire. The winding was continuous to the edges of the rectangular opening that was cut into the core to permit entrance of the viewing port. A booster winding of the same wire positioned on the outer alumina core, as indicated in the figure, was used to compensate for the large heat losses at the center.

The water-cooled viewing port was machined from copper, and its inner surface was curved to the same radius as the specimen. A shield of platinum foil, 0.002 -in thick, surrounded the outer surfaces of the port, including the edges that faced the specimen. This helped to thermally isolate the viewing port from the furnace interior. The inner surfaces of the viewing port and the portion of the platinum shield nearest the specimen were blackened to minimize the possibility of errors from reflected radiation. The opening at the inner end of the port was $1 / 8$-in wide by $1 / 2$-in high.

The specimens were prepared so as to be cylindrical to \pm 0.002 in. The alumina support tube (fig. 1) was surface ground to this same tolerance. The spindle was driven by a $1 / 8$ HP motor that was coupled to a gear reducer. With the arrangement used, the rotation of the specimen could be adjusted to any speed in the range 1 to $300 \mathrm{rpm}$.

The design of the furnace shell was such that the furnace could be operated in an inert atmosphere, as well as in air. Glass-metal seals were used for power leads and "O"-ring seals were used for the shell ends, as well as for a sodium chloride viewing windows. The thrust bearings were designed to provide a reasonably gas-tight seal at the point where the spindle shaft entered the shell. Although this controlled-atmosphere feature had been incorporated into the design, all measurements included in this report were made in air with no windows. Also, although the furnace was capable of being operated at $1800^{\circ} \mathrm{K}$, no measurements were made at this temperature, because of temperature control difficulties (see sec. 3.3).

${ }^{3}$ Difference in temperature between specimen and blackbody has the greatest effect on emittance at wavelengths below the energy peak of blackbody radiation. The selected setting of $1.4 \mu$ is well below this peak for the selected test temperatures. 


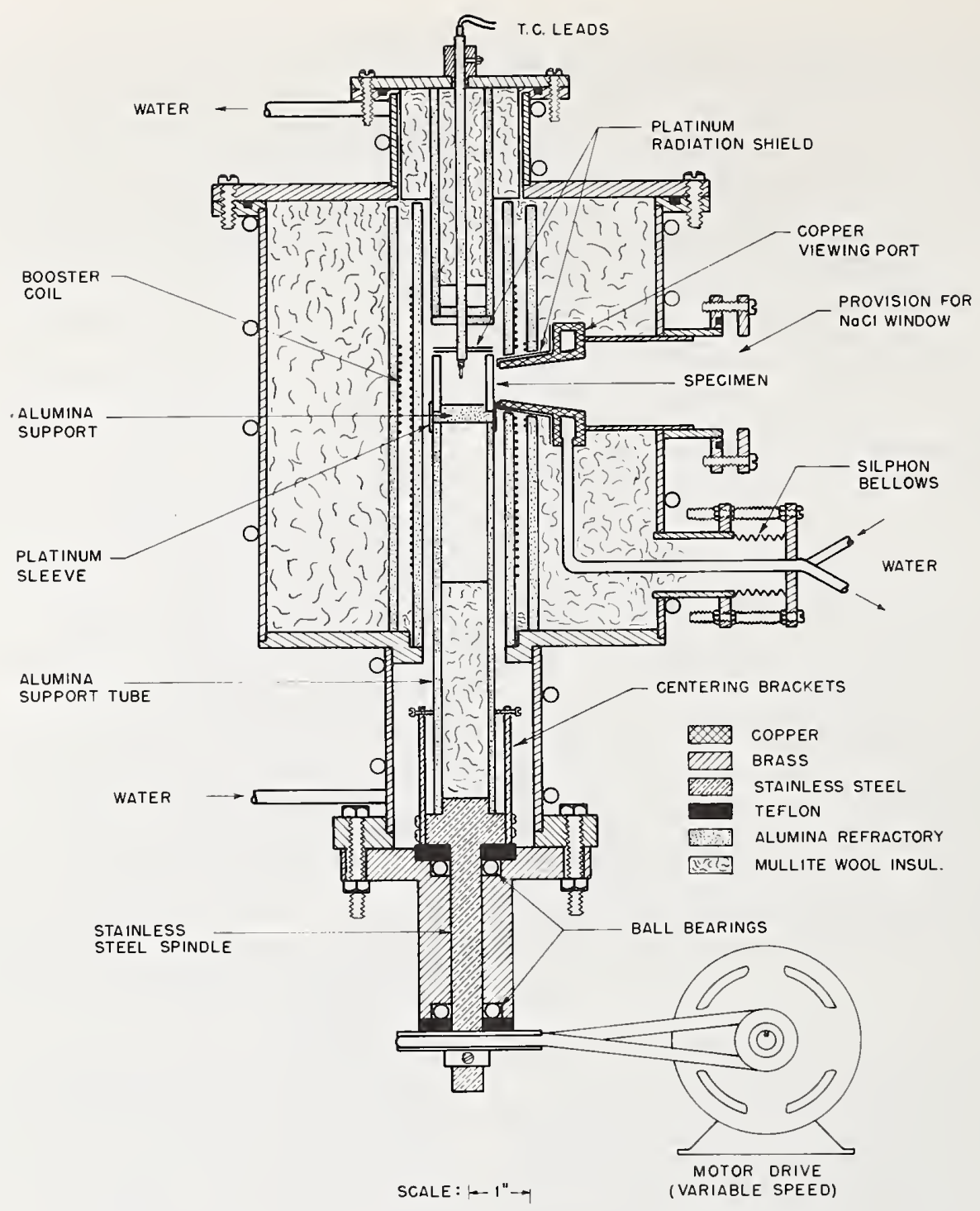

Figure 1. Specimen furnace.

Axial temperature gradients in the specimens were reduced to low values by adjusting the power to the booster coil. The gradients were measured by sighting a micro-optical pyrometer on a rotating specimen through the viewing port. With the settings used, no temperature difference from top to bottom was observed for any of the specimens at $1200{ }^{\circ} \mathrm{K}$. At $1400^{\circ} \mathrm{K}$, the top may have been as much as $2^{\circ} \mathrm{K}$ higher than the bottom, while at $1600^{\circ} \mathrm{K}$, the top was as much as $6^{\circ} \mathrm{K}$ higher. The precision to which the pyrometer could be read was about $2^{\circ} \mathrm{K}$. The specimen thermocouple was located in the ces ter of the specimen cavity and was shielded from turnace radiation as shown in figure 1 .

\subsection{Blackbody Furnaces \\ a. Details of Construction}

Figure 2 is a schematic of the blackbody furnace. Two of these were required in the measurement system, and both were made to be as nearly identical as possible. The inner cavity of the furnaces was formed of fused alumina bonded with 20 percent by weight of a calcium aluminate cement. This mixture, which sets hydraulically, was mixed with 17.5 percent by weight of water and vibrated into a greased plaster mold. The mold was made by using an alumina core as a pattern: a brass mandrel was 


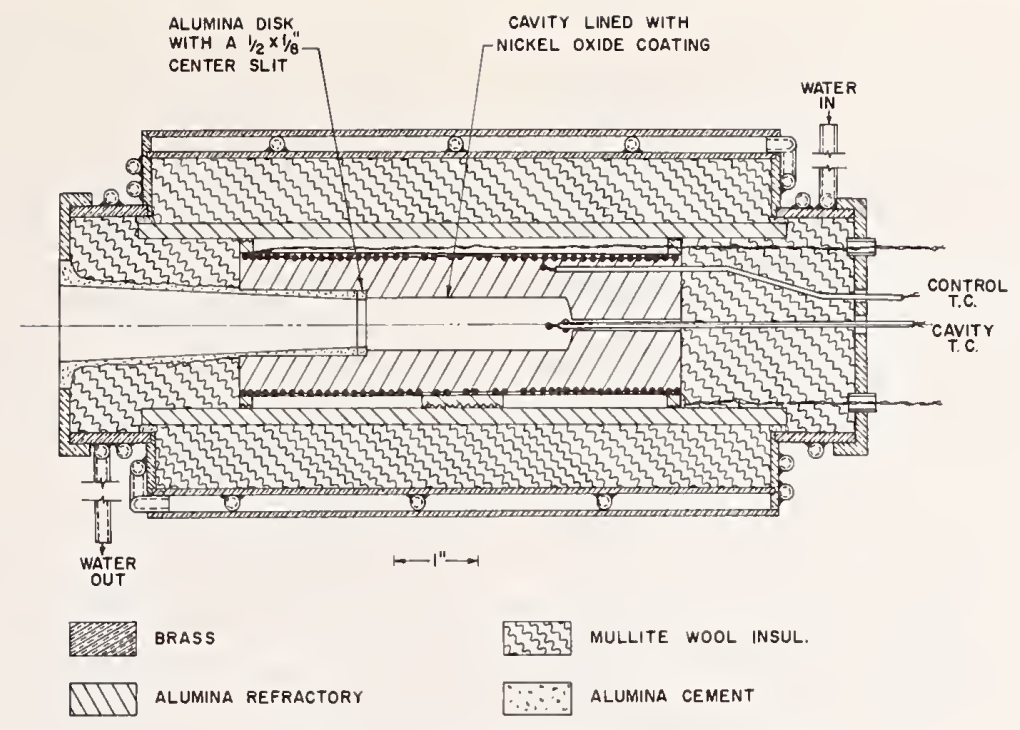

W/D alumina BONDED WITH CALCIUM aluminate

Figure 2. Blackbody furnace.

positioned at the center of the mold to form the outline of the cavity. After allowing $20 \mathrm{hr}$ for curing, the core was removed from the mold, dried in an oven for $24 \mathrm{hr}$, and then heated to $1925^{\circ} \mathrm{K}$ for l hr. It was later wound with 0.032 -in-diam platinum-40 percent rhodium resistance wire.

An alumina disk with a $1 / 2$-in by $1 / 8$-in center slit was placed over the front end of the cavity to reduce heat losses by radiation. The viewing port was formed with mullite wool, over which was placed a thin layer of alumina cement. The shaping of the port was facilitated by use of a tapered aluminum mandrel that was machined especially for the purpose.

All furnace thermocouples (including the one for the specimen furnace) were made of 0.020-in-diam Pt:Pt-10 percent Rh wire that had been calibrated by the Temperature Physics Laboratory at the National Bureau of Standards. Two-hole alumina tubing was used to insulate the leads. This tubing was inserted into an alumina sleeve that was cemented into the core so that when the thermocouple was inserted, it would be nearly flush with the side wall of the cavity. With this arrangement, the spectrophotometer did not "see" the thermocouple bead, but rather was "looking" at the back of the cavity, which was largely isothermal. Of course, no important deviation from blackbody conditions would result from the presence of the thermocouple at the center of the cavity if the cavity was isothermal and if there was no separation between the alumina sleeving and the thermocouple tubing. However, a small clearance was present, and this clearance showed up as a tiny dark crescent when looking into the heated cavity. The presence of a dark area of this type was undesirable, in that it would tend to generate low flux readings.

The alumina core material has a low emittance in the wavelength region 1 to $5 \mu$. Inasmuch as the cavity emittance depends, in part, on the emittance of the walls, it was desirable to line the cavity with a coating that would have a high emittance at the short, as well as the long wavelengths. The coating developed for this purpose consisted of black nickel oxide $\left(\mathrm{Ni}_{2} \mathrm{O}_{3}\right)$ bonded with 7.5 percent by weight of Florida kaolin. This coating was prepared as a coating slip and applied at a thickness of about 0.005 in to the inner wall surfaces of the cavity by a dipping operation. The core was then heated to $1850{ }^{\circ} \mathrm{K}$ for $1 \mathrm{hr}$, to bond the coating to the alumina. The spectral emittance of a cylindrical specimen of the core material coated in this way was found to be above 0.875 at all wavelengths from 1 to $15 \mu$ when measured at $1200{ }^{\circ} \mathrm{K}$. Hence, the lowest possible cavity emittance at any wavelength from 1 to $15 \mu$ was 0.990 when computed by an expression derived by Gouffé [11]. This expression assumes that the walls are diffusely reflecting and that the cavity is isothermal. The expression has been shown to predict slightly low values of emittance for cavities with large depth-to-diameter ratios [12].

\section{b. Temperature Gradients in Blackbody Furnaces}

Several furnace designs were tested early in the study in an attempt to minimize temperature differences within the cavity. To test for gradients, a Pt: Pt-10 percent Rh thermocouple made with 0.010 -indiam wire was inserted into the viewing port and moved 
from front to back with the hot junction within $1 / 8$ in of the interior wall. A record was made of the response at each position. In almost all cases, the indicated wall temperature near the center of the cavity was higher than at either end. In some instances, power was shunted from the central region of the furnace windings, while in other tests, varying numbers of turns were omitted from the heater coil. The final configuration was that shown in figure 2. No shunts were used and four loops were omitted in the central region. With this arrangement, temperature probing revealed that the cavity walls near the back were colder than those in the center by the amounts indicated below. ${ }^{4}$

\begin{tabular}{c|c}
\hline Approximate cavity temperature & Temperature difference \\
\hline${ }^{\circ} K$ & ${ }^{\circ} K$ \\
1200 & 18 \\
1400 & 14 \\
1600 & 8 \\
\hline
\end{tabular}

The presence of these gradients was a potential source of error in the measurements because of (a) uncertainty in determining the temperature to assign to the "blackbody" radiation, and (b) deviations from the Planck distribution of energy caused by the non. isothermal conditions. Naturally, it would be desirable if the spectral distribution of radiated energy, $E(\lambda)$, was proportional to the Planck function, $W(\lambda, T)$; i.e., $E(\lambda)=\epsilon_{\text {cav. }} W(\lambda, T)$. It was not immediately evident, however, that any single temperature could be assigned to the nonisothermal cavity. Temperatures that might be considered are the temperature of a thermocouple placed in the center of the cavity, the temperature of the back wall of the cavity, or the temperature indicated by an optical pyrometer sighted on the mouth of the cavity. This latter temperature was selected, since it represented the temperature of a perfect blackbody whose radiance at $0.65 \mu$ was the same as that of the nonisothermal laboratory furnace. A theoretical analysis is presented in appendiz A to show that when a radiance match is achieved at $0.65 \mu$ then the spectral distribution of radiated energy can be approximated by a Planck function over the 1 to 15 $\mu$ range with no more than a 0.5 percent error.

The procedure used for positioning the furnace thermocouple so that its response would agree with the temperature measured with a calibrated optical pyrometer was as follows:

(1) The furnace was first heated to the desired operating temperature and allowed to stabilize.

\footnotetext{
"Large uncertainties existed in temperature measurements made near the front of the cavity, because of excessive heat losses by conduction along the thermocouple leads and the protection tubing.
}

(2) A micro-optical pyrometer was then sighted at the front opening of the furnace and the "optical" temperature of the furnace cavity was determined.

(3) The furnace thermocouple was next moved to a position where its response indicated the same temperature as that measured with the optical pyrometer. This position was always within 1 in of the rear wall of the cavity.

(4) Finally, a mark was placed on the thermocouple tubing at the back of the furnace shell so that the positioning of the junction could be duplicated in subsequent determinations at the same test temperature. This positioning was checked periodically to be certain that no changes in the temperature behavior of the furnace had occurred.

The calibration procedure as outlined needed to be applied to only one blackbody furnace. The position of the thermocouple in the second blackbody furnace could then be adjusted by noting the shape of the spectral curve that compared the energy from these two furnaces (100 percent line, see sec. 4). At long wavelengths, small temperature differences between the furnaces lead to negligible differences in energy content of the two beams, but at short wavelengths they had a pronounced effect. Thus, if the 100 percent curve was higher at $1 \mu$ than at, say, $9 \mu$, it indicated that furnace No. 2 was at a higher temperature than the reference blackbody (No. 1). The thermocouple in No. 1 was then moved to a position where it indicated a lower temperature. The automatic control equipment would then lower the temperature of No. 2 until it matched that of No. 1 and a new spectral curve was determined. This procedure was repeated until a flat 100 percent line was obtained. When this condition was achieved, the furnace No. 1 was at the same effective temperature as No. 2 in that it was supplying an equivalent flux to the spectrophotometer.

\subsection{Temperature Control System}

A differential thermocouple control system similar to that described by Harrison et al. [13], was used to maintain temperature differences of less than $1^{\circ} \mathrm{K}$ between blackbody No. 1 and either the specimen furnace or blackbody No. 2. The No. 1 furnace was controlled, in turn, by a separate temperature controller, which maintained its temperature to $\pm 0.5^{\circ} \mathrm{K}$. A double-pole, double-throw switch was used for switching the differential thermocouple controller from blackbody No. 2 to the specimen furnace.

The differential thermocouple controller was actuated by the difference in signal between the thermocouple in blackbody furnace No. 1 and the thermocouple in either blackbody No. 2 or the specimen furnace. This signal was amplified by a d-c amplifier and fed to a center-zero recorder-controller. The output of the recorder-controller, which was proportional to the temperature difference between the two furnaces, was fed to a current-actuating mechanism that provided three types of current control. One type was proportional to the input signal, a second to the first 


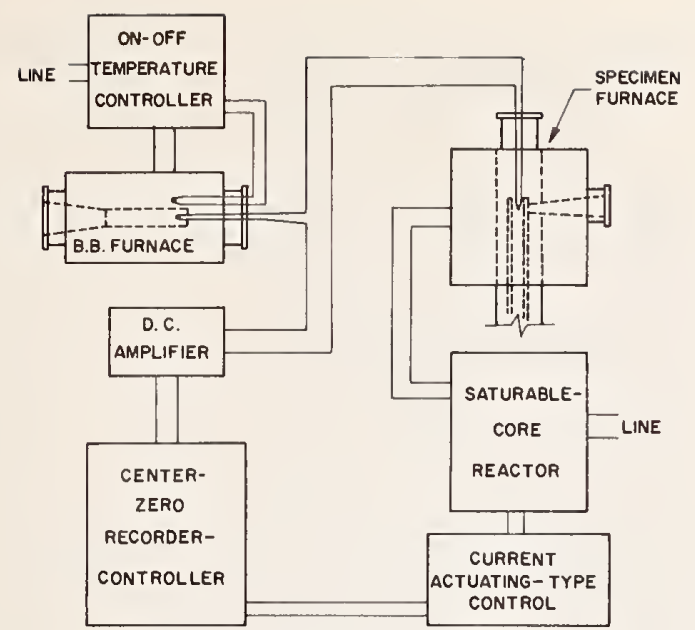

Figure 3. Block diagram of temperature control equipment Controller is actuated by signal from differential thermornuble.

time derivative of the input signal, and a third selected the frequency at which the first correction was repeated. The output from this current actuating device was fed to the coil of a saturable-core reactor, which then varied the power input to either blackbody No. 2 or the specimen furnace, so as to bring the temperature into agreement with the reference blackbody (No. 1).
A block diagram of the temperature control equipment is shown in figure 3 . The system gave almost trouble-free operation at 1200,1400 , and $1600{ }^{\circ} \mathrm{K}$. However, at $1800^{\circ} \mathrm{K}$, it became largely inoperative because of excessive noise in the thermocouple signal. This may have originated from electrical leakage through the alumina core material, whose electrical conductivity becomes appreciable at these very high temperatures. For this reason, no measurements at $1800^{\circ} \mathrm{K}$ were possible, even though all of the furnaces could be operated at this temperature.

\subsection{Spectrophotometer and Transfer Optics}

A double-beam infrared spectrophotometer with a sodium chloride prism was used for obtaining the radiance comparisons. This instrument, which utilizes a chopper operating at $10 \mathrm{~Hz}$, was modified to operate from 1 to $15 \mu$ rather than in the intended 2 to $16-\mu$ range. A second modification consisted of reducing the angle of the attenuator-comb in the reference beam from blackbody No. 1, so that the maximum opening of the comb did not exceed the height of the furnace apertures. This required only a small reduction in the comb angle.

The double-beam optical system of the instrument was used for all measurements. When operating in this mode, the spectrophotometer achieves a null balance through use of the aforementioned mechanically-driven attenuator, or comb, in the reference

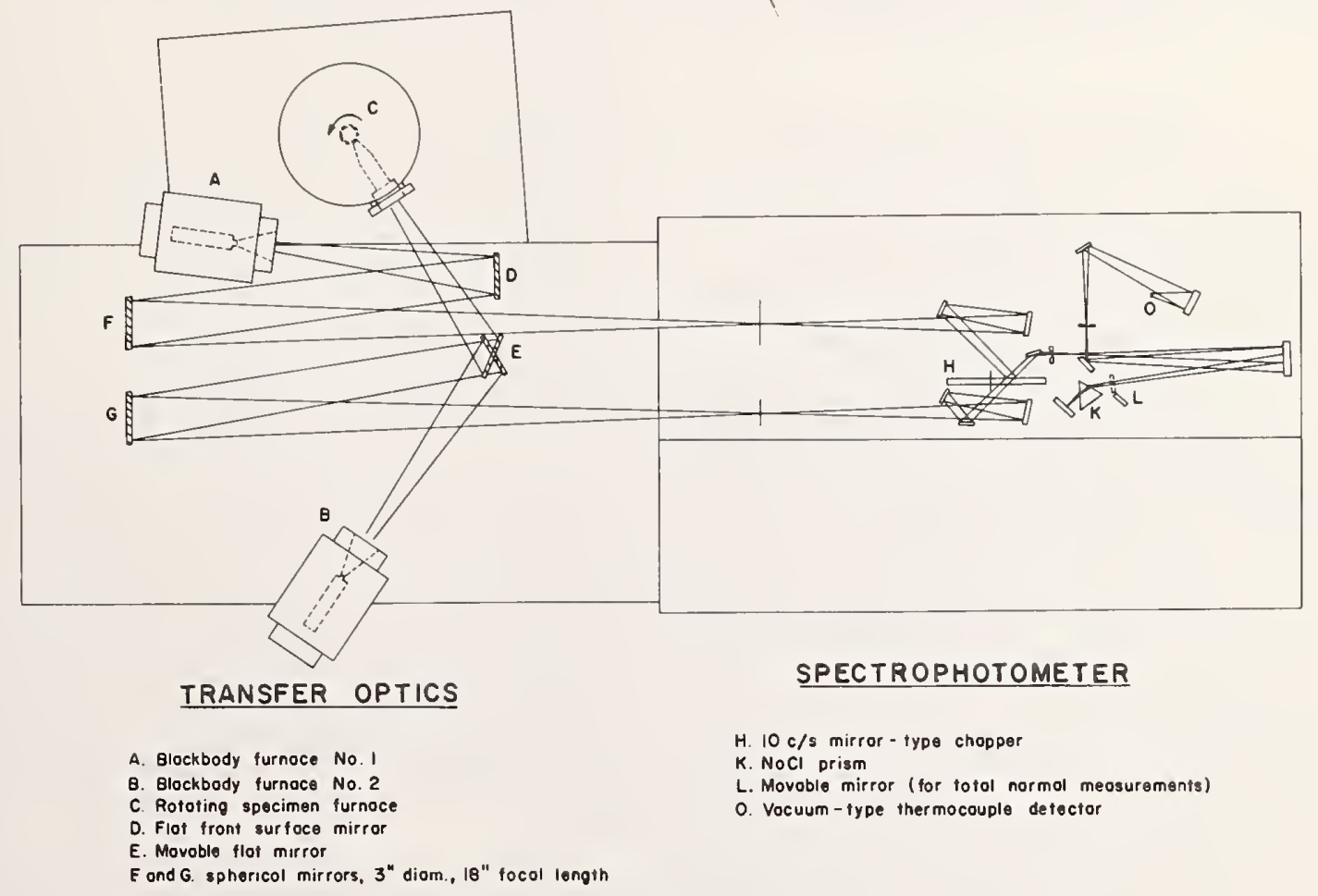

FIGURE 4. Arrangement of equipment showing optical paths of the two beams. 


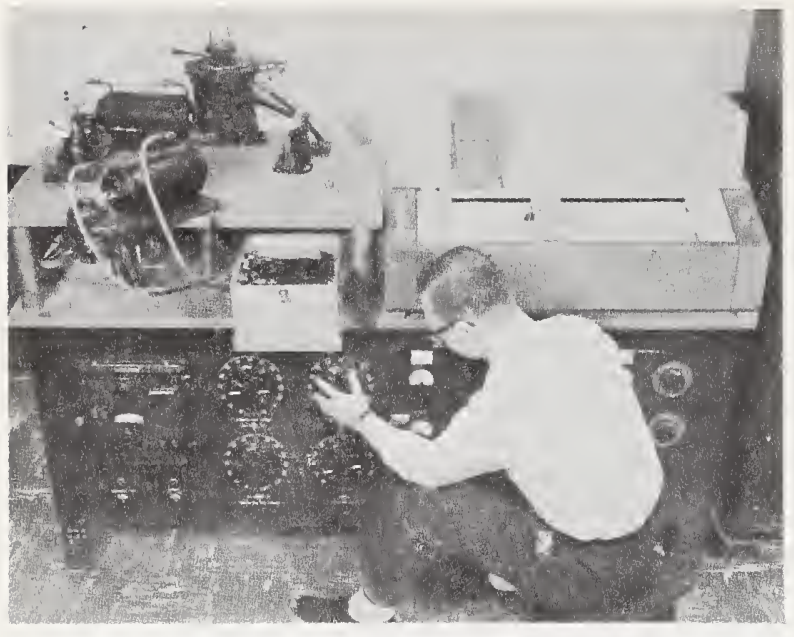

Figure 5. Equipment in operation.

beam. A recorder pen, coupled mechanically to the comb, permits the ratio of the radiances of the two beams to be continuously recorded with respect to wavelength. In all measurements reported herein, the instrument was set to scan the $1-$ to $15 \cdot \mu$ range in $16 \mathrm{~min}$.

Prior to using the instrument, the source optics were removed and replaced with the transfer optics shown schematically in figure 4 . The off-axis angle of the two spherical mirrors was approximately $5^{\circ}$.

Figure 5 is a photograph of the equipment in operation.

\subsection{Calibration for Linearity of Response}

The response of the modified instrument to known attenuations of flux in the specimen beam was determined through use of sectored-disk attenuators. To conduct this test both blackbodies were first adjusted to some temperature in the range 1200 to $1600^{\circ} \mathrm{K}$. A sectored-disk attenuator was then inserted into the specimen beam and rotated at high speed (greater than $500 \mathrm{rpm}$ ). Six disks were used with measured transmission factors of 75.1, 50.1, 25.3, 12.7, and 5.1 percent, respectively. The resulting spectral curves, which were found to be reproducible to within the precision of measurement, showed that the instrument response was always lower than the actual beam attenuation. This lowering was the same at all wavelengths. Wide variations in disk speed above $500 \mathrm{rpm}$ had no effect on the instrument response, which showed that resonance coupling between the chopper and attenuator was not affecting the results.

Since no method could be devised to restore linearity to the modified instrument, a correction curve (fig. 6) was prepared. This curve was then used to correct the spectral emittances that were obtained by the procedure outlined in section 4 . The dashed line in figure 6 shows the type of behavior expected from an instrument with a completely linear response.

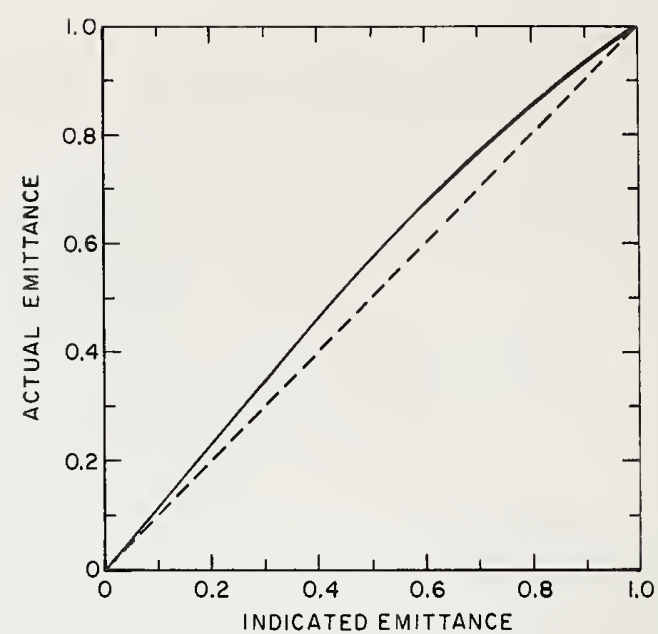

Figune 6. Calibration curve (solid curve) for correcting for lack of instrument linearity.

Dashed line shows type of behavior desired of an instrument (linear response).

\section{Measurement Procedure}

In making a determination, a specimen was first placed on the top of the refractory pedestal, carefully centered, and then fixed in position with high-temperature alumina cement. This centering and mounting operation was performed outside the furnace with the aid of a specially prepared jig. After the mounting had been completed, the pedestal was inserted into the furnace from the bottom and carefully positioned radially by a screw adjustment of the bottom flange, so that the specimen rotated at an average distance of 0.010 in from the inner face of the viewing port. The separation distance was measured optically by a telescope with a calibrated reticle. The telescope was sighted through the top of the furnace tube while the furnace was at approximately $1400^{\circ} \mathrm{K}$. Specimen wobble did not exceed \pm 0.005 in.

After positioning of the specimen was completed, the top insulation was replaced, the motor drive was started, and the specimen was brought to a rotational speed of $100 \mathrm{rpm}$. The controller on blackbody No. 1 was then adjusted to $1600^{\circ} \mathrm{K}$ and the differential thermocouple controller actuated to bring blackbody No. 2 to the same temperature. As soon as the temperatures had equilibrated, the flux from blackbody No. 2 was compared to that from blackbody No. 1 by scanning from 1 to $15 \mu$. The resulting curve, a representative section of which is shown in figure 7 , was termed the 100 percent line. Next, the beam from blackbody No. 2 was blocked, and a second curve was obtained on the same chart paper. This is the zero line shown in figure 7 .

After the zero line had been obtained, the automatic control was switched to the specimen furnace, the movable mirror (see fig. 4) was shifted to the specimen 


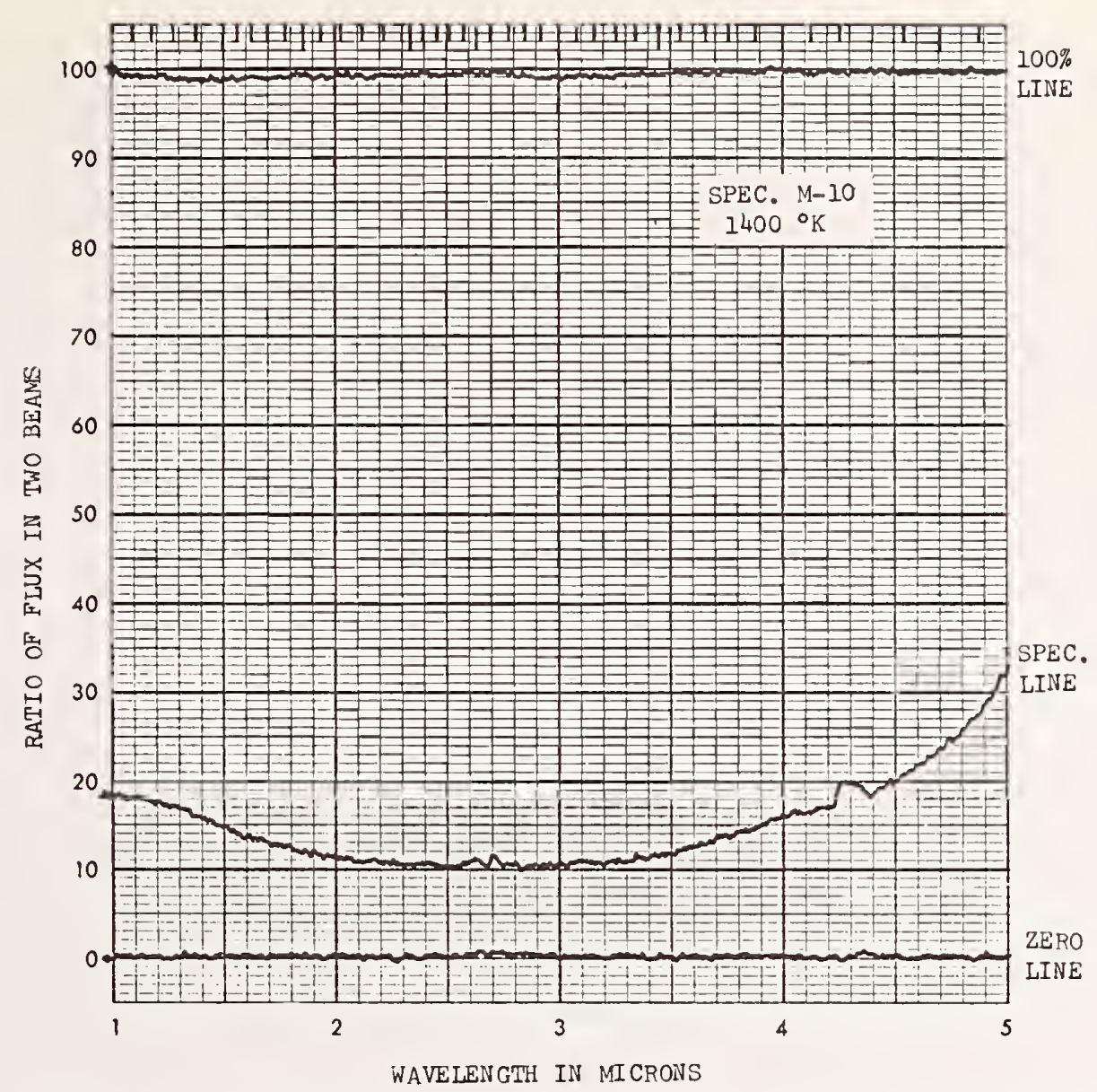

FIGURE 7. Section of recorder chart showing segments of a "100 percent Line," "Specimen Line," and "Zero Line."

position, and after an equilibrium condition had been attained a specimen curve was plotted, again on the same chart paper.

Curves such as those shown, in part, in figure 7 represent raw data for one determination at one temperature. To transform these data to an actual or true spectral emittance curve for the specimen, two operations were performed:

(1) The height of the specimen curve at preselected wavelengths was obtained as a ratio of the respective heights of the zero and 100 percent lines. Stated mathematically, if $Z_{\lambda}$ is the height of the zero line, $S_{\lambda}$ the height of the specimen curve, and $H_{\lambda}$ the height of the 100 percent line, then $\epsilon_{\lambda i}$, the uncorrected or "indicated" emittance, is

$$
\epsilon_{\lambda i}=\frac{S_{\lambda}-Z_{\lambda}}{H_{\lambda}-Z_{\lambda}}
$$

Values for $\epsilon_{\lambda i}$ were, in most cases, obtained at intervals of $0.5 \mu$.

(2) The values for $\epsilon_{\lambda i}$ were next converted to true or actual emittances, $\epsilon_{\lambda}$ through use of the calibration curve shown in figure 6 . These values were then plotted against wavelength to obtain the curves shown in later sections of the report.

In all data reported herein, measurements on each specimen were made first at $1600^{\circ} \mathrm{K}$, next at $1400^{\circ} \mathrm{K}$, and finally at $1200{ }^{\circ} \mathrm{K}$; all three measurements being made during a single working day. Three specimens of each type were measured, and two completely independent determinations were made on each specimen. These duplicate determinations on the same specimen were always made on different working days, and in each case, the specimen had been removed from the furnace between the first and second series of measurements. 


\section{Discussion of Errors}

\subsection{Transfer Optics}

An important consideration in the design of any double beam system is the requirement of complete optical equivalence of the two beams. Failure to meet this requirement would cause inflections in the spectral curves due to differential absorption by the atmosphere or by the mirrors in the system. In the present study the use of the two blackbodies to obtain a 100 percent line provided a convenient means to check for these effects.

\section{a. Atmospheric Absorption}

In most double-beam infrared spectrophotometers, no irregularities are observed when scanning through the absorption bands for carbon dioxide and water vapor, since the flux attenuation is the same for both beams. However, the flux available for measurement drops off drastically at the major absorption bands. In the present measurements, no appreciable inflection in the curve was observed at the strong water absorption band at $2.7 \mu$, since at this wavelength there was apparently sufficient energy in the beams to allow the instrument to compensate for the sudden change in flux. At longer wavelengths, however, there was considerably less energy in the beams, and whenever the instrument scanned through the strong absorption band for carbon dioxide at $4.2 \mu$, a small discontinuity in the curve was observed. However, since this represented a deficiency of the instrument rather than an abrupt change in specimen emittance, a smooth curve was drawn through the minor inflection at $4.2 \mu$. Any error resulting from this procedure would be extremely small and would apply to this one wavelength only.

\section{b. Preferential Mirror Absorption}

A thin protective layer of silicon oxide was present on the aluminum front surface mirrors procured for the transfer optics in the present equipment. Coating layers of this type normally consist of mixtures of such compounds as $\mathrm{SiO}, \mathrm{SiO}_{2}$, and $\mathrm{Si}_{2} \mathrm{O}_{3}$, all of which have absorption bands in the 8 - to $10-\mu$ region [14].

The presence of the silicon oxide layer on the mirrors would have no appreciable effect on the measurements if (a) the same number of mirrors were in both optical paths, (b) the coating thicknesses were the same on all mirrors, and (c) the respective beams struck the mirrors at the same angle for the determination of both the 100 percent and specimen curves. Unfortunately, this last requirement was not fulfilled since the beam from blackbody No. 1 strikes the plane mirror D (see fig. 4) at an angle of incidence of $9^{\circ}$ while the beams from blackbody No. 2 and from the specimen strike the mirror $\mathrm{E}$ at angles of $19^{\circ}$ and $32^{\circ}$, respectively. These differences in angle caused different attenuations in the respective beams at the wavelengths where the silicon oxide layer is highly absorbing but not at other wavelengths.
Because of this differential absorption effect, all 100 percent lines and specimen curves showed a small dip in the 8 to $10 \mu$ region. The emittance curves included in this report have been smoothed out over the 8 - to $10-\mu$ region. Since this smoothing operation is equivalent to taking no data points in this region, the emittances indicated on the curves at both $8.5 \mu$ and at $9 \mu$ have greater uncertainty than those at other wavelengths.

\subsection{Extraneous Transmitted and Reflected Radiation}

In the paragraphs which follow, an attempt is made to place an upper limit on the magnitude of the errors that can arise due to extraneous radiation, i.e., energy which contributes to the radiance of the specirnen beam but does not originate at the specimen.

\section{a. Nonopacity of Specimen Wall}

As mentioned in the introduction, many polycrystalline ceramic oxide specimens are partially transparent to radiation in the visible and the near infrared. Hence, when measuring emittance, if the specimen wall is not sufficiently thick to be opaque, energy from within the specimen cavity will pass through the specimen wall and enter the specimen beam. Since with the present measurement method this energy will approximate blackbody radiation, high emittance rearlings will result unless the wall thickness is sufficient to provide the required opacity.

Earlier work had suggested that a wall thickness of $3 / 16$ in would be adequate for the particular materials selected for study [9]. However, to be certain that the $3 / 16$-in thickness was sufficient in all cases, specimens with $5 / 16$-in walls were also measured. If the emittances were the same for both, then it would be almost positive evidence that the transmittance through the $3 / 16$-in wall was too small to cause an error.

Tests of this type were made for each material. The agreement between specimens with $3 / 16$-in and $5 / 16$-in-thick walls was, in each case, within the precision of measurement; hence, it was concluded that there was no measurable error from wall transmission when the specimens with $3 / 16$-in-thick walls were used.

\section{b. Furnace Radiation Reflected Into Specimen Beam}

The purpose of the water-cooled viewing port in the specimen furnace is to reduce extraneous flux, both emitted and reflected, from entering the specimen beam. Emitted flux is reduced to an insignificant level by water-cooling the walls of the port. To diminish reflected flux, however, the specimen must rotate in close proximity to the inner face of the port; otherwise, as illustrated in figure 8 , flux from the furnace walls will strike the face of the specimen and be diffusely reflected into the specimen beam thereby giving high values of emittance.

It was recognized early in the investigation that large errors could result if sizeable separations were permitted between specimen and viewing port [15]. A theoretical computation of errors caused by various 


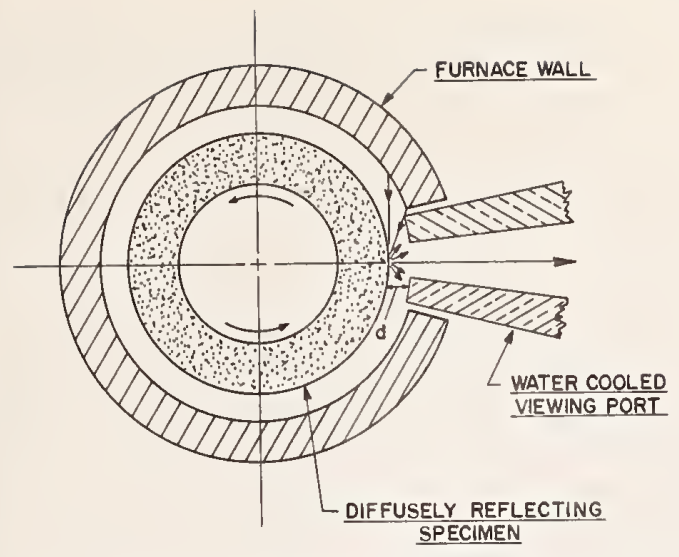

FIGURE 8. Illustration of how fux from furnace walls may be diffusely reflected into specimen beam.

separations was not feasible because of uncertainties in (a) the local furnace wall temperatures, and (b) the optical properties of the specimens. Thus an attempt was made to arrive at their magnitude experimentally.

The experiment consisted of determining the indicated emittance of various specimens at carefully measured distances of separation. To accomplish this, a screw adjustment was incorporated on the brass flange at the bottom of the specimen furnace. By turning this screw, the rotating pedestal could be moved in known increments toward or away from the viewing port. A microscope with a calibrated reticle was used to measure the distance of separation to \pm 0.001 in.

To evaluate the effect of separation distance, the specimen was first brought to a temperature near $1400{ }^{\circ} \mathrm{K}$ with the microscope mounted above the top furnace opening. Next, the specimen was moved forward so that the average separation between the surface of the specimen and the viewing port was $0.010 \mathrm{in.}$ In all of these particular tests, specimen wobble was less than \pm 0.002 in. After completing the distance measurement, the microscope was moved out of position, the top cover reinserted, the furnace temperature allowed to stablize, and a spectral curve determined. Then, by adjusting the calibrated screw, the specimen was moved back from the viewing port in known increments. After each step, the emittance was determined at certain selected wavelengths. Finally, when the limit of travel of the adjusting screw had been reached, the microscope was again used to verify the final separation distance.

The first measurements of this kind were made with a polished platinum specimen. As might be predicted, no change occurred with separation distance, since all wall radiation that struck the viewed area of the specimen was specularly reflected away from the viewing port and did not enter the specimen beam.

When the same platinum specimen was grit-blasted, however, its surface became a partially diffuse reflector and the measured emittance was no longer insensitive to separation distance. This is illustrated by the solid curves in figure 9. At each of the four wavelengths, the rate of change of measured emittance with distance decreased as the distance approached zero. In fact, the shape of the curves suggests that if all measurements had been made with a separation of $0.01 \mathrm{in}$, which is as small as was practical with the present system, the measured values would be high by no more than about 0.005 in emittance.

Platinum, like other metals, is substantially opaque to radiation in the 1 - to $15 \mu$ region. This means that the reflection occurs at or very near the surface. In the case of polycrystalline ceramics, however, reflection occurs largely by internal scattering. Flux striking the surface can penetrate to appreciable distances and then reemerge, after multiple internal reflections, at a point on the surface that may be appreciably separated from the point where it entered. This particular reflection behavior is believed to be responsible for the type of results obtained with the ceramic oxide specimens. The data for an alumina specimen plotted as dashed lines in figure 9 are typical. It will be noted that the rate of change of measured emittance with distance is largely unchanged as the specimen approaches the viewing port. This behavior at least implies that even if a zero separation was possible, radiation from the furnace walls would still be penetrating the surface at some distance from the viewing port and then reemerg. ing at the port to enter the specimen beam. However, when emittances as low as 0.04 (Alumina, $2 \mu, 1200^{\circ} \mathrm{K}$ ) were measured, it seems unlikely that they could be high by more than 0.02 , notwithstanding any compensating errors. Various attempts were made either to eliminate or precisely evaluate the magnitude of this error, but none were successful.

\subsection{Errors From Temperature Uncertainties}

The comparison method of measuring emittance requires that the specimen and blackbody furnaces all be maintained at the same temperature. If this condition is not fulfilled, an error is introduced that is dependent upon (a) the magnitude of the temperature difference, (b) the wavelength, and (c) the test temperature. Since the thermocouples were prepared from wires taken from the same spools and were checked periodically, and since the controller maintained the thermocouples at the same temperature to within better than $1.0^{\circ} \mathrm{K}$, no sizeable error from temperature difference would be expected unless the thermocouples failed to indicate the "true" temperatures of the specimen and the reference blackbody.

With a specimen speed of $100 \mathrm{rpm}$, the analysis described in section 2 indicated that since radial gradients are small, the specimen thermocouple should be in close agreement with the specimen temperature and, hence, no important error should result from this source.

As mentioned in section 3.2.a, temperature gradients existed in the blackbody furnaces. However, 


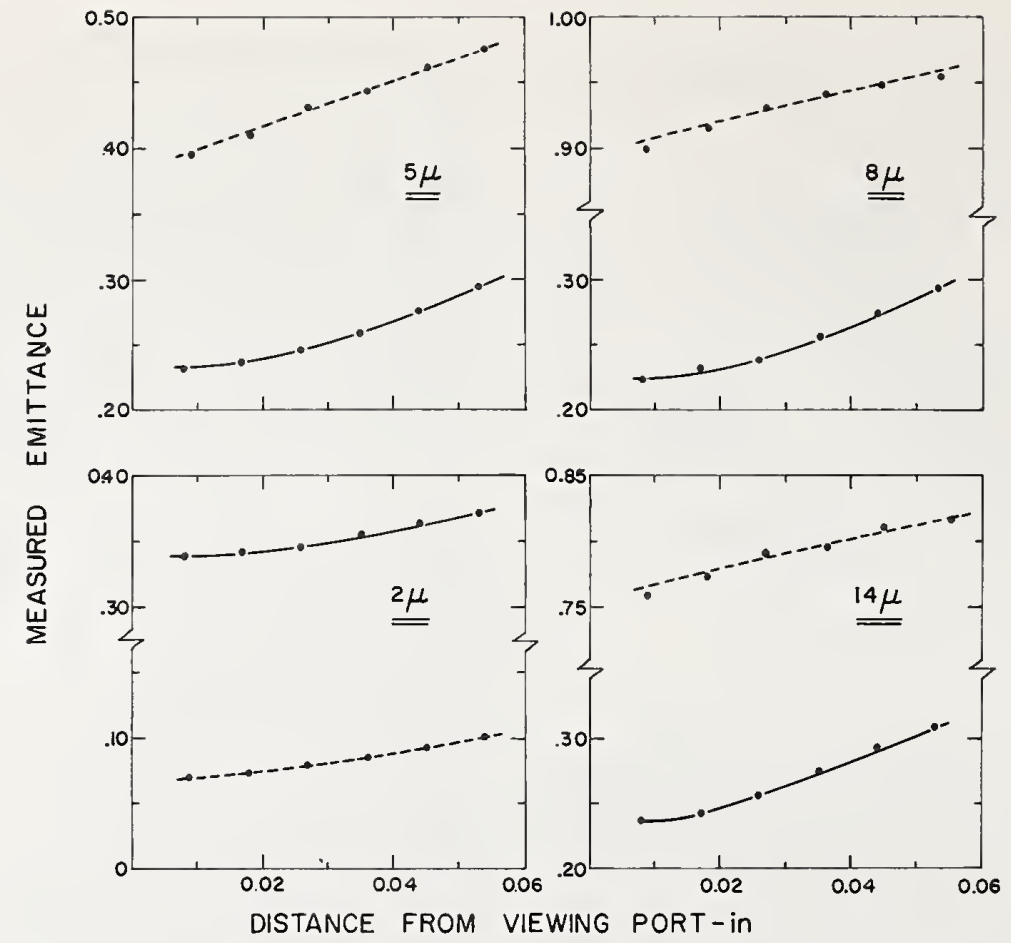

FIGURE 9. Effect of distance of specimen from viewing port on measured emittance of grit-blasted platinum specimen (solid lines) and a polycrystalline alumina specimen (dashed lines).

as shown in appendix $A$, when the thermocouple is located so that its response agrees with an optical pyrometer sighted on the cavity, the distribution of radiated flux at $1 \mu$ differs from a Planck distribution by less than $1 / 2$ percent at $1200^{\circ} \mathrm{K}$. The size of this error decreases with both increasing temperature and wavelength. If the true emittance of the specimen was 0.10 , then an error of the magnitude discussed above would change the measured emittance by only 0.0005 , a completely negligible quantity.

From the foregoing discussion it follows that any uncertainty in temperature measurements will depend primarily upon the accuracy and precision of the optical pyrometer used for positioning the thermocouple. Since the instrument had been carefully calibrated and periodically checked by the National Bureau of Standards Temperature Physics Section, it is unlikely that the combined uncertainty due to inaccuracy of the pyrometer and imprecision introduced by the operator would exceed $\pm 5{ }^{\circ} \mathrm{K}$.

At wavelength $\lambda$, temperature $T$, and uncertainty $d T$, a sample whose true emittance was $\epsilon_{t}$ would appear to have an emittance $\epsilon_{m}$ given by

$$
\epsilon_{m}=\frac{\epsilon_{t} W(\lambda, T)}{W(\lambda, T+d T)}=\frac{\epsilon_{l} W(\lambda, T)}{W(\lambda, T)+d W}
$$

where $W(\lambda, T)$ is the Planck function. Dividing both numerator and denominator by $W(\lambda, T)$ one obtains

$$
\epsilon_{m}=\epsilon_{t} \frac{1}{1+\frac{d W}{W}}=\epsilon_{t} \frac{1}{1+F \frac{d T}{T}}
$$

where the factor $F=F(\lambda T)$ relating $\frac{d W}{W}$ to $\frac{d T}{T}$ is a tabulated function [16].

For the purpose of estimating errors it is more useful to have the true emittance in terms of a measured value. Thus eq (3) is written

$$
\epsilon_{t}=\epsilon_{m}\left(1+F \frac{d T}{T}\right)
$$

If the uncertainty in temperature is $\pm 5{ }^{\circ} \mathrm{K}$ at $T=1200^{\circ} \mathrm{K}$ and $\lambda=2 \mu$, then $F=6.2$ and

$$
\epsilon_{t}=\epsilon_{m} \pm 0.026 \epsilon_{m} \text {. }
$$

Thus, a sample whose measured emittance was 0.050 would be in error by no more than $\epsilon_{t}-\epsilon_{m}= \pm 0.026$ $\times 0.050= \pm 0.0013$. Similarly, if the measured emittance was 0.30 at $2 \mu$ and $1200^{\circ} \mathrm{K}$, the error would be $\epsilon_{t}-\epsilon_{m}= \pm 0.0078$. The function $F$ is a monotonically decreasing function of the product $\lambda T$; emittance, on 
the other hand, generally increases. The net result is that errors of this type are not greatly influenced by changes in either wavelength or temperature.

The effect of a temperature gradient along the axis of the specimen may be treated in much the same way. As pointed out in section 3.1 this gradient amounts to little more than $5^{\circ} \mathrm{K}$ so that some sort of "average" temperature $T^{\prime}$ may be assigned to the specimen. When this is done, it then becomes possible to approximate the flux from the specimen, $N(\lambda)$, by $\epsilon_{t} W\left(\lambda, T^{\prime}\right)$ with very little error. Only if the thermocouple bead was located at the extreme top or bottom of the specimen cavity, would its temperature differ from $T^{\prime}$ by even $3{ }^{\circ} \mathrm{K}$. Since the bead is carefully positioned in the center of the viewed area, it is reasonable to assume, on the basis of the previous discussion, that any errors from temperature differences which may exist along the axis of the specimen are negligible.

\subsection{Random Error of Measurement}

When expressed in terms of emittance, the pooled estimate, $\bar{S}_{w}$, of the standard deviation due to random error of measurement at $\lambda=2 \mu$ was 0.00427 . $\left(\bar{S}_{w}\right.$ is the root mean square value for the $S_{w}$ 's tabulated in table 3). This estimate is based on two completely independent measurements at each of three temperatures on three polycrystalline ceramic oxide specimens of three different materials. ${ }^{5}$ In effect, this standard deviation is an index to the reproducibility of the measurement, and takes into consideration such factors as the ability to mount the specimen on its pedestal with a minimum amount of wobble, the ability to mount each specimen at the same distance from the viewing port, and the ability to return the blackbody thermocouples to the proper position each time the temperature is changed. It also considers the reproducibility of the spectrophotometer, the precision with which the data are read from the charts and corrected for linearity, the day-to-day variations in moisture content of the air and numerous other variables which could affect the reproducibility of the measurements; and finally, its value is influenced by the stability of the specimens, i.e., their ability to resist changes caused by the high temperatures (see sec. 6.2c).

More important, however, is the fact that pooled estimates of the standard deviation due to measurement error, like the one above, may be used to establish a confidence interval for the true averages corresponding to the measured emittances given in table 2 . Thus, using the standard statistical formulas, one may conclude with 95 percent confidence that the values listed in this table at $2 \mu$ are within

$$
\Delta=t \cdot \frac{\bar{S}_{w}}{\sqrt{n p}}=2.052 \frac{0.00427}{\sqrt{6}}=0.00358 \approx 0.004
$$

of the true average values (for these three specimens)

\footnotetext{
"The values of $S_{w}$ for zirconia are abnormally bigh because of the extreme structural instability of this material (see sec. $6.2 \mathrm{c}$ ). For this reason the zirconia data were omitted from this particular analysis.
}

that would be obtained if an infinite number of measurements were made. In the foregoing expression, $\bar{S}_{w}$ is the pooled estimate of the standard deviation due to random measurement errors, and the value of $t_{0.975}$ was obtained from reference [17] for the case of $9 \times 3=27 \mathrm{deg}$ of freedom.

\subsection{Estimated Accuracy}

An estimate of the accuracy of measurements made with the rotating-cylinder equipment on nonmetals can be obtained by considering both types of error; i.e.. those that cause a bias and those that cause random errors. Let us now assume one of the worst conditions from the accuracy standpoint; i.e., data at $2 \mu$ taken at $1200^{\circ} \mathrm{K}$. Error from extraneous radiation would certainly not exceed +0.02 in emittance. Temperature uncertainties would contribute \pm 0.008 as a maximum while random error of measurement will be on the order of \pm 0.004 (see sec. 5.4). Thus, the true values of emittance at $2 \mu$ and $1200^{\circ} \mathrm{K}$ for the particular specimens measured might be as much as 0.032 lower or 0.012 higher than those listed in table 2 .

The uncertainty in the listed values could, of course, be decreased to \pm 0.012 if the data were corrected for the extraneous radiation that entered the specimen beam. However, as mentioned earlier, the uncertainties as to the exact magnitude of this error were too great to justify this procedure.

\section{Measurements on Polycrystalline Oxide Specimens}

\subsection{Description of Specimens}

The ceramic oxide specimens were obtained commercially. They consisted of hollow cylinders $1 \frac{1}{16} 16$-in high and $1 \frac{1}{32}$-in o.d. with a wall thickness of $3 / 16$-in, although wall thickness of $5 / 16$-in were also furnished. All specimens were cylindrical to $\pm 0.002 \mathrm{in}$. The outer surfaces were smooth but not polished.

The sintering treatment for all specimens was $27 \mathrm{hr}$ at $1865^{\circ} \mathrm{K}$. Chemical analyses of the raw materials, as provided by the manufacturer, are shown in table 1 .

The measured porosity of the alumina specimens was about 30 percent; of the thoria 13 percent; of the magnesia 30 percent, and of the zirconia 20 percent. All are given as percentage by volume. Grain sizes were not measured.

No attempts were made to characterize the surface finish of the specimens since it was found that roughening the initially smooth surface of an alumina specimen by grit blasting had no measurable effect on emit. tance in the 1 to $15 \mu$ range. This observation is in agreement with the recent results of Gannon and Linder [18] as well as those reported by Cox [19]. Also, it agrees with the predictions made recently by Richmond as a result of a theoretical analysis of the possible effects of surface roughness on the thermal radiation properties of nonmetals [20]. 
TABLE 1. Vendor's chemical analysis of specimen materials in percentage by weight

\begin{tabular}{|c|c|c|c|c|}
\hline & Alumina a & Thoria " & Magnesia & Zirconia \\
\hline 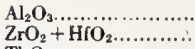 & 99. + & & 0.1 & $\begin{array}{l}0.15 \\
94.2\end{array}$ \\
\hline 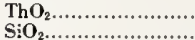 & & $99 .+$ & & \\
\hline $\mathrm{TiO}_{2 \ldots}$ & 0.10 & 0.05 & 0.4 & $\begin{array}{l}0.60 \\
0.30\end{array}$ \\
\hline $\mathrm{CaO} \ldots \ldots \ldots \ldots \ldots \ldots$ & 0.07 & trace & 0.1 & 4.50 \\
\hline $\mathrm{Fe}_{2} \mathrm{O}_{3} \ldots$ & 0.40 & & $\begin{array}{r}99.2 \\
0.1\end{array}$ & $\begin{array}{l}0.07 \\
0.10\end{array}$ \\
\hline
\end{tabular}

Also, 0.02 percent $\mathrm{Na}_{2} \mathrm{O}$

- Also, trace amounts of the oxides of phosphorus, iron, and the rare earths as well as a minor amount of alkali oxides which was expressed in the analysis as 0.10 percent of combined $\mathrm{Na}, \mathrm{K}$, and $\mathbf{L}$.

\subsection{Results and Discussion}

\section{a. Spectral Emittance Data}

Results of measurements on the four oxide materials are given in table 2 and in figures $10,11,12$, and 13. In each case the values listed or plotted are the averages of six determinations, two each on three specimens, taken at $0.5 \mu$ intervals over the 1 to $15 \mu$ range. The small dip in the magnesia curve (fig. 12) at $6.4 \mu$ was observed for all specimens at all three temperatures.

\section{b. Statistical Scatter of Data}

The statistical scatter of the data at 1200, 1400, and $1600{ }^{\circ} \mathrm{K}$ is listed in tables 3 and 4 . The sample esti- mate of the standard deviation due to error of measurement, $S_{w}$ (i.e., due to differences within specimens), was computed from the expression given below [21].

$$
S_{w}=\sqrt{\frac{\sum_{1}^{n}\left(x-\bar{x}_{1}\right)^{2}+\sum_{1}^{n}\left(x-\bar{x}_{2}\right)^{2} \ldots+\sum_{1}^{n}\left(x-\bar{x}_{p}\right)^{2}}{(n-1) p}}
$$

where $x$ is an individual measurement, $\bar{x}_{1}$ is the average of $n$ measurements on one specimen, and $p$ is the number of specimens. For data in this report, $n=2$, $p=3$, and $S_{w}$ corresponds to $(n-1) p=3 \mathrm{deg}$ of freedom.

A sample estimate of the overall standard deviation due to differences in specimens and random errors of measurement $S_{\bar{x}}$ was also computed. The expression used for this computation was

$$
S_{\bar{x}}=\sqrt{\frac{\sum_{1}^{p} \overline{(\bar{x}-\overline{\bar{x}})^{2}}}{p-1}}
$$

where $\overline{\bar{x}}$ is the overall average of the $n \times p$ measurements, $n$ each on $p$ specimens. $S_{\bar{x}}$ corresponds to $p-1=2 \mathrm{deg}$ of freedom. One could, if it were desired, use the values of $S_{\bar{x}}$ given in table 4 to set up a confidence interval for the true mean of the entire population of specimens similar to those described

TABLE 2. Normal spectral emittance of ceramic oxide specimens

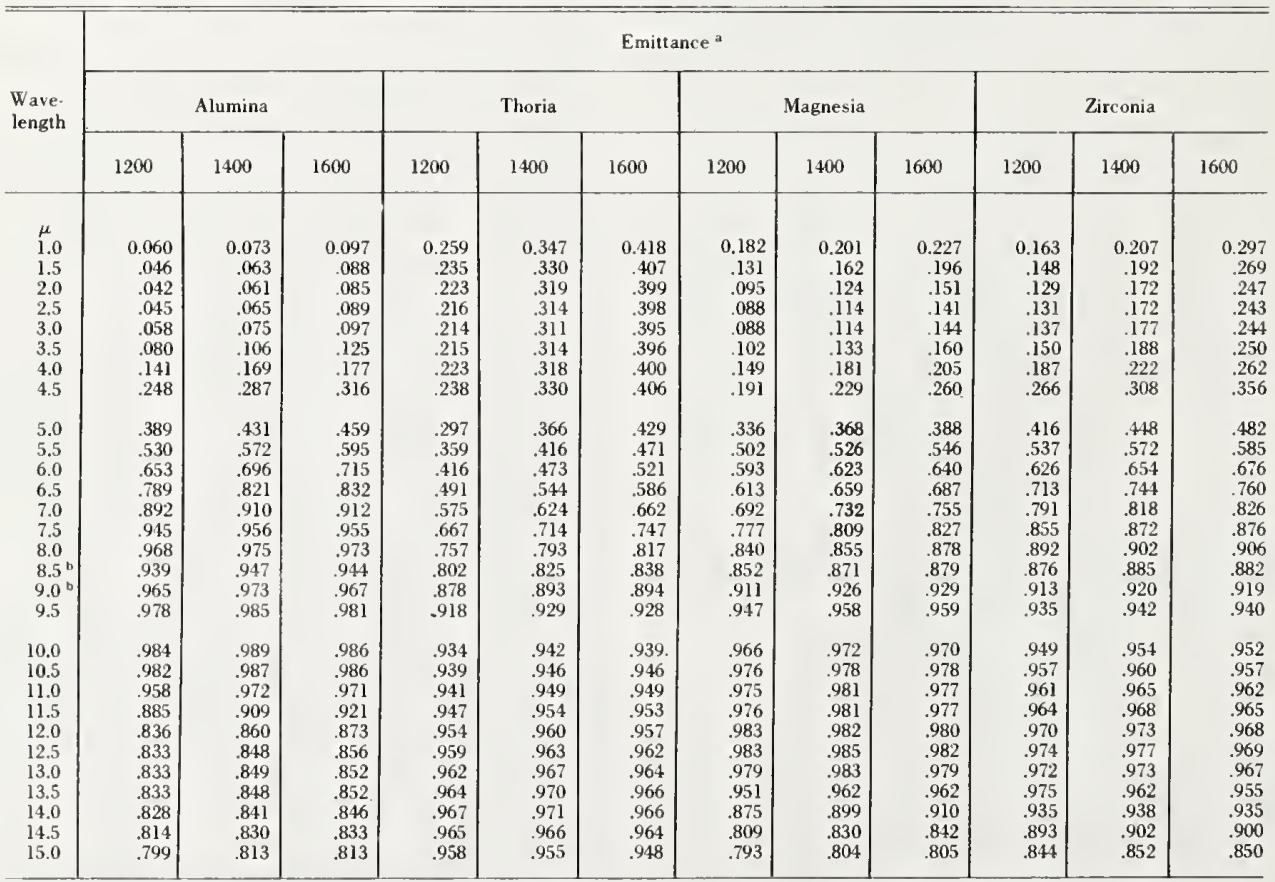

${ }^{B}$ Average of two readings each on three specimens.

${ }^{b}$ Emittance values low because of error from preferential mirror absorption (sect, 5.1). 


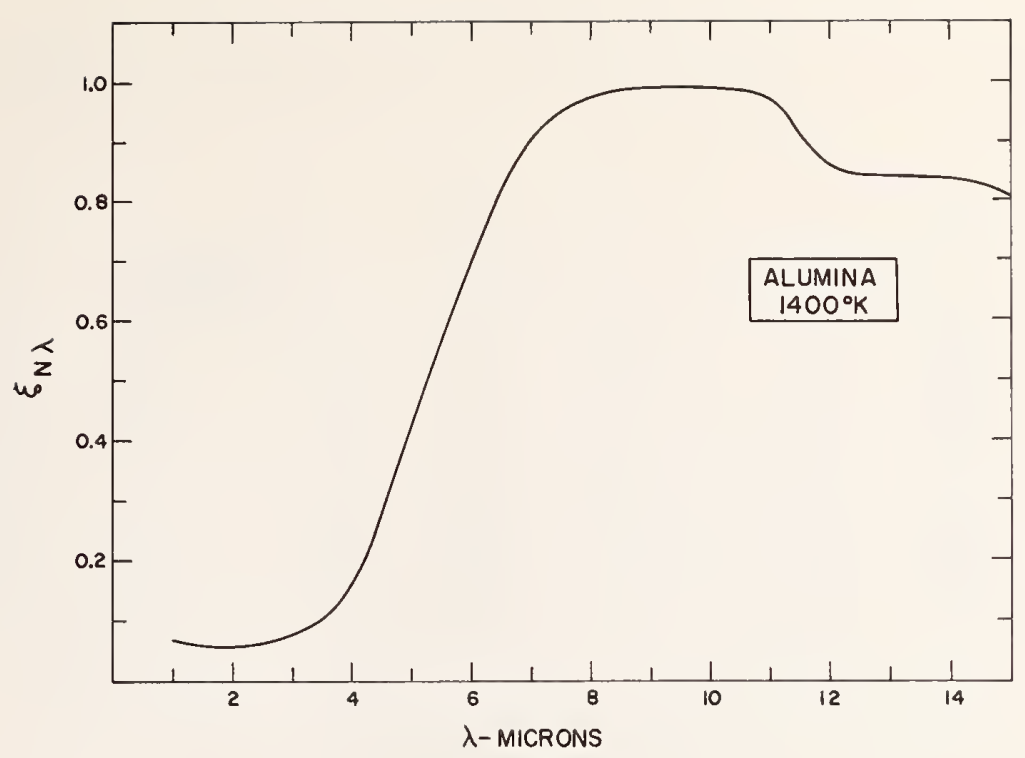

FIGURE 10. Normal spectral emittance of commercially pure alumina.

Curve represents average from six determinations; two each on three specimens.

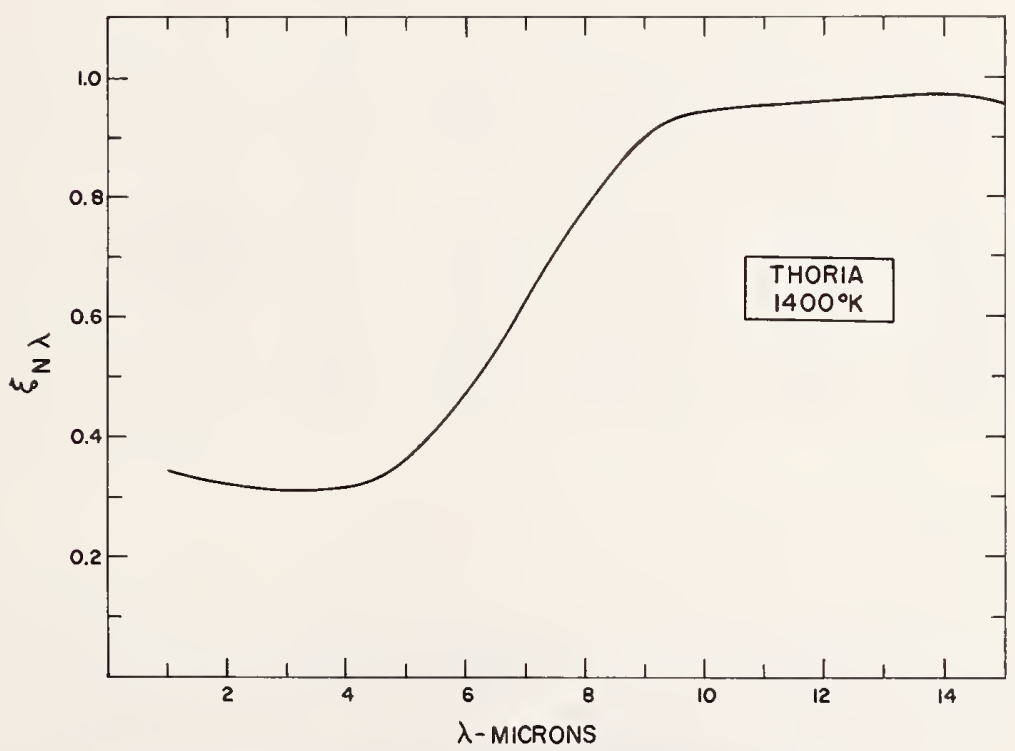

FIGURE 11. Normal spectral emittance of commercially pure thoria.

Curve represents average from six determinations; two each on three specimens.

in this report. Also, it is interesting to note that the two standard deviations are related in the following way

$$
S_{\bar{x}}^{2}=\frac{S_{w}^{2}+n S_{b}^{2}}{n}
$$

where $S_{b}$ is the sample estimate of the standard deviation due to differences between specimens.
As shown in table 3 , the values of $S_{w}$ fall in the range 0.001 to 0.021 and are largely independent of temperature. Their magnitude, however, is a function of wavelength, the values being appreciably larger at the short wavelengths than at the long. The values of $S_{\bar{x}}$, on the other hand, are less well behaved due to the manner in which they depend upon differences between specimens. For example, the average value of $S_{\bar{x}}$ is about 0.008 in the wavelength region 1 to $6 \mu$ 


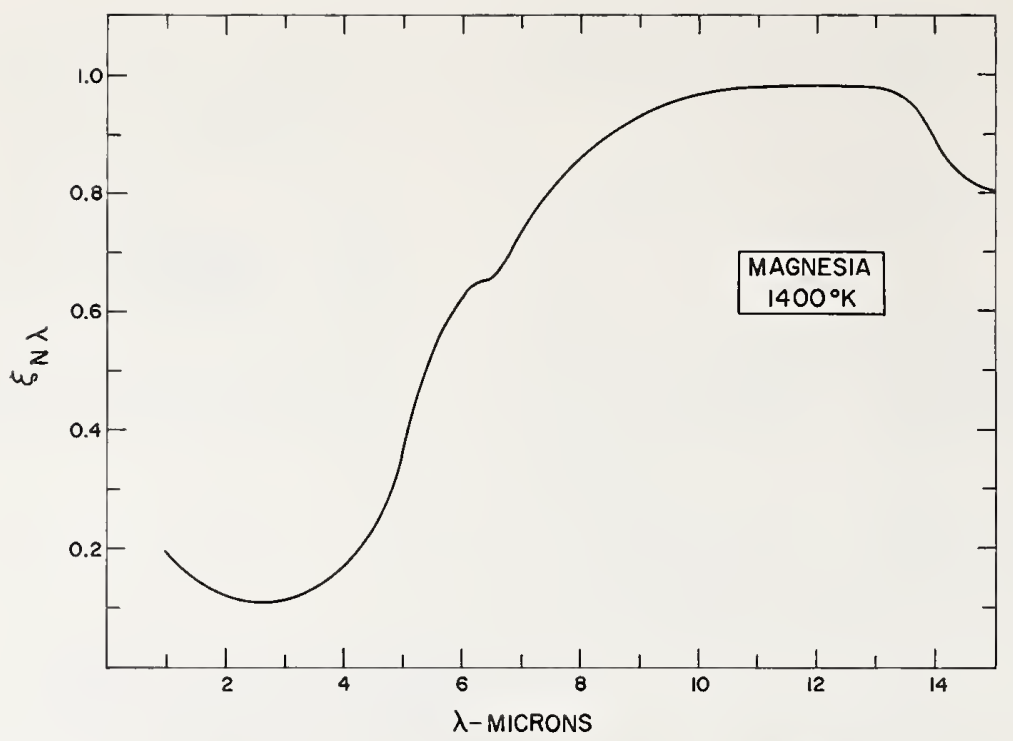

FigURE 12. Normal spectral emittance of commercially pure magnesia. Curve represents average from six determinations; two each on three specimens.

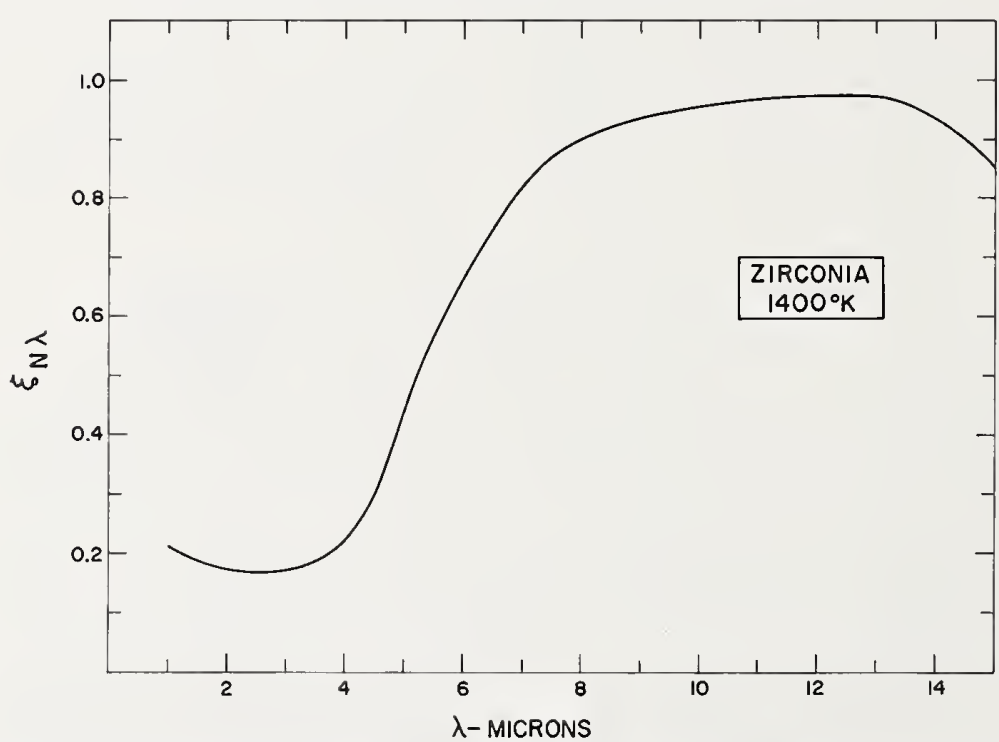

FIGURE 13. Normal spectral emittance of commercially pure, lime-stabilized zirconia. Curve represents average from six determinations; two each on three specimens.

for the alumina, magnesia, and zirconia specimens, but for thoria the average is approximately 0.041 over this same region. Visual examination of the thoria specimens revealed that one of the three $(\mathrm{T}-10)$ was darker in color than the other two. This was the same specimen that yielded high emittance in the short wavelength regions. For example, at $2 \mu$ the average emittances from the two determinations on the three thoria specimens were as follows:

\begin{tabular}{|c|c|c|c|}
\hline \multirow[t]{2}{*}{ Spec. No. } & \multicolumn{3}{|c|}{ Ave. $\epsilon_{n \lambda}$ at $2 \mu$} \\
\hline & $1200^{\circ} \mathrm{K}$ & $1400^{\circ} \mathrm{K}$ & $1600^{\circ} \mathrm{K}$ \\
\hline $\begin{array}{l}\mathrm{T}-10 \ldots \ldots \ldots \ldots \\
\mathrm{T}-11 \ldots \ldots \ldots \ldots \\
\mathrm{T}-12 \ldots \ldots \ldots \ldots \ldots\end{array}$ & $\begin{array}{r}0.276 \\
.201 \\
.193\end{array}$ & $\begin{array}{r}0.367 \\
.299 \\
. .292\end{array}$ & $\begin{array}{r}0.437 \\
.382 \\
.377\end{array}$ \\
\hline
\end{tabular}


TABLE 3. Standard deviations due to error in measurement

Sample estimate, $S_{w,}$ of standard deviation due to error in measurement ${ }^{*}$

\begin{tabular}{|c|c|c|c|c|c|c|c|c|c|c|c|c|}
\hline \multirow{2}{*}{ Wavelength } & \multicolumn{3}{|c|}{ Alumina } & \multicolumn{3}{|c|}{ Thoria } & \multicolumn{3}{|c|}{ Magnesia } & \multicolumn{3}{|c|}{ Zirconia } \\
\hline & 1200 & 1400 & 1600 & 1200 & 1400 & 1600 & 1200 & 1400 & 1600 & 1200 & 1400 & 1600 \\
\hline $\begin{array}{l}1 \\
2 \\
3 \\
4 \\
5\end{array}$ & $\begin{array}{r}0.00376 \\
.00058 \\
.00684 \\
.00689 \\
.00793\end{array}$ & $\begin{array}{r}0.00163 \\
.00372 \\
.01470 \\
.00801 \\
.00696\end{array}$ & $\begin{array}{r}0.00464 \\
.00212 \\
.01390 \\
.00954 \\
.00793\end{array}$ & $\begin{array}{r}0.00582 \\
.00570 \\
.00493 \\
.00777 \\
.00927\end{array}$ & $\begin{array}{r}0.00862 \\
.00404 \\
.00464 \\
.00919 \\
.01099\end{array}$ & $\begin{array}{r}0.00367 \\
.00706 \\
.01173 \\
.01358 \\
.01389\end{array}$ & $\begin{array}{r}0.00141 \\
.00314 \\
.00346 \\
.00671 \\
.00757\end{array}$ & $\begin{array}{r}0.00091 \\
.00319 \\
.00684 \\
.01021 \\
.00933\end{array}$ & $\begin{array}{r}0.00408 \\
.00520 \\
.00890 \\
.01390 \\
.01507\end{array}$ & $\begin{array}{r}0.00780 \\
.01511 \\
.01674 \\
.02071 \\
.01618\end{array}$ & $\begin{array}{r}0.00523 \\
.01185 \\
.01380 \\
.01652 \\
.01363\end{array}$ & $\begin{array}{r}0.00410 \\
.00700 \\
.00889 \\
.01266 \\
.00881\end{array}$ \\
\hline $\begin{array}{r}6 \\
7 \\
8 \\
9 \\
10\end{array}$ & $\begin{array}{l}.00850 \\
.00153 \\
.00091 \\
.00220 \\
.00187\end{array}$ & $\begin{array}{l}.00478 \\
.00289 \\
.00220 \\
.00235 \\
.00242\end{array}$ & $\begin{array}{l}.00635 \\
.00507 \\
.00396 \\
.00449 \\
.00465\end{array}$ & $\begin{array}{l}.01002 \\
.00389 \\
.00534 \\
.00351 \\
.00402\end{array}$ & $\begin{array}{l}.01179 \\
.01797 \\
.00456 \\
.00379 \\
.00361\end{array}$ & $\begin{array}{l}.01488 \\
.00751 \\
.00346 \\
.00416 \\
.00329\end{array}$ & $\begin{array}{l}.00814 \\
.00332 \\
.00297 \\
.00349 \\
.00376\end{array}$ & $\begin{array}{l}.00458 \\
.00615 \\
.00370 \\
.00204 \\
.00300\end{array}$ & $\begin{array}{l}.0075] \\
.00809 \\
.00464 \\
.00238 \\
.00314\end{array}$ & $\begin{array}{l}.01081 \\
.00835 \\
.00292 \\
.00420 \\
.00297\end{array}$ & $\begin{array}{l}.01034 \\
.00523 \\
.00168 \\
.00300 \\
.00204\end{array}$ & $\begin{array}{l}.00498 \\
.00493 \\
.00394 \\
.00204 \\
.00402\end{array}$ \\
\hline $\begin{array}{l}11 \\
12 \\
13 \\
14 \\
15\end{array}$ & $\begin{array}{l}.00277 \\
.00518 \\
.00513 \\
.00670 \\
.00780\end{array}$ & $\begin{array}{l}.00321 \\
.00520 \\
.00451 \\
.00513 \\
.00822\end{array}$ & $\begin{array}{l}.00385 \\
.00486 \\
.00754 \\
.00802 \\
.00883\end{array}$ & $\begin{array}{l}.00406 \\
.00458 \\
.00332 \\
.00389 \\
.00058\end{array}$ & $\begin{array}{l}.00208 \\
.00372 \\
.00351 \\
.00449 \\
.00614\end{array}$ & $\begin{array}{l}.00523 \\
.00274 \\
.00300 \\
.00442 \\
.00890\end{array}$ & $\begin{array}{l}.00332 \\
.00385 \\
.00416 \\
.00574 \\
.00518\end{array}$ & $\begin{array}{l}.00337 \\
.00332 \\
.00321 \\
.00505 \\
.00596\end{array}$ & $\begin{array}{l}.00122 \\
.00231 \\
.00122 \\
.00480 \\
.01122\end{array}$ & $\begin{array}{l}.00422 \\
.00168 \\
.00129 \\
.00071 \\
.00464\end{array}$ & $\begin{array}{l}.00258 \\
.00410 \\
.00311 \\
.00178 \\
.00297\end{array}$ & $\begin{array}{l}.00245 \\
.00306 \\
.00041 \\
.00387 \\
.00367\end{array}$ \\
\hline
\end{tabular}

"Each entry corresponds to three degrees of freedom.

TABLE 4. Overall standard deviation

Standard deviation, $S_{\bar{x}}$, dūe to differences between specimens and random error of measurement ${ }^{\text {a }}$

\begin{tabular}{|c|c|c|c|c|c|c|c|c|c|c|c|c|}
\hline \multirow{2}{*}{ Wavelength } & \multicolumn{3}{|c|}{ Alumina } & \multicolumn{3}{|c|}{ Thoria } & \multicolumn{3}{|c|}{ Magnesia } & \multicolumn{3}{|c|}{ Zirconia } \\
\hline & 1200 & 1400 & 1600 & 1200 & 1400 & 1600 & 1200 & 1400 & 1600 & 1200 & 1400 & 1600 \\
\hline $\begin{array}{l}1 \\
2 \\
3 \\
4 \\
5\end{array}$ & $\begin{array}{r}0.00407 \\
.00486 \\
.00597 \\
.00541 \\
.00937\end{array}$ & $\begin{array}{r}0.00721 \\
.00764 \\
.01169 \\
.01125 \\
.00854\end{array}$ & $\begin{array}{r}0.01077 \\
.01258 \\
.01473 \\
.01136 \\
.00975\end{array}$ & $\begin{array}{r}0.04497 \\
.04612 \\
.04418 \\
.04571 \\
.04258\end{array}$ & $\begin{array}{r}0.03311 \\
.04102 \\
.04526 \\
.04592 \\
.04217\end{array}$ & $\begin{array}{r}0.02943 \\
.03362 \\
.03597 \\
.04024 \\
.03892\end{array}$ & $\begin{array}{r}0.00624 \\
.00300 \\
.00400 \\
.00530 \\
.00624\end{array}$ & $\begin{array}{r}0.00673 \\
.00687 \\
.00776 \\
.01025 \\
.00889\end{array}$ & $\begin{array}{r}0.00757 \\
.00660 \\
.00889 \\
.01159 \\
.01002\end{array}$ & $\begin{array}{r}0.00465 \\
.00553 \\
.00676 \\
.00912 \\
.00879\end{array}$ & $\begin{array}{r}0.00416 \\
.00525 \\
.00633 \\
.00725 \\
.00606\end{array}$ & $\begin{array}{r}0.00679 \\
.00939 \\
.01225 \\
.01334 \\
.01190\end{array}$ \\
\hline $\begin{array}{r}6 \\
7 \\
8 \\
9 \\
10\end{array}$ & $\begin{array}{l}.00705 \\
.00597 \\
.00650 \\
.00375 \\
.00568\end{array}$ & $\begin{array}{l}.00701 \\
.00247 \\
.00475 \\
.00401 \\
.00379\end{array}$ & $\begin{array}{l}.00752 \\
.00626 \\
.00633 \\
.00626 \\
.00425\end{array}$ & $\begin{array}{l}.03744 \\
.02948 \\
.01779 \\
.00275 \\
.00293\end{array}$ & $\begin{array}{l}.03783 \\
.03248 \\
.02019 \\
.00729 \\
.00355\end{array}$ & $\begin{array}{l}.03646 \\
.02980 \\
.01473 \\
.00404 \\
.00325\end{array}$ & $\begin{array}{l}.00407 \\
.00391 \\
.00419 \\
.00401 \\
.00377\end{array}$ & $\begin{array}{l}.00541 \\
.00379 \\
.00650 \\
.00377 \\
.00189\end{array}$ & $\begin{array}{l}.00695 \\
.00486 \\
.00333 \\
.00318 \\
.00300\end{array}$ & $\begin{array}{l}.00621 \\
.00318 \\
.00306 \\
.00150 \\
.00236\end{array}$ & $\begin{array}{l}.00797 \\
.00551 \\
.00362 \\
.00176 \\
.00257\end{array}$ & $\begin{array}{l}.00855 \\
.00525 \\
.00161 \\
.00050 \\
.00132\end{array}$ \\
\hline $\begin{array}{l}11 \\
12 \\
13 \\
14 \\
15\end{array}$ & $\begin{array}{l}.00527 \\
.00454 \\
.00605 \\
.00825 \\
.00813\end{array}$ & $\begin{array}{l}.00425 \\
.00522 \\
.00725 \\
.00759 \\
.00978\end{array}$ & $\begin{array}{l}.00548 \\
.00625 \\
.00737 \\
.00804 \\
.00709\end{array}$ & $\begin{array}{l}.00321 \\
.00444 \\
.00275 \\
.00586 \\
.00391\end{array}$ & $\begin{array}{l}.00180 \\
.00153 \\
.00328 \\
.00225 \\
.00501\end{array}$ & $\begin{array}{l}.00200 \\
.00229 \\
.00257 \\
.00202 \\
.00306\end{array}$ & $\begin{array}{l}.00375 \\
.00506 \\
.00404 \\
.00506 \\
.00501\end{array}$ & $\begin{array}{l}.00306 \\
.00325 \\
.00247 \\
.00701 \\
.00477\end{array}$ & $\begin{array}{l}.00202 \\
.00351 \\
.00278 \\
.00506 \\
.00666\end{array}$ & $\begin{array}{l}.00100 \\
.00029 \\
.00318 \\
.00058 \\
.00350\end{array}$ & $\begin{array}{l}.00153 \\
.00229 \\
.00126 \\
.00321 \\
.00104\end{array}$ & $\begin{array}{l}.00000 \\
.00058 \\
.00293 \\
.00328 \\
.00450\end{array}$ \\
\hline
\end{tabular}

a Each entry corresponds to two degrees of freedom.

At $10 \mu$ and beyond all three thoria specimens were in excellent agreement as indicated by the $S_{\vec{x}}$ values plotted in figure 14. This observation suggests that minor variations in composition can have appreciable effects on the emittance at low wavelengths without causing any significant changes in the longer wavelength region.

\section{c. Lack of Stability of Zirconia Specimens}

The first shipment of zirconia specimens showed poor reproducibility of the emittance curves for repeated determinations on the same specimen. Since the degree of reproducibility seemed to be associated with the time of testing at $1600{ }^{\circ} \mathrm{K}$, a series of hourly measurements was made on a zirconia specimen maintained at that temperature. The emittances were found to progressively increase at the short wavelengths and progressively decrease at the longer wavelengths. The observed changes after $4 \frac{1 / 2}{2} \mathrm{hr}$ at $1600{ }^{\circ} \mathrm{K}$ are shown in figure 15 . X-ray diffraction patterns made before and after the $1600^{\circ} \mathrm{K}$ treatment showed that the zirconia had become partially destabilized. Before testing the crystal structure was primarily cubic; however, after $5 \mathrm{hr}$ at $1600{ }^{\circ} \mathrm{K}$, the percentage of the monoclinic form had increased by a factor of about three. Thus, although calcium oxide had been added to the zirconia to "stabilize" it as a cubic solid-solution crystal [22], the degree of stabilization actually achieved was inadequate. A later shipment of zirconia showed less significant changes in emittance after a similar test at $1600^{\circ} \mathrm{K}$. The data reported in the tables were obtained with zirconia specimens from the latter shipment. 


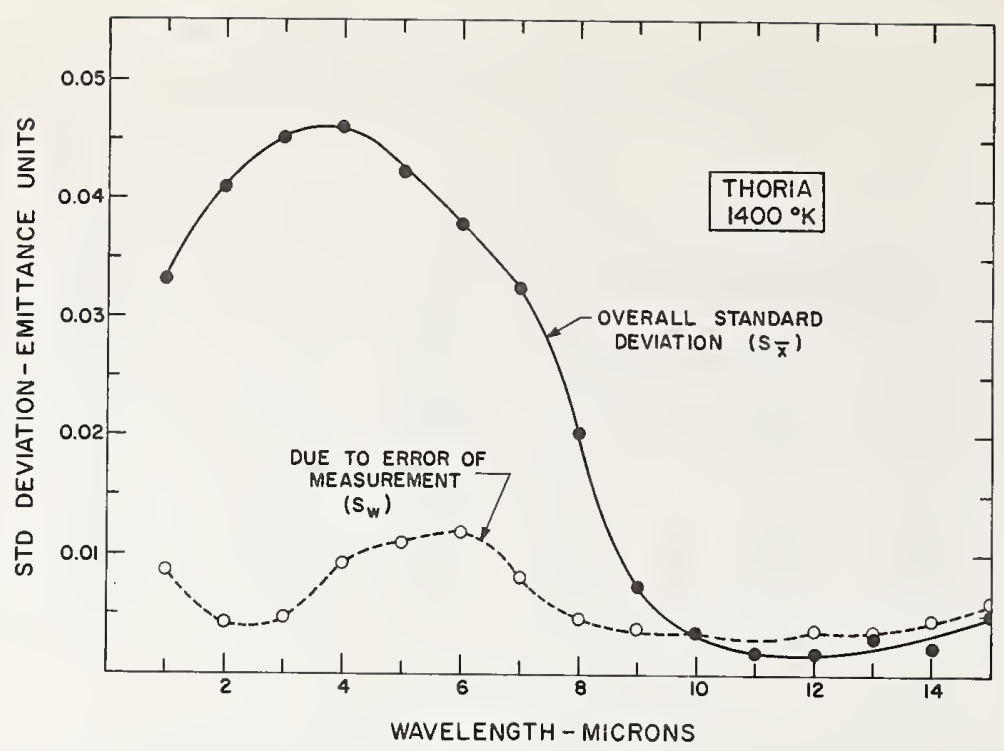

Figure 14. Standard deviation due to error of measurement $\left(\mathrm{S}_{\mathrm{w}}\right)$ and overall stand. ard deviation $\left(S_{\overline{\mathrm{x}}}\right)$ for the six thoria determinations.

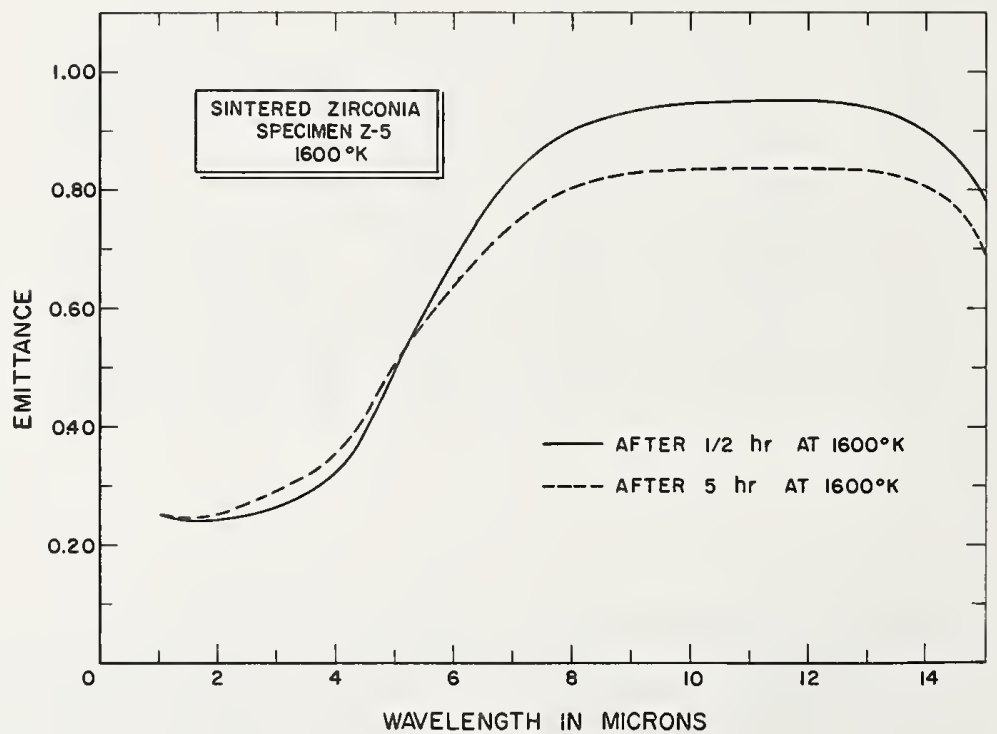

FIGURE 15. Effect of $5 \mathrm{hr}$ heating at $1600^{\circ} \mathrm{K}$ on emittance of poorly stabilized zirconia specimens (first lot).

Later shipment did not show this behavior.

\section{d. Effect of Temperature on Emittance}

Inspection of table 2 shows that the emittance of all four materials increases with increasing temperature. As shown in table 5 the magnitude of the increase is much less at the longer wavelengths, where the emittances are high, than at shorter wavelengths where the emittances are low. In fact, in a few cases at the long wavelengths no measurable change was observed, and for three cases in table 5 a small negative coefficient is reported, although these negative values are not believed to be real. 
TABLE 5. Average increases in emiltance per $100^{\circ} \mathrm{K}$ over 1200 to $1600^{\circ} \mathrm{K}$ temperature range

\begin{tabular}{|c|c|c|c|c|}
\hline \multirow{2}{*}{$\begin{array}{l}\text { Wavelength } \\
\text { in microns }\end{array}$} & \multicolumn{4}{|c|}{ Average increase in $\epsilon_{\wedge}$ per $100^{\circ} \mathrm{K}$. } \\
\hline & Alumina & Thoria & Magnesia & Zirconie \\
\hline 1 & 0.009 & 0.040 & 0.011 & \\
\hline 2 & .011 & .044 & .014 & .030 \\
\hline 3 & .010 & .045 & .014 & .027 \\
\hline 4 & .009 & .044 & .014 & .019 \\
\hline 5 & .018 & .033 & .013 & .017 \\
\hline 6 & .016 & .026 & .012 & .012 \\
\hline 7 & .005 & .022 & .016 & .009 \\
\hline 8 & .001 & .015 & .010 & .004 \\
\hline 9 & 0 & .004 & .005 & .002 \\
\hline 10 & 0 & .001 & .001 & .001 \\
\hline 11 & .003 & .002 & .001 & 0 \\
\hline 12 & .009 & .001 & -.001 & -.001 \\
\hline 13 & .005 & 0 & 0 & -.001 \\
\hline 14 & .004 & 0 & .009 & 0 \\
\hline 15 & .004 & -.002 & .003 & .002 \\
\hline
\end{tabular}

a Computed by subtracting emittance at $1200^{\circ} \mathrm{K}$ from emittance at $1600^{\circ} \mathrm{K}$ and dividing by four.

Some metals have been shown to have a negative temperature coefficient of spectral emittance in the very short wavelength regions and a positive coefficient at the longer wavelengths [23]. This means that a "crossover" or " $x$-point" exists in the emittance curves, and at the wavelength of this " $x$-point" the spectral emittance is independent of temperature. Comparison of the rotating-cylinder data for a platinum-13 percent rhodium alloy with other types of measurements made in the same laboratory by other methods $[13,24,25]$ strongly suggests that an " $x$ point" exists for this alloy also. This comparison is shown in figure 16.

No such crossover points were observed for the oxide specimens in the particular wavelength and temperature ranges investigated. At the long wavelengths the temperature dependence approaches zero but since the emittances in this region are already above 0.90 , it would obviously be impossible for the emittances to increase by any appreciable extent with temperature.

Figures 17 and 18 compare the changes in emittance with temperature obtained by the rotating-cylinder with those reported by other investigators $[7,26,27$, and 28]. The agreement between the reported values is. better at $10.0 \mu$ than at $2.0 \mu$. At both wavelengths, however, the trend is toward positive coefficients.

For this relatively narrow temperature range the data points obtained from the present measurements are roughly linear with temperature. This relation. ship can not be expected to apply in general. In fact, it does not even extend downward to room temperature as indicated by the curves plotted in figure 19 . The room-temperature $\left(300^{\circ} \mathrm{K}\right)$ points were computed from reflectance measurements made with an ellipsoidal reflectometer that measures absolute reflectance of specimens irrespective of whether they are specular, diffuse, or only partially diffuse in their reflection behavior [24]. The specimens were prepared with the same materials and processing used for the cylinders. Since the measurements were made under near-normal illumination and hemispherical viewing, the reflec-

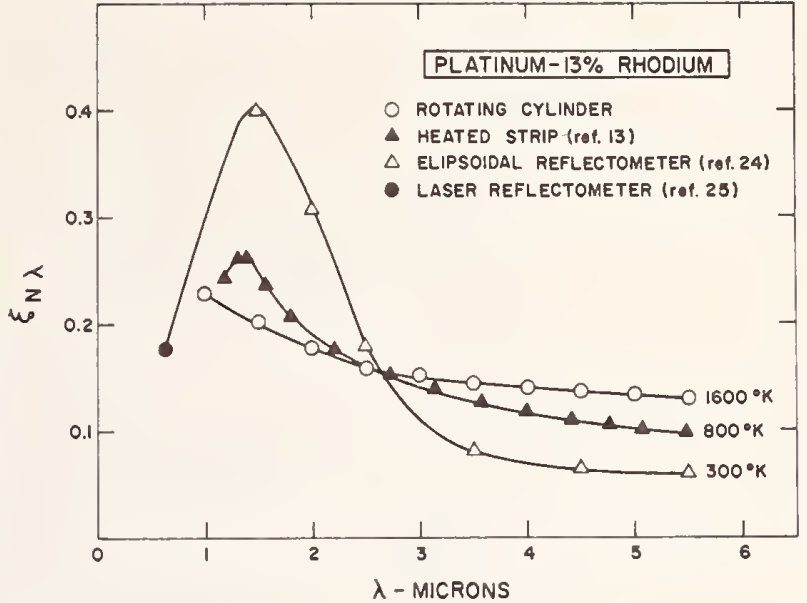

FIGURE 16. Emittances of a platinum-13 percent rhodium alloy at three temperatures.

Determinations by all three methodo were made in air on specimens that had been heated for $1 \mathrm{hr}$ at $1850^{\circ} \mathrm{K}$ prior to measurement.

tances can, in accordance with Kirchhoff's law, be converted to spectral normal emittance by subtracting the reflectances from unity. The fact that the points computed from the room-temperature reflectances fall reasonably well in line with the rotating-cylinder points tends to lend credence to both sets of data.

\section{e. Comments on Use of Data for Heat Transfer Computations}

In the past many heat transfer computations have been made with the assumption that the spectral emittance does not change greatly with temperature. The present results indicate that this is not a safe assumption for the polycrystalline oxide materials. Thoria, for example, shows almost a fourfold increase in emittance at $2 \mu$ when the temperature is raised from 300 to $1600{ }^{\circ} \mathrm{K}$. Many more data of this type on temperature dependence are needed before completely reliable heat transfer analyses will be possible. Temperature dependence data for both metals and nonmetals below room temperature are practically nonexistent and emittance data suitable for computing temperature coefficients are notably absent. The reflectance approach has been suggested as a means of obtaining these data because of its potentially greater accuracy $[13,24]$. Much can be said for this approach at low temperatures, but at high temperatures difficulties can be expected because of noise in the signal caused by the high background of radiant flux that is emitted from the specimen. Therefore, it seems likely that at temperatures above about 1200 ${ }^{\circ} \mathrm{K}$, direct measurements of emittance will continue to provide the most reliable data.

The emittances presented in the tables and figures of this report are for specimens of the compositions and structures outlined in section 6.1. Specimens prepared by different processing from materials of different purity, or from materials with different types of impurities, would be expected to show somewhat 


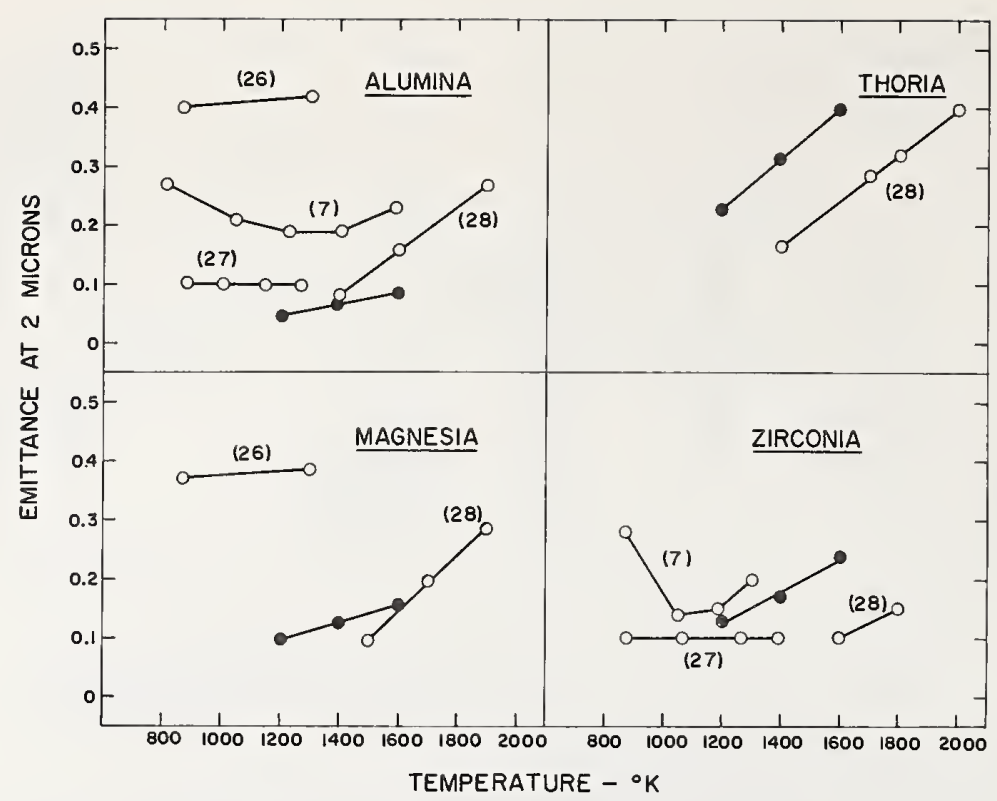

FIGURE 17. Comparison of reported effects of temperature on normal spectral emittance at $2 \mu$.

Source of data is given by reference number in brackets.

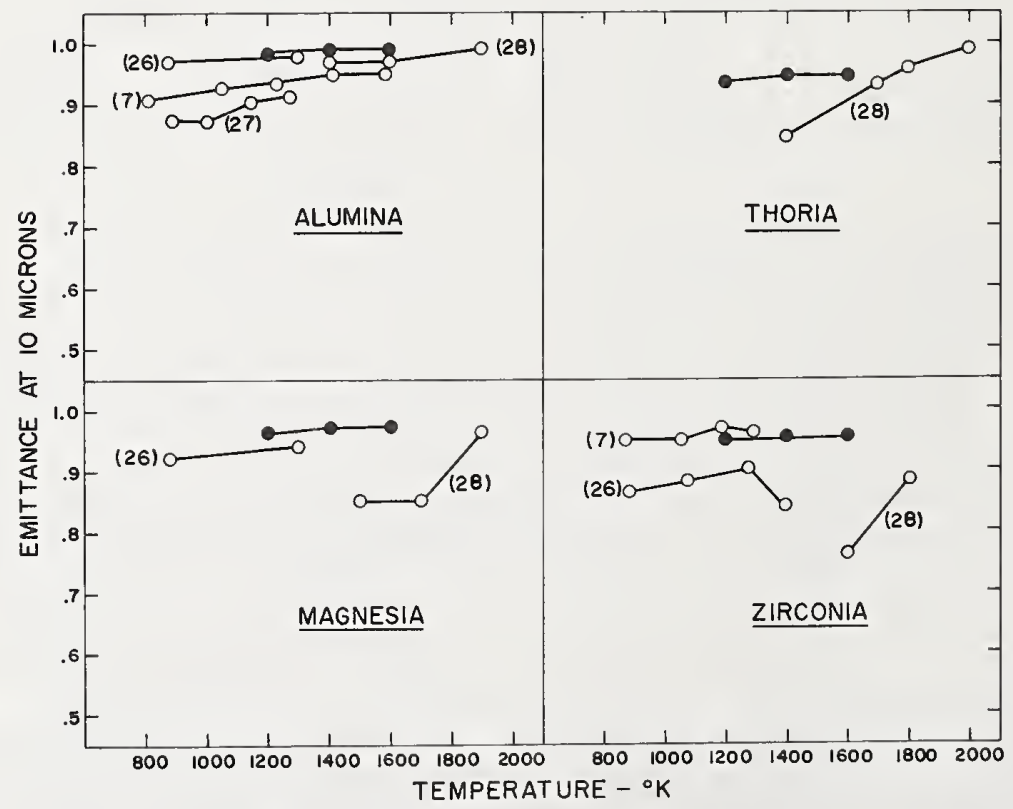

FIGURE 18. Comparison of reported effects of temperature on normal spectral emittance at $10 \mu$.

Source of data is given by reference number in brackets. 


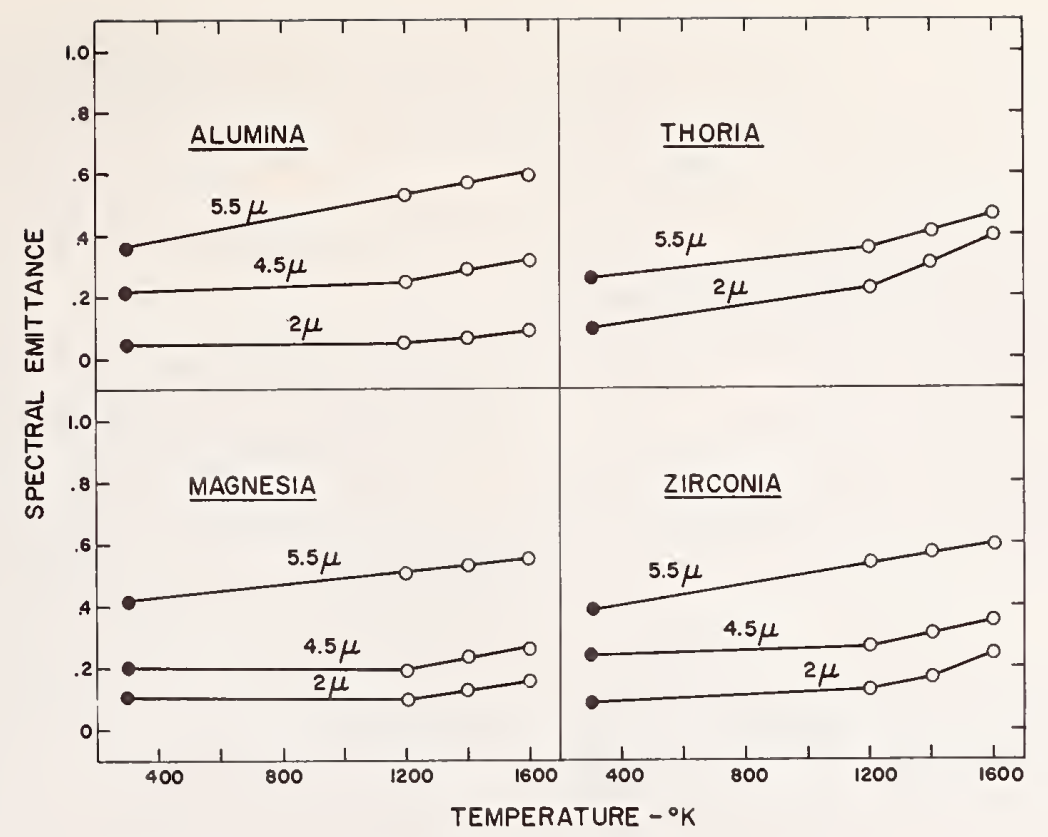

FIgURE 19. Temperature dependence of normal spectral emittance.

Open circles are rotating specimen data; closed circles were computed from reflectance data measured with ellipsoidal mirror reflectometer (reference 24 ).

different emittances, especially in the short wavelength region. For this reason, the emittances as reported herein should not be taken as the emittance of all alumina, thoria, magnesia, or zirconia materials. The only method that will define the thermal radiation properties of any given engineering material is actual measurement by a reliable technique.

Temperature gradients will normally exist in oxide parts used in engineering applications inasmuch as these parts are usually heated in such a way that one or more of the surfaces is free to radiate to ambient. Emittances measured for a specimen with no temperature gradients could of course be used for heat transfer calculations for such parts if the gradients were small. In those cases where the gradients are large, however, the emittances determined for isothermal specimens should be used with caution because of the sizable changes of $\epsilon_{n \lambda}$ with temperature that occur in some wavelength regions (table 3 ).

\section{f. Correlation of Data for Alumina With Reported Absorption Coefficients}

The changes observed in normal spectral emittance, $\epsilon_{n \lambda}$, with both wavelength and.temperature undoubt. edly reflect changes that are occurring in the optical properties of the materials. Recently Gryvnak and Burch [29] measured the absorption coefficient of single crystal alumina (sapphire) from room-temperature to the melting point. Figure 20 is copied from their data. It will be noted that the absorption coefficient of sapphire increases with increasing tem-

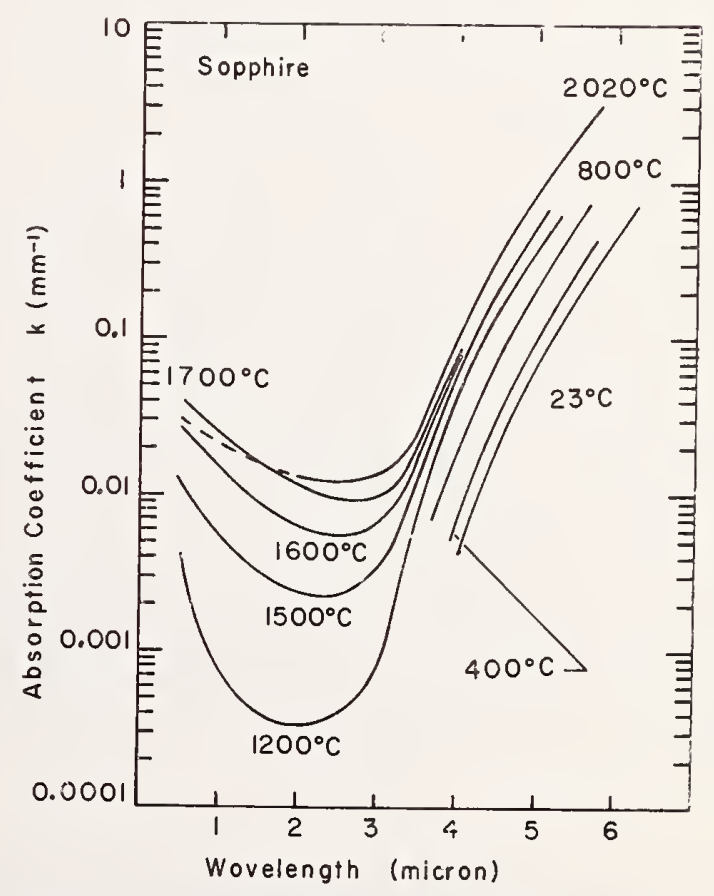

Figure 20. Absorption coefficient-wavelength curves for single crystal alumina as reported by Gryvnak and Burch (reference 29). 
perature and that the temperature dependence is greater at the shorter than at the longer wavelengths. The temperature dependence of emittance shows a somewhat similar behavior (table 3 ). This similarity is to be expected since, from a reflectance analogy, as absorption within the material increases the amount of the incident energy that emerges from the surface as reflected energy decreases. Therefore, the reflectance will decrease with increasing absorption coeffcient, and the emittance will increase.

Figure 20 shows a dip in the absorption coefflcientwavelength curves in the range 1 to $3 \mu$. As shown in figure 10 , a slight dip was also found when $\epsilon_{n \lambda}$, for the polycrystalline alumina, was plotted against wavelength. This dip, however, was not as pronounced for alumina as it was for magnesia (fig. 12), and zirconia (fig. 13).

\section{Summary}

Equipment is described for measurement of the normal spectral emittance of polycrystalline ceramics in the temperature range $1200-1600^{\circ} \mathrm{K}$. Specimens consisted of hollow cylinders that were rotated at $100 \mathrm{rpm}$ in a furnace enclosure before a water-cooled viewing port.

Various sources of error were investigated. The major source was determined to be furnace radiation that was reflected and/or scattered by the specimen into the specimen beam. At $2 \mu$ and $1200^{\circ} \mathrm{K}$, this effect could cause measured values of emittance to be as much as +0.02 higher than the true value. Temperature uncertainties would contribute \pm 0.008 as a maximum while the random error of measurement will be on the order of \pm 0.004 . Thus, the true values of emittance at $2 \dot{\mu}$ and $1200^{\circ} \mathrm{K}$ for the particular specimens measured might be as much as 0.032 lower or 0.012 higher than the reported values.

Measurements were made on commercially pure, polycrystalline specimens of alumina, thoria, magnesia, and zirconia. All showed similar behavior in that the emittance was low in the shorter wavelength regions and high at the longer wavelengths. Over the temperature range investigated the temperature coefficients of spectral emittance were positive for all four materials. The coefficients, however, varied with the material, those for thoria being much higher than for the other three. In all cases coefficients were greater at short wavelengths than at long.

The data were shown to be consistent with those obtained by several other measurement methods and in the case of alumina, the data were consistent with recently reported absorption coefficients for sapphire. Temperature coefficients appeared plausible on the basis of room-temperature reflectance measurements made on the same materials.

The authors gratefully acknowledge the assistance of A. G. Eubanks, who contributed to the solution of some of the early design and construction problems: of J. C. Richmond, for many helpful suggestions on method and procedure; of Francis J. Kelly, who prepared the computer programs for obtaining the standard deviations; and of Dr. David Hogben for guidance in preparing the statistical analysis.

\section{References}

[1]. Féry, C., Ann. Phys. Paris 27, 443 (1902).

[2]. Michaud, M., Silicates Ind. 19, 248 (1954).

[3]. Pattison, J. R., Trans. Brit. Cer. Soc. 54,698 (1955).

[4]. McMahon, H. O., J. Am. Cer. Soc. 34 (3), 91 (195\%).

[5]. Slemp, W. S. and Wade, W. R., Sym. on Measurement of Thermal Radiation Properties of Solids, Dayton, Ohio, NASA SP-31, 433 (1963) (available from U.S Govt. Printing Office, Washington, D.C., 20402, \$3.50).

[6]. Olson, O. H. and Katz, S., First Sym. Surface Effects on Spacecraft Materials, Palo Alto, Calif., 164 (1959).

[7]. Folweiler, R. C., Thermal Radiation Characteristics of Transparent, Semi-transparent, and Translucent Materials under Non-Isothermal Conditions, ASD-TDR-62-719 (April, 1964).

[8]. Clayton, W.A., Sym. on Measurement of Thermal Radiation Properties of Solids, Dayton, Ohio, NASA SP-31, 445 (1963), (Available from U.S. Govt. Printing Office, Washington, D.C., 20402, \$3.50).

[9]. Clark, H. E. and Moore, D. G., Equipment for Measuring Thermal Emittance of Ceramic Oxides to $1800^{\circ} \mathrm{K}$, Sym. on Thermal Radiation of Solids, San Francisco, Calif., NASA SP-55, 241 (1965). (Available from U.S. Govt. Printing Office, Washington, D.C., $20402 \$ 3.50$.)

[10]. Peavy, B. A. and Eubanks, A. G., Sym. on Measurement of Thermal Radiation Properties of Solids, Dayton, Ohio, NASA SP-31, 553, (1963). (Available from U.S. Govt. Printing Office, Washington, D.C. 20402, \$3.50.)

[11]. Gouffé, A., Rev. d'Opt. 24, 1 (1945).

[12]. Sanders, C. L. and Stevens, B. A., Rev. d'Opt. 33, 179 (1954).

[13]. Harrison, W. N., Richmond, J. C., Shorten, F. J., and Joseph, H. M., Standardization of Thermal Emittance Measurements, WADC TR-59-510, Pt. IV (Nov. 1963).

[14]. Hass, George and Salzberg, Calvin D., J. Opt. Soc. Am. 44, 181 (1954).

[15]. Clark, H. E., On Avoiding Errors From Stray Radiation in Measuring the Spectral Emittance of Diffusely Reflecting Specimens, J. Appl. Opt. 4, 1356 (1965).

[16]. Richmond, J. C., Radiative Transfer from Solid Materials, 142, ed. H. Blau \& H. Fischer (MacMillan Co., New York, 1962).

[17]. Natrella, M. G., Experimental statistics, National Bureau of Standards Handbook 91, Table A-4 (1963). (Available from USGPO, Wash., D.C. 20402, \$4.25.)

[18]. Gannon, R. E. and Linder, B., J. Am. Cer. Soc. 4 7, 592 (1964).

[19]. Cox, R. L., Fundamentals of Thermal Radiation in Ceramic Materials, Sym. on Thermal Radiation of Solids, San Francisco, Calif., NASA SP-55, 83 (1965). (Available USGPO, W ash., D.C. 20402, \$2.75.)

[20]. Richmond, J. C., Effect of Surface Roughness on Emittance of Non-metals, J. Opt. Soc. Am. 56, 253 (1966).

[21]. Eisenhart, C., Realistic Evaluation of the Precision and Accuracy of Instrument Calibration Systems, J. Res. NBS 67C (Engr. and Inst.) No. 2, 161 (1963).

[22]. Weber, B. C., Garrett, H. J., Mauer, F. A., and Schwartz, M. A., J. Am. Cerm. Soc. 39, 197 (1956).

[23]. Emslie, A. G., A Review of Some Problem Areas in the Theory of Thermal Radiation of Solids, Sym. on Thermal Rad. of Solids, San Francisco, Calif., NASA SP-55, p. 3 (1965). (Available from USGPO, Washington, D.C. 20402 \$2.75.)

[24]. Dunn, S. T., Design and Analysis of an Ellipsoidal Mirror Reflectometer, Ph.D. Thesis, Okla. State Univ., May 1965. (Available from University Microfilms, Inc., Ann Arbor, Mich.)

[25]. DeWitt, D. P. and Richmond, J. C., Design and Calibration of a Laser Source Integrating Sphere Reflectometer. (Presented at Am. Inst. Astr. and Aeronautics Meeting in New York City on Jan. 27, 1965 - to be published.) 
[26]. Blau, H. H., Marsh, J. B., Jasperse, J. R., and Chaffee, E. Infrared Spectral Emittance Properties of Solid Materials. AFCRL TR-60-416 (Oct. 1960).

[27]. Schatz, E. A., Counts, C. R. and Burks, T. L., Coatings for Energy Utilization, Control, and Protective Functions, AFML ML TDR 64-146 (1964).

[28]. Ritzow, G., Ann. der Physik 19, 769 (1934).

[29]. Gryvnak, D. A. and Burch, D. E., Optical and Infrared Properties of $\mathrm{Al}_{2} \mathrm{O}_{3}$ at Elevated Temperatures. J. Opt. Soc. Am. 55, 625 (1965).

\section{Appendix A. Analysis of Error Caused by Nonisothermal Blackbody Furnace}

Consider a cylindrical blackbody furnace cavity, whose back wall is maintained at $1200^{\circ} \mathrm{K}$ and whose sides are at $1225^{\circ} \mathrm{K}$. Further, assume that the cavity is constructed of an opaque material with an emittance $\epsilon_{\lambda}$ of 0.50 at all wavelengths. As a first approximation, the energy radiated along the axis of the cavity will consist of two components, an emitted component, $\epsilon_{\lambda} W\left(\lambda, T_{b}\right)$, and a reflected component, $\rho_{\lambda} W\left(\lambda, T_{s}\right)$, where $W(\lambda, T)$ is Planck's expression for the energy radiated by a blackbody at temperature $T$. $\mathrm{T}_{b}$ is the temperature of the back wall, and $T_{s}$ is the temperature of the sides. Since the reflectance of the back wall will be $\rho_{\lambda}=1-\epsilon_{\lambda}$, its reflectance will be 0.50 . One can then write for the energy distribution of radiant flux, $W_{\lambda}$

$$
\begin{aligned}
W_{\lambda} & =0.50 W\left(\lambda, T_{b}\right)+0.50 W\left(\lambda, T_{s}\right) \\
& =0.50 W\left(\lambda, 1200^{\circ} \mathrm{K}\right)+0.50 W\left(\lambda, 1225^{\circ} \mathrm{K}\right)
\end{aligned}
$$

When an optical pyrometer is sighted on the furnace opening, as was done in the present measurements, the indicated temperature, $T^{\prime}$, at $\lambda=0.65 \mu$ is assumed to be that of a perfect blackbody. Thus, one can write:

$$
\begin{aligned}
W\left(0.65 \mu, T^{\prime}\right)=0.50 W( & \left.0.65 \mu, 1200^{\circ} \mathrm{K}\right) \\
+ & 0.50 W\left(0.65 \mu, 1225^{\circ} \mathrm{K}\right) .
\end{aligned}
$$

The error $E_{\lambda}$ introduced by assuming that the nonisothermal furnace can be approximated by a perfect blackbody at the optical temperature $T^{\prime}$ is

$$
E_{\lambda}(\%)=\frac{W_{\lambda}-W\left(\lambda, T^{\prime}\right)}{W_{\lambda}} \times 100
$$

A computer program, prepared to calculate $T^{\prime}$ and $E_{\lambda}$ at $0.25 \mu$ intervals over the range 1 to $15 \mu$, showed a maximum error of only 0.44 percent. This occurred at a wavelength of $1.25 \mu$. At $10 \mu$ the error is only 0.14 percent.

The blackbody furnaces used in this investigation had gradients less severe than those assumed in the calculation, and the emittance of the actual cavity walls is significantly higher than 0.50 . Thus, the energy radiated along the axis of the laboratory furnaces should even more closely approximate blackbody radiation. At higher temperatures, 1400 and 1600 ${ }^{\circ} \mathrm{K}$, the Planck Function $W(\lambda, T)$ is less sensitive to temperature differences, and the measured gradients are smaller, so that the errors will be even less significant than at $1200^{\circ} \mathrm{K}$. 


\title{
Apparatus for the Measurement of the Normal Spectral Emissivity in the Infrared
}

\author{
Arthur G. Maki, Ralph Stair, and Russell G. Johnston
}

(December 21, 1959)

\begin{abstract}
Apparatus and methods are described for measuring in the infrared the normal spectral emissivity of metals and coatings or oxides which tightly adhere to metals. Examples of the use of this apparatus are given in measurements of the emissivity of platinum and of oxidized Inconel within the spectral region of 1.5 to 15 microns. Measured values were reproduced to better than 5 percent.
\end{abstract}

\section{Introduction}

The measurement of the spectral emissivity of heated substances may be accomplished by comparing the energy emitted by the substance under study with that emitted by a standard substance for radiation at various wavelengths. For such a standard one usually uses a blackbody, that is, a hollow body provided with a hole for viewing the radiation inside. The inside surface of the blackbody must be maintained at a uniform temperature. The wavelength separation may be accomplished using a double-beam monochromator or a singlebeam monochromator.

The specimen under investigation may be formed into a rod, bar, ribbon, or other shape. Some workers have even formed the specimen into a blackbody. For such a specimen both the inside surface (the blackbody) and the outside surface (the specimen) are observed.

The double-beam monochromator and associated method have certain advantages especially with relation to the elimination of the effects of absorption by carbon dioxide and water vapor, but the optical adjustment is critical as compared with a single-beam instrument. Furthermore through the use of a single set of optics and equal path lengths in the single-beam monochromator the effects of both atmospheric absorption and differential reflectivity in the auxiliary optics are practically eliminated. The apparatus described herein is of the latter type.

The specimen may vary in temperature from point to point depending upon its shape, size, and method of heating. If made into the form of a blackbody the inside temperature may differ from that on the outside. A convenient shape is that of a long thin ribbon heated electrically by contacts at the two ends. The data presented in this paper apply to samples of this type for which the temperature is measured by means of thermocouples in metallic contact with the opposite face from the point of radinnt energy measurement.

\section{Apparatus and Method}

This apparatus was set up as shown in figure 1 . The blackbody and sample under investigation were mounted side by side on an optical bench (constructed from a lathe bed and table) so they could be alternately placed at the focal point of the optical system. The auxiliary optics, consisting of a plane and a spherical mirror (65-cm radius of curvature), were mounted rigidly on the monochromator base. The placing of the sample and blackbody aperture alternately at the same position ensured essentially equal light paths and the use of identical opties.

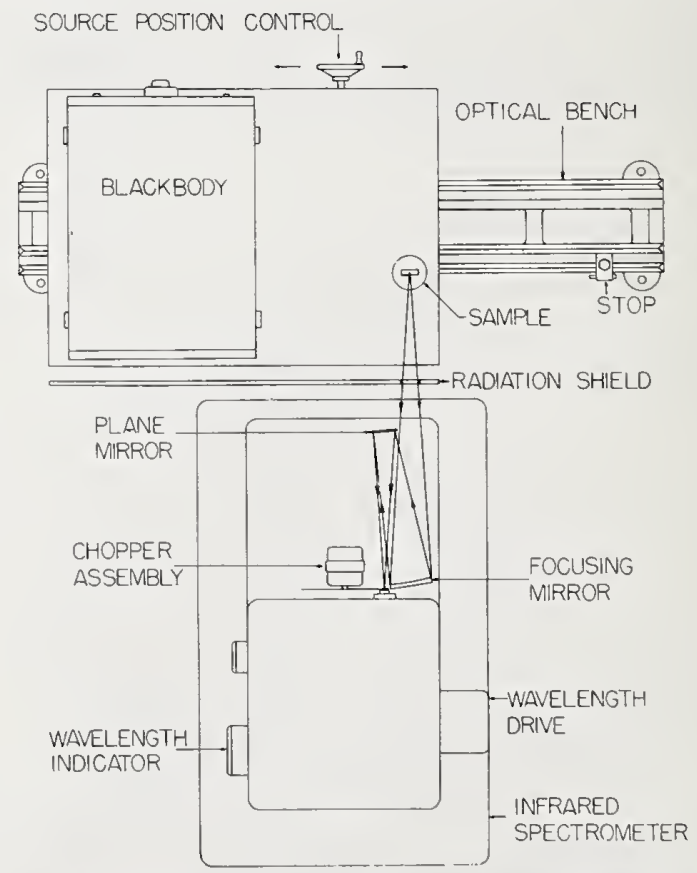

FIGURE 1. Instrumental setup for the measurement of the normal spectral emissivity of heated substances. 
A single-beam monochromator incorporating a sodium chloride prism and thermocouple was modified to vield the arrangement shown in figure 1 for use in this work. It was calibrated for wavelength in the usual manner by using atmospheric and polystyrene absorption bands. The light beam was chopped at $10 \mathrm{cps}$ and the thermocouple voltage amplified through the use of a ten-cycle thermocouple amplifier giving an approxinately linear output.

The linearity of the amplifier was of importance as the emissivity was obtained as the ratio of the measured outputs for the blackbody and specimen, both being maintained at the same temperature. For the high emittance measurements the linearity of the amplifier was cliecked using filters of known transmission. In the case of the low-emissivity measurements an attenuator switch was used to bring the blackbody and specimen readings within a comparable range. This attenuator was then calibrated by comparing the measured values of the radiant energy from a blackbody at a high temperature with those from the blackbody at a lower temperature. Using the Planck blackbody radiation equation the ratio of the radiation from these two sources was calculated and used to calibrate the amplifier and attenuator.

In this work the wavelength bandwidth (half intensity width) averaged about $0.15 \mu$ although it varied somewhat with the wavelength setting.

The blackbody cavity was constructed of an alloy casting of essentially 80 percent nickel-20 percent cliromium ( $3 \mathrm{in}$. outside diam. by 6 in. in length, with a wall thickness of about $1 / 2 \mathrm{in}$.). The low reflectivity of this (oxidized) metal coupled with the fact that the aperture was only 0.3 percent of the interior surface area resulted in a blackbody of extremely high effective emissivity (probably 0.999 or above). The high heat capacity of the furnace employed in heating the blackbody gave it a high thermal stability and made manual temperature regulation easy (see fig. 2). The large size of the furnace, the method of heating, and the small furnace aperture used to permit viewing the blackbody yielded a very uniform temperature within the blackbody.

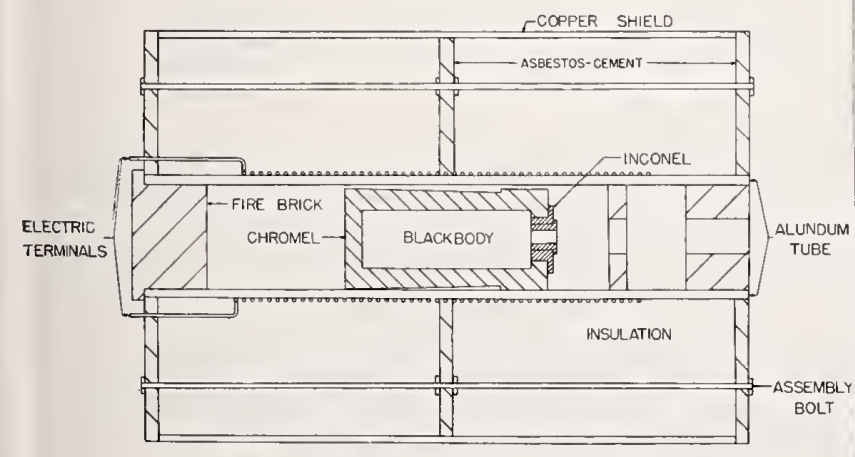

Figure 2. Blackbody and furnace cross section.
The temperature of the blackbody, as well as that of the sample under investigation, was measured through the use of platinum versus platinum-10 percent-rhodium thermocouples. Checks against standard thermocouples indicated that the absolute temperatures of the blackbody recorded herein are correct to within about $\pm 6^{\circ} \mathrm{K}$.

The specimen consisted of a metal strip about $83 \frac{1}{2}$ in. long by $1 / 4$ in. wide and about $1 / 32 \mathrm{in}$. thick. The thermocouple used to measure the specimen temperature was welded to the back side of it directly behind the surface area from which the measurements were made. The thermocouple used was of rather small diameter wire $(5 \mathrm{mils})$ so that the cooling of the surface which resulted from conduction along the thermocouple wire was quite small. The useful radiating surface area was about $3 / 8$ in. by $1 / 16$ in. and the radiation used was essentially normal to the surface. Observations with an optical pyrometer indicate that the temperature gradient over the observed surface was less than $\sim 4^{\circ} \mathrm{K}$.

The specimen under examination was heated by passing through it an electric current of about 80 amp at $5 \mathrm{v}$. It was clamped rigidly at the two electrodes and held centrally located within a watercooled metal tube, about $1 \frac{11}{4} \mathrm{in}$. inner diameter. Although the inner surface of this tube was highty oxidized, reflection from its inner surface affected the emissivity measurements by about 1 percent. However, its use reduced air currents and added greatly to the stability of the temperature of the sample so that its temperature could be manually held constant within about $\pm 1.5^{\circ} \mathrm{K}$ even at $1,400^{\circ} \mathrm{K}$.

\section{Examples of the Use of This Apparatus}

As examples of the use of this apparatus, the emittances of two very different substances, platinum and oxidized Inconel, were measured. "The oxide coating on Inconel has a very high spectral emittance while the surface of platinum being highly reflective has a very low emissivity.

\subsection{Oxidized Inconel}

The Inconel strips were prepared by first cleaning the surface by sandblasting, and then heating in air for $6 \mathrm{hr}$ at a ligh temperature in order to form an opaque oxide coating.

The spectral emittance of oxidized Inconel is given in table 1 and figure 3 . These data are the average of the values obtained on three separate specimens each of which was run several times at each temperature.

The oxide coating has a very high emissivity as is characteristic of many oxides. The most prominent features of the emissivity curve are two minimums at around 6 and $6.5 \mu$. In this same wavelength region there are two strong absorption maximums for atmospheric water vapor. Although care was exercised to make the pathlengths for blackbody and specimen equal, the atmospheric absorption may be the cause of these minimums. 
TABLE 1. Normal spectral emittance of oxidized Inconel at $1,400^{\circ} \mathrm{K}, 1,050^{\circ} \mathrm{K}$, and $780^{\circ} \mathrm{K}$ from 1.5 to $15 \mu$

\begin{tabular}{|c|c|c|c|}
\hline $\begin{array}{l}\text { Wave- } \\
\text { length }\end{array}$ & $1,400^{\circ} \mathrm{K}$ & $1,050^{\circ} \mathrm{K}$ & $780^{\circ} \mathrm{K}$ \\
\hline & & & \\
\hline $\begin{array}{l}1.5 \\
2.0\end{array}$ & $\begin{array}{r}0.93 \\
92\end{array}$ & $\begin{array}{r}0.88 \\
.88\end{array}$ & $\begin{array}{r}0.77 \\
.79\end{array}$ \\
\hline 2.5 & .91 & .86 & .78 \\
\hline 3.0 & .90 & .86 & .77 \\
\hline 3.5 & .90 & .85 & .78 \\
\hline 4. 0 & .90 & .85 & .78 \\
\hline 4.5 & .90 & .84 & .78 \\
\hline 5.0 & .90 & .84 & .77 \\
\hline 5. 5 & .89 & .83 & .77 \\
\hline 6.0 & .86 & .80 & \\
\hline 6.5 & .86 & .80 & .74 \\
\hline 7. 0 & .88 & .84 & .78 \\
\hline 7.5 & .90 & .87 & .82 \\
\hline 8.0 & .91 & .90 & .85 \\
\hline 8.5 & .91 & .90 & .87 \\
\hline 9.0 & .92 & .90 & .87 \\
\hline 9.5 & .92 & .90 & .86 \\
\hline 10.0 & .92 & .89 & .86 \\
\hline 10.5 & .92 & .90 & .87 \\
\hline 11.0 & .92 & .90 & .88 \\
\hline 11.5 & .93 & .91 & .88 \\
\hline 12. 0 & .94 & .92 & .89 \\
\hline 12.5 & .95 & .93 & .91 \\
\hline 13.0 & .95 & .94 & .92 \\
\hline 13.5 & .96 & .94 & .93 \\
\hline 14. 0 & .96 & .94 & .90 \\
\hline 14.5 & .96 & .94 & .89 \\
\hline 15. 0 & .96 & .94 & .87 \\
\hline
\end{tabular}

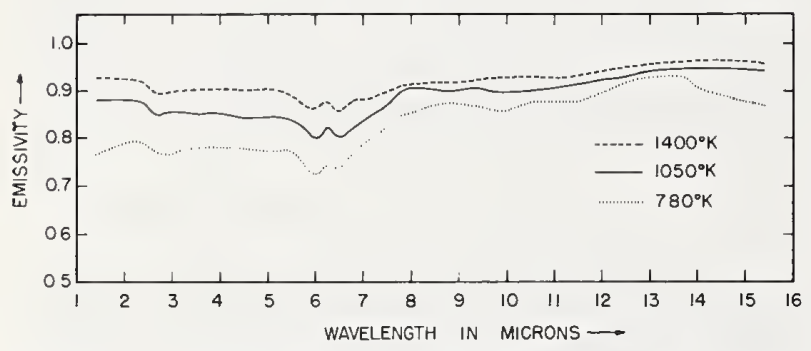

Figure 3. Normal spectral infrared emissivity of oxidized Inconel at selected temperatures.

Figure 3 shows the very distinct trend for the emissivity to increase with temperature. Many nonmetals seem to show very little change in emissivity with temperature. The change which we have observed, however, is attributed to the oxide coating because previous experiments indicate that coatings formed in the manner described are opaque to any radiation from the underlying metal. Moreover, although metals are expected to show such an increase in emissivity with temperature, the emissivity of metals is too low to be a contributing factor.

\subsection{Platinum}

The two platinum strips examined were first annealed by heating to about $1,525^{\circ} \mathrm{K}$. One specimen was held at that temperature for $1 \mathrm{hr}$ while the other was heated for only $10 \mathrm{~min}$. Both specimens, although initially smooth surfaced, acquired a crystalline appearance after the annealing process. This crystalline surface would probably have a slightly different emissivity from a perfectly smooth surface, but infrared radiation, since it has relatively large wavelength, is rather insensitive to small surface irregularities.

The emissivity of the platinum strip is given in table 2 and the results are shown in figure 4 . The low emissivity is characteristic of most metals. This follows from the fact that the reflectivity of metals is very high.

Price [1] ${ }^{1}$ measured the spectral emissivity of platinum at $1,400^{\circ} \mathrm{K}$ over the wavelength range of 0.65 to $4.75 \mu$. Price reported that due to the nonperfect blackbody which he used his emissivity values, if in error, were probably too high. This may account for the fact that our values are everywhere lower than Price's.

TABLE 2. Calculated and observed values of the normal spectral emissivity of platinum at $1,400^{\circ} \mathrm{K}$ from 1.5 to $15 \mu$

\begin{tabular}{r|r|r}
\hline \hline $\begin{array}{r}\text { Wave- } \\
\text { length }\end{array}$ & $\begin{array}{c}\text { Calculated } \\
\text { emissivity }\end{array}$ & $\begin{array}{c}\text { Observed } \\
\text { enissivity }\end{array}$ \\
\cline { 1 - 2 } 1.5 & & \\
2.0 & 0.189 & 0.197 \\
2.5 & .165 & .179 \\
3.0 & .149 & .154 \\
3.5 & .127 & .139 \\
4.0 & .119 & .128 \\
4.5 & .112 & .117 \\
5.0 & .107 & .108 \\
5.5 & .102 & .090 \\
6.0 & .098 & .094 \\
6.5 & .094 & .088 \\
7.0 & .091 & .090 \\
7.5 & .088 & .092 \\
8.0 & .085 & .093 \\
8.5 & .083 & .091 \\
9.0 & .081 & .091 \\
9.5 & .078 & .090 \\
10.0 & .077 & .087 \\
10.5 & .075 & .083 \\
11.0 & .073 & .080 \\
11.5 & .072 & .078 \\
12.0 & .072 & .075 \\
12.5 & .069 & .072 \\
13.0 & .067 & .068 \\
13.5 & .066 & .066 \\
14.0 & .065 & .064 \\
14.5 & .064 & .063 \\
15.0 & .063 & .065 \\
\hline & & \\
\hline
\end{tabular}

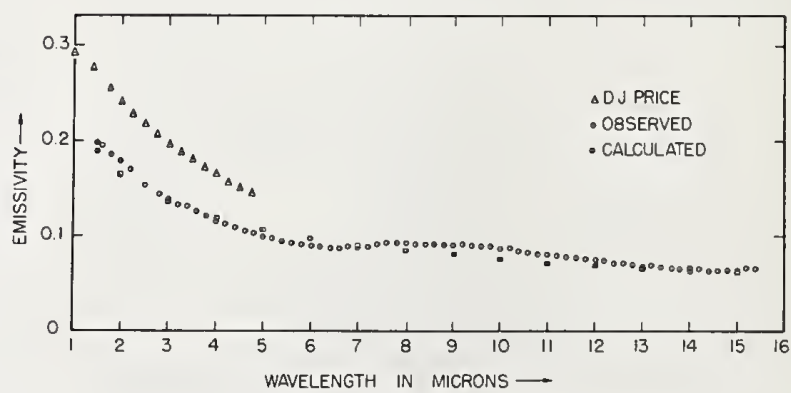

FIgURE 4. Normal spectral infrared emissivity of commercial grade platinum at $1,400^{\circ} \mathrm{K}$.

Observations by D. J. Price showu by triangles $(\Delta)$; caleulated emissivity shown by squares (๑).

1 Figures in brackets indicate the litcrature referenees at the end of this paper. 
In the long wavelcngth region the emissivity of metals increases witl increase in tempcrature. This faet has been verified for platinum by Hagen and Rubens [2]. Thus our results should be higher than measurements made at lower temperatures. As expeeted the reflectivity values for platinum at lower temperatures reported by other workers yiekd emissivity values lower than ours.

The mcasured spcetral emissivity values for platinum may be compared with the thcoretieal emissivity ealculated by using the Drude-Zener relation. This is valid only for metals for which the electrons are perfectly free. According to this relationship [3] the long wavelength spectral emissivity is given by

$$
\epsilon=0.365\left(\frac{\rho}{\lambda}\right)^{\frac{1}{2}}-0.0464 \frac{\rho}{\lambda} .
$$

Here $\rho$ is the clcetric resistivity of the metal in ohm centimeters and $\lambda$ is the wavelength in centimeters. Table 2 and figure 4 include the theoretical emissivities calculated using this equation and the resistivity value $46.52 \mu \mathrm{ohm}$ em measurcd at $1,400^{\circ}$ K by Wm. F. Rocser [4].

At long wavclengths the measured emissivity is in excellent agreement with the theoretical values. There is, however, an unexpected rise in the obscrved values around 8,9 , and $10 \mu$. This may be due to very small amounts of impurities on thic surface of the metal. The deviation at shortcr wavelengths may be partially due to the fact that the theoretical curve is expeeted to apply only to the long wavelength region.

\section{Conclusion}

The apparatus described in this paper may be used to measure the emissivity of metallic substances or substances eapable of forming tightly adhering eoatings on metals to within about \pm 3 percent for substances with a high emittance and to about \pm 5 percent (of measured value) for substances with a low emittance. For highly emissive substances the temperaturc measurement is probably the principal source of error while for low-cmittance spceimens the amplifier stability becomes a major source of error. The reprodueibility of the results has been found to be very good. It was checked by making repeated measurements on the same speeimen and on diffcrent spceimens of the same material. In addition a different operator was used to check some of the work.

One eould also measure the emissivity by changing the blackbody temperature so as to equate the radiation of sample and blackbody. The principal source of error would then be due to the tempcrature measurement for all emissivity values. A big disadvantage of this procedure is the slowness of making measurements due to the large thermal inertia of the blackbody.

In conclusion the authors express their gratitude to Wm. F. Roeser of the Building Technology Division for helpful suggestions; also to Fred Shorten for assistance in taking some of the observations.

\section{References}

[1] D. J. Price, Proc. Phys. Soc. (London) 59, 118 (1947).

[2] E. Hagen and H. Rubens, Ann. Physik 11, 873 (1909)

[3] Max Jakob, Heat transfer, vol. I (John Wiley and Sons, Inc., New York, N.Y.).

[4] Temperature; its measurement and control in science and industry, p. 1312 (Reinhold Publishing Corp., New York, N.Y.).

Washington, D.C.

(Paper 64C2-29) 


\title{
Approximate Normal Emissivity Spectra in the Infrared at Elevated Temperatures of Single-Crystal and Polycrystalline Calcium Fluoride
}

\author{
W. Fussell and J. Geist
}

\begin{abstract}
A single-beam ir spectroradiometric system has been used to measure the normal spectral emissivity of specimens of single-crystal and polycrystalline calcium fluoride in the ir at elevated temperatures. The wavelength range was $2-12 \mu$, and data were taken at sample temperatures of $500^{\circ} \mathrm{C}$ and $600^{\circ} \mathrm{C}$. Room temperature data on the index of refraction of single-crystal calcium fluoride and its rate of change with temperature were extrapolated to $500^{\circ} \mathrm{C}$ and $600^{\circ} \mathrm{C}$; the normal spectral reflectivities were computed from the extrapolated indices at these temperatures. Then the computed reflectivities were used to extract normal spectral absorption coefficients from the emissivity data. It was possible to compute absorption coefficients with reasonable accuracy at wavelengths of $6 \mu, 8 \mu$, and $10 \mu$; the smallest estimated error was about $2 \%$ at $8 \mu$. The absorption coefficients increased markedly with temperature at these three wavelengths. The absorption coefficients of the polycrystalline material were consistently higher than the corresponding values for the single-crystal calcium fluoride. It was found that the spectral absorption coefficients at $8 \mu$ and $10 \mu$ microns varied approximately as the 2.1 and 1.6 power of the absolute temperature, respectively, between room temperature and $600^{\circ} \mathrm{C}$. These exponents lie within the range predicted by theory.
\end{abstract}

\section{Introduction}

The normal spectral emissivity of a sample, at a given wavelength and temperature, is defined as the ratio of its spectral radiance, in a direction perpendicular to its surface, to the corresponding spectral radiance of a blackbody at the same temperature. Therefore, the normal spectral emissivity of the sample can be found by measuring alternately the normal spectral radiance from the blackbody and from the sample, both at the same temperature, with a single-beam spectroradiometer. This method of measurement was employed to procure the data presented in this paper. Figure 1 shows the basic elements of the system used. The sample and blackbody ovens are hollow, electrically heated cylinders. The blackbody is placed in one oven, and the sample mounted in its holder is placed in the other. Both the blackbody and the sample holder are eylindrical in shape, with an outside diameter slightly less than the inside diameter of the ovens (see Fig. 2). Both ovens are mounted on a lathe bed by means of which either the sample or the blackbody can be placed in the field of view of the spectroradiometer. The

Both authors were with the National Bureau of Standards, Gaithersburg, Maryland when this work was done; W. Fussell is now with the U.S. Weather Bureau, Silver Spring, Maryland. Received 25 August 1966.

This work was supported by the Advanced Research Projects Agency, U.S. Department of Defense. external optics of the system consist of one flat mirror and one spherical mirror, positioned to form a full-size image of the entrance slit of the spectrometer at a point centered either in the blackbody radiant aperture or on the sample. To prevent the spectrometer from viewing the interior walls of the oven or the sample holder, an aperture stop was placed over the spherical mirror. The opening in the stop is off-center near the rim of the mirror so that off-axis angle at which it operates is minimized.

The temperatures of the blackbody and the sample are measured by platinum- $10 \%$ rhodium thermocouples which have been calibrated by the NBS Temperature Physics Section.

It has been shown that the normal spectral emissivity of a plane parallel slab is a function of the normal spectral absorption coefficient of the material of which the slab is composed, its normal spectral surface reflectance, and the slab thickness. Therefore, if the normal spectral emissivity of a plane parallel sample of known thickness is measured at a given wavelength, and if the normal spectral surface reflectance is either computed or measured at the same wavelength, the corresponding normal spectral absorption coefficient can be computed also.

The normal spectral surface reflectance of the sample can be computed from the index of refraction, if this is known. Unfortunately, most index of refraction data have been taken near room temperature and in the 


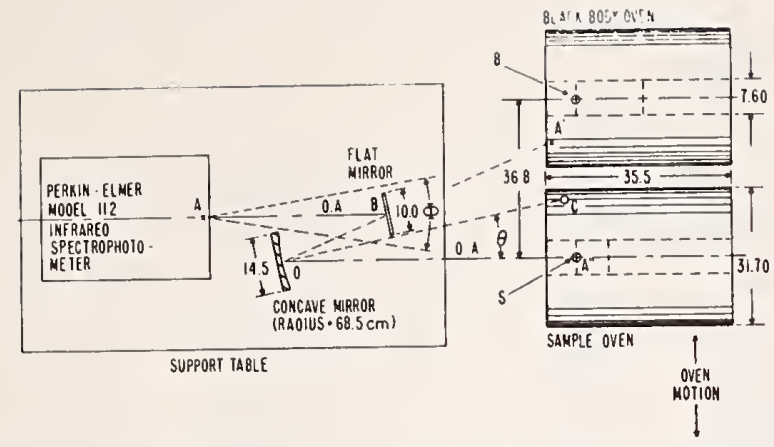

Fig. 1. Schematic plan view of spectroradiometric system for measuring the normal spectral emissivity of optical materials. (Note: all dimensions in centimeters.) $A B=43.8 \mathrm{~cm} . \quad B D=$ $26.3 \mathrm{~cm}$. $D A=69.9 \mathrm{~cm}$.

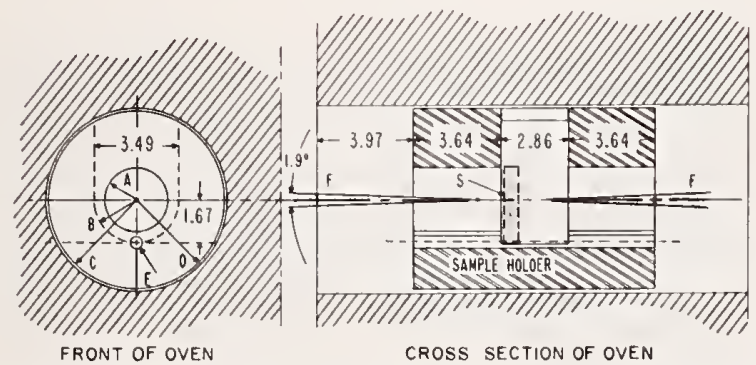

Fig. 2. Detail views of the sample holder and the spectrometer field of view for a point at the entrance slit center (dimensions in inches).

spectral range over which the material under consideration has at least fair transmission in reasonable thicknesses. Thus, to compute the index of refraction at elevated temperatures and at wavelengths for which the material absorbs appreciably, it is usually necessary to extrapolate with respect to wavelength from data in the region of good transmission, and then to extrapolate with respect to temperature from room temperature by using data on the rate of change of index with temperature. However, in the waveleng th range studied, $2 \mu$ to $12 \mu$, calcium fluoride has a low index of refraction, and also a low rate of change of index with temperature so that comparatively larger errors in the computed index produce negligible errors in the transmittance computed from the measured emissivity.

A major source of uncertainty in the emissivity data was the background correction; due to the nonperfect alignment of the optical system, the spectrometer viewed a significant amount (about $15 \%$ compared with the blackbody) of radiation from the empty sample holder. This radiation was corrected for when computing the sample transmittance, but the correction neglected possible variations in the background due to the presence of the sample.

It is felt that the normal spectral absorption coefficient of a material is more useful than the normal spectral emissivity of a particular sample of the material, so this coefficient was computed from the emissivity data wherever the calculated values had reasonable accuracy. It has been shown that for a constant error in the value of the normal spectral internal transmittance of a sample-as computed from the measured normal spectral emissivity and the computed normal spectral surface reflectance - the error in the computed normal spectral absorption coefficient varies as the reciprocal of the product of the optical thickness and the transmittance. (The optical thickness is the product of the physical thickness of the sample and its normal spectral absorption coefficient.) This error function has a minimum when the optical thickness is one. Therefore, for a given estimated value of the spectral absorption coefficient, there corresponds a sample thickness that will enable the value of the coefficient to be measured with higher accuracy than with thinner or thicker samples. This also means that, if the spectral absorption coefficient of a material increases rapidly in a wavelength region, a sample of given thickness will provide emissivity data from which the spectral absorption coefficient can be computed accurately only over a limited wavelength band. For example, at $500^{\circ} \mathrm{C}$ and $600^{\circ} \mathrm{C}$, an $8-\mathrm{mm}$ thick, singlecrystal calcium fluoride sample yielded emissivity data useful only for computing the spectral absorption coefficient near $8 \mu$ wavelength.

In accordance with the preceding considerations, samples of a wide range of thicknesses were used to obtain normal spectral emissivity data for both singlecrystal and polycrystalline hot-pressed calcium fluoride at $500^{\circ} \mathrm{C}$ and $600^{\circ} \mathrm{C}$, and from $2 \mu$ to $12 \mu$ wavelength. The normal spectral surface reflectance of both types of calcium fluoride at these temperatures was estimated from data on the index of refract on of the single-crystal material at room temperature. ${ }^{1}$ The error in the emissivity data was estimated to be less than 0.02 after correction for background radiation. The resulting error in the corresponding normal spectral absorption coefficients was less than $5 \%$ in all cases.

Table I. Normal Spectral Absorption Coefficients of SingleCrystal and Polycrystalline Calcium Fluoride at Room Temperature, $500^{\circ} \mathrm{C}$ and $600^{\circ} \mathrm{C}$ and at $6 \mu, 8 \mu$, and $10 \mu$ Wavelength

\begin{tabular}{cccccc}
\hline \multicolumn{5}{c}{$\begin{array}{c}\text { Normal spectral absorption } \\
\text { coefficients }\left(\mathrm{cm}^{-1}\right)\end{array}$} \\
$\begin{array}{c}\text { Wave- } \\
\text { length } \\
(\mu)\end{array}$ & $\begin{array}{c}\text { Temper- } \\
\text { ature } \\
\left({ }^{\circ} \mathrm{C}\right)\end{array}$ & $\begin{array}{c}\text { Single } \\
\text { crystal }\end{array}$ & $\begin{array}{c}\text { Est. } \\
\text { error }\end{array}$ & $\begin{array}{c}\text { Poly- } \\
\text { crystal- } \\
\text { line }\end{array}$ & $\begin{array}{c}\text { Est. } \\
\text { error }\end{array}$ \\
\hline 6 & R.T. $^{a}$ & 0.0024 & - & - & - \\
& 500 & - & - & - & - \\
& 600 & 0.23 & 0.01 & 0.24 & 0.01 \\
8 & R.T. ${ }^{b}$ & 0.16 & - & - & - \\
& 500 & 1.16 & 0.03 & 1.35 & 0.02 \\
10 & 600 & 1.56 & 0.02 & 1.63 & 0.02 \\
& R.T. ${ }^{b}$ & 1.8 & - & - & - \\
& 500 & 8.4 & 0.2 & 8.6 & 0.2 \\
& 600 & 10.1 & 0.3 & 10.4 & 0.3 \\
\hline
\end{tabular}

a Data extrapolated from Ref. 2.

${ }^{b}$ Data from Ref. 2. 


\begin{tabular}{|c|c|c|c|c|c|c|c|c|}
\hline \multirow{2}{*}{$\begin{array}{c}\text { Wavelength } \\
(\boldsymbol{\mu})\end{array}$} & \multirow{2}{*}{$\begin{array}{l}\text { Temperature } \\
\left({ }^{\circ} \mathrm{C}\right)\end{array}$} & \multicolumn{3}{|c|}{$\begin{array}{l}\text { Single crystal } \mathrm{CaF}_{2} \\
\text { thickness }(\mathrm{mm})\end{array}$} & l spectral emi & \multicolumn{3}{|c|}{$\begin{array}{l}\text { Polycrystalline } \mathrm{CaF}_{2} \\
\text { thickness }(\mathrm{mm})\end{array}$} \\
\hline & & 2.08 & 8.09 & 16.11 & Background & 1.07 & 3.18 & 6.54 \\
\hline \multirow[t]{2}{*}{2} & 500 & 0.20 & 0.18 & 0.18 & 0.20 & - & - & - \\
\hline & 600 & 0.20 & 0.18 & 0.19 & 0.21 & - & - & - \\
\hline \multirow[t]{2}{*}{4} & 500 & 0.16 & 0.16 & 0.12 & 0.16 & 0.17 & 0.18 & 0.20 \\
\hline & 600 & 0.15 & 0.15 & 0.14 & 0.16 & 0.17 & 0.19 & 0.20 \\
\hline \multirow[t]{2}{*}{6} & 500 & 0.16 & 0.17 & 0.19 & 0.15 & 0.17 & 0.17 & 0.19 \\
\hline & 600 & 0.17 & 0.20 & 0.25 & 0.16 & 0.17 & 0.19 & 0.23 \\
\hline \multirow[t]{2}{*}{8} & 500 & 0.31 & 0.67 & 0.85 & 0.14 & 0.24 & 0.41 & 0.64 \\
\hline & 600 & 0.37 & 0.74 & 0.92 & 0.16 & 0.28 & 0.49 & 0.70 \\
\hline \multirow[t]{2}{*}{10} & 500 & 0.83 & 0.99 & 0.99 & 0.12 & 0.63 & 0.94 & 0.97 \\
\hline & 600 & 0.87 & 0.99 & 0.99 & 0.14 & 0.71 & 0.95 & 0.96 \\
\hline \multirow[t]{2}{*}{12} & 500 & 0.97 & 0.97 & 1.00 & 0.09 & 0.94 & 0.98 & 0.98 \\
\hline & 600 & 0.96 & 1.00 & 0.98 & 0.11 & 0.98 & 0.98 & 0.98 \\
\hline
\end{tabular}

The spectral absorption coefficients and their estimated errors, computed from the emissivity data, are tabulated in Table I. This table also lists room temperature spectral absorption coefficients for singlecrystal calcium fluoride taken from the American Institute of Physics Handbook ${ }^{2}$ at $8 \mu$ and $10 \mu$ and extrapolated to $6 \mu$. Table I also shows the estimated maximum errors in the absorption coefficients. Table II presents the measured normal spectral emissivity data from which the absorption coefficients were computed. Table III lists the room temperature indices of reirraction of single-crystal calcium fluoride from $2 \mu$ to $12 \mu^{*}$, the corresponding computed indices at $500^{\circ} \mathrm{C}$

* Taken from Ref. 1 out to $8 \mu$ and then extrapolated to $10 \mu$ and $12 \mu$ by the dispersion formula of Ref. 1 . and $600^{\circ} \mathrm{C}$, the computed normal surface reflectances corresponding to these indices, and the rate of change of surface reflectance with index. It is clear that even an error of as much as \pm 0.01 in the computed index will produce a reflectance error of less than 0.001 . Table IV presents the exponents to which the ratios of the absolute temperatures must be raised in order to fit approximately the ratios of the observed spectral absorption coefficients.

\section{Experimental Procedure}

Three samples of single-crystal calcium fluoride and three samples of polycrystalline calcium fluoride were used. All samples were cylindrical disks about $3.2 \mathrm{~cm}$ in diam with polished, parallel faces. The single-crystal thicknesses were $2.08 \mathrm{~mm}, 8.09 \mathrm{~mm}$, and $16.11 \mathrm{~mm}$,

Table III. Index of Refraction and Normal Spectral Surface Reflectance of Single-Crystal Calcium Fluoride from $2 \mu$ to $12 \mu$ Wavelength

\begin{tabular}{|c|c|c|c|c|c|}
\hline $\begin{array}{l}\text { Wavelength } \\
\qquad(\mu)\end{array}$ & $\begin{array}{l}\text { Temperature } \\
\left({ }^{\circ} \mathrm{C}\right)\end{array}$ & $\begin{array}{l}\text { Index } \\
\text { of refr. }\end{array}$ & $\begin{array}{l}\text { Rate of change } \\
\text { of index with } \\
\text { temperature } \\
\left({ }^{\circ} \mathrm{C}\right)^{-1}\end{array}$ & $\begin{array}{l}\text { Computed } \\
\text { surface } \\
\text { reflectance }\end{array}$ & $\begin{array}{c}\text { Rate of change } \\
\text { of surface reflectance } \\
\text { with index }\end{array}$ \\
\hline \multirow[t]{3}{*}{2} & R.T. & 1.4238 & $-5.9 \times 10^{-6}$ & 0.030 & 0.12 \\
\hline & 500 & 1.4195 & & 0.030 & 0.12 \\
\hline & 600 & 1.4186 & & 0.030 & 0.12 \\
\hline \multirow[t]{3}{*}{4} & R.T. & 1.4096 & $-7.6 \times 10^{-6}$ & 0.029 & 0.12 \\
\hline & 500 & 1.4060 & & 0.028 & 0.12 \\
\hline & 600 & 1.4052 & & 0.028 & 0.12 \\
\hline \multirow[t]{3}{*}{6} & R.T. & 1.3856 & $-7.0 \times 10^{-6}$ & 0.026 & 1.11 \\
\hline & 500 & 1. 3822 & & 0.026 & 0.11 \\
\hline & 600 & 1.3815 & & 0.026 & 0.11 \\
\hline \multirow[t]{3}{*}{8} & R.T. & 1.3498 & $-6.2 \times 10^{-6}$ & 0.022 & 0.11 \\
\hline & 500 & 1.3468 & & 0.022 & 0.11 \\
\hline & 600 & 1.3462 & & 0.022 & 0.11 \\
\hline \multirow[t]{3}{*}{10} & R.T. & 1. 2996 & $-5.5 \times 10^{-6}$ & 0.017 & 0.10 \\
\hline & 500 & 1.2970 & & 0.017 & 0.10 \\
\hline & 600 & 1. 2964 & & 0.017 & 0.10 \\
\hline \multirow[t]{3}{*}{12} & R.T. & 1.2189 & $-4.7 \times 10^{-6}$ & 0.010 & 0.08 \\
\hline & 500 & 1.2166 & & 0010 & 0.08 \\
\hline & 600 & 1.2162 & & 0.010 & 0.08 \\
\hline
\end{tabular}




\begin{tabular}{|c|c|c|c|c|c|c|}
\hline $\begin{array}{l}\text { Wave- } \\
\text { length } \\
(\mu)\end{array}$ & $\begin{array}{c}\text { Absolute } \\
\text { temperature } \\
\left({ }^{\circ} \mathrm{K}\right)\end{array}$ & $\begin{array}{c}\text { Temperature } \\
\text { ratio } \\
\text { (to } 293^{\circ} \mathrm{K} \text { ) }\end{array}$ & $\begin{array}{l}\text { Normal } \\
\text { spectral } \\
\text { absorption } \\
\text { coefficient } \\
\left(\mathrm{cm}^{-1}\right)\end{array}$ & $\begin{array}{c}\text { Normal } \\
\text { spectral } \\
\text { absorption } \\
\text { coefficient } \\
\text { ratio (to } 293^{\circ} \mathrm{K} \text { ) }\end{array}$ & \multicolumn{2}{|c|}{$\begin{array}{c}\text { Exponent defined as: } \\
{[\log (\text { ratio N.S. absorp. coeff. })]} \\
[\log (\text { ratio }) \text { A. temperature })]\end{array}$} \\
\hline \multirow{3}{*}{ S } & & & & & & Average \\
\hline & 293 & 1.00 & 0.16 & 1.00 & - & - \\
\hline & 773 & 2.64 & 1.16 & 7.25 & 2.04 & \\
\hline \multirow{4}{*}{10} & 873 & 2.98 & 1.56 & 9.75 & 2.08 & 2.06 \\
\hline & 293 & 1.00 & 1.8 & 1.00 & - & - \\
\hline & 773 & 2.64 & 8.4 & 4.66 & 1.59 & - \\
\hline & 873 & 2.98 & 10.1 & 5.61 & 1.58 & 1.58 \\
\hline
\end{tabular}

while the polycrystalline thicknesses were $1.07 \mathrm{~mm}$, $3.18 \mathrm{~mm}$, and $6.54 \mathrm{~mm}$. The procedure for each sample was as follows-(1) A sample was placed in the sample lolder, which was then inserted into the sample oven so the front face of the sample was about $8 \mathrm{~cm}$ within the oven. The ealibrated platinum ( $10 \%$ rhodium) thermorouple was inserted into the sample holder with the hot junction touching the sample. (2) The blackbody was inserted into the blackbody oven so its radiant aperture was also about $8 \mathrm{~cm}$ within the oven, and the blackbody thermocouple was inserted into the blackbody cavity with the hot junction touching the rear of the cavity. The sample and blackbody thermocouples were made of platinum, and platinum- $10 \%$ rhodium, wires taken from the same spools. The cold junctions of the thernocouples were maintained at $0^{\circ} \mathrm{C}$ within $0.05^{\circ} \mathrm{C}$ by means of a thermoelectric ice-point. (3) Both sample and blackbody were heated to $500^{\circ} \mathrm{C} \pm$ $3^{\circ} \mathrm{C}$. And after approximately a half-hour period of stahilization, their exact temperatures were measured with a potentiometer to a precision of $0.1^{\circ} \mathrm{C}$. If the difference between the sample and blackbody temperatures exceeded $0.5^{\circ} \mathrm{C}$, one of the automatic oven temperature control systems was adjusted slightly to make the difference less than $0.5^{\circ} \mathrm{C}$. (4) Several alternate readings of blackbody and sample radiance were made at wavelengths of $2 \mu, 4 \mu, 6 \mu, 8 \mu, 10 \mu$, and $12 \mu$. Spectroradiometer slit width and gain were held constant for each series of readings at each wavelength and temperature for each sample. The slit widths were such that they gave maximum spectral bandwidth of about $0.15 \mu$ (at a wavelength of $12 \mu$ ).

The above procedure was repeated for a temperature of $600^{\circ} \mathrm{C}$. Some data were taken at $700^{\circ} \mathrm{C}$, but sample deterioration was severe at this temperature; therefore, these data are not presented here.

The spectroradiometer gain and time constant settings were set to maintain the signal-to-noise ratio at better than 30:1 at all times (with respect to the blackbody reading). In addition, each reading was extended for a period of time equivalent to perhaps 10:20 time constants, so the average signal-to-noise ratio for each blackbody reading was better than 100:1.
The external optical system formed a full-size image of the entrance slit of the spectroradiometer at either the blackbody aperture or the sample. Due to a slight misalignment of the optic axis of the external optics, the spectroradiometer viewed a small portion of the sample oven wall. This background radiation was corrected for by measuring the emissivity of the empty sample holder at the wavelengths and temperatures at which sample emissivities were also measured.

\section{Analysis and Conclusions}

We introduce the nomenclature: $\epsilon(t, T, \lambda)=$ normal spectral emissivity of a plane parallel sample of thickness $t$ at wavelength $\lambda$ and absolute temperature $T$ (we drop the explicit expression of the dependence on $t, T$, $\lambda$, etc., wherever it is possible to do so without confusion); $\tau(t, T, \lambda)=$ normal spectral transmittance of the sample; $\rho(t, T, \lambda)=$ normal spectral reflectance of the sample; $\tau_{i}(t, T, \lambda)=$ normal spectral internal transmittance of the sample, defined as exp $(-K \times t)$, where $K(T, \lambda)=$ normal spectral absorption coefficient of the sample material; $\rho_{s}(T, \lambda)=$ normal spectral surface reflectance of the sample material; $\epsilon_{\beta}(T, \lambda)=$ normal spectral background emissivity; $n(T, \lambda)=$ index of refraction of the sample material; $\epsilon^{\prime}\left(t, T, \lambda, \epsilon_{\beta}\right)=$ normal spectral emissivity of the sample plus background; $\Delta \epsilon=$ estimated errors in $\epsilon^{\prime}, \Delta \epsilon^{\prime}=$ estimated error in $\epsilon^{\prime} ; \Delta K=$ estimated error in $K ; \Delta \rho_{s}=$ estimated error in $\rho_{s} ; \Delta n=$ estimated error in $n ; T_{\dot{B} \dot{B}}=$ blackbody temperature (absolute); $T_{S P}=$ sample temperature (absolute); $T_{R M}=$ room temperature (absolute) $; x=c_{2}(\lambda T)^{-1} ; c_{2}=$ second radiation constant, $1.44 \mathrm{~cm}^{\circ} \mathrm{K} ; x_{B B}=c_{2}\left(\lambda T_{B B}\right)^{-1} ;$ and $x_{R M}=c_{2}\left(\lambda T_{R M}\right)^{-1}$.

We next introduce McMahon's Relation ${ }^{3}$ which is

$$
\begin{aligned}
& \epsilon=\left(1-\rho_{s}\right)\left(1-\tau_{i}\right)\left(1-\rho_{s} \tau_{i}\right)^{-1}, \\
& \tau=\tau_{i}\left(1-\rho_{s}\right)^{2}\left(1-\tau_{i}^{2} \rho_{s}^{2}\right)^{-1}, \\
& \rho=\rho_{s}\left(1+\tau \tau_{i}\right) .
\end{aligned}
$$

Furthermore, we know that ${ }^{4}$

$$
\begin{aligned}
\rho_{s}=\left[(n-1)^{2}+\lambda^{2} K^{2}(4 \pi)^{-2}\right] & {\left[(n+1)^{2}\right.} \\
& \left.+\lambda^{2} K^{2}(4 \pi)^{-2}\right]^{-1} .
\end{aligned}
$$


We rewrite $\epsilon$ from Eq. (1) in the form

$$
\begin{aligned}
\epsilon_{1} & =A\left(t_{1}, n, K\right) \\
\epsilon_{2} & =A\left(t_{2}, n, K\right)
\end{aligned}
$$

for the emissivity values, $\epsilon_{1}$ and $\epsilon_{2}$, obtained from two specimens of the same material, but different thicknesses $t_{1}$ and $t_{2}$.

We know that the pair of simultaneous equations, Eqs. (5a) and (5b), can be solved for $n$ and $K$ in terms of $\epsilon_{1}, \epsilon_{2}, t, t_{2}$, if the Jacobian,

$$
\left\{\partial\left[A\left(t_{1}, n, K\right), A(t, n, K)\right]\right\} / \partial(n, K)
$$

is not zero ${ }^{5}$, which is the case here.

However, there is a simpler approach which is valid for materials for which: (1) values of $n$ and $d n / d T$ are known at room temperature; (2) $d n / d T$ is small and $n$ is near one so the extrapolated value of $\rho_{s}$ at the temperatures of measurement is small; and (3) the errors in the measured values of $\epsilon$ are larger than the errors in the computed values of $\rho_{s}$.

These conditions (1)-(3) were fulfilled for the calcium fluoride data presented in this paper.

This simpler approach consists in computing extrapolated values of $\rho_{s}$ at the wavelengths and temperatures for which it is possible to compute reasonably accurate values of $K$ from the emissivity data. Then we substitute the appropriate values of $\rho_{s}$ and $\epsilon$ into the equation

$$
\tau_{i}=\left(1-\epsilon-\rho_{s}\right)\left(1-\rho_{s}-\epsilon \rho_{s}\right)^{-1},
$$

and compute $\tau_{i}$ from Eq. (6). Since the specimen thickness $t$ is known, $K$ can be computed from the definition of $\tau_{i}$ :

$$
\tau_{i} \equiv \exp (-K \times t) .
$$

The presence of the background $\epsilon_{\beta}$ complicated the mathematics somewhat for the data of this paper. In the presence of a background, Eq. (6) is modified to

$\tau_{i}=\left\{\begin{array}{c}-\left(1-\rho_{s}\right)^{2}\left(1-\epsilon_{\beta}\right)+\left[\left(1-\rho_{s}\right)^{4}\left(1-\epsilon_{\beta}\right)^{2}\right. \\ \left.+4\left(1-\epsilon^{\prime}-\rho_{s}\right) \rho_{s}\left(1-\rho_{s}-\rho_{s} \epsilon^{\prime}\right)\right]^{1 / 2} \\ 2 \rho_{g}\left(1-\rho_{s}-\rho_{s} \epsilon^{\prime}\right)\end{array}\right\}$.

If one discards terms of order $\rho_{s}^{2}, \epsilon_{\beta}{ }^{3}, \rho_{s} \epsilon_{\beta}{ }^{2}$ or higher, Eq. (8) reduces to

$$
\begin{aligned}
\tau_{i}=\left(1-\epsilon^{\prime}\right)\left(1+\epsilon_{\beta}+\epsilon_{\beta}{ }^{2}\right)-\rho_{\theta}\left(\epsilon^{\prime}\right)^{2} & -\rho_{s} \epsilon_{\beta}\left[2-2 \epsilon^{\prime}+3\left(\epsilon^{\prime}\right)^{2}\right] .
\end{aligned}
$$

Since we are particularly interested in computing $K$, it is helpful to compute the error in the computed values of $K$ due to an error $\Delta \epsilon$ in the measured value of $\epsilon$. $K t$ is given by

$$
K t=-\ln \tau_{i},
$$

so

$$
\Delta(K t)=\left[2(K t) / 2\left(\tau_{i}\right)\right] \Delta \tau_{i}
$$

or

$$
\Delta(K t)=-\tau_{i}^{-1} \Delta \tau_{i} .
$$

Thus, it is seen that (assuming that the sample thick-

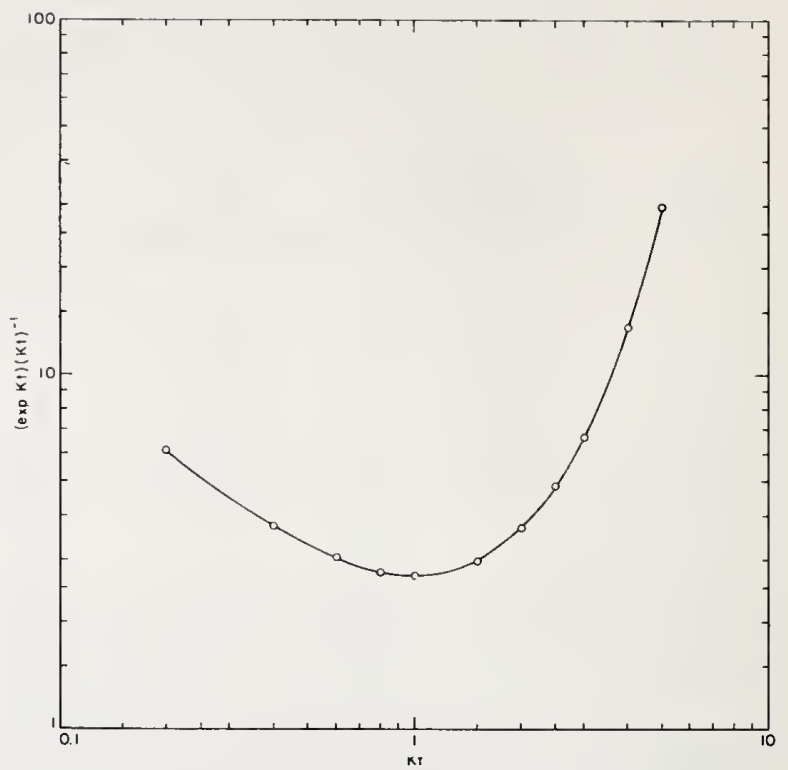

Fig. 3. Hamilton's error coefficient $(\exp K t)(K t)^{-1}$ vs $K t$.

ness is known accurately)

$$
t \Delta K=\tau_{i}^{-1}\left(2 \tau_{i} / 2 \epsilon\right) \Delta \epsilon
$$

or

$$
K^{\prime} \Delta K=\Delta \epsilon\left\{\begin{array}{c}
\frac{\left(1-\rho_{s}\right)^{2}}{\left[\ln \left(1-\epsilon-\rho_{s}\right)-\ln \left(1-\rho_{s}-\epsilon \rho_{s}\right)\right]} \\
\times\left[\left(1-\epsilon-\rho_{s}\right)\left(1-\rho_{s}-\epsilon \rho_{s}\right)\right]
\end{array}\right\} .
$$

It is clear from Eq. (14) that $\Delta K$ becomes infinite at both $\epsilon=0$ and $\epsilon=1-\rho_{s}$ and hence that there is a value $\epsilon_{0}$ of $\epsilon$ for which $\Delta K$ is minimum. Omitting the detailed calculations (which consist in differentiating the right side of Eq. (14) with respect to $\epsilon$, setting the derivative equal to zero, and solving for $\epsilon_{0}$, we find (to order $\rho_{s}$ )

$$
\epsilon_{p}=0.623-0.968 \rho_{s},
$$

so the sample thickness $t_{0}$ for minimum error in $K$ is (again to order $\rho_{s}$ ) given by

$$
K t_{0}=1-0.087 \rho_{s}
$$

Hamilton ${ }^{6}$ has shown that $K t_{0}=1$ for $\rho_{s}=0$; Hamilton's error coefficient, $(\exp K t)(K t)^{-1}$, is plotted in Fig. 3.

We see, then, that a sample of given thickness allows only a comparatively narrow range [roughly, $0.3<$ $K t<3.0$, for $2.7<(\Delta \epsilon K)^{-1} \Delta K<5.4$ ] of $K$ values to be accurately calculated from emissivity data. This is the reason for the limited range of wavelengths $(6-10 \mu)$ for which accurate $K$ values for calcium fluoride were computed.

The major sources of error in the system used to obtain the data presented in this paper are, in order of decreasing importance, as follows-

(1) The background emissivity $\epsilon_{\beta}$ (which is actually a slowly varying function of sample thickness); as 


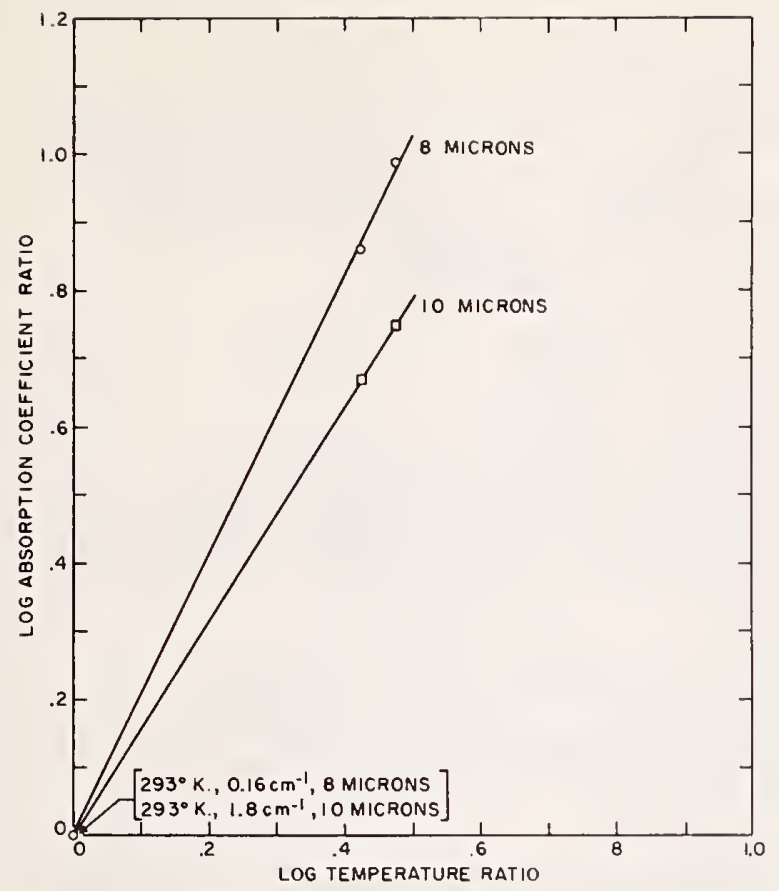

Fig. 4. Logarithm of normal spectral absorption coefficients of single-crystal calcium fluoride at room temperature, $500^{\circ} \mathrm{C}$ and $600^{\circ} \mathrm{C}$, at $8 \mu$ and $10 \mu$ wavelength vs logarithm of temperature.

mentioned earlier this was due to misalignment of the sample holder.

(2) A slight vertical displacement between the optic axes of the sample holder and the blackbody produced emissivity values roughly $4 \%$ too large; it was assumed that this was a geometrical effect and that a constant correction should be applied to all the data obtained, the amount of the correction being determined by the reasonable assumption that the $8 \mathrm{~mm}$ and $16 \mathrm{~mm}$ thick samples were opaque at $12 \mu$ wavelength at $500^{\circ} \mathrm{C}$ and $600^{\circ} \mathrm{C}$, and that their corresponding normal spectral emissivities were equal to $1-\rho_{s}$.

(3) The difference between the effective sample temperature and the measured sample temperature was a source of error. Since the sample temperature is measured at the rim of the sample, and the spectroradiometer field of view is in the central portion of the sample, it is clear that the effective sample temperature will be less than the measured temperature. An approximate theoretical treatment yields the formula

$$
\alpha=\delta\left\{1-\exp \left[-2 \sigma \epsilon_{T} r^{2} T^{3}{ }_{S P}\left(\sigma_{T} \times t\right)^{-1}\right]\right\},
$$

where

$$
\begin{array}{ll}
\alpha & =\Delta T_{S P}\left(T_{S P}\right)^{-1}, \\
\delta & =\text { twice the fraction of a hemisphere viewed by one side } \\
& \text { of the sample, } \\
\Delta T_{S P}= & \text { difference between the sample measured temperature } \\
& \text { and the sample effective temperature, } \\
\sigma \quad & \text { Stcfan-Boltzmann constant }\left[0.57 \times 10^{-11} \mathrm{~W} \mathrm{~cm} \mathrm{~cm}^{-2}\right. \\
& \left.\left({ }^{\circ} \mathrm{K}\right)^{-4}\right], \\
\epsilon_{T} & =\text { total normal emissivity of sample, } \\
r & =\text { radius of sample holder apcrture, and } \\
\sigma_{T} & =\text { thermal conductivity of sample. }
\end{array}
$$

The above formula neglects room temperature radiation. It assumes that the sample is heated by conduction through the rim and radiation from the sample holder, and that $\alpha$ and $\delta$ are both much less than one.

If we apply the formula to the $2-\mathrm{mm}$ thick calcium fluoride sample at $600^{\circ} \mathrm{C}$, we find that $\alpha \approx 0.002$. This produces roughly $0.5 \%$ change in the measured emissivity at $6 \mu$.

(4) The measured emissivity also depends on variations in the shutter temperature, the ambient temperature, and the spectrometer temperature. The ideal, noerror situation is for all these temperatures to be equal and constant during a series of sample and blackbody spectral radiance measurements. This is normally not the case. The exact error analysis is complicated, but the magnitude of the error may be estimated from the formula

$$
\begin{array}{r}
\Delta \epsilon=\Delta\left(\ln T_{R M}\right) \times x_{R M}\left[1-\exp \left(-x_{R M}\right)\right]^{-1} \tau\left[1-\exp \left(-x_{B B}\right)\right] \\
\times\left[\exp \left(x_{R M}+x_{B B}\right)-1\right]^{-1},
\end{array}
$$

where $\Delta T_{R M}=$ maximum temperature difference over a series of measurements between the shutter, the ambient, and the spectrometer temperatures. For constant $\Delta T_{R M}, T_{S P}$, and $T_{B B}$, and varying the wavelength $\lambda$, it is clear that $\Delta \epsilon$ increases monotonically with increasing $\lambda$ to an asymptotic limit for $x_{B B}, x_{R M} \ll 1$ (Rayleigh-Jeans region) of

$$
\Delta \epsilon_{\max }=\Delta\left(\ln T_{R M}\right)\left[x_{R M}\left(x_{B B}\right)^{-1}-1\right]^{-1} \times \tau .
$$

Crudely estimating the value of $\Delta T_{R M}$ as $10^{\circ} \mathrm{C}$, then for $\lambda=10 \mu, T_{R M}=293^{\circ} \mathrm{K}$ and $T_{B B}=773^{\circ} \mathrm{K}$; and taking $\tau=0.5$, we find $\Delta \epsilon \approx 0.004$.

As Table IV and Fig. 4 show, the normal spectral absorption coefficient of single-crystal calcium fluoride varies approximately with the 2.06 power of the absolute temperature at $8 \mu$ wavelength and with the 1.58 power at $10 \mu$. Born and Huang ${ }^{7}$ show theoretically that the absorption coefficient of an ionic crystal near a reststrahlen band varies as the third power of the temperature at the wings of the band, and as the reciprocal of the third power at the band center, there being a continuous transition between these extremes. Thus, the data of Table IV are consistent with the Born and Huang theory.

\section{References}

1. I. H. Malitson, Appl. Opt. 2, 1103 (1963).

2. D. E. Gray, Ed., American Institute of Physics; Handbook (Mcliraw-Hill Book Co., New York, 1963), 2nd ed., Chap. 6, p. 53 .

3. H. O. McNahon, J. Opt. Soc. Am. 40, 376 (1950).

4. Reference 2, Chap. 6, p. 103.

5. D. V. Widder, Advanced Calculus (Prentice-Hall, Inc., New York, 1947), Chap. 1, p. 47

6. R. H. Hamilton, Ind. and Eng. Chem. (Anal. Ed.) 16, 123 (1944).

7. M. Born and K. Huang, Dynamical Theory of Crystal Lattices (Oxford University Press, London, 1954), Chap. 7, pp. 357361 .

January 1967 / Vol. 6, No. 1 / APPLIED OPTICS 


\title{
56-PERIODIC HEAT FLOW IN A HOLLOW CYLINDER ROTATING IN A FURNACE WITH A VIEWING PORT
}

\author{
BY B. A. PEAYY AND A. G. EUBANKS
}

NATI ONAL BUREAU OF STANDARDS, WASHINGTON, D.C.

A rotating-specimen furnace has been designed and constructed for use in determining the thermal emittance, both spectral and total, of materials in the temperature range $1300^{\circ}$ to $1800^{\circ} \mathrm{K}$. In this apparatus, a hollow cylindrical specimen is rotated at constant speed in a concentric cylindrical furnace with heated walls, and the radiant flux from the specimen surface is measured as it passes a small viewing port.

An analytical method is given for estimating the surface temperature of the specimen as a function of rotation speed, thermal properties of the specimen material, and system geometry.

The apparatus is described, and data obtained with the specimen rotating at slow speeds are compared with values predicted by the analytical method.

A rotating-specimen furnace has been designed and constructed at the National Bureau of Standards for measuring the thermal emittance (both spectral and total) of nonmetallic solids in the temperature range from $1300^{\circ}$ to $1800^{\circ} \mathrm{K}$. In this apparatus, a hollow cylindrical specimen is rotated continuously in a concentric cylindrical furnace with heated walls, and the radiant flux from the specimen surface as it passes a small viewing port is measured with a suitable radiometer. In passing the viewing port, the surface is lowered in temperature by heat transfer to surfaces at or near room temperature and the magnitude of this reduction is dependent upon both the speed of rotation and the thermal properties of. the specimen. Previous investigations (ref.1, $2,3,4$, and 5) employing the principles of the rotating specimen furnace did not present an analysis of this temperature variation.

The present paper gives an analytical method for estimating the surface temperature of the specimen as a function of angular velocity and thermal properties of the specimen material. The purpose of this investigation was to determine reasonable speeds of rotation at which surface temperature changes become negligible. The surface temperature must be determined quite accurately for computing emittances and consideration must be given principally to radial temperature gradients in the specimen, especially if the specimen material is of low thermal conductivity or diffusivity.

As the specimen rotates in the furnace (fig. $56-1$ ), an area of its surface gains heat principally by radiation from the furnace walls, and loses heat at the viewing port during the portions of the cycle when the respective surfaces are seen. Because this is a continuous opération, a steady periodic heat flux boundary condition, as illustrated in figure $56-2$, is assumed at the outer surface of the hollow cylindrical specimen. A zero heat flux is assumed at its inner surface.

Concurrently with the analytical treatment, experiments were conducted with the specimen rotating at slow speeds, and the temperature changes thus obtained were compared with the values predicted by the analytical method.

\section{MATHEMATICAL DEVELOPMENT}

\section{General Analysis}

For conduction heat transfer in the hollow cylindrical specimen it is assumed that there is no flow of heat at the inner radius $r=a$, and that the heat flux at the outer radius $r=b$ is a 


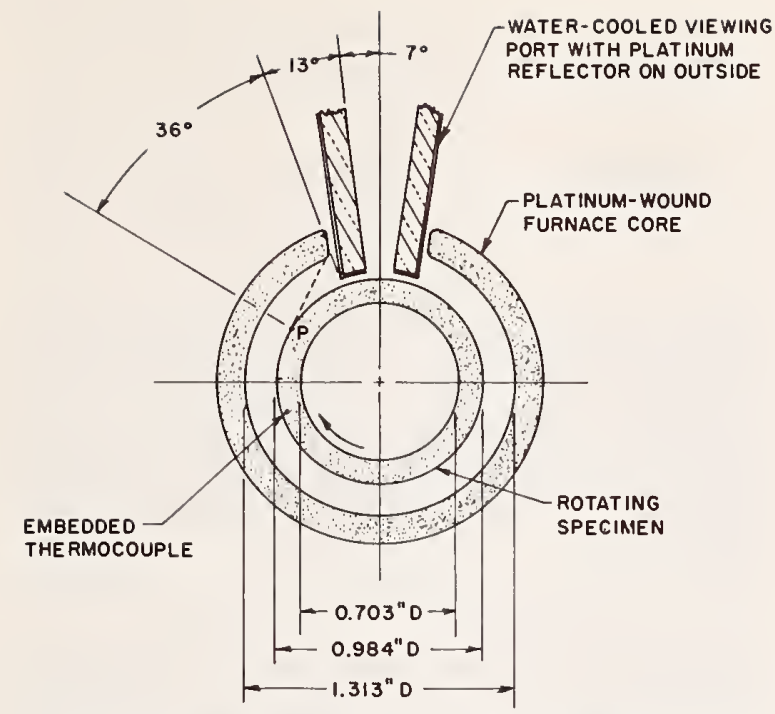

Ficure 56-1.-Cross section through rotating specimen furnace and viewing port with dimensions and angular positions.

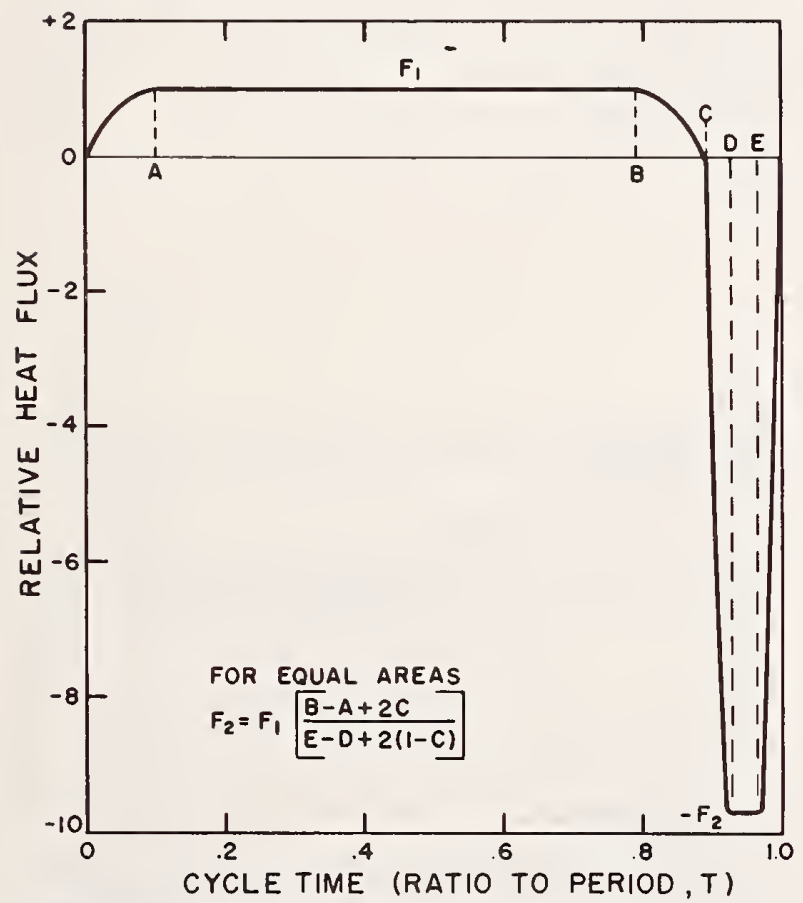

FiguRe 56-2.-Assumed heat flux history of a point on the surface of the rotating specimell.

periodic function of time. As shown in general terms in figure $56-2, F_{1}$ represents the average heat flux radiated to the specimen surface from the furnace walls during the time period $A$ to $B$, and $F_{2}$ is that radiated from the specimen as it passes the viewing port from $l)$ to $E$. The times $A, B, C, D$, and $E$, are arbitrary and are proportioned to the period $T$ of one revolution of the specimen.

The curved lines between $O$ and $A, B$ and $C$, $C$ and $D$, and $E$ and 1.0 are quadratic curves chosen to give heat flux continuity throughout, and slope continuity at $A, B, C$, and $E$. By selecting the designated times, changes in the contour can be made, and the continuity conditions used give faster convergence of the Fourier series involved in the analysis. The amplitudes of the positive and negative fluxes are adjusted so that the summation over the cycle of the areas above and below the zero flux line is \%ero. By this adjustment, a steady periodic condition is assured.

As the cylinder rotates, a point $P \quad(r=b$, $\varphi=\varphi_{1}$ ) on the surface experiences a flux cycle. Assuming the cylinder stationary and the furnace rotating with a period $T$, a complex form of a Fourier series

$$
\begin{array}{r}
k\left(\frac{\partial \theta}{\partial r}\right)_{r=0}=F_{1}\left\{\sum_{n=1}^{\infty}\left(P_{n}+i Q_{n}\right) \operatorname{Exp}\left[i\left(\varphi+\frac{2 n \pi t}{T}\right)\right]\right. \\
+ \text { conjugate }\}
\end{array}
$$

can be used to describe the arbitrary periodic flux function which serves as a boundary condition. $k$ is the thermal conductivity of the specimen, $\theta$ is the temperature departure from the mean temperature of the system, $\varphi$ is an arbitrary angular position within the specimen and

$$
\begin{aligned}
P_{n}= & \frac{2}{(2 n \pi)^{2}}\left[\frac{\psi}{1-E}-\frac{1}{A}+\left(\frac{\psi}{D-C}\right.\right. \\
& \left.\left.-\frac{1}{C-B}\right) \cos 2 n \pi C\right]+\frac{2}{(2 n \pi)^{3}}\left[\frac{\sin 2 n \pi A}{A^{2}}\right. \\
& -\frac{\sin 2 n \pi B}{(C-B)^{2}}+\left\{\frac{\psi}{(D-C)^{2}}\right. \\
& \left.+\frac{1}{(C-B)^{2}}\right\} \sin 2 n \pi C+\psi\left\{\frac{\sin 2 n \pi E}{(1-E)^{2}}\right. \\
Q_{n}= & \frac{2 \sin 2 n \pi C}{(2 n \pi)^{2}}\left[\frac{1}{C-B}-\frac{M}{D-C}\right] \\
& \left.-\frac{2}{(2 n \pi)^{3}}\left[\frac{1-\cos 2 n \pi A}{A^{2}}+\frac{\cos 2 n \pi B}{(C-B)^{2}}\right\}\right], \\
& -\left\{\frac{\psi}{(D-C)^{2}}+\frac{1}{(C-B)^{2}}\right\} \cos 2 n \pi C \\
& \left.\left.+\psi \frac{\cos 2 n \pi D}{(E-D)^{2}}-\frac{1-\cos 2 n \pi E}{(1-e)^{2}}\right\}\right] .
\end{aligned}
$$


To yield a zero net flux over the cycle, $F_{2}=\psi F_{1}$ where

$$
\psi=\frac{B-A+2 C}{E-D+2(1-C)} .
$$

The partial differential equation for conduction heat transfer in a homogeneous material, where heat flow in the axial direction is neglected, is for polar coordinates $(r, \varphi)$

$$
\frac{\partial^{2} \theta}{\partial r^{2}}+\frac{1}{r} \frac{\partial \theta}{\partial r}+\frac{1}{r^{2}} \frac{\partial^{2} \theta}{\partial \varphi^{2}}-\frac{1}{\alpha} \frac{\partial \theta}{\partial t}=0
$$

where $\alpha$ is the thermal diffusivity. Substituting $\theta=f_{ \pm n}(r) \operatorname{Exp}\left[ \pm i\left(\varphi+\frac{2 n \pi t}{T}\right)\right], n=1,2,3, \ldots$

in equation (2) gives

$$
\begin{aligned}
f_{ \pm}^{\prime \prime}+\frac{1}{r} f_{ \pm n}^{\prime}-f_{ \pm n}\left[\frac{1}{r^{2}} \pm \frac{2 n \pi i}{\alpha T}\right]=0 . & +\frac{\left[P_{n}-i Q\right]}{z} \\
L_{ \pm n}= & \frac{I_{1}\left(\frac{r z}{a} \sqrt{ \pm i}\right) K_{1}^{\prime}(z \sqrt{ \pm i})-K_{1}\left(\frac{r z}{a} \sqrt{ \pm i}\right) I_{1}^{\prime}(z \sqrt{ \pm i})}{\left.\sqrt{ \pm i}\left[I_{1}^{\prime}\left(\frac{b z}{a} \sqrt{ \pm i}\right) K_{1}^{\prime}(z \sqrt{ \pm i})-K_{1}^{\prime}\left(\frac{b z}{a} \sqrt{ \pm i}\right) I_{1}^{\prime} z \sqrt{ \pm i}\right)\right]} \\
= & \frac{R_{n}\left(\frac{r z}{a} ; z\right) \pm i S_{n}\left(\frac{r z}{a} ; z\right)}{X_{n}\left(\frac{b z}{a} ; z\right) \pm i Y_{n}\left(\frac{b z}{a} ; z\right)}
\end{aligned}
$$

where $z^{2}=2 n \pi /\left(\alpha T / a^{2}\right)$, and $I_{1}$ and $K_{1}$ are first order modified Bessel functions of the first and second kind, respectively. Substitution of boundary conditions $d \theta / d r=0$ at $r=a$, and equation (1) at $r=b$, in the first derivative of equation (3) gives a solution for $M$ and $N$, and vields

$$
\begin{aligned}
\frac{\theta k}{F_{1} a} & =\sum_{n=1}^{\infty}\left\{\frac{\left(P_{n}+i Q_{n}\right)}{z} L_{n} \operatorname{Exp}\left[i\left(\varphi+\frac{2 n \pi t}{T}\right)\right]\right. \\
& \left.+\frac{\left[P_{n}-i Q\right]}{z} L_{n} \operatorname{Exp}\left[-i\left(\varphi+\frac{2 n \pi t}{T}\right)\right]\right\}
\end{aligned}
$$

where

$$
\begin{aligned}
X_{n}(x ; y)=b e r_{1}{ }^{\prime} x k e r_{1}{ }^{\prime} y-b e i_{1}^{\prime} x k e i_{1}^{\prime} y- \\
\operatorname{ber}_{1}^{\prime} y k e r_{1}^{\prime} x+b e i_{1}^{\prime} y k e i_{1}{ }^{\prime} x
\end{aligned}
$$$$
Y_{n}(x ; y)=b e r_{1}{ }^{\prime} x k e i_{1}{ }^{\prime} y+b e i_{1}{ }^{\prime} x k e r_{1}{ }^{\prime} y-
$$$$
b e r_{1}^{\prime} y k e i_{1}^{\prime} x-b e i_{1}^{\prime} y k e r_{1}^{\prime} x
$$

$R_{n}(x ; y)=b e r_{1} x k e r_{1}^{\prime} y-b e i_{1} x k e i_{1}^{\prime} y-$

$$
k e r_{1} x b e r_{1}^{\prime} y+k e i_{1} x b e i_{1}^{\prime} y
$$

$S_{n}(x ; y)=b e r_{1} x k e i_{1}^{\prime} y+b e i_{1} x k e r_{1}{ }^{\prime} y-$

$$
k e r_{1} x b e i_{1}^{\prime} y-k e i_{1} x b e r_{1}^{\prime} y
$$

which are obtained from the identities (6):

$$
\begin{gathered}
i^{ \pm 1} I_{1}(x \sqrt{ \pm i})=b e r_{1} x \pm i b e i_{1} x \\
i^{ \pm 3 / 2} I_{1}{ }^{\prime}(x \sqrt{ \pm i})=b e r_{1}{ }^{\prime} x \pm i b e i_{1}{ }^{\prime} x \\
i^{ \pm 1} K_{1}(x \sqrt{ \pm i})=k e r_{1} x \pm i k e i_{1} x \\
i^{ \pm 1 / 2} K_{1}{ }^{\prime}(x \sqrt{ \pm i})=k e r_{1}{ }^{\prime} x \pm i k e i_{1}{ }^{\prime} x
\end{gathered}
$$

Rationalizing the denominators of $L$, equation (6) becomes

$\frac{\theta k}{F_{1} a}=2 \sum_{n=1}^{\infty} \frac{U_{n} \cos \left(\frac{2 n \pi t}{T}+\varphi\right)-V_{n} \cdot \sin \left(\frac{2 n \pi t}{T}+\varphi\right)}{z\left(X_{n}^{2}+Y_{n}^{2}\right)}$

where

$$
\begin{aligned}
& U_{n}=R_{n}\left(X_{n} P_{n}+Q_{n} Y_{n}\right)-S_{n}\left(Q_{n} X_{n}-Y_{n} P_{n}\right) \\
& V_{n}=R_{n}\left(Q_{n} X_{n}-Y_{n} P_{n}\right)+S_{n}\left(X_{n} P_{n}+Q_{n} Y_{n}\right)
\end{aligned}
$$

'l'he expressions for $P_{n}$ and $Q_{n}$ contain, and their values are fixed by, the arbitrary values chosen for $A, B, C, D$, and $E$ (fig. 56-2). Accordingly, equation (7) can be represented using dimensionless parameters in the following functional form,

$$
\frac{\theta k}{F_{1} a}=g\left(\frac{\mathrm{A} T}{a^{2}}, \frac{b}{a}, \frac{r}{a}, \frac{t}{T}\right)
$$

assuming the angular position $\varphi=0$. 


\section{Radiation Geometry Considerations}

The convection and conduction heat transfer across the air space between the specimen and furnace surfaces is assumed to be negligible, and heat transfer by radiation only is considered in the determination of $F_{1}$ for nse in equation (7).

Referring to figure $56-1$, a point $P$ on the rotating specimen surface will see the walls of the water-cooled viewing port when within an angular displacement of about $36^{\circ}$ before and after passing these walls. The geometry of the arrangement and the different emittances of the surfaces involved make an exact analysis of this problem virtually impossible; so it is assumed that $F_{1}$ is equal to the average heat flux between the two surfaces when the point $P$ of the cycle sees only the furnace walls, involving a total angle of about $248^{\circ}$ of the cycle. The net heat flux to the specimen within the two $36^{\circ}$ angles will be somewhat less due to radiation to the colder veiwing port walls. The time dimensions as shown on figure 56-2 are assumed from the above consideration to be: $A=0.1, B=0.79, C=0.89$, $D=0.927, E=0.963$, and $\Psi=9.65$.

The computation for $F_{1}$ then becomes

$$
F_{1}=\frac{\sigma}{\frac{1}{\epsilon_{1}}+\left(\frac{1}{\epsilon_{2}}-1\right) \frac{A_{1}}{A_{2}}}\left[T_{2}^{4}-\frac{\sum_{n=1}^{p} T_{1, n}{ }^{4}}{p}\right]
$$

$\sigma$ is the Stefan-Bolizmann constant, $5.67 \times 10^{-12}$ $w / \mathrm{cm}^{2} \mathrm{~K}^{4}, \epsilon$ is the emittance, $T$ is absolute temperature, $A$ is the surface area, and subscripts 1 and 2 refer to properties of the specimen and furnace surfaces, respectively. The summation is taken from temperature determinations for $p$ intervals in the region where a point on the specimen sees only the furnace wall. The temperature of the furnace wall is assumed to remain constant.

\section{Numerical Solutions}

Numerical solutions to equation (7a), with the time dimensions as given above and with ap.

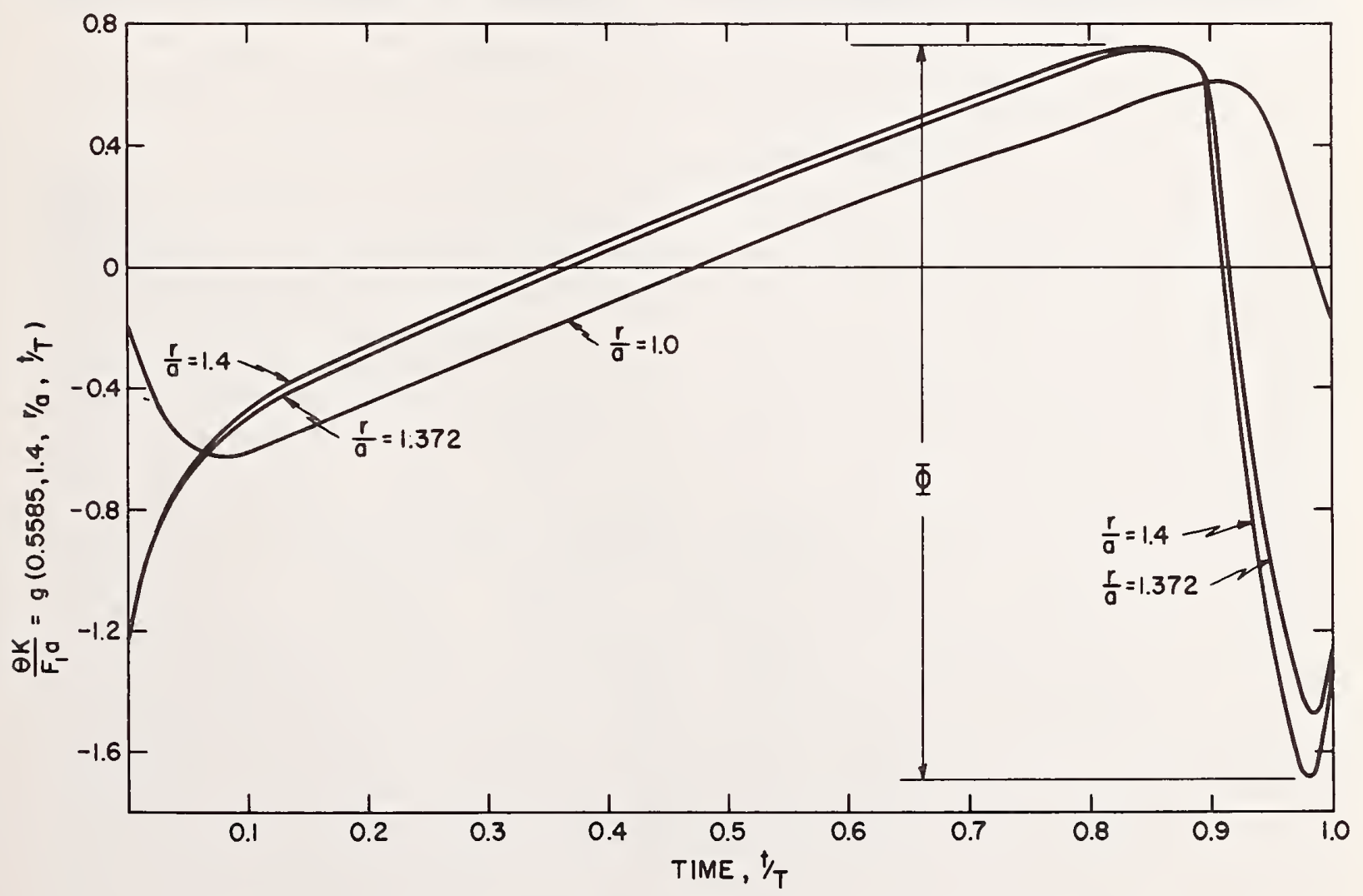

Figure 56-3.-Plot of $\Theta k / F_{1} a$ versus time for $\alpha T / a^{2}=0.5585$ and $r / a=1.4,1.372$, and 1.0. 
propriate experimental dimensions, were computed by a digital computer. Figure $56-3$ is a plot of $\theta k / F_{1} a$ against $t / T$ for $\alpha T / a^{2}=0.5885$ (corresponding to the experimental speed of 1 $\mathrm{rpm}$ ), and for $r / a=1.4$ (specimen outer surface), 1.372 (an embedded thermocouple), and 1.0 (specimen inner surface). For a particular specimen with $k, a$, and $F_{1}$ remaining constant, the temperature difference $\theta$ varies above and below the mean temperature of the cycle represented by $\theta=0$.

The maximum change in $\theta k / F_{1} a$ during a cycle of period $T$ is denoted by $\Phi\left[=f\left(\alpha \mathrm{T} / a^{2}\right.\right.$, $b / a, r / a)$ ], illustrated on figure 56-3. Figure 56-4 shows $\Phi$ versus $a T / a^{2}$ for $b / a=1.4$ and various values of $r / a$. Figure 56-4 shows that a reduction of the temperature fluctuation occurs with distance into the specimen, and with an increase in the rotational speed, which is inversely proportional to the period $T$.

\section{EXPERIMENTAL APPARATUS AND PROCE- DURE}

Figure $56-5$ is a vertical cross section of the rotating-specimen furnace, and figure $56-6$ is a photograph of the furnace and accessory parts.
The $15 / 1$-inch ID alumina tube surrounding the specimen is wound with platinum- $20 \%$ rhodium wire, with taps to adjust power input into the center and end sections. The center winding is continuous to the edges of the hole that is cut into the tube to permit entrance of the viewing port. An outer "booster coil" is provided, as shown in the figure, to supply additional heat to the central portion of the furnace. The temperature of the furnace is thermostatically controlled by a recorder-controller actuated by a platinum:platinum-10\% rhodium (Pt:Pt-10\% Rh) thermocouple embedded in the wall of the furnace tube. The heater and apparatus were designed to operate in the temperature range from $1300^{\circ}$ to $1800^{\circ} \mathrm{K}$.

The viewing port was machined from solid copper. A radiation shield of platinum foil surrounds the outside surfaces of the viewing port, including the edges that face the specimen. The inside surfaces are coated witn a black heatresistant paint. The inner face of the port was machined to a cylindrical surface of 0.503 -inch radius.

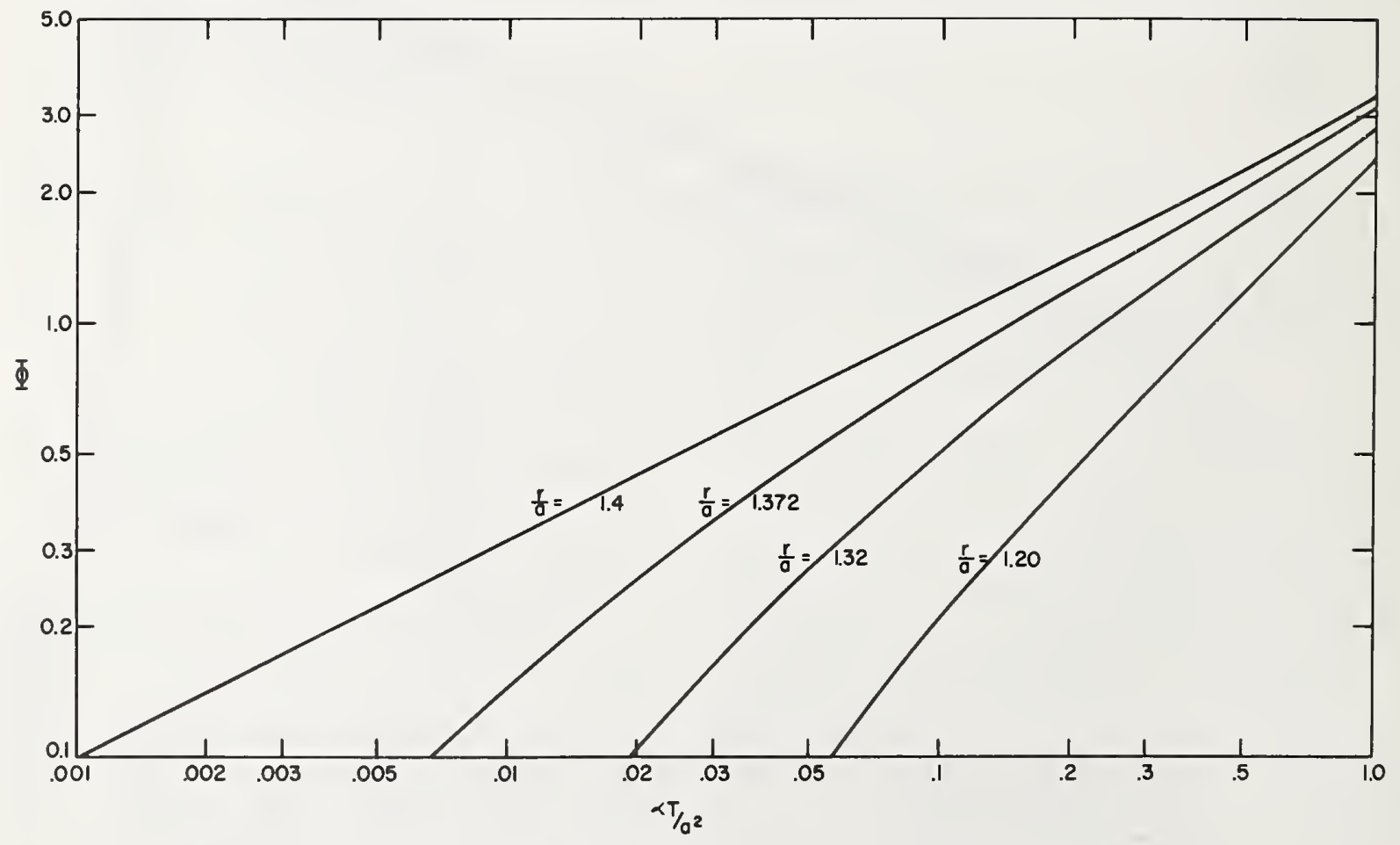

Figure 56-4.-Plot of $\Phi$ versus $\alpha T / a^{2}$ for $r / a=1.4,1.372,1.32$, and 1.2. 


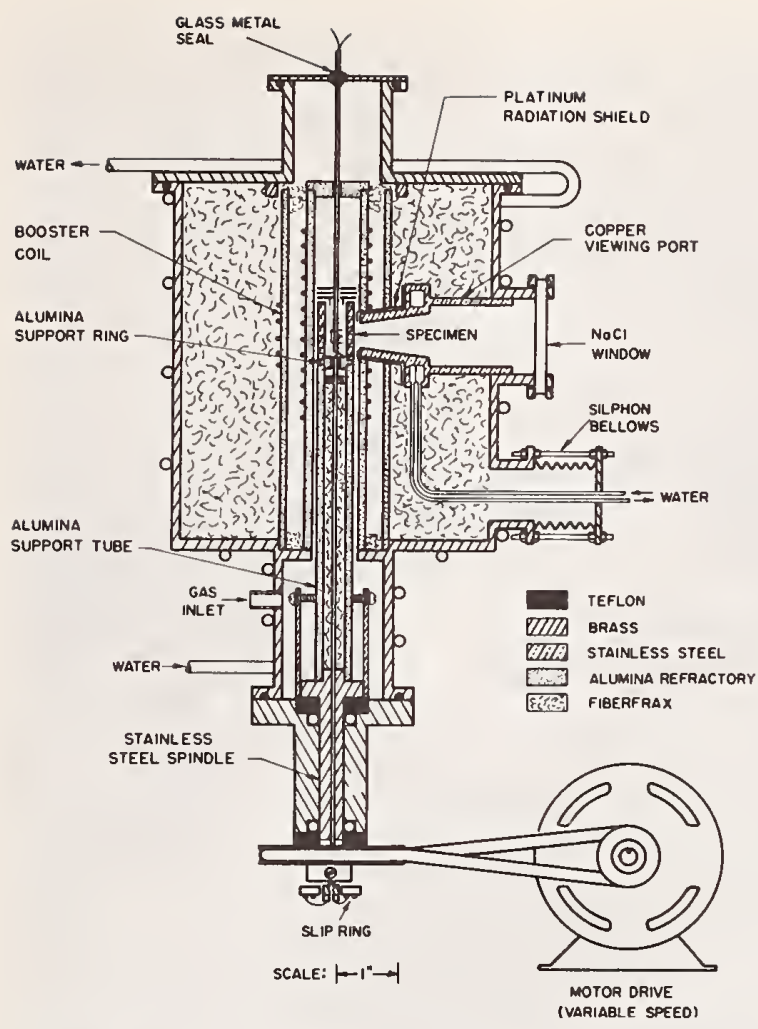

Frgure 56-5.-Vertical section through rotating specimen furnace with viewing port.

The furnace shell is sealed so that the specimen may be heated in an inert atmosphere, as well as in air. Power leads and thermocouple leads are brought out through glass-metal seals, while O-ring seals are used for the shell ends, as well as the viewing window. The Teflon thrust bearings provide a gas-tight seal at the point where the spindle shaft which supports the specimen enters the shell. The entire furnace shell is water cooled.

The spindle is driven through a gear reducer by a //8-hp motor. The rotation of the specimen can be adjusted to any speed in the range from 1 to $300 \mathrm{rpm}$.

The specimen is a hollow cylinder with nominal dimensions of 1 -inch outer dimeter by 1 inch high, with 3 -inch walls. Prior to test, each specimen is surface ground so as to be cylindrical to \pm 0.002 inch. The alumina support tube, or pedestal, was surface-ground to the same tolerance. The inner surfaces of this tube are surface-ground near both ends so that the alumina support plug can be inserted with a slip fit in the top and the stainless steel spindle could be inserted into the support tube with close tolerances at the bottom. Three stainless steel strips, spaced $120^{\circ}$ apart, project up from the spindle outside the alumina support tube. Each has a steel centering screw at the upper end that bears against a strip of stainless steel foil attached to the tube. These three screws are used for the final centering of the specimen. The temperature in the center cavity of the hollow specimen is observed by means of a calibrated thermocouple suspended from above and shielded from extraneous radiation.

Experiments were carried out to determine how the temperature near the surface of a non-metallic specimen fluctuated as the specimen rotated in the furnace and also to determine the rotational speed that was required to virtually eliminate temperature differences from the inner wall of the specimen and at a point near the outer wall.

A coarse-grained alumina refractory was selected for this investigation as a suitable nonmetallic material. A specimen was prepared of this material by first forming a cylinder and then firing it to about $1250^{\circ} \mathrm{K}$ to give a soft machinable structure. After finishing to the desired size and shape, as specified above, a small groove was machined circumferentially in the outer wall surface midway between the two ends. A butt-welded Pt:Pt-10\%Rh thermocouple made of calibrated wire, 0.005 inch in diameter, was placed in this groove, with the leads passing through two holes that had been drilled through the specimen wall at points nearly diametrically opposite to the bead position. The leads from the thermocouple were then brought down through the support tube and spindle assembly to a slip ring with silverto-sliver contacts at the bottom of the spindle.

The depth of the thermocouple bead was measured and the center-line of the bead was found to be 0.010 inch below the outer wall surface of the specimen. Next, the groove with the thermocouple wire in position was filled with an excess of the alumina refractory and the specimen fired at $1800^{\circ} \mathrm{K}$. The final operation consisted of surface grinding the outside of the specimen until the excess material had been ground flush with the original surface. 


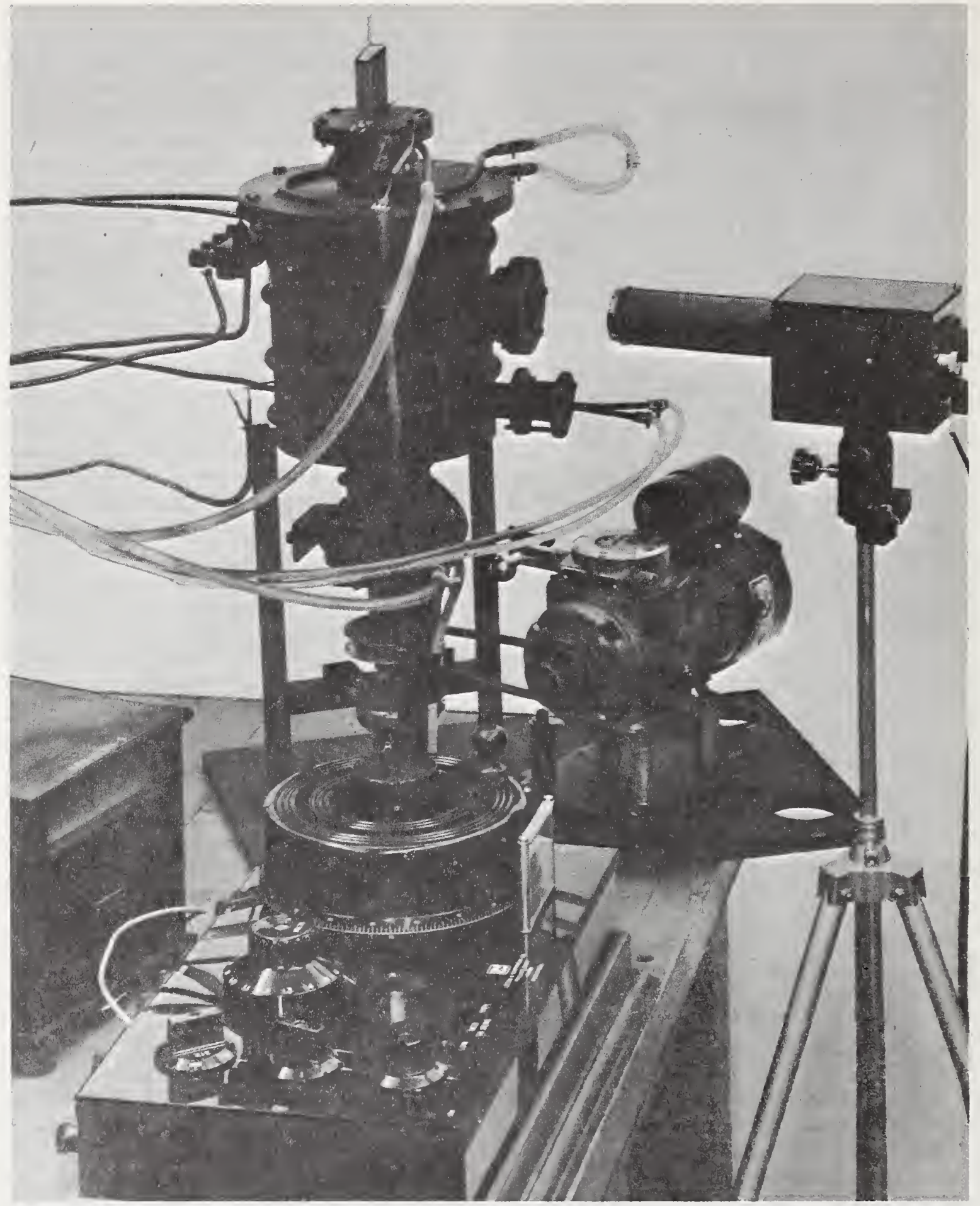

FigURE 56-6.-Rotating specimen furnace and accessory equipment. 
The finished specimen was uniform in appearance and free from surface cracks.

Data with the alumina specimen were obtained at various speeds of rotation. The temperature of the embedded thermocouple changed as the specimen rotated through $360^{\circ}$. The termperatures were obtained with a Leeds \& Northrup type $\mathrm{K}$ potentiometer by observing the deflection of the galvanometer spot as a function of the angular position of the thermocouple and converting these readings to temperature.

\section{RESULTS}

The average observed temperature variation of the embedded thermocouple as a function of angle is shown in figure 56-7 for speeds of 1 , $1.5,2$, and $2.5 \mathrm{rpm}$, with the furnace wall temperature maintained at $1430^{\circ} \mathrm{K}$. The dotted vertical lines show the angular positions of the outside edges of the viewing port. The maximum temperature change of the thermocouple as a result of passing the viewing port decreased systematically from $100^{\circ} \mathrm{K}$ at $1.0 \mathrm{rpm}$ to $64^{\circ} \mathrm{K}$ at $2.5 \mathrm{rpm}$, which was the maximum speed at

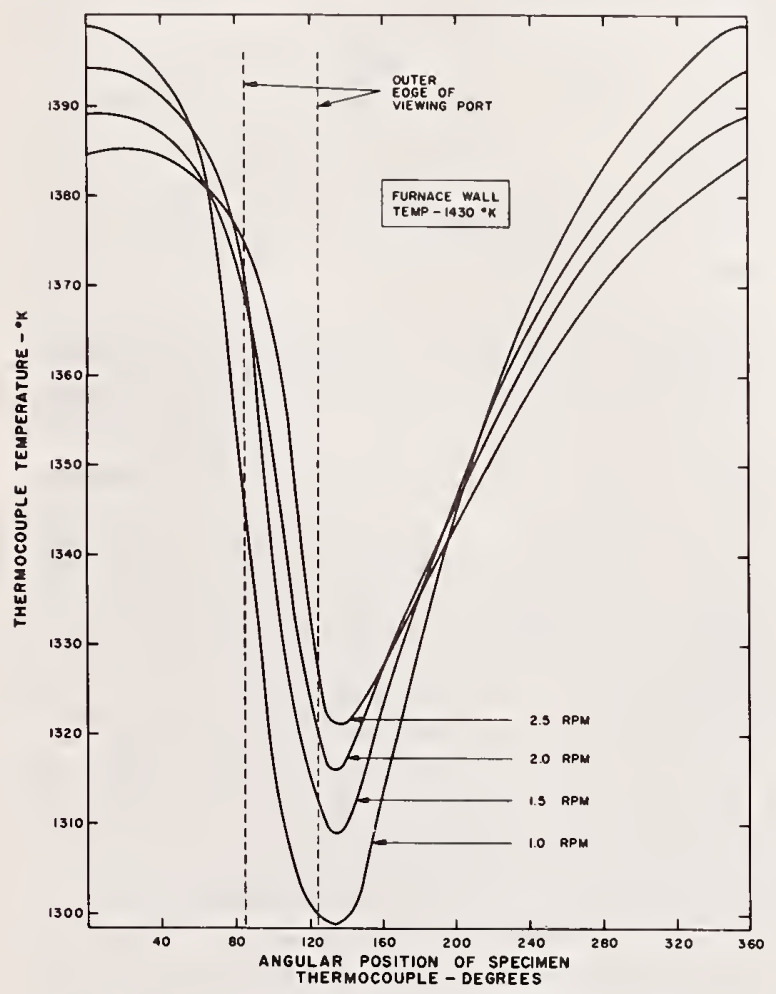

Figure 56-7.-Temperature of embedded thermocouple versus angular position of thermocouple. which measurements of this type could be made by the galvanometer method.

The maximum temperature reached by the embedded thermocouple while it was in the hot zone of the furnace decreased as the rotation speed was increased, but the mean temperature during a cycle remained constant within $\pm 1^{\circ} \mathrm{K}$ for all rotation speeds, at approximately $1362^{\circ}$ $\mathrm{K}$. The mean temperature during a cycle was above the minimum temperature by approximately $63 \%$ of the maximum temperature change.

Other tests were performed on this specimen over a wide range of rotational speeds and at different furnace temperatures where temperatures of the embedded thermocouple and shielded thermocouple in the center cavity of the hollow specimen were observed. For rotational speeds from 10 to $360 \mathrm{rpm}$, the mean temperature of the embedded thermocouple was within $\pm 1^{\circ} \mathrm{K}$ of the temperature of the shielded thermocouple. These results indicated that the shielded thermocouple will give the mean temperature of the specimen within reasonable limits of speeds. Below $10 \mathrm{rpm}$, the temperature of the shielded thermocouple was $3-5^{\circ} \mathrm{K}$ above the mean temperature of the embedded thermocouple, the latter computed by adding $63 \%$ of the maximum temperature change to the minimum temperature.

\section{ANALYSIS}

For the purpose of the mathematical analysis the pertinent properties of the alumina test specimen were determined, or estimated from the literature, as follows:

thickness, b-a

$=0.357 \mathrm{~cm}(0.141 \mathrm{in}$.

density, $\rho$

porosity

$=2.16 \mathrm{~g} / \mathrm{cm}^{3}$

specific heat_eq. (7), $c=1.3 \mathrm{w}$-sec/g. ${ }^{\circ} \mathrm{K}$ thermal conductivity _. -

$$
\text { eq. (8), } k=0.0208 \mathrm{w} / \mathrm{cm}^{\circ} \mathrm{K}
$$

thermal diffusivity, $\alpha=0.0074 \mathrm{~cm}^{2} / \mathrm{sec}$

emittance, $\epsilon_{1}$ (estimated) $=0.4$

outside radius, $b \quad=1.25 \mathrm{~cm}(0.492 \mathrm{in}$.)

inside radius, $a \quad=0.893 \mathrm{~cm}(0.352 \mathrm{in}$.)

radius at thermocouple $=1.225 \mathrm{~cm}(0.482 \mathrm{in}$.)

From these data, $\Phi=(0.00931 T, 1.4,1.372)$ at the position of the embedded thermocouple. Values of $\Phi$ are shown in figure 56-4, and the maximum change in temperature at any radial position during any cycle is $\Delta \theta=42.92 \Phi F_{1}$. 
$F_{1}$ was computed using equation (8), in which the emittance of the furnace wall was assumed to be the same as that of the specimen. The temperatures $T_{1, n}$ used in the summation of equation (8) were the observed temperatures of the embedded thermocouple. Actually, the temperature of the surface in passing through the heating part of the cycle should slightly exceed the embedded thermocouple temperature, as can be seen in figure 56-3. The slight difference between these two temperatures can be calculated using the value of $F_{1}$ obtained above, and applied as a correction to $T_{1, n}$ for computation of a better value of $F_{1}$. This correction was not considered for this paper. This is shown in the following table, in which parameters for the four tests are given, and also the analytically calculated, and the experimental, values of $\Delta \theta$.

\begin{tabular}{|c|c|c|c|c|c|}
\hline $\begin{array}{c}\text { Rotation } \\
\text { Speed, } r p m\end{array}$ & $\begin{array}{c}F_{1}(\mathrm{eq} . \\
(8)) \text { w/ } \mathrm{cm} \mathrm{cm}^{2}\end{array}$ & $\alpha T / a^{2}$ & $\begin{array}{l}\Phi,(f i g . \\
56-4)\end{array}$ & $\begin{array}{c}\Delta \theta,{ }^{\circ} K(r \\
\text { Analysis }\end{array}$ & $\begin{array}{l}\quad a=1.572) \\
\text { Experiment }\end{array}$ \\
\hline 1.0 & 1. 084 & 0.558 & 2. 18 & 101 & 101 \\
\hline $1.5 \ldots$ & 1. 087 & .372 & 1. 70 & 79 & 85 \\
\hline 2.0 & 1. 081 & .279 & 1. 46 & 68 & 73 \\
\hline $2.5 \ldots$ & 1. 108 & .223 & 1. 27 & 61 & 64 \\
\hline
\end{tabular}

Figures $56-8$ and $56-9$ were plotted from the analytical solution assuming $F_{1}$ was constant irrespective of rotational speed. Curves A and $B$ of figure $56-8$ show the maximum temperature change, $\Delta \theta$, at the surface and the position of the embedded thermocouple, respectively, versus the rotation speed. Curve $C$ of figure 56-8 shows the temperature change of the specimen surface in passing from one edge

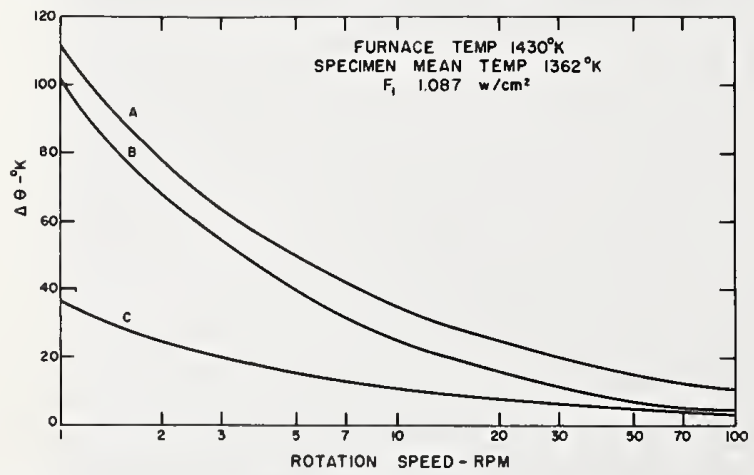

Figure 56-8.-Temperature departure versus rotation speed: A-Maximum temperature change in one revolution at specimen surface, $r / a=1.4$; $\mathrm{B}-$ maximum temperature change in one revolution at position of embedded thermocouple, $r / a=1.372$; and $\mathrm{C}$-temperature change at specimen surface from inside edge of viewing port to other inside edge.

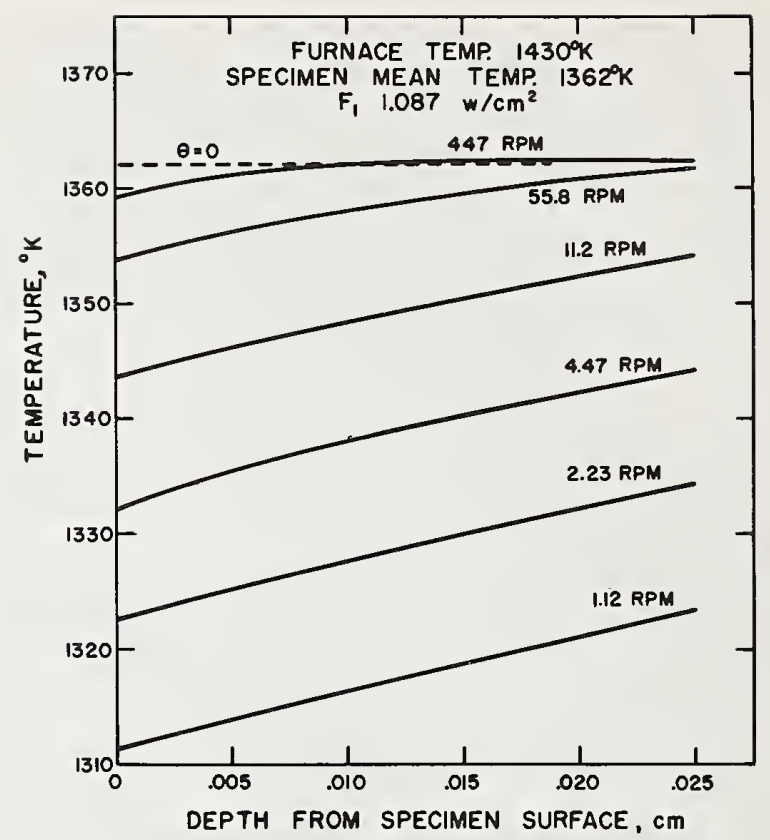

FIGURE 56-9.- Specimen temperature at center of viewing port opening versus depth from specimen surface for various rotation speeds.

of the viewing port to the other edge. An optical pyrometer focused on the specimen at the two edges of the viewing port opening should see the temperature difference indicated by curve $C$, if the specimen surface is opaque to radiation. The temperature difference across the viewing port, when determined from measurements made with an optical pyrometer, was found to be approximately $32^{\circ} \mathrm{K}$ at $2 \mathrm{rpm}$ and $2^{\circ} \mathrm{K}$ at $50 \mathrm{rpm}$.

Figure 56-9 shows the specimen temperature as a function of depth from the surface for six rotation speeds for a position at the center of the viewing port, assuming a mean temperature $(\theta=0)$ of $1362^{\circ} \mathrm{K}$. According to this analysis, fairly high rotation speeds are required for the porous alumina specimen before the temperature of the shielded cavity thermocouple can be accepted as a good approximation to the true temperature at or near the specimen surface.

The factor, $F_{1}$, of equation ( $7 \mathrm{a}$ ) was computed from equation (8), where the summation term was determined from experimental temperatures of a thermocouple embedded in the specimen. For the emittance tests to be conducted in this apparatus there will not be an embedded thermocouple. The experimental data seem to show that the summation term can be approxi- 
mated, for this apparatus, by

$$
\frac{\sum_{n=1}^{p} T_{1, n^{4}}}{p}=\left(T_{m}+T_{d}\right)^{4}
$$

where $T_{n}$ is the mean temperature, approximated by the temperature of the shielded cavity thermocouple, and $T_{d}=4.0$, for experiments with speeds from 1 to $2.5 \mathrm{rpm}$. With $F_{1}$ computed from this approximation, the temperature at the center of the viewing port opening, $T_{c}=T_{m}-g F_{1} a / k$, can be computed from figure 56-10 for a specimen whose thermal properties are known and which has a rotation speed of period $T$.

\section{SUMMARY}

Equations were developed analytically for computing cyclic temperatures of a hollow

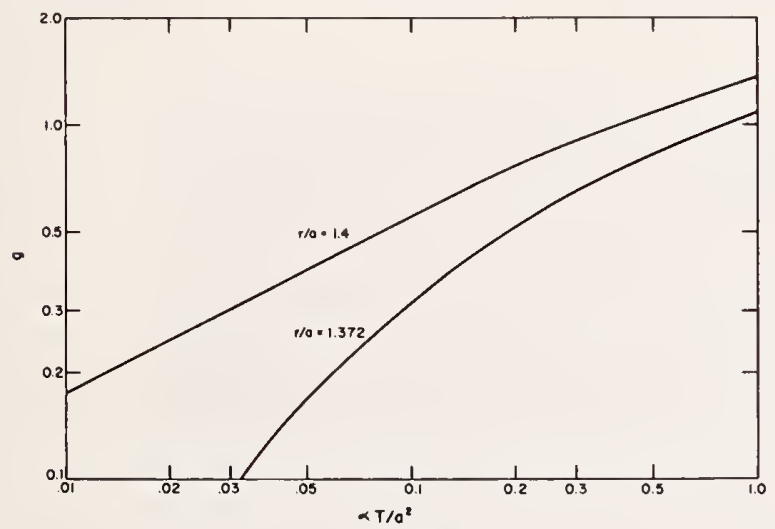

Figure 56-10.-Plot of $\theta k / F_{1} a=g\left(\alpha T / a^{2}, 1.4, r / a\right.$, 0.945 )-eq. (7a)-versus $\alpha T / a^{2}$ for $r / a=1.4$ and 1.372 , where $t / T=0.945$ is the center of the viewing port opening. cylindrical specimen rotating in a furnace with a viewing port as a function of system geometry, rotational speed, thermal properties of the specimen material, and a derived average heat flux for the portion of the cycle when the specimen is being heated by the furnace. Simplifying assumptions were that (1) the heat flux history at the outside surface of the specimen for one cycle is as shown in figure 56-2, (2) there is no heat flow at the inside surface of the specimen, and (3) there is no longitudinal heat flow in the specimen.

A comparison was made between specimen temperatures computed analytically and those obtained experimentally at low speeds of specimen rotation. Agreement within 6\% was obtained, at rotational speeds from 1 to 2.5 rpm, in regard to the maximum temperature change occurring at the position of an embedded thermocouple 0.01 inch under the surface. This agreement seems well within possible experimental error and uncertainty as to the thermal properties of the specimen material.

The analysis showed that the surface temperature of the specimen, at the center of the viewing port opening, was substantially lower than the mean temperature of the specimen at low rotational speeds, but the departure was reduced to within $3^{\circ} \mathrm{K}$ at about $450 \mathrm{rpm}$ for this case. Because the error in surface temperature acceptable for emittance determinations is less than $1^{\circ} \mathrm{K}$, a function is given graphically (figure 56-10) with which the departure can be estimated for various speeds in this apparatus.

\section{REFERENCES}

1. Fery, C.: Rayonnement Calorifique et Lumineux de Quelques Oxydes, Ann. Chim. de Phys., 27, 1902, p. 433.

2. Kingery, W. D.: Property Measurements at High Temperatures, John Wiley \& Sons, Inc., New York, N.Y., pp. 119-120 (1959).

3. Plunkett, J. D.; and Kingery, W. D.: The Spectral and Integrated Emissivity of Carbon and Graphite, Proc. of Fourth Conf. on Carbon, Pergamon Press, Belfast, England, pp. 457-472 (1960).

4. Olsen, H. O.; and Morris, J. C.: Determination of Emissivity and Reflectivity Data on Aircraft Structural Materials: Part III, Techniques of Measurement. WADC Tech. Rpt. 56-222, Jan. 1959.

5. Clayto v, W. A.: Thermal Property Measurement Techniques, Boeing Co. (Seattle Div.) Report No. DZ-80281 to Wright Air Dev. Div., on Contract AF33(600)-41517, Dec. 1961. 
6. Dwight, H. B.: Tables of Integrals and Other Mathematical Data. The Macmillan Co., 1934.

7. Russell, A. S.: Alumina Properties. Tech. Paper 10, Aluminum Company of America, Pittsburgh, Pa., 1953.

8. Goldsmith, A.; and Waterman, T. E.: Thermophysical Properties of Solid Materials, Section VII-M-1. Armour Research Foundation, WADC Tech. Rpt. 58-476, Wright Air Dev. Center, Wright-Patterson Air Force Base, Ohio, 1959. 


\title{
Survey of Infrared Measurement Techniques and Computational Methods in Radiant Heat Transfer
}

\author{
S. Thomas Dunn and Joseph C. Richmond \\ National Bureau of Standards, Washington, D. C. \\ AND \\ Jerome F. PARMer \\ University of West Virginia, Morgantown, W. Va.
}

\author{
Nomenclature
$A=$ area
$B \quad=$ radiosity (emitted and reflected flux per unit area)
$D \quad=$ subscript implying diffuse
$E \quad=$ energy function
$F_{(i-j)}=$ view factor from surface $i$ to surface $j$
$F_{(i-j)_{\tau}}=$ total image view factor
$H \quad=$ irradiation (irradiance)
$i, k \quad=$ index
$L \quad=$ radiance

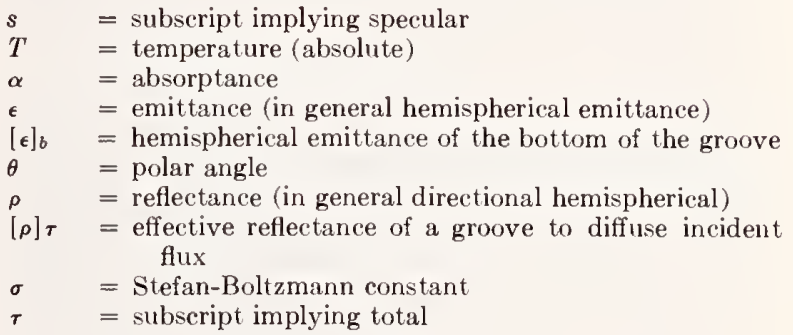

Stuart Thomas Dumn is a Mechanical Engineer with a B.S. from the University of Missouri at Rolla in 1962; an M.S. in 1963 and Pl.D. in 1965, both from Oklahoma State University. He was employed in the Photometry and Colorimetry Section of the National Burcau of Standards from May 1964 through December 1965, whére he completed his Ph.D. thesis in the field of infrared reflectance. He is now president of Dunn Associates Inc., of Silver Spring, Md. Dr. Dunn has several publications in the general arca of themal radiation neasurements.

Jerome F. Parmer is a Mechanical Engineer with a B.S. from the University of Notre Dame in 1957, an M.S. from West Virginia University in 1960, and a Ph.D. from Oklahoma State University in 1965. At West Virginia University, he was an lnstructor from 1957-1960, an Assistant Professor of Mechanical Engineering from 1960-1962, and has been Associa te Professor of Mechanical Engineering and Assistant Director of the Engineering Experiment Station since 1965. Dr. Parmer has several publications dealing with spacecraft thermal control.

Joseph C. Richmond is a Ceramics Engineer with a B.S. from the State University of New York, College of Ceramics, in 1935; a B.S. with a major in mathematics from Alfred University in 1935; and an M.S. from North Carolina State University in 1939. He has been employed at the National Bureau of Standards since 1939, and is currently Leader of the Thermal Radiation Group of the Photometry and Colorimetry Section. He was Editor of the book Measurement of Thermal Radiation Properties of Solids (NASA SP-31), and General Chairman of the Symposium on Thermal Radiation of Solids held in San Francisco in March 1964. He is currently a member of the Thermophysics Committee of AIAA, and Vice-Chairman elect of the American Society for testing materials (ASTM) Committee E-21 on Space Simulation. Mr. Richmond has over 60 publications, mostly in the areas of porcelain enamels and high-temperature ceramic coatings for metals, and evaluation of thermal radiation properties of materials.

Presented as Preprint 65-657 at the AIAA Thermophysics Specialist Conference, Monterey, Calif., September 13-15, 1965; revision received May 9, 1966. The authors express their appreciation to the following persons for their help with the paper and questionnaire: C. P. Mook, Headquarters, NASA; D. F. Stevison, U. S. Air Force Materials Laboratory; J. Geist and G. J. Kneissl, National Bureau of Standards. Part of the work of this paper was done under NASA Contract R-09-022-032, and Air Force Contract (33-616) 65-1005. Certain commercial materials are identified in this paper to adequately specify the materials employed. In no case does such identification imply recommendation or endorsement by the National Bureau of Standards, nor does it imply that the materials identified are necessarily the best available for the purpose. 


\section{Introduction}

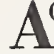
CTIVITY in the field of radiant heat transfer has increased greatly in recent years because, in the vacuum of space, radiation is the only mode of heat transfer between a vehicle and its environment that does not also involve mass transfer. It is used extensively for temperature control of satellites and space vehicles, generation of power from solar energy, and heat rejection systems for nuclear- or solarpowered generating equipment based on heat engines. As a result, techniques for both radiant heat transfer and thermal radiation property measurements have been greatly advanced. However, not all of the workers have taken advantage of the improved techniques that are now available and, in some cases, they may not even be aware of their existence.

To establish the state of the art, a questionnaire (National Bureau of Standards NBS-194)* was circulated to collect information on current practices in and future requirements for 1) measurements and 2) computations in the field of radiant heat transfer, particularly in relation to the national space program and military applications. This paper presents an analysis of the 133 replies received as of September 15,$1965 ; 112$ of them answered questions dealing with experimental techniques, and 79 responded on computational methods. These results from the questionnaire are supplemented to give a broad picture of the current status and future requirements in 1) measurements of thermal emittance, 2) measurements of infrared reflectance, and 3) computational methods for radiant heat transfer. A brief review of the Russian literature in the field is included.

\section{General Information}

Several questions were asked to provide general information about the responders, their reasons for making measurements, their participation in cooperative testing programs (round robin tests), and the configurations and assumptions used in computations. Table 1 gives the major regional locations of the responders, their years of experience in the field of thermal radiation, their levels of education, and their society affiliations and preferences. It is interesting to note that many of them are not members of the groups they feel are most important to the field. The East and West Coast Thermal Radiation Discussion Groups were not included in this tabulation.

The purposes listed for making measurements were: theoretical studies, 40 ; material studies, 77 ; to obtain optical properties, 46; for use by heat transfer analysts, 51; for commercial use, 7; for thermal control surfaces, 17; for radiometric instrumentation, 7; for degradation studies, 15; and miscellaneous, 12. Property measurements were made at temperatures below $100^{\circ} \mathrm{K}$ by 21 laboratories, and at temperatures above $2500^{\circ} \mathrm{K}$ by 24 laboratories.

A number of the responders had participated in previous round-robin tests: 9 in the Ames test, 4 in the Aerospace Industries Association test, 8 in J. Triolo's NASA test, and 9 in private tests. Significantly, 30 reported that more round-robin tests are needed, whereas 6 said that no more were needed. The properties suggested for measurement in future round robins were spectral directional reflectance, 14; ultraviolet degradation, 6 ; total emittance, 8 ; directional spectral emittance, 4 ; total emittance above $1366^{\circ} \mathrm{K}\left(2000^{\circ} \mathrm{F}\right)$, 5 ; solar absorptance, 6; transmittance, 4; scattering, 2; gonio-reflectance, 4 ; absorptance as a function of temperature, 1 ; and specular reflectance of mirrors, 5 .

* Copies of the questionnaile are available upon request from J. C. Richmond, Photometry and Colorimetry Section, Metrology Division, National Bureau of Standards, Washington, D. C.
Major problems remaining to be solved in the field of thermal radiation property measurements were identified as follows: material characterization, 27; techniques, 15; instrumentation, 11; surface temperature measurements, 11; accuracy, 9; property standards, 10; goniometric measurements, 8 ; spectral directional reflectance, 10 ; emittance at temperatures below $273^{\circ} \mathrm{K}\left(0^{\circ} \mathrm{C}\right)$, 7 ; near and far infrared sources, 14 ; terminology, 8 ; ultraviolet degradation, 6; specular reflectance standards, 10; detectors, 5; and blackbodies, 3 .

Table 2 indicates the relative importance of various geometrical surface configurations to the responders of the questionnaire, and Table 3 gives responses to the questions on analysis.

In response to other questions, the responders indicated that: 1) they were equally interested in the prediction of surface flux and surface temperature; 2) they use their own laboratory for data more than the literature; 3) they have a strong interest (but little experience) in thermal modeling; 4) they consider terminology to be a very serious problem that should be solved by National Bureau of Standards, AIAA, Office of the Secretary of the Army, and American Society for Testing Materials; 5) they believe the error in their temperature predictions is $6 \%$ (average of all answers); and 6) they would like to reduce errors in surface flux by an order of magnitude in the next five years. A detailed listing of the remaining responses to the questionnaire is available from the authors.

\section{Computational Methods}

During the past ten years mathematical techniques have developed far in excess of the ability of our educational system to incorporate the new material into the undergraduate curriculum. However, despite the lack of an adequate text, many universities recently have introduced graduate course work in radiant heat transfer. Another problem is the lack of readily usable data, which hampers the engineer even if he is aware of new techniques at his disposal.

If the heat-transfer analyst, by virtue of the system configuration and materials used, is allowed the assumptions that the surfaces are diffuse, the surfaces are isothermal, and the radiosities of the surfaces are uniform, then the methods of Hottel, ${ }^{1}$ Oppenheim, ${ }^{2}$ and Gebhart ${ }^{3}$ give quite satisfactory results. In the approach to a more exact analysis, one may relax the foregoing assumptions and allow for temperature and radiosity variations over the surfaces; this leads to the formulation of integral equations that yield more accurate answers. However, for complicated multisurface configurations, the integral solution demands an effort in computation that may be prohibitive, and it is unable to accommodate within the system a surface that has a specular reflecting quality. Unfortunately, few materials of practical importance are diffuse. ${ }^{5}$ Even with the limitations of the diffuse assumptions, things are not quite as bad as they might seem, since, in some cases, the specularity of a surface will not affect the final answer to any great extent. ${ }^{6}$ However, for the case in which surface specularity plays an important role, mathematical solutions and methods are now available which should be of considerable help. The mirror-imaging technique works quite well for transfer systems in which the surfaces are either totally diffuse or totally specular. However, one does not find many that are totally specular, eitherl. At best, then, one can analyze and design for performance between these limits. "Thus, results obtained from the present formulation are to be regarded as limiting values in the same sense as are the results obtained from the presently standard diffuse enclosure theories."6

In the following discussion of the specular-diffuse calculation problem, it is implied that this analysis may be used for either total or monochromatic calculations, where, for monochromatic calculations, one suns the heat transfer in 


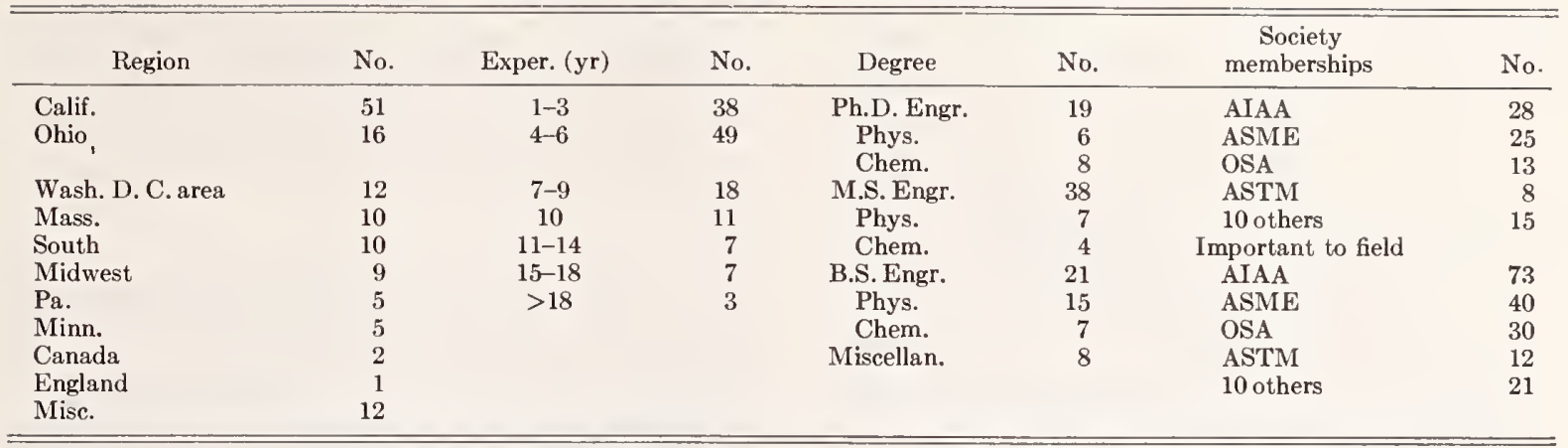

each of the small wavelength bands for all wavelengths of importance.

In his discussion of Ref. 4 , Seban ${ }^{8}$ suggests a useful method, i.e., separation of the reflectance in to two components, diffuse and specular, such that $\rho=\rho_{D}+\rho_{s}$ where specular flux is the amount of flux reflected in the specular direction over and above the diffuse component of flux in the specular direction. This, of course, is only a crude approximation to the real geometrical distribution of the reflected flux, but it is obviously better than assuming surfaces to be either perfect specular or diffuse reflectors. Bevans and Edwards, ${ }^{9}$ Sparrow and Lin,,$^{93}$ and Corlett ${ }^{9 b}$ also illustrate this approach. The diffuse radiosity of a surface now can be defined in terms of its diffuse emitted and reflected flux, so that

$$
B_{D}=\epsilon \sigma T^{4}+\rho_{D} H
$$

If one assumes that the radiosities are uniform over each of the surfaces in the radiation system, then the irradiation of each surface is given by

$$
A_{i} H_{i}=\sum_{k=1}^{N} B_{k} A_{k} F(k-i)_{\tau}
$$

where $F_{(i-k)_{\tau}}$ (total imaged view factor) is the fraction of the radiosity of surface $A_{k}$ that strikes surface $A_{i}$ by both the direct view and via specular reflections. This is similar to the factor $\Phi$ in Ref. 7, differing only in the fact that use is made of the partial specularity of the surfaces involved. Seban wrote his equations in integral form, which is a higher level of exactness, but for practical utilization the following assumptions may be applicable: 1) all surfaces are uniformly irradiated; 2) $\rho=\rho_{D}+\rho_{s}$; 3) $\alpha=\epsilon=1-\rho$; 4) all surfaces are isothermal; and 5) $\rho_{D}$ and $\rho_{S}$ are independent of the directional distribution of the incident radiant flux and of the surface temperature.

The total radiosity of a surface in the system (diffuse plus specular) is

$$
\epsilon \sigma T^{4}+\rho H=\epsilon \sigma T^{4}+\left(\rho_{D}+\rho_{S}\right) H
$$

\begin{tabular}{|c|c|c|c|c|c|c|c|c|c|c|}
\hline \multirow[b]{2}{*}{ Config. } & \multicolumn{9}{|c|}{ Order of choice } & \multirow{2}{*}{$\begin{array}{l}\text { Wtd. } \\
\text { avg. }\end{array}$} \\
\hline & 1 & 2 & 3 & 4 & 5 & 6 & 7 & 8 & 9 & \\
\hline Sphere & 10 & 11 & 5 & 4 & 4 & 4 & $\ldots$ & 1 & $\ldots$ & 20 \\
\hline Platform & 11 & 6 & 5 & 2 & 1 & 2 & 2 & 3 & 1 & 17 \\
\hline$V$ groove & 4 & 4 & 3 & 1 & 5 & 3 & 4 & 5 & $\ldots$ & 10 \\
\hline Cavities & 15 & 9 & 5 & 6 & 3 & 5 & $\ldots$ & 1 & $\ldots$ & 24 \\
\hline Wall enclos. & 18 & 9 & 6 & 2 & 1 & $\ldots$ & 1 & & $\ldots$ & 25 \\
\hline Grooves & 1 & 3 & 1 & 7 & 1 & 5 & 5 & 1 & 1 & 5 \\
\hline Louvers & 3 & 1 & 3 & 4 & 6 & $\ldots$ & 4 & & 3 & 8 \\
\hline Opposing plates & 11 & 7 & 8 & 5 & 2 & $\ldots$ & $\ldots$ & 2 & $\ldots$ & 19 \\
\hline
\end{tabular}

Table 2 Frequency of investigation of various configurations and the net radiant heat transfer at a surface is

$$
Q / A=B_{D}+\left(\rho_{s}-1\right) H
$$

Since $B_{\tau}=B_{D}+\rho_{s} H$, substituting for $H$ by use of Eq. (1) yields

$$
Q / A=B_{D}+\left(\rho_{s}-1\right)\left[\left(B_{D}-\epsilon \sigma T^{4}\right) / \rho_{D}\right]
$$

\begin{tabular}{|c|c|c|c|c|c|c|}
\hline \multirow{2}{*}{$\begin{array}{l}\text { Assumption } \\
\text { or technique }\end{array}$} & \multicolumn{3}{|c|}{ Use } & \multicolumn{3}{|c|}{ Familiarity $^{a}$} \\
\hline & Reg. & Occas. & Seldom & $A$ & $B$ & C \\
\hline \multicolumn{7}{|l|}{ Surface assumptions } \\
\hline Diffuse & 71 & 6 & 2 & $\ldots$ & 1 & 2 \\
\hline Specular & 33 & 25 & 7 & $\ldots$ & 2 & 3 \\
\hline $\begin{array}{l}\text { Diffuse with } \\
\text { specular } \\
\text { component }\end{array}$ & 28 & \multicolumn{3}{|c|}{ Diffuse with } & & \\
\hline \multicolumn{7}{|l|}{ Homogeneous over } \\
\hline area & 49 & 7 & 4 & 2 & 4 & 2 \\
\hline Known goniometric & 5 & 11 & 8 & 19 & 13 & 7 \\
\hline Nonpolarizing & 24 & 2 & 8 & 10 & 12 & 3 \\
\hline \multicolumn{7}{|l|}{ Independent of } \\
\hline $\begin{array}{l}\text { angle } \\
\text { Other }\end{array}$ & 27 & 7 & 6 & 4 & 6 & 4 \\
\hline Other & 4 & 2 & $\cdots$ & 1 & 1 & 2 \\
\hline \multicolumn{7}{|l|}{ Other assump tions } \\
\hline Gray-body & 51 & 9 & 9 & $\cdots$ & $\cdots$ & 1 \\
\hline Uniform radiosity & 44 & 5 & 7 & 2 & $\cdots$ & 1 \\
\hline Monochromatic & 33 & 16 & 13 & $\cdots$ & $\cdots$ & 1 \\
\hline Opaque & 64 & 4 & 3 & $\cdots$ & 1 & 1 \\
\hline Isotropic surface & 50 & 4 & 4 & 3 & 2 & 1 \\
\hline Anisotropic surface & 6 & 14 & 27 & 5 & 8 & 2 \\
\hline Isothermal surface & 51 & 11 & $\cdots$ & $\cdots$ & 1 & 1 \\
\hline \multicolumn{7}{|l|}{$\begin{array}{l}\text { No radiation } \\
\text { coupling with } \\
\text { conduction and }\end{array}$} \\
\hline convection & 24 & 13 & 15 & 1 & 4 & 3 \\
\hline Transmitting & 14 & 16 & 17 & $\cdots$ & 2 & 2 \\
\hline \multicolumn{7}{|l|}{ Nonisothermal } \\
\hline Other & 4 & $\ldots$ & $\ldots$ & $\ldots$ & 1 & 2 \\
\hline \multicolumn{7}{|l|}{ Techniques } \\
\hline Oppenheim network & 25 & 5 & 10 & $\cdots$ & 13 & 1 \\
\hline Monochromatic & & 3 & 8 & & 13 & 1 \\
\hline $\begin{array}{c}\text { network } \\
\text { Monte-Carlo }\end{array}$ & 10 & 3 & 0 & $\cdots$ & 13 & 1 \\
\hline $\begin{array}{l}\text { goniometric or } \\
\text { diffuse }\end{array}$ & 8 & 6 & 11 & 11 & 16 & 2 \\
\hline $\begin{array}{l}\text { Specular mirror } \\
\text { imaging }\end{array}$ & 18 & 19 & 11 & & & 1 \\
\hline Variational calculus & 18 & 20 & 13 & 2 & 8 & 1 \\
\hline Other & 12 & 1 & $\ldots$ & $\ldots$ & ... & 1 \\
\hline
\end{tabular}

Table 3 Responses on assumptions and techniques in analysis

a $A$, not familiar with; $B$, would like to know more about; $C$, should be stressed in literature. 


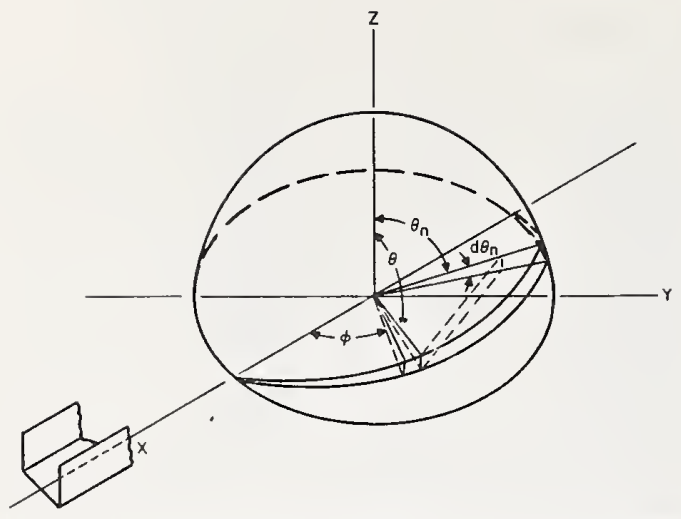

Fig. 1 Deseription of eonditions for specular-diffuse groove ealeulation.

or

$$
Q / A=\left[\left(\rho_{D}+\rho_{s}-1\right) B_{D}-\epsilon \sigma T^{4}\left(\rho_{s}-1\right)\right] / \rho_{D}
$$

which reduces to

$$
Q / A=\left[\sigma T^{4}\left(1-\rho_{s}\right)-B_{D}\right] \epsilon / \rho_{D}
$$

Unfortunately, Eq. (7) is indeterminate for surfaces that are absolutely specular, since for that case $B_{D}=\epsilon \sigma T^{4}$, and $1-$ $\rho_{s}=\epsilon$. In that case,

$$
Q / A=+\epsilon \sigma T^{4}-\alpha H
$$

where $H$ is calculated by Eq. (2).

The $B_{D}$ 's needed in Eqs. (2, 7, and 8 ) may be calculated by solving a simultaneous set of linear algebraic equations, which may be formulated on the basis that $B_{D}$ for a totally specular surface is equal to its diffuse emission. Thus,

$$
B_{D_{i}}=\epsilon_{i} \sigma T_{i}^{4}+\rho_{D_{i}} \sum_{k=1}^{N} B_{D_{k}} \frac{A_{k}}{A_{i}} F(k-i)_{\tau}
$$

For example, consider the case of a $90^{\circ} \mathrm{V}$ groove with equal length legs and infinite in extent. Assumptions are that 1) the surfaces are isothermal, $\left.T_{1}=T_{2} ; 2\right) \rho_{1}=\rho_{2}=1-\epsilon_{2}=$ $\rho_{D}+\rho_{s}$; 3) the surface is infinite in extent; and 4) the surfaces have uniform radiosities. From the geometry of the system, the view factors are calculated to be

$$
\begin{aligned}
& F_{2-1}=F_{1-2}=0.293 \quad F_{2-3}=F_{1-3}=0.707 \\
& F_{2,}{ }_{1-3}=F_{1,{ }^{\prime}-3}=0.293 \\
& F_{(2-3) \tau}=F_{2-3}+\rho_{s 1} F_{\left(2 .{ }^{\prime}{ }_{1-3)}\right.}=0.707+\rho_{s 1} 0.293 \\
& F_{(1-3) \tau}=F_{1-3}+\rho_{s 2} F_{\left(1,{ }^{\prime}-3\right)}=0.707+\rho_{s 2} 0.293
\end{aligned}
$$

By Eq. (9)

$$
\begin{aligned}
& B_{D 2}=\epsilon_{2} \sigma T_{2}{ }^{4}+B_{D 1} F_{(1-2) \tau} \rho_{D 2} \\
& B_{D 1}=\epsilon_{1} \sigma T_{1}{ }^{4}+B_{D 2} F_{(2-1) \tau} \rho_{D 1}
\end{aligned}
$$

Since the system is geometrically and thermally symmetric, $B_{D 1}=B_{D 2}$, and therefore

$$
B_{D 2}=\epsilon_{2} \sigma T_{2}{ }^{4} /\left[1-F_{(1-2)} \rho_{D 2}\right]
$$

writing Eq. (7) for surface 2 and substituting Eq. (11),

$$
\frac{Q_{2}}{A_{2} \sigma T^{4}}=\frac{\left(1-\rho_{D 2}\right)-\epsilon_{2} /\left[1-F_{(1-2) \tau} \rho_{D 2}\right]}{\rho_{D 2} / \epsilon_{2}}
$$

For the totally diffuse case, this reduces to

$$
\frac{Q_{2}}{A_{2} \sigma T^{4}}=\frac{\epsilon_{2}}{\rho_{D 2}}-\frac{\epsilon_{2}{ }^{2}}{\rho_{D 2}-F_{(1-2) \tau} \rho_{D 2}{ }^{2}}=\frac{\left[1-F_{(1-2) D}\right] \epsilon_{2}}{1-F_{(1-2) \tau} \rho_{D 2}}
$$

which is identical to the expression given in Ref. 10 for the simplified analysis, which yields answcrs within $1 \%$ of the exact analysis.

If the surfaces are completely specular, then Eq. (7) is indeterminate and Eq. (8) must be used. For this case,

$$
Q_{2} / A_{2} \sigma T^{4}=\psi H_{2} / \sigma T^{4}-\epsilon_{2}
$$

but $H_{2}+A_{1} / A_{2} B_{D 1}\left(F_{12}\right)_{\tau}$ and $B_{D 1}=\epsilon_{1} \sigma T^{4}$ since $A_{1}=A_{2}$, and $\alpha_{1}=\epsilon_{1}=\epsilon_{2}$

$$
Q_{2} / A_{2} \sigma T^{4}=\epsilon\left[\epsilon F_{(12) \tau}-1\right]
$$

which is logically an exact solution for this case because none of the radiant energy that is emitted by the isothermal surfaces can be reflected back to the surface as long as the angle between the adjacent surfaces is greater than $90^{\circ}$.

The solution to Eqs. $(12,13$, and 15) for a material whose emittance is 0.5 is given in Table 4 . It is apparent that for this particular geometry there is not much difference in emission between the fully diffuse and the fully specular case. However, if one were interested in the equilibrium temperature of a surface in the solar environment, the specularity would play a far greater role. The following example for a right angle $V$ groove irradiated by sunlight normal to the groove opening makes use of some of the calculations from Ref. 11. One side of the groove will absorb energy in the amount $q a=A_{1}(442)(\cos \pi / 4) \alpha_{s}$, where $\alpha_{s}$ is the solar absorptance of the groove which depends upon the diffuseness of the groove material. ${ }^{11}$ Two cases will be considered.

Case 1 (groove diffuse to solar radiation and specular with respect to its own emission).

$$
\begin{array}{r}
\epsilon=0.5 \quad \alpha_{s}=0.58 \text { (Ref. 11) } \\
Q / A \alpha T^{4}=0.427 \text { (Table } 4 \text { ) }
\end{array}
$$

Heat absorbed equals heat emitted; therefore

$$
Q=A \alpha T^{4}(0.427)=A_{1}(442)(\cos \pi / 4)(0.58)
$$

from which

$$
\begin{gathered}
\frac{T}{100}=\left[\frac{442(0.707)(0.58)}{0.1714(0.427)}\right]^{1 / 4}=(2470)^{1 / 4} \\
T=707^{\circ} \mathrm{R}\left(393^{\circ} \mathrm{K}\right)
\end{gathered}
$$

Case 2 (groove specular with respect to solar radiation and specular with lespect to its own emission):

$$
\begin{gathered}
\epsilon=0.5 \quad \alpha_{s}=0.75(\text { Ref. 11) } \\
\left.A / \Lambda \alpha T^{4}=0.427 \text { (Table } 4\right) \\
Q=\Lambda \alpha T^{4}(0.427)=.1(442)(\cos \pi / 4)(0.75)
\end{gathered}
$$

from which

$$
T=752^{\circ} \mathrm{R}\left(418^{\circ} \mathrm{K}\right)
$$

Furthermore, Sarofim and Hottel, ${ }^{12}$ using an approach similar to the one just illustrated, show the same kind of discrepancies for the cube with specular-diffuse walls. Thus it is apparent that large temperature errors can be made if one assumes a surface to be diffuse when, in truth, it is specular.

Another interesting aspect of specular-diffuse analysis is that many of the existing calculations on the reflectance of specular-walled or diffuse grooves to parallel irradiation may be uscd in a simple formulation to calculate the effective hemispherical emittance of the same isothermal groove.

Table 4 Typical values of $Q / \Delta T^{4}$ for materials with $\epsilon=0.5$

\begin{tabular}{ccc}
\hline$\rho_{D}$ & $\rho_{8}$ & $Q / A \sigma T^{4}$ \\
\hline 0.5 & 0.0 & 0.413 \\
0.2 & 0.3 & 0.422 \\
0.0 & 0.5 & 0.427 \\
\hline
\end{tabular}


One of the properties of a groove is that for irradiation by a parallel bundle of rays only one directional parameter need be specified, that is, the dihedral angle between the plane formed by the incoming rays and the plane perpendicular to the groove opening and parallel to its length (Ref. 11, pp. 1 and 2). Thus, the directional reflectance of a groove may be expressed as a function of the angle $\theta_{n}$ only, as in Fig. I.

If one assumes the groove to be in a hohlraum, then

$$
\epsilon_{H}=1-\rho_{H}
$$

where $\rho_{H}$, its hemispherical reflectance, is the quotient of two integrations over a hemisphere

$$
\rho_{H}=\int \rho(\theta) L \cos \theta d \omega / \int L \cos \theta d \omega
$$

For the groove, however, it is easier to evaluate the incoming energy in terms of the amount of energy contained in the wedge-shaped section defined by $\theta_{n}$ and $d \theta_{n}$, as in Fig. 1 .

If $H$ is the irradiation of the groove-opening in the hohlraum, one may write

$H=\pi L=\int_{\text {Hemisphere }} L \cos \theta d \omega=2 \int_{0}^{\pi / 2} E\left(\theta_{n}\right) d \theta_{n}$

in which $E\left(\theta_{n}\right)$ is an energy function deseriptive of the amount of energy contained in the wedge $d \theta_{n}$. Now we can redefine $\rho_{H}$ in terms of Eq. (18).

$$
\rho_{H}=2 \int_{0}^{\pi / 2} \rho\left(\theta_{n}\right) E\left(\theta_{n}\right) d \theta_{n} / 2 \int_{0}^{\pi / 2} E\left(\theta_{n}\right) d \theta_{n}
$$

$E\left(\theta_{n}\right)$ is known from the following derivation: let

$$
\cos \theta=f_{0}\left(\theta_{n}, \varphi\right) \quad d \omega=f_{1}\left(\theta_{n}, \varphi\right) d \theta_{n} d \varphi
$$

Thus,

$\int_{\text {Hemisphere }} L \cos \theta d w=2 \int_{0}^{\pi} \int_{0}^{\pi / 2} L f_{0}\left(\theta_{n}, \varphi\right) f_{1}\left(\theta_{n}, \varphi\right) d \theta_{n} d \varphi$

By comparing (18) and (21) then,

$$
E\left(\theta_{n}\right)=\int_{0}^{\pi} L f_{0}\left(\theta_{n}, \varphi\right) f_{1}\left(\theta_{n}, \varphi\right) d \varphi
$$

From the geomctry of the system, one obtains

$$
\cos \theta=\cos \theta_{n} \sin \varphi \text { and } d \omega=\sin \varphi d \varphi d \theta_{n}
$$

Using Eqs. (23) in (22) and integrating yields

$$
E\left(\theta_{n}\right)=\left(\pi-L^{\prime} \cos \theta_{n}\right) / 2
$$

The hemispherieal reflectance of a groove in a hohlraum,

$$
\rho_{H}=2 \int_{0}^{\pi / 2} E\left(\theta_{n}\right) \rho\left(\theta_{n}\right) d \theta_{n} / 2 \int_{0}^{\pi / 2} E\left(\theta_{n}\right) d \theta_{n}
$$

upon integration reduces to

$$
\rho_{H}=\int_{0}^{\pi / 2} \cos \theta_{n} \rho\left(\theta_{n}\right) d \theta_{n}
$$

Refs. 11 and 17 contain data for several groove configuration and reflectance properties that may be used to illustrate the usefulness of Eq. (26) and compare it to results obtained by use of Seban's method Eqs. (7) and (8).

For the rectangular groove of Ref. 17 with specular walls of reflectance $\rho_{v}$ and a diffuse bottom of reflectance $\rho_{b}$, one obtains the data shown in Table 5 after evaluating Eq. (26)

Table 5 Hemispherical emittances from Eq. (28) and Seban's method

\begin{tabular}{cclc}
\hline & & \multicolumn{2}{c}{$\epsilon_{H}$} \\
\cline { 3 - 4 }$\rho_{\omega}$ & $\rho_{b}$ & Eq. 28 & Seban's $^{-}$ \\
\hline 0.7 & 0.5 & 0.296 & 0.295 \\
0.2 & 0.5 & 0.1266 & 0.126 \\
\hline
\end{tabular}

by the trapezoidal rule and evaluation of Seban's equations for the system. Since the calculation of $\rho\left(\theta_{n}\right)$ in Ref. 17 was analytically exact, the evaluation of Eq. (26) is in error only by the amount generated by numerical evaluation. The comparison with Seban's method shows only a trivial difference.

The problem of calculating the total image view factor for complex multinode configurations is almost insurmountable without the use of Monte Carlo techniques such as illustrated in Refs, 13-16. This technique results in very long computer runs for a system of any degree of complexity. Furthermore, any heat-transfer approach of the sophisticated degree recommended here and in Refs. 9 and 12 demands data beyond the present state of the art of reflectance measurements, espccially since, for the specular-diffuse approach to be most effcctive, it is essential that the heat-transfer analysis be on a spectral basis. Almost no reliable specular-diffuse spectral reflectance data cxist over the spectral range of interest. Furthermore, data recently presented by E. M. Sparrow and K. E. Torrance ${ }^{17 a}$ call into question the speculardiffuse model for reflection at angles very far from the normal. Thus, it appears that the necessary computational techniques are available to provide very excellent predictions of radiant heat transfer; however, the necessary data on common engineering materials are almost completely lacking. Two additional references of interest in the area of radiant heat transfer are Sparrow ${ }^{17 b}$ and Wiebelt. ${ }^{17 \mathrm{c}}$

\section{Thermal Emittance Measurements}

Thermal emittance $\epsilon$ is defined as the ratio of the radiant flux emitted by a specimen at a given temperature and under given geometric and spectral conditions to that emitted by a blackbody radiator at the same temperature and under the same conditions.

The geometric conditions refer to the size of the einitting area, the size of the solid angle $\omega$ over which the emitted flux is measured, and the direction $\theta, \omega$ of the centroid of that solid angle from the emitting surface. The angle $\theta$ is measured from the given direction to the normal to the emitting surface, and the angle $\varphi$ is the azimuth angle of the given direction from some reference on the specimen. When the solid angle is a complete hemispherc, $2 \pi$ sterad, the emittance is referred to as hemispherical emittance $\epsilon_{I I}$. When the solid angle is small, its size usually is not specified, and the emittance is referred to as directional emittance $\epsilon(\theta, \varphi)$, or if the direction is normal to the emitting surface, as normal emittance $\epsilon_{N}$. The azimuth angle $\varphi$ is specified only for surfaces in which $\epsilon$ varies with azimuth angle, such as fiblous materials, textiles, oriented crystals, and grooved surfaces.

The spectral conditions refer to the wavelength range of the radiant flux. If all wavelengths are measured, the emittance is referred to as total emittance $\epsilon_{\tau}$. Since emittance, and particularly total emittance, varies with temperature, total emittance is frequently plotted as a function of temperature to give a total emittance curve. If only a narrow wavelength band of flux is measured, centered about a wavelength $\lambda$, the emittance is referred to as spectral emittance $\epsilon(\lambda)$ at wavelength $\lambda$. Spectral emittance at a given temperature is frequently plotted as a function of wavelength to give a spectral emittance curve.

In order to define completely the measured emittance, it is necessary to specify the temperature and the geometric conditions and spectral conditions of measurement. Hence, two or more modifiers are usually needed, such as total hemispherical emittance at $800^{\circ} \mathrm{K}, \epsilon_{T H}\left(800^{\circ} \mathrm{K}\right)$, or normal spectral emittance at $5 \mu$ and $1000^{\circ} \mathrm{K}, \epsilon_{N}\left(5 \mu, 1000^{\circ} \mathrm{K}\right)$.

There are two basic techniques for measuring emittance: calorimetric and radiometric. However, there are many variations of these two basic techniques which will be discussed in more detail later. There were 60 replies to the questionnaire that described emittance measurement tech- 
niques, and a total of 97 techniques were listed. These included 33 for measuring total hemispherical emittance, 28 for measuring total normal emittance, and 37 for measuring spectral emittance.

\section{Total Hemispherical Emittance Measurements}

Of the 33 methods described for measuring total hemispherical emittance, 15 can be classified as the hot filament method, 15 as the temperature decay method, and 3 as radiometric techniques.

In the hot filament method, the sample is in the form of a long strip of small and uniform cross-sectional area. It is mounted in a vacuum chamber with cool walls, and heated by passing a current through it. The power input is held constant until the sample comes into thermal equilibrium with its surroundings, and under conditions such that there is essentially no axial thermal gradient near the midlength of the sample. The power input to and temperature of the specimen, a shiort segment of known surface area near the midlength of the sample, is measured. In variations of this method used for dielectric materials, the specimen in the form of thin foil was wrapped around a cylindrical heating element, or a specimen in the form of flat sheet was cemented in good thermal contact with a heater plate, and the heater plate and specimen were surrounded on back and edges by a guard heater maintained at the same temperature as the specimen. In this case, the heat loss by radiation was confined to the front surface of the specimen.

In every case, heat losses from the specimen by modes other than radiation either were assumed to be negligibly small, or were corrected for; and the total hemispherical emittance was computed as the ratio of the measured radiant heat dissipation rate per unit area to that for a blackbody radiator at the same temperature and in the same surioundings. The methods varied in details such as the temperature and method of cooling the vacuum chamber walls (liquid nitrogen, water or air), the method of measuring the specimen temperature (specimen resistance, thermocouple, or optical pyrometer sighted on an integral blackbody cavity), and the method of heating (internal resistance, internal filament heater, or attached guarded heater block). The over-all error of the method varied from about 2 to $20 \%$ or more, depending upon the relative temperatures of specimen and chamber walls, adequacy of corrections for conductive heat loss from the specimen, and accuracy of temperature measurement.

In the temperature decay method, a specimen of relatively large surface area and small mass is suspended in a vacuum chamber with cooled walls, and is heated to a temperature appreciably above that of the walls. The power input to the specimen is turned off and the rate of cooling of the specimen is measured. The rate of heat dissipation by radiation is computed from the measured cooling rate and the known surface area, mass and specific heat of the specimen, and the total hemispherical emittance is computed as deseribed for the hot filament method. The method of heating the sample varied for the different procedures described (by radiation from a coil heater, a carbon are, an arc-image furnace, or a solar simulator, by induction, and by an internal heating coil). When the specimen is heated by a solar simulator, the equilibrium temperature is related to the ratio of the solar absorptance to the total hemispherical emittance, and the cooling rate is related to total hemispherical emittance; hence, both properties can be evaluated in a single series of measurements. The over-all error of the method varies from about 2 to $20 \%$, with most of the reported errors being $5 \%$ or more.

The radiometric procedure for measuring total hemispherical emittance depended upon use of a relatively large specimen and a much smaller detector placed close to the heated specimen, in vacuum. The space between the specimen and detector was occupied by a light pipe of polished metal, so that the detector saw the hot specimen over essentially a whole hemisphere. The equipment was calibrated against a large-aperture conical blackbody cavity or a specimen of known total hemispherical emittance. Errors in this method are seldom less than $5 \%$, and may exceed $10 \%$.

\section{Total Normal Emittance Measurements}

Of the 28 procedures reported for measuring total normal emittance, 6 were by use of a portable emissometer, one was by integration of spectral data, and 21 by comparative radiometric techniques. The portable emissometer consisted of a small vacuum chamber with a bismuth-silver thermopile detector mounted so that the back side viewed a cooled platen, and the front side viewed the specimen through a KRS-5 window. The detector viewed the specimen over a large field of view that was limited by a collimating channel with blackened walls. The conical solid angle of view had an included central plane angle of somewhat less than $90^{\circ}$ at the center of the detector. Because of this large solid angle, the measured emittance was intermediate between the usual normal emittance that is measured over a small solid angle and the hemispherical emittance. The instrument was used to measure specimens at ambient temperatures, and the scale was calibrated to read directly in total normal emittance. It was calibrated by means of standards at ambient temperature that have high $(0.97)$ and low (0.03) emittance, respectively, immediately before use. The error is reported to be on the order of 5 to $15 \%$, but the precision is somewhat better. The instrument was designed as an inspection tool rather than as a research instrument.

In the comparative radiometric procedures, the flux per unit area emitted by a hot specimen and by a blackbody at the same temperature were measured by means of a spectrally nonselective detector, and the total normal emittance was computed as the ratio of the two measured values. The reported methods varied in the type of comparison blackbody used (separate heated cavity, integral cylindrical cavity, integral wedge, and hole in tubular specimen), the type of detector, method of heating the specimen (by radiation, by internal resistance, by induction, by a flame, and by conduction from a heating element), temperature (room temperature to $3300^{\circ} \mathrm{K}$ ), method of measuring the temperature of the specimen (thermocouple or optical pyrometer), and type of blackbody reference (separate laboratory blackbody furnace, integral blackbody cavity). The reported error of measurement varied from $10 \%$ for room temperature measurements with the inspection instrument to an optimistic 2 or $3 \%$ in some cases at high temperatures.

\section{Normal Spectral Emittance Measurements}

All of the 37 methods reported for measuring spectral emittance were radiometric procedures. One was for hemispherical spectral emittance, obtained by integration from directional spectral measurements made over a hemisphere. Three procedures measured only directional spectral emittance, four measured both normal and directional spectral emittance, and 28 measured only normal spectral emittance. One procedure was for measuring the spectral emittance of a gas.

In general, the radiometric procedure for measuring directional spectral emittance consisted of collecting the radiant flux emitted over a small solid angle by a specimen at a given temperature, dispersing this flux by means of a monochromator, and measuring the flux in a narrow wavelength band centered about a given wavelength. This measured value was then divided by the measured value obtained from a blackbody radiator under the same conditions. The reported techniques varied in the following ways: 1) the mothoi of heating the specimen (internal resistance, radi- 
auon, conduction from an internal or attached resistance heater, in an enclosed furnace and by convection); 2) the type of spectrometer (prism, grating or filter instrument), 3 ) the wavelength range covered (from a single wavelength at $0.65 \mu$ to a range from 0.15 to $120 \mu$ ) method of taking data (point by point with a single-beain instrument or with a double-beam instrument or continuous scanning over a large wavelength range); 4) method of temperature measurement and control (thermocouple, optical, or radiation pyrometer, and manual or automatic control); and 5) type of reference blackbody used (separate laboratory blackbody furnace, the furnace in which the specimen is heated, or an integral blackbody cavity in the specimen). The accuracy claimed varied from 1 to $2 \%$ for metallic specimens heated by internal resistance to $15 \%$ for measurements made on nometallic materials at temperatures above $2000^{\circ} \mathrm{K}$.

\section{Summary of Emittance Measurement Techniques}

Calorimetric techniques probably are most satisfactory for measuring total hemispherical emittance, and radiometric techniques are probably most satisfactory for measuring normal or directional emittance, either total or spectral. The errors in calorimetric measurements were discussed previously. Assuming that a suitable optical system is used, including a monochromator for spectial measurements, and that the detector-amplifier system has linear response, the error of radiometric measurements is determined largely by the temperature difference between specimen and blackbody reference, and the degree to which the blackbody reference departs from a true blackbody radiator. The over-all errors probably are lowest for measurements on metallic specimens, which are highly opaque at all wavelengths at which measurements are normally made, and which in general have high thermal conductivity, and at temperatures below about $1800^{\circ} \mathrm{K}$, at which conventional thermocouples give accurate readings. With metallic specimens at temperatures below $1800^{\circ} \mathrm{K}$, accuracies of $2 \%$ or better are attained with careful work.

Errors in measurement of thermal radiation properties of nometallic materials are likely to be larger than for metallic materials measured under the same conditions, for two reasons. First, nonmetallic materials, in general, tend to have lower thermal conductivities than metallic materials, and hence thermal gradients are likely to be larger. Second, nonmetallic materials in general tend to be more translucent than metallic materials, and hence emit, reflect, and absorb radiant energy within a surface layer of appreciable thickness; whereas metals reflect at the surface only, and absorb and emit within a very thin surface layer. If the nonmetallic specimen is heated from the back and emits from the front surface, there will be a large thermal gradient normal to the surface, and it is difficult to define the effective temperature of the specimen as an emitter, and even more difficult to measure it. As a result, if accurate measurements of the emittance of nonmetallic specimens are to be made, the specimen must be heated under conditions such that there is essentially no thermal gradient normal to the surface. This is attained in many cases by use of a rotating specimen that is heated in a furnace during a large fraction of a revolution and passes in front of a water-cooled port through which it is viewed. Under such conditions, if the speed of rotation is fast enough, the specimen will reach an equilibrium condition where there is essentially no thermal gradient normal to the surface. Because of the problems associated with thermal gradients and temperature measurements on nonmetallic materials, the expected errors are somewhat larger than for metallic specimens. However, with careful work, it should be possible to attain accuracies of 3 to $5 \%$ in such measurements.

At temperatures at which an optical or radiation pyrometer must be used for temperature measurement, errors are likely to be much larger. These temperature measurement techniques are quite accurate for blackbody radiators, but for nonblackbody radiators the measured brightness or radiance temperature must be corrected for the emittance of the specimen, which is unknown. Hence, emittance measured under these conditions may be in error by 10 to $20 \%$, even with careful work.

\section{Reflectance Tcchniques}

The inherent problems in measuring normal and hemispherical emittance (especially below $800^{\circ} \mathrm{K}$ ) have led to the development of a myriad of instruments to measure various kinds of reflectance. However, before discussing these methods, it is necessary to discuss terminology. To describe reflectance adequately, one must specify the incident and leaving conditions fairly precisely. The following definitions, all on a monochromatic basis, are presented as a basis for discussion and consideration.

Bi-directional reflectance is defined by

$$
\rho\left(\theta, \varphi ; \theta^{\prime}, \varphi^{\prime}\right)=\left[L^{\prime}\left(\theta^{\prime}, \varphi^{\prime}\right)\right] /[L(\theta, \varphi) \cos \theta d \omega]
$$

where $\theta$ is the polar angle, and $\varphi$ is the azimuthal angle with respect to a convenient reference. The primes denote leaving directions, and the unprimed letters denote the incident directions. Term $L(\theta, \varphi)$ denotes radiance as a function of $\theta, \varphi$. Further, $\rho\left(\theta, \varphi ; \theta^{\prime}, \varphi^{\prime}\right)$ has been shown by reciprocity considerations to be symmetrical with respect to $\theta, \varphi$ and $\theta^{\prime}$, $\varphi^{\prime}{ }^{18}$ In addition, this definition of radiance (i.e., $\partial^{2} P / \cos$ $\theta \partial A \partial \omega$ where $P$ is power) has been shown to be invariant in a lossless system. ${ }^{20}$ Other authors, at various times, have called $\rho\left(\theta, \varphi, \theta^{\prime}, \varphi^{\prime}\right)$ biangular reflectance, reflectance function, and partial remission factor. Further, there are at least two other definitions for this term in the literature that tend to define the measurement technique but do not follow the laws of reciprocity. They are

$$
\begin{aligned}
& \rho_{1}\left(\theta, \varphi ; \theta^{\prime}, \varphi^{\prime}\right)=L^{\prime}\left(\theta^{\prime}, \varphi^{\prime}\right) / L(\theta, \varphi) \\
& \rho_{2}\left(\theta, \varphi ; \theta^{\prime}, \varphi^{\prime}\right)=\frac{L^{\prime}\left(\theta^{\prime}, \varphi^{\prime}\right) \cos \theta^{\prime} d \omega^{\prime}}{L(\theta, \varphi) \cos \theta d \omega}
\end{aligned}
$$

Many times it is convenient to reduce data by normalizing to the specular direction, resulting in the plotting of

$$
\frac{\rho\left(\theta, \varphi ; \theta^{\prime}, \varphi^{\prime}\right)}{\rho(\theta, \varphi ; \theta, \varphi+\pi)}=\frac{L^{\prime}\left(\theta^{\prime}, \varphi^{\prime}\right)}{L^{\prime}(\theta, \varphi+\pi)}
$$

for the first two definitions for $\rho\left(\theta, \varphi ; \theta^{\prime}, \varphi^{\prime}\right)$, whereas $\mathrm{E}_{(1}$. (27b) yields

$$
\frac{\rho_{2}\left(\theta, \varphi ; \theta^{\prime}, \varphi^{\prime}\right)}{\rho_{2}(\theta, \varphi ; \theta, \varphi+\pi)}=\frac{L\left(\theta^{\prime}, \varphi^{\prime}\right) \cos \theta^{\prime}}{L(\theta, \varphi+\pi) \cos \theta}
$$

which results in an energy ratio and may be more useful in the correlation of experimental data.

Directional hemispherical reflectance $\rho(\theta, \varphi)$ is the ratio of the flux reflected into the hemipshere above the surfare to that incident from a direction $\theta, \varphi$. Thus,

$$
\rho(\theta, \varphi)=\frac{\int_{H} L^{\prime}\left(\theta^{\prime}, \varphi^{\prime}\right) \cos \theta^{\prime} d \omega^{\prime}}{L(\theta, \varphi) \cos \theta d \omega}
$$

This also could be written as

$$
\rho(\theta, \varphi)=\int_{H} \rho\left(\theta, \varphi, \theta^{\prime}, \varphi^{\prime}\right) \cos \theta^{\prime} d \omega^{\prime}
$$

Of course we measure the reflectance for a small group of directions centered on $\theta, \varphi$. Thus we depend on $\rho(\theta, \varphi)$ to change only slowly with small changes in $\theta, \varphi$. In general, the study of this variable has only begun. Furthermore, it is true that the past experiences in the visible spectrum 


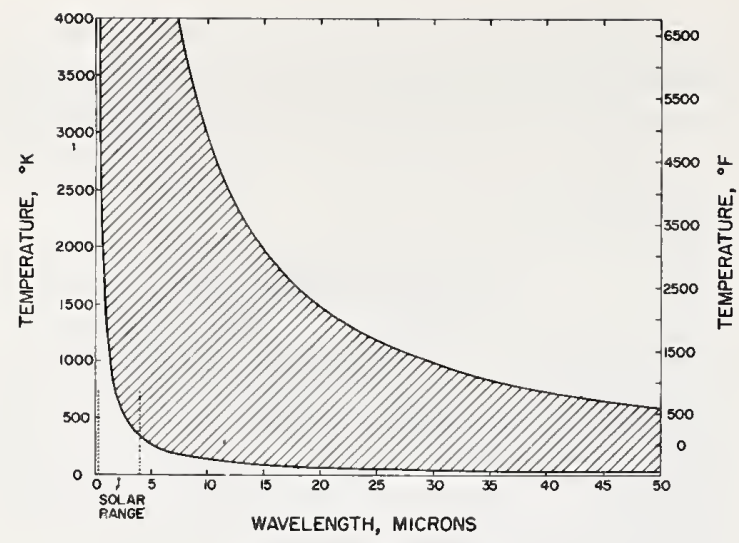

a) High temperature

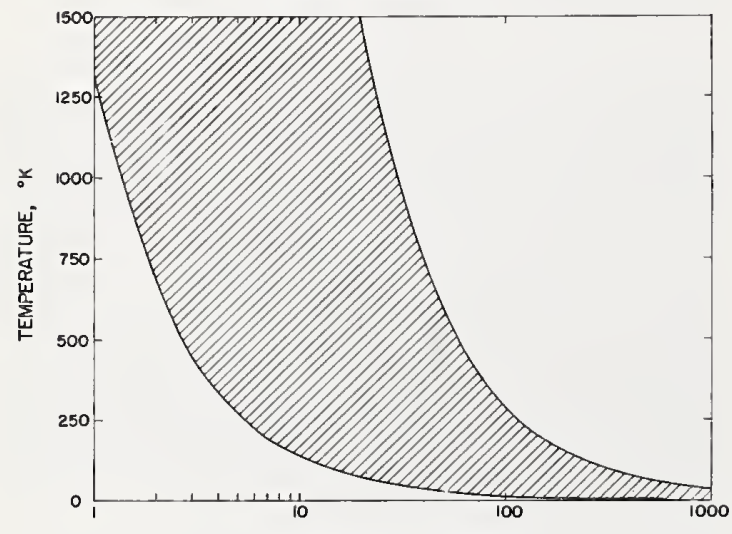

WAVELENGTH, MICRONS

b) Low temperature

Fig. 2 99\% energy bands for blackbody emission.

$(0.4-0.7 \mu)$ are not directly applicable either to the ultraviolet or the infrared region of the spectrum.

The specular component of reflectance $\rho\langle\theta, \varphi)$ is the ratio of the flux reflected in the specular direction $\left(\theta^{\prime}=\theta\right.$ and $\varphi^{\prime}=180^{\circ}+\varphi$ ) over and above the diffuse component in that direction to the flux incident from the direction $\theta, \varphi$. This is a very difficult term to assess realistically for common engineering materials, since the separation of specularly reflected flux from the diffusely reflected flux is difficult.

The diffuse component of reflectance $\rho_{D}(\theta, \varphi)$ is the directional hemispherical reflectance minus the specular component of reflectance [i.e., $\rho_{D}(\theta, \varphi)=\rho(\theta, \varphi)-\rho_{s}(\theta, \varphi)$ ]. The problems in determining the directional specular component of reflectance similarly affect the definition of the diffuse component of reflectance.

Hemispherical directional reflectance $\rho\left(\theta^{\prime}, \varphi^{\prime}\right)$ is the ratio of flux reflected in the direction $\theta^{\prime}, \varphi^{\prime}$ to that incident diffusely over the hemisphere,

$$
\rho\left(\theta^{\prime}, \varphi^{\prime}\right)=\left[L^{\prime}\left(\theta^{\prime}, \varphi^{\prime}\right) \cos \theta^{\prime} d \omega^{\prime}\right] / \pi L
$$

Further, $\rho\left(\theta^{\prime}, \varphi^{\prime}\right)$ is not equal to $\rho(\theta, \varphi)$; however, considerations of reciprocity lead us to the result that

$$
\rho(\theta, \varphi)=\rho\left(\theta^{\prime}, \varphi^{\prime}\right) \pi / \cos \theta d \omega
$$

where $\theta, \varphi$ are equal to $\theta^{\prime}, \varphi^{\prime}$, respectively. Some authors have assigned a new symbol to this term, $\overline{\rho(\theta, \varphi)}$; others have suggested that it be called directional hemispherical reflectance because of the equivalcnce; and still others have promoted the term hemispherical reflectance factor $[r(\theta$, $\varphi)$ ]. This latter term is important since most infrared reflectometers measure this quantity. It is the ratio of flux reflected into the direction $\theta, \varphi$ [for conditions of diffuse irradiation, i.e., $L(\theta, \varphi)=$ constant for all $\theta, \varphi]$ to the flux reflected by the diffuse complete reflector into the direction $\theta, \varphi$ for the same irradiation conditions. This is equivalent to

$$
\rho(\theta, \varphi)=\left[L_{s}{ }^{\prime}(\theta, \varphi)\right] /\left[L_{0}(\theta, \varphi)\right]
$$

where $L_{s}{ }^{\prime}(\theta, \varphi)$ is reflected radiance of sample, and $L_{0}(\theta, \varphi)$ is the reflected radiance of the diffuse complete reflector. $L_{0}(\theta, \varphi)$ is equal to the incident radiance.

Bi-hemispherical reflectance $\rho_{H}$ is the ratio of the flux reflected into the hemisphere to that which is incident over the hemisphere. Note that this definition is considerably more general than the others, since nothing is specified about the incoming or outgoing flux; further, this term probably has the least use in accurate heat-transfer calculations and only limited use to the theoretical investigator.

Diffuse hemispherical reflectance $\rho_{D H}$ is the ratio of flux reflected into the hemisphere above the surface to the incident flux, which is perfectly diffuse over the hemisphere. This is

$$
\rho_{D H}=\left[\int_{H} L^{\prime}\left(\theta, \varphi^{\prime}\right) \cos \theta^{\prime} d \omega\right] / \pi L
$$

or

$$
\rho_{D H}=\frac{1}{\pi} \int_{H} \overline{\rho\left(\theta^{\prime}, \varphi^{\prime}\right)} \cos \theta^{\prime} d \omega^{\prime}
$$

These definitions lead to the following well-known conclusions:

1) In a hohlraum,

$$
\epsilon(\theta, \varphi)=1-\overline{\rho(\theta, \varphi)}
$$

2) From conservation of energy,

$$
\alpha(\theta, \varphi)=1-\rho(\theta, \varphi)
$$

3) From reciprocity $[\overline{\rho(\theta, \varphi)}=\rho(\theta, \varphi)]$,

$$
\therefore \alpha(\theta, \varphi)=\epsilon(\theta, \varphi)
$$

The foregoing definitions frequently consider the unidirectional case, while in practice we almost always measure a reflectance for a small solid angle centered in the direction of $\theta, \varphi$ or $\theta^{\prime}, \varphi^{\prime}$. The solid angle that is small enough to measure $\rho(\theta, \varphi)$ depends on $\rho\left(\theta, \varphi ; \theta^{\prime}, \varphi^{\prime}\right)$; this is dependent on so many surface and material variables that a general statement on the size of measurement solid angle is impossible. The experimentalist must exhibit care in choosing the solid angle for measurement, depending on the samples to be measured, wavelength range of interest, energy limitations, information desired, etc. Furthermore, the foregoing definitions do not discuss the problem of the solid angles lying between the hemispherical case and the unidirectional case. Thus it is necessary to integrate the incident intensity function over the solid angle of interest, using the appropriate weighting factor, such as $\rho(\theta, \varphi)$.

References 9, and 18-22 discuss the nomenclature problem. It is discouraging to note that these references do not agree between themselves, and only Refs. 9 and 18-20 discuss the many kinds of reflectances needed by the heat-transfer analyst.

With the preceding types of reflectance defined on a monochromatic basis, it is necessary to know the wavelengths at which these reflectances should be measured. If one considers the problems of heat transfer, it is apparent that the wavelengths of interest for a reflectance measurement are those at which the principal amount of energy is transmitted. The cross-hatched areas of Figs. $2 \mathrm{a}$ and $2 \mathrm{~b}$ indicate the wavelength band encompassing $99 \%$ of the flux emitted by a blackbody source at the indicated temperature. For high 
temperatures the band is small and centered near the visible $(0.4$ to $0.7 \mu)$; as the temperature decreases, the center of the band becomes wider and shifts to longer wavelengths, and at very low temperatures it is extremely wide and centered far out in the infrared. For satellite temperature control, one is concerned primarily with the 0.35 to $100 \mu$ region, which encompasses the principal flux from the sun, that emitted and reflected by the earth, and that cmitted by the spacecraft. The theorist usually is more concerned with having a wide band from which to choose wavelengths, rather than with the wavelengths measured.

\section{Measurement Capabilities in the 0.3 to $2.6 \mu$ Wavelength Range}

The measurement of directional hemispherical reflectance [or its optical equivalent, diffuse directional reflectance factor $r(\theta, \varphi)]$ in the 0.3 to $2.6 \mu$ range is performed very conveniently by using an integrating sphere reflectometer. The wavelength range is restricted for two reasons: 1) the diffuse sphere coatings in use today have high reflectances only over the 0.3 to $2.6 \mu$ range; and 2) because sphere efficiency is very low, the energy available from common sources is not sufficient to activate common detectors outside of this general range even if good high-reflecting sphere coatings existed for the longer wavelengths. Of the people responding to the questionnaire, 36 reported use of integrating spheres, 20 of which were commercially procured. It is encouraging that about half of the respondents using an integrating sphere are using an absolute reading instrument like the one described by Edwards, ${ }^{23}$ which appears to be the most accurate and versatile of its class. This type of sphere, with the sample suspended in the middle, is probably the most completely analyzed integrating sphere reflectometer. ${ }^{17,23}$

References 24-28 provide historical background, whereas Ref. 29 indicates the use of a laser as a source for a 14-in.diam integrating vacuum sphere. Most integrating spheres have the basic capability of excluding or including the specular component of reflectance, but only for a specific, systcm-defined, solid angle, which may or may not be relevant to the real specular component of reflectance. Other reflectometers commonly used as the shorter wavelength's are used also in the infrared and thus are discussed in the next section. None of these instruments appears at this time to compete with the Edwards type sphere in the 0.3 to $2.6 \mu$ range.

\section{Measurement Capabilities in the Range above $2 \mu$}

There exist two basic methods of measuring directional hemispherical reflectance in this range: 1) Coblentz-hemisphere type instruments ${ }^{33}$ ( 7 responses indicated use of this method), and 2) Gier-Dunkle heated-cavity reflectometers ${ }^{34}$ (10 responses indicated their use). These two instruments, with individual variations, are presented briefly in chronological order of development. For more detailed discussions see Refs. 30-32.

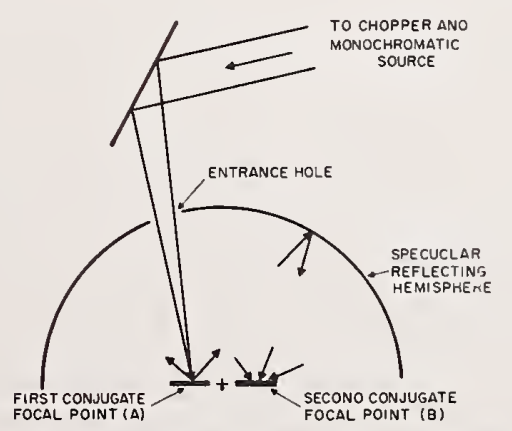

Fig. 3 Coblentz-hemisphere reflectometer.

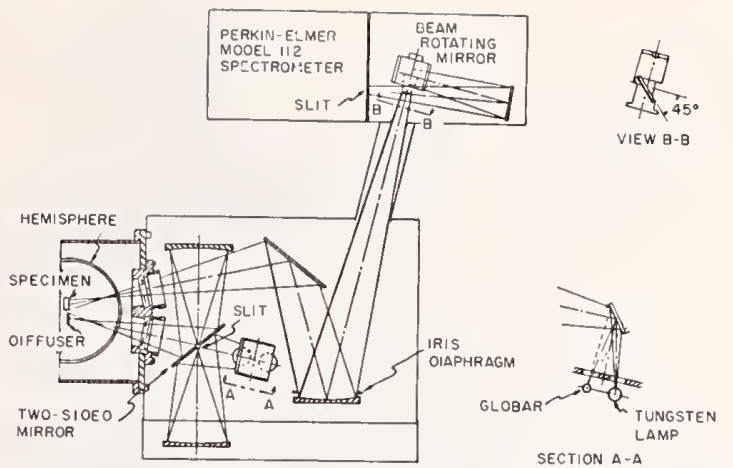

Fig. 4 The optical system of the Janssen-'Torborg hemispherical refleetometer (reprinted from Ref. 41 by permission).

\section{Coblentz-hemisphere}

Around 1910, several investigators ${ }^{33}, 35-37$ measured $\rho(\theta$, $\varphi)$ by use of this instrument (Fig. 3), which is based on the concept of conjugate focal points existing about the center of curvature of a hemisphere. Thus, if a light source is moved to the right of the center of curvature, its image appears to the left of the center or curvature. Because of spherical aberrations that increase with distance from the center ${ }^{38}$ the imagc is smeared out and enlarged; if a sample is placed at the first conjugate focal point, an enlarged image of the illuminated area appears at the second conjugate point. Absolute data are obtained with this instrument by measuring the incident flux and the reflected flux and calculating reflectance from a knowledge of system losses; relative data are taken by measuring the reflected flux from the sample and calculating the reflectance of the sample from knowledge of relevant system losses and the reflectance of a standard. In the absolute mode, the detector is placed at the first conjugate focal point to read the incident flux, and then the sample is placed at the first conjugate focal point and the detector at the second conjugate to measure the reflected flux. The ratio of these two fluxes, corrected for system losses, is then $\rho(\theta, \varphi)$, where generally $\varphi$ is close to the normal. In the comparison mode, two measurements are made with the detector at the second conjugate focal point, one with a sample at the first conjugate focal point, and the other with a standard of known reflectance at the first conjugate focal point.

Major flux losses in this system are due to a) atmospheric absorption, b) losses out the entrance hole, c) mirror absorption (which varies with position on the mirror), and d) the angular and spatial sensitivity of the detector. Because of the spherical aberrations, a large image is formed at the second conjugate focal point, which requires the use of a large detector (such as a ten junction thermopile). Thus, large errors caused by detector area sensitivity are probable. ${ }^{39},{ }^{40}$ In the relative modc of operation, the foregoing effects can be greatly reduced if the distribution of the reflected flux from the sample and from the standard are similar. If not, losses from effects $(b-d)$ are not equivalent in the two cases. The hole loss is very dependent on the distribution of reflected flux; for a specular surface there is no hole loss, but for a perfectly diffuse reflection, the hole loss is identical to the diffuse configuration factor between the image and the hole. The correction for the distribution of reflected flux between perfectly specular and perfectly diffuse reflection can be almost any fraction of the reflectance up to unity for a perfect back scatterer. Furthermore, the detector is more sensitive to energy from the top of the hemisphere than from the edges. An additional problem is that the locations of the sample and detector are quite critical. If they are not both in the focal plane, reflected energy may miss the detector entirely. 

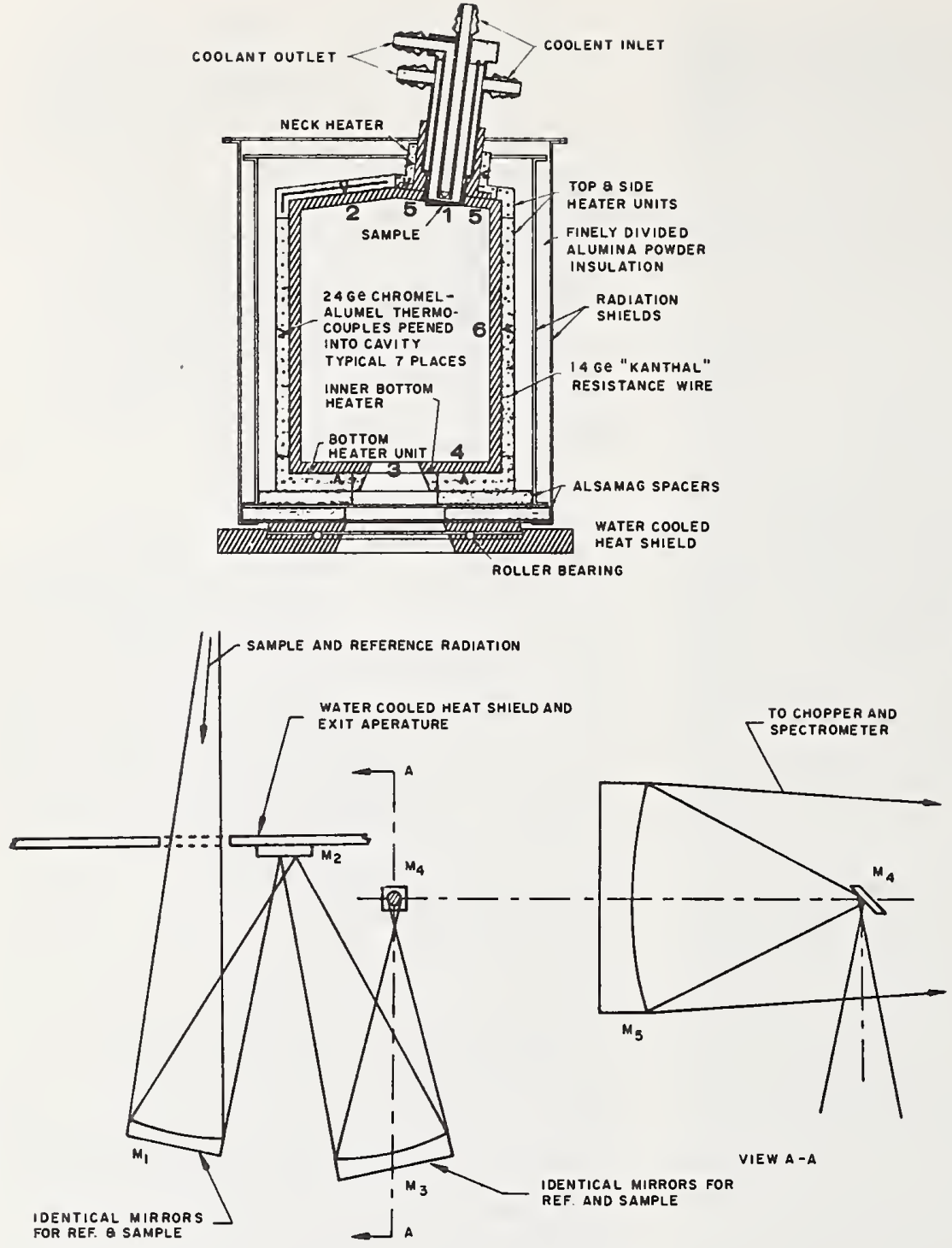

Fig. 5 A typical heated-cavity reflectometer with associated optics (reprinted from Ref. 4] by permission).
The wavelength range of this instrument is normally 0.5 to $15 \mu$, with a probable accuracy for any general engineering surface of, at best, $10 \%$. During the $50-\mathrm{yr}$ history of this instrument, many innovations have been made. Janssen and Torborg ${ }^{41}$ modified the original concept to measure $r\left(\theta^{\prime}, \varphi^{\prime}\right)$, as shown in Fig. 4, to allow sample heating and relative reflectance measurements from 0.4 to $20 \mu$, with probable accuracy of $5 \%$ for general engineering materials. This modification essentially eliminated the detector problem, doubled the hole correction problem, introduced the problem of obtaining a good infrared diffuser, introduced the effect of sample-diffuser interreflection, and did not affect other principal errors. For accurate use of this instrument, the sample and standard must have the same distribution of reflected flux. Under these specific conditions, it is probable that an accuracy on the order of $2 \%$ is obtainable. Furthermore, $r\left(\theta^{\prime}, \varphi^{\prime}\right)=\rho\left(\theta^{\prime}, \varphi\right)$ only if a perfect diffuser is used in the first conjugate focal point and an accurate correction can be made for the hole losses.

White ${ }^{42}$ built an automatic recording instrument for $\mathrm{Na}$ tional Bureau of Standards that uses a specular standard and a diffuse standard and makes relative measurements of $r\left(\theta^{\prime}, \varphi^{\prime}\right)$ over the 2.5 to $22.5 \mu$ range with an accuracy on the order of $10 \%$. It is questionable whether this instrument irradiates the sample diffusely, so that it is doubtful that data from this instrument can be used easily in the reciprocity relations to yield $\rho\left(\theta^{\prime}, \varphi^{\prime}\right)$. The main innovation was placing the source at the first conjugate focal point and viewing the source and sample through a very large hole in the hemisphere.

Birkebak and Hartnett ${ }^{43}$ extended the hemisphere to about $\frac{3}{4}$ the size of a sphere, which allowed the sample and detector to be tilted so that $\theta$ was no longer restricted to near normal irradiation. Kozyrev and Vershinin ${ }^{44}$ extended the use of the instrument to a directional annular cone reflectance measurement by use of carefully constructed baffles. References 45-48 illustrate some of the many other varied applications of this instrument.

With proper procedures, any of the foregoing Coblentzhemisphere instruments can measure the reflectance with and without the specular component; however, they are all very restricted in the size of solid angle which can be eliminated as the specular component. 
In the late 1940's the heated cavity reflectometer (Fig. 5) was developed, and it offered a means of measuring $\rho(\theta, \varphi)$ from 1 to $35 \mu$, with a probable àccuracy of 2 to $5 \%$ for general engineering materials. ${ }^{32,34,47,49,50}$ The sample is placed in the middle of a blackbody cavity (i.e., equal radiance from all directions) and irradiated diffusely. The sample and a spot on the wall are viewed alternately through a small hole in the cavity to obtain a measure of the incident and reflected flux, and hence absolute reflectance. The angle of viewing $\theta^{\prime}$ for measurement of $\rho(\theta, \varphi)$ can be varied by tilting the sample. ${ }^{51}$ The major unique problems with this instrument are eliminating the temperature gradients and hole effects in the cavity, so that it is indeed a blackbody cavity, and accounting for the energy emitted by the sample. In the Coblentz-type instruments, the incident energy is chopped so that its a.c. signal can be distinguished from the d.c. signal of the energy emitted by the sample; whereas in the heated cavity, no distinction can be made between the two. Furthermore, in the heated cavity, it is difficult to control the sample temperature, except for samples of high thermal conductivity. As for Coblentz-type reflectometers, the effect of the hole loss is similarly dependent (through the reciprocity relations) on distribution of the reflected flux. The specular component can be eliminated by viewing at $\theta^{\prime}=0$, so that the specular component comes from the hole, which emits no energy into the cavity. (Again, however, the solid angle for the specular component is limited by the hole size and its distance from the sample.) It seems apparent that the heated cavity reflectometer offers the largest wavelength range and the highest accuracy of the instrument discussed previously for use in infrared reflectance measurements.

\section{New reflectometers}

In the past two years, two new reflectometers have been utilized successfully: 1) the paraboloidal reflectometer ${ }^{30,52}$ and 2) the ellipsoidal reflectometer. ${ }^{40,55}$ Figures 6 and 7 illustrate the use of these mirrors in place of the hemispherical mirror of the Cohlentz-type reflectometers. These new reflectometers were initially identical in concept; however, under use as presently indicated in the literature, there are significant differences in their capabilities.

Ellipsoidal mirror reflectometer (one response indicated its use): Figure 7 illustrates the basic design of this instrument and shows a Globar as the source. The flux from the Globar is chopped by an 11.3-cps chopper before entering a monochromator. The monochromatic beam then is refocused through a small entrance hole onto the first focal point of an ellipsoidal mirror that is $12 \frac{1}{4}$ in. in diameter and $3 \frac{5}{8} \mathrm{in}$. high. The first focal point is in the plane of the edges of the mirror, and the second is $17 \mathrm{in}$. below the first. The detector signal is amplified by a thermocouple synchronous amplifier. To measure the incident flux, the detector is placed at the first focal point. The reflected flux is measured by placing the detector at the second focal point and the sample at the first focal point; the reflected flux leaves the sample and is reflected by the ellipsoidal mirror to the detector at the second focal point. Thus, after correcting for system losses (quantitatively described in Ref. 40), the absolute directional hemispherical reflectance is measured for $\theta=7^{\circ}$. When the instrument is operated in the relative mode, the detector is always at the second focal point and two measurements are made, one with a sample at the first focal point, and the other with a specular reflectance standard at the first focal point. As in other methods of reflectance measurements, the relative measurement tends to eliminate the atmospheric absorption correction and reduce the magnitude of the other corrections.

$\dagger$ This was purchased from Strong Electric Corporation, City Park Avenue, Toledo, Ohio.

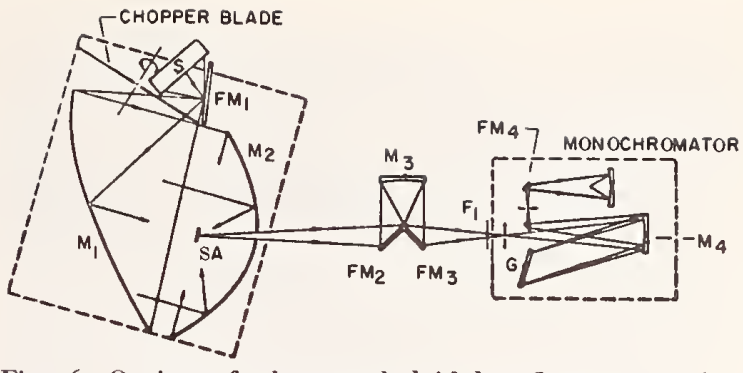

Fig. 6 Optics of the parabaloidal reflectometer (reprinted from Ref. 52 by permission).

This instrument has the same inherent losses and problems as the other systems, with the following exceptions: 1) aberrations are reduced to a minimum, since all measurements are centered around true focal points rather than conjugate focal points ${ }^{38} ; 2$ ) the reflected energy is concentrated in a small cone ( $24^{\circ}$ half-angle), hence the problem of losses caused by low sensitivity of the detector to flux incident at angles near grazing is eliminated; 3 ) the detector and sample are widely separated, which will allow heating and cooling of the sample over large temperature ranges (an advan tage shared with the Janssen-Torborg system); and 4) the optics (also true for the paraboloidal mirror) allow accurate calibration of mirror and hole losses for all but the most radically distributed reflected flux (such as from a diffraction grating).

The large magnification of the ellipsoidal mirror causes large images to be formed at the second focal point, necessitating the use of an averaging device over the detector (since large area detectors are prohibitively spatially sensitive). ${ }^{53}$ A 2-in. diam sphere coated with $\mathrm{BaSO}_{4}$ for the 0.3 to $2.5 \mu$ range and sulfur for the 1.5 to $8 \mu$ range has been used ${ }^{40}$ for the averaging device.

The spatial distribution of the reflected energy crossing the first focal plane is related precisely to the goniometric distribution of the reflected flux. That is, every direction $\theta^{\prime}, \varphi^{\prime}$ in the hemisphere above the surface is represented by a point $P$ in the first focal plane, and every solid angle centered in the direction $\theta^{\prime} \varphi^{\prime}$ is represented by an area

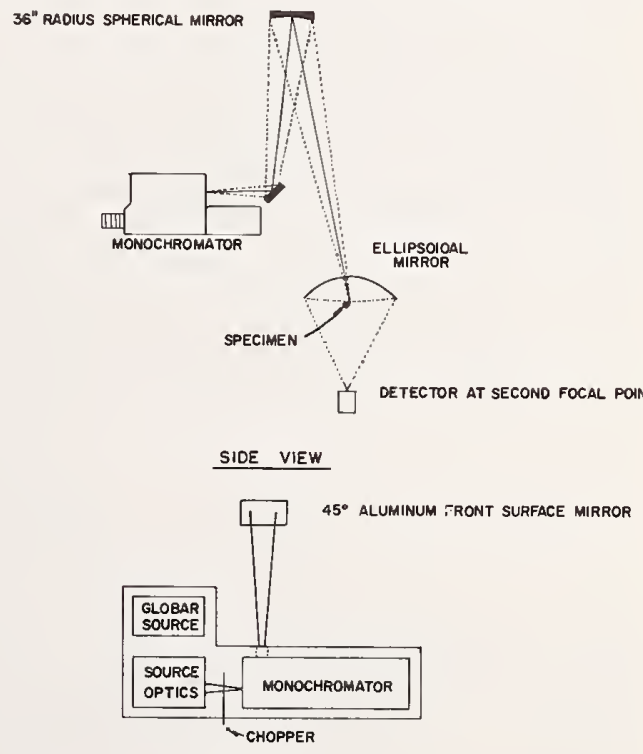

PARTIAL TOP VIEW

Fig. 7 General optical system of the ellipsoidal mirros reflectometer. 


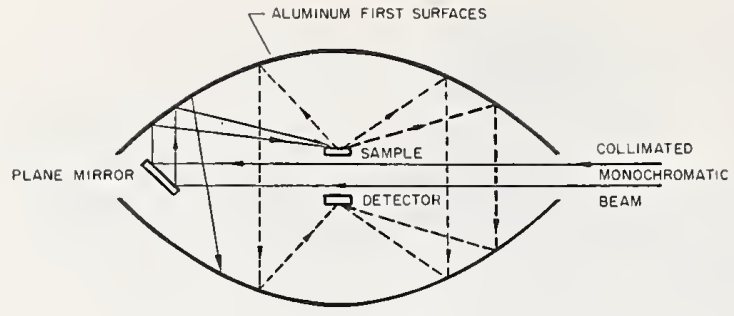

- INCIDENT ENERGY

Fig. 8 Parabaloidal reftectometer (reprinted from Ref. $30)$.

about $P$. This implies the ability to select the energy that the detector views by blanking out the unwanted energy with a shield placed in the first focal plane. With this procedure, a specular component can be measured which has a solid angle determined by the open area of the shield placed in the first focal plane. Similarly, the bidirectional reflectance can be measured by varying the position of the hole in the shield. By placing rings in the first focal plane, one can measure the directional annular cone reflectance, which is extremely useful for studying the diffuseness of samples. ${ }^{40}$ The ability to measure the distribution of the reflected flux aids greatly in making precise corrections for the system losses.

The angle of incidence can be varied by the construction of a movable mirror-source assembly similar to that shown in Fig. 8; however, the construction of this device is not as simple as with the paraboloid, since the angle of the flat mirror with respect to the source must change as the mirror is moved in and out, so that the source always appears to be at the second focal point.

Total measurement error is estimated to be less than $2 \%$ and probably less than $1 \%$ for all engineering materials regardless of the distribution of the reflected flux. However, the present lack of comprehensive data on the goniometric distribution of reflected flux from common engineering materials precludes a positive general statement of accuracy at this time. High accuracy is possible because 1) errors caused by variations in a real and angular sensitivity of the detector have been minimized by use of the sulfur and $\mathrm{BaSO}_{4}$ coated averaging spheres; 2 ) a technique for correcting the entrance and/or exit hole loss has been effectively utilized ${ }^{33}$; 3 ) the use of the ellipsoidal mirror reduces aberrations in the system and reduces the size of the solid angle of the flux incident on the detector; 4) the effective reflectance of the ellipsoidal mirror is measured as a function of position, providing an accurate correction for variations in the mirror's reflectance; 5) the reflectometer needs only a specular reference standard that is easy to calibrate accurately; and 6) the system losses can be evaluated by establishing the flux involved in each loss through the use of shields placed in or near the first focal plane.

The averaging device severely limits the useful wavelength range of this instrument to about $0.3-8 \mu$ with conventional techniques. In addition, the sample's size is restricted, since the larger the sample, the higher the uncertainty in the measurement, because the sample blocks part of the reflected beam. Also, the magnification in the ellipsoid requires a small incident image on the sample.

Paraboloidal reflectometer (two responses indicated its use): Figure 8 illustrates the original design. ${ }^{30}$ If the paraboloid over the detector had a much longer focal length than the paraboloid over the sample (thus reducing the solid angle of energy incident on the detector), it would be conceptually identical to the ellipsoidal mirror reflectometer just discussed. However, increasing the focal lengtl of the paraboloid over the detector implies the same image magnification problem as experienced with the latter, with the inherent wavelength restrictions of averaging devices. Reversing the optics in Fig. 8 and placing the diffuse source at the focal point of one paraboloid and the sample at the focal point of the other (identical) paraboloid would provide the diffuse irradiation necessary for the measurement of $r\left(\theta^{\prime} \varphi^{\prime}\right)$. However, then the source would be in the incident optical path, and chopping would be difficult. To facilitate the use of the paraboloid mirrors in the 30-100 $\mu$ range, Neher ${ }^{52}$ used an off-axis paraboloid (of $25 \mathrm{~cm}$ focal length) to bring the flux from an extended source on to a $10-\mathrm{cm}$ focal length parabola that focused the flux on the sample. A sample and specular standard are viewed by the monochrometer to measure $r\left(\theta^{\prime} \varphi^{\prime}\right)$. Bevans ${ }^{54}$ also used a similar configuration for the shorter wavelengths. This configuration has allowed the use of the reflectometer for reasonably accurate measurements over wavelength ranges previously unattained in this country; however, this was at the expense of versatility and accuracy.

A disadvantage of this mode of operation is that the flux from the source is focused on the sample before it goes to the monochromator. Sample heating problems, similar to those experienced with heated cavity reflectometers, are minimized by filtering the short wavelengths in the $30-100 \mu$ instrument. ${ }^{52}$ The use of a specular mirror standard tends to accentuate the possible error connected with nondiffuse incident conditions for comparison with diffusing samples, whereas a diffusing reference tends to accentuate the error for near-specular samples.

Neher ${ }^{52}$ obtained data in the $30-100 \mu$ region with an estimated accuracy of $5 \%$. The use of the double paraboloid reflectometer to measure $\rho(\theta, \varphi)$ as in Fig. 8 (modified to reduce the solid angle on the detector) has the same versatility of measurement and the same capability of accuracy as the ellipsoid, since shields (to determine flux density distribution) can be used in the plane between the mirrors. When it is used in the reverse mode, its wavelength range is increased, essentially extending the heated cavity type measurement (with synchronous chopping and amplification) to $100 \mu$.

In conclusion, these two types of reflectometers minimize the problems previously experienced in Coblentz-type and heated-cavity instruments. It is anticipated that they will both be improved and extended in the next year.

\section{Review of USSR Literature}

References $44,56-61$, and 72 are included to indicate the ap. proach of the other major space-oriented country to the problems of measuring thermal radiation properties. Reference 72 indicates the trend to measure spectral reflectance to determine spectral emittance (as in this country). Reference 44 illustrates a much more sophisticated approach to the use of the Coblentz hemisphere, which includes the ability to measure directional annular cone reflectance (defined in Ref. 40). This instrument has been used with diffraction gratings over the far infrared spectrum, up to $200 \mu .^{56-60}$ A similar configuration of this instrument is uscd in the shorter wavelength region with a prism monochromator. ${ }^{59,61}$ This instrument seems to provide the major backbone of their measurement program and has an estimated accuracy of $8-10 \%$ in the far infrared and a precision of $3-5 \%$. Reference 59 describes the reflectometer as follows:

The monochromator (IKS-12) of the apparatus was set up as an autocollimator. A Globar lamp was used as the radiation source up to $100 \mu$, but for the longer wavelengths a PRK-4 mercury quartz lamp was used. The detectors were two compensated radiation thermal elements (RTE) with windows of KRS-5 (for the $20-55 \mu$ region) and crystalline quartz (for wavelengths longer than $55 \mu$ ). These operated in conjunction with an FEOU-18 photoelectrical optical amplifier and an EPP-09 
electronic potentiometer. The radiation was filtered by means of a combination of reflection and transmission filters. The window of the RTE served as a transmission filter. Filtering the radiation by means of reflection and transmission filters, however, proved inadequate, especially when working with the echellettes near the blaze angle...

It is significant that in almost all of the articles reviewed, the reflectometer was used out to $100-200 \mu$, whereas in this country only Neher ${ }^{52}$ and Camus et al. ${ }^{73}$ have been successful in measuring spectral directional hemispherical reflectance (or its reciprocal) at these long wavelengths. And Camus' effort (which used a Michelson interferometer) was only partially successful because of 1) insufficient collection optics, and 2) troubles with the Golay cell. In addition, Aronson ${ }^{74}$ has made specular reflectance measurements to about $300 \mu$.

\section{Measurement of Specular Reflectance}

The measurement of specular reflectance was reported by eight responses to the questionnaire. In general, the Strong ${ }^{66}$ and the Bennett ${ }^{63}$ specular reflectometers account for the entire range of instrumentation for the accurate measurements. References 40 and 62-71 represent the various approaches to the measurement of specular reflectance and the data available in this area. Accuracies on the order of $0.1 \%$ have been reported, ${ }^{40,63,67-69}$ making this measurement the most accurate of thermal radiative property measurements. The wavelength range of the Bennett reflectometer is 0.3 to about $32 \mu$, whereas this useful wavelength range of the Strong multiple reflection specular reflectometer has been extended recently to $10 \mu$ by use of a sulfur-coated averaging sphere over the detector ${ }^{53}$ Aronson $^{74}$ is making specular reflectance measurements to $300 \mu$ with a Perkin-Elmer attachment.

\section{Summary}

The following conclusions are based on a) the foregoing discussion on heat-transfer calculation technique and on thermal radiation property measurement techniques, and b) the responses to the thermal radiation questionnaire.

1) The major problems of the general field seem to be nomenclature and appropriate society affiliation. Both stem from the same background, that is, the diverse educational and experience backgrounds of the personnel in this field. It is hoped that the new affiliation with AIAA will provide solutions to both these problems.

2) The practicing engineer, in general, uses opaquediffuse-gray heat-transfer calculations. He is interested primarily in the goniometric distribution of reflected and emitted flux, the coupling between conduction-convection and radiant heat transfer, and the calculation of emittance for semiopaque bodies with a temperature gradient normal to the surface. Sufficient computational methods are available to enable the heat-transfer analyst to perform very accurate calculations. The immediate need is for more work on the specular-diffuse calculation technique to improve its usefulness to the practicing engineer and to reduce the required computer time for the Monte Carlo technique for complex systems. Furthermore, tabulation of the total image view factor for common configurations now is possible. The major deterrent to these types of calculations is the lack of data on the spectral directional specular and diffuse component of reflectance over the appropriate wavelength ranges. It also is possible to use Monte Carlo techniques to calculate the heat transfer between surfaces whose complete goniometric distributions are known.

3) Satisfactory equipment and procedures are available for measuring total hemispherical emittance and spectral and total normal emittance to an accuracy between 3 and $8 \%$ at temperatures from $100^{\circ}-1800^{\circ} \mathrm{K}$; errors are likely to be much larger at temperatures above $1800^{\circ} \mathrm{K}$ or below $100^{\circ} \mathrm{K}$.
4) It appears that measurement of reflectance is still an art, and the estimated accuracy as given herein for each instrument is intended to imply the use of the best possible measurement procedures for surfaces of unknown distribution of reflected flux. Most of the data in the literature (going back to the work of Coblentz in 1913) do not reflect the best measurement procedures available today and may be in error on the order of $30 \%$.

The Edwards type integrating sphere is the most generally used reflectometer for measurements at short wavelengths $(<2.6 \mu)$, and the heated cavity is the most used reflectometer in the infrared part of the spectrum. The extension of the Coblentz concept to the ellipsoidal and paraboloidal reflectometers provides increased versatility, accuracy, and wavelength range. It is anticipated that both of these instruments will be developed more fully and, possibly, available commercially in the next year.

The following appear to be the major remaining problems in the measurement of reflectance.

1) Accuracy of the measurement should be increased to the order of $1-2 \%$.

2) The wavelength range of the measurement should cover at least the range of 0.2 to $100 \mu$ and hopefully 0.2 to $500 \mu$ (for bodies at cryogenic temperatures in space).

3) Reflectance should be measured as a function of sample temperature from $4^{\circ}$ to approximately $1000^{\circ} \mathrm{K}$. Above this temperature emittance techniques seem appropriate.

4) More data are needed on the effects that polarization and angle of incidence have on reflectance.

5) More goniometric data are needed, both in and out of the plane of incidence.

6) Diffuse standards of spectral directional hemispherical reflectance of $1 \%$ accuracy are needed over at least the 0.2 to $35 \mu$ range.

7) Standards of spectral specular reflectance are needed to cover the 0.2 to $35 \mu$ range, and of various levels of reflectance (about five, distributed equally between 0 and 1 ).

8) More accurate ultraviolet degradation studies should be made.

9) The effect of the size of the measurement solid angle on the measured value of the directional specular component of reflectance should be established.

10) Data on the spectral specular and diffuse components of reflectance are needed for the 0.2 to $35 \mu$ range.

11) There is great need for new radiant sources. It is suggested that the continuous wave laser would be a superb source since it has a high-intensity collimated beam. ${ }^{29}$ Furthermore, the developments in this field indicate that wavelengths of laser emission will be available (in the next five years) to cover much of the spectrum of interest. Salisbury effect devices ${ }^{78}$ seem to offer a possible far infrared source of high intensity.

12) A study of the experimentally necessary and sufficient conditions for $r(\theta, \varphi)$ should be made.

13) New techniques are needed for measurements in the far infrared. A possible solution is the Michelson interferometer approach, which, for the same elapsed measurement time, provides marked increases in the signal to noise ratio. ${ }^{73,75-77}$

14) Probably most important of all, methods of characterizing the measured sample are needed desperately.

\section{References}

'Hottel, H. C., "Radiant heat transmission," Heat Transmission, edited by W. H. McAdams (McGraw-Hill Book Co. Inc., New York, 1954), 3rd ed., Chap. 4.

2 Oppenheim, A. K., "The network method of radiation analysis," American Society of Mechanical Engineers Paper 54-A75 (1954).

${ }^{3}$ Gebhart, B., Heat Transfer (McGraw-Hill Book Co. Inc., New York, 1961), pp. 117-122.

${ }^{4}$ Münch, B., "Die Richtungsverteilung bei der Reflexion von 
Wärmestrahlung und ihr Einfluss auf die Wärmeübertragung," Mitt. Inst. Thermodynamik Verbrenmung. Eidgenoss. Techn. Hoch. Zürich, no. 16 (1955).

${ }^{5}$ Birkebak, R. C., "Monochromatic directional distribution of reflected thermal radiation from roughened surfaces," Ph.D. Thesis, University of Minnesota (1962).

${ }^{6}$ Sparrow, E. M., Eckert, E. R. G., and Jonsson, V. K., "An enclosure theory for radiative exchange between specularly and diffusely reflecting surfaces," J. Heat Transfer 84, 294-300 (1962).

${ }^{7}$ Bobco, R. P., "Radiation heat transfer in semigray enclosures with specularly and diffusely reflecting surfaces," American Society of Mechanical Engineers Paper 63-AHGT-10 (1963).

${ }^{8}$ Seban, R. A., "Discussion," J. Heat Transfer 84, 299-300 (1962).

${ }^{9}$ Bevans, J. T..and Edwards, D. K., "Radiation exchange in an enclosure with directional wall properties," American Society of Mechanical Engineers Paper 64-WA/HT-52 (1964).

${ }^{9}$ Sparrow, E. M. and Lin, S. L., "Radiation heat transfer at a surface having both specular and diffuse reflectance components," Intern. J. Heat Mass Transfer 8, 769-779 (1965).

${ }^{a b}$ Corlett, R. C., "Direct numerical simulation of thermal radiation in vacuum," American Society of Mechanical Engineers Paper 65-WA/HT-40 (1965).

${ }^{10}$ Sparrow, E. M., Gregg, J. L., Szel, J. V., and Manos, P., "Analysis, results, and interpretation for radiation between some simply-arranged grey surfaces," J. Heat Transfer 83, 207-214 (1961).

11 Sparrow, E. M. and Lin, S. H., "Absorption of thermal radiation in V-groove cavities," Office of Technical Services, U.S. Dept. of Commerce Publication N6210610 (November 5, 1962).

${ }_{12}$ Sarofim, A. F. and Hottel, H. C., "Radiative exchange among non-Lambert surfaces," American Society of Mechanical Engineers Paper 65-HT-7 (August 1965).

${ }^{13}$ Kahn, H., "Applications of Monte Carlo," Rand Corp., Research Memo. RM-1237-AEC (April 27, 1956).

${ }^{14}$ Cashwell, E. D. and Everett, C. J., "A practical manual on the Monte Carlo method for random walk problems," Los Alamos Science Laboratory, LA-2120 (1957).

${ }^{15}$ Howell, J. R. and Perlmutter, M., "Monte Carlo solution of thermal transfer through radiant media between gray walls," J. Heat Transfer 86, 116 (1964).

${ }_{15}$ Perlmutter, M., "Monte Carlo solution of a highly rarefied ionized gas flowing through a channel with a transverse magnetic field," Advances in Applied Mechanics, Rarefied Gas Dynamics, Fourth Symposium (Academic Press Inc., New York, 1965).

17 Parmer, J. F., "The thermal radiation characteristics of specular walled grooves in solar space environment," Ph.D. Thesis, Oklahoma State University (May 1965).

17a Torrance, K. E. and Sparrow, E. M., "Off-specular peaks in the directional distribution of reflected thermal radiation," American Society of Mechanical Engineers Paper 65-WA/HT-19 (1965).

${ }^{17 \mathrm{~b}}$ Sparrow, E. M. and Cess, R. D., Radiant Heat Transfer (Wadsworth Publishing Co. Inc., Belmont, Calif., 1966).

170 Wiebelt, J. A., Engineering Radiation Heat Transfer (Holt, Rinehart and Winston Inc., New York, 1966).

${ }^{18}$ Fragstein, C. V., "On the formulation of Kirchhoff's law and its use for a suitable definition of diffuse reflection factors," Optik 12,60-70 (1955).

${ }^{19}$ MeNicholas, H. J., "Absolute methods in reflectometry," J. Res. Natl. Bur. Std. 1, 29-73 (1928).

${ }^{20}$ Nicodemus, F. E., "Directional reflectance and emissivity of an opaque surface," Appl. Opties 4, 767-773 (1965).

${ }^{21}$ International Lighting Vocabulary (International Commission on Illumination, Paris, 1957), 3rd ed.

${ }^{22}$ Unpublished reports of various nomenclature committees of American Society for Testing and Materials, American Institute for Aeronautics and Astronautics, Optical Society of America, and National Bureau of Standards.

${ }^{23}$ Edward, D. K., Gier, J. T., Nelson, K. E., and Roddick, R. D., "Integrating sphere for imperfectly diffuse samples," Appl. Optics, 51, 1279-1288 (1961).

${ }^{24}$ Jacques, J. A. and Kuppenheim, H. F., "Theory of the integrating sphere," J. Opt. Soc. Am. 45, 460-470 (1955).

${ }^{26}$ Karrar, E., "The use of the Ulbright sphere in measuring reflection and transmission factors," J. Opt. Soc. Am. 11, 96-120 (1921).

${ }^{26}$ Jacques, J. A. and Kuppenheim, H. F., "Effect of elimination of the specular component on efficiency and error of the integrating sphere," J. Opt. Soc. Am. 46, 428-429 (1956).

${ }^{27} \mathrm{Hardy}, \mathrm{A}$. C. and Pineo, O. W., "The errors due to the finite size of holes and sample in integrating sphere," J. Opt. Soc. Am. 21, 502-506 (1931).

${ }^{28}$ Taylor, A. H., "Errors in reflectometry," J. Opt. Soc. Am. $25,51-56$ (1935).

${ }^{29}$ Richmond, J. C., Dunn, S. T., DeWitt, D. P., and Hayes, W. D., Jr., "I'rocedures for precise determination of thermal radiation properties," National Bureau of Standards TN 267 (1965).

${ }^{30}$ Dunkle, R. V., "Spectral reflectance measurements," Surface Effects on Spacecraft Materials (John Wiley \& Sons Inc., New York, 1960), pp. 117-137.

${ }^{31}$ Clayton, W. A., "Comments on measurement techniques," Symposium on Thermal Radiation of Solids, edited by S. Katzoff, NASA SP-55 (1965).

${ }^{32}$ Streed, E. R., McKellar, L. A., Rollings, R., Jr., and Smith, C. A., "Errors associated with Hohlraum radiation characteristics determinations," Measurement of Thermal Radiation Properties of Solids, edited by J. C. Richmond, NASA SP-31, pp. 237-252 (1963).

${ }^{33}$ Coblentz, W. W., "The diffuse reflecting power of various substances," Bull. Natl. Bur. Std., 9, 283-325 (1913).

${ }^{34}$ Gier, J. T., Dunkle, R. V., and Bevans, J. T., "Measurement of absolute spectral reflectivity from 1.0 to 15 microns," J. Opt. Soc. Am. 44, 558-562 (1954).

${ }^{35}$ Royds, T., "The reflective power of lamp- and platinumblack," Phil. Mag. 21, 167-172(1911).

${ }^{35}$ Royds, T., "Das Reflexionvermögen schwarzer Flächen," Physik. Z. 11, 316-319 (1910).

${ }^{37}$ Paschen, F., "Uber die Vertheilung der energie im Specktrum des Schwarzen Korpers bei niederen temperaturen," Sitzungsberichte Akademie der Wissenschaften zu Berlin 27, 405-420 (1899).

${ }^{38}$ Brandenberg, W. M., "Focusing properties of hemispherical and ellipsoidal mirror reflectometers," J. Opt. Soc. Am. 54, 1235-1237 (1964).

${ }^{39}$ Stair, R. and Schneider, W. E., "Standards, sources, and detectors in radiation measurements," Symposium on Thermal Radiation of Solids, edited by S. Katzoff, NASA SP-55 (1965).

${ }^{40}$ Dunn, S. T., "Design and analysis of an ellipsoidal mirror reflectometer," Ph.D. Thesis, Oklahoma State University (May 1965).

${ }^{41}$ Janssen, J. E. and Torborg, R. H., "Measurement of spectral reflectance using an integrating hemisphere," Measurement of Thermal Radiation Properties of Solids, edited by J. C. Richmond, NASA SP-31, pp. 169-182 (1963).

${ }^{42}$ White, J. U., "New method for measuring diffuse reflectance in the infrared," J. Opt. Soc. Am. 54, 1332-1337 (1964).

${ }^{43}$ Birkebak, R. C. and Hartnett, J. P., "Measurements of the total absorptivity for solar radiation of several engineering materials," Trans. Am. Soc. Mech. Engrs. 80, 373-378 (1958).

${ }^{44}$ Kozyrev, B. P. and Vershinin, O. E., "Determination of spectral coefficients of diffuse reflection of infrared radiation from blackened surfaces," Opt. Spectry. (USSR) (Engl. Transl.) 6, 345-350(1959).

${ }^{45}$ Derksen, W. L., Monahan, T. I., and Lawes, A. J., "Automatic recording reflectometer for measuring diffuse reflectance in the visible and infrared regions," J. Opt. Soc. Am. 47, 995999 (1957).

${ }^{45}$ Derksen, W. L. and Monahan, T. I., "A reflectometer for measuring diffuse reflectance in the visible and infrared regions," J. Opt. Soc. Am. 42, 263-265 (1952).

${ }^{47}$ Sanderson, J. A., "The diffuse spectral reflectance of paints in the near infrared," J. Opt. Soc. Am. 37, 771-777 (1947).

48 Martin, W. E., "Hemispherical spectral reflectance of solids,' Measurement of Thermal Radiation Properties of Solids, edited by J. C. Richmond, NASA SP-31, pp. 183-192 (1963).

${ }^{49}$ Dunkle, R. V., Ehrenburg, F., and Gicr, J. T., "Spectral characteristics of fabrics from 1 to 23 microns," J. Heat Transfer 82, 64-70(1960).

${ }_{50}$ Reid, D. C. and McAlister, E. D., "Measurement of spectral emissivity from $2 \mu$ to $15 \mu$," J. Opt. Soc. Am. 49, 78-82 (1959).

${ }^{51}$ Dunkle, R. V., Edwards. D. K., Gier, J. T., Nelson, K. E., and Roddick, R. D., "Heated cavity reflectometer for angular reflectance measurement," Progress in Inlernational Rescarch on Thermodynamics and Transport Properties (American Society of Mechanical Engineers, New York, 1962).

${ }^{52}$ Neher, R. T. and Edwirds. D. K., "Far irfrared reflecton- 
eter for imperfectly diffuse specimens," Appl. Optics 4, 775780 (1965).

53 Dunn, S. T., "Flux averaging devices for the infrared;" National Bureau of Standards TN 279 (1965).

${ }^{64}$ Bevans, J., personnel communication (1965).

${ }^{65}$ Blevin, W. R. and Brown, W. J., "An infrared reflectometer with a spheroidal mirror," J. Sci. Inst. 42, 1-5 (1965).

${ }^{56}$ Kropotkin, M. A. and Kozyrev, B. P., "Apparatus for the determination of the diffuse reflection coefficients in long wavelength infrared part of the spectrum," Leningradskii Electroteknicheskii Institute Bull. XLIV; XLIV, 87-99 (1960).

${ }^{67} \mathrm{Kozyrev}$, B. P. and Kropotkin, M. A., "Study of spectral reflection coefficients of thermal radiation receiver coatings for the wavelength range from 10 to $200 \mu$," Opt. Spectry. (USSR) (Engl. Transl.) 10, 345-347 (1949).

ss Kryzhanovskii, V. P., Kuznetsov, A. Y., and Pafomova, L. A., "Reflection in long-wavelength region of semiconducting films of silicon monoxide containing silver and gold," Opt. Spectry. (USSR) (English Transl.) 14, 447-448 (1963).

${ }^{60}$ Kropotkin, M. A. and Kozyrev, B. P., "Study of the reflection spectra of natural and synthetic materials in the $0.7-100 \mu$ range," Opt. Spectry. (USSR) (Engl. Transl.) 17, 136-139 (1964).

${ }_{60}$ Kropotkin, M. A. and Kozyrev, B. P., "Methods and results of investigation obtained on the apparatus for determining the spectral reflection of scattering materials in long-wavelength infrared part of the spectrum," Izv. Vysshikh. Uchebn. Zavedentii Bull. USSR-App. Construct. 7, 122-127 (1964).

${ }^{61}$ Kozyrev, B. P. and Kropotkin, M. A., "Study of diffuse reflection from white coatings which strongly absorb infrared radiation," Opt. Spectry. (USSR) (Engl. Transl.) 12, 78-79 (1961).

${ }^{62}$ Gates, D. M., Shaw, C. C., and Beaumont, D., "Infrared reflectance of evaporated metal films," J. Opt. Soc. Am. 48, 8889 (1958).

${ }^{63}$ Bennett, H. E. and Koehler, W. F., "Precision measurement of absolute specular reflectance with minimized systematic errors," J. Opt. Soc. Am. 50, 1-6 (1960).

${ }^{64}$ Weeks, R. F., "Simple wide range specular reflectometer," J. Opt. Soc. Am. 48, 775-777 (1958).
${ }^{65}$ Ramsey, W. Y., "Specular spectral reflectance of paints from 0.4 to 40.0 microns," Meteorlogical Satellite Laboratory Rept. 31 (April 1964).

${ }_{66}$ Strong, J., Procedures in Experimental Physics (PrenticeHall Inc., Englewood Cliffs, N. J., 1938), p. 376.

${ }^{67}$ Bennett, H. E., Silver, M., and Ashley, E. J., "Infrared reflectance of aluminum evaporated in ultra-high vacuum," J. Opt. Soc. Am. 53, 1089-1095 (1963).

${ }^{68}$ Bennett, H. E., Bennett, J. M., and Ashley, E. J., "Infrared reflectance of evaporated aluminum films," J. Opt. Soc. Am. 52, $1245-1250$ (1962).

${ }^{69}$ Bennett, J. M. and Ashley, E. J., "Infrared reflectance and emittance of silver and gold evaporated in ultra-high vacuum," Appl. Opt.4, 221-224 (1965).

${ }^{70}$ Hass, G., "Optics section," American Institule of Physics Handbook, edited by Gray (McGraw-Hill Book Co. Inc., New York, 1957), pp. 6-19.

${ }^{71}$ Hass, G., "Filmed surfaces for reflecting optics," J. Opt. Soc. Am. 45, 945-952 (1955).

${ }^{72}$ Kropotkin, M. A. and Kozyrev, B. P., "Determining the emissivity of materials on the basis of their infrared reflection spectra," Inzh. Fiz. Zh. Akad., Nauk. Belorussk SSR VII, 108112 (1964).

${ }^{33}$ Camus, J., Gunderson, R. B., Holl, F. F., and Spade, G. L., "Radiation properties of materials-detailed refined measurements," Space Systems Div. TDR-64-224 or DDC 450295 (September 1964).

${ }^{74}$ Blau, H., personal communication; also Aronson, J. R. and McLinden, H. G., "Far infrared spectra of solids," Symposium on Thermal Radiaition of Solids, edited by S. Katzoff, NASA SP-55, pp. 29-38 (1965).

${ }^{75}$ Bobo, S. W., "Block associates model I-4 spectrometers," J. Am. Opt. 51 (August 1961).

${ }^{76}$ Wyntjes, G., "Rapid scanning interferometer spectrometer stabilization," J. Opt. Soc. Am. XIII 52 (November 1962).

${ }^{77}$ Connes, J., "Spectroscopic studies using fourier transformation," Rev. Opt. 40, 45-72, 116-140, 171-190, 231-265 (1961).

${ }^{78}$ Salisbury, W. W., personal communication (1965). 


\title{
Thermal Radiation Property Measurement Techniques
}

\author{
S. T. Dunn, J. C. Geist, D. G. Moore, \\ H. E. Clark, and J. C. Richmond
}

This is an annual summary report of work completed on NASA Contract R-09-022-032. The work comprised (1) completion of the development and calibration of a rotating cylinder procedure for measurjing normal spectral emittance of non-conducting materials at temperatures in the range of 1200 to $1600{ }^{\circ} \mathrm{K},(2)$ analysis and calibration of an ellipsoidal mirror reflectometer, and (3) a study of the relation between surface roughness and geometric distribution of flux reflected from a surface.

Key Words: Averaging spheres, diffuse reflectance, emissivity, emittance, flux averaging devices, infrared reflectance, spectral reflectance, spectral emittance, specular reflectance. 


\title{
NATIONAL BUREAU OF STANDARDS Technical Note 252
}

ISSUED NOVEMBER 20, 1964

\section{PROCEDURES FOR PRECISE DETERMINATION \\ OF THERMAL RADIATION PROPERTIES \\ NOVEMBER 1962 TO OCTOBER 1963}

\author{
Joseph C. Richmond, Davis P. DeWitt, \\ and Warren D. Hayes, Jr.
}

\section{ABSTRACT}

The preliminary design of an intergrating-sphere reflectometer, utilizing a helium-neon continuous-wave gas laser as the source, for measuring the reflectance of specimens at high temperature, was completed. Development work on an ellipsoidal mirror reflectometer for measuring spectral reflectance in the wavelength range of 2 to 15 microns of specimens at room temperature was continued. The study of equations relating spectral emissivity of metals to other properties was continued. Platinum-13\% rhodium and oxidized Inconel working standards of normal spectral emittance were calibrated over the wavelength range of 1 to 15 microns at temperatures of 800,1100 and $1300^{\circ} \mathrm{K}$. Several modifications of the normal spectral emittance equipment were made to permit operation in the 15-35 micron range. 
$+0 \wedge \infty \infty+\infty \infty \pi$ ษ ต.

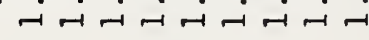

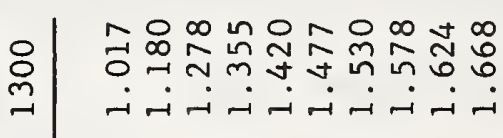

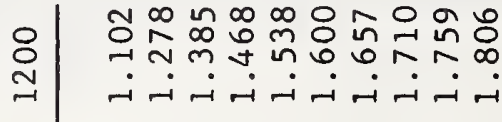

ำ -

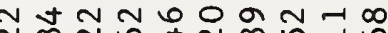

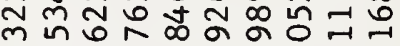
-i-i-iનi $\dot{\sim} \dot{\sim}$

a t

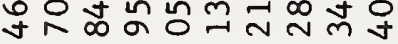
-in-inanin

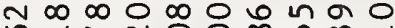
กำ

$\stackrel{\circ}{\circ}$ -ininanina

$\stackrel{\circ}{\circ}$

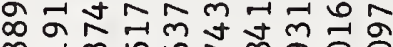
$\infty$ ำ -iÑN N N

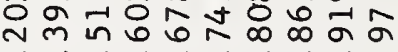

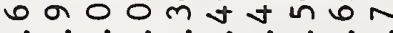
ง น

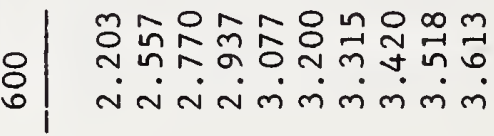

-
ஸิ ปี兀 ก 0 ช 0 ก $\infty \infty$ - - - -

옹ㅇำ

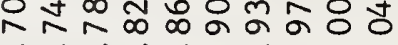
-

ำกำ

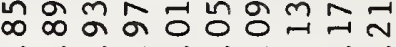
th-

어네

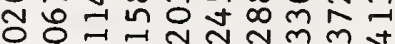

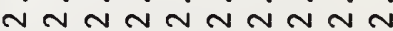

ㅊำㄴำ을

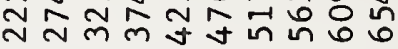
กั่

anm $m$ N ชิ๊ิ nivinanana

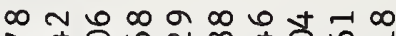
సิ ก نं $\dot{\sim} \dot{\sim} \dot{m} \dot{m} \dot{m}$

ง ปัก - Nmm $\dot{m} \dot{m} \dot{m} \dot{m} \dot{m} \dot{m} \dot{m} \dot{m}$

ㅇํㅇำㄴำ

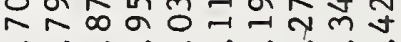
लंmंmं $\dot{y} \dot{j} \dot{y} \dot{j} \dot{j} \dot{j}$

ㄱำㄴำㅇำ N $\mathrm{m} m$ ษ กัก

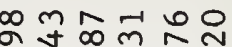

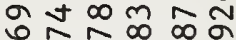
NลN N N

กิศี สूสু ठิ $\operatorname{rnn} N$

n $0+\infty \sim 0$ ธีむฏテั NกN N N

$\infty \infty$ 중ำ N $\mathrm{mm}$ m NNNNNN

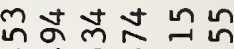
I t in in 0 NNNN N N

$\infty m n-100$ ชิำ ก ก ก N

$\infty \infty N \varphi \bullet$ बㅎํ웜 तेंलं लंm

Natano № $m$ a n $m$ ง เก เn लंmं mं $\dot{m}$

ํํำำำำ $\infty$ नूने चै mंmं

유ำ ง

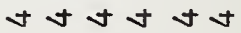

un un un un un un un un in in

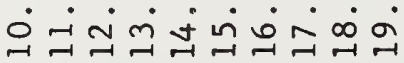

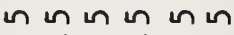
ํำ ขิ 


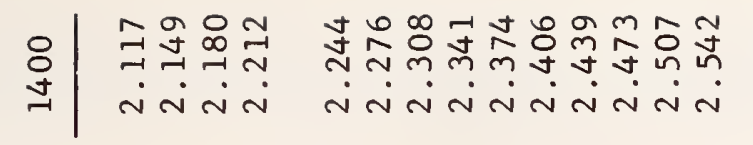

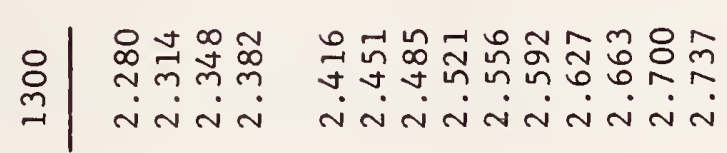

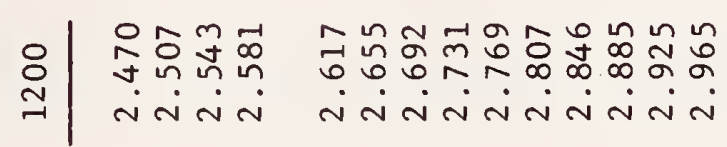
ஸ ફ

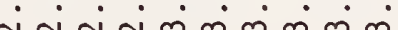
NNN Nmmm m m

-

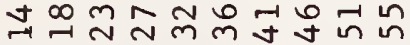
लं लं लं लं $\dot{m} \dot{m} \dot{m} \dot{m}$ तं लंm

$m \sim-r$ ๙ิ

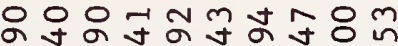
ป นี้

$\dot{m} \dot{m} \dot{m} \dot{m} \dot{m} \dot{m} \dot{m} \dot{m}$

लं लं ले

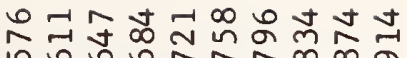
in 6 ठ 0 त nNNNNNNNNT

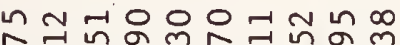

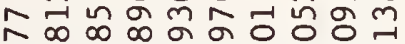
Nं

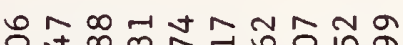
ㅇํㅇำ

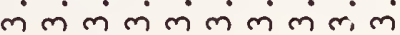

ก

ㅊำ่ ฟ $\mathrm{m}$ ง $\dot{m} \dot{m} \dot{m} \dot{m} \dot{m} \dot{m} \dot{m} \dot{m}$

nขल

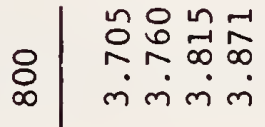

ด สำ mं $\dot{j} \dot{j} \dot{j} \dot{j} \dot{j} \dot{j}$

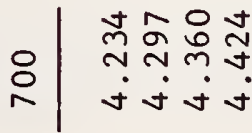

กิ ง กิ

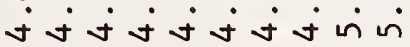

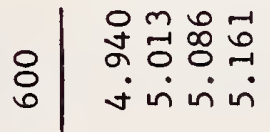

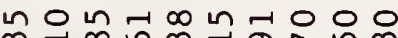
ฟ ค่
กิมกษด ๆ ำ نे نं

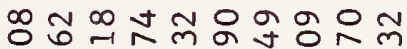
ㅇํㅇำ ન adiajajiajad

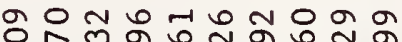

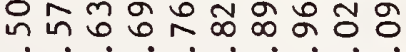
ádiaja

-1m $0-1 \infty$ in $m m$ in $\infty$ -19 ด. ก. ن

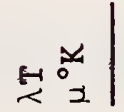

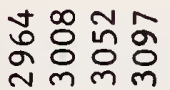

-

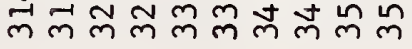

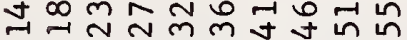

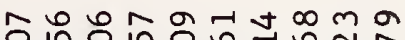

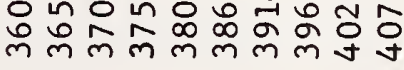

थก थ नl| |

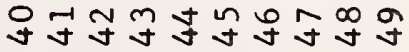




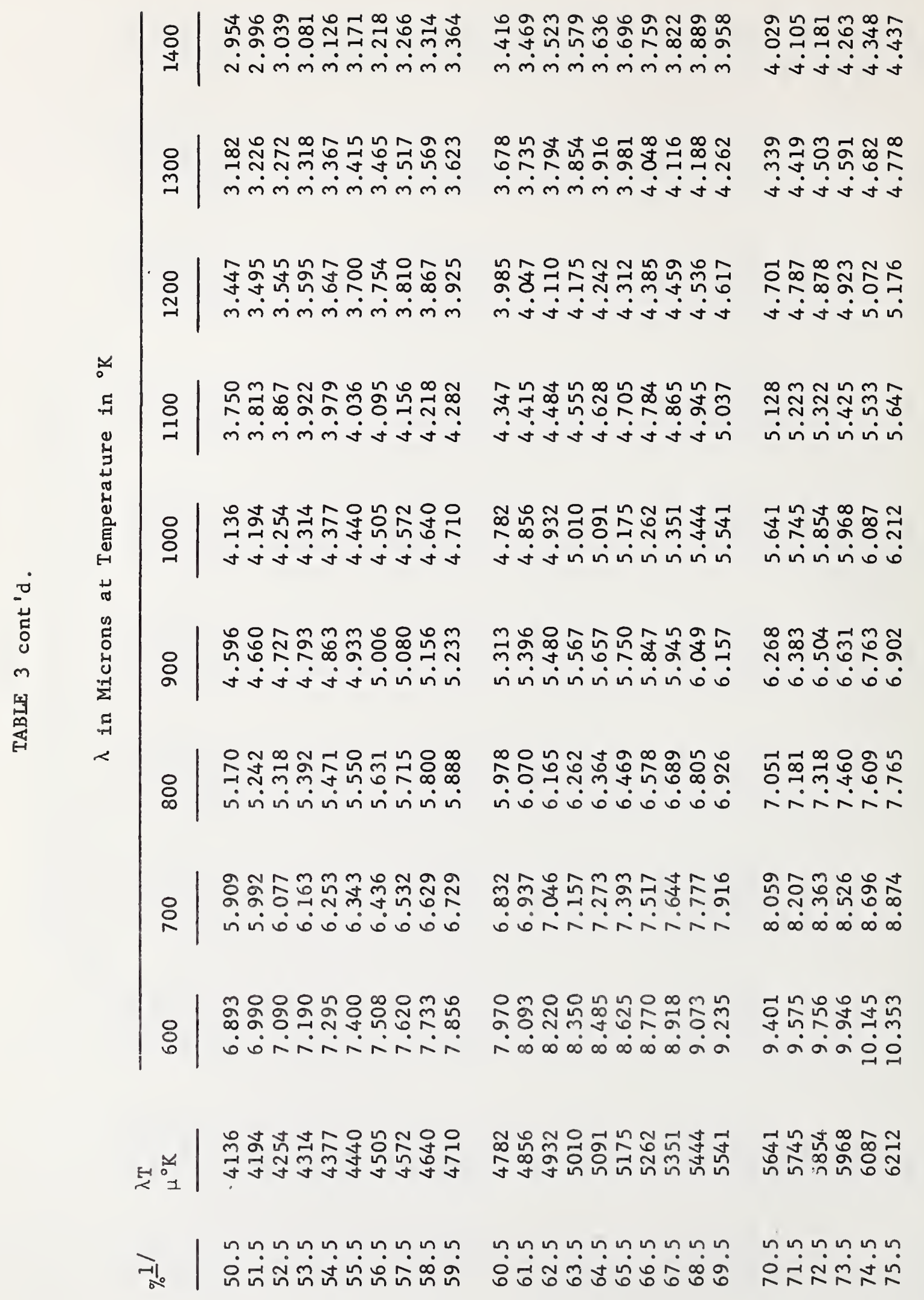




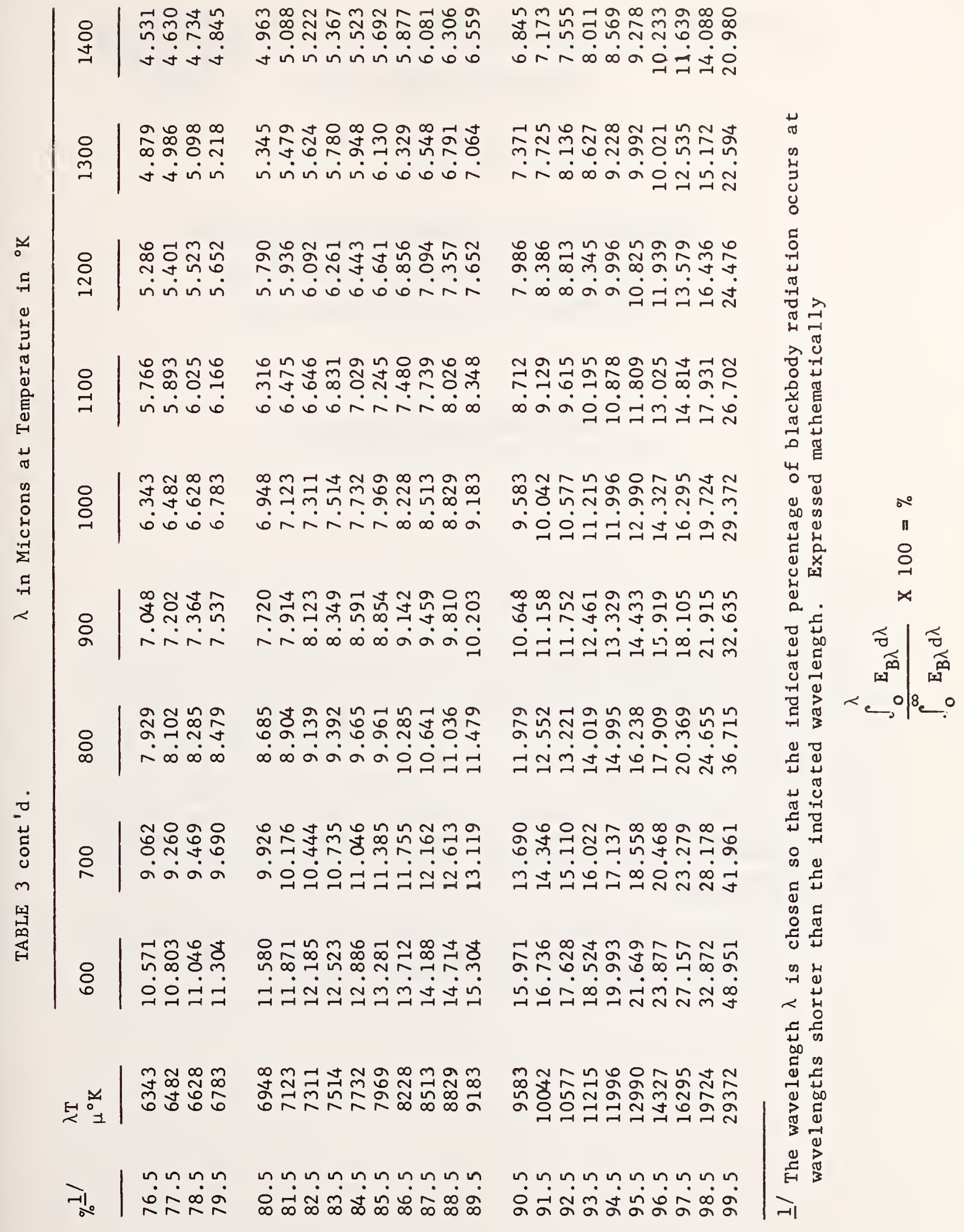




\title{
NATIONAL BUREAU OF STANDARDS Technical Note 267
}

ISSUED DECEMBER 17,1965

\section{PROCEDURES FOR PRECISE DETERMINATION \\ OF THERMAL RADIATION PROPERTIES \\ NOVEMBER 1963 TO OCTOBER 1964}

Joseph C. Richmond, S. Thomas Dunn, David P. DeWitt, and Warren D. Hayes, Jr.

\begin{abstract}
A laser-source integrating sphere reflectometer was designed and built to measure the reflectance of specimens at high temperatures. The sphere was calibrated for linearity of response at 0.632 by means of a shallow cylindrical cavity with a variable depth-to-radius ratio, having a lining of known reflectance. Preliminary tests showed that the flux emitted by a hot specimen at temperatures up to $2500^{\circ} \mathrm{K}$ will not invalidate the reflectance measurement. An ellipsoidal mirror reflectometer was calibrated for all known errors in the 1 to $7.5 \mu$ range. A preliminary analysis indicates that the errors in measurement of absolute reflectance with this equipment should not exceed 2 percent. A review of the literature on relation of thermal radiation properties to other properties of materials is presented, together with a summary of the work done in an effort to compute the spectral emittance of rhodium.
\end{abstract}

Key word8: Emissivity, emittance, high temperature reflectance, infrared reflectance, radiation properties, reflectance, spectral emittance, spectral reflectance, thermal radiation. 


\title{
NBBS TECHNICAL NOTE 292 \\ ISSUED FEBRUARY 10, 1967
}

\section{Procedures for Precise Determination of Thermal Radiation Properties November 1964 to October 1965}

\author{
J. C. Richmond, G. J. Kneissl, \\ D. L. Kelley, and F. J. Kelly
}

\begin{abstract}
The broad overall objective of this continuing program is to develop equipment and procedures for measuring the important thermal radiation properties of materials, particularly those used in aircraft, missiles and space vehicles, at temperatures up to the melting point of the most refractory material, and to develop physical standards for checking such equipment and procedures. During the period covered by this report the specific objectives were (1) continued development of the laser-source integrating sphere reflectometer, (2) an error analysis of the shallow cavity technique for measuring normal spectral emittance, and (3) a study of the feasibility of preparing emittance standards for use at temperatures above $1400{ }^{\circ} \mathrm{K}$ (about $2000^{\circ} \mathrm{F}$ ).

An error analysis of the shallow cavity technique for measuring total normal emittance of ceramic materials at very high temperatures showed that there was an error due to the translucency of the specimens that was as much as $+60 \%$ for alumina, and a second error due to thermal gradients in the specimen that was on the order of $-10 \%$. Two new techniques were devised in the hope of greatly reducing the translucency error. Progress was made in developing codes to compute and correct for the thermal gradients present in the specimen. The lasersource integrating sphere reflectometer for measuring reflectance of specimens at very high temperatures was extensively redesigned to eliminate errors due to flux reaching the detector on the first reflection, and to convert the reflectometer from the substitution to the comparison mode A literature search was made of techniques for measuring thermal radiation properties of solids at temperatures above $2500^{\circ} \mathrm{K}\left(4000^{\circ} \mathrm{F}\right)$.
\end{abstract}





\section{Material Properties}

Papers

4.1. Effect of Surface Roughness on Emittance of Nonmetals.

J. C. Richmond, J. Opt. Soc. Am. 56, No. 2, 253-254 (Feb. 1966).

Key words: Emittance; nonmetals; surface roughness_-_-

4.2. Thermal Radiation Properties of Ceramic Materials.

J. C. Richmond, Proc. Symposium on Mechanical and Thermal

Properties of Ceramics, Gaithersburg, Md., Apr. 1-2, 1968,

Nat. Bur. Stand. (U.S.) Spec. Publ. 303, pages 125-137 (May

1969).

Key words: Alumina; ceramics; emittance; magnesia ; reflectance; temperature coefficient of emittance; thermal radiation properties; thoria; zirconia______._._._._._.

4.3. Relation of Emittance to Other Optical Properties.

J. C. Richmond, J. Res. Nat. Bur. Stand. (U.S.) 67C, No. 3, 217-226 (Sept. 1963).

Key words: Emittance; layer properties; mathematical relations

4.4. Total Hemispherical Emittance of Coated and Uncoated Inconel and Types 321 and 430 Stainless Steel.

J. C. Richmond and W. N. Harrison, J. Res. Nat. Bur. Stand. (U.S.) 66C, No. 3, 261-269 (Sept. 1962).

Key words: Alloys; ceramic coatings; emittance; Inconel ;

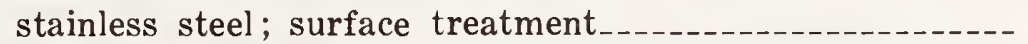

4.5. Spectral Emittance of Ceramic-Coated and Uncoated Specimens of Inconel and Stainless Steel.

J. C. Richmond, and J. E. Stewart, J. Am. Ceram. Soc. 42, No. 12, 633-640 (Dec. 1959).

Key words: Ceramic-coated materials ; emittance; Inconel ; spectral emittance; stainless steel

\section{Abstract}

4.6. Theory and Measurement of the Thermal Radiative Properties of Metals.

D. P. DeWitt and J. C. Richmond, Techniques of Metals Research, Vol. VI, (John Wiley \& Sons, New York, N.Y., 1970).

Key words: Absorptance; electromagnetic theory; emittance; measurement techniques; metals ; reflectance ; surface effects; thermal radiation properties............... 



\title{
Effect of Surface Roughness on Emittance of Nonmetals
}

\author{
JOSEPH C. RICHMOND \\ Institule for Basic Standards, National Bureau of Standards, Washington, D. C. 2023-t
}

(Received 27 July 1965)

\begin{abstract}
It has been observed experimentally, both at NBS and elsewhere, that the emittance of polished metals can be markedly increased by roughening the surface, by as much as a factor of 2 or 3 . For nonmetals and particularly white ceramic materials, on the other hand, the emittance appears to be essentially independent of surface roughness, at least for wavelengths below 7 or $8 \mu$. This apparant anomaly is explained on the basis of the differences in the optical properties of the two types of materials.
\end{abstract}

INDEX HEADINGS : Emissivity; Reflectance.

\section{INTRODUCTION}

$\mathrm{P}$ REVIOUS work in this laboratory has shown that the normal spectral emittance of Inconel and type 321 stainless steel was increased by a factor of 2 to 3 by sandblasting, ${ }^{1}$ and also that the same treatment increased the total hemispherical emittance of Inconel and types 321 and 430 stainless steel by similar amounts. ${ }^{2}$ Gannon and Linder ${ }^{3}$ report that the total normal emittance of alumina is not a function of surface roughness, and ascribe this effect to the transparency of alumina. Similar data taken at NBS on the normal spectral emittance of alumina are shown in Fig. 1. The demonstrated difference in the effect of surface roughness on the emittance of metals as compared to nonmetals calls for an explanation.

The difference can be explained qualitatively on the basis of the properties of the materials involved. The phenomena can be more easily explained in terms of reflectance than in terms of emittance. This is legitimate because for opaque specimens, the emittance and reflectance sum to one, by Kirchhoff's law.

When a beam of radiant flux strikes an interface between two media of different indices of refraction, part of the beam is reflected and part is transmitted across the interface. Since the interface has no thickness, there can be no absorption at the interface, and all of the energy of the incident beam is accounted for in the reflected and transmitted beams. The fraction of the incident beam that is reflected can be computed from the Fresnel reflection equation.

If the interface is the surface of a polished metal, a large fraction (on the order of 0.9 ) of the incident radiant flux is reflected, and that portion that is transmitted is quickly absorbed by the metal. Essentially none of it is internally reflected. When the metal is roughened, the effective reflectance of the interface is reduced because of multiple reflections from the surface, and

1 J. C. Richmond and J. B. Steward, J. Am. Ceram. Soc. 42, 633 (1959). "Emittance" as used in that paper and this is defined as the ratio of flux emitted by a specimen to that emitted by a blackbody at the same temperature and under the same spectral and geometric conditions.

${ }^{2}$ J. C. Richmond and W. N. Harrison, J. Res. Natl. Bur. Stds. C66, 261 (1962).

R. E. Gannon and B. Linder, J. Am. Ceram. Soc. 47, 592 (1264) the transmitted portion of the incident flux is again absorbed by the metal. Hence the absorptance and, by Kirchhoff's law, the emittance, is markedly increased, as has been observed experimentally.

If the interface is the surface of a polished dielectric that has high internal scattering, the fraction of the incident flux that is reflected by the interface is small (on the order of 0.1 ), the portion that is transmitted is internally scattered by the dielectric, and most of it ultimately is reflected back through the interface. When the surface is roughened, the reflectance of the surface is reduced, but the flux that is not reflected by the surface is internally reflected within the specimen and back through the interface, and the net effect of the roughness on the reflectance, and, hence, on the emittance, is very small.

\section{MATHEMATICAL TREATMENT}

The case of the dielectric material can be treated mathematically. The reflectance $R$ for diffuse incidence of a metal specimen coated with a light-scattering dielectric coating was shown in a previous paper ${ }^{4}$ to be

$$
\begin{aligned}
& R=\rho_{\mathrm{e}}+\left(1-\rho_{\mathrm{e}}\right)\left(1-\rho_{\mathrm{i}}\right) \\
& \quad \times\left\{\left[(1-\beta) M e^{\sigma D}-(1+\beta) O e^{-\sigma D}\right] /\left(M N e^{\sigma D}-O P e^{-\sigma D}\right)\right\},
\end{aligned}
$$

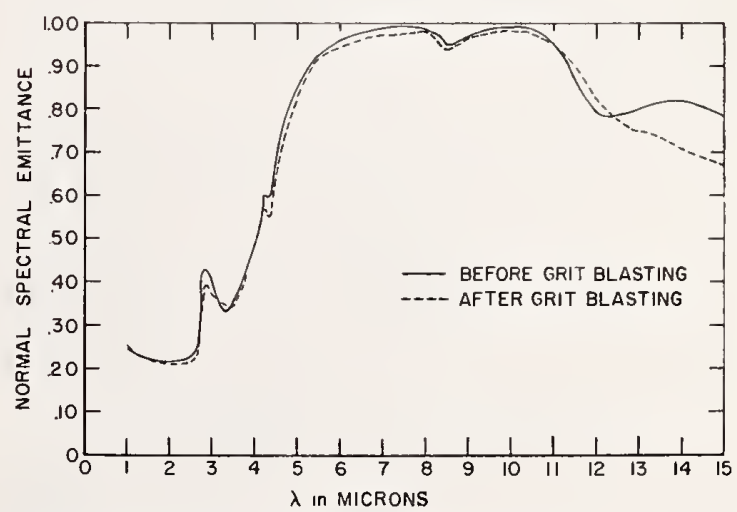

Fig. 1. Normal spectral emittance of a specimen of AD-5 alumina, measured at NBS by the rotating cylinder method. The effect of grit blasting is considered not significant at wavelengths below $12 \mu$.

' J. C. Richmond, J. Res. Natl. Bur. Stds. C67, 217 (1963). 
in which $\rho_{\mathrm{e}}$ is the external reflectance of the coating-air interface, $\rho_{\mathrm{i}}$ is the internal reflectance of the same interface, both for diffuse incident flux, $\beta=[\alpha /(\alpha+2 S)]^{\frac{3}{3}}$ where $\alpha$ is the absorption and $S$ is the backscattering coefficient of the coating material for diffuse flux, $e$ is the base of natural logarithms $\sigma=[\alpha(\alpha+2 S)]^{\frac{1}{3}}, D$ is coating thickness, and $N=(1+\beta)-\rho_{\mathrm{i}}(1-\beta) . M, O$, and $P$, which are defined in Ref. 4 , are eliminated in the following derivation and need not be defined here.

For a completely opaque coating, $\sigma D$ is very large, and $e^{-\sigma D} \cong 0$. Under these conditions, Eq. (1) reduces to

$$
\begin{aligned}
R=\rho_{\mathrm{e}} & +\left(1-\rho_{\mathrm{e}}\right) \\
& \times\left[(1-\beta)-\rho_{\mathrm{i}}(1-\beta)\right] /\left[(1+\beta)-\rho_{\mathrm{i}}(1-\beta)\right] .
\end{aligned}
$$

For a specimen with an optically smooth surface, $\rho_{\mathrm{e}}$ and $\rho_{\mathrm{i}}$ can be computed from the index of refraction from the Fresnel equation. For diffusely incident flux, the integrated Fresnel equation ${ }^{5}$ gives

$$
\begin{aligned}
\rho_{\mathrm{e}} & =\frac{1}{2}+\frac{(n-1)(3 n-1)}{6(n+1)^{2}}+\left[\frac{n^{2}\left(n^{2}-1\right)^{2}}{\left(n^{2}+1\right)^{3}}\right] \ln \frac{n-1}{n+1} \\
& -2 n^{3} \frac{\left(n^{2}+2 n-1\right)}{\left(n^{2}+1\right)\left(n^{4}-1\right)}+\left[\frac{8 n^{4}\left(n^{4}+1\right)}{\left(n^{2}+1\right)\left(n^{4}-1\right)^{2}}\right] \ln n,
\end{aligned}
$$

in which $n$ is the index of refraction. For metals, the complex index of refraction $n^{*}$ must be substituted for $n$. $\rho_{\theta}$ is related to $\rho_{\mathrm{i}}{ }^{5}$ by

$$
\rho_{\mathrm{i}}=1-1 / n^{2}+\rho_{\mathrm{e}} / n^{2}=1-\left(1-\rho_{\mathrm{e}}\right) / n^{2} .
$$

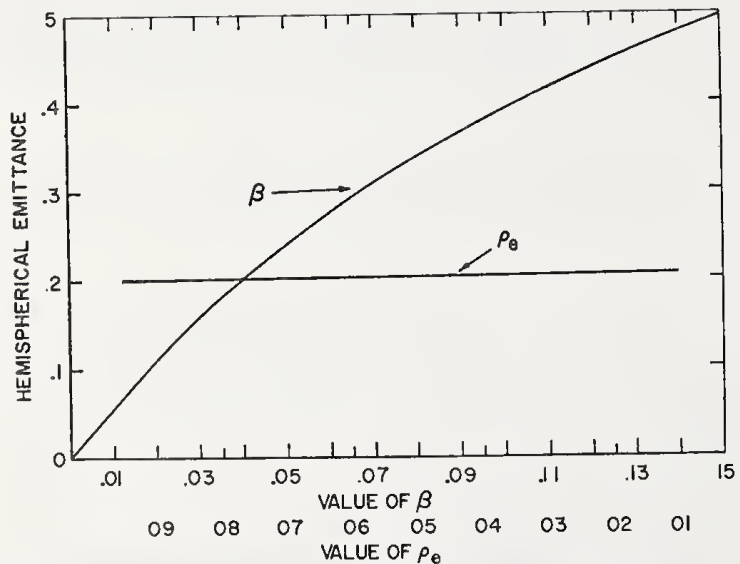

FIG. 2. Computed hemispherical emittance of a specimen of a material for which $n=1.76, \beta=0.04$, when $\rho_{\mathrm{e}}$ is varied, for and $\rho_{\mathrm{e}}=0.095$, when $\beta$ is varied, showing effect of variations in $\rho_{\mathrm{e}}$ and $\beta$.

${ }^{5}$ J. W. T Walsh, Dept. Sci. \& Ind. Res. Illumination Res. Tech. Paper No. 2 (London, England, 1926), p. 10.
If we substitute in Eq. (2) the value for $\rho_{\mathbf{i}}$ given in Eq. (4) we obtain

$$
\begin{aligned}
R=\rho_{\mathrm{e}}+ & \left(1-\rho_{\mathrm{e}}\right) \\
& \times\left(1-\rho_{\mathrm{e}}\right)(1-\beta) /\left[\left(1-\rho_{\mathrm{e}}\right)(1-\beta)+2 n^{2} \beta\right] .
\end{aligned}
$$

It can be seen from the form of Eq. (5) that small variations in $\rho_{\mathrm{e}}$ should not appreciably affect $R$. First, the equation has the form $R=\rho_{\mathrm{e}}+\left(1-\rho_{\mathrm{e}}\right) K$, which could be rewritten $R=K+\rho_{\mathrm{e}}(1-K)$. The term $K$ is the fraction in the second term of $\mathrm{Eq} .(5) ; \rho_{\Theta}$ appears as the term $\left(1-\rho_{\mathrm{e}}\right)$ in both numerator and denominator of this fraction, and since $\beta$ is usually less than 0.05 , and $\rho_{\mathrm{e}}$ is usually less than 0.1 , variations in $\rho_{\mathrm{e}}$ have only minor effects on the value of $K$. Variations in $\beta$, however, have large effects on the value of $K$.

Consider the case of polislied alumina, for which $n=1.76$ in the visible; $\beta$ varies with purity, grain size, porosity, processing, etc., but a representative value is 0.04. From Eq. (3), $\rho_{\mathrm{e}}=0.095$. Substituting these values in Eq. (5) gives $R=0.799$, from which the hemispherical emittance, $\epsilon_{\mathrm{H}}=0.201$. This agrees with the normal spectral value at about $2 \mu$ shown in Fig. 1 .

In the case of metals, all of the reflectance is due to surface or interface reflection. Data in Ref. 2 show that the total hemispherical emittance at $800^{\circ} \mathrm{C}$ of type 430 stainless steel was about 0.30 in the electropolished condition, and 0.65 in the sandblasted condition. The reflectance for diffusely incident flux from a blackbody at $800^{\circ} \mathrm{C}$ would thus be 0.70 for the polished and 0.35 for the sandblasted metal. Since $0.7^{3}=0.343$, we estimate that the effect of the sandblasting was to increase from one to about three the average number of reflections from the surface for each incident ray. If the effect of sandblasting the alumina was equivalent, then its surface reflectance would be reduced to $0.095^{3}=0.00086$.

Substituting this value in Eq. (5), and retaining the same values for $\beta$ and $n$, we get $R=0.796$ and $\epsilon_{\mathrm{H}}=0.204$. Thus, by this analysis, the increase in emittance expected to be produced by sandblasting is only 0.003 ; or about $1.5 \%$, which is too small to be evaluated in most measurements. The results of actual measurements indicate no significant change. See Fig. 1.

Figure 2 shows the effect of changes in $\rho_{\mathrm{e}}$ and $\beta$ on the computed hemispherical emittance of a material having $n=1.76, \beta=0.04$ when $\rho_{\mathrm{e}}$ is varied and $\rho_{\mathrm{e}}=0.095$ when $\beta$ is varied. Note that the emittance is quite sensitive to changes in $\beta$, but is quite insensitive to changes in $\rho_{\mathrm{e}}$. These calculations confirm the experimental results.

Reprinted from Journat, of the Optical Society of America, Vol. 56, No. 2, 253-254, February 1966 Printed in U. S. A. 


\title{
Thermal Radiation Properties of Ceramic Materials
}

\author{
Joseph C. Richmond \\ Institute for Basic Standards, \\ National Bureau of Standards, Washington, D.C. 20234
}

\begin{abstract}
The thermal radiation properties of materials are defined, and the basic physical laws governing the amount of thermal radiation emitted by a blackbody radiator, and its geometric and spectral distribution, are reviewed. Equations are given relating the thermal radiation properties of emittance, reflectance, transmittance, and absorptance to the optical properties of index of refraction, absorption coefficient, and scattering coefficient. The effect of impurities and processing variables such as grain size and porosity on the optical properties is discussed. The influence of thermal gradients normal to the surface of ceramic specimens on the observed thermal radiation properties is reviewed. Several methods of measuring thermal radiation properties of ceramic materials are discussed briefly, and representative normal spectral emittance data for alumina, thoria, magnesia, and zirconia are given.
\end{abstract}

Key Words: Alumina; ceramics; emittance; magnesia; reflectance; temperature coeffcient of emittance; thermal radiation properties; thoria, zirconia.

\section{Introduction}

Thermal radiation is electromagnetic radiation that is generated by the thermal motion of the particles of which a material is composed, such as molecules, atoms, ions, and electrons. All matter is constantly emitting thermal radiation, and in turn is constantly being irradiated by thermal radiation from its surroundings. Both processes occur simultaneously, and the net rate of heat transfer to or from a body is determined by the relative rates of the two processes.

The thermal radiation properties of a body are those properties that determine the rates of absorption and emission of thermal radiation, and hence the rates of heat transfer to or from the body by radiation. When thermal radiation is incident on a body, it is either reflected, absorbed or transmitted. Nothing else can happen to it. Hence, we can write

$$
\Phi_{r}+\Phi_{a}+\Phi_{t}=\Phi_{i}
$$

where $\Phi$ is flux, the time rate of flow of radiant energy, and the subscripts $r, a, t$ and $i$ designate the reflected, absorbed, transmitted, and incident flux, respectively. If we divide both sides of equation (1) by $\Phi_{i}$ and define the reflectance, $\rho$, as $\Phi_{r} / \Phi_{i}$, the absorptance, $\alpha$, as $\Phi_{a} / \Phi_{i}$ and the transmittance, $\tau$, as $\Phi_{t} / \Phi_{i}$, we get

$$
\rho+\alpha+\tau=1 \text {. }
$$

For an opaque specimen, $\tau=0$, hence

$$
\rho+\alpha=1 \text {. }
$$

Kirchhoff's law states that the absorptance, $\alpha$, is equal to the emittance, $\epsilon$,

$$
\alpha=\epsilon,
$$

hence, for an opaque body the reflectance and the emittance sum to one

$$
\rho+\epsilon=1 \text {. }
$$

We thus see that for an opaque body the thermal radiation properties are fully defined by either the emittance or the reflectance.

Fluorescent and phosphorescent materials absorb radiant energy at one wavelength and reemit it at another, usually longer, wavelength. Because of this behavior, they may appear to deviate from the above relationships when irradiated with heterochromatic flux, since at the wavelength of fluorescent or phosphorescent emission the reflected or transmitted flux may exceed the incident flux. For such materials it is necessary to measure the spectral reflectance or transmittance by a technique in which the specimen is irradiated by monochromatic flux, and the reflected or transmitted flux is measured by means of a detector that senses only flux of the same wavelength as the incident flux. The flux emitted by phosphorescent or fluorescent emission is not considered to be reflected flux.

\section{Definition of Terms}

Up to this point we have described thermal radiation, thermal radiation properties, and flux. It will be appropriate at this time to more rigor- 
ously define a number of additional terms that we shall be using. ${ }^{1}$

Reflectance, $\rho$, is the fraction of the incident flux that is reflected without change in frequency.

Absorptance, $\alpha$, is the fraction of the incident flux that is absorbed and converted to another form of energy.

Transmittance, $\tau$, is the fraction of the incident flux that is transmitted without change in frequency.

All of these properties are influenced by the wavelength, direction of incidence, and degree and direction of polarization relative to the plane of incidence of the incident flux, and, for reflectance and transmittance, by the size and direction of the solid angle over which the reflected or transmitted flux is collected for measurement. They may also vary somewhat with the temperature of the specimen. Hence, these terms are modified to indicate these conditions.

Spectral means at a given wavelength, or in a narrow wavelength band centered about a given wa velength.

Directional means in a given direction, usually measured from the normal to the surface.

Normal is a special case of directional, and means in a direction normal to the surface.

Specular, referring to reflectance, means in the direction of mirror reflection.

Diffuse, or hemispherical, referring to incident flux, means incident uniformly from all possible directions.

Hemispherical, referring to reflected, transmitted, or emitted flux, refers to flux summed over a hemisphere.

The terms reflectance, absorptance, and transmittance are defined as fractions of incident flux, and are dimensionless numbers. The numerical value, being a ratio, is independent of the magnitude of the incident flux, but for equations (2) and (3) to be valid, the conditions of irradiation must be the same for each term in the equation. This means that the spectral or wavelength composition and state of polarization of the incident flux and its direction of incidence must be the same for each term.

A blackbody radiator absorbs all radiant energy incident upon it, and emits radiant energy at the maximum possible rate for any body at its temperature. The amount of flux emitted by a blackbody radiator is given by the StefanBoltzmann equation, its spectral distribution by the Planck equation, and its geometric distribution by Lambert's cosine law. These are the fundamental laws of thermal radiation.

The Stefan-Boltsmann equation is

$$
M=\sigma T^{4}
$$

1 There has been much confusion in nomenclature in this area. Judd [1] has published a recommended system of nomenclature for reflectance terms. (Figures in brackets indicate the literature references at the end of this paper). where $M$ is the radiant exitance, or total flux emitted per unit surface area by a blackbody emitter in units of $\mathrm{Wm}^{-2}, \sigma$ is the StefanBoltzmann constant, $5.6697 \times 10^{-8} \mathrm{Wm}^{-2} \mathrm{~K}^{-4}$, and $T$ is the absolute temperature in kelvins.

Lambert's cosine law may be expressed as follows:

$$
I_{\theta}=I_{0} \cos \theta
$$

in which $I_{\theta}$ is the intensity per unit area in units of $W \mathrm{Wr}^{-1} \mathrm{~cm}^{-2}$ of a blackbody radiator in a direction $\theta, \theta$ being the angle between the given direction and the normal to the emitting surface, and $I_{0}$ is the intensity per unit area of the blackbody radiator normal to its surface.

Planck's radiation equation is

$$
M_{\lambda}=c_{1} \lambda^{-5}\left[\exp \left(c_{2} / \lambda T\right)-1\right]^{-1}
$$

where $M_{\lambda}$ is the spectral radiant exitance of a blackbody radiator per unit wavelength interval at wavelength $\lambda$, in units of watts per square meter and meter wavelength interval, $c_{1}$ is the first radiation constant, $3.7415 \times 10^{-16} \mathrm{Wm}^{2}, \lambda$ is the wavelength in meters, $c_{2}$ is the second radiation constant, $1.43879 \times 10^{-2} \mathrm{mK}$, and $T$ is the absolute temperature, in kelvins.

No real material is a true blackbody radiator; hence, for all real materials, the flux emitted is less than that emitted by a balckbody radiator. However, the radiation laws can still be used for real materials by using the emittance as a proportionality factor.

Emittance, $\epsilon$, is defined as the ratio of the radiant exitance of a specimen (radiant flux per unit area emitted) to that of a blackbody radiator at the same temperature and under the same conditions.

Emissivity is defined as the emittance of a specimen having an optically smooth surface and a thickness sufficient to be completely opaque, and is a property of the material of which the specimen is composed.

Emittance varies with the direction of emission relative to the surface of the specimen, the wavelength of the emitted radiation, and the temperature of the specimen. The emitted radiation may be elliptically polarized, particularly if emitted at large angles from the normal to the surface of the specimen. The terms directional, normal, hemispherical and spectral apply to emittance as well as to all of the other terms previously defined. The term total, referring to emittance, means at all wavelengths. This term has no specific meaning for reflectance, absorptance, and transmittance, unless the spectral distribution of the incident flux, or source, is specified. For emittance, however, it is only necessary to specify the temperature, since the spectral distribution of flux emitted by a blackbody radiator is given by the radiation laws as a function of temperature.

For Kirchhoff's law, equation (4), to be rigorously correct, ${ }^{2}$ it is necessary that the spectral

\footnotetext{
'See reference [2] for \& proof of Kirchhofl's law
} 
composition, degree of polarization, and direction of incidence of the incident flux for the absorptance evaluation should be the same as the spectral composition, degree of polarization, and direction of emission for the emitted flux in the emittance evaluation. This would appear to impose a severe restriction upon the general applicability of Kirchhoff's law. However, there are no polarization effects for normally incident flux, and the effects become of appreciable significance only for specularly reflecting materials at angles near the Brewster's angle. This point will be discussed in more detail later. For spectral incident flux the spectral distribution within the narrow wavelength interval approximates that of blackbody radiation sufficiently closely that the effect of the deviation is negligible. Hence equation (3) applies to normal spectral values of absorptance and emittance, and can be used to convert one to the other.

Normal spectral emittance over a wide wavelength range, at a given temperature, can be converted to total emittance at the same temperature by multiplying the spectral values, wavelength by wavelength, by the value of the Planck distribution function at the same wavelength to produce a curve of emitted flux as a function of wavelength. This curve is integrated to obtain the total emitted flux, which is then divided by the total integrated flux for a black body at the same temperature to obtain the total normal emittance. For most nonconducting materials, the emittance, either total or spectral, does not change appreciably with angle of emission at angles of emission less than about $50^{\circ}$ from the normal. For conductors such as metals, the effect begins to appear at angles greater than 10 to $15^{\circ}$ from the normal.

Spectral emittance and absorptance are not strong functions of temperature, hence values obtained at one temperature can be used to predict values at other temperatures with only small errors, if the difference in temperature is not great. Total emittance, on the other hand, may or may not vary appreciably with temperature depending upon the shape of the spectral emittance curve.

Figure 1 shows the variation with temperature of the total hemispherical emittance of Inconel coated with two different ceramic coatings, and figure 2 shows the variation with temperature of total hemispherical emittance of electropolished and of oxidized type 321 stainless steel. Figure 3 shows variation with temperature of normal spectral emittance of alumina, thoria, magnesia and zirconia.

Reflectance is usually easier to measure than absorptance, and spectral absorptance is frequently computed from spectral reflectance by use of equation (3). In this case, the reflectance must be measured under conditions of hemispherical viewing, and the resulting absorptance is for

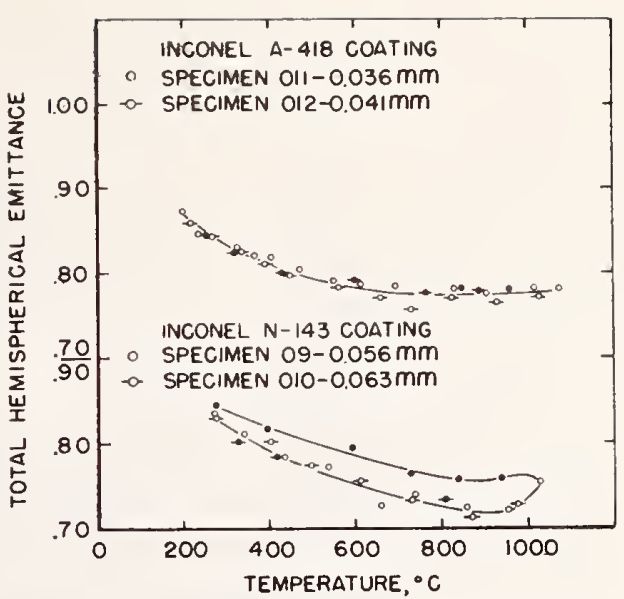

Figure 1. Total hemispherical emittance of composite specimens consisting of Inconel coated with $N B S$ coating $A-418$, top curve, and $N B S$ coating $N-143$, bottom curve.

open points represent values obtained during heating; closed points, during cooling.

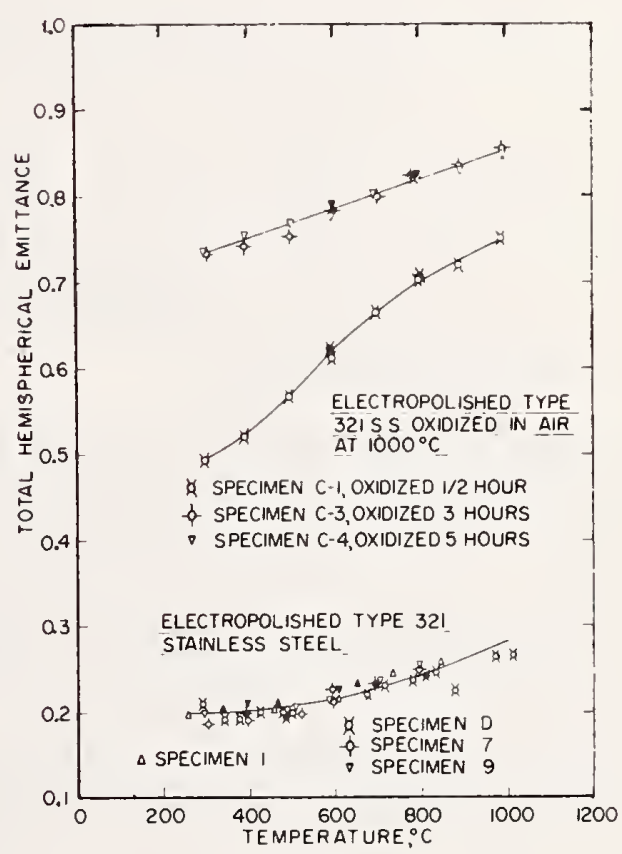

FIgURE 2. Total hemispherical emittance of type 321 stainless steel specimens in the electropolished condition, bottom curve, and after oxidation in air at $1000{ }^{\circ} \mathrm{C}$ for various times, top curves.

Open points represent values obtained during heating; closed points, during cooling.

the same conditions of irradiation as the reflectance.

Thermal radiation properties are frequently referred to as surface properties. This is obviously 


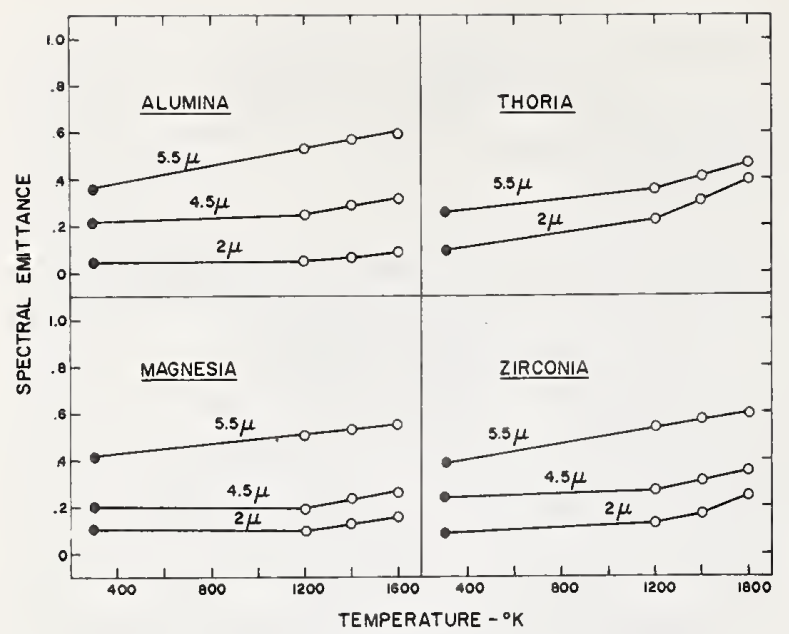

FIGURE 3. Temperature dependence of normal spectral emittance of four polycrystalline ceramic oxides.

a gross approximation, and is true only to the extent that the thermal radiation properties can be markedly affected by the surface condition of the specimen being measured, particularly its roughness. The processes of absorption and emission involve interaction of the electromagnetic waves with material particles, and hence cannot occur at a surface, which is only a twodimensional interface. While in one sense reflection occurs at an interface, even this process involves penetration of the incident electromagnetic waves beyond the interface to a depth that may be several times the wavelength.

The function of the surface, or interface, in determining thermal radiation properties is that it affects the amount and geometric distribution of the reflected flux, and the amount and distribution of the flux that penetrates the interface to where it can be absorbed and transmitted. It also affects the fraction of the internally generated flux that penetrates the interface and is emitted, and to a lesser extent, its geometric distribution. This will be discussed in more detail later.

Surface contamination, even in amounts that are difficult to detect by normal chemical means, can also significantly affect thermal radiation properties.

For metals, all of which have a very high absorption coefficient over most of the wavelength range of thermal radiation, the processes of emission, absorption and reflection occur in a very thin surface layer, perhaps only a few hundred atomic diameters in thickness. Hence for metals, it is a fair approximation to say that the thermal radiation properties are surface properties. Ceramic materials, on the other hand, usually have much lower absorption coefficients than do metals, at least at some wavelengths, and for such materials the thermal radiation properties must be considered to be volume properties.
The thermal radiation properties of a specimen can be affected by the contour of its surface. The specimen will have the highest reflectance or the lowest emittance if its surface is completely smooth. Increasing surface roughness will decrease reflectance and increase emittance. In the case of a metal, the effect can be large. For instance the total hemispherical emittance of type 321 stainless steel at $400^{\circ} \mathrm{C}$ increased from about 0.2 in the electropolished condition to about 0.55 when sandblasted [3]. The normal spectral emittance of polycrystalline ceramics, on the other hand, has been shown to be nearly independent of surface roughness [4].

Bennett [5] has derived the following equation relating reflectance of a metal at normal incidence to surface roughness:

$$
\rho / \rho_{0}=\exp -(4 \pi \sigma / \lambda)^{2}+32 \pi^{4}(\sigma / \lambda)^{4}(\Delta \alpha / m)^{2},
$$

in which $\rho$ is the reflectance of a rough metal surface for normally incident flux reflected into an optical system having an acceptance solid angle with a half angle $\Delta \alpha(\Delta \alpha=\pi / 2$ for hemispherical collection), $\rho_{0}$ is the reflectance of a perfectly smooth surface of the same metal under the same conditions, $\sigma$ is the root-mean-square (rms) roughness of the surface, $\lambda$ is the wavelength of the incident radiation, and $m$ is the rms slope of the surface.

The first term, $\exp -(4 \pi \sigma / \lambda)^{2}$, accounts for interference effects, and predominates when $\sigma / \lambda$ $<<1.0$. The second term accounts for geometric effects, and predominates when $\sigma / \lambda>>1.0$.

The treatment becomes almost hopelessly complex at oblique incidence, because of polarization effects. If such effects are neglected, equation (9) can still be used by substituting for $\sigma$ the term $\sigma$ $\cos \theta$, where $\theta$ is the angle of incidence, measured from the normal to the gross plane of the surface.

\section{Wavelength Range of Interest}

The spectral distribution of the radiance (the flux per unit wavelength interval, unit solid angle and unit area of source projected normal to the direction of emission) of a blackbody radiator is given by the Planck equation:

$$
\begin{aligned}
L_{\lambda}=1.19096 \lambda^{-5} & \\
\times & {[\exp (1.43879 / \lambda T)-1]^{-1} \times 10^{-16}, }
\end{aligned}
$$

where $L_{\lambda}$ is the radiance in watts per square meter, steradian, and meter wavelength interval $\left(\mathrm{Wm}^{-2} \mathrm{sr}^{-1}\right) \mathrm{m}^{-1}, \lambda$ is wavelength in meters and $T$ is temperature in kelvins. A plot of $L_{\lambda}$ as a function of $\lambda$ for a blackbody at $1000 \mathrm{~K}$ is shown in figure 4.

The nature of equation (10) is such that there is some energy emitted at all wavelengths between zero and infinity, but the amount at very short and very long wavelengths is so small that it can be neglected. At any temperature, 99 percent of 


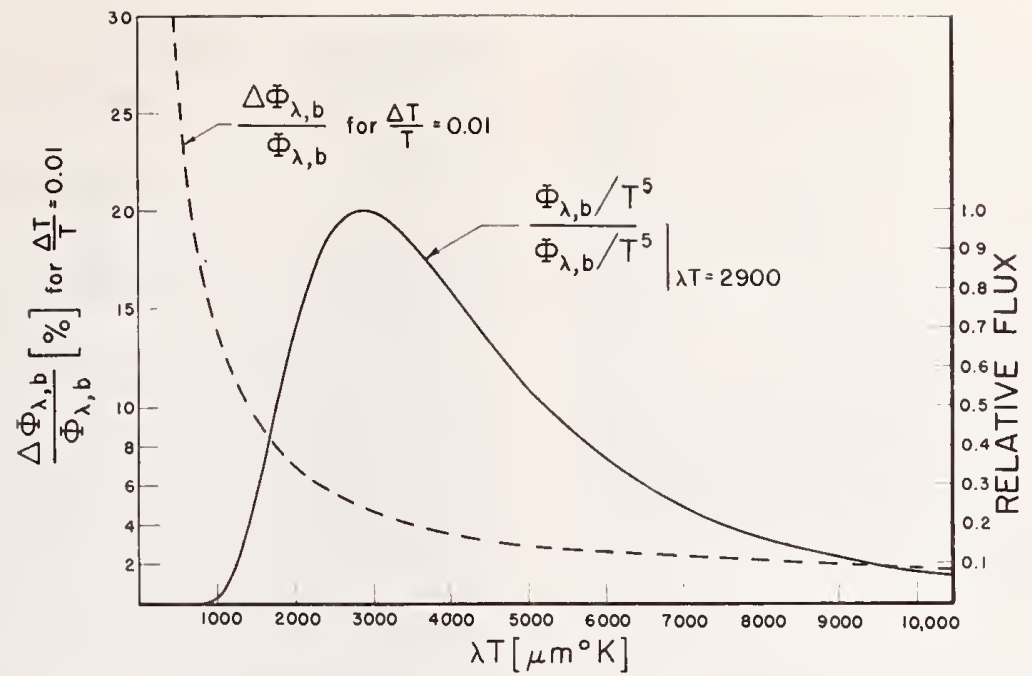

FIGURE 4. Relative spectral radiance of a blackbody radiator, plotted as a function of $\lambda \mathrm{T}$ in $\mathrm{cmK}$, curve $A$, and percentage increase in spectral radiance produced by $a$ one percent increase in temperature, plotted as a function of $\lambda \mathrm{T}$ in $\mathrm{cm} K$, curve $B$.

the energy is emitted between wavelengths represented by values of $\lambda T$ of 1322 and $29372 \mu \mathrm{mK}$, and about 25 percent of the energy is at wavelengths shorter than the peak. At room temperature $(300 \mathrm{~K}) 99$ percent of the energy is at wavelengths between 4.4 and $97.9 \mu \mathrm{m}$. As a practical matter, most determinations of thermal radiation properties cover the wavelength range of 1 to $15 \mu \mathrm{m}$, with others covering the extended range from about 0.25 to $40 \mu \mathrm{m}$. There is considerable interest in spectral emittance at 0.65 $\mu \mathrm{m}$, the wavelength often used in optical pyrometry. Most of our discussion will be confined to the 1 - to $15-\mu \mathrm{m}$ range. Figure 5 shows how the wavelength limits for 99 percent of the radiant energy change with temperature.

\section{Theoretical Considerations}

When two transparent media of different indices of refraction are separated by an optically smooth interface, a beam of radiation striking the inter. face will be refracted according to Snell's law, and reflected according to Fresnel's law. Snell's law is expressed as:

$$
n_{1} \sin \theta_{i}=n_{2} \sin \theta_{r},
$$

where $n_{1}$ is the index of refraction of the medium from which the beam is incident, $\theta_{i}$ is the angle of incidence, measured from the normal to the interface, $n_{2}$ is the index of refraction of the medium into which the beam is refracted, and $\theta_{T}$ is the angle of refraction, again measured from the normal to the surface. The equation is valid over the entire range of $\theta_{1}$ when $n_{2}>n_{1}$. When $n_{1}>n_{2}$, the equation is still valid at values of $\theta_{1}$ less than the critical angle. At angles greater than the critical angle, total internal reflection will occur. The critical angle, $\theta_{c}$, is defined by the equation

$$
\theta_{c}=\sin ^{-1}\left(n_{2} / n_{1}\right) \text {. }
$$

Fresnel's law, in its general form, is expressed as:

$$
\begin{aligned}
& \rho=1 / 2\left[\frac{n_{1} \cos \theta_{1}-n_{2} \cos \theta_{r}}{n_{1} \cos \theta_{i}+n_{2} \cos \theta_{r}}\right]^{2} \\
& +1 / 2\left[\frac{n_{2} \cos \theta_{i}-n_{1} \cos \theta_{r}}{n_{2} \cos \theta_{i}+n_{1} \cos \theta_{r}}\right]^{2}
\end{aligned}
$$

where $\rho$ is the reflectance of the interface for unpolarized incident flux. Equation (13) can be reduced, by substituting for $n_{1}$ or $n_{2}$ from equation (11), to

$$
\rho=1 / 2\left[\frac{\sin ^{2}\left(\theta_{i}-\theta_{\tau}\right)}{\sin ^{2}\left(\theta_{i}+\theta_{\tau}\right)}\right]+1 / 2\left[\frac{\tan ^{2}\left(\theta_{i}-\theta_{\tau}\right)}{\tan ^{2}\left(\theta_{i}+\theta_{\tau}\right)}\right]
$$

In equations (13) and (14) the first term gives the reflectance for radiation polarized perpendicular to the plane of incidence, and the second term that for radiation polarized parallel to the plane of incidence. The place of incidence is the plane containing the direction of incidence and the normal to the interface (surface). There is some disagreement in the literature as to what constitutes the plane of polarization. In this paper it is defined as the plane containing the $\mathbf{E}$ (electric) vector.

It can be seem from equation (14) that when $\left(\theta_{i}+\theta_{\tau}\right)=\pi / 2, \tan \left(\theta_{i}+\theta_{\tau}\right)$ goes to infinity, and the reflectance for radiation polarized parallel to the plane of incidence goes to zero. The angle at 

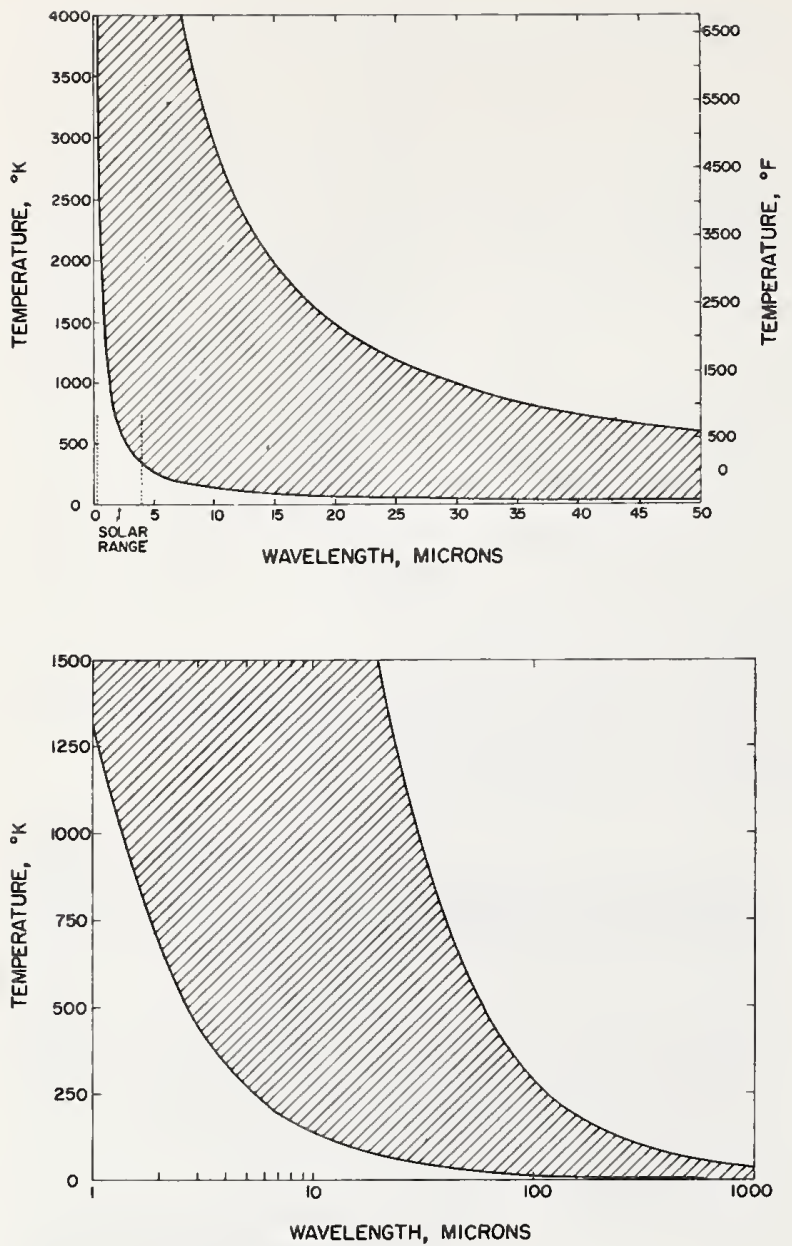

FIGURE 5. Wavelength limits encompassing 99 percent of the flux emitted by a blackbody radiator at the indicated temperature, for high temperatures, top curve; and low temperatures, bottom curve.

which this occurs is known as the Brewster's angle, $\theta_{B}$, and is defined as:

$$
\theta_{B}=\tan ^{-1} n_{2} / n_{1}
$$

Equations (11) through (15) were derived for a perfect dielectric that is optically homogeneous. Such a material has no absorption. Although no real material conforms completely to this model, the equations can be applied with no significant error to transparent dielectric materials in general. For materials of which the absorption coefficient is not negligible, the complex index of refraction, $n^{*}$, must be substituted for the simple index of refraction, $n$, in the equations.

$$
n^{*}=n+i k
$$

where $i=\sqrt{ }-1$, and $k$ is the absorption index.

$$
k=a \lambda / 4 \pi
$$

where $a$ is the absorption coefficient, and $\lambda$ is wavelength.

The absorption coefficient, $a$, is defined by the equation

$$
\Phi_{x}=\Phi_{0} \exp (-a x)
$$

where $\Phi_{x}$ is the flux remaining after traversing a thickness $x$ of nonscattering material, and $\Phi_{0}$ is the flix at $x=0$.

Let us assume that we have an optically homogeneous specimen of a dielectric material, having two parallel faces, $A$ and $B$, that are optically smooth and essentially infinite in extent, so that edge effects can be neglected. We will further assume that interference effects can be neglected. This is a reasonable model for a sheet of polished plate glass, or a single crystal of quartz or sodium chloride, for instance. When a beam of radiation is incident on surface $A$ of such a specimen, it is partly reflected and partly refracted, according to the Fresnel equation (13) or (14). The radiation that is refracted is transmitted undeviated until it hits the second surface, $B$, where it again undergoes reflection and refraction according to the Fresnel equation. In its course through the material, it is partially absorbed according to equation (18). By following the beam until it is completely absorbed or has left the specimen, we can compute the reflectance, transmittance, and absorptance of the specimen. All of the flux initially reflected by surface $A$ plus that leaving the specimen through surface $A$ makes up the reflected portion, that leaving through surface $B$ makes up the transmitted portion, and that absorbed makes up the absorbed portion. These portions divided by the incident flux are the reflectance, transmittance, and absorptance.

Gardon [6] gives a very complete account of the emittance of isothermal transparent materials. He derives a spectral volume emissive power, $j_{\lambda}$, defined by the equation:

$$
j_{\lambda}=a_{\lambda} n^{2} M_{B, \lambda} \pi^{-1}
$$

where $M_{B, \lambda}$ is the spectral exitance of a blackbody radiator at the temperature of the specimen. $M_{B, \lambda}$ can be obtained from the Planck equation. His final equation for the spectral radiance on the inside of the surface, $L_{i \lambda} \varphi_{X}$, at wavelength $\lambda$ and angle $\varphi$ for a specimen of thickness $X$ is:

$$
L_{\mathrm{I} \lambda \mathrm{X}}=n_{2} \frac{M_{B}, \lambda}{\pi}[1-\exp (-a X / \cos \varphi) \cos \varphi],
$$

and for the internal spectral irradiance on the inside of the surface, $E_{i \lambda x}$, at wavelength $\lambda$ for a specimen of thickness $X$,

$E_{\imath \lambda \mathrm{X}}=n^{2} M_{b, \lambda}\left[2-\exp \left(-a_{\lambda} X\right)-\left(a_{\lambda} X\right)^{2}-\xi_{1}\left(-a_{\lambda} X\right)\right]$ 
where the exponential integral $\xi_{t}(-p)$ is defined as $\xi_{i}(-p)=\int_{p}^{\infty} e^{-q} q^{-1} d q$. The flux that is incident internally on the surface of the specimen undergoes reflection and refraction. All of the flux that is incident at angles greater than the critical angle is totally reflected. For a specimen thick enough to be opaque, this is a fraction $1-1 / n^{2}$ of the total flux. For a transparent specimen, the fraction is larger. The critical angle, $\theta_{c}$, is defined by equation (12). The fraction of the internally incident flux that is incident at angles less than $\theta_{c}$ is refracted to fill the entire hemisphere on the external side of the surface, and the fraction transmitted at any angle can be computed from equation (14) by setting $\theta_{r}$ as the internal angle of incidence and $\theta_{i}$ as the external angle of refraction.

For transparent birefringent single crystals each ray appears as two plane polarized components with their planes of polarization normal to each other, and the fraction of each component is determined by the orientation of the ray relative to the crystal axes of the specimen. For such a specimen the index of refraction will be different for the two portions of the ray, and because the two portions are plane polarized, the two parts of equation (14) must be used separately. As a further complication, unless the plane of incidence happens to include a crystallographic axis of the crystal, neither of the planes of polarization will be in the plane of incidence. The situation where there is a thermal gradient normal to the surface of the specimen has been treated in a second paper by Gardon [7].

For an optically inhomogeneous specimen, the situation becomes so complex that Gardon's approach is no longer valid. A single ray undergoes so many internal reflections and refractions that it soon loses its identity. Practically all polycrystalline materials fall into the category of intensely scattering materials, which have been treated by Kubelka [8] and by Hamaker [9]. The scattering occurs by reflection and refraction whenever the index of refraction of the material changes along the path of the ray, primarily at pores, grain boundaries, or inclusions. For materials of this type, an entirely different mathematical treatment is required.

Consider a specimen of very large area, so that edge effects may be neglected, that is irradiated diffusely over its entire area. Under steady-state conditions, the incident flux not reflected at the surface will penetrate into the specimen, diminishing with the depth of penetration; the amount of the diminution at any point along a plane parallel to the surface will be a function only of the distance of the plane from the surface. Under these conditions a one-dimensional analysis can be used. All flux lost by lateral scattering will be compensated by an equal gain through similar scattering from the surrounding area.
The flux is separated into two portions, $I$, traveling outward from the interior of the specimen, and $J$, traveling inward from the surface, both traveling normal to the plane of the surface. Both are attenuated by scattering and absorption. A diffuse absorption coefficient, $K$, is defined by equating $K I d x$ to the reduction in $I$ by absorption within an infinitesimal layer $d x$. It should be noted that the diffuse absorption coefficient, $K$, is exactly twice the absorption coefficient, $a$, referred to previously. A diffuse backscattering coefficient $S$ is similarly defined by equating $S I d x$ to the flux scattered backwards from $I$ and added to $J$ in the layer $d x$.

Within the distance $d x$ the flux $I$ will be diminished by absorption and backscattering, and reinforced by the flux backscattered by $J$. Hence we can write:

$$
\begin{aligned}
& d I / d x=-(K+S) I+S J, \text { and } \\
& d J / d x=(K+S) J-S I .
\end{aligned}
$$

These are the basic differential equations used by Kubelka [8], Hamaker [9], and others.

Klein [10] derives the following equations for those materials where there is essentially no specular reflection at the surface. These equations should apply to most unglazed ceramic specimens, particularly if the surface is not polished:

$$
\begin{aligned}
& \rho=\frac{\left(1-\beta_{0}{ }^{2}\right) \sinh \sigma_{0} D}{\left(1+\beta^{2}\right) \sinh \sigma_{0} D+2 \beta_{0} \cosh \sigma_{0} D} \\
& \alpha=\frac{2 \beta_{0}\left(B_{0} \sinh \sigma_{0} D+\cosh \sigma_{0} D-1\right)}{\left(1+\beta^{2}\right) \sinh \sigma_{0} D+2 \beta_{0} \cosh \sigma_{0} D} \\
& \tau=\frac{2 \beta_{0}}{\left(1+\beta_{0}{ }^{2}\right) \sinh \sigma_{0} D+2 \beta_{0} \cosh \sigma_{0} D}
\end{aligned}
$$

in which $\beta_{0}=[K /(K+2 S)]^{1 / 2}$,

$$
\sigma_{0}=[K(\dot{K}+2 S)]^{1 / 2} \text {, and }
$$

$D=$ thickness of specimen.

Equation (24) has been further developed [11] to include the effects of external specular reflectance of the specimen and internal specular reflection at both surfaces of the specimen. The final equation is :

$$
\begin{aligned}
\rho(2 \pi ; 2 \pi)= & \rho_{e}+\left(1-\rho_{e}\right)\left(1-\rho_{\imath}\right) \\
& \times \frac{\left(1-\beta_{0}\right) M e^{\sigma_{0} D}-\left(1+\beta_{0}\right) O e^{-\sigma_{0} D}}{M N e^{\sigma_{0} D}-O P e^{-\sigma_{0} D}}
\end{aligned}
$$

where $\rho(2 \pi ; 2 \pi)$ is the hemispherical reflectance of the specimen for completely diffuse irradiation, $\rho_{e}$ is the specular reflectance of the specimen front surface for externally incident diffuse flux, $\rho_{1}$ is the specular reflectance of the specimen front surface for internally incident diffuse flux, $\rho_{s}$ is 
the specular reflectance of the specimen back surface for internally incident diffuse flux, and

$$
\begin{gathered}
M=\left(1+\beta_{0}\right)-\rho_{s}\left(1-\beta_{0}\right), \\
N=\left(1+\beta_{0}\right)-\rho_{\imath}\left(1-\beta_{0}\right), \\
O=\left(1-\beta_{0}\right)-\rho_{s}\left(1+\beta_{0}\right), \\
P=\left(1-\beta_{0}\right)-\rho_{\imath}\left(1+\beta_{0}\right) .
\end{gathered}
$$

For a specimen thick enough to be completely opaque, equation.(27) reduces to:

$$
\rho(2 \pi ; 2 \pi)=\rho_{e}+\frac{\left(1-\rho_{e}\right)\left(1-\rho_{i}\right)\left(1-\beta_{0}\right)}{\left(1+\beta_{0}\right)-\rho_{i}\left(1-\beta_{0}\right)}
$$

and for such a specimen:

$$
\epsilon(2 \pi)=1-\rho(2 \pi ; 2 \pi)
$$

which states that the hemispherical emittance is equal to one minus the hemispherical reflectance for diffuse irradiation.

The absorption coefficient, either $a$ or $K$, is primarily a function of composition, and is usually not greatly affected by the structure. However, for some impurities, the effect on the absorption coefficient will depend on whether they are present in solution in the crystals of the primary constituents or as separate crystallites at grain boundaries, and if present as crystallites, by their size. The scattering coefficient, on the other hand, is markedly affected by the structure of the specimen, particularly the grain size and porosity.

A few years ago we observed an interesting example of the effect of the form in which impurities are present on the thermal radiation properties of a ceramic material. We are making some measurements on sintered magnesium oxide. The material as received was a dirty brown color, which we assumed was due to contamination by iron oxide. A specimen was treated with hydrochloric acid in an effort to remove the contaminant, then washed and heated with a torch to drive off any remaining chlorides. After this treatment the specimen was white, and we assumed that the contaminant had been removed. However, when the specimen was heated in a furnace for measurement of its emittance, it regained its dirty brown color on cooling to room temperature. We then discovered that the color change was reversible. When the specimen was cooled rapidly from a high temperature, it was white; when cooled slowly, it was dirty brown. The explanation offered was that the impurity, presumably iron oxide, went into solution in the $\mathrm{MgO}$ crystals at high temperature, and remained in solution on ripid cooling, where it had little effect on reflectance. On slow cooling, the impurity precipitated out at grain boundaries, where it had an appreciable effect on reflectance.
Typical values of $\beta_{0}, \sigma_{0}, S$ and $K$ are as follows. For a highly opaque white porcelain enamel with a reflectivity of about $0.9, \beta_{0}=0.05$ and $\sigma_{0}=50$ $\mathrm{mm}^{-1}, K=2.5 \mathrm{~mm}^{-1}$ and $S=500 \mathrm{~mm}^{-1}$. For a polycrystalline ceramic with a reflectivity of about 0.82 that is essentially opaque at a thickness of $3 \mathrm{~mm}$, the values would be $\beta_{0}=0.1$, $\sigma_{0}=1.67 \mathrm{~mm}^{-1}, K=0.167 \mathrm{~mm}^{-1}$ and $S=8.25$ $\mathrm{mm}^{-1}$. For a material with a reflectivity of about 0.6 that is essentially opaque at a thickness of $25 \mathrm{~mm}, \beta_{0}=0.25, \sigma_{0}=0.2 \mathrm{~mm}^{-1}, K=0.05 \mathrm{~mm}^{-1}$ and $S=0.375 \mathrm{~mm}^{-1}$. A material such as an opalescent glass might have these properties. In general, $K$ can vary from near zero for materials composed of highly transparent crystals, to a value as high as $50,000 \mathrm{~mm}^{-1}$ for some metals at some wavelengths. $S$ will vary from near zero for an optically homogeneous material such as an optical glass or a pure metal to a high of perhaps $1000 \mathrm{~mm}^{-1}$ for some white paints. It should be emphasized that for light-scattering materials the reflectivity is related to $\beta_{0}$, which is a function of the ratio of $K$ to $S$, and not to the absolute value of either $K$ or $S$, while the extinction coefficient, which determines the opacity of a material, is related to the product $S K$.

The usual laws of reflection and refraction at an interface between two materials of different indices of refraction, equations (14) and (11) above, apply only if there is a finite thickness of material on each side of the interface. This finite thickness is of the same order of magnitude as the wavelength of the radiation involved. The phenonemon can be explained quantitatively in terms of frustrated internal reflectance [12]. If two $45^{\circ}$ totally reflecting prisms are placed so that their totally reflecting surfaces are parallel, and are then brought slowly together, the totally reflecting surfaces will begin to tranşmit as they approach each other. The transmission for polarized radiation with its electric vector in the plane of incidence is given by

$$
\frac{\rho}{\tau}=\frac{\left(N^{2}-1\right)^{2}}{N^{2}\left(N^{2}-2\right)} \sinh ^{2}\left[\pi \frac{\sqrt{2 d}}{\lambda} \sqrt{N^{2}-2}\right]
$$

where $\rho$ is the reflectance of the interface, $\tau$ is its transmittance $(\rho+\tau=1), N$ is the index of refraction ratio, $d$ is the distance separating the prisms and $\lambda$ is the wavelength of the radiation involved. For these conditions, $\rho / \tau=3 / 4$ that given in equation (30) when the electric vector is normal to the plane of incidence. The index of refraction ratio is $N=n_{1} / n_{2}$, where $n_{1}$ is the index of the prisms and $n_{2}$ is that of the layer. When the layer is vacuum, $N=n_{1}$. When it is air, $N=n_{1}$ with an error of about 3 parts in 10,000 .

Because of this phenomenon, there is a marked effect of the size of particles such as inclusions, crystallites, or pores on the scattering efficiency of each particle. The scattering efficiency per particle will decrease markedly with diameter 
below a critical diameter. As a rough approximation, the total scattering power will be proportional to the total interface area, and from this standpoint the scattering particles should be as small as possible. The net result of the two effects is that there is an optimum size for scattering efficiency. A study of rutile pigments in paint [13] has shown that in this case the optimum diameter of the pigment particles is about 0.4 times the wavelength of the light being scattered.

The scattering power of a particle diminishes as the distance separating it from its nearest neighbors becomes less than the critical distance mentioned above. On this basis the maximum scattering power per particle would occur when particles of the optimum size are separated by a distance at least equal to their diameter. If spherical particles were arranged in a cubic array, this would occur at volume concentrations of particles of less than about 6.6 percent. Actually the total scattering for all particles increases as the number of particles increases, because of the increase in total interface area, until the particles get so closely packed that the decrease in effective interface area is greater than the increase in total interface area. Reference [13] indicates that for the rutile pigment studied, the maximum total scattering occurred at a pigment volume concentration of about 25 percent.

Radiant heat transfer within and from optically inhomogeneous materials with no specular reflection at the surface is covered by Hamaker [9] and Klein [10], for the case where there is a thermal gradient in the material.

\section{Thermal Gradient Problems}

The general physical properties of ceramic materials combine to make thermal gradients a serious problem in the evaluation and use of thermal radiation properties of ceramics. Ceramic materials in general tend to be somewhat translucent, and hence emit and absorb thermal radiation within a surface layer of appreciable thickness. Ceramic materials in general also have low thermal conductivity and high total emittance as compared to metals. These properties combine to produce thermal gradients within a heated specimen unless careful precautions are taken to minimize such gradients. The gradients tend to be normal to a surface that is emitting or absorbing thermal radiation. As a further complication, the gradients tend to be nonlinear near such a surface.

When a specimen is emitting from a surface layer of appreciable thickness with a large thermal gradient normal to the surface, it has no unique temperature, and it is difficult to define an effective temperature for the emitting layer. Emittance is defined as the ratio of the flux emitted by a specimen to that emitted by a blackbody radiator at the same temperature and under the same conditions. It is thus necessary to define an effective temperature for the nonisothermal specimen before its emittance can be evaluated. If the effective temperature is defined as that of the surface, a specimen with a positive thermal gradient (surface cooler than interior) will emit at a greater rate than an isothermal specimen at the same temperature, and in some cases may even have an "emittance" greater than 1.0. If the gradient is negative (surface hotter than interior) it will emit at a lesser rate. If the "effective temperature" is defined as that of an isothermal specimen that emits at the same rate as the nonisothermal specimen, we find that the "effective temperature" is difficult to evaluate even if the extinction coefficient and thermal gradient are accurately known. Also, if spectral emittance is desired, we find that the extinction coefficient, and hence the thickness of the emitting layer, varies with wavelength, and we have the anomolous situation of a specimen whose effective temperature varies with wavelength. There is no completely satisfactory solution to this problem. The Planck equation does not apply to a nonisothermal specimen, and any attempt to apply it to such specimens will result in errors. Because of these considerations, all measurements of emittance of ceramic specimens should be made under conditions where thermal gradients within the specimen are as small as possible.

\section{Methods of Measurement}

The properties of reflectance, absorptance, and transmittance for most materials, and particularly ceramic materials, are not strong functions of temperature, and hence these properties can be measured on nonisothermal specimens with but little error. However, such measurements are not easy to make on hot specimens. First, all of the reflected or transmitted flux must be measured, which involves collection over a hemisphere. Such measurements are difficult at wavelengths beyond about $2.5 \mu \mathrm{m}$, where conventional integrating sphere coatings decrease markedly in reflectance. Second, the small amount of reflected or transmitted flux must be measured in the presence of a much larger amount of flux emitted by the hot specimen. Such measurements require the use of chopped incident flux and synchronous amplification of the signal from the detector.

Because of the difficulties mentioned above, most measurements of thermal radiation properties of ceramic materials have been direct emittance measurements, where the flux emitted by a hot specimen is measured and compared to that emitted by a blackbody reference at the same temperature.

Any temperature difference between the specimen and reference blackbody will cause an error in the measured emittance that is much larger than the percentage difference in absolute temperature. For total emittance measurements, the percentage error in emittance is approximately four times the percentage difference in tempera- 
ture. This is illustrated in figure 4 for a one percent difference in temperature. As seen in figure 4, curve $B$, the error in spectral emittance can be much larger at wavelengths shorter than the peak in the Planck curve, A.

In most cases where direct emittance measurements of ceramic specimens have been made, an effort has been made to heat the specimen under conditions where the thermal gradients are kept as small as possible. This may involve alternate heating and viewing of a moving specimen. The specimen is enclosed in a furnace where it is heated then moved in front of a cooled port where it is viewed briefly. It may also involve alternate heating by radiation and viewing of a stationary specimen, where the periods of heating and viewing are controlled by rotating shutters. Movable specimens have been tested in the form of a rotating disc $[14,15,16,17]$, a rotating cylinder $[18,19]$, or as discs mounted on an oscillating beam [20]. Stationary specimens have been heated in an arcimage furnace $[21,22,23,24]$ and in a solar furnace [25].

In the case of a moving specimen that is alternately heated and cooled, the specimen will reach a steady-rate temperature, and if the motion is rapid enough that the viewing time is short, the temperature fluctuation of the viewed surface will be so small that it can be neglected.

Photon conduction does not generate a thermal gradient in a material. The photons pass through the material without affecting it in any way until they are absorbed. As a result, a specimen that is heated by radiation tends to have a small thermal gradient normal to the heated surface. The radiation is absorbed within the surface layer from which the emitted radiation originates, and after the specimen has reached a steady state condition, there is little phonon conduction within the surface layer. If the time periods during which the specimen is alternately heated and viewed are brief, the temperature fluctuation of the specimen will be small. Usually these periods are only a fraction of a second. With both moving specimens and radiantly heated stationary specimens the thermal gradients normal to the surface can be reduced to a point where they do not introduce a significant error into the measurement.

The rotating cylinder method used at the $\mathrm{Na}-$ tional Bureau of Standards [18] will be described briefly. The specimen was a hollow cylinder $25 \mathrm{~mm}$ (one inch) in diameter and $25 \mathrm{~mm}$ (one inch) high, with an outer surface ground to be round to about $.05 \mathrm{~mm}(0.002 \mathrm{in})$. A cross sectional view of the furnace is shown in figure 6 . The specimen was mounted on top of an alumina pedestal, which revolved inside a platinum-wound resistance furnace. The winding of the furnace was designed so that there was no axial thermal gradient along the specimen. The specimen revolved in front of a water-cooled viewing port. A theoretical analysis [26], confirmed by temperature measurements at a point near the surface of a rotating specimen, indicated (1) that the temperature drop at a point on the surface of the rotating specimen as it passed in front of the viewing port was inversely proportional to the speed of rotation of the specimen, and was only about $2 \mathrm{~K}$ at a speed of $50 \mathrm{rmp}$, and (2) that the temperature measured by a stationary, radiation-shielded, thermocouple located at the center of the hollow rotating specimen was the same within about $1 K$ as that measured by the thermocouple imbedded near the surface of the rotating specimen.

The flux from the hot specimen was focused on the entrance slit of one beam of a double beam infrared spectrometer, and the flux from a laboratory blackbody furnace was focused on the entrance slit for the second beam of the spectrometer. The blackbody furnace was controlled to the same temperature as the specimen. The spectrometer automatically scanned over the spectral range of 1 to $15 \mu \mathrm{m}$, and plotted the ratio of the signals from the two beams, which after correction for spectrometer errors, was the normal spectral emittance of the specimen.

Typical data at $1400 \mathrm{~K}$ for alumina, thoria, magnesia and zirconia are shown in figures $7,8,9$, and 10 . The curves are similar in several respects. The emittance is low at short wavelengths, where the absorption coefficient for these materials is low, and high at long wavelengths, where the absorption coefficient is high. The location of the absorption edge, the steep part of the curve connecting the high and low portion, and its slope, is different for different materials. The height of the low-emittance and high-emittance portions of the curve is also different for the different materials. The effect of temperature on the spectral emittance at several wavelengths for these materials is shown in figure 3. In general, the spectral emittance increases with an increase in temperature, and the percentage increase is greater at short wavelengths, where the emittance is low, than at long wavelengths, where the emittance is high. The absorption edge also tends to move toward a shorter wavelength as the temperature is increased, but the change is not large.

\section{Summary}

The terminology in the field of thermal radiation properties and radiant heat transfer has been reviewed, and the equations relating the thermal radiation properties of ceramics to their optical properties of index of refraction, absorption coefficient, and scattering coefficient have been given. The problems involved in measuring the thermal radiation properties of ceramic materials have been discussed, one specific method has been briefly described, and data on alumina, thoria, magnesia, and zirconia presented. 


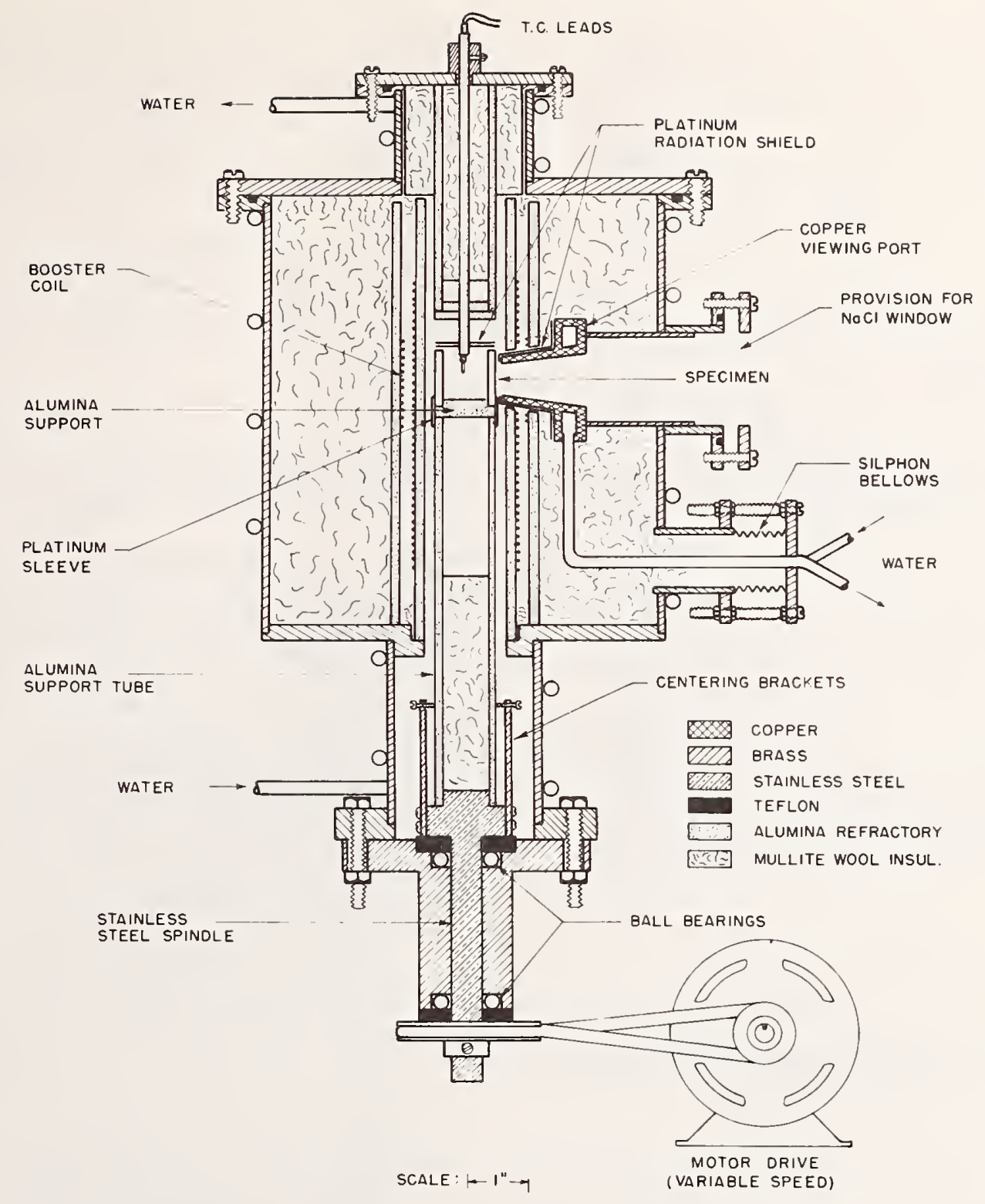

Figdre 6. Cross-sectional view of the rotating cylinder specimen furnace used in measuring normal spectral emittance of ceramic materials. 


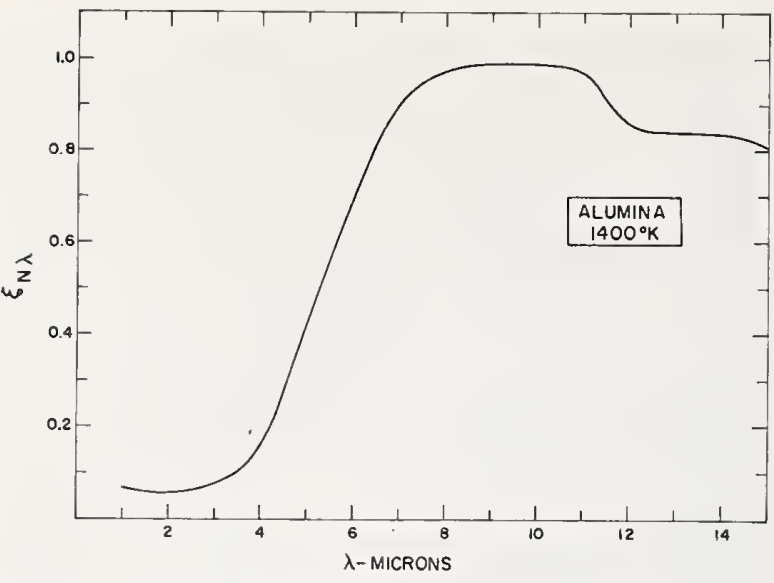

FIGURE 7. Normal spectral emittance of commercially pure alumina at $1400 \mathrm{~K}$.

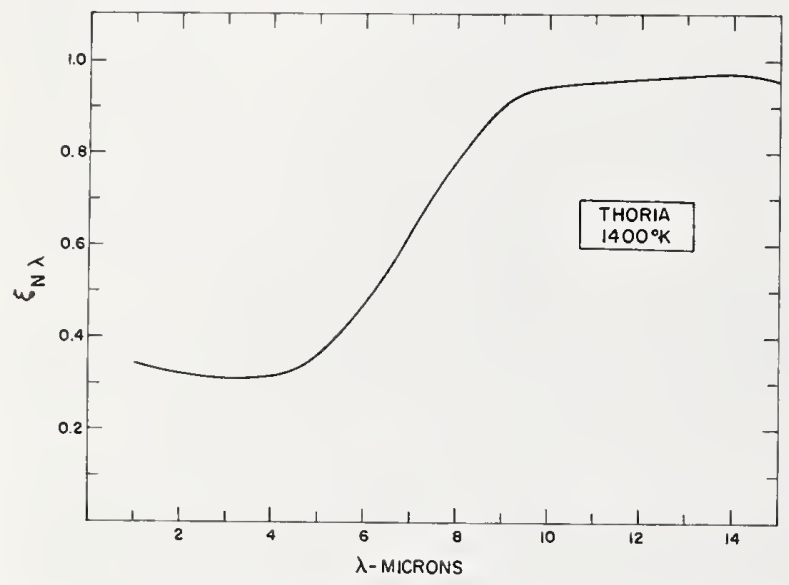

Figdre 8. Normal spectral emittance of commercially pure thoria at $1400 K$.

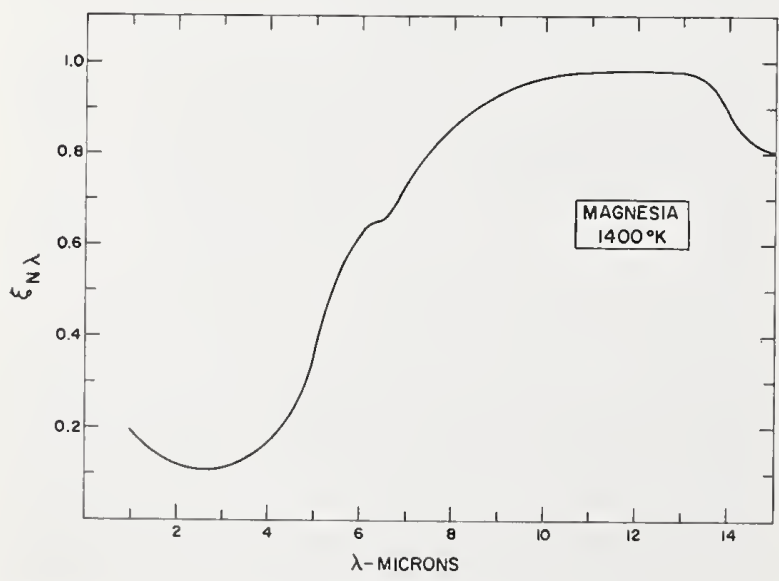

FIGURE 9. Normal spectral emittance of commercially pure magnesia at $1400 K$.

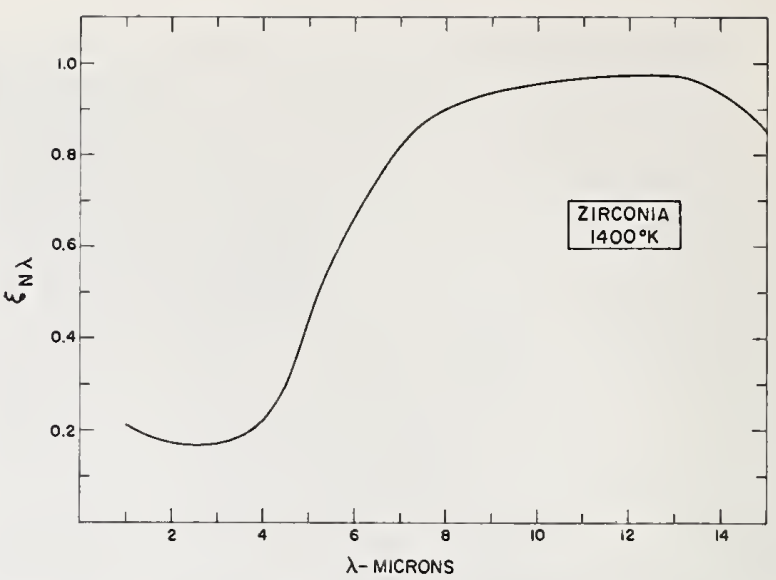

FIGURE 10. Normal spectral emittance of commercially pure zirconia at $1400 K$.

\section{References}

[1] Judd, D. B., Terms, definitions, and symbols in reflectometry, J. Opt. Soc. Am. 5\%, No. 4, 545-552 (April 1967).

[2] Kneissl, G. J., and Richmond, J. C., A laser-source integrating sphere reflectometer, NBS Tech. Note $439,1968$.

[3] Richmond, J. C., and Harrison, W. N., Total hemispherical emittance of coated and uncoated Inconel and types 321 and 430 stainless steel, J. Res. NBS 66C (Engineering and Instrumentation), No. 3, 261-269 (1962).

[4] Richmond, J. C., Effect of surface roughness on emittance of nonmetals, J. Opt. Soc. Am. 56, No. 2, 253-254 (Feb. 1966).

[5] Bennett, H. E., Influence of surface roughness, surface damage, and oxide films on emittance, Proceedings of Symposium on Thermal Radiation of Solids, San Francisco, 1964, ed. by S. Katzoff (NASA Special Publication SP-55, Washington, Superintendent of Documents, GPO 1965) pp. 145152.

[6] Gardon, R., The emissivity of transparent materials, J. Am. Ceram. Soc. 39, No. 8, 278-287 (August 1956).

[7] Gardon, R., A review of radiant heat transfer in glass, J. Am. Ceram. Soc. 44, No. 7, 305-312 (July 1961).

[8] Kubelka, P., New contributions to the optics of intensely light-scattering materials, J. Opt. Soc. Am. 38, No. 5, 448-457 (May 1948).

[9] Hamaker, H. C., Radiation and heat conduction in light scattering materials, Philips Research Reports 2, 55-67, 103-111, 112-125, 420-425 (1947).

[10] Klein, J. D., Heat transfer, by radiation, in powders, Doctoral Dissertation, Massachusetts Institute of Technology (1960) Available from University Microfilms.

[11] Richmond, J. C., Relation of emittance to other optical properties, J. Res. NBS 67C (Engin eering and Instrumentation), No. 3, 217-226 (1963).

[12] Strong, John, Concepts of Classical Optics, p. 124 (W. H. Freeman and Co., San Francisco, 1958).

[13] Bruehlman, R. J., Thomas, L. W., and Gomek, E., Effect of particle size and pigment volume concentration on hiding power of titanium dioxide, Federation of Societies for Paint Technology Official Digest, 33, No. 433, 252-267 (Feb. 1961).

[14] Slemp, W. S., and Wade, W. R., A method for measuring the spectral normal emittance in air of a variety of materials having stable emittance characteristics, Proceedings of Symposium on Measurement of Thermal Radiation Properties of Solids, Dayton, 
O., 1962, ed. by J. C. Richmond (NASA Special Publication SP-31, Washington, Superintendent of Documents, GPO, 1963) pp. 433-439.

[15] Clayton, W. A., A $500^{\circ}$ to $4500^{\circ} \mathrm{F}$ thermal radiation test facility for transparent materials, Proceedings of Symposium on Measurement of Thermal Radiation Properties of Solids, Dayton, O., 1962, ed. bv J. C. Richmond (NASA Special Publication SP-31, Washington, Superintendent of Documents, GPO, 1963) pp. 445-460.

[16] Kjelby, A. S., Emittance measurement capability for temperatures up to $3000^{\circ} \mathrm{F}$, Proceedings of Symposium on Measurement of Thermal Radiation Properties of Solids, Dayton, O., 1962, ed. by J. C. Richmond (NASA Special Publication SP-31, Washington, Superintendent of Documents, GPO, 1963).

[17] McMahon, H. O., Thermal radiation characteristics of some glasses, J. Am. Ceram. Soc. 34 No. 3, 91-96 (1951).

[18] Clark, H. E., and Moore, D. G., A rotating cylinder method for measuring normal spectral emittance of ceramic oxide specimens from $1200^{\circ}$ to $1600^{\circ} \mathrm{K}$, J. Res. NBS roA (Physics and Chemistry), No. 5, 393-415 (1966).

[19] Folweiler, R. C., Thermal radiation characteristics of transparent, semi-transparent and translucent material under non-isothermal conditions, Air Force Systems Command, Wright-Patterson Air Force Base, Aeronautical Systems Division Technical Documentary Report ASD-TDR-62-719 (April, 1964).

[20] Betz, H. T. Olson, O. H., Schurin, B. D., and Morris, J. C., Determination of emissivlty and reflectivity data on aircraft structural materials, Part II, Techniques for measurement of total normal emissivity, solar absorptivity and presentation of results, Wright Air Development Center Technical Report TR 56-222 Part II (Oct. 1958).

[21] Comstock, D. F., Jr., A radiation technique for determining the emittance of refractory oxides Proceedings of Symposium on Measurement of
Thermal Radiation Properties of Solids, Dayton O., 1962, ed. by J. C. Richmond (NASA Special Publication SP-31, Washington, Superintendent of Documents, GPO, 1963) pp. 461-468.

[22] Evans, R. J., Clayton, W. A. and Fries, M. A very rapid $3000^{\circ} \mathrm{F}$ technique for measuring emittance of opaque solid materials, Proceedings of Symposium on Measurement of Thermal Radiation Properties of Solids, Dayton, O., 1962, ed. by J. C. Richmond (NASA Special Publication SP-31, Washington, Superintendent of Documents, GPO, 1963) pp. $483-488$.

[23] Null, M. R., and Lozier, W. W., Measurement of reflectance and emittance at high temperature with a carbon arc image furnace, Proceedings of Symposium on Measurement of Thermal Radiation Properties of Solids, Dayton, O., 1962, ed. by J. C. Richmond (NASA Special Publication SP-31, Washington, Superintendent of Documents, GPO, 1963) pp. 535-539.

[24] Wilson, R. G., Hemispherical spectral emittance of ablation chars, carbon and zirconia (to $3700^{\circ} \mathrm{K}$ ), Proceedings of Symposium on Thermal Radiation of Solids, San Francisco, 1964, ed. by S. Katzoff (NASA Special Publication SP-55, Washington, Superintendent of Documents, GPO, 1965) pp. $259-275$.

[25] Laszlo, T. S., Gannon, R. E. and Sheehan, P. J., Emittance measurements of solids above $2000^{\circ} \mathrm{C}$, Proceedings of Symposium on Thermal Radiation of Solids, San Francisco, 1964, ed. by S. Katroff, (NASA Special Publication SP-55, Washington, Superintendent of Documents, GPO, 1965) pp. $277-286$.

[26] Peavy, B. A., and Eubanks, A. G., Periodic heat flow in a hollow cylinder rotating in a furnace with a viewing port, Proceedings of Symposium on Measurement of Thermal Radiation Properties of Solids, Dayton, O., 1962, ed. by J. C. Richmond, (NASA Special Publication SP-31, Washington, Superintendent of Documents, GPO, 1965) pp. $553-563$. 


\title{
Relation of Emittance to Other Optical Properties *
}

\section{J. C. Richmond}

(March 4, 1963)

\begin{abstract}
An equation was derived relating the normal spectral emittance of an optically inhomogeneous, partially transmitting coating applied over an opaque substrate to the thickness and optical properties of the coating and the reflectance of the substrate at the coatingsubstrate interface.
\end{abstract}

\section{Introduction}

The $45^{\circ}$ to $0^{\circ}$ luminous daylight reflectance of a composite specimen comprising a partially transparent, spectrally nonselective, light-scattering coating applied to a completely opaque (nontransmitting) substrate, can be computed from the reflectance of the substrate, the thickness of the coating and the reflectivity and coefficient of scatter of the coating material. The equations derived for these conditions have been found by experience $[1,2]^{1}$ to be of considerable practical usefulness, even though several factors are known to exist in real materials that were not considered in the derivation. For instance, porcelain enamels and glossy paints have significant specular reflectance at the coating-air interface, and no real coating is truly spectrally nonselective.

The optical properties of a material vary with wavelength, but in the derivation of the equations referred to above, they are considered to be independent of wavelength over the rather narrow wavelength band encompassing visible light. The condition of spectral nonselectivity is met sufficiently well by a number of materials to permit use of the pertinent equations with but a single set of optical properties for visible light. Over the enormously wider range of wavelengths within which the emission and absorption of radiant energy are important, few if any materials are sufficiently nonselective to permit use of the pertinent equations with a single set of optical properties applicable to all wavelengths. Hence the optical properties as a function of wavelength are required for computation of wide-range spectral reflectance or emittance of such composite specimens.

The present study was undertaken to derive an equation relating the spectral reflectance (or emittance) of a composite specimen comprised of a partially transmitting, light-scattering coating applied to a completely opaque (nontransmitting) substrate, from the thickness of the coating and the spectral optical properties of the coating and substrate.

\section{Review of the Literature}

The relationship between the thickness and reflectance of a layer of light-scattering materials as a function of the optical constants of the material was developed by Kubelka and Munk [3]. Judd [1] developed graphical methods for the solution of the Kubelka-Munk equation, and demonstrated that it was useful in studying real materials which departed somewhat from the ideal material postulated in development of the equation. Kubelka [4] derived more exact equations in a form that was capable of relatively easy solution. In all of these cases, the materials considered were assumed to be spectrally nonselective, so that a single value of each optical constant could be used throughout the visible range of wavelengths.

Gardon [5] considered the case of an optically homogeneous material with a three-dimensional analysis. Hamaker [6] and Kilein [7] worked with powders, which may be considered as a special case of optically inhomogeneous (light-scattering) materials, and derived equations for computing heat transfer by radiation in powders. In these cases a single value applicable to total blackbody radiation was used for each optical property.

- This work was sponsored and financed by the George C. Marshall Spaee Flight Center of NASA.

1 Figures in brackets indiente the literature references at the end of this paper. 
Saunderson [9] worked with partially transmitting plastics, and used equations for transmittance and reflectance derived by Kubelka and Munk [3], that are similar but not identical to those of Klein [7]. However, he did incorporate a correction for the specular reflection at the plastic-air interface.

\section{Derivation of Equation}

Because of the relationships

$$
A+T+R=1
$$

in which $A=$ absorptance, $T=$ transmittance, and $R=$ reflectance, and Kirchoff's law,

$$
A=E
$$

in which $E$ is emittance, ${ }^{2}$ it is possible to compute emittance from reflectance for completely opaque specimens. This method will therefore be used, because of its simplicity as compared to the approacl used by Gardon [5].

Consider a flux of completely diffuse radiant energy, incident in uniform geometric distribution over the entire area of an optically flat layer of inhomogeneous isotropic dielectric material having infinite area and uniform thickness. Upon attainment of a steady state condition, the incident flux that is not reflected at the surface will penetrate into the material, diminishing with the depth of penetration, the amount of diminution at any internal plane parallel to the surface being a function only of the distance of the plane from the surface.

Attenuation of the inward-bound diffuse flux will result from absorption of radiant energy within the specimen, and from backscattering by the dispersed particles in the material. The backscattered flux will proceed as completely diffuse flux propagated in a direction normal to the surface outward through the coating. In both cases, the radiant flux lost by lateral scattering will be compensated by an equal gain through similar scattering from adjoining portions of the specimen. The outward-bound flux will also be attenuated by absorption and backscattering. This backscattered flux will reinforce the incoming flux.

Under the postulated conditions, a one-dimensional mathematical analysis can describe the variation in diffuse radiant flux density along a line normal to the surface, due to absorption and scattering within the material, as a function of distance from the coating-substrate interface.

In using the one-dimensional analysis, the diffuse radiant flux traversing unit area normal to the direction of propagation is considered as being made up of two directionally opposed portions, one $I$, outward from the interior of the specimen and normal to the flat surface, and the other $J$, in the opposite inward direction. A spectral absorption coefficient, $K$, is defined by equating $K I d x$ to the reduction in $I$ by absorption within a layer of infinitesimal thickness, $d x$. A scattering coefficient, $S$, is similarly defined by equating $S I d x$ to the flux scattered backwards from $I$ in the unidirectional beam (and therefore included in $J$ ) within a layer of infinitesimal thickness, $d x$.

Within the distance $d x$ the flux, $I$, will be not only diminished by absorption and scattering, but also augmented by the backscattering from $J$. Hence we can write

$$
\begin{aligned}
& d I / d x=-(K+S) I+S J \\
& d d J / d x=(K+S) J-S I .
\end{aligned}
$$

These are the general differential equations first used by Kubelka and Munk [3] as a starting point, and subsequently by many other investigators.

2 Emittance, as used in this paper, is defined as follows: Emittance is a property of a specimen; it is the ratio of its emissive power to that of a blackbody radiator at the same temperature and under the same spectral and geometric conditions of viewing.

Emissive power is the rate of thermal emission expressed as radiant flux per unit surface area. 
Hamaker's [6] solutions for eqs (3) and (4), considering both absorption and scattering, are

$$
\begin{aligned}
& I=L_{1}(1-\beta) e^{\sigma x}+L_{2}(1+\beta) e^{-\sigma x} \\
& J=L_{1}(1+\beta) e^{\sigma x}+L_{2}(1-\beta) e^{-\sigma x}
\end{aligned}
$$

where $L_{1}$ and $L_{2}$ are constants determined by the boundary conditions, and where

and

$$
\sigma=\sqrt{K(K+2 S)}
$$

$$
\beta=\sqrt{K /(K+2 S)} .
$$

\subsection{Equations Applicable to Coatings}

Most coatings, such as paint, porcelain enamel, and ceramic coatings, unlike the materials postulated by Kubelka [4] and dealt with by Hamaker [6] and Klein [7], have a disperse medium other than air, usually of a glassy nature, in which the scattering particles are distributed. Hence specular reflectance at the coating-air and coating-substrate interfaces must be considered. This structural difference will give different boundary conditions than were considered by previous authors.

In the systems under consideration, the reflectance at the interfaces will be considered, as follows:

$\rho_{e}=$ specular reflectance at the coating-air interface for externally incident, completely diffuse radiant flux $\left(J_{e}\right)$.

$\rho_{s}=$ reflectance of the substrate for completely diffuse radiant flux $\left(J_{0}\right)$ incident upon the substrate from the coating.

$\rho_{i}=$ specular reflectance of the coating-air interface for completely diffuse radiant flux $\left(I_{D}\right)$ incident from within the coating.

Let $x$ be the perpendicular distance from the coating-substrate interface to a point in the coating, and $D$ be the thickness of the coating.

If only the disposition of incident flux be considered, and if radiant energy emitted by the specimen be ignored, the boundary conditions for substitution in eqs (5) and (6) are

$$
\begin{gathered}
I=J_{o} \rho_{s} \text { at } x=0 \\
J=J_{e}\left(1-\rho_{e}\right)+I_{D} \rho_{i} \text { at } x=D
\end{gathered}
$$

when $x=0, e^{\sigma x}=e^{-\sigma x}=1$, hence from eqs (5), (6), and (9)

$$
L_{1}(1-\beta)+L_{2}(1+\beta)=\rho_{s}\left[L_{1}(1+\beta)+L_{2}(1-\beta)\right],
$$

and from eqs (5), (6), and (10),

$$
L_{1}(1+\beta) e^{\sigma D}+L_{2}(1-\beta) e^{-\sigma D}=J_{e}\left(1-\rho_{e}\right)+\rho_{i}\left[L_{1}(1-\beta) e^{\sigma D}+L_{2}(1+\beta) e^{-\sigma D}\right] .
$$

${ }^{3}$ It may be worth while to mention the physical significance of $\sigma$ and $\beta$. As can be secn in eq (7), $\sigma$ is a type of extinction coefficient. If $S=0$, $\sigma=K$; if $K=0, \sigma=0$. The combination $\sigma x$ is a dimensionless optical thickness; all layers having the same $\sigma x$ product will produce the same attenuation in a bcam of radiant energy. $\sigma$ is dctermined by the absolutc values of $S$ and $K$. $\beta$, on the other hand, is a function of the relative magnitudcs of $S$ and $K$. If $S=0, \beta=1$, and if $K=0, \beta=0 . \beta$ is shown by Klein [7] to be rclated to emissivity, $E_{\infty}$, and reflcctirity, $R_{\infty}$, by the equations

$$
E_{\infty}=\frac{2 \beta}{1+\beta} \text { and } R_{\infty}=\frac{1-\beta}{1+\beta},
$$

provided there is no specular reflection at the surface. For glossy materials, these relationships are modificd by specular reflection at the surface. 
Solving eqs (11) and (12) for $L_{1}$ and $L_{2}$, we get:

$$
\begin{gathered}
L_{1}=\frac{J_{e}\left(1-\rho_{e}\right)\left[(1+\beta)-\rho_{s}(1-\beta)\right]}{\left[(1+\beta)-\rho_{i}(1-\beta)\right]\left[(1+\beta)-\rho_{s}(1-\beta)\right] e^{\sigma D}-\left[(1-\beta)-\rho_{i}(1+\beta)\right]\left[(1-\beta)-\rho_{s}(1+\beta)\right] e^{-\sigma D}} \\
L_{2}=\frac{-J_{e}\left(1-\rho_{e}\right)\left[(1-\beta)-\rho_{s}(1+\beta)\right]}{\left[(1+\beta)-\rho_{i}(1-\beta)\right]\left[(1+\beta)-\rho_{s}(1-\beta)\right] e^{\sigma D}-\left[(1-\beta)-\rho_{i}(1+\beta)\right]\left[(1-\beta)-\rho_{s}(1+\beta)\right] e^{-\sigma D}} .
\end{gathered}
$$

The equations can be simplified by making the following substitutions:

Let

$$
\begin{aligned}
M & =(1+\beta)-\rho_{s}(1-\beta) \\
N & =(1+\beta)-\rho_{i}(1-\beta) \\
O & =(1-\beta)-\rho_{s}(1+\beta) \\
P & =(1-\beta)-\rho_{i}(1+\beta) .
\end{aligned}
$$

Then eqs (13) and (14) become

$$
\begin{aligned}
& L_{1}=\frac{J_{e}\left(1-\rho_{e}\right) M}{M N e^{\sigma D}-O P e^{-\sigma D}} \\
& L_{2}=\frac{-J_{e}\left(1-\rho_{e}\right) O}{M N e^{\sigma D}-O P e^{-\sigma D}} .
\end{aligned}
$$

The overall specular plus diffuse reflectance, $R$, of the specimen is defined as the reflected flux divided by the incident flux. The reflected flux will include the fraction of $J_{e}$ that is specularly reflected at the coating-air interface. That is

$$
R=\frac{J_{e} \rho_{e}+\left(1-\rho_{i}\right) I_{D}}{J_{e}}
$$

From eqs (5), (19), and (20),

$$
I_{D}=\frac{J_{e}\left(1-\rho_{e}\right)\left[(1-\beta) M e^{\sigma D}-(1+\beta) O e^{-\sigma D}\right]}{M N e^{\sigma D}-O P e^{-\sigma D}},
$$

hence

$$
R=\rho_{e}+\left(1-\rho_{e}\right)\left(1-\rho_{i}\right) \frac{(1-\beta) M e^{\sigma D}-(1+\beta) O e^{-\sigma D}}{M N e^{\sigma D}-O P e^{-\sigma D}}
$$

$R$ in eq (23) is the reflectance of the composite specimen under conditions of completely diffuse illumination and hemispherical viewing. The equation has been written in terms of the coating parameters $\beta, \sigma, \rho_{e}, \rho_{i}, \rho_{s}$, and the thickness of the coating, $D$. The values of these parameters vary with wavelength. The equation applies only to spectral reflectance, $R_{\lambda}$, under the given geometric conditions, and spectral values of the coating and coatingsubstrate parameters, $\beta_{\lambda}, \sigma_{\lambda}, \rho_{e \lambda}, \rho_{i \lambda}$, and $\rho_{s \lambda}$ are required. The subscript $\lambda$ indicates that the symbol applies to the spectral value at wavelength $\lambda$.

When transmittance is zero, as it is in the case of the coated specimens under consideration, the emittance is equal to one minus the reflectance, as is indicated by eqs (1) and (2), if consistent geometric conditions of incident, reflected, and emitted flux are used. $R$ has been defined as the spectral reflectance under conditions of diffuse illumination and hemispherical viewing. The emittance corresponding to $R$ is $E_{H}$, the hemispherical spectral emittance. Hence we can write

$$
E_{H}=1-\left\{\rho_{e}+\left(1-\rho_{e}\right)\left(1-\rho_{i}\right) \frac{(1-\beta) M e^{\sigma D}-(1+\beta) O e^{-\sigma D}}{M N e^{\sigma D}-O P e^{-\sigma D}}\right\}
$$


It is legitimate also to apply eqs (1) and (2) to evaluate the normal spectral emittance, $E_{N}$, if, and only if, the geometric conditions of irradiation and viewing under which the external reflectance, $\rho_{e}$, is measured are (1) normal incidence and hemispherical viewing or (2) perfectly diffuse incidence and normal viewing.

If we consider that the internal reflectance of the coating plus substrate, neglecting the effect of specular reflectance at the coating-air interface, is $R_{i}$, then with diffuse illumination and hemispherical viewing

$$
R=\rho_{e}+\left(1-\rho_{e}\right) R_{1}\left(1-\rho_{i}\right) .
$$

Equation (25) is another way of writing eq (24), with $R_{i}$ replacing the fraction containing exponential terms.

$$
R_{i}=\frac{(1-\beta) M e^{\sigma D}+(1+\beta) O e^{-\sigma D}}{M N e^{\sigma D}-O P e^{-\sigma D}} .
$$

For normal illumination and hemispherical viewing, the equation becomes

$$
R_{N}=\rho_{N}+\left(1-\rho_{N}\right) R_{i}\left(1-\rho_{i}\right)
$$

in which $R_{i}$ is again the internal reflectance, neglecting the effect of specular reflectance at the coating-air interface, of the coating plus substrate. If $R_{i}$ is the same for normal and diffuse incident flux, ${ }^{4}$ we can write

$$
E_{N}=1-\left\{\rho_{N}+\left(1-\rho_{N}\right)\left(1-\rho_{i}\right) \frac{(1-\beta) M e^{\sigma D}-(1+\beta) O e^{-\sigma D}}{M N e^{\sigma D}-O P e^{-\sigma D}}\right\}
$$

which is the desired equation.

Substituting for $M, N, O$, and $P$ from eqs (15), (16), (17), and (18), and making use of the relationship

and

$$
2 \sinh x=e^{x}-e^{-x}
$$

eq (28) reduces to

$$
2 \cosh x=e^{x}+e^{-x}
$$

$$
E_{N}=\left(1-\rho_{N}\right)\left\{1-\left(1-\rho_{i}\right) \frac{\left[\left(1-\rho_{s}\right)-\beta^{2}\left(1+\rho_{s}\right)\right] \sinh \sigma D+2 \rho_{s} \beta \cosh \sigma D}{\left[\left(1+\beta^{2}\right)\left(1+\rho_{i} \rho_{s}\right)-\left(1-\beta^{2}\right)\left(\rho_{i}+\rho_{s}\right)\right] \sinh \sigma D+2 \beta\left(1-\rho_{i} \rho_{s}\right) \cosh \sigma D}\right\} .
$$

The specular reffectances at the coating-air interface, $\rho_{e}$ and $\rho_{i}$, are for diffuse illumination and hemispherical viewing. The value to be used for $\rho_{s}$ must be determined experimentally, because the coating-substrate interface is normally rough, and the reflectance may be affected by interaction between coating and substrate at the interface. For an optically smooth interface, which may be approximated by the coating-air interface for some coatings at some wavelengths, $\rho_{e}$ and $\rho_{i}$ can be computed from the index of refraction of the coating ${ }^{5}$ by integration of the Fresnel equation over a hemisphere. In its general form, the equation for dielectrics can be written

$$
2 \rho_{d}=\frac{\sin ^{2}(\varphi-\theta)}{\sin ^{2}(\varphi+\theta)}+\frac{\tan ^{2}(\varphi-\theta)}{\tan ^{2}(\varphi+\theta)}
$$

in which $\rho_{d}$ is the directional specular reflectance for unpolarized parallel flux, $\phi$ is the angle of incidence, and $\theta$ is the angle of refraction, both angles measured from the normal to the interface. The angles $\phi$ and $\theta$ are related to index of refraction, $n$, by Snell's law. In its most general form, this is $n_{1} \sin \phi=n_{2} \sin \theta$, in which $n_{1}$ is the index of refraction of the medium on the

\footnotetext{
4 This condition will be approximated if the incident fux is completely diffused after traversing a small thickness of the coating.

3 Most optically inhomogeneous coatings consist of opacif ying particles dispersed in a continuous medium or vehicle. The scat tering is due to the difference in indices of refraction of the opaeifier and vehicle. For glosş coatings, the opacifying particles are not usually exposed at the surface of the coating, and the effective index of refraction for specular reflection is that of the vehicle.
} 
side of the interface from which the flux is incident, and $n_{2}$ is that on the opposite side of the interface. In the case of flux incident from vacuum, $n_{1}=1$. In the case of air, no serious error is introduced by considering $n_{1}=1 \quad\left(n_{1}=1.0003\right.$, approximately, for air under normal conditions).

The reflectance of an optically smooth surface of index of refraction $n$, for completely diffuse flux incident from vacuum (or air) can be expressed as

$$
\rho_{e}=\frac{\int_{0}^{\pi / 2} \sin \varphi \cos \varphi\left\{\frac{\sin ^{2}(\varphi-\theta)}{\sin ^{2}(\varphi+\theta)}+\frac{\tan ^{2}(\varphi-\theta)}{\tan ^{2}(\varphi+\theta)}\right\} d \varphi}{2 \int_{0}^{\pi / 2} \sin \varphi \cos \varphi d \varphi}
$$

This expression has been integrated by Walsh [8] to give

$$
\begin{aligned}
\rho_{e}=\frac{1}{2}+\frac{(n-1)(3 n-1)}{6(n+1)^{2}}+\left\{\frac{n^{2}\left(n^{2}-1\right)^{2}}{\left(n^{2}+1\right)^{3}}\right\} \ln \frac{(n-1)}{(n+1)} & \\
& -2 n^{3} \frac{\left(n^{2}+2 n-1\right)}{\left(n^{2}+1\right)\left(n^{4}-1\right)}+\left\{\frac{8 n^{4}\left(n^{4}+1\right)}{\left(n^{2}+1\right)\left(n^{4}-1\right)^{2}}\right\} \ln n .
\end{aligned}
$$

For completely diffuse flux incident on the interface from within the material of index $n$, all of the radiant energy that is incident at angles greater than the critical angle. $\varphi_{c}$, will be totally reflected. The critical angle is that at which $\theta=\pi / 2$, or $\sin \varphi_{c}=\frac{1}{n}$. The fraction of flux incident at angles from $\sin ^{-1} \frac{1}{n}$ to $\pi / 2$ can be obtained by integration and divided by the total flux to give the fraction $1-\frac{1}{n^{2}}$ of the incident flux that is totally reflected. Hence only $\frac{1}{n^{2}}$ of the total flux will be incident at angles at which it is refracted, and a fraction $\rho_{e}$ of that flux will be internally reflected, as indicated in eq (33). Hence we can write

$$
\rho_{i}=1-\frac{1}{n^{2}}+\frac{\rho_{e}}{n^{2}} \quad \text { or } \quad n^{2}\left(1-\rho_{i}\right)=\left(1-\rho_{e}\right)
$$

The expression for $\rho_{e}$ given in eq (34) is somewhat complex. Judd [10] gives computed values of $\rho_{e}$ and $\rho_{i}$ for indices of refraction from 1.00 to 1.60 in increments of 0.01 .

The geometric distribution of the emitted flux will be determined by the directional reflectance at the coating-air interface, and can be computed from the refractive index by means of the Fresnel equation. For normally incident parallel flux, the Fresnel equation reduces to

and

$$
\rho_{N}=\left(\frac{n-1}{n+1}\right)^{2}
$$

$$
1-\rho_{N}=\frac{4 n}{(n+1)^{2}}
$$

where $\rho_{N}$ is specular reflectance for normally incident flux.

The normal spectral emittance, $E_{N}$, of any specimen can be computed from the hemispherical spectral emittance by use of the Fresnel equations for reflectance of internally incident normal and diffuse flux. This conversion is not affected by the coefficient of scatter of the coating.

\section{Discussion}

In the case of paper, layers of powder and unglazed porous ceramic materials, air may be considered the continuous phase, and there will be no specular reflection at the interfaces. If, in addition, there is no substrate, or if the reflectance of the substrate is zero, the conditions will 
be identical to those postulated by Klein [7]. If zero is substituted for $\rho_{e}, \rho_{s}$, and $\rho_{i}$ in eq (23), it reduces to

$$
R=\frac{\left(1-\beta^{2}\right) \sinh \sigma D}{\left(1+\beta^{2}\right) \sinh \sigma D+2 \beta \cosh \sigma D}
$$

which is identical to Klein's [7] eq (46).

\subsection{Properties of Nonabsorbing, Nonscattering Layers}

It may be of interest to consider how the emittance and reflectance of a coating will vary as $S$ and $K$ approach zero. Obviously, if both $S$ and $K$ are zero, the material will have perfect transmittance, and both reflectance and absorptance (or emittance) will be zero. This is true for a perfect vacuum, and is closely approximated by a gas at those wavelengths at which no absorption occurs.

\section{a. Properties of Nonscattering Layers}

If there is no scattering, $S=0$, and eqs (3) and (4) become

and

$$
d I / d x=-K I
$$

hence

$$
d J / d x=K J
$$

$$
I_{(x+\Delta x)}=I_{x} e^{-K \Delta x},
$$

where $I_{x}$ represents the flux density at a level $x$ within the material, and $I_{(x+\Delta x)}$ represents the flux density after traversing a thickness $\Delta x$ of the material. Equation (41) differs from the familiar Bouguer's law only in that the attenuation of completely diffuse flux is twice as rapid as that of unidirectional flux.

If the scattering coefficient, $S$, is zero, which will be approached by optically homogeneous materials, such as optical glass and many single crystals, then $\sigma=K$ and $\beta=1$, hence (24) reduces to

$$
E_{H}=\left(1-\rho_{e}\right)\left\{1-\frac{\left(1-\rho_{i}\right) \rho_{s} e^{-K D}}{e^{K D}-\rho_{i} \rho_{s} e^{-K D}}\right\}
$$

However, when $S$ becomes small, the internally reflected flux will not be rediffused, if reflected from optically smooth surfaces. Thus (42) is only valid if the coating-substrate interface is so rough that perfectly diffuse reflection occurs. For the more general case, where this interface approaches optical smoothness, a three-dimensional analysis of the type used by Gardon [5] is more nearly valid.

\section{b. Properties of Nonabsorbing Layers}

If the absorption coefficient, $K$, is zero, as will be approximated at some wavelengths by a freshly smoked layer of magnesium oxide or by freshly fallen snow, both $\sigma$ and $\beta$ become zero, and eq (24) becomes indeterminate, which would be expected for an opaque coating of the specified materials, but not for a composite specimen. However, under these conditions, eqs (3) and (4) become

$$
d I / d x=-S(J-I)=d J / d x .
$$

This equation can be solved by a procedure similar to those used for eqs (3) and (4) to give

$$
\begin{gathered}
I=\frac{J_{e}\left[S x\left(1-\rho_{e}\right)\left(1-\rho_{s}\right)+\rho_{s}\left(1-\rho_{e}\right)\right]}{S D\left(1-\rho_{i}\right)\left(1-\rho_{s}\right)+1-\rho_{i} \rho_{s}} \\
J=\frac{J_{e}\left[S x\left(1-\rho_{e}\right)\left(1-\rho_{s}\right)+\left(1-\rho_{e}\right)\right]}{S D\left(1-\rho_{i}\right)\left(1-\rho_{s}\right)+1-\rho_{i} \rho_{s}} .
\end{gathered}
$$


Substituting eq (44) in eq (21) we get

$$
R=\rho_{e}+\left(1-\rho_{i}\right)\left(1-\rho_{e}\right) \frac{S D\left(1-\rho_{s}\right)+\rho_{s}}{S D\left(1-\rho_{i}\right)\left(1-\rho_{s}\right)+\left(1-\rho_{i} \rho_{s}\right)} .
$$

If $\rho_{e}, \rho_{i}$, and $\rho_{s}$ are zero, as they were under the conditions postulated by Klein [7], eq (46) reduces to

which is identical to Klein's eq (48).

$$
R=\frac{S D}{S D+1}
$$

Under the conditions postulated for the derivation of eq (47), the emittance will approach zero and the reflectance will approach one as the coating approaches a thickness at which it is completely opaque, which will only occur at infinite thickness. Since $K=0$, the absorptance is zero by definition, and the transmittance is one minus the reflectance. Hence we can write

$$
T=\frac{1}{S D+1}
$$

which is identical to Klein's eq (a7).

\subsection{Example of Computed Emittance}

Figure 1 is a plot of emittance, computed from eq (31), as a function of thickness for composite specimens with optically smooth surfaces comprised of a coating having an index of refraction of 1.40 (from which $\rho_{e}=0.028, \rho_{i}=0.53$ ), a value of $\beta$ of 0.8 , and values of $\sigma$ of 40 , $20,13.3$, and $10.0 \mathrm{~mm}^{-1}$, respectively, applied over a mat substrate having a reflectance into the coating of 0.9 . (The values of $S$ and $K$ corresponding to a $\beta$ of 0.8 and $\sigma$ of $40,20,13.3$, and $10 \mathrm{~mm}^{-1}$ are as follows: $S=8.0,4.0,2.65$, and $2.0 \mathrm{~mm}^{-1}$ and $K=28.44,14.22,9.42$, and $7.11 \mathrm{~mm}^{-1}$, respectively.) These conditions might be approximated at wavelengths in the visible by a glossy gray paint applied over a white backing. All of the coating materials have the same emissivity (about 0.918 ), but the coating with a $\sigma$ value of $40 \mathrm{~mm}^{-1}$ reaches 99 percent of this value at a thickness of $0.05 \mathrm{~mm}$, while the coating with a $\sigma$ value of $10 \mathrm{~mm}^{-1}$ requires a thickness of $0.2 \mathrm{~mm}$ to reach the same value.

The plotted emittance at zero thickness of the coating is greater than the emittance $(0.10)$ of the substrate because the coating has a lower index of refraction than the substrate, and hence reduces reflection at the interface. 'The plotted value is what would be obtained with a very thin, perfectly transparent glossy coating having an index of refraction of 1.40 .

FIGURE 1. Computed normal spectral emittance, plotted as a function of coating thickness, for a composite specimen comprised of partially transmitting coatings with optically smooth surfaces having an index of refraction of $1.40, \beta$ value of 0.80 , and $\sigma$ values of $40,20,13.3$, and $10 \mathrm{~mm}^{-1}$, respectively, applied over a substrate having a reflectance of 0.90 , plotted as a function of coating thickness. These conditions would be approximated by a glossy gray paint applied over a polished metal or white substrate.

Note that the emittance at zero coating thickness is not that of the substrate. It was assumed that even at zcro thickness, there would be an effect of the index of refraction of the coating. Actually, such effect wourd be produced by a transparent coating of $40 \mathrm{~mm}^{-1}$ beconies of 1.40 . The coating having a $\sigma$ value of other coatings at successively greater thickncsses.

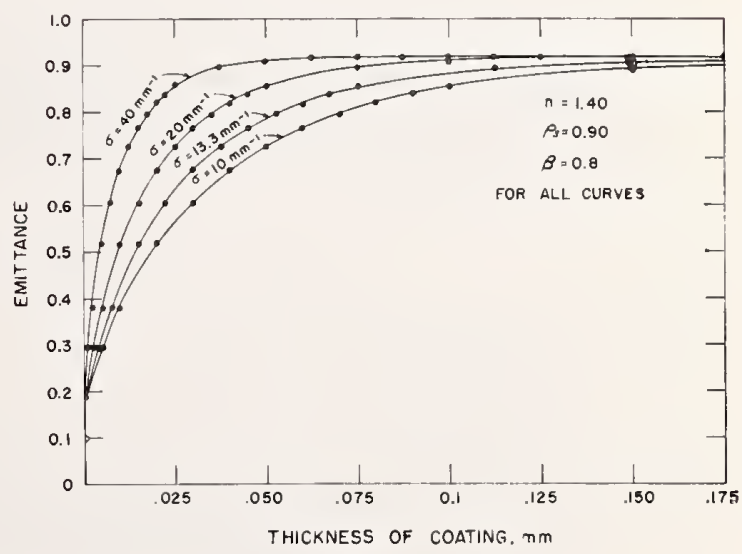




\subsection{Limitations to Equations}

There are a number of conditions encountered in practice that deviate from the conditions postulated in the derivation of eq (24). There will always be a thermal gradient normal to the surface in any situation where a body is heated internally and is dissipating radiant energy, and the effect of such a gradient has been omitted in the derivation. In the case of thick ceramic coatings (typically $0.5 \mathrm{~mm}$ or more), significant errors may be introduced by ignoring the effect of thermal gradients, especially at high temperatures. The gradients will be negligibly small when the specimen and surroundings are at room temperature or below, and the effect of the gradients in thin coatings, typically less than $0.1 \mathrm{~mm}$, is considered to be negligibly small even at high temperatures.

During firing of porcelain enamels and some other types of ceramic coatings, on some metals, there is appreciable chemical reaction at the coating-metal interface, and some of the reaction products diffuse into the coating for measurable distance. The reaction products may change the reflectance of the substrate into the coating, and the reaction products diffusing into the coating may significantly change its optical properties near the interface. The extent and importance of these effects depend upon the particular materials involved, their thicknesses, the firing, and the service conditions. When the total effect of these factors is large, appropriate adjustments in the values substituted into the equation are required for its useful application. Most types of organic and flame-sprayed ceramic coatings, however, will be essentially free from these effects.

In the case of some paints, there may be segregation of the pigment particles within the velicle during drying, which will also tend to invalidate the equation. For mat coatings, where the coating-air interface is not optically smooth even on a micro scale, it may be necessary to measure $\rho_{e}$ and $\rho_{i}$ experimentally, instead of computing them from the index of refraction.

\section{Summary}

The thermal radiation properties of a composite specimen, comprised of a partially transmitting coating applied over an opaque substrate, usually vary significantly with the thickness of the coating. An equation was derived relating these properties to the thickness of the coating, the reflectance of the substrate, and the optical properties of the coating material. If the optical properties of a coating and the reflectance of the substrate are known as functions of wavelength, the equation can be used to compute (1) the normal spectral emittance (or reflectance) of any thickness of coating over the substrate or (2) the thickness of the coating over the substrate required to give any normal spectral emittance (or reflectance) within any given wavelength interval intermediate between the emittance of the substrate and of an infinitely thick coating.

The author gratefully acknowledges the assistance of Louis Joseph, of the Applied Mathematics Division, in checking the mathematics, and of Deane B. Judd, of the Optics and Metrology Division, for his advice and helpful suggestions.

\section{List of Symbols}

$A=$ absorptance.

$T=$ transmittance.

$R=$ reflectance.

$E=$ emittance.

$I=$ diffuse radiant flux proceeding outward from the interior of a specimen.

$J=$ diffuse radiant flux proceeding inward toward the interior of a specimen.

$K=$ absorption coefficient. 
$S=$ backscattering coefficient.

$x=$ distance from the coating-substrate interface to a point in the coating.

$L=$ a constant that depends upon boundary conditions.

$\sigma=\sqrt{K(K+2 S)}$.

$\beta=\sqrt{K /(K+2 S)}$.

$\rho_{e}=$ reflectance of the coating-air interface for externally-incident diffuse radiant flux.

$\rho_{s}=$ reflectance of the substrate for diffuse radiant flux incident on the coating-substrate interface from within the coating.

$\rho_{i}=$ reflectance of the coating-air interface for internally incident diffuse radiant flux.

$J_{e}=$ externally incident diffuse radiant flux.

$J_{0}=$ flux $J$ at $x=0$ (at coating-substrate interface).

$I_{D}=$ flux $I$ at $x=D$ (at coating-air interface).

$D=$ thickness of coating.

$M=(1+\beta)-\rho_{s}(1-\beta)$.

$N=(1+\beta)-\rho_{i}(1-\beta)$.

$O=(1-\beta)-\rho_{s}(1+\beta)$.

$P=(1-\beta)-\rho_{i}(1-\beta)$.

$n=$ index of refraction.

$n_{1}=$ index of refraction in medium from which flux is incident.

$n_{2}=$ index of refraction in medium into which flux is refracted.

$\varphi=$ angle of incidence, from the normal.

$\theta=$ angle of refraction, from the normal.

$\varphi_{c}=$ critical angle of incidence for total internal reflectance.

$E_{H}=$ hemispherical spectral emittance.

$\rho_{N}=$ reflectance of the coating-air interface for normally incident parallel flux.

$E_{N}=$ normal spectral emittance.

$R_{i}=$ internal reflectance of a coating.

\section{References}

[1] Judd, D. B., Optical specification of light-seattering materials, J. Res. NBS 19, 287 (1937) RP1026.

[2] ASTM Method C 347-57, Reflectivity and Coefficient of Scatter of White Porcelain Enamels, 1961 AsTM Book of Standards, pp. 612-615.

[3] Kubelka, P., and F. Munk, Ein Beitrag zur Optik der Farbanstriche, Z. tech. Physik, 12, 593 (1931).

[4] Kubelka, P., New contributions to the optics of intensely light-scattering materials, Part I. J. Opt. Soc. Am. 38, 448 (1948).

[5] Gardon, R., The emissivity of transparent materials, J. Am. Ceram. Soc. 39 [8], 278-285 (1956),

[6] Hamaker, H. C., Radiation and heat conduction in light-scattering material, Philips Res. Reports, 2, $55-67,103-111,112-125,420-425$ (1947).

[7] Klein, J. D., Heat transfer by radiation in powders, Doctor's Dissertation, Mass. Inst. of Technology (1960).

[8] Walsh, J. W. T., The reflection factor of a polished glass surface for diffused light, Dept. Sci. and Ind. Res. Illumination Research, Tech. Paper No. 2, p. 10 (1926).

[9] Saunderson, J. L., Calculation of the color of pigmented plastics, J. Opt. Soc. Am. 32 [12], 727-736.

[10] Judd, D. B., Fresnel reflection of diffusely incident light, J. Research NBS 29, 329-332 (1942) RP1504.

(Paper 67C3-132) 


\title{
Total Hemispherical Emittance of Coated and Uncoated Inconel and Types 321 and 430 Stainless Steel
}

\author{
Joseph C. Richmond and William N. Harrison
}

(February 27, 1962)

\begin{abstract}
The total hemispherical emittance of specimens of three alloys, Inconel and types 321 and 430 stainless steel, in six different surface conditions, was evaluated at temperatures in the range 200 to $1,000{ }^{\circ} \mathrm{C}$ by a modification of the hot filament method. The surface conditions were (1) polished, (2) sandblasted, (3) polished and then oxidized, (4) sandblasted and then oxidized, (5) coated with NBS coating A-418, and (6) coated with NBS. coating $\mathrm{N}-143$.

The total hemispherical emittance was markedly affected by the surface treatment. In general emittance was low for polished specimens, intermediate for sandblasted specimens, and high for oxidized or ceramic-coated specimens of each alloy.
\end{abstract}

\section{Introduction}

Total hemispherical emittance is the amount of radiant energy emitted, per unit area and time, expressed as a fraction of that emitted by a blackbody or complete radiator under the same conditions. When this property of a specimen has been measured, the rate of heat dissipation by radiation of the specimen at any temperature for which data are available can be computed.

The total hemispherical emittance of a specimen is affected by its chemical composition, internal structure and surface texture, and if a specimen of a given material is not completely opaque to the emitted radiant energy, its emittance is also a function of its thickness.

The present investigation was undertaken to study the effect of surface treatments, including the application of several thicknesses of ceranic coatings $\mathrm{A}-418$ and $\mathrm{N}-143$, on the total hemispherical emittance of specimens made from three alloys.

\section{Description of Material}

The three alloys studied were Inconel, type 321 stainless steel, and type 430 stainless steel. Inconel is an austenitic alloy, nominally 80 percent nickel, 14 percent chromium, and 6 percent iron, that is used for those high-temperature applications in which an alloy of good oxidation and corrosion resistance is required, and moderate high-temperature strength is acceptable. Type 321 stainless steel is an austenitic alloy, nominally containing 18 percent chromium and 8 percent nickel, that is stabilized with titanium to inhibit intergranular corrosion at elevated temperatures. Type 430 stainless steel is a ferritic alloy that contains noininally 17 percent chromium.

Two ceramic coatings were used, NBS coatings $\mathrm{A}-418$ and $\mathrm{N}-143$. These have been previously described $[1,2] .{ }^{1}$ 'The frit and batch compositions are

\footnotetext{
1 Figures In brackets indicate the liturature references at the end of this paper.
}

given in table 1 . Coating A-418 consists of an alkalifree barium borosilicate frit, with a refractory mill addition of chromic oxide. Coating N-143 consists of a boron-free barium beryllium silicate frit with a refractory mill addition of cerium oxide.

TABLE 1

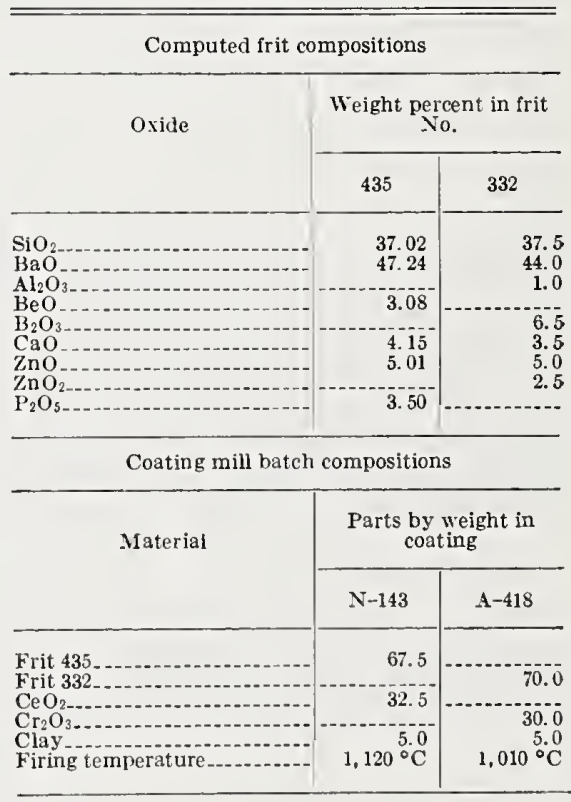

\section{Preparation of Specimens}

The Inconel and type 321 stainless steel were received in the form of dull-finished sheets approximately $30 \mathrm{in}$. wide by $8 \mathrm{ft}$ long. The type 430 stainless steel was received in the form of coiled strip, 2 in. wide. The strips had a highly polished surface. Several different thicknesses were used. 
The sheet metal was sheared into strips $20 \mathrm{~cm}$ long by $6.35 \mathrm{~mm}$ wide ( 8 by $1 / 4 \mathrm{in}$.). These strips were then mounted in a special jig, in lots of 20 to 100, and the edges were ground on a surface grinder until the sides were parallel to within $\pm 0.013 \mathrm{~mm}$ over the center $10 \mathrm{~cm}$ of each strip.

\subsection{Polished and Sandblasted Specimens}

Polished spccimens of Inconel and type 321 stainless steel were prepared by elcctropolishing; the procedure used is outlined in table 2. Because the type 430 stainlcss steel was received in the form of highly polished strip, no further polishing was required.

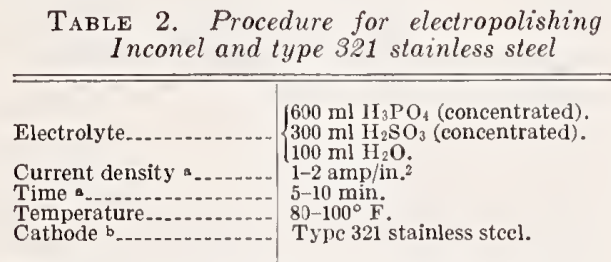

a Time and current density vary with initial condition of the surface.

b Two cathodes, onc on each side of specimen.

Sandblasted specimens of each alloy were prepared by blasting with $60-\mathrm{mesh}$ fused alumina grit, at an air pressure of approximately 75 psi. Care was taken in blasting to avoid excessive warpage of the thinner specimens.

Three thermocouples were welded to each uncoated, unoxidized specimen. Very shallow grooves were inscribed perpendicular to the axis of the specimen, one at the midlength of the specimen, and one $13 \mathrm{~mm}$ from the midlength on each side. Wires were laid in these grooves in order to position them accurately before welding. The $0.127 \mathrm{~mm}$ diam Chromel and Alumel thermocouple wires were separately welded to the specimen by means of an electronic condenser-discharge type of spot welder. The welds forming each thermocouple were about $3 \mathrm{~mm}$ apart. The Chromel leads from the outside thermocouples were used as the voltage taps.

\subsection{Oxidized Specimens}

Eight specimens of each alloy, in the polished and sandblasted condition, respectively, were prepared and marked for identification. The width, length, and thickness of each specimen was accurately measured, and it was weighed to the nearest $0.1 \mathrm{mg}$. these specimens were then oxidized in air in an electrically heated furnace at a temperature of $1,000 \pm 5{ }^{\circ} \mathrm{C}$ for times of $1 / 2,1,3,5,10,20,40$, and $80 \mathrm{hr}$. One specimen of each alloy in each condition was removed at the end of each period of oxidation. All of the specimens remaining in the test were weighed at the end of each oxidation period.

After oxidation, the oxide layer was removed from both sides by grinding each specimen for a distance of about $25 \mathrm{~mm}$ at each end, to ensure good contact with the electrodes. Also, a light scratch was made through the oxide at the three areas where the thermocouples were to be attached. The thermocouple wires were then welded in place, as described above.

\subsection{Coated Specimens}

The ceramic coatings were applied, by dipping, to sandblasted specimens to which thermocouples had been welded. It was found that when the coated specimens were fired in a furnace to mature the coatings, the thermocouple leads oxidized excessively. To eliminate this difficulty the coated specimens that were used were fired in air, heated by an electric current passed through them.

\section{Experimental Procedure}

The equipment and procedure for evaluation of total hemispherical emittance has been described in a previous publication [3]. Only a brief summary will be given here.

The specimen was heated in a vacuum of $4 \times 10^{-5}$ $\mathrm{mm}$ of $\mathrm{Hg}$ or less by passing a current through it. The test conditions were such that essentially all of the heat dissipation from the area at and near the midlength of the specimen was by radiation. The power input to, and temperature of, a small length (approximately $25 \mathrm{~mm}$ ) of known dimensions near the midlength of the specimen and the temperature of the walls of the chamber surrounding the hot specimen were measured. The total hemispherical emittance was then computed from these values by means of the following relationship derived from the Stefan-Boltzmann equation:

$$
E=\frac{V \cdot I}{A \sigma\left(T_{1}^{4}-T_{2}^{4}\right)}
$$

in which $E=$ total hemispherical emittance

$V=$ voltage drop between voltage taps

$I=$ current flowing through specimen

$A=$ surface area of specimen between voltage taps

$\sigma=$ Stefan-Boltzmann constant

$T_{1}=$ temperature of specimen, ${ }^{\circ} \mathrm{K}$

$T_{2}=$ temperature of shell, ${ }^{\circ} \mathrm{K}$.

In these determinations the specimen was held at one power input only long enough for its temperature to stabilize, and the changes in emittance that occurred on long-time heating in vacuum were not followed. The time that each specimen was held at each power input usually was less than $10 \mathrm{~min}$, and in many cases the time was less than $5 \mathrm{~min}$. While some changes in emittance undoubtedly did occur in these short times, the magnitude of the changes was less than 2 percent, except where noted.

\section{Results and Discussion}

Data for Inconel are shown in figures 1, 4, and 7 ; for type 321 stainless steel in figures 2,5 , and 8 ; and for type 430 stainless steel in figures 3,6 , and 9 . In 


\title{
Total Hemispherical Emittance of Coated and Uncoated Inconel and Types 321 and 430 Stainless Steel
}

\author{
Joseph C. Richmond and William N. Harrison
}

(February 27, 1962)

\begin{abstract}
The total hemispherical emittance of specimens of three alloys, Inconel and types 321 and 430 stainless steel, in six different surface conditions, was evaluated at temperatures in the range 200 to $1,000{ }^{\circ} \mathrm{C}$ by a modification of the hot filament method. The surface conditions were (1) polished, (2) sandblasted, (3) polished and then oxidized, (4) sandblasted and then oxidized, (5) coated with NBS coating A-418, and (6) coated with NBS, coating N-143.

The total hemispherical emittance was markedly affected by the surface treatment. In general emittance was low for polished specimens, intermediate for sandblasted specimens, and high for oxidized or ceramic-coated specimens of each alloy.
\end{abstract}

\section{Introduction}

Total hemispherical emittance is the amount of radiant energy emitted, per unit area and time, expressed as a fraction of that emitted by a blackbody or complete radiator under the same conditions. When this property of a specimen has been measured, the rate of heat dissipation by radiation of the specimen at any temperature for which data are available can be computed.

The total hemispherical emittance of a specimen is affected by its chemical composition, internal structure and surface texture, and if a specimen of a given material is not completely opaque to the emitted radiant energy, its emittance is also a function of its thickness.

The present investigation was undertaken to study the effect of surface treatments, including the application of several thicknesses of ceramic coatings $\mathrm{A}-418$ and $\mathrm{N}-143$, on the total hemispherical emittance of specimens made from three alloys.

\section{Description of Material}

The three alloys studied were Inconel, type 321 stainless steel, and type 430 stainless steel. Inconel is an austenitic alloy, nominally 80 percent nickel, 14 percent chromium, and 6 percent iron, that is used for those high-temperature applications in which an alloy of good oxidation and corrosion resistance is required, and moderate high-temperature strength is acceptable. Type 321 stainless steel is an austenitic alloy, nominally containing 18 percent chromium and 8 percent nickel, that is stabilized with titanium to inhibit intergranular corrosion at elevated temperatures. Type 430 stainless steel is a ferritic alloy that contains nominally 17 percent chromium.

Two ceramic coatings were used, NBS coatings $\mathrm{A}-418$ and $\mathrm{N}-143$. These have been previously described $[1,2] .^{1} \quad$ The frit and batch conıpositions are

\footnotetext{
- Figures in brackets indleate the llturature references at the end of this paper.
}

given in table 1 . Coating $\mathrm{A}-418$ consists of an alkalifree barium borosilicate frit, with a refractory mill addition of chromic oxide. Coating N-143 consists of a boron-free barium beryllium silicate frit with a refractory mill addition of cerium oxide.

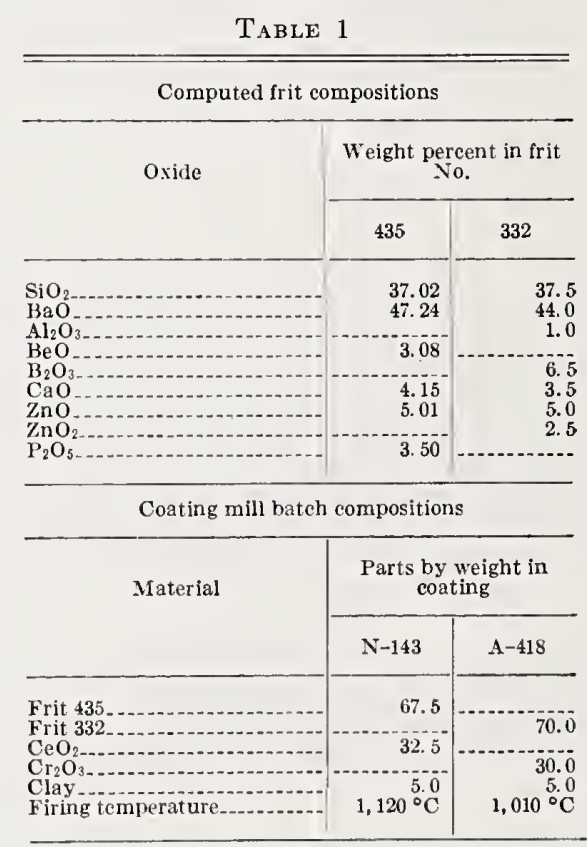

\section{Preparation of Specimens}

The Inconel and type 321 stainless steel were received in the form of dull-finished sheets approximately $30 \mathrm{in}$. wide by $8 \mathrm{ft}$ long. The type 430 stainless steel was received in the form of coiled strip, 2 in. wide. 'The strips had a highly polished surface. Several different thicknesses were used. 
The sheet metal was sheared into strips $20 \mathrm{~cm}$ long by $6.35 \mathrm{~mm}$ wide ( 8 by $1 / 4 \mathrm{in}$.). These strips were then mounted in a special jig, in lots of 20 to 100 , and the edges were ground on a surface grinder until the sides were parallel to withm $\pm 0.013 \mathrm{~mm}$ over the center $10 \mathrm{~cm}$ of each strip.

\subsection{Polished and Sandblasted Specimens}

Polished specimens of Inconel and type 321 stainless steel were prepared by electropolishing; the procedure used is outlined in table 2. Because the type 430 stainless steel was received in the form of highty polished strip, no further polishing was required.

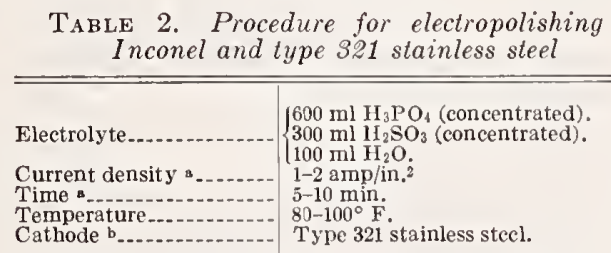

a Time and current density vary with initial condition of the surface.

b Two cathodes, one on each side of specimen.

Sandblasted specimens of each alloy were prepared by blasting with 60 -mesh fused alumina grit, at an air pressure of approximately 75 psi. Care was taken in blasting to avoid excessive warpage of the thinner specimens.

Three thermocouples were welded to each uncoated, unoxidized specimen. Very shallow grooves were inscribed perpendicular to the axis of the specimen, one at the midlength of the specimen, and one $13 \mathrm{~mm}$ from the midlength on each side. Wires were laid in these grooves in order to position them accurately before welding. The $0.127 \mathrm{~mm}$ diam Chromel and Alumel thermocouple wires were separately welded to the specimen by means of an electronic condenser-discharge type of spot welder. The welds forming each thermocouple were about $3 \mathrm{~mm}$ apart. The Chromel leads from the outside thermocouples were used as the voltage taps.

\subsection{Oxidized Specimens}

Eight specimens of each alloy, in the polished and sandblasted condition, respectively, were prepared and marked for identification. The width, length, and thickness of each specimen was accurately measured, and it was weighed to the nearest $0.1 \mathrm{mg}$. these specimens were then oxidized in air in an electrically heated furnace at a temperature of $1,000 \pm 5^{\circ} \mathrm{C}$ for times of $1 / 2,1,3,5,10,20,40$, and $80 \mathrm{hr}$. One specimen of each alloy in each condition was removed at the end of each period of oxidation. All of the specimens remaining in the test were weighed at the end of each oxidation period.

After oxidation, the oxide layer was removed from both sides by grinding each specimen for a distance of about $25 \mathrm{~mm}$ at each end, to ensure good contact with the electrodes. Also, a light scratch was made through the oxide at the three areas where the thermocouples were to be attached. The thermocouple wires were then welded in place, as described above.

\subsection{Coated Specimens}

The ceramic contings were applied, by dipping, to sandblasted specimens to which thermocouples had been welded. It was found that when the coated specimens were fired in a furnace to mature the coatings, the thermocouple leads oxidized excessively. To eliminate this difficulty the coated specimens that were used were fired in air, heated by an electric current passed through them.

\section{Experimental Procedure}

The equipment and procedure for evaluation of total hemispherical emittance has been described in a previous publication [3]. Only a brief summary will be given here.

The specimen was heated in a vacuum of $4 \times 10^{-5}$ $\mathrm{mm}$ of $\mathrm{Hg}$ or less by passing a current through it. The test conditions were such that essentially all of the heat dissipation from the area at and near the midlength of the specimen was by radiation. The power input to, and temperature of, a small length (approximately $25 \mathrm{~mm}$ ) of known dimensions near the midlength of the specimen and the temperature of the walls of the chamber surrounding the hot specimen were measured. The total hemispherical emittance was then computed from these values by means of the following relationship derived from the Stefan-Boltzmann equation:

$$
E=\frac{V \cdot I}{A \sigma\left(T_{1}^{4}-T_{2}^{4}\right)}
$$

in which $E=$ total hemispherical emittance

$V=$ voltage drop between voltage taps

$I=$ current flowing through specimen

$A=$ surface area of specimen between voltage taps

$\sigma=$ Stefan-Boltzmann constant

$T_{1}=$ temperature of specimen, ${ }^{\circ} \mathrm{K}$

$T_{2}=$ temperature of shell, ${ }^{\circ} \mathrm{K}$.

In these determinations the specimen was lield at one power input only long enough for its temperature to stabilize, and the changes in emittance that occurred on long-time heating in vacuum were not followed. The time that each specimen was held at each power input usually was less than $10 \mathrm{~min}$, and in many cases the time was less than 5 min. While some changes in emittance undoubtedly did occur in these short times, the magnitude of the changes was less than 2 percent, except where noted.

\section{Results and Discussion}

Data for Inconel are shown in figures 1, 4, and 7 ; for type 321 stainless steel in figures 2,5 , and 8 ; and for type 430 stainless steel in figures 3,6 , and 9 . In 


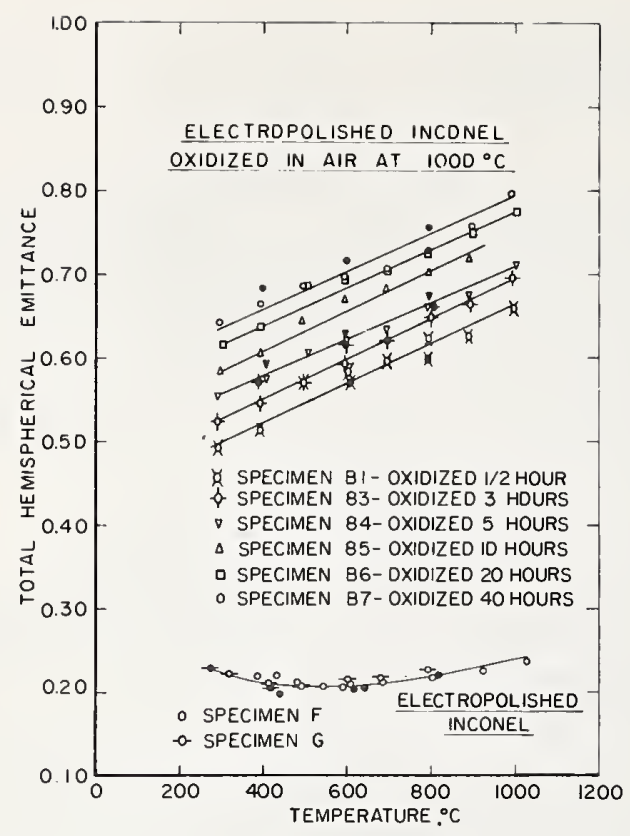

FIGIRE 1. Total hemispherical emittance of Inconel in the electropolished condition, bottom curve, and after oxidation at $1,000{ }^{\circ} \mathrm{C}$ for various times subsequent to electropolishing, top curves.

Open points represent values obtained during heating; closed points, during cooling.

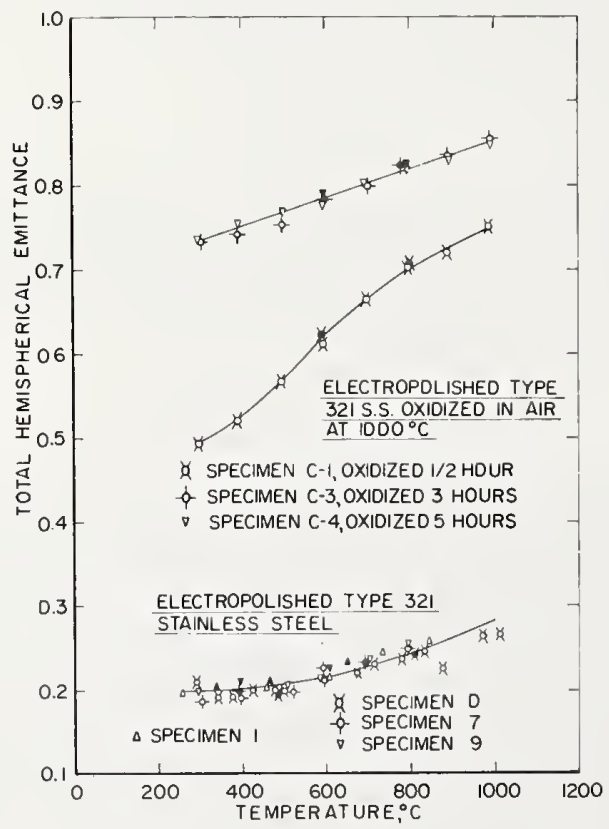

FiguRE 2. Total hemispherical emittance of lype 321 stainless steel specimens in the electropolished condition, bottom curve, and after oxidalion in air at $1,000{ }^{\circ} \mathrm{C}$ for various times subsequcnt to clectropolishing, top curves.

open points represent values obtained during heating; closed points, during cooling.

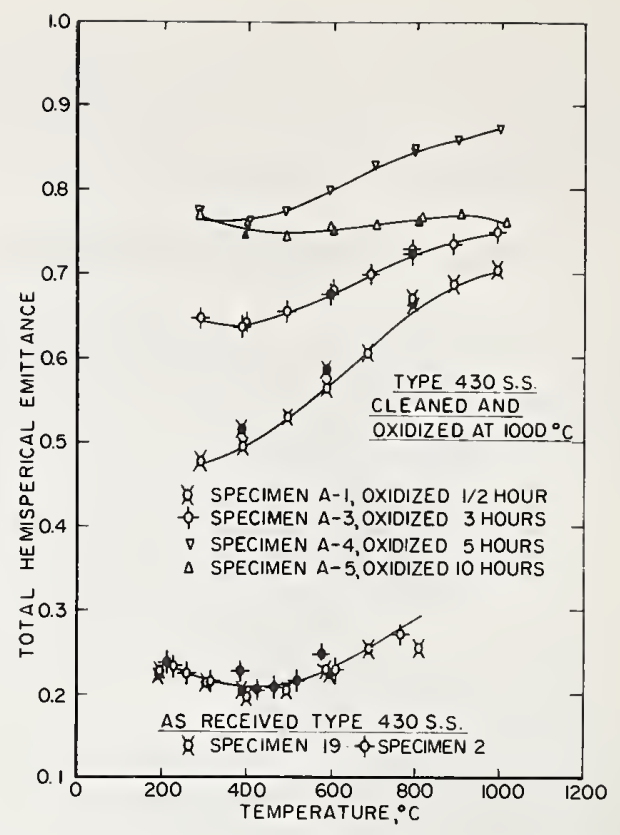

Finure 3. Total hemispherical emittance of type 430 stainless steel in the as-received condition (polished) bottom curve, and after oxidation in air at $1,000{ }^{\circ} \mathrm{C}$ for various times, top curves.

open points represent values obtained during heating: closed points, during cooling.

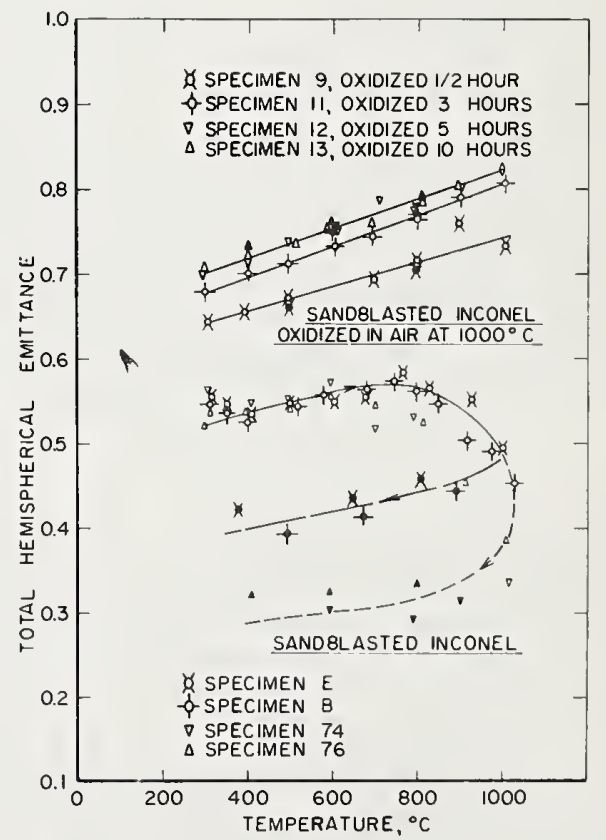

Figure 4. Total hemispherical cmittance of Inconel in the sandblasted condition, bottom curves, and after oxidation at $1,000{ }^{\circ} \mathrm{C}$ for various times subsequent to sandblasting, top curves.

Opu points represent values obtained during heating; closed points, during cooling. 


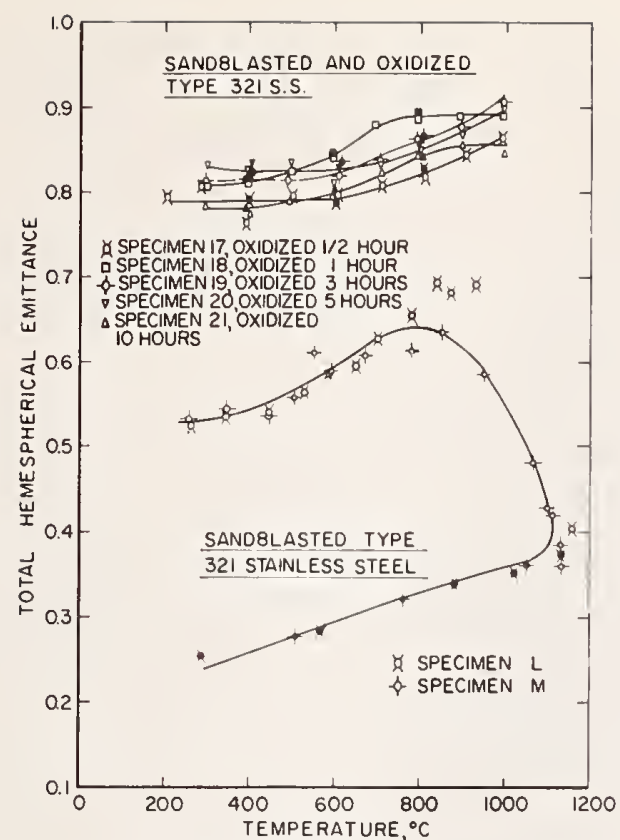

Figure 5. Tolal hemispherical enittance of type 321 stainless steel specimens in the sandblasted condition, bottom curves, and after oxidation in air at $1,000{ }^{\circ} \mathrm{C}$ for various times subsequent to sandblasting, top curves.

Open points represent values obtained during heating; closed points, during cooling.

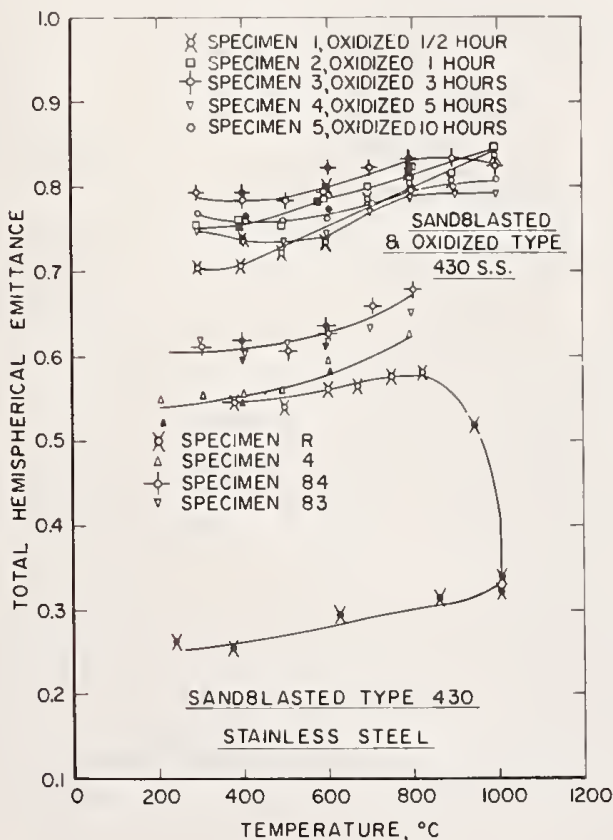

Figure 6. Total hemispherical emittance of type 430 stainless steel in the sandblasted condition, bottom curves, and after oxidation in air at $1,000{ }^{\circ} \mathrm{C}$ for various times subsequent to sandblasting, top curves.

Open points represent values obtained during heating; closed points, during cooling.

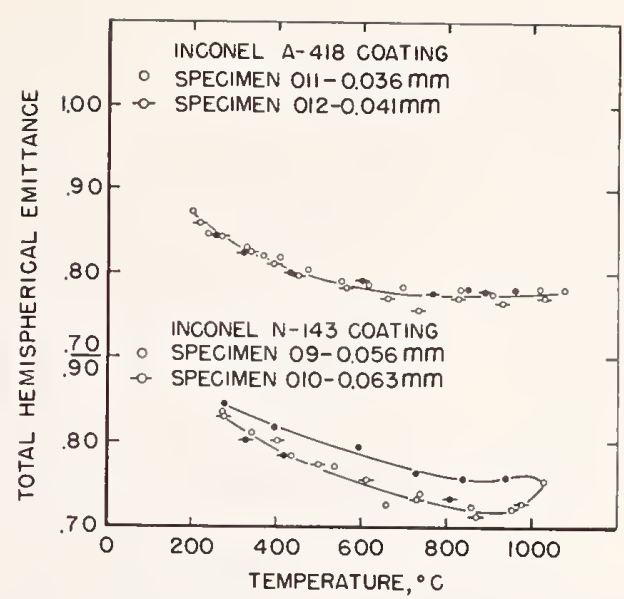

Figure 7. Total hemispherical emittance of composite specimens consisting of Inconel coated with NBS coating A-418, top curve, and NBS coating $N-143$, bottom curve.

Open points represent values obtained during heating; elosed points, during cooling.

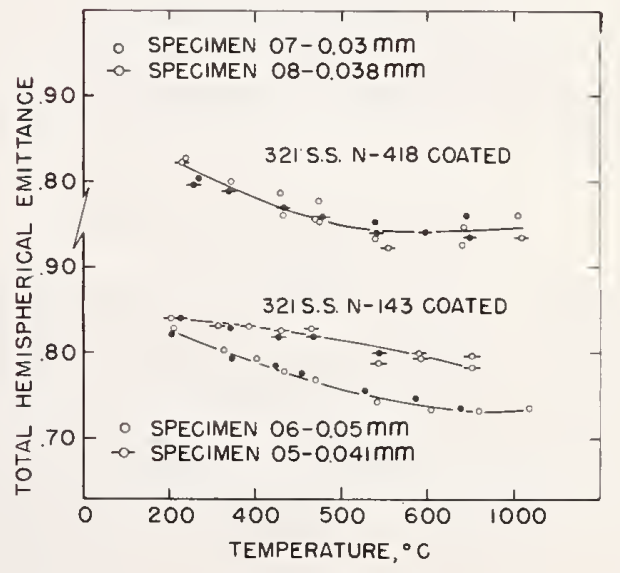

Figure 8. Total hemispherical emittance of composite specimens consisting of type 321 stainless steel coated with NBS ceramic coating $A-418$, top curves, and NBS ceramic coating $N-143$, bottom curves.

Open points represent values obtained during heating; elosed points, durlng cooling. 


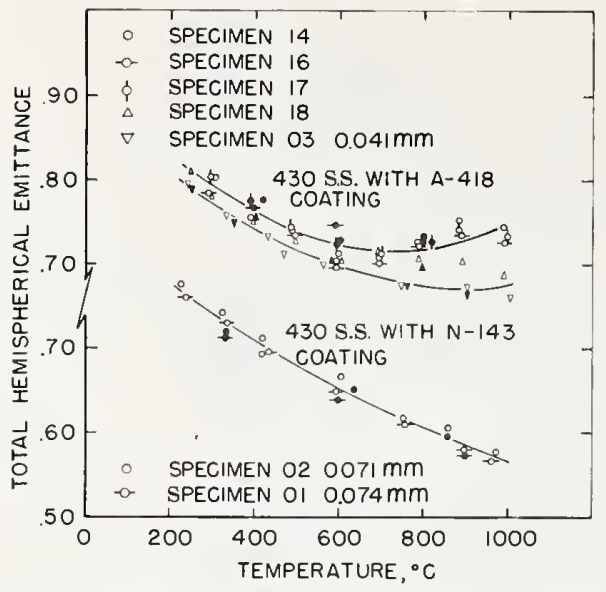

FiguRE 9. Total hemispherical emittance of composite specimens consisting of type 430 stainless steel coated with NBS ceramic coating $A-418$, top curves, and NBS ceramic coating $N-143$, bottom curve.

open points represent values obtained during heating; closed points, during cooling.

each case open symbols indicate data points that were obtained during the heating cycle, and filled symbols indicate data points that were obtained during the cooling cycle.

\subsection{Polished Specimens}

The data for the polished specimens, figures 1,2 , and 3 , show that there was very little difference in the emittance of the three alloys in this condition. They all had total hemispherical emittance on the order of 0.20 to 0.28 , increased in emittance with an increase in tcmperature, and showed no evidence of a permanent change in emittance on heating in vacuum for the times required to complete the evaluations. The total hemispherical emittance of the polished Inconel was slightly higher at $400{ }^{\circ} \mathrm{C}$ and below, and slightly lower at $600{ }^{\circ} \mathrm{C}$ and above, than that of the type 321 and type 430 stainless steel specimens.

\subsection{Sandblasted Specimens}

The data for the sandblasted specimens are shown in figurcs 4,5 , and 6 . In general there was little difference between the emittances of the different alloys in this condition. 'They all had total hemispherical emittance on the order of 0.5 to 0.65 , and the emittance of each alloy increased with tcmperature. There was no indication that heating in vacuum at temperatures bclow $800{ }^{\circ} \mathrm{C}$ for the times rcquired to complete the evaluations produced any permanent change in cmittance. None of these sandblasted specimens showed any visual evidence of an oxide film formation after completion of the tests.

The total hemispherical emittance of each of the alloys in the sandblasted condition was permanently reduced by heating in vacuum at temperatures above $800{ }^{\circ} \mathrm{C}$, even for the short times required to complete the evaluations. When a time of approximately" 10 min elapsed bctween successive evaluations, as in specimens 74 and 76 in figure 4 , specimens $L$ and $M$ in figure 5, and specimen $\mathrm{R}$ in figure 6 , the total hemisplierical emittance was reduced on the order of 0.25 to 0.30 . When the time between successive readings was reduced to about $5 \mathrm{~min}$, specimens $\mathrm{E}$ and $B$, figure 4 , the amount of the reduction in emittance was on the order of 0.15 .

The observed reduction in total hemispherical emittance of sandblasted metal specimens on heating in vacuum was previously reported [3] to be due to volatilization of chromium, which was accompanied by a polishing action on the surface, somewhat similar in appearance to electropolishing. At temperatures below $620^{\circ} \mathrm{C}$, specimens oxidized even at a pressure of $9 \times 10^{-5} \mathrm{~mm}$ of mercury and emittance increased; at temperatures of $795^{\circ} \dot{\mathrm{C}}$ or above, the oxide dissociated and chromium volitalized, and the emittance decreased.

\subsection{Oxidized Specimens}

The data for the specimens that had been polished and then oxidized are shown in figures 1,2 , and 3 , and for the specimens that had been sandblasted and then oxidized in figures 4,5 , and 6 . In every case the oxidation treatment greatly increased the total hemispherical emittance. Also in every case but one the emittance of each oxidized specimen increased with an increase in temperature.

Apparently the oxide film formed during oxidation at $1,000{ }^{\circ} \mathrm{C}$ in air for $30 \mathrm{~min}$ was not completely opaque for any of the alloys studied, in either the polished or sandblasted condition. This is indicated by the observation that emittance increased with time of oxidation, at least for the first few hours.

The weight gain, in $\mathrm{mg} / \mathrm{cm}^{2}$, is plotted as a function of oxidation time for Inconel in figure 10, for type 321 stainless steel in figure 11, and for type 430 stainless steel in figure 12 . The computed thickness of the oxide layer, in microns, is also indicated on these figures. The computation was made on the following assumptions: (1) The oxide has a density of $5.2 \mathrm{~g} / \mathrm{cm}^{3}{ }^{2}$ (2) The specimen is flat, i.e., the area of the oxide layer was computed from the measured length, width, and thickness of each specimen, and (3) the oxide layer is of uniform thickness. All of these assumptions are in error, usually in the direction that would tend to give high values for the computed thicknesses, but the computed thicknesses nevertheless serve to give a rough approximation of the thickness of the oxide layers, and a much better approximation of the relative thicknesses of the oxide layers on different specimens of any one alloy in a single surface condition.

In the case of electropolished Inconel, figure 1, the emittance increased with time of oxidation for at

2 The exaet density of the oxide layer is not easily determined. It may rary with the time of oxidation, and it is probably different for the three alloys used in this investigation. The ralue of 5.2 was selected as a lower limit on the basis of published values of the densities of oxides that may be present. 


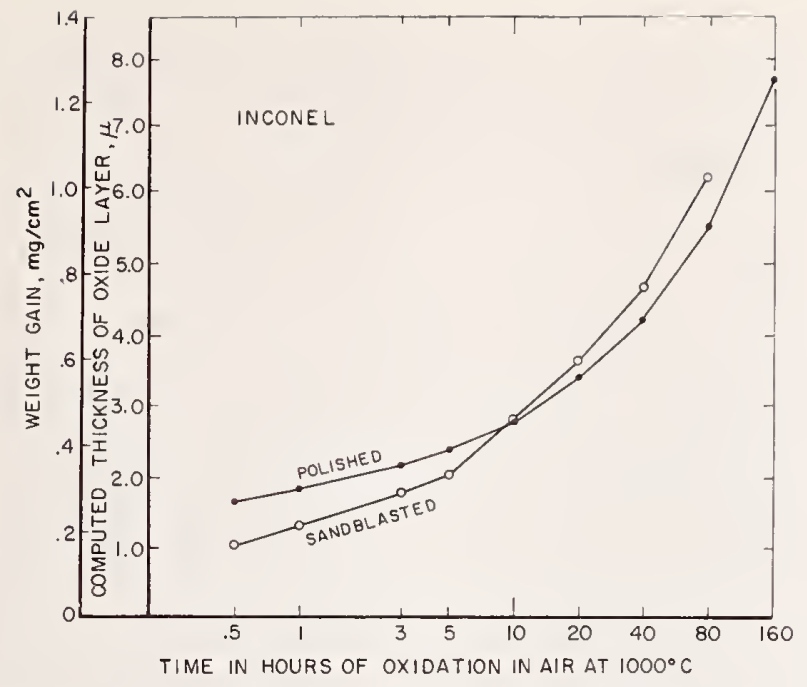

FIGURE 10. Weight gain, in $\mathrm{mg} / \mathrm{cm}^{2}$, and computed thickness of the oxide layer, in microns, of specimens of Inconel, in the as-received and sandblasted conditions, plotted as a function of time of oxidation in air at $1,000^{\circ} \mathrm{C}$.

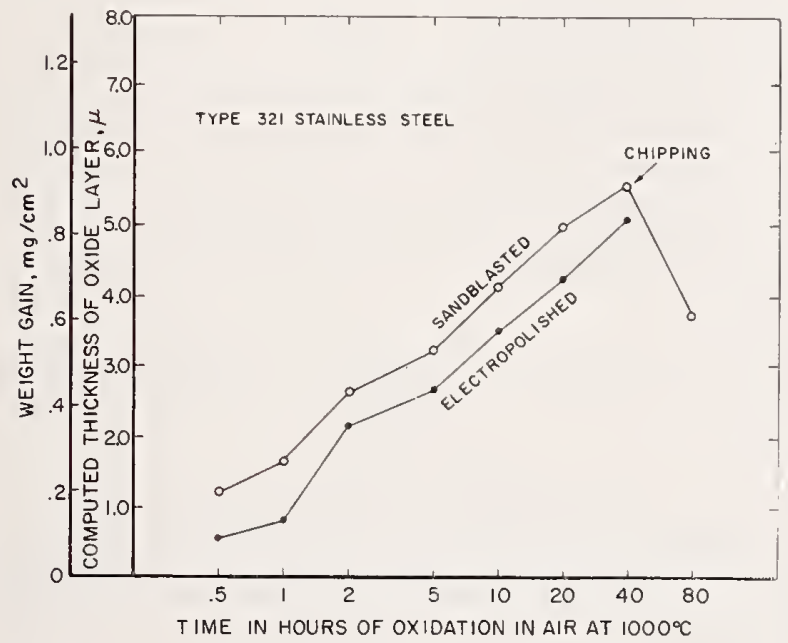

Frgure 11. Weight gain, in $\mathrm{mg} / \mathrm{cm}^{2}$, and computed thickness of the oxide layer, in microns, of specimens of type 321 stainless steel in the electropolished and sandblasted conditions, plotted as a function of time of oxidation in air at $1,000^{\circ} \mathrm{C}$.

least $40 \mathrm{hr}$. The emittance of specimens oxidized for times longer than $40 \mathrm{hr}$ was not determined. The computed thickness of the oxide laver produced by oxidation of electropolished Inconel in air at $1,000^{\circ} \mathrm{C}$ for $40 \mathrm{hr}$ was slightly over $4 \mu$.

The increase in emittance with time of oxidation of the electropolished Inconel could be due to the increase in thickness of the oxide layer, to a gradual increase in roughness of the surface and/or a gradual change in the composition, crystal form, or physical condition of the oxide layer. X-ray diffraction patterns were made of the specimens that had been oxidized for periods of $1 / 2,5,10$, and $40 \mathrm{hr}$. All of the patterns showed the oxide to consist of a nickel-

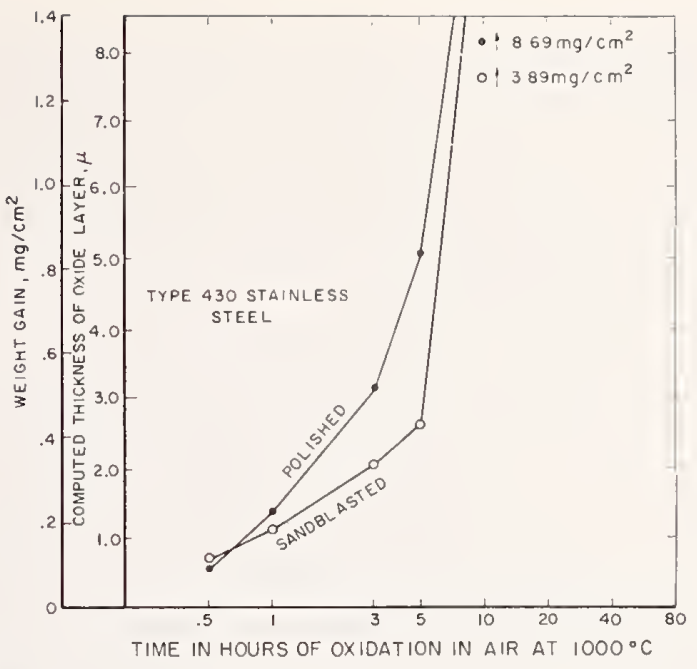

FIGURE 12. Weight gain, in $\mathrm{mg} / \mathrm{cm}^{2}$, and computed thickness of the oxide layer, in microns, of specimens of type 430 stainless steel in the as-received and sandblasted conditions, plotted. as a function of time of oxidation in air at $1,000^{\circ} \mathrm{C}$.

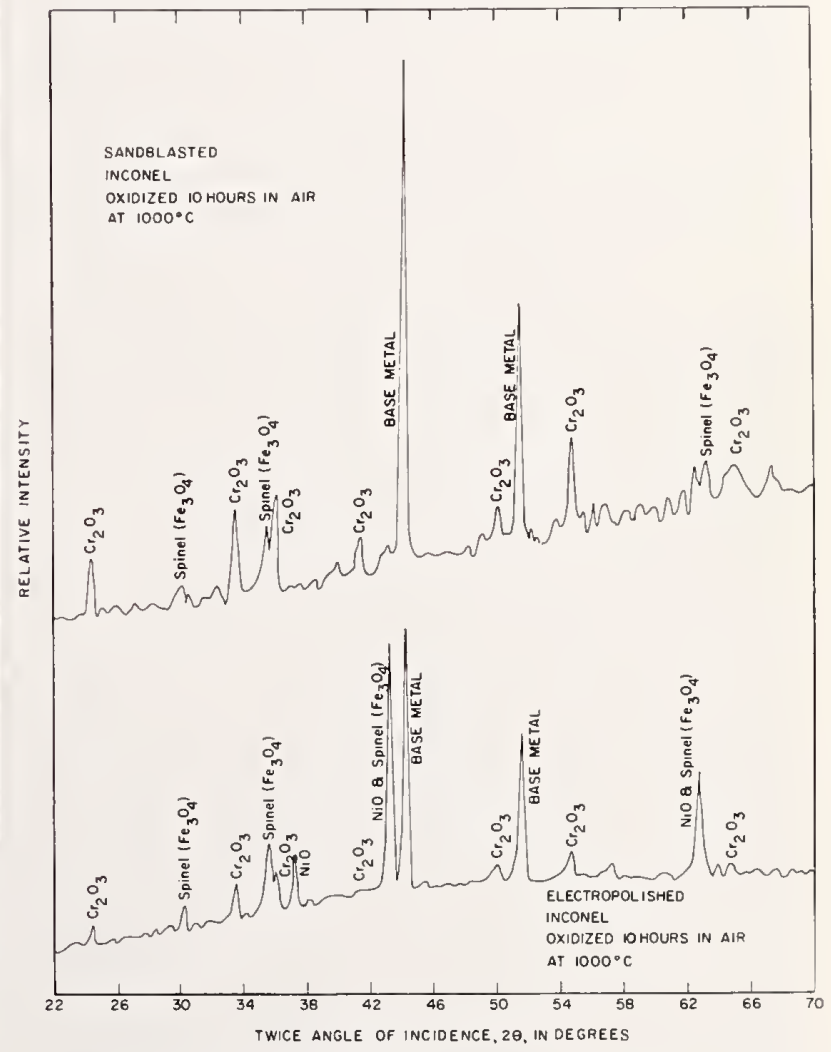

Figure 13. X-ray diffraction patterns of oxidized specimens of electropolished and sandblasted Inconel.

$\mathrm{NiO}$ was found to be a major constituent of the oxide on the electropolished spacimen, but was not found in the oxide on the sandblasted specimen. 
chrome spinel, $\mathrm{NiO}$, and $\mathrm{Cr}_{2} \mathrm{O}_{3}$. The $\mathrm{X}$-ray patterns for the spinel and for $\mathrm{Fe}_{3} \mathrm{O}_{4}$ are practically indistinguishable; however, from thermodynamic considerations, the presence of $\mathrm{Fe}_{3} \mathrm{O}_{4}$ is unlikely. No significant differences in composition or crystal form were found and no marked increase in roughness was observed; hence it is believed that for electropolished Inconel the increase in emittance with an increase in time of oxidation is due to the increase in thickness of the oxide layer.

In the case of sandblasted Inconel, figure 4, there was an increase in emittance with time of oxidation up to $5 \mathrm{hr}$, but there was no significant increase in emittance on increasing the time of oxidation from 5 to $10 \mathrm{hr}$. 'This difference in behavior of the oxide layers on electropolished and sandblasted Inconel was somewhat puzzling, and called for an explanation.

The weight-gain oxidation data in figure 10 shows that the computed thickness of the oxide laver on the electropolished Inconel that had been oxidized for $40 \mathrm{hr}$ was on the order of $4 \mu$ while that of the sandblasted Inconel that had been oxidized for 5 hir was only about $2 \mu$. Actually the difference in true layer thickness is much greater than is indicated by these computed values. Neasurements made in another study [4] showed that the sandblasting treatment increased the surface area by about 50 percent; hence, on this basis, the average thickness of the oxide laver on the sandblasted specimen would be more nearly 1.3 than $2 \mu$. Obviously thickness differences do not account for all the observed difference in emittance behavior between specimens that har been electropolished and sandblasted before oxidation. 'The effect of surface roughness on emittance noted on the unoxidized specimens will, of course, persist in the oxidized specimens.

An X-ray diffraction pattern was made of a specimen of sandblasted Inconel that had been oxidized in air at $1,000{ }^{\circ} \mathrm{C}$ for $10 \mathrm{hr}$. This is compared in figure 13 to a similar pattern from a specimen of electropolished Inconel that had been oxidized in air at $1,000{ }^{\circ} \mathrm{C}$ for $10 \mathrm{hr}$. The curves show that $\mathrm{NiO}$ was a major constituent of the oxide layer on the electropolished Inconel, but was not identified as such in the oxiche layer on the sandblasted specimen. Similar differences in the $\mathrm{NiO}$ content of the respective oxide scales were found in a previous study [5].

It is believed that these observed differences in composition of the oxirie formed on sandblasted and polished Inconel satisfactorily account for the observed difference in emittance behavior. Apparently the oxide layer containing $\mathrm{NiO}$ as a major constituent requires a much greater thickness to become opaque than does the NiO-f'ree oxide layer.

The data for oxidized trpe 321 stainless steel sug'gest that the oxide layer on the electropolished specimens, figure 2 , became essentially opaque after 3 hr of oxidation. The total hemispherical emittance of a specimen oxidized for 3 hr was appreciably greater than that of a specimen oxidized for $1 / 2$ hr, but no difference in emittance was found between specimens oxidized for 3 and $5 \mathrm{hr}$, respectively.
In the case of the sandblasted type 321 stainless steel, figure 5 , the picture becomes more complex. The total hemispherical emittance of all sandblasted oxidized specimens was greater than 0.75 . The emittance of the specimen oxidized for $1 \mathrm{hr}$ was appreciably greater than that of the specimen oxidized for $1 / 2 \mathrm{hr}$, but the emittance decreased on continued oxidation until the emittance at some temperatures after $10 \mathrm{hr}$ of oxidation was lower than that after. $1 / 2 \mathrm{hr}$ of oxidation. In this case there was a change in the appearance of the oxide layer with continued oxidation, which suggests that the composition or structure of the oxide was changing. Small flakes of oxide began chipping from the corners of the specimens after about $3 \mathrm{hr}$ of oxidation, and severe flaking occurred after $40 \mathrm{hr}$ of oxidation. Such a decrease in emittance could also result from a decrease in roughness of the sandblasted surface with continued oxidation, which did occur to some extent.

In the case of type 430 stainless steel rather marked changes in the oxide layer occurred as oxidation progressed. The as-received specimens, figure 3, increased in emittance with time of oxidation up to $5 \mathrm{hr}$, but the enittance decreased appreciably, especially at the higher temperatures, on further oxidation for an additional $5 \mathrm{hr}$. It is interesting to note that the total hemispherical emittance of specimen $\mathrm{A}-5$ that had been oxidized in air at $1,000{ }^{\circ} \mathrm{C}$ for $10 \mathrm{hr}$ showed very little variation with temperature over the range of 300 to $1,000{ }^{\circ} \mathrm{C}$. 'The weight-gain oxidation data in figure 12 show that rate of oxidation increased markedly after about $5 \mathrm{hr}$ of oxidation.

'The general pattern for the sandblasted specimens of type 430 stainless steel that had been oxidized is similar to that for the as-received specimens, but in this case the maximum emittance was obtained after $3 \mathrm{hr}$ of oxidation. The type of oxidation on both as-received and sandblasted type 430 stainless steel changed after $5 \mathrm{hr}$ of oxidation from uniform attack, with a smooth, dense oxide layer, to a pitting type of attack with tubercle formation in the oxide.

There was no indication in the data that significant changes in emittance of any of the oxidized specimens were produced by heating in vacuum for the times required to complete the eraluations.

\subsection{Ceramic-Coated Specimens}

The data for the ceramic-coated specimens, figures 7,8 , and 9 , show that in every case the total hemispherical emittance of the coated specimens decreased with an increase in temperature, in contrast to that of the unoxidized and oxidized metal specimens. 'The effect of increasing temperature in increasing' total normal emittance of bare and oxidized metal, on the one hand, and decreasing the total normal emittance of ceramic-coated metal on the other hand, has been explained [5] on the basis of the differences in the spectral distribution of the emitted energ'r. The same explanation applies to the differences observed in the total hemispherical enittance.

The Inconel and type 430 stainless steel specimens coated with A-418 had higher emittances than the corresponding specimens coated witl $N-143$. In the 
case of the coated type 321 stainless steel specimens, the emittance was nearly the same for specimens 07 and 08 coated with 0.03 and $0.038 \mathrm{~mm}$ respectively of A-418 and specimen 06 coated with $0.05 \mathrm{~mm}$ of $\mathrm{N}-143$. Specimen 05 coated with $0.041 \mathrm{~mm}$ of N-143 had slightly higher emittance than the specimens coated with $\mathrm{A}-418$.

An effect of the base metal on the total hemispherical emittance was to be expected, because the previous report [5] showed that these coatings are partially transparent at thicknesses of less than about $0.13 \mathrm{~mm}$. There was less effect of base metal composition on total hemispherical emittance of specimens coated with $\mathrm{A}-418$ than on those coated with $\mathrm{N}-143$.

Of the specimens coated with $\mathrm{A}-418$, the Inconel specimens had the highest emittance, and the type 430 stainless steel the lowest emittance at each temperature, and the maximum difference was on the order of 10 percent. Of the specimens coated with $\mathrm{N}-143$, emittance was about the same for Inconel and type 321 stainless steel, and appreciably lower for type 430 stainless steel. Also the slope of the curve over the 200 to $600{ }^{\circ} \mathrm{C}$ temperature range for the type 430 stainless steel specimens was steeper than that for the other alloys.

There is no evidence that heating in vacuum at temperatures up to $1,040{ }^{\circ} \mathrm{C}$ for the time required to complete the determinations produced any appreciable change in emittance of the specimens coated with $\mathrm{A}-418$, or in the type 321 or 430 stainless steel specimens coated with $\mathrm{N}-143$. One specimen of Inconel to which coating $\mathrm{N}-143$ was applied at a thickness of $0.056 \mathrm{~mm}$ showed a permanent increase in emittance of 2 to 3 percent after heating in vacuum at $1,015^{\circ} \mathrm{C}$.

\section{Comparison of Results With Published Data}

Total normal emittance determinations were reported by De Corso and Coit [6] for as-rolled and sandblasted Inconel, both in the initially unoxidized condition and after oxidation in air at $982{ }^{\circ} \mathrm{C}(1,800$ ${ }^{\circ} \mathrm{F}$ ) for $15 \mathrm{~min}$, and for Nichrome $\mathrm{V}$ coated with NBS ceramic coating A-417. NBS ceramic coatings $\mathrm{A}-417$ and $\mathrm{A}-418$ are similar but not identical in composition. Similar results were reported by Snyder, Gier, and Dunkle [7] for 18-8 stainless steel, type not specified, in the as-received condition and after mechanical polishing, sandblasting and weathering, and "oxidized 1,500 'F and weathered"; and by Wilkes [8] for types 316 and 347 stainless steels, Inconel $\mathrm{X}$ and $\mathrm{A}$ Nickel in the polished condition. The agreement with the total hemispherical data shown in this paper is only fair in most cases. The differences may be ascribed to (1) inherent differences in normal and hemispherical emittance of materials, and (2) differences in the composition or surface preparation of specimens.

The directional emissivity of a material varies with the angle of emission, due to variations in the transmittance of the material-air interface with angle of incidence. Jakob [9] has computed the theo- retical ratio of normal to hemispherical emissivity of dielectrics to vary from 1.00 at a normal emissivity of 0.50 to a low of 0.935 at a normal emissivity of 0.90 and 0.95 . He also computed [10] the same ratio for electrical conductors, and found it to vary from a high limit of 1.33 for a normal emissivity approaching zero to 1.055 for a normal emissivity of 0.35 . All of these values apply only to completely opaque specimens with optically smooth surfaces, as the term emissivity implies. The trend would be in the same direction for surfaces that are not optically smooth, but it is not possible to compute the ratio for such surfaces, unless the surface contour is known with precision.

The data in figures $1,2,3,4,5$, and 6 show that differences in surface preparation or oxidation treatment can cause appreciable differences in emittance, even for specimens of identical composition. Also, the differences in composition among the three alloys are reflected in differences in emittance. These differences are small for the unoxidized specimens, but become greater after oxidation.

A previous report [5] gave data for total normal emittance computed by integrating normal spectral data obtained on specimens of Inconel and type 321 stainless steel. The data obtained show only fair agreement with the measured values of total hemispherical emittance shown in figures $1,2,4,5,7$, and 8 . Bevans, Gier, and Dunkle [11] found appreciable differences in the total normal emittance of supposedly identical specimens obtained (1) by direct measurement, and (2) by computation from spectial data. It is not surprising that even larger differences were found in the present study between (1) the measured total hemispherical emittance and (2) the total normal emittance computed from spectral data.

O'Sullivan and Wade [12] reported data on the total hemispherical emittance of Inconel that had been mechanically polished and then oxidized in air at $1,093{ }^{\circ} \mathrm{C}\left(2,000{ }^{\circ} \mathrm{F}\right)$ for $13 \mathrm{~min}$. They measured the directional emittance at angles of $0,30,45$, and $60^{\circ}$ to the normal, corrected the values for the actual area of the specimen viewed, plotted the points in polar coordinates, drew a smooth curve through the points and extrapolated it to $90^{\circ}$ to the normal. The total hemispherical emittance was obtained by integrating the resulting curve. Their values were about 0.04 higher than the highest values reported in figure 1 for oxidized Inconel. Iater, Wade [13] reported similar data for the total hemispherical emittance of type 347 stainless steel specimens that were mechanically polished and then oxidized in air at $1,093{ }^{\circ} \mathrm{C}\left(2,000^{\circ} \mathrm{F}\right)$ for $30 \mathrm{~min}$. His reported values are higher than any of the values for oxidized electropolished type 321 stainless steel shown in figure 2 .

The values reported by these authors for the directional emittance of the oxidized Inconel and type 347 stainless steel showed practically no deviation from values that would have been computed from the total normal emittance by the cosine law. Hence, the total hemispherical emittance computed from these values was identical to the total normal emit- 
tance. Jakob [5] showed that for dielectric materials appreciable deviations from the cosine law do not occur at angles of $60^{\circ}$ or less from the normal The values reported by O'Sullivan and Wade [12] and Wade [13] agree with theory, but their method of computation tends to give high values, because all of their measurements were made at angles at which no appreciable deviation from the cosine law would be expected.

\section{Summary and Conclusions}

The total hemispherical emittance of Inconel and types 321 and 430 stainless steel specimens in six different surface finishes was evaluated by a modification of the hot-filament method. The six surface finishes were (1) polished, (2) polished and then oxidized, (3) sandblasted, (4) sandblasted and then oxidized, (5) coated with A-418, and (6) coated with $\mathrm{N}-143$. The findings may be summarized briefly as follows:

(1) For the three alloys studied, the surface condition of the metal had much more effect on the total hemispherical emittance of the unoxidized and uncoated specimen than did the composition of the alloys. Each of the polished specimens had total hemispherical emittance in the range 0.20 to 0.28 , and the emittance increased with an increase in temperature. Each of the sandblasted specimens had total hemispherical emittance in the range 0.50 to 0.65 and again the emittance increased with an increase in temperature.

(2) Oxidation in air at $1,000{ }^{\circ} \mathrm{C}$ greatly increased the total hemispherical emittance of each uncoated specimen tested, and the emittance increased with time of oxidation for at least several hours. The total hemispherical emittance of the oxidized specimens increased with an increase in temperature.

(3) All ceramic-coated specimens evaluated had relatively high total hemispherical emittance at low temperatures, and the emittance decreased with an increase in temperature. The coatings were not opaque, as indicated by the fact that the composition of the substrate metal affected the total hemispherical emittance of the composite specimens. The ceramic-coated specimens of Inconel had the highest total hemispherical emittance and the ceramic-coated type 430 stainless steel specimens had the lowest emittance in each case.

(4) The total hemispherical emittance of Inconel and type 430 stainless steel specimens coated with A-418 was higher than that of similar specimens coated with $\mathrm{N}-143$.

(5) The total hemispherical emittance of sandblasted specimens of all three alloys decreased markedly on heating in vacuum at temperatures above $900{ }^{\circ} \mathrm{C}$, even for the short times required to complete the evaluations. No appreciable changes were noted in the emittance of polished, oxidized or ceramic-coated specimens as a result of heating in vacuum for the times required to complete the evaluations, even at temperatures up to $1,000{ }^{\circ} \mathrm{C}$, except for one specimen of Inconel coated with $\mathrm{N}-143$, that showed a slight increase in emittance on heat in vacuum to $1,015^{\circ} \mathrm{C}$.

The work described in this report was completed under the sponsorship and with the financial assistance of the Army Ballistic Missile Agency, now the George C. Marshall Space Flight Center of the National Aeronautical and Space Agency, Redstone Arsenal, Huntsville, Ala.

\section{References}

[1] A. G. Eubanks and D. G. Moore. Development of erosion resistant coatings for aircraft engine components, WADC Technical Report 56-129 (Wright Air Development Center, January 1956) 34 pp.

[2] J. C. Richmond, H. G. Lefort, C. N. Williams, and W. N. Harrison, Ceramic coatings for nuclear reactors, J. Am. Ceram. Soc. 38, [2] 72 (1955).

[3] J. C. Richmond and W. N. Harrison, Equipment and procedures for evaluation of total hemispherical emittance, Am. Ceram. Soc. Bull. 39, [11] pp. 668-673 (Nov. 1960).

[4] A. G. Eubanks, D. G. Moore, and W. A. Pennington, The effect of surface contour on the oxidation rate of ingot iron J. Electrochem. Soc., [5] 109, 382-389 (1962).

[5] J. C. Richmond and J. E. Stewart. Spectral emittance of uncoated and ceramic-coated Inconel and type 321 stainless steel, NASA Memorandum 4-8-59W (Apr. 1959) $30 \mathrm{pp}$.

[6] S. M. DeCorso and R. L. Coit. Measurement of total emissivities of gas turbine combustor materials, Trans. ASME 7\%, [8] pp. 1189-1197 (Nov. 1955).

[7] N. W. Synder, J. T. Gier, and R. V. Dunkle, Total normal emissivity measurements on aircraft materials between 600 and $800^{\circ} \mathrm{F}$, Trans. ASME \%7, [7] 10111019 (Oct. 1955).

[8] G. B. Wilkes, Total normal emissivities and solar absorptivities of materials, WADC Technical Report $54-42$.

[9] Max Jakob, Heat Transfer, Vol. I, p. 43 (J. Wiley \& Sons, Inc., N.Y., 1949).

[10] Ibid-p. 51 .

[11] J. T. Bevans, J. T. Gier, and R. V. Dunkle. Comparison of total emittances with values computed from spectral measurements, Trans. ASME 80, (7) 1405-1416 (Oct. 1958).

[12] W. J. O'Sullivan, Jr. and W. R. Wade, Theory and apparatus for measurement of emissivity for radiative cooling of hypersonic aircraft with data for Inconel and Inconel X, NACA Technical Note 4121 (Oct. 1957) $48 \mathrm{pp}$.

[13] W. R. Wade, Measurements of total hemispherical emissivity of several stably oxidized metals and some refractory oxide coatings, NASA Memorandum 1-20-59L (Jan. 1959) 30 pp.

(Paper 66C3-102) 


\title{
Spectral Emittance of Ceramic-Coated and Uncoated Specimens of Inconel and Stainless Steel
}

\author{
by JOSEPH C. RICHMOND and JAMES E. STEWART
}

Notionol Bureas of Standards, Washington, D. C.

\begin{abstract}
The normal spectral emittance of Inconel and type 321 stainless steel specimens with six different surface treatments, including application of National Bureau of Standards ceramic coatings $\mathrm{A}-418$ and $\mathrm{N}-143$, was determined at $900^{\circ}$, $1200^{\circ}, 1500^{\circ}$, and $1800^{\circ} \mathrm{F}$. over the wave-length range 1.5 to $15 \mu$. The specimens with different surface treatments had markedly different spectral emittance curves. The bare polished specimens had the lowest spectral emittance at all wave lengths, and the specimens coated with A-418 had the highest spectral emittance over most of the range 5 to $15 \mu$. The spectral emittance of all specimens increased with temperature at all wave lengths.
\end{abstract}

\section{Insroduction}

M ODERN aircraft and missiles operating at supersonic speeds in the earth's atmosphere develop high temperatures, due to skin friction, that may cause undesirable creep and corrosion of metallic components. There is a tendency for such aerodynainic heating to be concentrated at areas of direct impingement, such as the leading edges of wings and the nose cones of missiles. As a result, large thermal gradients develop, causing distortion and impairment of aerodynamic efficiency. In severe cases, this heating may cause total destruction of structural components. Heattransfer processes in such structures are obviously important because they affcct the temperature and the thermal gradients.

Radiation plays an increasingly important role in heat transfer as the temperature of the hot body increases, and as the density of the atmosphere decreases. Hence there is an urgent need for information on the thermal-emission properties of aircraft structural materials at elevated temperatures, and the possibilities of their improvement by the use of coatings.

Some data on total emittance of metals and coatings have been reported in the literature. ${ }^{1}$ The amount of radiant energy emitted by a hot body at a known uniform temperature can be computed from its total hemispherical emittance. Computation of heat transfer between two bodies, however, requires consideration of the geometric relations of the bodies and the spectral distribution of the radiant energy emitted by, and incident upon, each body. Spectral emittance values are required for such computations, and few such values are to be found in the literature.

A gray body has the same spectral emittance at all wave lengths, and the spectral emittance at any wave length is the same as the total emittance. Assumptions have been made that some materials conform approximately to these conditions except at very short wave lengths. Ceramic materials and painted or oxidized metals, however, are not gray bodies, but show variation in emittance with wave length. Most of the metals used in aircraft structures are pairited, or soon accumulate an oxide film in service and lose the radiation characteristics of the metal.

Goodman, ${ }^{2}$ in computing radiant heat transfer between parallel plates of polished aluminum and asi-rolled Inconel, found that the moderate deviations of these materials from true gray-body emission caused the heat transfer rates computed from total emittance values to be as much as $29 \%$ lower than those computed from spectral emittance data. Errors for ceramic materials and painted or oxidized metals which in general depart significantly from gray-body emission, would undoubtedly be much larger.

\section{Experimental Procedure}

Two different metals were studied, type 321 stainless steel and Inconel. Type 321 stainless steel is a titanium-stabilized $18 \%$ chromium, $8 \%$ nickel austenitic stainless steel. Inconel is nominally $80 \%$ nickel, $14 \%$ chromium, and $6 \%$ iron.

The metal specimens were in the form of 0.050 -in. strips, 6 in. long and $3 / 4$ in. wide at the ends. The center portion of each specimen was necked down to a width of $0.300 \mathrm{in}$. for a distance of $2.4 \mathrm{in.}$ A hole $0,040 \mathrm{in}$. in diameter and about $0.045 \mathrm{in}$. deep was drilled near the center of the necked-down portion of each specimen. A base-metal thermocouple having a bead about 0.038 in. in diameter was inserted into this hole and peened in before the final surface treatment of the specimen.

Surface treatments included (1) electropolishing, (2) sandblasting, accomplished by means of a 40 -mesh glass sand and air at a pressure of $40 \mathrm{lb}$. per sq. in., (3) oxidation in air at $1800^{\circ} \mathrm{F}$. for $1 / 2$ hour following electropolishing, (4) oxidation in air at $1800^{\circ} \mathrm{F}$. for $1 / 2$ hour following sandblasting, (5) finishing with NBS coating $\mathrm{N}-143$, and (6) finishing with NBS coating A-418.

The electropolishing and coating finishes were applied only to the necked-down portion of a specimen. The ends of the oxidized and coated specimens were sandblasted following the respective treatments to provide good contact for the electrodes.

The two ceramic coatings have been described in previous reports. ${ }^{3}$ Coating $\mathrm{N}-143$ consists of a boron-free barium

Presented at the Fifty-Ninth Annual Meeting, The American Ceramic Society, Dallas, Texas, May 8, 1957 (Enamel Division, No. 18). Received April 9, 1959; revised copy received July 13 1959 .

This work was performed at the National Bureau of Standards under the sponsorship and with the financial assistance of the National Advisory Committee for Aeronautics (now the National Aeronautics and Space Administration).

At the time this work was done, the writers were, respectively, ceramic engineer, Enameled Metals Section, and chemist, Gas Chemistry Section, National Bureau of Standards. J. E. Stewart is now with Beckman Instruments, Incorporated, Fullerton, California.

${ }^{\prime}$ G. G. Gubareff, Shao-Yen Ko, and P. E. McNall, Jr., "Review of Thermal Radiation Property Values for Metals and Other Materials," GR 2462-R3, Minneapolis-Honeywell Regulator Co., Hopkins, Minn., August 1957.

2 Stanley Goodman, "Radiant-Heat Transfer Between Nongray Parallel Plates,"'J. Research Natl. Bur. Standards, 58 [1] 37-40 (1957); RP 2732.

${ }^{3}$ (a) J. C. Richmond, H. G. Lefort, C. N. Williams, and W. N. Harrison, "Ceramic Coatings for Nuclear Reactors," J. Am. Ceram. Soc., 38 [2] 72-80 (1955).

(b) A. G. Eubanks and D. G. Moore, "Development of Erosion Resistant Coatings for Aircraft Engine Components," WADC (Wright Air Develop. Center) Tech. Rept., No. 56-129. $34 \mathrm{pp}$. (January 1956). 
beryllium silicate frit, with a refractory mill addition of cerium oxide. It is light cream in color. Coating A-418 consists of an alkali-free barium borosilicate frit with a refractory mill addition of chromic oxide. It is dark green.

The coatings are normally applied at a thickness of approximately 0.002 in., and most of the coated specimens for test were prepared at this thickness. For a few tests, coatings of greater thickness (up to 0.0085 in.) were used.

The equipment and procedure used to evaluate normal spectral emittance have been described previously ${ }^{4}$ and are briefly outlined here.

A recording double-beam infrared spectrophotometer with a sodium chloride prism was used for all measurements. The external optics of the instrument were modified as indicated in footnote 4 to permit the radiant energy from either the specimen or the standard to be compared with that from a Nernst glower source. A recrystallized silicon carbide heating element was used as the standard in all measurements. The normal spectral emittance of this element had been determined previously ${ }^{4}$ by comparison with a laboratory blackbody furnace.

The specimen and the comparison standard were heated by passing a 60-cycle current through them. The temperature was held to $\pm 3^{\circ} \mathrm{F}$. of that desired by manual adjustment of the power input. The temperature of the standard was measured by means of a Chromel-Alumel thermocouple, the head of which was wedged into a 0.040 -in.-in-diameter hole near the center of the element, extending from one side to within about 0.015 in. of the opposite side. The temperature of a specimen was measured by means of the Chromel-Alumel thermocouple, the bead of which was peened in to the hole at the center of the specimen.

In making a determination, the specimen and standard were mounted side by side near the instrument, in a position such that an image of either could be focused on the entrance slit of the monochromator, and were brought to the same temperature. A curve representing the ratio of the radiant flux of the standard to that of the Nernst glower was obtained over the range 1.25 to $15 \mu$, after which a second curve was obtained representing the corresponding ratio for the specimen. The ratio of the heights of the two curves was then computed at $0.25-\mu$ wave-length intervals over the entire range. Each value thus obtained was multiplied by the emittance of the standard, at that wave length, as previously determined. to obtain the normal spectral emittance of the specimen. Determinations were made at temperatures of $900^{\circ}, 1200^{\circ}, 1500^{\circ}$, and $1800^{\circ} \mathrm{F}$.

No correction was made for the thermal gradient through the coating of normal $(0.002$-in.) thickness, because it was estimated that the gradient was smaller than the normal $\pm 3^{\circ} \mathrm{F}$. fluctuation in temperature of the metal specimen. Sully et al. ${ }^{5}$ reported measured thermal gradients in ceramic coatings on the order of $1{ }^{\circ} \mathrm{F}$. per mil of thickness.

Several techniques were considered for measuring the surface temperature directly on specimens with the thicker coatings, but the experimental difficulties of obtaining accurate surface temperatures of coatings in this thickness range were believed to be too great. It was finally decided to maintain the specimen at a definite metal temperature, and then to compute the temperature drop through the coating from a knowledge of the thermal conductivity of the coating layer, its thickness, the surface area of the specimen, and the heat dissipation rate.

4 J. E. Stewart and J. C. Richmond, "Infrared Emission Spectrum of Silicon Carbide Heating Elements," $J$. Research Nall. Bur. Standards, 59 [6] 405-409 (1957); RP 2810; Ceram. Abstr., 1958, April, p. $104 f$.

${ }^{\circ}$ A. H. Sully, E. A. Brandes, and R. B. Waterhouse, "Radiation-Sinppressing Coatings for Metals at Elevated Temperatures," Fulmer Research Inst. Spec. Rept., No. 1, 24 pp. (April 1953).
The heat dissipation rate was computed from the measured power input. Power-input measurements were made on metal specimens of the same design as that of those used for emittance measurements. These specimens also contained a thermocouple peened into a hole near the center. Two small areas, about $1 \frac{1}{2}$ in. apart, about equally spaced on either side of the center of the necked-down portion of the specimen, were cleaned by sandblasting, and light scratches, perpendicular to the axis of the specimen, were made in each cleaned area. Fine Nichrome wires for potential taps were laid in the scratches and spot welded to the specimen. Before making a test the distance between these two wires was measured to the nearest 0.01 in. by means of a vernier caliper, and the width and thickness of the specimen was measured to the nearest $0.001 \mathrm{in}$. by means of a micrometer califer.

In making a test, the specimen was heated by passing an electric current through it, as in the emittance measurements. The power input was adjusted until the temperature of the specimen was stabilized at the desired temperature, the current in the circuit was recorded to the nearest $0.1 \mathrm{amp}$, and the potential across the measured gauge length was recorded to the nearest 0.01 volt. Tests were made at temperatures of $900^{\circ}, 1200^{\circ}, 1500^{\circ}$, and $1800^{\circ} \mathrm{F}$

The power input to the specimen over the meacured gauge length was computed from the measured current and potential drop. The surface area of the measured gauge length was computed, and the power input was expressed as B.t.u. per sq. foot per hour. There was some heat loss due to conduction through the ends of the specimens, but means were not available to compute its magnitude and it was neglected.

The thermal conductivity of the coatings was not accurately known, but thermal conductivities of other ceramic coatings had been measured previously, and were found to be in the range 6 to 9 B.t.u. per sq. ft. per hour ( ${ }^{\circ} \mathrm{F}$. per in.). A value of 7.5 was assumed for the coatings, and the thermal gradient, in ${ }^{\circ} \mathrm{F}$. per mil, was computed from the measured rate of heat flow. In the emittance determinations on specimens having thick coatings, the measured temperature of the metal specimen was corrected for the computed gradient to obtain the surface temperature of the coating.

\section{Presenfation and Discussion of Dafa}

The data on spectral emittance of the metal specimens at $900^{\circ}, 1200^{\circ}, 1500^{\circ}$, and $1800^{\circ} \mathrm{F}$. for wave lengths at $0.5-\mu$ intervals from 1.5 to $15 \mu$ are shown in Table I.

The spectral emittance curves for type 321 stainless steel at $900^{\circ} \mathrm{F}$. are shown in Fig. 1. The general features of interest in this figure are the relatively flat, low curve for the electropolished metal, the relatively flat but somewhat higher curve for the sandblasted metal, the much higher curves for the oxidized specimens that fall to lower values at the longer wave lengths, and the rapid increase in emittance between 2 and $5 \mu$ for the coated specimens and the relatively high emittance of those specimens at longer wave lengths. The emittance of the specimen coated with $\mathrm{A}-418$ was higher than that of the specimen coated with $\mathrm{N}-143$ at all wave lengths.

All the curves for stainless steel at $1800^{\circ} \mathrm{F}$. (Fig. 2) are displaced upward relative to those at $900^{\circ} \mathrm{F}$. The polished and sandblasted specimens oxidized too rapidly at this temperature to permit evaluation in the unoxidized condition.

The curves for Inconel at $900^{\circ} \mathrm{F}$. are shown in Fig. 3 . The curves for the polished and sandblasted metal are approximately the same as those for the type 321 stainless steel, and the curves for the coated specimens are similar in shape to the corresponding curves for stainless steel, but are displaced upward, particularly the curve for the specimen coated with $\mathrm{N}$ 143. The curves for the oxidized specimens of Inconel are quite different from the corresponding curves for oxidized type 321 stainless steel. The specimen oxidized after polishing had higher emittance at all wave lengths than did the specimen oxidized after sandblasting and the curves for the oxidized specimens, particularly the prepolished one, do not show 
Table I. Spectral Emittance Data for Metal Specimens

\begin{tabular}{|c|c|c|c|c|c|c|c|c|c|c|c|c|c|c|c|}
\hline & & & & & & Spectra & l emitta & nce $\langle\mu\rangle$ & t indica & ted wave & length & & & & \\
\hline $\begin{array}{l}\text { 1ype or } \\
\text { specimen }\end{array}$ & & 1.5 & 2.0 & 2.5 & 3.0 & 3.5 & 4.0 & 4.5 & 5.0 & 5.5 & 6.0 & 6.5 & 7.0 & 7.5 & 8.0 \\
\hline Electropolished stainless steel & 900 & & 0.12 & 0.15 & 0.15 & 0.15 & 0.15 & 0.15 & 0.14 & 0.14 & 0.14 & 0.13 & 0.13 & 0.13 & 0.13 \\
\hline & 1200 & 0.29 & .27 & .25 & .24 & .22 & .21 & .19 & .18 & .17 & .15 & .15 & .15 & .14 & .14 \\
\hline Sandblasted stainless steel & 900 & & & .34 & .36 & .38 & .39 & .39 & .40 & .41 & .42 & .41 & .40 & .39 & .38 \\
\hline & 1200 & .34 & .36 & .37 & .38 & .41 & .41 & .41 & .40 & .41 & .40 & .28 & .38 & .38 & .39 \\
\hline Electropolished oxidized stainless & 900 & & .63 & .66 & 68 & 69 & .69 & .67 & .64 & .65 & .68 & .68 & .69 & .70 & .73 \\
\hline steel & 1200 & & .60 & .66 & .69 & .70 & .69 & .68 & .65 & .68 & .69 & .71 & .72 & .73 & .73 \\
\hline & 1500 & .70 & .76 & .78 & .79 & 78 & .76 & .74 & .68 & .73 & .74 & .76 & .77 & .78 & .78 \\
\hline & 1800 & .67 & .73 & .75 & .77 & .77 & .77 & .76 & .70 & .77 & .78 & .80 & .78 & .77 & .76 \\
\hline Sandblasted oxidized stainless & 900 & & .52 & .59 & .65 & .67 & 69 & .69 & .69 & .70 & .71 & .70 & .70 & .71 & .73 \\
\hline steel & 1200 & .65 & 65 & 68 & .72 & .73 & .74 & .75 & .75 & .74 & .74 & .74 & .73 & .75 & .76 \\
\hline & 1500 & 65 & .65 & 68 & 72 & .73 & .74 & .75 & .76 & .76 & .76 & .75 & .75 & .75 & .76 \\
\hline & 1800 & 65 & .71 & .77 & .79 & .82 & .82 & .80 & .76 & .77 & .76 & .75 & .75 & .75 & .77 \\
\hline Stainless steel coated A-418 & 900 & & .29 & .35 & .41 & .49 & .56 & .63 & .69 & .74 & .77 & .79 & .81 & .83 & .84 \\
\hline (approx. 2 mils) & 1200 & .31 & .36 & .41 & .46 & .53 & .58 & .61 & .70 & .74 & .77 & .79 & .81 & .83 & .84 \\
\hline & 1500 & .38 & .45 & .49 & .55 & .64 & 69 & .71 & .78 & .82 & .84 & .86 & .88 & .89 & .89 \\
\hline & 1800 & .39 & .46 & .54 & .60 & .66 & .71 & .75 & .78 & .82 & .85 & .86 & .89 & .89 & .90 \\
\hline Stainless steel coated $\mathrm{N}-143$ & 900 & & .08 & .14 & .20 & .25 & .31 & .35 & .44 & .50 & .52 & .54 & .55 & .58 & .60 \\
\hline (approx. 2 mils) & 1200 & .04 & 14 & .22 & .27 & .31 & .34 & .39 & .49 & .54 & .56 & .59 & .60 & .63 & .65 \\
\hline & 1500 & .08 & .16 & .23 & .29 & .34 & .38 & .43 & .54 & .60 & .63 & .65 & .66 & .67 & .69 \\
\hline & 1800 & .15 & .22 & .30 & .36 & .43 & .47 & .51 & .58 & .63 & .66 & .67 & .68 & .69 & .70 \\
\hline Electropolished Inconel & 900 & & .20 & .18 & .17 & .17 & .17 & .16 & .16 & .15 & .15 & .15 & .14 & .14 & .13 \\
\hline & 1200 & & .30 & .24 & .21 & .19 & .18 & .17 & 16 & .17 & .16 & .15 & .15 & .15 & .15 \\
\hline Sandblasted Inconel & 900 & & .34 & .34 & .35 & .36 & .37 & .36 & .35 & .34 & .33 & .33 & .33 & .33 & .33 \\
\hline & 1200 & .41 & .38 & 41 & .41 & .40 & .39 & .39 & .38 & .37 & .36 & .36 & .36 & .35 & .35 \\
\hline Electropolished oxidized 1 nconel & 900 & & & $.5 \overline{7}$ & .59 & .60 & .60 & .59 & .59 & .59 & .58 & .57 & .62 & .70 & .75 \\
\hline & 1200 & .58 & .59 & .60 & 61 & .61 & .63 & .62 & .61 & .60 & .59 & .59 & .64 & .71 & .75 \\
\hline & 1500 & .68 & .71 & .72 & .69 & .71 & .69 & .66 & .65 & .65 & .64 & .66 & .68 & .74 & .78 \\
\hline & 1800 & .70 & .75 & .76 & .75 & .79 & .76 & .72 & .71 & .69 & .68 & 68 & .70 & .74 & .77 \\
\hline Sandblasted oxidized Inconel & 900 & & & .44 & .47 & .49 & .51 & .53 & .55 & .55 & .56 & .56 & .62 & .66 & 68 \\
\hline & 1200 & & .50 & .51 & .51 & .53 & .54 & .55 & .57 & .56 & .56 & .56 & .63 & .66 & .67 \\
\hline & 1500 & & .49 & .51 & .53 & .54 & .56 & .57 & .57 & .58 & .58 & .59 & .65 & .67 & .69 \\
\hline & 1800 & .52 & .55 & .56 & .57 & .60 & .60 & .58 & .58 & .59 & .60 & .59 & .63 & .64 & .65 \\
\hline Inconel coated A-418 (approx. 2 & 900 & .22 & .22 & .39 & .46 & .53 & .58 & .64 & .73 & .78 & .82 & .84 & .86 & .88 & .90 \\
\hline mils) & 1200 & .33 & .39 & .44 & .51 & .55 & .61 & .66 & .76 & .78 & .82 & .85 & .87 & .88 & .89 \\
\hline & 1500 & .40 & .46 & .51 & .57 & .64 & .68 & .70 & .78 & .83 & .85 & .88 & .89 & .90 & .90 \\
\hline & 1800 & .44 & .51 & .58 & .62 & .69 & .73 & .75 & .80 & .84 & .87 & .88 & .91 & .90 & .92 \\
\hline 1nconel coated N-143 (approx. 2 & 900 & & .17 & .26 & .34 & .39 & .43 & .48 & .60 & .65 & .68 & 69 & .71 & .75 & .79 \\
\hline mils & 1200 & .18 & .24 & .31 & .37 & .42 & .48 & .54 & .66 & .69 & .73 & .76 & .77 & .80 & .83 \\
\hline & 1500 & .23 & .28 & .35 & 42 & .46 & .49 & .55 & .70 & .76 & .78 & .81 & .83 & .84 & .85 \\
\hline & 1800 & .26 & .33 & .41 & .46 & .53 & .58 & .61 & .72 & .78 & .81 & .83 & .85 & .86 & .88 \\
\hline & & 8.5 & 9.0 & 9.5 & 10.0 & 10.5 & 11.0 & 11.5 & 12.0 & 12.5 & 130 & 13.5 & 14.0 & 14.5 & 15.0 \\
\hline Electropolished stainless steel & 900 & 0.13 & 0.13 & 0.12 & 0.12 & 0.11 & 0.11 & 0.11 & 0.11 & 0.11 & 0.11 & 0.11 & 0.10 & 0.10 & 0.09 \\
\hline & 1200 & .14 & .14 & .13 & .13 & .13 & .13 & .12 & .12 & .12 & .12 & 11 & .11 & .11 & .10 \\
\hline Sandblasted stainless steel & 900 & .39 & .41 & .41 & .40 & .39 & .39 & 40 & .40 & .41 & .41 & .42 & .42 & .41 & .40 \\
\hline & 1200 & .40 & .41 & .41 & .41 & .42 & .41 & 41 & .40 & .42 & .44 & .44 & .44 & .43 & .42 \\
\hline Electropolished oxidized stainless & 900 & .76 & .77 & .75 & .69 & .65 & .63 & .59 & .54 & .49 & .43 & .38 & .34 & .34 & .34 \\
\hline steel & 1200 & .74 & .75 & .74 & .73 & .70 & .67 & .64 & .59 & .52 & .46 & .41 & .37 & .36 & .36 \\
\hline & 1500 & .79 & .80 & .78 & .76 & .74 & .71 & .68 & .63 & .58 & .51 & .46 & .42 & .40 & .39 \\
\hline & 1800 & .76 & .77 & .75 & .72 & 69 & .66 & .64 & .61 & .57 & .54 & .49 & .44 & 42 & .40 \\
\hline Sandblasted oxidized stainless & 900 & .77 & .83 & .82 & .82 & .84 & .86 & .85 & .81 & .78 & .75 & .72 & .68 & .65 & .63 \\
\hline steel & 1200 & .78 & .83 & .84 & .84 & .85 & .86 & .86 & .86 & .83 & .80 & .77 & .75 & .72 & .69 \\
\hline & 1500 & .78 & .83 & .84 & .84 & .84 & .84 & .84 & .84 & .81 & .78 & .76 & .73 & .70 & .67 \\
\hline & 1800 & .81 & .84 & .85 & .85 & .85 & .86 & .87 & .87 & .86 & .83 & .82 & .81 & .76 & .71 \\
\hline ted $A-418$ & 900 & .85 & .85 & .82 & .78 & .77 & .77 & .78 & .80 & .84 & .86 & .88 & .89 & .89 & .88 \\
\hline (approx. 2 mils) & 1200 & .85 & 85 & .81 & .78 & .77 & .77 & .78 & .82 & .86 & .86 & .88 & .93 & .93 & .90 \\
\hline & 1500 & .90 & .90 & .88 & .85 & .83 & .82 & .82 & .83 & .87 & .89 & .90 & .89 & .92 & .92 \\
\hline & 1800 & .93 & .95 & .94 & .88 & .86 & .83 & .82 & .84 & .89 & .93 & .95 & .91 & .89 & .87 \\
\hline d N-143 & 900 & .61 & 61 & .59 & .56 & .57 & .60 & .63 & .65 & .68 & .69 & .69 & 69 & .68 & .66 \\
\hline (approx. 2 mils) & 1200 & .66 & .66 & .62 & .60 & .60 & .61 & .64 & .66 & .68 & .69 & .70 & .70 & .59 & .67 \\
\hline & 1500 & .71 & .70 & .66 & .63 & .62 & .64 & .66 & .67 & .68 & .70 & .71 & .72 & .71 & 69 \\
\hline & 1800 & .73 & .73 & .69 & .65 & .65 & .65 & .67 & .70 & .73 & .76 & .78 & .75 & .74 & .71 \\
\hline Electropolished 1nconel & 900 & .14 & .13 & .12 & .11 & .11 & .11 & .11 & .10 & .10 & .10 & .10 & .09 & .09 & .08 \\
\hline & 1200 & .15 & .15 & .15 & .14 & .14 & .14 & .14 & .14 & .13 & .13 & .13 & 13 & .12 & .12 \\
\hline Sandblasted Inconel & 900 & .34 & .36 & .35 & .35 & .35 & .36 & .36 & .36 & .35 & .35 & .35 & .35 & .35 & .35 \\
\hline & 1200 & .36 & .37 & .36 & .36 & .36 & .36 & .37 & .37 & .37 & .37 & .39 & .39 & .39 & .38 \\
\hline Electropolished oxidized 1nconel & 900 & .78 & .78 & .73 & .70 & .72 & .73 & .73 & .74 & .75 & .76 & .79 & .81 & .81 & .80 \\
\hline & 1200 & .78 & .79 & .79 & .80 & .78 & .77 & .77 & .77 & .78 & .79 & .81 & .84 & .83 & .82 \\
\hline & 1500 & .78 & .79 & .78 & .78 & .77 & .76 & .76 & .78 & .79 & .80 & .81 & .82 & .83 & .83 \\
\hline & 1800 & .79 & .80 & .80 & .78 & .78 & .79 & .81 & .83 & .85 & .87 & .87 & .87 & .85 & .84 \\
\hline Sandblasted oxidized 1 nconel & 900 & .69 & .70 & .67 & 61 & .59 & .58 & .56 & .54 & .52 & .52 & .52 & .52 & .52 & .52 \\
\hline & 1200 & .68 & .67 & .65 & .61 & .60 & .58 & .57 & .53 & .52 & .51 & .52 & .53 & .53 & .53 \\
\hline & 1500 & .70 & .70 & .61 & .65 & .63 & .61 & .58 & .56 & .54 & .53 & .53 & .53 & .53 & .53 \\
\hline & 1800 & .65 & .66 & .66 & .62 & .59 & .57 & .55 & .54 & .55 & .53 & .53 & .52 & .51 & .51 \\
\hline Inconel coated A-418 (approx. 2 & 900 & .92 & .93 & .88 & .85 & .84 & .83 & .83 & .84 & .88 & .91 & .94 & .95 & .96 & .96 \\
\hline mils) & 1200 & .90 & .90 & .87 & .82 & .81 & .80 & .82 & .83 & .87 & .90 & .91 & .92 & .93 & .92 \\
\hline & 1500 & .91 & .91 & .88 & .84 & .83 & .82 & .82 & .86 & .88 & .90 & .91 & .93 & .93 & .91 \\
\hline & 1800 & .94 & .96 & .93 & .88 & .86 & .83 & .83 & .85 & .91 & .94 & .95 & .93 & .91 & .88 \\
\hline Inconel coated N-143 (approx. 2 & 900 & .82 & .82 & .72 & 69 & .71 & .74 & .77 & .80 & .83 & .84 & .85 & .85 & .84 & .81 \\
\hline & 1200 & .85 & .84 & .78 & .73 & .74 & .77 & .80 & .86 & .89 & .87 & .88 & .88 & .87 & .86 \\
\hline & 1500 & .88 & .86 & .81 & .77 & .77 & .78 & .80 & .83 & .86 & .87 & .87 & .86 & .86 & .85 \\
\hline & 1800 & .91 & .91 & .85 & .79 & .77 & .78 & .81 & .87 & .90 & .92 & .92 & .88 & .87 & .86 \\
\hline
\end{tabular}




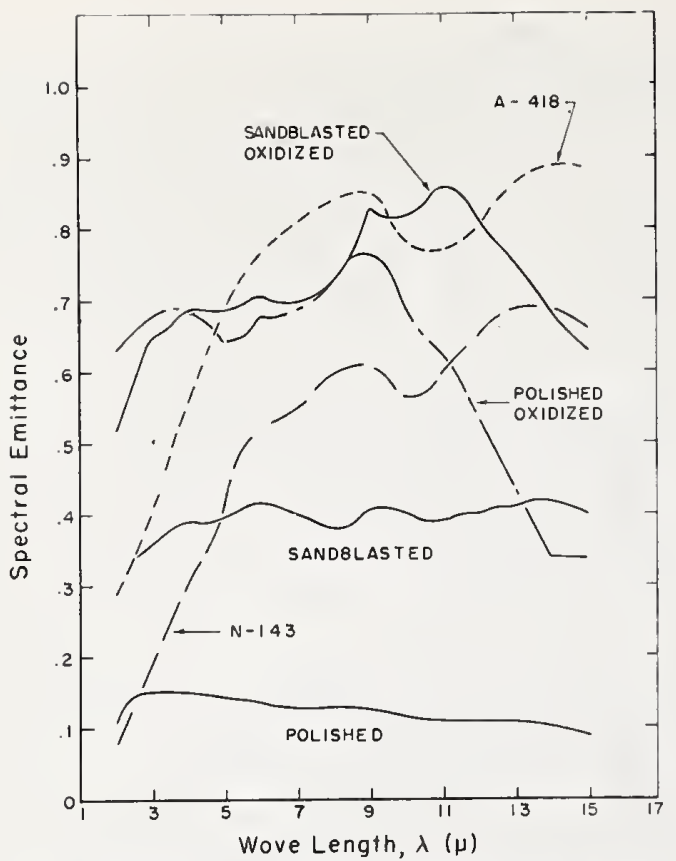

Fig. 1. Spectrol emittance curves at $900^{\circ} \mathrm{F}$. for type 321 stoinless steel specimens with different surfoce treatments; coating thickness approximately 2 mils.

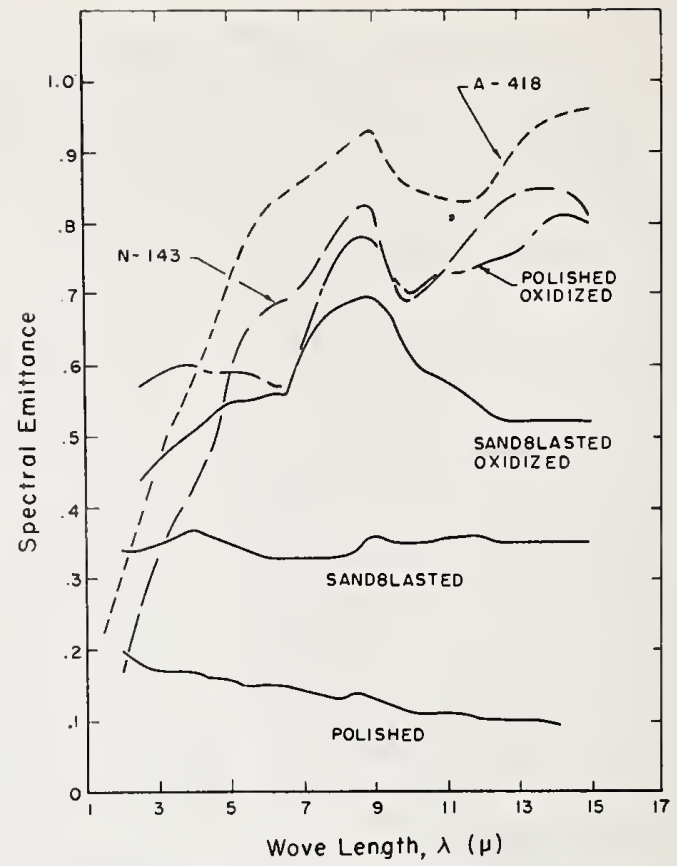

Fig. 3. Spectral emittonce curves of $900^{\circ} \mathrm{F}$. for Inconel specimens with different surface treotments; cooting thickness opproximotely 2 mils.

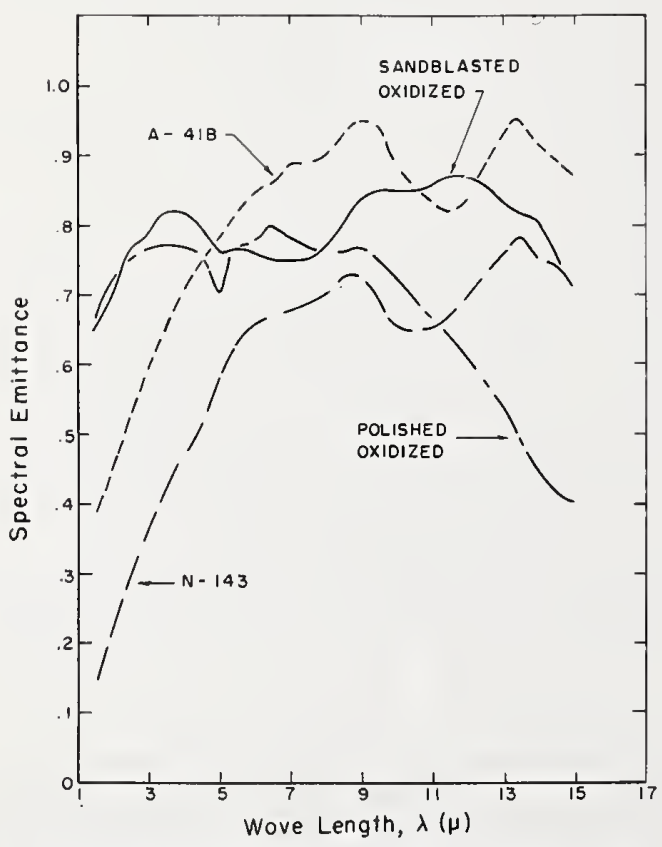

Fig. 2. Spectrol emittance curves of $1800^{\circ} \mathrm{F}$. for type 321 stainless steel specimens with different surfoce treotments; coating thickness opproximotely 2 mils.

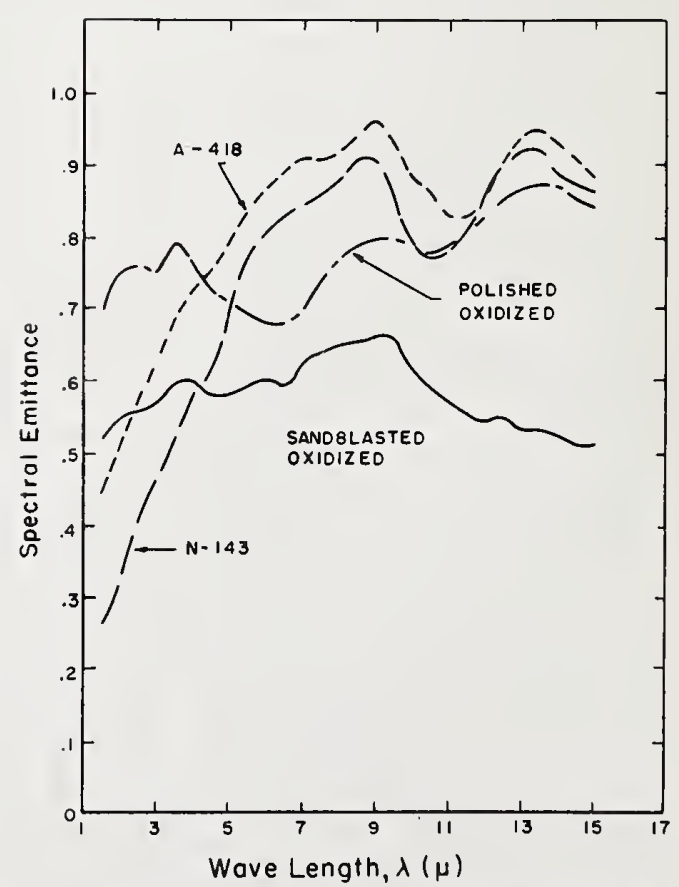

Flg. 4. Spectrol emittance curves at $1800^{\circ} \mathrm{F}$. for Inconel specimens with different surface treotments; cooting thickness opproximotely 2 mils. 
the marked decrease in emittance at longer wave lengths noted for the type 321 stainless steel specimens.

The curves for Inconel at $1800^{\circ} \mathrm{F}$. are shown in Fig. 4 . Again the curves are roughly similar in shape to the corresponding curves at $900^{\circ} \mathrm{F}$. but, in general, are displaced upward. The polished and sandblasted specimens oxidized too rapidly at this temperature to permit evaluation in the unoxidized condition. The Inconel specimen coated with $\mathrm{N}-143$ had appreciably higher emittance at all wave lengths than did the corresponding stainless steel specimen tested at this temperature, but the Inconel specimen coated with A-418 had only slightly higher emittance than did the corresponding stainless steel specimen. The specimen oxidized after polishing had appreciably higher emittance at all wave lengths than did the specimen oxidized after sandblasting, as was also observed at $900^{\circ} \mathrm{F}$.

The shape of the spectral emittance curves of the coated specimens in general agrees with those found by De Corso and Coit $^{6}$ for Inconel and coating $A-417 / 235$, but the spectral bands used by these investigators were too broad to reveal any fine structure. The curves are also in qualitative agreement with the spectral reflectance curve for white porcelain enamel published by Smith. ${ }^{7}$

The shape and general height of the spectral emittance curves for the polished metals are in qualitative agreement with that computed from the spectral reflectance curve for polished aluminum presented by Smith. ${ }^{7}$ None of the spectral emittance curves for Inconel agree even qualitatively with that of De Corso and Coit ${ }^{6}$ for as-rolled Inconel. Their curve shows emittance of 0.8 at about $2 \mu$ falling to about 0.2 at about $12 \mu$. This might indicate that a thin film of oxide was present on their specimens.

The lower emittance of the sandblasted oxidized Inconel as compared with the polished oxidized Inconel called for an explanation. The oxide layers were examined by $\mathrm{X}$-ray diffraction. Only $\mathrm{Cr}_{2} \mathrm{O}_{3}$ was identified in the oxide layer on the sandblasted specimen, but $\mathrm{Cr}_{2} \mathrm{O}_{3}, \mathrm{Fe}_{2} \mathrm{O}_{3}$, and $\mathrm{NiO}$ were found in the oxide layer on the electropolished specimen. From the relative heights of the peaks of the diffraction curves it was estimated that both $\mathrm{Fe}_{2} \mathrm{O}_{3}$ and $\mathrm{NiO}$ were present in substantially greater amounts than was $\mathrm{Cr}_{2} \mathrm{O}_{3}$. Published values ${ }^{1}$ for total emissivity at $2000^{\circ} \mathrm{F}$. are 0.73 for $\mathrm{Cr}_{2} \mathrm{O}_{3}, 0.89$ for $\mathrm{Fe}_{2} \mathrm{O}_{3}$, and 0.86 for $\mathrm{NiO}$. On this basis, the difference in composition between the oxide layers on the two types of specimens might account for part, but not all, of the observed difference in emittance, although it is recognized that any conclusions based on total emissivity are questionable.

Examination of tapered sections of the oxidized specimens revealed that the oxide-metal interface was much rougher on the sandblasted than on the electropolished specimen, as would be expected. The oxide layer on the sandblasted specimen appeared to be very much thinner than that on the electropolished specimen. This observation was confirmed by weight-gain oxidation tests, results of which are shown in Fig. 5. After 30 minutes in air at $1800^{\circ} \mathrm{F}$, the sandblasted specimen had gained only about half as much weight as the electropolished specimen. When the much larger surface area per unit of nominal area of specimen is considered, it is apparent that the ratio of thicknesses of the oxide coatings on the two types of specimen indicates a more strongly selective oxidation effect than is indicated by the ratio of the weights of oxide. Computations indicated that the oxide layer on the sandblasted specimen would not exceed about $3 \mu$ in thickness, even if the true surface area were no greater than the nomina

${ }^{6} \mathrm{~S}$. M. De Corso and R. L. Coit, "Measurement of Total Emissivities of Gas-Turbine Combustor Materials," Trans. Am. Soc. Mech. Engrs., 77 [8] 1189-97 (1955).

'A. D. Smith, "Surface Emissivity as a Factor in Appliance Design," Elec. Eng., 74 [12] 1054-58 (1955).

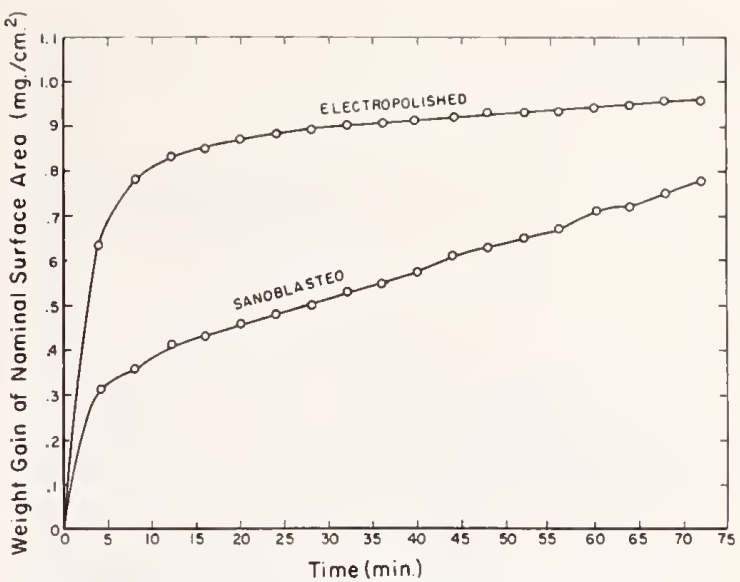

Fig. 5. Weight gain in oir of $1800^{\circ} \mathrm{F}$. for electropolished and sondblosted specimens of Inconel.

specimen area. Thin oxide films are known to be partly transparent to infrared radiation; hence it is believed that the observed difference in thickness of the oxide layers on the sandblasted and electropolished specimen satisfactorily accounts for the observed difference in spectral emittance, since this property was influenced to varying degrees by emission from the substrate metal.

Each value in Table I is the result of four different comparisons made on the spectrophotometer: (1) The Nernst glower vs. the black body, (2) the Nernst glower vs. the silicon carbide heating element, both used in calibrating the silicon carbide standard, (3) the Nernst glower vs. the specimen, and (4) the Nernst glower vs. the silicon carbide standard, both used in making a determination. The reproducibility of each comparison, determined from duplicate runs, was on the order of $\pm 2 \%$ at wave lengths below about $5 \mu$, and $\pm 1 \%$ at longer wave lengths. If all the errors should be in the same direction, this could result in a maximum error of $\pm 8 \%$ in the values in Table I. If the errors are random, as is believed to be the case, the errors would not be expected to exceed $\pm 4 \%$. These random errors may arise from any of the following causes, among others: (1) temperature fluctuations in the Nernst glower, due to slight variations in the line voltage, (2) temperature fluctuations in the specimen or silicon carbide standard, within the $\pm 3^{\circ} \mathrm{F}$. zone to which it is controlled, (3) temperature gradients over the surface of the specimen or standard, in the area from which radiant energy is accepted for measurement, and (4) slight variations in the sensitivity of the detector or in the amplifier of the spectrophotometer. There also may be present some small constant errors due to such factors as failure of the energy emitted by the black-body furnace to conform exactly to that from an ideal black body, slight differences in absorption or reflection along the two optical paths in the instrument, and temperature differences due to errors in the calibration of thermocouples. Such errors, however, are believed to be small.

The radiant flux of a black body, expressed as a fraction of the radiant flux at the maximum point, was computed from the Planck radiation equation and plotted as a function of wave length times absolute temperature $(\lambda T)$ as shown in Fig. 6. A corresponding curve for each specimen was computed by multiplying the spectral emittance at each value of $\lambda T$ by the corresponding radiant flux of the black body. The total emittance was then computed from the area under the curve of the specimen, measured with a planimeter and expressed as a fraction of the area under the black-body curve. The total emittance data are shown in Table II. 


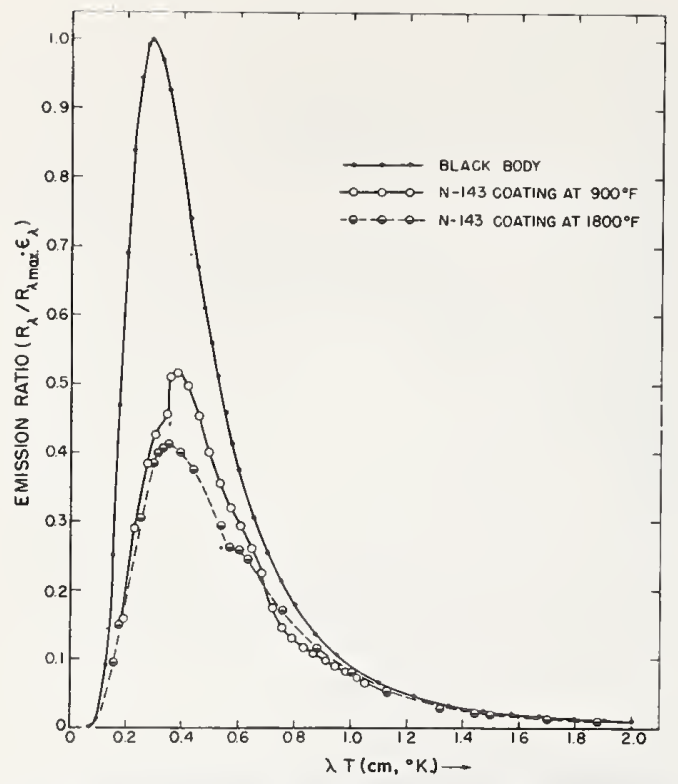

Fig. 6. Planck distribution curve for o black body, plotted as a function of absolute temperature times wave length $(\lambda T)$, ond similar curves for two cooted specimens. The totol emittance of the coated specimen is the ratio of the area under the curve for the specimen ta the area under the curve for the black body.

The data in Table I. plotted in Figs. 1, 2, 3, and 4, show a consistent trend toward an increase in spectral emittance with an increase in temperature, regardless of surface finish or wave length. The data for total emittance (Table II) show a corresponding trend for the uncoated bare or oxidized specimens. This is in agreement with the findings of Betz, Olson, Schurin, and Morris ${ }^{8}$ De Corso and Coit, ${ }^{6}$ Sully, Brandes, and Waterhouse, ${ }^{5,9}$ and Snyder, Gier, and Dunkle. ${ }^{10}$ The coated specimens, however, show a slight but definite trend toward decrease in total emittance with an increase in temperature. This is in agreement with the findings of Sully, Brandes, and Waterhouse ${ }^{5,9}$ but is in conflict with the data reported by Bennett. ${ }^{11}$ Bennett's data, however, covered only the wavelength range 0.7 to $1.2 \mu$ and hence can be considered to be closer to spectral than to total emittance, and on this basis his findings check the data in Table I.

It may appear as a contradiction in the data on the emittance of coated specimens that, on the one hand, spectral emittance increases with temperature at all wave lengths and, on the other hand, total emittance decreases with an increase in temperature. This apparent anomaly can be explained by

${ }^{8}$ H. T. Betz, O. H. Olson, B. D. Schurin, and J. C. Morris, "Determination of Emissivity and Reflectivity Data on Aircraft Structural Materials: I, Technique for Measurement of Total Normal Emissivity and Reflectivity with Some Data on Copper and Nickel," WADC (Wright A ir Develop. Center) Tech. Rept., No. 56-225, Part 1, 43 pp. (October 1956).

9 A. H. Sully, E. A. Brandes, and R. B. Waterhouse, "Some Measurements of Total Emissivity of Metals and Pure Refractory Oxides and Variation of Emissivity with Temperature," Brit. J. Appl. Phys., 3 [3] 97-101 (1952).

${ }_{10}$ N. W. Snyder, J. T. Gier, and R. V. Dunkle, "Total Normal Emissivity Measurements on Aircraft Materials Between $100^{\circ}$ and $800^{\circ}$ F.," Trans. Am. Soc. Mech. Engrs., 77 [7] 1011-1019 (1955).

${ }_{11}$ D. G. Bennett, "Suppression of Radiations at High Temperatures by Means of Ceramic Coatings," J. Am. Ceram. Soc., 30 [10] 297-305 (1947).
Table II. Total Emittance Data for Metal Specimens

\begin{tabular}{|c|c|c|c|c|}
\hline \multirow[b]{2}{*}{ Specimen type } & \multicolumn{4}{|c|}{ Total emittance $(\%)$ at } \\
\hline & $900^{\circ} \mathrm{F}$. & $1200^{\circ} \mathrm{F}$. & $1500^{\circ} \mathrm{F}$. & $1800^{\circ} \mathrm{F}$. \\
\hline $\begin{array}{l}\text { Electropolished } \\
\text { stainless steel }\end{array}$ & 10 & 18 & & \\
\hline $\begin{array}{l}\text { Sandblasted stain- } \\
\text { less steel }\end{array}$ & 34 & 38 & & \\
\hline $\begin{array}{l}\text { Electropolished } \\
\text { oxidized stainless } \\
\text { steel } \\
\text { Sandblasted oxi- }\end{array}$ & 65 & 69 & 74 & 72 \\
\hline $\begin{array}{l}\text { dized stainless } \\
\text { steel } \\
\text { Stainless steel }\end{array}$ & 68 & 75 & 70 & 74 \\
\hline & 63 & 58 & 63 & 61 \\
\hline $\begin{array}{l}\text { coated N-143 } \\
\text { Electropolished In- }\end{array}$ & 38 & 37 & 34 & 36 \\
\hline $\begin{array}{l}\text { conel } \\
\text { Sandblasted Inco- }\end{array}$ & 11 & 18 & & \\
\hline & 31 & 37 & & \\
\hline & 60 & 61 & 68 & 73 \\
\hline $\begin{array}{l}\text { dized Inconel } \\
\text { Inconel coated A- }\end{array}$ & 52 & 52 & 54 & 55 \\
\hline $\begin{array}{l}418 \\
\text { Inconel coated } \mathrm{N}-\end{array}$ & 68 & 63 & 64 & 64 \\
\hline 143 & 55 & 50 & 51 & 49 \\
\hline Coating A-418 & & & & \\
\hline Inconel, 20 mils & & 64 & & \\
\hline Inconel, 4.8 mils & & 71 & & \\
\hline Inconel, 5.8 mils & & 69 & & \\
\hline Stainless, 2.2 mils & & 59 & & \\
\hline Stainless, 5.5 mils & & 70 & & \\
\hline Coating N-143 & & & & \\
\hline Inconel, 2.6 mils & & 55 & & \\
\hline Inconel, 4.9 mils & & 66 & & \\
\hline Inconel, 8.6 mils & & 61 & & \\
\hline Stainless, 2.2 mils & & 40 & & \\
\hline Stainless, 5.1 mils & & 62 & & \\
\hline
\end{tabular}

the shape of the spectral emittance curves. The spectral emittance of the coated specimens increased sharply with wave length over the range 1.5 to $5 \mu$. At the temperatures used in this investigation the peak of the Planck distribution curve for a black body lies in this spectral region. The shift of this peak with temperature toward shorter wave lengths, where the spectral emittance of the coated specimen is lower, is more than enough to compensate for the small increase with temperature of the spectral emittance at all wave lengths. Hence for these specimens the total emittance decreases with an increase in temperature, not as a result of the change in spectral emittance with temperature, but as a result of the shift with temperature in the relative spectral distribution of the energy emitted by the specimen and by a black body at the same temperature.

Figure 6 shows the distribution curves for the radiant energy for a black body and for specimens coated with coating $\mathrm{N}$ 143 , at temperatures of $900^{\circ}$ and $1800^{\circ} \mathrm{F}$., plotted as a function of $\lambda T$. The total emittance is proportional to the area under the curve for each specimen, and it can be seen that this area is appreciably larger for the specimen at $900^{\circ} \mathrm{F}$. than for the specimen at $1800^{\circ} \mathrm{F}$.

The data on the thermal gradients computed from the power-input measurements are shown in Table III. The indicated difference in thermal gradients in coating N-143 as applied to Inconel and to type 321 stainless steel is undoubtedly due to the effect of the underlying metal on the emittance of the coating. All the power-input measurements were made on specimens having a coating approximately 2 mils thick. The curves in Figs. 1, 2, 3, and 4 indicate that at this thickness the emittance of the coated specimens was affected by the 
Table III. Thermal Gradients for Ceramic Coatings, Computed from Power-Input Measurements

\begin{tabular}{|c|c|c|c|c|}
\hline \multirow[b]{3}{*}{$\underset{\left({ }^{\circ} \mathrm{F} .\right)^{\text {Specimen }}}{ }$} & \multicolumn{4}{|c|}{ Thermal gradient $\left({ }^{\circ} \mathrm{F} . / \mathrm{mil}\right)$ for speci mens } \\
\hline & \multicolumn{2}{|c|}{$A-418$ on } & \multicolumn{2}{|c|}{$N-143$ on } \\
\hline & Inconel & $\begin{array}{c}\text { Stainless } \\
\text { steel }\end{array}$ & Inconel & $\begin{array}{c}\text { Stainless } \\
\text { steel }\end{array}$ \\
\hline 900 & 1.02 & 1.03 & 0.97 & 1.02 \\
\hline 1200 & 2.00 & 1.95 & 1.74 & 1.95 \\
\hline 1500 & 3.37 & 3.39 & 3.10 & 3.33 \\
\hline 1800 & 5.55 & 5.47 & 5.11 & 5.19 \\
\hline
\end{tabular}

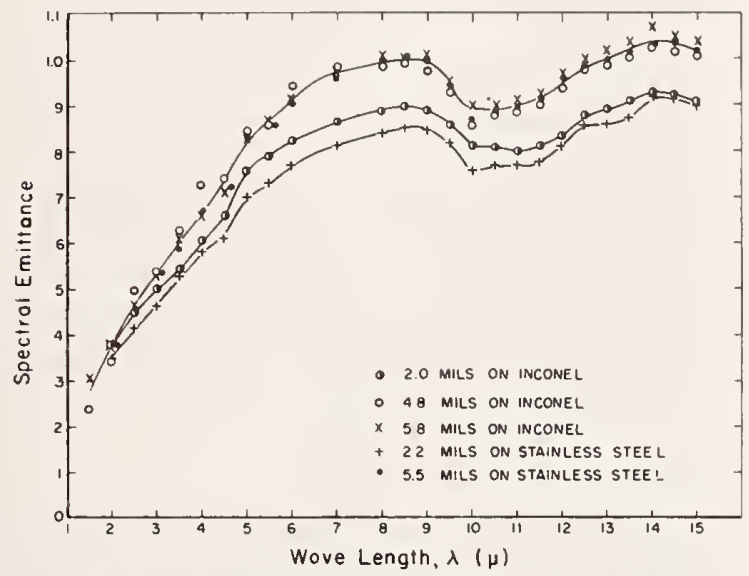

Fig. 7. Spectral emittance curves of $1200^{\circ} \mathrm{F}$. for metal specimens with coating $A-418$ applied in different thicknesses.

alloy to which the coating was applied as well as by the composition of the coating, and that the effect of the underlying metal was greater for coating N-143 than for coating A-418.

The question arises as to whether the assumption that the thermal gradients in the 0.002 -in. coatings were negligible was justified. Computations based on an estimated value for the extinction coefficient indicate that the "effective temperature" of the coated specimens was much closer to the temperature at the eramel-metal interface than to that of the outer surface of the coating. The indicated error in temperature measurement resulting from gradients in the 0.002 -in. coatings is less than the experimental error of the determination.

The power-input measurements showed that the power required to maintain an Inconel specimen at $1800^{\circ} \mathrm{F}$. was about $20 \%$ greater for a specimen coated with $\mathrm{A}-418$ than for a bare, as-rolled specimen of the same alloy. This indicates that the coating increased heat dissipation from the coated specimen as compared with a bare specimen of the same alloy. When the coated and bare Inconel specimens were subjected to the same power input of 40,000 B.t.u. per sq. ft. per hour, the coated specimen reached an equilibrium temperature $105^{\circ} \mathrm{F}$. lower than that reached by the bare specimen.

The effect of thickness of coating on the spectral emittance of coated specimens is shown in Figs. 7 and 8 . Figure 7 shows that of the specimens coated with A-418 the stainless steel specimen with a coating 2.2 mils thick had the lowest emittance at all wave lengths, the Inconel specimen with a coating 2.0 mils thick had the next lowest emittance, and there was little difference in the emittance of the Inconel specimens having coatings 4.8 and 5.8 mils thick and the

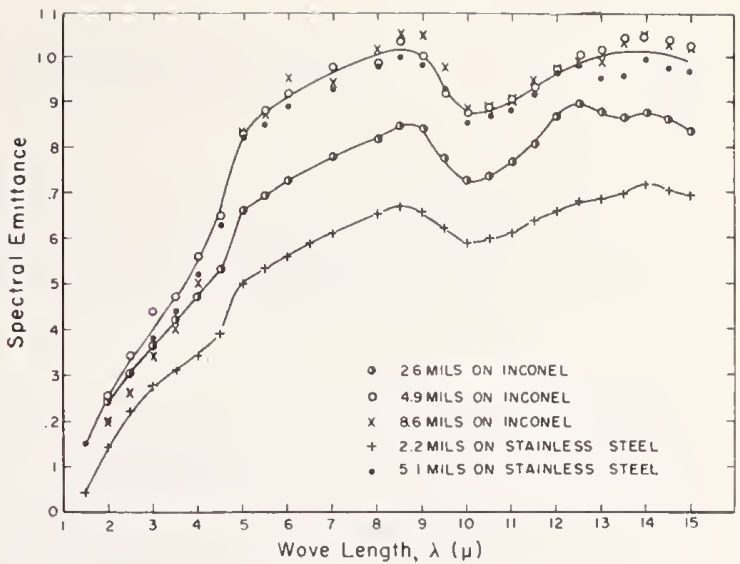

Fig. 8. Spectral emittance curves at $1200^{\circ} \mathrm{F}$. for metal specimens with coatling $\mathrm{N}-143$ applied in different thicknesses.

stainless steel specimen having a coatıng 5.5 mils thick. This indicates that this coating is opaque when applied at a thickness of about 5 mils, because the effect of increasirg the thickness from 4.8 to 5.8 mils is very slight. Figure 8 shows similar data for specimens coated with $\mathrm{N}-143$, and again the effect of the underlying metal is very marked when the coating is applied at a thickness of about 2 mils, but nearly disappears at thicknesses of about 5 mils or greater. Specimens coated with 5 mils or more of coatings $A-418$ and $N-143$, respectively, had nearly the same spectral emittance at wave lengths greater than about $5 \mu$, whereas at wave lengths below $5 \mu$ the $\mathrm{A}-418$ coated specimens had the higher emittance.

The specimens coated with 5 mils or more of $\mathrm{A}-418$ or $\mathrm{N}-$ 143 had indicated spectral emittances of more than 1.0 at wave lengths near 8.5 and $14 \mu$. This result obviously is in error, since by definition a black body emits the maximum possible amount of radiant energy at all wave lengths at a given temperature. It might be due to an accumulation of errors of the types mentioned previously or to errors in correcting the surface temperature for the thermal gradient through the coating, but part of it undoubtedly is due to the fact that some of the radiant energy emitted by the coated specimen originated beneath its surface, where it was at a temperature higher than its surface temperature, and hence was emitting at a higher rate. This error could be eliminated by measuring the emittance of specimens held at a uniform temperature, under conditions such that no thermal gradient exists in the coating. Suitable precautions will be taken to avoid this error in future work.

\section{Summary}

The normal spectral emittance of coated and uncoated Inconel and type 321 stainless steel with different surface treatments was measured at temperatures of $900^{\circ}, 1200^{\circ}, 1500^{\circ}$, and $1800^{\circ} \mathrm{F}$. over the wave-length range 1.5 to $15 \mu$. The measurements involved comparison of the radiant energy emitted by the heated specimens to that emitted by a comparison standard at the same temperatures, by means of a recording double-beam infrared spectrophotometer. The silicon carbide comparison standard had previously been calibrated against a laboratory black-body furnace. Surface treatments included (1) electropolishing, (2) sandblasting, (3) electropolishing followed by oxidation in air for $1 / 2$ hour at $1800^{\circ} \mathrm{F}$., (4) sandblasting followed by oxidation in air for $1 / 2$ hour at $1800^{\circ} \mathrm{F}$., (5) application of NBS ceramic coating A-418, and (6) application of NBS ceramic coating $\mathrm{N}-143$ 
The normal spectral emittance of both alloys in the electropolished condition was low (on the order of $15 \%$ ) and decreased slightly with increasing wave length. The normal spectral emittance of both alloys in the sandblasted condition was considerably higher (on the order of $38 \%$ ) and varied only slightly with wave length. The oxidation treatment greatly increased the normal spectral emittance of type 321 stainless steel specimens, both electropolished and sandblasted; it had a similar effect on the electropolished Inconel specimens. It also introduced some spectral selectivity into all the curves. However, the oxidation treatment brought about only a moderate increase in the normal spectral emittance of the sandblasted Inconel.

Of the specimens to which coatings were applied at a thickness of 0.002 in., those with $\mathrm{A}-418$ had higher emittance at all wave lengths than did those with $\mathrm{N}-143$, and the specimens of coated Inconel had higher emittance at all wave lengths than did the corresponding specimens of coated type 321 stainless steel. Both of the coatings were found to be partly transparent to the emitted energy at a thickness of 0.002 but essentially opaque at a thickness of 0.005 in. Specimens with 0.005 in. or more of coating did not show the effect of the underlying metal on spectral emittance, and there was no significant difference at wave lengths greater than about $5 \mu$ in the normal spectral emittance of specimens having the different coatings, but at shorter wave lengths the normal spectral emittance of specimens with coating $\mathrm{A}-418$ was greater than that of specimens coated with $\mathrm{N}-143$.

In general, the shape of the spectral emittance curves for specimens with coating $\mathrm{A}-418$ was similar to that for specimens with coating N-143. The normal spectral emittance increased rapidly with wave length from $1.5 \mu$ to a maximum at about $9 \mu$, decreased to a minimum at about $11.5 \mu$, and then increased to a second maximum at about $13.5 \mu$.

The normal spectral emittance of all specimens measured tended to increase with temperature at all wave lengths.

\section{Appendix}

The terminology used in this paper follows the recommendation of Worthing, ${ }^{12}$ in that terms ending in "ivity" are fundamental properties of a material that are not influenced by the size, shape, or surface condition of the specimen, and terms ending in "ance" are properties of a specimen and are influenced by the size, shape, and surface condition of the specimer. In addition, terms ending in "ion" refer to the fundamental processes involved.

Emission is the act or process by which radiant energy is emitted by a hot body as a consequence of its temperature only.

Emittance is a property of a specimen, influenced by its composition, thickness, and surface texture, and is the ratio of the rate of emission of radiant energy from the specimen to that from a black body at the same temperature, under the same conditions.

Emissivity is a special case of emittance; i.e., it is the emittance of a specimen of material having an optically smooth surface and having sufficient thickness to be opaque.

These terms are modified to indicate specific geometric and spectral conditions. The term "hemispherical emittance" pertains to emittance in all possible directions from a flat surface. "Directional emittance" is used with reference to emittance at a specified angle to the surface. "Normal emittance" is a special case of directional emittance, the direction being normal to the surface. The term "total emittance" signifies that all wave lengths are involved. "Spectral emittance" is the term used when restricted wave-length intervals are involved

12 A. G. Worthing, "Temperature Radiation Emissivities and Emittances"; pp. 1164-87 in Temperature, Its Measurement and Control in Science and Industry. American Institute of Physics, Reinhold Publishing Corporation, New York, 1941.

\section{P. DeWitt and J. C. Richmond}

Abstract: This is a general review of the thermal radiation properties of metals, and includes (1) description and definition of the properties, and a discussion of their interrelationships, (2) a brief review of the physical laws relating to blackbody radiation, (3) discussion of the theory of the interaction of electromagnetic waves with electrical conductors, (4) the effect of surface condition-- profile and surface films -- on thermal radiation properties of metals, and (5) a review of methods of measuring thermal radiation properties of metals.

Key words: Absorptance; electromagnetic theory; emittance; metals; reflectance; surface effects; thermal radiation properties.

* Techniques of Metals Research, Volume VI, (John Wiley \& Sons, New York, N. Y., 1970). 


\section{Irradiance Standards}

Papers

5.1. Memorandum on a Procedure for Obtaining Spectral Radiant Intensities of Tungsten-Filament Lamps, 400-700 $\mathrm{m} \mu$.

L. E. Barbrow, J. Opt. Soc. Am. 49, No. 11, p. 1122, (Nov. 1959).

Key words: Irradiance; spectral irradiance; standard_----

5.2. The New Tungsten-Filament Lamp Standards of Total Irradiance.

Ralph Stair, William E. Schneider and William B. Fussell, Appl. Opt. 6, No. 1, 101-106 (Jan. 1967).

Key words: Carbon-filament lamp; irradiance; standard; total irradiance; tungsten-filament lamp_.........-...-.-.

5.3. Instructions for Using the NBS Tungsten-Filament Lamp Standards of Total Irradiance, (March 1966).

Key words: Irradiance; standard; total irradiance; tung-

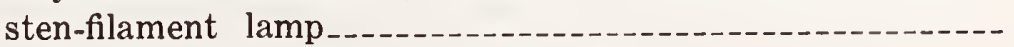

5.4. A New Standard of Spectral Irradiance.

Ralph Stair, W. E. Schneider, and John K. Jackson, Appl. Opt. 2, No. 11, 1151-1154 (Nov. 1963).

Key words: Irradiance; quartz-iodine lamp; spectral irradiance; standard

5.5. Instructions for Using the NBS 1000-Watt Quartz-Iodine Lamp Standards of Spectral Irradiance, (Nov. 1964).

Key words: Irradiance; quartz-iodine lamp; spectral irradiance ; standard

5.6. A Standard for Extremely Low Values of Spectral Irradiance. Ralph Stair, William B. Fussell and William E. Schneider, Appl. Opt. 4, No. 1, 85-89 (Jan. 1965).

Key words: Irradiance; spectral irradiance; standard....-

5.7. A One-Solar-Constant Irradiance Standard.

William E. Schneider, Appl. Opt. 9, No. 6, 1410-1418 (June 1970)

Key words: Calibration techniques; radiometric standards; spectral irradiance; solar constant; total irradiance 



\section{Memorandum on a Procedure for Obtaining \\ Spectral Radiant Intensities of Tungsten- Filament Lamps, $400-700 \mathrm{~m} \mu$}

LOUIS E. BARRROW

Viational Bureau of Standards, Washington 25, D. C.

(Received July 15, 1959)

W

ITHIN recent months there has been evidence of increased need for standards of spectral radiant intensity. The purpose of this memorandum is to point out that within the spectral range 400 to $700 \mathrm{~m} \mu$, a tungsten-filament lamp calibrated for luminous intensity and for color temperature can be used as such a standard. The values of spectral radiant intensity of such a calibrated lamp can be calculated by the procedure to be described in which the following constants are used,

$$
\begin{aligned}
& C_{1}=374 \times 10^{-14} \text { watt-cm }{ }^{2}, \\
& C_{2}=1.438 \mathrm{~cm}-\mathrm{deg} .
\end{aligned}
$$

Luminance of a blackbody at the freezing point of platinum $=60$ candelas $/ \mathrm{cm}^{2}$. Temperature of freezing platinum $=2042^{\circ} \mathrm{K}$.

Table 75 of the Smithsonian Physical Tables, ninth revised edition, gives for a series of blackbody temperatures the spectral luminous intensity per $10 \mathrm{~m} \mu$ band per unit total luminous intensity. For example at $2800^{\circ} \mathrm{K}$, it lists at $550 \mathrm{~m} \mu$ a value of 0.08517 candle $/ 10 \mathrm{~m} \mu$ which is equivalent to $0.08517 \mathrm{lu} / \mathrm{sterad}$ $10 \mathrm{~m} \mu$. Since the maximum spectral luminous efficacy* of radiant flux is $680 \mathrm{lu} / \mathrm{w}$, and since 0.995 is the luminous efficiency* of radiant flux of wavelength $550 \mathrm{~m} \mu, 1 \mathrm{w}$ at $550 \mathrm{~m} \mu$ yields $0.995 \times 680$ $=677 \mathrm{lu} / \mathrm{w}$. The radiant power corresponding to $0.08517 \mathrm{lu}$ is thus $0.08517 / 677=126 \times 10^{-6} \mathrm{~W}$, and the spectral radiant intensity at $550 \mathrm{~m} \mu$ of a lamp of color temperature $2800^{\circ} \mathrm{K}$ is $126 \times 10^{-6}$ w/sterad per $10 \mathrm{~m} \mu$ for each candela of the lamp. The spectral radiant intensities at other wavelengths may then be found by the use of tables of relative spectral radiant intensities of blackbodies such as Table $\mathbf{5 5}$ of the Smithsonian Physical Tables referred to previously (100 $\mathrm{m} \mu$ intervals) or Harvard University Computation Laboratory "Tables of blackbody radiation functions" (10 $\mathrm{m} \mu$ intervals).

The uncertainty of spectral data computed by this procedure is probably no greater than $3 \%$ but may be as much as $5 \%$. This estimate of total uncertainty is based upon the following estimates of contributing uncertainties: $2 \%$ from the assumption of a blackbody distribution within the specified range of wavelengths, $2 \%$ from uncertainty in the determination of color temperature, and $1 \%$ from photometric uncertainty.

For convenience Table I gives values of spectral radiant
TABLE I. Microwatts ler (sterad-10 in $\mu$-candela) at the

\begin{tabular}{|c|c|c|c|c|c|c|c|}
\hline $\begin{array}{l}\text { Wabe- } \\
\text { lengtl } \\
\text { ni } \mu\end{array}$ & 2300 & 2400 & 2500 & 2600 & 2700 & 2800 & 2854 \\
\hline 400 & 8.0 & 9.8 & 11.7 & 13.9 & 16.2 & 18.6 & 20.0 \\
\hline 410 & 10.4 & 12.5 & 14.7 & 17.2 & 19.8 & 22.5 & 24.1 \\
\hline$\$ 20$ & 13.3 & 15.7 & 18.2 & 21.0 & 23.9 & 26.9 & 28.6 \\
\hline 430 & 16.7 & 19.4 & 22.3 & 25.4 & 28.5 & 31.8 & 33.6 \\
\hline 440 & 20.7 & 23.7 & 27.0 & 30.3 & 3.3 .7 & 37.2 & 39.1 \\
\hline 450 & 25.3 & 28.7 & 31.2 & 35.8 & 39.4 & 43.1 & 45.1 \\
\hline 460 & 30.7 & 34.4 & 38.1 & 41.9 & 45.7 & 49.5 & 51.5 \\
\hline 470 & 36.8 & 40.7 & 44.6 & 48.6 & 52.5 & 56.3 & 58.4 \\
\hline 480 & 43.7 & 47.8 & 51.9 & 55.9 & 59.8 & 63.7 & 65.7 \\
\hline 490 & 51.4 & 55.6 & 59.7 & 63.7 & 67.6 & 71.4 & 73.5 \\
\hline 500 & 60.0 & 64.2 & 68.3 & 72.2 & 76.0 & 79.6 & 81.6 \\
\hline 510 & 69.4 & 73.5 & 77.5 & 81.2 & 84.3 & 88.2 & 90.0 \\
\hline 520 & 79.8 & 83.7 & 87.4 & 90.8 & 94.1 & 97.2 & 98.8 \\
\hline 530 & 91.0 & 94.5 & 97.9 & 101 & 104 & 106 & 108 \\
\hline 540 & 103 & 106 & 109 & 111 & 114 & 116 & 117 \\
\hline 550 & 116 & 118 & 121 & 123 & 124 & 126 & 127 \\
\hline 560 & 130 & 132 & 133 & 134 & 135 & 136 & 136 \\
\hline 570 & 145 & 145 & 146 & 146 & 146 & 146 & 146 \\
\hline 580 & 160 & 160 & isy & 158 & 157 & 156 & 156 \\
\hline 590 & 177 & 175 & 173 & 171 & 169 & 167 & 166 \\
\hline 600 & 194 & 190 & 187 & 183 & 1s0 & 177 & 176 \\
\hline 610 & 212 & 206 & 201 & 196 & 192 & 188 & 186 \\
\hline 620 & 230 & 223 & 216 & 210 & 204 & 198 & 196 \\
\hline 630 & 249 & 240 & 231 & 223 & 210 & 209 & 206 \\
\hline 640 & 269 & $25 i$ & 246 & 236 & $22 \pi$ & 219 & 215 \\
\hline 650 & 200 & 275 & 262 & 250 & 23i) & 2.30 & 225 \\
\hline 660 & 311 & 29.3 & $2 \pi$ & 263 & 251 & 240 & 234 \\
\hline 670 & 332 & 311 & 29.3 & $27 \%$ & 262 & 2511 & 244 \\
\hline 680 & $35 \overline{3}$ & 324 & 308 & 290 & 274 & 200 & 253 \\
\hline 690 & 375 & 348 & 324 & 30.3 & 285 & 209 & 262 \\
\hline 700 & 34) & 366 & 340 & $31 \%$ & 296 & 279 & 270 \\
\hline
\end{tabular}
designated color temneratures $\left({ }^{\circ} \mathrm{K}\right)$.

intensities per candela computed by the procedure described previously for seven color temperatures. Wavelengths slightly longer than $570 \mathrm{~m} \mu$ will be recognized from the table as the Crova wavelengths for the color temperatures tabulated.

* These two terms are used herein in accord with their definitiuns as follows recently adopted by the Illuminating Engineering Society (see IES Lighting Handbook, 3rd edition, pp. 3-6). Spectral luminous effcacy, $K_{\lambda}$ : the ratio of the luminous flux at a given wavelength to the radiant flux at that wavelength. It is expressed in $l u / w$ and is equal to $680(\mathrm{lu} / \mathrm{w})$ times the spectral luminous efficiency data. The spectial luminous efficiency for a particular wavelength is the ratio of the spectral luminous efficacy for that wavelength to the value at the wavelength $(555 \mathrm{~m} \mu)$ of maximum spectral luminous efficacy.

I Deane B. Judd, J. Research, Natl. Bur. Standards 44, 5 (1950), RP2053.

Reprinted from Joursal of the Optical Society of America, Vol. 49, No. 11, 1122, November, 1959 


\title{
The New Tungsten-Filament Lamp Standards of Total Irradiance
}

\author{
Ralph Stair, William E. Schneider, and William B. Fussell
}

\begin{abstract}
The National Bureau of Standards standard of total irradiance as presently issued in the form of a 50-W carbon filament lamp was originally calibrated more than fifty years ago. Recently, needs for higher accuracy and wider ranges of total irradiance have necessitated the setting up of three sizes (100 $\mathrm{W}, 500 \mathrm{~W}$, and $1000 \mathrm{~W}$ ) of tungsten-filament lamp standards of total irradiance. These standards operate at a higher temperature than was possible with the carbon-filament lamps, and are shielded, except for a narrow area of the bulb in front of the filament, so the reception of long wavelength flux from the lamps is reduced to a minimum. The new lamps were calibrated by the use of a blackbody at a known temperature together with a quartz plate whose spectral transmittance was accurately determined. The quartz plate limits the flux received from the blackbody to the spectral region below about $4.5 \mu$ and thus reduces errors resulting from water vapor absorption at $6 \mu$ and longer wavelengths. Comparisons show the new standards to be in close agreement with the carbon-filament lamp standard.
\end{abstract}

\section{Introduction}

The accurate measurement of thermal radiation requires the use of either a standard source or a standard detector. Most of the early work in this area was centered around meteorological problems and was based upon two independent approaches initiated by Ångström $^{1}$ of Sweden and Abbot ${ }^{2}$ of America. Emphasis was placed upon the development of a detector that could be calibrated in an absolute manner directly into response as a function of the radiant flux incident upon its surface. Prior to 1900, Ångström began work on an electrical compensation instrument later known as the Angström pyrheliometer. This instrument became the basis of most European work, not only in meteorology but in other fields as well. Meanwhile, in the Simithsonian Institution under the direction of the pioneer astrophysicist Langley, Abbot and his coworkers developed the silver disk and the water-flow pyrheliometers. The water flow instrument was the primary or absolute standard, but the silver disk instrument became the working standard because of its greater simplicity in operation and use.

Most of the meteorological measurements throughout the world are still based upon either the Angström type pyrheliometer or the Abbot silver disk instrument. Since neither of these instruments can be considered truly absolute, it is not surprising that through the years differences in results through the use of the two instrumentations have occurred. These differences of about

The authors are with the National Bureau of Standards, Washington, D.C. 20234.

Received 25 July 1966.
$1 \%$ to $2 \%$ between the two scales have not been completely resolved ${ }^{3}$, thereby emphasizing the need for a new standard in this area.

For other than meteorological measurements, a standard of total irradiance originally set by Coblent $z^{4}$ in 1913 in the form of a 50-W carbon-filament lamp and operating at a color temperature of around $1800^{\circ} \mathrm{K}$ to $2000^{\circ} \mathrm{K}$ has been employed to the present time. This standard has served well for more than fifty years in many laboratories not only in America but throughout the world.

This standard is based upon the Stefan-Boltzmann Law of Radiation which relates the flux per unit area $(M)$ from a blackbody to its absolute temperature $T$ : $M=\sigma T^{4}$. In the original measurements by Coblentz the value of $\sigma$ was taken as $5.7 \times 10^{-12} \mathrm{~W} \mathrm{~cm}^{-2} \mathrm{deg}^{-4}$. The blackbody was usually operated near $1400^{\circ} \mathrm{K}$ and set at a distance of approximately $70 \mathrm{~cm}$. (Other distances and temperatures were also employed.) No correction was made for water vapor absorption for either the blackbody or the lamp. While the value of $\sigma$ employed by Coblentz (namely, $5.7 \times 10^{-12} \mathrm{~W} \mathrm{~cm}^{-2}$ $\mathrm{deg}^{-4}$ ) represented the best information at that time, it may be noted that later experimental values are much higher.

These data ${ }^{5}$ (see Table I), obtained between 1916 and 1933, scatter around a mean of 5.767. In addition to the values of Table I, a recently unpublished value of 5.77 was obtained by E. J. Gillham. ${ }^{6}$ On the other hand, the theoretical value of $\sigma$ based upon $\sigma=\left(2 \pi^{5} c^{2} h\right)$ $\left.15 c_{2}^{4}\right)$ or $\left(2 \pi^{5} k^{4} / 15 c^{2} h^{3}\right)$ results in a value of 5.669 , about $0.5 \%$ below the value employed by Coblentz in 1913 . Thus the proper value of $\sigma$ still remains to be determined. 


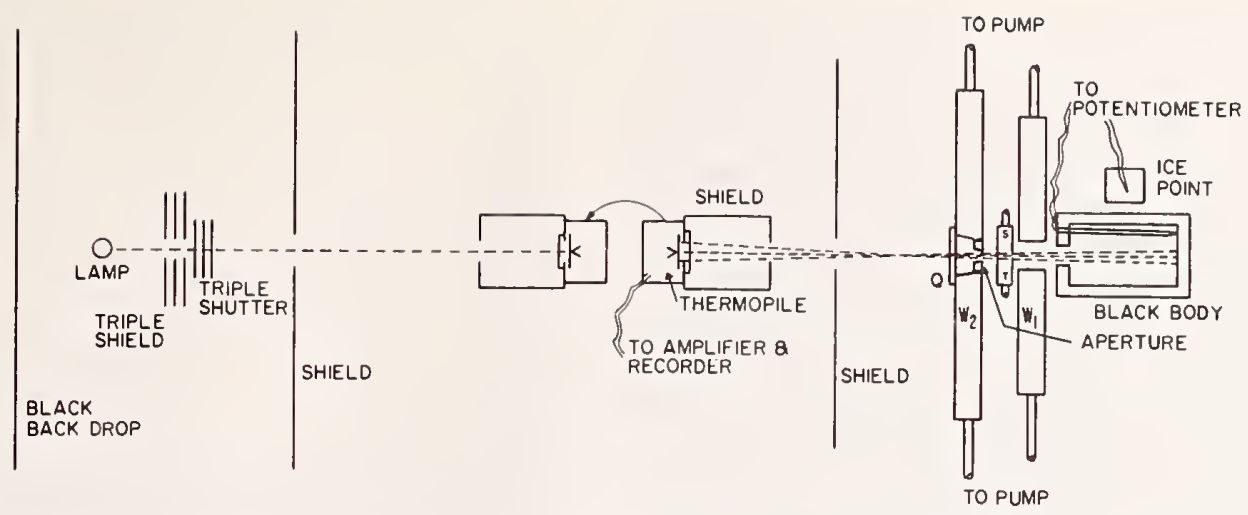

Fig. 1. Layout of blackbody; water-cooled shields, shutter, and aperture; thermopile; lamp, and lamp shutter and shields; and auxiliary equipment employed in the comparison of lamps with blackbody. $Q$, quartz plate. $W_{2}$, water-cooled shield containing aperture. $W_{1}$, secondary water-cooled shield. $S$, water-cooled shutter. $T$, thermometer.

Nevertheless, since recent needs for higher accuracy and wider ranges of total irradiance have arisen, we have proceeded to set up lamp standards of total irradiance in the form of tungsten-filament lamps of three sizes (100 $\mathrm{W}, 500 \mathrm{~W}$, and $1000 \mathrm{~W}$ ) operated at color temperatures between $2700^{\circ} \mathrm{K}$ and $2850^{\circ} \mathrm{K}$, and based upon the spectral radiance $L_{\lambda}$ of a blackbody: $L_{\lambda}=c_{1} / \lambda^{5}\left(e^{c_{2} / \lambda T}-1\right)$, where $c_{1}=1.19096 \times 10^{-12} \mathrm{~W} \mathrm{~cm}{ }^{2} / \mathrm{sr}, c_{2}=1.43879 \mathrm{~cm}$ $\operatorname{deg}, \lambda=$ wavelength in centimeters, and $T=$ temperature in degrees K (TKTS).

\section{Instrumentation and Methods}

In order to eliminate problems that arise because of significant water vapor and $\mathrm{CO}_{2}$ absorption beyond $4 \mu$, a quartz plate calibrated for spectral transmittance was interposed between the blackbody and the receiver. Atmospheric absorption of the energy from the lamp standard is almost insignificant, first because of the operation of the lamp at a relatively high temperature and second because the glass envelope is shielded except for a narrow area $(2.5 \mathrm{~cm}$ in height) of the bulb in front of the filament. Most of the radiant energy emitted by the glass is excluded by the shielding. In Fig. 1 is shown the experimental setup employed in comparing the irradiance from the blackbody with that from a tungsten-filament lamp. In this case for the $100-\mathrm{W}$ lamp, the blackbody is set at a distance of about $33 \mathrm{~cm}$ and the lamp at about $1.3 \mathrm{~m}$ from the detector. Calibrated, water-cooled apertures* determine the area of the blackbody source as seen by the detector at the set temperatures that are controlled to $\pm 0.2^{\circ} \mathrm{C}$ over extended periods of time. The thermopile (or thermocouple) is rotated to face alternately the blackbody and the lamp under study. The thermoelectric outputs are evaluated through the use of a nanovoltmeter and strip

* Three apertures having diameters of approximately $2 \mathrm{~mm}$, $3 \mathrm{~mm}$, and $4 \mathrm{~mm}$ were carefully calibrated by David Spangenberg of the Engineering Metrology Section of the Bureau. Most of our measurements were made with the 4-mm aperture. chart recorder. To keep the two thermoelectric voltages comparable when calibrating the $100-\mathrm{W}, 500-\mathrm{W}$, and $1000-\mathrm{W}$ lamps, the blackbody temperature is altered, the distance of the lamp is varied, or an attenuating chopper is set up between the lamp and detector as required. Extensive shielding is incorporated into the setup to eliminate any effects from extraneous irradiation reaching the thermoelectric detector. The blackbody furnace (and blackbody) is shielded by a double water-cooled shield. The detector is covered by a shield limiting its view to a small angle. A triple (aluminum) metal shield (also triple shutter), each piece blackened on the side facing the detector and placed about $25 \mathrm{~cm}$ from the standard lamp, ensures a near room temperature condition on all surfaces visible to the detector. A supplementary shield placed midway between the multiple shields and the detector further reduces stray irradiances and air currents in the vicinity of the detector.

Although several thermoelectric detectors were set up and employed in the early measurements in this investigation, the final results are based primarily upon the use of two conical cavity detectors of the type previously described $^{7}$ and illustrated in Fig. 2. This detector is constructed in the form of a cone of small angle and coated with carbon or other black on the inside surface. To reduce heat capacity and thereby increase response rate, the cone is made of the thinnest gold foil having sufficient strength to ensure adequate support under

Table I. Experimental Values of the Stefan-Boltzmann Constant, 1916-1933

\begin{tabular}{lll}
\hline W. Gerlach & 1916 & $5.80 \times 10^{-12} \mathrm{~W} \mathrm{~cm}^{-2} \mathrm{deg}^{-4}$ \\
W. W. Coblentz & 1917 & 5.72 \\
K. Hoffman & 1923 & 5.76 \\
A. Kussmann & 1924 & 5.74 \\
F. Hoare & 1928,1932 & 5.74 \\
C. F. Mendenhall & 1929 & 5.79 \\
C. Müller & 1929,1933 & 5.77 \\
\hline
\end{tabular}


Iaboratory conditions. It is the nearest approach to a blackbody detector of any studied in this laboratory. Certainly over the narrow spectral range of about $0.3 \mu$ to about $5.0 \mu$, which includes above $99 \%$ of the irradiance from both the blackbody and the standard tungsten-filament lamps; the spectral response of this detector may be considered to be neutral.

The blackbody, which was operated up to $1400^{\circ} \mathrm{K}$, is constructed of a casting of an alloy of $80 \%$ nickel and $20 \%$ chromium and has a $7.6 \mathrm{~cm} \mathrm{o.d.,} \mathrm{a} 15.2 \mathrm{~cm}$ length, and a wall thickness of $1.27 \mathrm{~cm}$. The low reflectivity of this oxidized metal coupled with the small aperture $(0.95$ $\mathrm{cm}$ diam) as compared with the internal surface area results in a blackbody eff ective emissivity (when applying the DeVos method of determining cavity emissivity) of 0.999. The high heat capacity of the associated furnace gives the blackbody a high thermal stability. The temperature of the blackbody was both measured and controlled by the voltage generated by a platinum-platinum ( $10 \%$ rhodium) thermocouple previously calibrated by the NBS Temperature Physics Section to an accuracy of $0.5^{\circ} \mathrm{K}$ (IPTS). To correct for thermocouple depreciation at the temperatures of operation $\left(1300^{\circ} \mathrm{K}\right.$ to $\left.1400^{\circ} \mathrm{K}\right)$, replacement or recalibration of this element was required from time to time. The thermocouple ice point was maintained at $0.0 \pm 0.05^{\circ} \mathrm{C}$ by means of a commercial thermoelectric ice point. Copper wires without soldered connections carried the thermocouple voltage to copper knife switches, by which it could be directed-alternately or simultaneously - to the temperature measuring or temperature controlling equipment.

The measuring equipment consisted of a Leeds \& Northrup K-3 potentiometer with accessory equipment. The standard cell had previously been calibrated by the NBS Electrochemistry Section.

The temperature controlling equipment consisted of a Leeds \& Northrup 10877 control system plus an FAG3 magnetic amplifier. The 10877 control system consists of a No. 10810 set point unit, a No. 9834-2 null detector, and a No. 10877 CAT (current adjusting type) control unit.

The mode of operation was as follows-

(A) The desired blackbody temperature was selected, and the corresponding thermocouple voltage was set into the set point unit by means of a $0-50 \mathrm{mV}$ coarse adjustment with $5-\mathrm{mV}$ steps and a ten-turn helipot fine

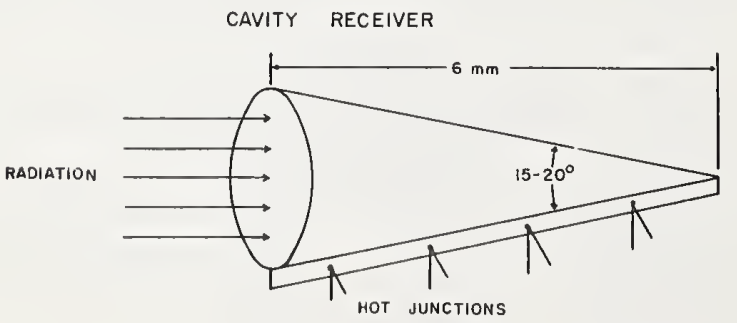

Fig. 2. Conical blackbody detector consisting of a formed gold foil cone blackened inside and having several thermojunctions attached along a single fold.

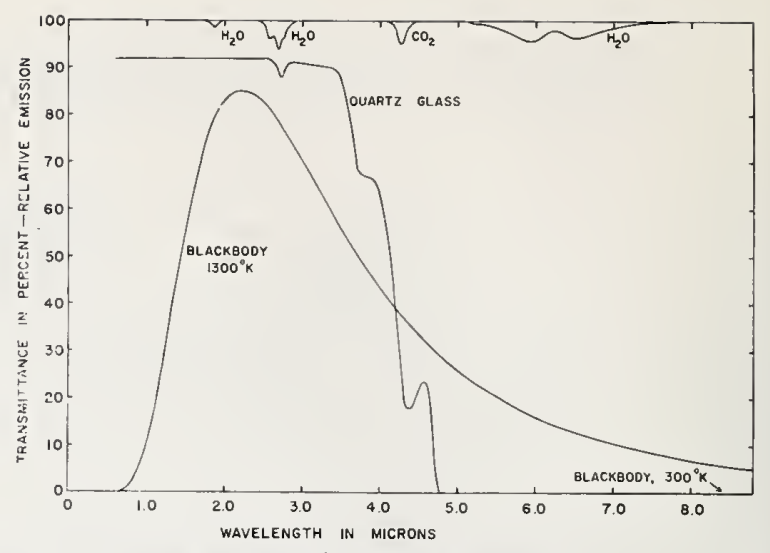

Fig. 3. Spectral emission of a $1300^{\circ} \mathrm{K}$ blackbody, and a $300^{\circ} \mathrm{K}$ blackbody; negligible transmittance of a quartz plate; and transmittance of a $33-\mathrm{cm}$ path for water vapor and carbon dioxide.

adjustment that divided each 5 - $\mathrm{mV}$ interval into one thousand $5-\mu \mathrm{V}$ divisions. The output of the set point unit was the difference: thermocouple voltage minus set point voltage. This output formed the input for the null detector, which amplified it; the output of the null detector was the input for the CAT unit, which transformed it to a current source; finally, the output of the CAT unit was the input for the FAG3 magnetic amplifier. The magnetic amplifier provided up to $20 \mathrm{~A}$ current at up to $90 \mathrm{~V}$ in order to heat the blackbody oven.

(B) To operate the blackbody at $1400^{\circ} \mathrm{K}$, for instance, the oven current was set manually at about $15 \mathrm{~A}$ when the oven was started from room temperature. When the null detector indicated the blackbody temperature was roughly $50^{\circ} \mathrm{C}$ below the desired temperature, the CAT unit was switched from manual to automatic control. As the blackbody temperature approached the desired value, the sensitivity of the null detector was slowly increased to a level sufficient to maintain the equilibrium temperature.

(C) If the equilibrium temperature differed from the set point, it could be corrected by a minor adjustment of the set point.

Figure 3 illustrates the relative spectral irradiance of a blackbody operated at $1300^{\circ} \mathrm{K}$, the temperature employed in some of the work. Also, there is indicated on the same scale the spectral irradiance from a $300^{\circ} \mathrm{K}$ blackbody - the temperature of the water-cooled shutter-which may be considered zero on this scale. The spectral transmittance of the quartz plate determines the longwave cutoff of the blackbody irradiance. Hence, the effects of the water vapor absorption at $6 \mu$ and longer wavelengths are eliminated, as is the case with much of the $\mathrm{CO}_{2}$ absorption at $4.2 \mu$. Only the $\mathrm{H}_{2} \mathrm{O}$ bands at shorter wavelengths need be considered. On this chart the water vapor absorption for the $33-\mathrm{cm}$ path length between the blackbody aperture and thermopile is indicated on the basis of an amount equivalent to 0.0001 precipitable $\mathrm{cm}$ at normal temperature and 


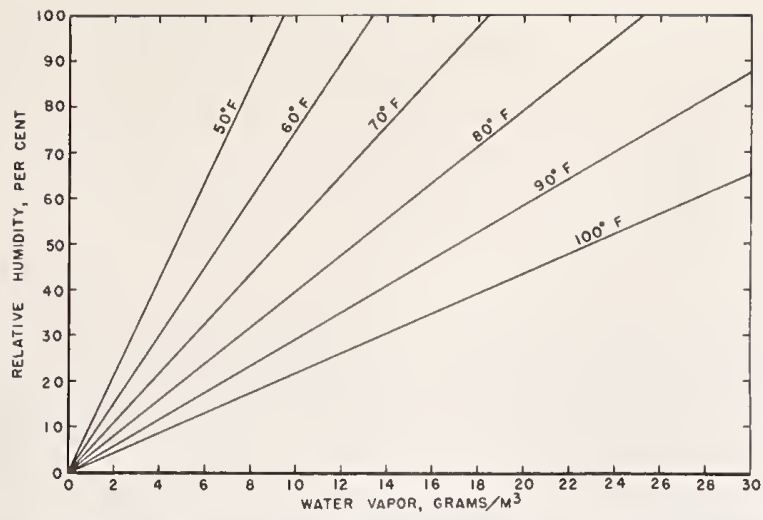

Fig. 4. Total atmospheric humidity as a function of relative humidity and temperature. (From Smithsonian Meteorological Tables.)

pressure. This amount of absorption by water vapor is based upon data recently published by Wyatt and associates. $^{8}$ The $\mathrm{CO}_{2}$ values are based upon the data by Stull and coworkers. ${ }^{9}$ The combined absorptions amount to approximately $0.5 \%$.

The water vapor content of the atmosphere was determined from measurements of the temperature and relative humidity of the laboratory during the course of the measurements which ranged (a few degrees or percent) around $75^{\circ} \mathrm{F}\left(24^{\circ} \mathrm{C}\right)$ and $70 \%$. And from the curves of Fig. 4, which were prepared from the Smithsonian meteorological tables ${ }^{10}$ giving the density of water vapor at saturation as a function of temperature, the moisture content (the absolute humidity of the atmosphere) was determined in grams per cubic meter.

\section{Results}

In Table II are shown data obtained on the three original carbon-filament lamp standards set up by Coblentz in 1913. As noted earlier, his data, shown in the fifth column, were obtained by using the StefanBoltzmann Law and making no corrections for water vapor absorption. Our data were based upon the Planck Law of Radiation with a correction of $+0.5 \%$ being made for water vapor absorption. His data incorporated a value for $\sigma$ of 5.7 , while a value of about 5.67 , as pointed out above, is consistent with the constants that we used in Planck's equation.

In Table III data on total irradiance are given for three groups of tungsten-filament lamps $(100 \mathrm{~W}, 500 \mathrm{~W}$, and $1000 \mathrm{~W}$ ) when operated at $0.75 \mathrm{~A}, 3.60 \mathrm{~A}$, and 7.70 A, respectively. These data were not corrected for any absorption of radiant flux by water vapor between the lamp and radiometer. Any correction in the original data is small. However, it is possible that some correction should be made if the lamps are used in a humid atmosphere. ${ }^{13}$

Since the new standards operate at much higher temperatures than the carbon-filment lamps, the peak of the spectral energy curve is shifted toward the shorter wave- lengths; consequently, they conform more closely with the spectral curves of the NBS standards of luminous intensity, spectral radiance, and spectral irradiance. The lamps employed are commercial projection type tubular-bulb lamps having C-13 type coiled filaments and may be operated on either ac or dc. The useful life for the $100-\mathrm{W}$ lamps (at least $50 \mathrm{~h}$ for $1 \%$ change) falls somewhat short of that previously available through the use of the carbon-filament lamps. A similar lamp life may be expected in the case of the $500-\mathrm{W}$ and $100-\mathrm{W}$ lamp standards since their operating color temperature (when operated at $3.60 \mathrm{~A}$ and $7.70 \mathrm{~A}$, respectively) lies between $2800^{\circ} \mathrm{K}$ and $2850^{\circ} \mathrm{K}$. Otherwise, their characteristics are similar, with outputs being about five and one-half and thirteen times those for the $1000-\mathrm{W}$ lamp standards.

An estimate of the accuracy of the values assigned to the lamps is based on the following factors: (1) the measurement of the blackbody temperature through the use of the thermocouple is determined to within $0.5^{\circ}$ at $1300^{\circ} \mathrm{K}$ based on the International Practical Temperature Scale (IPTS). However, in order to obtain the best blackbody radiance values, the thermocouple calibrations were corrected to the Thermodynamic Kelvin Temperature Scale (TKTS) which, at $1300^{\circ} \mathrm{K}$, required an addition of $1.4^{\circ}$ to the IPTS temperature. The standard deviation uncertainty of the correction is

Table II. New and Original Calibrations on Three Primary Carbon-Filament Lamps, in $\mu \mathrm{W} / \mathrm{cm}^{2}$ at $1.00 \mathrm{~m}$

\begin{tabular}{cccccc}
\hline & \multicolumn{3}{c}{ 1965 Values } & & \\
\cline { 2 - 5 } $\begin{array}{c}\text { Lamp } \\
\text { no. }\end{array}$ & Trial 1 & Trial 2 & Mean & Values & $\begin{array}{c}\text { Difference } \\
(\%)\end{array}$ \\
\hline C-1 & 358.9 & 361.4 & 360.15 & 359.6 & +0.15 \\
C-2 & 330.9 & 330.0 & 330.45 & 333.2 & -0.83 \\
C-3 & 367.9 & 367.5 & 367.7 & 368.0 & -0.08 \\
Average & & & & & -0.25 \\
\hline
\end{tabular}

Table III. Total Irradiances from Three Groups of TungstenFilament Lamp Reference Standards

Irradiance at $1 \mathrm{~m}$, Lamp No.

Volts ${ }_{\mu} \mathrm{W} / \mathrm{cm}^{2}$

100-W Lamp Standards Operated at 0.75 A

$\begin{array}{lll}7741 & 97.57 & 570.2 \\ 7745 & 96.56 & 583.9 \\ 7746 & 94.94 & 549.5 \\ 7749 & 94.04 & 542.8\end{array}$

500-W Lamp Standards Operated at $3.60 \mathrm{~A}$

$\begin{array}{lll}1 & 90.18 & 2963 \\ 3 & 90.00 & 3007 \\ 4 & 89.51 & 3072 \\ 5 & 89.24 & 2967\end{array}$

1000-W Lamp Standards Operated at 7.70 A

$\begin{array}{lll}1 & 100.94 & 7234 \\ 2 & 101.04 & 7398 \\ 3 & 101.16 & 7350 \\ 4 & 101.03 & 7238\end{array}$


about $0.25^{\circ}$. These uncertainties in the blackbody temperature produce uncertainties of $\pm 0.2 \%$ and $\pm 0.1 \%$, respectively, in the radiance values. (2) The distance measurements are known to within $0.5 \mathrm{~mm}$, thereby producing an uncertainty of $\pm 0.2 \%$. (3) The transmittance of the quartz plate is known with an uncertainty of $\pm 0.4 \%$. (4) The area of the blackbody aperture produces an uncertainty of $\pm 0.1 \%$. (5) The precision of measurement in terms of a standard deviation is $\pm 0.3 \%$. (6) The uncertainty of the blackbody emissivity is $0.1 \%$.

Combining the above factors in quadrature results in an estimated standard deviation of about $0.6 \%$ of the values of total irradiance assigned to the lamps.

\section{Operation of the Standards}

The carbon-filament lamp was employed as a standard of total irradiance for half a century largely because of its proved stability. ${ }^{11}$ The requirements for higher irradiances at higher temperatures has necessitated the switch to the tungsten-filament type lamps. At this Bureau, considerable experience over many years with commercial projection lamps as standards of luminous intensity and color temperature has proved their adaptability for use as standards of total irradiance. Although their useful life may not equal that of the early carbon-filament lamps, proper care and handling should ensure a life of at least $50 \mathrm{~h}$ with a change in total irradiance not exceeding about $1 \%$.

These standards have been set up with operation on de and with all current and voltage measurements made with potentiometric equipment. It is recommended that they be operated at the fixed current values listed, with voltage measurements made as power input checks from time to time. They may be cperated on ac or de as desired. A change in the current-voltage relationship is a definite signal that the lamp standard requires recalibration or replacement; but if it is necessary to use the standard in further measurements, operation at calibrated wattage would be preferable. To conserve the calibration, which always gradually changes with use, these lamps should be kept as reference standards only, and other lamps used as working standards in all cases where extensive radiometric comparisons are made.

Most thermal detectors are not neutral in response over wide spectral ranges ${ }^{12}$; and if a window is used on the radiometer, its transmittance may not be uniform through the entire spectral range of wavelengths emitted by the standard lamp or source under study, in which case correction will be required for the window absorp$\operatorname{tion}^{13,14}$ and the selective response of the detector. For example, a quartz window or quartz glass, may be found to have a transmittance for the total standard lamp irradiance of about $90 \%$ while its spectral transmittance is near $92 \%$ through much of the uv, visible, and ir to near $3 \mu$. For another source, for example a low temperature heater or blackbody, the total transmittance of the quartz may be as low as $10 \%$ to $50 \%$. Similarly, a $\mathrm{CaF}_{2}$ window having a spectral transmittance of around $92 \%$ from the uv to near $7 \mu$ (for the total irradiance of the standard lamp) may be found to have a $50 \%$ to $80 \%$ transmittance for the irradiance of a low temperature source. A KBr window having a high spectral transmittance from the uv to near $20 \mu$ (total transmittance for the standard lamp of about $89 \%$ ) may be found to have a $60 \%$ to $80 \%$ transmittance for the total flux from a low temperature source.

The work reported in this paper was supported by the National Aeronautics and Space Administration.

\section{References}

1. K. Ångström, Astrophys. J. 9, 332 (1899); H. L. Callendar, Proc. Phys. Soc. 23, 1 (1910); A. K. Ångström, Astrophy. J. 40, 274 (1914); A. K. Ångström, Tellus 10, 342 (1958); A. K. Ångström, Monthly Weather Rev. 47, 798 (1919).

2. C. G. Abbot, Smithson. Misc. Coll. 56, No. 19 (1911); C. G. Abbot and L. B. Aldrich, Smithson. Misc. Coll. 87, No. 15 (1932); C. G. Abbot, L. B. Aldrich, and A. G. Froiland, Smithson. Misc. Coll. 123, No. 5 (1954); L. B. Aldrich, Smithson. Misc. Coll. 111, No. 14 (1949).

3. A. J. Drummond, Solar Energy 5, 19 (1961).

4. W. W. Coblentz, Bull. Bur. Std. 11, 87 (1914).

5. A. J. Drummond, unpublished data.

6. E. J. Gillham, private communication.

7. Ralph Stair and William E. Schneider, Symposium on Thermal Radiation of Solids, S. Katzoff, Ed., NASA Report SP-55, Washington D.C. (1965), p. 217.

8. Philip J. Wyatt, V. Robert Stull, and Gilbert N. Plass, Appl. Opt. 3, 229 (1964).

9. V. Robert Stull, Philip J. Wyatt, and Gilbert N. Plass, Appl. Opt. 3, 243 (1964).

10. Robert L. List, Smithsonian Meteorological Tables (Government Printing Office, Washington, D.C., 1951), 6th ed., Table 108, p. 382.

11. E. B. Rosa and G. W. Middlekauff, Proc. Am. Inst. Elec. Engrs. 29, 1191 (1910).

12. Ralph Stair, W. E. Schneider, W. R. Waters, and J. K. Jackson, Appl. Opt. 4, 703 (1965).

13. W. W. Coblentz, Bull. Bur. Std. 11, 471 (1913); Bur. Std. Sci. Papers 16, 701 (1920).

14. Ralph Stair and Russell G. Johnston, J. Res. Natl. Bur. Std. 53, 211 (1954). 
INSTRUCTIONS FOR USING THE NBS TUNGSTEN-FILAMENT LAMP

STANDARDS OF TOTAL IRRADIANCE

Issued Narch 1966

These instructions cover the use of 100-watt, 500-watt, and 1000watt tungsten-filament lomp standards of thermal radiation. The lamps employed (types 100T8-1/2,120V, 500T20 115-120 V, and 1000T20. 115-120V, each with a $\mathrm{Cl} 3$ filament) have been seasoned, marked for orientation, and calibrated for density of radiant flux at fixed distances in a specified direction from the lamp filament. Suitable markings ( a line on one side and a line through a circle on the opposite side) have been etched into the glass of the lamp bulbs to assist the operator in setting up the lamps relative to the radiometer in a position identical to that under which the standards were calibrated.

In operation the lamp is to be screwed into a suitable socket that is held upright by a support, which cannot reflect radiant energy into the radiometer. The lamp is to be oriented so that the lamp bulb is vertical and so that the etched line on one side and the line and circle on the opposite side are in line with the radiometer. The circle on the lamp is to be situated away from the radiometer with the center of the filament on the horizontal axis through the radiometer. The lamp bulb and mounting are shielded from the radiometer through the use of a triple-plate shield described below. Sufficient time must be given (say five minutes for the 100-watt lamp and ten minutes for the 500-watt and 1000-watt lamps) for the lamp to become thoroughly warmed, otherwlse errors will be introduced into the irradiance measurements. The distance of the lamp is measured from the radiometer to the center of the lamp filament.

A black cloth, about 1 meter by 1 meter, should be placed about 1 meter to the rear of the lamp. A triple metallic shield about $30 \mathrm{~cm}$ by $30 \mathrm{~cm}$, having an opening $7.5 \mathrm{~cm}$ wide and $2.5 \mathrm{~cm}$ high, is placed at a distance of about $25 \mathrm{~cm}$ in front of the lamp. To screen this opening, a shutter, about $10 \mathrm{~cm}$ by $10 \mathrm{~cm}$ and of construction similar to that of the shield, is placed between this shield and the radiometer. Facing the opening in the shield, the radiometer is placed at a distance of 1.0 or 2.0 meters from the lamp. In this manner, constant extraneous conditions are maintained between the lamp and the radiometer when the shutter is opened and closed. The shield and shutter are each made of air-separated sheets (spacing $2 \mathrm{~cm}$ ) of aluminum, painted black on three sides toward the radiometer, but left with the bright metallic surfaces toward the lamp.

Before the lamp is lighted, the shutter should be opened and closed to determine the amount of stray thermal radiation falling upon the radiometer. This test may be applied at any time provided the lamp has been given sufficient time to come to room temperature. The screen to the rear of the lamp may be cooler than the shutter, which will cause a negative deflection. The correction to the observed lamp deflection is in that case, positive. 
It is desirable to make the calibration in a dimly lighted room to avoid errors from sunlight which is continually varying with cloudiness, thus varying stray radiant energy within the room as well as the temperature of the screens and also causing air currents near the radiometer. The insertion of diaphragms between the shutter and the radiation detector may be helpful in minimizing this background energy.

The values of total irradiance assigned to the tungsten fllament reference standards of the National Bureau of Standards are based upon numerous comparisons of the total irradiances from these lamps with the integrated spectral irradiance from a blackbody, with a quartz plate interposed between it and the receiver. These values are accurate to about 1 percent. The values of the issued standards, compared with the reference standards, are in agreement within about 0.5 percent. The overall accuracy in the use of these standards is somewhat dependent upon the conditions of temperature and humidity existing during their operation. Highest reproducibility will be obtained at a room temperature of about $25^{\circ} \mathrm{C}$ and at a relative humidity of about 60 percent, near which the orlginal calibrations were made. For extreme conditions of temperature or of relative humidity, corrections may be required, but in ordinary practice may be neglected (see Journal of Research, NBS, Vol. 23, p. 211, 1954).

During the calibration of each standard the current through the lamp is set(at $0.75,3.60$, or 7.70 amperes respectively) and the voltage across the base of the lamp is determined. If the measurement of current is made when a voltmeter is in the circuit with the lamp, a correction will usually have to be made to the observed current. The setting of the current through the lamp is, of course, sufficient to determine the irradiance of the lamp at the specified distances of 1 and 2 meters, the voltage being useful mainly to determine whether the lamp characteristics have remained constant.

To conserve the calibration, which gradually changes with use, these lamps should be kept as reference standards only, and other lamps used as working standards in all cases where extensive radiometric comparisons are made.

These instructions apply to the use of standards of irradiance with radiometers in air. If a window is used on the radiometer, as for example in a vacuum radiometer, then a correction has to be made for the radiant flux absorbed by the window, for the particular lamp used as a standard (since absorption is a function of the temperature of the lamp filament) and for the source measured. It was found that for a glass or quartz window about $2.2 \mathrm{~mm}$ in thickness, the transmittance was about 90 percent when a certain standard lamp was enployed. With a fluorite window, the transmittance is higher (about 91.6 percent) and varies but little from lamp to lamp.

The transmittance of the window varies also with the spectral quality of the radiant flux emitted by the source under investigation. This must also be taken into consideration. 
The thermal radiation sensitivity of a thermopile varies with the degree of evacuation; with high evacuation this sensitivity may be several times as great as in air. Since at low air pressures the sensitivity is variable with the pressure, great care must be taken to test the thermopile's sensitivity under the exact conditions existing during its use. Generally, there is no further change in the sensitivity of a thermoelectric detector at pressures below $10^{-5}$ torr.

The entire radiometer receiver should be exposed to the standard of irradiance as well as to the unknown source.

Example of a Thermopile Calibration The thermopile was exposed, at $1.0 \mathrm{~m}$, to the standard of irradiance (operated at $0.750 \mathrm{~A}$ ) under which conditions the irradiance was $540 \times 10^{-6}$ watt per $\mathrm{cm}^{2}$. Since the thermopile was covered with of fluorite window only 91.6 percent was transmitted, or $0.916 \times 540 \times 10^{-6}=495 \times 10^{-6}$ watt per $\mathrm{cm}^{2}$ was the transmitted irradiance. Under these conditions, the measuring instrument gave a scale deflection of 3.15 units. Hence, a deflection of 1 unit indicates that ( $495 \times 10^{-6}$ ) $3.15)=157 \times 10^{-6}$ watt per $\mathrm{cm}^{2}$ is incident on the thermopile element.

Suppose that the irradiance from a source rich in infrared energy is being measured, for which the fluorite window transmits only 80 percent of the total. Under this condition an instrumental deflection or reading of 1 unit indicated that $\left(157 \times 10^{-6} / 0.80\right)=196 \times 10^{-6}$ watt per $\mathrm{cm}^{2}$ was incident on the thermopile window.

If the radiometer window is of quartz, then the spectral correction for losses by reflection is about 8.5 percent for all wavelengths from the ultra-violet to $2 \mu$ in the infrared where absorption of quartz begins(B.S. Scientific Papers Nos. 237 and 401).

Owing to the large heat capacity of the filament there is no appreciable difference in the radiation emitted by this type of lamp on $60 \mathrm{~Hz}$ alternating current and on direct current. The use of low frequencies is questionable. In order to maintain a constant voltage, it is preferable to use a storage battery if one is available. However, through the use of a small voltage regulator and a small variable voltage transformer satisfactory voltage and current control may be obtained on standard 120-volt, 60-herz circuits. 


\title{
A New Standard of Spectral Irradiance
}

\author{
Ralph Stair, William E. Schneider, and John K. Jackson
}

\begin{abstract}
The National 13ureau of Standards has made available a new standard of spectral irradiance in the form of a $200-\mathrm{W}$ quartz-iodine lamp with a coiled-eoil tungsten filument operating at about $3000^{\circ} \mathrm{K}$ and calibrated over the spectral range of 0.25 to $2.6 \mu$. The ealibration of this standard is based upon the radiance of a blackbody as rlefined by the Planck law of radiation, since it was done by eomparisons with the NBSS standards of speetral radianee, of luminous intensity, and of total irradiance, each of which was established through the use of blackbodies. This standard is used without auxiliary optics. Because of its small physical size and high operating temperature, rekatively high spectral irradiances may be obtrined through its use.
\end{abstract}

\section{Introduction}

The precise measurement of irradiance from a souree requires the use of a calibrated detector or a calibrated source which may be employed in a transfer ealibration. The need for a ealibrated source was originally met by Coblentz ${ }^{1}$ in 1914 with the development of a carbon filament standard of total irradiance. Calibration and use of this type of standard have been subsequently reported by Coblentz and other personnel of the NBS Radiometry Laboratory. ${ }^{2-4}$ Aecurate calibration with this type of standard requires a detector with a nonselective spectral response. A thermopile heavily eoated with dull lampblack or earbon black meets this requirement reasonably well from the near ultraviolet through the visible and near infrared portions of the spectrum. However, the relatively low sensitivity of thermopiles and other thermal deteetors together with the faet that they are not entirely nonselective spectrally makes their use difficult for the evaluation of the speetral distribution of radiant flux.

Considerable work has been done on the emissivity of tungsten ribbon and wire filament lamps, ${ }^{5-11}$ and, coupled with measurements of temperature, an approximate standard of spectral radiance or of spectral irradiance can be set up. A number of laboratories including the National Bureau of Standards have followed this proeedure. ${ }^{12-17}$ In 1960, the National Burcau of Standards developed a standard of spectral radiance $^{18}$ in the form of a tungsten ribbon-filament

The authors are in the Radiometry Laboratory, National Bureau of Standards, Washington 25, D.C.

Received 18 June 1963.

This researeh was supported by a grant from the U.S. Public Health Serviee, Department of Health, Edueation, and Welfare. lamp calibrated by direct comparison with a blackbody, the speetral radianee of which is computed from Planck's radiation equation. These lamp standards have found wide use in the ealibration of spectroradiometric and other equipment in whieh a small area, like a slit, is to he irradiated. However, the use of this standard is limited by the small area which ean be irradiated, by the low irradiance which the standiud provides in the ultraviolet, and by the auxiliary opties required. For many types of spectrorarliometric calibration, a standard of speetral radiance has been found to be very useful and will no doubt continue to be useful. However, in many cases, a standard of spectral irradiance is needed. To fulfill this need and to provide a souree of higher irradianec in the ultraviolet, a new standard of spectral irradianee has been devcloped.

\section{Apparatus and Method}

l3efore proceeding with the establishment of a standard of spectral irradianee for the region of 0.25 to 2.6 $\mu$ based upon the radianee of a blackbody at a known temperature, we made a study of possible lamp desigus. First thought was given to the specially constructed tungsten-in-quart\% lamp previously cmployed in this laboratory ${ }^{12,13}$ as a standard of spectral irradianee based upon eolor temperature and the published spect ral emissivity data for tumgsten. It was recognized that this lamp had three principal defieiencies: its low operating temperature, poor optical quality ancl bulkiness of the quart\% envelope, and relatively larese filament area. Only very low ultraviolet irradiuness could be realized at a spectrometer slit. Mlso, sinec each tungsten filament differs somewhat in emissivity as a function of its specifie shape and surface eondition, 


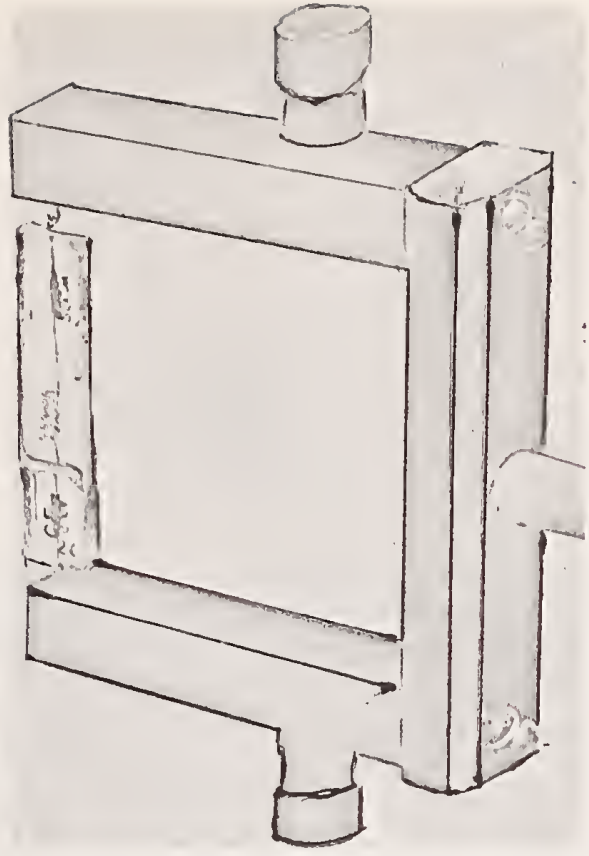

Fig. 1. Quartz-iodine lamp standard of spectral irradiance.

the eomputed values of spectral irradiance were uncertain by an indefinite amount.

Attention was given next to lamps being made eommereially. The General Electric (GE) Model 6.6A/T4Q/1CL-200-W (puartz-iodine lamp (see Fig. 1) was cxamined and found to have aceeptable characteristics for use as a standard. It is of robust construction, in a sinall (quart\% envelope of relatively good optical (puality so that the intensity usually varies but little over a considerable solid angle eentered normal to the axis of the lamp. The filament is a compact eoiled eoil with over-all dimensions approximating $3 \mathrm{~mm} \times 1: 3 \mathrm{~mm}$. The small size of the lamp envelope (about $13 \mathrm{~mm} \times$ 5) cm) together with the small area of the filament permits plaeing the lamp witlin a few eentimeters of the slit of a spectrometer. Sinee this lamp is being set up as a standard of spectral irradianee to be employed without auxiliary opties, relatively high irradiance at a slit cun be realized simply by placing the souree elose to it.

Because of its high operating temperature, the cuartziodine lamp enits a relatively large pereentage of ultraviolet radiation. The high temperature is made possible through the unicue ehemieal action of the iodine vapor ${ }^{19,21}$ which results in the return of evaporated tungsten from the bulb to the lamp filament, therelyy keeping the envelope clean and prolonging the useful life of the lanp. The design life of this lamp when operated at $6.6 \mathrm{~A}$ is $500 \mathrm{~h}$. For ealibration as a standard, the current is set at $6.50 \mathrm{\Lambda}$ which usually gives a color temperature betweer 3000 and $3100^{\circ} \mathrm{K}$, corresponcling to a filament temperature around $3000^{\circ} \mathrm{K}$.
The establishment of the new standard of speetral irradiance was aceomplished by comparing a group of quart\%-iodine lamps with three other types of NBS standards, each of which is based upon the speetral radianee of a blackbody computed from Planck's radiation equation, wherein the radiation eonstants $c_{1}=$ $.1 .9088 \times 10^{-12} \mathrm{~W} \mathrm{em} 2 / \mathrm{ster}$ and $c_{2}=1.4380 \mathrm{em}^{\circ} \mathrm{K}$.

The group of (quarty-iodine lamps was first compared spectroradiometrically with the NBS standards of spectral radianee. ${ }^{18}$ The optieal arrangement is shown in lig. 2. Additional details may be found in the reference cited. To eover the eomplete speetrum from 0.25 to $2.6 \mu$, three detectors were employed. An RCA type 11'-28 multiplier phototube was used to eovel' the range from 0.25 to $0.75 \mu$. lior the range of 0.35 to $1.2 \mu$, an RCA type 7102 multiplier phototube was employed, while an Eastman l'bS cell was used through the range of 0.5 to $2.6 \mu$. To eliminate the effeet of spectral reflectanee of the two aluminized mirrors employed with the ribbon-filament standards of speetral radianee, two plane mirror's having identically prepared surfaces were used with the quart\%-iodine lamps. Aetually, plane mirror $M_{1}$ was common to both optical systems (see Fig. 2). By this procedure, all instrumental characteristies were identieal or elosely similar for the two types of lamps. Hence, the optical and electronic factors should eancel out in the procedure. However, it was found that the light beam from the quartz-iodine lamp passed through the spectroradiometer' somewhat differently and irradiated a slightly different area of the detector from that irradiated by the ribbon-filament standard. The beam of flux from the ribbon filament converged on entering the spectrometer, then diverged filling an appreciable part of the instrumental aperture, and finally at the exit slit emerged as a divergent bean. The flux from the quartz-iodine lamp passed through the spectrometer and emerged as

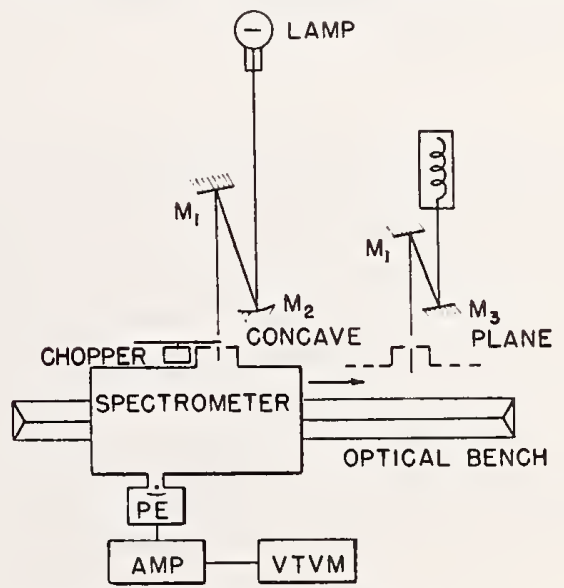

Fig. 2. Optical arrangement of spectrometer, lamp, mirrors, and associated equipment for (ompalling the (fuartz-iodine lamp) st:indards of spectral irradiance with the ribbon-filament standards of spectral radiance. 
Table 1. Spectral irradiance of Three 200-W Quartz-lodine Tungsten-Filament Lamps"

\begin{tabular}{|c|c|c|c|}
\hline $\begin{array}{l}\text { If:avelongth } \\
\text { (nm) }\end{array}$ & Lamp) ( & Jаmp Q Ql & L:Imp $\left(Q \mathrm{I}_{-10}\right.$ \\
\hline $2 ; 0$ & $0.00 \mathrm{il}$ & 0.00522 & 0.0051 \\
\hline $2(i 0$ & $0.00 ! 93$ & $0.00(0) 3$ & 0.0090 \\
\hline 270 & 0.01 .5 & $0.015 !)$ & 0.0155 \\
\hline 250 & 0.0253 & 0.0252 & $0.02 \cdot 1 \cdot 4$ \\
\hline 290 & 0.0380 & $0.03 \times 0$ & 0.0369 \\
\hline 300 & 0.0 .545 & 0.0548 & 0.05332 \\
\hline 320 & 0.104 & $0.10 \mathrm{i}$ & 0.102 \\
\hline 350 & 0.237 & 0.242 & 0.234 \\
\hline 370 & 0.366 & 0.374 & 0.363 \\
\hline 400 & 0.643 & 0.647 & 0.630 \\
\hline 4.50 & 1.26 & 1.26 & 1.23 \\
\hline 500 & 2.04 & 2.04 & 2.02 \\
\hline 5.50 & 2.93 & 2.96 & 2.91 \\
\hline 600 & 3.85 & 3.94 & 3.85 \\
\hline 6.50 & $4.7 !)$ & 4.91 & 4.80 \\
\hline 700 & 5.54 & 5.72 & $5.5 \mathrm{~s}$ \\
\hline 750 & 6.11 & 6.32 & 6.14 \\
\hline 800 & 6.51 & 6.69 & 6.49 \\
\hline 900 & 6.72 & 6.94 & 6.71 \\
\hline 1000 & 6.51 & 6.73 & 6.53 \\
\hline 1100 & 6.07 & $6.2 \bar{i}$ & 6.11 \\
\hline 1200 & 5.53 & 5.67 & 5.55 \\
\hline 1300 & 4.97 & $5.09)$ & 4.98 \\
\hline 1400 & 4.44 & 4.52 & 4.44 \\
\hline 1500 & 3.93 & 4.00 & 3.93 \\
\hline 1600 & 3.46 & 3.51 & 3.45 \\
\hline 1700 & 3.03 & 3.06 & 3.01 \\
\hline 1800 & 2.63 & $2.6 \bar{j}$ & 2.61 \\
\hline 1900 & 2.29 & $2.2 S$ & 2.26 \\
\hline 2000 & 1.98 & $1.9 \bar{t}$ & 1.95 \\
\hline 2100 & 1.73 & 1.71 & 1.70 \\
\hline 2200 & 1.52 & 1.51 & 1.50 \\
\hline 2300 & 1.36 & 1.34 & 1.33 \\
\hline 2400 & 1.22 & 1.21 & 1.21 \\
\hline 2500 & 1.12 & 1.10 & 1.11 \\
\hline 2600 & 1.04 & 1.03 & 1.04 \\
\hline
\end{tabular}

${ }^{a}$ In $\mu \mathrm{W}$ per $\mathrm{em}^{2}$ per $\mathrm{nm}$ at a distance of $43 \mathrm{em}$ (measured from the axis of the lamp filament and normal to the plane of the lamp press) when operated at $6.50 \mathrm{~A}$.

an essentially narrow "pencil" of flux, thereby being confined to a small part of the instrumental aperture and falling upon a small area of the detector. As a result, differential transmission within the spectrometer and variations in the sensitivity of the detector over its surface area* resulted in small crrors of unknown magnitude. Hence, the resulting spectral cnergy curves for the quartz-iodine lamps, obtained by this method, require adjustment.

Three methods for determining the correction for the obscrved data were pursued. They are:

(a) Direct comparison, without the use of a spec-

\footnotetext{
- Much of the variation of the detertor sensitivity was elimintaled by plueing in front of it a guartz plate finely ground on loth faces.
}

trometer, of the quartz-iorline lamps with the ribusnfilament lamps by a thermopike and filter methorl, wherein sclected spectral regions were comparerl ractiometrically. 'This comparison was accomplished l,y focusing an image of the ribbon filannent on the receiver of the thermopile by means of the two aluminum mirrors usually employed with the standard of spectral radiance. The quartz-iodine lamp directly irradiated the thermopile receiver. Correction was made for the spectral reflectance of the two aluminum mirrors. The ratio between the two radiometric readings gave a measure of the relative spectral intensitics of the two sources for the different spectral recions defined loy the filters cmployed.

(b) Measurement of luminous intensity as compared to calculated luminous intensity based upon the spectroradiometrically determined curve and the spectral luminous efficacy of the CIE standard observer. This method gave an independent cvaluation of the spectral irradianee of the quartz-iodine lamps in the visible spectrum based upon NBS standards of luminous intensity, which had been assigned values by measurements relative to a blackbody at the freczing point of platinum. ${ }^{21}$

(c) Measurement of irradiances over selected spectral regions. by cmploying optical filters and a thermopile found by comparison with a cavity detector to have a relatively flat spectral response and calibraterl through the use of the NBS carbon-filament standard of total radiation. This method afforded an independent evaluation of the spectral irradiance from the riuartziodine lamps based upon the NBS standard of total irradiance and the blackbodics used in its establishment. ${ }^{1,2}$ Corrections ranging from 4.2 to $5.3 \%$ and nonsclective with wavelength werc indicated by the three methods. The original spectroradiometric data were accordingly corrected by $5.0 \%$.

\section{Resuits}

Spectral irradiance data obtained on three quart\%iodine lamps are given in Table I in $\mu \mathrm{W} / \mathrm{cm}^{2}$ at a distance of $43 \mathrm{~cm}$ (measured from the axis of the lamp filament and normal to the plane of the lamp press) for a wavelength interval of $1 \mathrm{~mm}$. It is estimated that the maximum unecrtainty in the results ranges from about $8 \%$ at the shortest wavelengths in the ultraviolet to about $3 \%$ in the visible and infrared. The data have becn corrected for the water-vapor absorption occurring at or near 1.1, 1.4, 1.9, and $2.6 \mu$. No significant radiant-energy absorption oceurs at other wavelengths because of other gases normally present in the usual laboratory environment.

The quart\%-iodine lamps may be cmployed at any convenient distance by eomputing or otherwise determining the irrarlianee at the new clistance. 'l'he inverse sequare law should not be applicel to these lampes 


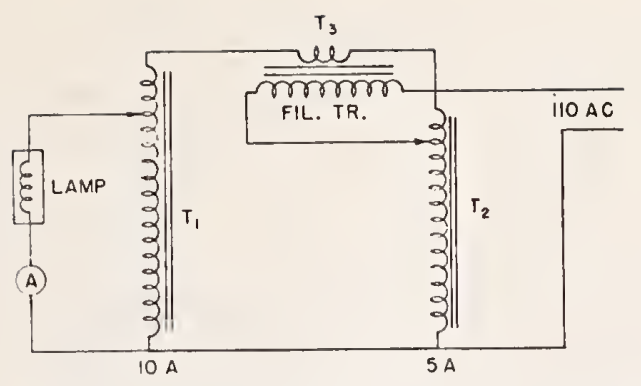

Fig. 3. Electricizl circuit for lamp operation to provide smooth current control.

for distanees shorter than about $40 \mathrm{~cm}$. In most laboratory atmospheres the correction for water-vapor absorption will be small for distanees of less than $50 \mathrm{~cm}$. However, for greater distances and under conditions of high humidity, a correction may be required. ${ }^{22-26}$ The relationship between the apparent absorption of water rapor (as indicated in a prism spectrum) and the amount of vapor is not a simple one, since each absorption band really consists of many fine lines of unequal intensity and irregular spacing. Furthermore, the attenuation at any wavelength due to water vapor is a complicated function of the pressure, temperature, and coneentration of the vapor per unit volume.

Aging tests conducted on three quartz-iodine lamps indicate after $100 \mathrm{~h}$ of operation an increase at $6.50 \mathrm{~A}$ of less than $1 \%$ in total irradiance, luminous intensity, ancl ultraviolet irradianee around $360 \mathrm{~nm}$.

\section{Use of the Standard of Spectral Irradiance}

liach cuartz-iodine standard is marked with an identifying number at one cul of the lamp. The lamp is mounted in a motal support and is calibrated with this narked cud down and with the plane of the front surface of the lower press seal set to contain the horizontal perpendicular to the line comeeting the lamp filament axis and detector or spectrometer slit. 'The lamp tip seal is positioned away from the detector or slit. Precise setting of the lamp as regards to verticality and to rotation about the filament as an axis are: important, since a few degrees displacement may result in an error of $1 \%$ or more.

The current is scet at 6.50 $\Lambda$ ac, and the lamp is allowed to operate for at least 5 min before data are recorded. Any convenient method may be employed to control the euresit; lowever, the circuit illustrated in lïg. 3 lias been found very useful in this laboratory. It consists of two variable autotransformers (10- $\Lambda$ and i) $A$ capacities) and a radio filament transformer. (Onc-lialf of a center-tapped 5- or (6.3-V secondary has bern found to be satisfactory for smooth current control.) If a $3(0) \mathrm{V}$ step-down transformer is available, it may profitably be used between the variable autotransformer, 'I'-1, and the lamp.

\section{Conclusions}

The quart\%-iodine lamp is a useful working standarl for use in spectral irradiance measurements within the region of 0.25 to $2.6 \%$. The methods of calib)ation are based upon indirect comparisons with the radiances of blackbodies and thus do not involve evaluation of filament temperature or tungsten emissivity. The prineipal uncertainties in the results are due to difficulties involved in aecurate blackbody high-temperature evaluation and procise current settings for the various lamps. The maxmum uncertainty in the results is estimated to range from about $8 \%$ at the shortest wavelengths in the ultraviolet to about $3 \%$ in the visible and infrared.

\section{References}

1. W. W. Collentz, Bull. Natl. Bur. Standards 11, 87, S227 (1914).

2. W. W. Coblentz and W. B. Emerson, Bull. Natl. Bur. Standiards 12, 503, S261 (1916).

3. W. W. Coblentz and R. Stair, J. Research Natl. Bur. Standards 11, 79 (1933):

4. R. Stair and R. G. Jolinston, J. Research Natl. Bur. Standards 53, 211 (1954).

5. A. G. Wortling, Physs. Rev. 10, 377 (1917); J. Opt. Soc. Am. 13, 635 (1926).

6. H. C. Hantaker, "Refleetivity and Emissivity of Tungsten" (In:ug. 1)iss., Utreclit, Holland, 1934).

7. J. C. 1)eVos, Physica 20, 669, 690, 715 (1954).

8. R. D. Latrrabee, J. Opt. Soc. Am. 49,619(1959).

9. F. Hoffman and II. Willenberg, Plysik \%. 35, 1, 713 (1934).

10. I. S. Ornstein, Plysiarı 3, 561 (1936).

11. C. "Zwikker, "Plysiche Jigenshappen Vau Wolfrum by Ilooge 'Temperaturem" (the'sis, Aunstcrelani, 1925).

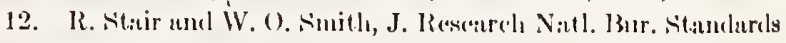
$30,+19)(19)[3)$.

13. R. Stair, J. Research Nittl. Jur. Standards 46, 4:37 (19:51).

14. W. J. Condefl and F. 'T. Byrne, J. ()pt. Soc. Am. 47, 1, 135i (1!):57).

15. L. Ji. I3:arlorow, J. (Opt. Soc. Am. 49, 1122 (1959).

16. W. J. Condell, Jr., T'ungsten spectral intensity culihration methols, U. MU. Repert RWMIP-3 (1959)).

17. F. (irum, $\Lambda_{\text {ppl) }}$ ()pt. 2, 2:37 (1963).

18. R.. Stair, R. (i. Jolnsten, and li. W. Halbuck, J. Reseure'l Natl. Bur. Stindiurds 64A, 291 (1960).

19. Li. ( . Zulser iud F. A. Moshy, Illum. Ling. LIV, 734 (1953)).

20. C. J. Allen ind R. L. P':ught, Illum. Fing. LIV, $7+1$ (1959).

21. R. P. Tede, J. (1pt. Sioc. An. 52, s.26 (1962).

22. F. W. Fowle, Astrophy's. J, 35, 1.19 (1912),

23. F. F. Fowle, Astrophy's. J. 37, 35!) (1913).

2.4. F. L. liowle, Astrophys. J. 42, 39.4 (1915).

25. L. B. Aldrich, Surithsomian lnst. Mise. Collections 111 (No. 12) I (1!).19).

26. R. Statir :und R. (i. Jolnston, J. Resentrela Natl. Bur. Standards $61,+1 !)(19.5 \%)$.

APPLIED OPTICS / Vol. 2, No. 11 / November 1963 
INSTRUCTIONS FOR USING THE NBS 1000-WATT QUARTZ IODINE LAMP STANDARDS OF SPECTRAL IRRADIANCE

November 30,1964

These instructions cover the use of tungsten-filament quartz-iodine lamps issued as standards of spectral irradiance for the wavelength range of 0.25 to 2.4 micrometres. The lamps employed are commercial G. E. type DXW-1000-watt lamps having a tungsten colled-coil filament enclosed in a small ( $3 / 8$ inch $\times 3$ inches) quartz envelope containing a small amount of iodine.

The spectral radiant intensity of the entire lamp as mounted in the manner prescribed below is measured and reported. The spectral irradiance from these lamps is based upon the spectral radiance of a blackbody as defined by Planck's equation and has been determined through comparison of a group of quartz lodine. lamps with (1) the NBS standards of spectral radiance, (2) the NBS standards of luminous intensity, and (3) the NBS standards of total irradiance.

The lamp is mounted vertically with the NBS-numbered end of the lamp down with number away from the detector. Measurements of distance (from lamp filament) are made along a horizontal axis passing through the center of the lamp filament. The correct vertical position is determined by setting the centers of the upper and lower seals along a plumb line as viewed from one side of the lamp. The plane of the front surface of the upper press seal is set to contain the horizontal perpendicular to the line connecting the lamp filament center and detector.

The lamp is mounted in the supplied holder which is constructed in such a manner as to reflect a negligible amount of radiant flux in the direction of the radiometer or spectrometer slit. A black shield should be placed at a distance of about 4 feet to the rear of the lamp to intercept stray radiant flux along the radiometric axis and adequate shielding should be provided to intercept stray flux from other directions.

If there is excessive water vapor in the laboratory atmosphere, errors may result at the wavelengths of water-vapor absorption bends. In the original calibrations the comparisons of the lamps with the other NBS standards were made in such a manner that the effect of water-vapor absorption was eliminated.

Values of spectral irradiance for these lamps are tabulated as a function of wavelength in microwatts per (square centimeter-nanometer) at a distance of 50 centimeters from center of lamp to receiver. Values of spectral irradiance for wavelength intervals other than one nanometer, say $x$ nanometers, may be found by multiplying the tabulated values by $x$. 
These standards require no audiliary optics. If any are employed proper correction must be made for their optical characteristics. The lamp is simply placed at a measured distance from the detector or spectrometer slit. If a distance other than 50 centimeters is used, the inverse-square law may be used to calculate the spectral irradiance (the inverse-square law should not, however, be used for distances shorter than about 45 centimeters).

In measurements wherein two sources (a standard source and a test source) are being compared by the direct substitution method (slit widths kept unchanged, use of the same detector) no knowledge of the spectral transmittance of the spectrometer, nor of the spectral sensitivity of the detector is required. It is necessary, however, to make sure that the entrance slit of the spectrometer is fully and uniformly filled with radiant flux both from the standard and from the test source; and it at any one wavelength the detector response for the standard is significantly different from that for the test source, the deviation from linearity of response of the detector must be evaluated and taken into account. Furthermore, if the standard and test source differ in geometrical shape, it must be ascertained that the instrument transmittance and detector response are not adversely affected thereby. Many detectors are highly variable in sensitivity over their surface area and may require diffusion of radiant flux over their surface to insure accurate radiant energy evaluations.

All calibrations were made by the use of alternating current and it is recommended that they be so used in service. To reduce the line voltage a 20-ampere variable autotransformer may be empolyed for coarse control. For fine control a second (5-ampere) variable autotransformer may be used to power a radio-filament transformer whose secondary (2.5-5volt) winding is wired in series with the primary of the 20-ampere transformer. It was found that this method is very effective for accurate control of the 8.30ampere current.

These standards of spectral irradiance are expensive laboratory equipment and it is suggested that they be operated sparingly and with care in order to prolong their useful Iife. They should be turned on and off at reduced current, and great care should be taken so that at no time will the current appreciably exceed 8.30 amperes. It is recommended that for general use, working standards be prepared by calibrating them relative to the laboratory standards supplied by NBS.

These lamps operate at high temperature such that the quartz envelope is above the flammable point of organic materials. They may thus cause fires, and also the burning of lint, etc. on the envelope which may result in optical damage to its surface. In no case should the fingers come into contact with the quartz envelope, either hot or cold, as the resulting finger prints will burn into its surface during lamp operation. 


\title{
A Standard for Extremely Low Values of Spectral Irradiance
}

\author{
Ralph Stair, William B. Fussell, and William E. Schneider
}

\begin{abstract}
To serve the interests of workers in certain fields of extremely low irradiation such as is present in the airglow and in phosphorescent, fluorescent, biochemical, and associated areas, a standard of spectral irradiance of extremely low intensity has been set up. This standard, in effect, combines the NBS standard of spectral radiance with a small aperture which approximates a point source of radiant energy of known spectral distribution. This combination, with one or more convex mirrors, will supply extremely Jow-spectral irradiances in the wavelength range $0.25 \mu$ to $2.6 \mu$ with an uncertainty of about $10 \%$.
\end{abstract}

\section{Introduction}

There has long been a need for a low-intensity standard of spectral irradiance. Actually, no convenient standard of spectral flux existed until the recent establishment of a standard of spectral radiance in the form of a tungsten strip lamp by direct comparisons with the radiances of blackbodies. ${ }^{1}$ This standard, however, yiclds irradiances for reasonable aperture sizes and distances that are many times too high for certain calibration work requiring photomultipliers, scintillators, or other high-sensitivity detectors for measuring low irradiances such as those from the airglow, or from weak phosphorescent, fluorescent, or biochemical sources. Although miniature diaphragms might be employed, the interference-fringe effects resulting therefrom, together with the necessity of working at great distances from this standard, rule out its use in this area without the use of auxiliary opties.

This paper does not present anything distinctly new, but rather brings together existing standards, methods, and instrumentation to producc practicable working standards of spectral and total irradiance.

\section{Description of New Standard}

The NBS standard of spectral radiance ${ }^{1}$ in the form of a tungsten strip lamp operated at a current of $25 \mathrm{~A}$, $30 \mathrm{~A}$, or $35 \mathrm{~A}$ (corresponding to color temperatures in the range $2000^{\circ} \mathrm{K}$ to $2700^{\circ} \mathrm{K}$ ) has been chosen as the source of radiant energy in this new standard. Through the use of an aluminum-coated spherical mirror (with or without a similarly coated plane mirror) an image of the lamp filament is focused upon a small opening (not less than a few tenths of a millimeter in diameter). The radiant flux entering this aperture ( $E_{1}$ in Fig. 1), as defincd by the radiance of the lamp filament and the

The authors are with the National Bureau of Standards, Washington, D.C.

Received 19 June 1964. optics employed, becomes the new source, effectively located at this aperture. The radiant flux is further spread out, in a manner previously employed by Engstrom, ${ }^{2}$ by the convex mirror $M_{2}$, so that the irradiance at distance $b$ is very greatly reduced. If additional reduction is required, a third mirror $M_{3}$, either plane or convex, may be employed, its curvature and the distance $c$ being adjusted to produce the desired level of spectral irradiance at the exit $E_{2}$. .

This standard is based on the radiance of a blackbody at a measured temperature, the accuracy of the attained standard depending primarily upon the accuracies in calibration of the lamp standard ${ }^{1}$ and the accuracy of the measurement of the various distances involved, and the curvature and reflectance of the coatings of the mirrors employed. Hence, the standard involves no assumptions regarding the emission rates associated with any radioactive, chemicoluminescent, thermoluminescent, electroluminescent, or other slow energy transformation, ${ }^{3}$ but is set up through physical measurements and calculations based solely upon established optical principles and laws.

The spectral limits of this standard are necessarily set by the limits of the NBS standard of spectral radiance, namely, $0.25 \mu$ to $2.6 \mu$. However, for longer wavelengths the substitution of a small blackbody for the strip lamp may be readily accomplished, and the long wavelength limit extended greatly thereby.

The standard may be used for total irradiance measurements simply by integrating the spectral irradiance through any desired filter covering a selected spectral range. An cxample is given in Sec. IV.

\section{Mathematical Analysis}

The mathematical analysis of the radiometric behavior of the low-intensity standard is based upon the work of Engstrom ${ }^{2}$ and Stephens. ${ }^{4}$

Figure 1 shows a schematic diagram of the complete system which includes the following: (1) a calibrated 


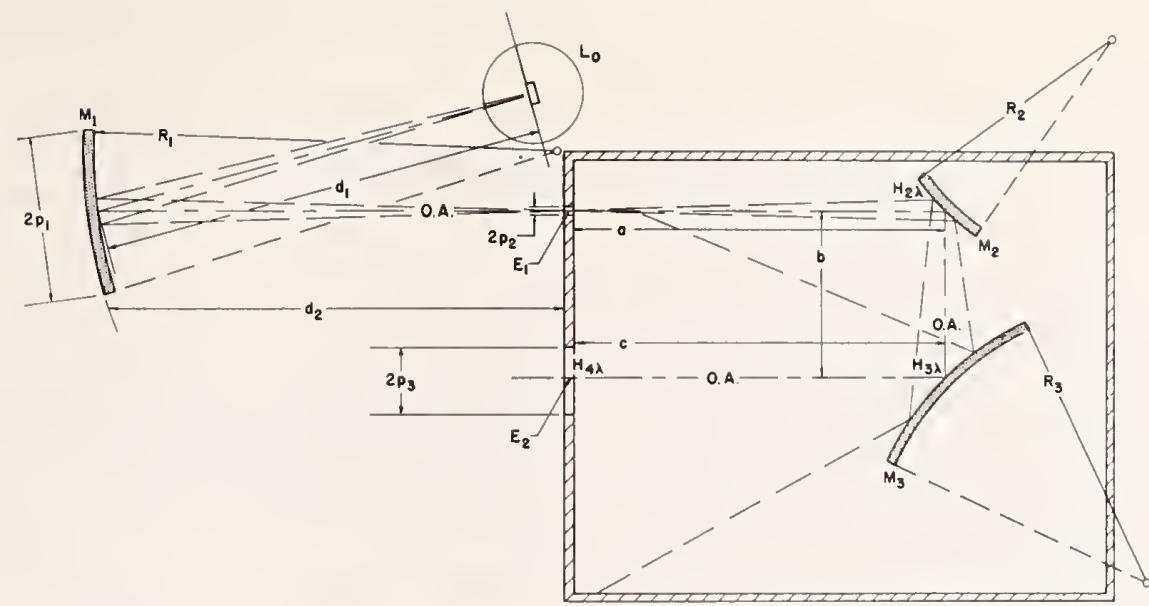

Fig. 1. Schematic diagram of the optical system of the low-intensity standard. $L_{0}-$ Standard of spectral radiance. $M_{1}-$ Concave spherical mirror imaging $L_{0}$ on $E_{1}$. $E_{1}$-Circular entrance. $M_{2}, M_{3}$-Convex spherical mirrors. $E_{2}-$ Circular exit. O.A.-Optic axis. $H_{2 \lambda}, H_{3 \lambda}, H_{4 \lambda}$-Spectral irradiance at $M_{2}, M_{3}$, and $E_{2}$, respectively.

standard $L_{0}$ of spectral radiance $N_{\lambda} ;(2)$ a concave spherical mirror $M_{1}$ of reflectivity $\rho_{1}(\lambda)^{*}$ at wavelength $\lambda$, diameter $2 p_{1}$, and radius of curvature $R_{1}$, separated from $L_{0}$ by a distance $d_{1}$; (3) a circular entrance aperture $E_{1}$ of radius $p_{2}$ at a distance $d_{2}$ from $M_{1}$; (4) a spherical convex mirror $M_{2}$ of reflectivity $\rho_{2}(\lambda)$ and radius of curvature $R_{2}$, separated from $E_{1}$ by a distance $a$; [the normal to the surface of $M_{2}$, at the point at which the optic axis (O. A.) intersects its surface, forms an angle of $45^{\circ}$ with the optic axis]; (5) a third spherical mirror $M_{3}$, also convex, of reflectivity $\rho_{3}(\lambda)$ and radius of curvature $R_{3}$, at a distance $b$ from $M_{2}$; (the normal to the surface of $M_{3}$, forms an angle of $45^{\circ}$ with the optic axis); finally, (6) the circular exit aperture $E_{2}$ of radius $p_{3}$, separated from $M_{3}$ by a distance $c$.

If $d_{1}=d_{2}$, it can be shown that they both equal $R_{1}$. In this case the filament of $L_{0}$ is imaged full-size on the entrance aperture $E_{1}$. Since $L_{0}$ is a standard of spectral

* In evaluating the spectral reflectivity of the aluminized mirrors through measurements on plane surfaces, account should be taken of both the angle of incidence and of the amount of polarization of the flux beam in the final instrumental setup. The measurement for $M_{1}$ is made with unpolarized flux at near normal incidence, in which case polarization in the reflected beam is negligible. Accordingly, for $M_{2}$, unpolarized flux should be employed, but with the angle of incidence set at $45^{\circ}$. As a result the reflected flux is partially elliptically polarized. Hence, in the evaluation of the reflectivity for $M_{3}$ the flux employed should first be reflected at $45^{\circ}$ to produce a partially elliptically polarized beam and, since the amount of polarization may be expected to vary with wavelength, these measurements should be made at selected intervals throughout the wavelength region employed. However, it is possible to eliminate the effects of polarization by one of two rearrangements of optical layout, namely, (a) by keeping the angles of incidence for $M_{2}$ and $M_{3}$ near normal, or (b) by having the planes defined by the angles of incidence and reflection at $M_{2}$ and $M_{3}$, respectively, at right angles. radiance, only a portion of the filament is used, and $E_{1}$ must be smaller in diameter than the width of the filament. Let the spectral radiance of $L_{0}$ at wavelength $\lambda$ be $N_{\lambda} \mu W$ per ( $\mu$ bandwidth $-\mathrm{cm}^{2}$ of filament area-sr). Then the entrance aperture $E_{1}$ acts as a source of spectral radiance $\rho_{1}(\lambda) N_{\lambda}$ for directions deviating from the optic axis by less than $p_{1} d_{2}^{-1}$ rad. Since $a$ is much larger than $p_{2}$, the spectral irradiance, $H_{2 \lambda}$, of a surface passing perpendicular to the beam through the center of mirror $\mathrm{M}_{2}$ may be computed for these directions simply by the inverse-square law as:

$$
H_{2 \lambda}=N_{\lambda \rho_{1}}(\lambda) \pi p_{2}{ }^{2} a^{-2} \text {. }
$$

The only restriction on $p_{1}$ is that it be large enough to fill exit aperture $E_{2}$ for the limiting case of plane mirrors (i.e., $R_{2}=R_{3}=\infty$ ), that is, $p_{1} d_{1}^{-1}$ must only fulfill the condition that it be greater than $\left(p_{2}+p_{3}\right)(a+b+c)^{-1}$, which is easily achieved.

It will be shown later that the ratio, $r_{3}(\lambda)$, of the spectral irradiance of a surface perpendicular to the beam at $M_{3}, H_{3 \lambda}$, to $H_{3 \lambda}$, is

$$
\begin{aligned}
r_{g}(\lambda)=\rho_{2 \lambda}\left[\left(1+b a^{-1}+\right.\right. & \left.2 \sqrt{2} b R_{2}^{-1}\right) \\
& \left.\times\left(1+b a^{-1}+\sqrt{2} b R_{2}^{-1}\right)\right]^{-1},
\end{aligned}
$$

so that

$$
H_{3 \lambda}=N_{\lambda \rho_{1}}(\lambda) \pi p_{2}{ }^{2} a^{-2} r_{3}(\lambda) .
$$

Equation (2) can be derived from Engstrom's formulas, ${ }^{2}$ which are more general than Eq. (2) in that the off-axis angle is not restricted to $45^{\circ}$. Similarly, the ratio, $r_{4}(\lambda)$, of the spectral irradiance at the exit aperture $E_{2}, H_{4 \lambda}$, to $H_{3 \lambda}$ is

$$
\begin{aligned}
r_{4}(\lambda)=\rho_{3}(\lambda) & {\left[1+\frac{c\left(R_{2}+2 \sqrt{2} a\right)}{b\left(R_{2}+2 \sqrt{2} a\right)+R_{2} a}+\frac{2 \sqrt{2} c}{R_{3}}\right]^{-1} } \\
\times & {\left[1+\frac{c\left(R_{2}+\sqrt{2} a\right)}{b\left(R_{2}+\sqrt{2} a\right)+R_{2} a}+\frac{\sqrt{2} c}{R_{3}}\right]^{-1}, }
\end{aligned}
$$


so that

$$
H_{4 \lambda}=N_{\lambda} \rho_{1}(\lambda) \pi p_{2}^{2} a^{-2} r_{3}(\lambda) r_{4}(\lambda) .
$$

\section{Proof of Equations (2) and (4)}

Figures 2(a) and (b) present a generalized view of the entrance aperture $E_{1}$ and the spherical mirror $M_{2}$. The angle between the optic axis and the normal to the spherical mirror is shown here as angle $\Phi$ instead of $45^{\circ}$, as in Fig. 1 . The points $P, P^{\prime}, P^{\prime \prime}$, and $P^{\prime \prime \prime}$ are the corners of a rectangular aperture stop on the mirror with the axis at corner $P$. The radiant energy leaving the entrance aperture so as to fall within the rectangular stop forms a pyramid of rectangular cross section. If $\delta \theta$ and $\delta \gamma$ are the horizontal and vertical angles of divergence, respectively, of this pyramid, then the ratio, $r_{3}(\lambda)$ of Eq. (2) to be evaluated is

$$
r_{3}(\lambda)=\rho_{2}(\lambda) a^{2} \delta \theta \delta \gamma(\delta X)^{-1}(\delta Y)^{-1},
$$

where $\delta X$ and $\delta Y$ are the lengths of the horizontal and vertical sides of the cone containing the reflected energy at the center of mirror $M_{3}$. The corners of this cross section are indicated by $Q, Q^{\prime}, Q^{\prime \prime}$, and $Q^{\prime \prime \prime}$ in Figs. 2(a) and (b).

If second-order terms are neglected, it is clear that $\delta X$ and $\delta Y$ can each be resolved into two components, $\delta X^{\prime}$ and $\delta X^{\prime \prime}$, and $\delta Y^{\prime}$ and $\delta Y^{\prime \prime}$. The components $\delta X^{\prime}$ and $\delta Y^{\prime \prime}$ are due to the mirror image of $E_{1}$ in the tangent plane to $M_{2}$ at $P$, denoted $T$. The component $\delta X^{\prime}$ is due to the dihedral angle between the vertical tangent planes at $P$ and $P^{\prime \prime}, \delta \beta$. The component $\delta Y^{\prime}$ is due to the dihedral angle between the tangent planes at $P$ and $P^{\prime \prime}, \delta \alpha$. It is seen that $\delta \beta$ is related to $\delta \theta$ by the equation

$$
R_{2} \delta \beta=a \delta \theta \sec \Phi,
$$

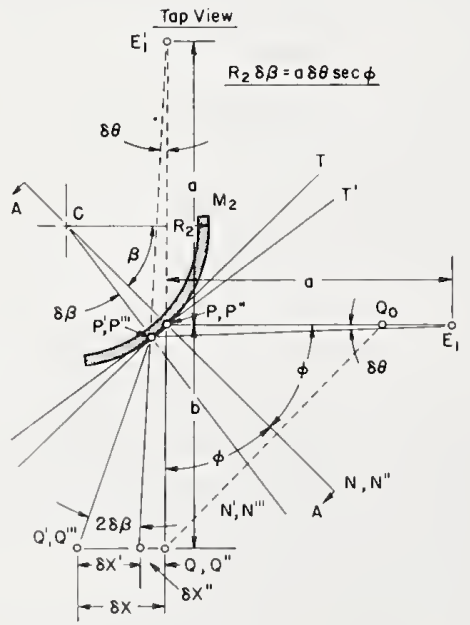

(a) and that $\delta \alpha$ is related to $\delta \gamma$ by the equation

$$
R_{i} \delta \alpha=a \delta \gamma,
$$

where $R_{2}$ is the radius of the mirror $M_{2}$.

It is also seen that $\delta X^{\prime \prime}$ and $\delta Y^{\prime \prime}$ are given by the equations

$$
\delta X^{\prime \prime}=(a+b) \delta \theta,
$$

and

$$
\delta Y^{\prime \prime \prime}=(a+b) \delta \gamma,
$$

where $a$ is the distance from $E_{1}$ to the point $P$ on $M_{2}$ and $b$ is the distance from $P$ to the point $Q$.

Furthermore, it is clear that

$$
\delta X^{\prime}=b 2 \delta \beta,
$$

and

$$
\delta Y^{\prime}=2 b \cos \Phi \delta \alpha .
$$

Substitute now the values of $\delta \beta$ and $\delta \alpha$ obtained from Eqs. (7) and (8) into Eqs. (11) and (12) above to obtain

$$
\delta X^{\prime}=2 b a R_{2}^{-1} \sec \Phi \delta \theta,
$$

and

$$
\delta Y^{\prime}=2 b a R_{2}^{-1} \cos \Phi \delta \gamma .
$$

Now add Eq. (9) to Eq. (13), and add Eq. (10) to Eq. (14) to get the following equations for $\delta X^{\prime} \equiv \delta X^{\prime}+$ $\delta X^{\prime \prime}$, and $\delta Y \equiv \delta Y^{\prime}+\delta Y^{\prime \prime}$ :

$$
\begin{aligned}
& \delta X=\left[a+b+2 b a R_{2}^{-1} \sec \Phi\right] \delta \theta, \\
& \delta Y=\left[a+b+2 b a R_{2}^{-1} \cos \Phi\right] \delta \gamma .
\end{aligned}
$$

Finally, to evaluate $r_{3}(\lambda)$ substitute Eqs. (15) and (16) into Eq. (6), and get

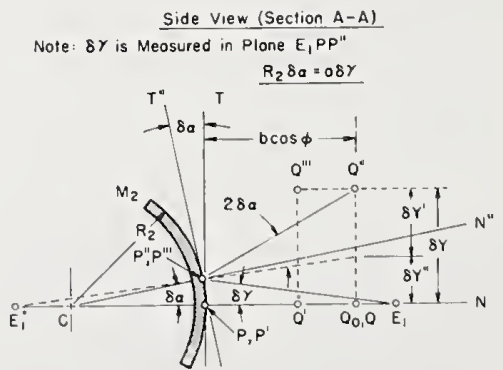

(b)

Fig. 2. Geometrical relationships between the rays emanating from a point source before and after reflection from a convex spherical mirror. $E_{1}$ - Source. $M_{2}$ - Convex spherical mirror of radius $R_{2}, P, P^{\prime}, P^{\prime \prime}, P^{\prime \prime \prime}$ - Points defining a rectangular stop on $M_{2}$. $T, T^{\prime}$, $T^{\prime \prime}$-Planes tangent to $M_{2}$ at $P, P^{\prime}, P^{\prime \prime}$, respectively. $E_{1}^{\prime}$-Image of $E_{1}$ in plane $T$. $Q, Q^{\prime}, Q^{\prime \prime}, Q^{\prime \prime \prime}$ - Illuminated area of spherical mirror $M_{3}$ (see Fig. 1). 


$$
\begin{aligned}
r_{3}(\lambda)= & \rho_{2}(\lambda)\left[1+b a^{-1}+2 b R_{2}^{-1} \sec \Phi\right]^{-1} \\
& \times\left[1+b a^{-1}+2 b R_{2}^{-1} \cos \Phi\right]^{-1},
\end{aligned}
$$

which reduces to Eq. (2) for $\Phi=45^{\circ}$, as required.

Equation (17) can also be derived by considering the astigmatic images of $E_{1}$ in $M_{2}$. Each of these astigmatic images then acts as a vertical (or horizontal) line source object which produces a vertical (or horizontal) line source inage in mirror $M_{3}$.

Figure 3 shows the positions of the astigmatic images of $E_{1}$ in $M_{2}$, denoted $S_{2}$ and $T_{2}$. Rays from $E_{1}$ and diverging in the plane of the drawing are focused at the tangential image $T_{2}$; rays diverging in a plane perpendicular to the plane of the drawing are focused at the sagittal image $S_{2}$.

It is seen that $r_{3}(\lambda)$ can now be expressed in terms of the astigmatic image distances, $s_{2}$ and $t_{2}$, as

$$
r_{3}(\lambda)=\rho_{2}(\lambda) s_{2}\left(b+s_{2}\right)^{-1} t_{2}\left(b+t_{2}\right)^{-1},
$$

where $s_{2}$ and $t_{2}$ are (see, for example, Jenkins and White $^{5}$ ) given by the equations

$$
s_{2}^{-1}=a^{-1}+2 R_{2}^{-1} \cos \Phi
$$

and

$$
t_{2}^{-1}=a^{-1}+2 R_{2}^{-1} \sec \Phi .
$$

Now substitute Eqs. (19) and (20) into Eq. (18). The result is Eq. (17).

To derive Eq. (4), proceed as follows. Compute the position of the sagittal image of $S_{2}$ in $M_{3}$, denoted $S_{3}$, and the position of the tangential image of $T_{2}$ in $M_{3}$, denoted $T_{3}$. The distance, $s_{3}$, that image $S_{3}$ lies inside $M_{3}$ is computed by substituting $b+s_{2}$ for $a$ and $R_{3}$ for $R_{2}$ in Eq. (19); similarly, the distance, $t_{3}$, that image $T_{3}$ lies inside $M_{3}$ is computed by substituting $b+t_{2}$ for $a$ and $R_{3}$ for $R_{2}$ in Eq. (20). The ratio of the spectral irradiance at the exit slit $E_{2}$ (see Fig. 1) to that at mirror $M_{3}, r_{4 \lambda}$, is clearly given by the equation

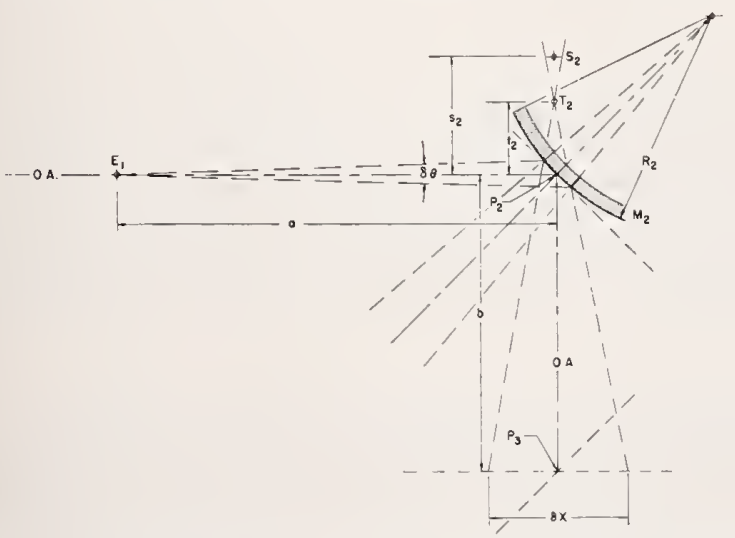

Fig. 3. Relationship between the sagittal and tangential images of a point source in a spherical mirror and the beam intensity. O.A.-Optic axis. $E_{1}$-Point source. $\delta \theta$-Divergence of a pencil of rays from $E_{1}$. $M_{2}$-Spherical mirror of radius $R_{2}$. $S_{2}$ - Sagittal image of $E_{1}$ in $M_{2} . T_{2}$-Tangential image of $E_{1}$. $P_{3}$-Intersection of the O.A. with spherical mirror $M_{3}$ (see Fig. 1).

$$
r_{4}(\lambda)=\rho_{3}(\lambda) s_{3}\left(c+s_{3}\right)^{-1} t_{3}\left(c+t_{3}\right)^{-1} .
$$

Now substitute the computed values of $s_{3}$ and $t_{3}$ into Eq. (21) and take $\Phi=45^{\circ}$. The result is Eq. (4) as desired.

\section{Use of the New Standard}

This standard requires great care in its construction and handling. Its accuracy, furthermore, depends greatly upon these factors. The lamp standard of spectral radiance has an estimated uncertainty of $3 \%$ to $5 \%$ when it is properly oriented and operated preciscly at the certified current. The entrance aperture must be constructed with high precision in order that its area be known to within $2 \%$; also, the radius of curvature of each spherical mirror should be known to within about 1\%. The error introduced in the measurement of all distances should be no more than $1 \%$. Furthermore, each mirror must have a high quality coating with a spectral reflectance that is known to within $1 \%$. When all of these requirements have been fulfilled, the uncertainty of this standard will be less than about $10 \%$.

Although the mathematical analysis of this standard is belicved to be sufficicntly complete for ready use, a sample calculation is included as guidance in not only its over-all use but in the design of the standard for a particular irradiance level. This example is carried out for a single wavelength and for a single set of mirrors set at specific distances. The irradiance at a particular position can be increased or dccreased through a design change of any of the elements between the standard lamp and that position. While the design calls for two internal convex mirrors, one or both of these may be omitted or replaced with plane mirrors if ligher irradiances are desired.

For the arithmetical example given, the following values have been assigned to the various components of the standard:

$$
\begin{aligned}
& N_{\lambda}=2.53 \mathrm{~W} \text { per }\left[\mathrm{sr}-\mu-\mathrm{cm}^{2} \text { of source] at } 0.5-\mu\right. \text { wavelength, } \\
& \left.R_{1} \quad=d_{1}=d_{2}\right)=71 \mathrm{~cm}, \text { radius of curvature of mirror } M_{1}, \\
& p_{1} \quad=1.25 \mathrm{~cm} \text {, one-half diam of aperture of mirror } M_{1}, \\
& p_{2}=0.05 \mathrm{~cm}, \text { radius of entrance aperture } E_{1}, \\
& a \quad=84 \mathrm{~cm}, \\
& b \quad=75 \mathrm{~cm}, \\
& c \quad=84 \mathrm{~cm}, \\
& R_{2}=7.5 \mathrm{~cm}, \\
& R_{3}=5 \mathrm{~cm}, \text { and } \\
& \rho_{1}(\lambda)=\rho_{2}(\lambda)=\rho_{3}(\lambda)=09 .
\end{aligned}
$$

We find, substituting these numerical values in Eq. (1), that $H_{2 \lambda}=2.534 \times 10^{-6} \mathrm{~W}-\mathrm{cm}^{-2}$ per $\mu$-wavelength bandpass, at $0.5-\mu$ wavelength. $H_{3 \lambda}$ is computed from Eq. (3) to be $4.714 \times 10^{-9} \mathrm{~W}-\mathrm{cm}^{-2} \mu^{-1}$, while $H_{4 \lambda}$ is found from Eq. (5) to be $3.313 \times 10^{-12} \mathrm{~W}-\mathrm{cm}^{-2} \mu^{-1}$.

The irradiance produced by this standard, if constructed following the example above, at $0.5-\mu$ wavelength, and for a bandwidth of $0.01 \mu$, is thus $3.313 \times$ $10^{-14} \mathrm{~W}-\mathrm{cm}^{-2}$; this is equal to an irradiance of $8.35 \times$ $10^{4}$ quanta $\mathrm{sec}^{-1} \mathrm{~cm}^{-2}$. In terms of "rayleighs," this irradiance is equal to $8.35 \times 10^{-2}$ rayleigh, a value 
much lower than the several hundred rayleighs produced by the average night-sky airglow. ${ }^{7}$

An examination of the several equations indicates that the flux density at a particular point varies directly as the square of the entrance aperture radius $p_{2}$, and inversely as the square of the optical distance, $(a+b+$ $c)$, between the entrance and exit apertures, for radii of curvature of the convex mirrors, $R_{2}$ and $R_{3}$, large compared to $(a+b+c)$. Also, if $R_{2}$ and $R_{3}$ are small compared to $b$ and $c$, respectively, the flux density at a particular point varies directly as the product of the squares of $R_{2}$ and $R_{3}$. Hence, through the proper choice of the mirror curvatures and spacings, any desired spectral flux density over an extremely wide range may be realized.

\section{References}

1. R. Stair, R. G. Johnston, and E. W. Halbach, J. Res. Natl. Bur. Std. A64, 291 (1960).

2. R. W. Engstrom, Rev. Sci. Instr. 26, 622 (1955).

3. J. W. Hastings and G. Weber, J. Opt. Soc. Am. 53, 1410 (1963).

4. R. E. Stephens, J. Opt. Soc. Am. 43, 704 (1953).

5. F. Jenkins and H. White, Fundamentals of Optics (McGrawHill, New York, 1950), 2nd ed., Chap. 6, p. 92.

6. D. Hunten, F. Roach, and J. Chamberlain, J. Atmospheric Terrest. Phys. 8, 345 (1956).

7. F. Roach, J. McCaulley, and E. Marovich, J. Res. Natl. Bur. Std. D63, 15 (1959). 


\title{
A One-Solar-Constant Irradiance Standard
}

\author{
W. E. Schneider
}

\begin{abstract}
A new high-intensity standard of total and spectral irradianee has been developed recently at the $\mathrm{Na}$ tional Buren of Standuds. Tho standurd consists of a 1000-W tumgsten-lualogen Imup) monnled in a

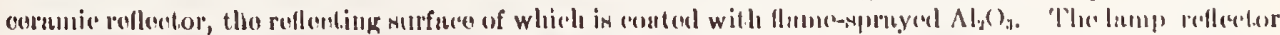

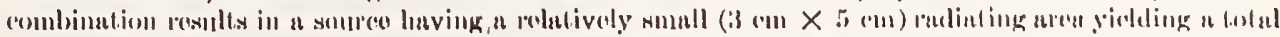

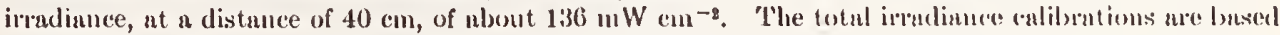
on the radianee of a $1400-\mathrm{K}$ blaekbody and have an estimated maximum systemutic errol of $0.9 \%$ and a maximum estimated stundard error of $0.19 \%$. The speetral ineasurements were made over the wuvelength range of $0.3 \mu \mathrm{m}$ to $2.5 \mu \mathrm{m}$ relative to the NBS $1000-\mathrm{W}$ tungsten-lualogen irradiunce standards. The estimated uncertainty in these measmements ranges form $4 \%$ in the visible and nea in to $8 \%$ in the 11 .
\end{abstract}

\section{Introduction}

The need for a high intensity standard of total and spectral irradiance has existed for some time. Although a number of radiometric standards are presently available, ${ }^{1-5}$ problems arise when using these standards for determining irradiances or detector responsivities at irradiance levels higher than those provided by the standard. In particular, the measurement of total and spectral irradiance of solar simulators requires, ideally, a radiometric standard that provides an irradiance comparable with that of the simulator. The problem of developing a standard that has both a total irradiance of one solar constant (reported to be about $136 \mathrm{~mW} \mathrm{~cm}^{-2}$ ) and a spectral irradiance similar to that of the sun is an extremely difficult one and has not yet been solved. However, there is also a need for a standard source having a total irradiance on the order of onc solar constant and a known, if not solar, spectral irradiance. This paper describes the establishment of such a standard.

\section{Evaluation of Sources}

Before the development of a high intensity irradiance standard was undertaken, a study was made of commercially avirialsle sources that might be suitable for use as a standind. The sources wero evaluated in terins of (1) cappilsility of producing total irradiances on the order of at least one solur constant at a reasonable

The author was with the Nutional Bureau of Standards when this work was done; he is now with Optronics lalooratories, 7676 fenton St., Silver Spring, Md. This work was supported by NASA.

Rereived $2 \times$ July 1969. working distance; (2) reproducibility; (3) stability; and (4) spectral distribution.

Previous experience ${ }^{6,7}$ with high pressure arc lamps such as $\mathrm{Hg}, \mathrm{Hg}-\mathrm{Xc}$, Xe, and krypton indicated that, although sufficient irradiance could be obtained, the reproducibility and long term stability of these ares did not mect the requirements necessary for use ats a radiometric standard. The above also applies to carbon ares. Consideration was given to 5000- $\mathrm{W}$ tungsten filament projection lamps, whose reproducibility is satisfactory. Unfortunately, the stability of these units over a period of $15 \mathrm{~h}$ is relatively poor, and the large size of the envelope and rather short distances required to produce sufficient irradiance canse serious difficulties in alignment. Another reason for ruling out the 5000-W lamp is its glass envelope, which docs not trunsmit below about $350 \mathrm{~nm}$. Thus a sulvey of commercially available sources indicated that, in order to obtain a reasonably stable irruliance on the order of a solar constant, a new source would hare to be developed.

An obvious approach to try first was the incorporation of the present $1000-\mathrm{WV}$ spectral irradiance standard in some type of diffuse reflector. Sever:al difterent pitrabolically shaped aluminum forms were constructed and coated with a high diffuse reflectsunce material such

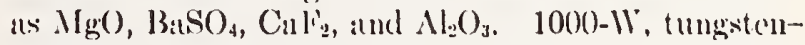
lualogen type limps mounted in the reflector produced relatively high irradiances. However, the time required for the unit to come to equilibrium wats about 30 min, which was considered inconemiently long. 'Therefore, a different type reflector male from slip-cist fused silica was tried. The lamps were originally positioned aloout $2 \mathrm{~mm}$ from the batek wall of the reflector, resulting in a very short lifo for the lamps (less than is h). 'The design of the unit wats accordingly' modified 


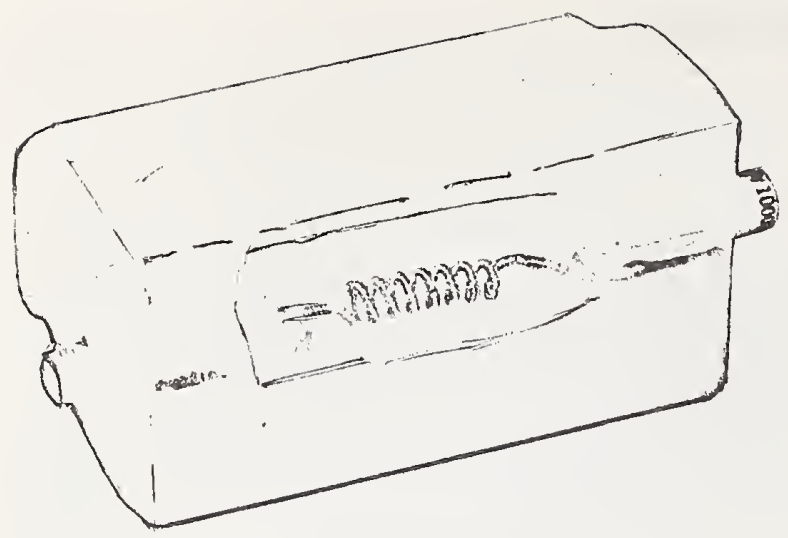

Fig. 1. High intensity source.

a number of times and the lamp repositioned within the reflector until an operating period of more than $150 \mathrm{~h}$ was obtained. The lamp, when mounted in the reflector (see lig. 1), has all effective radiating area of only $3.2 \mathrm{~cm} \times 5 \mathrm{~cm}$. The presence of the reflector increases the radiant intensity in a direction normal to the front surface of the unit by a factor of about three and results in a source having an irradiance of about a solar constant $\left(136 . \mathrm{mW} \mathrm{cm}^{-2}\right)$ at a reasonable distance of $40 \mathrm{~cm}$. A check was also made of the uniformity of the irradiance at $40 \mathrm{~cm}$ by moving a $1-\mathrm{mm}$-diam detector first vertically and then horizontally at $1-\mathrm{mm}$ increments and noting the associated detector output for each position. These measurements indicated that the uniformity was better than $\pm 0.25 \%$ over a $2 \mathrm{~cm} \times$ $2 \mathrm{~cm}$ alrea.

One of the experimental arrangements used to determine both the total and the spectral stability of these units is shown in Fig. 2. Two detectors (a phototube and a PhS cell) were mounted on an adjustable table within a special dual-detector housing so that, by means of a verniel drive, either detector could be positioned directly behind the entrance port of the housing. Provisions were matle to place narrow-band interference fillers direter overe the entrance port. Thus, through the selection of a number of interferenee filters and two detectors, a simple spectrornaliometer was constructed that was eapable of making diserete spectral irradiance measurements over the wavelength region of $0.25-2.5$ $\mu \mathrm{m}$. The total irradiance measurements were made by using a thermopile without a eover window. The test souree operated eontinuously, whereas a reference lamp, which consisted of a clear 1000-W, tungsten-halogen lamp, wis turned on only during the measurements. The ratios of the spectral or total detector output when irradiated by the test source to the output when irradiated by the reference source, afford a measure, over any period of time, of any relative change of the test source that might occur.

In order to expedite the stability studies, a second

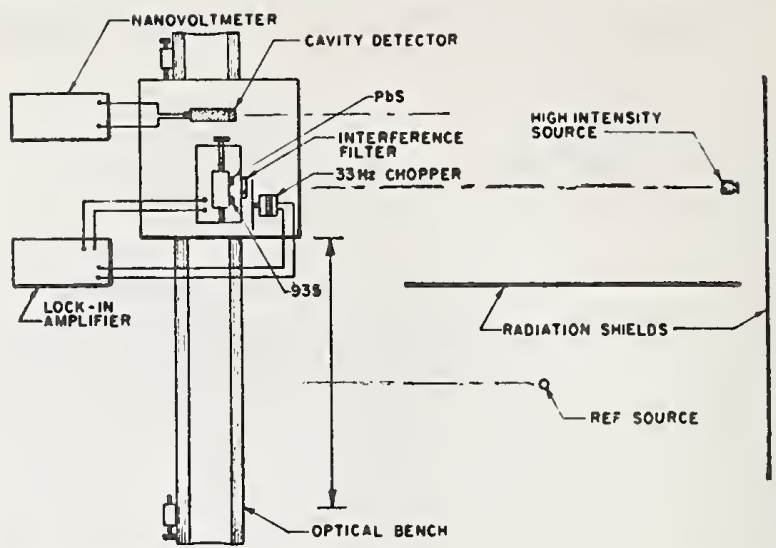

Fig. 2. Experimental apparatus for conducting total and spectral stability tests.

system was constructed. This system was identical in principle to the first, except for the means of dispersion. In this case, for obtaining discrete spectral bands, a quartz double-prism monochromator rather than the interference filters was employed. The monochromator was mounted on a second optical bench along with a second thermopile. Thus two systems, operating simultaneously, were available for studying the stability properties of various high intensity sources.

Initial tests on the 1000-W, tungsten-halogen lamp, ceramic reflector combination indicated that degradation of the fused silica reflective coating was occurring. Although these lamps are not overly rich in uv radiation, their close proximity to the reflecting surface (about $6 \mathrm{~mm}$ ) caused sufficient uv energy to be incident upon the reflector to result in degradation of the reflector. Table I gives the results for a $1000-\mathrm{W}$, tungsten-halogen lamp when mounted in a ceramic reflector having a fused silica reflecting surface. Note that, in the uv region at $270 \mu \mathrm{m}$, the change in ratios over a $40-\mathrm{h}$ interval is $13 \%$, whereas with increasing wavelength, the degradation decreases. At the long wavelength limit of 2.5 $\mu \mathrm{m}$ the change over $40 \mathrm{~h}$ of operation decreased to $2 \%$ and the change in the total irradiance of the souree is nlise about $2 \%-$ too large a change for the present requirements.

\section{See Journal for complete article pages 1410 to 1418}

June 1970 / Vol. 9, No. 6 / APPLIED OPTICS 


\section{Radiometric Measurement Techniques}

Papers

6.1. Spectral Irradiance as Determined Through the Use of Prism Page and Filter Spectroradiometric Techniques.

William E. Schneider, Ralph Stair, and John K. Jackson, Appl. Opt. 6, No. 9, 1479-1486 (Sept. 1967).

Key words: Filter spectroradiometry; measurement techniques; prism spectroradiometry; radiometry; sources ; spectral irradiance

6.2. The Measurement of Solar Radiation with Principal Emphasis on the Ultraviolet Component.

Ralph Stair, Air and Wat. Pollut. Int. J. 10, pp. 665-688 (1966).

Key words: Solar radiation measurement; ultraviolet radiation

6.3. Some Factors Affecting the Sensitivity and Spectral Response of Thermoelectric (Radiometric) Detectors.

Ralph Stair, William E. Schneider, William R. Waters, and John K. Jackson, Appl. Opt. 4, No. 6, 703-710 (June 1965).

Key words: Area sensitivity; detector surface uniformity; spectral response; thermal detectors; thermopiles_.......--

6.4. Radiometric Methods for Measuring Laser Output.

D. A. McSparron, C. A. Douglas, and H. L. Badger, Nat. Bur. Stand. (U.S.) Tech. Note 418, 16 pages (Nov. 1967).

Key words: Laser; radiometry ; laser energy measurement; radiometric calibration; photoelectric photometry; optical attenuator

6.5. Effects Upon Radiant Intensity Measurements due to Scattering by Optical Elements.

William H. Venable, Jr., Appl. Opt. 9, No. 3, pp. 609-615 (March 1970).

Key words: Instrument function; radiance; scattering_--- 



\title{
Spectral Irradiances as Determined Through the Use of Prism and Filter Spectroradiometric Techniques
}

\author{
William E. Schneider, Ralph Stair, and John K. Jackson
}

\begin{abstract}
Two spectroradiometers, one based on a conventional prism monochromator and the other on a system employing narrow band pass interference filters, have been set up and independently used in the determination of the spectral irradiances of a number of sources over the wavelength range from $0.25 \mu$ to $2.5 \mu$. Basically, the method of calibration for each system consists of comparing the spectral irradiance of the source under investigation with that of an NBS standard of spectral irradiance. The results obtained with each system on a number of continuous sources agree to about $1 \%$ whereas the differences in the spectral irradiances obtaincd with the two setups on a number of line sources range up to several percent.
\end{abstract}

\section{Introduction}

'The methods presently employed at the National Bureau of Standards for measuring the spectral irradiances of various sources over the solar spectrum consist of comparing the spectral irradiance of the source under investigation with that of an NBS standard of spectral irradiance. ${ }^{1}$ Two spectroradiometers, ${ }^{2}$ one based on a conventional prism monochromator and the other a system employing narrow band pass interference filters, have been set up and independently used in the determination of the spectral irradiances of a number of sources.

In recent years considerable interest has developed in the methods and techniques employed in the accurate measurement of the spectral energy distribution of various lamp or arc sources over the solar spectrum. The methods principally employed consist of using either (1) a standard of spectral irradiance or of spectral radiance $^{3}$ in conjunction with a conventional monochromator, (2) a standard of spectral irradiance or of spectral radiance together with a set of narrow band pass interference filters and photoelectric detectors, or (3) a thermal detector which is calibrated through the use of a standard of total irradiance ${ }^{4-6}$ or an absolute radiometer ${ }^{7}$ used in conjunction with a set of medium to wide band pass filters. It is also possible to determine the spectral radiance of tungsten filament lamps over a limited spectral range by using the published values for the emissivity of tungsten ${ }^{8,9}$ along with a knowledge of

The authors were with the National Bureau of Standards, Washington, D.C., when this work was done; R. Stair is now with Electro Mechanical Research, Incorporated.

Received 30 March 1967. the brightness temperature at a particular wavelength ${ }^{10}$ and over the visible range, to calculate the spectral radiant intensity from a knowledge of the luminous intensity and the color temperature. ${ }^{11}$ Variations in the above methods ${ }^{12,13}$ have been made and information has been obtained on the energy distribution of a number of sources. However, little information is available on the agreement of the spectral irradiance values obtained through the use of two or more of the above methods.

\section{Prism Spectroradiometer (Instrumentation)}

As shown in Fig. 1, the basic component of the conventional spectroradiometer is the quartz double-prism monochromator. The use of a double-prism instrument reduces the scattered flux to a minimum and also offers a relatively high degree of resolution. The monochromator is light in weight and compact, such that it may be mounted on a rotary turntable. Thus, the instrument can be rotated to view first one source and then another, one of which is a standard of spectral irradiance. The use of Infrasil quartz prisms allows the monochromator to be used over the entire spectral range of interest $(0.25 \mu$ to $2.5 \mu)$.

In this instrument, a single wavelength drum is geared with linkage controls which rotate the two prisms, making possible a slow continuous scan through the spectrum. A three-speed reversible synchronous motor drive has been constructed and geared to the wavelength drum. During each revolution of the wavelength drum, three unevenly spaced contacts, built into the mechanical drive, produce wavelength index marks on the recorder chart. The wavelength calibration of the instrument is performed through the use of known emission lines of various arc lamps. 


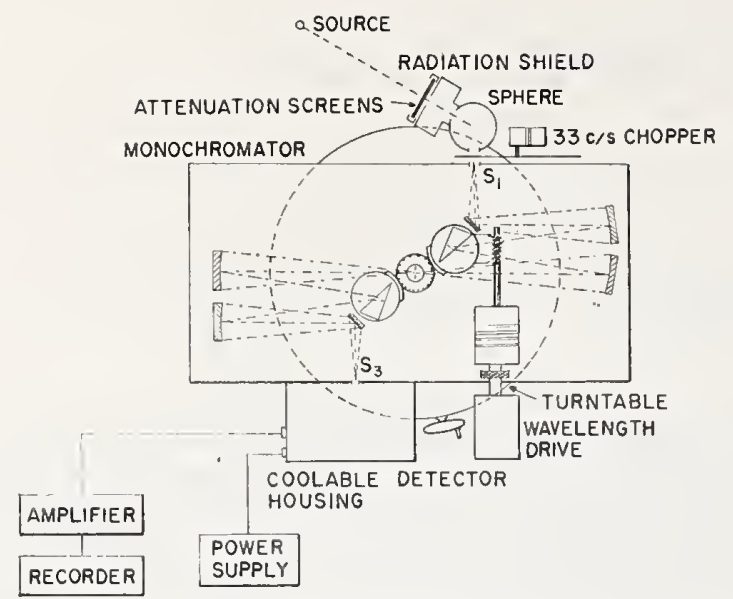

Fig. 1. Optical layout of monochromator and block diagram of prism spectroradiometer.

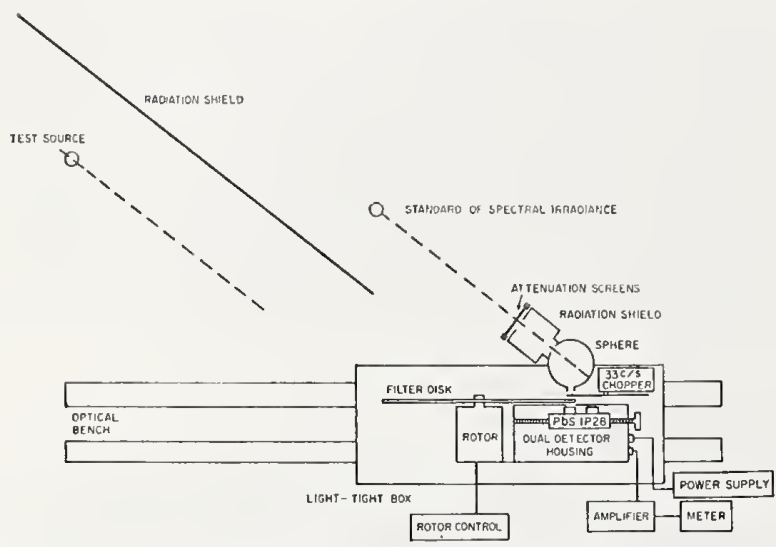

Fig. 2. Block diagram of photoelectric filter spectroradiometer.

Since the detectors used with the setup vary significantly in sensitivity over their receiving surfaces, and the transmittance of the monochromator varies over its optical aperture, an integrating sphere is placed at the entrance slit of the instrument in order to ensure that the detector always views the same source. The sphere which is $7.6 \mathrm{~cm}$ in diameter has a circular entrance port $(19 \mathrm{~mm}$ in diameter) and a rectangular $(19 \mathrm{~mm} \times 6.35$ $\mathrm{mm}$ ) exit port. The entrance port is situated far enough off the normal to the exit port that it cannot be seen by the detector.

A number of sphere coatings were examined for relative efficiency with the resultant use of $\mathrm{MgO}$ reagent grade powder. The powder is applied to the inner surface of the sphere by merely pressing the powder directly onto the sphere surface by hand and then smoothing the surface with a fine camel's hair brush. Although the coating is rather fragile, the $\mathrm{MgO}$ powder-coated sphere was found to be a factor of at least two greater in efficiency than the other sphere coatings tested over the spectral range of about $2.0 \mu$ to $2.5 \mu$ and equal to or greater in efficiency than the other coatings over the remainder of the solar spectrum.

Although the reflectance of the $\mathrm{MgO}$ powder is quite high (about 98\% throughout the visible region), ${ }^{14}$ the the actual efficiency of the integrating sphere as used with the setup is very low and it should be noted that a small decrease in reflectance results in a significantly larger decrease in sphere efficiency. Depending on the wavelength, the presence of the integrating sphere at the entrance slit of the monochromator reduces the flux as seen by the detector by a factor of about 400 to 4000 . Therefore, the detectors and amplifying system must be very sensitive and low in noise in order to obtain satisfactory results, especially over the uv range from $0.25 \mu$ to $0.30 \mu$ and in the ir beyond $1.5 \mu$.

Two detectors are employed to cover the entire spectral range from $0.25 \mu$ to $2.5 \mu$. An S-20 response photomultiplier mounted in a coolable $\left(193^{\circ} \mathrm{K}\right)$ housing is used from $0.25 \mu$ to $0.8 \mu$, and a $2-\mathrm{mm}$ by $10-\mathrm{mm}$ l'bS detector also cooled to $193^{\circ} \mathrm{K}$ is used from about $0.65 \mu$ to $2.5 \mu$. The cooling of the photomultiplier increases the detectivity by a factor of two to three, whereas the detectivity of the cooled PbS detector is a factor of fifty greater than the room temperature $\mathrm{PbS}$ detector previously used. Both of the detector housings are constructed to permit either one to be rigidly mounted at the exit slit of the prism instrument. Horizontal and vertical slots provided on separate mounting plates enable the detectors to be positioned for greatest selısitivity.

Various amplifying systems were tested not only for optimum signal-to-noise ratios, but also for stability and convenience in actual use. Of those examined the lock-in type amplifier was found to best meet the above requirements and was accordingly used in most of the work. In actual use the incident flux is chopped at 33 $\mathrm{Hz}$ and the output of the amplifier is fed directly to a strip chart recorder which can be operated at a chart speed of $\sim 2.5 \mathrm{~cm} / \mathrm{sec}$ (360 inches/hour). This relatively fast chart speed is especially helpful when analyzing data obtained on sources containing a number of emission spectra.

In an attempt to reduce stray radiation effects to a minimum, a cylindrical shield $(11.4 \mathrm{~cm}$ in length and $11.4 \mathrm{~cm}$ in diameter) with a flat black interior is mounted to the integrating sphere directly in front of the entrance aperture. The front end of the shield is removable and, depending upon the geometry involved, a diaphragm having the appropriate size opening is used. In all cases, however, care must be taken that the limiting aperture of the system is the entrance port of the integrating sphere.

When the spectral irradiance of a standard lamp is compared with that of the source under investigation, large differences in the majority of cases exist between the irradiances of the two sources. These differences can range up to a few orders of magnitude and usually vary with wavelength. Therefore, either a linearity check of the detection system must be performed, or some means of accurately attenuating the more intense 
source to a value roughly equivalent to that of the other source is necessary.

In our case, it was found both convenient and accurate to use neutral density screens as a means of both performing linearity checks on the detection system and as a means of obtaining equivalent irradiances. The transmittances of the screens were determined through the use of a set of sector disks calibrated by the Length Measurement Section at NBS with the screens positioned exactly as used. The screens $(7.6 \mathrm{~cm} \times 7.6 \mathrm{~cm})$ are placed directly over the opening of the cylindrical shield by sliding the screens into a set of grooves mounted on the shield's diaphragm.

\section{Prism Spectroradiometer (Measurements)}

When measurements with the prism instrument are made, the standard of spectral irradiance is placed at a distance of either $30 \mathrm{~cm}$ or $50 \mathrm{~cm}$ from the entrance port of the sphere. In most cases a standard calibrated for

Table I. Filter Band Passes

\begin{tabular}{rc}
\hline$\lambda(\mathrm{nm})$ & Band pass $(\mathrm{nm})$ \\
\hline 260 & 20 \\
290 & 16 \\
360 & 12 \\
436 & 5 \\
500 & 8 \\
600 & 12 \\
800 & 19 \\
1000 & 17 \\
1200 & 14 \\
1500 & 14 \\
1800 & 17 \\
2400 & 50 \\
\hline
\end{tabular}

Table II. Effective Wavelengths of Filters when Used with a Photomultiplier and Several Sources

\begin{tabular}{cccc}
\hline$\lambda_{W}$ & $\lambda_{\mathrm{Kr}}$ & $\lambda_{\mathrm{Xe}}$ & $\lambda_{\mathrm{HgXe}}$ \\
\hline 296.6 & 294.7 & 294.8 & 298.2 \\
361.3 & 361.4 & 361.4 & 363.9 \\
391.3 & 391.0 & 391.2 & 390.7 \\
437.0 & 436.9 & 437.0 & 438.1 \\
451.5 & 451.5 & 451.5 & 451.0 \\
550.1 & 550.2 & 550.1 & 549.1 \\
579.0 & 579.0 & 579.0 & 579.2 \\
640.4 & 640.4 & 640.3 & 640.4 \\
680.1 & 680.0 & 680.0 & 680.3 \\
\hline
\end{tabular}

Table III. Effective Wavelengths of Filters when Used with a PbS Cell and Several Sources

\begin{tabular}{rrrr}
\hline \multicolumn{1}{c}{$\lambda_{W}$} & \multicolumn{1}{c}{$\lambda_{\mathrm{Kr}}$} & \multicolumn{1}{c}{$\lambda_{\mathrm{Xe}}$} & \multicolumn{1}{c}{$\lambda_{\mathrm{H}_{\mathrm{B}} \mathrm{Xe}_{\mathrm{e}}}$} \\
\hline 640.8 & 640.8 & 640.7 & 640.8 \\
681.6 & 681.5 & 681.4 & 681.8 \\
699.1 & 699.1 & 699.0 & 699.0 \\
799.6 & 800.2 & 800.1 & 799.7 \\
900.7 & 900.1 & 900.6 & 900.4 \\
1001.6 & 1001.1 & 1001.2 & 1001.8 \\
1193.9 & 1194.3 & 1194.0 & 1194.1 \\
1299.6 & 1300.0 & 1299.2 & 1299.6 \\
1697.8 & 1698.4 & 1697.8 & 1698.9 \\
\hline
\end{tabular}

irradiance at $30 \mathrm{~cm}$ is used in order to obtain higher irradiances which are required in the uv and ir for satisfactory signal-to-noise ratios. The spectrometer, along with its auxiliary components, is then rotated to a preset position and the source under investigation is aligned. Both sources (standard and unknown) are continuously scanned over the entire spectral range of each detector. Usually two or more sets of curves are obtained for each source where one set consists of a complete scan of each source over the entire spectral range from $0.25 \mu$ to $2.5 \mu$.

It is then possible to plot the irradiance of the unknown source as a function of wavelength by reading the data at as many wavelengths as are needed to give a good representation of the shape of the irradiance curve. That is, when determining the spectral irradiance of a line source, the irradiance is computed every $2 \mathrm{~nm}$ in the uv where the resolution of the monochromator is relatively good (about $1 \mathrm{~nm}$ to $2 \mathrm{~nm}$ ), every $4 \mathrm{~nm}$ in the visible (resolution about $4 \mathrm{~nm}$ to 6 $\mathrm{nm}$ ), every $10 \mathrm{~nm}$ in the ir out to about $1.5 \mu$ where the monochromator's resolution becomes increasingly less (from $10 \mathrm{~nm}$ to $50 \mathrm{~nm}$ ), and every $20 \mathrm{~nm}$ from $1.5 \mu$ to 2.5 $\mu$. By using the irradiance values at these wavelengths and the actual recorder tracings, the spectral irradiance of the unknown source is determined.

The time required to reduce the data is significantly reduced by tabulating the recorder readings, amplifier gain setting, and the transmittance for each screen directly onto Fortran coding forms. The defining equation for determining the spectral irradiance $E_{\lambda}$ at wavelength $\lambda(\mathrm{nm})$ is given as

$$
E_{\lambda}=E_{\lambda, S} D G \tau_{S} / D_{S} G_{S \tau},
$$

where $E_{\lambda S}=$ spectral irradiance of standard $\left(\mu \mathrm{W} \mathrm{em}^{-2}\right.$ $\left.\mathrm{nm}^{-1}\right) ; D=$ recorder deflection for test source; $G=$ amplifier setting for test source; $\tau=$ transmittance of screen used with test source; $D_{S}=$ recorder deflection for standard of spectral irradiance; $G_{S}=$ amplifier setting for standard of spectral irradiance; and $\tau_{\mathcal{S}}=$ transmittance of screell used with standard of spectral irradiance.

However, it should he noted that although the resulting curve gives a true representation of the continuum, a true representation of the spectral emission lines is not obtained. Since line widths may vary considerably, especially when a source is operated at very high pressures, the lines are arbitrarily reported in one of three ways: (1) as plotted by the particular instruments; (2) as triangles of arbitrary base; or (3) as rectangles of arbitrary base. For most of our work, the first method is used for best representing the data since most of the line sources examined contain spectral lines which are relatively broad and which tend to extend into overlapping bands; this method also affords greater ease in the reduction of the data.

\section{Photoelectric Filter Spectroradiometer (Instrumentation)}

The photoelectric filter spectroradiometer which is 


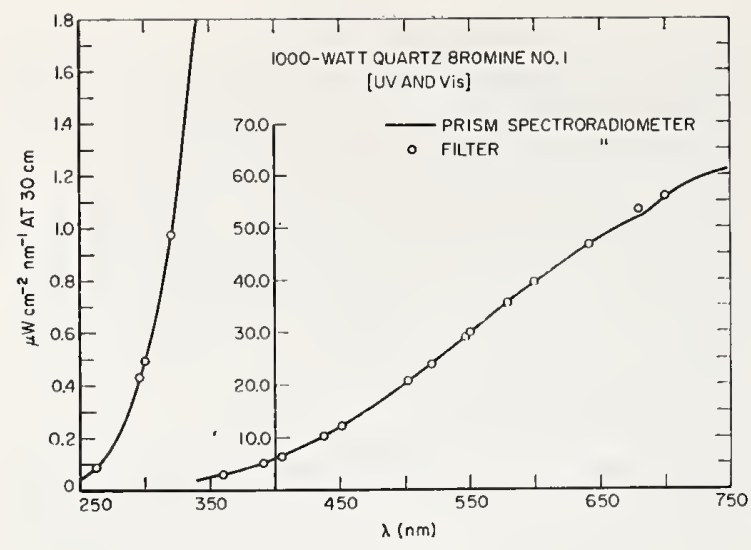

(a)

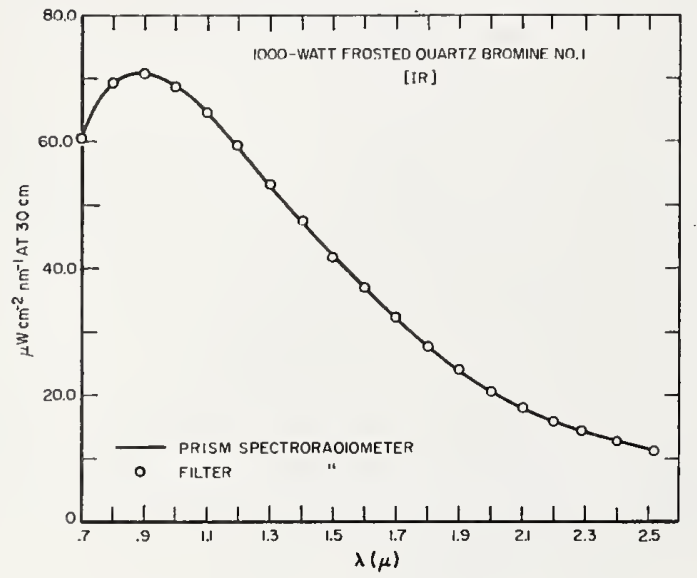

(b)

Fig. 3. (a) Spectral irradiance of $1000-W$ quartz bromine No. 1 from $250 \mathrm{~nm}$ to $750 \mathrm{~nm}$. (b) Spectral irradiance of $1000-\mathrm{W}$ quartz bromine No. 1 from $0.7 \mu$ to $2.5 \mu$.

shown diagramatically in Fig. 2 contains a set of thirtysix narrow band pass interference filters that are mounted at $10^{\circ}$ intervals on an aluminum disk which can be rotated by a 36-position servomotor to any one of the thirty-six positions. A spring and cam arrangement ensures that each filter is positioned directly in front of the detector. A particular filter is set in position merely by adjusting a dial to the corresponding filter setting.

The two detectors, a photomultiplier and a $\mathrm{PbS}$ detector, are mounted on an adjustable table so that by means of a screw either detector can alternately be brought into proper horizonal position after the dual detector housing is mounted and adjusted for correct vertical positioning.

While an integrating sphere is not absolutely necessary for this instrument, its use is recommended to avoid small errors due to variations in detector surface sensitivity and in determining the true optical distance from the source to the detector. A 10.2-cm diam integrating sphere having a $19-\mathrm{mm}$ diam entrance aperature and a $12.7-\mathrm{mm}$ diam exit aperture has accordingly been incorporated into the instrument. $\mathrm{A} \mathrm{BaSO}_{4}$ coating was used in perference to the less durable $\mathrm{MgO}$ coating since higher energies are available with the filter instrument.

In order to eliminate stray radiation, the entire system is enclosed in a light tight box. Again, to facilitate comparison of the source under investigation with the standard of spectral irradiance, the entire setup is mounted on an optical bench (lathe bed) that allows a rapid interchange of position between the two sources.

The electronies employed with the filter setup along with the readout system, light shield, and attenuating screens are interchangeable with the prism spectroradiometer. However, a duplicate chopper is required since the chopper itself is an integral part of both systems.

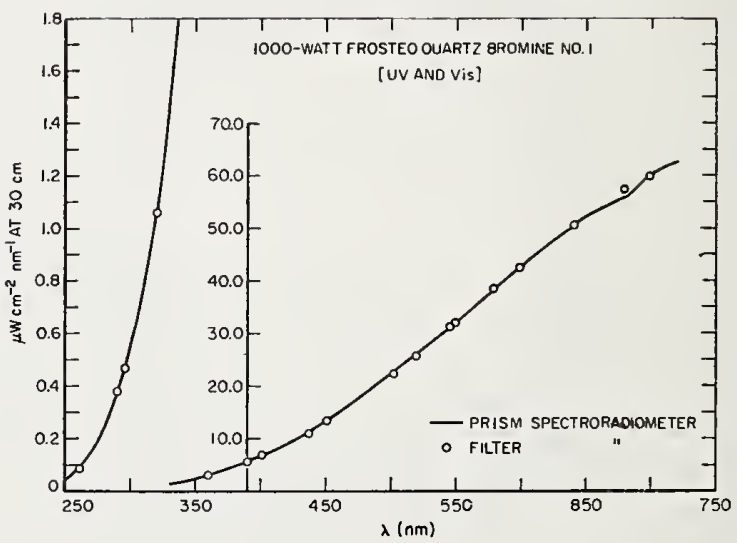

(a)

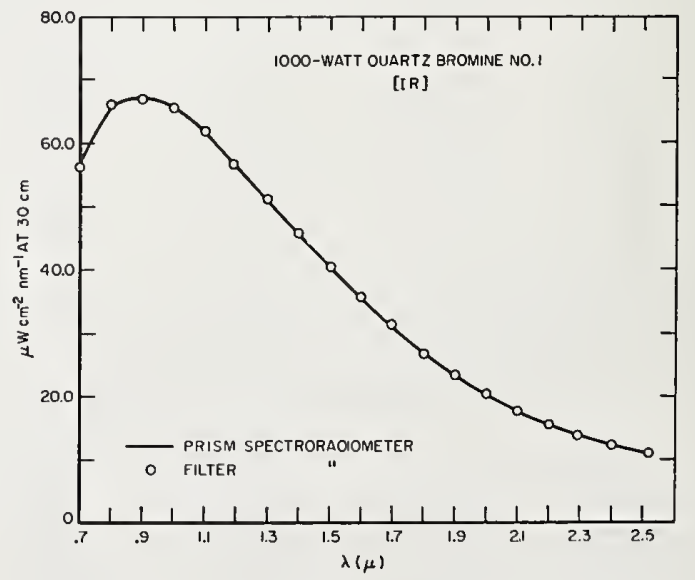

(b)

Fig. 4. (a) Spectral irradiance of $1000-W$ frosted quartz bromine No. 1 from $250 \mathrm{~nm}$ to $750 \mathrm{~nm}$. (b) Spectral irradiance of $1000-W$ frosted quartz bromine No. 1 from $0.7 \mu$ to $2.5 \mu$. 


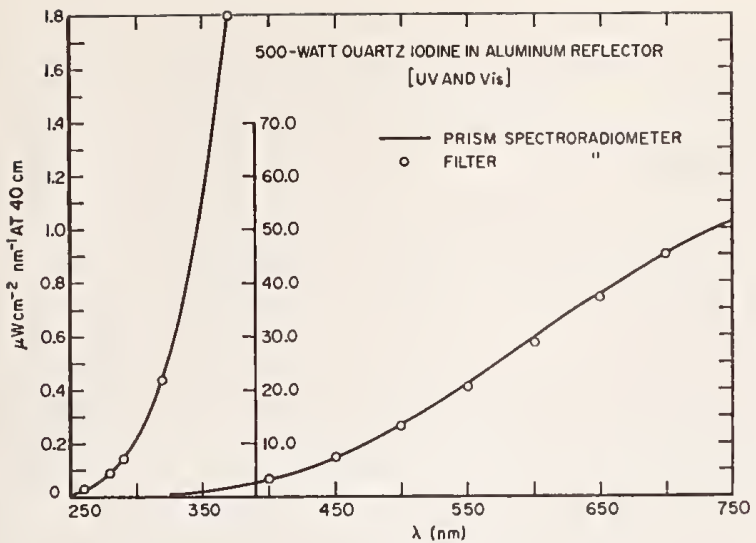

(a)

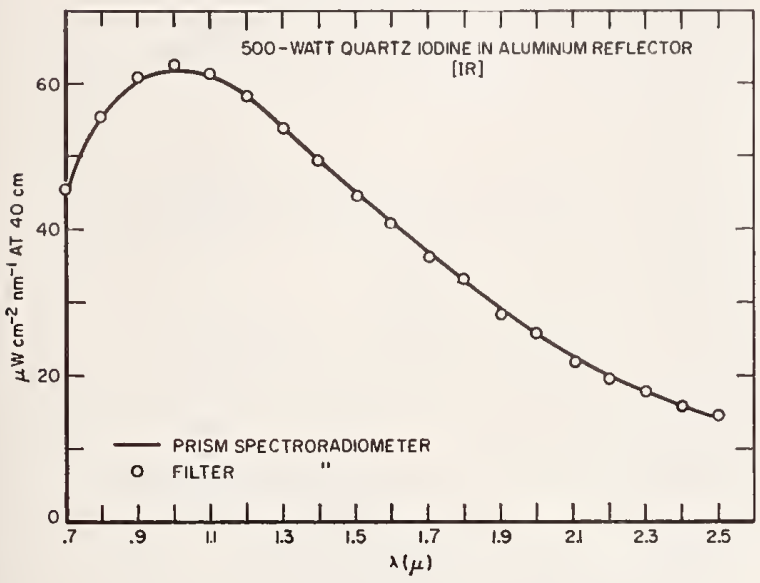

(b)

Fig. 5. (a) Spectral irradiance of 500-W quartz iodine in aluminum reflector from $250 \mathrm{~nm}$ to $750 \mathrm{~nm}$. (b) Spectral irradiance of $500-\mathrm{W}$ quartz iodine in aluminum reflector from $0.7 \mu$ to $2.5 \mu$.

\section{Photoelectric Filter Spectroradiometer (Measurements)}

Even though the spectral band passes of the interference filters shown in Table I are relatively narrow for various portions of the spectrum, it is necessary to determine the effective wavelength for each filterdetector-source combination. From a knowledge of the spectral transmittance of the filter, the relative spectral response of the detector, and the relative spectral energy distribution of the source, the effective wavelength $\lambda_{\text {efs }}$ can be calculated through the use of the following equation:

$$
\lambda_{\text {eff }}=\frac{\int_{\lambda_{1}}^{\lambda_{2}} R(\lambda) \tau(\lambda) E_{\lambda} \lambda d \lambda}{\int_{\lambda_{1}}^{\lambda_{2}} R(\lambda) \tau(\lambda) E_{\lambda} d \lambda} .
$$

where $\lambda_{\text {eff }}=$ effective wavelength; $R(\lambda)=$ relative spectral response of detector; $\tau(\lambda)=$ spectral transmittance of interference filter; $E_{\lambda}=$ relative spectral irradiance of source; and $\lambda=$ wavelength. The limits of integration $\lambda_{1}$ and $\lambda_{2}$ are assigned as the wavelengths at $0.1 \%$ transmittance.

In most eases the effective wavelength of the standard lamp-filter-detector combination is very nearly equal to that of the unknown source-filter-detector combination throughout the continuum whereas in the vicinity of strong emission lines these differences in $\lambda_{\text {oft }}$ for each source may be significant. However, once the effective wavelength for a particular type of source (filterdetector-source combination) such as a xenon are lamp has been determined, it is not essential that $\lambda_{\text {eff }}$ be redetermined for other xenon lamps since only large differences between the relative energy distribution of the lamps will significantly change $\lambda_{\text {eff }}$. In Tables II and III are shown the effective wavelengths for a number of filters when used in conjunction with various sources over the photomultiplier spectral range (Table II) and when used over the $\mathrm{PbS}$ spectral range (Table III).

Measurements with the filter spectroradiometer are made with the standard of spectral irradiance at a

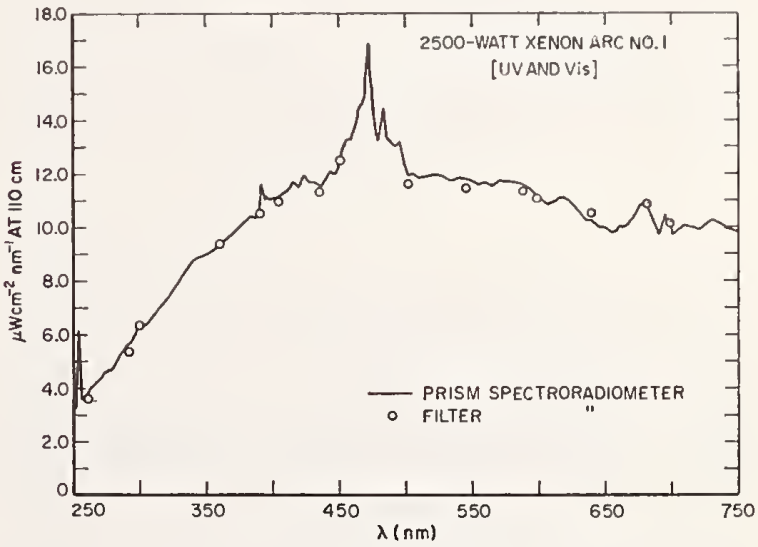

(a)

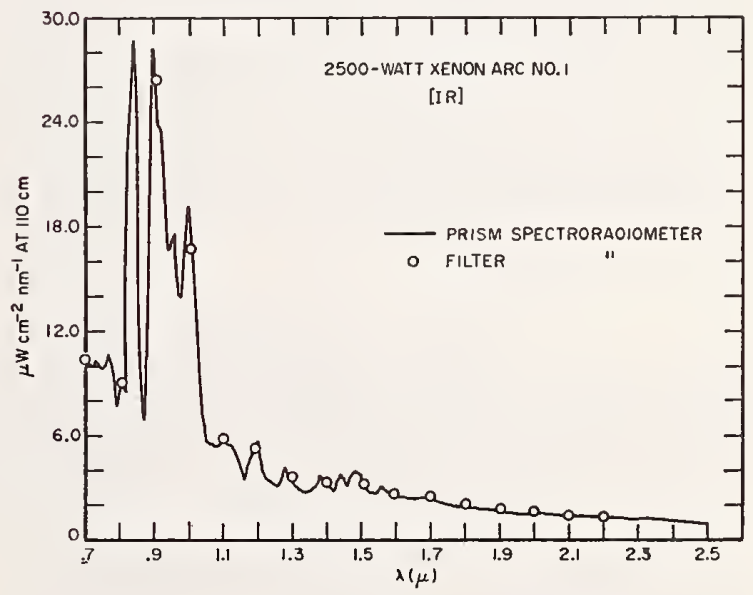

(b)

Fig. 6. (a) Spectral irradiance of $2500-W$ xenon arc No. 1 from $250 \mathrm{~nm}$ to $750 \mathrm{~nm}$. (b) Spectral irradiance of $2500-\mathrm{W}$ xenon arc No. 1 from $0.7 \mu$ to $2.5 \mu$. 


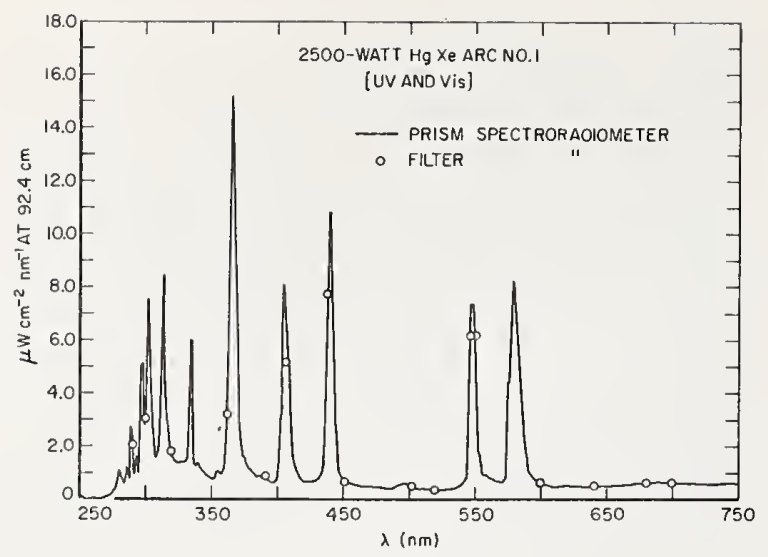

(a)

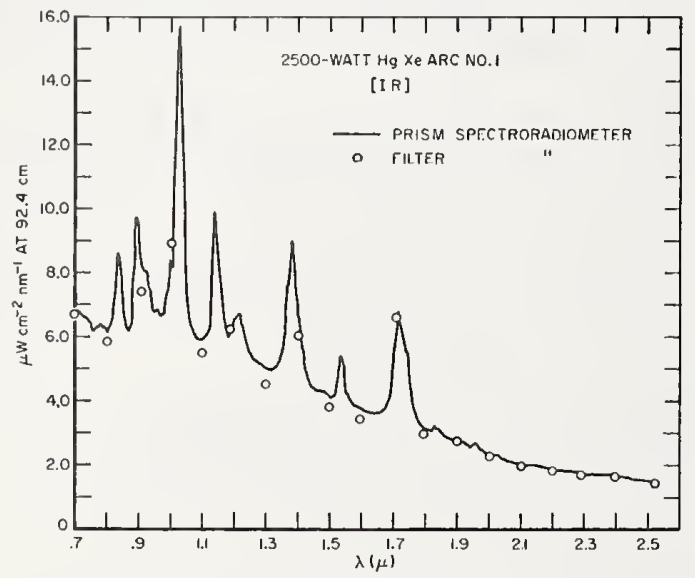

(b)

Fig. 7. (a) Spectral irradiance of $2500-\mathrm{W}$ HgXe arc No. 1 from $250 \mathrm{~nm}$ to $750 \mathrm{~nm}$. (b) Spectral irradiance of $2500-\mathrm{W} \mathrm{HgXe}$ arc No. 1 from $0.7 \mu$ to $2.5 \mu$.

distance of $50 \mathrm{~cm}$ from the entrance port of the sphere. The signal-to-noise ratio when looking at the standard lamp is at least 100 or better at the various wavelengths. The irradiance of the unknown source is compared directly with that of the standard by alternately moving the filter spectroradiometer to view one source and then the other at each wavelength of interest. The spectral irradiance of the unknown source is calculated from the following relationship:

$$
E^{\prime}{ }_{(\lambda \mathrm{eff})^{\prime}}=\left(R_{x} / R_{\mathrm{std}}\right) E_{\lambda \mathrm{eff}},
$$

where $E^{\prime}{ }_{(\lambda e f f)^{\prime}}=$ spectral irradiance at $(\lambda \text { eff })^{\prime}$ for the unknown source; $E_{\lambda \text { eff }}=$ spectral irradiance at $\lambda$ eff for the standard of spectral irradiance; $R_{x}=$ detector response for unknown; and $R_{\text {std }}=$ detector response for standard.

\section{Comparison of Prism Spectroradiometric with Photoelectric Filter Spectroradiometric Results}

The spectral irradiances of a number of sources have been measured by using both the prism and filter spec- troradiometers and the comparison of the results obtained on a few of the sources measured is shown in Figs. 3-9. In all cases, the solid lines represent the spectral irradiance values as determined with the prism spectroradiometer and the circles are representative of the spectral irradiances as determined with the filter spectroradiometer. Both the prism and the filter instrument results are the averages of two or more sets of measurements with each method having a spread of less than $1 \%$ over most of the spectral range. Each figure is divided into parts (a) and (b); the (a) section includes the spectral irradiance of the source from 0.25 $\mu$ to $0.75 \mu$ and the (b) section from $0.7 \mu$ to $2.5 \mu$. Since Figs. 3, 4, and 5 are representative of continuous sources, section (a) has two ordinate scales, $0-1.8 \mu \mathrm{W}$ $\mathrm{cm}^{-2} \mathrm{~nm}^{-1}$ from $250 \mathrm{~nm}$ to about $340 \mathrm{~nm}$ and $0-70 \mu \mathrm{W}$ $\mathrm{cm}^{-2} \mathrm{~nm}^{-1}$ from about $340 \mathrm{~nm}$ to $750 \mathrm{~nm}$.

As shown in Figs. 3, 4, and 5, good agreement is obtained between the two methods of measurement, the

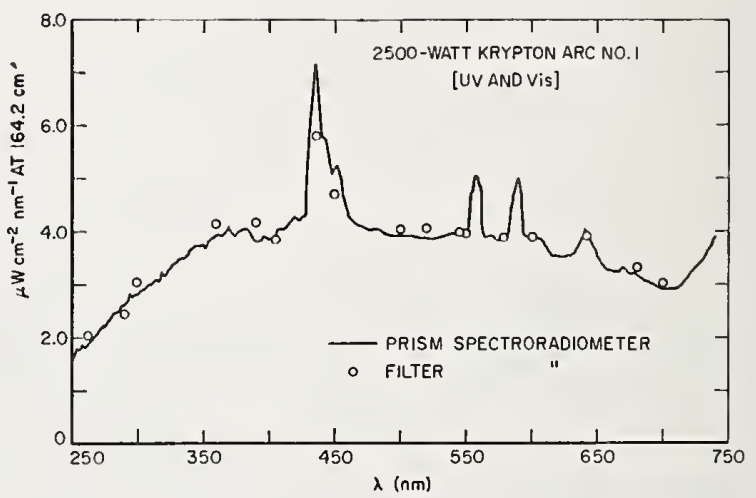

(a)

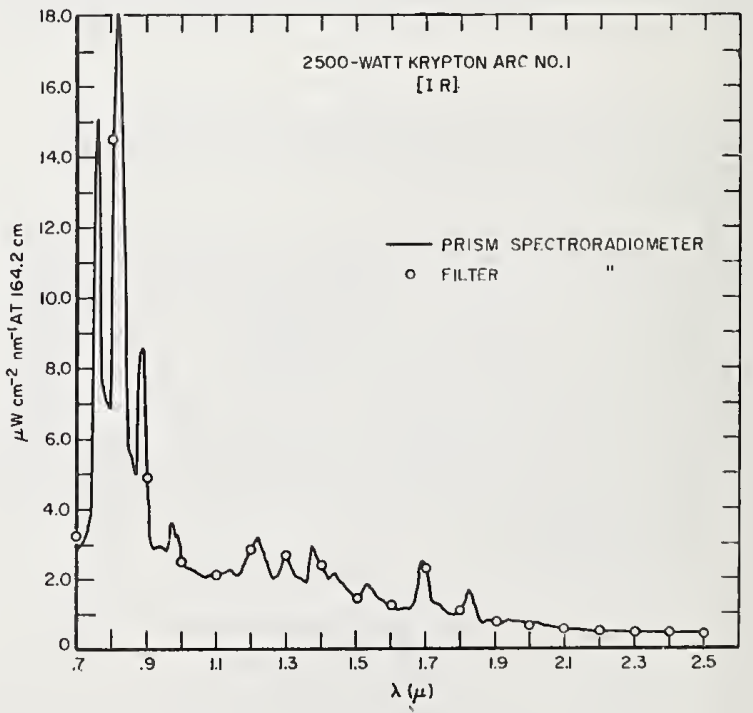

(b)

Fig. 8. (a) Spectral irradiance of 2500-W krypton are No. 1 from $250 \mathrm{~nm}$ to $750 \mathrm{~nm}$. (b) Spectral irradiance of $2500-\mathrm{W}$ krypton arc No. 1 from $0.7 \mu$ to $2.5 \mu$. 


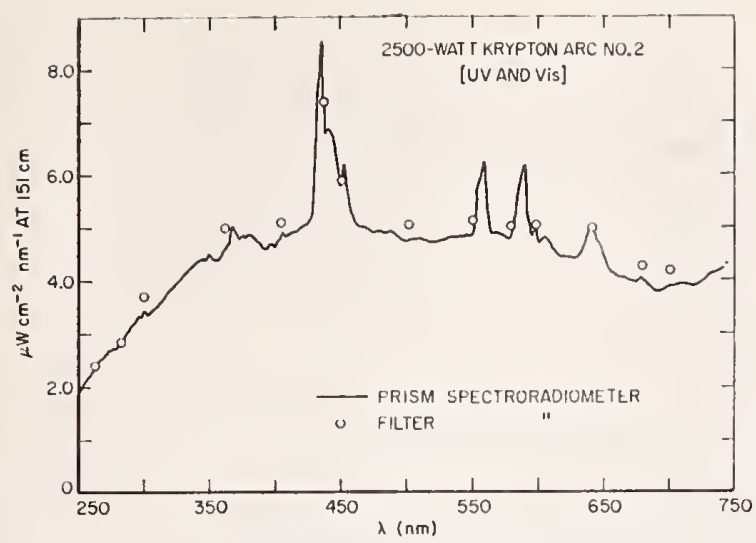

(a)

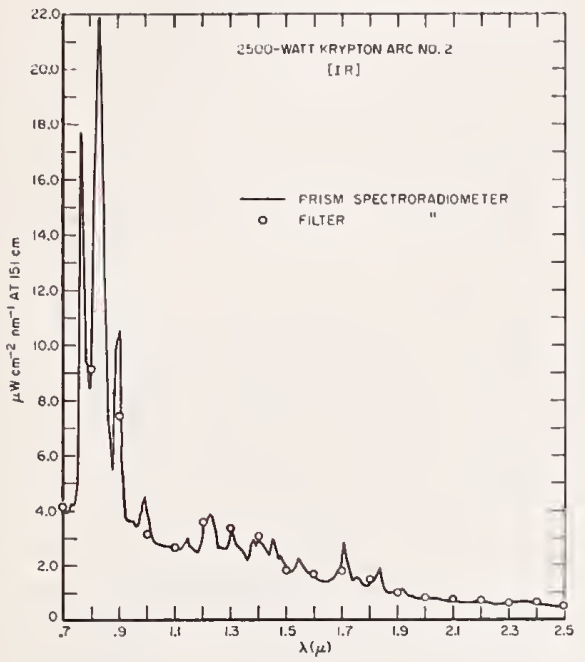

(b)

Fig. 9 (a) Spectral irradiance of 2500-W krypton are No. 2 from $250 \mathrm{~nm}$ to $750 \mathrm{~nm}$. (b) Spectral irradiance of 2500-W krypton arc No. 2 from $0.7 \mu$ to $2.5 \mu$.

difference being less than $1 \%$ in most cases and the largest difference being about $3 \%$. When high pressure arc sources are measured, the general agreement between the two methods of measurement is not so good as the agreement for the con tinuous sources; the results, nevertheless, are encouraging. In Figs. 6-8 and 9 are shown the spectral irradiances of various high pressure arcs which are representative of the sources commonly used in a number of solar simulators. In Figs. 6(a) and 6 (b), the filter values at the majority of wavelengths for a $2500-\mathrm{W}$ high pressure xenon arc agree within $1 \%$ to $2 \%$ of the curve derived via the prism instrument. However, there is a $7 \%$ discrepancy at $260 \mathrm{~nm}$ and a $5 \%$ discrepancy at $290 \mathrm{~nm}$. The agreement between the two methods is also good in the spectral region of 0.8 $\mu$ to $1.5 \mu$ where a number of emission bands are present. In Figs. 7-9 are shown the spectral irradiances of a $2500-\mathrm{W}$ high pressure $\mathrm{HgXe}$ arc and two $2500-\mathrm{W}$ high pressure krypton arcs. The agreement between the values for the krypton ares is not so good as that for the xenon arc. This can primarily be attributed to the instability of the krypton ares.

It should be noted that in the majority of cases in the ir past $1.0 \mu$, the spectral band passes of the interference filters are narrower than the band pass of the monochromator; in the uv and visible, the situation is reversed. This effect is quite noticeable in regions where the filters transmit at wavelengths of strong line emission or transmit at wavelengths between two lines that are relatively close together. For example, in Fig. 7 (a) the filter spectroradiometer measured lower values for the $365-\mathrm{nm}$, 405-11m, and 546-nm emission lines where the resolution of the filters is not so good as that of the prism monochromator. In the ir at $1.1 \mu, 1.3 \mu$, and 1.5 $\mu$ [see Fig. 7(b)], the filter spectroradiometer measures lower values since these wavelengths are situated between two strong emission lines that are close to each other. In the first case, the prism spectroradiometer values are more nearly correct, whereas in the second case the filter spectroradiometer better represents the actual irradiance of the source. Also, in Figs. 3(a) and 4 (a) at $682 \mathrm{~nm}$ the prism results are slightly lower than the values obtained with the filter setup. This difference in all probability is due to a combination of absorption by the bromine at this wavelength region and to the narrower band pass of the prism instrument relative to the interference filters.

\section{Conclusions}

The close agreement of the spectral irradiances of the sources examined by means of both the prism spectroradiometer and the photoelectric filter spectroradiometer suggests that repeated spectral irradiance determinations of solar simulators, weatherometers, etc., using the monochromator method are not always essential. Once the spectral irradiance of the source has been carefully determined by means of the more elaborate prism spectroradiometer, a simple photoelectric filter spectroradiometer can be used for periodically checking the irradiance at as many wavelengths as desired.

The work reported in this paper was supported by the National Aeronautics and Space Administration.

\section{References}

1. R. Stair, W. E. Schneider, and J. K. Jackson, Appl. Opt. 2, 1151 (1963).

2. R. Stair, W. E. Schneider, W. R. Waters, J. K. Jackson, and R. E. Brown, "Some Developments in Improved Methods for the Measurement of the Spectral Irradiances of Solar Simulators", NASA Report No. CR-201, A pril 1965.

3. R. Stair, R. G. Johnston, and E. W. Halbach, Natl. Bur. Std. J. Res. 64A, 291 (1960).

4. W. W. Coblentz, Bul. Bur. Std. 11, 98 (1941) S 227.

5. W. W. Coblentz and W. B. Emerson, Bull. Bur. Std. 12, 503 (1916) S 261.

6. R. Stair, W. E. Schneider, and W. B. Fussell, Appl. Opt. 6, 101 (1967).

7. A. K. Angström, Tellus 10, 342 (1958). 
8. J. C. DeVos, Physica 20, 690 (1954).

9. Robert D. Larrabee, J. Opt. Soc. Am. 49, 619 (1959).

10. R. Stair and W. O. Smith, J. Res. Natl. Bur. Std. 30, 449 (1943) RP 1543.

11. Louis E. Barbrow, J. Opt. Soc. Am. 49, 122 (1959).

12. J. R. Hickey, "Correlation of Monochromator and Filter Radiometry Determinations of the Spectral Distribution in Large Simulators," Proceedings IES and ASTM Interna- tional Symposium on Solar Radiation, Los Angeles, January 1965. (Institute of Environmental Sciences, Mount Prospect, Ill.)

13. G. C. Goldman, "Spectral Measurements by the Filter Method on Lewis Carbon-Arc Solar Simulators," NASA Lewis Research Center Report, 1964.

14. David G. Goebel, B. Patrick Caldwell, and Harry K. Hammond, III, J. Opt. Soc. Am. 56, 783 (1966).

APPLIED OPTICS / Vol. 6, No. 9 / September 1967 


\title{
THE MEASUREMENT OF SOLAR RADIATION, WITH PRINCIPAL EMPHASIS ON THE ULTRAVIOLET COMPONENT*
}

\author{
RALPH STAIR \\ National Bureau of Standards, Washington, D.C., U.S.A \\ (First received 17 January 1966 and in final form 23 February 1966)
}

\begin{abstract}
A review is presented covcring much of the scientific work of the past half-century relating to the measurement of the spectral distribution of radiant energy (in particular in the u.v. region) from the sun. Mention is made of those special solar disturbances that are expected to result in large variations of the u.v. solar emission as well as the absorbing medium, ozone, which through its turbulence and distribution further affects greatly the spectral distribution of the solar radiation reaching the earth's surface at any locality. Various instrumentations and the standards employed by the different laboratories are discussed in some detail with emphasis placed upon some of their shortcomings and the great lack of accurate solar spectral data, in particular within the u.v. region. Finally, new instrumentation offering promise of considerable usefulness in obtaining rapidly more accurate data in this area is described.
\end{abstract}

THE SUN, our nearest neighbor of the stars of the milky way, consists of a large, hot, gaseous mass (principally hydrogen). When viewed through a dark glass, without magnification, it appears as a brilliant, quiescent, smooth, circular disk. However, when examined with the aid of refined techniques, the photospheric surface is highly granulated and is surrounded by a gaseous envelope, the chromosphere. Beyond this and often extending many millions of miles into space is the more tenuous corona.

The sun's surface and its surrounding atmosphere are by no means static. The short-lived photospheric grains (see FIG. 1) constantly change in size and shape from minute to minute while the dark sunspots and bright areas change more slowly as they cover larger areas indicating more massive disturbances. These, together with the erupting prominences, filaments, and flares such as those illustrated in the coronal photograph (FIG. 2), indicate that the entire observable sun is in a state of considerable turmoil. This turmoil is associated with the magnitude and spectral distribution of the solar radiation reaching the outer limits of the earth's atmosphere.

FIGURE 3 illustrates some loop prominences. These are very interesting phenomena, but their character and cause are not clearly understood at this time. They are no doubt associated in some way with magnetic fields in the solar atmosphere, since their formation and velocities seem to defy the usual laws of gravitation and the expulsion velocity of matter. This and the previous two photographs were made by and supplied through the courtesy of the Sacramento Peak Observatory, Air Force Cambridge Research Laboratories, Sunspot, New Mexico.

The solar corona (see FIG. 4), while a very interesting phenomenon, adds little to the solar radiation reaching the earth's surface or outer atmosphere. It is rather an index to other activities in progress in the solar atmosphere, and results from the

\footnotetext{
* Contribution of the National Bureau of Standards, not subject to copyright.
} 
exciting particles (principally protons) comprising the solar wind which give the corona its radial character. The solar corona may be considered to extend throughout the solar system as evidenced by the appearance of comet tails (which always extend away from the sun), but its intensity is so weak at the earth that its only effects are the production of such things as the aurorae and certain magnetic disturbances. This photograph, taken by the U.S. Navy on 24 January 1925, shows the corona with the sun approaching a period of maximum sunspot activity. At such times the corona is more uniform and has approximate, but not exact, circular symmetry (MENZEL, 1959; KUIPER, 1953). At times of minimum solar activity the corona exhibits striking series of bright filaments emerging from the sun's polar regions strongly resembling the effects of magnetic fields, while at the solar equatorial regions unusually long streamers appear, giving the corona a very unsymmetrical shape.

The largest sunspot groups ever photographed (see FIG. 5) are shown in these pictures from the U.S. Naval Observatory. The upper left view was observed in February 1946. The upper right vielv was photographed in July 1946, the middle left in March 1947, and the middle right in May 1947. The latter represents the highest monthly sunspot number in more than 100 vears. The lower left is an enlarged view of the sunspot group of March 1947. The lower right is a large group visible in April 1947 (maybe a later phase of the March group).

While the sunspots themselves consist of cool material in a relatively calm state, usually associated with them are very bright and unstable areas (MENZEL, 1959; KUIPER, 1953) emitting excessive u.v. radiation at very short wavelengths. The emission of u.v. from such areas and from various flares, prominences, etc., no doubt varies considerably from time to time. While no instrumentation has yet been set up to prove this statement through monitoring such changes, sufficient rocket data are available to establish high shortwave u.v. emission during times of extreme solar activity.

The source of solar energy production within the sun was a puzzle to the scientific world for many years until the mechanism accounting for the conversion of hydrogen into helium at elevated temperatures and pressures was discussed and essentially solved by Bethe and others (Bethe, 1939; BetHe and Critchfield, 1938; von WEIZSÄCKER, 1938) in 1938/9. It is now well established that the solar energy production is primarily a consequence of various thermonuclear processes through which hydrogen is converted into helium. It is indicated that these processes operate near the solar center where the temperature is in equilibrium near $15,000,000^{\circ}$ and result in the emission of intense gamma radiation and the release of particles which later recombine or leave the sun as corpuscular radiation. The gamma radiation is rapidly transformed into local thermal energy which after multiple radiation, absorption, and re-radiation reaches the solar surface. Since the solar interior is in relative equilibrium, and since the solar material has a low coefficient of heat conductivity, the temperature gradient outward is high. But since heat-travel outward must be the same as its rate of generation, the outflow will be independent of the properties of the solar materials. Hence, it is to be expected that the total solar radiation emitted should be closely uniform over long periods of time.

Measurements by Abbot and others (АBBOT, 1939; HARDIE and GiCLAS, 1955) have shown that the total solar radiation reaching the earth's atmosphere varies not more than 1 to 2 per cent. The variation for wavelengths longer than about $3000 \AA$ pene- 


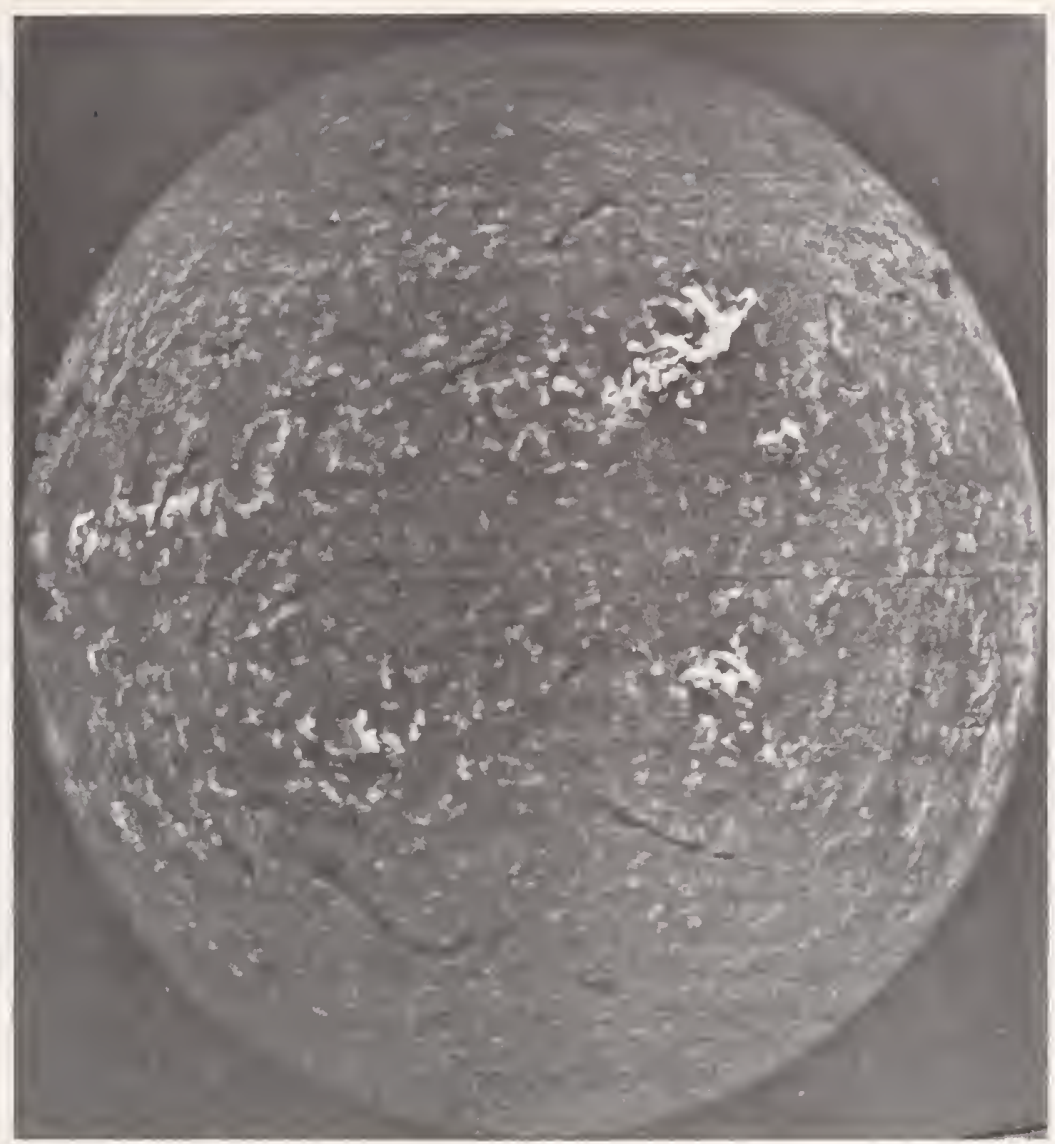

FIG. 1. A spectroheliogram of the sun showing the photospheric grains. (Courtesy Sacramento Peak Observatory). 


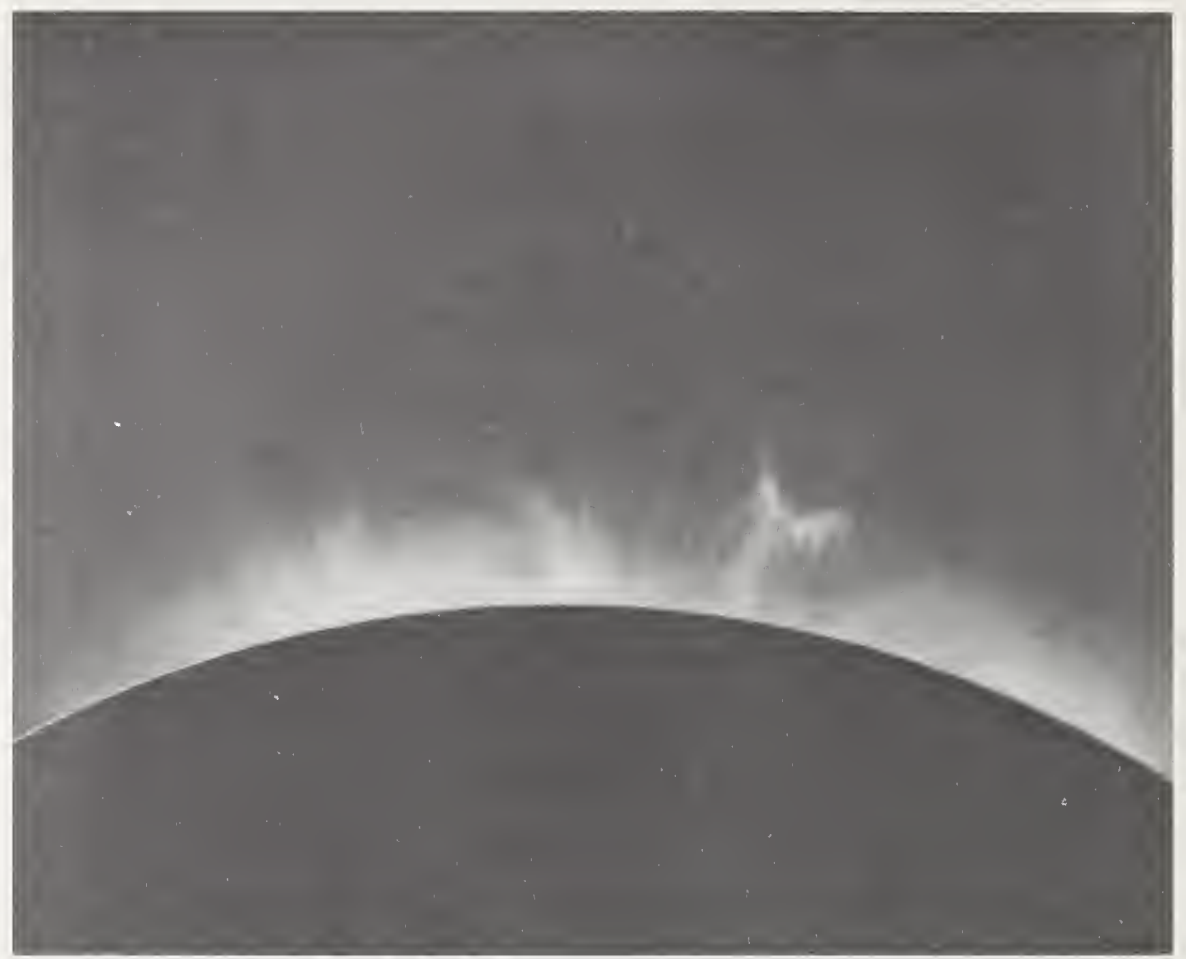

FiG. 2. Coronal photograph showing erupting prominences, filaments and flares. (Courtesy Sacramento Peak Observatory). 


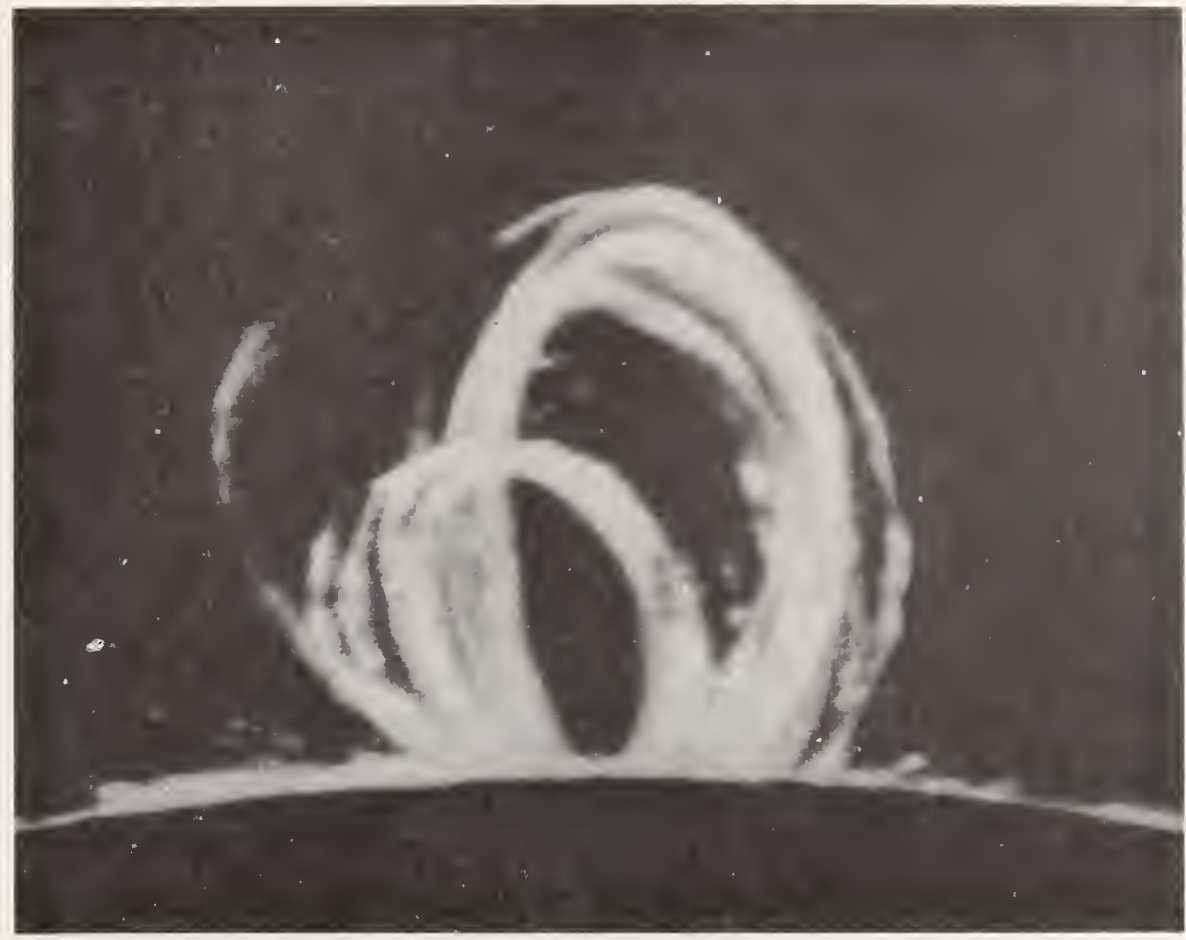

FIG, 3. Coronal photograph showing intense loop prominences. (Courtesy Sacramento Peak Observatory). 


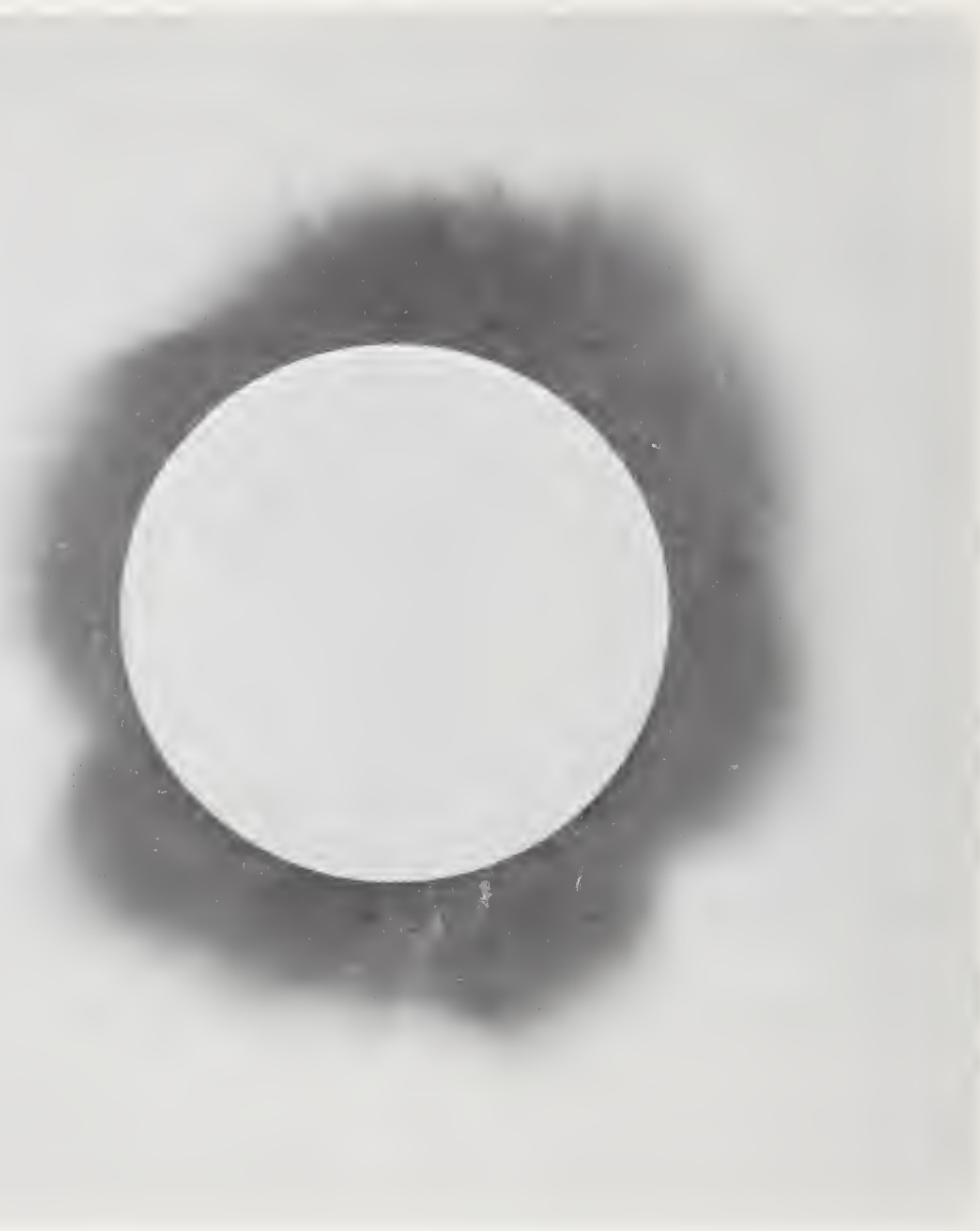

FIG. 4. Solar Corona, 24th January 1925. (Courtesy U.S. Navy). 

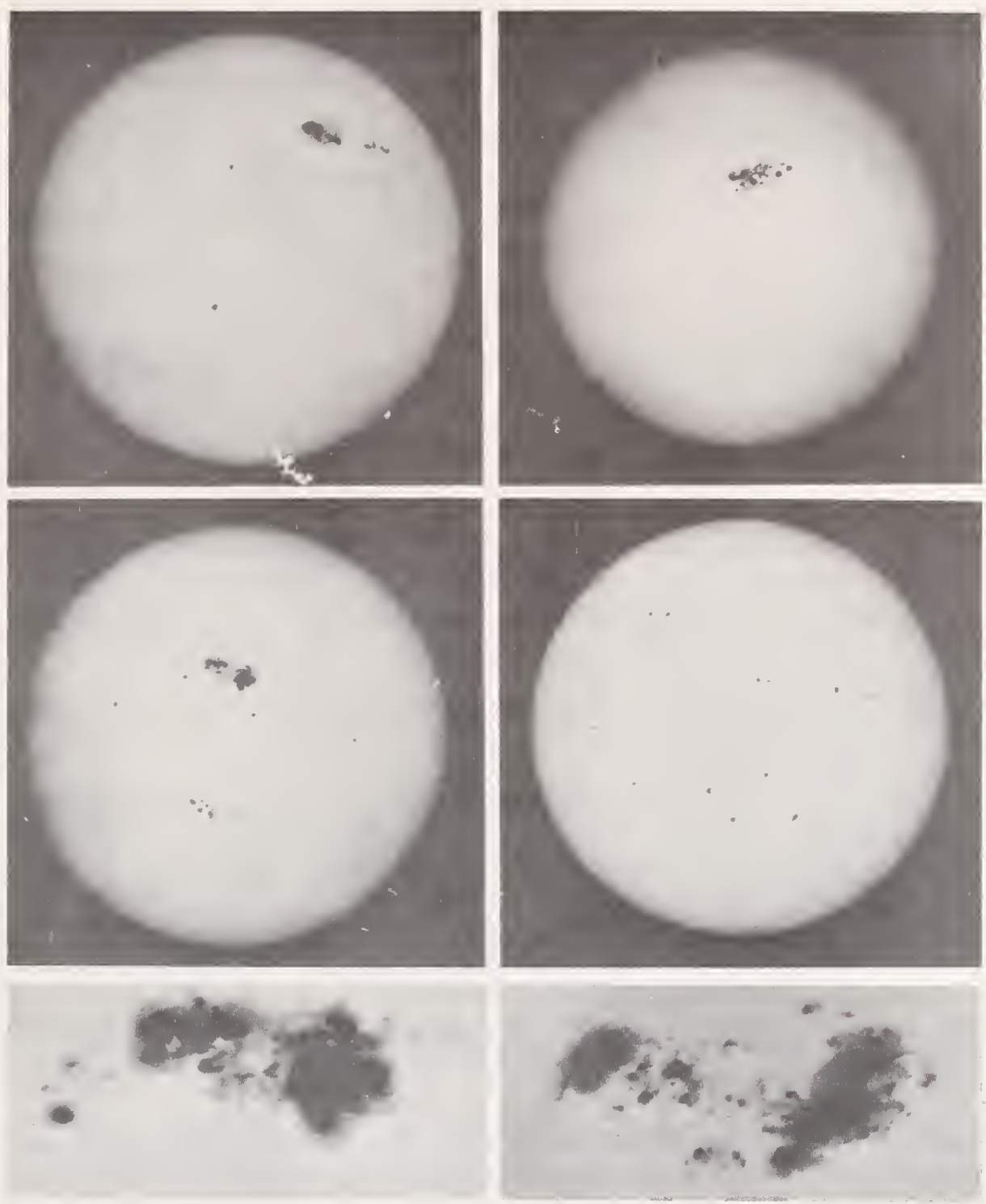

Fig. 5. Large sunspot groups, 1946-1947. Upper left, February 1946; Upper right, July 1946; Middle left, March 1947; Middle right, May 1947; Lower left, enlarged view of sunspot group of March 1947; Lower right, large group of April 1947. (Courtesy U.S. Naval Observatory). 
trating the clearest mountain atmosphere is small since ozone and other atmospheric gases absorb all the shortwave u.v. associated with extreme solar activity, and water vapor content is relatively constant on clear days.

The absorption of ozone (of which there is a total of about $0.25 \mathrm{~cm}$ (S.T.P.) in the atmosphere above the earth at any point) shown in Fig. 6, is complete for all practical purposes for all wavelengths from about $3000 \AA$ to below $2000 \AA$ (TsI-Ze and SHINPiaw, 1932, 1933; INN and TANAKA, 1959; AFRCL HANDBOOK, 1960). At shorter

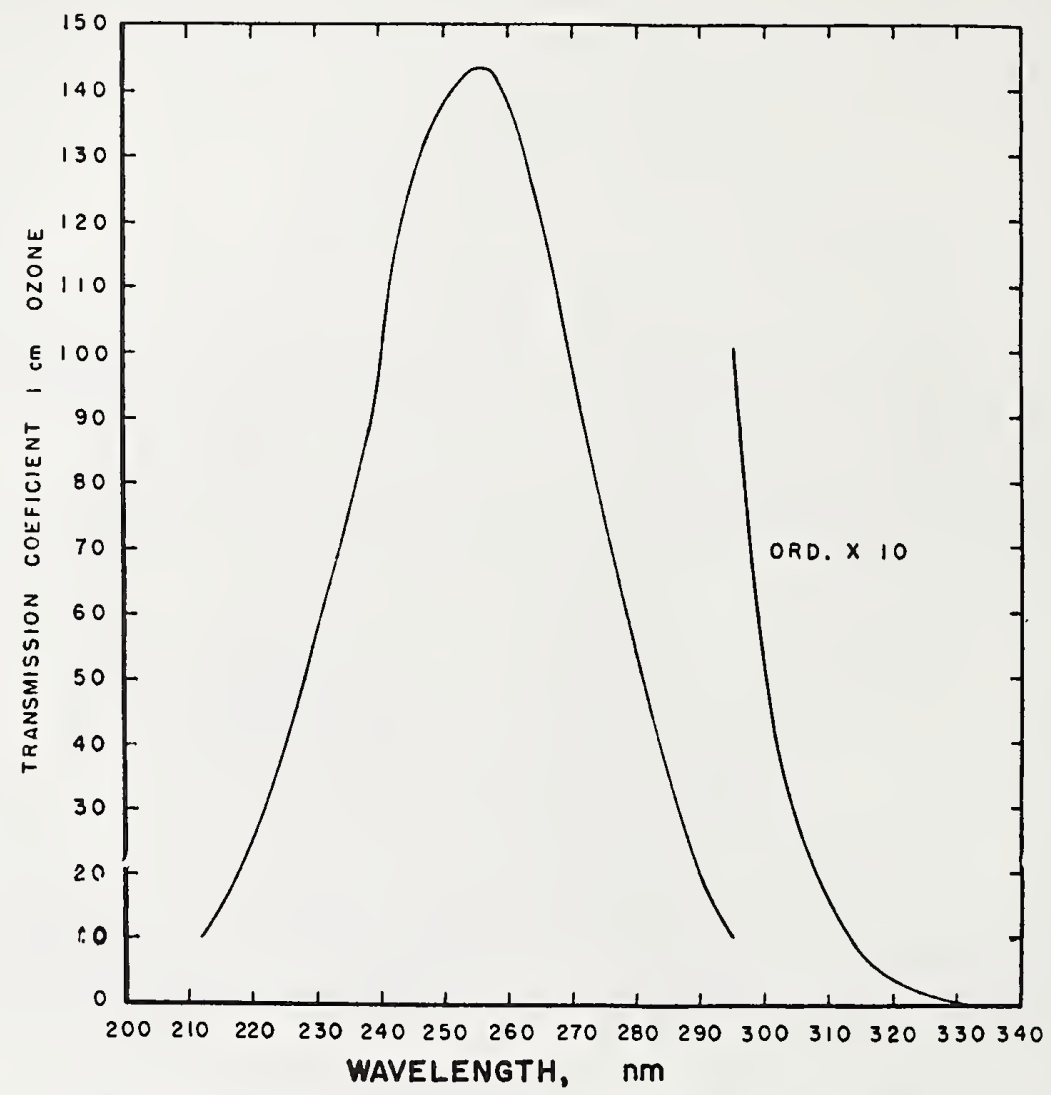

FiG. 6. Absorbance of ozone. After TsI-Ze and Shin-PIAw (1932).

wavelengths the other atmospheric gases may be expected to block out all u.v. solar radiation. Ozone is produced in the upper atmosphere where the shortwave radiation of sunlight transforms the oxygen into the atomic state. At lower atmospheric levels the presence and amount of ozone are greatly dependent upon atmospheric turbulence and circulation.

From many measurements made throughout the world, the total amo $t$ of atmospheric ozone in a vertical column usually present is between 0.2 and $1, \mathrm{~cm}$ (S.T.P.). Measurements at Climax (StaIR, 1952), Colorado, (altitude, 11190 ) in 
September 1951 shown in Fig. 7 indicate ozone existed in the stratosphere above the station at that time in the amount of $0.21 \mathrm{~cm}$. Similar measurements (see Fig. 8) at Sunspot, New Mexico (Stair and Johnston, 1956) (altitude $9200 \mathrm{ft}$ ) indicated ozone in the amount of $0.22 \mathrm{~cm}$ above that station in June 1955. These results are in very close agreement with those obtained at Climax four years earlier. As is to be expected, a slightly higher atmospheric scattering is observed at Sunspot because of its lower altitude.

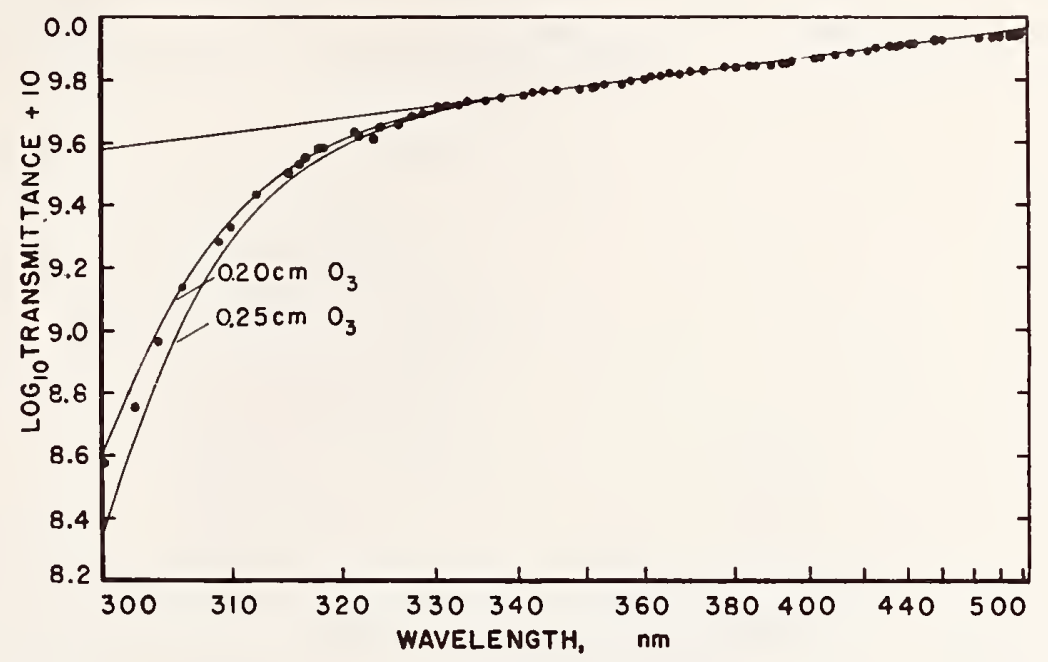

FIG. 7. Atmospheric transmittance at Climax, Colorado (altitude $11,190 \mathrm{ft}$ ), and determination of total ozone above observing station; mean of data for 4 clear days in September 1951.

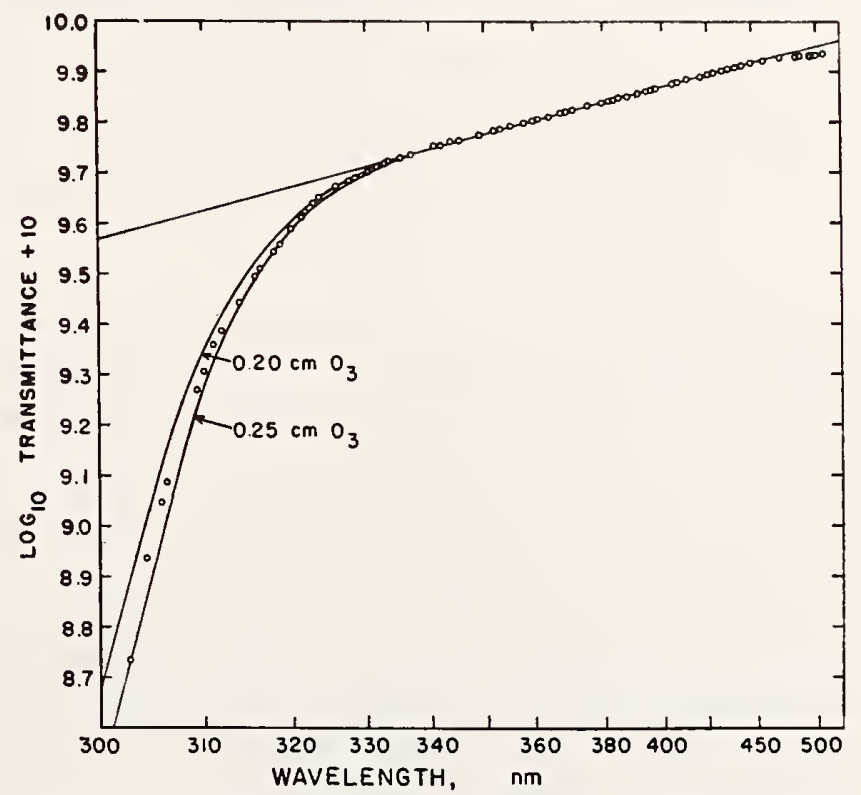

Fig. 8. Atmospheric transmittance at Sacramento Peak (Sunspot), N.M. (altitude $9200 \mathrm{ft}$ ), and determination of total ozone above observing station; mean of data for 4 clear days in June 1955. 
When the total amount of upper atmospheric ozone is measured from day to day over a period of years as shown in FIG. 9, a considerable variation results. These data are from Washington, D.C., between 1934 and 1943 (STAIR, 1949). It is noted that during August a minimum value occurs, with little change from day to day, or from year to year. During this period, upper air circulation and turbulence are at a minimum. Hence, the larger values at other seasons may be considered to result primarily from changes in the upper atmosphere. For example, if the turbulence is such as to bring part of the normal ozone to a lower level, additional oxygen will be exposed to shortwave u.v. with the result that additional ozone will be produced at the higher level.

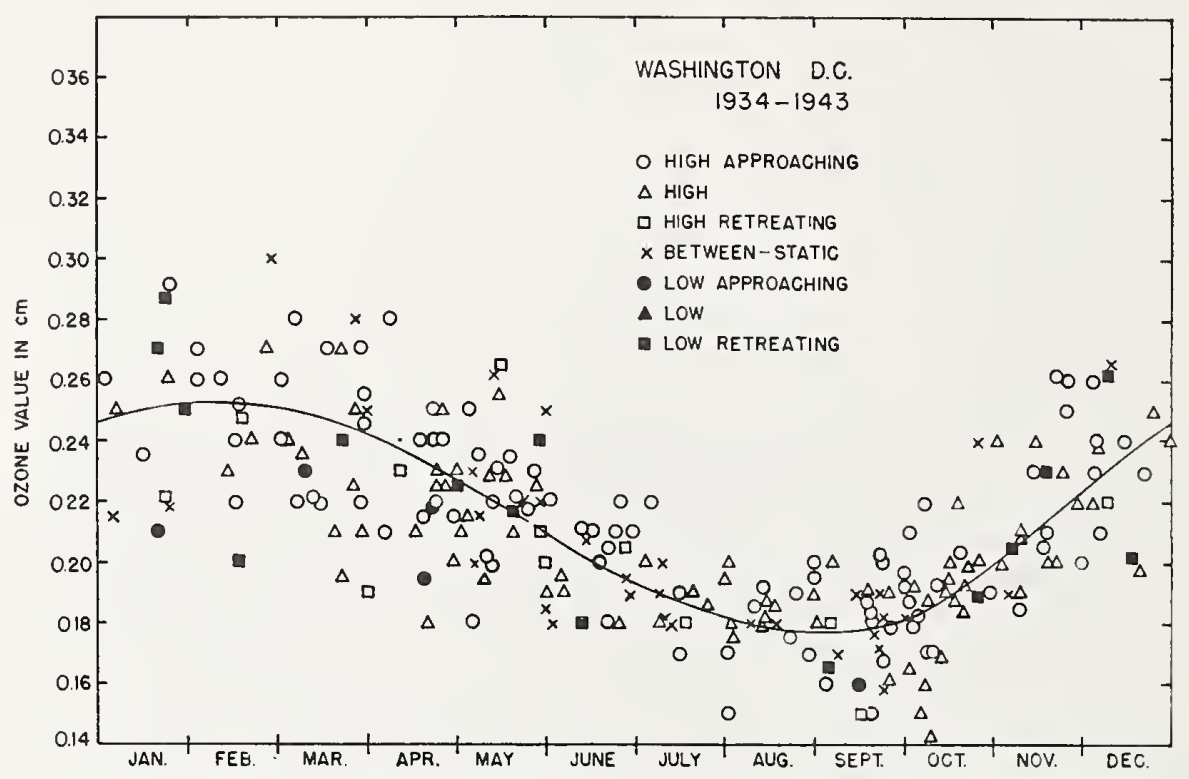

Fig. 9. Seasonal variation of ozone at Washington, D.C. (altitude $300 \mathrm{ft}$ ), using phototube and filter ozone meters.

FIGURE 10 illustrates data on the vertical distribution of ozone as obtained by the NBS (STAIR, 1959), by REgenER and REgener (1934), REgENER (1939), and by the National Geographic Society (1936). Differences are evident -but one could surmise that they result in part from the use of the different methods and instrumentations employed by the different observers. But when a number of measurements (see FIG. 11) are made by the same observer-in this case by REGENER (1951) employing a spectrograph carried aloft by balloons-significant variations in the vertical distribution of ozone are observed. At least two maxima at different altitudes may occur. Shifting of the single maximum to higher or lower altitudes is evident from these data. Other observers employing other types of instrumentation have observed similar shifts in the vertical distribution of ozone at a fixed location or from place to place (DüTSCH, 1959; GREEN, 1964).

Since the solar rays contact the earth's atmosphere at inclined angles as the polar regions are approached, and since upper air circulation patterns differ with latitude 


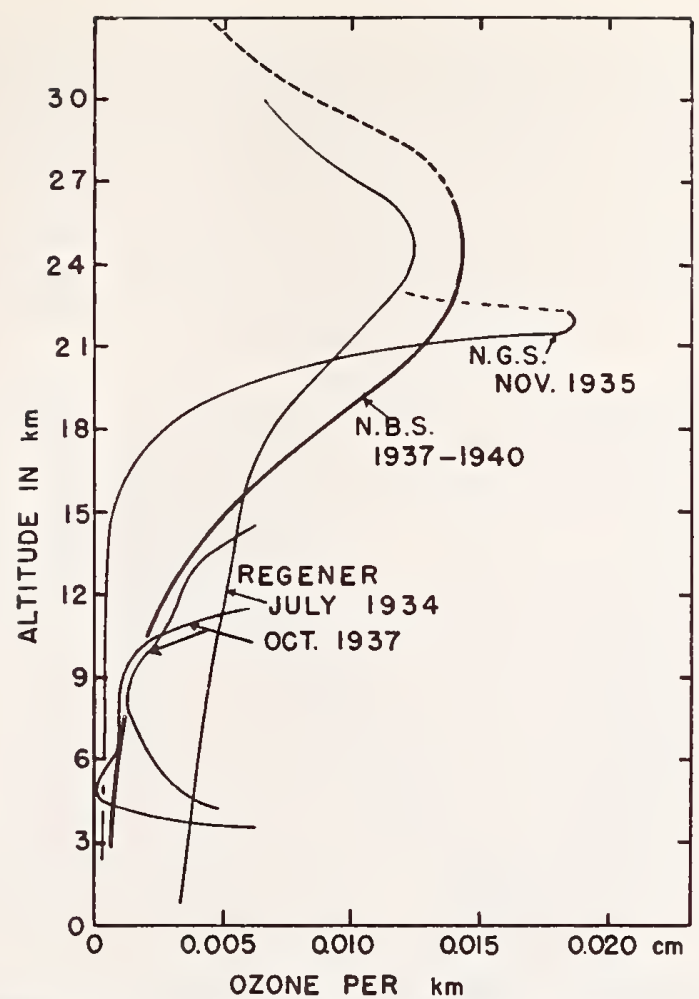

FIG. 10. Vertical distribution of ozone in the stratosphere. NBS curve is mean June and July measurements obtained in 18 unmanned balloon flights using phototube and filter ozone meters. NGS data obtained with spectrograph in manned balloon. Regener measurements obtained with spectrograph in unmanned balloons.

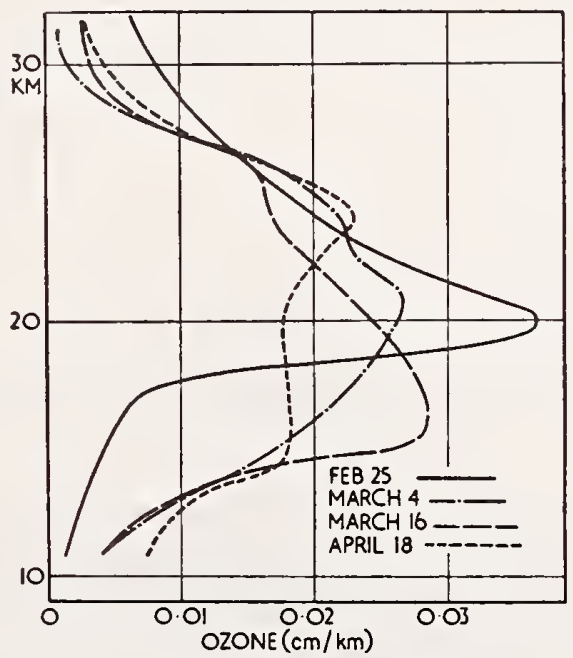

FIG. 11. Vertical distribution of ozone (indicating double maxima) over New Mexico in 1950 using spectrographs in unmanned balloons. After Regener (1951). 
and season of the year, general variations in the amount of ozone with season and with latitude occur over the earth's surface. Variations in ozone also occur from day to day in most latitudes (except possibly in equatorial regions). FIGURE 12 illustrates some variations at Washington D.C., during several days in March 1939 as related

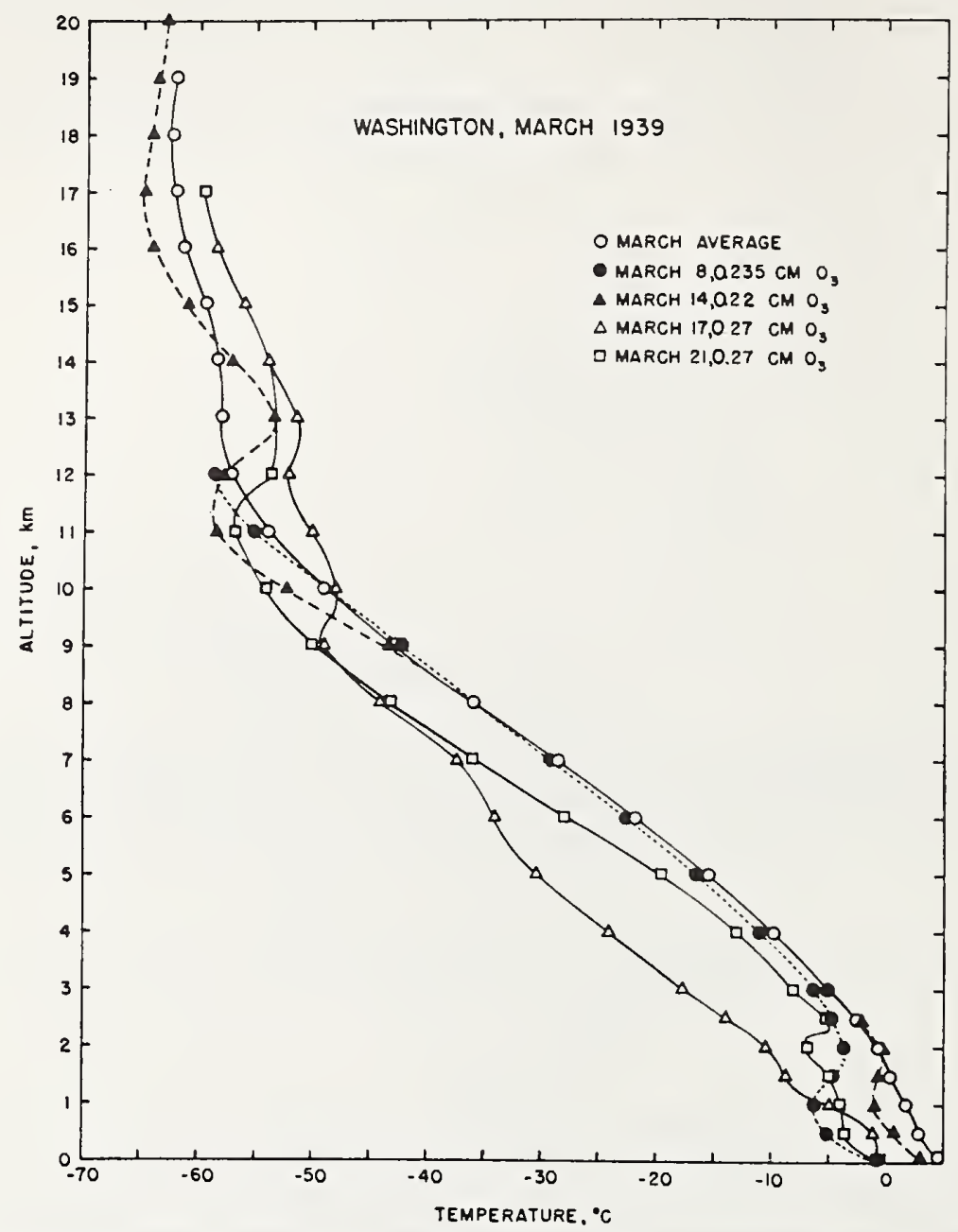

FIG. 12. Upper atmosphere temperatures over Washington, D.C. during March, 1939. (Courtesy U.S. Weather Bureau).

to the upper atmospheric temperatures. The temperature shifts with altitude reflect atmospheric circulation trends. Hence, the presence of ozone in the upper atmosphere acts both as a smoothing control (similar to a current or voltage regulator) and as a modulating factor in affecting the amount of ultraviolet (at wavelengths shorter than $3400 \AA$ ) penetrating the earth's atmosphere.

Although the effects of atmospheric turbidity in the form of water vapor, dust, smoke, and other air pollutants (WYATT et al., 1964; PENNDORF, 1962; DEIRMENDJIAN, 
1964; Deirmendian and Sekera, 1956; Deirmendilan et al., 1961; Danzer and BULLRICH, 1965) affect the penetration of solar radiation (in particular the u.v.) in the lower atmosphere, much more than does ozone, it was thought expedient to include the above-mentioned facts about ozone before continuing with the presentation of data on solar radiation measurements as such. Scattering effects can become very complicated and, although many analyses and calculations have been made on Mie and other types of scattering only elaborate radiant energy measurements will suffice to answer the question as to what and how much solar energy transverses the atmosphere.

Some of the earlier measurements (see FIG. 13) of the spectral distribution of radiant energy from the sun were made by FABRY and BuIsson (1921), PeTTIT (1932),
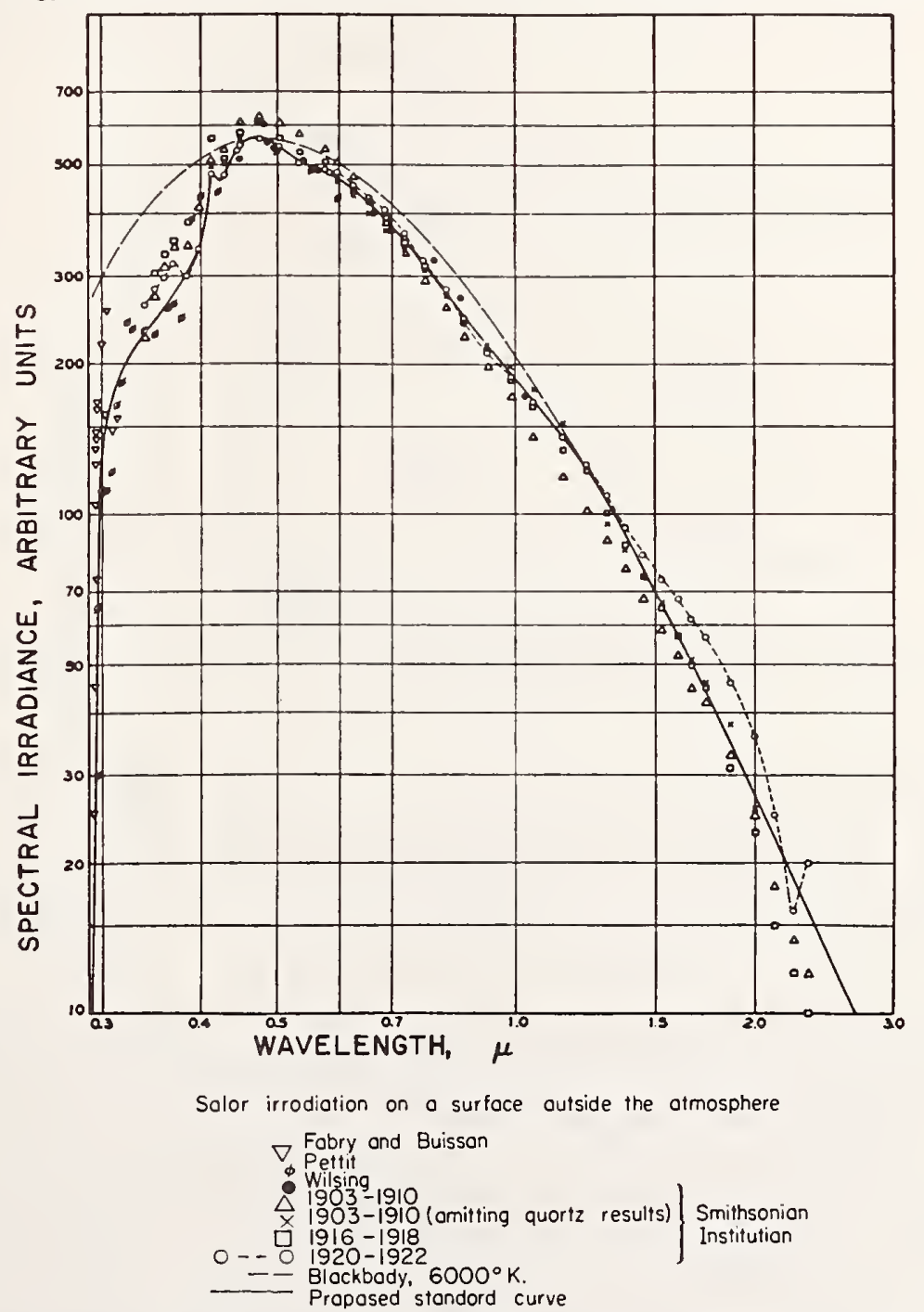

FIG. 13. Relative spectral distribution of solar radiation outside the earth's atmosphere. After Moon (1940). 
AвBOT and AldRICH $(1913 a, 1913 b, 1922)$. This figure gives the mean values for these data as summarized in 1940 by Moon (1940) for use by scientists and engineers. They are given on a relative scale only and do not portray any Fraunhofer structure in the u.v., nor do they suggest the presence of shortwave u.v. Little information regarding the Fraunhofer structure was obtainable with most of the equipment employed because wide spectral slit widths were usually required in order to obtain sufficient spectral energy to permit measurement. In fact, most of Abbot's measured data extended down to only about $3200 \AA$.

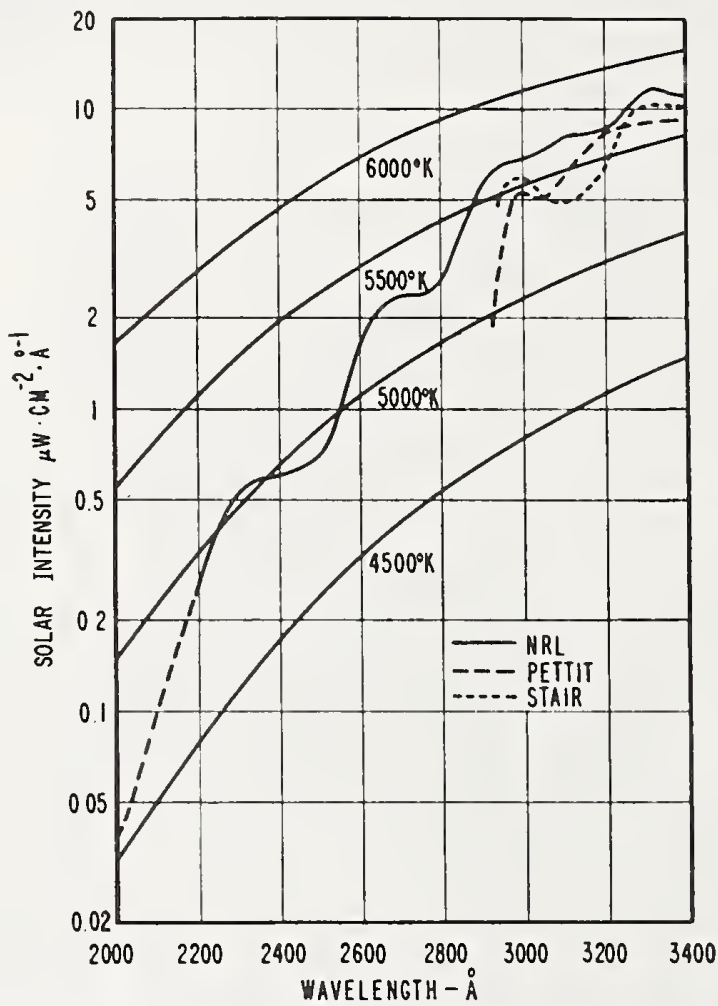

FIG. 14. Ultraviolet solar spectral irradiance outside the earth's atmosphere, averaged over $100 \AA$ intervals. After JoHNson et al. (1954).

In Fig. 14 are shown extraterrestrial spectral solar u.v. irradiance data obtained by the Naval Research Laboratory (JoHNSON et al., 1954) together with blackbody spectral irradiance curves for several temperatures, over the spectral range of about $2000 \AA$ to $3400 \AA$. For comparative purposes, there is also shown some of the early solar data obtained by PeTtit (1932) and by Stair (1952) through the use of spectroradiometers at mountain stations. Again, the data are shown without appreciable Fraunhofer structure. It did not exist for the Pettit data and was averaged out for the other two curves over $100 \AA$ intervals. In general, the agreement may be considered good. The data, furthermore, show a departure from a blackbody curve. This is to be expected since the interior of the sun is much hotter than the surface layers which 
are visible from the earth. Since the surface layers have strong Fraunhofer absorption in the u.v. and since they vary in transmission with wavelength-the outer layers being most important as radiators in the ultraviolet-no fixed temperature can be assigned to represent the solar emission. Furthermore, since the sun is a sphere, the effective depths of the various layers become a function of their position on the sun relative to their distance from the center of the disk.

It is not the purpose of this report to enter into an extended discussion of the extreme u.v. solar radiation which does not penetrate the earth's atmosphere. This has been reported on in considerable detail by TOUSEY (1962), DETwILER et al. (1961), JoHnSON et al. (1958), who have made many measurements with rockets and by other means in the upper atmosphere. The use of their data, however, is most important in arriving at extraterrestrial spectral solar intensities in the ultraviolet region.

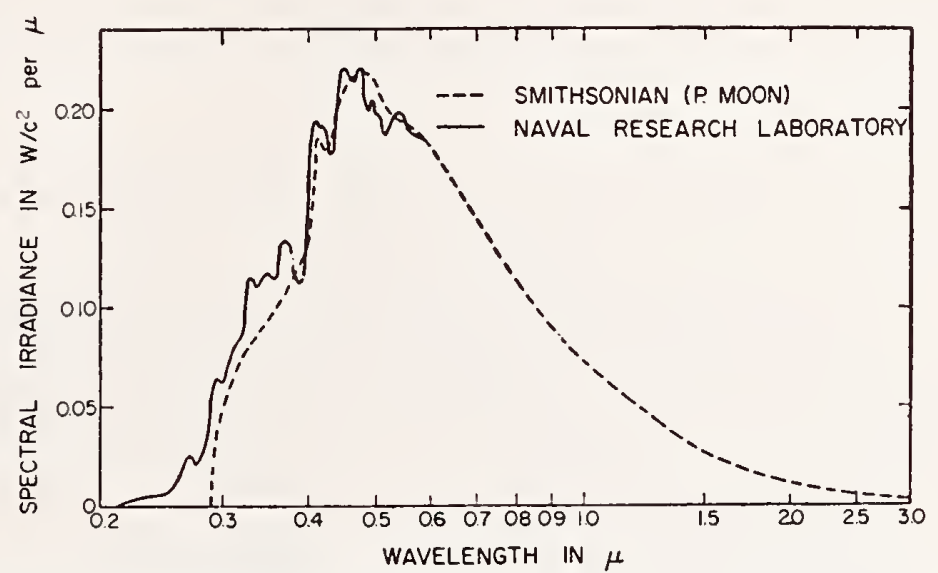

Fig. 15. Solar spectral-irradiance curve outside earth's atmosphere, corrected to mean solar distance. After JoHnson (1954).

JoHNSON (1954b) summarized the available data on the extraterrestrial spectral distribution of solar radiation in absolute value as illustrated by the solid line in FIG. 15. For purposes of comparison, the data previously summarized by Moon are included through the u.v. Both summaries follow the same curve in the infrared. In Fig. 16 the solar curve obtained by Nicolet (1951) (as compiled by DRUMMOND and LAUE, 1965) is shown by the dotted line in comparison with the Johnson (NRL) curve. Significant differences appear in certain spectral regions, in particular with the u.v. from 300 to $380 \mathrm{~nm}$. These differences are much larger than the probable errors indicated by the two observers, indicating that instrumental error may be much larger than either anticipated. However, within the greater part of the solar spectral range, the agreement between the curves is very good (since they are essentially based on Abbot's data). In those spectral regions wherein they differ only more and better measurements will determine the true spectral solar energy curve. Data obtained by STAIR and JoHNSTON (1956) are shown along with the Johnson curve in FIG. 17. The NBS values are shown as obtained through the use of a fixed slit-width double quartz prism spectroradiometer. This instrumentation employed in this manner 
results in sufficient dispersion within the u.v. spectral region to display appreciable Fraunhofer structure. There is, in general, good agreement between the two curves, except within certain spectral regions, in particular for wavelengths shorter than about $330 \mathrm{~nm}$. The data illustrated in this and the previous figures represent the best

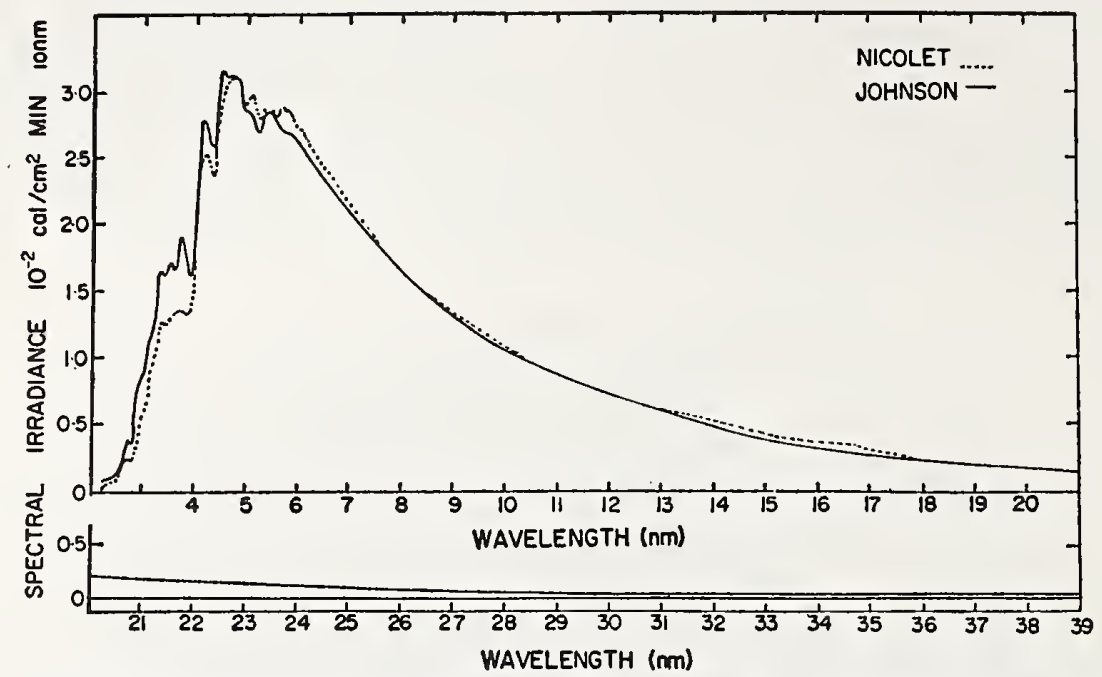

FIG. 16. Spectral distribution of the extraterrestrial solar irradiance, according to Johnson and to Nicolet (plotted from data referred to $\Delta y=10 \mathrm{~nm}$ ), from DRUMmOND (1965a).

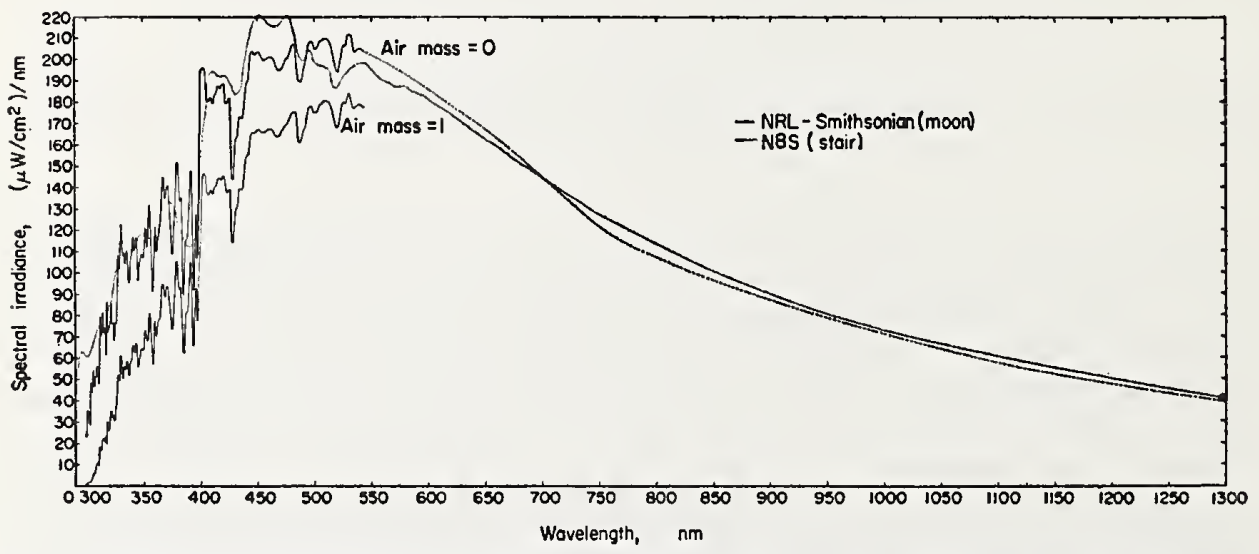

FIG. 17. Solar spectral-irradiance curve outside the earth's atmosphere, corrected to mean solar distance. After JoHnson (1954); also NBS corrected data. STAIR and JoHNSTON (1956).

information available on the spectral distribution and the total irradiance from the sun. None of these data were obtained recently, however, and thus were not obtained with the advanced electronic and optical equipment and methods presently available. Different standards were employed by different observers. While the Smithsonian work by Abbot and his associates was based solely upon the silver-disk pyrheliometers, 
the efforts of many European workers have been based principally upon the Ångstrom type instrument. Often there has been considerable confusion in comparing data based upon the two scales. The work with these instrumentations of the previous thirty years or more in terms of the best estimates for the proper correction to the two scales (see FIG. 18) was reviewed by DRUMMOND (1961) in considerable detail immediately prior to the International Geophysical Year (1957). Although corrections have been made to the two scales various intercomparisons continue to show disagreement.

Johnson's solar curve (JoHnson, 1954) (although set up earlier than the survey by Drummond) contained most of the scale corrections but, in the short-wavelength u.v., had incorporated into it data obtained by NRL wherein a carbon arc whose assigned values of spectral irradiance are highly questionable was employed as the standard. The original NBS data on solar radiation of Stair and his associates were based upon the irradiance of an incandescent lamp standard set up in terms of color temperature (STAIR and SMITH, 1943) and tungsten emissivity to give the relative spectral distribution, and the carbon filament lamp standard of total irradiance to

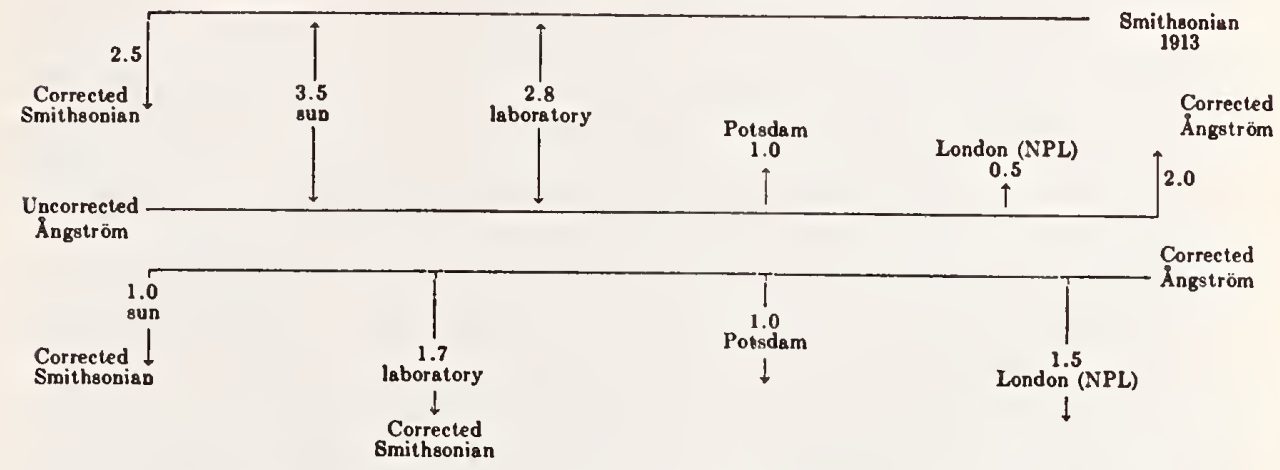

FIG. 18. Diagram illustrating the relationship (in per cent) of the classical Ångstrom and Smithsonian pyrheliometric scales and the basis for the introduction of the International Pyrheliometric Scale. After Drummond (1961).

give the absolute value at one or more wavelengths. The data presented in Fig. 17 have been revised (reduced about 4.5 per cent in the u.v:, visible and near infrared) as the result of indicated discrepancies between the color temperature standard and the new quartz-iodine lamp standard of spectral irradiance (STAIR et al. 1963) illustrated in FIG. 19. While the proven accuracy of this standard falls far short of that desired for use in solar radiation measurements, it is hoped that as new checks can be set up, its spectral irradiance can be established within an accuracy of 1 to 2 per cent over the entire solar spectral range. Present comparisons against standards of luminous intensity indicate an accuracy of this level within the visible spectrum. Unfortunately, no other national (or other) laboratory has a comparable standard against which checks might be made at this time.

Recent studies on variations in the sensitivity of detectors (STAIR et al., 1965a), both thermal and photoelectric, and their effect upon the performance of double monochromators have indicated that it is not possible to obtain accurate or precise results in the measurement of the spectral irradiance from the sun by using such detectors as the exit slits of such instruments unless special precautions are taken. First 


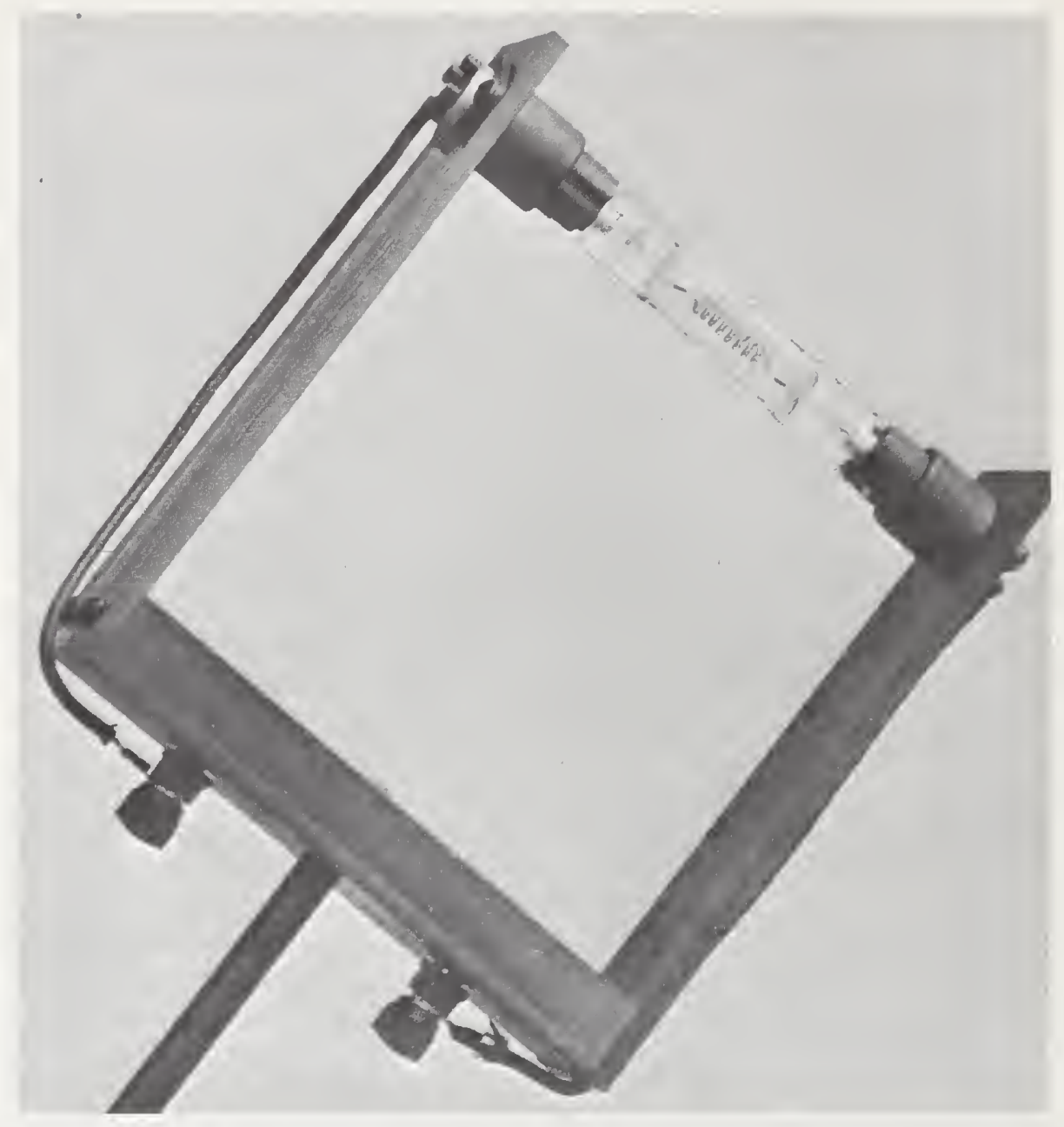

FIG. 19. The 1000-W quartz-iodine lamp standard of spectral irradiance. The $200-\mathrm{W}$ standard is of similar appearance. 


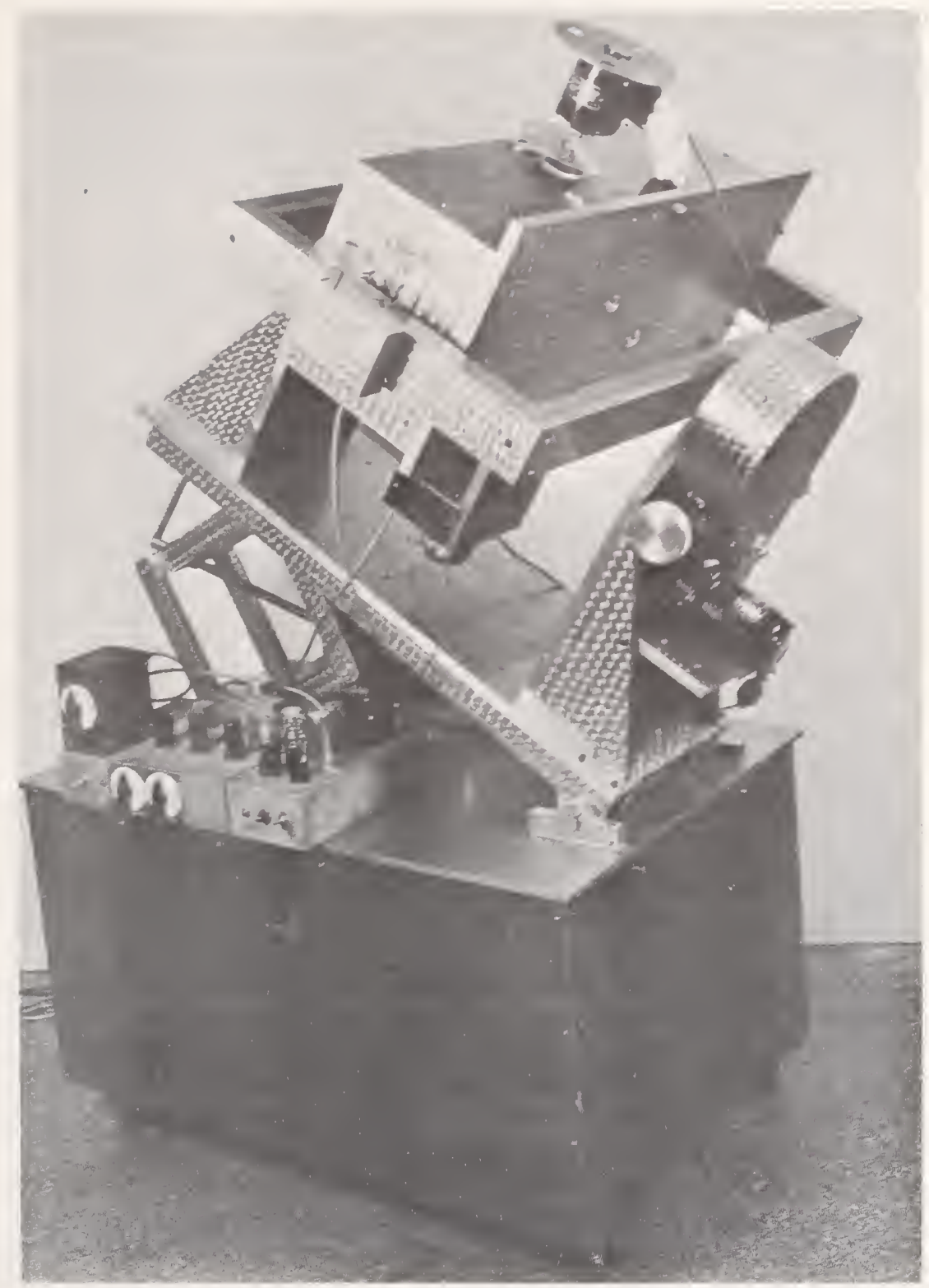

Fig. 21. Photograph of solar spectroradiometer mounted on a polar axis. 
of all, if a heliostat is employed to reflect a beam of sunlight into the entrance slit of a spectroradiometer, the flux is thereby modified not only by the spectral selectivity of the mirrors employed but by polarization of the light beam itself. This may amount to as much as 10 per cent as illustrated in FIG. 20 for a particular heliostat (STAIR, 1952) for different mirror angular positions during the day. Since polarization is always present in a spectroradiometer, the combination of the two polarizations through various phases during the day as the instrument rotates may result in errors of several per cent in the spectral measurements.

In our work the polarization problem was solved by eliminating the heliostat, by placing the spectroradiometer (STAIR, 1955) on an equatorial mount (polar axis) (see FIG. 21). But this involves certain difficulties also, especially with most double

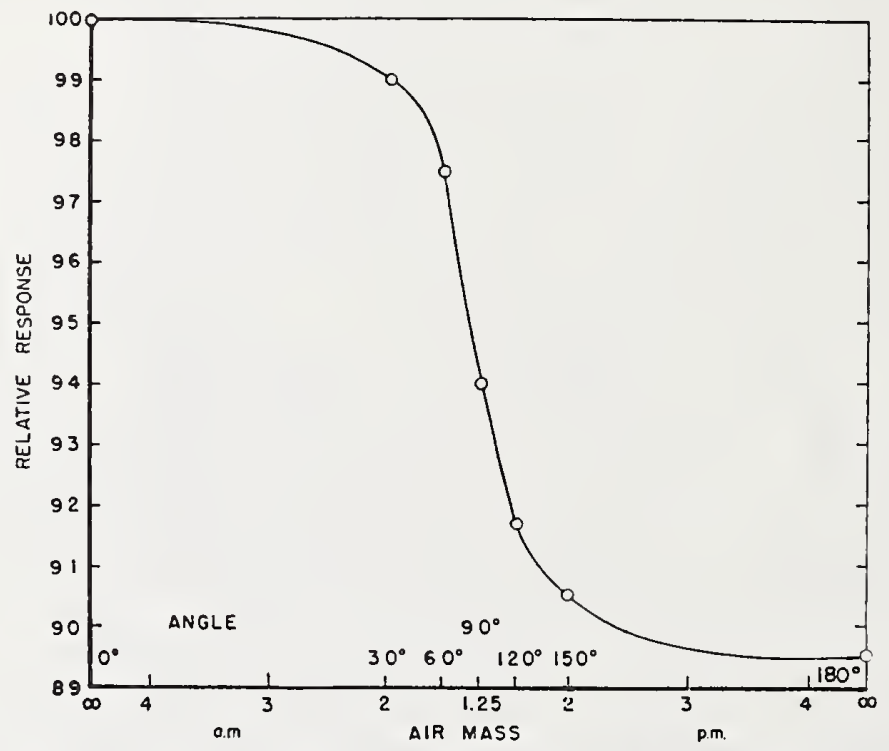

FIG. 20. Relative response of complete solar spectroradiometer as affected by light polarization by the heliostat and the spectrometer.

monochromators. Their rigidity is usually insufficient for this type of manipulation. For the instrumentation illustrated, the optical members were made sufficiently rigid through the addition of supplementary braces within the instrument. Even then, for highest accuracy, all standard lamp calibrations must be made with the instrument in its operating position. While this particular instrument has been operated successfully under these adverse conditions, serious difficulties have been experienced in operating similar instruments on polar axes.

While the polarization effects are eliminated through mounting the spectroradiometer on a polar axis, the problems of variation in sensitivity over the detector surface coupled with variations in effective instrumental transmission and shift of the flux beam at the exit slit of the spectroradiometer as the spectrum is scanned remain unsolved. A recent modification (STAIR et al., 1965b) of this instrumentation incorporates the use of an integrating sphere (see FIG. 22) at the entrance slit. This ensures that 
the flux from the sun or from the standard lamp source irradiates the spectroradiometer in precisely the same manner. Although the sun and lamp subtend different angular sizes and shapes as "seen" by the integrating sphere, the sphere irradiates in each case the full aperture of the spectrometer in the same manner. Hence, although the detector will vary in sensitivity over its surface, it is always irradiated in the same manner in each case. Furthermore, since the integrating sphere will depolarize the radiation, its use should again permit the employment of a heliostat thereby leaving the spectroradiometer on a firm table inside the laboratory. The use of an integrating sphere has recently been employed by ADHAV (1963) wherein measurements of the spectral irradiance from the sun and sky on a horizontal surface (over the spectral

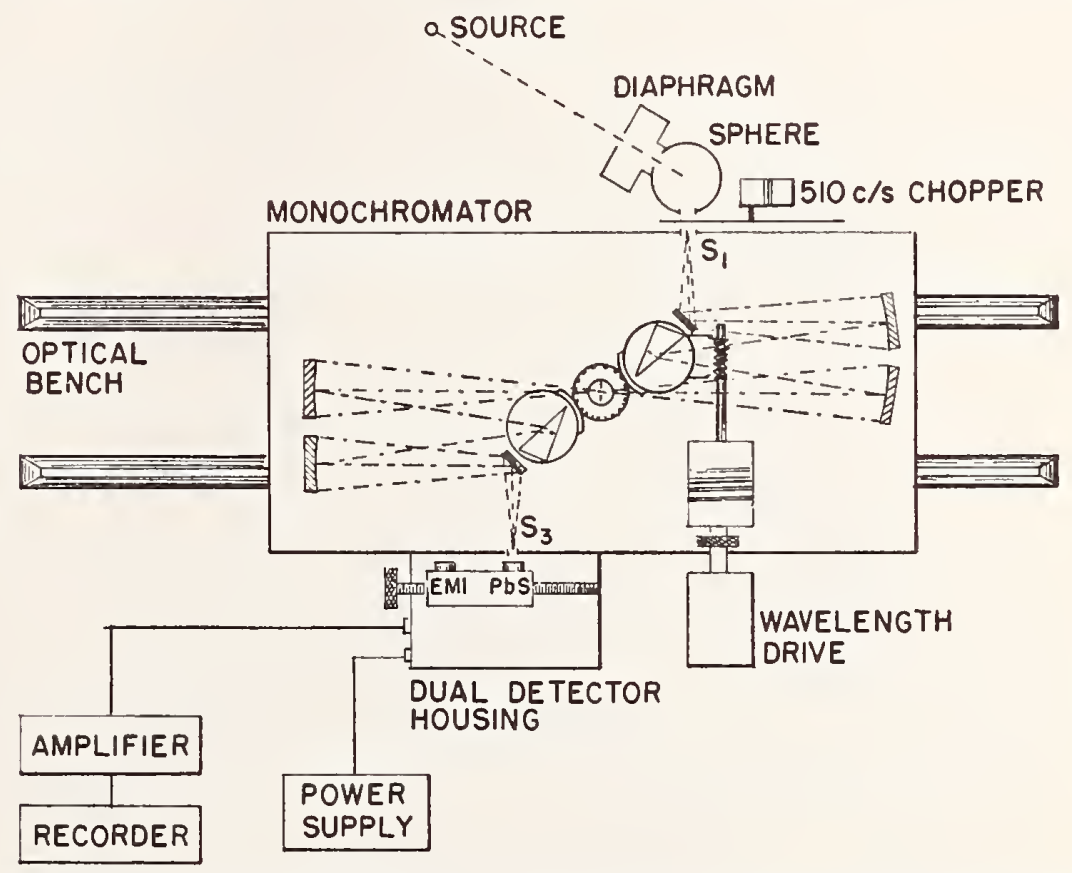

FIG. 22. Optical layout of Carl Leiss double monochromator and block diagram of complete spectroradiometer as employed with an integrating sphere in solar radiation and solar simulator spectral measurements.

range $420-1110 \mathrm{~nm}$ ) was measured through the use of a single grating spectroradiometer. LARCHÉ and SCHULZE (1942) were the first to describe the use of similar instrumentation for measuring both the direct solar spectrum and the integrated value (for sun and sky) on a horizontal surface for selected spectral regions. A flat plate of white Vitrolite was recently employed by HENDERson and HodGKiss (1963) as a diffuser of sky light in measurements of the spectral distribution of energy in daylight.

Apparatus employing an integrating sphere and presently set up at the National Bureau of Standards is arranged to measure either the direct solar radiation with the instrumentation mounted on a polar axis to follow the sun or to remain stationary with the aperture of the sphere set in a horizontal plane to measure the spectral 
distribution of the sun plus the sky. In either case, the spectral range covers the u.v. and visible spectrum through the use of a multiplier phototube as the detector. This instrumentation has been designed for use in precise measurements of spectral solar radiation.

Data obtained with this instrumentation on direct solar irradiance at Washington, D.C., on 1 April 1965 are shown in FIG. 23(a). This was a cold, windy day, with little indication of atmospheric smog. The wind was from the north-usually north-east. Some local smoke was occasionally present. Atmospheric water vapor was relatively high, with a thin veil of cloudiness present much of the time. Rain followed for the next few days.

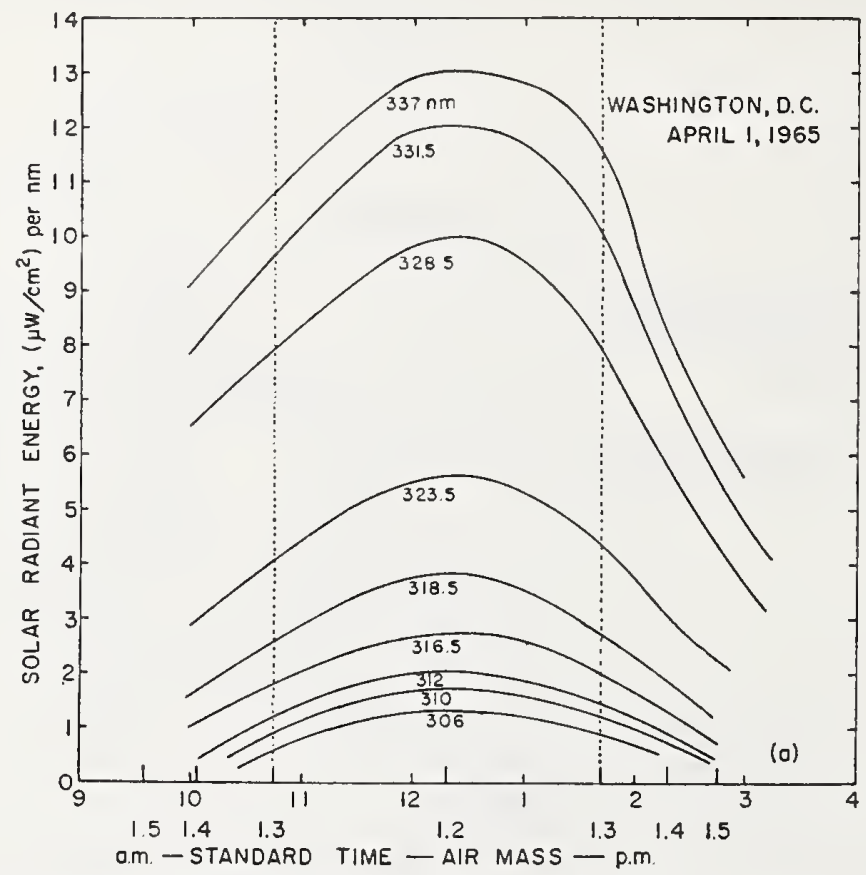

Fig. 23a. Spectral irradiance (measured at normal incidence) from the sun at selected wavelengths as a function of time of day (and air mass), at Washington, D.C. on (a clear day) April, 1965.

For comparative purposes, data obtained with the same instrumentation on sun plus sky irradiance incident on a horizontal surface on 28 June 1965 are shown in FIG. 23(b). The day was relatively cool for the season and very clear following a week-end shower. There was a light west wind, and the sky remained clear all day, except for a few scattered clouds on occasion-principally in the afternoon.

To illustrate the effect of smog, FIG. 24, representing a typical October day in the Los Angeles area, is included (STAIR, 1955). As was usually the case, the amount of smog increased after about $10.00 \mathrm{a} . \mathrm{m}$. Without smog, the morning and afternoon curves should be symmetrical about solar noon. It is noted that on this particular day, the direct u.v. irradiance was reduced by approximately one-half. On extremely smoggy days, the reduction may amount to as much as 90 per cent or more, as occurred on "Black Thursday", 18 October, 1954 (see Fig. 25). 
Through the years, various types of u.v. and light meters have been developed for use in rapid observations (COBLENTZ et al., 1933; WEYDE, 1930: ZWORYKIN and RAMBERG, 1949; RENTSCHLER, 1937; TAYLOR and KERR, 1933). Some have used chemical solutions or chemically impregnated papers. Others have employed liquid photosensitive cells. Some have employed barrier-type photosensitive surfaces. And many have had incorporated into them photoemissive cells of varying grade. DRummond and MARCHGRABer (1959) have introduced instrumentation for the automatic recording of total sun and sky u.v. radiation, using a differential thermopile approach. A simplified version of this instrument (employing a selenium photocell detector and with provision for the isolation of narrow bandpasses as well as the integrated u.v.) has recently been constructed at Newport (DRUMMOND, 1965).

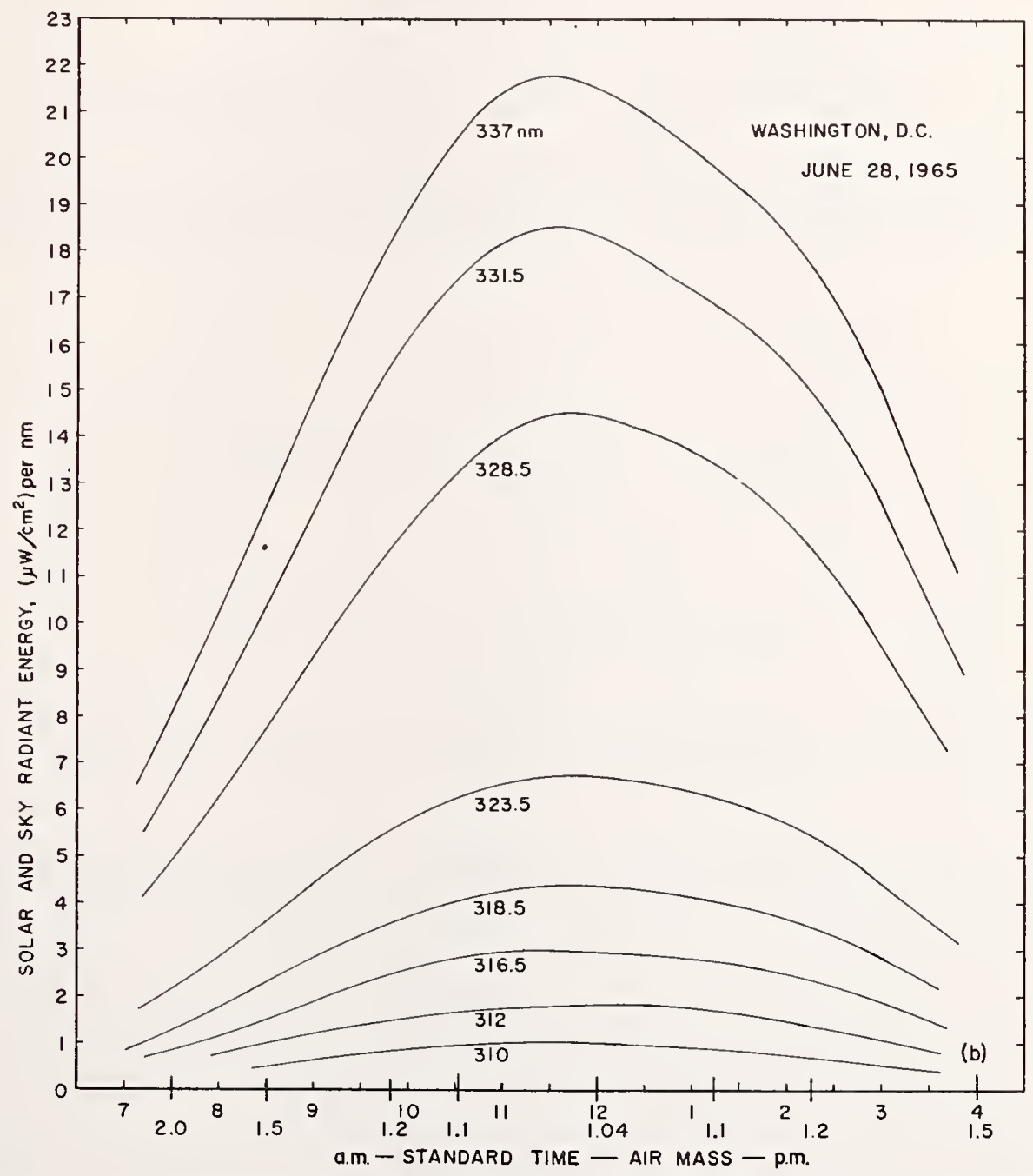

F1G. 23b. Spectral irradiance (measured at normal incidence) from the sun at selected wavelengths as a function of time of day (and air mass), at Washington, D.C. on (a clear day) June. 1965. 


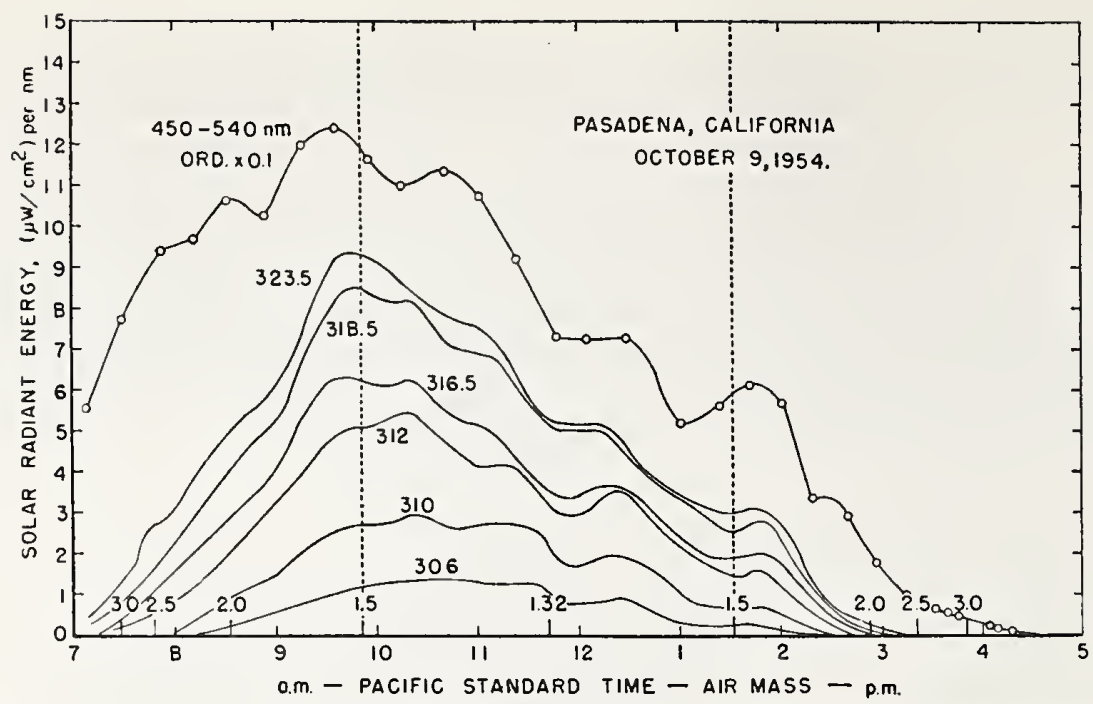

Fig. 24. Spectral irradiance (measured at normal incidence) from the sun at selected wavelengths as a function of time of day (and air mass) at Pasadena, California, on October 9, 1954.

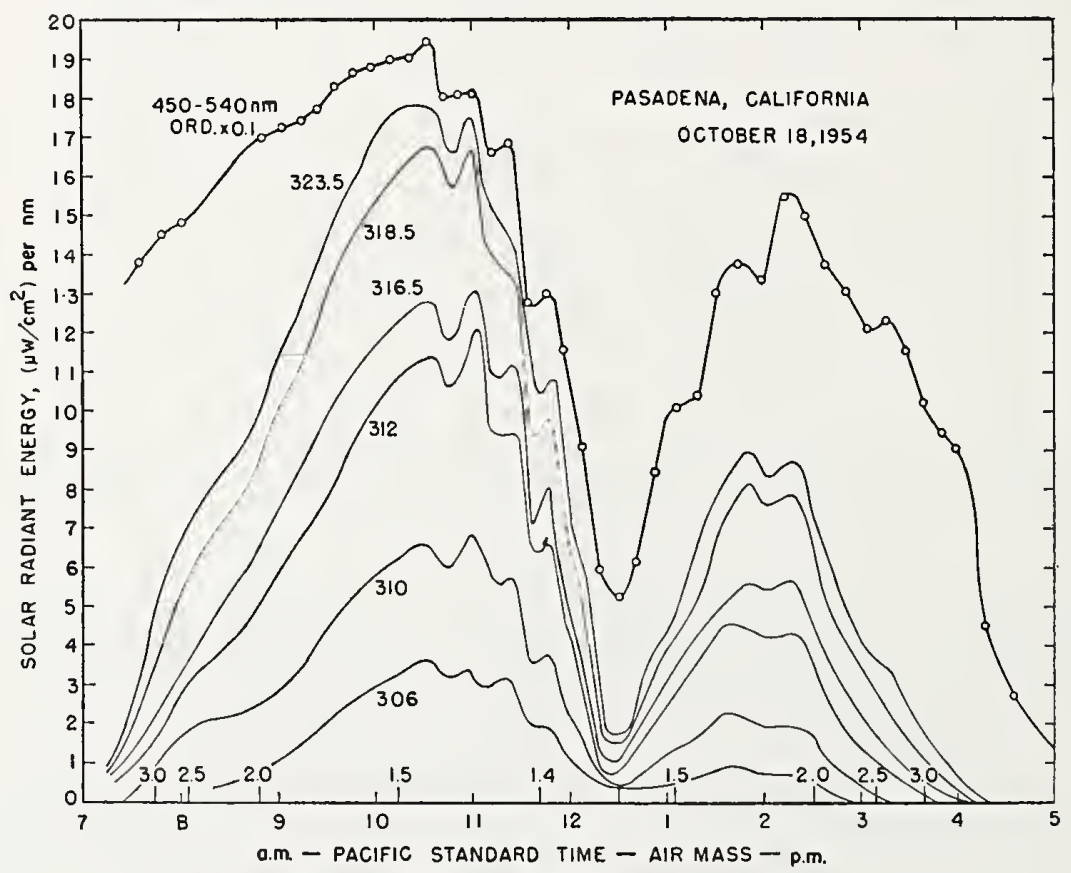

Fig. 25. Same as Fig. 24, on October 18, 1954. 
FIGURE 26 illustrates the use of particular photoemissive cells combined with a number of filters arranged for measuring irradiances at selected spectral regions in the u.v. (CoBlentZ and StaIR, 1935). This particular instrument was employed quite successfully in the evaluation of ozone-the data of FIG. 9 giving the variation in ozone over a number of years at Washington, D.C. Such an instrumentation is quite useful when carefully calibrated. With similar phototubes incorporated into impulse counters and arranged for automatic recording, CoBlentZ and STAIR (1944) found that the u.v. component of sunlight (wavelengths less than about $315 \mathrm{~nm}$ ) varied

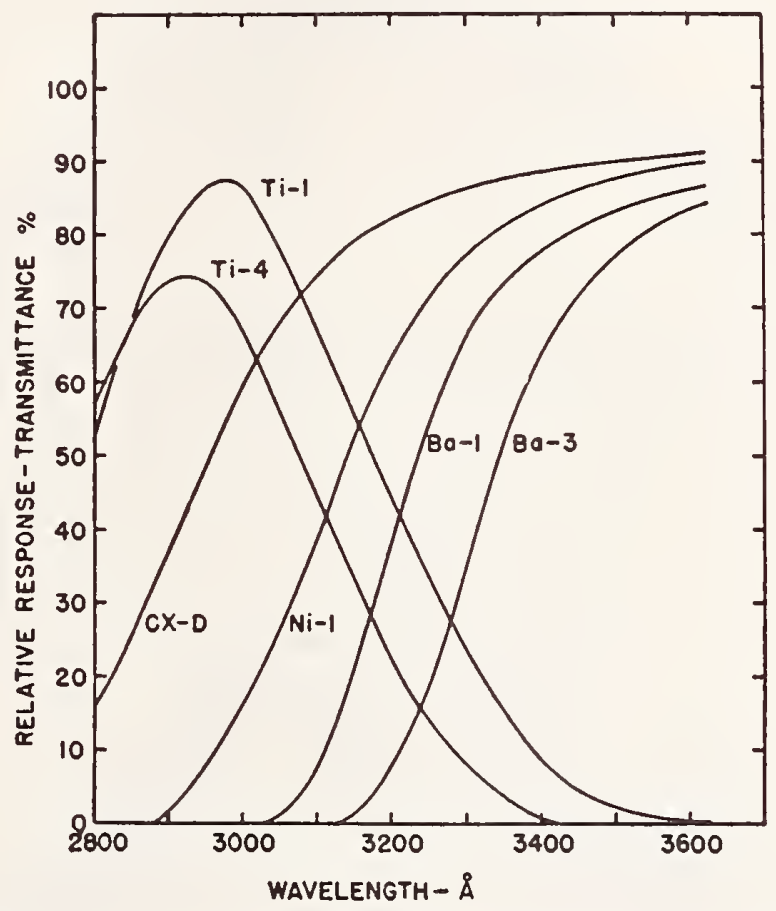

FIG. 26. Relative spectral response curves for three titanium phototubes; transmittance curves for 4 U.V. filters. See StarR (1949) and Drummond and MARChGraber (1959).

about 10 to 1 between summer and winter (see Fig. 27). Similar contrasts were observed between cloudy and clear days. These data were obtained at Washington, D.C., in 1942. FIGURE 28 shows similar data obtained in 1943. In each case, the integrated total daily values are plotted.

Some representative curves illustrating the daily and seasonal variation in u.v. solar and sky irradiance ( 300 to $315 \mathrm{~nm}$ ) for four clear days during 1942 and 1943 are plotted in FIG. 29. FIGURE 30 illustrates the relationship between the shortwave u.v. (300 to $315 \mathrm{~nm}, \mathrm{UVQ}$ ) and total solar and sky irradiance (Q) for a typical clear day in October at Washington (CoBlenTZ, 1945). This shows also that the early morning and late afternoon sunlight is relatively devoid of shortwave u.v. These measurements were relatively crude but represent the best available information in this area. The u.v. component isolated was that for the $300-315 \mathrm{~nm}$ region only; 
it is unfortunate that these data did not include values over a wider spectral range since from the standpoint of photochemical air pollution, the region of 3400 to $4000 \AA$ is often considered of primary importance. But the fact is, the longer wavelengths were not included, either in this or in most other studies through the years.

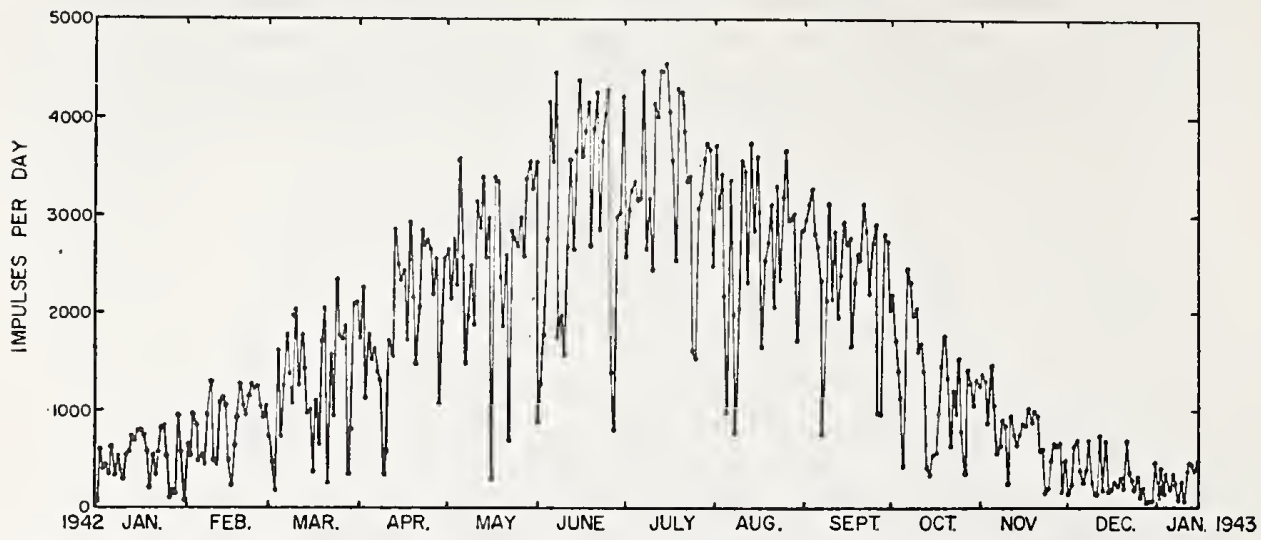

FIG. 27. Relative total integrated daily amount of "biologically effective" ultraviolet solar and sky radiation incident in Washington, D.C. for 1942. From CoBlenTZ and STAIR (1944).

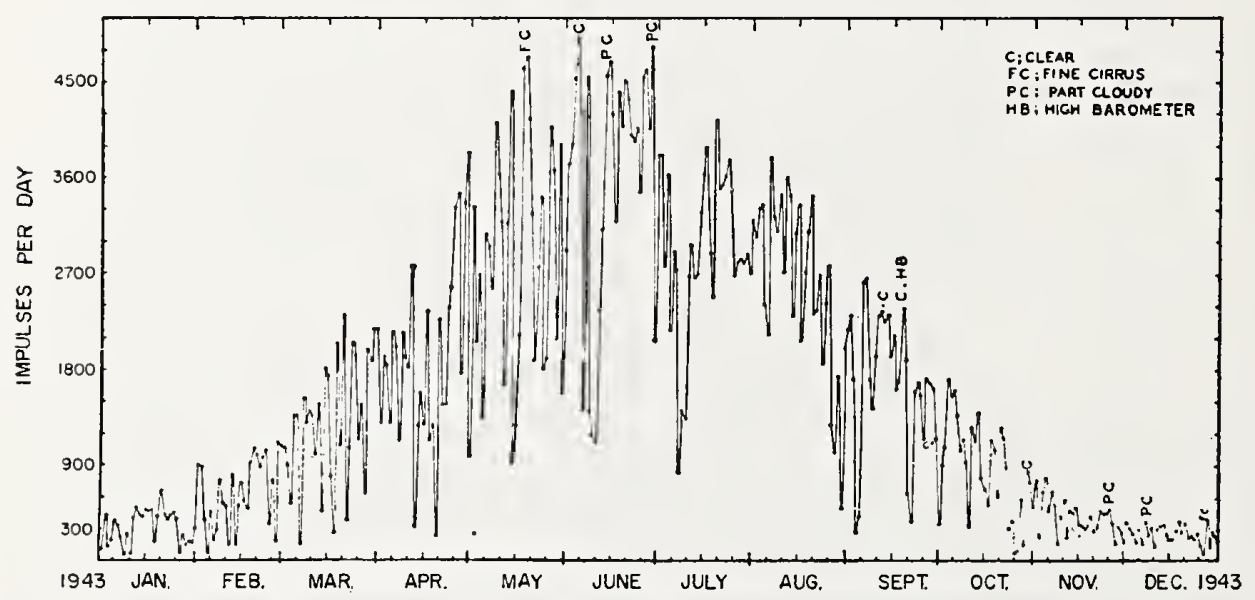

Fig. 28. Same as Fig. 27. For 1943, from Coblentz and StaIR (1944).

Calculations made by Mr. T. H. MacDonald and based upon some of the previously illustrated data on shortwave u.v., in combination with U.S. Weather Bureau data for the continental U.S., result in the chart of FIG. 31 illustrating the expected mean daily u.v. irradiance ( 300 to $315 \mathrm{~nm}$ ) at various localities. In this figure, the south-west has the highest values. It may well be, however, that Florida and the Gulf Coast are underweighted in view of the lower ozone concentration at low latitudes, but accurate data are not available at this time. However, with the recent development of excellent narrow-band interference filters in the u.v. in combination with new high precision electronics and high sensitivity phototubes and photomultipliers covering wide 
spectral ranges, the design of new photoelectric filter spectroradiometers has been made possible. FIGURE 32 shows such an instrument mounted on an optical bench for source comparison. Several have been constructed to cover various spectral ranges. For measuring u.v. solar radiation, a disk carrying 9 filters, each having a half-band width of $100 \AA$ and spaced at $100 \AA$ intervals between $3100 \AA$ and $3900 \AA$, provides a practical filter spectroradiometer covering the solar u.v. Two such instruments have been constructed for evaluating solar irradiances (sun plus sky) under varying conditions of weather and smog. Data obtained with these instrumentations in the Los Angeles area (Mt. Wilson and downtown Los Angeles) in October 1965 will be published in a forthcoming report. Accurate results are assured through calibrations against the solar spectroradiometer supplemented by the use of standards of spectral irradiance.

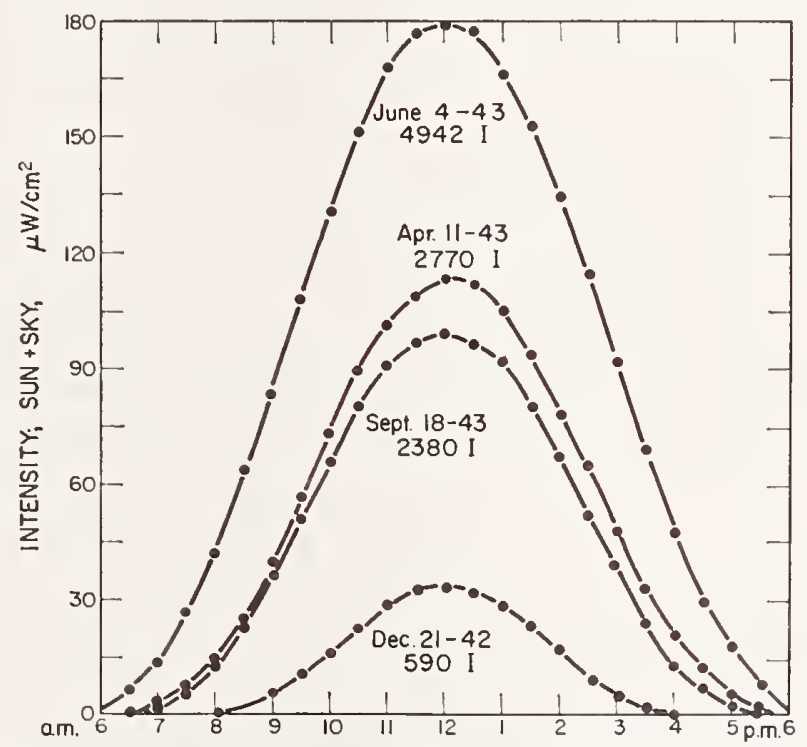

FIG. 29. Daily and seasonal ultraviolet solar and sky irradiances ( 300 to $315 \mathrm{~nm}$ ) on a horizon surface in $\mu \mathrm{W}$ per $\mathrm{cm}^{2}$ observed on 4 very clear days in Washington, D.C. From Coblentz and StaIR (1944).

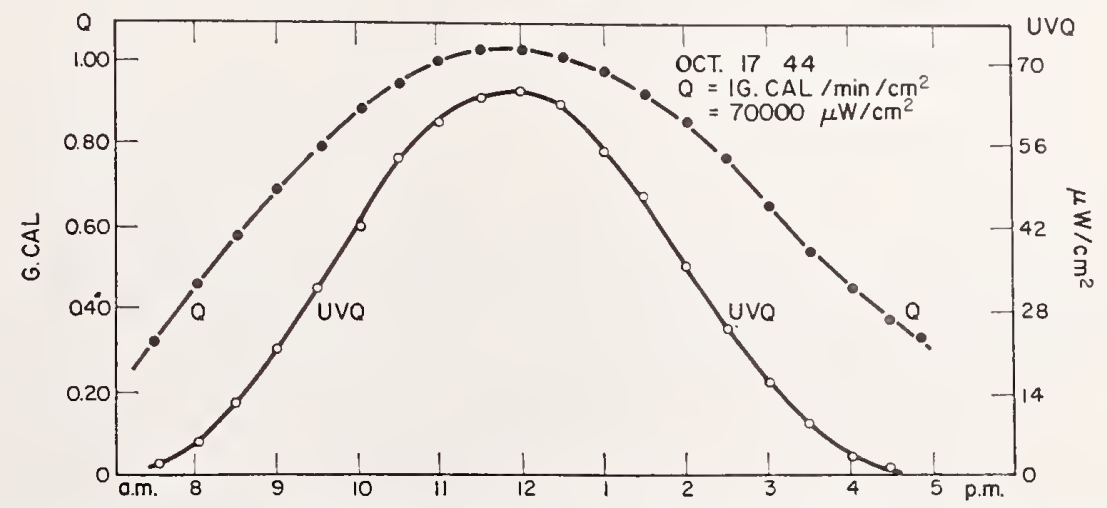

FIG. 30. Diurnal variations in ultraviolet ( 300 to $315 \mathrm{~nm}$ ) and total solar and sky irradiances on a horizontal surface on a clear day in Washington, D.C. After CoBlentz (1945). 


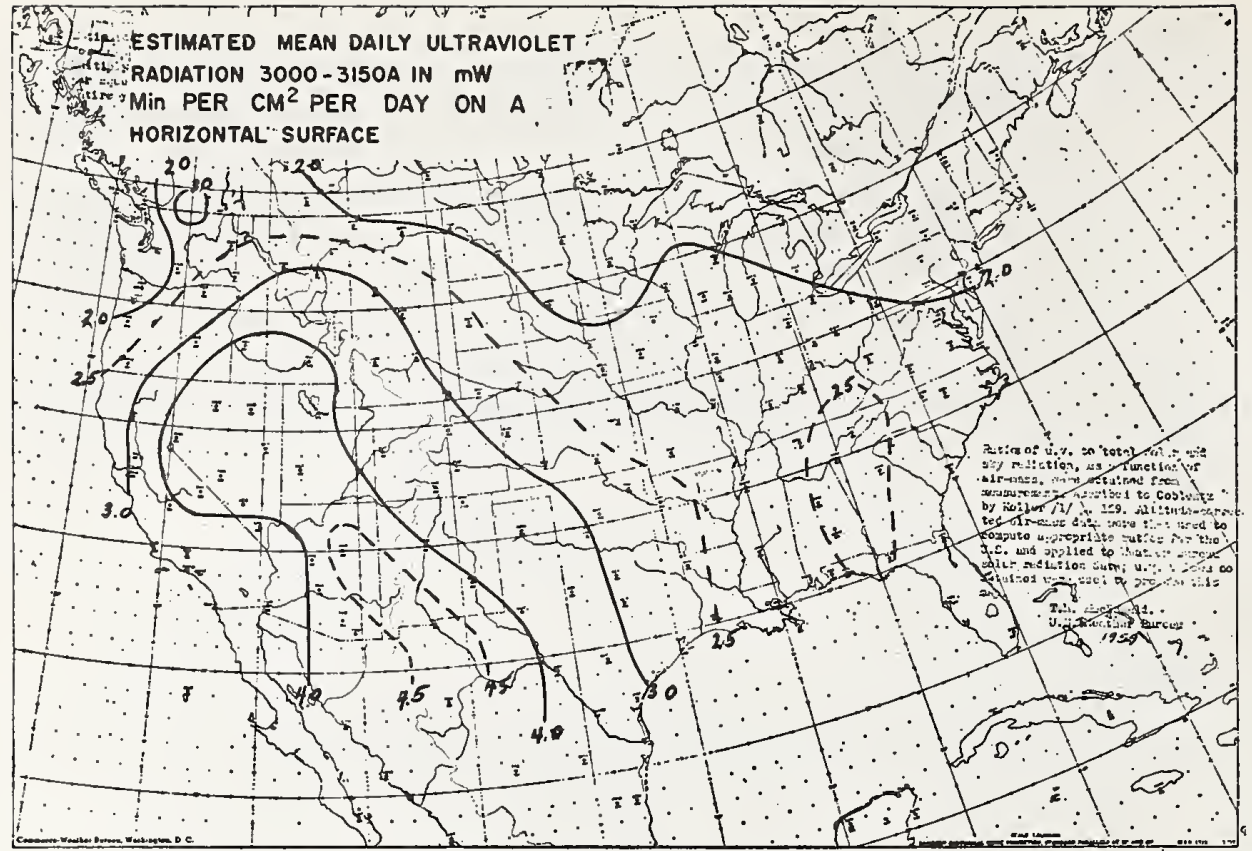

Fig. 31. Estimated mean daily ultraviolet ( 300 to $315 \mathrm{~nm}$ ) solar and sky irradiances on a horizontal surface for the continental United States. (After T. H. MACDonALD, U.S. Weather Bureau).

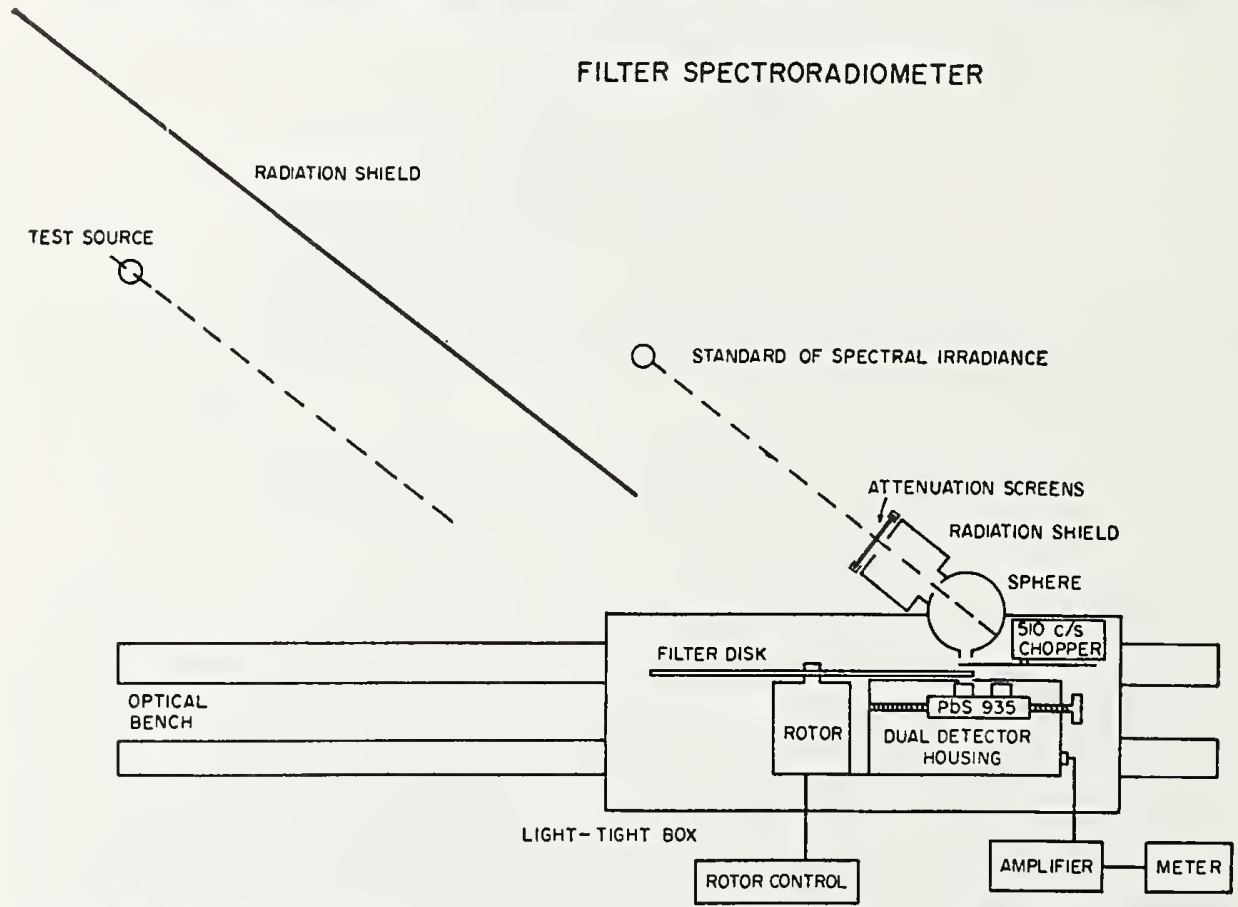

FIG. 32. Block diagram of photoelectric filter spectroradiometer. For use in solar irradiance measurements the opening in the integrating sphere is set horizontal or else normal to the direct solar beam. 
Acknowledgements-The author expresses his appreciation to present and past associates for much assistance in making this presentation possible. Acknowledgment is due especially to WILLIAM $\mathbf{R}$. WATERS for the data presented in FIo. 23(a) and 23(b). The author is indebted to the Sacramento Peak Observatory of Air Force Cambridge Research Laboratories for the use of Figs. 1 to 3 and to the U.S. Navy for Figs. 4 and 5.

The development of the new instrumentation described and employed in obtaining some of the new data presented was supported in the major part by funds transferred from the National Aeronautics and Space Administration and from the United States Public Health Service.

\section{REFERENCES}

Aввот C. G. and Aldrich L. B. (1913a). Aim and method of principal investigation, Ann. Astrophys. Obs. Smithson. Instn 3, 21.

Aввот C. G. and AlDRICH L. B. (1913b). Results of measurements of the intensity of solar radiation. Ann. Astrophys. Obs. Smithson. Instn 3, 73.

Aввот C. G. and AlDRich L. B. (1922). Results of measurements of the intensity of solar radiation. Ann. Astrophys, Obs, Smithson. Instn 4, 99.

Aввот C. G. (1939) The variations of the solar constant and their relation to weather. Q.J. R. Met. Soc. 65, 215.

ADHAV R. S. (1963) Wide angle spectroradiometer. J. Scient. Instrum. 40, 455.

AFCRL HANDBOOK of Geophysics (1960) Macmillan, New York.

Bethe H. A. and Critchfield C. L. (1938) The formation of deuterons by proton combination. Phys. Rev. 54, 248.

BetHe H. A. (1939) Energy production in stars. Phys. Rev. 55, 434.

Coblentz W. W., Stair, R. and Hogue J. M. (1933) Measurements of ultraviolet solar radiation in various localities. B.S. J. Res. $10,79$.

Coblentz W. W. and StaiR R. (1935) Factors affecting ultraviolet solar-radiation intensities. J. Res. natn. Bur. Stand. 15, 123.

Coblentz W. W. and Stair R. (1939) Distribution of ozone in the stratosphere. J. Res, natn. Bur. Stand. 22, 573.

Coblentz W. W. and Stair R. (1941) Distribution of ozone in the stratosphere: Measurements of 1939 and 1940. J. Res. natn. Bur. Stand., 26, 161.

Coblentz W. W. and StatR R. (1944) A daily record of ultraviolet solar and sky radiation in Washington, 1941 to 1943. J. Res. natn. Bur. Stand. 33, 21.

CoBlentz W. W. (1945) Bioclimatic measurements of u.v. solar and sky radiation in Washington D.C., 1941-1944. Bull. Am. Met. Soc. 26, 113.

DANZER K. H. and Bullrich K. (1965) The influence of absorption on the extinction of solar and sky radiation. Appl. Opt. 4, 1500.

Deirmendian D. and Sekera Z. (1956) Atmospheric turbidity and the transmission of ultraviolet sunlight. J. Opt. Soc. Am. 46, 565.

Deirmendian D., Clasen R. and Vifzee W. (1961) Mie scattering with complex index of refraction. J. Opt. Soc. Am. 51, 620.

DeIRMENDJAN D. (1964) Scattering and polarization properties of water clouds and hazes in the visible and infrared. Appl. Opt. 3, 187.

Detwiler C. R., Garrett D. L., Purcell J. D. and Tousey -R. (1961) The intensity distribution in the ultraviolet solar spectrum. Annls. Géophys. 17, 263.

Drummond A. J. and Marchgraber R. M. (1959) Instrumentation for the automatic recording of total sun and sky ultraviolet radiation. I.U.G.G. Monograph No. 4, 10.

DRUMmond A. J. (1961) Current developments in pyrheliometric techniques. Solar Energy, 5, 19.

Drummond A. J. and LAUE E. (1965) The extra-terrestrial solar spectrum. Proceedings of Joint Symposium on Solar Radiation, Los Angeles, California, 18-20 January.

DRUMmond A. J. (1965) Private communication.

DüTsch H. U. (1959) Vertical ozone distribution from Umkehr observations. Arch. Met. Geophys. Bioklim. (A), II, 240.

FABRY CH. and Buisson H. (1921) A study of the ultraviolet end of the solar spectrum. Astrophys. $J$. 54, 297. 
Green Alex. E. S. (1964) Attenuation by ozone and the earth's albedo in the middle ultraviolet. Appl. Opt. 3, 203.

Hardie R. H. and Giclas H. L. (1955) A search for solar variation. Astrophys. J. 122, 460.

HeNderson S. T. and Hodgkiss D. (1963) The spectral energy distribution of daylight. Br. J. Appl. Phys. 14, 125.

INN EdWARD C. Y. and TANAKA Yosho (1959) Ozone absorption coefficients in the visible and ultraviolet regions. Advances in Chemistry Series, No. 21, p. 263, American Chemical Society.

Johnson F. S., Purcell J. D., Tousey R. and Wilson N. (1954) The ultraviolet spectrum of the sun. Rocket Exploration of the Upper Atmosphere (Ed. BoYd and SeAton), p. 279. Pergamon Press, London.

Johnson F. S. (1954) The solar constant. J. Meteorol. 11, 431.

Johnson F. S., Malitson H. H., Purcell J. D. and Tousey R. (1958) Emission lines in the extreme ultraviolet spectrum of the sun. Astrophys. J. 127, 80.

KUIPER G. P. (Ed.) (1953) The Sun, University of Chicago Press, Illinois.

LARChÉ KURT und SCHUlZe ReINHART (1947) Úber ein Ultraviolett-Messgerät mit Vorsatzkugel für Strahlungseinfall unter grossem Winkel. Z. tech. Phys. 4, 114.

Menzel D. H. (1959) Our Sun, Revised edition. Harvard University Press, Massachusetts.

Moon P. (1940) Proposed standard solar-radiation curves for engineering use. J. Franklin Instn 230, 583.

Nicolet M. (1951) Sur le probléme de la constante solaire. Ann. Astrophys. 14, 249.

PENNDORF RUDOLF B. (1962) Scattering and extinction coefficients for small absorbing and nonabsorbing aerosols. J. Opt. Soc. Am. 52, 896.

PetTIT E. (1932) Measurements of ultraviolet solar radiation. Astrophys. J. 75, 185.

Regener E. and Regener V. H. (1934) Aufnahmen des Ultravioletten Sonnenspektrums in der Stratosphere und Vertikale Ozonverteilung. Physik. Z. 35, 788.

Regener V. H. (1938) Neue Messungen der Vertikalen Verteilung des Ozons in Atmosphäre. $Z$. Physik 109, 642.

REGENER V. H. (1951) Vertical distribution of atmospheric ozone. Nature, Lond. 167, 276.

Rentschler H. C. (1930) An ultra-violet light meter. Trans. illum. engng. Soc., N. Y. 25, 406.

Statr R. and Coblentz W. W. (1938) Radiometric measurements of ultraviolet solar intensities in the stratosphere. J. Res. natn. Bur. Stand. 20, 185.

StaIR R. and SMITH W. O. (1943) A tungsten-in-quartz lamp and its applications in photoelectric radiometry. J. Res. natn. Bur. Stand. 30, 449.

StaIR R. (1948) Measurement of ozone over the Organ Mountains, New Mexico. J. Res. natn. Bur. Stand. 40, 9.

StaIR R. (1949) Seasonal variations of ozone at Washington, D.C. J. Res. natn. Bur. Stand. 43, 209.

STAIR R. (1952) Ultraviolet radiant energy from the sun observed at 11,190 feet. J. Res. natn. Bur. Stand. 49, 227.

STAIR R. (1955) The spectral radiant energy from the sun through varying degrees of smog at Los Angeles. Proc. 3rd Natn Air Pollut. Symp., April 18-20, Pasadena, California.

Stair R. and Johnston Russell G. (1956) Preliminary spectroradiometric measurements of the solar constant. J. Res. natn. Bur. Stand. 57, 205.

StaIR R. (1959) Measurement of ozone in terms of its optical absorption. Advances in Chemistry Series, No. 21, p. 269, American Chemical Society, Washington, D.C.

Stair R., SCHNeIDER William E. and Jackson John K. (1963) A new standard of spectral irradiance. Appl. Opt. 2, 1151.

Stair R., Schneider William E., Waters William R. and Jackson John K. (1965a) Some factors affecting the sensitivity and spectral response of thermoelectric (radiometric) detectors. Appl. Opt. 4, 703.

Stair R., Schneider William E., Waters Willitam R., Jackson John K, and Brown Roger E. (1965b) Some Developments in Improved Methods for the Measurement of the Spectral Irradiances of Solar Simulators. NASA Report No. CR-201, Washington, D.C. April 1965.

TAYLOR A. H. and KERR G. P. (1933) A direct current amplifier with good operating characteristics. Rev. Scient. Instrum. 4, 28. 
The National Geographic Society (1936) U.S. Army Air Corps Stratosphere Flight of 1935, in the balloon Explorer II. Natn. Geogr. Soc. Contrib. Tech. Papers. Stratosphere Series No. 2, Washington.

Tousey R. (1962) The extreme ultraviolet-past and future. Appl. Opt. 1, 679.

TsI-Ze Ny and ShIn-PIAw Choong (1932) L'absorption de la lumière par l'ozone entre 3050 et $3400 \AA$ (region des bandes de Huggins). C.r. hebd. Séanc. Acad. Sci., Paris, 195, 309.

TSI-Ze Ny and SHIN-PIAw ChOoNg (1933) L'absorbtion de la lumière par l'ozone entre 3050 et $2150 \AA$ C.r. hebd. Séanc. Acad. Sci., Paris, 196, 916.

WeIZSÄCKER F. vON (1938) Über Elementumwandlungen im Innern der Sterne II. Phys. Z. 39, 633.

WeYdE E. (1930) Ueber die Grundlagen eines neuen U.V. Messinstrumentes, Strahlentherapie, 38, 378.

Wyatt Phulip J., Stul V. Robert and Plass Gilbert N. (1964) The infrared transmittance of water vapor. Appl. Opt., 3, 229.

Zworykin V. K. and RAmberg E. G. (1949) Photo-electricity. John Wiley, New York.

Air \& Wat. Pollut. Int. J. Pergamon Press 1966. Vol. 10, 


\title{
Some Factors Affecting the Sensitivity and Spectral Response of Thermoelectric (Radiometric) Detectors
}

\author{
Ralph Stair, William E. Schneider, William R. Waters, and John K. Jackson
}

\begin{abstract}
A number of thermal detectors (thermopiles) has been studied for variations in sensitivity which exist over their surfaces and also for variations of sensitivity with wavelength. These variations differ depending upon whether the thermoelectric output is measured with dc instrumentation, with conventional ac instrumentation, or with ac synchronous rectifier instrumentation. In general, lower responses for the longer wavelengths are observed when commercial thermopiles are compared with a new cavity blackbody detector-and the difference may be greater when measured with ac synchronous rectifier instrumentation. When the output of a detector is measured through the use of a conventional tuned ac amplifier, variation in sensitivity of as much as 5 to 1 has been found for different areas of the surface. When a phase-sensitive amplifier is employed, first set for maximum response with the entire thermopile irradiated, even larger variations in sensitivity may be observed.
\end{abstract}

\section{Introduction}

While it is generally known that photoelectric detectors vary in sensitivity over their surfaces and with wavelength, thermoelectric detectors are often assumed to be uniformly sensitive in these respects. Some data on this subject were presented previously,,$^{1,2}$ but only recently have extensive measurements in this area been made at the National Bureau of Standards. It was shown from preliminary measurements on thermopiles that, in addition to the effects of selective transmission of detector windows, the black coating may vary widely in effective blackness as a function of wavelength, the surface may vary widely in sensitivity from place to place, and when the measurements were made with an ac synchronous rectifier-type amplifier, the apparent sensitivity varied with the chopping frequency. It was further shown in connection with the development of a blackbody cavity-type detector that, although some information relating to the spectral response of a detector can be obtained through a study of the spectral reflectance and transmittance of the black coating, the final answer with regard to the performance of a particular detector comes only through a direct measurement of its spectral response. This response is affected not only by the many parameters entering into the design of the detector and detector housing such as: window transmittance, thermal conductivity and transmittance of the black coating, emissivity of the receiving sur-

The authors are with the National Bureau of Standards, Washington, D.C.

Received 10 February 1965. face, reflectance of the underlying receiver metal, electrical resistance, etc., of the unit, but also by the manner in which the thermoelectric signal is amplified and recorded. More specifically, if the radiant flux is chopped, the recorded signal is highly dependent on the chopping frequency and the type of amplifying equipment employed: This report deals primarily with the apparent variation in spectral and surface sensitivities of three thermopiles picked at random, but representative of fast, slow, and very slow types (time constants approximately $0.05 \mathrm{sec}, 0.25 \mathrm{sec}$, and $1.0 \mathrm{sec}$ ) when the outputs are measured with conventional de, conventional ac, and with synchronously rectified ac instrumentation.

\section{Methods, Instrumentation, and Results}

In this investigation the relative spectral responses of the thermopiles weru determined by direct comparison with a blackbody cavity detector. ${ }^{2-4}$ This detector, constructed as indicated in Fig. 1, is in the form of a cone and is coated with carbon or other black on the inside surface. When formed with small angle and from the thinnest gold foil having sufficient strength (about $2 \mu$ ) to ensure support under laboratory conditions, and coated with the best available black, it provides the nearest approach to a nonselective detector of any we have examined. Among elements coated with gold black, carbon black (painted on), and camphor black (smoked on), the cavity detector No. 3 having a coating of smoked camphor black has the nearest neutral or flat response with wavelength. ${ }^{5}$ Although the spectral selectivity to be expected from this detector may be calculated approximately $y^{6,7}$ from a knowledge of its geometry and surface reflectance, other factors, as 


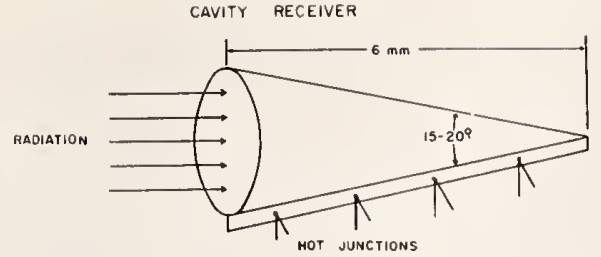

Fig. 1. Preliminary design of conical blackbody detector consisting of a formed gold foil cone blackened inside and having several thermojunctions attached (in series or parallel) along the single fold.

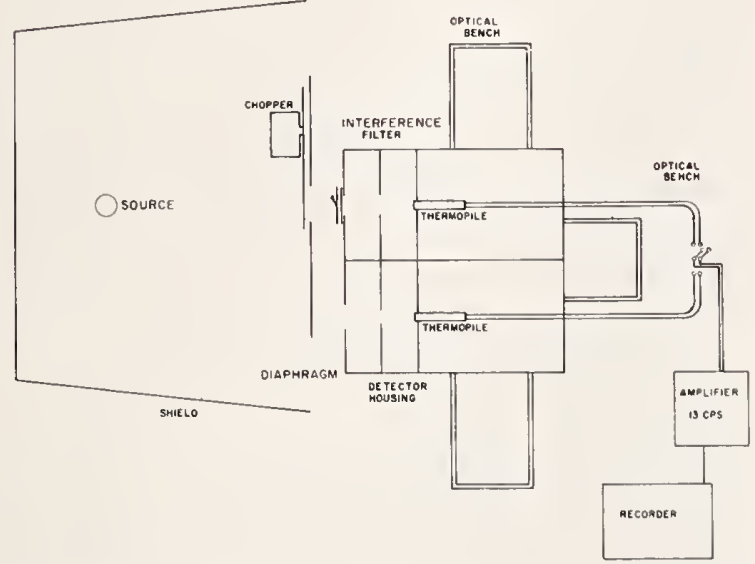

Fig. 2. Block diagram of filter spectroradiometer setup for use with chopper. The source, chopper, and interference (or other) filter remain fixed while the metal box containing the thermopiles is moved laterally along the (lathe bed) optical bench to a stop at each end, defining the exact optical position of each detector.

indicated above, greatly affect its performance. As a result, two cavity detectors coated with blacks differing by not more than a few percent in spectral reflectance may differ appreciably in spectral response.

When a thermopile was compared with cavity detector No. 3 , both detectors were placed in a metal box mounted on an optical bench (see Fig. 2). Each detector is positioned alternately in the path of a spectrally narrow beam of flux. Isolated energy bands spaced at approximately equal intervals from $0.25 \mu$ to $20 \mu$ were obtained through the combination of about 30 narrow bandpass interference filters and various light sources. Response ratios were obtained for different detectors relative to NBS cavity detector No. 3 at different wavelengths. Data for gold-black thermopile No. R-9371 are shown in Fig. 3. Data for the de curve were obtained by measuring the output of the detector with a nanovoltmeter. For the other two sets of data the same dc amplifier was employed with the cavity detector, but for thermopile No. R-9371 the radiant flux was chopped at $13 \mathrm{cps}$ and the outputs were evaluated with either a synchronous rectifier amplifier* or a

* This instrumentation consists of a conventional ac amplifier followed by a cam-operated full-wave rectifier which is integral with the chopper shaft. conventional ac-tuned amplifier. All data for the three types of instrumentation are in relatively close agreement. However, in some earlier measurements, ${ }^{2}$ wherein the flux incident upon both detectors was chopped, some spectral differences were noted, especially when the synchronous rectifier amplifier was employed with heavily coated detectors. Possibly the depth of penetration of the radiation into the receiver coating is different at longer wavelengths, thereby causing a change in time response of the detector. This could produce a phase shift in the detector output, thereby resulting in a change in the magnitude of the rectified signal. An alternate explanation might be differential emission from the blackened surface resulting from differential depths of radiant energy absorption. This subject will be discussed further in a later section of this paper. Other surface thermopiles coated with gold black or lamp black have been found to perform similarly in that the relative response at longer wavelengths falls below that in the ultraviolet and visible regions of the spectrum. Only those detectors coated with Parsons' black have been found to be nearly nonselective with wavelength as compared with cavity detector No. 3. The latter measurements were necessarily made only with de instrumentation, since the use of Parsons' black results in a thermopile of extremely slow response.

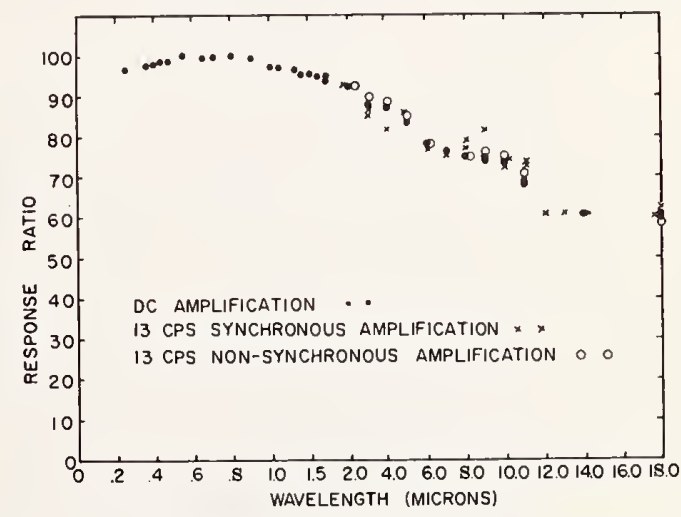

Fig. 3. Spectral sensitivity of the gold-black coated thermopile No. R-9371 vs NBS cavity detector No. 3 measured with de instrumentation, with conventional ac instrumentation, and with ac synchronous rectifier instrumentation.

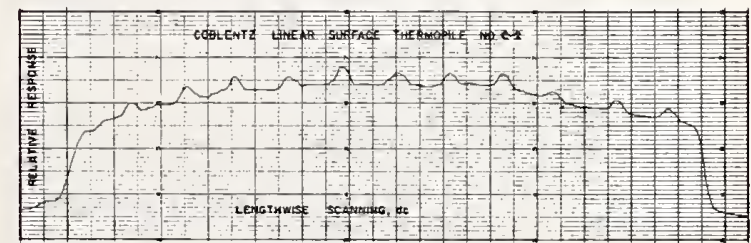

Fig. 4. Lengthwise sensitivity variations of Coblentz twelvejunction lamp-black coated thermopile No. C-2 for an incandescent lamp beam of flux with the output signal measured with dc instrumentation. The positions of all twelve junctions are indicated. 


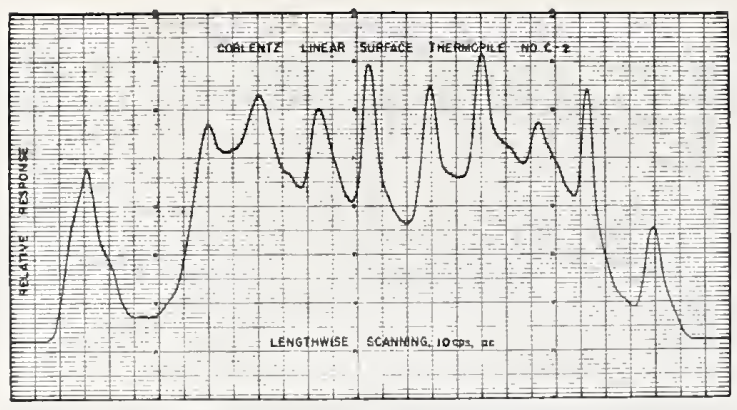

Fig. 5. Lengthwise sensitivity variations of Coblentz twelvejunction lamp-black coated thermopile No. C-2 for an incandescent lamp beam of flux chopped at $10 \mathrm{cps}$ and the output signal measured with conventional ac instrumentation. The precise locations of the twelve junctions are not evident.

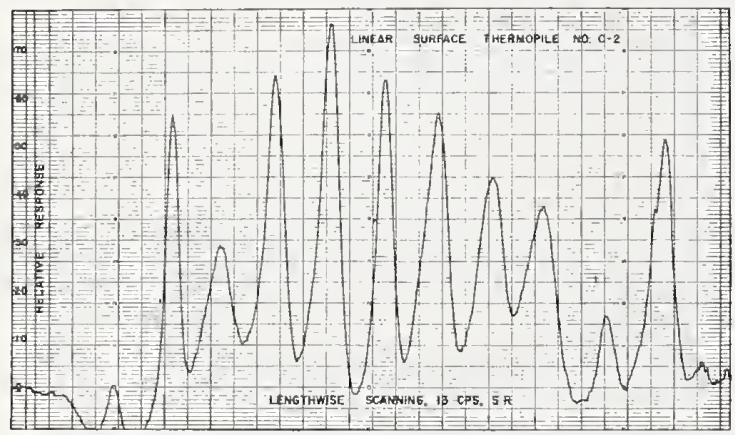

Fig. 6. Lengthwise sensitivity variations of Coblentz twelvejunction lamp-black coated thermopile No. C-2 for an incandescent lamp beam of flux chopped at $13 \mathrm{cps}$ and the output signal measured with synchronous rectifier instrumentation. Again the precise locations of the twelve junctions are not evident, and for some areas the signal is negative.

Figures 4-17 illustrate the observed variations in sensitivity over the surfaces of three thermopiles as indicated by de and ac outputs. The detector was mounted with its receiving surface perpendicular to the beam of flux on a motor-driven carriage immediately back of a fixed narrow slit (width set near $0.25 \mathrm{~mm}$ ). The carriage was moved horizontally (with the slit vertical, and the detector set with its long dimension horizontal for lengthwise scanning, and vertical for crosswise scanning) by means of a synchronous-motor gear-and-screw drive at a speed of $2.12 \mathrm{~mm}$ per $\mathrm{min}$. This corresponds to a detector movement or scanning rate of $0.529 \mathrm{~mm}$ per recorder chart scale division, each abscissa scale division thereby representing $0.529 \mathrm{~mm}$ along the detector.

Figure 4 illustrates the variable dc output of a heavily lamp-black coated Coblentz thermopile. This is a twelve-junction bismuth-silver unit having a time constant of about 1 sec. Each junction is soldered to a 2 $\mathrm{mm} \times 2 \mathrm{~mm}$ tin receiver. These are mounted in a manner similar to overlapping shingles on a roof, but insulated from one another with shellac. The sensitive surface (about $2 \mathrm{~mm} \times 14 \mathrm{~mm}$ ) is heavily coated with lamp black, painted on, then smoked. Figure 5 shows the ac output of the same thermopile as measured with a conventional ac amplifier and meter when the beam of flux is chopped at $10 \mathrm{cps}$. At other chopper frequencies only minor differences appear-principally lower signals for higher frequencies. But with the synchronous rectifier amplifier, major differences result, especially if exact adjustment of chopper position relative to commutator for this particular thermopile has not been made. And these differences change with chopper frequency, probably also being amplified by large variations in the thickness of the lamp-black coating. Variations in sensitivity along this thermopile, when the signal is chopped at $13 \mathrm{cps}$ and the output is measured with an ar synchronous rectifier amplifier, are shown in Fig. 6.

Figure 7 illustrates the de output when the beam of flux is scanned along an intermediate-speed (time constant about $0.25 \mathrm{sec}$ ) gold-black linear thermopile designated No. R-1. This thermopile has four junctions in thermal contact with a flat receiver strip (about

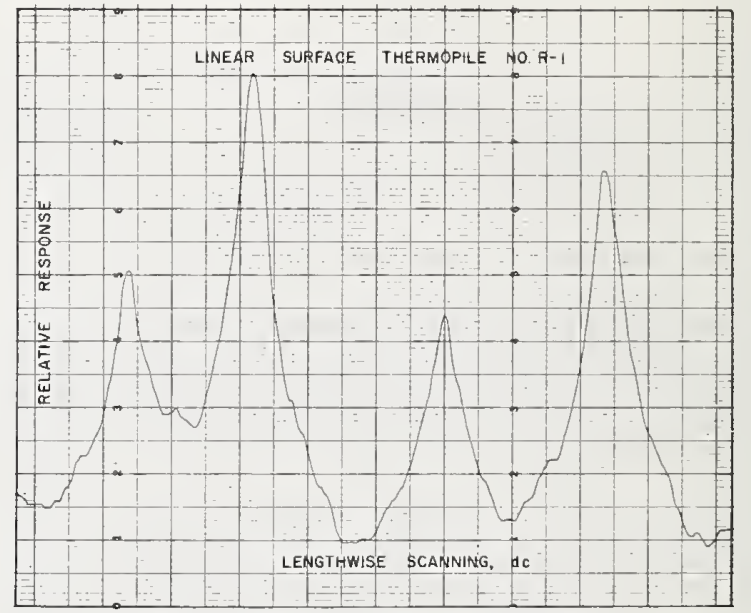

Fig. 7. Lengthwise sensitivity variations of the four-junction gold-black coated thermopile No. R-1 for an incandescent lamp beam of flux with the output signal measured with de instrumentation. Four distinct peaks of (uneven) sensitivity are indicated. Areas between peaks have near zero sensitivity. The small irregularities are noise.

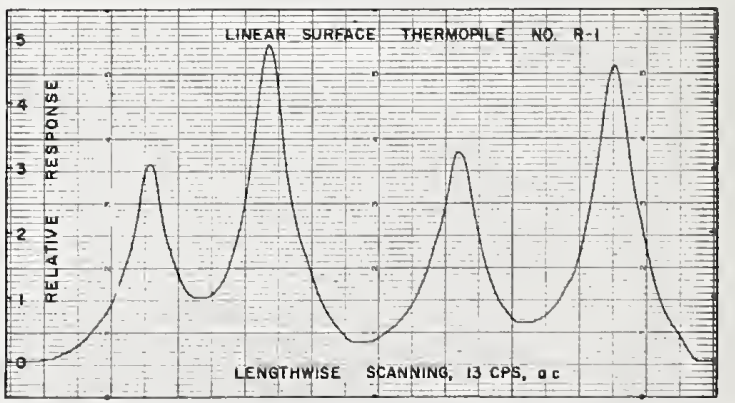

Fig. 8. Lengthwise sensitivity variations of the four-junction gold-black coated thermopile No. R-1 for an incandescent lamp beam of flux chopped at $13 \mathrm{cps}$ and the output signal measured with conventional ac instrumentation. 


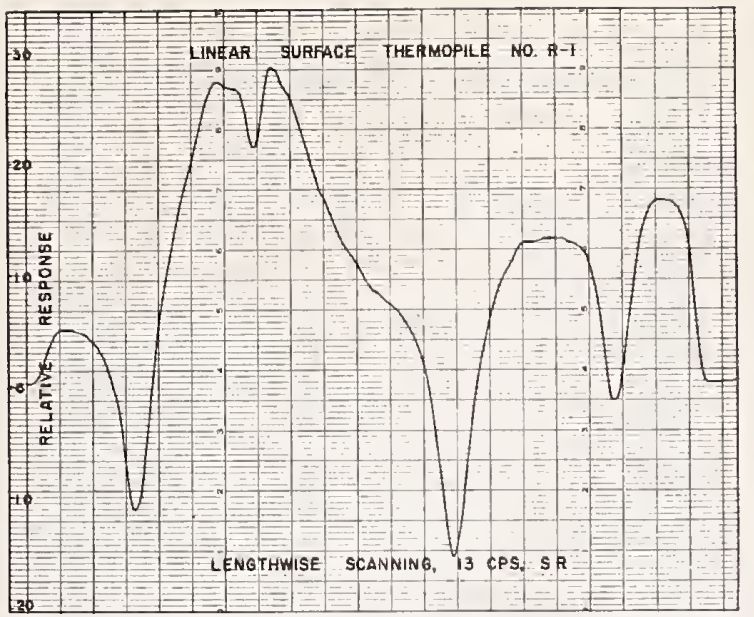

Fig. 9. Lengthwise sensitivity variations of the four-junction gold-black coated thermopile No. R-1 for an incandescent lamp beam of flux chopped at $13 \mathrm{cps}$ and the output signal measured with synchronous rectifier instrumentation. The precise locations of the four junctions are not evident-and in some areas the signal is of high-magnitude negative.

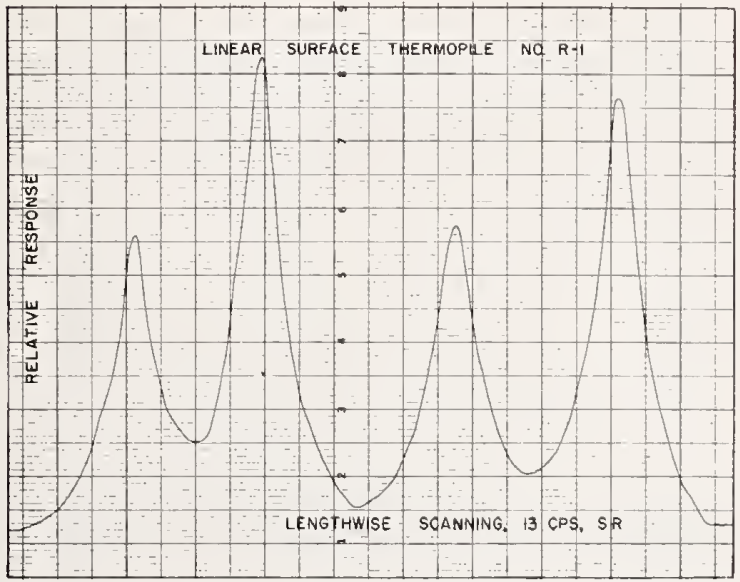

Fig. 10. Same as Fig. 9 except that chopper phasing was reset for maximum response when entire surface of detector was irradiated.

$1 \mathrm{~mm} \times 10 \mathrm{~mm})$. Each junction is represented by a definite peak. Also, the observed sensitivity between junctions is relatively low. Figure 8 illustrates the response of the same thermopile as measured with a conventional ac amplifier and meter. The differences are small but real. However, when the flux was chopped at $13 \mathrm{cps}$, the output signal amplified, synchronously rectified, and recorded, the curve shown on Fig. 9 was obtained. Investigation revealed an improper phase relation between the chopped and the synchronous rectifier. When this was corrected the curve of Fig. 10 was obtained.

When the slitlike beam was set parallel to the long dimension of thermopile R-1 and slowly moved across it, the variation in sensitivity pattern also changed for the three methods of measurement. In Fig. 11 are shown the variations in response across this thermopile observed when the measurement was made in terms of the de output. The small variations result because of air currents, temperature fluctuations, noise, and other factors. The dip on each side of the response peak indicates that some of the beam was reflected onto the cold junctions of the thermopile when the beam was at the edge of the receiver. The sensitivity pattern indicated the distinct changes shown in Fig. 12 when the beam was chopped at 13 cps and the output was amplified by the conventional ac amplifiel. Further changes were observed when the synchronous rectifier amplifier was employed as shown in Fig. 13. The positive deflections ob-

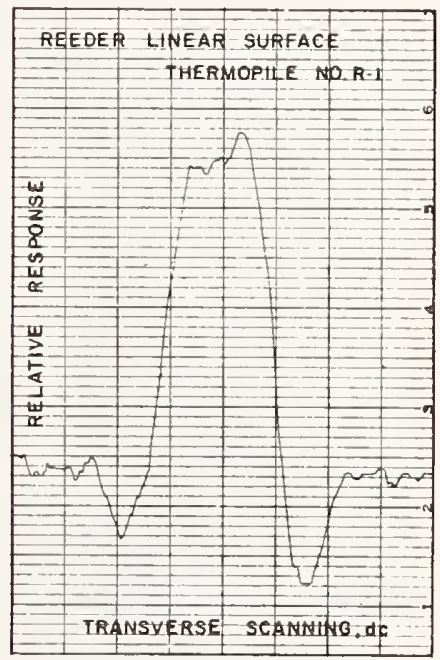

Fig. 11. Crosswise sensitivity variations of the four-junction gold-black coated thermopile No. R-1 for an incandescent lamp beam of flux with the output signal measured with de instrumentation.

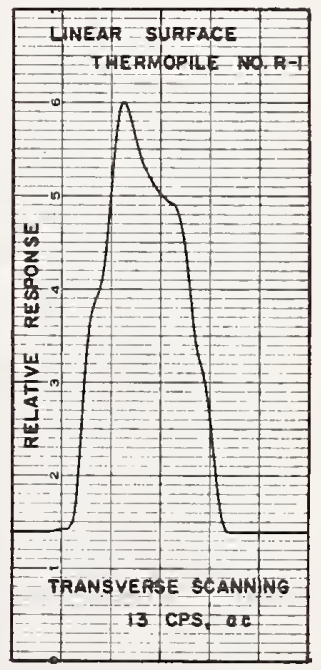

Fig. 12. Same as Fig. 11 except that beam of flux is chopped at $13 \mathrm{cps}$ and the output signal is measured with conventional ac instrumentation. 
served at the edges of the detector are interesting, but not explainable at this time. No edge effects are evident in Fig. 12 when conventional ac amplification is enployed. These variations in sensitivity across the detector are most troublesome ${ }^{2}$ in the spectroradiometric evaluation of spectral radiance and spectral irradiance, especially the latter, because the receiver is irradiated by two geometrically different sources to make these measurements.

Figure 14 illustrates the variations in sensitivity along thermopile No. R-9371 when the dc output is measured. This is a rapid-response (time constant about 0.05 sec) gold-black coated unit having five junctions. The five junctions (each attached to a rectangular receiver) are in line and form a flat receiving surface about $2 \mathrm{~mm} \times 10 \mathrm{~mm}$ in area. Each contact is again discernible by a peak in the curve. A very similar curve was obtained for ac amplification since this is a fast-responding detector (see Fig. 15). The

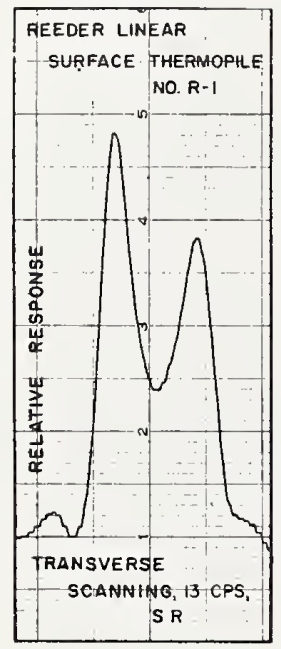

Fig. 13. Same as Fig. 11 except that beam of flux is chopped at $13 \mathrm{cps}$, and the output signal is measured with synchronous rectifier instrumentation.

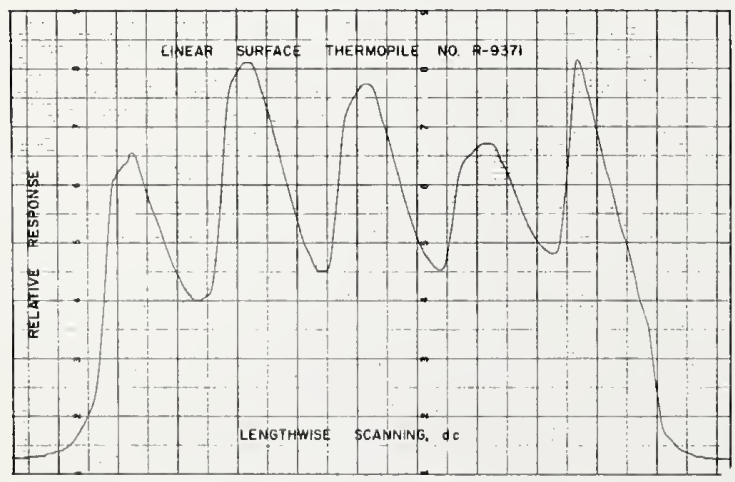

Fig. 14. Lengthwise sensitivity variations of the five-junction gold-black coated thermopile No. R-9371 for an incandescent lamp beam of flux with the output signal measured with dc inst.rumentation.

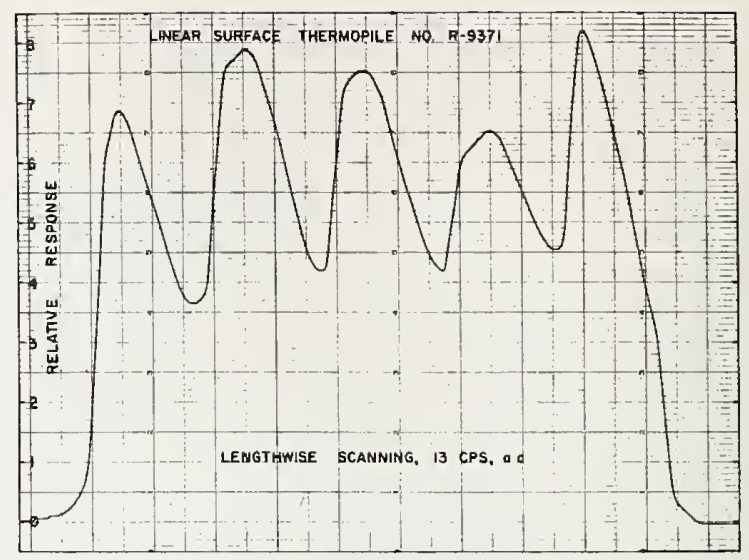

Fig. 15. Same as Fig. 14 except that beam of flux is chopped at $13 \mathrm{cps}$ and the output signal is measured with conventional ac instrumentation.

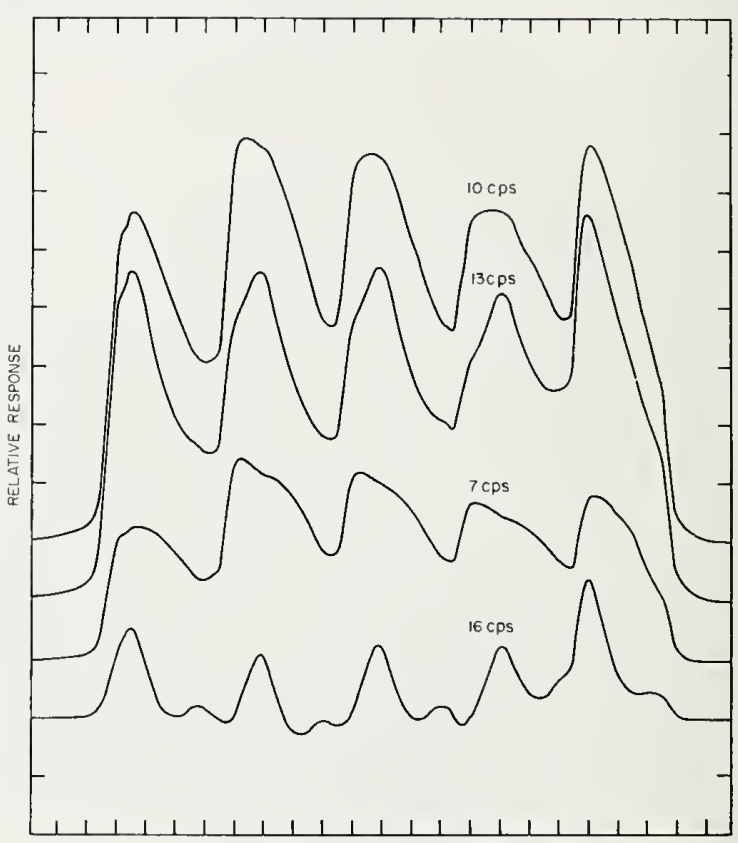

Fig. 16. Lengthwise sensitivity variations of the five-junction gold-black coated thermopile No. R-9371 for an incandescent lamp beam of flux chopped at $7 \mathrm{cps}, 10 \mathrm{cps}, 13 \mathrm{cps}$, and $16 \mathrm{cps}$, with the output signal measured with synchronous rectifier instrumentation. Chopper blade was set at $20^{\circ}$ (see Fig. 18) for all chopping frequencies.

beam was chopped at $13 \mathrm{cps}$ for the data in the figure, but little difference was evident when the chopping frequency was varied from $7 \mathrm{cps}$ to $16 \mathrm{cps}$.

Now, if we take thermopile R-9371 and, employing a synchronous rectifier amplifier, make a number of changes in either the chopping rate or phase synchronization of the chopper blade, some interesting effects occur. Figure 16 shows the effect of changing the chopping frequency. For these curves the angular position 


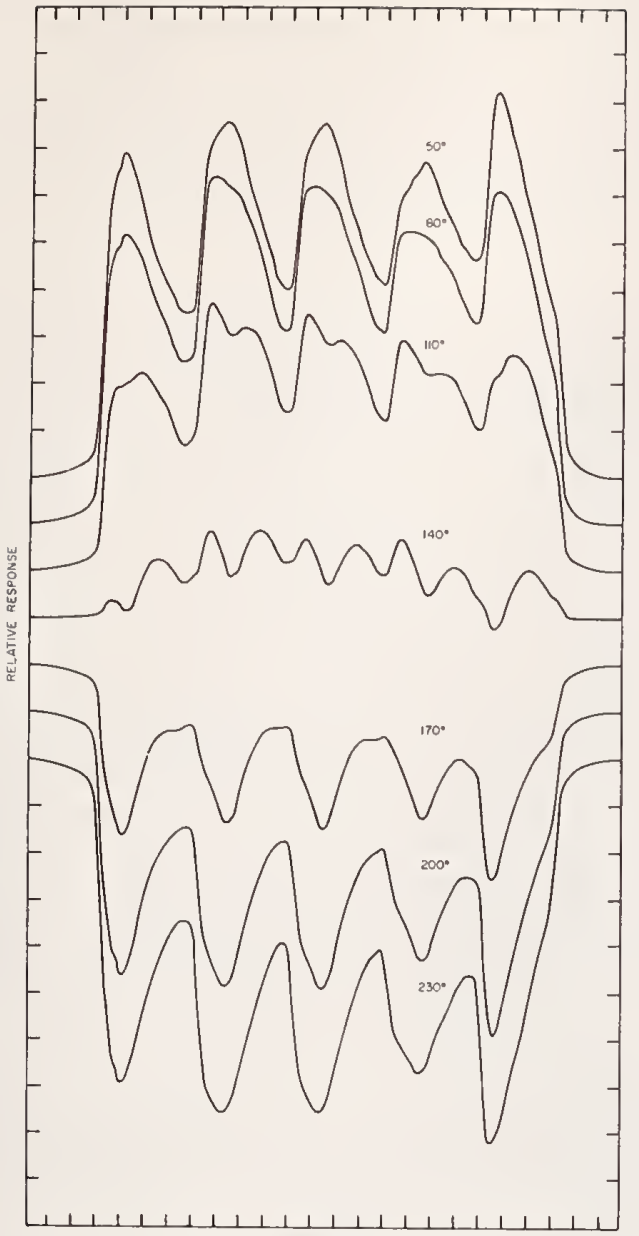

(a)

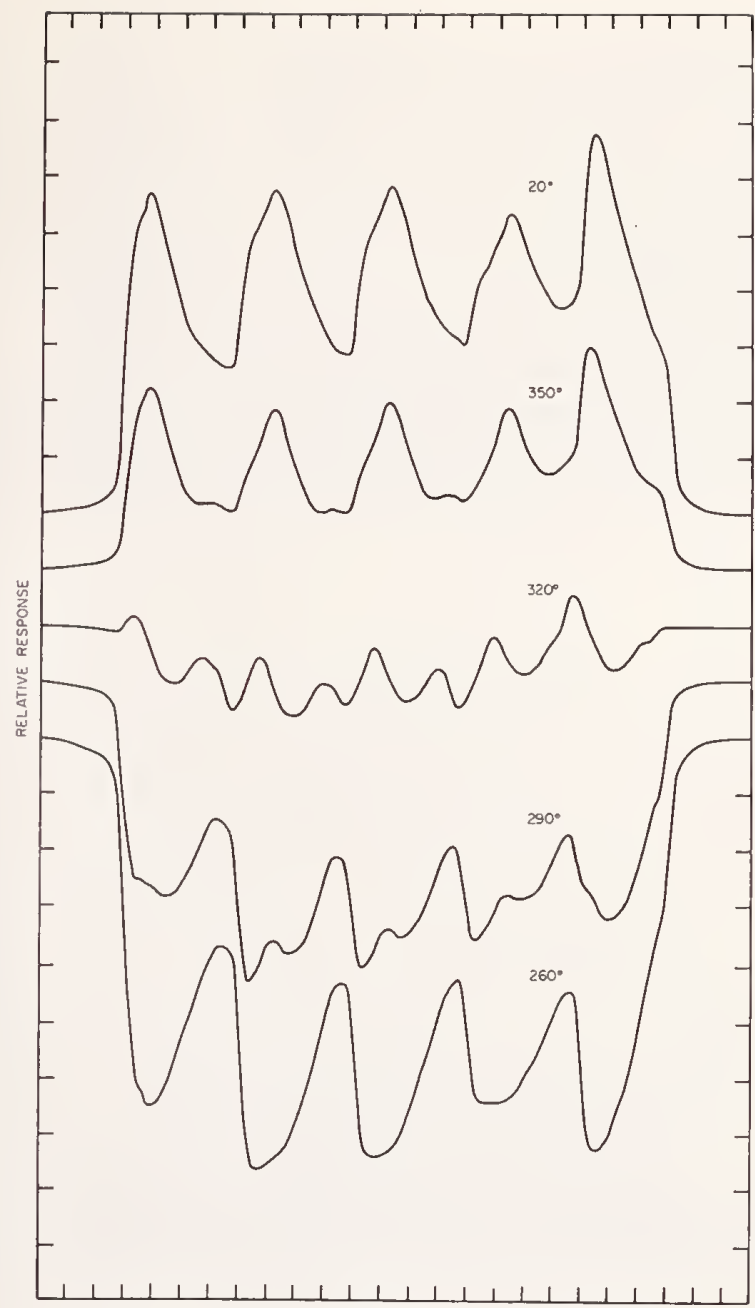

(b)

Fig. 17. (a) and (b) Lengthwise sensitivity variations of the five-junction gold-black coated thermopile No. R-9371 for an incandescent lamp beam of flux chopped at $13 \mathrm{cps}$ with the output signal measured with synchronous rectifier instrumentation. The chopper blade was set at intervals of $30^{\circ}$.

of the chopper blade was set at $20^{\circ} *$ (see Fig. 18) for the four frequencies, $7 \mathrm{cps}, 10 \mathrm{cps}, 13 \mathrm{cps}$, and $16 \mathrm{cps}$.

The effect of changing the phase of the chopper blade relative to the synchronous rectifier commutator is shown in Figs. 17 (a) and (b). In all cases the chopper frequency was set at $13 \mathrm{cps}$ and for successive curves the blade was simply advanced $30^{\circ}$. Note that as the chopper angle is advanced the signal changes from a maximum (near $50^{\circ}$ ) through zero to a reversed maximum (near $230^{\circ}$ ) and then returns again to its original value as the chopper blade is rotated through $360^{\circ}$ (as would be expected).

In the use of the synchronous rectifier amplifier the proper chopper blade setting for a particular thermopile or thermocouple is that which produces maximum signal. This angular setting differs from one detector

* Angular scale for chopper blade position was arbitrarily laid out through $360^{\circ}$. to another as shown in Fig. 18 and may differ also for different areas of the same detector. The angular position for maximum response appears to be related to the time constant of the detector as might be expected. The time constants of the four detectors illustrated in Fig. 18 are approximately $0.05 \mathrm{sec}, 0.25 \mathrm{sec}$, $0.5 \mathrm{sec}$, and $1.0 \mathrm{sec}$, in the order of increasing angular position for maximum response.

For slow-response detectors the time constant was observed to be a function of wavelength. No wavelength effect was observed in the case of No. R=9371 or for No. R-1 (as its window did not permit measurement at long wavelengths). However, the effect was observed for cavity detector No. 3 and for Coblentz thermopile No. C-2. Data on the latter are illustrated in Fig. 19 and indicate an angular shift of about $10^{\circ}$ between visible and infrared radiation. This shift (corresponding to a longer time constant) is in the expected direction for absorption of visible flux near the surface 
of the coating, while infrared flux is absorbed at some depth below the surface of the coating.

There also appears to be a relationship between flux density and angular position of chopper for maximum response. For example, when thermopile R-9371 was uniformly irradiated at various intensities by using different sources and density filters, as well as by varying source distance, the observed angle of maximum response was found to shift to various positions between about $17^{\circ}$ and $33^{\circ}$. The angular setting for maximum response at about $20^{\circ}$ illustrated in Fig. 18 was the mean of three determinations using flux of low uniform density in spectrally narrow bands at $2 \mu, 9 \mu$, and $18 \mu$. When a very narrow beam (width $0.25 \mathrm{~mm}$ ) of intense

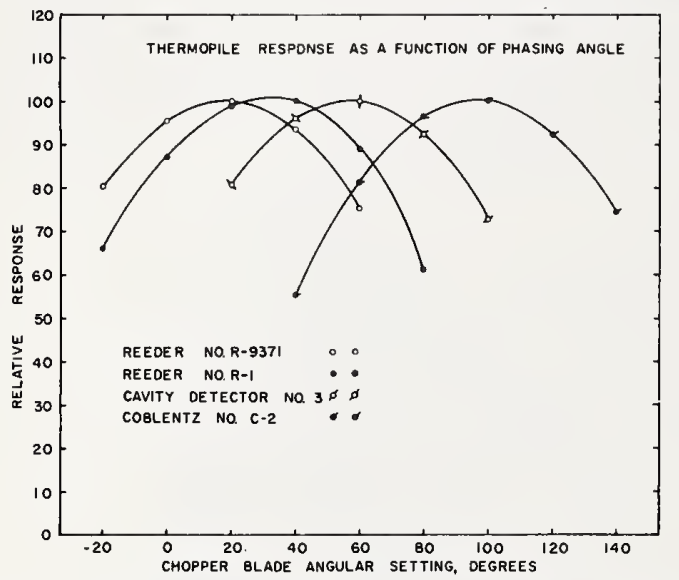

Fig. 18. Relative responses of four thermal detectors as a function of chopper blade setting for low flux filtered irradiance (usually mean of $2 \mu, 9 \mu$, and $18 \mu$ ). The angular settings for maximum response for the various detectors correlate closely with their time constants, namely, $0.05 \mathrm{sec}, 0.25 \mathrm{sec}, 0.5 \mathrm{sec}$, and $1.0 \mathrm{sec}$.

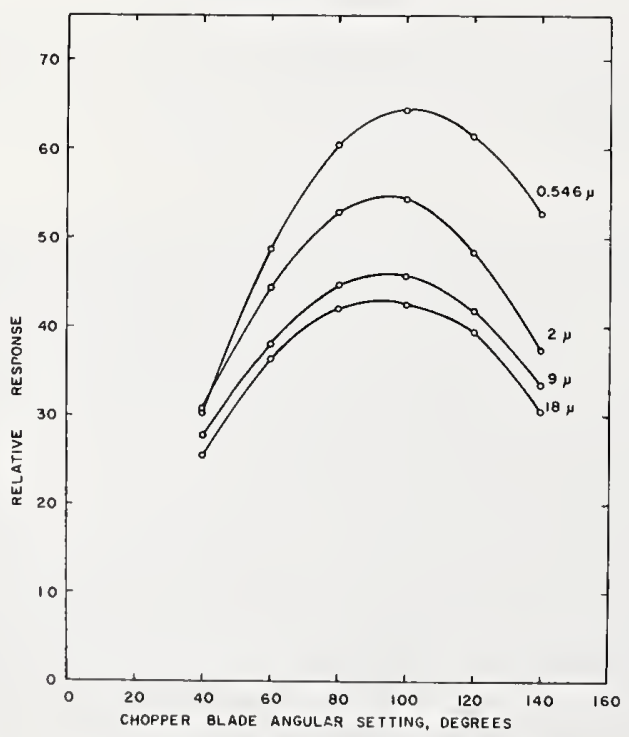

Fig. 19. Relative response of Coblentz thermopile No. C-2 as a function of chopper blade setting for low flux filtered irradiance at selected wavelengths.

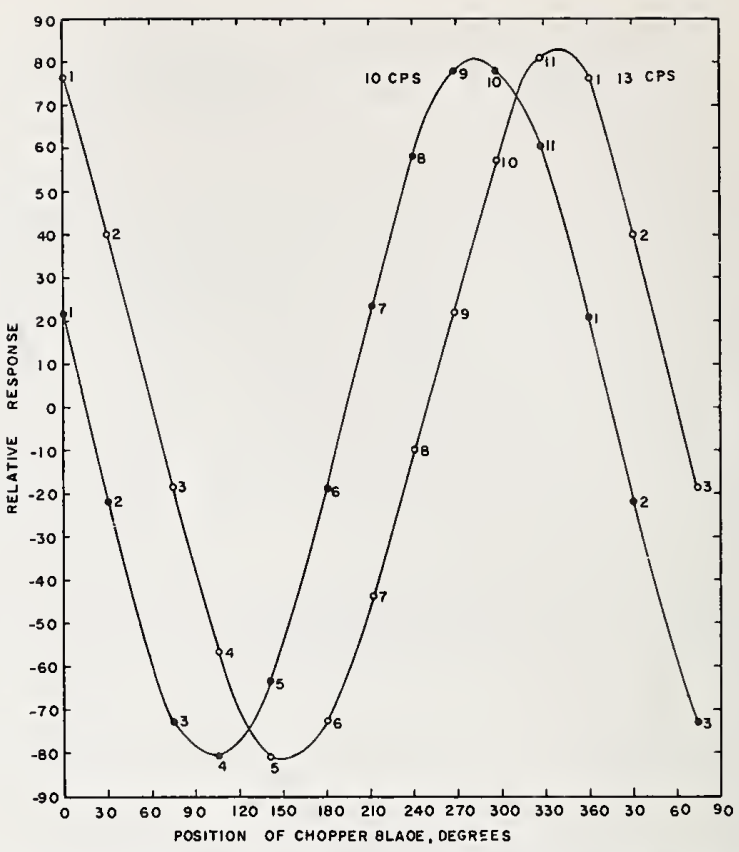

Fig. 20. Curves illustrating the relationships between different chopper blade settings and different chopper frequencies as regard phase and magnitude of recorded signal for a specific area of thermopile No. R-9371. Chopper rates of lower (or higher) frequencies would result in similar curves to the left (or right) of those illustrated. [Zero angle was set arbitrarilybut not the same as for Figs. 17(a) and 17(b).]

flux fell upon the element the maximum response (as determined by integrating the areas under the curves) was shifted to a larger angular setting (about $50^{\circ}$ ) as illustrated in Figs. 17(a) and (b) or to a lower chopping frequency as illustrated in Fig. 16. A similar effect occurred in the case of thermopile R-1 (see Figs. 10 and 18) where the maximum response shifted from about $33^{\circ}$ to $60^{\circ}$. It is possible that this effect results because of some electronic factor, but such a cause is not readily evident.

The curves in Fig. 20 illustrate the relationships between different chopper blade settings and two chopper frequencies for thermopile R-9371. The plotted points on these curves represent the recorded deflections when a certain area on the thermopile was irradiated while the angular position of the chopper blade and the chopping frequencies were varied. Note that each point on the family of curves, for which two are shown in Fig. 20, corresponds to a characteristic variation-in-sensitivity curve similar to those shown in Figs. 17 (a) and (b). The variation-in-sensitivity curve represented by any position on one of the curves of Fig. 20 matches closely the variation-in-sensitivity curve represented by a corresponding phase position on another curve. For example, the variation-in-sensitivity curve obtained at position 1 on the 13-cps curve is matched closely by that for position 10 on the 10 -cps curve. The results of these experiments indicate the effects in the recorded results of phase changes in the 
amplifier chopper associated with the time constants of the various areas of the detector. If the signal reaches the synchronous rectifier in phase a large signal results, if out of phase a small or negative reading is recorded. Because of variations in time constant over the surface of available detectors, all sorts of values can result unless the phase of the synchronous rectifier is adjusted to provide maximum signal for the particular detector in use.

\section{Conclusions}

The data presented herein indicate two important difficulties involved in performing accurate radiometric work with the conventional spectrometer when a thermoelectric detector is placed at the exit slit. Detector sensitivity has been found to be a function of wavelength and of irradiated area of the detector surface. To eliminate the effect of variation in sensitivity as a function of wavelength, the detector can be calibrated by comparison with a detector of known spectral response although this still leaves the problem of calibration of the reference detector'. 'To eliminate the effect of variation in sensitivity as a function of area of irradiation the detector must always be irradiated in an identical manner, preferably by providing uniform irradiation by utilizing a flux mixing mechanism, such as a small integrating sphere, rather than focusing the images of sources, such as lamp filaments, directly on the detector. Further difficulties may be encountered in radiometric work, whether in conjunction with a spectrometer or not, when measurements are made by any method in which the beam of flux is chopped. The difficulties may be increased if a synchronous rectifier type of amplifier is employed, especially if the rectifier is improperly phased for the particular thermopile in use. Optimum phasing, however, may be difficult with an extended receiver because the phase adjustment will be a function of the irradiated area of the receiver surface and possibly also of wavelength. Some of the diffculties encountered in amplification can be eliminated by the use of de instrumentation if accompanying drift is not intolerable. The use of de instrumentation is highly recommended if its use is suitable to the problem at hand. In any case in the radiometric use of a thermoelectric detector the receiving surface should be as uniformly and completely irradiated as possible. Otherwise, the results will be clouded by the uncertainties associated with local and irregular heating of a nonuniformly sensitive surface wherein such things as emission and conduction losses and time constant variations cannot be assessed.

\section{References}

1. W. E. Schneider and R. Stair, J. Opt. Soc. Ana. 53, 1354 (1963).

2. R. Stair and W. E. Schneider, "Standards, Sources, and Detectors in Radiation Measurements", Symposium on Thermal Radiation of Solids, San Francisco, California (4-6 March 1964) (NASA SP-55, to be published May 1965).

3. R. Stair, W. E. Schneider, and J. K. Jackson, Appl. Opt. 2, 1151 (1963).

4. W. L. Eisenman, R. L. Bates, and J. D. Merriam, J. Opt. Soc. Am. 53, 729 (1963).

5. R. Stair and W. E. Schneider, "The NBS Instrumentation for Use in the Measurement of Spectral Irradiances of Solar Simulators", Proceedings of Joint Symposium on Solar Radiation Simulation, Los Angeles, California, 18-20 January 1965 (to be published).

6. A. Gouffé, Rev. Opt. 24, Nos. 1-3, 1 (1945).

7. E. M. Sparrow and V. K. Jonsson, J. Opt. Soc. Am. 53, 816 (1963). 


\section{NBS TECHNICAL NOTE 418 \\ ISSUED NOVEMBER 1967}

\section{Radiometric Methods for Measuring Laser Output}

D. A. McSparron, C. A. Douglas, and $H$. L. Badger

Metrology Division Institute for Basic Standards National Bureau of Standards Washington, D.C. 20234 means for making available scientific data that are of transient or limited interest. Technical Notes may be listed or referred to in the open literature. 


\section{CONTENTS}

PAGE

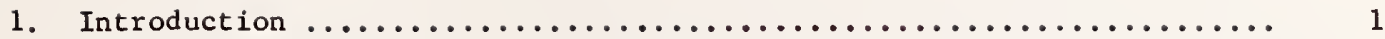

2. Experimental setup $\ldots \ldots \ldots \ldots \ldots \ldots \ldots \ldots \ldots \ldots \ldots \ldots \ldots \ldots \ldots \ldots \ldots \ldots$

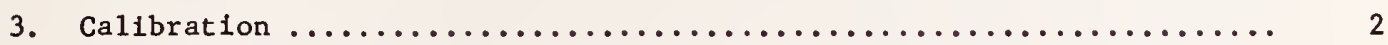

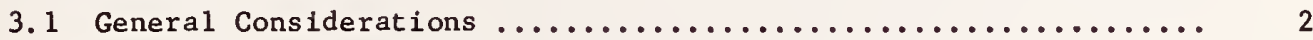

3. 2 Phototube Calibration $\ldots \ldots \ldots \ldots \ldots \ldots \ldots \ldots \ldots \ldots \ldots \ldots \ldots \ldots \ldots \ldots \ldots \ldots \ldots$

4. Laser Measurements with the Phototubes $\ldots \ldots \ldots \ldots \ldots \ldots \ldots \ldots \ldots \ldots$

5. Thermop lle Calibration $\ldots \ldots \ldots \ldots \ldots \ldots \ldots \ldots \ldots \ldots \ldots \ldots \ldots \ldots \ldots \ldots . \ldots$

6. Comparison with Calorimeter $\ldots \ldots \ldots \ldots \ldots \ldots \ldots \ldots \ldots \ldots \ldots \ldots \ldots \ldots \ldots$

7. Discusston of Possible Sources of Error $\ldots \ldots \ldots \ldots \ldots \ldots \ldots \ldots \ldots$

8. Conclusions $\ldots \ldots \ldots \ldots \ldots \ldots \ldots \ldots \ldots \ldots \ldots \ldots \ldots \ldots \ldots \ldots \ldots \ldots \ldots$

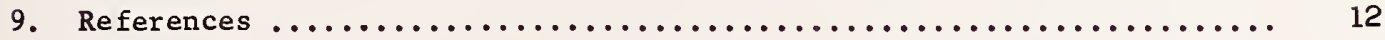




\section{LIST OF ILLUSTRATIONS}

1. Experimental Setup for Measuring Laser Energy...................

2. Experimental Setup for Calibrating Recelvers....................

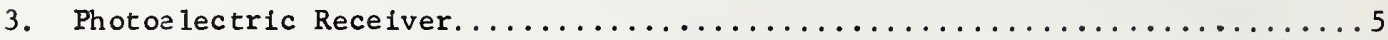

4. Block Circuit Diagram of the Phototube Measuring Circuit............

5. Block Circuit Diagram of the Thermopile Measuring Circuit............ 10

6. Experimental Setup for Measuring Laser Energy with a Calorimeter.........10 
D. A. McSparron, C. A. Douglas, and H. L. Badger

The output of pulsed lasers may be measured with reasonable accuracy by modifications of established radiometric methods. The receiver, thermopile or phototube, is used ballistically. It is calibrated by an incandescent-lamp standard of spectral irradiance. The laser beam is attenuated to make its irradiation on the receiver comparable to that from the calibrating source. Attenuation is accomplished by intercepting the entire laser beam on a diffusely reflecting surface. The attenuation factor is determined from direct measurement of the reflectance of this diffusing surface and the geometric constants of the system. The accuracy of the measurement of laser output is dependent upon the accuracy of calibration of the standard of spectral irradiance and the accuracy with which the spectral sensitivity of the receiver, the spectral transmittance of the band-pass filters, and the reflectance of the diffuser can be determined. Measurements made with different photoelectric receivers agree to about $1.5 \%$ using a $1 / 4$-joule pulsed ruby laser as a source. Total uncertainty is estimated to be about $5 \%$. Measurements made using these radiometric methods have been compared with calorimetric measurements and a discrepancy of $9 \%$ was observed.

Key Words: Laser, radiometry, laser energy measurement, radiometric calibration, photoelectric photometry, optical attenuator.

\section{Int roduction}

The rapid development of laser technology has left workers in the field without recognized methods of measuring the parameters of interest. The situation is particularly bad in the measurement of the energy emitted from pulsed lasers. Discrepancies of $25 \%$ or more have been common. Two widely differing approaches to the problem have been popular, namely, radiometric and calorimetric. A common calorimetric approach entails the absorption of the entire laser beam in a liquid. The temperature rise of the liquid is then a measure of the total energy contained in the pulse. Calorimetric measurements are difficult to perform because of the low signal levels obtained from low energy pulsed lasers, usually a few microvolts, and because of the long recycling times, thirty minutes or more. In addition, no information can be obtained from calorimetric measurements on the peak power levels of the pulse. The work described in this paper employs a radiometric approach. In addition to the advantages of relatively high signal levels and fast recycling times, a radiometric method can provide information about the peak power levels in the pulse through the display of the receiver output on an oscilloscope. Although the work to date has involved only a relatively low powered, approximately $1 / 4-$ joule, conventional ruby 1 aser operated at 694.3 $\mathrm{nm}$, the measurement techniques and procedures should be readily applicable to a wide range of energies and wavelengths.

A pulsed laser beam differs from conventional sources in that it is highly monochromatic, extremely intense, of short time duration, and in some cases polarized. These properties require modification of conventional radiometric measurement techniques if existing standards are to be used for the calibration. Such receivers as vacuum phototubes, photo multipliers and thermopiles can tolerate maximum incident power levels only of the order of milliwatts $/ \mathrm{cm}^{2}$ for conventional tubes or at most watts $/ \mathrm{cm}^{2}$ for present biplanar photodiodes. Beyond these levels physical damage occurs. Since instantaneous power levels in laser beams run to megawatts/ $\mathrm{cm}^{2}$ and higher, some attenuation device is necessary. An ideal 
attenuator should be simple, rugged, and readily standardized through existing techniques. Attenuation factors that range from $10^{-4}$ to $10^{-8}$ are necessary. A diffusing surface of high reflectance meets these requirements.

\section{Experimental Setup}

The experimental setup used is shown in Figure 1. Energy from the laser is incident normally on a pressed block of USP grade magnesium carbonate. Receivers are placed at a distance $d$ from the diffuser, in tracks that form a $20^{\circ}$ angle with the incident beam. The attenuation factor, $\alpha$, is given by:

$$
\alpha=\frac{\beta a \cos 20^{\circ}}{\pi d^{2}}
$$

where $a$ is the area of the receiver, $\beta$ is the $0^{\circ}: 20^{\circ}$ (incidence angle: reflectance angle) reflectance factor $1 /$ of the $\mathrm{MgCO}_{3}$ and $\mathrm{d}$ is the distance from the $\mathrm{MgCO}_{3}$ to the receiver. $\mathrm{MgCO}_{3}$ was chosen for the diffusely reflecting surface and has proved satisfactory for the wavelength and energy used as will be discussed later. $2 /$ An angle of $20^{\circ}$ was chosen to be sufficiently far from the normal that the effects of specular components of the reflected beam are negligible and yet to be sufficiently small so that $\cos \theta$ is not a rapidly varying function. The $\beta$ of this equation was measured at $20^{\circ}$ on a goniophotometer with substantially monochromatic light of the same wavelength as the ruby laser used in this work. Thus, no assumptions about the diffuse reflection properties of $\mathrm{MgCO}_{3}$ have been made. For typical values, a one-centimeter-radius receiver and a receiving distance of $100 \mathrm{~cm}, \alpha$ is approximately $10^{-4}$. Increased attenuation may be obtained by increasing $\mathrm{d}$ or, if necessary, by introducing a second diffuser.

Two types of receivers have been used in this work. The first is a type PJ14B vacuum phototube. These phototubes have very low dark currents and an S-7 response which is relatively flat at the ruby wavelength. This tube is no longer being manufactured. It was originally developed for use in the Hardy (General Electric) recording spectrophotometer. The second type of receiver, so far used for preliminary investigations only, is a Moll large-surface thermopile. It has 80 constantan-manganin junctions placed in a circular array one centimeter in radius with a platinum black surface.

\section{Calibration}

\section{1 General Considerations}

Typically in radiometry employing a photoelectric or thermoelectric receiver, the primary problem is not the measurement of the electrical output of the receiver to the desired degree of accuracy and precision. Instead the primary problem is the calibration of the receiver in terms of electrical output per unit of incident energy (or power). The problem is particularly acute when the sensitivity of the receiver is not constant but varies with the wavelength of the incident energy as does the sensitivity of a phototube, and a calibrating source having a spectral energy distribution identical with that of the source to be measured is not available. The calibration procedure is as follows.

I/ Reflectance factor is the ratio of the reflected flux to that reflected by a perfect diffuser identically irradiated.

2/ Although $\mathrm{MgCO}_{3}$ was the chosen material other diffusers of high reflectance such as $\mathrm{Mg} 0$ and $\mathrm{BaSO}_{4}$ should also prove satisfactory. These materials would be more stable chemically when irradiated by high-energy laser pulses. $\mathrm{MgCO}_{3}$ was chosen mainly because of the ready availability of pressed blocks with large surface areas. 


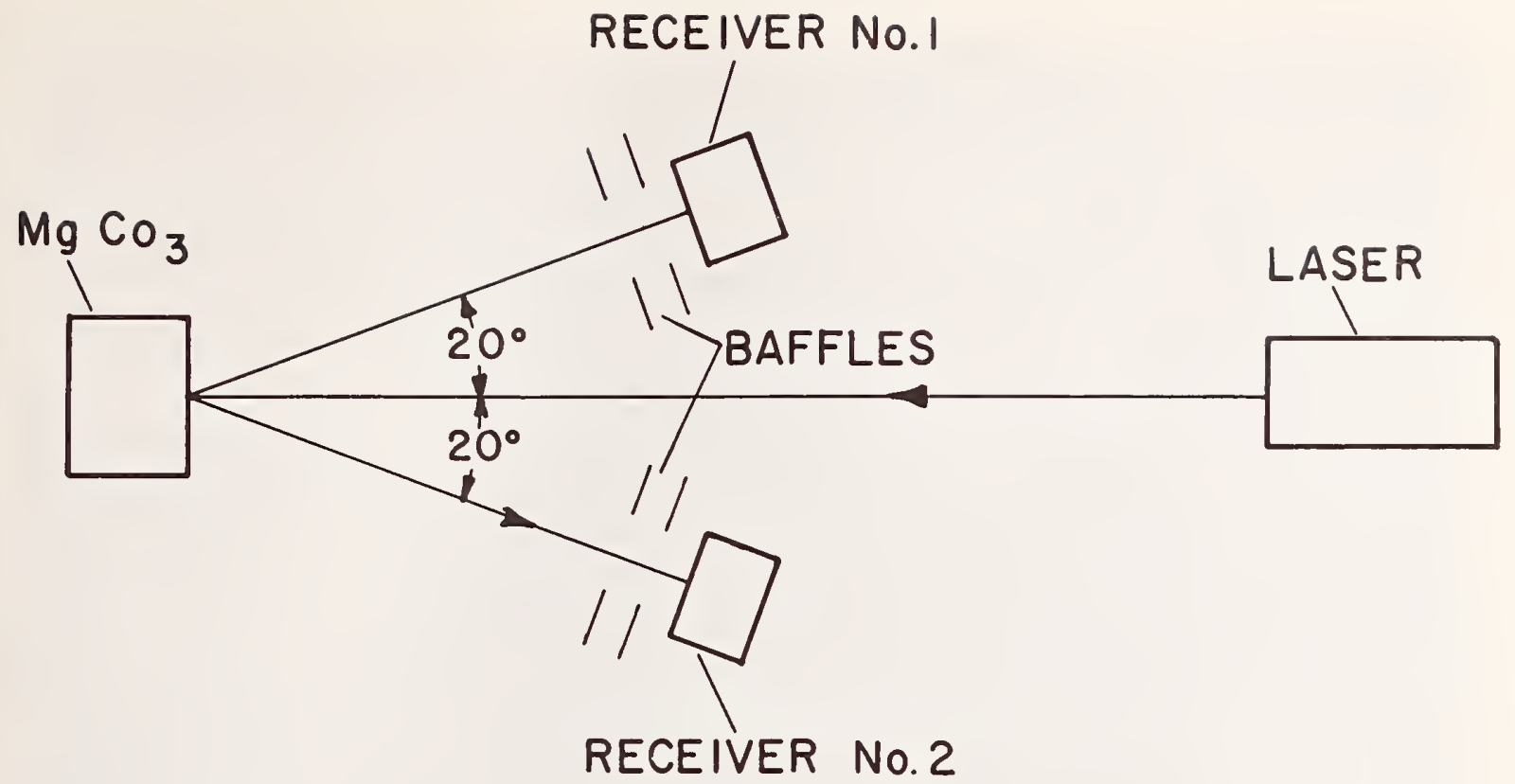

Figure 1. Experimental Setup for Measuring Laser Energy

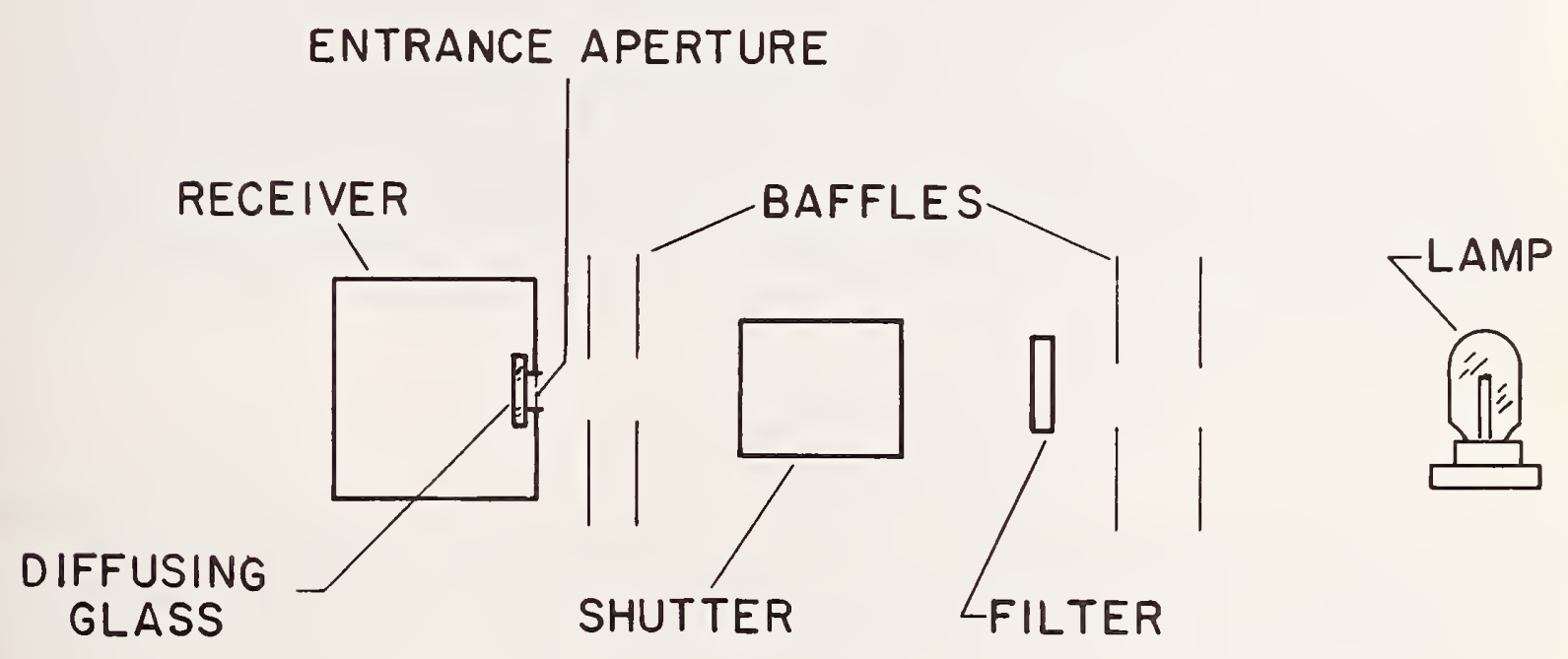

Figure 2. Experimental Setup for Calibrating Receivers 
The receivers were calibrated in a separate experiment. The calibration setup is shown in Figure 2. A lamp calibrated for spectral irradiance is used as an energy source. Energy from this lamp is passed through a filter, then a shutter and finally on to the receiver. The filter is introduced in order to spectrally limit the output of the lamp. A special two-part shutter was used. The first part consists of a $60-\mathrm{r} / \mathrm{min}$. synchronous motor which drives a sector disk through a multiple-speed reduction gear-head. The disk is thus rotated at various known slow speeds to provide series of light pulses of accurately known time durations. The second part is a simple open-close shutter that allows selection of a single pulse from the series of pulses provided by the sector disk. The combination thus simulates the pulsed characteristic of the laser beam except that the pulse lengths are longer.

Care was taken to fully and uniformly irradiate the sensitive surface of the receiver because receivers might exhibit large sensitivity variations across the receiving surface. $[1,2] *$ The requirement for uniform irradiance was met by using source-to-receiver distances which were large in comparison to the size of the source and the receiver aperture. In addition, since the available energy and phototube sensitivity allowed it, a diffusely transmitting glass was incorporated as part of each phototube receiver as shown in Figure 3. The baffling shown in Figures 1 and 2 is required to eliminate the effects of stray light and interreflections. The laser head emits stray light in two ways, namely flashtube light escaping through the vent holes and light emitted through the rod which has not undergone laser action. In contrast to the laser beam proper, the flux incident on the $\mathrm{MgCO}_{3}$ diffuser from these sources of stray light obeys the inverse-square-law and hence, can be reduced to any degree desired by the use of large laser-head-to-diffuser distances. In this work a distance of five meters was used.

\subsection{Phototube Calibration}

Figure 4 is a block circuit diagram of the measuring circuit used with the phototubes. The photocurrent charges a capacitor, C. An electrometer-amplifier is used to determine the voltage to which the capacitor is charged and the result is displayed on a chart recorder.

At a wavelength $\lambda$, a steady-burning lamp with a spectral radiant intensity, $I_{\lambda}, \underline{3} /$ and at a distance, $d$, from the receiver aperture, will produce a spectral irradiance, $\lambda_{\mathrm{E}}$, on the receiver aperture where $E_{\lambda}=I_{\lambda} / d^{2}$. If an interference filter of spectral transmittance, $T(\lambda)$, is placed between the lamp and the phototube and if the phototube has a relative spectral response, $R(\lambda)$, the phototube output will be proportional to the triple product: $E \tau(\lambda) R(\lambda)$. In the calibration setup (Figure 2) the shutter allows irradiation of the phototube recelving surface for a measured time, T. Hence, the voltage to which the capacitor is charged, $V_{P}$, is given by:

$$
\mathrm{v}_{\mathrm{P}}=\mathrm{K}_{\mathrm{PT}} \mathrm{aT} \int_{\lambda_{1}}^{\lambda_{3}} \mathrm{E}_{\lambda} T(\lambda) \mathrm{R}(\lambda) \mathrm{d} \lambda
$$

where $a$ is the area of the receiver aperture, $k_{p T}$ is the receiver calibration factor, and $\lambda_{1}$ and $\lambda_{3}$ are the lower and upper limits of the pass band of the filter. Thus:

* Numbers in [] refer to similarly numbered references at the end of the paper.

3/ In this paper quantities which are restricted to a narrow wavelength band are indicated by adding the word spectral. The corresponding symbols are indicated by a subscript, e.g., $E_{\lambda}$, for a spectral concentration or a $\lambda$ in parenthesis, e.g., $T(\lambda)$, for a function of wavelength. This usage is in accord with the recomendations of ISO, IEC, CIE, and SUN Commission of IUPAP and in forthcoming American Standard Y10.6. 


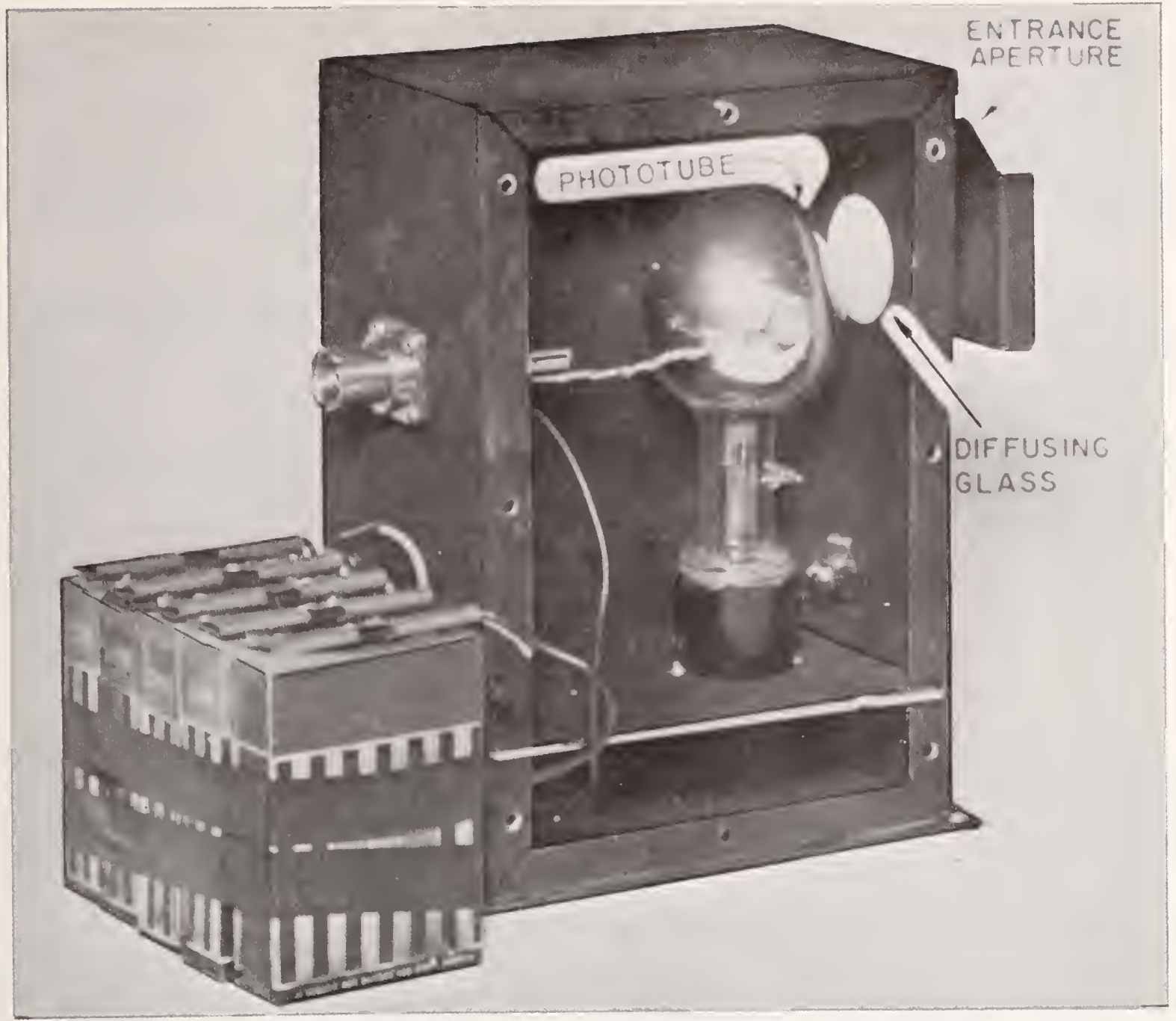

Figure 3. Photoelectric Receiver

ELECTROMETER
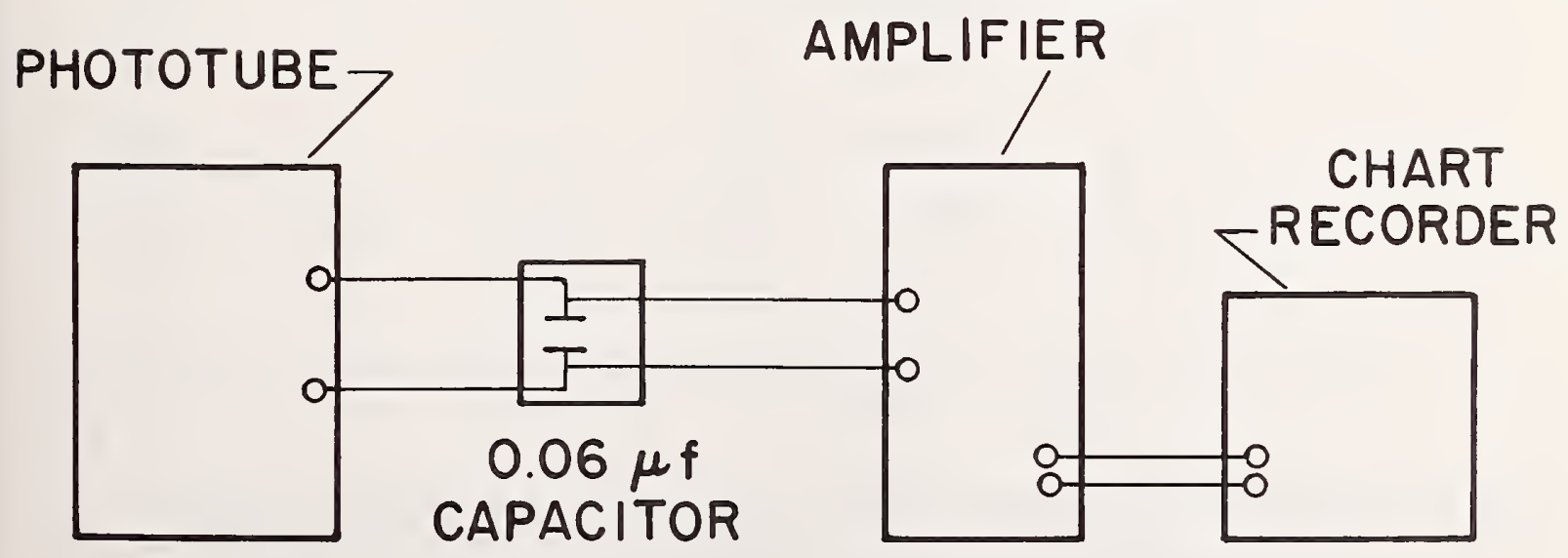

Figure 4. Block Circuit Diagram of the Photetube Measuring Circuit 


$$
\mathrm{K}_{\mathrm{PT}}=\frac{\mathrm{V}_{\mathrm{P}}}{\mathrm{aT} \int_{\lambda_{1}}^{\lambda_{2}} E_{\lambda^{2}} \tau(\lambda) R(\lambda) \mathrm{d} \lambda}
$$

This factor is a function of the transmittance of the diffusing glass, the size of the capacitor, and the absolute sensitivity of the phototube at the wavelength at which $R(\lambda)$ is unity.

In the laser-measuring setup (Figure 1), the voltage, $V_{L}$, developed on the capacitor as the result of a laser shot will be:

$$
\mathrm{V}_{\mathrm{L}}=\mathrm{K}_{\mathrm{PT}} \alpha \mathrm{R}_{(694.3)^{\mathrm{Q}}}
$$

where $\alpha$ is the attenuation factor given by Equation 1 , and $R_{(694.3)}$ is the relative spectral response of the phototube at $694.3 \mathrm{~nm}$. Hence, the laser energy is:

$$
Q=\frac{V_{L}}{K_{P T} \alpha R_{(694.3)}}
$$

\section{Laser Measurements with the Phototubes}

In order to establish the validity of the phototube calibrations, a complete factorial calibration experiment was performed. Systematically, all combinations of three phototubes, two interference filters, two exposure times, two lamps, and two receiver housings with diffusers were used to determine six calibration factors, one

\begin{tabular}{|c|c|c|c|c|}
\hline Phototube & $\begin{array}{l}\text { Receiver } \\
\text { Housing }\end{array}$ & $\frac{\text { Average } \mathrm{K}_{\mathrm{PT}}^{\mathrm{a}}}{\text { (Volts/Joule }^{\mathrm{b}}}$ & $\begin{array}{c}\text { S.D. of a } \\
\text { Single Measurement } \\
(\%)\end{array}$ & $\begin{array}{l}\text { S.D. of } \\
\text { Avg. } c \\
(\%)\end{array}$ \\
\hline \multirow[t]{2}{*}{2134} & B & 9,528 & 2.44 & 0.85 \\
\hline & A & 6,982 & 0.91 & .32 \\
\hline \multirow[t]{2}{*}{2136} & B & 8,049 & 2.41 & .85 \\
\hline & A & 5,893 & 2.59 & .92 \\
\hline \multirow[t]{3}{*}{2138} & B & 12,493 & 2.11 & .75 \\
\hline & A & 8,892 & 1.47 & .53 \\
\hline & & Average & 1.99 & 0.70 \\
\hline
\end{tabular}
each for each phototube-housing combination. The results are shown in Table I.

\section{Table I: Phototube Calibrations}

a Based upon the relative response of the phototube at $694.3 \mathrm{~nm}\left(\mathrm{R}_{694.3}\right)=1.00$

b Volts per joule incident on the receiver aperture.

c Based on 8 independent determinations of $\mathrm{K}_{\mathrm{PT}}$. 
Each calibration factor, $\mathrm{K}_{\mathrm{PT}}$, shown in $\mathrm{Table} \mathrm{I}$ is an average of eight different combinations of lamp, filter, and exposure time. The standard deviations quoted are indicative of the precision with which the various measured parameters, $V_{p}, R(\lambda), \tau(\lambda)$, and $E_{\lambda}$ can be determined. Analysis of the data shows no systematic variation with filter, exposure time, or receiver housing. A shift of $1.3 \%$ was traceable to the standard lamp used. However, the direction and magnitude of this shift are such as to be explained by the fact that one of the lamps has functioned as a working standard while the other has been held as a reference standard.

In order to check the consistency of the calibration factors thus determined, an additional experiment was performed. Laser energy was measured by using the arrangement shown in Figure 1. Each phototube-housing combination was used in each of the 2 positions shown to measure the energy of three laser shots. Since only two phototube-housing combinations could be used at one time, an experimental design involving a total of 36 laser shots was employed. This design allowed separation of the effects due to varying laser energy from those due to calibration uncertainties. The data from these two experiments were analyzed statistically. The results for the various factors involved in the calibration experiment were as stated above. Two additional effects were found. Measurements made with one of the phototubes, Number 2136, appear to differ from the other two by about $1 \%$. The exact cause has not been traced as yet. The second effect was a $4 \%$ shift depending only on whether the receiver was located at position 1 or position 2 of Figure 1. This effect was traced to interreflected energy reaching the receiver at one position and not the other, emphasizing again the need for adequate shielding of the equipment. Table II shows the experimental design and the results of the energy measurements. (All results obtained by measurements at receiver position 1 have been corrected by subtracting $4 \%$ from the measured value.)

Table II: Laser Energy Measurements

\begin{tabular}{|c|c|c|c|c|c|c|}
\hline $\begin{array}{l}\text { Phototube } \\
\text { (Position 1) }\end{array}$ & $\begin{array}{l}\text { Receiver } \\
\text { Housing } \\
\end{array}$ & $\begin{array}{l}\text { Laser } \\
\text { Output } \\
\text { Joules }\end{array}$ & $\begin{array}{l}\text { Phototube } \\
\text { (Position 2) }\end{array}$ & $\begin{array}{l}\text { Receiver } \\
\text { Housing } \\
\end{array}$ & $\begin{array}{l}\text { Laser } \\
\text { Output } \\
\text { Joules a }\end{array}$ & $\begin{array}{c}\text { Difference } \\
\text { (Pos. 1-Pos. 2) }\end{array}$ \\
\hline 2134 & A & .2245 & 2138 & B & . 2190 & 2.3 \\
\hline 2136 & A & .2157 & 2138 & B & .2189 & -1.4 \\
\hline 2136 & A & . 2199 & 2134 & B & . 2264 & -2.7 \\
\hline 2138 & A & .2249 & 2134 & B & .2279 & -1.3 \\
\hline 2138 & A & .2322 & 2136 & B & .2300 & 0.9 \\
\hline 2134 & A & .2339 & 2136 & B & .2346 & -0.4 \\
\hline 2136 & B & .2328 & 2134 & A & .2353 & -1.7 \\
\hline 2138 & B & .2327 & 2134 & A & .2381 & -2.1 \\
\hline 2138 & B & .2316 & 2136 & A & .2204 & 4.9 \\
\hline 2134 & B & .2305 & 2136 & A & .2157 & 6.8 \\
\hline 2134 & B & .2227 & 2138 & A & .2312 & -3.5 \\
\hline 2136 & B & .2269 & 2138 & $\mathrm{~A}$ & .2312 & -1.8 \\
\hline
\end{tabular}

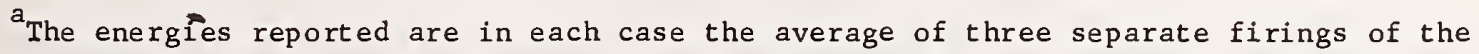
laser.

The results of this statistical analysis show that the physical parameters, exposure time, lamp energy, relative spectral response of the receivers, and particularly notable, interference filter transmittances, have been evaluated and combined in a mutually consistent manner. The experimental parameters, such as receiver housing and receiver position, are also under adequate control. Hence, a set of mutually consistent measurements has 
been achieved under widely varied conditions.

Several experiments were run to check on possible sources of systematic error. An investigation was made to determine if the phototubes were saturating under the high power density produced by the laser. The usual aperture was replaced with a larger aperture (area ratio 11.6) and the supply voltage across the phototube was varied. A second phototube was used to monitor the laser output. Table III indicates that phototube saturation was not biasing the experiments.

Table III: Phototube Saturation Experiment

\begin{tabular}{cccc}
$\begin{array}{c}\text { Supply } \\
\text { Voltage }\end{array}$ & $\begin{array}{c}\text { Test Phototube } \\
\text { Output }\end{array}$ & $\begin{array}{c}\text { Monitor } \\
\text { Output }\end{array}$ & $\begin{array}{c}\text { Ratio } \\
\text { (Volts) }\end{array}$ \\
\cline { 2 - 2 } 520 & 2.06 & 0.189 & 10.90 \\
270 & 2.08 & .193 & 10.78 \\
180 & 2.02 & .188 & 10.74 \\
100 & 1.81 & .177 & 10.22 \\
$100^{\mathrm{b}}$ & 1.82 & .188 & 9.68
\end{tabular}

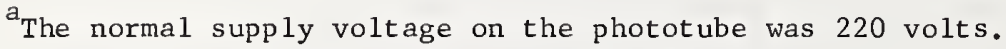

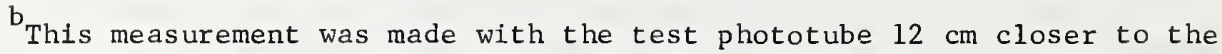
$\mathrm{MgCO}_{3}$ block than the previous ones. The inverse-square law has been applied to put this measurement on the same basis as the others.

In order to check for possible polarization effects, a linear polarizer was placed in front of one of the two receivers. No change in the measured values was observed as the plane of polarization was rotated.

Several experiments were run to check on the stability and suitability of $\mathrm{MgCO}_{3}$ for the diffusing surface. The reflectance factor, $\beta$ of Equation 1, was remeasured one year after the original measurement. The measurements agreed to within $1 / 2 \%$, the precision of the gonioreflectometer. A converging lens was introduced into the laser beam in order to focus the beam on the $\mathrm{MgCO}_{3}$ block. Although the energy density was concentrated by a factor of $10^{3}$, no effects were observed either in the measured energy or in the physical condition of the block. Finally the usual measuring angle of $20^{\circ}$ was varied and the phototube responses noted. Although the angle was varied from $15^{\circ}$ to $45^{\circ}$ no anamalous effects were observed.

\section{The rmopile Calibration}

A block circuit diagram of the thermopile measuring circuit is shown in Figure 5. The circuit is one that allows for calibration against an external emf and thus obviates the necessity of relying on amplifier or recorder stability. The output of the thermopile is stated in terms of the microvolts developed at the amplifier input terminals.

For a thermopile, $R(\lambda)$ in Equation $2 b$ is 1.00 and hence no significant error is introduced by the relative spectral sensitivity parameter. However, in contrast with the PJ14B phototubes whose spectral sensitivity is negligible beyond one micron, thermopiles respond to energy far into the infrared. Since the spectral irradiance values, $E_{\lambda}$, of the standard lamps become uncertain beyond 2.5 microns, it is necessary to introduce a filter to absorb long-wavelength energy. A "heat-absorbing" glass with a transmittance of $1 \%$ or less for wavelengths beyond $1.1 \mathrm{microns}$ was used. However, even when the filter is used, $16 \%$ of the transmitted energy from the standard lamps is contained in the region 
beyond 1.1 microns.

With the modification that $R(\lambda)=1.00$ the calibration procedure described for the phototubes is applicable. When this was tried experimentally, difficulties were encountered. The lamp energies available during calibration were such that exposure times in the range $1 / 2$ to 3 seconds were required to keep the thermopile response far enough above the noise level for accurate measurements. Experiments showed that the thermopile had a $1 /$ e time constant of about 3 seconds. The amplifier-recorder system also had a response time on the order of seconds. The effects due to the thermopile and those due to the measuring system proved inseparable and new equipment having a faster response time is being obtained. It is expected that when this equipment is put in operation thermopile measurements of the laser energy will be possible.

Although accurate calibration of a thermoplle is uncertain at this time, the short time duration of the laser pulse allows self-consistent measurements. This experiment was performed by placing a phototube at one of the receiver positions shown in Figure 1 and the thermopile at the other one. The ratio of their readings should be constant. Experimentally the standard deviation of a single measurement was $2.8 \%$. A similar experiment performed with two phototubes gave a standard deviation of a single measurement of $0.77 \%$. This internal consistency of thermopile energy measurements indicates that future work with the thermopile will be profitable.

\section{Comparison with Calorimeter}

A check of the accuracy of the calibrations was made by making a limited comparison of results with a calorimeter built at the NBS Boulder Laboratory. [3] Since the calorimeter accepts the entire laser beam, an auxiliary monitoring system is required. Figure 6 shows the arrangement employed. The monitor was first calibrated relative to the phototubes by removing the calorimeter and allowing the laser beam to impinge on the $\mathrm{MgCO}_{3}$ block as in Figure 1. The calorimeter was then put in place and a transfer comparison obtained. In placing the calorimeter, care was taken not to align it with its entrance window perpendicular to the incoming laser beam. The calorimeter was canted with respect to the laser beam enough to insure that the energy reflected from the entrance windows did not proceed back along the beam track to the laser head. A simple calculation (assuming $16 \%$ reflectance from the calorimeter windows, $90 \%$ transmittance and $8 \%$ surface reflectance at the beam splitter) shows that an additional energy increment amounting to $12 \%$ of the calibrating energy can reach the monitor if this procedure is not observed.

When the transfer comparison between the calorimeter and the phototubes was carried out, a discrepancy of about $9 \%$ was observed. The calorimeter indicated lower energies than the phototubes.

\section{Discussion of Possible Sources of Error}

In view of the discrepancies observed between data on laser energies obtained with the phototubes and with the calorimeter, consideration has been given to possible systematic arrors in the calibration of the photoelectric receivers.

The estimated uncertainty of the $E_{\lambda}$ values is $3 \%$ in the spectral region of interest, 650 to $750 \mathrm{~nm}$. Since the laser wavelength, $694.3 \mathrm{~nm}$, lies in the visible region a check on the irradiance values of the standard lamps was possible through a measurement of luminous intensity and color temperature. [4] The irradiance values thus obtained checked those obtained directly within $1 / 2 \%$ and $3 \%$ for the two lamps.

The transmittance values, $T(\lambda)$, of the interference filters used to approximate the monochromaticity of the laser beam were measured at NBS on a Cary 14 recording spectrophotometer. Since filters having narrow pass bands centering approximately on the laser wavelength were used, the calibration procedure is spectrally a substitution method. The narrower of the two interference filters employed has a half-band width of $1.1 \mathrm{~nm}$. The spectrophotometer has a maximum resolution of $0.2 \mathrm{~nm}$ and thus will not completely resolve this filter spectrally. Instead the readout will be an average transmittance value for a $0.2-\mathrm{nm}$ bandwidth. 


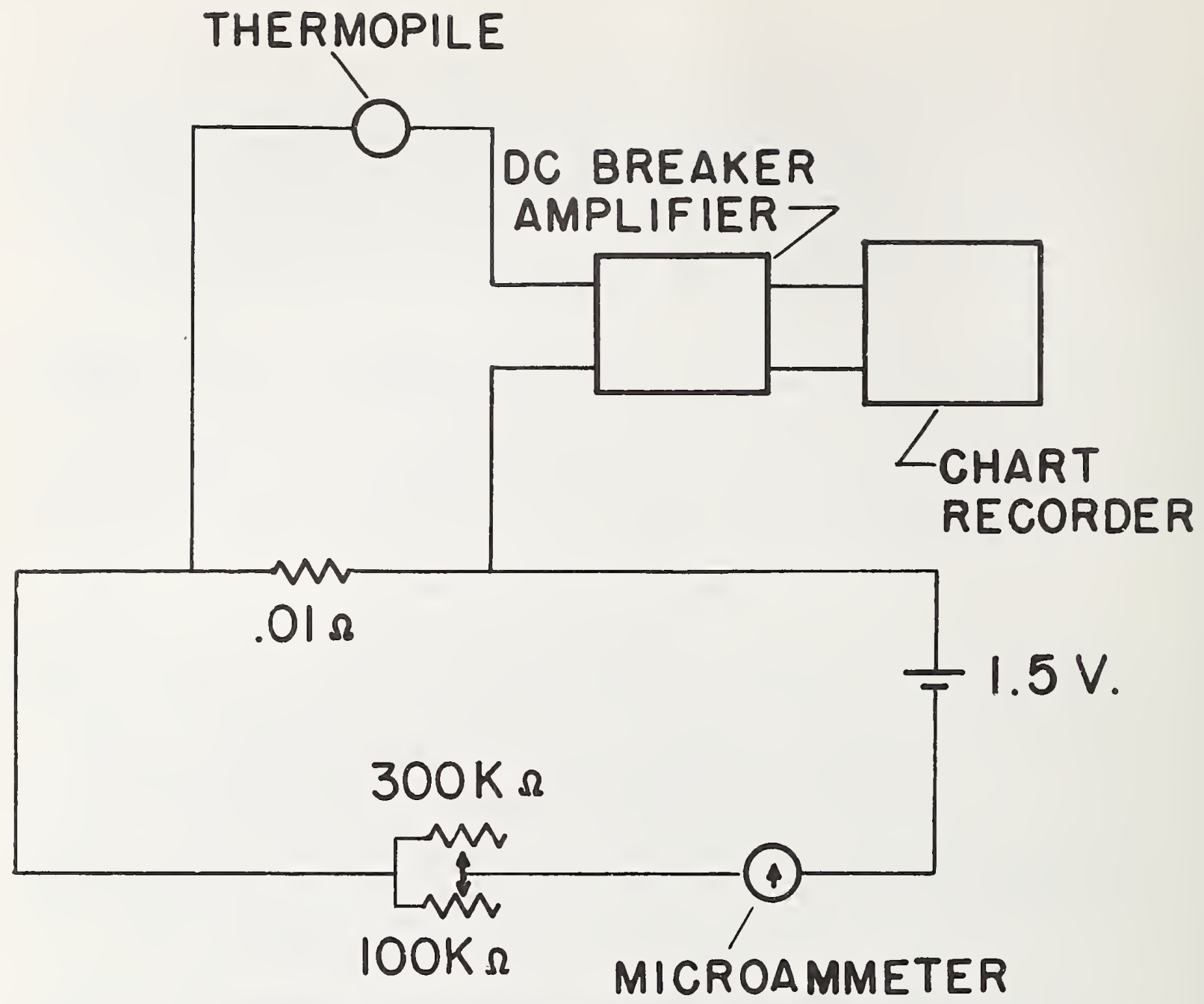

Figure 5. Block Circuit Diagram of the Thermopile Measuring Circuit

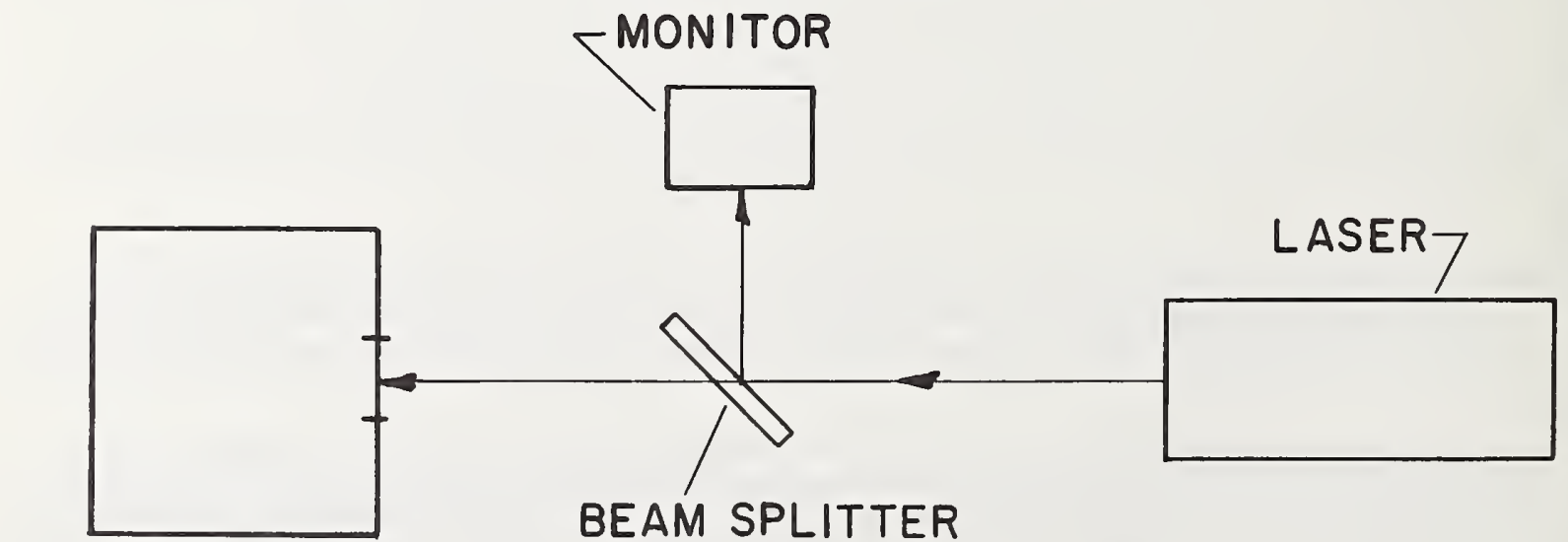

CALORIMETER

Figure 6. Experimental Setup for Measuring Laser Energy with a Calorimeter 
Although the measured spectral transmittance values at any given wavelength will be in error, the area under the transmission curve should be constant for a wide range of resolutions. Further, since $E_{\lambda}$ and $R(\lambda)$ are slowly varying functions across the pass band of the filter, the energy reaching the phototube as computed in the integral in Equation $2 \mathrm{~b}$ should be very nearly correct because of the self-compensating nature of this error in the $\tau(\lambda)$ values. Hickey's experiments confirm this directly. [5]

The relative spectral response, $R(\lambda)$, of the phototube-diffusing-glass combinations was measured on a detector-spectrum recorder. This instrument consists of an electronically-controlled incandescent lamp which, together with a single-grating monochromator, gives a constant spectral radiant flux with a band width of approximately $10 \mathrm{~nm}$. The instrument is similar to the one described by Mitsuhashi and Nakayama. [6] Measurements made on this instrument are believed to be uncertain by no more than $\pm 5 \%$ for the slope of the $R(\lambda)$ curve in the spectral region of interest. Note that since the laser wavelength lies within the narrow band passed by the interference filter it is the slope of $R(\lambda)$ within the pass band of the interference filter that affects the accuracy of the calibration rather than the values of $R(\lambda)$.

The narrow pass band interference filters were used to insure that the shape of the $\tau(\lambda)$ curve would be the dominant factor in the integral in Equation $2 b$ since $T(\lambda)$ can be measured with higher accuracy than $R(\lambda)$ or $E_{\lambda}$. Both the $E_{\lambda}$ and the $R(\lambda)$ functions are slowly varying in the narrow spectral region of the pass band of the interference filters. Analytic studies show the dominance of $T(\lambda)$ over $E_{\lambda}$ and $R(\lambda)$ on the calibration accuracy. For example, a $5 \%$ shift in the slope of the $R(\lambda)$ curve makes only a $1 / 2 \%$ change in the value of the integral.

Systematic errors in $\tau(\lambda), R(\lambda)$ and the exposure times would have been detected immediately in the course of the analysis of the factorial calibration experiment. Systematic errors in the reflectance factor, $\beta$ (Equation 1), or the irradiance, $E_{\lambda}$ (Equation 2b) could still be present and would not necessarily have been so detected. As indicated above, experience and direct measurement indicate that $E_{\lambda}$ is known to within $3 \%$. Past experience Indicates that reflectance factor measurement $\hat{s}$ should be in error by no more than 1\%. As indicated previously interreflections can be a source of error. However, the magnitude of the residual interreflections, after correction for the positional effect, is believed to be much too small to account for the difference between the phototube and calorimeter calibrations. Lack of experimental precision is not likely to be the cause. The 3-sigma limit for the agreement of two independent and simultaneous measurements of laser output based on the difference column of Table II is 4.5\%. Since the average of 12 sets of 3 measurements each with the photoelectric receivers was used in the comparison with the calorimeter, the 3-sigma limit for the mean of the photoelectric measurements is insignificant in comparison to the possible systematic errors. Thus the discrepancy between the phototubes and calorimeter is difficult to explain.

\section{Conclusions}

The output of low energy, ruby lasers has been measured using photoelectric radiometers. Attenuation of the laser beam was accomplished by intercepting the entire laser beam on a diffusely reflecting surface and viewing this surface from a distance with the photoelectric receiver. Measurements made with different photoelectric receivers agree to about 1.5\%. Total uncertainty is estimated to be about $5 \%$. Measurements made using this radiometric method have been compared with calorimetric methods and a discrepancy of $9 \%$ was observed.

Future plans call for continued research to eliminate the discrepancy between the calorimeter and phototube measuring systems. Comparisons with a thermopile calibration and measuring system are also planned. The results reported above have been obtained with a relatively low-powered, conventional ruby laser. Present plans call for applying these measurement techniques to lasers of higher energy, to Q-switched lasers, and to lasers operating at wavelengths other than $694.3 \mathrm{~nm}$. Once agreement is established among the various measuring systems, transfer calibrations simflar to the one described for the monitor used in the calorimeter work can be used to calibrate any number of additional measuring systems. 
The authors wish to express their thanks to Mr. R. P. Teele, [7] whose preliminary studies led to the present work, and to Mr. Hsien Ku of the NBS Statistical Engineering Section for his help in analyzing the results of the calibration and laser-measuring experiments. In addition, the authors wish to thank their other colleagues, too numerous to mention individually, for their valuable assistance.

\section{References}

[1] R. Stair, W. E. Schneider, W. R. Waters, and J. K. Jackson, Some Factors Affecting the Sensitivity and Spectral Response of Thermoelectric (Radiometric) Detectors, Applied Optics, Vol. 4, pp. 703-710 (June 1965).

[2] R. Stair, W. E. Schneider, W. R. Waters, J. K. Jackson, and R. E. Brown, Some Developments in Improved Methods for the Measurement of the Spectral Irradiances of Solar Simulators, NASA CR-201.

[3] D. A. Jennings, Calorimetric Measurement of Pulsed Laser Output Energy, IEEE Transactions on Instrumentation and Measurement, Vol. IM-15, No. 4, Pp. 161-164 (Dec. 1966).

[4] L. E. Barbrow, Memorandum on a Procedure for Obtaining Spectral Radiant Intensities of Tungsten-Filament Lamps, 400-700 mu, Journal of the Optical Society of America, Vol. 49, p. 112? (Nov. 1959).

[5] J. R. Hickey, Correlation of Monochromator and Filter Radiometry Determinations of the Spectral Distribution in Large Solar Simulators (The Eppley Laboratory, Inc., Reprint Series No. 26), Institute of Environmental Sciences and American Society for Testing and Materials International Symposium on Solar Radiation Proceedings, pp. 95-105.

[6] H. Mitsuhashi and T. Nakayama, Apparatus for the Study of Spectral Response of Photoelectric Surface, Journal of the Physical Society of Japan, Vol. 11, Pp. 308-311 (March 1956).

[7] R. P. Teele, Measurement of Pulsed Laser Output, presented at the 1965 Spring Meetings, Optical Society of America, Dallas, Texas, Paper TE12, 31 March - 2 Apri1 1965. 


\title{
Effects upon Radiant Intensity Measurements Due to Scattering by Optical Elements
}

W. H. Venable, Jr.

\begin{abstract}
Measurements to determine the radiance of one portion of a nonuniform source can be strongly influenced by radiation from other portions of the source scattered by the optical system used to transmit the radiation to the detector. Conditions under which such scattering is important and procedures to correct for it are discussed. An illustration is provided from measurements made on a nitrogen arc source.
\end{abstract}

\section{The Scattering Problem}

The object of the experiments with which this paper is concerned is to obtain a spatial mapping of the radiance of a source of radiation as it is observed by a given detector. For many sources of radiation, conditions in the source such as high temperature, strong electric field, or pressure differences make it impractical to mask the source itself in order to make measurements of the radiation from a small area. Instead, an optical system is used to produce an easily maskable image of the source. Ideally, a mapping of the irradiation of the image would be proportional to a mapping of the radiance of the source. In this paper, such an ideal mapping will be denoted by $T$ and called the actual radiance.

In reality, some of the transmitted radiation from a given small portion of the source fails to fall on the corresponding portion of the image but instead, because of scattering and diffraction in the imaging optics, is spread out over the rest of the image. Likewise, some radiation from outside that portion of the source which one wishes to observe falls into the observed portion of the image. The net result is that the actual radiance is smoothed-brightened in the dim portions and dimmed in the bright portions. This smoothed mapping will be denoted by $F$ and called the measured radiance.

The actual radiance can be related to the measured radiance through a function which will be denoted by $S$ and which will be called the scattering function. This function relates $F$ to $T$ by means of an integral equation of the form,

The author is with the National Bureau of Standards, Washington, D.C. 20234.

Received 1 August 1969.

$$
F(r)=\int^{\infty} S\left(\mathbf{r}, \mathbf{r}^{\prime}\right) T\left(\mathbf{r}^{\prime}\right) d A\left(\mathbf{r}^{\prime}\right)
$$

where $\mathbf{r}$ and $\mathbf{r}^{\prime}$ each represent position in the image plane, and the integral with respect to the primed coordinates is taken over an infinite range of area. The scattering function $S\left(\mathbf{r}, \mathbf{r}^{\prime}\right)$ can be thought of as the measured radiance at the location $\mathbf{r}$ which would be produced by a unit point source, ${ }^{*}$ the image of which would appear at $\mathbf{r}^{\prime}$ in the absence of scattering. For this discussion, $S$ will be normalized so that

$$
\int^{\infty} S\left(\mathbf{r}, \mathbf{r}^{\prime}\right) d A(\mathbf{r})=1 .
$$

From this normalization and Eq. (1) it follows that

$$
\int^{\infty} F^{\prime}(\mathbf{r}) d A(\mathbf{r})=\int^{\infty} T\left(\mathbf{r}^{\prime}\right) d A\left(\mathbf{r}^{\prime}\right) .
$$

This normalization, although quite arbitrary, is consistent with the notion that the introduction of scattering changes the pattern of irradiation in the image but does not affect the total irradiation of the image plane.

Solving Eq. (1) for the $F$ corresponding to a particular $T$ can be greatly simplified by taking advantage of symmetries usually found in $S$. For example, in cases in which the scattering is due mainly to random irregularities in the surfaces of the optical components it often follows that

$$
S\left(\mathbf{r}, \mathbf{r}^{\prime}\right)=S\left(\left|\mathbf{r}-\mathbf{r}^{\prime}\right|\right) .
$$

In another large class of measurements, the radiance of the source is essentially uniform in one direction. To map such a source, one could arrange an observation slit

\footnotetext{
$* T\left(\mathbf{r}^{\prime}\right)=\delta\left(\mathbf{r}-\mathbf{r}^{\prime}\right)$, a Dirac delta function.
} 


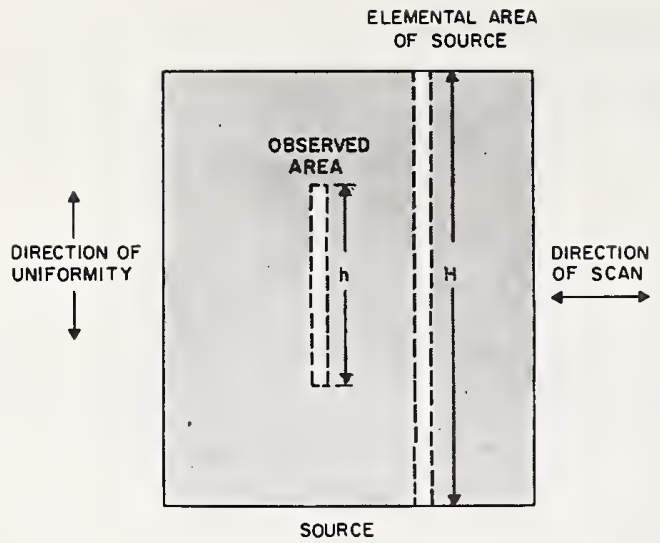

Fig. 1. A schematic illustration of a uniform source of length $H$ being scanned by a slit detector of length $h$.

parallel to the direction of uniformity and move the slit and source relative to one another to scan the source normal to the direction of uniformity as shown in Fig. 1. Thus, the only dimension involved explicitly in the functions $T, F$, and $S$ will be distance in the direction in the image plane of scan (denoted by $x$ ). If, in addition, the scattering function is the same for all $x^{\prime}$, one can rewrite Eq. (1) in the form,

$$
F(x)=\int_{-\infty}^{\infty} S\left(x^{\prime}-x\right) T\left(x^{\prime}\right) d x^{\prime}
$$

In the remainder of this paper, the discussion is confined to a one-dimensional scattering problem as described in the foregoing discussion, although the techniques used in determining a scattering function and solving for an actual radiance can be readily extended to a twodimensional case in which the scattering function remains the same over the portion of the image plane in which there is significant irradiation, e.g., the case described in the discussion leading up to Eq. (4).

The relationship between a particular set of functions $F, S$, and $T$ is illustrated in Fig. 2. A trapezoidal $T(x)$ (such as would be expected when a uniform ribbon source is scanned with a uniform detector masked with a slit) and the normalized scattering function,

$$
S\left(x^{\prime}-x\right)=\left\{\pi\left[1+\left(x^{\prime}-x\right)\right]^{2}\right\}^{-1},
$$

were used in Eq. (5) to calculate the corresponding $F(x)$. Notice that the measured radiance at the center of this source is less than the actual radiance. For a given optical system, this difference is a larger fraction of the actual radiance for narrower sources and, therefore, errors can result when one compares the radiance of uniform sources of different sizes by viewing only their centers. If the direct comparison of the radiance of several different uniform sources is the only consideration, a size-of-source correction such as that discussed by $L_{e}{ }^{1}$ can be made. If, on the other hand, one is interested in measuring the relative radiance of different parts of a nonuniform source, it is necessary to deter- mine the scattering function $S$ under the conditions of the measurement and then to solve an integral equation for the $T$ corresponding to each $F$.

It is apparent from Eq. (5) that determining the scattering function [solving for $S\left(x^{\prime}-x\right)$ with given $T\left(x^{\prime}\right)$ and $F(x)$ ] and determining the actual radiance [solving for $T\left(x^{\prime}\right)$ with given $S\left(x^{\prime}-x\right)$ and $F(x)$ ] are the same mathematical problem, for which methods of solution by transformation theory ${ }^{2-4}$ or series solution ${ }^{5,6}$ appear in the literature. However, the nature of the function involved and the presence of experimental error in the data appear to call for a different approach to the solution for each of the two cases. A practical approach to solving the problem in each case is presented in Secs. II and III.

\section{Determining the Scattering Function}

In the one-dimensional case illustrated in Fig. 1, the scattering function would be the measured radiance from a uniform strip source of length $H$ and infinitesimal width as seen by a uniform strip detector of length $h$ and infinitesimal width. Although much work has been done on scattering from surfaces and films, ${ }^{7,8}$ it is usually not practical to determine the parameters by which the scattering function can be predicted for even a rather simple optical system. Therefore, the $F(x)$ corresponding to a known $T\left(x^{\prime}\right)$ must be obtained experimentally under conditions as close as possible as those encountered in the measurement to be corrected,* and

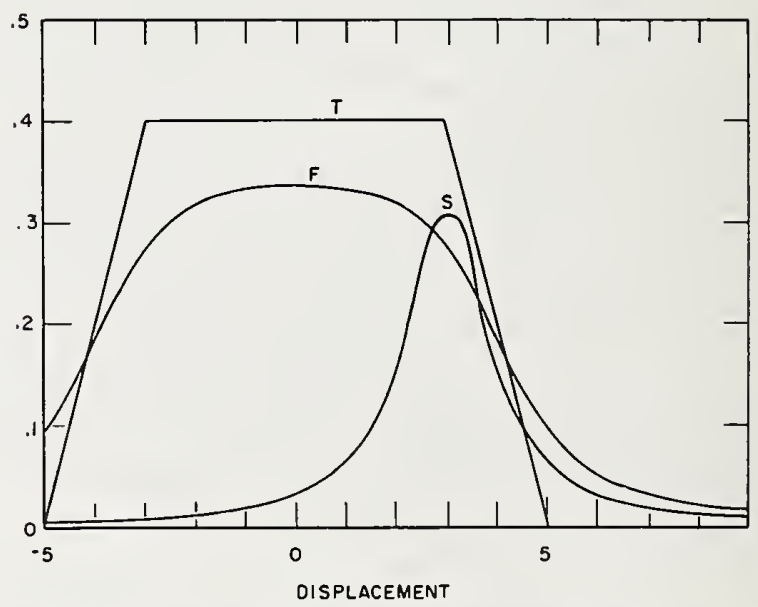

Fig. 2. An illustration of the distorted mapping $F$ of a radiance profile $T$ produced by an instrument with scattering function $S$. The $F$ and $T$ functions are drawn to the same arbitrary scale. The vertical scale is numbered to indicate the values of the normalized $S$ function.

* Since there is also scattering in a direction normal to the direction of scan, $S\left(x^{\prime}-x\right)$ is an implicit function of $H$ and $h$. Therefore, these should remain the same as in the measurement to be corrected. 
these functions can then be used in Eq. (5) to determine $S\left(x^{\prime}-x\right)$.

In many cases a slit of length $H$ arranged parallel to the direction of source uniformity and illuminated from behind by a uniform source can be placed in the source position with the measuring system set up as it is to be used. If the illuminating source is broad enough and close enough to the slit, so that the edges of it are not visible along any path which light passing through the optical system could take, such a slit would resemble a uniform element of source as shown in Fig. 1 and would be subject to the same scattering of its light. From the geometry of the situation, one can conclude that $T\left(x^{\prime}\right)$ is a trapezoid with major base equal to the sum of the source and observation slit widths and minor base equal to the absolute value of their difference. (If the magnification of the optical system is not unity, the width of the projection of the observation slit into the plane of the source should be used in place of the actual width of the observation slit.) The height of the $T\left(x^{\prime}\right)$ trapezoid is adjusted with regard to the experimental $F(x)$ data so that

$$
\int_{-\infty}^{\infty} T\left(x^{\prime}\right) d x^{\prime}=\int_{-\infty}^{\infty} F(x) d x .
$$

With $T\left(x^{\prime}\right)$ so chosen, the resulting scattering function will satisfy the normalization condition:

$$
\int_{-\infty}^{\infty} S\left(x^{\prime}-x\right) d x=1
$$

Once the corresponding $F(x)$ and $T\left(x^{\prime}\right)$ have been obtained, Eq. (5) can be solved for $S\left(x^{\prime}-x\right)$. In the present work, an iterative solution is used which takes advantage of the fact that the domain of $T\left(x^{\prime}\right)$ is finite, and that far outside the region in which $T\left(x^{\prime}\right)$ is nonzero, the entire actual radiance appears as a slit source so that the measured radiance and the scattering function are proportional. Therefore, the measured radiance is normalized and used as a zeroth approximation to the scattering function. For each value of $x$, beginning in the region far from where $T\left(x^{\prime}\right)$ is nonzero and working toward the region of large $T\left(x^{\prime}\right)$, one then uses the approximate scattering function in Eq. (5) to solve for $F(x)$, compares this value to the experimentally obtained value of $F(x)$, and adjusts the approximate scattering function to bring the calculated $F(x)$ into closer agreement with the experimental one. Details of this calculation are given in Appendix A of this paper.

Figure 3 illustrates this calculation as applied to the $T\left(x^{\prime}\right)$ of Fig. 2 and the $F(x)$ which was obtained from it analytically. By the seventh iteration, the original, known $S\left(x^{\prime}-x\right)$ was reproduced to within $5 \%$ at every point.

\section{Solving for the Actual Radiance}

Once the scattering function has been obtained, Eq. (5) can be solved for the unknown actual radiance corresponding to a given measured radiance. The series solution to be presented in this section is designed to take advantage of the fact that much of the light is scat-

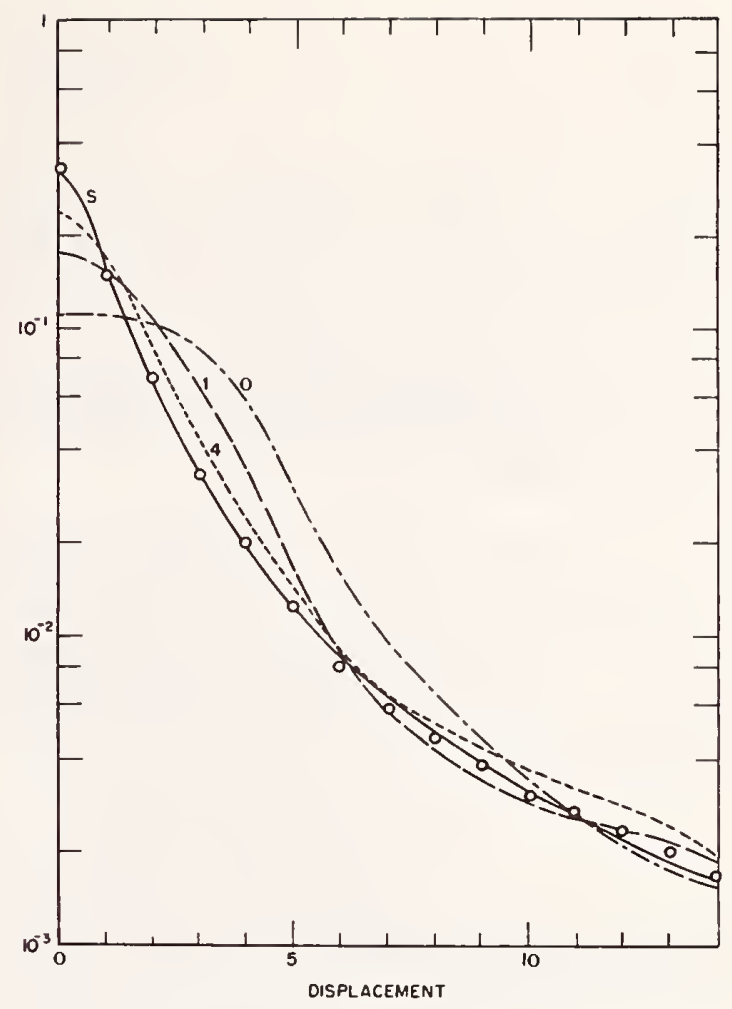

Fig. 3. An illustration of the convergence toward a known solution for $S$. The $O$ th approximation is $F$, scaled to a unity integral. Curves 1 and 4 represent the solution after 1 and 4 iterations, respectively, and the circles indicate values taken from the solution after 7 iterations. The vertical scale indicates the value of the normalized functions.

tered only slightly, i.e., $S\left(x^{\prime}-x\right)$ is large only in a small part of its domain surrounding $x^{\prime}-x=0$. It is obtained in a manner analogous to solving for $B$ in the algebraic equation

$$
A=B D+B d \quad(D>d)
$$

by substitution. One can rearrange Eq. (8) as

$$
B=A / D-B d / D
$$

and by $N$ repeated substitutions of the entire right side of Eq. (9) for $B$ in the right side of an expression obtained by beginning with Eq. (9), one can obtain the series

$$
B=\sum_{i=0}^{N}(A / D)(-d / D)^{i}+B(-d / D)^{N+1} .
$$

For $d<D$ and large enough $N$, the final term can be dropped to yield a series of the form

$B=A / D-(d / D)(A / D)$

$$
-(d / D)[-(d / D)(A / D)]-\ldots .
$$

To apply this procedure to solve Eq. (5) for $T(x)$, one replaces the portion of the integral in the region near $x^{\prime}-x=0$ with the aoproximate value $s T(x)$ defined by 


$$
\int_{x-\epsilon}^{x+\epsilon} S\left(x^{\prime}-x\right) T\left(x^{\prime}\right) d x^{\prime} \simeq\left[\int_{-\epsilon}^{\epsilon} S(z) d z\right] T(x) \equiv s T(x) .
$$

This approximation is exact for linear $T\left(x^{\prime}\right)$ and symmetric $S\left(x^{\prime}-x\right)$, and is very good when the slope of $T\left(x^{\prime}\right)$ varies only slightly in the distance $2 \epsilon$. This latter condition is realized in the large class of measurements in which the resolution is governed by the width of the observation slit rather than by the width of the centra] portion of the scattering function. ${ }^{*}$ With this approximation, the algebraic analogy is followed, in correspondingly numbered steps, by first separating the integral in Eq. (5) into two portions as

$$
F(x)=\int_{x-\epsilon}^{x+\epsilon} S\left(x^{\prime}-x\right) T\left(x^{\prime}\right) d x^{\prime}+\int_{-\infty}^{\infty} S\left(x^{\prime}-x\right) T\left(x^{\prime}\right) d x^{\prime}
$$

where the prime before the integral indicates that the region $x-\epsilon<x^{\prime}<x+\epsilon$ is excluded from the range of integration. Using the approximation in Eq. (11) this can be rewritten as

$$
T(x)=F(x) / s-(1 / s)^{\prime} \int_{-\infty}^{\infty} S\left(x^{\prime}-x\right) T\left(x^{\prime}\right) d x^{\prime} .
$$

By repeated substitution of the entire right side of Eq. (13) into the right side of an equation obtained by starting with Eq. (13), one obtains

$$
\begin{array}{r}
T(x)=F(x) / s-(1 / s)^{\prime} \int_{-\infty}^{\infty} S\left(x^{\prime}-x\right)\left[F^{\prime}\left(x^{\prime}\right) / s\right] d x^{\prime} \\
-(1 / s)^{\prime} \int_{-\infty}^{\infty} S\left(x^{\prime \prime}-x\right)\left\{-(1 / s)^{\prime} \int_{-\infty}^{\infty} S\left(x^{\prime}\right.\right. \\
\left.\left.\quad-x^{\prime \prime}\right)\left[F\left(x^{\prime}\right) / s\right] d x^{\prime}\right\} d x^{\prime \prime} \ldots .
\end{array}
$$

For a bounded $F(x)$, a sufficient condition for convergence is $s>\frac{1}{2}$. The choice of $s$ involves a compromise between obtaining more rapid convergence with a larger $s$ and higher resolution with a smaller $s$. In the work from which the example at the end of this paper was taken, $s=\frac{4}{5}$ was found to be a practical compromise. $\dagger$ From the alternating nature of the series in Eq. (14) it can be shown that in the solution for $T(x)$ at any point the fractional error introduced by the approximation of Eq. (11) will be less than the largest fractional error in

\footnotetext{
* It should be emphasized at this point that the need for a scattering correction of this type arises because the total amount of radiation scattered out of a given region and the amount of light scattered into that region from the entire remaining part of the source are relatively great. The procedure described in this paper is not intended to be a correction for the short-range loss of resolution due to the width of the central portion of the scattering
} function.

$\dagger$ Since $S\left(x^{\prime}-x\right)$ is not easily evaluated near $x^{\prime}-x=0$, it is usually more practical to determine $\epsilon$ so that

$$
\int_{-\infty}^{-\epsilon} S(z) d z+\int_{\epsilon}^{\infty} S(z) d z=1-s .
$$

this approximation and that the fractional error introduced by experimental errors in the $F(x)$ data will be less than the greatest fractional error in this data. Since the function in braces in each integral of Eq. (14) is simply the entire preceding term, this series is readily evaluated on a computer. Details of the solution appear in Appendix B.

Figure 4 illustrates the result of applying this calculation to the analytic $S\left(x^{\prime}-x\right)$ and calculated $F(x)$ of Fig. 2. If computer error is negligible, the infinite series solution will be bracketed between solutions obtained by truncating the series at consecutive terms. Thus, in the example, the infinite series solution will lie in the shaded region bounded by the seven- and eight-term solutions. In two ways, this illustrative example demands more of the method of solution than experimental situations normally would. First, the $F(x)$ was derived from a $T(x)$ which was arbitrarily defined in a way which could only be realized with perfect resolution. Therefore the resolution of the solution reached is characteristic of the $\epsilon$ chosen, which in this case was 1.25 , and the sharp corners are rounded. In many cases $T\left(x^{\prime}\right)$ would be smoothed already by an instrument function determined, for example, by slits which are wider than the $\epsilon$ used in the scattering solution. Second, in this example $s$ was deliberately chosen small at 0.573 in order that the convergence be slow enough to be easily illustrated.

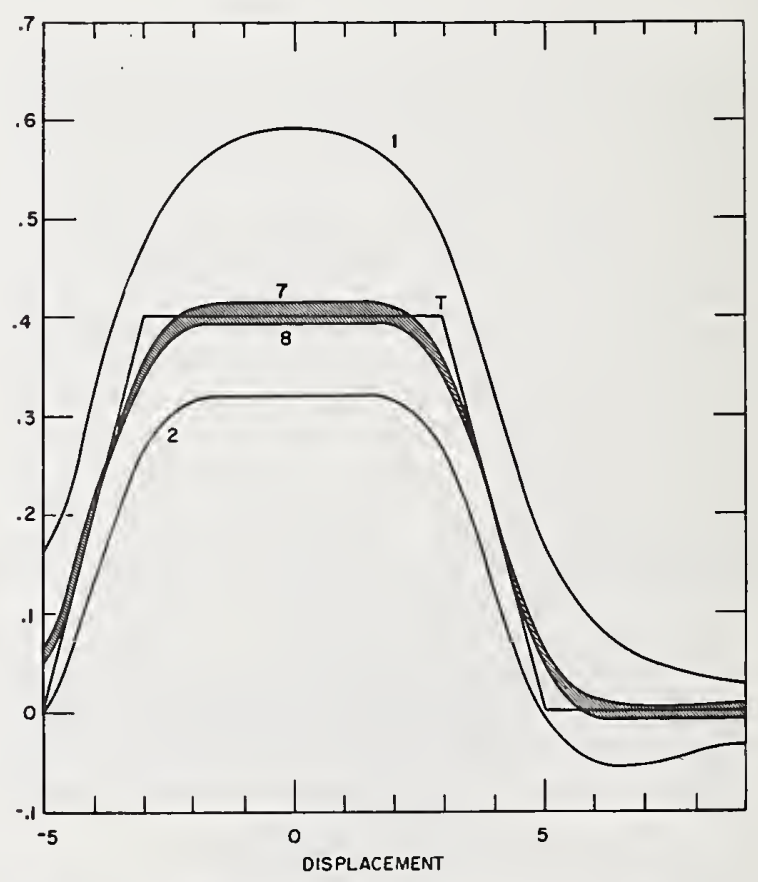

Fig. 4. An illustration of convergence toward a solution for $T$. Each curve is numbered according to the number of terms in the series of Eq. (11). The solution with an infinite number of terms would fall within the shaded region. The functions are plotted to the same arbitrary scale as that of $F$ and $T$ in Fig. 2. 


\section{Application to Experimental Data}

The results illustrated in Figs. 2, 3, and 4 were obtained from mathematically contrived functions $F(x)$, $T\left(x^{\prime}\right)$, and $S\left(x^{\prime}-x\right)$. In a final illustration, the results of applying these solutions to experimental data obtained in observing a nitrogen $\operatorname{arc}^{9}$ will be shown.

The optical system which was used is shown in Fig. 5 . A slit, illuminated from behind by a mercury lamp, was used as a known source in determining $S\left(x^{\prime}-x\right)$. The resulting functions for two different wavelengths are shown in Fig. 6. It can be seen that the wings of the $S\left(x^{\prime}-x\right)$ approximate an inverse square function in each case, a rather common occurrence which has been noted by Eaglesfield. ${ }^{10,11}$ From measurements made in the 300-600-nm region, a suitable expression for $S\left(x^{\prime}-\right.$ $x$ ) for use in the primed integral in Eq. (14) was found to be

$$
S\left(x^{\prime}-x\right)=1.49\left(x^{\prime}-x\right)^{-2} \lambda^{-0.89} \text { per } \mathrm{mm},
$$

where $\lambda$ is the wavelength in nanometers and the displacement $x^{\prime}-x$ is in millimeters.

The experimental data to be corrected were taken from side-on radiance observations of a cylindrical are, i.e., the observation slit was parallel to the are axis and the direction of scan and the optic axis were perpendicular to the arc axis. The intensity of an atomic line was to be compared to the intensity of a group of lines from a molecular band in the arc. In general, the atomic lines were most intense near the center of the arc while the molecular bands were most intense near the edge of the arc, and it was suspected that scattering might distort the comparison. The measured radiance values from the two features were each corrected for scattering, and the resulting intensity profiles* with and without correction for scattering are shown in Fig. 7. The importance of the correction in this case is obvious.

This paper is a Contribution of the National Bureau of Standards, not subject to copyright.

\section{Appendix A}

Computer programs for the two solutions described in this paper are presented here as an aid for those who wish to understand the computations in detail. These programs are written in the $\mathrm{BASIC}^{13}$ language and, in order to keep them simple, they are written for the case of symmetric functions $S, T$, and $F$ in one dimension for which the data are taken at equal intervals. Once the procedure is understood, extension of the program to handle nonsymmetric functions, two dimensions, and unequal spacing of datum points is straightforward. Although the programs listed can be used in the form

* In this particular case intensity (power per unit volume) as a function of radius was the quantity of interest, so that an inverse Abel transformation ${ }^{12}$ was performed on the corrected radiance (power per unit area) as a function of scan displacement to obtain the results shown in Fig. 7.

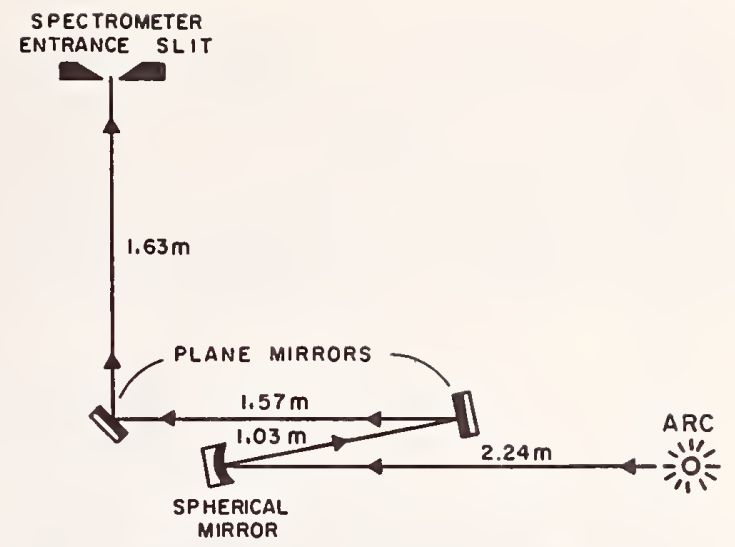

Fig. 5. A schematic representation of the optical system used for measuring arc profiles. All mirrors are front surface.

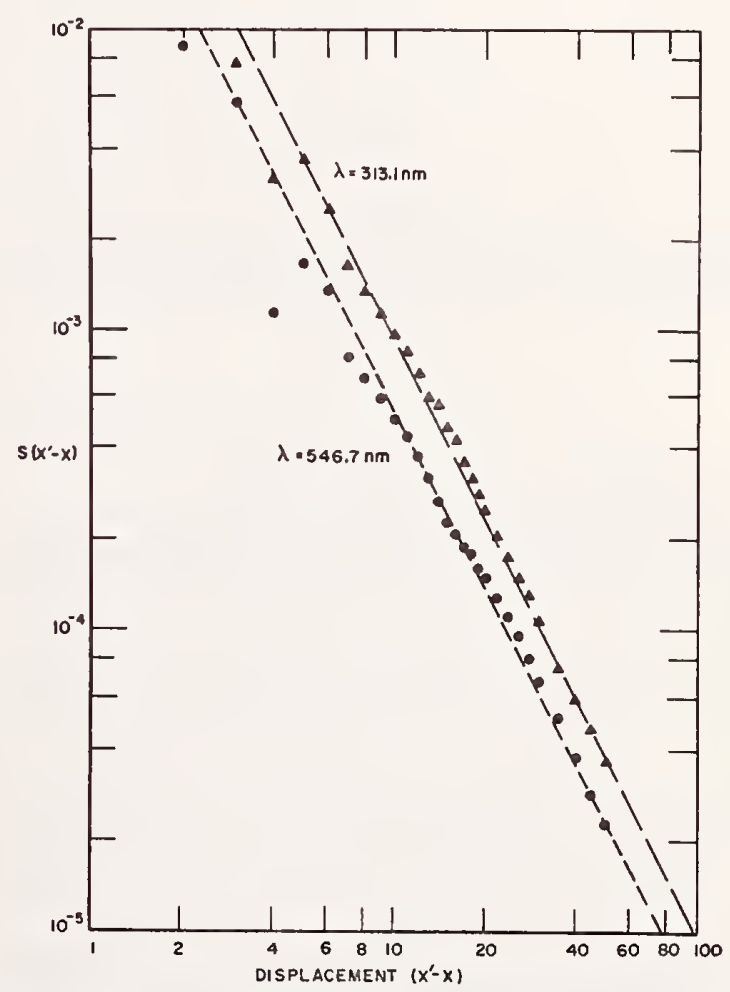

Fig. 6. The scattering function per unit displacement for the optical system illustrated in Fig. 5 as obtained at two different wavelengths. The dashed lines represent a best fit inverse square function. (1 displacement unit $=95 \mu \mathrm{m}$ ).

given, they are designed for ease of understanding rather than for efficient use of the computer. Therefore, they should be modified before being applied to extensive problems. 


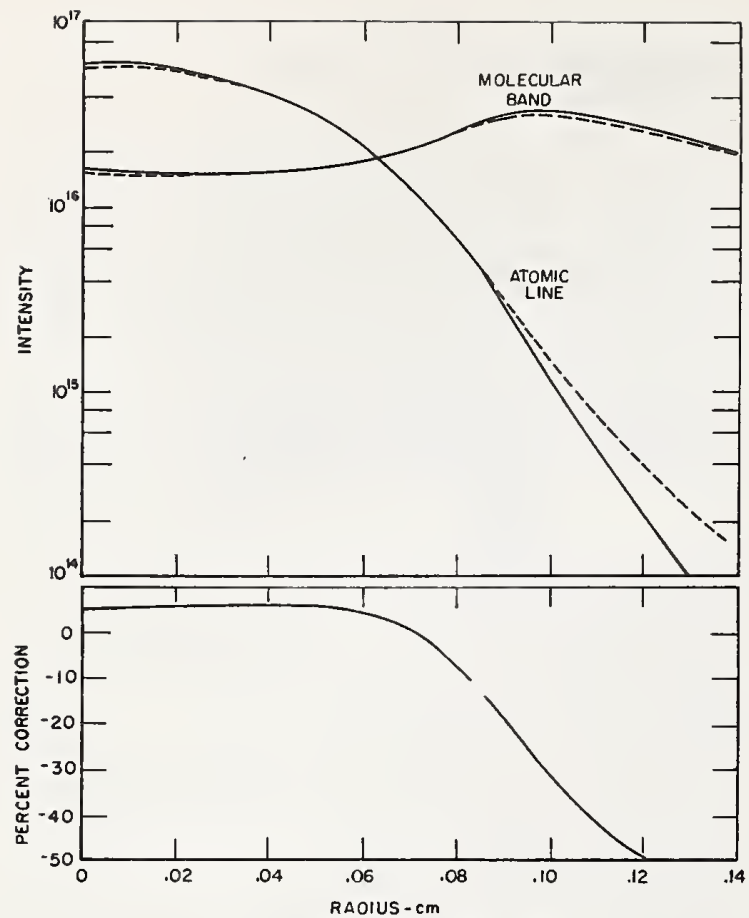

Fig. 7. The effect of scattering upon the observed profile of a 1atm nitrogen are for an atomic line and a molecular band. Where the scale permits, the uncorrected profile is shown as a dotted line. The lower curve indicates the percentage error in the ratio of the band intensity to the line intensity caused by scattering.

A program for carrying out the type of calculations described in Sec. II is presented in Fig. 8.

Several parameters are read into the program in step 200. The distance between experimental datum points is $W$ meters. An acceptable solution for $S\left(x^{\prime}-x\right)$ will be one for which the $F(x)$ determined by using that solution and the known $T\left(x^{\prime}\right)$ an Eq. (5) agrees with the experimental data to within a fractional error $E$ at each point. There are $N+1$ datum points from the center of $T\left(x^{\prime}\right)$ to the edge of its nonzero range and $N+1$ experimental datum points taken from the center out for $F(x)$. If an acceptable solution is not reached in $L$ iterations, the computation will be concluded anyhow. In steps 300-310, the actual intensity function is scaled to satisfy Eq. (6), and in steps $400-410$ a zeroth approximation to the scattering function is calculated. In both of these calculations, as well as in the subroutine for evaluating Eq. (5) which appears in lines 900-920, a simple sum is used in place of an integral, since for reasonably small $W$ the error so introduced is much smaller than the expcrimental errors involved. In lines 500-545, Eq. (5) is evaluated using the best current value of $S\left(x^{\prime}-x\right)$ in each step. The corrections, applied in steps 530-540, are weighted heaviest for smaller $x^{\prime}-x$ since the corresponding $S\left(x^{\prime}-x\right)$ have not been corrected as often and are therefore more probably in error. After each iteration has been completed, the resulting $S\left(x^{\prime}-x\right)$ approximation is evaluated in steps 610-630. The evaluation is begun at the edge and proceeds toward the center, and $M$ points before the first point at which the solution is found to be unsatisfactory another iteration is begun. In the concluding steps, $800-825$, the scattering function is normalized and scaled. The change in $S(0)$ introduced by the normalization prodedure is to correct for computation errors and will be slight if the calculation has gone well.

The value of the tolerable error $E$ introduced in line 200 may have to be altered in order to obtain a solution. If $E$ is unrealistically small in light of the experimental error in the $F(x)$ data and $L$ is too large, there will be a tendency for the solution of become oscillatory. Although such a solution can produce $F(x)$ values within the experimental error, it is probably not realistic physically unless the oscillations can be correlated with a diffraction pattern which could be produced in the optical system.

\section{Appendix B}

A program for carrying out the type of calculations described in Sec. III is presented in Fig. 9.

In line 105, several parameters are read in, beginning with $W$, the spacing between datum points. An ac-

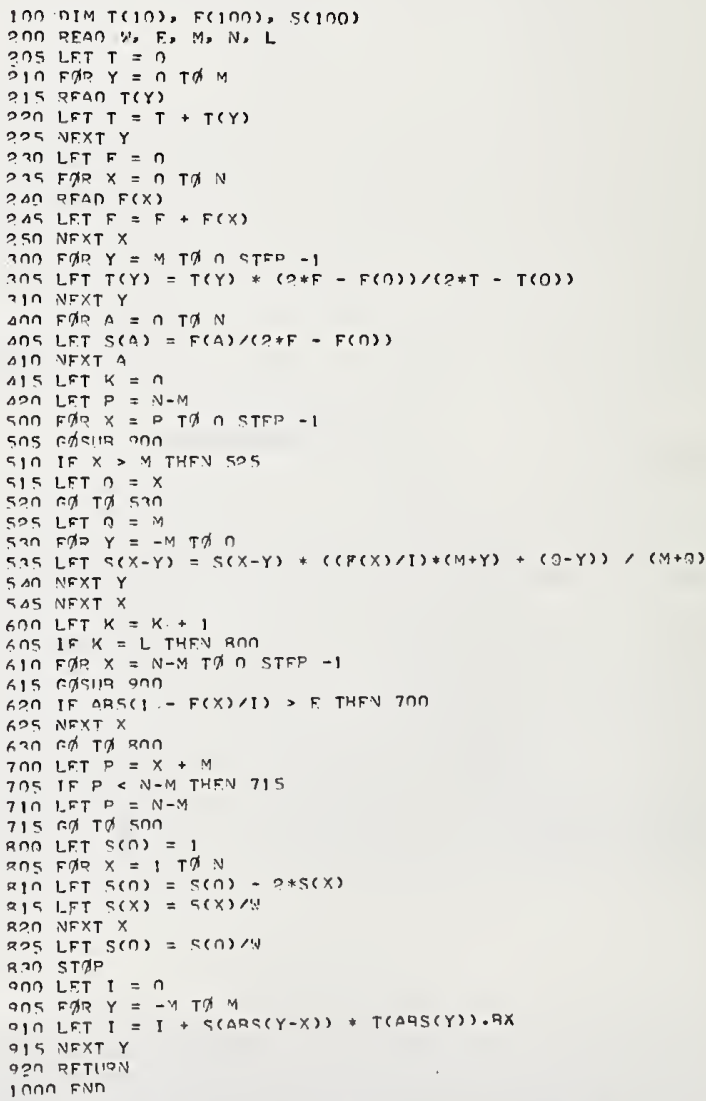

Fig. 8. A BASIC program for determining the scattering function. 
inn nIM $F(200), T(10 n), U(? 00), v(200)$

inS RFAO W, F, N, L, Z

110 LFT $M=2 * N$

1 is LF.T $K=1$

गก $F \not R$ R $A=n T \not M$

2กS LFT $U(A)=$

2. In NEXT A

IS LET $r:=-z$

गD LFT $n=$

गS LFT $A=$

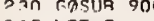

2.75 LF.T $S=1$

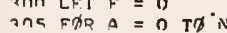

?I PFAO $F(A)$

1) 5 LFT $V(A)=F(A) / S$

3 3ก LET $T(A)=V(A)$

305 LFT $F=F+F(A)$

จ ח N NEXT A

$\triangle D \cap$ LFT $F=$ ? $F-F(n)$

$\triangle O S$ LFT $\&=F(n)$

$\triangle 10 F d R A=0 T D N$

$\triangle 1 S$ LET $F=6+3 * F(A)$

$\triangle 2 N$ IF $F>=.9 *$ F THE.N 4.30

$\triangle 2.5$ NFXT A

$\triangle$ an LET $r_{0}=-A * b$

$\triangle 3$ S LET $D=A * W$

$\triangle \Delta \cap F \not R A=N+1$ TQ $M$

$\triangle \triangle 5$ EXSIJP 900

$\triangle S D$ LET $V(A)=1 / C$

$\triangle 55$ NFXT A

SON $F \not D A_{A}=$ ก Td $M$

SnO FOR $A=0$ TA $M$
SnS LFT $C=-M * W$

5 S LFT $C=-M * N$
$\sin$ LFT $n=A * N-Z$

510 LFT $n=A$
515 IF $C<0$ THEN $5.3 n$

515 IF $C<0$
Son L.F.T I = ?

5ว5 R.dT 5.25

530 roxsur on

5?5 LFT $\|(\Delta)=-I$

$5 \Delta n$ LFT $C=A * W+Z$

$5 \Delta 5$ LFT $n=M *$ N

$55 n$ IF $C<n$ THFN 565

555 LFT I $=0$

san ad $T$ Td 570

565 roxGUIR ono

570 LF.T $U(A)=(U(A)-I) / S$

575 NFXT

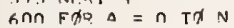

Gก5 LFT $T(A)=T(A)+U(A)$

GIN NFXT Q

$70 n$ IF $K>=$ L THFN 915

$705 \mathrm{FDR} A=0 \mathrm{~T}$ (d $\mathrm{N}$

7 In IF AP5(U)(A)/T(A)) > E THEN BDO

715 NFXT A

700 GdT 915

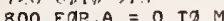

ROก FP.T $U(A)=U(A)$

SIO NF.XT A

815 LFT $K=K+1$

son $F \phi T \phi$ TnO

ONO REM IN THIS SURRQUTINF: THE INTEGRAL DF S(Y-A) * V(Y) WITH

905 REM RESPECT TI $Y$ IS EVALUATED FRDM $Y=C$ T $Y=D$.

9 II RFTURN

I $O$ OO F.NO

Fig. 9. A BAsic program for solving for the actual radiance.

ceptable solution for $T\left(x^{\prime}\right)$ will be one for which the fractional error introduced by dropping the remaining terms in Eq. (14) is less than $E$.at every point. There are $N+1$ datum points in the measured radiance being evaluated and the calculation is to proceed for no more than $L$ terms. Z presents the $\epsilon$ introduced in Eq. (11). In steps 200-225, the value of $s$ in Eq. (7) is determined. The $F(x)$ data are read in step 310 and the first term of the series solution is determined in steps 315 and 320 . [The vector $V(A)$ is used to store the integrand of the term of Eq. (11) which is currently being evaluated and $U(A)$ is used to store the integral.] In steps 400-455, additional $F(x)$ data are evaluated in order to double the range over which data are available for calculation. In this way, the approximation of an infinite integral by a finite one will introduce only a small error in the $T\left(x^{\prime}\right)$ solution even as $x^{\prime}$ approaches $W^{*} N$. To obtain these data, $T\left(x^{\prime}\right)$ in Eq. (5) is approximated by scaling the central portion of the $F(x)$ data. In steps $500-575$, the primed integrals in Eq. (14) are evaluated for the cur- rent term, and in steps 600-610 these are added to the sum of the preceding terms. The test for a satisfactory solution given in line 710 is based upon the result that the absolute value of each term in a uniformly converging alternating series is greater than the sum of the remaining terms. The form of the integral calculation in the subroutine indicated by lines 900-910 will depend upon the form in which the $S\left(x^{\prime}-x\right)$ data are available.

\section{References}

1. R. D. Lee, Metrologia 2, 150 (1966).

2. E. C. Titchmarsh, Introduction to the Theory of Fourier Integrals (Oxford University Press, New York, 1948).

3. J. S. Rollet and L. A. Higgs, Proc. Phys. Soc. London 79, 87 (1962).

4. A. F. Jones and D. L. Misell, Brit. J. Appl. Phys. 18, 1479 (1967).

5. H. C. Burger and P. H. vanCittert, Z. Physik 79, 722 (1932).

6. H. W. Drawin, Z. Physik 172, 181 (1963).

7. P. Beckmann and A. Spizzichino, The Scattering of Electromagnetic Waves from Rough Surfaces (Macmillan Company, New York, 1963).

8. Richard Blazey, Appl. Opt. 6, 831 (1967).

9. W. H. Venable, Jr., and J. B. Shumaker, Jr., J. Quant. Spect. Radiative Transfer 9, 1215 (1969).

10. C. C. Eaglesfield, Proc. Phys. Soc. London 82, 406 (1963).

11. C. C. Eaglesfield, Electron. Lett. 1, 237 (1965).

12. M. P. Freeman and S. Katz, J. Opt. Soc. Amer. 53, 1172 (1963).

13. J. G. Kemeny and T. E. Kurtz, Basic (Dartmouth Publications, Hanover, New Hampshire). 



\section{Radiance Standards}

Papers

7.1. Standard of Spectral Radiance for the Region of 0.25 to 2.6 Microns.

Ralph Stair, Russell G. Johnston, and E. W. Halback, J. Res. Nat. Bur. Stand. (U.S.) 64A, No. 4, 291-296 (July-Aug. 1960).

Key words: Blackbody; spectral radiance; standard; tungsten strip lamp._._-_._-_._-

7.2. Spectral Radiance of a Low Current Graphite Arc.

Albert T. Hattenburg, Appl. Opt. 6, No. 1, 95-100 (Jan. 1967)

Key words: Arc; graphite; spectral radiance; spectroradiometer; temperature blackbody

7.3. High-Accuracy Spectral Radiance Calibration of Tungsten-Strip Lamps.

H. J. Kostkowski, D. E. Erminy, A. T. Hattenburg, Ch. 4, Advances in Geophysics, Vol. 14, Academic Press, N.Y. (1970).

Key words: Blackbody; high temperature measurement; radiometry; spectral irradiance; tungsten-strip lamp_...--

7.4. The NBS Photoelectric Pyrometer and its Use in Realizing the International Practical Temperature Scale above $1063^{\circ} \mathrm{C}$.

R. D. Lee, Metrologia, 2, No. 4, 150-162 (Oct. 1966).

Key words: Blackbody; freeze; gold point; temperature scale

Abstracts

7.5. Construction and Operation of a Simple High-Precision CopperPoint Blackbody and Furnace.

R. D. Lee, Nat. Bur. Stand. (U.S.) Tech. Note 483, 15 pages (May 1969).

Key words: Blackbody; copper-point temperature; optical pyrometry ; radiometry

7.6. Radiance Temperature at $6550 \AA$ of the Graphite Arc.

R. D. Lee and E. Lewis, Appl. Opt. 5, No. 11, p. 1858 (Nov. 1966).

Key words: Arc; graphite; spectral radiance; radiance temperature

7.7. Theory and Methods of Optical Pyrometry.

H. J. Kostkowski and R. D. Lee, Nat. Bur. Stand. (U.S.) NBS Monogr. 41, 28 pages, (Reprinted Sept. 1962).

Key words: Blackbodies; gold point; optical pyrometry ; tungsten strip lamps 



\title{
Standard of Spectral Radiance for the Region of 0.25 to 2.6 Microns
}

\author{
Ralph Stair, Russell G. Johnston, and E. W. Halbach
}

(March 1, 1960)

\begin{abstract}
This paper contains information relating to the setting up of standard blackbodies for use through the temperature range of about $1,400^{\circ}$ to $2,400^{\circ} \mathrm{K}$ and their use in the calibration of tungsten strip lamps as laboratory standards of spectral radiance for the wavelength region of 0.25 to 2.6 microns. A graphite blackbody is described and representative data are given on the spectral characteristics of the new lamp standard as compared to blackbodies at several selected temperatures.
\end{abstract}

\section{Introduction}

Fifty years ago the principal need in the evaluation of radiant energy was a convenient standard against which a radiometer might be calibrated. Although several crude standards of total radiance in the form of oil lamps or candles existed about 1900, the establishment of the carbon filament lamp $[1,2,3]^{1}$ provided a convenient working standard for a limited range of total radiance. Then, as today, the blackbody was considered the reference standard, but its use was relegated only to the few primary scientific laboratories. The carbon filament standard has received wide acceptance in scientific research, not only in this country but throughout the world. It has been, and remains, extremely useful, but within recent years has been recognized as being inadequate to cover many new uses wherein spectral energy distributions were required.

It is to fulfill this latter requirement that a new secondary standard of spectral radiance in the form of a tungsten strip lamp has been set up. Again the blackbody has been employed as the reference source in the development of the new standard.

It has been established that the total and spectral radiation characteristics of a blackbody may be defined in terms of certain equations or laws. Planck's radiation law relates the radiance $N_{\lambda}$ at a particular wavelength to the absolute temperature $T$ by the relationship

$$
N_{\lambda}=\frac{C_{1}^{\gamma} \lambda^{-5}}{{ }_{e} / \lambda T_{-1}}
$$

wherein $C_{1}$ and $C_{2}$ are the first and second radiation constants having the dimensions watts $-\mathrm{m}^{2}$ and cm-degree, respectively. The exact values attached to these depend not only on the units employed in expressing $N_{\lambda}$, but also upon the most probable values of the various fundamental atomic constants.

In the present case the following values have been employed for the various parameters in the Planck equation:

\footnotetext{
1 Figures in brackets indicate the literature references at the end of this paper.
}

$$
\begin{aligned}
\lambda & =\text { wavelength in } \mathrm{cm} ; \\
C_{1} & =1.19088 \times 10^{-12} \text { wat }-\mathrm{cm}^{2} / \mathrm{stcr} \\
C_{2} & =1.4380 \mathrm{~cm}-\mathrm{K} ; \\
N_{\lambda} & =\text { watt } \mathrm{cm}^{-2} \mathrm{ster}^{-1} / \mathrm{cm} \text { wavelength interval. }
\end{aligned}
$$

Much research has been carried out on tungsten lamps, in particular regarding the spectral emissivity of tungsten $[4,5,6,7,16,17,18]$. Although all the results are not in perfect agreement, it is generally arreed that with reasonable caution the emissivity of pure clean tungsten ribbon remains fairly constant throughout the life of the lamp. Recently, in many laboratories $[8,10,11,12,15]$ the determination of the spectral distribution of radiant energy from a tungsten filament lamp has been obtained through making use of the published values of the emissivity of tungsten and the observed color temperature or brightness temperature of the filament. These calculations are based upon a doubtful assumption that all samples of tungsten are identical in emissivity. No account was taken of the effects of impurities present, or of the size and shape of the filament or of its mechanical or crystalline structure $[4,6]$. All these properties affect markedly the true spectral and total emissivity. Furthermore, it has been found that interreflections within the lamp envelope [6] affect the total spectral radiation from a particular tungsten strip. In order to obtain the correct spectral radiance of a lamp, it becomes necessary that the particular lamp as set up for use be calibrated against a blackbody.

\section{Apparatus and Method}

Before discussing the apparatus and method employed in comparing a group of tungsten ribbon strip lamps with blackbodies at various temperatures a discussion is in order relative to the type of lamp chosen.

Investigations by Worthing [4a] many years ago resulted in the accumulation of considerable information relating to the radiation characteristies of tungsten, in particular in regard to the effects of the angle (polarization, etc.) from which the filament is 
viewed. These results proved the neerssity of using a flat-ribbon filament to insure a reproducible source. A lamp was designed at the National Burenu of Standards a number of rears ago which entailed the for'egoing qualities and was accepted for commercial production by the General Electric Co. as their type G.E. 30A/T24/77 Th is lamp was chosen for use as the new standard (see fig. 1). It has a mogul bipost base and a nominal rating of $30 \mathrm{amp}$ at $6 \mathrm{v}$. Radiant energy is emitted from the flat strip filament through al 1/4-in. fused silica window placed parallel to and at a distance of about 3 to $4 \mathrm{in}$. from the plane of the filament. This separation of the window (necessitated by a graded seal) assists greatly in reducing the deposit of metallic tungsten on the lamp window as the lamp ages.

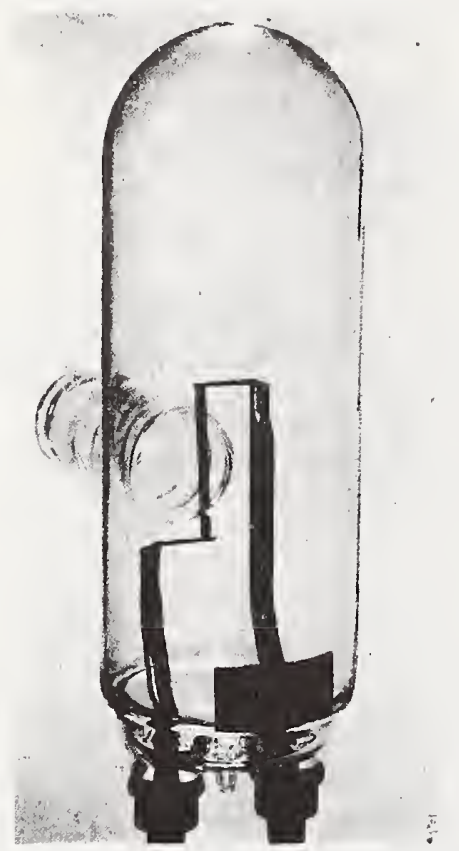

FigURE 1. Tungsten ribbon strip lamp standard of spectral radiance.

The principal apparatus employed in comparing the radiant energy from the lamp with that of the blackbody was set up as shown in figure 2 to cover the spectral region from 0.7 to $2.6 \mu$. The lamp and the blackbody were mounted side by side on an optical bench (constructed from a lathe bed and table) so they could alternately be placed at the focal point of the auxiliary optical system. In figure 3 is shown the monochromator mounted on the optical bench with the auxiliary opties rigidly secured on the front of the monochromator. This arrangement was employed for the spectral region of 0.25 to $0.75 \mu$ wherein a high-temperature graphite blackbody was used. It was possible to move the monochromator so that the lamp and the blackbody would be alternately at the correct object distance of the auxiliary optics.

The auxiliary optics consisted of a plane mirror and a spherical mirror having a $71-\mathrm{cm}$ radius of curvature. The placing of the lamp and the blackbody alternately at the samo position insured equal light paths and the use of identical optics.

The blackborly indicated in figure 2 , which was operated up to $1,400^{\circ} \mathrm{K}$, was constructed of a casting of an alloy of 80 pereent nickel and 20 percent chromium and had a 3 -in. outside diameter, was 6 in. in length, and had a wall thickness of $1 / 2$ in. The

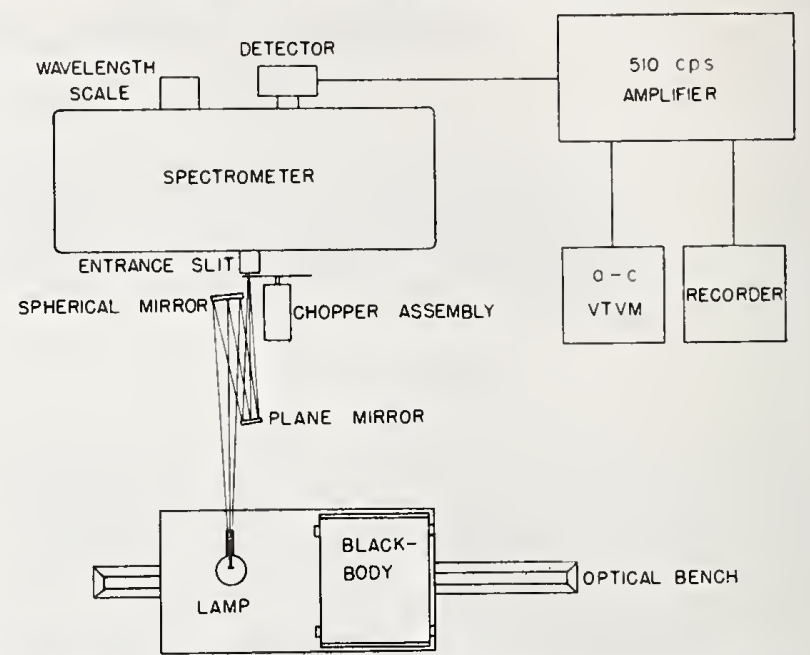

FIGURE 2. Instrumental set-up of blackbody, monochromator, lamp and associated equipment for the wavelength region of 0.7 to 2.6 microns.

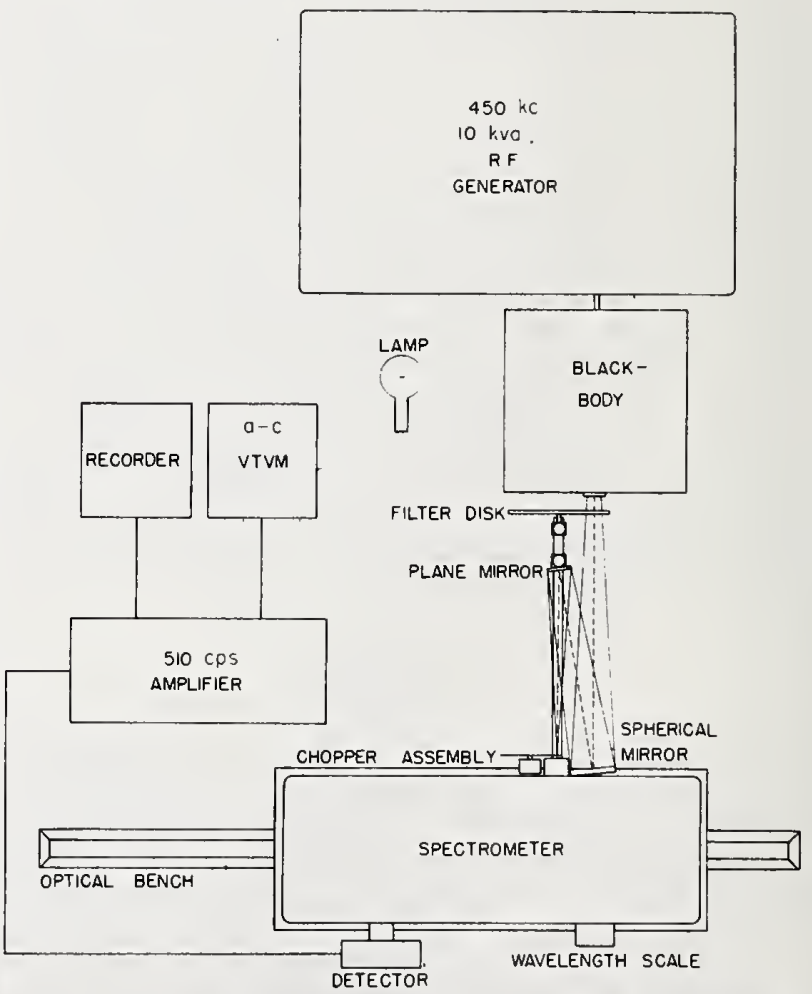

FIGURE 3. Instrumental setup of blackbody, monochromator, lamp and associated equipment for the wavelength region of 0.25 to 0.75 micron. 
low reflectivity of this oxidized metal coupled with the small aperture (3/16 in. by $1 / 2 \mathrm{in}$.) as compared with the internal surface area resulted in a blackbody of extremely high effective emissivity. The high heat capacity of the associated furnace gave the blackbody a very high thermal stability, making manual temperature regulation easy. Further information on a similar blackbody employed as a standard at longer wavelengths has been published elsewhere [14].

The blackbody employed for the shorter wavelengths and shown in figures 3 and 4 was constructed of high purity graphite. It consists of a cylindrical enclosure $4 \frac{1}{2}$ in. long and $1 \frac{1}{2}$ in. in diameter, having walls $3 / 16$ in. thick. The exit end of the tube has a 3/8-in. opening shielded by a conical graphite endpiece $3 / 4$ in. long (see fig. 4.) The very low reflectivity of the graphite (rough machined surface) and the relatively small aperture (as compared to the total internal surface) resulted in a blackbody of high effective emissivity.

This graphite blackbody is heated by induction inside a water-cooled coil by a radiofrequency generator operating at $450 \mathrm{kc}$. The blackbody tube is insulated by firmly packed boron nitride powder inside a high temperature porcelain tube (closed at one end) $4 \mathrm{in}$. in outside diameter and $6 \mathrm{in}$. in length. An alundum ceramic tube placed midway between the graphite core and the high temperature porcelain tube increased the mechanical stability of the unit.

Depreciation of the graphite at high temperature was reduced by enclosing the blackbody unit in an airtight chamber (see fig. 4) through which dry helium was passed. The concentration of oxygen was further reduced by heating copper coils within the chamber preceding each operation of the blackbody.

The spectral transmittance of the fused silica window of the enclosure was measured and the necessary corrections were made for temperature measurements and spectral radiance.

This blackbody is many times larger than the small tungsten enclosures often used in high temperature work. A much larger opening can be employed thereby making possible the use of the entire slit of the usual spectroradiometer.

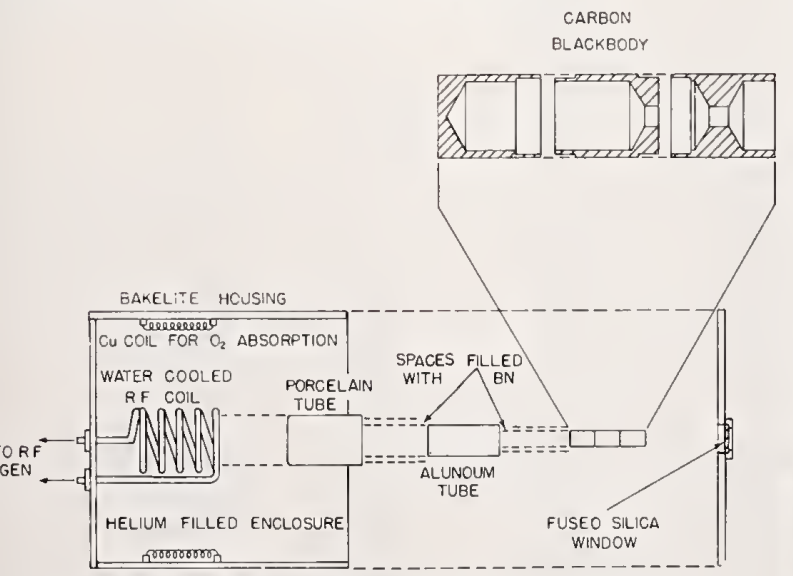

FIGURE 4. Graphite high temperature blackbody.
Leiss double quartz prism monochromators with relative aperture ranges of $F / 6.4$ to $F / 7.2$ were employed as shown in figures 2 and 3 . The detector used at the exit slit of the monochromator for the wavelengths from 0.7 to $2.6 \mu$ was an uncooled lead sulfide cell, while for the wavelength range of 0.25 to $0.75 \mu$ a photomultiplier, type 1 P28 was employed [9]. In all cases care was taken (through the use of filters to reduce the light intensity when required, and by other means) in using the photomultiplier to make certain that the detector was not exposed to excessive radiation which might produce a change or nonlinear character in the photoelectric response.

The radiant energy beam was mechanically chopped at $510 \mathrm{cps}$ and the detector signal amplified by a special tuned amplifier [15]. Usually the output signal was read on an a-c vacuum tube voltmeter and manually recorded. However, equipment was available (and occasionally used) for recording the data. with a strip chart recorder connected to the d-c output of the tuned amplifier.

The temperature of the blackbody used at $1,400^{\circ} \mathrm{K}$ (and below) was determined by a platinum versus platinum 10 percent rhodium thermocouple calibrated by the heat division of the National Bureau of Standards. The same laboratory calibrated the optical pyrometer employed to determine the temperatures from $1,400^{\circ}$ to $2,400^{\circ} \mathrm{K}$ in the graphite blackbody. Check measurements between the optical pyrometer and the thermocouple usually were in agreement within $2^{\circ}$ to $3^{\circ} \mathrm{K}$. Calibrations of the optical pyrometer by the heat division before and following the measurements reported in this paper were in agreement to about $1^{\circ} \mathrm{K}$.

Tests using thermocouples placed at various positions and by observations with the optical pyrometer indicated closely uniform temperatures within the low temperature blackbody. Similar tests with the optical pyrometer indicated a similar uniform condition within the high-temperature enclosure.

Each lamp was seasoned by operation on alternating current at $35 \mathrm{amp}$ (about $2,470^{\circ} \mathrm{K}$ ) for $2 \mathrm{hr}$. During calibration the lamp current was controlled manually through the use of variable and stepdown transformers as described in a later paragraph (see fig. 5).

Each lamp was set up with the envelope vertical and with the section of the filament viewed horizontally through the center of the lamp window being employed in the measurements (no note was made of a notch placed in one edge of the lamp filament for another use). Through the use of the external optics (plane mirror and spherical mirror) a full size image of this section of the lamp filament was focused upon the entrance slit of the spectroradiometer. Hence, the physical dimensions of the spectrometer slit (set at about $0.5 \mathrm{~mm}$ by $5 \mathrm{~mm}$ ) determined the source area employed in the measurements. Since the optical arrangement was identical for the lamp and the blackdody equal areas of the two sources were always being compared. 


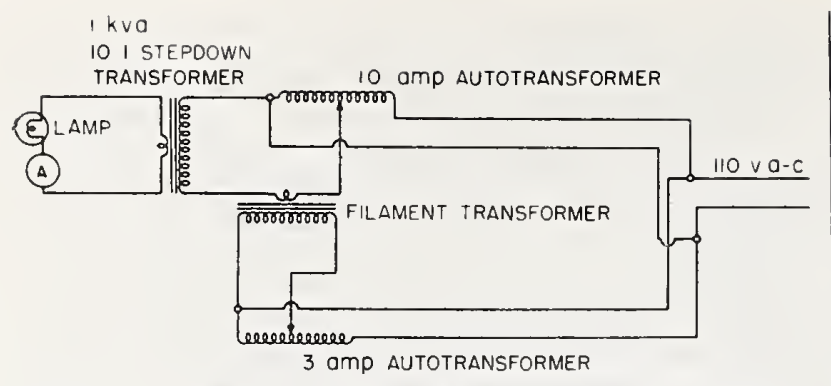

FiguRE 5. Electrical circuit for lamp operation to give smooth current control.

Readings were alternately taken on the blackbody and on the lamp for ench wavelength setting throughout the spectral range. The blackbodies were operated at several selected temperatures so that the radiance from the lamp and the blackbody were approximately equal in a given region of the spectrum. At each wavelength a number of readings were taken (with changes in the temperature of the blackbody) such that in some cases the radiance of the lamp was greater than that of the blackborly while in others it was less than that of the blackbody. This procedure enabled us to keep the effect of any departure from linearity within the electronics in the detector circuitry as small as practicable.

\section{Results}

Data obtained upon two lamps as compared with the blackbodies are given in table 1. Other lamps result in slightly higher or lower values depending upon the characteristics of the particular lamp chosen. For the laboratory lamp standards more than 20 comparisons between the lanps and blackbodies were made within the shorter wavelength region $(0.25$ to $0.75 \mu)$. About 10 to 12 independent comparisons were made at the longer wavelengths. Due to the sinall magnitued of scatter of the results, little is to be gained by additional measurements.

The data of table 1 are tabulated in terms of radiance, mierowatts per square centimeter at $u$ distance of $1 \mathrm{in}$ from a square millimeter area source, and for a wavelength interval of $0.1 \mu$. Hence the radiant energy values are in a form directly applicable for use with a spectroradiometer of fixed entrance slit. Those who desire to inake solid angle measurements may change the tabulated values to erg $\mathrm{cm}^{-2} \operatorname{ster}^{-1} \mu^{-1} \mathrm{sec}^{-1}$ as reported by DeVos [6] simply by nultiplying the recorded value by $10^{8}$. Similarly, the data recorded in the DeVos tables may be reduced to the units employed herein by multiplying by $10^{-8}$ (within about 0.1 percent resulting from slightly different atomic constants employed in the DeVos calculations).

Although the data for all wavelengths are given on the same basis as regards the wavelength interval of $0.1 \mu$, in the experimental setup the actual instrumental spectral slit widths ranged from about 0.001 $\mu$ at $0.25 \mu$ to $0.060 \mu$ at $2.6 \mu$ (being about $0.020 \mu$ at $1.0 \mu$ ). The use of the $0.1 \mu$ interval affects the absolute values but little for wavelengths longer than about $1 \mu$. However, for the shorter wavelengths where the spectral energy curve exhibits a greater degree of curvature, higher accuracy may be obtained

TABLE 1. Spectral radiance of blackbodies and tungsten lamps $=N_{\lambda}=P \times 10^{-a}$ expressed in microwatts per square centimeter al 1 meter for a $1-\mathrm{mm}^{2}$ source and for a wavelength interval of 0.1 micron

\begin{tabular}{|c|c|c|c|c|c|c|c|c|c|c|c|c|c|c|c|c|c|c|}
\hline \multirow{2}{*}{$\begin{array}{c}\text { Wrave. } \\
\text { length } \\
-\cdots \\
0.250 \\
.260 \\
.270 \\
.280 \\
.290\end{array}$} & \multicolumn{2}{|l|}{$\begin{array}{c}1,4 \% 0^{\circ} \mathrm{K} \\
\text { blackbody }\end{array}$} & \multicolumn{2}{|l|}{$\begin{array}{c}1,600^{\circ} \mathrm{K} \\
\text { blackbody }\end{array}$} & \multicolumn{2}{|l|}{$\begin{array}{l}1,800^{\circ} \mathrm{K} \\
\text { blackbody }\end{array}$} & \multicolumn{2}{|l|}{$\begin{array}{c}2,000^{\circ} \mathrm{K} \\
\text { blackbody }\end{array}$} & \multicolumn{2}{|l|}{$\begin{array}{c}2,200^{\circ} \mathrm{K} \\
\text { blackbody }\end{array}$} & \multicolumn{2}{|l|}{$\begin{array}{c}2,400^{\circ} \mathrm{K} \\
\text { blackbody }\end{array}$} & \multicolumn{2}{|l|}{$\begin{array}{c}\text { Lamp } \# 20 \\
25 \text { amp }\end{array}$} & \multicolumn{2}{|l|}{$\begin{array}{c}\text { Lamp } \# 20 \\
30 a m p\end{array}$} & \multicolumn{2}{|l|}{$\begin{array}{l}\text { Lamp \#16 } \\
35 \text { amp }\end{array}$} \\
\hline & $\begin{array}{l}P \\
\text { 1. } 7493 \\
6.9821 \\
2.4973 \\
\text { 8. } 1013 \\
2.4084\end{array}$ & $\begin{array}{c}q \\
12 \\
12 \\
11 \\
11 \\
10\end{array}$ & $\begin{array}{c}P \\
\text { 2. } 9736 \\
9.7412 \\
\text { 2. } 9018 \\
7.9434 \\
2.0160\end{array}$ & $\begin{array}{r}q \\
10 \\
10 \\
9 \\
9 \\
8\end{array}$ & $\begin{array}{l}P \\
\text { 1. } 6145 \\
\text { 4. } 5360 \\
\text { 1. } 1720 \\
\text { 2. } 8113 \\
\text { 6. } 3092\end{array}$ & $\begin{array}{l}q \\
8 \\
8 \\
7 \\
7 \\
7\end{array}$ & $\begin{array}{l}P \\
\text { 3. } 9432 \\
\text { 9. } 7969 \\
\text { 2. } 2592 \\
\text { 4. } 8756 \\
9.9166\end{array}$ & $\begin{array}{l}q \\
7 \\
7 \\
6 \\
6 \\
6\end{array}$ & $\begin{array}{c}P \\
\text { 5. } 3971 \\
1.2103 \\
2.5429 \\
5.0331 \\
9.4458\end{array}$ & $\begin{array}{l}q \\
6 \\
5 \\
5 \\
5 \\
5\end{array}$ & $\begin{array}{c}P \\
4.7597 \\
9.8342 \\
1.9119 \\
\text { 3. } 5211 \\
6.1797\end{array}$ & $\begin{array}{l}q \\
5 \\
5 \\
4 \\
4 \\
4\end{array}$ & \begin{tabular}{c}
$P$ \\
\\
\hdashline
\end{tabular} & $q$ & $\begin{array}{c}P \\
P\end{array}$ & $q$ & $\begin{array}{l}P \\
4.13 \\
9.59 \\
1.88 \\
3.40 \\
5.84\end{array}$ & $\begin{array}{l}q \\
5 \\
5 \\
4 \\
4 \\
4\end{array}$ \\
\hline $\begin{array}{l}.300 \\
.320 \\
.350 \\
.400 \\
.450 \\
.500 \\
.550 \\
.600 \\
.650 \\
.700 \\
.750\end{array}$ & $\begin{array}{l}6.6199 \\
4.0740 \\
4.0766 \\
8.1943 \\
7.8861 \\
4.5642 \\
1.8812 \\
5.6287 \\
1.4227 \\
3.0045 \\
5.6866\end{array}$ & $\begin{array}{r}10 \\
9 \\
8 \\
7 \\
6 \\
5 \\
4 \\
4 \\
3 \\
3 \\
3\end{array}$ & $\begin{array}{l}4.7810 \\
2.2519 \\
1.5976 \\
2.0301 \\
1.3677 \\
5.9508 \\
1.9255 \\
4.7836 \\
1.0214 \\
1.8808 \\
3.1435\end{array}$ & $\begin{array}{l}8 \\
7 \\
6 \\
5 \\
4 \\
4 \\
3 \\
3 \\
2 \\
2 \\
2\end{array}$ & $\begin{array}{l}1.3340 \\
5.1031 \\
2.7707 \\
2.4646 \\
1.2582 \\
4.3847 \\
1.1764 \\
2.5267 \\
4.7345 \\
7.8326 \\
1.1889\end{array}$ & $\begin{array}{l}6 \\
6 \\
5 \\
4 \\
3 \\
3 \\
2 \\
2 \\
2 \\
2 \\
1\end{array}$ & $\begin{array}{l}1.9128 \\
6.1953 \\
2.7157 \\
1.8160 \\
7.4259 \\
2.1670 \\
5.0096 \\
9.5681 \\
1.5838 \\
2.4523 \\
3.4470\end{array}$ & $\begin{array}{l}5 \\
5 \\
4 \\
3 \\
3 \\
2 \\
2 \\
2 \\
1 \\
1 \\
1\end{array}$ & $\begin{array}{l}1.6901 \\
4.7772 \\
1.7577 \\
9.3067 \\
3.1737 \\
8.0096 \\
1.6399 \\
2.8441 \\
4.4118 \\
6.2392 \\
8.2366\end{array}$ & $\begin{array}{l}4 \\
4 \\
3 \\
3 \\
2 \\
2 \\
1 \\
1 \\
1 \\
1 \\
1\end{array}$ & $\begin{array}{l}1.0386 \\
2.6206 \\
8.3334 \\
3.6326 \\
1.0648 \\
2.3808 \\
4.4073 \\
7.0506 \\
1.0192 \\
1.3586 \\
1.7026\end{array}$ & $\begin{array}{l}3 \\
3 \\
3 \\
2 \\
1 \\
1 \\
1 \\
1\end{array}$ & $\begin{array}{l}8.00 \\
1.88 \\
3.64 \\
6.24 \\
9.56 \\
1.35\end{array}$ & $\begin{array}{l}3 \\
2 \\
2 \\
2 \\
2 \\
1\end{array}$ & $\begin{array}{l}4.04 \\
8.14 \\
1.39 \\
2.13 \\
2.99 \\
3.74\end{array}$ & $\begin{array}{l}2 \\
2 \\
1 \\
1 \\
1 \\
1\end{array}$ & $\begin{array}{l}9.72 \\
2.34 \\
7.00 \\
2.76 \\
7.27 \\
1.56 \\
2.72 \\
4.05 \\
5.66 \\
7.17 \\
8.59\end{array}$ & $\begin{array}{l}4 \\
3 \\
3 \\
2 \\
2 \\
1 \\
1 \\
1 \\
1 \\
1 \\
1 \\
1\end{array}$ \\
\hline $\begin{array}{r}.800 \\
.900 \\
1.000 \\
1.100 \\
1.200 \\
1.300 \\
1.400 \\
1.500\end{array}$ & $\begin{array}{l}9.6470 \\
2.2294 \\
4.1215 \\
6.5110 \\
9.1769 \\
1.1883 \\
1.4428 \\
1.6672\end{array}$ & $\begin{array}{l}3 \\
2 \\
2 \\
2 \\
2 \\
1 \\
1 \\
1\end{array}$ & $\begin{array}{l}4.8017 \\
9.2845 \\
1.4883 \\
2.0924 \\
2.6762 \\
3.1923 \\
3.6135 \\
3.9295\end{array}$ & $\begin{array}{l}2 \\
2 \\
1 \\
1 \\
1 \\
1 \\
1 \\
1\end{array}$ & $\begin{array}{l}1.6731 \\
2.8163 \\
4.0409 \\
5.1891 \\
6.1679 \\
6.8898 \\
7.3864 \\
7.6649\end{array}$ & $\begin{array}{l}1 \\
1 \\
1 \\
1 \\
1 \\
1 \\
1 \\
1\end{array}$ & $\begin{array}{l}4.5420 \\
6.8433 \\
8.9872 \\
1.0736 \\
1.1992 \\
1.2761 \\
1.3103 \\
1.3101\end{array}$ & $\begin{array}{l}1 \\
1 \\
1\end{array}$ & $\begin{array}{l}1.0284 \\
1.4153 \\
1.7290 \\
1.9472 \\
2.0713 \\
2.1154 \\
2.0974 \\
2.0349\end{array}$ & & $\begin{array}{l}2.0323 \\
2.5938 \\
2.9841 \\
\text { 3. } 2003 \\
\text { 3. } 2694 \\
\text { 3. } 2273 \\
\text { 3. } 1089 \\
\text { 2. } 9425\end{array}$ & & $\begin{array}{l}1.66 \\
2.22 \\
2.74 \\
3.12 \\
3.32 \\
3.36 \\
3.30 \\
3.16\end{array}$ & $\begin{array}{l}1 \\
1 \\
1 \\
1 \\
1 \\
1 \\
1 \\
1 \\
1\end{array}$ & $\begin{array}{l}4.38 \\
5.42 \\
6.12 \\
6.38 \\
6.41 \\
6.24 \\
5.94 \\
5.55\end{array}$ & $\begin{array}{l}1 \\
1 \\
1 \\
1 \\
1 \\
1 \\
1 \\
1\end{array}$ & $\mid$\begin{tabular}{l} 
\\
\hdashline
\end{tabular} & $\begin{array}{l}-- \\
-- \\
-- \\
-- \\
- \\
- \\
-\end{array}$ \\
\hline $\begin{array}{l}1.600 \\
1.700 \\
1.800 \\
1.900 \\
2.000 \\
2.100 \\
2.200 \\
2.300 \\
2.400 \\
2.500 \\
2.600\end{array}$ & $\begin{array}{l}1.8534 \\
1.9982 \\
2.1024 \\
2.1690 \\
2.2023 \\
2.2072 \\
2.1888 \\
2.1516 \\
2.0999 \\
2.0372 \\
1.9667\end{array}$ & $\begin{array}{l}1 \\
1 \\
1 \\
1 \\
1 \\
1 \\
1 \\
1 \\
1 \\
1 \\
1\end{array}$ & $\begin{array}{l}\text { 4. } 1431 \\
\text { 4. } 2641 \\
4.3055 \\
4.2819 \\
4.2071 \\
\text { 4. } 0941 \\
\text { 3. } 9531 \\
\text { 3. } 7930 \\
3.6214 \\
3.4432 \\
\text { 3. } 2633\end{array}$ & $\begin{array}{l}1 \\
1 \\
1 \\
1\end{array}$ & $\begin{array}{l}7.7585 \\
7.7037 \\
7.5363 \\
7.2874 \\
6.9828 \\
6.6434 \\
6.2857 \\
5.9212 \\
5.5593 \\
5.2060 \\
4.8657\end{array}$ & $\begin{array}{l}1 \\
1 \\
1 \\
1 \\
1 \\
1 \\
1 \\
1 \\
1 \\
1 \\
1\end{array}$ & $\begin{array}{l}1.2839 \\
1.2393 \\
1.1825 \\
1.1185 \\
1.0508 \\
9.8224 \\
9.1466 \\
8.4935 \\
7.8705 \\
7.2833 \\
\text { 6. } 7335\end{array}$ & $\begin{array}{l}1 \\
1 \\
1 \\
1 \\
1 \\
1 \\
1\end{array}$ & $\begin{array}{l}1.9429 \\
1.8331 \\
1.7144 \\
1.5929 \\
1.4731 \\
1.3576 \\
1.2482 \\
1.1458 \\
1.0508 \\
9.6317 \\
8.8277\end{array}$ & $\begin{array}{l}1 \\
1\end{array}$ & $\begin{array}{l}2.7499 \\
2.5465 \\
2.3427 \\
2.1454 \\
1.9585 \\
1.7842 \\
1.6235 \\
1.4764 \\
1.3425 \\
1.2211 \\
1.1113\end{array}$ & & $\begin{array}{l}2.96 \\
2.74 \\
2.45 \\
2.20 \\
1.97 \\
1.78 \\
1.58 \\
1.40 \\
1.26 \\
1.13 \\
1.02\end{array}$ & $\begin{array}{l}1 \\
1 \\
1 \\
1 \\
1 \\
1 \\
1 \\
1 \\
1 \\
1 \\
1\end{array}$ & $\begin{array}{l}5.04 \\
4.52 \\
3.94 \\
3.45 \\
\text { 3. } 03 \\
2.65 \\
2.37 \\
2.13 \\
1.92 \\
1.66 \\
1.52\end{array}$ & $\begin{array}{l}1 \\
1 \\
1 \\
1 \\
1 \\
1 \\
1 \\
1 \\
1 \\
1 \\
1 \\
1\end{array}$ & 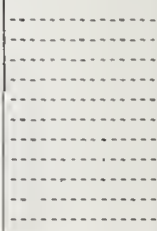 & $\begin{array}{l}\because \\
\because \\
\because \\
\cdots \\
\cdots \\
\cdots \\
-- \\
-\end{array}$ \\
\hline
\end{tabular}


if the tabulated values be converted to a shorter wavelength interval corresponeling more nearly to that employed in ohtaining the data.

In the original comparisons with the blackbody the cone of radiant energy from the lamp was linnted to approximately $5^{\circ}$ and near normal to the plane of the lamp filament. If, in use, a larger angle is required it should be ascertained that the flux density is uniform and free of polarization throughout the added aporture.

Although the calibrations of the stanklurels are given in terms of radiant power per ('m2 for the various waveleirgths at a distance of $1 \mathrm{ml}$, the lamps nuy be used at any convenient distance providerl proper correction is made for the new distance. However, if there is excessive water vapor in the laboratory atmosphere, error's may result at the wavelengths of water vapor absorption. In the original ealibrations any water vapor absolption effects cancelled out for the greater part since tho measurements wore mache using essentially identical air paths.

The quality of the two blackbolies used in this work was determined by the method outlined by André Gouffé [13] and the first and second orkler approximations of the quality of the low temperature blackbody was found to be 0.999 . The quality of the high temperature graplite blackbody was 0.996 .

The certification of the optical pyrometer was given as $\pm 2^{\circ}$ from $1,000^{\circ}$ to $1,400^{\circ} \mathrm{K}$ and $\pm 6^{\circ}$ from $1,400^{\circ}$ to $2,300^{\circ} \mathrm{K}$. This gives rise to an uncertainty of $1 / 2$ to 2 percent in the longer wavelengths while in the shorter wavelengths the uncertainty increases to 1 to 4 percent. The uncertaint $y$ for the thermocouple was less than $\pm 3^{\circ}$ which would result in an uncertainty in the radiance not in excess of 2 percent.

If one assumes an ability to read the meters employed as two-fifths of the smallest division, this would lead to an uncertainty never more than 1.0 percent. The ability of the electronies to reproduce an output when a given signal is applied to the input is of the order of 1.0 percent.

It is estimated that the maximum uncertainty in the results ranges from about 8 percent at the shortest wavelengths to about 3 percent at the longest wavelengths.

\section{Use of the Standards of Spectral Radiance}

The auxilin opties employed with this standard may be composed of two units such as those employed in the original calibrations (see figs. 2, 3, and 6), namely a plane mirror and a spherical mirror (each aluminized on the front surface). If the spherical mirror is placed at a distance from the lamp filament equal to its radius of curvature and the plane mirror set about one-third to two-fifths this distance from the spherical mirror, and facing it (at an angle of $10^{\circ}$ or less), an image of the filament equal in size to that of the filament itself may be focused upon the spectrometer slit. Little distortion of the filament image occurs provided good optical surfaces are employed and all reflection angles are kept to less than $10^{\circ}$.
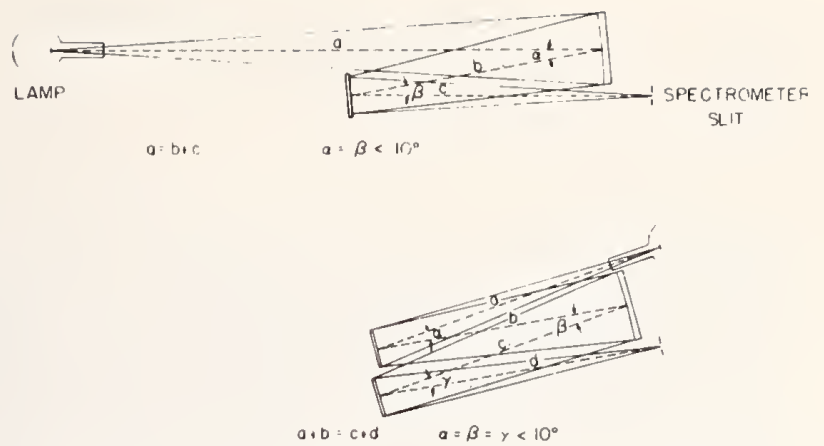

FIr:IRE 6. Auxiliary optics when using a lan $p$ standard of spectral radiance in the calibration of a spectroradiometer; and for determining the spectral reflectivity of the aluminized mirrors employed.

In general, the radius of curvature of this a uxiliary spherienl mirror should be greater than the focal length of the spectrometer employed so that no loss of radiant energy will result through overfilling the spectrometer opties. Furthermore, as noted above, the aperture of this mirror should be kept within a total conical angle of about $5^{\circ}$

No diaphragm or other shiclding is required in the use of these standards, except for a shield to prevent direct radiation from the lamp, not falling on the concave inirror from entering the spectrometer, since in their use an optical image of the filament is focused upon the spectrometer slit.

In order to calibrate a spectroradiometer with this standard lamp, a knowledge of the spectral reflective characteristics of the mirror surfaces is required in order to evaluate the radiant energy properly at the spectrometer slit. A good aluminized surface should have a spectral reflectivity above 87 percent throughout the spectral region of 0.5 to $2.6 \mu$ but which increases slightly with wavelength except for a slight dip in the regron of 0.8 to $1.0 \mu$. In practice the proper reflectance losses can best be determined through the use of a third mirror (a second plane mirror) which may be temporarily incorporated into the optical setup from time to time. (See fig. 6 for a possible urrangement of the auxiliary optics when including the third inirror to determine its spectral reflectince.)

In experiments where sources of radiation are being comprred, no knowledge of the spectral reflectance of the auxiliary mirrors, the spectrometer transmission characteristics, or the spectral sensitivity of the detector is required. Furthermore when the same auxiliary optics are employed no measure need be taken of the spectrometer slit widths, or slit areas, provided the slit is fully and uniformly filled in both cases.

Operation of these standards should be on alternating current to obviate filament crystallizing effects which occur when the operation is on direct current. To reduce line voltage a stepdown transformer (1-kva capacity) having a ratio of 10 to 1 or a 50-amp variable transformer nay be employed. (See fig. 5.) Then to give fine control a second variable transformer (10-amp capacity) is wired into the circuit to control the input of the heavy duty 
transformer. For still finer control a third variable transformer may be employed with a radio filament transformer to add (or subtract) a small voltage $(0$ to $2.5 \mathrm{v})$ to the primary voltage fed into the stepdown transformer. It was found that this method was very effective in accurately controlling the larger lamp currents. The heavy-duty (1 kva) stepdown transformer is preferred to that of a 50 -amp variable transformer since the latter is subject to contact damage when operated for long intervals of time at high current values.

These lamp standards are expensive laboratory equipment and it is suggested that they be operated at the lower current value in order to prolong their usual life. Only for short intervals should they ever be operated above 30 amp (about 2,200 ${ }^{\circ} \mathrm{K}$ ), and then only to calibrate a similar lamp as a working standard. In general even at lower currents a working standard should be prepared and used, except for purposes of checking the operation of such working standard.

\section{Conclusions}

The tungsten strip lamp is a useful working standard for use in spectral radiance measurements within the region of 0.25 to $2.6 \mu$. At wavelengths shorter than $0.25 \mu$ the available radiant energy from heated tungsten is too low for practical uses. At longer wavelengths than about $2.6 \mu$ the low emissivity of tungsten, together with window absorption and reradiation effects render the use of this type of lamp impractical. Some other source, in air or employing a special window, will be found more practical. The recent measurenents on the spectral emissivity of platinum from 2.0 to $15.0 \mu$ provides one possible standard for radiance at long wavelengths [14].

The method of calibration against a blackbody is direct and leaves no question relating to filament temperature or tungsten emissivity. The energy of the lamp is equated to that of the blackbody. The principal uncertainty in the results lie within the accurate determination of the blackbody temperatures and the measurement of the current througl? the lamp filament.

The authors thank Milton Burdick and H. S. Parker of the Engineering Ceramies Section of the Bureau for useful suggestions in developing the high temperature blackbody, and also William F. Roeser of the Building Technology Division for constructive criticisms.

\section{References}

[1] W. W. Coblentz, Measurements on standards of radiation in absolute value, Bull. Eur. Stds. 11, 87 (1914) SP227.

[2] IV. IW. Coblentz and R. Stair, The present status of the standards of thermal radiation maintained by the Bureau of Standards, BS J. Research 11, 79 (1933) RP578.

[3] Ralph Stair and Russell G. Johnston, Effects of recent knowledge of atomic constants and humidity on the calibrations of the National Bureau of Standards Thermal Radiation Standards, J. Research NBS 53, 211 (1954) RP2535.

[4] A. G. Worthing, The true temperature scale of tungsten and its emissivity powers at incandescent temperatures, Phys. Rev. 10, 377 (1917); Deviation from Lambert's law and polarization of light emitted by incandescent tungsten, tantalum and molybdenum and changes in the optical constants of tungsten with temperature, J. Opt. Soc. Am. 13, 635 (1926).

[5] H. C. Hamaker, Reflectivity and emissivity of tungsten (Inaug Diss, Utrecht, Holland, 1934).

[6] J. C. DeVos, Evaluation of the quality of a blackbody, Physica 20, 669 (1954). A new determination of the emissivity of tungsten ribbon, physica 20,690 (1954); relation between brightness, temperature, true temperature and colour temperature of tungsten. Luminance of tungsten, Physica 20, 715 (1954).

[7] Robert D. Larrabee, Spectral emissivity of tungsten, J. Opt. Soc. Am. 49, 619 (1959).

[8] R. Stair and W. O. Smith. A tungsten-in-quartz lamp and its applications in photoelectric radiometry, J. Research NBS 30, 449 (1943) RP1543.

[9] Ralph Stair, Thomas C. Bagg, and Russell G. Johnston, Continuous measurement of atmospheric ozone by an automatic photoelectric method, J. Research NBS 52, 133 (1954) RP2481.

[10] W. J. Condell, Jr., Tungsten spectral intensity calibration methods, U. Md. Report, REMP-3 1959.

[11] William J. Condell and Francis T. Byrne, Spectral intensity of ac operated tungsten lamps, J. Opt. Soc. Am. 4\%, 1, 135 (1957).

[12] Louis E. Barbrow, Memorandum on a procedure for obtaining spectral radiant intensity of tungsten filament, J. Opt. Soc. Am. 49, 1,122 (1959).

[13] André Gouffé, Aperture corrections for artificial blackbodies, Revue d'Optique, 24, Nos. 1-3 (1945).

[14] A. Maki, Ralph Stair, and Russell G. Johnston, Apparatus for the measurement of the normal spectral emissivity in the infrared, J. Research NBS 64C, 99 (1960).

[15] Ralph Stair, Photoelectric spectroradiometry and its application to measurement of fluorescent lamps, J. Res. NBS 46, 437 (1951) RP2212.

[16] F. Hoffman and H. Willenberg, Uber Messungen an Temperaturstrahlen im Ultraviolett, Phvsik Z. 35, 1 (1934). Das Emissionsvermogen des Wolframs im Ultraviolett bei hohen Temperature, Physik Z. 35, 713 (1934).

[17] L. S. Ornstein, Tables of the emissivity of tungsten as a function of wavelength from $0.23-2.0 \mu$ in the region of temperature $1,600^{\circ}-3.000^{\circ} \mathrm{K}$, Physica 3, 561 (1936).

[18] C. Zwikker, Physiche Eigenshappen Van Wolfraam by Hooge Temperaturen (thesis, Amsterdam, 1925).

Washington, D.C.

(Paper 64A4-51) 


\title{
Spectral Radiance of a Low Current Graphite Arc
}

\author{
Albert T. Hattenburg
}

The spectral radiance of the anode of a low current graphite arc has been determined throughout most of the $850-210 \mathrm{~nm}$ region by utilizing a recently developed high accuracy spectroradiometer. The estimated standard deviation uncertainty varies from about $1.5 \%$ at the longer wavelengths to about $5 \%$ at the shorter. Results are given at twenty wavelength points in the region and also as a continuous function of wavelength throughout most of the region, excluding some areas of high molecular band radiation originating in the arc stream.

\section{Introduction}

The low current graphite are has long been of interest as a bright, reproducible source of radiation. A number of workers ${ }^{1-6}$ have investigated the spectral radiance of this source using graphite and/or carbon (lampblack base) electrodes, and employing various techniques to yield absolute values. Nevertheless, at 400 nanometers $(4000 \AA)$, differences between two of the more widely used results ${ }^{4,5}$ are as high as $15 \%$; below $400 \mathrm{~nm}$ there have been fewer investigations and the uncertainties are greater. The recent development of a high accuracy spectroradiometer ${ }^{7}$, employing a high quality blackbody and accurate temperature measurement capability, made possible a more accurate determination of the are spectral radiance from $850 \mathrm{~mm}$ to $210 \mathrm{~nm}$. This paper presents the results of this determination at twenty fixed wavelength points in this region and, with somewhat less accuracy, the spectral radiance as a continuous function of wavelength throughout most of the region.

\section{Apparatus}

The spectroradiometer consists of a stable high temperature blackbody, a double monochromator with photomultiplier detector and associated electronics, and a set of auxiliary sources and filters used to determine the temperature of the blackbody. The blackbody is a resistively heated tube operated in an argon atmosphere, and capable of steady operation in the $3000{ }^{\circ} \mathrm{K}$ region for long periods of time. The emissivity is estimated to be about 0.999. The double monochromator is a quartz prism grating instrument

The author is with the National Bureau of Standards, Washington, D.C. 20234.

Received 5 August 1966.

This work was supported in part by the National Aeronautics and Space Administration. featuring negligible scattered light, a dispersion figure of 15-40 $\AA / \mathrm{mm}$ throughout the range employed, and a relative aperture of $f / 8$ in the vertical plane and $f / 16$ in the horizontal plane. The fourteen-stage photomultiplier tube employs a trialkali cathode and a quartz window. The tube current is amplified by a linear de amplifier and displayed on a potentiometric recorder. A portion of the spectroradiometer operates as a photoelectric pyrometer and has been given a primary calibration on the International Practical Temperature Scale (IPTS) (Ref. 8).

Table i. Spectral Radiance of the Graphite Arc at Selected Wavelengths ${ }^{a}$

\begin{tabular}{lc}
$\begin{array}{c}\lambda \\
(\mathrm{nm})\end{array}$ & $\begin{array}{c}L_{\lambda} \\
\left(\mathrm{W} / \mathrm{cm}^{3} \mathrm{sr}\right)\end{array}$ \\
\hline 210 & $4.72 \times 10^{4}$ \\
220 & 3.83 \\
230 & 3.98 \\
240 & 3.21 \\
250 & 4.11 \\
260 & 5.39 \\
280 & 9.69 \\
300 & 16.6 \\
330 & 32.6 \\
365 & 61.1 \\
395 & 92.3 \\
430 & 123 \\
480 & 175 \\
525 & 220 \\
600 & 279 \\
654.6 & 306 \\
700 & 318 \\
750 & 324 \\
800 & 324 \\
850 & 315 \\
\hline
\end{tabular}

a The spectral bandpass varied from about $1.5 \AA$ to $4 \AA$ at the short and long wavelengths respectively. The standard deviation uncertainties range from about $4 \%$ at $210 \mathrm{~nm}$ to $1.5 \%$ at $850 \mathrm{~nm}$. 


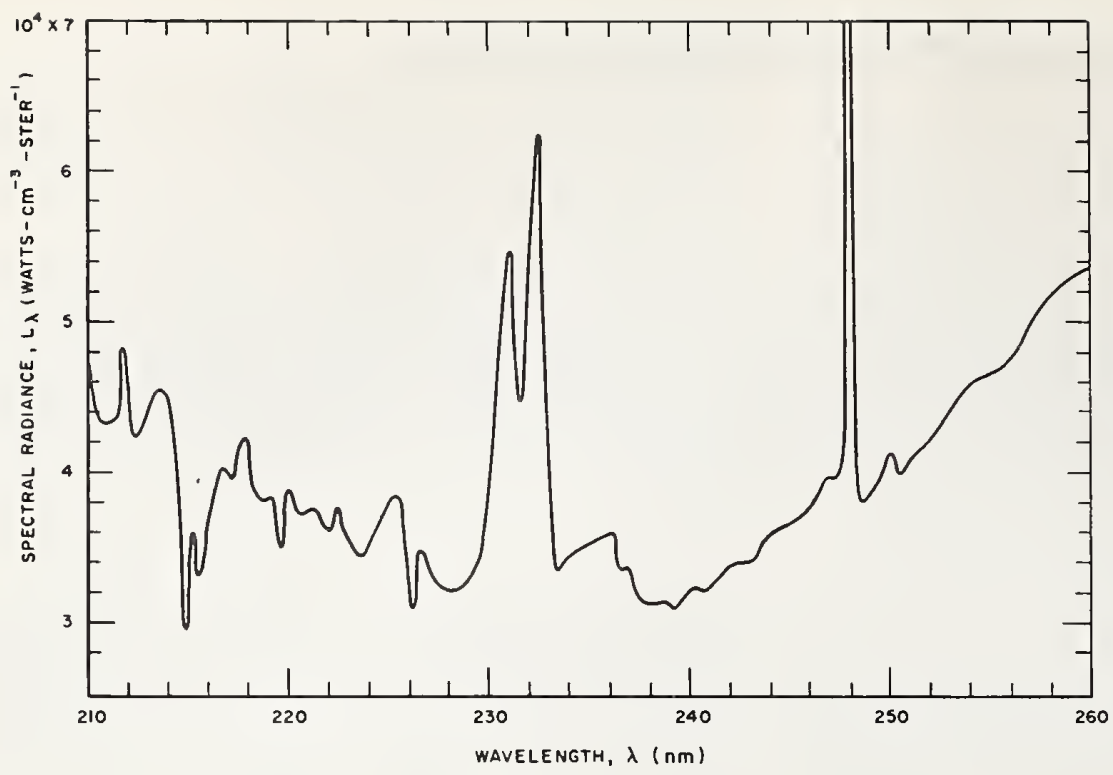

Fig. 1. Spectral radiance of the arc at the shorter wavelengths. The spectral band-pass varied from about $1.5 \AA$ at $210 \mathrm{~nm}$ to 2 $\AA$ at $260 \mathrm{~nm}$. The standard deviation uncertainties were about $5 \%$ to $3 \%$ at the short and long wavelengths, respectively.

The arc employed was a commercial device manufactured by the Mole-Richardson Company, and designated as the Pyrometric Molarc Lamp type 2371. It was operated in a manner recommended by the manufacturer. It employs a rate adjustable continuous feed mechanism for both electrodes as well as the necessary manual adjustments, a $120^{\circ}$ angle between electrodes, and an imaging screen to make possible continuous monitoring of the electrode positions. The arc power supply consisted of a $120-\mathrm{V}$ battery, a suitable resistor for ballast, and a carbon pile rheostat for fine current adjustment. The anodes were National Carbon SPK graphite, $0.63 \mathrm{~cm}$ diam, and the negative electrodes were National Carbon AGKS graphite, 0.32 $\mathrm{cm}$ diam. The anode was burned for at least $10 \mathrm{~min}$ before any data were taken. The arc was operated at the current approximately $0.25 \mathrm{~A}$ below the overload point. The electrode spacing was constantly monitored to maintain the anode face in the plane normal to the anode axis, and focused on the slit of the monochromator. The target area observed was $0.1 \mathrm{~mm}$ wide and $0.8 \mathrm{~mm}$ high, centered on the anode.

All sources were mounted on a motor-driven lathe bed to permit rapid and precise positioning of each image on the slit.

\section{Experimental Procedure}

The spectral radiance of the anode, as viewed through the arc stream, was determined at a number of selected wavelengths from $850 \mathrm{~nm}$ to $210 \mathrm{~nm}$. These measurements were made at fixed wavelengths so that short term variations and noise in the are signal could be averaged for each wavelength, and the arc current could more conveniently be maintained near the over-

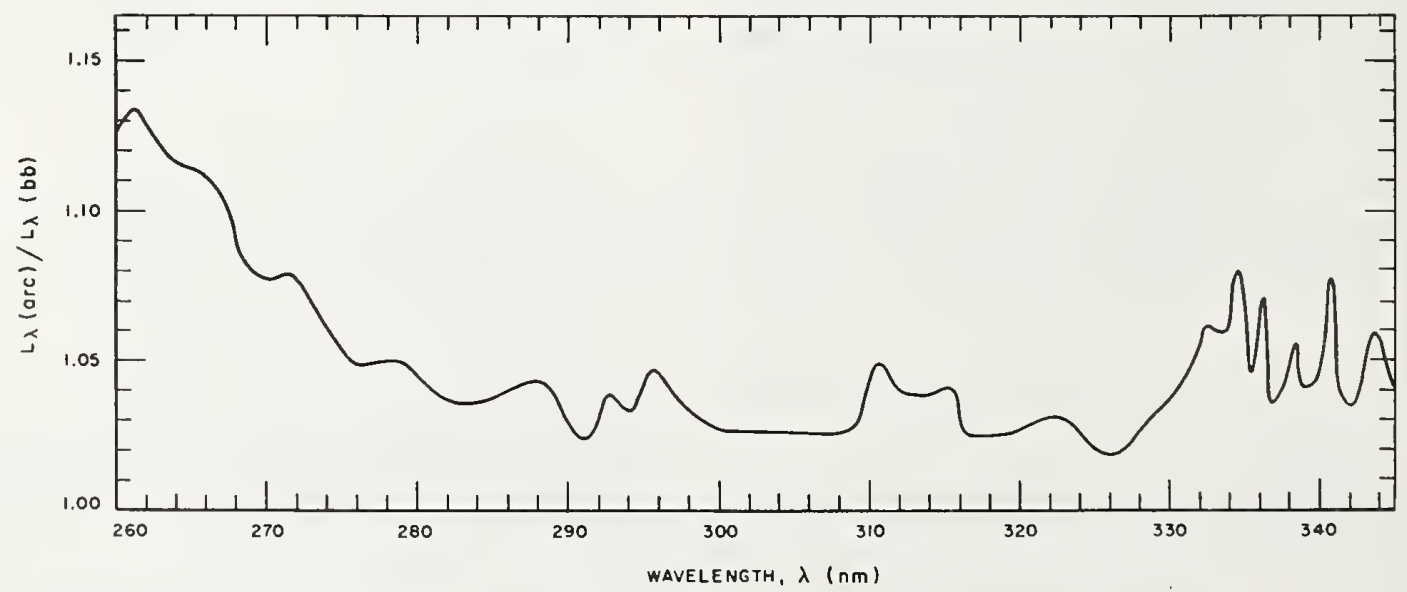

Fig. 2. Spectral radiance of the are relative to a $3792^{\circ} \mathrm{K}$ (IPTS) blackbody. The spectral bandpass varied from about $2 \AA$ at $260 \mathrm{~nm}$ to $3 \AA$ at $345 \mathrm{~nm}$. The standard deviation uncertainty was about $2.5 \%$ in this region. 


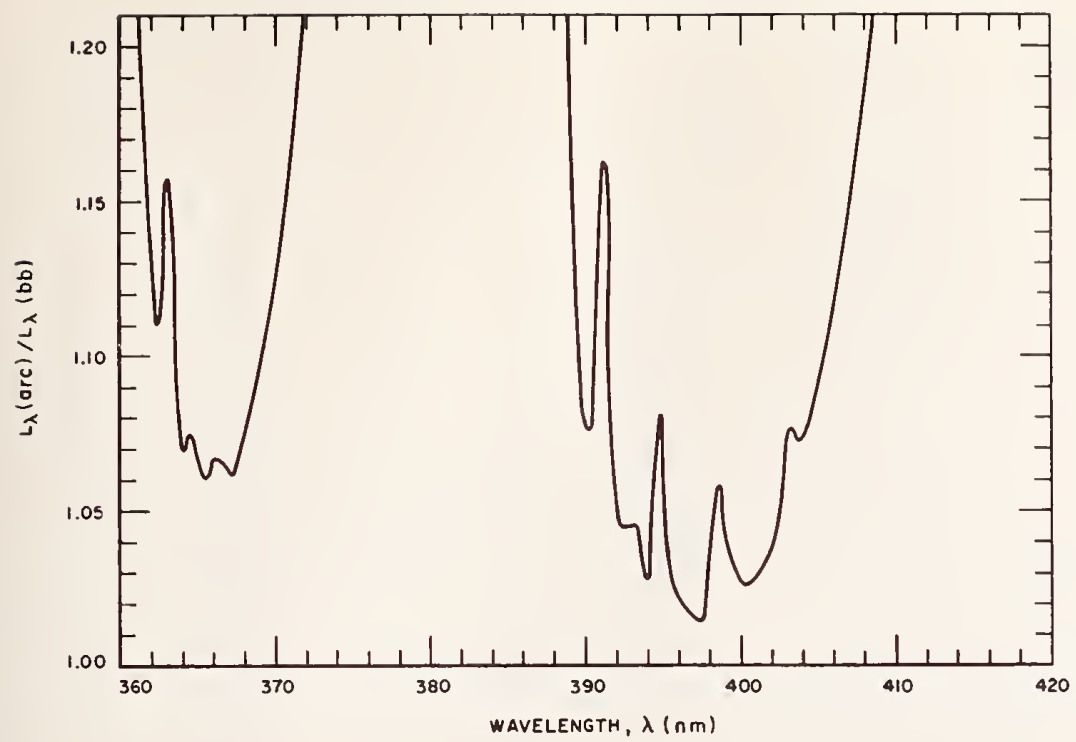

Fig. 3. Spectral radiance of the arc relative to a $3792^{\circ} \mathrm{K}$ (IPTS) blackbody. The spectral bandpass varied from $3 \AA$ to $360 \mathrm{~nm}$ to $3.5 \AA$ at $420 \mathrm{~nm}$. The standard deviation uncertainty was about $2 \%$ in this region.

load point. The wavelengths longer than $270 \mathrm{~nm}$ were selected on the basis of a small radiance contribution from the arc stream, and therefore from line or band spectra. Continuous scans of the are stream alone, with a spectral slit width of $0.2 \AA$, defined these areas. At wavelengths shorter than $270 \mathrm{~nm}$, where the arc stream contributed significantly at all wavelengths, the measurements were made at $10 \mathrm{~nm}$ intervals.

The normal use of the spectroradiometer involves comparing the source to be calibrated with the high temperature blackbody, which is adjusted to be equal to the source at each wavelength. Since the spectral radiance of the arc exceeded that of the blackbody by factors as large as four thousand at $210 \mathrm{~nm}$, this pro- cedure was modified to permit determination of the spectral radiance ratio of two unequal sources.

The blackbody was set at a high temperature (about $\left.2850^{\circ} \mathrm{K}\right)$. This temperature was measured before and after each set of comparisons. The arc was compared with the blackbody at each chosen wavelength by alternately centering the images of the two sources on the monochromator slit. Then the ratio of spectral radiances was obtained from the ratio of the electrical currents of the photomultiplier tube corresponding to the two radiation signals, after being amp ified and presented on the recorder. The departure from linearity of the detector-amplifier response was determined at two wavelengths, and found to be independent of

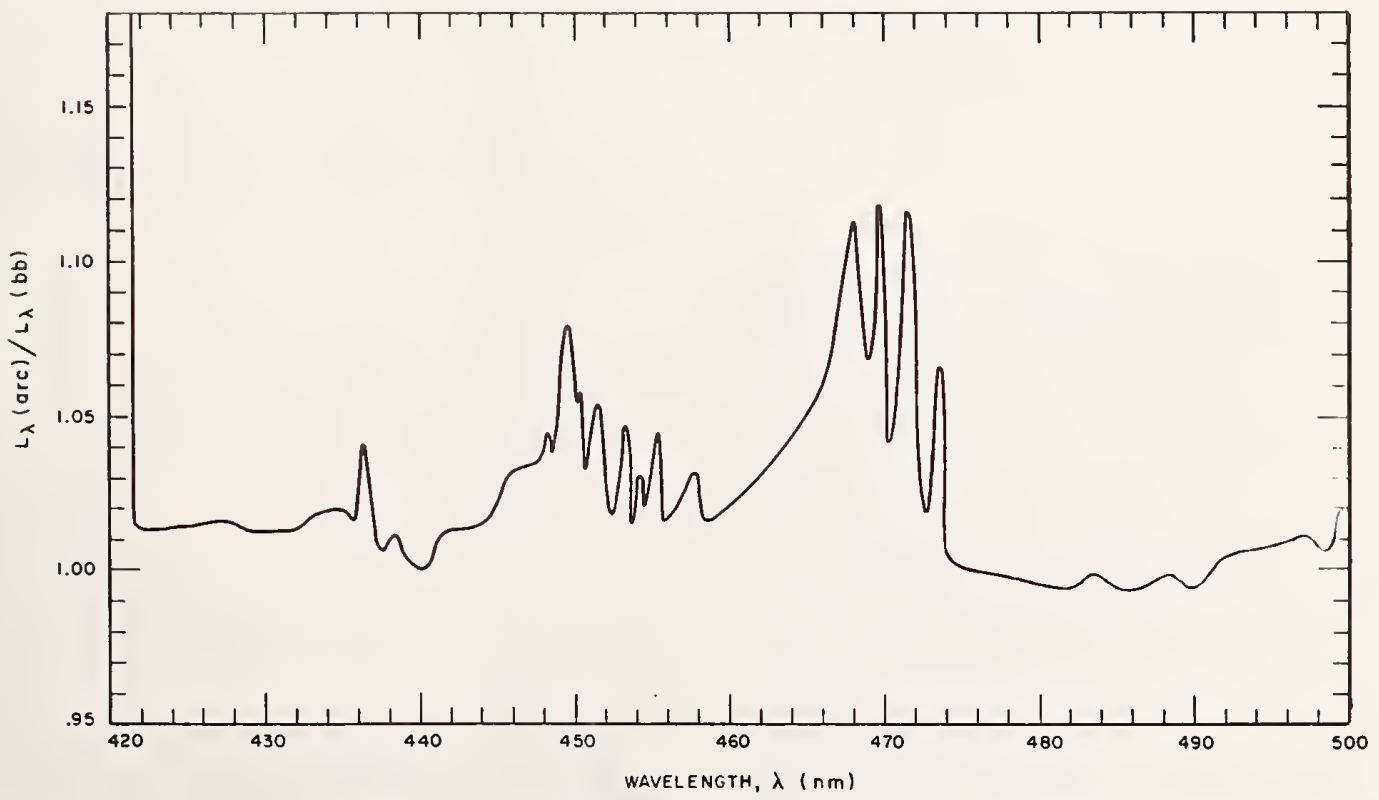

Fig. 4. Spectral radiance of the arc relative to a $3792^{\circ} \mathrm{K}$ (IPTS) blackbody. The spectral bandpass was about $4 \AA$. The standard deviation uncertainty was about $2 \%$. 


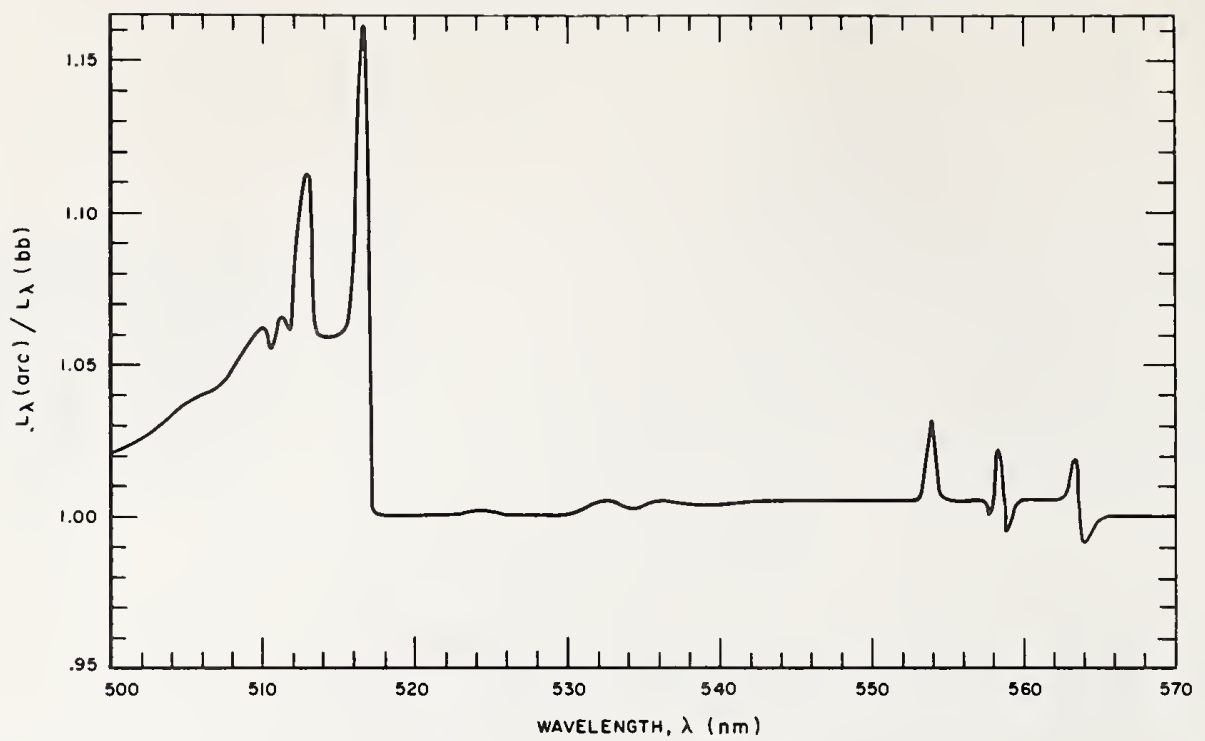

Fig. 5. Spectral radiance of the arc relative to a $3792^{\circ} \mathrm{K}$ (IPTS) blackbody. The spectral bandpass was about $4 \AA$. The standard deviation uncertainty was about $2 \%$.

wavelength and to vary from about $0.5 \%$ at the lower ratios to $4 \%$ for those at about 4400 .

The spectral radiance of the arc was determined as a continuous function of wavelength by averaging three successive wavelength scans of the arc over regions of 50-100 nin-each of which included one or more of the fixed points-and comparing the result to a similar scan with the blackbody or a spectrally calibrated tungsten lamp. The two sets of curves were normalized through the absolute calibration of the arc at the fixed wavelengths. Regions of high molecular band radiation between $345 \mathrm{~nm}$ and $425 \mathrm{~nm}$ were excluded. The proximity of the arc to the overload current was checked at intervals throughout the scans.

\section{Results}

The spectral radiance values of the arc at the selected wavelengths are presented in Table I. Although these values were derived from a comparison with a blackbody whose temperature was determined on the IPTS, they represent values based on a best approximation to the Thermodynamic Kelvin Temperature Scale (TKTS). The normal practice of reporting temperatures on the IPTS ${ }^{\circ}$ is followed here because of the international acceptance of this seale and the relative ease of realizing and maintaining it. Nevertheless, when using a temperature to calculate another physical parameter in an equation from thermodynamics or statistical mechanics, it is necessary, for the most accurate result, to convert to the Thermodynamic Kelvin Temperature Scale.

The ratio of spectral radiances of two blackbodies does not depend upon a temperature scale. It follows that for two such blackbodies, one of which is at the temperature of freezing gold $\mathrm{T}_{\mathrm{Au}}$,

$$
L_{\lambda T}=L_{\lambda} T_{\mathrm{Au}}\left(L_{\lambda} T / L_{\lambda} T_{\mathrm{Au}}\right)=L_{\lambda} T_{\mathrm{Au}}\left(L_{\lambda} T^{\prime} / L_{\lambda} T_{\mathrm{Au}}{ }^{\prime}\right) .
$$

Using the Planck radiation equation and rearranging,

$$
L_{\lambda T}=L_{\lambda T^{\prime}}\left[\frac{\exp \left(c_{2}{ }^{\prime} / \lambda_{T_{A u}}{ }^{\prime}\right)-1}{\exp \left(c_{2} / \lambda_{T A u}\right)-1}\right] .
$$

If the primed and unprimed quantities represent values on the IPTS and the TKTS, respectively, the expression in brackets is the factor that converts values of spectral radiance calculated from an IPTS temperature to those calculated from a TKTS temperature. The required IPTS values are $c_{2}{ }^{\prime}=1.438 \mathrm{~cm}{ }^{\circ} \mathrm{K}$ and $T_{\mathrm{Au}}{ }^{\prime}=1336.15^{\circ} \mathrm{K}$; the TKTS values chosen here are $c_{2}=1.43879 \mathrm{~cm}{ }^{\circ} \mathrm{K}$ (Ref. 10) and $T_{\mathrm{Au}}=1337.65^{\circ} \mathrm{K} .{ }^{11}$ Using the Wien approximation to the Planck function in the ratio of gold point radiances, expanding the resulting exponential, and retaining only the first two terms, we have

$$
L_{\lambda} T(\mathrm{TKTS})=L_{\lambda} T(\mathrm{IPTS})\left[1+\left(0.616 \times 10^{-6} / \lambda\right)\right],
$$

where $\lambda$ is in centimeters. The spectral radiance values of Table I were calculated from Eq. 3.

The spectral radiance of the arc as a function of wavelength from $210 \mathrm{~nm}$ to $260 \mathrm{~nm}$ is presented in Fig. 1. The ratio of the arc spectral radiance to that of a $3792^{\circ} \mathrm{K}$ (IPTS) blackbody over most of the $260-570 \mathrm{~nm}$ region is given in Figs. 2, 3, 4, and 5. From $570 \mathrm{~nm}$ to $850 \mathrm{~nm}$ the ratio is equal to one; i.e., the arc radiance is equivalent to the radiance of a $3792^{\circ} \mathrm{K}$ (IPTS) blackbody to well within the experimental error. This presentation of the data was chosen because the arc deviates in radiance from a fixed temperature blackbody rather slowly in the region above $260 \mathrm{~nm}$. Table II lists the spectral radiance for the $3792^{\circ} \mathrm{K}$ (IPTS) blackbody as ealculated from Eq. 3. Thus, the spectral radiance of 
Table II. Spectral Radiance of a $3792^{\circ} \mathrm{K}$ (IPTS) Blackbody ${ }^{\circ}$

\begin{tabular}{|c|c|c|c|}
\hline$\stackrel{\lambda}{\lambda}$ & $\begin{array}{c}L_{\lambda} \\
\left(\mathrm{W} / \mathrm{cm}^{3} \mathrm{sr}\right)\end{array}$ & $\begin{array}{c}\lambda \\
(\mathrm{nm})\end{array}$ & $\begin{array}{c}L_{\lambda} \\
\left(\mathrm{W} / \mathrm{cm}^{3} \mathrm{sr}\right)\end{array}$ \\
\hline 260 & $4.753 \times 10^{4}$ & 560 & $250.8 \times 10^{4}$ \\
\hline 265 & 5.687 & 565 & 254.7 \\
\hline 270 & 6.749 & 570 & 258.5 \\
\hline 275 & 7.945 & 575 & 262.2 \\
\hline 280 & 9.284 & 580 & 265.7 \\
\hline 285 & 10.77 & 585 & 269.2 \\
\hline 290 & 12.42 & 590 & 272.6 \\
\hline 295 & 14.22 & 595 & 275.8 \\
\hline 300 & 16.20 & 600 & 278.9 \\
\hline 305 & 18.34 & 605 & 281.9 \\
\hline 310 & 20.65 & 610 & 284.8 \\
\hline 315 & 23.15 & 615 & 287.6 \\
\hline 320 & 25.81 & 620 & 290.3 \\
\hline 325 & 28.66 & 625 & 292.9 \\
\hline 330 & 31.58 & 630 & 295.3 \\
\hline 335 & 34.87 & 635 & 297.7 \\
\hline 340 & 38.23 & 640 & 299.9 \\
\hline 345 & 41.77 & 645 & 302.1 \\
\hline 350 & 45.47 & 650 & 304.1 \\
\hline 355 & 49.32 & 655 & 306.0 \\
\hline 360 & 53.33 & 660 & 307.8 \\
\hline 365 & 57.49 & 665 & 309.5 \\
\hline 370 & 61.80 & 670 & 311.1 \\
\hline 375 & 66.23 & 675 & 312.6 \\
\hline 380 & 70.79 & 680 & 314.0 \\
\hline 385 & 75.48 & 685 & 315.4 \\
\hline 390 & 80.27 & 690 & 316.6 \\
\hline 395 & 85.16 & 695 & 317.7 \\
\hline 400 & 90.15 & 700 & 318.7 \\
\hline 405 & 95.23 & 705 & 319.7 \\
\hline 410 & 100.4 & 710 & 320.5 \\
\hline 415 & 105.6 & 715 & 321.3 \\
\hline 420 & 110.9 & 720 & 322.0 \\
\hline 425 & 116.2 & 725 & 322.6 \\
\hline 430 & 121.6 & 730 & 323.1 \\
\hline 435 & 126.9 & 735 & 323.5 \\
\hline 440 & 132.4 & 740 & 323.9 \\
\hline 445 & 137.8 & 745 & 324.2 \\
\hline 450 & 143.2 & 750 & 324.4 \\
\hline 455 & 148.7 & 755 & 324.5 \\
\hline 460 & 154.1 & 760 & 324.6 \\
\hline 465 & 159.5 & 765 & 324.6 \\
\hline 470 & 164.9 & 770 & 324.5 \\
\hline 475 & 170.2 & 775 & 324.4 \\
\hline 480 & 175.5 & 780 & 324.2 \\
\hline 485 & 180.8 & 785 & 323.9 \\
\hline 490 & 186.0 & 790 & 323.6 \\
\hline 495 & 191.1 & 795 & 323.2 \\
\hline 500 & 196.2 & 800 & 322.8 \\
\hline 505 & 201.2 & 805 & 322.3 \\
\hline 510 & 206.2 & 810 & 321.8 \\
\hline 515 & 211.1 & 815 & 321.2 \\
\hline 520 & 215.8 & 820 & 320.6 \\
\hline 525 & 220.5 & 825 & 319.9 \\
\hline 530 & 225.2 & 830 & 319.2 \\
\hline 535 & 229.7 & 835 & 318.4 \\
\hline 540 & 234.1 & 840 & 317.6 \\
\hline 545 & 238.4 & 845 & 316.7 \\
\hline 550 & 242.6 & 850 & 315.8 \\
\hline 555 & 246.8 & & \\
\hline
\end{tabular}

a The values in this table are calculated from Eq. 3 and therefore represent values based on the Thermodynamic Kelvin Temperature Scale. the arc can be obtained by selecting the ratio for the desired wavelength (unity above $570 \mathrm{~nm}$ ) and multiplying by the spectral radiance from Table II.

These results were obtained under specific conditions of $f$ number, spectral bandpass, and target area. Experimental checks should be performed when deviating significantly from these values. Use of an arc stand of different geometry may also alter the results, particularly below $280 \mathrm{~nm}$. The barometric pressure was about $760 \mathrm{~mm} \mathrm{Hg}$ for this work. Chaney et al. ${ }^{12}$ report a value of about $0.2^{\circ} \mathrm{K} / \mathrm{mm} \mathrm{Hg}$ for the variation in anode brightness temperature with pressure.

\section{Uncertainties}

The uncertainties in the spectral radiance for the fixed wavelength points are caused primarily by the variations in the are itself (including variations between anodes of the same and different lots), the response characteristic of the photomultiplier-electronics system, the calibration of the spectroradiometer, and the uncertainty of the Thermodynamic Kelvin Temperature Scale. Additional uncertainties arise in the case of the continuous scans because of the lack of signal averaging and the greater difficulty in maintaining the arc current near overload.

The arc variations are reflected in the standard deviations of the individual measurements, and vary from about $1.3 \%$ to $2.5 \%$ from $850 \mathrm{~nm}$ to $210 \mathrm{~nm}$. The calibration of the system response is uncertain to about $0.5 \%$ for values down to $240 \mathrm{~nm}$, and increases to about $3 \%$ at $210 \mathrm{~nm}$. The spectroradiometer has a standard deviation uncertainty of about $0.4 \%$ at $850 \mathrm{~nm}$ and $1.1 \%$ at $210 \mathrm{~nm}$ relative to the IPTS. Conversion to the TKTS adds about $0.25 \%$ at $850 \mathrm{~nm}$ and $1 \%$ at $210 \mathrm{~nm}$. The additional error for the continuous scans is estimated to be about $1 \%$ at the long wavelengths and $2 \%$ at the short. Combining these results in quadrature yields a standard deviation uncertainty of $1.4 \%$ at $850 \mathrm{~nm}, 2.6 \%$ at $240 \mathrm{~nm}$, and $4.2 \%$ at $210 \mathrm{~nm}$ for the fixed points, while the corresponding figures for the continuous scans are $1.7 \%, 3.0 \%$ and $4.7 \%$.

The author wishes to thank H. J. Kostkowski, under whose general direction this work was performed, for his many suggestions and criticisms, and R. C. Hughes for his contributions to the experimental setups, data collection, and reduction.

\section{References}

1. H. G. MacPherson, J. Opt. Soc. Am. 30, 189 (1940).

2. D. M. Packer and C. Lock, J. Opt. Soc. Am. 42, 879 (1952).

3. F. S. Johnson, J. Opt. Soc. Am. 46, 103 (1956).

4. J. Euler and R. Ludwig, Arbeitsmethoden der Optischen Pyrometrie (Verlag Braun, Karlsruhe, 1960).

5. M. R. Null and W. W. Lozier, J. Opt. Soc. Am. 52, 1156 (1962).

6. H. Magdeburg, Z. Naturforsch. 20a, 980 (1965).

7. H. J. Kostkowski, C. R. Yokley, D. E. Erminy, and A. T. Hattenburg, NBS Report 9099, 1965. 
8. H. J. Kostkowski and R. D. Lee, in Temperalure, Its Measurement and Control in Science and Industry, C. F. Herzfeld, Ed. (Reinhold Publishing Corp., New York, 1962) Vol. 3, Part 1, p. 449.

9. H. F. Stimson, J. Res. Natl. Bur. Std. 65a, 139 (1961).

10. Natl. Bur. Std. Tech. News Bull. 47, 175 (1963).
11. H. Moser, in Temperature, Its Measurement and Control in Science and Industry, C. F. Herzfeld, Ed. (Reinhold Publishing Corp., New York, 1962) Vol. 3, Part 1, p. 167.

12. N. K. Chaney, V. C. Hamister, and S. W. Glass, Trans. Am. Electrochem. Soc. 67, 107 (1935).

APPLIED OPTICS / Vol. 6, No. 1 / January 1967 


\title{
HIGH-ACCURACY SPECTRAL RADIANCE CALIBRATION OF TUNGSTEN-STRIP LAMPS*
}

\author{
H. J. Kostkowski, D. E. Erminy, and A. T. Hattenburg
}

\subsection{INTRODUCTION}

The capability of determining spectral radiance of tungsten-strip lamps with an uncertainty of less than $1 \%$ has recently been reported (Kostkowski et al., 1965, work to be published). This chapter is a summary of this work, particularly those aspects that might be generally useful in radiometry.

The approach that was used in the spectral radiance calibration was the classical method of comparing the unknown to a blackbody. This method was undertaken because it is the basis of radiation pyrometry where, at least at one wavelength and relative to the International Practical Temperature Scale, considerable success has been achieved (Lee, 1966).

It is generally accepted that the spectral radiance of a blackbody is given by the Planck radiation equation

$$
L_{\mathrm{b} \lambda}=C_{1} \lambda^{-5} /\left[\exp \left(C_{2} / \lambda T\right)-1\right]
$$

Experimental verification of this equation has not been realized to better than about $5 \%$. Nevertheless, because of its strong theoretical base, it has been assumed that the above relation is exact. Therefore, a spectral comparison of a source with a blackbody will result in the determination of the spectral radiance of the source with an accuracy which depends on the accuracy of $C_{1}, C_{2}, \lambda$, and $T$; the quality of the blackbody; and the accuracy of the comparison.

The initial goal in our effort was a total uncertainty of $1 \%$ or less. Therefore, an attempt was made to limit individual sources of error in terms of spectral radiance to about $0.1 \%$. The question arises as to how well the abore constants are known and how well the other parameters would have to be determined to achieve this criterion.

* The research associated with this chapter (which is an official contribution of the U.S. National Bureau of Standards and not subject to copyright) was supported in part by the National Aeronautics and Space Administration. 


\subsection{Accuracy Requirements}

4.2.1. Accuracy of $C_{1}$

The first radiation constant $C_{1}$ is related to the velocity of light $c$ and the Planck constant $h$ by the equation

$$
C_{1}=2 c^{2} h
$$

The Committee on Fundamental Constants of the National Academy of Science-National Research Council has recommended (1963) the value $C_{1} \doteq 1.19096 \times 10^{-12} \mathrm{~W} \mathrm{~cm}^{2}(\mathrm{sr})^{-1}$ and estimates an uncertainty in $C_{1}$ in terms of one standard deviation of $0.0027 \%$. Thus, $C_{1}$ is reported to be known about 40 times more accurately than required.

\subsubsection{Accuracy of $\mathrm{C}_{2}$}

The second radiation constant $C_{2}$ is related to the atomic constants by the equation

$$
C_{2}=h c / k
$$

where $k$ is the Boltzmann constant. The above mentioned committce has also rccommended a value of $C_{2}=1.43879 \mathrm{~cm}$ deg with an uncertainty in terms of one standard deviation of $0.0042 \%$. This corresponds to an error of $0.045 \%$ for the spectral radiance in Eq. (4.1) at a wavelength of $550 \mathrm{~nm}$ and $2500^{\circ} \mathrm{K}$, a typical strip-lamp brightness temperature. Thus, $C_{2}$ is also known sufficiently well for the $0.1 \%$ criterion.

\subsubsection{Wavelength Accuracy}

The wavelength accuracy required in comparing a blackbody to a strip lamp can easily be obtained from the equation

$$
\frac{T_{1}-T_{2}}{T_{1} T_{2}}=\frac{\lambda_{2}-\lambda_{1}}{\lambda_{1}}\left(\frac{1}{T_{1}}-\frac{1}{T_{\mathrm{c}}^{\prime}}\right) \cong \frac{\Delta T}{T_{1}{ }^{2}}
$$

which is commonly used in optical pyrometry (Kostkowski and Lee, 1962) to relate the brightness temperature $T_{1}$ at a wavelength $\lambda_{1}$ to the brightness tempcrature $T_{2}$ at another wavelength $\lambda_{2}$, where $T_{\mathrm{c}}$ is the color temperature as defined in the reference given above. Using Wien's equation as an approximation to Planck's equation, i.e., dropping the -1 in Eq. (4.1) and differentiating with respect to $T$, one obtains

$$
\frac{d L_{\mathrm{b} \lambda}}{L_{\mathrm{b} \lambda}}=\frac{C_{2}}{\lambda T} \frac{d T}{T}
$$


Combining this with Eq. (4.4)

$$
\frac{\Delta L_{\mathrm{b} \lambda}}{L_{\mathrm{b} \lambda}} \cong \frac{C_{2}}{\lambda_{2}}\left(\frac{1}{T_{1}}-\frac{1}{T_{\mathrm{c}}}\right) \Delta \lambda
$$

Assuming $\Delta L / L=0.001, T_{1}=2500^{\circ} \mathrm{K}, \lambda=550 \mathrm{~nm}$, and using $T_{\mathrm{c}} \simeq 2860^{\circ} \mathrm{K}$ (obtained for tungsten from the "Handbook of Physics and Chemistry"), one obtains $\Delta \lambda \simeq 0.5 \mathrm{~nm}$. This then is the wavelength uncertainty permitted at $550 \mathrm{~nm}$ in spectrally comparing the blackbody to a tungsten-strip lamp.

\subsubsection{Accuracy of Blackbody Temperature}

The uncertainty permitted for the temperature of the blackbody can be obtained from Eq. (4.5). Solving for $\Delta T$ for $\lambda=550 \mathrm{~nm}, \Delta L / L=0.001$, and $T=2500^{\circ} \mathrm{K}$, results in $\Delta T=0.24^{\circ}$.

\subsubsection{Blackbody Quality}

To satisfy our criterion, the blackbody must have an emissivity of about 0.999 and a stability of $0.1 \%$. A rough estimate of the permissible temperature nonuniformity can be obtained as follows. Assume that the emissivity of the cavity material is 0.9 and the walls of the cavity have either a tempera. ture $T$ or $T-\Delta T$, with each irradiating the wall target area equally as illustrated in Fig. 4.1. To a first approximation

$$
\begin{gathered}
L_{\lambda}\left(\begin{array}{c}
\text { vargel } \\
\text { tarel }
\end{array}\right) \cong 0.9 L_{\mathrm{b} \lambda}(T)+0.1 \times 0.5 L_{\mathrm{b} \lambda}(T)+0.1 \times 0.5 L_{\mathrm{b} \lambda}(T-\Delta T) \\
\cong 0.95 L_{\mathrm{b} \lambda}(T)+0.05\left[L_{\mathrm{b} \lambda}(T)-\frac{\Delta L_{\mathrm{b} \lambda}}{\Delta T} \Delta T\right]
\end{gathered}
$$

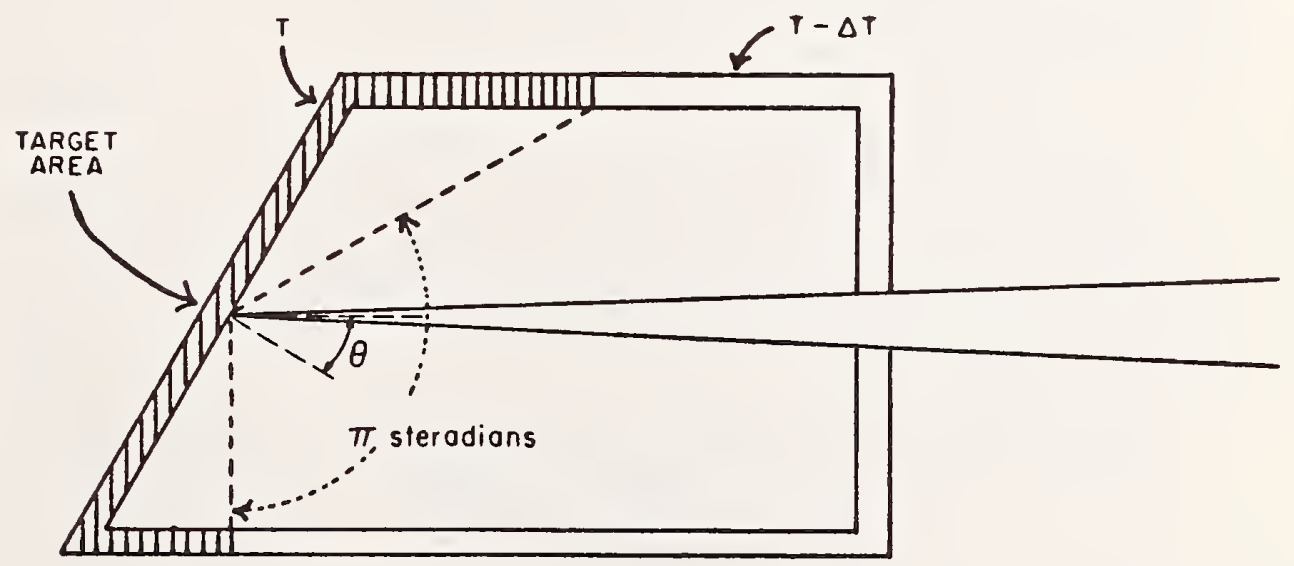

Fia. 4.1. Cavity with nonuniform wall temperaturo. 
and using Eq. (4.3)

$$
\begin{aligned}
L_{\lambda}\left(\begin{array}{c}
\text { wall } \\
\text { target }
\end{array}\right) \cong & 0.95 L_{\mathrm{b} \lambda}(T)+0.05\left[L_{\mathrm{b} \lambda}(T)-\frac{C_{2}}{\lambda T^{2}} L_{\mathrm{b} \lambda}(T) \Delta T\right] \\
\cong & L_{\mathrm{b} \lambda(T)}\left[1-0.05 \frac{C_{2}}{\lambda T^{2}} \Delta T\right] \\
& \frac{L_{\lambda}\left(\begin{array}{c}
\text { wall } \\
\text { (argef })
\end{array}\right)}{L_{\mathrm{b} \lambda}(T)}=\varepsilon_{\text {effectivc }}=1-0.05 \frac{C_{2}}{\lambda T^{2}} \Delta T
\end{aligned}
$$

assuming $\varepsilon_{\text {effective }}=0.999, T=2500^{\circ} \mathrm{K}$, and $\lambda=550 \mathrm{~nm}, \Delta T \simeq 5^{\circ}$.

\subsubsection{Accuracy of Comparison}

The accuracy of the blackbody-strip lamp comparison must be 0.999 and $L_{\lambda}$ should not drift by more than $0.1 \%$ in the length of time required for the calibration and its application.

These then are the general requircments. The remainder of the chapter will consider the design and techniques utilized in trying to realize these requirements and the results that have been achieved.

\subsection{Tile BLACKBOdy}

\subsubsection{Description}

The blackbody used for this work consisted of a graphite tube resistively heated in an argon atmosphere. The major features of the blackbody and control system are shown in Figs. 4.2 and 4.3, respectively. With this control system, it has been possible to limit the instability and drift to about $0.1 \%$ ov(r n 30-10 45-min perior. 'I'his is sufficient time in which to determine the blackbody temperature, make a comparison at some wavelength, and again check the temperature.

\subsubsection{Temperature Uniformity}

Limiting the temperature nonuniformity to about $5^{\circ}$ was achieved by modifying the shape of the graphite tube until, when sighting with a visual optical pyrometer at the cavity hole on the side or at the various holes along the axis of the tube from the top, this condition was confirmed.

\subsubsection{Emissivity}

The emissivity of the blackbody cavity depends on the geometry and dimensions of the cavity and on the partial reflectivity (deVos, 1954) of the 
material, particularly the wall opposite the cavity sighting hole. To a first approximation, which is usually quite good in high quality cavities,

$$
\varepsilon=1-r \Omega
$$

where $r$ is the partial reflectivity and $\Omega$ is the solid angle originating at the wall target and subtended by the cavity opening.

The partial reflectivity of the wall target, rclative to an incident and reflected angle $\theta$ (as shown in Fig. 4.1), was minimized by having the wall sliaped like a coarse thread. Laboratory measurements of the room temperature partial reflectivity, which could easily be performed with a visual pyrometer, resulted in a value of $0.02(\mathrm{sr})^{-1}$. Using Eq. (4.8), an $\varepsilon=0.999$, and $r=0.02(\mathrm{sr})^{-1}$, the required $\Omega$ is $0.033 \mathrm{sr}$. Thus, since $\Omega \simeq \pi R^{2} / D^{2}$ and the depth of the cavity $D$ is about $10 \mathrm{~mm}$, the radius of the cavity hole $R$ required to realize an emissivity of 0.999 is $1 \mathrm{~mm}$. This is the size of the cavity opening adopted and usually used.

A more direct ineasurement was employed to confirm the 0.999 emissivity. The method consists of making changes in $\Omega$ and determining the relative change of spectral radiance at two widely spaced wavelengths, say 325 and $650 \mathrm{~nm}$. A convenient equation (Kostkowski, 1966) to use in predicting the change is

$$
\frac{L_{\lambda^{\prime}}^{(2)}}{L_{\lambda^{\prime}}^{(1)}}=\left(\frac{\varepsilon_{1}(\lambda) \tau_{1}(\lambda)}{\varepsilon_{2}(\lambda) \tau_{2}(\lambda)}\right)^{\lambda / \lambda^{\prime}} /\left(\frac{\varepsilon_{1}\left(\lambda^{\prime}\right) \tau_{1}\left(\lambda^{\prime}\right)}{\varepsilon_{2}\left(\lambda^{\prime}\right) \tau_{2}\left(\lambda^{\prime}\right)}\right)
$$

Equation (4.9) gives the ratio of spectral radiance of source 2 to that of source 1 at a wavelength $\lambda^{\prime}$, when the two sources are adjusted to have the same spectral radiance at wavelength $\lambda$. The $\varepsilon$ 's are the spectral emissivities of the emitters and the $\tau$ 's are transmittances of any windows surrounding the emitters. If there are no windows and if we nssume that the sources are gray bodies, Eq. (4.9) becomes

$$
\frac{L_{\lambda^{\prime}}^{(2)}}{L_{\lambda^{\prime}}^{(1)}}=\left(\frac{\varepsilon_{1}}{\varepsilon_{2}}\right)^{\lambda / \lambda^{\prime}} /\left(\frac{\varepsilon_{1}}{\varepsilon_{2}}\right)=\left(\frac{\varepsilon_{1}}{\varepsilon_{2}}\right)^{\left(\lambda-\lambda^{\prime}\right) / \lambda^{\prime}}
$$

Thus, if it is assumed that the graphite cavity is at least a gray body, the ratio of the spectral radiances at $\lambda^{\prime}$ of two sucli cavitics, with exiting angles of $\Omega_{1}$ and $\Omega_{2}$, is

$$
\frac{L_{\lambda^{\prime}}^{(2)}}{L_{\lambda^{\prime}}^{(1)}}=\left(\frac{1-r \Omega_{1}}{1-r \Omega_{2}}\right)^{\left(\lambda-\lambda^{\prime}\right) / \lambda^{\prime}}
$$

provided that the power input is adjusted so that their spectial radiances are equal at wavelength $\lambda$. Determination of this ratio for ineasured values 
of $\Omega_{1}$ and $\Omega_{2}$ thus permits a determination of the partial reflectivity. These measurements were performed, and within the total precision available (Std $\sim 0.3 \%$ ) the partial reflectivity was determined to be $0.02 \pm 0.02(\mathrm{sr})^{-1}$. An $r=0.04(\mathrm{sr})^{-1}$, together with a $1-\mathrm{mm}$ radius opening, would result in a cavity emissivity of 0.9987 .

\subsubsection{Absorption}

Gas absorption is another factor that could affect the blackbody quality of the radiation. The radiation leaving the cavity hole could be partially absorbed by gases in the vicinity of the hole but at a lower temperature than the cavity walls. The major gases of concern are $C, C_{2}$, and $C_{3}$, it being determined from the literature that the concentration of $C_{4}, C_{5}$, and the higher polyatomics would be significantly less (Drowart et al., 1959). Other molecules such as $\mathrm{O}_{2}, \mathrm{~N}_{2}$, and $C N$ should not be a problem as a result of the argon flushing.

From the energy level diagram of atomic carbon (Herzberg, 1944) and the selection rules governing atomic transitions, one would not expect any carbon lines to appear in absorption within our spectral region of 200 to $850 \mathrm{~nm}$ and temperatures of $3000^{\circ} \mathrm{K}$ or below. Nevertheless, a spectral search was made for the strong $247.8 \mathrm{~nm}$ line and the forbidden line, at $462.1 \mathrm{~nm}$, using narrow slits. No evidence could be found of the presence of either line.

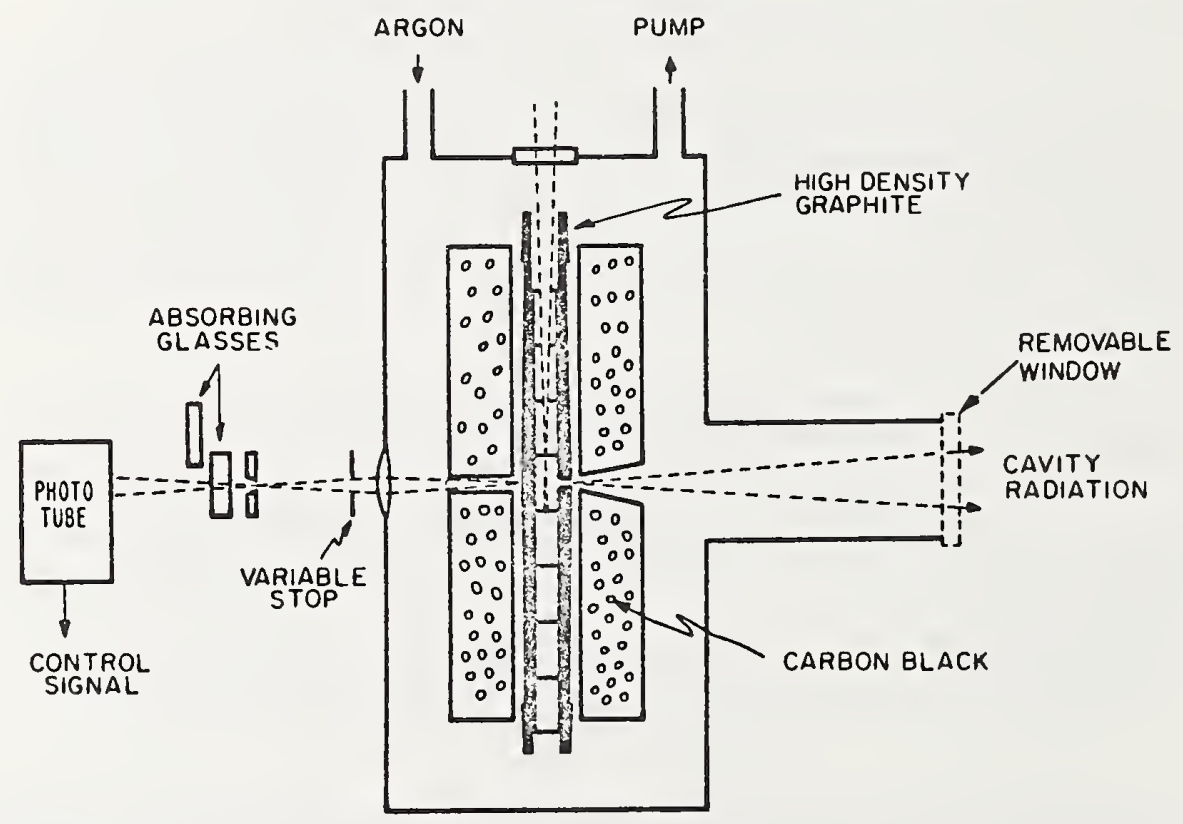

Fia. 4.2. High-temperature blackbody. 


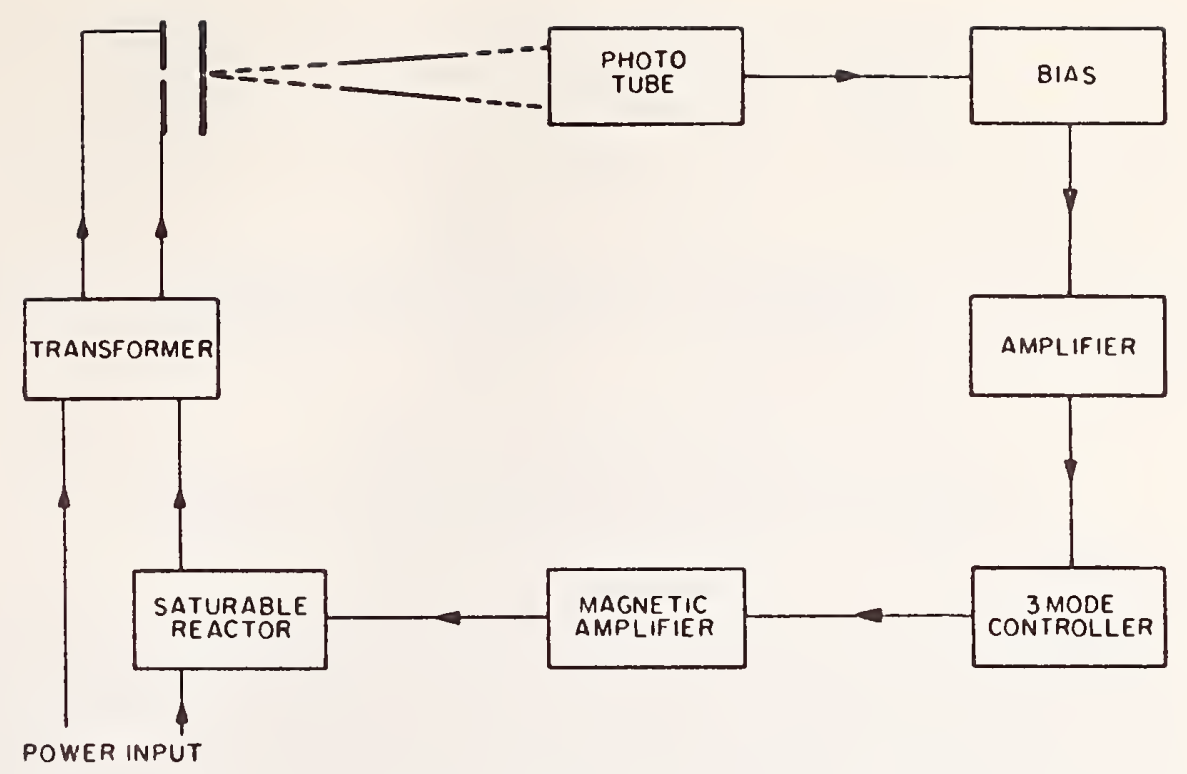

Fia. 4.3. High-temperature blackbody control system.

The molecule $C_{2}$ has very strong spectral bands in the blue and green referred to as the Swan bands (Herzberg, 1950; Clementi, 1960). If a significant portion of the cavity radiation was being absorbed by this moleculc, absorption lines characteristic of this band would be detectable in a spectral scan of the cavity radiation. No such absorption spectra could be detected.

Little is known about the energy levels of spectra of $C_{3}$. However, there is a broad, largely continuous band in the neighborhood of $400 \mathrm{~m}$ n that has been attributed to $C_{3}$ (Kiess and Broida, 1956). Such a band, especially if it were present to the extent of 1 or $2 \%$ in absorption, would not be casily noted. The following sensitive technique was evolved for determining the possible presence of this band.

The relative emissivity of a tungsten ribbon, at least over 20 or $30 \mathrm{~nm}$, appears to be well known. For example, the relative emissivity between 430 and $400 \mathrm{~nm}$ is the same within about $0.2 \%$ whether calculated from deVos' (1954) or Larrabee's (1959) published values. Therefore, if a strip lamp is adjustcd to have the same spectral radiance at $430 \mathrm{~nm}$ as the cavity, the ratio of spectral radiances at $400 \mathrm{~nm}$ can be calculated fiom Eq. (4.6) and should agree with an experimentally determined ratio if the cavity radiation at $400 \mathrm{~nm}$ has not been partially absorbed. The ealculations and the experimental measurements were performed and the two differed by $0.2 \%$ with a total uncertainty of $0.3 \%$. Thus, it is unlikely that $C_{3}$ absorption at $400 \mathrm{~nm}$ is a significant factor in affecting the blackbody quality. 
The only absorption lines present in the cavity radiation throughout the spectral range $200-850 \mathrm{~nm}$ were those of sodium, at 589.0 and $589.6 \mathrm{~nm}$, and of potassium at $766.5 \mathrm{~nm}$. These are probably produced by impurities in the graphite. Though rather weak, the blackbody cavity should not be used near these wavelengths.

As a result of the various calculations and experimental measurements described, it is believed that the graphite high-temperature blackbody largely satisfies the initial requirements.

\subsection{The Spectroradiometer}

\subsubsection{Design Criteria}

The uncertainty of an NBS calibration of a commercial visual optical pyrometer at $2500^{\circ} \mathrm{K}$ is about $5^{\circ}$ relative to the International Practical 'Iemperature Scale, IPTS (Kostkowski and Lee, 1962). The smallest uncertainty that has been reported for a calibration at $2500^{\circ} \mathrm{K}$ is $0.6^{\circ}$, and this is for the specially designed NBS photoclectric pyrometer (Lee, 1966). Thus, it appears that the measurement of the blackbody temperature will be the major uncertainty in the speetral radiance determination, and that the instrumentation should be designed to minimize this uncertainty. As a result, the following measures were taken.

(1) Use a photoclectric pyrometer for the temperature measurement.

(2) Design the photoelectric pyrometer as an integral part of the spectroradiometer utilizing similar opties, detectors, target areas, etc. This should minimize any errors in transferring the temperature scale to the blackbody.

(3) Calibrate the pyrometer portion of the speetroradiometer from basic principles, i.e., a primary ealibration.

\subsubsection{Description}

Figure 4.4 shows a block diagram of the spectroradioneter that has been developed. At $654.57 \mathrm{~nm}$ (the wavelength of a convenient speetral line in thorium) it is used as a photoelectric pyrometer. A tungsten-strip, vacuum pyrometer lamp, selected for maximum stability, is used as the equivalent of the small lamp in an ordinary pyrometer. Absorbing glasses are employed for temperatures above about $1320^{\circ} \mathrm{C}$, as in an ordinary pyrometer. The pyrometer lamp is compared to the blackbody by alternately positioning the two sources on the entrance slit of the monochromator.

In front of the entrance slit is one of two metal slit masks, limiting the slit height to either 0.2 or $0.8 \mathrm{~mm}$. The masks are engra ved with two perpendicular 


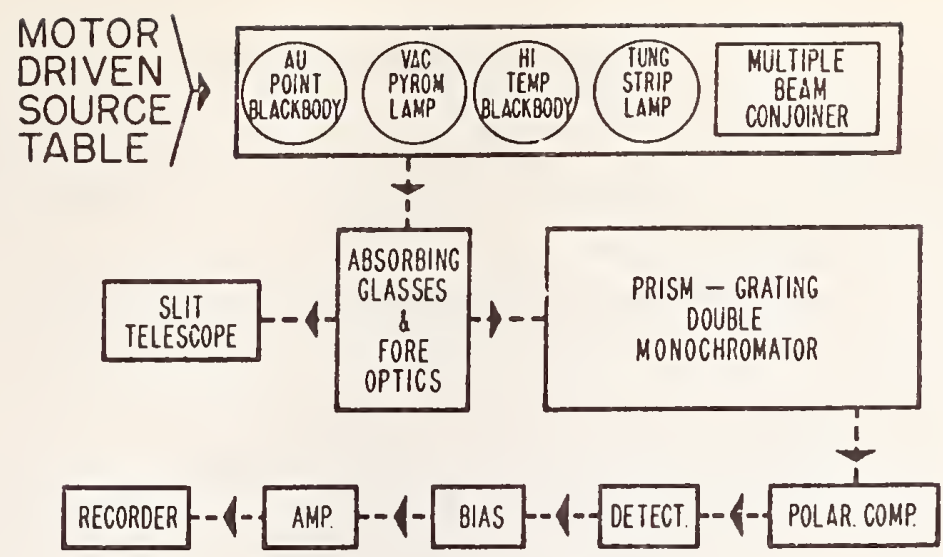

FIc. 4.4. Spectroradiometer.

scales having 0.1 mın divisions. The vacuum pyrometer lamp and other sources are placed in double gimbal mounts providing 6 degrecs of frecdom with mierometer-type motion. With the aid of the slit telescope, it is thus possible to position or to investigate the effects of changing position or oricntation of the sources, to about $0.02 \mathrm{~mm}$ or a few minutes of arc. The gradients present in some lamps make this capability highly desirable.

A double monochromator is uscd to ensure that scattered radiation from wavelengths other than that of interest will be sufficiently small, so as not to introduce a significant unccrtainty in comparing sources having different spectral distributions. This is equivalent to keeping the wings of the slit function small. The prism-grating rather than a prism-prism type double monochromator was sclected in order to have greater dispersion and greater wavelength reproducibility.

The elcetronics shown in Fig. 4.4 is that with which, through pyrometry, wc have had considerable success in high-precision $(<0.1 \%)$, low-level current $\left(<10^{-8} \mathrm{~A}\right)$ measurements. The radiation is not chopped and a de amplifier is used. With the biasing system cmployed, $0.1 \mathrm{in}$. on a recorder chart is equivalent to $0.2 \%$ spectral radiance. Generally, we are able by integrating over $1-2$ in. on the recorder chart to obtain a precision in terms of a standard deviation of 0.05 to $0.15 \%$, depending on the wavelength and tempcrature, when comparing a lamp to the blackbody. Since the two radiations are adjusted to be almost identical, i.c., we have essentially a null-type instrument, no errors arise due to nonlinearity of the photomultiplier or clectronics. The photomultiplicr has an $\mathbf{S 2 0}$ response and a quartz window. When it is used at a current level of about $10^{-8} \mathrm{~A}$ and is in operation for about $45 \mathrm{~min}$, fatigue produces at most a very small linear change with time which has a negligible effect on the measurements $(<0.05 \%)$. 


\subsection{Temperature Calibration}

Calibrating the pyrometer portion of the spectroradiometer from basic principles (a so-called primary calibration) means performing experiments that reflect the definition of the IPTS. The IPTS above the melting point of gold is defined (Stimson, 1961) in terms of the ratio of two blackbody radiances, one being at the temperature of equilibrium between liquid and solid gold (gold point) and the other at the unknown temperature T. Mathematically,

$$
\frac{L_{\mathrm{b} \lambda}(t)}{L_{\mathrm{b} \lambda}\left(t_{\mathrm{Au}}\right)}=\frac{\exp \left[C_{2} / \lambda\left(t_{\mathrm{Au}}+T_{0}\right)\right]-1}{\exp \left[C_{2} / \lambda\left(t+T_{0}\right)\right]-1}=\mathscr{R}
$$

where $t_{\mathrm{Au}} \equiv 1063^{\circ} \mathrm{C}, T_{0} \equiv 273,15^{\circ}$, and $C_{2} \equiv 1.438 \mathrm{~cm}$ deg are defined values.

\subsubsection{Low-Range Calibration}

The low-range calibration consists of determining electrical currents for the vacuum pyrometer lamp, which correspond to spectral radiance ratios of the lamp relative to the gold point. These ratios, and the wavelengths associated with them, can then be used to calculate blackbody or brightness temperatures from Eq. (4.11). The brightness temperature is usually limited to about 1300 to $1350^{\circ} \mathrm{C}$ in order to reduce lamp drift.

The starting point for such a low-range calibration is a gold-point blackbody. The one used is shown in Fig. 4.5 and was developed for calibrating the NBS photoelectric pyrometer (Lee, 1966). Various tests and calculations previously reported (loc. cit.) indicate that the emitted radiation in the vicinity of $654.5 \mathrm{~nm}$ is within $0.02 \%$ of that from a perfect blackbody at the temperature of freezing gold. The gold-point radiance could be transferred to the vacuum pyrometer lamp; i.e., a ratio of one, with a standard deviation uncertainty of about $0.05 \%$. However, in this transfer, a correction is necessary due to the effect of scattered radiation (originating outside of the target area) wheii comparing two sources of significantly different sizes. The gold-point blackbody has an effective radiating diameter of $25 \mathrm{~mm}$ and the strip lamp a similar area of about $1.3 \times 30 \mathrm{~mm}$. The magnitude of this effect was determined by performing the complementary experiment. A hole in an illuminated diffuse surface was imaged on the slit, of the spectroradiometer. For any particular condition of the entrance optics, the radiation detected depends on the size and shape of the illuminated surface. This was changed by placing black velvet paper in front of the illuminated surface simulating the gold-point blackbody and the strip lamp. The correction varied from about 0.05 to $0.3 \%$ in spectral radiance, depending primarily on the age and condition of the coating on the first mirror of the spectroradiometer optical system. 


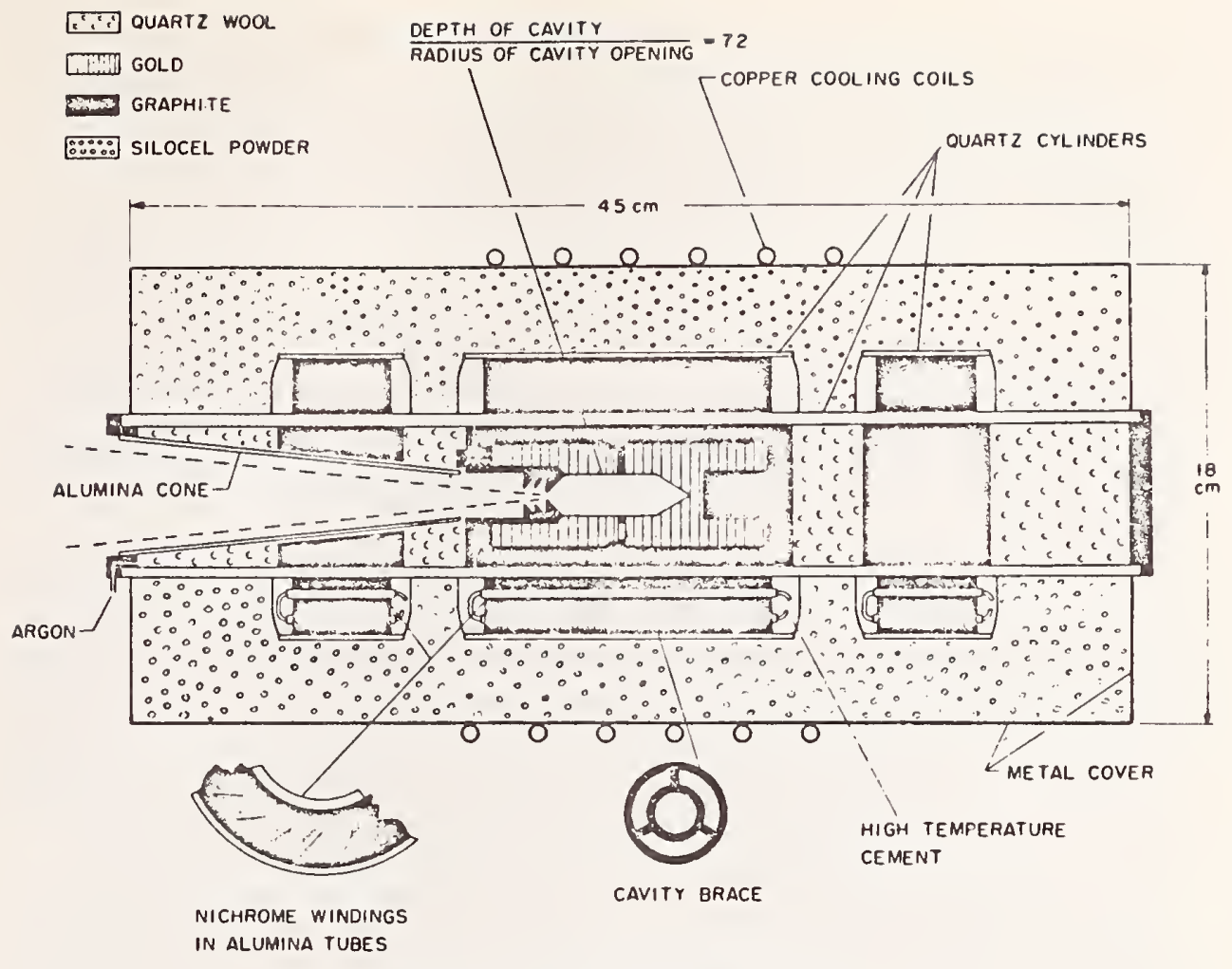

Fig. 4.5. Gold-point blackbody.

The wavelength in Eq. (4.11) is required to be known to at least $2.5 \AA$ $(0.25 \mathrm{~nm})$ if $0.12^{\circ}\left(0.1 \%\right.$ in $\left.L_{\lambda}\right)$ accuracy is desired at about $1300^{\circ} \mathrm{C}$. From the theory of optical pyrometry (Kostkowski and Lee, 1962), the wavelength in Eq. (4.11) is the mean effective wavelength between temperatures $t_{\mathrm{Au}}$ and $t$, designated $\lambda_{t_{A B}-t}$, where

$$
\frac{1}{\lambda_{l_{\mathrm{Aa}}-l}} \cong \frac{1}{2}\left(\frac{1}{\lambda_{l}}+\frac{1}{\lambda_{l_{\mathrm{Aa}}}}\right)
$$

and

$$
\frac{1}{\lambda_{t}}=\frac{\int\left[L_{\mathrm{b} \lambda}(t) \sigma\left(\lambda_{0} \lambda\right) R(\lambda) / \lambda\right] d \lambda}{\int L_{\mathrm{b} \lambda}(t) \sigma\left(\lambda_{0} \lambda\right) R(\lambda) d \lambda}
$$

The wavelength $\lambda_{t}$ is called the effective wavelength, $\sigma\left(\lambda_{0} \lambda\right)$ is the slit or apparatus function, and $R(\lambda)$ the relative spectral response of the entire system. The slit function was experimentally determined by spectrally scanning over a narrow emission line. The relative response $R(\lambda)$ was obtained by spectrally scanning the positive crater of a low-current graphite arc, the 
spectral radiance of which is largely equivalent to a $3800^{\circ} \mathrm{K}$ blackbody, from about 640 to $670 \mathrm{~nm}$ with narrow shits. The relative response is then the spectroradiometer output divided by the produet of the arc spectral radiance (Hattenburg, 1967) and the spectral slit width of the monoehromator.

With the $0.6 \mathrm{~mm}$ slit width $(2.4 \mathrm{~nm}$ spectral width at $650 \mathrm{~nm})$ normally used, the mean effective wavelength differed from the wavelcngth setting of the monochromator when using a narrow slit and spectral line by only $0.01 \mathrm{~nm}$ with an uncertainty of about $0.05 \mathrm{~nm}$. It was quite adequate, thercfore, when realizing the low range of the IPTS, to use the wavelength corresponding to the peak of a narrow spcctral line determined with a narrow slit. The maximum uncertainty due to mean effective wavelength in the calibration is estimated to be about $0.03^{\circ}$.

The ratio of speetral radiance $\mathscr{R}$ in Eq. (4.11) was obtained with the beam conjoiner shown in Fig. 4.6 (Erminy, 1963). By adjusting each source in the conjoiner to independently match the gold-point blackbody, all three sources together would represent an $\mathscr{R}$ of 3 . By adjusting two sources to be equal to each other and together equal to three times the gold point, eaeh would represent an $\mathscr{R}=3 / 2$. In this manner, many different integral and fraetional values of $\mathscr{R}$ were obtained without any assumption of linearity of the photomultiplier or electronie system.

Spectral radiance ratios relative to the gold point of $\frac{3}{4}, 1, \frac{3}{2}, 2,3,4,6,8,12$, and 14 were realized on the vacuum pyrometer lamp as a function of the lamp current. Using these $\mathscr{R}$ 's and the correct mean effective wavelength, Eq. (4.11) was used to determine the corresponding blackbody temperatures. A fourth-order equation of lamp current, as a function of temperature, was then fitted by a least squares criterion to the rcsulting ten temperatures and corresponding currents. The standard deviation of the fit was about $0.05^{\circ}$.

Three low-range calibrations have been performed to date, the second after $260 \mathrm{hr}$ of pyrometer lamp burning time and the third after $710 \mathrm{hr}$. The first and second calibrations differed by a constant of $0.83^{\circ}$, with a standard

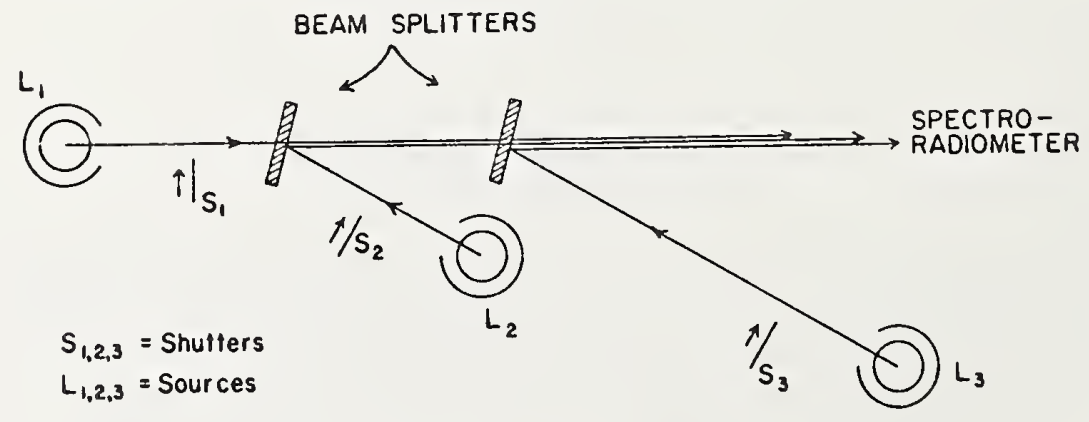

Fra. 4.6. Beam conjoiner. 
deviation of the differences of the unfitted data of $0.061^{\circ}$; and the seennd and third differed by $0.91^{\circ}$ with a standard deviation of $0.04^{\circ}$. In the $710 \mathrm{hr}$ in which this lamp has been used, its ealibration has ehanged by the same amounl. (in temperature) at all points in the low range. This makes it possible to eheek the entire low range with only one ratio determination, e.g., the goid point itself.

\subsubsection{High-Range Calibration}

The upper ranges of the pyrometer were ealibrated by determining transmittanees for the individual absorbing glasses using the low-range ealibration. This was aceomplished by matehing a vaeuun lamp at a brightness temperature of about $1320^{\circ} \mathrm{C}$, observed through an absorbing glass, with a vaeuum lanip observed direetly, so that

$$
\tau(\lambda) L_{\mathrm{b} \lambda}\left(T_{\mathrm{H} 1}\right)=L_{\mathrm{b} \lambda}\left(T_{\mathrm{L}}\right)
$$

Using Wien's equation, whieh has an error of only about $0.0001 \%$ for the maximum value of $\lambda T$ used in the low range, one obtains

$$
\tau(\lambda)=\exp -\left(C_{2} / \lambda\right)\left(1 / T_{\mathrm{L}}-1 / T_{\mathrm{H}}\right)=\exp -\left(C_{2} A / \lambda\right)
$$

where $A$ is the so-ealled " $A$ " value of the absorbing glass.

In pyrometry, absorbing glasses are usually seleeted whieh have an exponential transmittanes, i.e.,

$$
\tau(\lambda)=\exp -(k / \lambda)
$$

In this ease, the $A$ value is independent of the mean effeetive wavelength and a eonstant with respeet to the temperature of the source that the pyrometer is observing. Thus, an $A$ value determined in the low range may be used at higher temperatures. Also from Eq. (4.15), it ean be seen that two or three absorbing glasses ean be used in series; and, negleeting refleetion, the resulting $A$ value is the sum of the individual $A$ values.

Using the above prineiples and eorreeting for refleetanees, which were experimentaliy determined $(\sim 0.16 \%$ eorreetion in transmittance for two absorbing glasses), the $A$ values were obtained for groups of one, two, and three absorbing glasses; and the higher-range ealibrations, eonsisting of these $A$ values and the low-range ealibration, were thus obtained.

One additional point, the glass usually used in pyrometers for absorbing glasses is Corning Pyrometer Brown. This glass was found to have a temperature eoeffieient, transmittanee deereasing with an inerease in glass temperature, making it diffieult to use with the preeision desired. Another glass was found, however, Jena (Sehott) NG-3, which also had an exponential transmittance but had a coeffieient with the opposite sign. The two glasses were 
eombined in a composite filter, with their thieknesses seleeted so that the temperature coeffieient was negligible.

The pyrometer portion of the speetroradiometer has been calibrated at $2500^{\circ} \mathrm{K}$ with an estimated standard deviation uncertainty of about $0.6^{\circ}$ relative to the IPTS, or the same uncertainty reported (loe. eit.) for the ealibration of the NBS photoeleetrie pyrometer.

\subsubsection{Correction to the Thermodynamic Scale}

In order to obtain absolute values of speetral.radianee, it is necessary to use the Thermodynamic Kelvin Temperature Seale (TKTS) rather than the IPTS. Table I gives the estimated differences between these seales for temperatures in cur range of interest. In applying these differenees as eorreetions to the IPTS, an additional uncertainty of the differenee, about $0.9^{\circ}$ at $2500^{\circ} \mathrm{K}$, is ineurred. The 0.9 and $0.6^{\circ}$ uneertainties are independent, so that the total uneertainty on the TKTS is the square root of the sum of the squares of these individual standard deviation uneertainties or about $1.1^{\circ}$.

As a result of an extensive program in gas thermometry now in progress at NBS, the TKTS eorreetion uneertainty is expected to be redueed by a faetor of at least five in a few years. Thus, though the best eurrently available aecuraey of a temperature measurement on the TKTS at $2500^{\circ} \mathrm{K}$ is about $4 \frac{1}{2}$ times poorer than our criterion, it is only about $2 \frac{1}{2}$ times poorer on the IPTS and there are high expeetations that, in the near future, the correction to the TKTS will introduee an insignifieant additional uneertainty.

TABIE I. Estimated differences between the Thermodynamic Kelvin Temperature Scale and the International Practical Temperature Scale. ${ }^{a}$

\begin{tabular}{ll}
\hline T(TKTS) & T(TKTS) $-\mathrm{T}$ (IPTS) \\
\hline $2100^{\circ} \mathrm{K}$ & $2.9^{\circ}$ \\
2200 & 3.1 \\
2300 & 3.3 \\
2400 & 3.5 \\
2500 & 3.8 \\
2600 & 4.0 \\
2700 & 4.3 \\
2800 & 4.6 \\
2900 & 4.8 \\
3000 & 5.1 \\
\hline
\end{tabular}

a Terrien and Preston-Thomas, 1967. 
Many of the features required for using the spectroradiometer as an accurate pyrometer, at $654.7 \mathrm{~nm}$, are obviously equally useful at other wa velengths where the high-temperature blackbody and a gas-filled tungstenstrip lamp are to be compared. These include rapid precise positioning of the sources on the slit mask, low scattering of radiation originating outside of the target area, and accurate wavelength determination. The insignificant spcetral scattering, resulting from the use of a double rather than a single monochromator, is even more important at other wavelengths, particularly at the extremes of the wavelength range of interest, where either the photomultiplier response or the raciance is very low and scattered radiation constitutes a larger percentage of the speetroradiometer output.

The major uncertainty in the comparison of the blackborly and lamp is the lamp itself. The best gas-filled lamps observed drift at a ratc of about $0.002 \%$ per hour at a $2500^{\circ} \mathrm{K}$ brightness teniperature in the red. However, some lamps have been observed to drift by a factor of 100 greater. Thus, only the best lamps available are stable enough not to limit the accuracy of the calibration. Even then their burning time should be kept to a minimum.

The gas-filled strip lamps normally used in these calibrations are manufactured by the General Electric Company (sole U.S. supplier) and are designated ultraviolet spectrun lamps. The tungsten-strip filament is about $3 \mathrm{~mm}$ wide and 30-50 $\mathrm{mm}$ long and has a small notch at about its midpoint. There is a quartz window permitting use down to about $200 \mathrm{~nm}$. For the temperatures required for the ultraviolet spectral radiance calibrations, a direct current and voltage of about $40 \mathrm{~A}$ and 7-12 $\mathrm{V}$ is typical.

Before calibration, the optimum orientation for the lamp is determined. The radiation from the target area is very noisy and even oscillatory unless the lamp filament is oriented close to vertical. This is believed to be caused by convection of the argon gas cyclically cooling the filament. When the most favorable orientation is determined, an arrow is etched on the rear surface of the lamp; a line through the center of the notch and tip of the arrow serves as a reference axis. This axis is usually made horizontal and is also approximately the optic axis of the spectroradiometer, thereby insuring not only a reproducible orientation but also a reproducible direction of sighting on the lamp.

The reproducibility of the blackbody and tungsten-strip lamp comparison, using the lamp and blackbody as described, has a standard deviation ranging from about $0.05 \%$ at $650 \mathrm{~nm}$ to $0.15 \%$ at $300 \mathrm{~nm}$ and $0.3 \%$ at $210 \mathrm{~nm}$.

If the strip-lamp radiation and the response of the spectroradiometer are both polarized, an error will be produced in the comparison. 
The spectroradiometer has a polarization $\left[\left(R_{\max }-R_{\min }\right) /\left(R_{\max }+R_{\min }\right)\right]$ of about 0.20 , with a maximum response in a dircetion parallel to the slit at $210 \mathrm{~nm} ; 0.0$ at $500 \mathrm{~nm}$; and about 0.15 at $850 \mathrm{~nm}$, with a maximum in a direction perpendicular to the slit. At intermediate wavelengths, the value of the polarization varies approximately linearly with wavelength. The absence of polarization at $500 \mathrm{~nm}$ is brought about by the compensator shown in Fig. 4.4. It consists of two plates of calcium fluoride placed between the exit slit and the detector and so oriented that the response at $500 \mathrm{~nm}$ is independent of the polarization of the incident radiation.

In order to minimize the error due to the polarization, only lamps with a polarization of $1 \%$ or less, as determined at $500 \mathrm{~nm}$, are used. Under these conditions, the effect of polarization is small and can be corrected with sufficient accuracy so that the uncertainty in the comparison due to this factor is, at most, about $0.1 \%$.

\subsection{Summary of UnCertainties}

Table II summarizes the estimatcd unccrtaintics, in terms of per cent of spcetral radiance, with which the best available tungsten-strip lamps have been calibrated. The various sources of uncertainty are listed separately. The uncertainties are given in terms of one standard deviation and combined in quadrature to give a total standard deviation uncertainty.

The reduction in the uncertainty of the correction to the TKTS by about five, expected in a few years, will result in lowering the total unccrtainties by about $30 \%$. The probability of further improvement in the accuracy of spectral radiance determinations, in the wavelength range $210-850 \mathrm{~nm}$, is

TABLE II. Estimated uncertainties, in per cent of speetral radianee, of calibrating a gas-filled tungsten-strip lamp.

\begin{tabular}{|c|c|c|c|c|}
\hline \multirow{2}{*}{ Source of uncertainty } & \multicolumn{4}{|c|}{ Wavelength (nm) } \\
\hline & 210 & 300 & 650 & 850 \\
\hline $\begin{array}{l}\text { Blackbody quality } \\
\text { (for } \varepsilon=0.999 \text { ) }\end{array}$ & 0.2 & 0.1 & 0 & 0.1 \\
\hline IPTS temperature & 1.0 & 0.5 & 0.2 & 0.2 \\
\hline Correction to TKTS & 1.0 & 0.7 & 0.3 & 0.2 \\
\hline $\begin{array}{l}\text { Transfer from blackbody } \\
\text { to lamp }\end{array}$ & 0.3 & 0.2 & 0.1 & 0.1 \\
\hline $\begin{array}{l}\text { Total standard deviation } \\
\text { uncertainty }\end{array}$ & 1.5 & 0.9 & 0.4 & 0.3 \\
\hline
\end{tabular}


high if the stability of vacuum pyrometer lamps or some other convenienty usable reference source is improved. Otherwise, the probability is small. At any rate, without a corresponding improvement in the stability of the sources to be calibrated (i.e., the gas-filled tungsten-strip lamps) any increase in calibration accuracy is of limited value.

\title{
References
}

- (1963). New values for the physical constants-recommencled by NAS-NRC. Nat. Bur. Sid. U.S. Tcih. Neu:s. Bull. 47, 175-177.

Clementi, E. (1960). Transition probabilities for low-lying electronic states in $\mathrm{C}_{2}$. Astrophys. J. 132, 898-904.

deVos, J. C. (1954). Evaluation of the quality of a blackbody. Physica, 20, 669-689.

de Vos, J. C. (1954). A new determination of tic emissivity of tungsten ribbon. Physica 20,690-714.

Drowart, J., Burns, R. P., Dellaria, G., and Inghram, M. G. (1959). Mass spectrometric study of carbon vapor. J. Chcm. Phys. 31, 1131-1132.

Erminy, D. L. (1963). Seheme for obtaining intcgral and fractional multiples of a given radiance. J. Opt. Soc. Am . 53, 1448-1449.

Hattenburg, A. T. (1967). Spectral radiance of a low-current graphite are, J. Appl. Opt. 6, 95-100.

Herzbrrg, G. (1914). "Atomic Spectrn and Atomic Structure." 257 pp. Dover, New York. Hrrzberg, G. (1950). "Molecular spectra and Moleculur Structurc." 658 pp. Van Nostrand, Prineeton, New Jersey.

Kostkowski, H. J. (1966) A new radionctrie equation and its application. J. Appl. Opt. 5, 1959 .

Kostkowski, H. J., Erminy, D. E., and Hattenburg, A. T. (1965). Accuracy of spectral rarlianec calibrations inerrased. Nut. Bur. Std. U.S. Tech. Neu's Bull. 49, 14.

Kostkowski, H. J., Erminy, D. E., and Hattenburg, A. T. Higher accuracy spectral racliance measurcments. $J$. Res. Nat. Bur. Std. (to be published).

Kostkowski, H. J., and Lce R. D. (1962). Theory and methods of optical pyrometry. In "Tcmperature, Its Measurement and Control in Seicnce and Industry," Vol. 3, Part I (F. G. Briekwedde, erl.), pp. 449-481. Reinhold, Now York.

Kiess, N. H., and Broide, H. P. (1956). Spectrum of the $\mathrm{C}_{3}$ inolecule betwecn $3600 \AA$ and 4200 A. Can. J. Phys. 31, 1471-1479.

Larrabee, R. D. (1959). The spectral emissivity and optical properties of tungsten. J. Opt. Soc. Am. 49, 619-625.

Lee, R. D. (1966). The NBS photoelcctric pyrometer and its use in rcalizing the International Praetical Temperature Scalc above $1063^{\circ} \mathrm{C}$. Melrologia 2, 150-162.

Stimson, H. F. (1961). International Practical Tempcrature Scale of 1948; Text revision of 1960. J. Res. Nat. Bur. Sld. 65A, 139-145.

Terrien, J., and Preston-Thomas, H. (1967). Progress in the definition and in the mcasure. ment of temperature. Metrologia 3, 29-31.

\author{
REPRITTED FROM \\ ADVANCES IN GEOPHYSICS, YOL. 14 \\ (C) 1970 \\ ACADEMIC PRESS INC., NEW YORK
}




\title{
The NBS Photoelectric Pyrometer and its Use in realizing the International Practical Temperature Scale above $1063^{\circ} \mathrm{C}$
}

\author{
R. D. LEE \\ National Bureau of Standards, Washington, D. C.
}

Received June 1, 1966

\begin{abstract}
I photoelectric pyrometer has been developed with which the International Practical Tempcrature Scale (IPTS) above the gold point. $1063^{\circ} \mathrm{C}$, is realized about 5 times more accurately than with the prevalently used disappearing filament visual pyrometer. Estimated standard deviation uncertainties of realizing the IPTS with the photoclcctric instrument are $0.06 \operatorname{deg} \mathrm{C}$ at $1063^{\circ} \mathrm{C}, 0.12 \operatorname{deg}\left(\mathrm{C}\right.$ at $1256^{\circ} \mathrm{C}$. 0.27 deg $\mathrm{C}$ at $1650^{\circ} \mathrm{C}, 0.7 \mathrm{deg} \mathrm{C}$ at $2330^{\circ} \mathrm{C}$. and $2 \mathrm{deg} \mathrm{C}$ at $3525{ }^{\circ} \mathrm{C}$. The design, evaluation. and calibration to realize the IPTs are discussed.
\end{abstract}

\section{Introduction}

The International Practical Temperature Scale (IPTS) [1] above $1063{ }^{\circ} \mathrm{C}$ is usually realized with a disappearing filament optical pyrometer [2]. The reproducibility and accuracy of the optical pyrometer is primarily limited by the contrast sensitivity of the human eye [3]. Photoelectric means of detecting the equality of spectral radiance along the same principles as those of the visual instrument have been demon. strated to be considerably more precise, and this increased precision has been accomplished with a narrower spectral passband.

During the past few years, photoelectrie pyrometers have been developed at the National Bureau of Standards (NBS) and at other national standards laboratories $[4,5]$. Progress toward evaluating the NBS instrument has been previously reported $[6,7]$. This instrument has been calibrated relative to the IPTS four different times during the past two years, and has been used for about $\mathbf{7 3 5}$ hours in calibrating strip lamps and pyrometers. The present paper is a comprehensive description of the NBS photoelectric pyrometer and of the results in realizing and maintaining the IPTS.

\section{Description}

The optical system of the photoelectric pyrometer is shown in Fig. 1. The major differences of this optical system from that of the visual pyrometer are the $1.3 \mathrm{~mm}$ (liameter field stop ${ }^{\star}$, the passband filters in addition to a red glass, and a specularly refleeting disk with a slit. The image reflected at the disk provides for aligmment and focusing. For this purpose the $1.3 \mathrm{~mm}$ field stop is temporarily removed. Small $(0.2 \mathrm{~mm})$ vertical displacements of the pyrometer lamp alternate its image on the slit with that of the: external rarliant source. This can be accomplished manually or automatically at intervals of 30 sees. The equality of the multiplier phototube response as it is irracliated alternately by the two images constituter a photoelectric match.

The optical system is largely corrected for spherical aberration and coma. Lenses at IA are telescope objectives and are used in the pyrometer at the object and image distances for which they are correeted. The remaining lenses were designed specifically for the photoelectric pyrometer. According to the manufactures report, lenses IIA and IIB together have a longitudinal spherical aberration of less than $0.15 \mathrm{~mm}$ for $6563 \AA$ (Hydrogen C line), $5893 \AA$ (Sodium $\mathrm{D}$ line), and $5461 \AA$ (Mercury $\mathrm{E}$ line). The lens system IIIA and IIIB, treated as one optical component, is reported to have a longitudinal spherical aberration of only $1.5 \mathrm{~mm}$ for the $\mathrm{C}$ line and less for the $\mathrm{D}$ and $\mathrm{E}$ lines. The resulting image quality appears to be adequate. Some defocusing (as a way to represent a poorer image) is possible without a detectable change in photoelectric response, thus indicating that the response is independent of the residual aberrations.

The absorbing glasses are made of Jena NG-3 filter glass, and are inserted into the optical path to extend the temperature range above $1256{ }^{\circ} \mathrm{C}$. Corning Pyrometer Brown glass is often used for this purpose; however, this glass was not available in small quan-

* Lenses at IA and the field stop were not in the original version of the instrument described in Ref. [6]. 


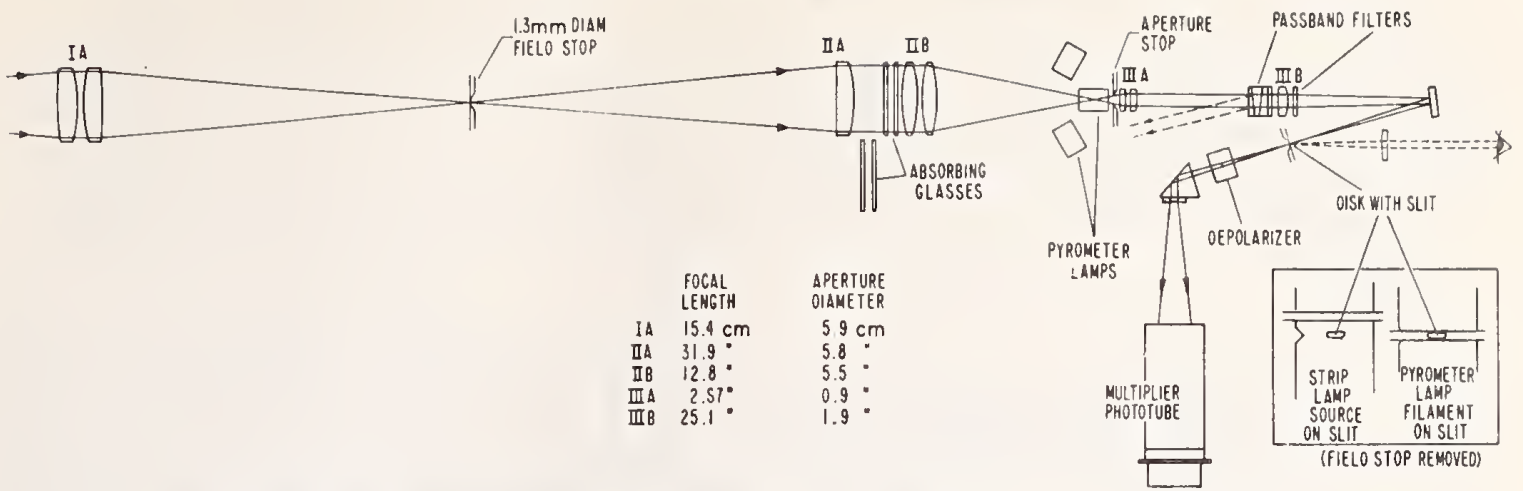

Fig. 1. Optical system of the NBS photoelectric pyrometer. The plane of the source is fixed $30.4 \mathrm{~cm}$ from the first lens, IA

tities at the time of construction of the pyrometer. The Jena NG-3 was selected for its similarity to the Corning Brown in its relative spectral transmittance within the spectral passband of the pyrometer. Both of these glasses have " $A$ " values [2] that vary only slightly with source temperature. This characteristic facilitates a more reliable determination of the " $A$ " value at all temperatures. The 4 glasses are nearly equal in " $A$ " value (approximately 135 mireds) and are used in tandem for the high temperature ranges. They are positioned in a parallel beam so that the image formed by the inter-reflected rays is superimposed on the primary image. An accurate correction can be made for this additional radiation using the experimentally determined reflectance of each glass surface.

The photoelectric pyrometer has three vacuum pyrometer lamps that are mounted so that they can be quickly and precisely interchanged. Thus the lamps may be accurately intercompared by using an external source which need be stable only for times of the order of 5 or 10 mins. The lamps have small tungsten ribbon filaments up to $0.1 \mathrm{~mm}$ wide. The flat filament provides a more uniform radiance than a round filament, and this makes positioning of the image over the slit less critical.

The aperture stop before the lenses at IIIA is not in the proper ratio to the objective aperture to fulfill Fatrchuld and Hoover's criterion [8]. This criterion was concerned with conditions for reducing diffraction at the edge of a pyrometer lamp filament, in order to provide good visual disappearance. The criterion is not applicable to the photoelectric pyrometer, since the slit is covered only by the central 0.8 of the filament image, and the edges are avoided. Both the entrance aperture and exit aperture are considerably larger than those of a conventional visual pyrometer, with the ratio, entrance to exit, being 1.23 .

The multi-element filter in front of lens IIIB plus an additional filter behind this lens isolates a spectral passband centered on $6545 \AA$ with a width at half the transmittance of $125 \AA^{\star}$. The multi-element filter consists of a multi-layer dielectric interference filter, a sideband suppression interference filter, and a red glass, all of which are cemented together. The remaining filter is another side-band suppression inter-

* Previously reported as $110 \AA$ from manufacturer's data. ference filter, addcd to suppress some radiation detected at $7700 \AA$. Fig. 2 compares the passband of the photoelectric pyrometer with that of the NBS laboratory visual pyrometer [2], which is typical, in this respect, of present day optical pyrometers.

Two mechanically interchangeable specular disks are provided. One contains a slit about $0.8 \mathrm{~mm}$ by $2.4 \mathrm{~mm}$ and the other a circular aperture $0.8 \mathrm{~mm}$ in diameter. The conjugate target dimensions are $0.2 \mathrm{~mm}$ by $0.6 \mathrm{~mm}$, and $0.2 \mathrm{~mm}$ diameter. The slit is used for regular work, while the smaller circular aperture is used for determining the temperature gradient of a source.

The optical system, chiefly the interference filters, polarize the radiation about $5 \%$. This is probably due to the orientation of the interference surfaces at 7 degrees to the optic axis (to direct the reflected radiation out of the optical train). This polarization, if not corrected, would cause errors in radiance temperature measurements of polarized or partially polarized sources. A tungsten strip lamp, which is frequently used as a secondary standard source, is an example of a partially polarized source when it is viewed at an angle away from the normal of the strip surface. The most general solution to the problem was to depolarize the instrument, and this was done rather simply by inserting a parallel plane glass plate in the optical path and adjusting its orientation until the photoelectric

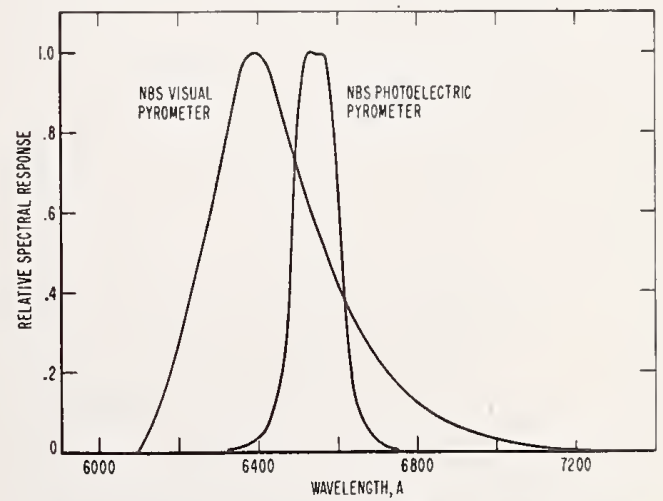

Fig. 2. Spectral passbands of the NBS visual and photoelectric pyrometer. The visual pyrometer curve is determined by a red glass and the visibility function of the observer, while the photoelectric curve is determined mainly by an interference fllter 
response to polarized radiation was constant regard. less of the direction of the polarization.

A block diagram of the electrical system of the photoelectric pyrometer is shown in Fig. 3. The system detects, amplifies, and records a de signal. The potential developed across the load resistor in the output of the multiplier phototube is largely canceled by an adjustable bias voltage in order to reducc the

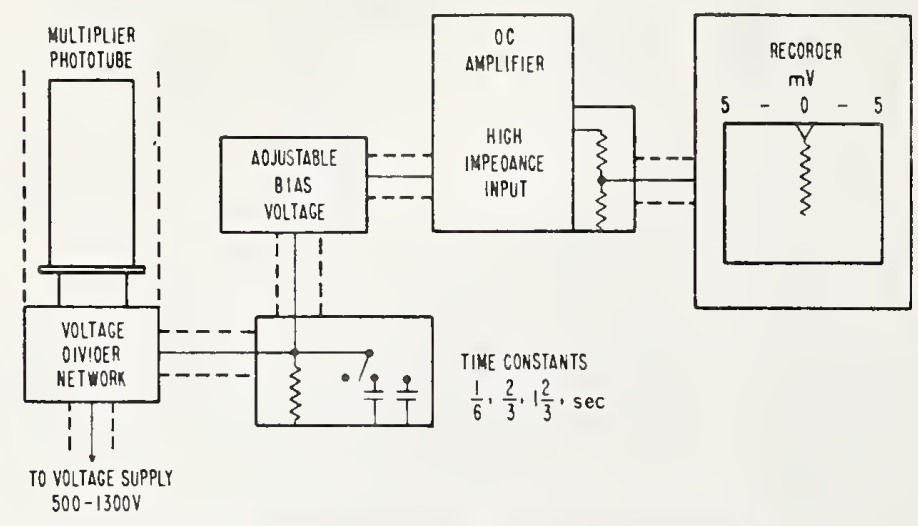

Flg. 3. Electrical system of the NBS photoelectric pyrometer

\section{Reproducibility}

\subsection{Precision of a radiance match}

The precision attainable in making a radiance match is limited by the fluctuations appearing on the recorder trace. Fig. 4 shows these in comparison to a 0.2 deg $\mathrm{C}$ change in radiance temperature of a source at $1063{ }^{\circ} \mathrm{C}$, using various elcctrical time responses.

When recorder traces 30 secs long are obtained, as has becn the practice, the higher frequency fluctuations or noise can be averaged fairly well by a straight line through the trace. The effect of longer period fluctuations is reduced by averaging 4 to 8 consecutive radiance matches. As a measure of the obtainable precision of a single radiance match, the sample standard deviation of a series of successive determinations of the radiance temperature of a vacuum tungsten strip lamp at a radiance temperature of about $1063^{\circ} \mathrm{C}$ was determined. A standard deviation of $0.02 \mathrm{deg} C$, largely independent of the electrical time re-

linearity and stability requirements of the amplifier and to increase the sensitivity of the recorder trace. Rather than to attempt to match completely the source and pyrometer lamp it is less time consuming to leave small differences in their recorder traces and interpolate to a match. These differences are usually less than the equivalent of $0.2 \mathrm{deg} \mathrm{C}$, and the interpolation does not add any additional significant uncertainty.

The multiplier phototube is an RCA 7265, 14. stage, end-on-illumination type with an S-20 spectral response. The tube data show a sensitivity at $6545 \AA$ of $35 \%$ of the maximum sensitivity at $4200 \AA$. The quantum efficiency at $6545 \AA$ is reported to be about $6 \%$. At present the multiplier phototube is operated at room temperature, but, if required, it can be cooled with a minor modification of the pyrometer. A voltage supply, reported by the manufacturer to regulate to $0.1 \%$ for an ae line change of 25 volts, supplies up to 1300 volts across a voltage divider resistance network for the phototube dynodes. Carbon resistors of $5 \%$ tolerance and 1 watt rating are used in the network. A battery supply has also been used with the individual batteries connected between the dynodes. Comparison of the performance of the phototube with the two voltage supplies has shown that the resistance network does not add a significant amount of noise to the signal.

Typical electrical parameters, used with a blackbody source of $1063^{\circ} \mathrm{C}$, are as follows. With 1150 volts on the multiplier phototube the tube has an output current of $0.058 \mu \mathrm{A}$ (with dark current $<0.0002 \mu \mathrm{A}$ ), which developes 192 millivolts across the $3.3 \mathrm{megohm}$ resistance. With this signal biased, and the amplifier plus attenuator at a gain of about 2 , the recorder has a 10 inch deflection for a change of signal of about $2.5 \%$. This change corresponds to about a $2 \mathrm{deg} \mathrm{C}$ change in the temperature of the $1063{ }^{\circ} \mathrm{C}$ source, or $0.02 \mathrm{deg} \mathrm{C}$ per chart division of 0.1 inch. sponse used, was obtained.

Since the dark current trace of Fig. 4 shows no significant noise, the noise appearing on the recorder trace under conditions of illumination must be due primarily to the statistical fluctuations of the electrons released at the cathode surface of the multiplicr phototube. In accord with theoretical considerations

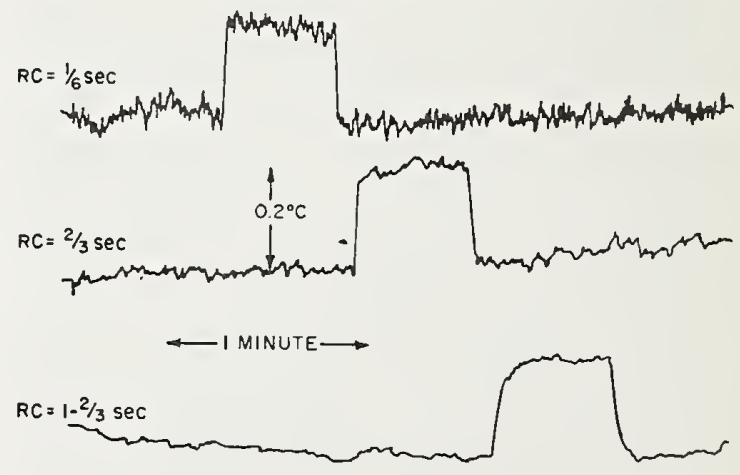

$R C=1 / 6 \sec$

DARK CURRENT

Fig. 4. Recorder traces showing the noise in comparison to 0.2 deg $\mathrm{C}$ change ia radiance temperature of a source at $1063{ }^{\circ} \mathrm{C}$, for various RC time constants of the electrical system

the expected rms value of this noise is proportional signal-to-noise ratio is also proportional to the square root of the signal. The characteristics which determine the signal-to-noise ratio are listed in Tab. 1. The entrance angle is the angle subtended at the source by the entrance pupil of the pyrometer. The target dimensions given are those of the conjugate image of the slit in the plane of the source. An improved signalto-noise ratio does not rapidly improve the precision of the radiance match over and above that obtainable to the square root of the signal [9], and therefore the 
Table 1. Characteristics which determine the signal to noise. ratio of the NBst photoelectric pyrometer

(1) Optical

Fintrance angle: 8 degrces

Target dimensions: $0.2 \mathrm{~mm} \times 0.6 \mathrm{~mm}$

Passband: 125 Angstroms at $6545 \AA$

Transmittance: $35 \%$

(2) Electrical

Multiplicr phototube: Quantum efficiency, $6 \%$ at $6545 \AA$ Time constants: $1 / 6,2 / 3,12 / 3 \mathrm{sec}$.

already from integrating $30 \mathrm{sec}$ traces. At the upper end of the low range, $1256^{\circ} \mathrm{C}$, the signal-to-noise ratio is greater by a factor of about threc; howcver, the precision of the match is only slightly improved. Therefore, considerablc change in the characteristics listed in Tab. 1 would be necessary to produce a significant improvement in the pyrometer.

\subsection{Stability of pyrometer lamps}

The frequency of recalibration and the accuracy to which the IPTS is maintained between calibrations depend in large measure on the reproducibility of the radiance of the pyrometer lamps for any given electric eurrent. New pyrometer lamps are aged before being calibrated in order to obtain a stable radianee $\mathrm{vs}$ current relationship. Eight lamps have been investigated for reproducibility during or after various schcdules of ageing. However the results have not shown a significant correlation between manner of ageing and stability. The instability of the pyrometer lamps is probably the major limitation in maintaining the IPTS.

The lamps tested have been labeled ph 1 to ph 8 . Lamps ph 1, 2, 7 and 8 are NBS designed lamps having tungsten filaments of $99.9 \%$ purity. The filaments are $0.1 \mathrm{~mm}$ wide, $0.01 \mathrm{~mm}$ thick, and $32 \mathrm{~mm}$ long. Lamps ph $3,4,5$, and 6 are commercial lamps with flat tungsten filaments about $0.05 \mathrm{~mm}$ wide and of $99.9+\%$ purity. All the lamps are vacuum type with an estimated bulb pressure of $10^{-5}$ torr.

Results of the stability tests are given in Figs. 5 to 9. Differences occurring as a function of time between pyrometer lamps left on continuously and a pyrometer lamp inoperative (except for the periodic comparisen) are plotted. The comparisons were made by using an external vacuum strip lamp as a transfer source.

Lamps ph 1 and ph 3 of Fig. 5 were aged prior to testing for 2000 hours at $1500{ }^{\circ} \mathrm{C}$ (tungsten temperature) and 1000 hours at $1450{ }^{\circ} \mathrm{C}$, respectively, while ph 4 was used as purchased.

Fig. 6 shows the behavior of a lamp which, because of its rapid decrease in radiance temperature $\left(\sim 1063{ }^{\circ} \mathrm{C}\right)$ for a constant current, was given additional treatment. After three hours at $1950{ }^{\circ} \mathrm{C}$ its radiance temperature at the initial current was about 8 deg $\mathrm{C}$ higher than just before the ageing, and was increasing rapidly. During 500 hours ageing at $1900{ }^{\circ} \mathrm{C}$ the radiance temperature decreased about $2 \mathrm{deg} \mathrm{C}$ but was found to increase with time on a subsequent run at $1063{ }^{\circ} \mathrm{C}$ radiance temperature. The transmittance of the windows of the lamp ph 2 was measured before and after the last ageing treatment in order to check the possible coating of the inner surfaces of the glass envelope. A decrease in the transmittance of about 1\%

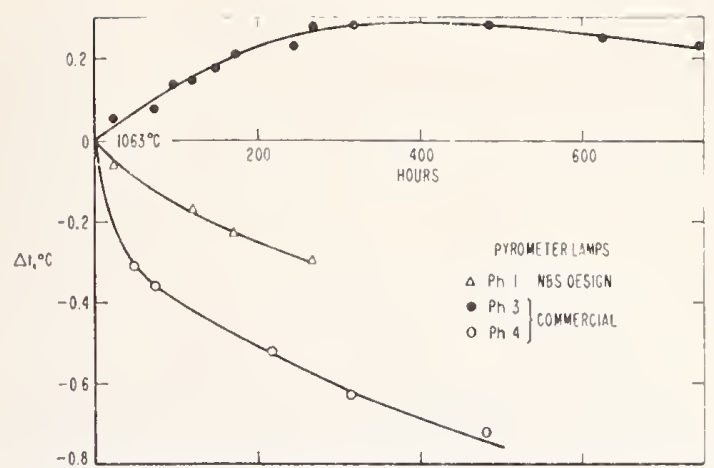

Fig. 5. Change in radiance tcmpcrature of pyrometer lamps initially at $1063{ }^{\circ} \mathrm{C}$ radiance temperature, versus hours at a constant lanip current

(for two windows) was observed. This aceounts for only $20 \%$ of the change observed after the 500 hour ageing treatment.

Fig. $7 \mathrm{~b}$ shows the results of two commercial lamps which were not given prior ageing at NBS. After 500 hours at lamp currents for a $1063{ }^{\circ} \mathrm{C}$ radiance temperature, the lamps were run at $1250{ }^{\circ} \mathrm{C}$ radiance temperature for an additional 300 hours, then finally set back to $1063{ }^{\circ} \mathrm{C}$ for the remainder of the test. The reproducibility of the radiance of the lamps was checked at $1063{ }^{\circ} \mathrm{C}$ throughout. Also shown (Fig. 7a) are the periodic comparisons between the pyrometer lamp used as a reference and the vacuum strip lamp used as the transfer source. These latter results show continual long term agreement between these infrequently used lamps.

Two new NBS lamps, ph 7 and ph 8, were checked during the process of ageing by periodic comparisons at $1063^{\circ} \mathrm{C}$ radiance temperature with a little used reference lamp. After 900 hours both lamps had become reasonably stable while they were kept on at $1550{ }^{\circ} \mathrm{C}$, Fig. 8. The stability was not maintained when the lamps were aged further at a lower temperature of $1130{ }^{\circ} \mathrm{C}\left(1063{ }^{\circ} \mathrm{C}\right.$ radiance temperature $)$, as Fig. 8 shows.

English vacuum tungsten strip lamps with strips $1.3 \mathrm{~mm}$ wide have been tested for stability with a spectroradiometer in another laboratory of the NBS Radiation Thermometry Section, and with a sensitivity within a factor of 2 or 3 of that of the photoelectric pyrometer. Some of these lamps, compared with a little-used reference lamp (another strip lamp), showed results similar to those pyrometer lamps of

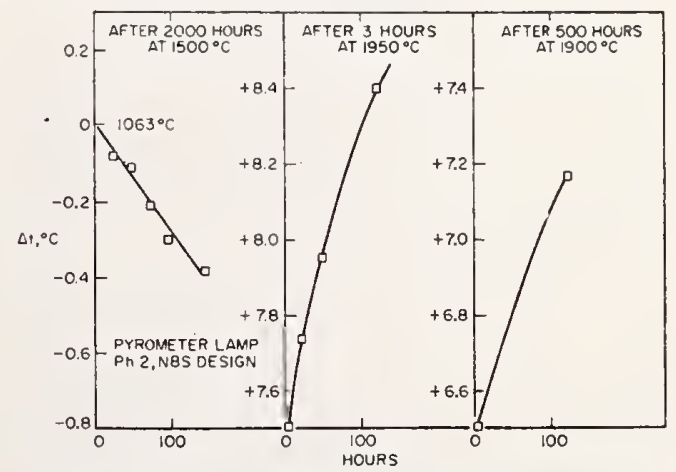

Fig. 6. Changes in radiance temperature of a pyrometer lamp versus hours at a constant lamp cturren, as a result of ageing the lamp 


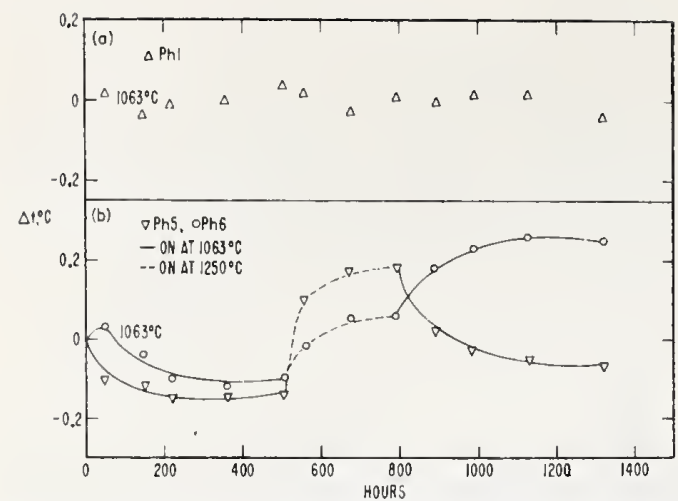

Fig. 7. (a) Comparisons of a pyrometer lamp and a $1.3 \mathrm{~mm}$ vacuum strip lanip both left off except for the brief comparisons. The pyrometcr and strip lamps were used to check lamps in b below. (b) Changes in radiance
temperature $1063^{\circ} \mathrm{C}$ versus hours at constant lamp currents for $1063^{\circ} \mathrm{C}$ or $1250{ }^{\circ} \mathrm{C}$ radiance temperatures

Fig. 7. Others behaved better while a few wcre substantially worse.

The photoelectric pyrometer was given a primary calibration, using the pyrometer lamp designated ph 1. This lamp, whose early history is shown in Fig. 5, had been frequently used thereafter, and therefore received additional ageing. After the calibration, comparisons were made at $1063{ }^{\circ} \mathrm{C}$ with the gold point furnace, as the most reliable way of ascertaining drifts and correcting the IPTS. Fig. 9 shows the results of these comparisons. After the first onehundred hours the trend has become fairly predictable.

Short term instabilities of pyrometer lamps were investigated by first matching to a strip lamp at $1063{ }^{\circ} \mathrm{C}$ radiance temperature, then turning the pyrometer lamps up to $1256{ }^{\circ} \mathrm{C}$ (highest temperature used) for an hour, and immediately thereafter rechecking at $1063{ }^{\circ} \mathrm{C}$ to see if the original matches were reproduced. Early in its history ph 1 showed a significant inertia, taking an hour to recover [6] in this treatment. After several years of use at various temperatures the lamp has improved and recent cycling tests show recovery within two minutes at $1063{ }^{\circ} \mathrm{C}$ after an hour at $1256{ }^{\circ} \mathrm{C}$.

Our experience with vacuum tungsten pyrometer lamps has not led to any conclusion as to the causes of observed instabilities. A new lamp may either increase or decrease in radiance with time and at an unpre-

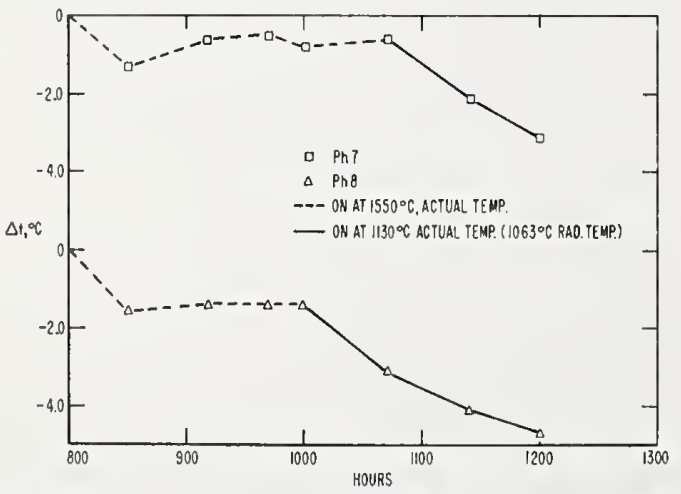

Fig. 8. Pyrometer ianps checked at $1063^{\circ} \mathrm{C}$ for final stablilty whlle on after the indleated period of ageing at $1550^{\circ} \mathrm{C}$, then checked for stability during the additional indicated ageing at $1083^{\circ} \mathrm{C}$ radiance tomperature

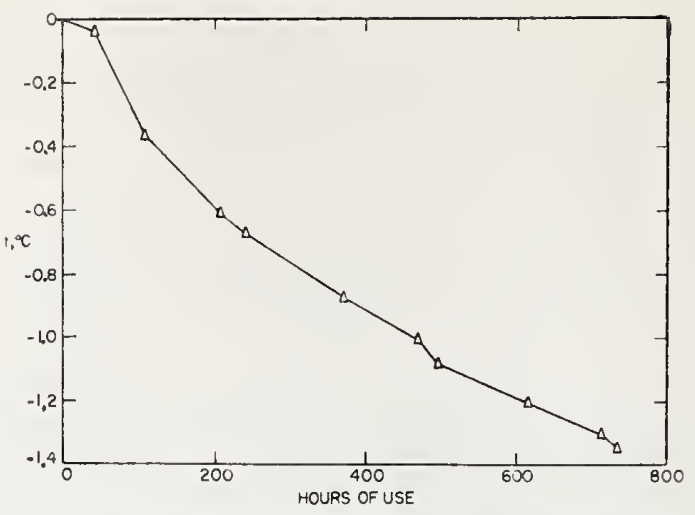

Fig. 9. Changes at $1063^{\circ} \mathrm{C}$ radiance temperature of pyrometer iamp, ph 1 , with hours of use in the calibrated pyrometer. The lamp becomes less radiant at a given current with continued usc

dictable rate. Lamps achieving good long tcrm stability have, on occasion, begun to change morc rapidly. Our present practice is to choose a lamp with a suitable history and check or recalibrate is after fifty to a hundred hours of use. In addition, a more comprehensive investigation of pyrometer lamps is bcing initiated.

\section{Effect of the size of the radiant source on accuracy}

Three different factors that could affect the accuracy of realizing the IPTS were related to the size of the radiant source. In the initial design of the pyrometer there were adverse effects caused by scattering of radiation in the optical system and heating of the pyrometer lamp filament by the incident image. In addition, there was heating of the absorbing glasses by the incident radiation, which was dependent on the source size (as well as its temperature); this heating was found to cause a change in absorptance. The effect on performance of all three factors has been considerably reduced by the addition of lenses IA and the $1.3 \mathrm{~mm}$ field stop (Fig. 1). Never. theless, prior investigation of these factors with lenses IIA and IIB as the objective had revealed several characteristics of interest.

\subsection{Radiation scatter in the optical system}

It had been observed that a significant amount of the radiation originating in the object plane (the conjugate image of the slit) outside the target area was detected by the pyrometer. Radiation from these areas on the source could arrive at the slit through diffraction, interreflections in the optical components, and scattering by dust or imperfections in the components. In the absence of detailed analysis of the causes and to simplify discussion, the combined effects will be referred to as a scattering of the radiation.

The effect of scatter on the radiance match with sources of the same radiance but differing in size was investigated. In the process of a primary calibration, a gold point furnace, which is a radiant source approximately $50 \mathrm{~mm}$ in diameter, and $1.3 \mathrm{~mm}$ wide tungsten strip lamps are used. An cxperimental source made of a $50 \mathrm{~mm}$ diameter disk coated with $\mathrm{MgO}$ paint was irradiated by a number of conventional incandescent lamps to represent the furnace. The flat disk at room temperature provided a convenient way to represent, 
also, a $1.3 \mathrm{~mm}$ by $50 \mathrm{~mm}$ strip source by positioning black velvet paper with a cut out of these dimensions in front of the disk. Upon comparing these two different sized and shaped surfaces at $1063{ }^{\circ} \mathrm{C}$ simulated tempcrature, the $50 \mathrm{~mm}$ disk. matched $0.1 \mathrm{deg} \mathrm{C}$ higher than the $1.3 \mathrm{~mm}$ strip.

There was some concern that the partial coherence of the reflected radiation from the $\mathrm{MgO}$ may not properly simulate a self-radiating source. Therefore, besides the $\mathrm{MgO}$ disk, a self-radiating $50 \mathrm{~mm}$ disk of $60 \%$ platinum, $40 \%$ rhodium alloy was used, with $1.3 \mathrm{~mm}$ graphite cut outs. Tests with both disks gave similar results, and indicated that the more convenient $\mathrm{MgO}$ source is suitable for additional work of this nature.

The amount of radiation detected from outside the target area is considerably more than that equivalent to the $0.1 \operatorname{deg} \mathrm{C}$ radiance temperature. Fig. 10 shows the relative photoelectric response in an experiment which reveals the total amount of scattered

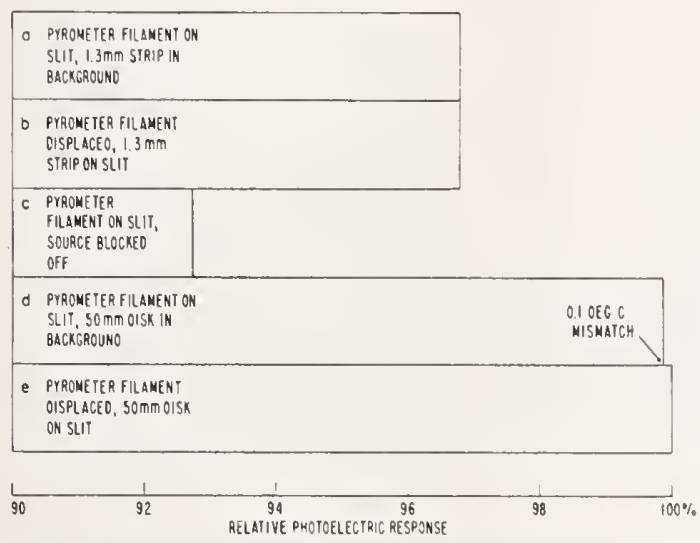

Fig. 10. Drop in photoelectric response to the pyrometer lamp flament when the $1.3 \mathrm{~mm}$ by $50 \mathrm{~mm}$ and the $50 \mathrm{~mm}$ disk sources are blocked of The pyrometer lamp current was constant throughout. Ony the 0.1 deg C mismatch is of significance to accuracy

radiation. The figure first represents a match ( $a$ and $b$ ) between the pyrometer lamp and the $1.3 \mathrm{~mm}$ strip. The drop in response at c occurs when the source is blocked off from the pyrometer. This shows that about $4 \%$ of the radiation (difference between a and c) arrived at the slit through scatter from the $1.3 \mathrm{~mm}$ strip source. Over $7 \%$ is seattered from the $50 \mathrm{~mm}$ disk (difference between $\mathrm{d}$ and $\mathrm{c}$ ).

For further explorations of scatter, an MgO disk was made with a $1 \mathrm{~mm}$ diameter hole through the center. In use the photoelectric pyrometer is sighted at the center of the dark hole. Thus, any photoelectric response under these conditions is that due to scatter alone.

By using this source it was determined that the interference filters were largely responsible for the radiation scatter. When the photoelectric pyrometer was tested without the filters the percentage scattered was reduced by a factor of three. The interference layers of the filters, when examined with oblique illumination, appear somewhat spotty.

By adding the $1.3 \mathrm{~mm}$ field stop and lenses IA to image the source at the stop, the effective size of the source was made invariant for the remainder of the pyrometer. The scattering under these conditions was about $3.5 \%$ of the radiation detected. The matching discrepancy between a $50 \mathrm{~mm}$ disk and a $1.3 \mathrm{~mm}$ strip was reduced from $0.1 \operatorname{dec} \mathrm{C}$ to $0.03 \mathrm{deg} \mathrm{C}$. This correction was made when comparing the gold point furnace and a $1.3 \mathrm{~mm}$ strip lamp.

\subsection{Heating of the pyrometer filament by incident radiation}

The heating of the pyrometer filament by the incident radiation could lead to errors in radiance temperature mcasurements. The filament ls horizontal, and of two sources of the same radiance the wider source gave rise to more heating of the filament, which would then require less lamp current for a match.

The heating of the filament was first noticed by a slight increase in pyrometer lamp current when a source was blocked off. This change indicated a decrease in the resistance of the lamp filament bronght about by its decrease in temperature. To check this further, an infrared transmitting filter with essentially complete absorption at $7500 \AA$ and shorter wavelengths was positioned between the source and the pyrometer. This filter eliminated any effects of the radiation scatter by absorbing most of the radiation which the photomultiplier could detect. Pyrometer lamp radiance changes were observed as the front lens (of the initial photoelectric pyrometer) was covered with a shutter. With a $1.3 \mathrm{~mm}$ strip lamp as a $1063{ }^{\circ} \mathrm{C}$ source the decrease in radiance temperature of the filament was about $0.05 \mathrm{deg}$ C. Before similar ohservations could be made with the gold point furnace, the pyrometer was modified with the additional lenses and field stop. It was estimated, that with the initial pyrometer design the gold point would have raised the pyrometer filament about $1 \mathrm{deg} \mathrm{C}$.

With the modification, as with the radiation scatter effect, the size (and width) of the source was made invariant, and there should be no unequal heating by different sources. Experiments, however, with the infra-red filter and the gold point furnace as well as the strip lamp showed a $0.03 \mathrm{deg} \mathrm{C}$ difference in temperature. This is believed to be caused by the greater infra-red content of the furnace radiation. The $0.03 \operatorname{deg} \mathrm{C}$ correction was made in the calibration process, where the gold blackbody and strip lamps were compared.

\subsection{Change of absorptance of the absorbing glasses through heating}

The change in the absorptance of the absorbing glasses due to heating by the incident radiation was indicated by matching a $1.3 \mathrm{~mm}$ strip lamp at $1350^{\circ} \mathrm{C}$, through an absorbing glass, before and immediately after sighting on a $3 \mathrm{~mm}$ by $45 \mathrm{~mm}$ strip lamp at $2400{ }^{\circ} \mathrm{C}$ for one hour. The difference in absorptance before and after heating by the high temperature lamp was observed as an erroneous change in the radiance temperature of the $1350{ }^{\circ} \mathrm{C}$ lamp of a little more than one deg C. Both the Jena NG-3 and the Corning Brown were tested in this manner. The Jena NG-3 glass decreased in absorptance with heating and the Corning glass increased by about the same amount.

Because the Jena and Corning glasses showed opposite temperature effects, a laminated absorbing 
glass of Jena NG-3 and Corning Brown was constructed in order to neutralize the heating effeet. Tests on the laminated glass showed this to be a satisfaetory solution. However, the addition of the $1.3 \mathrm{~mm}$ field stop, whieh was desirable for other reasons also, eut down the radiant energy incident on the glasses from a $3 \mathrm{~mm}$ strip lanp by a factor of about 100. Further tests showed this to be an adequate solution to climinating undue heating by radiant sourees, and the Jena NG-3 glasses were continued in use.

The pyrometer is used in a laboratory where the temperature is known to vary over a range of $2 \mathrm{deg} C$. To test the effect of the temperature of the glass as it changes with ambient temperature, a $6 \operatorname{deg} \mathrm{C}$ change in ambient temperature was induced while the pyrometer was matched to a $1.256^{\circ} \mathrm{C}$ strip lamp using an absorbing glass. Comparison of temperature measure-
$1063{ }^{\circ} \mathrm{C}, C_{2}$ as 1.438 centimeter degrecs, and $T_{0}$ as 273.15 degrees. $\lambda$ is the wavelength in centimeters. The realization of the II'TS above $1063{ }^{\circ} \mathrm{C}$ can be divided into four parts. These eonsist of (1), a calibration at the gold point, (2), a low range ealibration obtained by ineasuring ratios of raiianees of sources at higher temperature, to the gold point radianee, (3), determination of the moan effective wavelengths of the pyrometer, and finaliy, (4), determination of the " $A$ " values of the absorbing glasses used for the higher tempcraturc ranges. The " $A$ " value is defined as

$$
\text { "A" }=\frac{1}{T_{\mathrm{a}}}-\frac{1}{T}
$$

where $T$ is the source temperature in ${ }^{\circ} \mathrm{K}$ and $T_{\mathrm{a}}$ is the lower apparent temperature $\left({ }^{\circ} \mathrm{K}\right)$ of the source as seen through the glass.
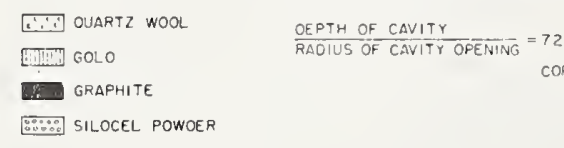

COPPER COOLING COILS

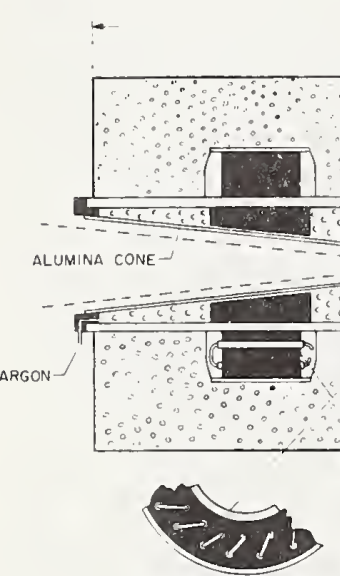

N!CHROME WINDINGS

IN ALUMINA TUBES

Fig. 11. Cross section of the NBS horizontal gold point blackbody and furnace

HIGH TEMPERATURE CEMENT

\subsection{Calibration \\ at the goid point, $106.3{ }^{\circ} \mathrm{C}$}

A gold stabilized hlackbody cavity and furnace that would be reliable enough to mcet the demands of the increased sensitivity of the photoelectric pyroneter were designed and constructed. Fig. 11 illustrates this design. Particular attention was devoted to achieving a uniform temperaturc over theinner walls of the cavity. The crucible containing 1800 grams of gold is shaped so that the mass of gold per unit length is about constant for the crucible's entire inner length. The furnacc has three independently controlled heater windings which are imbedded in cylindrical graphite muffles. The power inputs to the two end windings are adjusted to maintain the two end sections to within $5 \mathrm{deg} \mathrm{C}$ of $1063{ }^{\circ} \mathrm{C}$ as determined by einbedded thermocouples (not shown). The center winding is used for controlling the rate of heat loss by the gold during a freeze, as well as for heating during a melt.

Gold with a purity of 0.99999 as determined by the Analytical Chemistry Division at NBS is used. The cavity and crucible for the gold are made of the highest purity graphite a vailable. The manufacturer claims that the inpurities do not exceed 20 parts per million. In

addition, the graphite has a high enissi-

ments with the glass and without (i.e. on the low range) showed there was about $0.1 \%$ ehange in trans. mittance per deg $\mathrm{C}$ change in glass tcmperature for the Jena NG-3. In terms of errors in radiant temperatures measurement at $1256{ }^{\circ} \mathrm{C}, 0.1 \%$ amounts to about $0.1 \mathrm{deg}$ C. This effect is accounted for by noting the ambient temperature when the absorbing glasses are calibrated and correcting this calibration for any other ambient temperature during use.

\section{Calibration of the photoelectric pyrometer}

The prineiples involved in calibrating a visual optical pyrometer [2], that is, in realizing the IPTS, also apply to the photoclectric pyrometer. The definition of the IPTS above the gold point is as follows [1].

$$
\frac{N_{\mathrm{b} \dot{ }(t)}}{N_{\mathrm{b} \lambda\left(t_{\mathrm{au}}\right)}}=\frac{e^{\frac{C_{2}}{\lambda\left(\mathrm{t}_{\mathrm{au}}+T_{\mathrm{o}}\right)}}-1}{e^{\frac{C_{2}}{\lambda\left(t+T_{\mathrm{o}}\right)}}-1}
$$

$N_{\mathrm{b} \lambda}(t)$ and $N_{\mathrm{b} \lambda}\left(t_{\mathrm{au}}\right)$ are the speetral radiances of blackbodies at temperatures $t$ and $t_{\mathrm{au}} \cdot t_{\mathrm{au}}$ is defined as vity, a fairly high thermal conductivity, and is easy to
machine. The latter characteristic has made possible the construction of cavities of $0.5 \mathrm{~mm}$ wall thickness.

Sightings werc made on the diaphragm at the edge of the cavity opening as a check for temperature uniformity. The the hole. Since the effective emissivity of the diaphragm is expected to be less than that of ihe hole, the temperature difference between the cavity and the diaphragm is less than $0.2 \mathrm{deg}$ C. Nevertheless, if the inner surface of the diaphragm were $0.2 \mathrm{deg} \mathrm{C}$ lower than $1063^{\circ} \mathrm{C}$ the emissivity of the cavity would be reduced by only 0.000001 .

The effective emissivity of the cavity was calculated by using the equations derived by DE Vos [10]. The partial reflectance used was determined at room temperature on a piece of graphite with a surface condition similar to that in the cavity. DE Vos' first approximation to the emissivity was calculated to be 0.99999 .

By the time the first full calibration was completed, the gold point furnace had been used a number of tines over a two-year span. In particular. comparisons of two different gold point blackbodies, of melts and freezes, of the constancy of the melting and freezing plateaus, and trends during a day's operation had been obtained. One of the crucibles contained a cavity of $0.5 \mathrm{~mm}$ wall thickrress and the other a cavity of $1.0 \mathrm{inm}$ wall. Tab. 2 summarizes these results. From these comparisons the gold blacklody appears to be as reproducible radiance temperature was about $0.2 \mathrm{deg} C$ lower than that of 
as the precision available $(0.02 \mathrm{deg}$ C), and has not detcriorated in use. In aldition, the negligible differcner between the two erucibles indicates that the correction for temperature gradicnts across the cavity wall is negligible.

With the main heater power off, the duration of a frecze is about 15 to 17 mins. This time is largely dependent on the degree of furnace insulation and the amount of gold used. Fig. 12 is i reproduction of an epproach to and departure from a freezing plateau. With some power on, the duration of a frecze has been prolonged to an hour, and there is no significant difference in radiance from that of a $17 \mathrm{~min}$ freeze. For the melts the power input is adjusted for approximately 17 mins duration of melting.

There was a question of whether there may be some absorption of the exit radiation by the furnace gases, which probably consist of $\mathrm{C}, \mathrm{CO}$, and $\mathrm{CO}_{\mathrm{g}}$. Argon is fed in from the front of the furnace but it cannot be relied on to completely clear away other gases from the optical path. To check whether there may be absorption within the spectral pass band of the pyrometer, the crucible and back section were removed and the crucible was replaced with a graphite cylinder. The eylinder was hollowed out to allow an image of a strip lamp to be formed within the furnace and sighted upon by the pyrometcr. Matches were made with the strip lamp image through the hot $\left(1063^{\circ} \mathrm{C}\right)$ furnace and with the furnace removed. A diaphragm was placed in front of the lamp enrelope so that radiation from the furnace did not reach the strip lamp and raise its temperature. Ray tracing from the hot furnace parts through the lens to the diaphragm showed that the radiation was propcrly blocked. This was realized by making a larger opening in the graphite at the image plane than was otherwise necessary.

A difference in matches with and without the furnace had to be corrected for the extra radiation known to be scattered in from the furnace, and also for radiation reflected from the

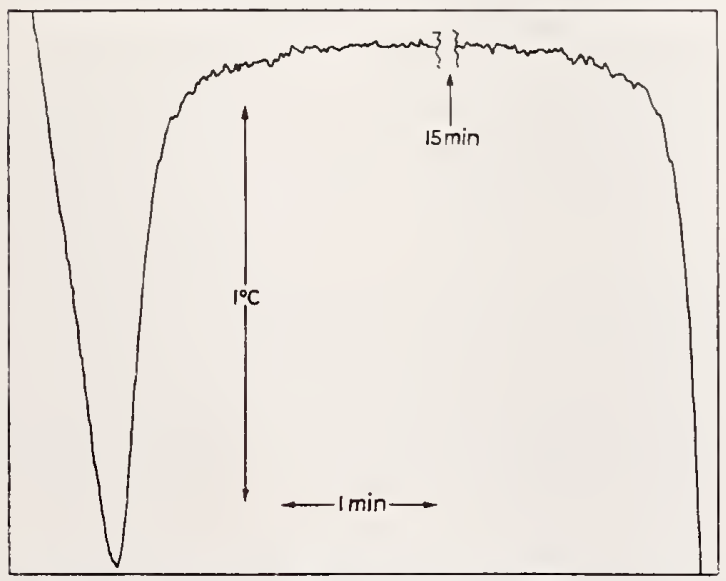

Fig. 12. Chart recording of approach to and termination of a gold point freeze plateau

lenses forming the strip image. To find this correction, the strip lamp was turned off, and the photoelectric response with the furnace in place was obtained while the pyrometer was focused on the image of the dark strip.

The corrected comparisons of radiance matches with and without the furnace should show whether furnace gases absorb a significant amount of the radiation. A number of comparisons averaged to a $0.002 \mathrm{deg} C$ difference with the standard deviation of the result being $0.015 \mathrm{deg} \mathrm{C}^{\star}$. Under the

* This number with other standard deviations yet to be discussed is listed in Table 3 .

Freezes vs. Melts

[Melt] - [Melt]

1 st half 2nd half

[Mclts] - [Melts] conditions of the experiment there was considerably more graphite surfacc exposed than with the gold-filled crucible. Aiso, therc was no discernible difference in the results with argon cither flowing in or turned off. It was concluded that to within thc cxperimental error of these neasureincrits there was no absorption by the furnace gascs in the spectral passband of the pyrometer.

A calibration of the photoelectric pyrometer at the gold point consists of radiance nutches with three melts and freezes.

Table 2. Analysis of the Gold Point Repreducibitity

n $\begin{array}{ll}\begin{array}{l}\text { Average of } \\ \text { Measurements }\end{array} & \begin{array}{l}\text { Number of S.D. of a single } \\ \text { Measure- Meagurement } \\ \text { ments }\end{array}\end{array}$

$0.5 \mathrm{~mm}$ Wall vs $1.0 \mathrm{~mm}$ Wall Cavity

[Melts and Freczes],

$+0.009 \mathrm{deg} \mathrm{C} \quad 14 \quad 0.015 \mathrm{deg} \mathrm{C}$

Trend of a Pliteau

[Freeze] - [Freeze]

$+0.001 \operatorname{deg} \mathrm{C} \quad 41 \quad 0.008 \mathrm{deg} \mathrm{C}$

$+0.010 \operatorname{deg} \mathrm{C} \quad 48 \quad 0.010 \mathrm{deg} \mathrm{C}$

Trend during a Day

[Freezes] - [Freezes

1 st half 2 nd half

$\begin{array}{lll}-3.012 \operatorname{deg} C & 23 & 0.015 \operatorname{deg} \mathrm{C} \\ -0.009 \operatorname{deg} \mathrm{C} & 21 & 0.015 \operatorname{deg} \mathrm{C}\end{array}$

Two measurcments are obtained for each platean with each measurement consisting of an average of eight matches. The standard deviation of a measurement is $0.023 \mathrm{deg}$ C. A correction of $0.03 \mathrm{deg} \mathrm{C}$ is made when the gold point radiance is transferred to a $1.3 \mathrm{~mm}$ strip lamp, to compensate for the effect of the difference in source size. An additional correction of $0.03 \mathrm{deg} \mathrm{C}$ is made for the difference in heating of the pyrometer lamps with the blackbody and strip lamp.

As far as it can be determined, the uncertainty in maintaining the IPTS at the gold point is preponderantly due to lack of sufficient stability of the pyrometer lamps rather thar. to the gold point apparatus or the calibration procedure. After each hundred hours of use the photoelectric pyrometer is recalibrated at the gold point to correct for the pyrometer lamp drift, which is shown in Fig. 9. Between gold point calibrations the amount of drift is predicted from an extrapola. tion in Fig. 9, and the necessary correction is made. The prediction is estimated to be uncertain by $0.05 \mathrm{deg} C$ by the end of a hundred hours. Taking this into aecount, along with the precision of the matches and the uncertainties of corrections made for transferrals to a strip lamp, the overall uncertainty of the gold point after a hundred hours was calculated to be $0.06 \mathrm{deg} \mathrm{C}$ (standard deviation). In the calculation, the various individual uncertainties are treated as independent standard deviations and are combined in quadrature.

\subsection{Realizing ratios of radiance}

Sectored disks are used in visual pyrometry to obtain radiance ratios required to realize the IPTS above the gold point [2]. They are feasible in visual work due to the persistence of vision of the observer. It did not appear desirable to usc sectored disks with the photoelectric pyrometer, because with their use a comparison would be made between a high, intermittent irradiance from the source and a low steady irradiance from the pyrometer lamp. Experience has indicatcd that the gain of the photomultiplier would not be constant with the accuracy desired for these two irradiances. Initially, a double beam splitter was used $[7,11]$ to obtain ratios relative to the gold point radiance. Ratios of $2^{n}$ where $n$ is an integer could be obtained by this derice. Erminy of NBS conceived a scheme for obtaining more numerous ratios and also fractional ratios [12] with a device for conjoining beams from three radiant sources. The method was implemented as shown in Fig. 13. Ratios of $1.5,2,3,4,6$, and 8 were used above the gold point to $1256^{\circ} \mathrm{C}$. A calibration was also made to $794^{\circ} \mathrm{C}$ 
using ratios of $0.67,5, .25$, cte.. with successive ratios of a factor of one half. This latter extension is needed in somo of the work in calibrating commercial opticel pyrometers. In all, 14 detcruninations of radiance temperature versus laup current from $794^{\circ} \mathrm{C}$. to $1256^{\circ} \mathrm{C}$ were obtained.

The sources used in the conjoiner were $1.3 \mathrm{~mm}$ tumpsten strip vacuum lamps. The lamj, currents were well regulated and essentially no lamp instability was detected over the thirty minutes that it took to obtain a calihration point. Typically, the lamps were matehed individually + times cach, then matehed in combination $x$ times, and finilly recherked individually with 4 matches cach. Some calibration points were taken with the thrce lamps set in different combinations of radiance ratios. For instance, the calibration at. $1 \cdot 56^{\circ} \mathrm{C}$ was obtained with lamps set at previously achieved ratios of 3,2 , and 3 and then at ratios of 4,2 . and 2 .

A ealibration at a given temperature was repented two or three tinıes, usually within a two hour period. Above $1063{ }^{\circ} \mathrm{C}$ the individual results differed by no more than 0.03 (leg $\mathrm{C}$, and below $1063^{\circ} \mathrm{C}$, except for $794^{\circ} \mathrm{C}$, there was a maximum of $0.04 \mathrm{deg} \mathrm{C}$ difference. At $794^{\circ} \mathrm{C}$ the calibration hall a rangc of $0.15 \operatorname{deg} C$. This was expected due 10 the poorer sensitivity at the low temperatures.

In order to provide arlequate interpolation between the calibrated points, least squares fits to virious equations werc

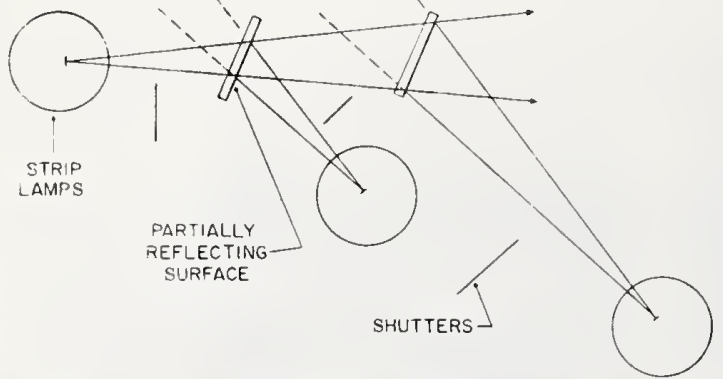

Fig. 13. Optical design of a beam conjoiner for providing radiance ratios rclative to the gold point

calculated. Following Kostrowski, Erminy, and HattenBURG [13], the equation

$$
\frac{1}{I}=\frac{A}{T_{r}^{2}}+\frac{B}{T_{r}}+C+D T_{\mathrm{r}}+E T_{r}^{2}+F T_{r}^{3}+\frac{G}{T_{r}^{s}}
$$

was derived by assuming that conduction losses in the pyro. meter lamp filament, to a first approximation, were proportional to the temperature. Tables were made using this equation. However, as found by Kostkowski, et al., a polynomial of the form $I=P\left(T_{\mathrm{r}}\right)$ fitted as well and required one less term. In both equations the least squares deriation of the points was less than $0.02 \mathrm{deg} \mathrm{C}$, and the standard deviation of the points along the curve was, on the avcrage, less than $0.02 \mathrm{deg} \mathrm{C}$.

Whenever a gold point calibration was made to check the pyrometer lamp drift, the low range was either recalculated on the basis of the new gold point and certain other assumptions discussed below, or (at alternate times) given a more complete calibration with the beam conjoiner. Ten-point calihrations were performed rather then fourteen-point calibrations as initially performed. The ten-point calibrations are less time consuming and are adequate to correct established tables.

Fig. 14 compares conjoiner calibrations performed after 260,495 , and 735 hours of pyrometer use with the initial conjoiner calihration. Each calibration is normalized to the original calibration by adding $\Delta t$, to the radiance temperature $t$ in the new calibration where

$$
\Delta t_{\mathrm{t}}=\Delta t_{\mathrm{au}}\left(\frac{t+273.15}{1336.15}\right)^{2}
$$

and $\Delta t_{t}$ and $\Delta t_{\mathrm{au}}$ are radiance temperature differences beiween the new calihration and the original calibration at the radiance tcmperature $t$ and the gold point, respectively. This normalization implies that the spectral radiance of the lamp has changed by the same percentage at all radiance temperatures. Fig. 14 shows that this has not been the case, at least in the earlier calibrations. The radiences corresponding to calibration points above the gold point appear to have changed less than the percent change at $1063^{\circ}{ }^{\prime}$ and vice vera below the gold point. However, departures from this simple model have cerreased with time as have changes in the gold point calibration. For example, the gold point changed by $0.7,0.4$ and 0.25 leg 6 " hetween successive calibrations at $0.260,495$, and 735 hours. At $1256 \%$, the change in terms of a departure from the above model has been $0.14,0.06$, and 0.05 deg. These epartures are in a direction to suggest using a model in which the radiance temperature changes are constant at all points. The various calihrations agrec with this latter model somewhat hetter but with an opposite sign. Thus the correct model is somewhere hetween the two simple ones discussed. However, the changes hetwcen the last two calihrations $(0.25 \mathrm{deg} C$ at the gold point and $0.28 \log \left(C^{\prime}\right.$ at $1256^{\circ} \mathrm{C}$ ) are sufficiently small that either simple model agrees with the experimental results within the short term reprolucibility of the low range calibration.

Calihrations will be performed every 200 hours of pyrometer operation, using the beam conjoiner, meanwhile retaking the gold point at 100 hour intervals. In addition to predicting trends in the lainp at $1063^{\circ} \mathrm{C}$, trends in the low range at

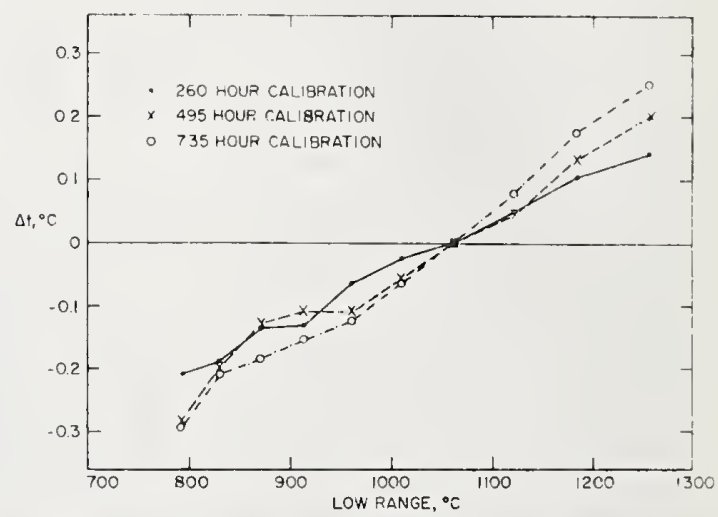

Fig. 14. Differences of three calibrations from the initial calibration after normalization to the initial gold point ralibration

temperatures away from $1063{ }^{\circ} \mathrm{C}$ are to be predicted, using the results in Fig. 14 as a guide.

In estimating uncertainties at $1256{ }^{\circ} \mathrm{C}$, a correction of $0.06 \mathrm{deg} \mathrm{C}$ is first to be made (in addition to one made for gold point changes) by the end of 200 hours. This extra correction is assessed as uncertain by $0.06 \mathrm{deg} C$, thus recognizing the possibility the correction may not be needed, or, on the other hand, that a $0.12 \mathrm{deg} \mathrm{C}$ difference may occur, corresponding (approximately) to the once observed difference of $0.14 \mathrm{deg} \mathrm{C}$ in 260 hours. Other sources of uncertainty at $1256^{\circ} \mathrm{C}$ are the uncertainty of the gold point, $0.06 \mathrm{deg} \mathrm{C}$, which increases to $0.08 \mathrm{deg} \mathrm{C}$ at $1256^{\circ} \mathrm{C}$, statistical variations of the beam conjoiner process of $0.046 \mathrm{deg} \mathrm{C}$ (standard deviation), and an estimated uncertainty of $0.03 \mathrm{deg} \mathrm{C}$ due to short-term prrometer lamp instabilities during the calibration process. In addition, there is a contribution due to the uncertainty of the mean effective wavelength, which is discussed later.

\subsection{Mean effective wavelength determination}

The mean effective wavelengths were derived from experimentally deternined curves of effective wavelength versus temperaturc [2]. The equation

$$
\frac{1}{\lambda_{\mathrm{e}}}=\frac{\int_{0}^{\infty} \frac{N_{\mathrm{b} \lambda} R_{\lambda} \mathrm{d} \lambda}{\lambda}}{\int_{0}^{\infty} N_{\mathrm{b} \lambda} R_{\lambda} \mathrm{d} \lambda}
$$

was used to obtain the effective wavelengths. $N_{\mathrm{b} \lambda}$ is the Planck radiation function, $\lambda$ the wavelength, $\lambda_{\mathrm{e}}$ the effective wavelength, and $R_{\lambda}$ is the relative spectral response of the photoclectric pyrouneter.

In the process of finding $R_{\dot{\lambda}}$, a small grating monochromator with an Ebert optical system was set up on its side to make the exit slit suitably horizontal for sighting with the 
pyrometer. The monochromator entrance optics were arranged so that a quiek and brief exchange of the image of tise regular source with that of a neon discharge sourec could be made. The monnchromator has a scauning drive, and the recorder anci the monoeliromator were run simultaneously. Thus a profile of photoelectric response versus wavelength was traeed. When a ehosen neon spectral line was approached, it was reeorded to provide a wavelength calibration on the reeorder chart.

Chart measurements were tiken at in $X$ intervals from 6000 to $6945 \AA$, at which wavelengths the response was less than $0.02 \%$ of the peak response. Corrections were inade for the relative spectral transmittance of the monochromator and the relative spectral radiance of the source in order to obtain $R \lambda$ of Eq. (5).

To find the relative transmittance of the monochromator (ineluding its external optics). sensitive vacuum thermopiles coated with gold black were used as approximately neutral detectors. A graphite are was used as a souree of sufficiently known speetral radiance [14] within the spectral passband. The optical beam was ehopperl at 11 cyeles per second and the output of a thermopile was fed to a tuncd amplifier. The spectral transmittance of the monochromator did not vary rapidly. Therefore it wits possible to use a relatively wide passband of $30 \AA$ which permitted a sufficient signal to woise ratio with the thermopile.

Twenty to thirty speetral transinittance curves were obtaned with each of three thermopiles. The average curve from eael thermopile was normalized to 1.00 at 6500 so that the three curves could be directly compared at other wavelengths. At $6800 \AA$ the transmittance was abont 0.9 of that at $6500 \AA$. and the range of the thrce values was 0.0035 . At $6300 \AA$ the relative transmittance was 0.95 and the range, 0.0075 . If these ranges were treated as errors, the range of 0.0075 would lead to an error in the effective wavelength of about $0.12 \AA$. This is not a significant error, and at any rate the average of the three curves should be in error less than the range, provide tliere are no systematic errors.

One thermopile was checked for its constancy of spectral ubsorptance by measuring the reflectance at several random spots on the gold black surface. There was a slight specular component, and measurements were taken at the specular angle as well as other angles. The absorptance (relative spectral) was calculated and was found to be independent of wavelength within the standard deviation of the measurement. This uncertainty had negligible effect on the effective wavelength.

Radiation is detectable at a low level at wavelengths well away from the main spectral passband of the pyrometer. It is of some significance to the accuracy of the effective wavelength to determine whether the wavelength of this radiation is that identified by the spectral setting, or otherwise scattered by the monochromator. While the amount scattered may be relatively insignificant, any scattered radiation at wavelengths of the main passband is preferentially detected by up to several orders of magnitude.

To test for speetral scatter, the spectral response of the photoelectric pyrometer was determined at monochromator settings of $6311 \AA, 6412 \AA$, and $6848 \AA$, with a strip lamp as a source. These are wavelengths away from the main passband, where the response is low, in the same order, $0.6 \%, 4.3 \%$, and $0.3 \%$ of the peak response. Three interference filters peaked at the given wavelengths were interposed in the optical path in turn as the monoehromator was set at the peak. The filters were spectrally narrow enough to reject the pyrometer's main passband and therefore any spectral scatter therefrom. A drop in response when a filter was interposed was corrected for the reduction of radiation at its peak transmittance. Any further drop would be attributed to scatter. However, less than $2 \%$ of the response at the tested wavelengths was accounted for in this way, and therefore scatter did not contribute to a significant error.

In determining the effective wavelengths several variations in experimental procedure were tried. A graphite arc $\left(3800^{\circ} \mathrm{K}\right)$ and a blackbody furnace at $1250^{\circ} \mathrm{C}$ were used as sources. Different sets of neon spectral lines were used. Wavelength scans were made in opposite speetral directions. The monochromator was fitted with different width slits although no greater than a $4 \AA$ passband was used. Various multiplier phototube voltage settings were used.

Of the different procedures only one gave eause for concern. A persistent difference of $0.6 \AA$ was found between scanning in the increasing and decreasing wavelength directions. The difference was opposite to that expected if the multiplier phototube were faliguing, which was primarily the factor the opposite scans were intended to check. The difference would he explained if the electronic response timcs $(1 / 6 \mathrm{sec})$ were not brief cnough to recorl a spectral line faithfully. Slower scans were limited by the burning time of the are and drifte of the 1250 ( $C$ furnacr. No reason could be found for a sean in one direction to result in a systematic error of $0.6 \AA$ while an opposite scan would contain no error. It was concluded that there was a systematic error in both cases of $0.3 \AA$, and that the average of oppositc scans was the most probably correct value.

The relative spectral response as found by the sruphite are and the $1250^{\circ} \mathrm{C}$ blackborly resulted in a difference in effective wavelength of $0.24 \AA$ at $1250^{\circ} \mathrm{C}$. This could be a statistical variation. Other variations in procedure did not reveal any systematic differences in results.

Effective wavelengths were taken a year apart to ascertain the stability of the spectral resporse. The photoelectric pyrometer was used frequently during this time. Initially 24 scans had been made and a year later 12 more. The averages of the two scts of values differed by $0.52 \AA$. The uncertainty of each average was estiniated to be about that amount. Since it is not readily disecrnible whether there was an actual change, the mean of both sets was used. In taking into account the uncertainties of the methods and also that ther: may be some change will time, an uncertainty of $0.8 \AA$ was assigned to the effective wavelength. This leads to an uncertainty of 0026 deg $\mathrm{C}$ in the calibration at $1256^{\circ} \mathrm{C}$.

The effective (and mean effecrive) wavelength of the pyrometer changres when absorbing glasses are used. To find the additional effective wavelength curves, the spectral transmittances of the glasses were determined, and the product of them and the previously found low range spectral response was taken as the overall spectral response with the glasses. The mean effective wavelengths of the pyrometer with absorbing glasses were not used in the calibration process. However, uncertainties of the relative transmittanee of the glasses figure in the calculations of " $A$ " values of the glasses.

\subsection{Determination of the " $A$ " values of the absorbing glasses}

Of the four Jena NG-3 absorbing glasses one is used singly to extend the temperature range from $1256^{\circ} \mathrm{C}$ to $1650^{\circ} \mathrm{C}$. The remaining glasses are successively added to provide tempera. ture ranges of $1650^{\circ} \mathrm{C}$ to $2330^{\circ} \mathrm{C}, 2330^{\circ} \mathrm{C}$ to $3730^{\circ} \mathrm{C}$ and, with all four glasses, $3730^{\circ} \mathrm{C}$ to $8570^{\circ} \mathrm{C}$. This procedure of aehieving the higher ranges with a series of glasses is used because the " $A$ " value of each glass, about 135 mireds, can be found separately and at the relatively low temperature of $1256^{\circ} \mathrm{C}$. In addition to this determination, however, the variation of the " $A$ " value with source temperature must be found. With the type glass used this variation should be small.

The " $A$ " values at $1256^{\circ} \mathrm{C}$ were obtained with a strip lamp source utilizing the low range ealibration. The glasses were inserted in turn and an apparent temperature of about $994{ }^{\circ} \mathrm{C}$ was measured. When a strip lamp rather than a blackbody radiator is used, its radiance temperature at the mean effective wavelength on the low range must be correeted for the mean effective wavelength on the higher range where the absorbing glass is used [2]. This correction is small, being only $0.04 \mathrm{deg} \mathrm{C}$ at $1256^{\circ} \mathrm{C}$.

The beam conjoiner was used to extend the calibration to $1383^{\circ} \mathrm{C}$ and $1533^{\circ} \mathrm{C}$ in an attempt to obtain the " $A$ " values at these temperatures, and therefore determine the variation of " $A$ " with temperature (trend). However, the " $A$ " value trend with temperature was too small to determine in this way. To establish the trend the equation,

$$
\int_{0}^{\infty} N_{\mathrm{b} \lambda}(T) R_{\lambda} T_{\mathrm{Bg}, \lambda} \mathrm{d} \lambda=\int_{0}^{\infty} N_{\mathrm{b} \lambda}\left(T_{\mathrm{a}}\right) R_{\lambda} \mathrm{d} \lambda
$$

was used. This equation expresses the radiance match between a blackbody source at the Kelvin temperature $T$, through an absorbing glass, and one at the resulting apparent temperature, $T_{\mathrm{B} .} T_{\mathrm{Bg}, \lambda}$ is the spectral transmittance of the glass. Values of $T$ were chosen and the equation was solved for $T_{\mathrm{a}}$. From $T$ and $T_{\mathrm{a}}$ the " $A$ " values were found. The increase of " $A$ " at $1650^{\circ} \mathrm{C}, 2330^{\circ} \mathrm{C}$, and $3730^{\circ} \mathrm{C}$ over that at $1256^{\circ} \mathrm{C}$, is in equivalent temperature, $0.05 \operatorname{deg} \mathrm{C}, 0.3 \mathrm{deg} \mathrm{C}$, and $0.7 \mathrm{deg} \mathrm{C}$. 
respcetively. The uncertainties of the values found are estimated to be $0.02 \mathrm{deg} C, 0.07 \mathrm{deg} C$, and $0.26 \mathrm{log} C$ in the same order.

Shortly after calibrations with the beam conjoiner the " $A$ " values were redeterinined at $1256^{\circ} \mathrm{C}$. The results from the first tliree calibrations, taken over a period of 14 months, show a linear increase of the " $A$ " valucs of about 0.035 mireds per year, or, in equivalent temperature at $1256{ }^{\circ} \mathrm{C}$. about $0.08 \mathrm{deg} C$ per year. The absorbing glasses were cleaned before the initial calibration, but they had not been clcaned thereafter, and the drift of the " $A$ " valucs indicated a need for recleaning. This was done but with variable results. Two absorbing glasses changed back to the original " $A$ " value and two did not. In cleaning glasses five months later for the fourth calibration, the " $A$ " values of the glasses changed significantly, about $0.2 \mathrm{deg} \mathrm{C} 1256^{\circ} \mathrm{C}$, from the previous calibration, and in the dircction of greater transmittance. The changes of the glasses and change betwcen glasses upon cleaning indicate our eleaning of them is not consistent. As a result, their " $A$ " values are more reproducible if thcy do not undergo the cleaning process. A slow drift of the glasses is tolerable when the drift is deterinined by recalibrations every 200 hours, and the correction predicted as a function of time. In the immediate future the " $A$ " values will be redetermined more often till a drift is discerncd. It is expected that the glasses may be cleaned at two year intervals, if the drift is no more than that previousiy observed.

The uncertainty of the " $A$ " values cletermined at $1256{ }^{\circ} \mathrm{C}$ is largely composed of uncertainties in the low range at $1256^{\circ} \mathrm{C}$ and at the resulting apparent temperature of $994^{\circ} \mathrm{C}$. (However, errors at these temperatures which are due to an error in the yold point determination cancel, and this is taken into account). When two or more glasses are used, errors in the " $A$ " values add. Inasmuch as the " $A$ " values are detcrmined at $1256^{\circ} \mathrm{C}$, when this temperature becomes the apparent temperature in a measurement, its error is additive to the errors in the " $A$ " values, i.e., they are not independent. The errors in other apparent temperatures are also likely to lack this independenee, inasmuch as errors in the low rance 2 way from the gold point arc probably in the same direction. Uncertainties are quoted for thosc high temperatures for which the apparent temperature is $1256{ }^{\circ} \mathrm{C}$ or near this temperature. The standard deviations of the non-independent uncertainties are $0.208 \mathrm{dcg} \mathrm{C}$ at $1650^{\circ} \mathrm{C}, 0.600 \mathrm{deg} \mathrm{C}$ at $2330^{\circ} \mathrm{C}$, and $1.755 \mathrm{deg} \mathrm{C}$ at $3525^{\circ} \mathrm{C}$ (which is in the vicinity of the graphite arc radiance temperaturc). These and other (indeperdent) standard deviations are listed in Tab. 3. It can be seen that the above given deviations are preponderant in affecting the accuracy of the IPTS at temperatures needing one or more absorbing glasses.

When two or more glasses are in position, interreflections add radiation to the image of the source. The reflected radiation superimposes an image upon the primary image. Thus a corrcction can be made which is good to the uncertainty of measuring the reflectance of the glass surfaces. Reflectance mcasurements were made to a precision of $0.4 \%$ standard deviation of a measurement), and this uncertainty is incorporated in the uncertainties of the high range temperatures.

\section{Estimated uncertainty in realizing and maiataining in IPTS.}

In developing the photoelectric pyrometer, a significant amount of time was spent on the investigation and elimination of possible systematic errors. The remaining errors, as far as can be determined, are the random errors in the various experimental processes in the calibration and the uncertainties of corrections for drifts of the pyrometer lamp.

In many cases the individual processes that coniribute to the IPTS were repeated often enough to yield a representative standard deviation. In other cases in which a process was not sufficiently repeated, standard deviations are estimated. In the case of trends, the

Table 3. Statistical errors and estimates of uncertainty in realizing and maintaining the IPTS

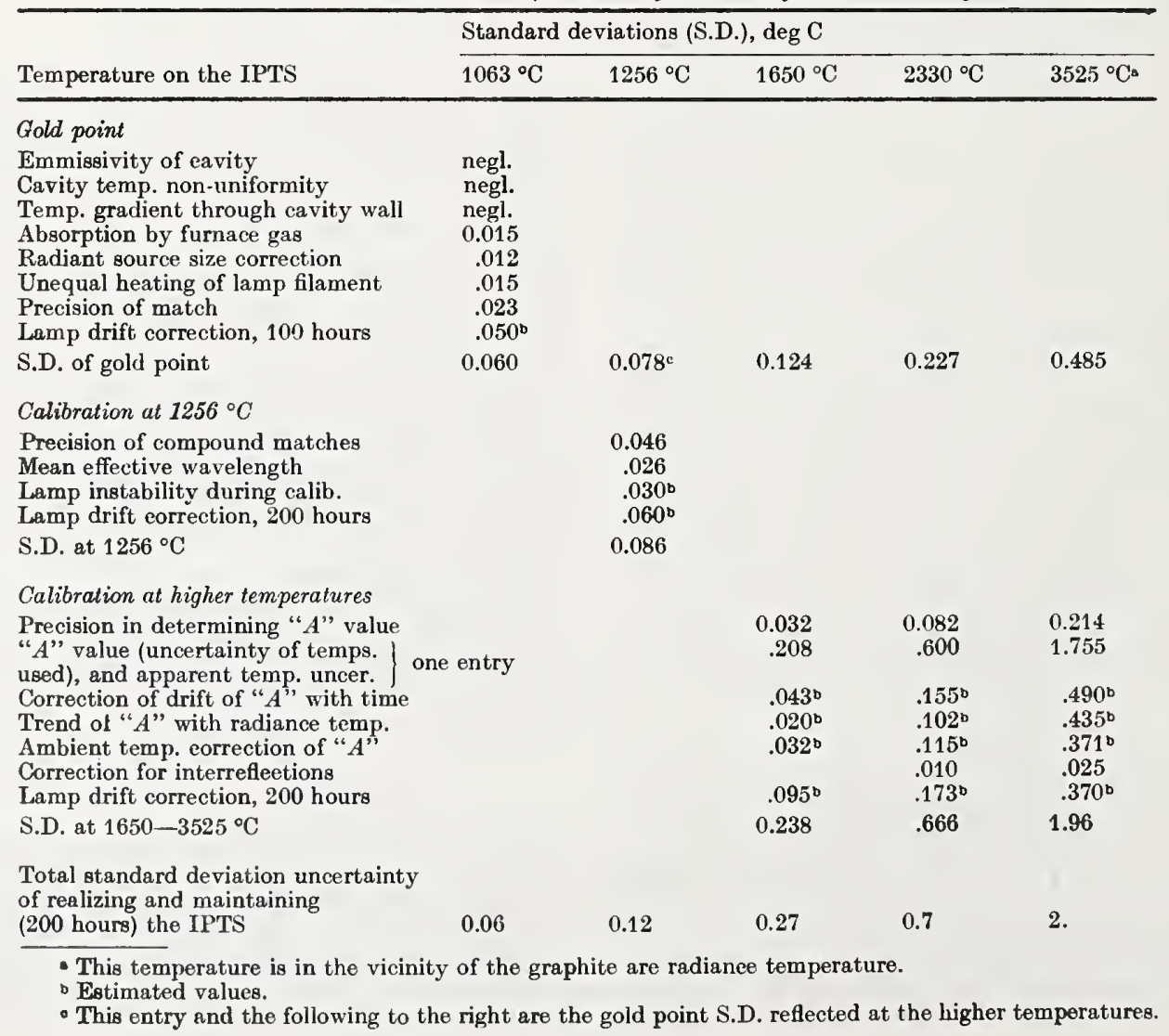


corrections for the trends are assigned estimates of standard deviations. It is our endea vor to combine in quadrature deviations of all the processes, to obtain crerall standard deviations at ehosen temperatures that are reasonably valid. Tab. 3 has been constructed tosummarize all the processes or elements that contribute to the IP'TS, along with their standari deviations (calculated or estimated). Some of the cntries are recognizable as related to a familiar process. Other entries originate when results are corrected for crrors which were experimentally determined. The corrections have statistical uncertainties which are passed on to the corrected result. The radiant source size correction in transferring a gold point calibration to a st rin lamp is an example.

'The final values at the end of Tab. 3 are the estimated standard deviation uncertainties of the IPTS at NBS after being maintained for 200 hours (photoclectric pyrometer use), with a gold point calibration at 100 hours. Immediately after a complet $\theta$ calibration the uncertainty up to $1256^{\circ} \mathrm{C}$ is reduced by about $40 \%$. At higher temperatures, where absorbing glasses are used, the improvement is not significant.

A spectroradiometer in the NBS Radiation Thermometry section of $w$ hich the photoelectric pyrometry project is a part, also requires the realization of the IPTS above $1063{ }^{\circ} \mathrm{C}$ [13]. The spectroradiometer provides a largely independent realization of the Scale, and with a temperature resolution of about 0.04 deg C. In IPTS calibrations with both instruments the same gold point furnace has bcen used, but from there on the instrumentation was signifieantly different. It was worthwhile to both projects to compare results as a measure of the uneertainties involved.

Comparisons were made on occasions about a year apart. On the first occasion a vacuum strip lamp on which the IPTS was newly realized in conjunction with the spectroradiometer was in turn checked with the recently calibrated photoelectric pyrometer. There was agreement at $1063^{\circ} \mathrm{C}$ within the precision of the pyrometer's radiance match, $0.02 \mathrm{deg}$ C. Of greater interest, the vacuum strip lamp was used with the spectroradiometer to ealibrate a gas-filled strip lamp at $2337^{\circ} \mathrm{C}$. This lamp was then calibrated with the photoelectric pyrometer and the two calibrations agreed to about $0.5 \mathrm{deg} \mathrm{C}$.

Recently, comparisons were made at $1040^{\circ} \mathrm{C}$, $1063{ }^{\circ} \mathrm{C}$ and $1256^{\circ} \mathrm{C}$ and differences were $0.03 \mathrm{deg} \mathrm{C}$, $0.06 \mathrm{deg} \mathrm{C}$, and $0.10 \mathrm{deg} \mathrm{C}$, respectively. A comparison at $3525^{\circ} \mathrm{C}$ using a graphite arc resulted in a difference of $0.2 \mathrm{deg}$ C. However, the reproducibility of the arc itself was only $3 \mathrm{deg}$ C.

In the course of making these comparisons, corrections of the strip lamp radiance temperatures were made for slightly different mean effective wavelengths, for different target areas over which temperature gradients existed, and for a partial polarization of the lamp. The spectroradiometer could not be completely depolarized at all wavelengths and there was a correction due to polarization at the mean effective wavelength of the pyrometer. The corrections were small, being about the same magnitude as the final differences observed.

The uncertainty inherent in the corrections made makes the comparisons less certain. But in allowing for this, the overall results, which reflect combined inaccuracies of both instrumente, are vicwed as supporting the uncertainty estimates of Tab. 3. A more regular schedule of comparisons is planned as a continuing check on the IPTS as realized. with the two different instruments.

The NBS laboratory visual pyrometer, which has been in service in calibrating strip lanps and visual optical pyrometers for many years, was compared with the photoelectric pyrometer through a simultaneous calibration of strip lamps. Up to $1300{ }^{\circ} \mathrm{C}$ a vacuum strip lamp was calibrated, and on up to $2300{ }^{\circ} \mathrm{C}$ a gas-filled lamp was calibraterl. Further, at about $2730{ }^{\circ} \mathrm{C}$ and $3525{ }^{\circ} \mathrm{C}$ a graphite are was used with both instruments. To attain $2730^{\circ} \mathrm{C}$ the are was used with an absorbing glass.

Differences in the calibrations are given in Fig. 15. Part of the differences may be real, but the results are of the same order of magnitude as the precision of the

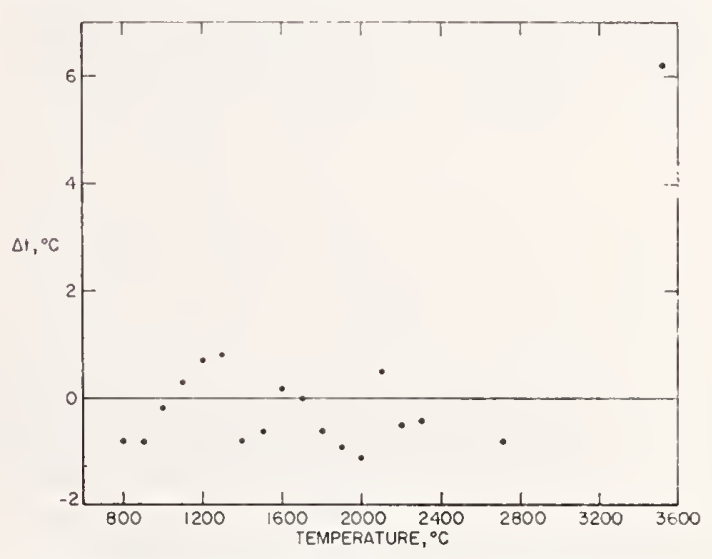

Fig. 15. Difference in the 1PTS as maintained on the NBS photoelectric pyrometer and the NBS laboratory vloual pyrometer

visual observations in performing the calibrations. The agreement is good considering the accuracy to which a visual pyrometer can be calibrated. 'The uncertainty (standard deviation) of the IPTS on the visual instrument has been estimated [15] to be $0.4 \operatorname{deg} \mathrm{C}$ at $1063{ }^{\circ} \mathrm{C}, 3 \operatorname{deg} \mathrm{C}$ at $2300^{\circ} \mathrm{C}$, and $7 \operatorname{deg} \mathrm{C}$ at $3525{ }^{\circ} \mathrm{C}$. Incidentally, the radiance temperature of the graphite are positive electrode measured with the photoelectric pyrometer was very close to $3525{ }^{\circ} \mathrm{C}$ or $3798^{\circ} \mathrm{K}$. However, differences between graphite electrodes up to as much as $12 \mathrm{deg} \mathrm{K}$ above or below $3798^{\circ} \mathrm{K}$ have been observed.

\section{Present status and future work}

In order to maintain the IPTS with an accuracy approaching that with which it can be realized, the photoclectric pyrometer must undergo frequent calibrations. A major limitation to the accuracy lies in the observed instabilities of the pyrometer lamps, unless they are frequently checked and corrected. Both the internal pyrometer lamps and the larger strip lamps, used as secondary temperature standards, lack the desired stability. There is a need for more reproducible temperature sources if the IPTS is to be transferred with a certainty approaching that attainable with the photoelectric pyrometer. As a result, an intensive investigation of lamps has been initiated. 


\section{Acknowledgments}

The author was privileged to complete the devclopment of a photoelectric pyromcter at NBS that was based on preliminary studies and experimental work of C. P. JoHnson, G. ZiEgLer and D. E. ERMinY. The photoelectric pyroncter was completed in the Radiation Thermometry section under the direction of H. J. Kostkowsku, Section Chief. The innumerable diseussions with him were of great value to the anthor. Most of the instrument was constructed by A. N. GRAEF and IV. Grote. Finally, the author wishes to thank E. L.w.w for his assistance in pyrometer calibrations and other work.

References: [1] Strmson, H. F.: The international practical temperature seale of 1948 . J. Research Nat. Bur. Standards (iid, No. 3,.139 (1961). - [2] Kos'rkowski. H.'J.. and R. D. LEE: Theory and methods of optical pyrometry. 'Temperature. its measurement and control in sciences and inclustry, Vol.3. Part 1, p. 449. New York: Reinhold Publishing Corporation 1961. - [3] LoveJoy, D. R.: Photometry of the optical pyrometer and its use below $800^{\circ} \mathrm{C}$. J. Opt. Soc. Am. 49, 249 (1959). - [4] K.andyba, V. V., and V. A. KovalevsKII: A photoelectric spcctropyrometer of high precision. Doklady Akad. Nauk. S.S.S.R. 10S, 633 (1956). - [5] MrDDLEHURST, J., and T. P. JoNES: A precision photoelectric optical pyrometer. Temperature, its measurement and control in seience and industry, Vol. 3. Part 1, p. 517. New York: Reinhold Publishing Corporation 1961. - [6] LEE, R. D.:
The NBS' photoclectric pyrometer of 1981. Temperature, its measurement and control in science and industry, Vol. 3, part 1, p. 507. New York: Reinhold Publishing Corporation 1961. - [7] LKE, R. D.: Some results and problems in calibrating the NBS photoelectric pyrometer of 1961. Comite Consultatif de Thermometrie, 6- Session (1962). - [8] FA r child. C. O., and W. H. Hoover: Disappearance of the filament and diffraction effects in improved forms of an optical pyrometer. .J. (Opt. Soc. Am. 7, 543 (1923). - [9] Enostroy, R. W.: Multiplier plototube characteristies: Application to low light levels. J. Opt. Soc. An. 37, 420 (1947). - [10] De Vos, I. C.: Evaluation of the quality of a blackbody. Physica 20, 669 (1954). - [11] BoJARski, L. A.: The use of the photoelectric methorl for precise measurements in the domain of the optical pyrometer. Procès.Verbaux des S'éances du Comité International des Poirls et Mesures 26A (1959). [12] Enminy, D. E.: Scheme for obtaining integral and fractional multiples of a given radiance. J. Opt. Soc. Am. 53, 1448 (1963). - [13] Kostkowsk1, H. J., D. E. Erminy, and A. T. HatTenbura: A high accuracy spectroradiometer, to be submitted to the Journal of the Optical Society of Amcriea. - [14] Null, M. R., and W. W. Lozier: Meisurement of reflectance and cmissivity at high temperatures with a carbon arc image furnace. Measurement of thermal radiation propert ies of solids. NAS.A SP-31 (1963). - [15] Kostkowskx, H. J., and G. W. BuRNs: Thermocouple and radiation thermometry above $900^{\circ} \mathrm{K}$. Mcasurement of thermal radiation propertirs of solids. NAS.A SP-31 (1963).

\author{
Offprint from ,Metrologia" \\ Journal International de Métrologie scientifique - International Journal of scientific Metrology \\ Internationale Zeitschrift für wissenschaftliche Metrologie. Vol. 2, No. 4, October 1966, Pages 150-162




\title{
Construction and Operation of a Simple High-Precision Copper-Point Blackbody and Furnace
}

\author{
R. D. Lee
}

NBS Technical Note 483, May 1969

Detailed instructions for the construction and operation of an inexpensive copper-point blackbody and furnace are presented. Such a source may be expected to realize the radiance temperature of $1083.3^{\circ} \mathrm{C}$ (Int. 48) with an uncertainty of $0.2^{\circ} \mathrm{C}$ and a variation in successive use of less than $0.033^{\circ} \mathrm{C}$. The furnace requires no inert gas or vacuum and utilizes only 225 watts to reach a melt in about an hour after turn on. Melts and freezes last about five minutes each and at least fifty melts and freezes are expected during the lifetime of the furnace. Key words: Blackbody; optical pyrometry; radiometry.

\section{Radiance Temperature at $6550 \AA$ of the Graphite Arc}

\section{R. D. Lee and E. Lewis}

National Bureau of Standards, Washington, D.C. 20234.

Received 1 August 1966.

Low current graphite arcs are frequently used as spectral radiance or radiance temperature standards, particularly in the vicinity of $6500-6600 \AA$, the spectral domain of the optical pyrometer. This letter reports on the results of a large number of radiance temperature measurements at $6550 \AA$ of the positive crater of a graphite arc. The measurements were made with the National Bureau of Standards photoelectric pyrometer ${ }^{1}$, which is estimated to realize the International Practical Temperature Scale (IPTS) ${ }^{2}$ with a standard deviation uncertainty of $2^{\circ} \mathrm{K}$ at $3800^{\circ} \mathrm{K}$, the approximate crater radiance temperature. The sensitivity of the pyrometer at $3800^{\circ} \mathrm{K}$ is $0.2^{\circ} \mathrm{K}$.

Spectroscopically pure graphite rods were used for the positive and negative electrodes (National Carbon grade SPK $\frac{1}{4}$ inch and AGKS $\frac{1}{8}$ inch, respectively). The negative electrode was inclined $-120^{\circ}$ to the positive electrode, which was mounted in a horizontal position, and the pyrometer sighting of the crater of the positive electrode was made along the axis of that electrode. The arc current (about $12.5 \mathrm{~A}$ ) was normally kept to within $0.2 \mathrm{~A}$ of

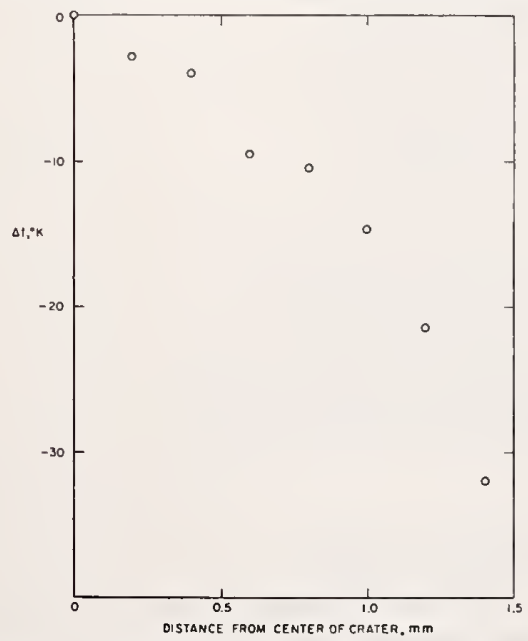

Fig. 1. Radial gradient in a graphite arc crater. the overload or hissing point. Occasionally, readings were made at $0.5 \mathrm{~A}$ below overload, and these showed a drop of less than $2^{\circ} \mathrm{K}$ in radiance temperature.

Two different lots of positive electrodes were used over a period of a year and a half. Out of one lot ten electrodes were burned, and the mean radiance temperature was found to be $3800^{\circ} \mathrm{K}$. The standard deviation of the radiance temperatures of the ten electrodes was $4^{\circ} \mathrm{K}$, the lowest and highest values being $3795^{\circ} \mathrm{K}$ and $3808^{\circ} \mathrm{K}$. Seven electrodes were burned from the other lot, with a resulting mean of $3793^{\circ} \mathrm{K}$ and a standard deviation of $7^{\circ} \mathrm{K}$. The range was from $3786^{\circ} \mathrm{K}$ to $3805^{\circ} \mathrm{K}$. Recently, one electrode was obtained from each of four additional lots. The radiance temperature of these four electrodes ranged from $3787^{\circ} \mathrm{K}$ to $3794^{\circ} \mathrm{K}$. Each of the radiance temperatures of the twenty-one electrodes was an average of several measurements over an interval of about $6 \mathrm{~min}$, beginning well after burn-in time. The atmospheric pressure was taken at the time of the measurements, and temperatures were corrected to the expected radiance temperature at a pressure of $760 \mathrm{~mm} \mathrm{Hg.}{ }^{3}$ The largest correction necessary was $1.6^{\circ} \mathrm{C}$ for a barometric pressure of $752 \mathrm{~mm} \mathrm{Hg}$.

The temperatures given are for a target area $0.2 \mathrm{~mm}$ in diam. and at the center of the crater. The temperature profile of a typical positive crater is shown in Fig. 1. The profile was obtained with an areal resolution of $0.2 \mathrm{~mm}$.

Additional studies have shown that a $30.5-\mathrm{cm}$ long SPK rod may vary in temperature over its useful burning length by an average of $7^{\circ} \mathrm{K}$, and occasionally by as much as $10^{\circ} \mathrm{K}$. Short term variations, fluctuations of a period of a few seconds, were about $3^{\circ} \mathrm{K}$ standard deviation from the mean. The fluctuations were recorded with an electronic time constant of $\frac{1}{6} \mathrm{sec}$.

From the present observations of twenty-one positive electrodes out of six different lots, the lowest observed radiance temperature was $3786^{\circ} \mathrm{K}$ and the highest was $3808^{\circ} \mathrm{K}$. All the observed radiance temperatures fall within $\pm 11^{\circ} \mathrm{K}$ of the nominal value of $3797^{\circ} \mathrm{K}$. This result agrees with Null and Lozier's ${ }^{4}$ radiance temperature of $3800^{\circ} \mathrm{K}, \pm 20^{\circ} \mathrm{K}$. The low current graphite arc as presently used, though useful as a radiometric fixed point, is significantly less reproducible than the accuracy with which the IPTS can be realized.

\section{References}

1. R. D. Lee, Metrologia (October 1966).

2. H. F. Stimson, J. Res. Natl. Bur. Std. 65A, 139 (1961).

3. N. K. Chaney, V. C. Hamister, and S. W. Glass, Trans. Am. Electrochem. Soc. 67, 107 (1935).

4. M. R. Null and W. W. Lozier, J. Opt. Soc. Am. 52, 1156 (1962). 
UNITED STATES DEPARTMENT OF COMMERCE • Luther H. Hodges, Secretary NATIONAL BUREAU OF STANDARDS - A. V. Astin, Director

\section{Theory and Methods of Optical Pyrometry}

\section{H. J. Kostkowski and R. D. Lee}

A detailed review of the theoretical methods of optical pyrometry and the application of these methods at the National Bureau of Standards in realizing, maintaining and distributing the International Practical Temperature Scale above $1063^{\circ} \mathrm{C}$ is presented. In the theoretical presentation, the concepts of effective and mean effective wavelengths are introduced, and various equations relating these parameters to each other and other physical quantities are derived. The important features of precision visual optical pyrometers are discussed and a number of blackbody sources and tungsten strip lamps described. Detailed experimental procedures and results of primary and secondary calibrations of optical pyrometers at NBS are given. Finally, recommendations for achieving high precision and accuracy and the fundamental limitations in visual optical pyrometry are presented.

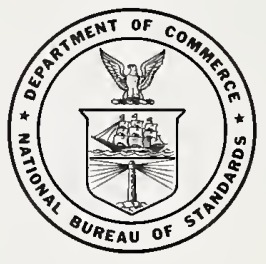

\section{National Bureau of Standards Monograph 41}

Issued March 1, 1962

\section{Reprinted with Corrections September 1962}

For sale by the Superintendent of Documents, U.S. Government Printing Office Washington 25, D.C. - Price 25 cents 


\section{Theory and Methods of Optical Pyrometry}

\section{Contents}

Introduction

Page

1. Theory

1.1. The Planck radiation equation...........................

1.2. The International Practical Temperature Scale

1.3. The primary calibration at the gold point

1.4. The primary calibration above the gold point..........

1.5. The absorbing glasses

1.6. The relation between effective and mean effective wavelengths . . . .

1.7. The use of non-blackbody sources in a primary calibration

2. Optical pyrometers

2.1. General characteristics of optical pyrometers

a. Pyrometer lamp

b. Apertures and filters......

c. Fairchild's criterion

2.2. The NBS optical pyrometer.

a. Objective lens assembly

b. Absorbing glasses, pyrometer lamp, and red filter...-

c. Microscope assembly

d. The electrical circuit

3. Sources.

3.1. Blackbodies

a. Gold-point blackbodies

b. Lesser quality blackbodies.

3.2. Tungsten strip lamps

a. Types of strip lamps.

3.3. Factors affecting the reproducibility of strip lamps

a. The carbon arc

a. The carbon arc

4. Primary calibration 4.1 . Calibration at the gold point

4.1. Calibration at the gold point

4.3. Estimated accuracy

5. Secondary calibrations...

5.1. Optical pyrometers

a. Inspection and cleaning

b. Determination of the effective wavelengths

c. Calibration procedure

d. Low-range calibration

e. High-range calibrations .

5.2. Tungsten strip lamps

a. Calibration range

b. Mounting and orientation.

c. The electrical system

d. Calibration

5.3. Precision and accuracy

a. Definition of precision and accuracy

b. Statistical model for calibration errors

c. Uncertainties on certificates

d. Differences between the IPTS and TTS

6. Applications

6.1. Temperature measurements

6.2. Spectrical radiance calibrations using strip lamps.......

6.3. Secondary standards

6.4. Recommendations for achieving high accuracy and precision

6.5. Fundamental limitations

6.6. Photoelectric optical pyrometers

7. References 



\section{Flux Measurement}

Papers

8.1. Theory, Construction, and Use of the Photometric Integrating

Sphere.

E. B. Rosa and A. H. Taylor, Bul. BS 18, 281-323, (1922-23) S447.

Key words: Integrating sphere; measurements of luminous flux; spherical candlepower

8.2. Application of Sulfur Coatings to Integrating Spheres.

S. Thomas Dunn, Appl. Opt. 4, No. 7, 877 (July 1965).

Key words: Integrating sphere; sulphur coating

Abstracts

8.3. Flux Averaging Devices for the Infrared.

S. Thomas Dunn, Nat. Bur. Stand. (U.S.) Tech. Note 279, 14 pages (Dec. 1965).

Key words: Angular sensitivity; flux averaging; infrared detector; integrating sphere; mixing mechanism; spatial sensitivity

8.4. A 5-Meter Integrating Sphere.

Mamoru Nonaka, Tokeo Kashima, and Yatoro Knodo, Appl. Opt. 6, No. 4, 757-771 (April 1967).

Key words: Integrating sphere; measurement of Iuminous flux 


\section{MEMORANDUM}

OF CALL

To:

YOU WERE CALLED BY-

YOU WERE VISITED BY-

OF (Organization)

$\square$ PLEASE CALL $\longrightarrow$ PHONE NO

$\square$ WILL CALL AGAIN $\square$ IS WAITING TO SEE YOU

$\square$ RETURNED YOUR CALL $\square$ WISHES AN APPOINTMENT MESSAGE
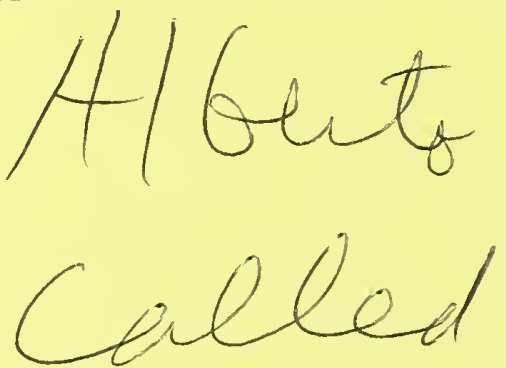

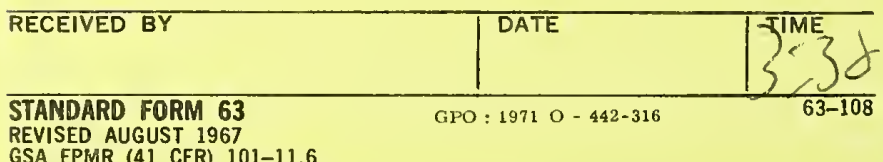




\title{
THEORY, CONSTRUCTION, AND USE OF THE PHOTONETRIC INTEGRATING SPHERE
}

\author{
By E. B. Rosa and A. H. Taylor
}

\begin{abstract}
ABSTRAC'T
Part I deals with the materials and construction of various splieres, and deseribes in detail the construction of a reinforeed conerete sphere at the Bureau of Stancards. It gives tests of the aecuracy of integration by this sphere, the absorption of light by the sphere eoating and by objects in the sphere, and the effeet of the position of lamps. Proper methods of operation are also outlined.

Part II gives a fairly complete resume of the general theory of the sphere, with the addition of a eonsiderable amount of new material, showing how to test the aceuracy of the sphere, and how to improve the accuracy of integration. It also gives a bibliography of the subject.
\end{abstract}

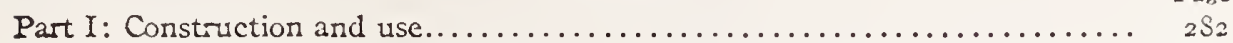

I. Introduction $\ldots \ldots \ldots \ldots \ldots \ldots \ldots \ldots \ldots \ldots \ldots \ldots \ldots \ldots \ldots \ldots \ldots$

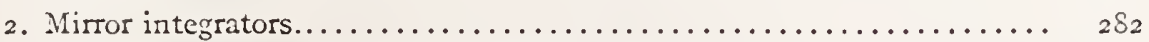

3. Ulbricht sphere.............................. $2 \delta_{3}$

4. Materials of which spheres have been eonstrueted............ $2 \varepsilon_{3}$

5. Other types of closed interrators. . . . . . . . . . . . . . . . $28_{4}$

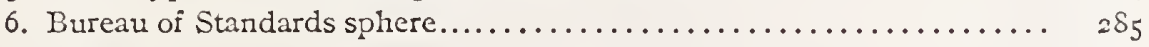

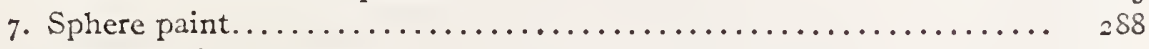

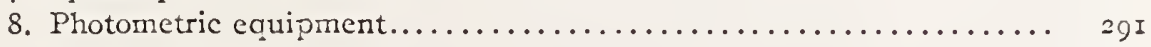

9. Costs of construction. . . . . . . . . . . . . . . . . . . . 292

Io. Advantages of burcau sphere........................ 292

II. Tests of bureaui sphere........................... 293

I2. Absorption of reflected light by foreign objects................ 295

I3. Reflection factor of spliere coating .................... 296

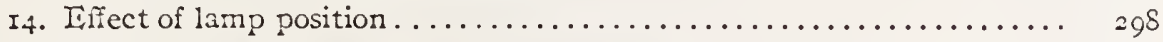

I5. Selective absorption by the sphere surface................. 299

I6. Method of operation................................ 3 I

I7. Special uses of integrating spheres..................... 304

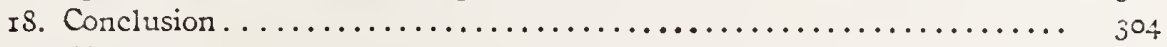

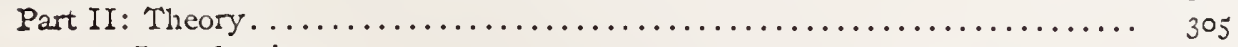

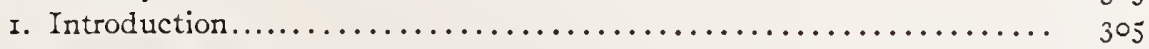

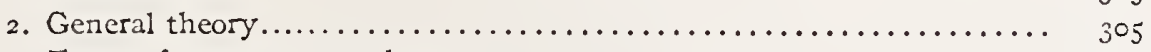

3. Errors of measurement due to sereen..................... 307

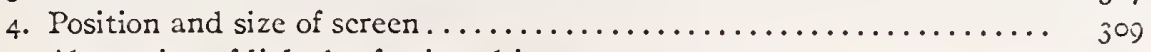

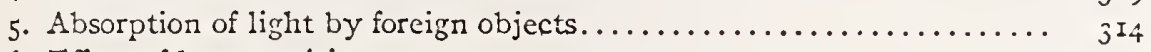

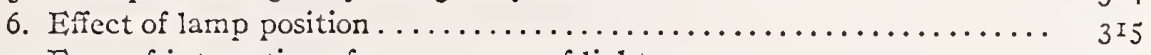

7. Error of integration of narrow cone of light................. 3 3r $_{7}$

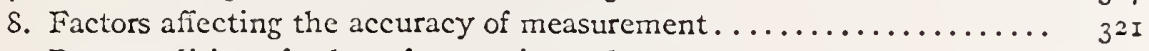

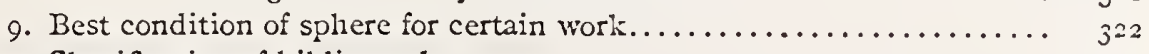

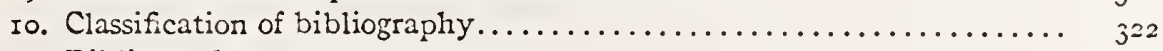

I . Bibliography..................................... $3^{22}$ 


\section{Scientific Papers of the Bureau of Standards}

\section{PART I.-CONSTRUCTION AND USE}

[By E. B. Rosa and A. H. Taylor]

\section{INTRODUCTION}

In the early history of photometry, as in most of the sciences, the apparatus was somewhat crude and simple in design. For a long time there was no need for other than simple photometers, which measured the light intensity in a single direction. With the development of various types of arc lamps and other improved illuminants the need for another type of photometric measurement, the measurement of relative intensities in various directions, became apparent. During the past 25 or 30 years the science of illumination has grown very fast, on account of the great strides made in the production of improved illuminants. This is especially true for the last decade. Since the light distribution from the various illuminants is so different, the only true basis of comparison is in terms of total light output, measured in lumens or spherical candlepower.

\section{MIRROR INTEGRATORS}

Probably the first instrument designed to give the spherical candlepower with only one measurement was the "lumenmeter" of Blondel.*1 It consisted of a small hollow sphere with two vertical $18^{\circ}$ sectors on opposite sides removed. This sphere was placed on the axis of an ellipsoidal mirror, at such a point that the light from a lamp placed at the center of the sphere (which had a black interior surface) fell on the mirror through the open sectors, and was reflected to the photometer. The use of this "lumenmeter" apparently was limited to the measurement of axially symmetrical light sources.

Other types of integrators which employ mirrors to reflect the light to the photometer from definite angular positions about the source have been designed by Blondel, Matthews, Russell, and Leonard. As it is not the purpose of this paper to describe mirror integrators, those who are interested are referred to the original articles, listed in the bibliography, for further details.

A study of the operation of integrators utilizing the reflections from multiple mirrors makes evident the fact that very accurate adjustment of the mirrors is necessary, and since they can not be depended upon to remain unchanged indefinitely the delicate adjustments must be checked frequently. In addition to this

*1 References are given in bibliography on p. 332 . 


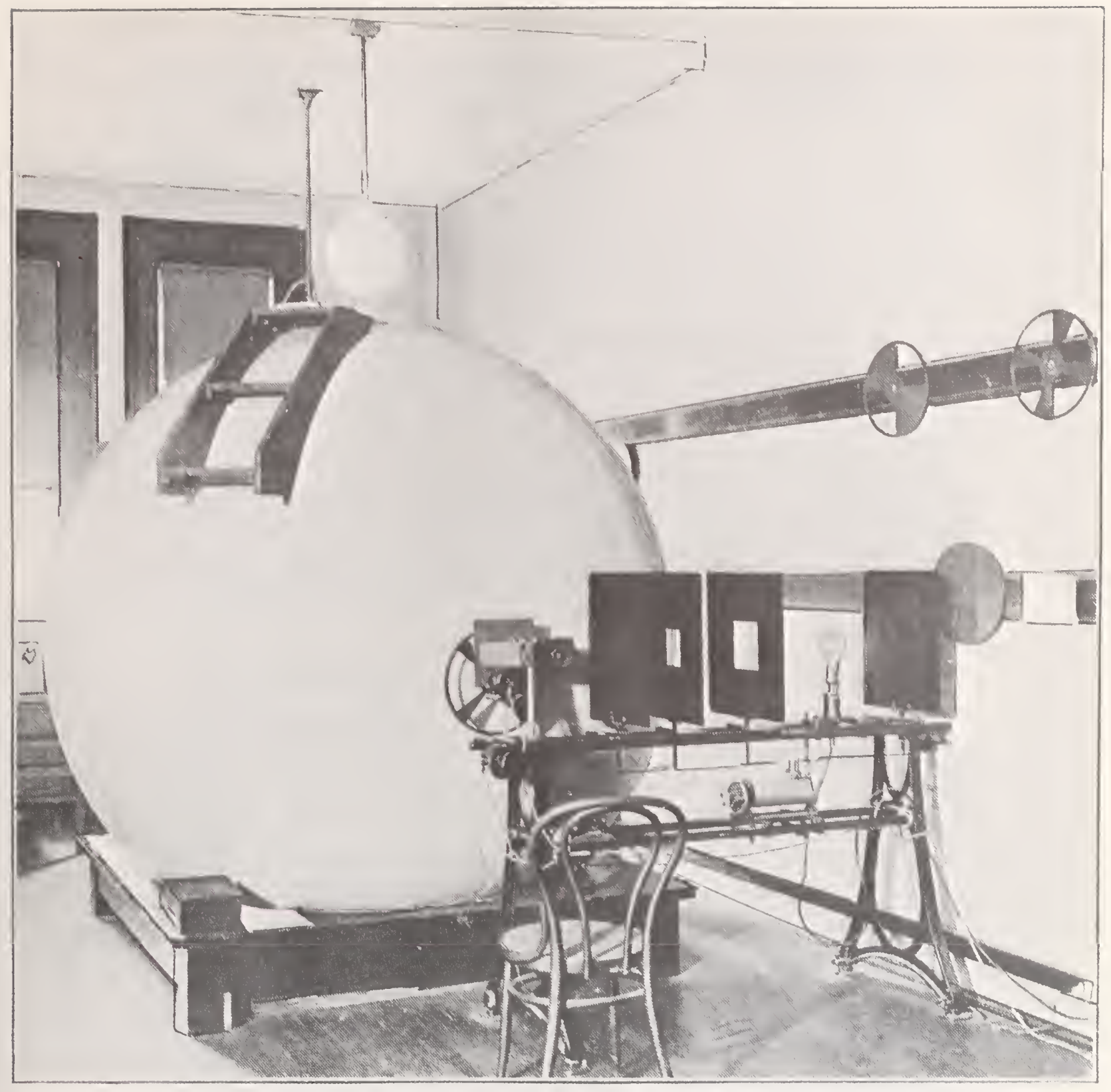

FIG. I.-General view of sphere and auxiliary equipment 


\section{Photombtric Integrating Sphere}

Gisadvantage, accurate integration of axially unsymmetrical liglit sources with such integrators is very dificult, if not inpossible.

\section{ULBRICIT SPFERE}

In Igoo Ulbrichts proposed the use of a hollow spinere, with difiuscly reflecting wlite walls, for light integration. His results and the investigations of others have shown that such a sphere, when properly designed and used, is capable of giving highly accurate results. Its great simplicity and usefulness have so strongly recommended it that it has superseded all other types of apparatus where it is desired to integrate the light flux from lamps.

Since the Ulbricht sphere has come into such general use, and is to be found in all well-equipped photometric laboratories of the present day, it has been thought desirable to give some structural details of spheres which have been described, as well as the methods which have been used in constructing a large sphere at the Burcau of Standards.

\section{MATERIALS OF WEICH SPEERES RAVE BEEI CONSTRUTCTED}

The first sphere with which Ulbricht ${ }^{8}$ made his experiments was a milk-glass ball, $50 \mathrm{~cm}$ in diameter, with an opaque covering. On one side a portion of the wall was unobscured, and the transmitted light, passing through an opening $8 \mathrm{~cm}$ in diameter in an opaque screen, illuminated the photometer screen.

Blondel was one of the first to realize a practical construction of the Ulbricht sphere. His sphere was $83 \mathrm{~cm}$ in diameter, and made of sheet metal shaped into hemispheres on a mold.

The smaller sizes of spheres in use to-day are usually made of sheet metal shaped on molds by pressure or hammering, or of sectors of sheet metal fastened together by riveting or welding. The Edison Lamp Works, Harrison, N. J., has an excellent large sphere of the latter type, in which the sheet-iron sectors are electrically welded. It is divisible into hemispleres. Bloch ${ }^{15}$ describes a sphere made of zinc plate $2 \mathrm{~mm}$ thick, the two halves of the globe being built up by pressure on a model, and subsequently connected by suitable screws and wing nuts. The sizes larger than I in diameter are usually made of sheet-metal segments fastened to structural steel or flat steel ribs. Such spheres have been described by Nionasch ${ }^{23}$ and various other writers. Marchant ${ }^{29}$ has described a sphere, at the University of Liverpool, 5 feet 3 inches $(1.6 \mathrm{~m}$ ) internal diameter, built of asbestos 
millboard one-fourth inch $(6 \mathrm{~mm})$ thicis fastened to 46 five-eighthincin $T$-iron ribs, bent into circular ares. It is made in halves, one of which is on rollers. Corsepius ${ }^{12}$ has described a sphere made of hair plaster, applied to a wire skeleton framework covered with gauze. Upon this plaster is a coat of plaster of Paris.

A fairly inexpensive spherical integrator can be made up by the use of a papier-mâché globe such as is used in instruction in geography.

\section{OTHER TYPES OF CLOSED INTEGRATORS}

Monasch ${ }^{13}$ has advocated the use of a large hemisphere with flat cover instead of a sphere for certain spherical candlepower measurements, on account of its lower cost and greater ease of manipulation. He described tests of a hemisphere of $2 \mathrm{~m}$ diameter which indicated that the illumination of the photometer window did not vary greatly when the lamp position was varied, the differences found being of the order of 2 per cent. This is too great a variation for precision work, however.

Sumpner ${ }^{26}$ has suggested the use of a rectangular box instead of a sphere on account of its simpler construction and lower cost. His article discusses the probable accuracy of such an integrator, but in the discussion several others disagreed with his conclusions. Details of the construction and use of cubical integrators have been described by other writers. Anyone interested is referred to the original literature. (See Bibliography.)

It seems doubtful that a cubical integrator would be very satisfactory where a higher accuracy than 2 to 4 per cent is desired for all types of light sources, but it might be quite useful where certain types of measurements were to be carried out, for example, change of efficiency of a gas lamp when the gas rate and air adjustment are changed. The box could be made to approach more nearly the spherical shape by blocking up the corners or filling them with cement. The principal advantage of the cubica! integrator lies in its low cost and simplicity of construction. A thorough study of such an instrument by a laboratory equipped to carry out exhaustive tests would perhaps be of consicierable value in showing the probable magnitude of the errors which might be encountered in service. The approach to spherical shape by blocking up the corners, which should be easy to accomplish, should be carried out in such an investigation. 


\section{BUREAU OT STANDARDS SPRERE}

During the summer of 1915 a reinforced concrete sphere, 88 inches $(2.23 \mathrm{~m})$ internal diameter, was built at the bureau. Since many details of its construction are unlike those of spheres
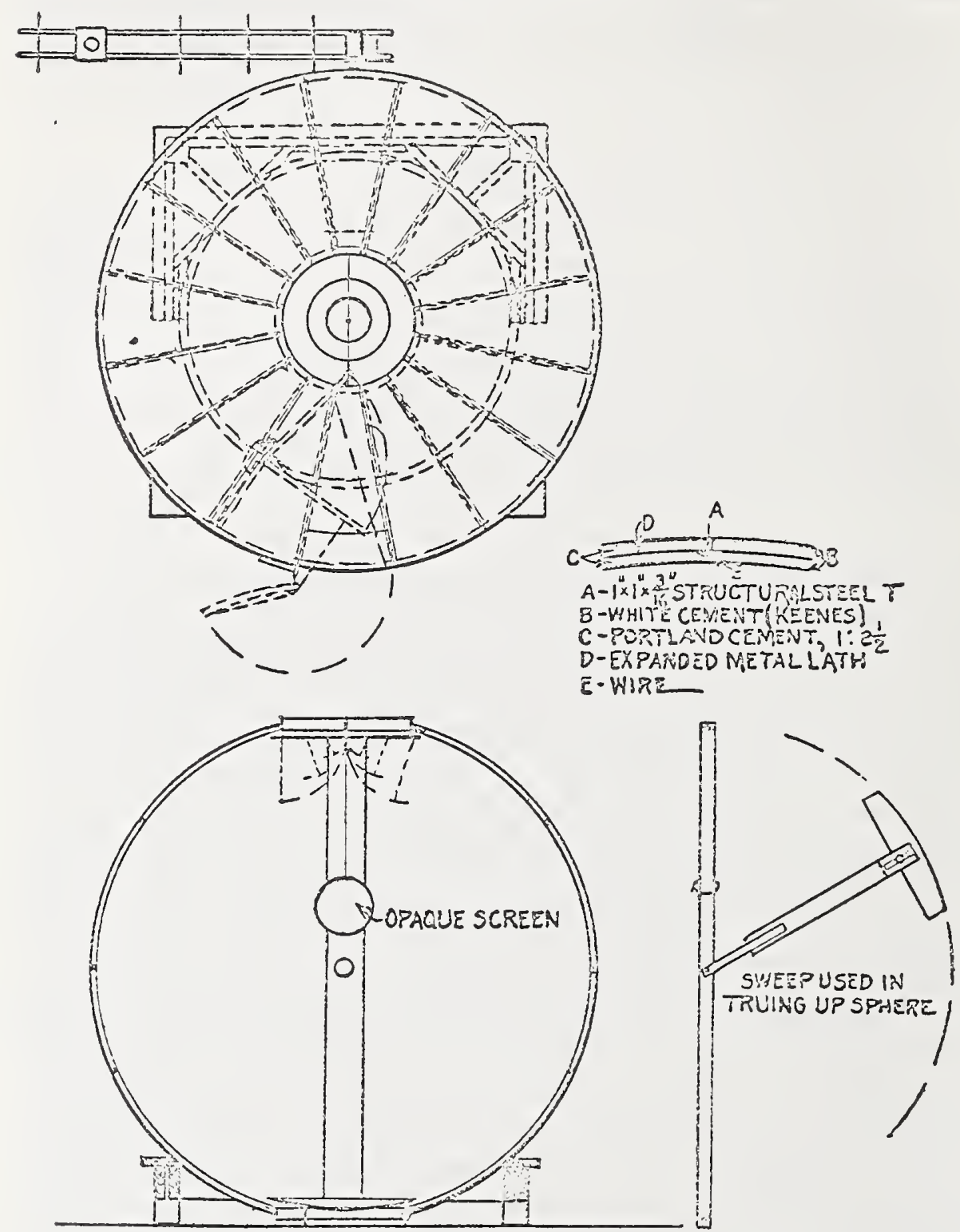

FIG. 2.-General plan of Bureau of Standards concrete sphere

previously described, it has seemed desirable to give a detailed description of it. The general plan of the sphere is shown in the sketches in Figure 2, while Figure I gives a general view of the sphere and its equipment. 
It has 16 vertical ribs of structural steel $T$, I by I by $1 / 8$ inch. (The ribs would be better if three-sixteenths insiead of oneeigith inch thick, as the latter have been found to be too flexible. Also, the structural framework should be supported above as well as below while putting on the expanded metal and applying the cement mortar and plaster, to prevent departure from the true spherical shape.) The ribs are fastened at top and bottom to steel rings, made up of 2 by $x, 1 / 2$ inch angle iron, bent and welded in rings of 2 feet diameter. Between each pair of ribs are three steel straps of I by. $1 / 4$ inch section, these being fastened with small bolts at each end to the top of the $T$, and butting up against its web. These strengthen the framework and make it rigid. Between the ribs are fastened sheets of expanded metal, such as is used in ordinary plastering work, cut to fit the spaces and fastened in place by wires passing over the top of the T's, as shown in the sectional sketch, Figure 2.

The body of the sphere is plastered inside and out with Portland cement mortar, of the proportions I part cement to $21 / 2$ parts of sand. This was applied to the expanded metal with a curved trowel, and swept out to a true spherical shape by a suitable sweep, as shown in Figure 2. The sweep was made up of a wooden circular arc fastened to an arm. Steel straps were bolted to this arm, and at the other end they were fastened by a horizontal bolt at the middle point of a 2 -inch pipe which passed through holes in boards forming bearings at the center of the top and bottom openings of the sphere. Hence the pipe could turn about a vertical axis, and the arm about a horizontal axis, thus allowing the sweep to reach all parts of the spherical surface. The pipe was threaded at both ends and fitted with lock nuts, so that a small adjustment up or down could be made. Provision was also made for a smail adjustment of the length of the arm carrying the sweep. After sweeping, the cement surface was scratched, and when dry the walls were coated inside and outside with a mixture of Keene's "fine" cement, with Io per cent hydrated lime added. This gives a hard white surface which can be made as smooth as desired. It is diffcult to obtain a pure white surface in this way, however, if a thin coating is used, as grains of the Portland cement work up to the surface in applying the white cement. In that case it is necessary to paint the surface later.

The bottom hole of the spinere is covered by a concave metal disk standing on three legs on the foor. This disk may be raised 
so that a space about I inch inigh is left for the admission of ar: whein tests of gas or arc lamips are being made. The top hole is covered by a fat aluminuin disk which may be let down from above far enough to allow an annular air space of about I inch. This disk is made up of annular rings, cut in inalf, and hinged so that any section can be let down, and any section can be entircly removed by drawing out the pin of the linge. Button latches hold the sections up. By means of this arrangement lamps suspended from an overhead track can be lowered into the sphere after dropping down the proper center sections. If it is cesired to have the lamp wholly in the sphere, these sections can be raised again, closing the opening except for a $\mathrm{I}$-inch nole througin which ine lamp suspension and wires pass. If it is desired to have only the globe of an arc lamp inside the sphere, the proper center sections can be removed entirely, and, if the mechanism casing does not fill the hole, white paper can be used to complete it. The top opening of the sphere is easily accessible by means of a stepladder and a short ladder fastened on the side of the sphere.

On one side of the sphere is a hinged door 37 inches high and $x 6 \frac{1}{2}$ inches wide at its widest point. When shut it forms part of a sector between two adjacent ribs. The hinges used are ordinary I6-inch strap hinges, bent to fit the curved surfaces to which they are fastened. The axes of the hinges are about 3 inches from the sphere surface and are braced by steel rods passing into the spherical shell. The rods have two nuts on the end passing through the hinge, so that it can be forced in either direction to take up sag due to the weight of the door.

While this method of adjustment has served satisfactorily to adjust such sags, some difficulty has resulted from the wear on the hinges which can not be compensated by this adjustment. These hinges carry a large weight and have to be opened and closed a great many times. It would be worth while to use specially constructed hinges with larger bearing surfaces, and the hinges should be kept well oiled.

In the wall diametrically opposite the door, on the equator, is a brass tube about $3 \frac{1}{s}$ inches in diameter. Into this telescopes another tube which carries the milk-glass window, with properdiaphragms for screening it. The milk glass is flusin with the inner surface of the sphere when in use, but can be removed for cleaning. A second thin milk-glass window, carried at the end of another telescoping tube, is outside of this window. Its 
use enables the operator to vary the intensity of the observation window through a limited range without a material change in the color of the light. It is much smalier than the other window, being only a little larger than the field of view of the photometer. It has been found to be very useful in practical operation of the sphere.

At a point about 27 inches in front of the window are two vertical rods upon which a runner may be moved up or down by a string passing through a small tube in the wall directly above. Four screens of diameters II, $2 \mathrm{I}, 3 \mathrm{O}$, and $3 \mathrm{~S} \mathrm{~cm}$ are provided to be attached to the runner, one at a time, so that for any lamp the smallest screen usable can be chosen.

The method of introducing the lamp socket into the sphere is unlike any heretofore described. At a point to the left of the door, just above the equator, a section of one-half-inch conduit passes into the sphere horizontally to a distance of $131 / 2$ inches, at which point another section about 23 inches long is hinged to it. At the end of this section are two lamp sockets, one above the other, so that lamps can be burned either tip up or tip down. Voltage and current leads are attached to the sockets in parailel, passing through the conduit and around the hinge. A short curved rod is attached to the stationary section just behind the hinge, and a spring is attached to this rod and to the movable section. One end of a flexible cord is fastened to the door and the other end to the conduit near the lamp sockets. When the door is opened the sockets are drawn forward to the opening by the cord to facilitate the change of lamps, and upon closing the door they are drawn by the spring to a point about ro inches from the center of the sphere.

The sphere is supported by a low oak platform. Before applying the cement all ribs were fastened to the table by long screws, so that sphere and table are rigidly connected.

\section{SPEERE PAINT}

One of the most important details of an integrating sphere is the use of the proper diffusely reflecting white paint. The use of the zinc white paint recommended by Utzinger ${ }^{33}$ had been contemplated, but it was not possible to find a coloriess glue called for in the formula. A number of sampies of so-called "flat white" paints were inspected, but none of them proved to be pure white initially, and it is a known characteristic of oil paints that they gradually become yellow with age. Hence it was finally decided 
that the Keene's cement used in surfacing the sphere was the whitest substance available at that time. Two thin brush coats were applied, allowed to dry, and then sandpapered. Even this coating is somewhat selective in absorption, though probably less so than many other coatings in use at present.

The requirements for a satisfactory paint for integrating spheres are unusually severe. To be entirely satisfactory it should fulnil the following conditions:

I. It should be a perfect difiuser, reflecting light in accordance with the cosine law.

2. It should be tenacious and somewhat elastic.

3. The paint should be a pure white-that is, its refiection should be nonselective-and it must not change in color with age or temperature.

4. The reflection factor should be high.

Of these requirements numbers $I$ and 3 are harciest to fulfill. No oil paint is colorless, and such paints will change in color with age. Although a perfect diffuser is unobtainable, it is possible to make up a paint which will fulfill this condition closely enough for all practical purposes.

When this paint problem arose, extensive experiments were made in the effort to find or develop a suitable paint. It appeared that the most serious defect of all the commercial paints examined was their color. In order to approximately reproduce the distortion of color which results from multiple reflections in the sphere, a test box was made up. It was cubical, with a colorless ground-glass window in one side, and contained a smail lamp. The reflecting walls were composed of removable cardboards painted with the paint to be tested. In making a test the walls were first lined with black velvet, and the lamp arranged to illuminate the window directly. This window illuminated the screen of a Lummer-Brodhun photometer. The comparison lamp voltage was then varied until a color match was obtained. Next the black velvet was replaced with the test cards, the direct light was screened from the window, and an effort was made to again obtain a color match.

After a number of sampies of commercial "flat white" paints had been tested in this manner, without satisfactory resuits, an efiort was made to deveiop a suitable paint. D. MacFarian Moore (discussion ${ }^{35}$ ) had stated that he had found a zinc-oxide paint, using a cellulose lacquer for a binder, to be entirely'satisfactory. 
His paint uses amyl acetate, which is expensive and also obnoxious because of the toxic effect of the vapor. In an endeavor to aroic the use of amyl acetate a lacquer was made up by dissolving celluloid and camphor in alcohol. Numerous pigments were tried, the most of the experiments being with magnesium carbonate and oxicie, zinc oxide, and barium sulphate. None of these were found to be nonselective, all except the barium sulphate resulting in a greenish-yellow hue which could not be colormatched very closely by a change of voltage of the comparison lamp. It was found that the barium sulphate gave the best results in this respect, and, although it was selective in its absorption, an almost perfect color match was obtainable by reducing the voitage of the comparison lamp. Other dificulties were encountered in trying to make up a paint with this pigment and lacquer and variable results were obtained, so that the effort was finaliy abandoned.

It was found that a fairly satisfactory paint could be made up with magnesium oxide, but the mixed paint, before being applied, gradually turns yellow, a perceptible change taking place within 24 to 48 hours. There is apparently no change in color after it is applied, however.

Although the zinc oxide paint is somewhat selective in its absorption, it is apparently satisfactory in all other respects, and is now being used in the spheres at the bureau. Before applying it to the concrete sphere the surface was given a priming coat of a thin solution of cabinetmakers' glue. Water glass would probably serve equally well, though this has not been tried.

In preparing the zinc oxide paint, first prepare a lacquer composed of the following:

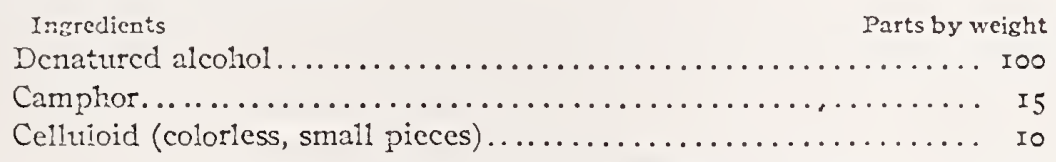

Dissolve the camphor in the alcohol before adding the celluloid, using a motor-operated stirrer. Add the small pieces of ciear, colorless celluloid slowly, while stirring, to prevent them from sticking together or to the wails of the vessel. Continue the stirritg (covering the vessei) intil all the celluloid is dissolved, which usually requires about ro or $\mathrm{I} 2$ hours. This makes a very thick, viscous lacquer. Keep a record of the weights used, so that the alcohol lost by evaporation can be replaced when the ceiluloid is dissolved. Use care to prevent any zinc oxide from 
getting into the lacquer while dissolving the celluloid, as this has apparently caused trouble at times.

The zinc oxide paint is tinen prepared by using the following:

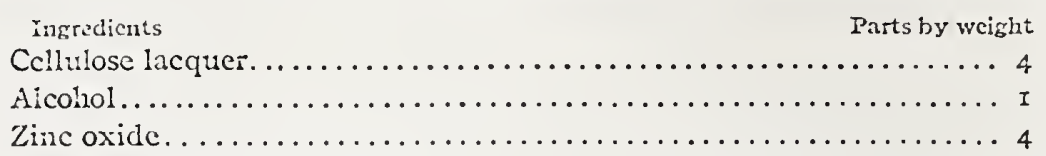

Add the zinc oxide slowly to the lacquer and alcohol, stirring constantly. Continue the stiring until a smooth, tinick paste is obtained. This requires about I hour or more. Then add slowly about 2 parts of alcohol and $x$ or 2 parts of water-white turpertine. If not thin enough, add more alcohol. The purpose cit the turpentine is to retard drying. It is probabie that glycerine could be used instead.

This paint dries very quickly, even on a nonporous surface, and should be brushed out as applied. Care should be taken not to brush over the fresh paint after it has been on Io or I5 seconds, else the brush will draw the paint. Avoid contact with any water, as water coagulates the paint. Best results mi1l De obtained if drying is retarded by preventing exposure to draits, etc. In painting a 30-inch metal spinere it has been found best to turn the hemispheres open sice up and cover with cloth as soon as the painting is completed. If the paint "checks," add some more camphor. If flaking occurs, make the paint less rich in lacquer. Allow about six or seven hours between successive coats.

This paint is tenacious, is permanent in color, and has a very high reflection factor.

The greenish-yeilow hue of the light on the sphere window can usually be almost perfectly matched by the introduction of greenish glass, such as ordinary window glass, on the comparison lamp side. (See sec. r6, "Method of operation.")

\section{PHOTOMETRIC EQUIPMENT}

The photometric equipment consists of a $1.5 \mathrm{~m}$ standard photometer bar, equipped with screens, lamp carriage, sectored disks, recording drum and printing magnet, and a Lummer-Brodhun contrast-type photometer head. The plotometar head is arranged so that the window of the sphere is viewed through a lens, to limit the field, and forms one side of the pinotometric field. The plaster of Paris screen iliuminated by the comparison lamp forms the other side of the field. The comparison lamp is shifted by a 
fiexible stranded wire belt and pulley arrangement which is operated by a wheel near the photometer head. The position of the comparison lamp ior a photometric balance is recorded on a sheet of paper on the recording drum under the lamp carriage by closing an electrical circuit by a push button in the center of the handwheel. This apparatus has been previously described in a publication of this bureau, ${ }^{42}$ but it may be of interest to give a description here. The long cylindrical drum is rotated by an electrical escapement clockwork, adapted to turn the drum o.I ${ }^{\circ}$ each time the circuit is closed. The axis of the drum is attached to the axis of the clockwork by a friction clutch, which can be released so that the drum can be rotated at will. A printing electromagnet is rigidly attached to the comparison lamp carriage and moves over the drum lengthwise. This magnet is provided with two revolving spools carrying a typewriter ribbon between the printing point and the sheet on the cylinder. The clockwork and printing magnet are operated in series by a low-voitage battery (io to I 6 volts), the magnet stamping a dot when the circuit is closed, the clock turning the cylinder a small amount when the key opens. The record ciots are thus prevented from falling together. In working up observations the average position of each bunch of dots is estimated and marked by a straight line, the position of which is determined by reference to an index line marked on the paper. The drum is turned a small amount between observations on different lamps.

A Brooks type defiection potentiometer is used for the electrical measurements.

\section{COSTS OF CONSTRUCTION}

There have been such large changes in costs of all materials since the construction of this sphere that it seems to be hardly possible to estimate present day costs. The materials used would not be a very large item of cost, but labor would be. It is uncertain whether this type of construction would be less expensive than all-metal construction for the smaller spheres, but for spheres larger than 5 feet in diameter it would very probably be less than for the all-metal sphere.

\section{ADVANTAGES OF RUREAU SPMERE}

Since this sphere differs in many ways from others in present use, it will be of interest to point out its advantages and disadvantages. Most of the large spheres are divisible into halves, one 
of which is on rollers, so that they can be separated for adjustment of arc lamps, etc., in the sphere, and for cleaning and painting. While this sphere is not divisible, it has been found that one can very quickly mount the ladder and make lamp adjustments from abore, in fact, probably more quickly than could be done if one lemisphere had to be rolled back. This method of construction also avoids the necessity for a track, which takes up valuable floor space. In order to permit work on the inner surface of the sphere, the bottom section can be lifted out. Dy means oi a stepladder the operator can step into the sphere, and, standing on the floor beneath the bottom opening, he can easily reach any part of the spherical surface.

The sphere is rigid and substantial in construction, has a good surface, continuous (except for necessary openings) and truly spherical, easily repaired or renewed if necessary, and may be ventilated if desired. It is not portable, however.

In operation the sphere has been found to possess distinct advantages due to the use of two sockets for buning lamps either tip up or tip down, and the arrangement for drawing the lamps near to the sphere center, good ventilation when desired, and the use of a standard photometer bar and its accessories.

After having been in use for seven years the surface has shown no signs of cracking or other imperfections.

\section{TESTS OF BUREAU SPEERE}

The principal tests of this sphere have been made to determine the accuracy of integration of sources of difierent types. For this purpose candlepower distribution measurements of various sources were made on a two-mirror selector, and calculated values of mean spherical candlepower were obtained. Direct measurements of the candlepower of each of the sources were then made in the sphere. The differences between the resuits obtained by these two methods are shown in the last column of Table $r$, in whici positive values indicate that the results in the sphere were higher than those obtained by distribution measurements, and negative values indicate that the sphere results were lower. The distribution curves for these sources are shown in Figure 3 . 


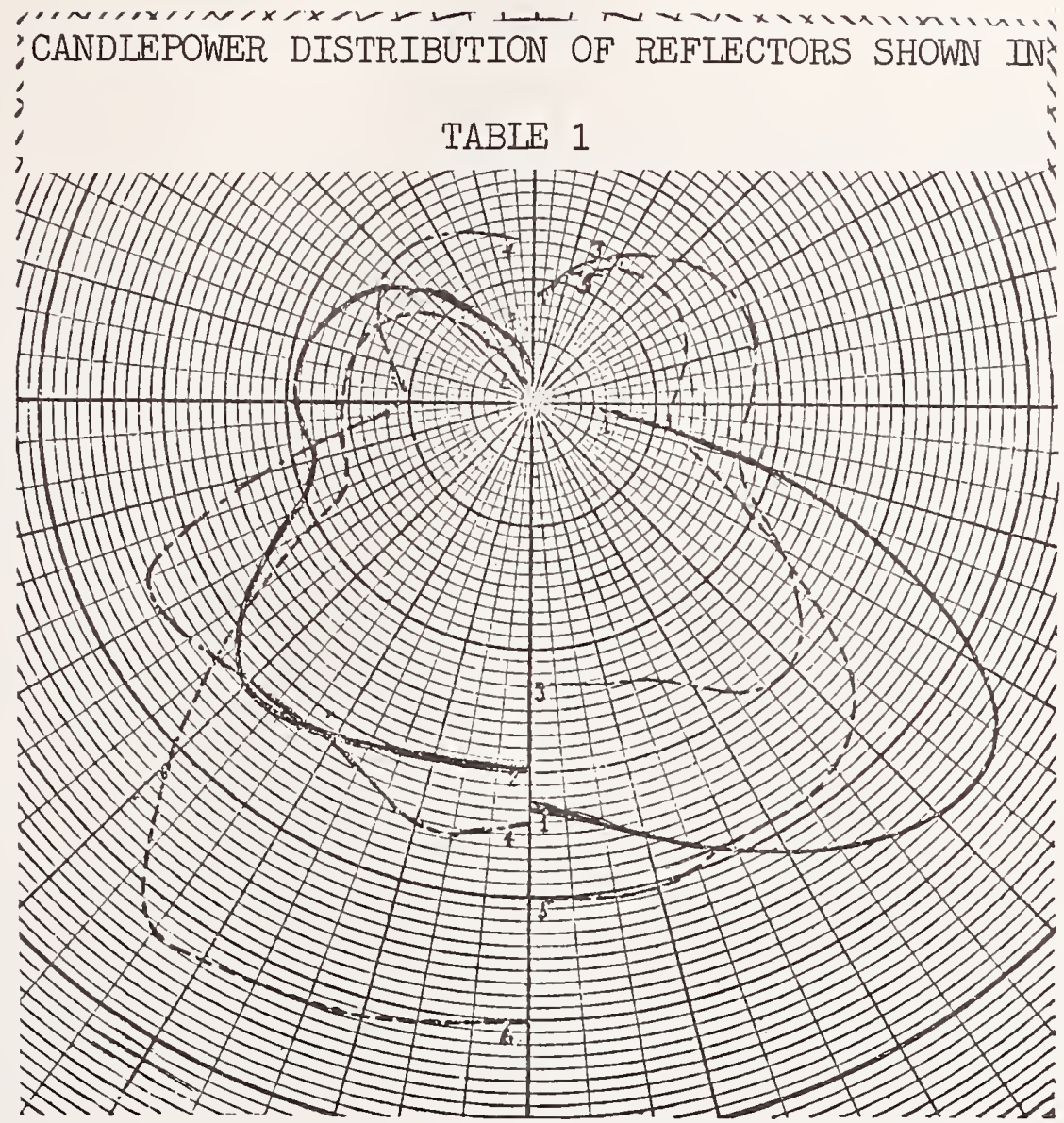

FIG. 3.-Light-distribution curves of reflectors used in tests of sphere

TABLE 1.-Comparison of Integration by Distribution Photometer and Integrating Sphere

\begin{tabular}{|c|c|c|c|}
\hline \multicolumn{3}{|c|}{ Source } & \multirow{2}{*}{$\begin{array}{l}\text { Percent } \\
\text { differ- } \\
\text { ence in } \\
\text { results }\end{array}$} \\
\hline Lamp number a & Accessory & Description & \\
\hline 1 ep up........... & Reflector No. 1............. & Porcelain enameled............ & 0.0 \\
\hline 1 tlp down....... & $\ldots . . . d 0 . . . . . . . .$. & [....do............................... & -.6 \\
\hline $2 \ldots \ldots \ldots$ & Reflector No. 2............. & Holophane prismatic, satin finish................... & +.6 \\
\hline $2 . .$. & Reflector No. 3............. & Ilght density opal, "Lucida"....................... & +.8 \\
\hline $2 \ldots$ & Reflector No. 4.... & Ilght density alba, flaring type..................... & +1.6 \\
\hline $2 \ldots$ & Reflector No. 5.... & Heavy density opal "Pheno"...................... & -1.0 \\
\hline 2.. & Reflector No.6......... & Holophane prismatic, clear, extensive............... & -.4 \\
\hline & Globe No. 1........... & 12-Inch opal globe, pollshed, "Polycase"........... & +.4 \\
\hline $1 . \ldots \ldots \ldots$ & Globe No. 2:............... & 12-Inch opal globe, depollshed, "Alabastine"..... & +.5 \\
\hline
\end{tabular}

- Ir:mp No. 1, roo-watt tungsten, clear. Lamp No. a, 40-watt tungsten, bowl frosted. 
The agreement of the values obtained in the two ways is good, except perhaps for refector No. 4. The distribution photometer measurements are dificult, on account of the low intensity of the light at many angles (photometer cistance was about Io feet) and the error is probably greater in those measurements than in the spicuial photometer. No value shown in the table is the result of many measurements, hence the differences are very probably dive partly to experimental errors.

\section{AESORPYION OF REFLECTED IIGEI BY FORETCN OSYECMS}

In order to measure the absorption of difisely refected light by black objects of various shapes and sizes, a number o: tíese were made up and painted with lampblack in shelac. Iamp To. : was burned tip down in reflector No. $r$, and the test object was suspended near the center of the sphere, where no direct light could fall on it. The test object was also, screened from the sphere window. The results are shown in Table 2. Where the test object is designated as "double disk," a disk blackened on both sicles was used, and where the term "single disk" is used, a circular disk blackened on one side, and fastened to the sphere screen, on the side away from the window, is indicated. The absorption values shown in the table indicate the amount by which the sphere window illumination was reduced when the objects were suspended in the sphere. The reduction to be expected is shown by equation (I8), to be $\frac{S a^{\prime}(\mathrm{I}-a)}{a A}$. Hence, if this equation is correct, the values in the last column of the table should all be equal, since $S$ is presumably the only variable in the equation. The differences found, with the exception of the last two, are probably errors of measurement, since the calculation is a severe test of the accuracy of the measurements. For example, if the observed absorption by the cylinder were 0.5 per cent higher, the value in the last column would be I I. 4 instead of I 0.7 . The lower values for the two globes were probably due to their having cut off some light from the opaque screen used to screen the window, thereby reducing the total amount of flux received and absorbed by them. Apparently the absorption is independent of the shape of the objects, as was to be expected. 
TABLE 2.-Absorption of Refiected Light by Dlack Objects of Various Skrpes and Sizes

\begin{tabular}{|c|c|c|c|c|}
\hline Object & Area & $\begin{array}{c}\text { Sphere } \\
\text { area }\end{array}$ & $\begin{array}{l}\text { Absorp- } \\
\text { tion }\end{array}$ & $\begin{array}{l}\text { Ratio of } \\
\text { percent- } \\
\text { age } \\
\text { absorp- } \\
\text { tion to } \\
\text { percent- } \\
\text { age of } \\
\text { sphere } \\
\text { area }\end{array}$ \\
\hline & $\mathrm{Cm}^{2}$ & Per cent & Per ceat & \\
\hline 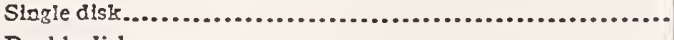 & 1152 & 0.738 & 7.89 & 10.7 \\
\hline 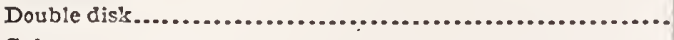 & 1078 & .690 & 7.65 & 11.1 \\
\hline 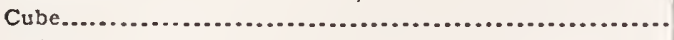 & 1110 & .711 & 8.20 & 11.5 \\
\hline 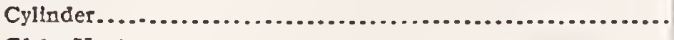 & 1122 & .719 & 7.69 & 10.7 \\
\hline 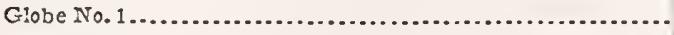 & 2012 & 1.288 & 12.9 & 10.0 \\
\hline 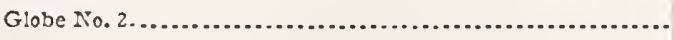 & 3007 & 1.925 & 18.4 & 9.6 \\
\hline
\end{tabular}

Tests to determine the amount of absorbtion of reflected light by clear lamps of sizes from 50-watt carbon to 250 -watt vacuum tungsten indicated that the absorption was negligible, being less than the uncertainty of measurement except in the case of large lamps with skirted bases.

\section{REFLECTION FACTOR OF SPEERE COATING}

The reflection factor of the sphere coating was determined in two ways, one determination being carried out on a separate sample of the material and one on the complete sphere. For the latter test the milk-glass window was removed from the sphere wall, placed on the photometer bar, and a portable photometer pointed at it. A standard lamp was arranged at a measured distance from the milk glass, and the photometer standardized to measure the illumination on the glass. The window was then replaced, and the illumination on the window measured when the same standard lamp was burned in place in the sphere.

Three measurements of illumination were made, namely, with window screened, window unscreened but screen remaining in the sphere, and screen removed from sphere. The difference between the first two measurements was the amount of direct illumination thrown on the window by the lamp alone, and this was subtracted from the illumination value obtained when the screen was removed from the sphere. The calculation of the absorption by the sphere walls is then as follows (for the development of this theory see Part II) : 
Let

$$
\begin{aligned}
E & =\text { average total illunination on sphere wall } \\
E_{d} & =\text { average direct illumination }=\frac{\text { m. s. ep. }}{\text { (radius }^{2}} \\
E_{m} & =\text { illimination by reflected light; } \\
& =\text { measured illumination }=E-E_{d} ; \\
m & =\text { reflection faetor; } \\
a & =\text { absorption faetor. } \\
E & =\frac{E_{d}}{I-m}=\frac{E_{d}}{a}=E_{d}+E_{m} \\
a & =\frac{E_{d}}{E_{d}+E_{m}}
\end{aligned}
$$

Calculations of the observed data on the Keene's eement surfaee, using this formula, gave an absorption value of 8 .I per cent, or refleetion faetor of 91.9 per eent.

These measurements indieated a very high reflection factor, much higher than had been believed to be possible, as a previous investigator had given 88 per cent as the refleetion factor of magnesium carbonate. ${ }^{11}$ Repetition of the measurements ehecked this result, and consequently steps were taken to verify it by another method. In this method the suriace brightness of a flat disk of the cement was measured at every $10^{\circ}$ when illuminated normally to a known value. These measurements gave further confirmation of this result. Later another eoat of the Keene's cement was applied and the absorption factor was then found to be 7.5 per cent, a distinct improvement over the previous coating.

A measurement of the absorption by the method suggested by Chaney (diseussion ${ }^{35}$ ), namely, direeting a narrow beam of ligint first at the screened area opposite the test plate, and, second, entirely within the unscreened area, gave a result in very close agreement with the above value.

This sphere has recently been palnted with the zinc-oxide paint deseribed above, and the absorption factor remeasured. It was found to be 6.0 per eent when the surface was fresh. This value was further verified by measurement by another method.

As an outgrowtin of this work one of the authors has given special attention to the improvement of methods for the measurement of refleetion faetors, and has devised two types of reflectometers ${ }^{40,}{ }^{11}$ in whieh the prineiples of the integrating sphere are applied. Results with these new instruments have eorroborated the high values of reflection factor mentioned above. 


\section{EREECT OT IAMP POSITION}

The enange of sphere winciow illumination when a clear lamp without refleetor was placed in various positions was measured, and the results are shown in Figure 4. The lamp used was a vacuum tungsten lamp. Two methods of varying the position were lised, namely, first, by setting the lamp-soeket arm at various distances from the door, and, seeond, by suspenaing the lamp from a cord at various distances from the door, in each case the direct light being sereened from the observation window by an opaque screen $38 \mathrm{em}$ in diameter. The results were practieally the same when a small lamp was tested by the suspension method. This apparently indieated that the change in window illumina-

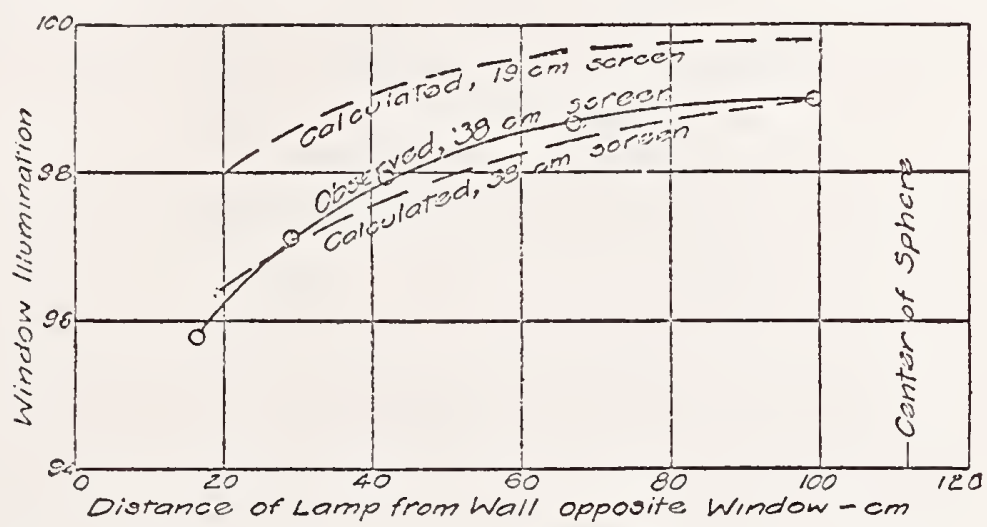

Fra. 4.-Effect of position of lamp on illumination of sphere window

Data for multiple vacuum tungsten lamp. The ordinates are pereentages of the illumination which the same lamp wouid produce in the empty sphere by reflected light only

tion was not due to absorption of light by the lamp. When the small lamp was moved toward either of the side walls $90^{\circ}$ from the window, being so arranged that no direct light reaehed the observation window, but without employing any large opaque screen, the window illumination did not decrease as the lamp approached the wall, but, on the contrary, inereased slightly. These and other tests definitely show that the observed decrease of window illumination as the lamp approached the screened wall was due to the fact that more and more of the direct light fell on the sereened areas and had to be reflected at leasi twice before reaching the window.

From the known values of the light distribution of a lamp of this type and the absorption of the sphere wall it is possible to ealculate the deerease to be expeeted. Such values have been calculated for two sizes of screen, of 38 and I $9 \mathrm{~cm}$ diameter, respec- 


\section{Phoionetric Integrating Sphere}

tively, and plotted as solid curves in Figure 4. For the $38 \mathrm{~cm}$ screen the oiserved curve has arbitarity been given the sane value as the calculated curve for the position nearest the center of the sphere. The close agreenent between caiculated and observed curves confirms the reason advanced above for the observed change. In neither case is the reduction of total ilimination ciue to absorption of multiple refiected light by the opaque screen taken into account, but it is not necessary to do so, since it is practically independent of the light distribution or position of the iamp. The method by which the calculated curve was obtained will be explained in the second part of this paper.

If a light source of extended area, such as a difiusing globe, is brought near the screened sphere wall, a decrease greater than that shown by Figure 4 will be observed, for in addition to the greater flux on the screened area there will be the additional effect of greater absorption by the globe itself, since it receives a greater fraction of the first reflected flux than when it is nearer the center of the sphere.

Probably the greatest error which occurs in the use of integrating spheres is caused by placing the lamps too near the sphere wall and making no correction for light absorbed by them. When testing life-test lamps in this manner, where the lamp may be appreciably blackened and consequently absorbs more reflected Iight than it did when new, the error is undoubtedly too large to be neglected.

\section{SELECTIVE ABSORPTION BY TER SPEZRE SURFACE}

In the bureau sphere, as is probably the case everywhere, there is some selective absorption by the sphere wall coating. Since this is such a general condition, it is desirabie to know whether or not it causes any error, and if so, approximately how much. A test to determine the amount of selectivity, or, in other words, the difference between the color of the light given out by a lamp and the color of the light emerging at the window, was made when the surface was coated with Keene's cement. For tinis purpose the sphere window was removed and placed on one side of the photometer. When a vacuum lamp of an efficiency of about r.2 watts per horizontal candle illuminated the window directiy, the comparison lamp gave the nearest color match at 98 volts, with an efficiency of $\mathrm{r} .33$ watts per horizontal candle. (This difference is due to selective absorption by the milk-glass window.) The first lamp was then piaced in the spinere, burned 
at the same voltage, and the voltage of the comparison lamp altered until the best color match was again obtained. Its voltage was then 70 , and its efficiency approximately 2.75 watts per horizontal cancile. A perfect color match is not obtainable in either case, partly because of selective absorption by the milk-glass window. Although this characteristic of milk glass probabiy does not cause any appreciable crror of measurement, it does make the photometric observations somewhat more difficult.

In order to determine whether or not any error of measurement results from selective absorption by the sphere walls, the amount of which has already been described, the following experiment was performed:

Two lamps of the same type were selected. One of these was used as the comparison lamp, and the other was put in the sphere. The voltages were set to give about the same efficiency, that is, about I.I wphc, and a reading taken. The voltages of both lamps were then altered until an efficiency of about 3.I5 wphc was obtained, and another reading taken. Since there is a large color difierence encountered in reading the photometer when the lamp is in the sphere, evidiently about equal for both conditions, precautions were taken to keep outside of the range of the Purkinje effect by making the illumination of the photometer screen practically equal in both cases.

The lamp was then removed from the sphere and arranged to illuminate the milk-glass window, which had been set up on the bar photometer. The measurements at the two efficiencies were then repeated.

If there is no error due to the selective absorption, the ratio of the illumination of the sphere window at the two voltages with one lamp in the spinere should be the same as that obtained when the lamp was measured on the bar photometer. If there is any error, it would be expected that the lamp in the sphere would be underrated at the higher efficiency on account of the greater absorption of the whiter light.

The ratios of the illumination in the two cases differed by only 0.4 per cent, which was within the error of measurement, since the measurements in the sphere were difficult because of the color difference. It is to be noted, also, that the difierence was in the direction opposite to that to be expected if there was any error introduced by the selectivity. Hence it seems safe to conclude that over this range of color difference no measurable error 
due to selective absorption applied to measurements made in this particular sphere.

At present it is very difficult to obtain for the winciow a dîfusing material which does not have some selective absorption. Milk glass usually has a higher absorption in the biue end of the spectrum, while clear glass in great thicknesses is usually green.

Experimental work at the bureau has shown that good milk glass loses practically nothing in diffusion properties by being ground down to a thickness of less tiran I $\mathrm{mm}$, while a very appreciable gain will be made in reduced selective absorption and increased transmission. The surface toward the sphere should be given the coarsest possible grinding with coarse carborundum or by sandblasting.

\section{MEETEOD OF CPERATION}

The "substitution" method which is usea in practically all the photometric work in the bureau makes possible the adjustment of the apparatus to the best condition of measurement in every case. This method also obviates any necessity for long-continued constancy of many factors in the measurements, such as the candlepower of the comparison lamp, the reflection factor of the shpere walls, etc.

The illumination on either side of the photometer screen is varied in part by the use of a set of sectored disks, depending on the well-known law that if the illumination of any surface is obstructed by means of a rapidly rotating sectored disiz, the apparent brightness of the surface is directily proportional to the angular opening of the disk. In certain cases neutrai absorption glass screens are used, though usually this is done only where the factor for the glass need not be known.

When the color of the light from the test lamps is very cifferent from that from the standard lamps, it is desirabie to use some method of altering the color of incident light from the comparison lamp in order to facilitate the measurements and free them from this element of uncertainty. The method used here is the interposition in the path of the light of glass cells filled with colored liquicis. These are made up by trial, and in cases where it is necessary to know the relative transmissions, this standardization is made by means of a flicker photometer. The solutions lised are water solutions of potassium bichromate and of copper suiphate. The desired color can be approximated by varying the ciensities o: the solutions or by mixing them. In the use of such cells, if the 
relative transmissions must be knom, the measurements should be made witin a lamp of the same color as the comparison lamp to be used. Aiso, it is undesirable to use the cells to alter the iight from the sphere, for this light varies in color according to the color of the lamps tested, and the transmission of a solution is known to be appreciabiy different for lights widely different in color.

Another method which has proved very successful is the use of orcinary window glass which has a greenish hue. When used of the proper thickness, on the comparison lamp side, it is usually possibie to obtain a nearly perfect color match with the paint described above, and in some cther cases. When used in this manner a correction should be macie for the change in length of the optical path. Insertion of the glass has the effect of shortering tire optical path, so that it is less than the measured distance by an amount approximately equal to one-third of the thickness of the glass.

To faciitate the work of evaluating the readings an inverse scuare scale, witi divisions expressing percentages, was ruled on a transparent triangic. Dy setting an index line on the triangle to concide with an index line on the sheet on which the photometer readings have been printed by the electromagnet, the proportionalities of the various readings can be read off. By the aid of a sicie rule these are easily convertibie into candlepower values.

In the case where lamps not wiciely different in candlepower are to be measurea, a candlepower scaile can be prepared. The variaticn of the intensity of the comparison lamp throughout a smail voltage range where it is to be used shouid be experimentally detemined, so that small adjustments can be made by varying its intensity. The method of measurement most easily carried out would then be somewhat as follows:

Put a stanciard lamp in the sphere, and set the comparison lamp at the point on the scale to make it read directly the spherical canciepower of the stanciard lamp, first having determined the voltage of the comparison lamp for a color match with the standard. Next alker the position of the secondary sphere winciow (if a couble window such as that described above is used) until an intensity match is obtained. Take readings on several standerd lamps, and if it is found that tincy read high or low by a small amount, change the voltage of the comparison lamp by the predetemined smali anount nccessary to make the read- 
ings correct. Rcadings on cre or more standard lamps stould be macie at frequent intervals throughont the ruin, to check the acewacy of the mastrenents. Whe high precision is desired, a number of stanciard lanps shouk be read both at the beginning ancine end of the observations, and if the results in the two cases do not agree, an adjustment of the values must be made or the run discorded if tire clisagreenent exceds a pernissibie amount.

The above method applies to the use of a sphere with which a bar photometer is used. With such a photometer bar a regular Iummer-Brodhun sight box can be used, one side of the sight box being removed so that the sphere winclow forms one side of the pinotometric fieid.

It is possible aiso to use a portable photometer, such as the Sharp-Millar, which has an inverse square scale, but it is evicict that ine use of such an instrument will not give an accuracy as high as that obtainable with a bar photometer and accessories.

In measurements of light sources having surfaces which absorb an appreciable amcunt of light, the following procedure, which is the general practice, is probably best:

The opacue screens to be used are placed in position, and a reading of the standard lamp, witinout the test lamp in the spiere, is taiken. Next, a small shield is arranged to screen from the test lamp when in position any direct light from the standard lamp, and another reading is taken. The test lamp is then brought into the sphere, in the position which it is to occupy, but unighted, and another reading is taken. The percentage difference between the readings in the last two cases is the reduction of spisere window illumination due to the absorption of reffected light by the test lamp. When the reacings of the test lamp are evaluated in terms of the reading of the standard lamp in the empty sphere this percentage should be added to the results. This is a very important corection, as appreciable errors may occur if it is nos taken into account. A modifiea procedure consists in taining reacings on the standard lamp when the test lamp is in the sphere, and evaluating the results on the test lamp directiy in tems of the readings on the standard lamp. In this method, as before, precautions should be taken to screen from the test lamp the cirect light from the standard lamp. The former method has an advantage if many lamps of the same kind are to be measmed, or if the same lamp is to be measured many times, since in that case a constant percentage correction can be applied to the obser- 
vations evaluated in terms of observations on standard lamps in the empty sphere.

It is good practice to cover large abso-bing surfaces, such as arc lamp mecirenism casings, with white paper or cardboard, whenever it car be dore in such a manner as not to cut off any light emitted by the lamp.

\section{SPECIAI JSES OF INTEGRATING SPEERES}

In addition to the measurement of mean spherical candlepowers the sphere may be used to make other important measurements. Its use for hemispherica! candlepower measurements has been extensively discussed in some of the articles listed in the bibiography. It can also be used for the measurement of the effective ligit fiux within a given solid angle. (See discussion of reference, ${ }^{35}$ by Chilias.) For these measurements a sphere divis:bie into hemispices is more useful. The use of the hemisprieres for measurements on searchlight beams has been described by Benfora. 3s 38

The integrating sphere principle has recently been applied to the measurement of diffuse reflection factors by one of the present autifors. $0^{40 \text { th }}$ Its use for this purpose promises to fill a long felt want.

The testing of jewels for the Panama-Pacife International Exposition is an interesting application of the spiere which has been described by S. I. E. Rose (discussion ${ }^{31}$ ). Many otiner applications of the apparatus will doubtless be made in future.

\section{CONCLUSION}

It may be said in conclusion that the authors believe that in the hands of careful and experienced photometricians a properly cesigned sphere will give very accurate results, and forms one of the most important parts of the equipment of a photometric laboratory.

Since the present types of lamps vary greatly in their light distribution, and change differently in distribution as they are bumed (as, for example, in a life test), the only true basis of comparison is in terms of their spherical intensity or total flux, and this is rapidly becoming the accepted practice.

The sphere described above has proved very satisfactory in practice, and is in more nearly constant use than any other apparatus in the photometric laboratory.

The authors are indebted to E. C. Crittenden for many helpful suggestions and for the selection of the photometric equipment. 


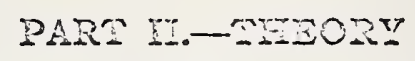

[Sy A. I.T. Tayio:]

\section{MrRoDJ0MON}

The theory of the Uibricht spinere has been extensively treated in the literature listed in the accompanying bibliography, but a great deal of the discussion is a repetition of certain pinases o: the general theory, and in no one place is there a complete discussion of all details of the theory. Hence it has been thought cesirable to give here a fairly complete résumé of the theory, togetie: win new developments of certain phases, with especial regarc to the best design of the sphere to increase the accuracy of measurement.

In preparing this paper the works of Uibricht, ${ }^{2} 15$ and Chaney and $\mathrm{Clark}^{31}$ have been freely consulted, as they have given the most complete discussions of the theory available until very recently, but a large part of the applied theory given here is new. In a number of other parts new methods of cevelopment which are believed to be less complex than those previousiy used have been followed.

\section{GENERA TEROSZ}

Theoretical photometry makes use of two general laws of radiation; first, that the intensity of illumination of a suriace is inversely proportional to the square of its distance from the ingth source, and, second, the cosine law of emission, often erioneousiy referred to as Lambert's cosine law, ${ }^{43}$ which states in suostance that the luminous intensity of an element of a periectly cistusing luminous suriace is directly proportional to the cosine of the arg:e between the normal to the surface and the line of emission. From the latter law it follows that the brightness of such a surface is independent of the angle of view and that the luminous intensity is directly proportional to the projected area. No iuminous surface obeys this law exactly, but it is possible to procuce a suriace which will approach fulfilment of the law. For simpicity in the following theoretical treatment exact fulfllment of the law is assumed, unless otherwise statec. The efiect of deviations from exact fulfilment is discussed later in this paper.

In considering the general theory of the sphere certain relations between brightness and the iuminous fiux emitted or received will be found very userul. The proofs can be found in another Scientific Paper of this bureau, ${ }^{27}$ or they can be cerived very simply 
by the metrods of the integral calculus. These relations are as follows:

Unit area cĥ̀ a plane disk radiating luminous fiux in accordance with the cosine law of emission-that is, one which has the same origitness $b$ (canciles per unit of projected area) at all angles of view-radiates a total flux of $\pi b$ lumens.*

A unit plane illuminated by a parallel infinite plane of uniform brightness $b$ receives $\pi b$ lumens.

Given a hollow sphere (Fig. 5) with a surface radiating light flux in accordance with the cosine law of emission, let

$r=$ radius of sphere;

aS $=$ area of small surface at $P$;

$b=$ irightress (candles per unit area) of $d S$;

$E_{1}=$ inimination (foot-candles) at $P_{1}$ due to $d S$.

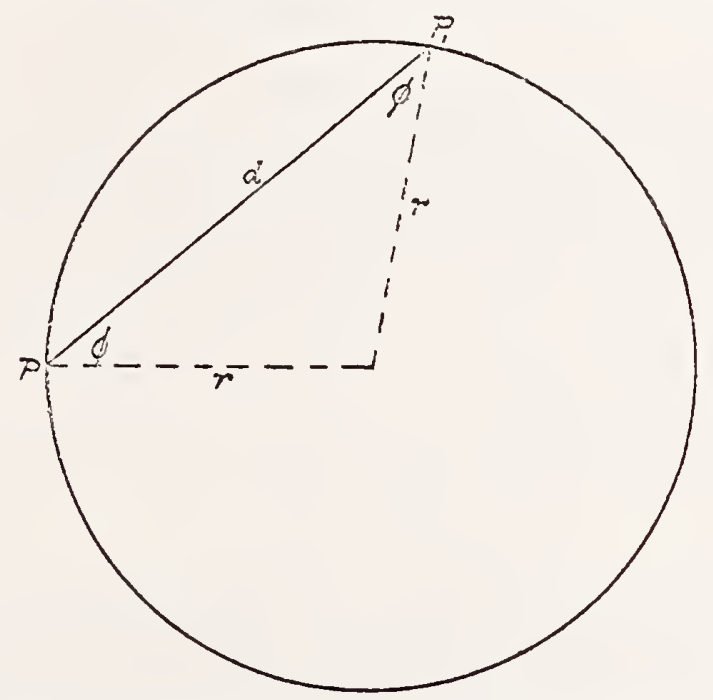

FIG. 5.-Relation between cmitting and illuminated clements of sphere

The candiepower of $d S$ in the direction $P P_{1}$ is $b d S \cos \phi$, and the illumination it would produce on a plane at $P_{1}$ normal to $P P_{1}$ would be $\frac{b d S \cos \phi}{d^{2}}$. Since the surface at $P_{1}$ is at an angle $\phi$ to the line $P P_{1}$, the illumination at $P_{1}$ due to $d S$ alone will be

$$
E_{1}=\frac{b d S \cos \phi}{d^{2}} \cos \phi
$$

But $d=2 r \cos \phi$, and therefore

$$
E_{1}=\frac{b d S \cos ^{2} \phi}{4 r^{2} \cos ^{2} \phi}=\frac{b d S}{4 r^{2}} .
$$

*The lumen is the unit of luminous flux, eduat to the Aux c:nited in a unit solid angle (steradian) by a point source of init canchepower. 
Since $P_{1}$ nuly be any point on the interior surface of the sphere, it is cviclent that any element of suface illuminates all other clements of the surface cqually. Hence, in a sphere having a surface which reflects light in accordance with the cosine law, the illumination of the surface by reflected light only is equal at all points, regardiess of any asymmetry of the light received directly from the source or of any variation of the reflection factor ci the surface from point to point.

$E_{\mathrm{d}}=$ the average illumination (foot-candles) of the sphere surface by light received directly from the lamp;

$E_{\mathrm{un}}=$ the illumination by diffuscly reflectcd light only;

$E=$ the average total illumination;

$m$ =reflection factor of the sphere suriace;

$a$ absorption factor of the sphere surface.

$=\mathrm{I}-\mathrm{m}$;

$F=$ total light flux (lumens) radiated by lamp in sphere;

$A$ = area of sphere surface.

$E_{\mathrm{m}}$ is composed of light of which a portion has been reflected a great many times, and hence $E_{\mathrm{m}}=m E_{\mathrm{d}}+m^{2} E d+\ldots$ etc.

$$
\begin{aligned}
& E=E_{\mathrm{d}}+E_{\mathrm{n}}, \text { or } \\
& \quad E=E_{\mathrm{d}}\left(\mathrm{I}+m+m^{2}+\ldots \ldots+m^{(\mathrm{n}-1)}\right)=E_{\mathrm{d}} \frac{\mathrm{I}-m^{\mathrm{n}}}{\mathrm{I}-m} .
\end{aligned}
$$

Since $n$ is infinite,

$$
E=\frac{E_{\mathrm{d}}}{\mathrm{I}-m}=\frac{E_{\mathrm{d}}}{a}
$$

This result can be obtained by other methods also, one of which is as follows:

Since the hollow sphere is a closed surface, all of the light flux radiated by the lamp will be absorbed. The intensity of the total illumination will depend on the absorption factor of the surface. An analogy to this is the strength of the current in a resonant electrical circuit.

Hence

$$
a E A=F *, E=\frac{F}{a A}=\frac{E_{\mathrm{d}}}{a}, \text { since } \frac{F}{A}=E_{\mathrm{d}} .
$$

\section{ERRORS OF MEASURENEYY DUN TO SCREEN}

The illumination of any particular point may difier fron $E$, since the direct illumination $E_{\mathrm{d}}$ varies with the ight distriburion of the source and its distance from the point considered. In order to measure $E_{\mathrm{m}}$, which is directly proportional to $I$, the spherica!

* Since an illumiuation of one foot-candie is produced by one lumen incident per square foot. 
candlepower of the lamp, it is necessary to insert in the sphere wail a difusely tronsmitting window and to screen from it the cirect ligit from the lamp in the sphere. Relative values of the brigintness of the window are then proportional to the relative spinerical intensities of the lamps tested. However, the necessary use of the screct to cut of the direct light from the window, and the insertion of the lamp into the sphere alter the above theoretical value of $E_{\mathrm{m}}$ somewhat oin account of obstruction and absorption of light by them, and it is important to know the magnitude of the pessible error which may occur.

In a sphere in which the direct light is screened from the observation window the direct light may be considered as having two components as regarás its effect on the brightness of the observation window, namely, the part $A$, which is incident on the surfaces visible from the observation window, and the part $B$, which falls on the areas screened from the window. The part $A$ can illuminate the window by the first reflection, but the part $B$ must suffer one extra reflection before any of it can reach the window. Any constant difference between the theoretical and observed values of $E_{\mathrm{m}}$ will be compensated for by the substitution method of photometry, but variable differences, due to different fractions of the total direct fiux of the two lamps being incident on the screen and screened area, will cause errors of measurement.

Let $\delta=$ fraction of total light fiux $F$ incicent on screen and screened area.

The portion of direct light refiected from the zone unscreened from the observation window (zone 3 , Fig. 6 ) is $m F(I-\delta)$. Its effect on the observation window is the equivalent of a secondary source radiating that amount of light fiux in an empty sphere. Eence the illumination of the observation window due to it is $\frac{m F(\mathrm{r}-\hat{o})}{a A}$. The portion $\delta F$ suffers two reflections before any of it reaches the observation window, and its effect on the window is the equivalent of a source radiating $m^{2} \delta F$ lumens. Fience the total illumination of the window is

$$
\begin{aligned}
E^{\prime} & =K\left[\frac{m F(\mathrm{I}-\delta)}{a A}+\frac{m^{2} \delta F}{a A}\right] \\
& =\frac{m \Gamma K}{a A}[(\mathrm{I}-\delta+m \delta)]=K^{\prime}(\mathrm{I}-\delta a),
\end{aligned}
$$

where $K$ is a proper fraction introduced to make allowance for the absorption of reflected ligint by the lamp and opaque screen. 
The factor $K$ is practically constant î̀ the screen is small, but $\delta$ is variable, being dependent on the distribution of ight from the source. Its value may be anything between o and $I$, being $o$ if no direct light is incident on these areas. Hence the maximum possible error is $a$, the absorption factor of the sphere surface.

If the substitution method of photometry is used, the error of measurement $e$ would be given by the equation

where

$$
e=\frac{\left(\delta^{\prime}-\delta^{\prime \prime}\right) a}{1-\delta^{\prime} a}
$$

$\delta^{\prime}=$ value of $\delta$ for standard lamp;

$\delta^{\prime \prime}=$ value of $\delta$ for test lamp.

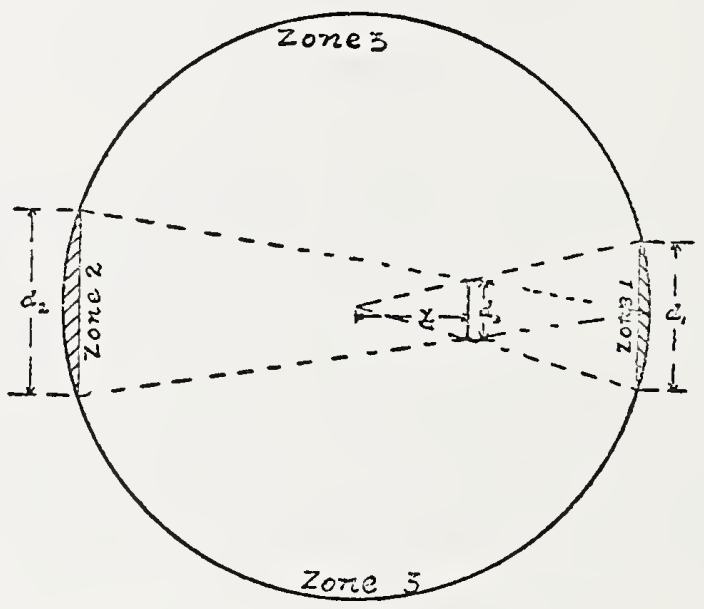

FIG. 6.-Screened and unscreened zoncs of sphere with light source at center

\footnotetext{
Zone I is screcncd from the source, and the cibscrvins window is placed in it. Zone 2 is therefore screened from the window. Zone 3 is not screcned from either source o: window.
}

\section{POSITION ATD SIZE OF SCREEN}

As has been incicated above, errors are caused by the screen only when different fractions of the direct flux of the two sources being compared fall on the screen and screened area. Assuming that the screen has the same refection factor as the sphere surface, its effect on the light which falls directly upon it is practically the same as if an equivalent zone (zone I, Fig. 6) were screened from the observation window. In general, it is evident that the smaller this equivalent zone and tine zone actuaily screened from the window (zone 2, Fig. 6) are made, the less chance there will be of errors resulting. 
The prope: position of the screen to make the screened areas a minimum can be determinec closely enough by drawing a diagram of the spincre, with different positions of a screen of constant size. anci fincing the point as which the sum of the squares of the ciameters of the screened areas on the sphcre wails will be a minimum. The postion can be more exantly detcrmined mathematically as follow:

Lamp at center of sphere. (See Fig. 6.)

İt $\vec{i}_{i}=$ diameter of zore $I$;

$d_{2}=$ diameter of zone 2 ;

$d_{3}=$ diameter of screen;

$x=$ distance of screen from center of sphere;

$y=$ sum of areas of zones I and 2 ;

$r$ = radius of sphere.

Zones I and 2 are the screened zones. It is desired to make $y$ a minimum. An approximate solution would be obtained as follows:

$$
\begin{aligned}
& \frac{d_{1}}{d_{3}}=\frac{r}{x}, d_{1}=\frac{a_{3}^{r} r}{x} . \\
& \frac{d_{2}}{\vec{a}_{3}}=\frac{2 r}{r-x}, d_{2}=\frac{2 d_{3} r}{r-x} .
\end{aligned}
$$

Area of zone I (approximately) $=\frac{\pi d_{1}^{2}}{4}$.

Area of zone 2 (approximately) $=\frac{\pi d_{2}^{2}}{4}$.

$$
\therefore y=\frac{\pi}{4}\left(d_{1}^{2}+d_{2}^{2}\right)=\frac{\pi d_{3}^{2}}{4}\left[\left(\frac{r}{x}\right)^{2}+\left(\frac{2 r}{r-x}\right)^{2}\right] \text {. }
$$

Differentiating and equating to o gives the condition for a minimum of screened area.

$$
\frac{d y}{d x}=\frac{\pi r^{2} d_{3}^{2}}{2}\left[\frac{4}{(r-x)^{3}}-\frac{1}{x^{3}}\right]=0, \frac{4}{(r-x)^{3}}-\frac{I}{x^{3}}=0 \text {, or } 4 x^{3}=(r-x)^{3} .
$$

Then $x=0.387^{r}$.

The value of $x$ when the lamp is not in the center of the sphere couid be calculated in a similar manner, though the calculation would be more complex because the screened zones would not be circular. In that case the solution is most easily obtained by graphical methods. 
If the imp is above or below the center of the spincre, so that the line from the lamp to the observation winciow makes an angic 0 with the line from the wincow to the center of the sphere, the winciow being on a horizontal dianeter of the sphere, the propen horizontal distance of the screen from the vertical axis of the sphere is given by Ulbricht as follows:

$$
\begin{aligned}
& \theta=30^{\circ}, x=0.32 r ; \\
& \theta=35^{\circ}, x=0.20 r
\end{aligned}
$$

By the graphical method suggested above the proper positions for the screen for varying positions of the lamp on a diameter

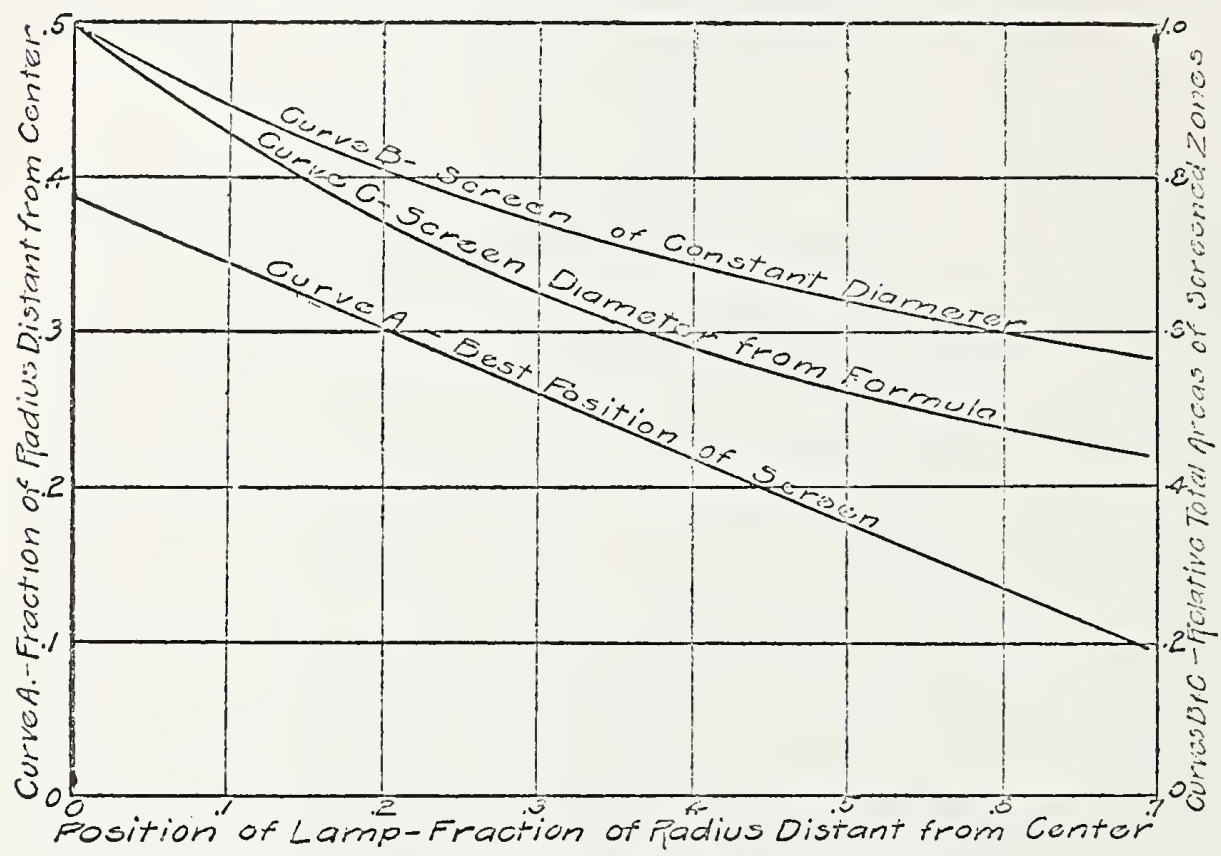

FIG. 7.-Best position of screcn, and relative areas screened by it with lamp in different positions

through the window have been determined. The result is shown by curve $A$ of Figure 7. The lamp and screen are assumed to be on opposite sides of the center of the sphere, with the screen or the side toward the wincow. The solution was obtained tor a screen whose diameter was o.I that of the spinere, but a checir calculation with a screen couble that ciameter gave substantially the same result. The sum of the screened areas decreases as the lamp is moved farther from the sphere center, the screen being placed in the position shown by cuve $A$. This result is shown by curve $B$. The necessary dianeter of the screen to give good screening also decreases. The diameter of the screen required 
when the screen and lamp are on opposite sicies of the sphere center may be determined as follows:

Let $l=$ the greatest dimcnsion of lamp to be screened, or length of locus of ligint source;

$r=$ racius of sphere;

$a^{\prime}=$ distance of lamp from center of sphere;

$b r=$ distance of screen from center of sphere;

$d=$ required diameter of screen.

Then

$$
\frac{d}{l}=\frac{(I-b) r}{(I+a) r}, \text { or } d=l \frac{I-b}{I+a} \text {. }
$$

Fence it is seen that tine diameter of the screen is independent of the size of the sphere, and depenas only on the positions of lamp

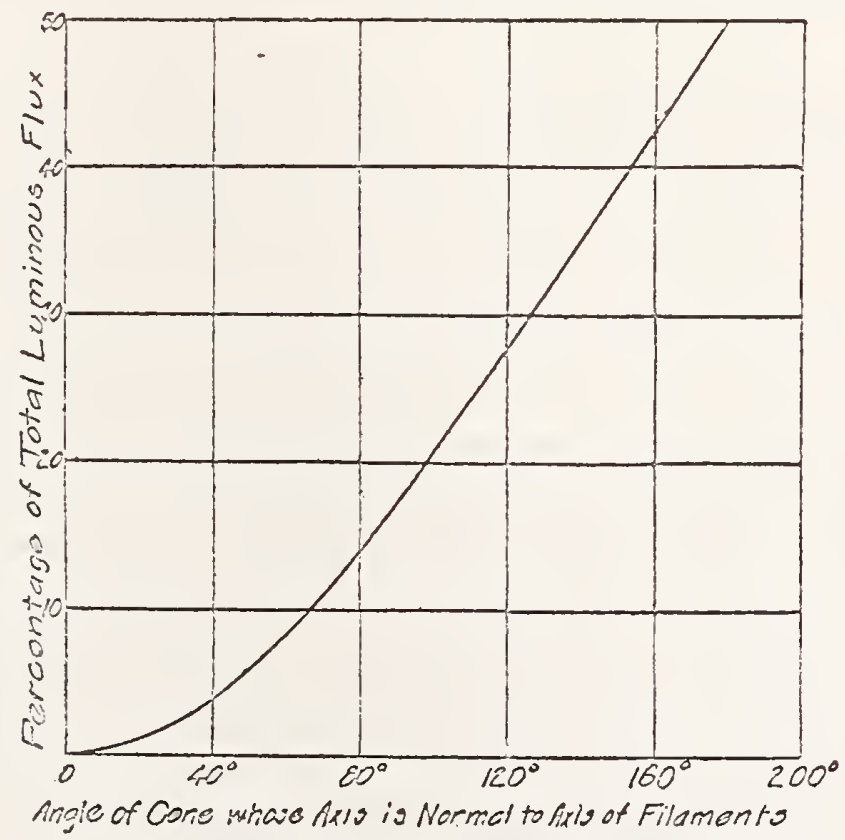

FIG. 8.-Percentage of total fux from vacuum tungsten lomp included in cones of various a perlures normal to lamp axis

and screen and the length of the largest lamp which it is desired to mcasure (asstiming that one screen is to serve for all lamps. if the lamps vary much in size, it may be desirable to liave a series of interciangeable screens). For safety the screen should be macie slightly larger than the soitution given by (13). Curve $C$ in Figure 7 shows the relative total areas of the screened zones for various positions of lamp and screen, when the screen diametcr is chosen by equation ( $I_{3}$ ) and the screen is located accorcing to cirve $A$, Figure 7. 
In Figure $S$ is shown, for the common type of vaculm tungsten lamp, the percentage of the total light which is emittad in cones $0 \hat{i}$ various angles with their axes normal to the axis of the filanents. The angle of the beam incident on each screcneci area is easily obtained, and by reference to this curve the percentage of the tota? flux which is incident on the screened area may be found. Yे when the lamp is in the center of the sphere the diameter of the proper screen is 0.2 or 0.4 of the sphere radius, its proper diametefor other positions of the lamp is shown by the soild curves of Figure 9, and the percentage of the total flux of a vacuum tungsten

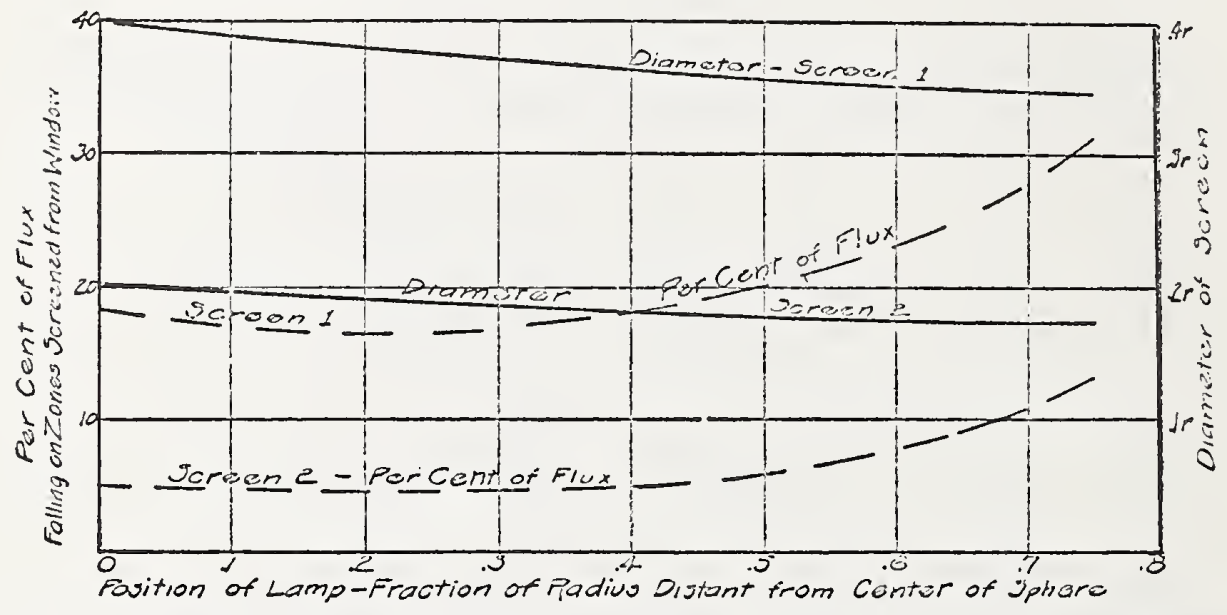

FIG. 9.-Diametcr of screens and percentage of light flux falling on parts screened from observing window with lamp in different positions

It is assumed that the lamp is such as to require a screcn dianetcr citior $(x) 0.4$ or (2) 0.2 of the spincre radius when the lamp is at the center of the spicre. Diameters for other positions are then calculated from equation $x_{3}$, and positions are determined by curve $A$, Fig. 7. The aux calculations apply to vactur turgsten lamps (sce Fig. S). The percentage of flux indicated falls oz the screen and on the zone screencd from the window, but both of thesc may be considerca as zones of the sphcre wall (sec Fig. 6)

lamp which is incident on the zones screened from the window is shown by the broken curves in the same figure.

This type of lamp gives more nearly tinan any other common type of incandescent lamp the light distribution which wouid be obtained from a straight luminous rod, and it is improbable that any other type of filament arrangement except the motion picture projection lamp would give a larger percentage of its total fiux in any solid angle normal to the lamp axis not exceeding $90^{\circ}$. Fence, if the best jocations of lamp and screen for measurements of lamps of this type are chosen, they would probably be approximately the best for measurements of lamps oi other types aiso.

From the curves in Figure 9 it is seen that the fiux on tine screened zones is a minimum for this type of lamp when the lamp is removed 
0.2 of the radius from the center of the sphere, though there is very littie chice between this position and any other between the center and 0.4 . Since the totai screened area is less at the $0.4=$ postion, that position woula probably be preferabie, at least in spheres larger than about I yard or I in in diameter. Other consierations nay make cesirabie a position nearer the center in the smailer spireres, but this must be judgec largeiy by the use to which the sphere is to be put. If the lamp is piaced too near the wall, there will be anotier factor to be reckoned with, namely, cosorption of first reflected light by the lamp. The effect of this wil be brought out more ciearly in the following paragraphs.

\section{ABSORPTION OE LEGEI BI TORETGN OBIECTS}

If any foreign object is brought into the spinere, it will absorb ingit and redice the illumination of the spliere window, even though it is screened from the window.

As before, let

$E_{m}=$ initinination by reflected light (foot-cancies);

$E_{\mathrm{c}}=$ average directillumination;

$a=$ absorption factor of sphere surface;

$a^{\prime}=$ absorption factor of foreign body in sphere;

$F=$ total igirt fiux from source, in lumens;

$r=$ racius of sphere, in feet,

$b=$ average bigitress of sphere wall (candles per square foot) when there is no foreign boay in the sphere;

$b^{\prime}=$ average b-ightness of sphere vall wien the foreign body is in the sphcre;

$S=$ surface area of foreign body;

$A=$ area of spicere.

As previously stated, the illumination at any point in front of an infinite plane of brightness $b$ is $\pi b$. Also, the flux given off by unit area is $\pi b$ lumens.

If the foreign bocy is far enough from the walls of the sphere so that it does not reduce the illumination of the walis unequally, and does not receive any direct light from the lamp, it will receive the average ilumination $\pi b^{\prime}$ foot candles (since the illuminating surface is equivalent to an infinite platne), and altogether the flux $\pi b^{\prime} S$, of winch it wili absorb the fraction $\pi b^{\prime} S a^{\prime}$ lumens.

The surface of the sphere raciates the light flux $\pi \dot{u}^{\prime} A$ lumens and $a b s o r b s$ the fraction $a$ of the total fitux received. Fience $x^{2}$ receives $\frac{\pi b^{\prime} A}{x-a}$ and absoibs $a \frac{\pi b^{\prime} A}{I-a}$ lumens. 


\section{Photometric Integrating Sphere}

Since all the light is absorbed, the flux absorbed must equal the total flux radiated by the lamp, or

$$
\begin{aligned}
& \pi b^{\prime} S a^{\prime}+\frac{\pi a b^{\prime} A}{\mathrm{I}-a}=F . \\
& b^{\prime}=\frac{F(\mathrm{I}-a)}{\pi S a^{\prime}(\mathrm{I}-a)+\pi a A} .
\end{aligned}
$$

If there is no foreign body in the sphere, then

$$
\begin{gathered}
\frac{\pi a b A}{\mathrm{I}-a} \text { must equal } F \text {, and } \\
b=\frac{F(\mathrm{I}-a)}{\pi a A} .
\end{gathered}
$$

Hence

$$
\frac{b^{\prime}}{b}=\frac{\frac{F(\mathrm{I}-a)}{\pi S a^{\prime}(\mathrm{I}-a)+\pi a A}}{\frac{F(\mathrm{I}-a)}{\pi a A}}=\frac{a A}{S a^{\prime}(\mathrm{I}-a)+a A^{\prime}}
$$

or

$$
b^{\prime}: b=\mathbf{I}: \mathbf{I}+\frac{S a^{\prime}(\mathbf{I}-a)}{a A} .
$$

If the foreign body is brought near the surface of the sphere (the foreign body is assumed to be something other than the lamp in this case), it cuts off some light from the wall approached, and hence its absorption of reflected light is less because the brightness of the wall approached is reduced. If the foreign body is the light source itself, the absorption is increased, because the lamp receives a greater fraction of the first reflected light. The value in (I8) is nearest correct when $a$ and $S$ are small, since in that case the brightness of the sphere wall is most nearly uniform.

The results shown in Table 2, page 296, apparently indicate that the absorption of reflected light by foreign objects is independent of their shape, which is to be expected from the theory as developed above.

\section{EFFECT OF LAMP POSITION}

In Figure 4, page 298, are given calculated and observed curves showing the reduction of sphere window illumination as the iamp approaches the portion of the wall screened from the observation window. The calculated curves were obtained in the following manner: 


\section{Scientific Papers of the Bureau of Standards}

A diagram of the sphere similar to Figure 6 was drawn, and the outline of the screened area, zone 2, was established. By the aid of a protractor the angles at the lamp subtended by the screen and zone 2 were measured, and from the curve in Figure 8 the percentages of the total flux which are incident on the screen and zone 2 were obtained. For example, take the case when the 38 $\mathrm{cm}$ screen is used and the lamp is $76 \mathrm{~cm}$ (30 inches) from the door. Then the angles at the lamp which are subtended by the screen and zone 2 are $27^{\circ}$ and $88^{\circ}$, respectively. Referring to Figure 8 , the flux incident on the screened areas is found to be 18. I per cent of the total delivered by the lamp. Since $a=7.5$ per cent, the illumination of the observation window is shown by equation (6) to be

$$
E^{\prime}=K^{\prime}(\mathrm{I}-\delta a)=K^{\prime}(\mathrm{I}-0 . \mathrm{I} 8 \mathrm{I} \times 0.075)=0.986 K^{\prime},
$$

which is 99.7 per cent of the illumination of the window when the lamp is $99 \mathrm{~cm}$ from the door, or 98.6 per cent of the theoretica value for the illumination of the empty sphere by reflected light only.

If a source giving all its light on the unscreened area is measured in terms of vacuum tungsten standards in this sphere, using the substitution method, the error of measurement to be expected would be given by the curves in Figure 4. For example, if the lamp and reflector were $100 \mathrm{~cm}$ from the sphere door, the error would be plus 0.2 per cent with a $19 \mathrm{~cm}$ screen, and $+\mathrm{I} .0$ per cent with a $38 \mathrm{~cm}$ screen. If this were the only factor affecting the accuracy of the results, it would be expected that higher values in terms of such standards would be obtained for an opaque reflector unit as it approached the screened wall, since practically all of the direct light falls on the unscreened area. As a matter of fact, however, measurements of such a unit, evaluated in terms of a standard lamp measured in the same positions, gave constant results at all positions up to about $20 \mathrm{~cm}$ from the sphere wall; that is, the sphere window illumination fell off in about the same proportion with the reflector unit as with the bare lamp. The probable reason for this was that the reflector unit absorbed more and more light as it approached the wall because it received more of the first reflected light from the wall, and the reduction due to this cause happened to be just the same in amount as was to be expected in the case of the bare lamp, where the reduction was due to absorption by the screened areas. It should be noted here 
that a screen of about $19 \mathrm{~cm}$ diameter is large enough for the measurement of all sizes of incandescent lamps in this sphere, and that the $38 \mathrm{~cm}$ screen would be used only in case a lamp of very extensive area was to be measured. Since nearly all other types of incandescent lamps and reflector units throw a still smaller proportion of their total flux on the screened areas in this sphere, the error of measurement due to the screened areas should not exceed 0.3 per cent when a $19 \mathrm{~cm}$ screen and vacuum tungsten standards are used, the lamp being in its normal position, $25 \mathrm{~cm}$ from the sphere center, and it would be as great as this only when measuring a source which threw all its light on the unscreened areas.

In case a lamp which threw all its light on the screened area was measured with the $19 \mathrm{~cm}$ screen, the lamp being at the 100 $\mathrm{cm}$ position, the error would be $-(\mathrm{a}-0.3$ per cent $)=-7.2$ per cent. This is an extreme case, however, since in such a measurement the logical method would be to direct the beam toward the unscreened area, when the error would be only +0.3 per cent.

This method of analyzing the operation of a sphere makes possible an estimate of the error which may be encountered in practice with a definite arrangement of sphere screen, lamp socket, etc., and such an analysis would be valuable in considering the accuracy of integration with any particular sphere. It should not be overlooked, however, that differences in absorption of reflected light by the standard and test lamps may cause errors greater than these.

\section{ERROR OF INTEGRATION OF NARROW CONE OF LIGHT}

In the theory of the sphere as developed above, and in all the literature on the subject, the assumption has been made that the sphere surface is a perfect diffuser. Also, it has been assumed that the translucent window is a perfectly diffuse transmitter of light, so that the brightness of the observed side is directly proportional to the illumination of the opposite side, regardless of the angle of incidence of the light contributing to this illumination. These ideal conditions are not realized in practice, and hence it is important to determine the amount of the departures and the error which may occur on account of them.

The illumination of any part of the surface of a sphere surfaced with rough white cement or a matt white paint may be analyzed into three components: (a) The light flux received directly from the lamp; (b) the flux contributed by the first reflection of the 
direct illumination of all other parts of the sphere surface; and (c) the light due to multiple reflections of (b). As previously stated, the part (a) may vary through a very wide range, and hence it is usually screened from the observation window. The surface is nearly enough a perfect diffuser to make the part (c) entirely uniform, and independent of the way in which the direct light is distributed over the sphere surface. Hence the departure from perfect diffuse reflection by the sphere surface may be assumed to affect only the part (b), though lack of perfect diffusion by the window affects both $(b)$ and $(c)$.

Let $A=$ area of sphere;

$F=$ light flux (lumens) radiated by lamp;

$E_{\mathrm{d}}=$ average direct illumination $=\frac{F}{A}$;

$E^{\prime}=$ average illumination by first reflection of $E_{\mathrm{d}}$;

$E=$ total illumination of observation window which is screened from $E_{\mathrm{d}}$.

Neglecting the effect of any direct light on the screened areas, and assuming perfect diffusion,

$$
\begin{aligned}
& E^{\prime}=m E_{\mathrm{d}}=(\mathrm{I}-a) E_{\mathrm{d}} ; \\
& E=\frac{E_{\mathrm{d}}}{a}-E_{\mathrm{d}}=\frac{E_{\mathrm{d}}(\mathrm{I}-a)}{a} .
\end{aligned}
$$

Then

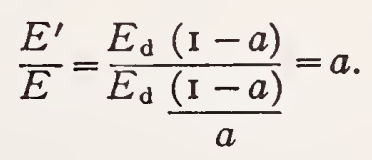

In Figure Io are shown experimental curves for the diffusion characteristics of the sphere window and the sphere surface. When a flat disk of the Keene's cement with which the sphere was originally surfaced is illuminated normally, its specific brightness is different at different angles of view.

If the radiated flux is integrated, it is found that at all angles except $50^{\circ}$ from the normal to the surface the brightness of the cement is greater or less than that of a perfect diffuser radiating the same total flux. The percentage deviations at various angles are shown by curve $A$ in Figure Io. The curve for the zinc oxide paint described in Part I of this paper is practically the same as curve $A$ for all angles from $15^{\circ}$ to $75^{\circ}$. It is about 5 per cent higher than curve $A$ at $5^{\circ}$ and 5 per cent lower at $80^{\circ}$.

If the window were a perfect diffuser, its brightness measured normally should vary as the cosine of the angle of incidence of 


\section{Photometric Integrating Sphere}

the light on the opposite side (angles being measured from the normal). Curve $B$ shows the deviation of a milk-glass window, surfaced with coarse carborundum (No. 6o), from the cosine law of transmission.

If a photometer is standardized to measure the normal illumination on the face of this milk glass opposite to the side viewed, calculations show that if the window was illuminated by a uniformly bright infinite plane the evaluation of the illumination would

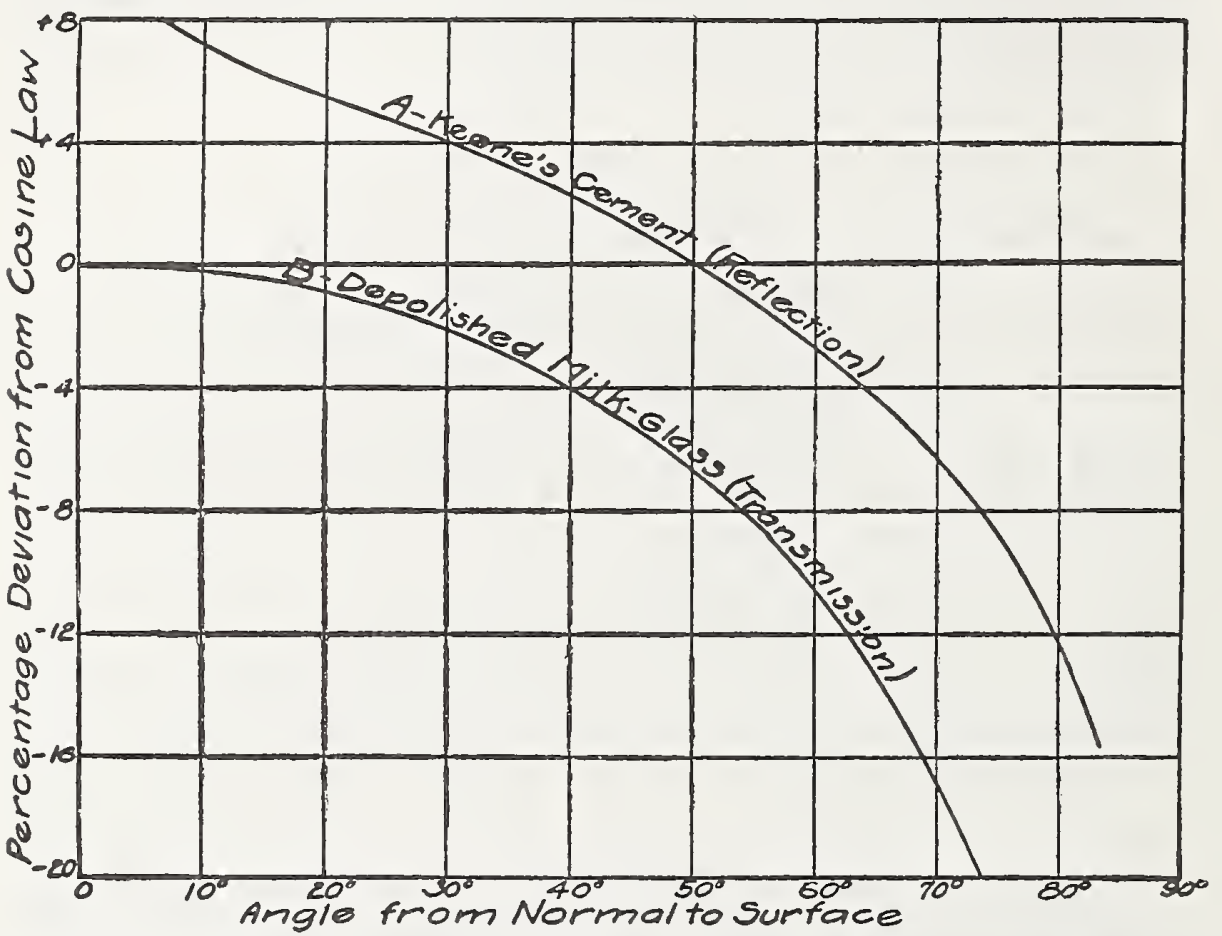

FIG. I0.-Diffusion characteristics of Keene's cement and depolished milk-glass

be too low by 7.6 per cent. This is practically the condition in the sphere as regards the portion (c) defined above. If the sphere surface is illuminated normally to a uniform amount, as would be the case if a point source of light was placed at the center of the sphere, the illumination due to the first reflected flux, not perfectly diffused (see curve $A$ ), would be evaluated too low by 8.8 per cent. Hence, since the absorption factor of this sphere is now 6.0 per cent, and since that also represents the ratio of $E^{\prime}$ to $E$ (see above), the error in the measured value of the window illumination would be

$$
\begin{aligned}
& e^{\prime}=0.94(-0.076)+0.06(-0.088)=-0.0714-0.0053=-0.0767 \\
& =-7.7 \text { per cent. }
\end{aligned}
$$




\section{Scientific Papers of the Bureau of Standards}

If the direct light is incident as a very narrow cone, the error of measurement of the first reflected flux depends on the relative positions of the illuminated spot and the window (assuming that the direct light is incident normally to the surface). The error of measurement of the diffusely reflected light from reflections after the first, which is $0.94 \times 0.076=7.14$ per cent, would remain the same. If the angle of incidence of this first reflected light on the window is $\theta$, the angle of emission from the sphere surface is also $\theta$. Referring again to Figure ro, the error of measurement of the first reflected light is the product of the algebraic sum of the deviations there shown by curves $A$ and $B$ for the particular angle considered into the absorption factor of the sphere surface. For example, if $\theta=45^{\circ}, \mathrm{e}^{\prime \prime}$, the error of measurement, would be

$\mathrm{e}^{\prime \prime}=6.0$ per cent $(+\mathrm{I} .3$ per cent -5.3 per cent $)=-0.25$ per cent;

$\therefore$ the total error would be $-7.14-0.25=-7.39$ per cent.

Other values have been calculated to be as follows:

0. angle of incidence

$0^{\circ}$.

22. 5

65.5
Error of measurement of window illumination* Per cent. $-6.55$ $-6.91$

$-7.39$ $-8.32$

The absolute value of this error is immaterial in the ordinary use of the sphere. The irnportant point is the agreement between the evaluation of the flux from a lamp when the direct light is uniformly distributed over the sphere surface and when the same amount of flux is concentrated in a narrow beam of light. It has been shown above that when the direct light is uniformly distributed the error in evaluating the illumination of the window in this sphere should be -7.7 per cent. If the narrow cone of light is directed at a point $90^{\circ}$ from the window-that is, $\theta=45^{\circ}$, the error of measurement would be only about 0.3 per cent less than the error made in integrating a point source radiating the same amount of flux-and this difference is the error made in evaluating a point source in terms of a narrow cone incident at a point $90^{\circ}$ from the observation window.

*Assuming no direct flux on screened areas. 


\section{FACTORS AFFECTING THE ACCURACY OF MEASUREMENT}

From equation (7) it is evident that increased accuracy may bc obtained by reducing $a$, the absorption factor, or decreasing $\delta$, the proportion of light on the screened area. This is accomplished by making the scrcen as small as possible and placing it in the position to make the screened area a minimum. Equation (I8) shows that the influencc of the presence of a foreign body may be decreased by making $a$, the absorption factor of the sphere, large, or by making the relative areas of forcign body and sphere surface small. Hence it is seen that in both cases the accuracy of integration is increased by making the sphere area large, assuming that no correction for the presence of the foreign body is to be made. This is ordinarily the case when incandescent lamps being life tested arc to be photometered.

The action of the absorption factor is opposite in the two cases, however, since in the former a small factor is best, whereas in the latter a large factor reduces the influence of the foreign body, and hence reduces the error of neasurement if no correction is made. In certain kinds of routine measurements, for example, the measurement of arc lamps, the effect of the presence of the lamp mechanism is easily corrected for by the method of measurement. This is not so easily done in the case of life test measurements of incandescent lamps, however, since the lamps may be of various sizes and degrees of blackening, and the measurement of the influence of each individual lamp in the sphere would involve a prohibitive amount of labor. On the other hand, if the absorption factor of the sphere walls is very low, this effect may cause appreciable errors if neglected.

In a particular case here, one life test lamp, a Iooo-watt type C Mazda which had fallen to about 20 per cent of its initial candlepower, reduced the sphere window illumination 8 per cent when it was introduced unlighted into the sphere. By calculation, using equation (18), it is found that the reduction of window illumination would have been $\mathbf{9 . 2}$ per cent for a sphere absorption factor of 6 per cent and 2.7 per cent for an absorption factor of 20 per cent. This lamp presented an extreme case, but it is not at allimprobable that lamps showing one-fifth of this degree of blackening would be frequently encountered. 


\section{Scientific Papers of the Bureau of Standards}

\section{BEST CONDITION OF SPHERE FOR CERTANN WORK}

In the case where a sphere is to be used for work such as life test measurements, or other work where determination of the allowance for absorption by the lamp would necessitate more labor than could reasonably be expended on the measurement, a sphere paint having a reflection factor of about 80 per cent would give better results than a highly reflecting paint, and the influence of the presence of the foreign body could usually be neglected in spheres about 6 feet and larger in diameter.

When a sphere is to be used most of the time for standardization work, it would be best to select a high reflecting power paint, and make allowance for the influence of the presence of the lamp, if measurable.

\section{CLASSIFICATION OF BIBLIOGRAPHY}

In order to assist in the examination of the articles listed in the following bibliography, a general summary of the subjects discussed in them has been prepared. The general classification is as follows:

(a) Mirror integrators: I to 6, 25 .

(b) Box integrators: 26, 28, 34 .

(c) Theory of the Ulbricht sphere: 7 to I0, I4, 15, 19, 24, 25, $27,29,3$ I $, 36,38,39,40$.

(d) Details of sphere construction: 8,9 , 10, 12, 13, 17, 18, 19, 2 I , 24, 30, 32, 35, 36 .

(e) Methods of measurement of spherical candlepower: 9, I0, I 2, I 3, I8, 2I, 24, 29, 3I, 35, 36 .

(f) Theory of hemispherical candlepower measurements: 9, I I, I 4, I 5 .

(g) Methods of measuring hemispherical candlepower: I 5, I 9, 20.

(h) Special tests of and with integrating spheres: 8, 9, I0, I2, I3, I5, 2I, 23, 29, 3I, 35, 36, 37, 38, 39, 40.

\section{BIBLIOGRAPHY}

I. Blondel, La détermination de l'intensité moyenne sphérique des sources de lumière, L'Éclair. Élect., 2, p. 385, I895; 3, p. 57, 406, 538, 583, I895; C. R.。 120, p. $31 \mathrm{I}, 550$, I895.

2. Blondel, Mesure directe de 1 'intensité lumineuse moyenne sphérique, L'Éclair. Élect., 8, p. 49; 1896 .

3. C. P. Matthews, An improved apparatus for arc-light photometry, Trans. A. I. E. E., 18, p. 671, 1901; 20, p. 59, 1902; L'Éclair. Élect., 40, p. 128, 1904; J. Inst. Elec. Engr., 32, p. 631, I903.

4. Blondel, Les integrateurs photométriques, mesophotometres èt lumenmètres, Bull. Societé Internationale des Électriciens, 4, p. 659, I904; L'Éclair. Élect., 42, p. 66, 1905; Sci. Abst. B, 8, abst. 487 and 1689, 1905. 


\section{Photometric Integrating Sphere}

5. E. P. Hydc, On the theory of the Matthews and the Russell-Leonard photometers for the mcasurcment of mcan spherical and mean hemispherical intensities. B. S. Bullctin 1, 1905 (or B. S. Sci. Papers, No. 12).

6. G. H. Stickncy and S. L. E. Rose, Photometry of large light sources, Trans. I. E. S., 6, p. 641 ; 1911 .

7. Sumpner, The diffusion of light, Phil. Mag., 35, p. 8r; r893.

8. R. Ulbricht, Die bestimmung der mittleren räumlịchen lichtintensität durch nur eine messung, ETZ, 21, p. 595, 1900; Sci. Abst., 8, abst. $2154,1900$.

9. Ulbricht, Dic vorange im kugelphotometer, ETZ, 26, p. 512, 1905; Sci. Abst., S, abst. 1160,1905 .

10. I. Bloch, Das kugelpliotometer in theorie und praxis, ETZ, 26, p. 1047, 1074, 1905: Illuminating Engineer (New York), 1, p. 421, 1906; Sci. Abst., 8, abst. 1688, 1905.

II. Ulbricht, Die hemisphärische lichtintensität und das kugelphotometer, ETZ, 27, p. 50, 1906; L'Éclair. Élect., 46, p. 432, rg06; Sci. Abst., 8, abst. 370, 1906.

32. M. Corsepius, Eine ausfuhrungsform des Ulbrichtschen kugelphotòmeters, ETZ, 27, p. 468, 1906; Illuminating Engineer (New York), 1, p. 482, 1906; Sci. Abst., 9, abst. 772, 1906.

13. B. Monasch, Vcrsuche mit hilfsapparaten zur bestimmung der mittleren sphärischen und der mittleren hemisphärischen lichtstürke, ETZ, 27, p. 669, 695, 803, 1906; Illuminating Engineer (New York), 1, p. 586, 700, 1906; Sci. Abst., 9, abst. $1233,1906$.

14. E. Presser, Die theorie der Ulbrichtschen kugel, Elektrotechnischer Anzeiger, p. $885,912,1906$.

15. Ulbricht, Zur anwendung des kugelphotometers, ETZ, 28, p. 777, 1907; Electrical World, 50, p. 418, 1907; Sci. Abst., 10, abst. 1086, 1907.

16. Schweizerischc Elektrotcchnische Zs., 4, p. 85, 97, 110, 1907; Sci. Abst., 10, abst. 437,1907 .

17. Modern photomctrical appliances, Illum. Engr. (London), 1, p. 228; 1908.

I8. I. Bloch, The globe photometer in practical photometry, Illum. Engr. (London), 1, p. 274; 1908.

19. On the theory of the globe photometer, Illum. Engr. (London), 1, p. 553; 1908.

20. M. Corsepius, The measurement of the mean hemispherical candlepower by the aid of the Ulbricht globe photometer, Illum. Engr. (London), 1, p. 801, 895: rgo8.

28. Sharp and Millar, The integrating sphere in industrial photometry, Trans. I. E. S., 8, p. 502, 1908; Illum. Engr. (London), 1, p. 1031, 1908.

22. Ulbricht, Zur lichtschwerpunkt-bestimmung, ETZ, 80, p. 322, 1909; Sci. Abst., 12, abst. 490,1909 .

23. A. A. Perrinc, Experimental study of the globe photometer, Electrical World, 54, p. 727; Ig09.

24. E. Winklcr-Buscher, Die Ulbrichtsche kugel zur bestimmung der mittleren sphärischen bezw. der mittleren hemisphärischen lichtstärke, Elektrotechnik und Machincnbau, 28, p. 659, 1910; Sci. Abst., 14, abst. 120, 1911.

25. The dctermination of mean spherical candlepower (by an engineering correspondent), Illum. Engr. (London), 3, p. 31, 265, 1910.

a6. W. E. Sumpner, The direct measurement of the total light emittcd from a lamp, Illum. Engr. (London), 3, p. 323, 1910; Discussion, p. 387; Electrician (London), 65, p. $72,1910$.

27. E. B. Rosa, Photometric units and nomenclature, B. S. Bulletin, 6, 1910 (or B. S. Sci. Papers, No. 14r).

28. I. W. Wild, The box integrating photometer, Illum. Engr. (London), 8, p. 549; Ioro. 


\section{Scientific Papers of the Bureau of Standards}

29. E. Dyhr, Nachtrage zur theorie des kugelphotometers, ET2, 31, p. I295, I9ro; Sci. Abst., 14, abst. 128, I9Ir.

30. E. W. Marchant, Globe photometer, Illum. Engr. (London), 4, p. 37; I9II.

31. Chaney and Clark, Notes on the integrating sphere and arc lamp photometry, Trans. I. E. S., 10, p. I; I9I5.

32. Integrating sphere equipped for testing gas-filled lamps, Electrical World, 65, p. I20; I9I5.

33. A. Utzinger, Innenanstrich für die Ulbrichtsche kugel, ETZ, 36, p. 137, I915; Sci. Abst., 18, abst. 724, I9I 5.

34. L. O. Grondahl, A. box photometer, Trans. I. E. S., 11, p. I52; 1916.

35. E. B. Rosa and A. H. Taylor, The integrating photometric sphere, its construction and use, Sci. Abst., B, 19, abst. 572, r916; Trans. I. E. S., 11, p. 453 , I9I6.

36. F. A. Benford, Jr., The integrating sphere and a new type of compensating screen, Trans. I. E. S., 11, p. 997 ; 1916.

37. Henry A. Gardner, The light-reflecting values of white and colored paints, J. Franklin Inst., 181, p. 99; 19r6.

38. F. A. Benford, An integrating hemisphere, Trans. I. E. S., 13, p. 323; 19 I8.

39. F. A. Benford, A universal photometric integrator, Trans. I. E. S., 15, p. I9; I920.

40. A. H. Taylor, Measurement of diffuse reflection factors, and a new absolute reflectometer, B. S. Sci. Papers No. 391; J. Optical Soc. of America, 4, p. 9, I920.

4I. P. G. Nutting, A new method and an instrument for determining the reflecting power of opaque bodies, Trans. I. E. S., 7, p. 412; I9I2.

42. Geo. W. Middlekauff, A new form of direct-reading candlepower scale and recording device for precision photometers, B. S. Bulletin, 7, p. II; IgII (or B. S. Sci. Papers No. 144).

43. A. P. Trotter, Diffused reflexion and transmission of light, Illum. Engr. (London), 12, p. 243; 19I9:

44. A. H. Taylor, A simple portable instrument for the absolute measurement of reflection and transmission factors, B. S. Sci. Papers No. 405; Trans. I. E. S., 15, p. 8I I, I920.

The following references have been added since the original manuscript was prepared. None of these have been consulted in the preparation of the paper.

A spherical photometer, Electrician, 57, p. I057; I906.

Pressner, Ulbricht sphere, Electrical World, 49, p. 806, 1907; Elektrolechnischer Anzeiger, March 3, I907.

W. Haensch, Die kugelbeleuchtungsapparate und ihre vielseitige verwendung, Deut. Mech. Zs., 1914, p. I.

N. A. Halbertsma, Die werkung der ringblende vor de milchglasscheibe der Ulbrichtschen kugcl, Zs. f. Beleuchtungswesen, 22, p. 43; rgi6.

The usc of the sphere plotometer in street lighting tests, (editorial), Electrical Review and Westem Electrician, 69, p. 93; r916.

Blondel, Zs. f. Beleuchtungswesen, April 30, 1906; Electrical World, 47, p. Ir53, 1906.

H. Krüss, Integrating photometer, J. f. Gasbeleuchtung., 51, 1908; Electrical World, 52, p. 307, 1908 .

A. Blondel, Blondcl-Krüss integrating photometer, Illum. Engr. (London), 2, p. 785 ; Ig09.

An improved spherical photometer, Electrical World, 48, p. 23; igo6.

R. von Voss, Ein neues kugelphotometer für betriebsmessung an glulılampen, ETZ, 38, p. 188; 1917. 


\section{Photometric Integrating Sphere}

Un nouveau photomètre sphérique pour lampes à incandescence, Rev. Gén. de l'Élect. 2, p. 807 ; 1917 .

R. von Voss, Siemens and Halske globe photometer, ETZ, 88, p. 605, 1917; Sci. Abst. $B, 21$, abst. 2 I 9 , I 918 .

R. von Voss, A new globe photometer for incandescent lamps, Electrician, 80, p. 630, I9r $8 ; 81$, p. 418 , 1918 .

Sphere pour la détermination des angles solides des flux lumineux, Rev. Gén. de

l'Élec., 5, p. I 54 D; Igrg.

F. E. Cady, Note on the effect of lack of whiteness in the paint and window of an Ulbricht sphere, J. Franklin Inst., 189, p. 787, 1920; Electrical World, 77, p. 368, $192 \mathrm{I}$.

F. A. Benford, A universal photometric integrator, Trans. I. E. S., 15, p. 19; 1920.

A. H. Taylor, A new portable reflectometer, Electrical World, 76, p. 467; 1920.

H. Buckley, The whitened cube as a precision integrating photometer, J. Inst. Elec. Engr. (London), 55, p. 143; 1921.

C. H. Sharp and W. F. Little, Measurement of reflection-factor, Trans. I. E. S., 15, p. $802 ; 1920$.

E. Karrer, The use of the Ulbricht sphere in measuring reflection and transmission factors, B. S. Sci. Papers No. 415; J. Optical Soc. of Amcrica, 6, p. 96, I921.

Von R. Ulbricht, Das kugelphotometer, published by R. Oldenbourg, München und Berlin; 1920.

WASHINGTON, September 26, I921. 


\section{Application of Sulfur Coatings to Integrating Spheres}

\section{S. Thomas Dunn}

National Bureau of Standards, Washington, D.C.

Received 19 April 1965.

This work was performed under a NASA contract.

In recent years there has been an increasing interest in sulfur as an infrared integrating sphere coating. ${ }^{1,2}$ This interest stems from (a) its high reflectance in the infrared, ${ }^{2,3}$ (b) its nonhygroscopic nature, and (c) its ability to function as a good diffuser when used in a finely divided state. ${ }^{1,2}$

No information exists in the literature as to suitable techniques for applying sulfur coatings to sphere walls. Three methods of application used in a recent investigation at NBS are described below.

\section{Hand Pressed}

Initially, sulfur was applied to the two halves of the splere over a thin coat of rubber cement by hand pressing (with the fingers). The sulfur was built up to a thickness of about $3 \mathrm{~mm}$, and contoured to conform roughly to the outline of the two hemispheres. The surface was then smoothed with an artist's brush. This surface had a fairly uniform appearance but the coating was extremely fragile.

Sprayed Coatings (The spray techniques were developed by John R. Anderson, a General Laboratory mechanic.)

a. Sulfur-Alcohol Slurry. To increase the uniformity of the coating over the surface of the sphere, and the reproducibility from one sphere to another, a spray application technique was investigated. One part sulfur was mixed with about two parts alcohol by volume to form a slurry which was sprayed from a vibrator-actuated spray gun. The slurry was sprayed so that most of the alcohol evaporated before the spray hit the roughened (approximately $2.5 \mu \mathrm{rms}$ ) sphere wall. To insure rapid evaporation of the remaining alcohol, the hemispheres were heated to $77^{\circ} \mathrm{C}$ before spraying. About ten spray applications were necessary to obtain a $3-\mathrm{mm}$ coating. The hemispheres were reheated to $77^{\circ} \mathrm{C}$ and the sulfur surface was smoothed with an artist's brush between applications. The resulting coating was uniform in appearance, but it tended to crack with time, and its adherence to the hemisplere was poor. The sulfur itself formed a quite hard surface. b. Benzene-Sulfur Slurry. To alkeviate the problems experienced with the sulfur-alcohol slurry, the alcohol was replaced by benzene. This slurry was applied to the roughened hemisphere wall over a thin coat of a benzene-soluble contact cement. During the first few seconds of spraying, the spray gun was held very close to the surface so that the benzene dissolved the contact cement, which migrated slightly into the sulfur coating. The thin coating was then dried, leaving the sulfur bonded to the sphere wall. For the subsequent spraying operation, the spray gun was moved further away from the sphere wall, the temperature of which was maintained at about $65^{\circ} \mathrm{C}$ by heat from two infrared lamps. This, and the fact that benzene is more volatile than alcohol, permitted the shurry to be sprayed continuously until a coating thickness of about $3 \mathrm{~mm}$ was obtained. The surface was then smoothed with an artist's brush. The surface produced by this technique was very uniform in appearance and mechanically strong enough to withstand normal laboratory handling. The surface hardened considerably with age.

All sulfur used in this work was Crystex brand sulfur (Stauffer Chemical Company, New York); however, Kronstein et al. ${ }^{3}$ indicate that ordinary flowers of sulfur has about the same reflectance as Crystex brand sulfur throughout the infrared. It should be noted that Stauffer Chemical Company also supplies a Crystex type sulfur which contains $20 \%$ by weight of oil and has more stable mechanical properties. However, information on its reflectance is not available.

The same methods were found suitable for applying barium sulfate to sphere walls.

Certain commercial materials are identified in this paper in order to specify adequately the materials employed. In no case does such identification imply recommendation or endorsement by the National Bureau of Standards, nor does it imply that the material identified is necessarily the best available for the purpose.

\section{References}

1. R. C. Birkebak, "Monochromatic Directional Distribution of Reflected Thermal Radiation from Roughened Surfaces", Ph.D. Thesis, University of Minnesota, 1962.

2. S. T. Dunn, "Design and Analysis of an Ellipsoidal Mirror Reflectometer", Ph.D. Thesis, Oklahoma State University, 1965.

3. M. Kronstein, R. J. Kraushaar, and R. E. Deacle, J. Opt. Soc. Am. 53, 458 (1963). 


\author{
ISSUED DECEMBER 9, 1965
}

FLUX AVERAGING DEVICES FOR THE INFRARED

\author{
S. Thomas Dunn
}

ABSTRACT

The spatial and angular sensitivity of infrared detectors recently has been investigated $(1,2)$. In order to eliminate this effect and obtain accurate measurements in the infrared, it is necessary to distribute the flux as uniformly as possible over the entire sensitive area of the detector. A solution to this problem is presented in the form of several averaging devices developed at the National Bureau of Standards. Among the devices investigated are roughened $\mathrm{NaCl}$ windows, diffusing light ducts, and spheres with different coatings. Each device was subjected to a series of tests to establish 1ts averaging capability and useful wavelength range. Results of these tests indicate that the use of a small sulfur-coated hollow sphere over the detector increased the accuracy of most types of infrared reflectance measurements and, at the same time, decreased the requirement for precise optical alignment of the detector in the wavelength range of 1.5 to 8 microns. The use of the sulfur-coated sphere over a thermopile extends the usefulness of the multiple-reflection specular reflectometer to about 10 microns. (Key Words: Detector, Averaging Device, Spatial Sensitivity, Angular Sensitivity, Sulfur, and Integrating Sphere).

*The work described in this paper was done under NASA Contract No. R-09-022-032.

\title{
A 5.m Integrating Sphere
}

\author{
Mamoru Nonaka,Takeo Kashima, and Yataro Kondo
}

\begin{abstract}
An integrating sphere with an inner diameter of $5 \mathrm{~m}$ was constructed with special attention in order to make a true sphere by welding together steel plates of thickness of $12 \mathrm{~mm}$. The deviations of radii from a true sphere are within $\pm 0.3 \%$. A special lift, which is installed below the sphere, is used for changing a lamp or painting the sphere. The inner surface of the sphere was coater with a water paint of $\mathrm{BaSO}_{4}$. Its spectral reflectance was measured by a method, in which a part of the spherical surface was substituted by a surface with low reflectance and spectrophotometric measurements were made on the luminance of the measurement window of the sphere before and after the substitution, operating a tungsten lamp in the sphere. The values of spectral refleetance obtained by this method were from 0.93 to 0.96 in the visible region, ten months after the conting.
\end{abstract}




\section{Reflectometry}

Papers

9.1. Terms, Definitions and Symbols in Reflectometry.

Deane B. Judd, J. Opt. Soc. Am. 57, No. 4, 445-452, (Apr. 1967).

Key words: Nomenclature; photometry; radiometry; reflectance --_---

9.2. Use of an Auxiliary Sphere with a Spectroreflectometer to Obtain Absolute Reflectance.

David G. Goebel, B. Patrick Caldwell and Harry K. Hammond III, J. Opt. Soc. Am. 56, No. 6, 783-788, (June 1966).

Key words: Absolute reflectance; auxiliary sphere; reflectance; spectrophotometry

9.3. Generalized Integrating-Sphere Theory.

David G. Goebel, Appl. Opt. 6, No. 1, 125-128, (Jan. 1967).

Key words: Absolute reflectance; integrating sphere; reflectance; sphere efficiency

9.4. Effect of Surface Texture on Diffuse Spectral Reflectance: A. Diffuse Spectral Reflectance of Metal Surfaces; H. J. Keegan, J. C. Schleter, and V. R. Weidner. B. Surface Texture Measurement of Metal Surfaces; D. B. Spangenberg, A. G. Strang, and J. L. Chamberlain, Symposium on Thermal Radiation of Solids, San Francisco, Calif. Mar. 4-6, 1964, pp. 165-177 (NASA SP-55, Nat. Aeron. and Space Admin., Washington, D.C. 1965).

Key words : Aluminum; beryllium ; diffuse reflectance ; interferograms; platinum; steel surface finish; surface texture; stylus technique

9.5. Ellipsoidal Mirror Reflectometer.

S. T. Dunn, J. C. Richmond, and J. A. Wiebelt, J. Res. Nat. Bur. Stand. (U.S.) 70C, No. 2, 75-88, (Apr.-June 1966).

Key words: Averaging spheres; bidirectional reflectance; diffuse reflectance; ellipsoidal reflectometer; infrared; infrared detectors; reflectance; reflectometer; spatial sensitivity; spectral reflectance; specular reflectance

9.6. High Purity Powdered CsI as a High Reflectance Infrared Diffuser.

Jon Geist, Gerhart J. Kneissl, and V. R. Weidner, Appl. Opt. 6, No. 7, 1280 (July 1967).

Key words: Cesium iodide; diffusing reflector; hemispherical reflectance factor

9.7. Preparation and Colorimetric Properties of a Magnesium Oxide Reflectance Standard.

Nat. Bur. Stand. (U. S.) Letter Circ. LC1050, 3 pp. (Revised June 1970).

Key words: Diffuse reflectance; CIE illuminants; luminous reflectance; magnesium oxide; chromaticity coordinates 


\section{Reflectometry-Continued}

Abstract

9.8. A Laser-Source Integrating Sphere Reflectometer.

G. J. Kneissl and J. C. Richmond, Nat. Bur. Stand. (U. S.) Tech. Note 439, 69 pp., (Feb. 1968).

Key words: Diffuse reflectance; graphite; high temperature reflectance; infrared reflectance; integrating sphere reflectometer; laser; reflectance; sodium chloride coating; synchronous amplification; thoria; tungsten.............. 


\title{
Terms, Definitions, and Symbols in Reflectometry
}

\author{
DeAne B. JudD* \\ National Bureau of Slandards, Washington, D. C. 20234
}

(Received 7 December 1966)

\begin{abstract}
Angular conditions of incidence are described as hemispherical, conical, or directional; the same adjectives are used to rlescribe the angular conditions of collection. This classification of angular conditions leads to nine kinds of reflectance; symbois for them are proposed in which $2 \pi, g$, and $\theta_{0}, \phi_{0}$ refer to hemispherical, conical, and directional incidence, $2 \pi, g^{\prime}$, and $\theta_{r}, \phi_{r}$ refer to the corresponding kinds of collection. Use of the perfectly reflecting mirror and of the perfectly reflecting diffuser as reference standards in reflectometry is discussed. Three of the nine reflectance ratios, specimen to perfect diffuser, in which the collection is directional have already been named radiance [luminance] factor. It is proposed to differentiate them by angular condition of incidence. It is also proposed to name the other six ratios: reflectance fact or qualified by the same adjectives identifying the type of incidence and collection as are used for reflectance. The interrelationships of these 18 concepts are shown both by formulas for computing one from another and by diagrams indlicating the process (integration, summation, averaging, equality, reflectance of perfect difluser, and reciprocity) by which values of one concept may be computed from those of another.
\end{abstract}

InDEX IIEAIINGS: Photometry; Reflectance; Radionetry.

Fi radiant encrgy is incident on an element of surface at an encrgy-flow rate $\Phi_{0}$, and is reflected by the element of surface at a rate $\Phi_{r}$, the ratio $\Phi_{r} / \Phi_{0}$ is called the reflectance of the surface. In general, this ratio depends on the wavelength and state of polarization of the incident energy. Furthermore, if the detector of radiant flux cmployed has, like the human eye, a spectral sensitivity not independent of wavelength, the ratio of fluxes so evaluated is still called reflectance with in (plalifying adjective (such as luminous, if the detector evaluates the flux in accord with the standard luminous -efficiency function). If, however, attention is confined to incident energy of fixed wavelength composition, or to detectors whose spectral sensitivities are strictly independent of wavelength, there still remain many problems of terminology and symbols. These problems arise because of the various angular conditions of incidence and collection used in reflectometry, and because most reffectometers do not measure the ratio of fluxes directly but employ specular surfaces or diffusing surfaces of known reflection characteristics as standards.

* With the collaboration of C. S. McCamy, J. C. Richmond, L. E. Barbrow, I. Nimeroff, and H. K. Hammond.
In a series of conferences among the staff of the National Bureau of Standards during the summer of 1965 , a set of terms and symbols was developed to indicate the various types of angular conditions used in reflectometry. All of us agreed to use this one set of terms and symbols to facilitate communication among us. These terms and symbols do not contradict those currently being considered in the nomenclature committees of various international bodies (International Commission on Illumination, CIE; International Standards Organization, ISO; International Union of Pure and Applied Plysics, IUPAP) nor of the nomenclature committees of the national bodies, American Standards Association, Optical Society of Anerica, and the Illuminating Engineering Society, but rather are extensions of those concepts and symbols.

The purpose of this paper is to present the NBS terminology and symbols for reflectometry, to give the arguments that shaped them, to give careful definitions of the terms, and to demonstrate the convenience of the symbols by using them to indicate the rather complicated interrelations among the concepts defined. We hope that this extension of accepted terminology, or some revision of it, will find acceptance in the 


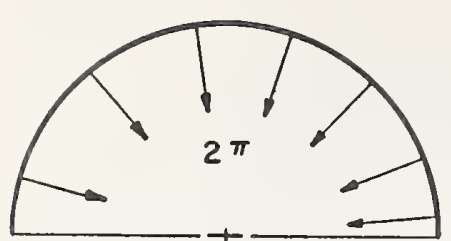

Hemispherical

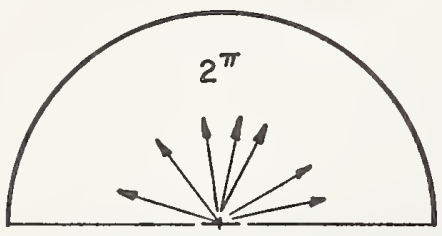

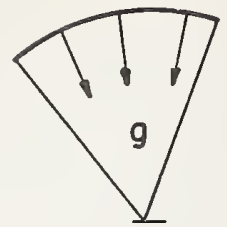

Conical

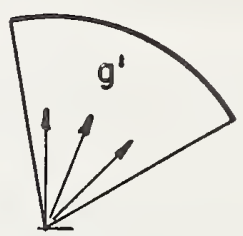

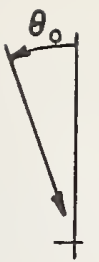

Directional

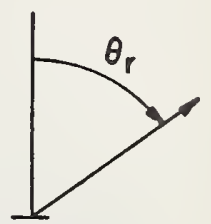

\section{Collection}

FIG. 1. The upper diagrams indicate hemispherical $(2 \pi)$, conical $(g)$, and directional $\left(\theta_{0}\right)$ incidence, respectively, and the lower dia grams indicate hemispherical $(2 \pi)$, conical $\left(g^{\prime}\right)$, and directional $\left(\theta_{r}\right)$ collection. The indications are for a particular pair of azimuths: $\phi_{0}$ and $\phi_{0}+\pi$, for incidence; and $\phi_{r}$ and $\phi_{r}+\pi$, for collection. The arrows in the upper diagrams all point toward the center of the specimen represented by a short horizontal line; those in the lower diagrams all point away from that center.

various national societies concerned with radiative transfer of which the Optical Society of America is one, and eventually also within international standardizing bodies. A preliminary version of these terms and symbols has been under consideration by CIE Committee E-1.1, Nomenclature, since December 1965, and has received active support from the Swedish National Committee of the CIE.

\section{REFLECTANCE}

Our point of departure lies in the customary definition of reflectance as "ratio of reflected radiant flux to incident flux" given, for example, in the book, The Science of Color, by the Committee on Colorimetry of the Optical Society of America. 'I 'Tis definition might be interpreted to mean "ratio of all of the reflected flux to incident flux," but we have not so interpreted it. Instead, we have taken a cue from the note appended to the definition currently being considered by Committee $\mathrm{E}-1.1$ on Nomenclature, International Commission on Illumination:

"20) -065 , Refleclance: Rattio of the reflected radiant or luminous llux to the incident flux."

1 Committee on Colorimetry, Optical Society of Anerica, The Science of Color (New York:'Thonuts Y. Crowell Company, 1953), 1). 37\%. (urrently a vailable from ()ptical Society of Anerica, 1155 Sixtecuth St., N.W., Washington D). (:. 20036.
"Note 1: Where mixed reflection occurs, the (total) reflectance may be divided into two parts, direct reflectance and diffuse reflectance, corresponding, respectively, to the modes of reflection."

Note that the ratio of the flux specularly reflected to the incident flux is still called direct reflectance even though not all of the reflected flux is included. We have accordingly agreed among ourselves to remove this ambiguity by the following more explicit definition of reflectance.

Reflectance: Ratio of some specified portion of the reflected flux to incident flux. Symbol: $\rho$.

Note 1: When qualified by one of the adjectives, hemispherical, conical, or directional, the term refers to all of the reflected flux, to the part within a finite solid angle less than $2 \pi s r$, or confined to essentially one direction, respectively. If no qualifying adjective is used, reflectance for hemispherical collection is meant. The qualifying adjective that specifies the angles of collection may be preceded by the adjective, hemispherical, conical, or directional to indicate whether the incident flux is uniformly distributed over the hemisplere, uniformly distributed over a finite solid angle less thin $2 \pi s r$, or confined to essentially one direction, respectively: 
Kinl of retlectance

Bi-hemisplerical

Hemispherical-conical

Ilemispherical-directional

Conical-hemispherical

Bi-conical

Conical-rlirectional

$\rho\left(g: \theta_{r}, \phi_{r}\right)$

l)irectional-hemispherical

t)irectional-conical

$\rho\left(\theta_{0}, \phi_{0}: 2 \pi\right)$

$\rho\left(\theta_{0}, \phi_{0}: \rho^{\prime}\right)$

[Bi-clirectiona]

$\rho\left(\theta_{0}, \phi_{0}: \theta_{r}, \phi_{r}\right)$
Evaluation of quantity

lor perfect mirror

lior perfect diffuser
Nole 2: 'The symbol $\rho$ may be followed by a iwo-part notation in parenthesis, the two parts being separated by a colon; the first part identifies the angles of incidence; the second, those of collection.

ligure 1 illustrates what is meant by hemispherical, conical, and directional incidence and collection, and Table I lists the terminology and symbols proposed for ninc kinds of reflectance inplicel by these terms. Hemispherical incidence is notated $2 \pi$ for the solid angle sultended by a hemisphere. Conical incidence is notatcel $g$ for the geometry of incidence. If the cone to be sperificd is a right-circular cone, $g$ may be written: $\alpha_{0}, \theta_{0}, \phi_{0}$, where $\alpha_{0}$ is the half-angle of the cone, $\theta_{0}$ and $\phi_{0}$ are angles specifying the direction of its axis; thus $\theta_{0}$ is the angle that the axis of the cone nakes with the normal to the element of surface and may be called zenith angle or polar angle or colatitude; $\phi_{0}$ is the aximuth angle or longitude. Most actual reflectometers irradiate the specinen along angles bounded by a right circular cone. If a reflectometer has more complicated angular conditions of incidence (single cone of noncirculal (ross-section or annular, which is the difference between two cones, or the sum of two or more cones), the symbol $g$ must stand for these angular conditions of incidence, however complicated they may be. For these complicated cases, as well as for the simple cases, $g$ must be explicitly defined. Directional incidence is notated simply by the zenith angle $\theta_{0}$ and the azimuth $\phi_{0}$ of this direction. If the direction of incidence is perpendicular to the surface of the specimen, $\theta_{0}=0$ and no value of azimuth angle exists; then the notation $\left(\theta_{0}, \phi_{0}\right)$ may be abbreviated as $(0)$.

The first column of Table I gives the adjectives by which the nine kinds of reflectance are differentiated. The seccnd column gives the corresponding symbols. Sone idea of the relations between the nine kinds of reflectance is indicated in columns 3 and 4 which give their values for the perfect mirror and the perfect diffuser, respectively

By perfect mirror is meant a surface such that, when irradiated within an elemental solid angle centering on the direction $\theta_{0}, \phi_{0}$ from specimen to source, it reflects all of the incident energy within an elemental solid angle, $d \omega=\sin \theta_{0} d \theta_{0} d \phi_{0}$, centered on the direction $\theta_{r}, \phi_{r}$, where $\theta_{r}=\theta_{0}$ and $\phi_{r}=\phi_{0}+\pi$. For these viewing angles, the bi-directional reflectance of a perfect mirror is unity; but for any other angles, zero. For henispherical collection, the reflectance of the perfect mirror is likewise unity; this is indicated in the third column for bi-hemispherical reflectance, conical-henispherical reflectance, and directional-hemispherical reflectance.

The directional-conical reflectance of a perfect mirror is unity if the direction $\theta_{0}, \phi_{0}$ coincides with any part of the mirrol image of $g^{\prime}$; otherwise it is zero. The conical-directional reflectance is similarly zero if the direction, $\theta_{r}, \phi_{r}$, fitils to coincide with any part of the mirror inage of $g$; otherwise it is $\cos \theta_{r} d \omega^{\prime} / \int_{\omega} \cos \theta_{0} d \omega$, which approathes zero. The bi-conical reflectance of the perfect nirror is the integral of $\cos \theta m \cdot \omega^{\prime}$ for the range of overlap between $g$ and the mirror image of 
TABLE II. Interrelationships among the nine kinds of reflectance.

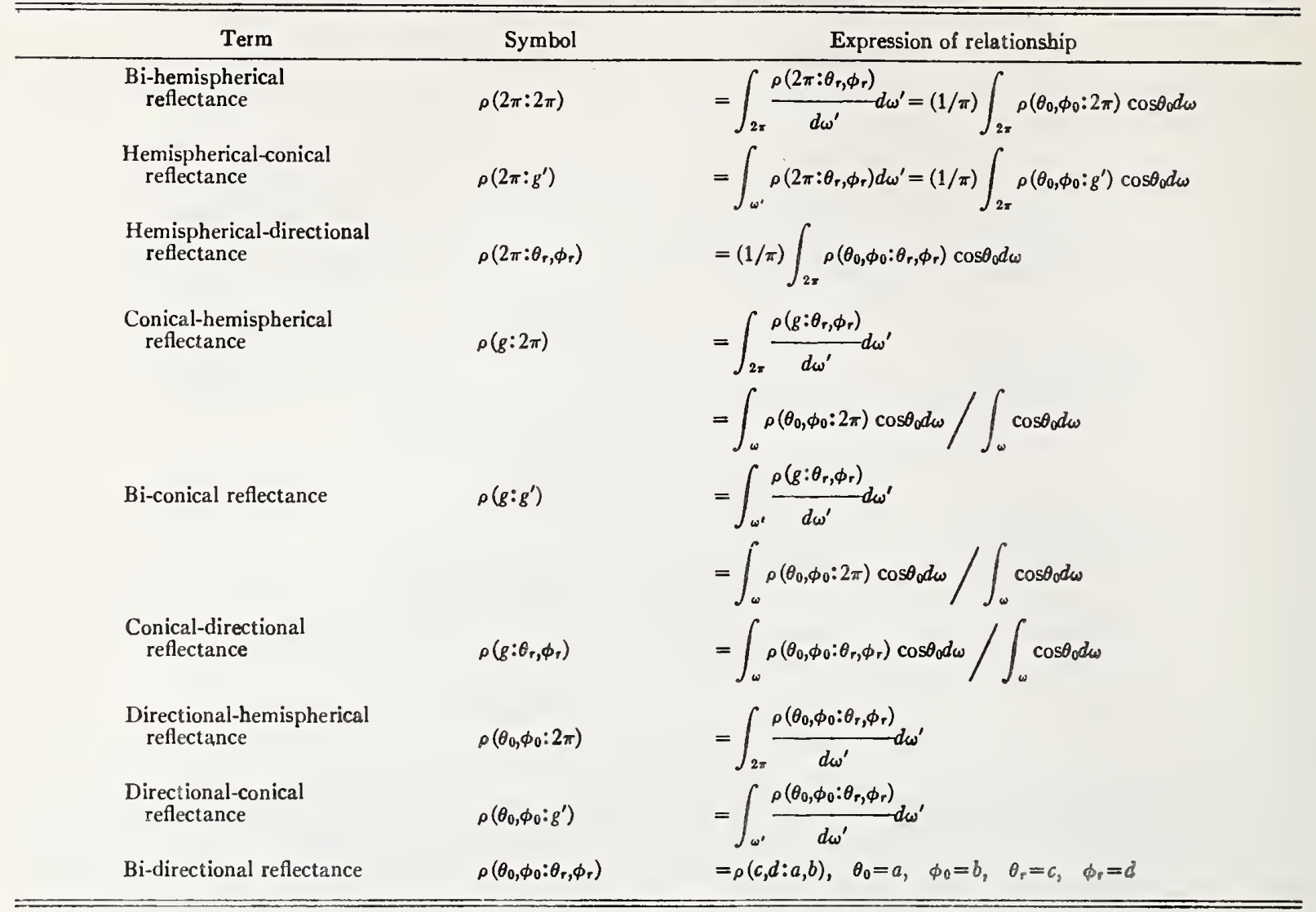

$g^{\prime}$, divided by the integral of $\cos \theta_{0} d \omega$ over the solid angle $\omega$ specified by $g$.

By perfect diffuser is meant a surface that, when irradiated according to any angular mode whatsoever, reflects all of the incident energy in accord with Lambert's law; that is, so that the radiance of the surface is constant, independent of the angle of view. For hemispherical collection the reflectance of the perfect diffuser is therefore unity, and column 4 of Table I shows this for bi-hemispherical, conical-hemispherical, and directional-hemispherical reflectance. Since it is impossible to discriminate between the perfect mirror and the perfect diffuser by any observations with hemispherical incidence, the expressions for bi-hemispherical, hemispherical-conical, and hemispherical-directional reflectances of the perfect diffuser are the same as those for the perfect mirror. Since the perfect diffuser distributes the reflected flux independent of angular conditions of incidence, the three expressions for the reflectance of the perfect diffuser for hemispherical incidence apply to conical and directional incidence also.

If reflectance of the perfect mirror is unity for a given set of angular conditions, the ratio of the flux reflected by any specimen to that reflected by the perfect mirror will be numerically identical to the re- flectance, itself, of the specimen. The perfect mirror thus may serve as a reflectance standard with a value of unity for these kinds of reflectance and actual mirrors may serve as standards with appropriate values less than unity. Table I shows that all kinds of reflectance involving hemispherical collection are eligible as are also some choices of angles for directional incidence. The other four kinds of angular conditions require evaluation of the reflectance of the specular surface used as standard for the particular condition: hemispherical-conical, hemispherical-directional, bi-conical, and conical-directional conditions; on this account mirrors are inconvenient standards for these kinds of reflectance. Only the kinds of conditions for which the mirror surface has zero reflectance, however, are actually ineligible: some choices of conical-directional, directional-conical, and bi-directional.

If reflectance of the perfect diffuser is unity for a given set of angular conditions, the ratio of the flux reflected by any specimen to that reflected by the perfect diffuser will be numerically identical to the reflectance, itself, of the specimen. The perfect diffuser may thus serve as a reflectance standard with a value of unity for these kinds of reflectince. Table 1 shows that these kinds of reflectance are bi-hemispherical, conical-hemispherical, and directional-hemispherical. 
Any diffusing surface may serve as a reflectance standard provided that its reflectance for the particular angular conditions has been cvaluated. For example, magnesiun oxicle is often used as a reflectance standard with the value, unity, assigned because, for a consiclerable wavelength range, it approximates rather cinely the perfect diffuser. Note from Table I that the ratio of reflectance of the perfect mirror to that of the perfect diffuser is unity for five kinds of reflectance, but for conical-elirectional and bi-directional conditions, the ratio is either zero or indefinitely great depending on the choice of angles.

If the bi-rlirectional reflectances of a specimen are known for all angles of incidence and collection, it is possible to compute values of all of the other eight kinds of reflectance of that specimen from those values. from the expressions representing these eight kinds of reflectance as functions of the bi-directional reflectances, relationships among these eight kinds may be derived. Table II gives the most important of these relationships. The bi directional reflectance of a diffusing specimen approaches zero and cannot be measured directly. The ratio $\rho\left(\theta_{0}, \phi_{0}: \theta_{r}, \phi_{r}\right) / d \omega^{\prime}$, however, can be found as the limit of $\rho\left(\theta_{0}, \phi_{0}: g^{\prime}\right) / \omega^{\prime}$ as $\omega^{\prime}$ approaches zero. On the other hand, the bi-directional reflectance of a glossy specimen is a finite number between 0 and 1 . It may be measured by using a source of small solidangular subtense and a detector of equal, or slightly greater, solid-angular subtense so placed as to receive all of the specularly reflected beam. McNicholas ${ }^{2}$ pointed out in 1928 that in any measurement of bidirectional reflectance, by virtue of the Helmholtz reciprocity relation, the same value is obtained by interchanging the souree and detector positions. The last equality in Table II expresses this reciprocity relation. It has sometimes been assumed erroneously that, because of this relation, the value of bi-conical reflectance is likewise unchanged if the source and collection angles are interchanged. The falsity of this assumption becomes conspicuously evident when the solid-angular subtense of $g$ is allowed to approach an elemental solid angle, and that of $g^{\prime}$ to approach $2 \pi$, in which case we have to ask whether the value of directional-hemispherical reflectance, $\rho\left(\theta_{0}, \phi_{0}: 2 \pi\right)$, is made equal to that of hemispherical-directional reflectance, $\rho\left(2 \pi: \theta_{r}, \phi_{r}\right)$, by setting $\theta_{r}$ and $\phi_{r}$ equal to the values previousty assigned to $\theta_{0}$ and $\phi_{0}$. Note, however, that the former is a finite quantity, while the latter approaches zero. If the specimen is the perfect diffuser, the former, as shown in Table $I$, is equal to 1 ; the latter is $(1 / \pi) \cos \theta_{r} d \omega^{\prime}$. There is no reciprocal relation between these two kinds of reflectance.

\section{RADIANCE FACTOR AND REFLECTANCE FACTOR}

Although the motive for measuring the ratio of the flux reflected by the specimen to that which would be

\footnotetext{
${ }^{2}$ H. J. McNicholas, J. Res. Natl. Bur. Std. (U. S.) 1, 29 (1928).
}

reflected into the same solid angle with the same conditions of incidence by an approximation to the perfect diffuser is often to obtain an evaluation of the reflectance, this ratio is important in its own right and has received the name radiance factor (symbol $\beta$ ) whenever the collection is accomplished within an elemental solid angle. The following definition of radiance [luminance] factor is that currently given by Committee E-1.1 on Nomenclature, International Commission on Illumination (CIE); but we have added the notes.

Radiance [luminance] factor, (at a given point on the surface of a non-self-radiating body, in a given direction, under specified conditions of irradiation): ratio of the radiance [luminance], in that direction, of the body to that of a perfect reflecting diffuser identically irradiated [illuminated]. Symbol: $\beta$.

Note 1: When qualified by the adjective, hemispherical, conical, or directional, radiance factor refers to incident flux uniformly distributed over the hemisphere, uniformly distributed over a finite solid angle less than $2 \pi s r$, or confined to essentially one direction, respectively.

Note 2: The symbol $\beta$ may' be followed by a two-part notation in parenthesis, the two parts being separated by a colon; the first part identifies the angles of incidence; the second, the angle of view. These notes provide names and symbols for three of the nine ratios of flux reflected by the specimen to that reflected by the perfect diffuser: hemispherical radiance factor, conical radiance factor, and directional radiance factor. If the collection is over a greater than elemental solid angle, the name radiance factor is not appropriate. We propose the name reflectance factor for these ratios as follows:

Reflectance factor (at a point of a surface, for the part of the reflected radiation contained in a given cone, and for incident adiation of given spectral and geometric distributions):

Ratio of the radiant flux reflected in the directions delimited by the cone to that reflected in the same directions by a perfect reflecting diffuser identically: irradiated. Symbol: $R .^{3}$

Note 1: The cross-section of the cone need not be circular nor even boundable by a single closed curve. It may, for example, be annular, or what in the ordinary restricted meaning of the term, cone, would be described as two or more cones.

Note 2: If the solid angle of the cone approaches zero, or $2 \pi s r$, the reflectance factor approaches radiance factor $\beta$, or reflectance for hemispherical collection, respectively.

Note 3: The adjectives, hemispherical, conical, and directional recommended for reflectance to designate the geometrical modes of incidence and collection are also appropriate for reflectance factor; and the corresponding notations: $2 \pi, g$, or $\theta_{0}, \phi_{0}$, respectively, are appropriate for use in parentheses following the symbol,

${ }^{3}$ C. S. McCamy, Phot. Sci. Fing. 10, 314 (Nov.-Dec. 1966). 

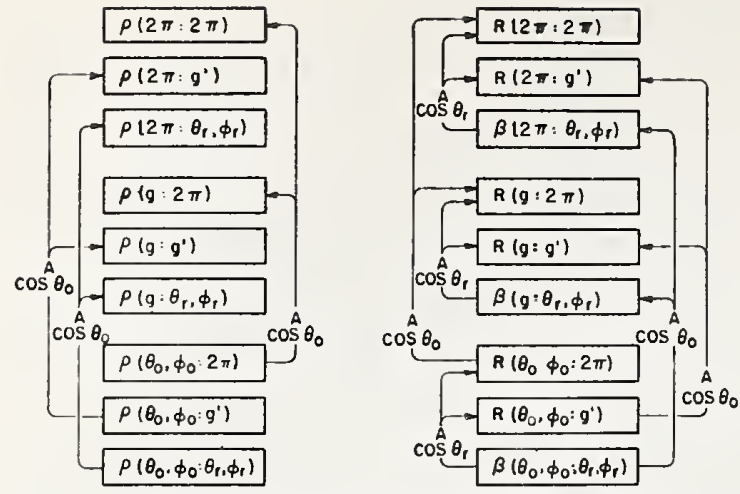

Fig. 2. The symbols refer to the nine kinds of reflectance $\rho$, the six kinds of reflectance factor ( $R$ with finite solid-angular subtense of collection), and the three kinds of radiance factor ( $\beta$ with elemental solid-angular subtense of collection). Jach arrow starts from the symbol for the concept whose average value corresponds (1) another of the concepts, and the arrow head indicates the symbol for that concept. In the labeling of the arrows, $A$ stands for average, and the appropriaie weighting factor (either $\cos \theta_{0}$ or $\cos \theta_{r}$ ) is also shown. All six kinds of reflectance faciors can be expressed as averages of directional radiance factor; but only two of the eight other kinds of reflectance can be expressed as averages of bi-directional reflectance.

$R$, with the notation for incidence preceding a colon, and that for collection: $2 \pi$, or $g^{\prime}$, following it.

Note 4: If the directions of incidence are confined to a solid angle $\omega$ not approaching $2 \pi s r$, and the cone of collection has a solid angle $\omega^{\prime}$ not approaching $2 \pi s r$, the term, bi-conical reflectance factor, is appropriate. The relation between bi-conical reflectance factor and conical-hemispherical reflectance is as follows: If several choices of the cone of collection are made such that no two of the cones overlap and such that together they sum to $2 \pi s r$, then the appropriately weighted average of the several bi-conical reflectance factors is numerically equal to the conical-hemispherical reflectance for the same incidence.

By dividing each of the expressions in Table II by their values for the perfect diffuser, given in Table I, it is possible to derive expressions indicating the chief interrelationships among the six kinds of reflectance factor and the three kinds of radiance factor, and also their relationships to the various kinds of reflectance. Table III gives the most important of these expressions. Many of these relationships have already been published. McNicholas ${ }^{2}$ published the first one listed in Table II, the relationship between bi-hemispherical reflectance, $\rho(2 \pi: 2 \pi)$, and directional-hemispherical reflectance, $\rho\left(\theta_{0}, \phi_{0}: 2 \pi\right)$; he also published the first one listed in Table III, between bi-hemispherical reflectance, $\rho(2 \pi: 2 \pi)$, and hemispherical radiance factor, $\beta\left(2 \pi: \theta_{r}, \phi_{r}\right)$, and the one listed third from the bottom in 'Table 111 , that between directional-hemisplerical reflectance, $\rho\left(\theta_{0}, \phi_{0}: 2 \pi\right)$, and directional radiance factor, $\beta\left(\theta_{11}, \phi_{11}: \theta_{r}, \phi_{r}\right)$. Brandenberg and $\mathrm{Neu}^{4}$ have recently

' W. M. Brandenlerg and J. T. Neu, J. Opt. Soc. Am. 56, 97 $(19)(3))$. drawn attention again to this last relationship. Doubtless others of these relationships have also already been published; the multiplicity of terms used to refer to them discourages a literature search. The derivations are all straight-forward, but without systematic terminology it has been very hard for an author to communicate what it is that he has derived. It will be noted from Table III that, without exception, these expressions correspond to averaging either with $\cos \theta_{0}$ or with $\cos \theta_{r}$ as the weighting factor. To compute hemispherical and conical reflectance factor from radiance factor the weighting factor is $\cos \theta_{r}$. To pass from directional incidence to conical and hemispherical incidence requires, of course, the weighting factor, $\cos \theta_{0}$. These twelve averages are shown diagrammatically by arrows in Fig. 2. The notation $A$ refers to average; below this the weighting factor is given. Figure 2 also shows in a similar way the kinds of reflectance that are connected by weighted averages, but note that there are only six such averages, those with weighting factors of $\cos \theta_{r}$.

Figure 3 shows most of the other interrelations among the nine kinds of reflectance, the six kinds of reflectance factor, and the three kinds of radiance factor. To pass from reflectance to reflectance factor or radiance factor for the same angular conditions of incidence and collection, and the reverse, requires application of a factor equal to the reflectance of the

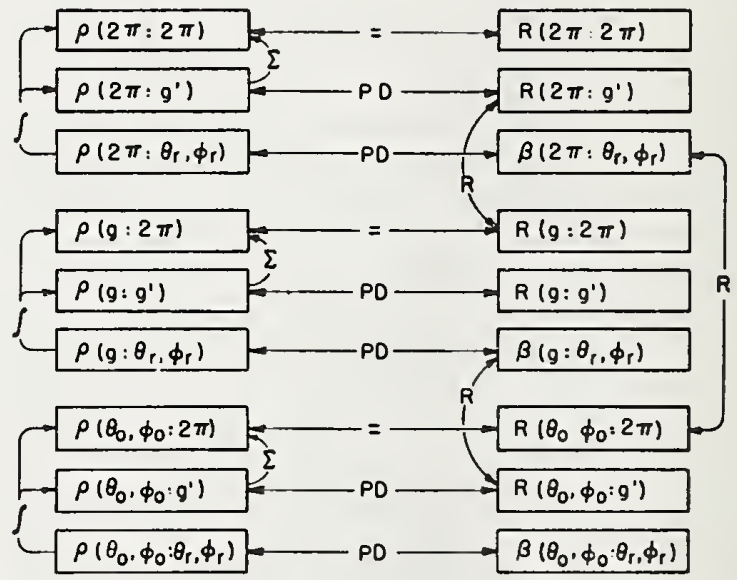

FIG. 3. Symbols shown are the same as those in Fig. 2. The arrows, like those in Fig. 2, pass from the symbols for concepts whose values may be processed so as to compute a value for the concept whose symbol is indicated by the arrow head. The arrows are labeled to indicate the process of computation: integral sign for integration, sumnation sign for summation, equal sign for numerical equality, $P D$ for multiplication or division by the reflectance of the perfect diffuser, and $R$ for numerical equivalents that, because of the Helnulit\% reciprocity relition, hold when the angular conditions of incidence and collection are interchanged. There exist three reciprocal relations that cannot be shown by this diagrammatic method:

$$
\begin{gathered}
\rho\left(\theta_{0}=m, \phi_{0}=n ; \theta_{r}=u, \phi_{r}=r^{\prime}\right)=\rho\left(u, s^{\prime}: m, n\right) \\
\beta\left(\theta_{0}=m, \phi_{0}=n ; \theta_{r}=u, \phi_{r}=s^{\prime}\right)=\beta\left(u, s^{\prime}: m, n\right) \\
\kappa^{\prime}\left(g^{\prime}=u: g^{\prime}=b\right)=\kappa^{\prime}(b: a) .
\end{gathered}
$$

The first of these three is the only reciprocul relation among the kinds of rellectance. 
TABLE III. Interrelationships among the six kinds of reflectance factor, the three kinds of radiance factor, and the nine kinds of reflectance.

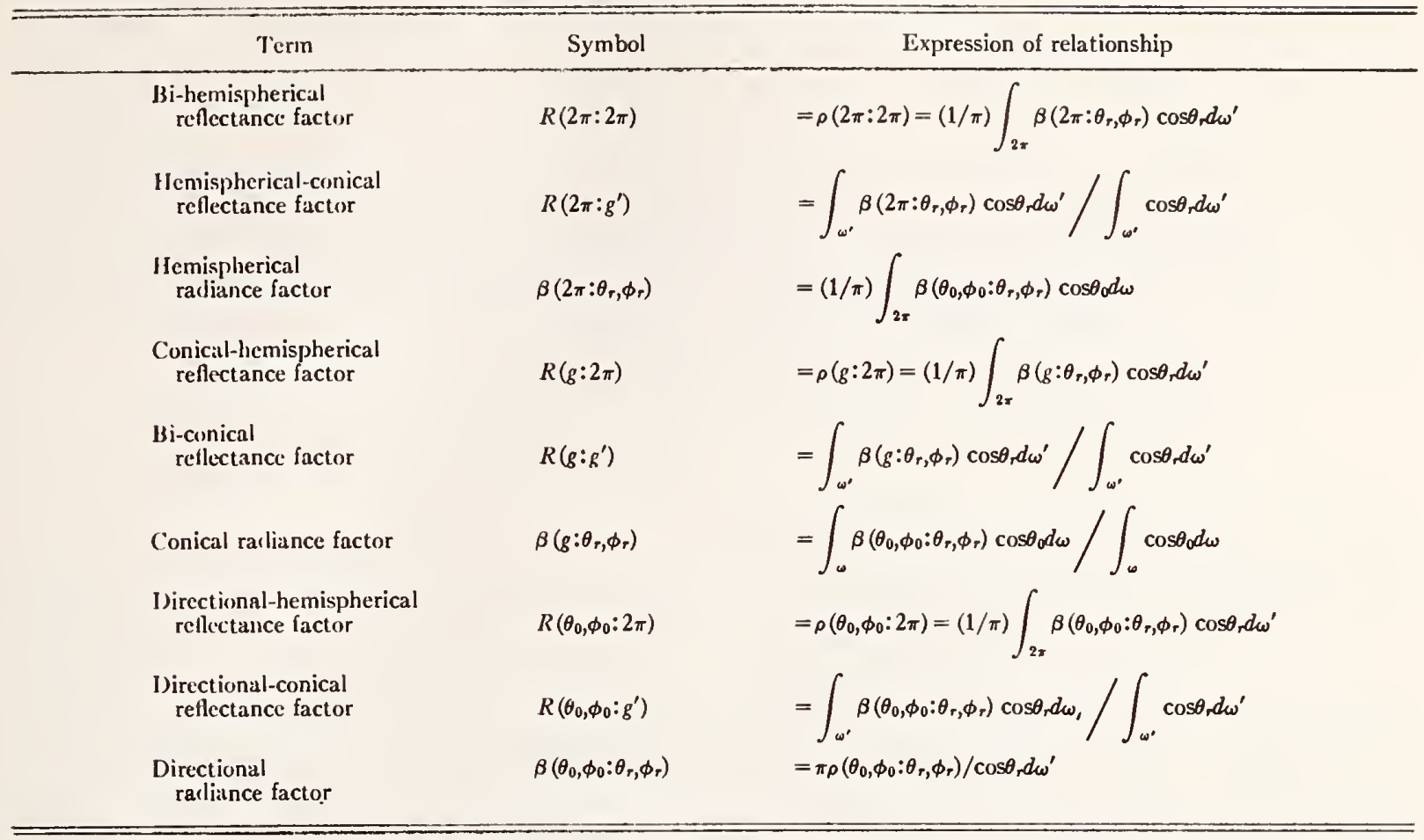

perfect diffuser for those angular conditions; see Table 1. Since, for hemispherical collection, the perfect diffuscr has a reflectance of $100 \%$, the relationship is that of numcrical equality; this is indicated on Fig. 3 by a double-headed arrow marked with an equal sign. For the other two types of collection, the double arrow is markel with " $P D$ " for perfect diffuser. For conical collection the fiuctor is $(1 / \pi) \int_{\omega^{\prime}} \cos \theta_{r} d \omega^{\prime}$; and for directional collection the factor is $(1 / \pi) \cos \theta_{r} d \omega^{\prime}$, as shown in Table 1. To pass from reflectance for directional collection to that for concal and hemispherical collection, requires as indicaled in Table II, integration. This is indicated in lig. 3 by single-headed arrows marked with an integral sign. To pass from reflectance for conical collection to that for hemispherical collection requires summation for the same choices of solid angles as are indicated in note 4 to the definition of reflectance factor. These three relationships are indicated on Iig. 3 by single-headed arrows marked with a summation sign. The corresponding relationships for reflectance factor are averages, but, to prevent Fig. 2 from becoming too confusing, arrows for these relationships have been left off of Fig. 2.

Although the Helmholtz reciprocity relation applies in reflectance only to the bi-directional angular condition, there are three pairs of such relations involving reflectance factor and radiance factor, indicated on Fig. 3 by double-hearled arrows marked with $R$ for reciprocity. I'or example, onc double-headed arrow indicates that conical radiance factor $\beta\left(g: \theta_{r}, \phi_{r}\right)$ of any specimen is numerically the same as directional-conical reflectance factor $R\left(\theta_{0}, \phi_{0}: g^{\prime}\right)$ of that specimen, provided that $g=g^{\prime}, \theta_{0}=\theta_{r}$, and $\phi_{0}=\phi_{r}$. Table III shows that making these substitutions in either of the expressions makes it identical to the other. Similarly, the numerical identity of hemispherical radiance factor $\beta\left(2 \pi: \theta_{r}, \phi_{r}\right)$ and directional-hemispherical reflectance factor $R\left(0_{0}, \phi_{0}: 2 \pi\right)$ provided $\theta_{0}=\theta_{r}$ and $\phi_{0}=\phi_{r}$ is obvious by inspection of Table III. The other reciprocal relation indicated in Fig. 3, that between hemisphericalconical rellectance fictor $R\left(2 \pi: g^{\prime}\right)$ and conical-hemispherical reflectance factor $R(g: 2 \pi)$ cannot be seen by inspection of Table III, because the expression for the former is in terms of $\beta\left(2 \pi: \theta_{r}, \phi_{r}\right)$, while the latter is in terms of $\beta\left(g: \theta_{r}, \phi_{r}\right)$; but substitution of the expressions given in Table III for these kinds of radiance factor in terms of directional radiance factor $\beta\left(\theta_{0}, \phi_{0}: \theta_{r}, \phi_{r}\right)$ readily shows the two to be equivalent provided that $g=g^{\prime}$. A similar substitution shows that $R\left(g=a: g^{\prime}=b\right)=R(b: a)$. McNicholas ${ }^{2}$ has already drawn attention to the reciprocal relation, shown in Fig. 3, between hemispherical radiance factor, $\beta\left(2 \pi: \theta_{r}, \phi_{r}\right)$, and directional-hemispherical reflectance factor, $R\left(\theta_{0}, \phi_{0}: 2 \pi\right)$. The numerical equality, shown in Fig. 3, between the latter and directional-hemispherical reflectance, $\rho\left(\theta_{0}, \phi_{0}: 2 \pi\right)$, permitted McNicholas to write:

$$
\rho\left(0_{0}, \phi_{0}: 2 \pi\right)=\beta\left(2 \pi: \theta_{r}, \phi_{r}\right)
$$

for $\theta_{r}$ and $\phi_{r}$ set equal to the values previously assigned to $\theta_{0}$ and $\phi_{0}$, respectively. 


\section{SUMMARY}

Names and symbols have been proposed for nine kinds of reflectance corresponding to the nine principal kinds of angular conditions. Names (reflectance factor, and radiance factor, with modifiers like those used for reflectance) and corresponding symbols have also been proposed for the ratios of the nine kinds of reflectance to that of the perfect diffuser. The convenience of the symbols has been demonstrated by using them to indicate the rather complicated, and little appreciated, relationships among the eighteen concepts defined (nine kinds of reflectance, six kinds of reflectance factor, and three kinds of radiance factor); see Tables II and III. These relationships have also been classified as integrals, summations, averages, ratio to perfect diffuser, and reciprocity relations, and have been shown diagrammatically in Figs. II and III. We submit that all eighteen of these concepts are important in reflectometry, and will eventually be given suitable names and symbols. Our proposal is a systematic extension of existing terminology and is presented as a suggestion to authors and nomenclature committees dealing with reflectometry.

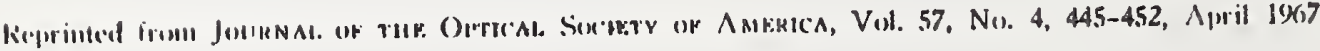




\title{
Use of an Auxiliary Sphere with a Spectroreflectometer to Obtain Absolute Reflectance
}

\author{
David G. Goebel, B. Patrick Caldwell, and Harry K. Hammond, III \\ Photometry E Colorimetry Section, National Bureau of Standards, W'ashington, D. C. 2023.
}

(Received 27 November 1965)

\begin{abstract}
Reflectance measurements that are made on a scale that is not relative to an arbitrary standard are often called "absolute" measurements. The method presented here uses an auxiliary sphere with a double-beam integrating-sphere spectrophotometer to make measurements on an absolute basis. The basic requirements are: (1) The auxiliary sphere must be uniformly coated with a highly-reflecting, highly-diffusing material; (2) a flat plate must be coated in an identical manner to provide a measure of reflectance of the coating;

(3) the interior-surface area of the sphere and the area of the entrance port must be measured.

The theory of the method is discussed and an error analysis is made. Reflectance data are reported for specimens of smoked $\mathrm{MgO}$ and pressed powders of $\mathrm{MgO}$ and $\mathrm{BaSO}_{4}$.

The precision of repeatability has been evaluated from measurements of a Vitrolite reference standard. More than a dozen measurements at each of eight wavelengths made over a 3-year period exhibited a standard deviation of 0.003 for the spectral reflectance.
\end{abstract}

Index Headings: Reflectance; Spectrophotometry.

\section{INTRODUCTION}

$\mathrm{S}^{\mathrm{P}}$ PECTRAL-REFLECTANCE measurements made on an "absolute" scale by use of an auxiliary sphere were described by Van den Akker and associates in 1956. ${ }^{1}$ Few laboratories have attempted to use the Van den Akker method, however, because it required a demountable auxiliary sphere equipped with 13 removable disks which had to be individually measured to obtain the average reflectance of the sphere coating and an index of its uniformity. If it were certain that the coating is uniform, measurement of only one disk would be sufficient. If the coating technique is sufficiently well controlled so that the reflectance of a separate flat plate, coated at the same time as the sphere, is essentially identical, then a removable disk is not required.

\section{THEORY}

Spectrophotometers designed for reflectance measurements are generally provided with integrating spheres to collect reflected flux. Two basic optical designs are used for specimen irradiation: double-beam and singlebeam. ${ }^{2}$ Only the double-beam design permits making absolute reflectance measurements with a high degree of accuracy by utilizing an auxiliary sphere with a single circular opening (See Fig. 1). When a given quantity of flux enters a sphere (or any enclosure) a certain fraction is reflected out through the opening. For a sphere with a uniform and perfectly diffusing coating, the "effective" reflectance of the opening due to multiple reflections of the flux within the sphere can be calculated from the measured reflectance of the coating and the areas of the sphere and the opening. ${ }^{1,3}$ In analyzing the exchange of flux between the instrument sphere and the

${ }^{1}$ J. A. Van den Akker, L. R. Dearth, and W. M. Shillcox, J. Opt. Soc. Am. 46, 378A (1956); 56, 250 (1966).

${ }^{2}$ Also referred to as the comparison mode, and the substitution mode, respectively.

${ }^{3} \mathrm{~J}$. A. Jacques and H. F. Kuppenheim, J. Opt. Soc. Am. 45, 460 (1955) auxiliary sphere, the important concept to keep in mind is that the opening of the auxiliary sphere appears the same to the measuring sphere as does a flat, diffusely reflecting specimen.

An integrating-sphere reflectometer can be used to measure the ratio of two reflectances. If the auxiliary sphere has as part of its wall a removable flat plate, then the ratio of the reflectance of the opening of the intact auxiliary sphere to the reflectance of the coating on the flat plate can be measured. From this ratio and the geometry of the sphere, the reflectance of the coating can be obtained on an absolute scale. The reflectance of the coating can then be used to obtain the reflectance of a reference standard of permanent material.

The effective reflectance of a port in a sphere $\rho_{s}$ is computed from the reflectance of the wall $\rho_{F}$ and the ratio $f$ of the area of the port to the area of the interior of the entire sphere, by using the following equation ${ }^{3}$ :

$$
\rho_{s}=\rho_{F} \quad\left[\left(1-\rho_{F}\right)(1-f)\right] .
$$

The spectrophotometer reading proportional to the ratio of the reflectance of the auxiliary sphere and a comparison specimen is

$$
Q_{s}=K\left(\rho_{s} / \rho_{c}\right) .
$$

The reading for the ratio of the removable plate to the

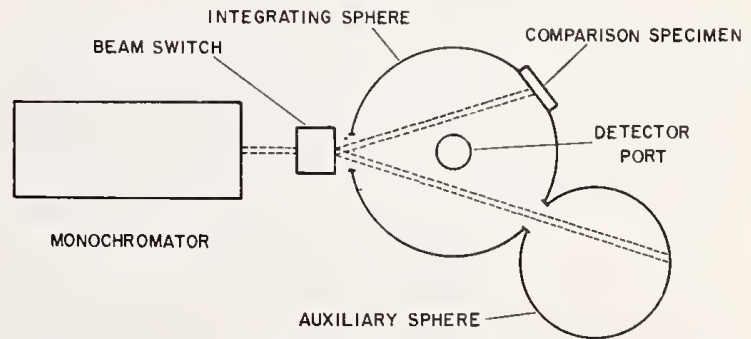

FIG. 1. Schematic diagram of double-beam spectrophotometer with integrating sphere and auxiliary sphere attached. 
Sphere coating reflectance $\rho_{F}$

Corresponding instrument reading $Q_{s}$
0.80

0.02
0.85

0.90

0.06

0.09

0.11

0.14

0.94

0.96

0.98

0.99

1.00

- Reading above one is obtained because comparison reflectance $\rho_{c}$ is assumed to be 0.95 .

comparison specimen is

$$
Q_{F}=K\left(\rho_{F} / \rho_{c}\right) .
$$

The ratio of $Q_{s}$ to $Q_{F}$ is

$$
Q_{s} / Q_{F}=f /\left[\left(1-\rho_{F}(1-f)\right]\right. \text {. }
$$

Therefore,

$$
\rho_{F}=(1-f)\left(Q_{F} / Q_{s}\right) /(1-f) .
$$

Note that Eq. (3) for $\rho_{F}$ is the same as the equation for $R_{d d}$ derived by Van den Akker. ${ }^{1}$ The ratio of the area on the sphere occupied by the port $A_{P}$ to the area of the sphere $A_{s}$ is

$$
f=A_{P} / A_{s}=1 / 2\left[1-\left(1-(r / R)^{2}\right)^{\frac{1}{2}}\right],
$$

where

$$
\begin{aligned}
& A_{P}=2 \pi R h \\
& A_{s}=4 \pi R^{2},
\end{aligned}
$$

in which $h$ is the height of the spherical segment, $h=R-\left(R^{2}-r^{2}\right)^{\frac{1}{2}}, r$ equals the radius of the port $A_{P}$, and $R$ equals the radius of the sphere.

There are several important implications in the foregoing equations. Of particular importance is the manner in which $\rho_{F}$ affects the value of $Q_{s}$.

$$
Q_{s}=K\left(\rho_{F} / \rho_{c}\right): f /\left[\left(1-\rho_{F}(1-f)\right] .\right.
$$

To obtain a reading $Q_{s}$ greater than 0.10 , it is necessary for the auxiliary-sphere coating to have a reflectance $\rho_{F}$ greater than 0.90 . See Table I for the effect of $\rho_{F}$ on $Q_{s}$ with $f=0.01, K=1.0$, and $\rho_{c}=0.95$. The effect of variation in the area ratio $f$ is shown in Fig. 2. To determine $Q_{\delta}$ for small values of $f$ different from 0.01 simply multiply the values for $Q_{s}$ in Table I by $f / 0.01$, neglecting the change in the $\rho_{F}(1-f)$ term; for example, if $f=0.007$, multiply $Q_{s}$ by 0.7 .

\section{ERROR ANALYSIS}

The uncertainty in $\rho_{F}$ is due to the uncertainties in the measured values in $Q_{F}, Q_{\theta}$, and $f$. The general equation for the uncertainty in a computed result $Z$ where the independent random variables $X_{i}$ are uncertain by
$\Delta X_{i}$ is given approximately by ${ }^{4}$

$$
(\Delta Z)^{2}=\sum_{i}\left(\frac{\partial Z}{\partial X_{i}} \Delta X_{i}\right)^{2} .
$$

The uncertainty in $\rho_{F}$ is therefore,

$$
\begin{aligned}
\left(\Delta \rho_{F}\right)^{2}=\left(\frac{1-Q_{F} / Q_{s}}{(1-f)^{2}} \Delta f\right)^{2}+\left(\frac{-f \Delta Q_{F}}{Q_{s}(1-f)}\right)^{2} & \\
& +\left(\frac{f Q_{F} \Delta Q_{s}}{Q_{s}{ }^{2}(1-f)}\right)^{2} .
\end{aligned}
$$

As an example, for $2.54-\mathrm{cm}$-diam hole in a $15.25-\mathrm{cm}$ diam sphere, $f$ has a value of approximately 0.007 . Assume that this value can be determined to an accuracy of $1 \%$. Assume further than $Q_{F}$ equals 0.99 and $Q_{s}$ equals 0.25 , yielding a value of 0.98 for $\rho_{F}$ from $\mathrm{Eq}$. (3). If $Q_{F}$ and $Q_{s}$ are each uncertain by \pm 0.002 , then the uncertainty in $\rho_{F}$ from Eq. (6) is

$$
\begin{gathered}
\left(\Delta \rho_{F}\right)^{2}=4.3 \times 10^{-8}+0.32 \times 10^{-8}+5.0 \times 10^{-8} \\
\Delta \rho_{F}=0.00031 .
\end{gathered}
$$

The uncertainty in $\rho_{F}$ can therefore be seen to be highly insensitive to the uncertainties in $Q_{F}, Q_{s}$, and $f$ for the above example. However, the uncertainty in the determination of $\rho_{F}$ does depend strongly on its magnitude. Take for example, a sphere with values of $f=0.007, \rho_{F}=0.90, Q_{F} \simeq 0.95$, and $Q_{s} \simeq 0.06$ ( $Q_{s}$ from Table I multiplied by 0.7 ) and with the same uncertainties assumed previously,

$$
\left(\Delta \rho_{F}\right)^{2}=1.1 \times 10^{-6}+5.5 \times 10^{-8}+13.8 \times 10^{-6},
$$

so that

$$
\Delta \rho_{F}=0.0039 .
$$

Note that a decrease in $\rho_{F}$ from 0.98 to 0.90 causes the uncertainty in its determination to increase by more than a factor of ten. Therefore, to obtain the most accurate value of $\rho_{F}$, the auxiliary-sphere coating should be as highly reflecting as possible; this requires the use of such coating materials as magnesium oxide, magnesium carbonate, or barium sulfate.

4 D. C. Baird, Experimentation: An Introduction to Measurement Theory and Experiment Design (Prentice-Hall, Inc., Englewood Cliffs, N. J., 1962). 
A more informative form of $\mathrm{Eq}$. (6) is oblatined by substituting licl. (2) for ()$_{s} /()_{F}$.

$$
\begin{aligned}
&\left(\Delta \rho_{F}\right)^{2}=\left(-\frac{1-\rho_{F}}{1-f} \frac{\Delta f}{f}\right)^{2}+\left(\frac{1-\rho_{F}(1-f)}{(1-f)} \frac{\Delta Q_{F}}{Q_{F}}\right)^{2} \\
&+\left(\frac{1-\rho_{H}(1-f)}{(1-f)} \frac{\Delta Q_{H}}{Q_{s}}\right)^{2} \\
& \simeq\left(\frac{1-\rho_{F}(1-f)}{1-f}\right)^{2}\left[\left(\frac{\Delta f}{f}\right)^{2}+\left(\frac{\Delta Q_{F}}{\rho_{F}}\right)^{2}+\left(\frac{\Delta Q_{s}}{Q_{*}}\right)^{2}\right], \\
& \text { for } f<0.04 .
\end{aligned}
$$

The percentage error in $\rho_{F}$ is therefore proportional, to a good approximation, to the square root of the sum of the squares of the percentage errors in the measurements,

$$
\underset{\rho_{F}}{\Delta \rho_{F}} \simeq \frac{\left(1-\rho_{F}\right)(1-f)}{\rho_{F}(1-f)} \Phi
$$

where

$$
\Phi=\left[\left(\frac{\Delta f}{f}\right)^{2}+\left(\frac{\Delta Q_{F}}{Q_{F}}\right)^{2}+\left(\frac{\Delta Q_{s}}{Q_{s}}\right)^{2}\right]^{\frac{1}{3}} .
$$

The dependence of the percentage error in $\rho_{F}$ on the magnitude of $\rho_{F}$ is well illustrated in a graph of Eq. (7)

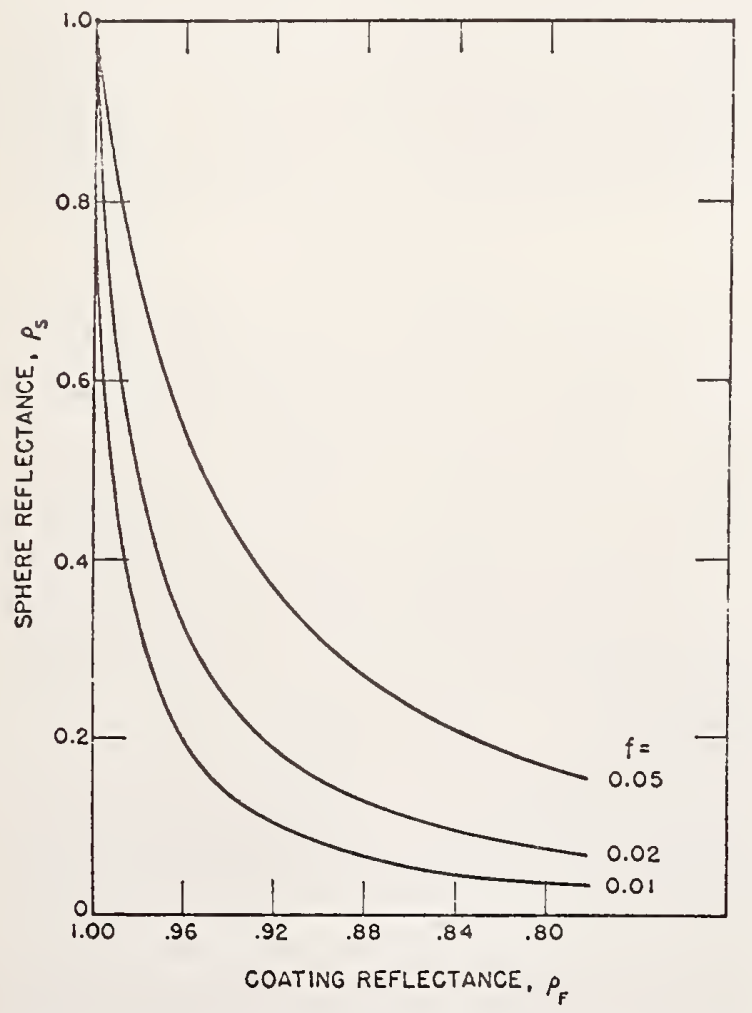

FIG. 2. Auxiliary-sphere refectance as a function of coating reflectanee for three values of $f$, the ratio of the area of the port to the area of the entire sphere.

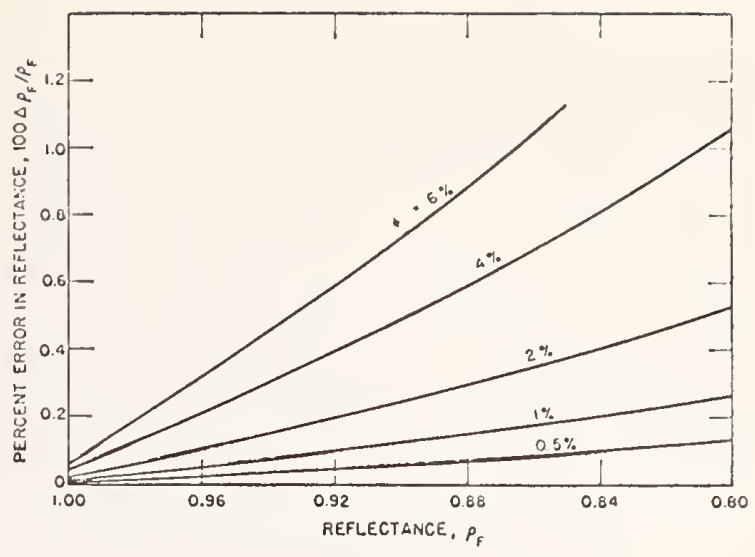

Fig. 3. Curves showing the percentane error in reflectance $100 \Delta \rho_{k} / \rho_{F}$ for various pereentarre errors in $/, Q_{F}$, and $Q_{n}$, computed from Eq. (7) for values of $\$$ equal to $0.5,1,2,4$, and $6 \%$ and for $f=0.01$.

where $\Delta \rho_{F} / \rho_{F}$ vs $\rho_{F}$ is plotted for $f=0.01$ and for various values for the square root of the sum of the sifuares of the percentage errors in the three measured parameters. See Fig. 3. The graph indicates that the percentige error in $\rho$ decreases rapidly with increasing reflectance even though the errors in the measured parameters are several percent.

The graph can also be used to determine the percentage error in $\rho_{F}$ for other values of $f$ which are slightly different from 0.01 .

If $f=0.01+\epsilon$, then Eq. (7) yields

$$
\Delta \rho_{F} / \rho_{F}=\left[\left(1-\rho_{F}\right)(1-0.01-\epsilon) / \rho_{F}(1-0.01-\epsilon)\right] \Phi .
$$

Thus,

$$
\begin{aligned}
\Delta \rho_{F} / \rho_{F} & \simeq\left[\left(1-\rho_{F}\right)(1-0.01) / \rho_{F}(1-0.01)+\epsilon\right] \Phi \\
& \simeq\left[\left(1-\rho_{F}\right)(1-0.01) / \rho_{H}(1-0.01)\right] \Phi+\epsilon \Phi,
\end{aligned}
$$

where the first term can be obtained from Firg. 3 and the second term is a small correction to this term. For example, if $f=0.005$, then $\epsilon=-0.005$. For $\rho_{F}=0.92$ and $\Phi=2 \%, \Delta \rho_{F} / \rho_{F} \simeq 0.002-0.005(0.02) \simeq 0.19 \%$.

Use of Eq. (4) shows that the percentage error in $f$ is approximately equal to twice the percentage error in $r$, the radius of the port, for

$$
\Delta f / f \simeq 2\left[(\Delta r / r)^{2}+(\Delta R / R)^{2}\right]^{2} .
$$

Since the percentage error in the determination of the radius of the sphere is likely to be an order of magnitude smaller than the percentage error in the ratius of the port, $\Delta f / f \simeq 2(\Delta r / r)$.

The theory of the integrating spinere is based on two assumptions concerning the coatling: (1) periect diffusion, and (2) periect uniformity of reticeiturec at all points on the surface of the splere. In achatiat, no surface is perfectly difiusing, and the rethectime of the sphere coating is likely to be somcwhat nomuniform. The uniformity of reflectance of a sphere coltibis should be tested, particularly smoked $\mathrm{MgO}$ coatings beciuse of the 
difficulty of depositing a uniformly thick layer of $\mathrm{MgO}$ in a sphere. To evaluate uniformity, Van den Akker, Dearth and Shillcox ${ }^{3}$ used a sphere with 13 disks which they removed for measurement to provide a representative sampling of the reflectance of the sphere coating. The effects on $\rho_{F}$ of lack of diffusion and lack of uniformity of the coating are difficult to evaluate theoretically, but our experience indicates that they may be the principal causes of the large discrepancies sometimes encountered in replicate determinations of absolute reflectance. Careful experimental technique in coating the sphere minimizes errors from these sources.

\section{EXPERIMENTAL METHODS}

All of the reflectance measurements with an auxiliary sphere were made on a Hardy-type spectrophotometer operated by the Spectrophotometry Group at NBS. Most of the measurements have been made from 0.4 to $0.75 \mu \mathrm{m}$; a few measurements have been made to 1.08 $\mu \mathrm{m}$. An auxiliary sphere with thirteen removable disks following the design of Van den Akker was measured in May 1962. The sphere was coated with $\mathrm{BaSO}_{4}$, approximately 1-mm thick, applied by spraying a suspension of the powder in alcohol without any binder. Considerable difficulty was encountered, however, in coating the sphere, in positioning it for measurement, and in removing the disks for measurement. Most of our measurements were made by using a 15 -cm-diam sphere with one removable plate since measurement of the thirteen disks indicated that the coating was uniform in reflectance to within approximately $\pm 0.2 \%$.

One 15 -cm-diam sphere was precision-made by machining hemispheres from blocks of aluminum to a tolerance of approximately $0.002 \mathrm{~cm}$ on the internal sphere diameter and the entrance-port diameter. Six other spheres were made from spun-aluninum hemispheres; three had nominal $10-\mathrm{cm}$ diameters and three had nominal $15-\mathrm{cm}$ diameters. All of these spheres were supplied with $2.54-\mathrm{cm}$-diam entrance ports and with separate flat plates to be coated simultaneously with the spheres.

Over the past three years twenty-two separate measurements were made utilizing various auxiliary spheres. Eight sets of data were discarded as being self-contradictory, the primary cause probably being nonuniformity in coating reflectance. Three of the discarded sets of data were obtained with a sphere coating of commercial, diffuse-white paint which had a reflectance of less than 0.90. The five other discarded sets of data were determined with spheres coated with $\mathrm{BaSO}_{4}$ or $\mathrm{MgO}$, applied by spraying powder suspended in alcohol. The coatings were rather thin (less than $1 \mathrm{~mm}$ ) and consequently had low reflectances, of the order of 0.90 . Of the fourteen usable sets of data, seven were obtained with coatings of $\mathrm{BaSO}_{4}$, applied by spraying, and seven were obtained with coatings of $\mathrm{MgO}$ powder pressed into the precision $15-\mathrm{cm}$-diam sphere.

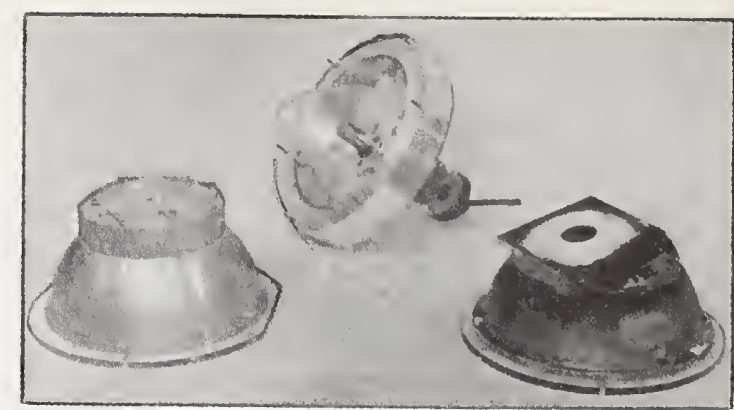

FIG. 4. Photograph of the disassembled precision-auxiliary sphere with jig used to provide accuratcly dimensioned interior coating for pressed-power specimens.

The pressed coatings were made by filling each hemisphere with powder and then packing it into the sphere wall. The excess powder was scraped out by using a Lucite disk mounted on a jig designed for the purpose (See Fig. 4). The coating layer was about $2.5 \mathrm{~mm}$ thick. Pressed coatings have three advantages over painted coatings: (1) higher reflectance, (2) better uniformity, and (3) more accurate sphere dimensions. Some of the powder removed while forming the sphere coating was pressed to form two flat samples, eliminating the need for a removable-flat sample in the sphere wall. The spectrophotometer curves for a sphere coated in this manner are shown in Fig. 5 for the visible spectrum and in Fig. 6 for the near-infrared spectrum. Note that the 100-percent curve is obtained from the two flat samples pressed from the material removed from the sphere and that the curve was rerun with the sample positions interchanged showing that the two were quite identical.

\section{RESULTS}

From each set of data the absolute reflectance of the sphere coating on the flat plate was obtained, and this was then used to calculate the absolute reflectance of a white-Vitrolite-reference standard. From the measurements of its reflectance a determination was made of the precision of the values. If $Q_{v}$ is the reading proportional to the ratio of the reflectance of the Vitrolite standard relative to the comparison specimen, and $Q_{F}$ is the reading for the flat plate relative to the same comparison specimen, then the absolute reflectance of the Vitrolite is given by

$$
\rho_{v}=\left(\rho_{F} / Q_{F}\right) Q_{v}
$$

A particular Vitrolite, V1-G3, has been used as the reference standard for reflectance measurements for a large number of specimens over the past twenty years. Thus, by now determining the absolute value of the reflectance of the Vitrolite we can compute the absolute reflectance of these specimens. In particular, a large number of freshly smoked $\mathrm{MgO}$ standards $1.0 \mathrm{~mm}$ thick has been prepared and measured by the Spectrophotometry Group. We have also measured the reflectance from 
0.4 to $0.75 \mu \mathrm{m}$ of a large number of pressed reagentgrade $\mathrm{MgO}$ and $\mathrm{BaSO}_{4}$ samples. The pressed samples were prepared in accordance with the ASTM recommended practice. ${ }^{5}$ Two pressed-MgO samples and two pressed- $\mathrm{BaSO}_{4}$ samples have been measured from 0.73 to $1.08 \mu \mathrm{m}$.

The uncertainties of the reflectance values for the samples are indicated in two ways: (1) The reproducibility of the samples is indicated in terms of the standard deviation of the individual measurements, ${ }^{6}$ (2) an estimate of the "accuracy" of the reflectance values is based on the combined uncertainty of the samples evaluated relative to the Vitrolite standard and the uncertainty of the Vitrolite absolute-reflectance calibration. Table II lists the absolute-reflectance values in the visible spectrum for an NBS-Vitrolite standard and the values for the average reflectance of a number of

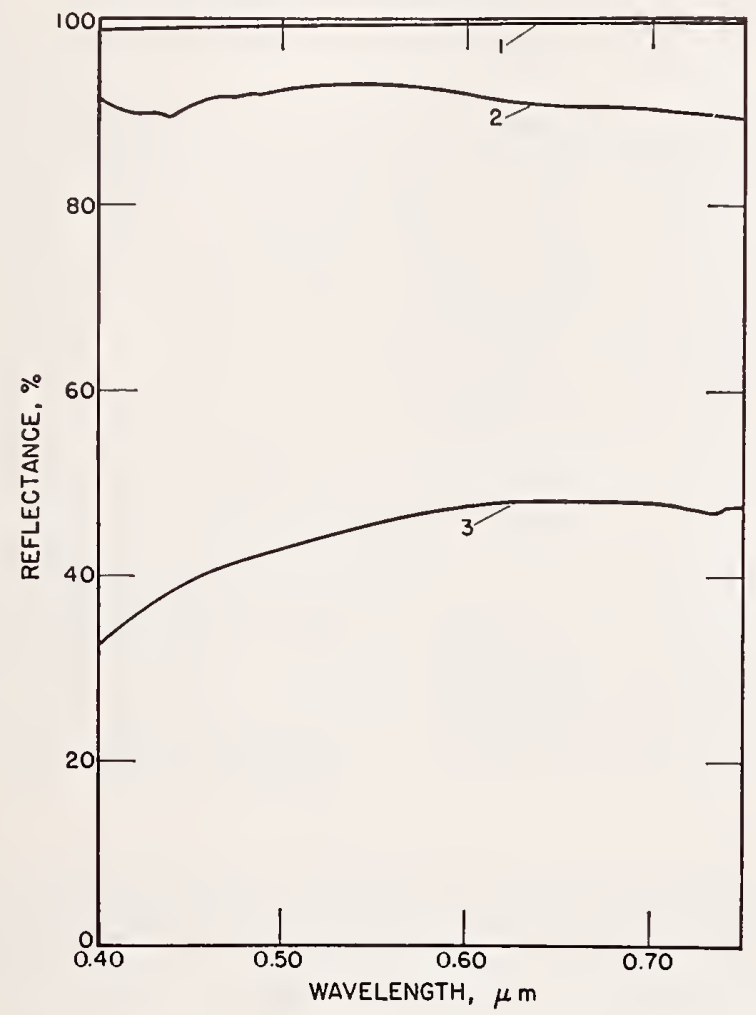

Fig. 5. Tracing of the curve sheet from a General Electric recording spectrophotometer used to compute absolute-reflectance data for the visible spectrum, 0.40 to $0.75 \mu \mathrm{m}$. All of the specimens were measured relative to the same comparison specimen, a specimen pressed from powder removed from the sphere: Curve (1) is the $100 \%$ curve obtained with the two pressed-powder specimens; Curve (2) was obtained with the Vitrolite-reference standard V1-G3; Curve (3) was obtained for a $15-\mathrm{cm}$ auxiliary sphere with a pressed-MgO coating and a $2.54-\mathrm{cm}$ port.

5 "Tentative Recommended Practice for Preparation of Reference White Reflectance Standards," ASTM Designation E 259 65T, Book of ASTM Standards, Part 30 (1966).

${ }^{6} \mathrm{~W}$. J. Dixon and F. J. Massey, Jr., Introduction to Statistical Analysis (McGraw-Hill Book Co., Inc., New York, 1957), 2nd ed.

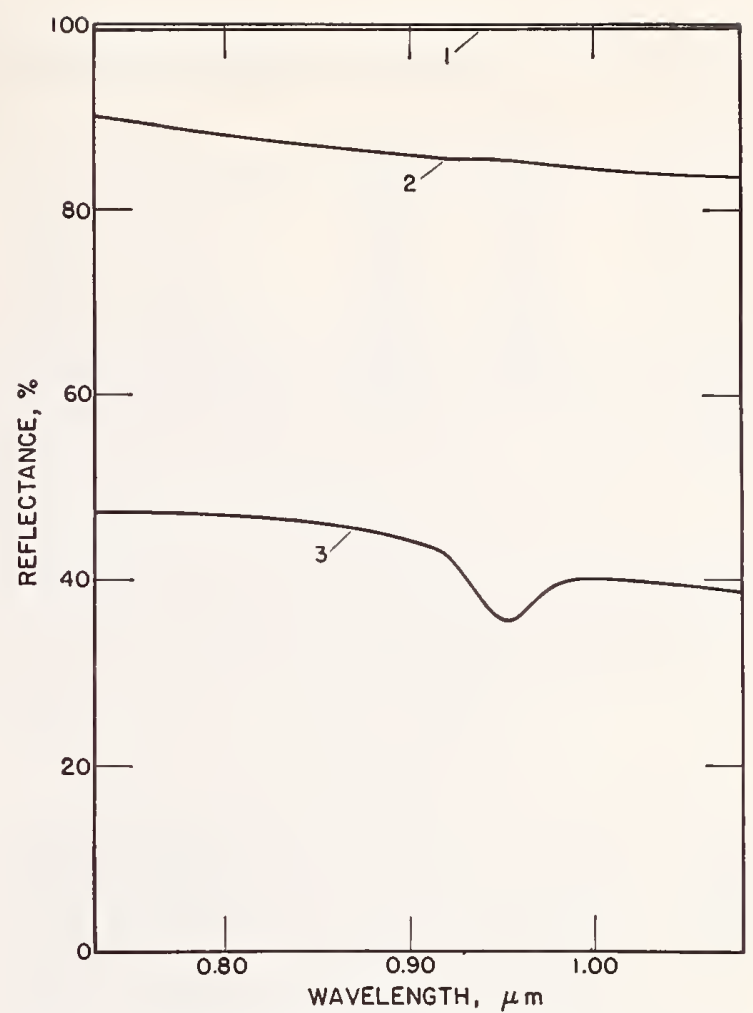

FIG. 6. Tracing of the near-infrared spectrum, 0.73 to $1.08 \mu \mathrm{m}$, for the same specimens as Fig. 5.

freshly smoked MgO specimens. This table also contains the values for the average reflectance of a number of pressed $\mathrm{MgO}$ and $\mathrm{BaSO}_{4}$ specimens together with values for the specimen of highest reflectance that we have measured, a smoked-MgO specimen prepared in an argon-oxygen atmosphere. Table III contains data for the spectral range 0.73 to $1.08 \mu \mathrm{m}$.

\section{CONCLUSIONS}

From the data presented, we draw the following conclusions:

(1) The auxiliary-sphere technique provides a simple method for determining the absolute reflectance of a reference standard if the coating is uniform and perfectly diffusing.

(2) The precision of the auxiliary-sphere technique is such that the reflectance of a reference standard can be determined from a relatively small number of measurements with a standard deviation of 0.003 .

(3) Absolute-reflectance values for pressed $\mathrm{MgO}$ and $\mathrm{BaSO}_{4}$ determined by the auxiliary-sphere technique are higher than 0.98 for the entire portion of the visible spectrum and are above 0.99 from 0.55 to $1.08 \mu \mathrm{m}$.

(4) The absolute-reflectance values for the average of 20 smoked-MgO specimens in Table II beyond 0.55 
TAlse II. Absolute reflectance values 0.4 to $0.75 \mu \mathrm{m}$.

\begin{tabular}{|c|c|c|c|c|c|c|c|c|c|}
\hline \multirow{2}{*}{$\begin{array}{l}\text { Wavelength } \\
(\mu \mathrm{m})\end{array}$} & \multicolumn{2}{|c|}{ Vitrolite V1-Cr.3n } & \multicolumn{2}{|c|}{ Smoked MgO'b } & \multirow{2}{*}{$\underset{\rho}{\text { Best } \mathrm{MgO}^{\mathrm{c}}}$} & \multicolumn{2}{|c|}{ Pressed $\mathrm{MgO}_{\mathrm{gl}}$} & \multicolumn{2}{|c|}{ Pressed ]3aSO() ${ }_{4}^{\mathrm{C}}$} \\
\hline & $\rho$ & $S_{0}$ & $\rho$ & $S$ & & $\rho$ & $S$ & $\rho$ & $S$ \\
\hline 0.40 & 0.9027 & 0.0046 & 0.9886 & 0.0021 & 0.9931 & 0.9841 & 0.0014 & 0.9828 & $0.0(125$ \\
\hline 0.43 & 0.880 .3 & $0.00,3.5$ & 0.9916 & 0.0014 & 0.99 .34 & 0.9877 & 0.0016 & 0.08 .57 & $0 .(x) 19$ \\
\hline 0.49 & 0.9101 & 0.0024 & $0.90(09$ & 0.0014 & 0.9970 & 0.9902 & 0.0012 & 0.0804 & 0.0016 \\
\hline 0.5 .5 & 0.9190 & 0.0021 & 0.9892 & $0.0(121$ & 0.99 .54 & 0.9908 & 0.0012 & 0.0006 & 0.01011 \\
\hline 0.61 & 0.906 .3 & 0.0022 & 0.9889 & 0.0018 & 0.9950 & 0.9014 & 0.0015 & $0.00(11$ & (0.0)14 \\
\hline 0.67 & 0.89 .52 & 0.002 .5 & 0.9874 & 0.0018 & 0.9948 & 0.9020 & 0.0018 & 0.900 .5 & $0.0)(018$ \\
\hline 0.72 & 0.8877 & 0.0028 & 0.9861 & 0.0021 & 0.9943 & 0.9912 & 0.0014 & 0.9906 & $0.0(1) 21$ \\
\hline 0.75 & 0.8804 & 0.0024 & 0.9851 & 0.0021 & 0.9949 & 0.9913 & 0.0015 & 0.9008 & 0.0017 \\
\hline \multirow{2}{*}{\multicolumn{3}{|c|}{$\begin{array}{l}\text { Pooled strl dev } S_{p} \\
\text { Uncertaint } \delta^{i}\end{array}$}} & & 0.0019 & & & 0.0015 & & 0.0018 \\
\hline & & & & 0.0035 & & & 0.0033 & & 0.00 .34 \\
\hline
\end{tabular}

n Average vilues of reflectance $\rho$ and the stanclircl rleviation of a single muasurement computed from 12 measurements.

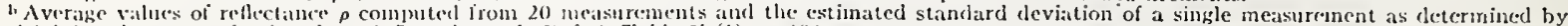

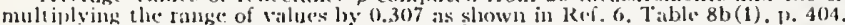

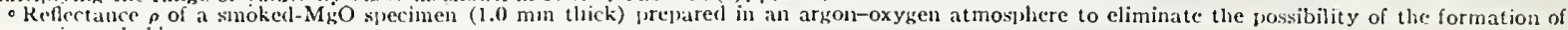
magnesium nitride.

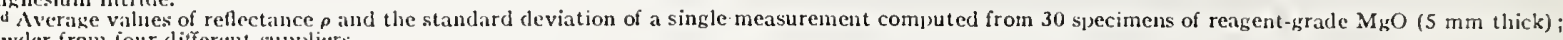
noweler from cour different sumpliers.

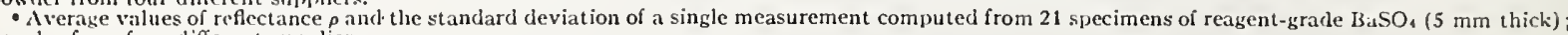
powsler from four different suppliers.

The accuracy of specinens uncasured relative to Vitrolite involves the uncertainty of both deterininations; $\delta$ is counputerl as $\left[\left(. S_{p r}\right)^{2}+S_{p}{ }^{2}\right]^{2}$.

TABLE III. Absolute reflectance values 0.73 to $1.08 \mu \mathrm{m}$.

\begin{tabular}{ccccccc}
\hline \hline $\begin{array}{c}\text { Wave- } \\
\text { length } \\
(\mu \mathrm{m})\end{array}$ & $\begin{array}{c}\text { Vitrolite V1-G3a } \\
\rho\end{array}$ & Range & $\rho$ & $S$ & $\rho$ & $\rho$ \\
\hline 0.73 & 0.8869 & 0.0000 & 0.9871 & 0.0024 & 0.9936 & 0.9956 \\
0.75 & 0.8811 & 0.0022 & 0.9860 & 0.0027 & 0.9937 & 0.9942 \\
0.80 & 0.8673 & 0.0002 & 0.9832 & 0.0028 & 0.9941 & 0.9946 \\
0.85 & 0.8543 & 0.0023 & 0.9813 & 0.0032 & 0.9934 & 0.9944 \\
0.90 & 0.8437 & 0.0023 & 0.9802 & 0.0032 & 0.9925 & 0.9946 \\
0.95 & 0.8366 & 0.0006 & 0.9812 & 0.0028 & 0.9882 & 0.9945 \\
1.00 & 0.8289 & 0.0005 & 0.9798 & 0.0033 & 0.9913 & 0.9924 \\
1.05 & 0.8213 & 0.0016 & 0.9767 & 0.0043 & 0.9913 & 0.9936 \\
1.08 & 0.8183 & 0.0000 & 0.9758 & 0.0043 & 0.9909 & 0.9938 \\
\hline \hline
\end{tabular}

- Average values of reflectance $\rho$ and range for two determinations.

$b$ Average values of reflectance $\rho$ and estimated standard deviation $S$ for 18 specimens.

- Reflectance values $\rho$ for one specimen. Note that this specimen has values ligher than the average values reported for pressed specimens in Table II.

d Average values of reflectance $\rho$ for two specimens; powder from two suppliers. $\mu \mathrm{m}$ are low compared to values for the average of 30 pressed-MgO specimens. This is probably due to the fact that the smoked-MgO specimens were only $1.0 \mathrm{~mm}$ thick whereas a thickness of $2.0 \mathrm{~mm}$ would be more likely to produce the maximum reflectance of the material with thermal deposition. Zeiss literature on their Elrepho reflectometer indicates that the critical-layer thickness is $1.5 \mathrm{~mm}$ in the red end of the spectrum and for this reason they use $1.8 \mathrm{~mm} .^{7}$

(5) Pressed- $\mathrm{MgO}$ and pressed- $\mathrm{BaSO}_{4}$ specimens have nearly the same reflectance on the average and differ in the visible spectrum from the average values for the $1 \mathrm{~mm}$ thick layers of freshly smoked $\mathrm{MgO}$ shown in Table II by less than 0.005 . In the near infrared the absolute-reflectance values of pressed $\mathrm{MgO}$ shown in Table III are $1 \%$ higher than those for smoked $\mathrm{MgO}$ at $0.8 \mu \mathrm{m}$ and $1.5 \%$ higher at $1.08 \mu \mathrm{m}$.

7 Calibration of the Reflectance Standard for the Elrepho (Carl Zeiss, Oberkochen, Germany and New York, 1963). 


\title{
Generalized Integrating-Sphere Theory
}

\author{
David G. Goebel
}

A general equation is developed for the efficiency of an integrating sphere with a nonuniform coating. The only assumptions are that the interior is a perfect sphere and that all areas reflect perfectly diffusely. Three special cases of the general equation are examined for the basic applications of integrating spheres as mixing mechanisms in hemispherical reflectance measurements and in absolute reflectance techniques.

\section{Introduction}

A sphere that is not uniform in the reflectance of its coating can be represented by a sphere with a number of areas of varying reflectance. The number and size of these areas can be chosen to approximate any nonuniform coating.

If $J$ is the intensity (flux-per-unit solid angle) of the reflected flux from an element $d a$, the resulting irradiance $H$ on any element $d A$ of the sphere wall is $H \equiv J \cos \alpha / r^{2}$ (see Fig. 1), where $J$ is the intensity in the given direction. Since $r=2 R \cos \theta$, and $\theta=\alpha$ for a sphere, $H=J \cos \theta / 4 R^{2} \cos ^{2} \theta$, where $R$ is the radius of the sphere. If the reflecting element $d a$ has a perfectly diffuse surface, $J=J_{0} \cos \theta$, where $J_{0}$ is the intensity in the perpendicular direction. Then the irradiance on any element of the sphere wall is

$$
H=J_{o} \cos ^{2} \theta / 4 R^{2} \cos ^{2} \theta=J_{0} / 4 R^{2} \text {. }
$$

Thus, the irradiance is constant, and the total flux reflected by the perfectly diffusing element $d a$ is simply $P=4 \pi R^{2} H=\pi J_{0}$.

Also, the fraction $f$ of the total reflected flux that is incident on an area $a$ of the sphere wall is simply equal to $a$ divided by $4 \pi R^{2}$, thus,

$$
f=a / 4 \pi R^{2} \text {. }
$$

If $a$ is a circular cap in the sphere wall with radius $r$, $a$ equals $2 \pi R h$ (see Fig. 2), where $h=R-\left(R^{2}-r^{2}\right)^{1 / 2}$. Therefore, for a circular cap $f=2 \pi R\left[R-\left(R^{2}-r^{2}\right)^{1 / 2}\right] /$ $4 \pi R^{2}$ or

$$
f=(1 / 2)\left\{1-\left[1-(r / R)^{2}\right]^{\frac{1}{2}}\right\}
$$

(see Table I).

Two other convenient forms for $f$ which are equivalent to Eq. (2) for a circular cap are $f=1 / 2(1-\cos 2 \theta)$ and $f=\sin ^{2} \theta$.

The author is with the Colorimetry and Spectrophotometry Section, National Bureau of Standards, Washington, D.C. 20234.

Received 11 July 1966.
Two useful approximate forms when $r \ll R$ are

$$
\left.f \simeq r^{2} / r^{2}+D^{2}\right)
$$

where

$$
D=2 R
$$

and

$$
f \simeq r^{2} / D^{2} \text {. }
$$

The last approximate form is the familiar relation obtained if the area of a flat disk $\pi r^{2}$ is used for $a$ instead of the spherical area. The error introduced by this approximation can be estimated by expanding Eq.

(2) in a power series in $l$, where $l=r^{2} / D^{2}$ :

$$
\begin{aligned}
f & =1 / 2\left[1-(1-4 l)^{1 / 2}\right] \\
& =1 / 2\left\{1-\left[1-1 / 2(4 l)-1 / 8(4 l)^{2}-1 / 16(4 l)^{3} \ldots\right]\right\} \\
& =1 / 2\left[2 l+2 l^{2}+4 l^{3}+\ldots\right] \\
& =l+l^{2}+2 l^{3}+\ldots
\end{aligned}
$$

If we approximate $f$ by $l$ to within a decimal error $\epsilon$,

$$
f-l=\epsilon l=l^{2}+2 l^{3}+\ldots
$$

or

$$
\begin{gathered}
\epsilon l \simeq l^{2} \\
\epsilon \simeq l=r^{2} / D^{2} \\
r^{2} \simeq \epsilon D^{2}
\end{gathered}
$$

or

$$
d^{2} \simeq 2 \epsilon D^{2}, \text { where } d=2 r,
$$

then

$$
d \simeq \sqrt{2 \epsilon} D .
$$

Use of the approximate form for $f$ results in an error greater than $1 \%$, if the diameter of $a$ is more than $20 \%$ of the sphere diameter. For an error of less than $0.1 \%$ the maximum value for $d$ is $0.06 \mathrm{D}$. For a 20 -cm sphere the use of $r^{2} / D^{2}$ for $f$ yields a $1 \%$ error for $d=4.0 \mathrm{~cm}$ and a $0.1 \%$ error for $d=1.2 \mathrm{~cm}$. 


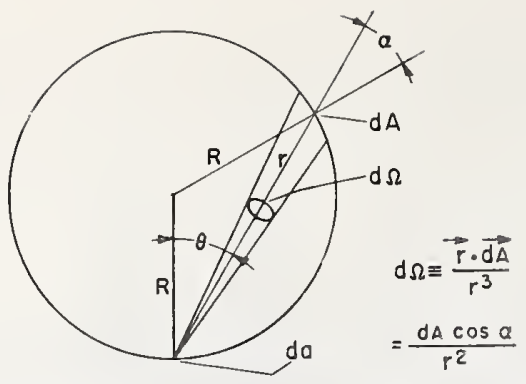

Fig. 1. Geometric relations in a sphere used for calculating the irradiance on $d A$ from $d a$.

\section{Theory}

Consider a sphere with total area $A_{s}$ equal to $\pi D^{2}$ which has $n+1$ areas $a_{i}$, with $\rho_{i}$ the reflectance of the $i$ th area. Openings are equivalent to areas with zero reflectance. The area of the sphere that is not covered by the $n+1$ areas has a reflectance $\rho_{w}$. The ratio of the area $a_{t}$ to the area of the sphere $A_{s}$ is called $f_{i}$. The average reflectance of the sphere wall is

$$
\bar{\rho}_{w}=\sum_{0}^{n} \rho_{i} f_{i}+\rho_{w}\left(1-\sum_{0}^{n} f_{i}\right)
$$

All surfaces are assumed to be perfectly diffuse reflectors. Flux $P_{o}$ is initially incident on the area $a_{0}$. An amount $\rho_{o} P_{0}$ reflected by $a_{o}$ is the total unabsorbed flux in the sphere after the first reflection. Of this flux $f_{i} \rho_{o} P_{o}$ is incident on $a_{i}$, and

$$
\left(1-\sum_{o}^{n} f_{i}\right) \rho_{o} P_{o}
$$

is incident on

$$
A_{s}-\sum_{o}^{n} a_{i}
$$

An amount $\rho_{i} f_{i} \rho_{o} P_{o}$ is reflected by $a_{i}$

$$
\sum_{o}^{n} \rho_{i} f_{i} \rho_{o} P_{o}
$$

is reflected by

$$
\sum_{0}^{n} a_{i}
$$

and

$$
\rho_{w}\left(1-\sum_{o}^{n} f_{i}\right) \rho_{0} P_{o}
$$

is reflected by

$$
A_{s}-\sum_{o}^{n} a_{i} .
$$

Therefore, the total unabsorbed flux in the sphere after the second reflection is $\bar{\rho}_{w} \rho_{o} P_{o}$, where

$$
\bar{\rho}_{w}=\rho_{w}\left(1-\sum_{o}^{n} f_{i}\right)+\sum_{o}^{n} f_{i} \rho_{i}
$$

is the average reflectance of the sphere wall. Of this flux

$$
\sum_{0}^{n} f_{i} \bar{\rho}_{w} \rho_{0} P_{o}
$$

is incident on

$$
\sum_{0}^{n} a_{i}
$$

and

$$
\left(1-\sum_{o}^{n} f_{i}\right) \bar{\rho}_{w} \rho_{o} P_{o}
$$

is incident on

$$
A_{s}-\sum_{0}^{n} \rho_{i}
$$

An amount

$$
\sum_{0}^{n} \rho_{i} f_{i} \bar{\rho}_{w} \rho_{0} P_{0}
$$

is reflected by

$$
\sum_{0}^{n} a_{i}
$$

and

$$
\rho_{w}\left(1-\sum_{o}^{n} f_{i}\right) \bar{\rho}_{w} \rho_{o} P_{o}
$$

is reflected by

$$
A_{s}-\sum_{0}^{n} a_{i}
$$

Therefore, the total unabsorbed flux in the sphere after the third reflection is

$$
\left[\rho_{w}\left(1-\sum_{0}^{n} f_{i}\right)+\sum_{0}^{n} \rho_{i} f_{i}\right] \bar{\rho}_{w} \rho_{o} P_{o}
$$

which equals $\bar{\rho}_{w}^{2} \rho_{0} P_{0}$. Similarly, the total unabsorbed flux in the sphere after the fourth reflection equals $\bar{\rho}_{w}{ }^{3} \rho_{o} P_{o}$, etc. The total flux $P_{i}$ which is incident on $a_{i}$ summed over an infinite number of reflections equals

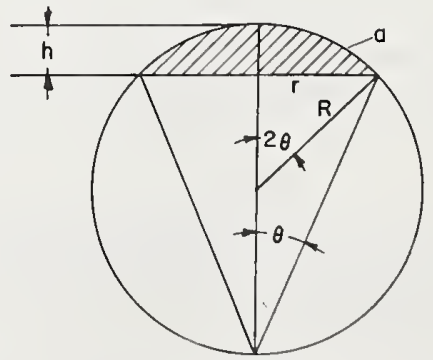

Fig. 2. Geometric relations used for calculating the area of a spherical cap. 


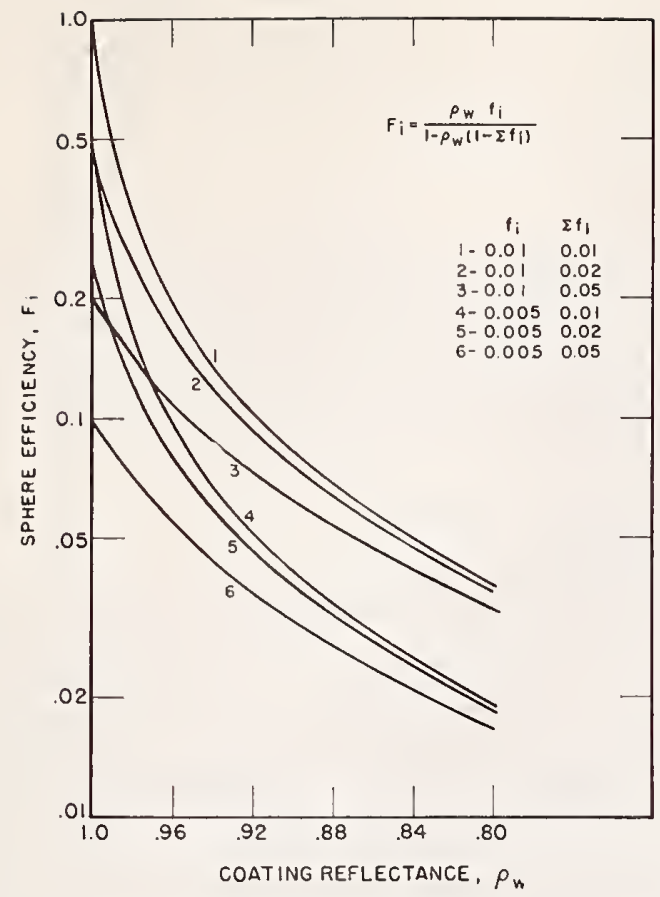

Fig. 3. Graph of Eq. 4; sphere efficiency vs coating reflectance.

$$
\begin{aligned}
& P_{i}=f_{i} \rho_{o} P_{o}+f_{i} \bar{\rho}_{w} \rho_{o} P_{o}+f_{i} \bar{\rho}_{w}{ }^{2} \rho_{o} P_{o}+f_{i} \bar{\rho}_{w}{ }^{3} \rho_{o} P_{o}+\ldots \\
& P_{i}=f_{i} \rho_{o} P_{o}\left(1+\bar{\rho}_{w}+\bar{\rho}_{w}{ }^{2}+\bar{\rho}_{w}{ }^{3}+\ldots\right) \\
& P_{i}=f_{i} \rho_{o} P_{o} f\left(\hat{j}-\bar{\rho}_{w}\right) .
\end{aligned}
$$

The fraction $F_{i}$ of the initial flux $P_{o}$ that is incident on $a_{t}$ is $P_{t} / P_{0}$. This is called the sphere efficiency: $F_{t}$ $=f_{i} \rho_{0} /\left(1-\bar{\rho}_{w}\right)$.

$$
\begin{aligned}
& \bar{\rho}_{w}=\rho_{w}\left(1-\sum_{o}^{n} f_{i}\right)+\sum_{o}^{n} f_{i} \rho_{i}, \\
& F_{i}=f_{i} \rho_{o}\left[1-\rho_{w}\left(1-\sum_{o}^{n} f_{i}\right)-\sum_{o}^{n} f_{i} \rho_{i}\right]^{-1} .
\end{aligned}
$$

Equivalent relations have been derived by Jacques and Kuppenheim ${ }^{1}$ using integral equation methods and by Hisdal ${ }^{2,3}$ using matrix relations. There are three cases to which the above equation is frequently applied.

Case I. The first case is that of the simple integrating sphere with $n$ holes in which the incident flux irradiates the sphere wall: $\rho_{o}=\rho_{v}$ and $\rho_{j}=0$ for $0<j \leq n$. The fraction of the incident flux that escapes out the $i$ th hole is

$$
F_{i}=f_{i} \rho_{u u}\left[1-\rho_{w}\left(1-\sum_{1}^{n} f_{j}\right)\right]^{-1}
$$
A simplification of Eq. (4), where $\sum_{1}^{n} f_{j}$ is much less
than one, is

$$
F_{i} \simeq \rho_{w} f_{i} / \alpha_{w} \text { for } \rho_{w} \neq 1.0,
$$

where $\alpha_{w}$, the absorptance of the sphere coating, equals $1-\rho_{t}$.

When written in this approximate form, $F_{i}$ can be seen to be strongly dependent on the absorptance $\alpha_{w}$. For large values of $\rho_{t}$, a small percentage change in $\rho_{w}$ yields a large percentage change in $\alpha_{w}$. More exactly, the percentage change in $F_{\imath}$ is from Eq. (5),

$$
\Delta F_{i} / F_{i} \simeq \Delta \rho_{w} / \rho_{w}+\Delta f_{i} / f_{i}-\Delta \alpha_{w} / \alpha_{w} ;
$$

since

$$
1-\rho_{w}=\alpha_{w}
$$

then

$$
\Delta \rho_{w}=-\Delta \alpha_{w}
$$

therefore,

$$
\Delta F_{i} / F_{i} \simeq 1 / \alpha_{w} \times \Delta \rho_{w} / \rho_{w}+\Delta f_{i} / f_{i}
$$

This means that $0.5 \%$ change in the reflectance of the sphere coating yields a $5 \%$ change in the sphere efficiency for a coating reflectance of approximately $90 \%$. A sphere coating that is only slightly selective spectrally can cause the sphere efficiency to be strongly selective as discussed above in Case I (see Fig. 3).

Case II. The second case is that of an integratingsphere reflectometer in which the incident flux irradiates one of two specimens at ports in the sphere wall:

$$
\rho_{o} \neq \rho_{w}, \rho_{1} \neq \rho_{w} \text { and } \rho_{j}=0 \text { for } j \neq 0,1
$$

$$
\begin{aligned}
& F_{i}=f_{i} \rho_{o}\left[1-\rho_{w}\left(1-\sum_{0}^{n} f_{j}\right)-\rho_{o} f_{0}-\rho_{1} f_{1}\right]^{-1} \\
& F_{i}=f_{i} \rho_{i}
\end{aligned}
$$

When an in fegfating-sphere reflectometer is operated in a double-beam or comparison mode, two specimens are placed at ports in the sphere wall and the change in the sphere efficiency is measured when first one and

Table I. $f=\frac{1}{2}\left\{1-\left[1-(d / D)^{2}\right]^{1 / 2}\right\}$

\begin{tabular}{llll}
\hline$d / D$ & \multicolumn{1}{c}{$f$} & $d / D$ & $f$ \\
\hline 0.00 & 0.000000 & 0.45 & 0.05349 \\
0.025 & 0.0001563 & 0.50 & 0.06699 \\
$1 / 24$ & 0.0004342 & 0.55 & 0.08242 \\
0.05 & 0.0006254 & 0.60 & 0.1000 \\
$1 / 16$ & 0.0009775 & $6 / 8$ & 0.1096 \\
0.075 & 0.001408 & 0.65 & 0.1200 \\
$1 / 12$ & 0.001739 & $2 / 3$ & 0.1273 \\
0.10 & 0.002504 & 0.70 & 0.1429 \\
$3 / 24$ & 0.003922 & 0.75 & 0.1693 \\
0.15 & 0.005657 & 0.80 & 0.2000 \\
$1 / 6$ & 0.006993 & $5 / 6$ & 0.2236 \\
$3 / 16$ & 0.008868 & 0.85 & 0.2366 \\
0.20 & 0.01010 & 0.90 & 0.2821 \\
$6 / 24$ & 0.01097 & 0.95 & 0.3439 \\
0.25 & 0.01588 & 1.00 & 0.5000 \\
0.30 & 0.02303 & & \\
$6 / 16$ & 0.02504 & & \\
$1 / 3$ & 0.02860 & & \\
0.35 & 0.03163 & & \\
$3 / 8$ & 0.03649 & & \\
0.40 & 0.04174 & & \\
$5 / 12$ & 0.04547 & & \\
\hline
\end{tabular}


then the other is directly irradiated. Since the average reflectance of the sphere is unchanged, the ratio of the two sphere efficiencies is directly equal to the ratio of the reflectances of the specimens. In the singlebeam or substitution mode, the change in the sphere efficiency is measured when one specimen is substituted for another in the sphere wall. Since the average reflectance of the sphere wall changes when the specimen is changed, the ratio of the sphere efficiencies is not exactly equal to the ratio of the specimen reflectances. The amount that the sphere efficiency is altered because of a change in the average reflectance of the wall can be quite large depending on the magnitude of the average reflectance (see Fig. 3).

Case III. In the third case the sphere wall is directly irradiated, and the areas of varying reflectance are diffusely irradiated:

$$
\begin{gathered}
\rho_{o}=\rho_{w} \\
F_{i}=f_{i} \rho_{w}\left[1-\rho_{w}\left(1-\sum_{1}^{n} f_{j}\right)-\sum_{1}^{n} f_{j} \rho_{j}\right]^{-1}
\end{gathered}
$$

An important application of Eq. (8) is the removable cap method of determining absolute reflectance first used by Taylor in $1920 .^{4}$ The sphere is constructed with an entrance port, a measurement port, and a removable cap with the same reflectance as the rest of the sphere wall. The sphere wall is directly irradiated by the entering flux. First, the fraction of the incident flux leaving the measurement port is measured with the cap on, and

$$
F=f_{m} \rho_{w}\left[1-\rho_{w}\left(1-f_{e}-f_{m}\right)\right]^{-1}
$$

The fraction of the incident flux measured with the cap removed is

$$
F^{\prime}=f_{m} \rho_{w}\left[1-\rho_{w}\left(1-f_{e}-f_{m}-f_{c}\right)\right]^{-1}
$$

where $f_{e}$ is the area ratio defined above for the entrance port, $f_{m}$ is the ratio for the measurement port, and $f_{c}$ is the ratio for the removable cap. Using these two measurements, one can obtain the absolute reflectance of the sphere coating from the following equation:

$$
\begin{aligned}
\rho_{w}=\left(Q-Q^{\prime}\right)\left[\left(Q-Q^{\prime}\right)(1\right. & \left.\left.-f_{e}-f_{m}\right)+Q^{\prime} f_{c}\right]^{-1} \\
& =\left[1-f_{e}-f_{m}+f_{c} Q^{\prime} /\left(Q-Q^{\prime}\right)\right]^{-1},
\end{aligned}
$$

where $Q=K F$ is the reading obtained with the cap on and $Q^{\prime}=K F^{\prime}$ is the reading obtained with the cap off. Another method of obtaining absolute reflectance by using an auxiliary sphere with an integating sphere reflectometer has been described by the author in a previous paper. ${ }^{5}$

\section{Conclusion}

By using the equations derived above together with Table I and Fig. 3, the designer should be able to construct an integrating sphere to obtain a desired efficiency; or, alternatively, one can determine the efficiency of a sphere built for a specific application. The equations will yield a good approximation for sphere efficiency even if the sphere coating is not perfectly diffuse, provided that the reflectance is high (greater than $\sim 0.9$ ), and provided that the first few specular reflections are confined to the sphere. The reason for this is that even for a slightly specular coating, after a few reflections in the sphere, the irradiance will become uniform.

\section{References}

1. J. A. Jacques and H. F. Kuppenheim, J. Opt. Soc. Am. 45, 460 (1955).

2. B. J. Hisdal, J. Opt. Soc. Am. 55, 1122 (1965).

3. B. J. Hisdal, J. Opt. Soc. Am. 55, 1255 (1965).

4. A. H. Taylor, Sci. Papers Bur. Std. S16, 421 (1920).

5. D. G. Goebel, J. Opt. Soc. Am. 56, 783 (1966). 


\title{
17. Effect of Surface Texture on Diffuse Spectral Reflectance
}

\section{A. Diffuse Spectral Reflectance of Metal Surfaces}

\author{
H. J. KEEGAN, J. G. SGHLETER, AND V. R. WEIDNER \\ NATIONAL BUREAU OF STANDARDS, WASHINGTON, D.C.
}

\section{B. Surface Texture Measurements of Metal Surfaces}

\author{
D. B. SPANGENBERG, A. G. STRANG, AND J. L. CHAMBERLIN
}

NATIONAL BUREAU OF STANDARDS, WASHINGTON, D.C.

\begin{abstract}
Part $\mathrm{A}$ describes an attempt to correlate the effect of surface finish (texture) on the diffuse spectral directional reflectance of metals; measurements were made over the wavelength range from 0.2 to $2.1 \mu$ for samples of sintered beryllium, steel gage blocks, steels machine-lapped with various types and sizes of abrasives, polished and sandblasted aluminum, platinum, and a chemically polished beryllium block.

Part $\mathrm{B}$ describes surface-texture measurements made on most of the same surfaces by interferometry. Several conventional parameters describing these surfaces were determined by stylus and interferometric techniques. Interferograms of the surfaces are included.
\end{abstract}

\section{A. Diffuse Spectral Reflectance of Metal Surfaces ${ }^{1}$}

In June 1963, the Flight Reentry Programs Office of the NASA Langley Research Center requested the NBS Spectrophotometry Unit of the Photometry and Colorimetry Section to measure the diffuse spectral directional reflectances of eight samples of sintered pressed beryllium over the wavelength range from 0.26 to $2.1 \mu$. Each beryllium block had one face polished, and the polished faces had different degrees of finish.

This study led to the investigation of other metals and a wider range of roughness; and the surface finishes were evaluated by several techniques, including microinterferometry.

\footnotetext{
1 Supported in part by the Advanced Research Projects Agency, Department of Defense.
}

Microinterferograms of 22 of these metal surfaces are included in part $B$ of this paper.

It was considered of particular interest to study the effect of the lay of the finish on the surface reflectance. In the field of color, the effect of the texture of woven dyed cloth on its spectral directional diffuse reflectance is well known and has been extensively investigated. Nutting (ref. 1) found that representative colorimetric specifications could be correlated with visual estimates by averaging the spectral directional diffuse reflectance measurements of each sample at two angles, one with the lay of the weave at $45^{\circ}$ and the other with the lay oriented $90^{\circ}$ from the first measurement. This method was used at NBS and correlated with other visual methods (ref. 2). A few studies have been made in other laboratories of the 
effect of lay of finish (direction of polishing) on the specular spectral reflectance (refs. 3, 4, and $5)$, and of the effect of electropolishing on specular reflectance (ref. 6). A study has also been made of the effect of lay on diffuse spectral reflectance of buffed silver surfaces as measured with the General Electric recording spectrophotometer for the visible spectrum only (ref. 7).

\section{SPECIMENS AND TESTS}

\section{Test Specimens}

The specimens studied were:

(1) Eight sintered beryllium samples, pressed and polished by manual lapping (no. 1, 2, 3, 4, 6, 7, 8, and 9a)

(2) One sintered beryllium sample chemically polished (no. 9b)

(3) Six commercial gage blocks made of steel

(4) Ten NBS steel samples, machine lapped

(5) One polished aluminum sample

(6) One sandblasted aluminum sample

(7) One polished platinum sample

(8) One sandblasted platinum sample

\section{Measurements}

Measurements of diffuse spectral directional reflectance were made for the following conditions of irradiation and reception:

(1) $6^{\circ}$ incidence, diffuse viewing (specular component included), 0.4 to $1.08 \mu$, on a GE spectrophotometer (ref. 8)

(2) $6^{\circ}$ incidence, diffuse viewing (specular component included), on a Cary model 14M spectrophotometer (ref. 9) equipped with a model 1411 diffuse reflectance integrating sphere attachment: 0.26 to $0.4 \mu$, hydrogen source, Dumont K1306 photomultiplier; and 0.36 to $0.7 \mu$, tungsten source, K1306 photomultiplier

(3) Diffuse illumination, $6^{\circ}$ viewing, on same equipment as described in (2): 0.36 to $0.7 \mu$, tungsten source, Hamamatsu R136 photomultiplier; and 0.6 to $2.1 \mu$, tungsten source, lead sulfide photocell

\section{Reference Standards}

For the visible and near infrared measurements, 0.4 to $1.08 \mu$, the reference standard used was freshly prepared magnesium oxide made by burning magnesium metal chips in air and depositing the $\mathrm{MgO}$ smoke on troughs $1 \mathrm{~mm}$ deep (ref. 10). All integrating spheres were also smoked with MgO. Working standards used were NBS V1-G3 Vitrolite standard (see ref. 11), a rhodium mirror used for some earlier measurements, and the diffuse side of a sample of Corning Thermometer White glass TW1-B1.

The same reference standards were used in the $\mathrm{i}$ ltraviolet, visible, and infrared measurements, 0.26 to 2.1 microns.

\section{RESULTS \\ Presentation of Results}

The results of these studies of the diffuse spectral reflectance of surfaces of metals are presented in figures 1 to 9 as follows:

Aluminum:

Figure

Machine lapped . . .

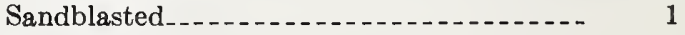

Platinum:

Machine lapped

Sandblasted....... 1

Beryllium:

Sample no. 1, hand lapped (about 4 microinches) - .

Sample no. 8, hand lapped (about 20 micro-

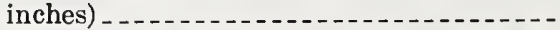

Sample no. 9a, hand lapped (about 20 microinches), in visible spectrum only.--

Sample no. $9 \mathrm{~b}$, chemically polished.

Steel:

5 commercial gage blocks_............ 5

Machine lapped, various sizes of grit_-..-- 6

Machine lapped, polished and depolished._ $\quad 7$

Rhodium-plated nickel:

Ruled roughness $(20 \mu$ in., arithmetic average)

Ruled roughness (125 $\mu$ in., arithmetic average)

\section{Discussion of Results}

\section{ALUMINUM}

'The diffuse spectral reflectance in the ultraviolet, visible, and infrared $(0.26$ to $2.1 \mu)$ of 


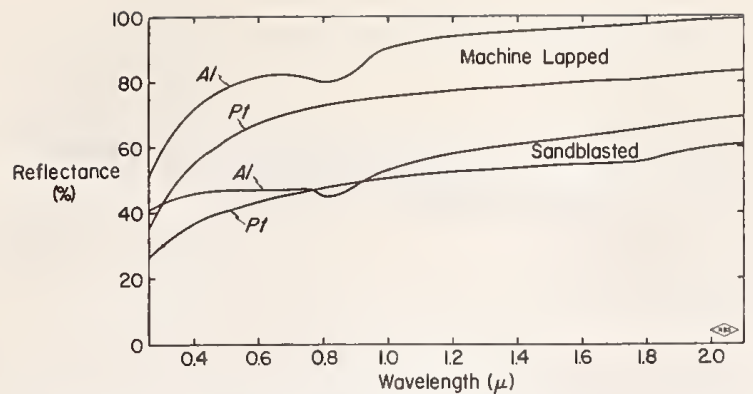

FIGURE 1.-Diffuse spectral reflectances $(0.26$ to $2.1 \mu)$ of machine-lapped and sandblasted samples of aluminum and of platinum. Each curve represents determinations at four orientations. There was no appreciable effect of orientation.

two samples of aluminum, one with a machinelapped finish and the other with a sandblasted finish, are shown in figure 1. Only a single curve is shown for each sample, since there was no observable separation of the curves obtained over the entire spectral range at four orientations of each sample $\left(0^{\circ}, 90^{\circ}, 180^{\circ}, 270^{\circ}\right.$, and a repeat measurement at $360^{\circ}$ between a fiducial mark on the sample and the vertical slit axis of the instrument), that is, the reflectance of the highly polished surface of aluminum and that of the highly depolished surface of aluminum were independent of angle of orientation of the sample. Spectrally the difference between the two reflectance curves varied from 0.10 at $0.26 \mu$ to 0.38 at $0.9 \mu$ and beyond.

\section{PLATINUM}

Diffuse spectral directional reflectance curves of polished and sandblasted platinum, similar to those for aluminum, are also shown in figure 1. These two curves are essentially parallel except in the ultraviolet.

\section{BERYLLIUM}

Figures 2, 3, and 4 show the diffuse spectral directional reflectance of sintered beryllium. Figures 2 and 3 are curves for hand-lapped finishes, at eight orientations each $\left(0^{\circ}, 45^{\circ}, 90^{\circ}\right.$, $135^{\circ}, 180^{\circ}, 225^{\circ}, 270^{\circ}, 315^{\circ}$, and a repeat measurement at $360^{\circ}$ between a fiducial mark on the sample and the vertical slit axis of the instrument). The most polished sample (fig. 2) showed no variation with orientation of the

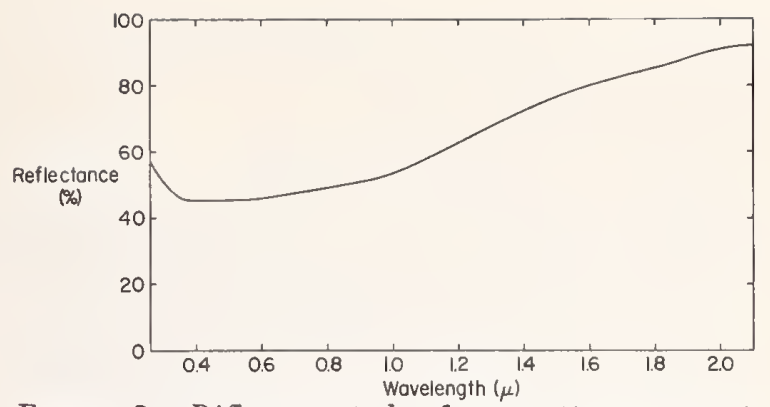

Figure 2.-Diffuse spectral reflectance (0.26 to $2.1 \mu)$ of surface of beryllium sample 1, hand-lapped to about 4 microinches. The curve represents determinations at eight orientations of the sample.

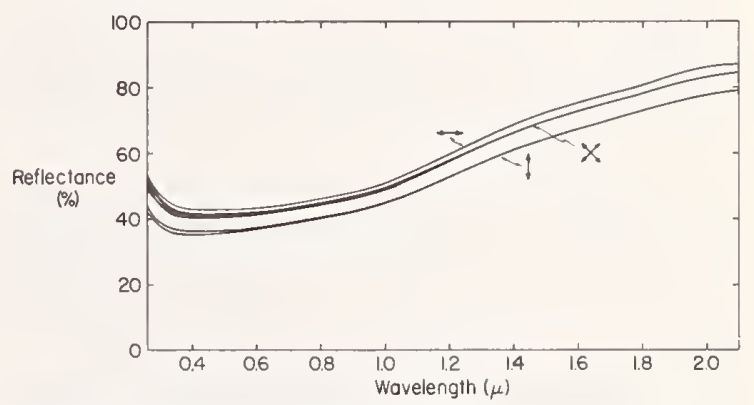

FIGURE 3.-Diffuse spectral reflectance (0.26 to $2.1 \mu)$ of surface of beryllium sample 8, hand-lapped to about 20 microinches, at eight orientations. The horizontal lay of finish (texture) yields highest reflectance throughout the spectrum and the vertical lay of finish yields lowest reflectance. The four $45^{\circ}$ orientations are in the middle but nearer to the horizontal. (Note: For the vertical lay of the finish, the projection of the incident beam on the surface is parallel to the lay.) The arrows indicate the direction of the lay of the finish rclative to the vertical slit axis of the instrument.

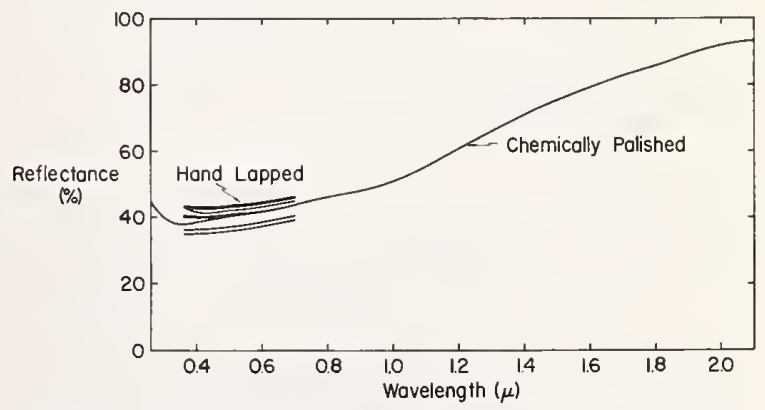

Figure 4-Diffuse spectral reflectances of beryllium samples $9 a$ and $9 b$, for eight orientations each. The curves for the hand-lapped surface $(9 a)$ are shown only for the visible spectral region $(0.36$ to $0.7 \mu)$. The curve for this surface after subsequent chemical polishing $(9 b)$ is shown for the range of 0.26 to $2.1 \mu$. 
sample. The least polished sample (fig. 3) showed approximately 8 percent variation of reflectance with orientation.

Figure 4 shows the variation of diffuse spectral reflectance for a hand-lapped beryllium specimen, similar to the one shown in figure 3 , for the visible spectral region, and for the same sample after chemical polishing, over the full spectral range $(0.26$ to $2.1 \mu)$. The hand-lapped surface showed an appreciable effect of orientation, but the chemically polished surface showed no variation in' reflectance with orientation.

\section{STEEL}

Figures 5, 6, and 7 show diffuse spectral directional reflectance of steel specimens with various finishes.

Figure 5 shows data for commercial steel gage blocks. Curves 2 and 4 are for eight orientations each, curves 1 and 3 are for two orientations each, and curve 5 is for six orientations. (The number of orientations was restricted because of the configuration of the gage block relative to the opening of the sphere.) The variations in diffuse spectral reflectance are due to differences in the types of steel used and possibly to oxide surface films.

In figure 6 are curves of diffuse spectral directional reflectance of nearly identical samples of steel with different finishes-a commercial gage block finish, a high polish obtained

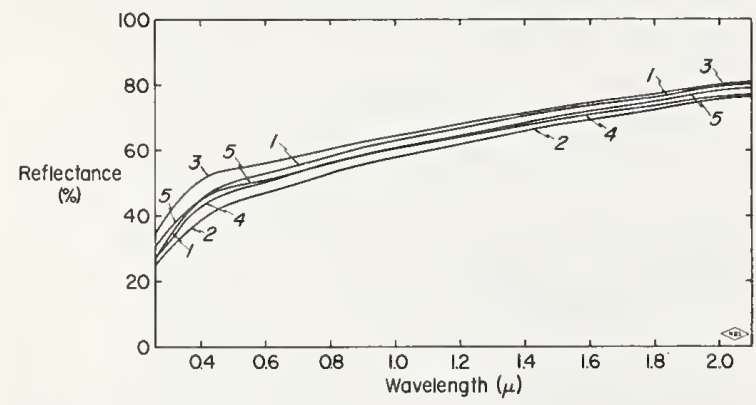

Figdre 5.-Diffuse spectral reflectances $(0.26$ to $2.1 \mu)$ of steel gage blocks with commercial finishes. The variation in reflectance is due to differences in the stecls used in the manufacture of the blocks and to oxide surface films.

1. Webber 5 min $M-4$

2. Webber $30 \mathrm{~min}$ $N B S \div 2$.

3. NPL 9 sec no. 5

4. Van Keuren $0.250 \mathrm{in.}$ 222.

5. Pratt and Whitney 0.500 in. Triangle.

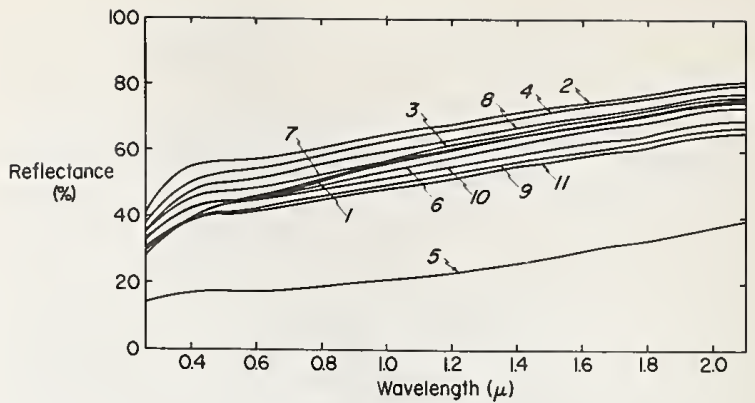

FIgURE 6.-Diffuse spectral reflectances (0.26 to $2.1 \mu$ ) of nearly identical steel samples with different finishes, for one orientation each. The finishes include a commercial finish, possibly with an oxide surface film, a high polish obtained with aluminum oxide on a pitch polisher, and a range of finishes obtained with different grades of diamond grit $(1 / 4$ to $60 \mu)$ on cast iron. The lowest curve is for a surface that was heat damaged during finishing.

1. No. 31: commercial finish 6. No. 54: $6 \mu$ diamond

2. No. 17: aluminum oxide 7. No. 40:14 $\mu$ diamond

3. No. 20: $1 / 4 \mu$ diamond 8. No. 86: $20 \mu$ diamond

4. No. 35: $1 \mu$ diamond 9. No. 85: $30 \mu$ diamond

5. No.77: $3 \mu$ diamond 10. No. 24: $45 \mu$ diamond

\section{No. $61: 60 \mu$ diamond}

from aluminum oxide on a pitch polisher, and a range of finishes obtained with various grades of diamond grit from $1 / 4$ to $60 \mu$ on cast iron. These curves are for a single orientation which is representative of the average reflectance for the eight orientations of the sample. The range of the measurements varied from approximately \pm 0.2 percent, for the specimen finished with the $1 / 4-\mu$ diamond grit, to approximately \pm 1.0

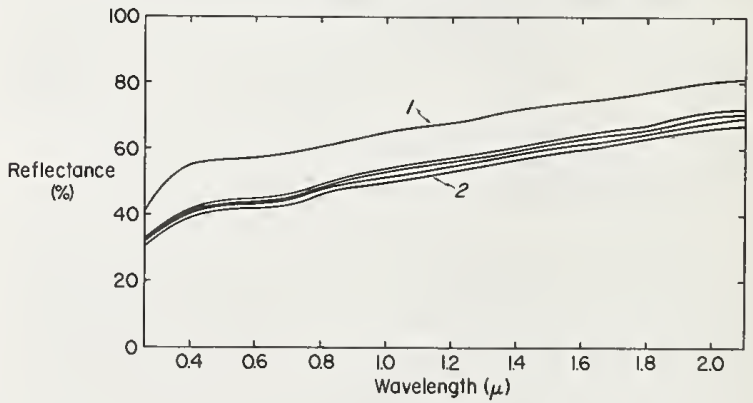

FiguRE 7.-Diffuse spectral reflectances (0.26 to $2.1 \mu$ ) of two of the specimens ( $\&$ and 10) shown in figure 6 for eight orientations.

1. Pitch polisher with aluminum oxide (Linde A)

2. Cast iron lap with $45 \mu$ diamond 


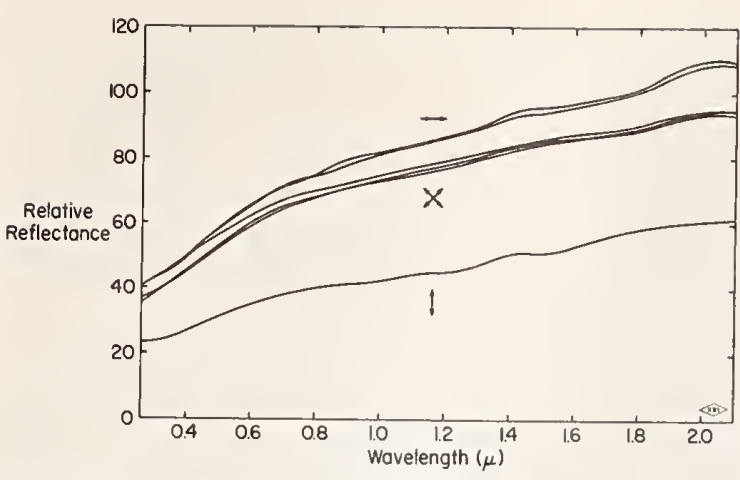

Figdre 8.-Diffuse spectral reflectance $(0.26$ to $2.1 \mu)$ of a rhodium-plated nickel ruled roughness specimen of 20-microinch AA surface roughness, for six orientations. The arrous indicate the direction of the lay of the finish relative to the vertical slit axis of the instrument.

percent, for the specimen finished with the $60-\mu$ diamond grit, from the average values indicated in figure 6 . Curve 5 is for a surface that was found to be heat damaged when removed from the block.

Curves for two of the specimens, no. 2 and 10 of figure 6, are shown in figure 7 for eight orientations.

\section{RHODIUM-PLATED NICKEL}

Curves for ruled roughness specimens of rhodium-plated nickel of 20 and 125 microinches AA (arithmetic average) for six orientations of the rulings are shown in figures 8 and 9 . The reflectance, relative to $\mathrm{MgO}$, at orientations of $90^{\circ}$ and $270^{\circ}$ exceeded 100 percent at wavelengths greater than about $1.8 \mu$. This is believed to be due to a focusing effect of the

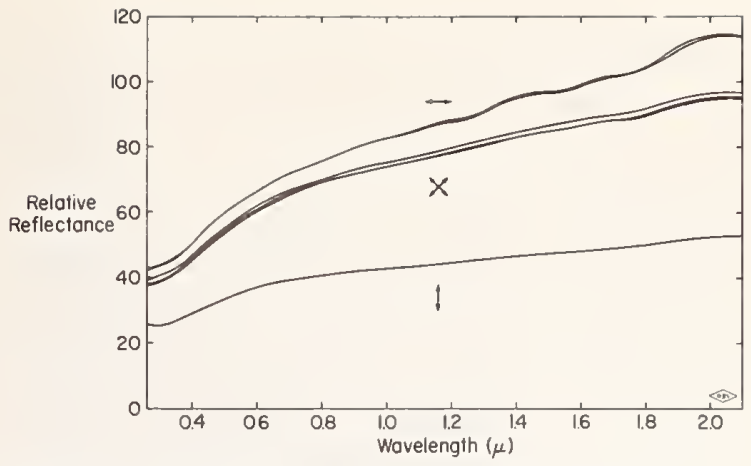

FIgURE 9.-Diffuse spectral refiectance $(0.26$ to $2.1 \mu)$ of a rhodium-plated nickel ruled roughness specimen of 125-microinch AA surface roughness, for six orientations. The arrows indicate the direction of the lay of the finish relative to the vertical slit axis of the instrument.

regular rulings of the Caliblock, the geometry of the integrating sphere, and the setting of the 100 percent curve relative to the diffusing $\mathrm{MgO}$ surface.

\section{CORRELATION WITH SURFACE TEXTURE}

Study of these spectrophotometric curves and of the corresponding 24 microinterferograms shown by Spangenberg, Strang, and Chamberlin in part B of this paper showed a possibility of a correlation between diffuse spectral directional reflectance and the surface texture (roughness) as therein evaluated. However, considerably more work on more accurately prepared specimens must be done before definite conclusions may be reached.

\section{B. Surface Texture Measurements of Metal Surfaces ${ }^{2}$}

Control of surface texture is usually based on a single roughness parameter of the surface. When such single parameter control is used, it is also necessary to specify the manufacturing process. The relationship between the control parameter and the resultant surface texture, for the defined process, is known either by experience or previous tests. No single parameter can, however, fully describe a surface. For

2 See also Paper 27. this correlation study, the surface textures of the finely finished specimens used were evaluated by measuring several conventional parameters.

\section{SPECIMENS}

Three sets of specimens were measured. These consisted of a set of specially prepared sintered beryllium specimens, a set of specially prepared tool steel specimens, and a set of commercially finished steel specimens. 
Six specimens of sintered beryllium were finished by a hand-polishing process similar to metallurgical polishing. These specimens were polished in one direction, so that there was a predominant lay to the surface texture of all but the finest finished specimen.

Ten specimens of gage block tool steel were finished by a lapping process. Specimen 17 was finished on pitch using a fine aluminum oxide abrasive (Linde A). All others were finished on a cast iron lap using various powdered diamond abrasives. These specimens were randomly finished and had no predominant lay.

Six commercially finished gage block steel specimens were also measured. These surfaces represent commercial machine lapping processes. Specimen 1 had a predominant lay; all others were randomly finished.

In addition, measurements on a commercial precision reference specimen (Caliblock) were also used in this study. This specimen is an electrolytic replica of ruled regular triangular wave patterns having apex angles of $150^{\circ}$. It had two patches with different peak-tovalley heights.

\section{MEASURED PARAMETERS}

The parameters measured are those in accord with the M system. This system is based on a mean line about which various roughness parameters are measured.

Although by no means complete, the following definitions should be sufficient for understanding the terminology used. Figure 10 graphically illustrates these parameters. A complete physical interpretation of the significance of these and other parameters is contained in references 12, 13, and 14 .

Mean line (center line): A mean line is a line parallel to the general direction of the measured surface profile and positioned such that the sums of the areas contained between it and those parts of the profile that lie on each side of it are equal.

Crest and root lines: For these measurements the crest and root lines were determined by the 10-point system, in which the crest line and root line are located parallel to the mean line and through the average height of the five extreme peaks and the average depth of the five extreme valleys of the profile, respectively.

Peak-to-valley height $R$ : The peak-to-valley height is the distance between the crest and root lines.

Leveling depth $R_{u}$ : The leveling depth is the distance between the crest and mean lines.

Mean depth $R_{m}$ : The mean depth is the distance between the root and mean lines.

Roughness height $R_{a}$ : The roughness height is the arithmetic average deviation of the profile measured perpendicular to the mean line, and is given by

$$
R_{a}=\frac{1}{L} \int_{0}^{L}|y| d x
$$

Roughness width $A_{r}$ : The roughness width is the average horizontal spacing between successive peaks of the predominant surface pattern.

Filling-out factor $k$ : The filling-out factor is the ratio of the mean depth $R_{m}$ to the peak-tovalley height $R$. Thus,

$$
k=\frac{R_{m}}{R}
$$

Profile angle factor $j$ : The profile angle factor is the ratio of the peak-to-valley height $R$ to the roughness width $A_{r}$. Thus,

$$
j=\frac{R}{A_{r}}
$$

Shape factor $l$ : The shape factor is the ratio of the roughness height $R_{a}$ to the leveling depth $R_{u}$. Thus:

$$
l=\frac{R_{a}}{R_{u}}
$$

Both stylus and interferometric methods were used for the examination of the specimens. Interferometric methods were used for all surfaces too fine for the stylus to penetrate to the full depth of the texture.

A Taylor, Taylor, and Hobson Talysurf, model 3 modified, was used for the stylus measurements (ref. 15). This instrument has a stylus with a tip radius of approximately 100 microinches which contacts the specimen with 


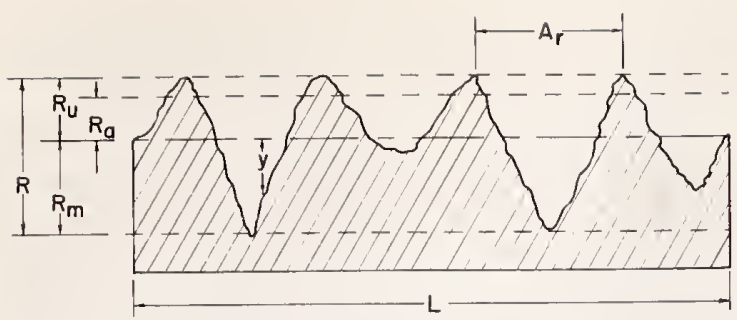

FiguRe 10.-Basic surface measures.

$$
\begin{aligned}
R_{a} & =\frac{1}{L} \int_{0}^{L}|y| d x & j & =\frac{R}{A_{r}} \\
k & =\frac{R_{m}}{R} & l & =\frac{R_{a}}{R_{u}}
\end{aligned}
$$

a static force of 30 milligrams. This low measuring force of the stylus permits the tracing of all hard materials without visibly marking them. The undulations of the specimen are measured relative to a straight datum and are graphed (profilogram) as the stylus is drawn over the surface. The desired parameters can be determined from this profilogram.
A Hilger and Watts surface microinterferometer, model TN 200, was used for the interferometric examination of the surfaces. This instrument is an interference microscope as proposed by Linnik (ref. 16). Photographs made of the interference patterns (microinterferograms) can be interpreted in the same manner as profilograms. All measurements were made using the mercury green spectral line (21.4-microinch wavelength).

To extend the usefulness of this instrument, the Zehender method of fringe demagnification $\lambda_{2}$ was used (ref. 17). Interferometric examination of rough surfaces, where the fringes are deflected to such an extent that they can not be resolved, can be performed by the technique. A transparent film replica of the surface is measured interferometrically while immersed in a fluid. The amount of demagnification is determined by the refractive indices of the replica and the fluid. Figure 11 graphically demonstrates this method. The microinterferograms shown are of the same specimen as measured directly and as measured by the Zehender method. Immersion fluids used were

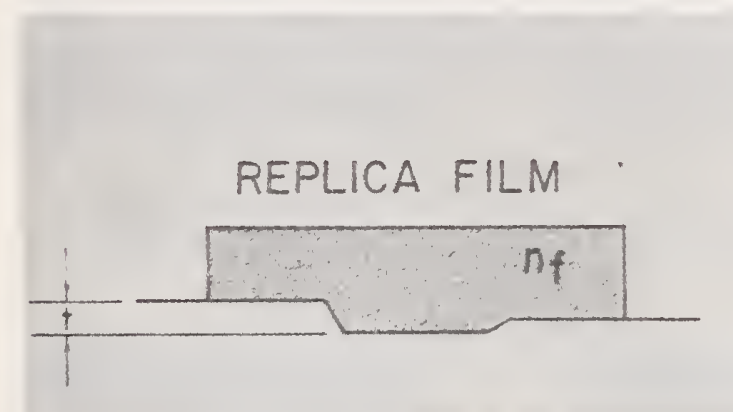

SURFACE TESTED

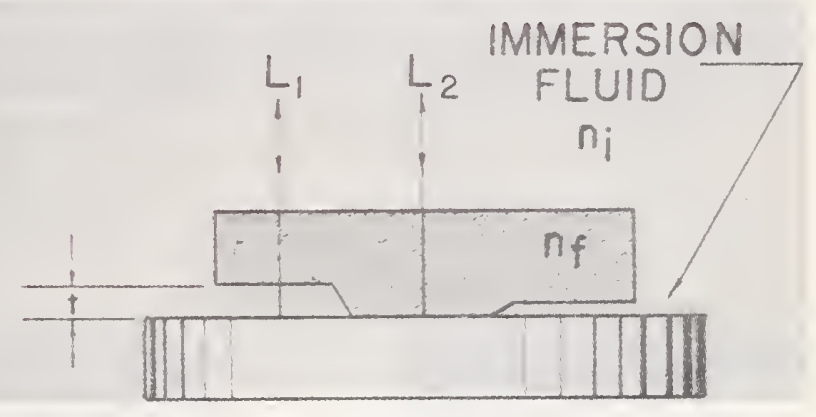

MIRROR

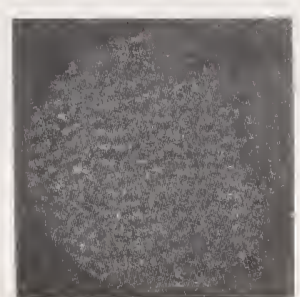

10.7

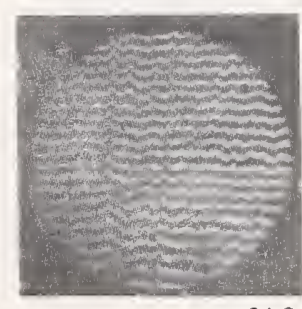

21.0

FIGURE 11. The Zehender method. $O P D=2 R\left(n_{f}-n_{i}\right) \quad \lambda_{z}=\frac{\lambda}{\left(n_{f}-n_{i}\right)}$. 
air, water, and oil, which gave, respectively, approximate demagnification ratios of $1 / 2,2 / 11$, and $1 / 11$.

\section{RESULTS}

Several profilograms and microinterferograms were made at random positions in the area of the specimen used for the spectral reflectance measurements. On those surfaces having lay, measurements were made both parallel and perpendicular to the direction of the lay. The measured parameters for the specimens are tabulated in tables I, II, and
III. The reported values are based on four random traces having traversing lengths of 0.030 inch.

Representative microinterferograms of the surfaces tested are presented in figures 12 , 13,14 , and 15 . The field size shown represents 0.0165 inch. The fringe spacing in microinches is given under each microinterferogram. This represents the half wavelength of the mercury green spectral line in the various immersion fluids used.

Profilograms and microinterferograms of the precision roughness specimen are presented for comparison in figure 16.
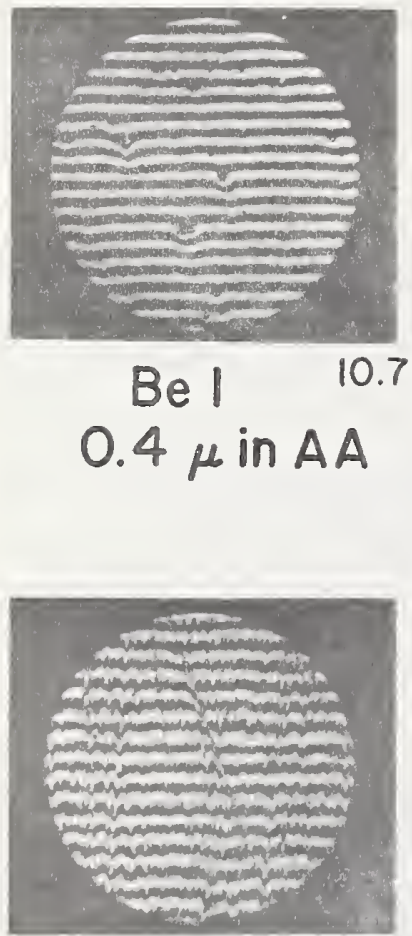

$\begin{array}{ll}\operatorname{Be} 6 & 10.7\end{array}$

1.I $\mu$ in AA
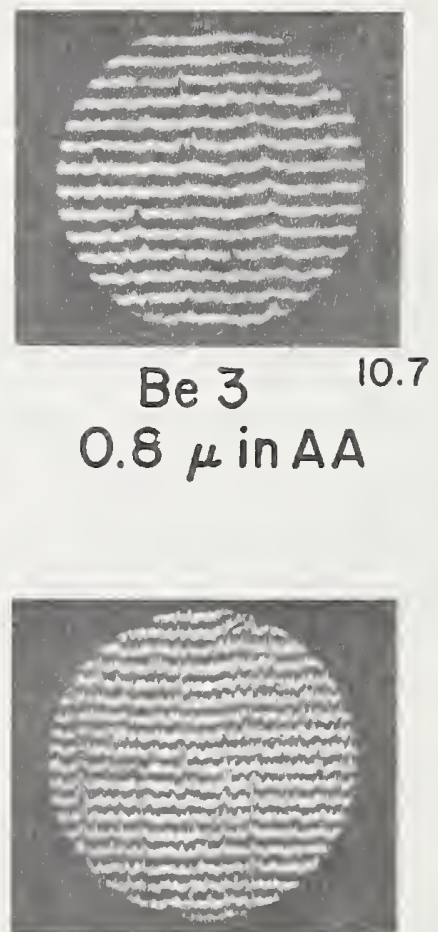

Be 7

10.7

I.I $\mu$ in AA
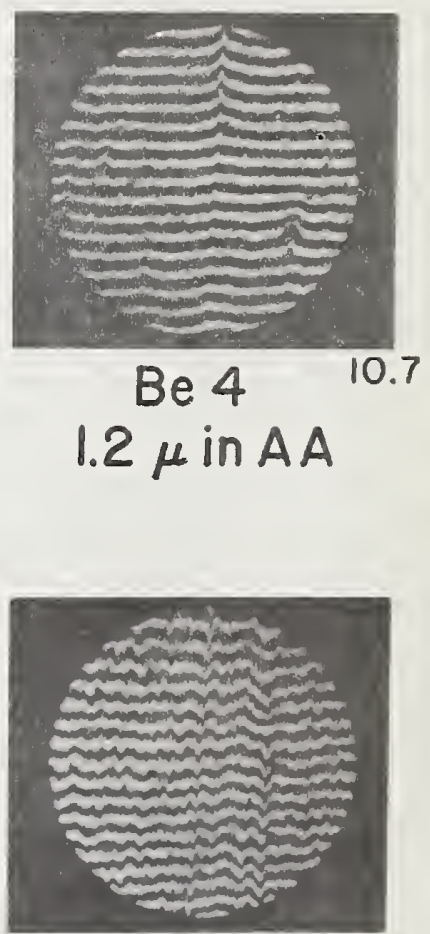

Be 8

21.0

$2.2 \mu$ in $A A$

FIGURE 12.-Microinterferograms of sintered beryllium specimens. Field size shown represents $0.0165-i n$. Fringe spacing in microinch is given below each microinterferogram. 
TABLE I.-Beryllium Specimens

\begin{tabular}{|c|c|c|c|c|c|c|c|c|c|}
\hline Sample & Lay & $\begin{array}{l}\text { Roughness } \\
\text { helght } R_{a}, \\
\mu \text { in. AA }\end{array}$ & $\begin{array}{c}\text { Peak to } \\
\text { valley } \\
\text { helght } R \\
\text { pln. }\end{array}$ & $\begin{array}{c}\text { Levellng } \\
\text { depth } R_{w}, \\
\mu \ln .\end{array}$ & $\begin{array}{c}\text { Mean } \\
\text { depth } R_{m}, \\
\mu \ln .\end{array}$ & $\begin{array}{l}\text { Roughness } \\
\text { width } A_{r,} \\
\qquad \boldsymbol{\mu l n} .\end{array}$ & $\begin{array}{c}\text { Fulling } \\
\text { out factor } \\
R_{\mathrm{m}} / R\end{array}$ & $\begin{array}{l}\text { Profile } \\
\text { angle } \\
\text { factor } \\
R / A \text {. }\end{array}$ & $\begin{array}{c}\text { Shape factor } \\
R_{a} / R_{u}\end{array}$ \\
\hline Be 1.. & Random & 0.4 & 2. 5 & 1.0 & 1.5 & + & 0.60 & $\ldots \ldots$ & 0.4 \\
\hline \multirow[t]{2}{*}{ Be 3} & $\perp$ & 0.8 & 6. 7 & 3. 7 & 3. 0 & 2300 & .45 & 0.003 & .2 \\
\hline & $\|$ & 1. 0 & 4. 5 & 1. 6 & 2. 9 & 5500 & .65 & .0008 & .62 \\
\hline \multirow{2}{*}{ Be $4 \ldots$} & $\perp$ & 1. 2 & 6.3 & 1. 9 & 4. 4 & 2600 & .70 & .002 & .63 \\
\hline & $\|$ & 1. 0 & 4. 7 & 2. 1 & 2. 6 & 15000 & .55 & .0003 & .48 \\
\hline \multirow[t]{2}{*}{ Be 6 6} & $\perp$ & 1. 1 & 7. 4 & 2. 2 & 5. 2 & 600 & .70 & .012 & .50 \\
\hline & $\|$ & 1. 1 & 7. 9 & 3. 7 & 4. 2 & 1600 & .53 & .005 & .30 \\
\hline \multirow[t]{2}{*}{ Be 7} & $\perp$ & 1. 1 & 9. 3 & 2. 9 & 6.4 & 600 & .69 & .016 & .38 \\
\hline & $\|$ & 0.7 & 5. 4 & 1. 3 & 4. 1 & 2200 & .76 & .002 & .5 \\
\hline \multirow[t]{2}{*}{ Be $8 \ldots$} & $\perp$ & 2. 2 & 15. 0 & 6.6 & 8. 4 & 700 & .56 & .021 & .33 \\
\hline & $\|$ & 2. 2 & 11. 9 & 6. 0 & 5. 9 & 2000 & .50 & .006 & .37 \\
\hline
\end{tabular}

+ Not applicable.
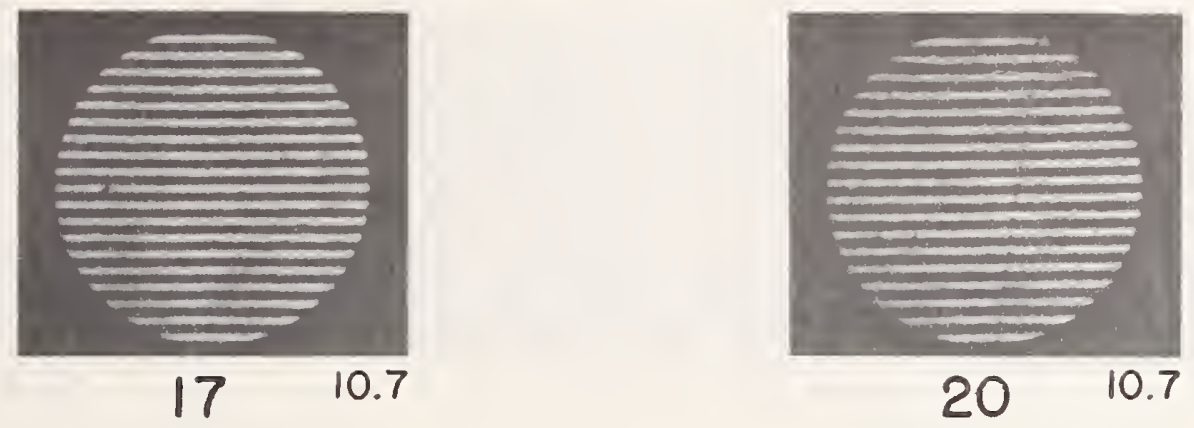
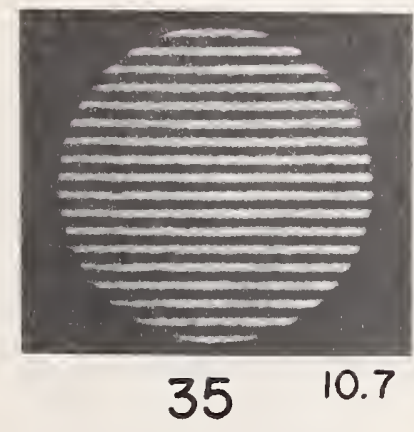

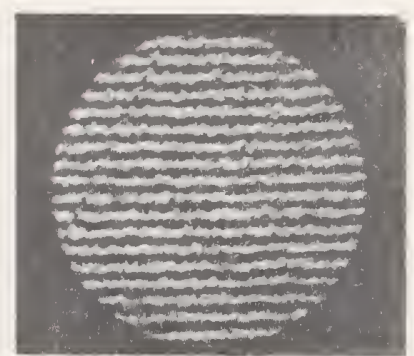

$\begin{array}{ll}77 & 10.7\end{array}$

$0.9 \mu$ in AA

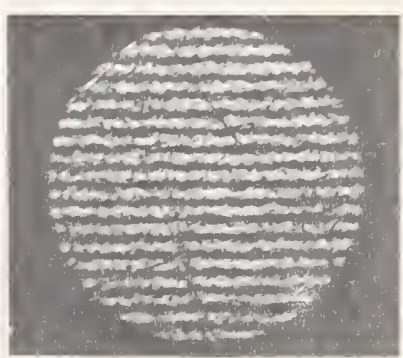

$54 \quad 10.7$ $1.4 \mu$ in AA

FIGURE 13.-Microinterferograms of prepared steel specimens. Field size shown represents 0.0165 -in. Fringe spacing in microinch is given below each microinterferogram. 
TABLE II.-Prepared Steel Specimens

\begin{tabular}{|c|c|c|c|c|c|c|c|c|c|}
\hline Sample & Grit & $\begin{array}{c}\text { Roughness } \\
\text { height } \\
R, \mu \text { in. AA }\end{array}$ & $\begin{array}{c}\text { Peak-to- } \\
\text { valley height } \\
R, \mu \text { in. }\end{array}$ & $\begin{array}{c}\text { Leveling } \\
\text { depth } \\
R_{u}, \mu \text { in. }\end{array}$ & $\begin{array}{c}\text { Mean } \\
\text { depth } \\
R_{m}, \mu \text { in. }\end{array}$ & $\begin{array}{c}\text { Roughness } \\
\text { width } \\
A_{r, \mu \mathrm{in}} .\end{array}$ & $\begin{array}{c}\text { Filling out } \\
\text { factor } \\
R_{m} / R\end{array}$ & $\begin{array}{c}\text { Profile } \\
\text { angle factor } \\
R / A_{r}\end{array}$ & $\begin{array}{c}\text { Shape factor } \\
R_{a} / R_{*}\end{array}$ \\
\hline 17 & Linde A & - & - & - & - & + & $\ldots$ & $\ldots \ldots$ & ....... \\
\hline 20 & $1 / 4 \mu$ & - & - & - & - & + & - . - - & -..... & - \\
\hline 35 & $1 \mu$ & - & 0.3 & - & - & 1400 & -..-- & 0.0002 & - \\
\hline 77 & $3 \mu$ & 0.9 & 5. 8 & 1. 6 & 4. 2 & 700 & 0.72 & .008 & 0.6 \\
\hline 54 & $6 \mu$ & 1. 4 & 8. 7 & 3. 2 & 5. 5 & 500 & .63 & .017 & .44 \\
\hline 40 & $14 \mu$ & 3. 9 & 18. 0 & 6. 6 & 11. 4 & 600 & .63 & .030 & .59 \\
\hline 86 & $20 \mu$ & 5. 0 & 25.5 & 12. 1 & 13. 4 & 700 & .53 & .036 & .41 \\
\hline 85 & $30 \mu$ & 13. 3 & 81.5 & 38. 2 & 43. 3 & 700 & .53 & .116 & .35 \\
\hline 24 & $45 \mu$ & 15. 5 & 96.0 & 34.5 & 61.5 & 800 & .64 & .120 & .45 \\
\hline 61 & $60 \mu$ & 15. 6 & 87. 0 & 38.5 & 48. 5 & 900 & .56 & .098 & .41 \\
\hline
\end{tabular}

- Below measurement capabilities.

+ Not applicable.
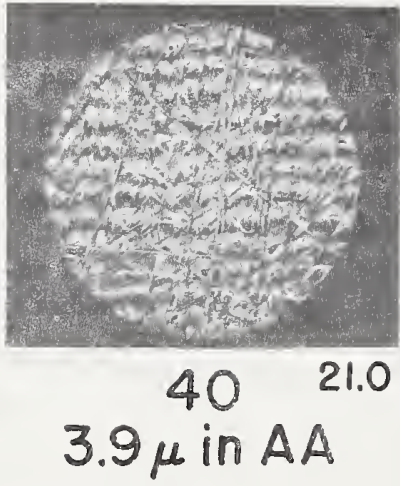

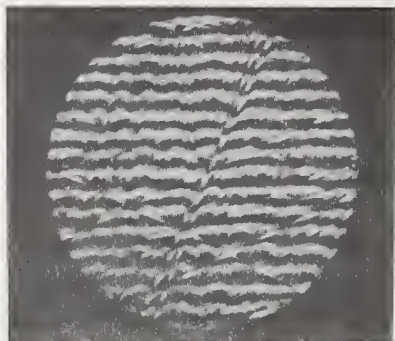

$86 \quad 60.5$

$5.0 \mu$ in $A$ A
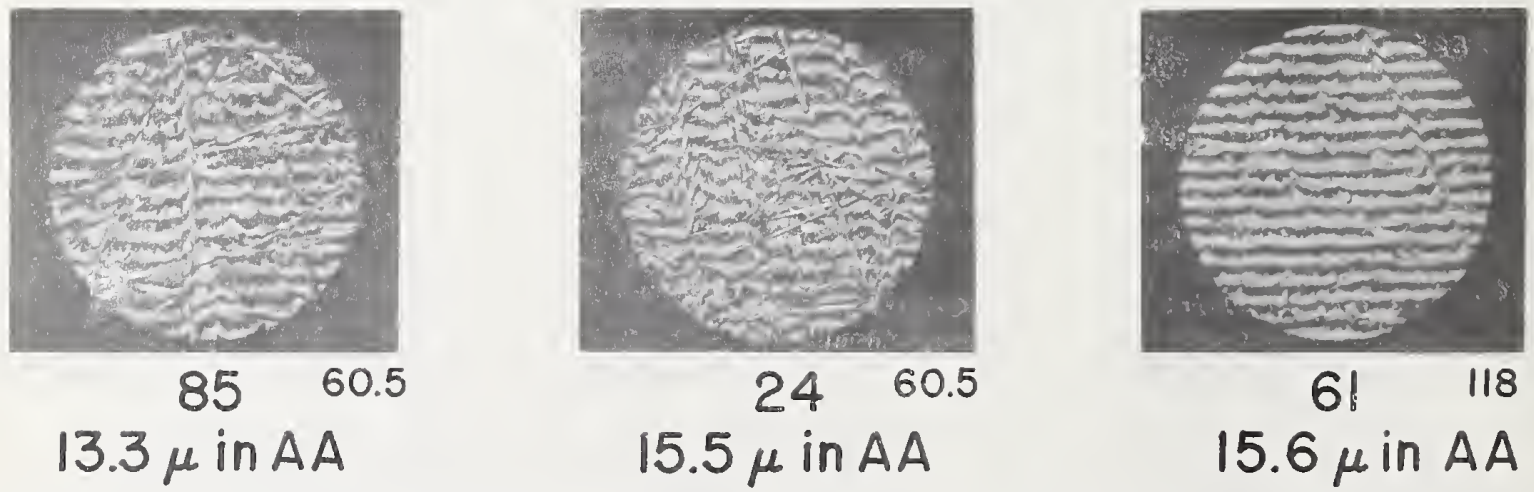

Figure 14.-Microinterferograms of prepared steel specimens. Field size shown represents 0.0165-in. Fringe spacing in microinch is given below each microinterferogram. 
TABLE III.-Commercial Steel Specimens ${ }^{a}$

\begin{tabular}{|c|c|c|c|c|c|c|c|c|}
\hline Sample & $\begin{array}{l}\text { Roughness } \\
\text { height } R_{a} \\
\mu \text { in. As }\end{array}$ & $\begin{array}{c}\text { Peak-to-valley } \\
\text { heights } R, \\
\mu \text { in. }\end{array}$ & $\begin{array}{l}\text { Leveling } \\
\text { depth } R_{u} \text {, } \\
\text { in. }\end{array}$ & $\begin{array}{l}\text { Mean depth } \\
R_{m} \mu \text { in. }\end{array}$ & $\underset{\text { width } A_{r} \mu \text { in. }}{\text { Roughness }}$ & $\begin{array}{l}\text { Filling out } \\
\text { factor, } R_{m} / R\end{array}$ & $\begin{array}{l}\text { Profle angle } \\
\text { factor } R / A \text {, }\end{array}$ & $\begin{array}{l}\text { Shape factor } \\
R_{a} / R_{u}\end{array}$ \\
\hline 1 & 0.7 & 3. 0 & $\mathrm{n}$ & $\mathrm{n}$ & 200 & $\mathrm{n}$ & 0.015 & - - - - - - - \\
\hline 2 & - & - & - & - & 1 & - & - & - - - - - - \\
\hline 3 & 0.6 & 3. 0 & $\mathrm{n}$ & $\mathrm{n}$ & 1000 & $\mathrm{n}$ & 0.003 & 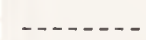 \\
\hline 4 & 0.5 & 2. 5 & n & $\mathrm{n}$ & 700 & $\mathrm{n}$ & 0. 004 & - - - - - \\
\hline 5 & 0.8 & 3.0 & 1. 1 & 1. 9 & 500 & 0. 63 & 0.006 & 0.7 \\
\hline 31 & 1. 9 & 8. 4 & 1. 3 & 7. 1 & 600 & 0.85 & 0.014 & 1.5 \\
\hline Caliblock & & & & & & & & \\
\hline $\begin{array}{c}20 \\
\text { Caliblock }\end{array}$ & 20 & 80 & 40 & 40 & 600 & 0.5 & 0.13 & 0.5 \\
\hline 125 & 120 & 480 & 240 & 240 & 3600 & 0.5 & 0.13 & 0.5 \\
\hline
\end{tabular}

- n Not determined.

- Below measurement capabllities.

+ Beyond measurement capabllitles.
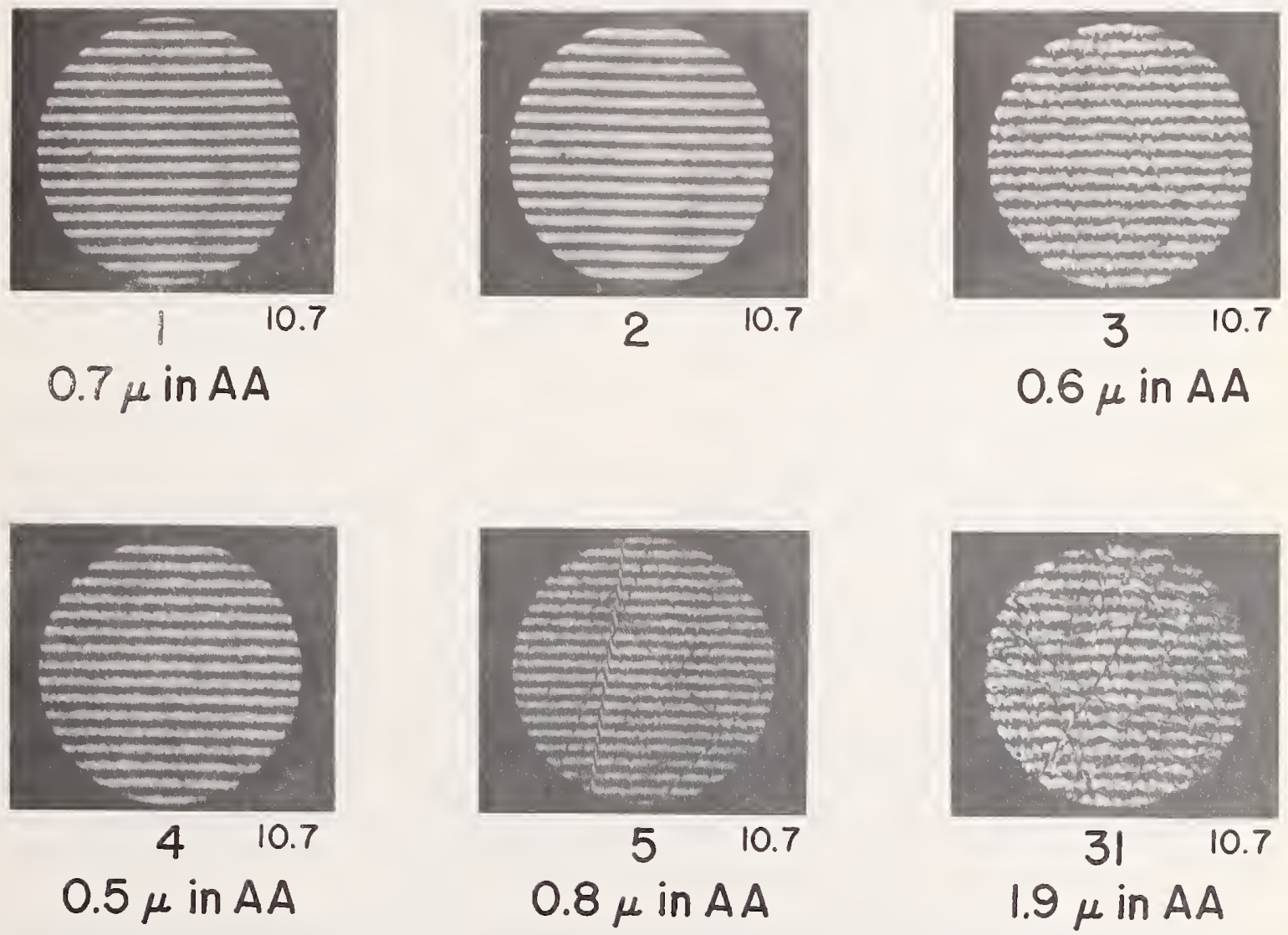

Figure 15.-Microinterferograms of commercial steel specimens. Fringe size shown represents $0.165-i n . \quad$ Fringe spacing in microinch is given below each microinterferogram. 


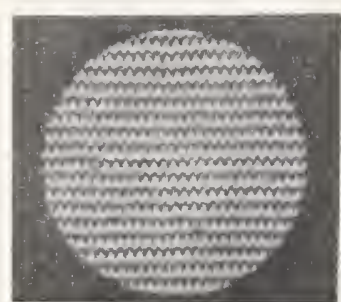

118

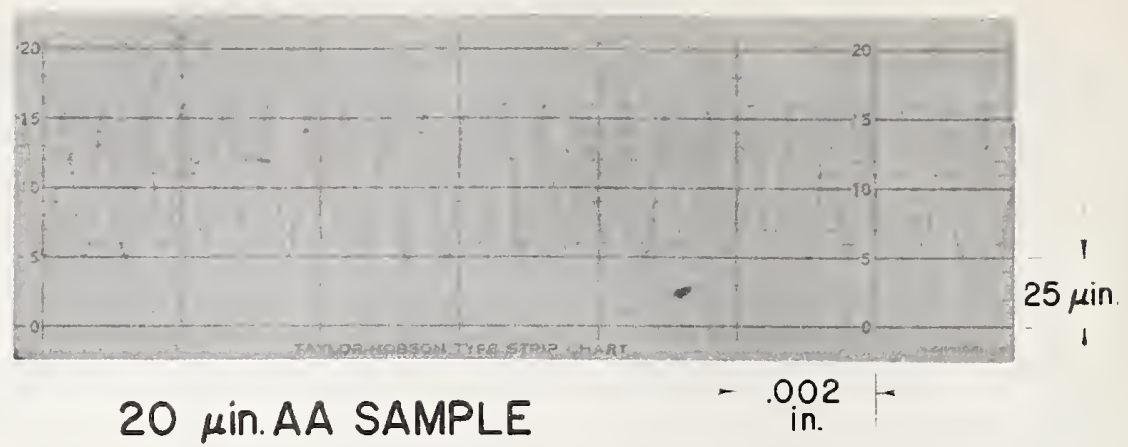

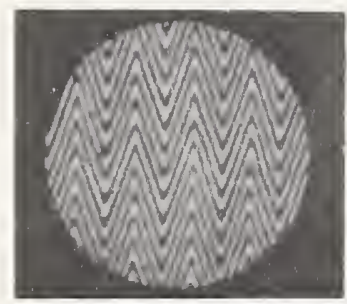

118

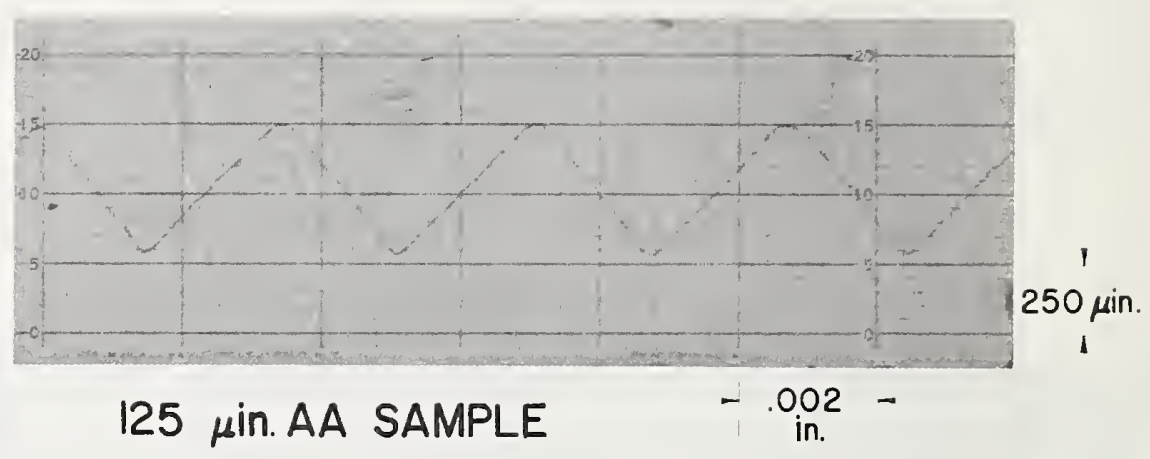

FIGURE 16.-Profilograms and microinterferograms of the two sample areas of the precision roughness specimen.

\section{REFERENCES}

1. Nutrung, R. D.: Interpretation of Data Obtained with Spectrophotometers of the Polarizing Type. Textile Research, vol. V, no. 9, pp. 391-400, July 1935.

2. Reimann, Genevieve, Judd, Deane B., and KeEgan, Harry J.: Spectrophotometric and Colorimetric Determination of the Colors of the TCCA Standard Color Cards. Jour. Optical Soc. of America, vol. 36, no. 3, Mar. 1946, pp. 128-159.

3. Bennetr, H. E., and Porteus, J. O.: Relation Between Surface Roughness and Specular Reflectance at Normal Incidence. Jour. Optical Soc. of America, vol. 51, no. 2, Feb. 1961, pp. 123-129.

4. Bennett, H. E.: Specular Reflectance of Aluminized Ground Glass and the Height Distribution of Surface Irregularities. Jour. Optical Soc. of America, vol. 53, no. 12, Dec. 1963, pp. 13891394.

5. Porteus, J. O.: Relation Between the Height Distribution of a Rough Surface and the Reflectance at Normal Incidence. Jour. Optical Soc. of America, vol. 53, no. 12, Dec. 1963, pp. 1394-1402.
6. Donovan, T. M., Ashley, E. J., and Bennett, H. E.: Effect of Surface Damage on the Reflectance of Germanium in the 2650-10 000-A Region. Jour. Optical Soc. of America, vol. 53, no. 12 , Dec. 1963 , pp. 1403-140'.

7. Olson, O. H., and Pontarelli, D. A.: Asymmetry of an Integrating Sphere. Appl. Optics, vol. 2, no. 6, June 1963, pp. 631-633.

8. Gibson, Kasson S., and KeEgan, Harry J.: Calibration and Operation of the General Electric Recording Spectrophotometer of the National Bureau of Standards. Jour. Optical Soc. of America, vol. 28, no. 10, Oct. 1938, pp. 372-385.

9. Keegan, Harry J., Schleter, John C., and Judd, Deane B.: Glass Filters for Checking Performance of Spectrophotometer-Integrator Systems of Color Measurement. Res. Paper 66A3154, Jour. Res. Nat. Bur. Standards, vol. 66A, no. 3, May-June 1962, pp. 203-221.

10. Anon: Preparation and Colorimetric Properties of a Magnesium-Oxide Reflectance Standard. NBS Letter Circular LC-547, U.S. Dept. Commerce, Mar. 17, 1939.

11. Keegan, Harry J., and Gibson, Kasson S.: On the Use of Working Standards of Didymium and Vitrolite Glasses for Spectrophotometric Measurements. Proc. Twenty-Ninth Annual Meeting 
of O.S.A., Jour. Optical Soc. of America, vol. 34, no. 12 , Dec. 1944 , p. 770 .

12. Olsen, K. V.: The Standardization of Surface Roughness, Brüel and Kjoer Technical Review No. 3, 1961.

13. American Standard B46.1-1962. Surface Texture.

14. British Standard 1134-1961. The Assessment of Surface Texture.

15. Reason, R. E., Hopkins, M. R., and Garrod, R. I.: Report on the Measurements of Surface
Finish by Stylus Method. Taylor-Hobson Research Dept., 1944.

16. Linnik, W.: Ein Apparat für Mikroskopisch-interferometrische Untersuchung Reflectierende Objecte. Compt. Rend. Acad. Science USSR S. 21 Bericht: A. Instr. 54, 1934, p. 462.

17. ZeHender, E.: An Interference Method for Examining Rough Surfaces. Z. VDI, vol. 94, no. $14-15,1952$, p. 456.

\section{DISCUSSION}

J. T. NEU, General Dynamics Astronautics: Mr. Keegan, you indicated that the Cary and the GE spectrophotometers were unsatisfactory for making reflectance measurements. Why is this?

KEEGan: The Cary model 14 spectrophotometer was designed and built for the measurement of spectral transmittance of solutions such as those prepared by chemists. To measure reflectance on the Cary 14 spectrophotometer an attachment must be purchased for diffuse reflection ineasurements and the instrument must be partially dismantled to install this attachment. Because the 100-percent curve is set from two diffusing standards, and because of the baffles in the integrating sphere, the results obtained for specular specimens depend upon the mode of illumination of the specimen.

The GE recording spectrophotometer accurately measures spectral directional reflectance of diffusing samples and samples that are nearly perfect mirrors so that the specular component may be excluded from the measurement. For semiglossy samples and nonplane surfaces the excluded measurement is in question because of the size of the specular cup. This instrument comes equipped with an integrating for measurements of transmittance and reflectance. It was designed and built for the measurement and specification of colored materials. Does this answer your question?

NEU: No. Is it not possible that the problem is with the integrating sphere that you attach to the spectrophotometer-rather than the spectrophotometer? I consider the spectrophotometer to be an excellent instrument.

KeEgan: Yes, it is good for diffuse reflectance if it has the proper integrating sphere.

NEU: That is the point! It is not the instrument, it is the device that you fasten to it.

KEEGAN: I rely on the Cary 14 for transmittance and diffuse reflectance measurements. It is the present reflectance attachment that does not measure specular reflectance accurately. 


\title{
Ellipsoidal Mirror Reflectometer*
}

\author{
S. Thomas Dunn ${ }^{1}$ and Joseph C. Richmond \\ Institute for Basic Standards, National Bureau of Standards, Washington, D.C. \\ and
}

John A. Wiebelt

Oklahoma State University

(January 6, 1966)

\begin{abstract}
A new ellipsoidal mirror reflectometer is described, in which radiant flux from an infrared monochromator is focused on a specimen placed at the first focal point of the ellipsoid and a thermopile detector is placed at the second. Errors associated with angular and areal variations in sensitivity of the detector and with aberrations in the optics were largely eliminated through use of a small averaging sphere placed over the detector. Losses caused by the presence of the entrance hole in the ellipsoidal mirror and from mirror absorption were evaluated both theoretically and experimentally. Corrections for these losses permitted absolute reflectance to be obtained for both diffuse and partially diffuse reflecting specimens. In addition, the unique optics of the ellipsoidal mirror provide more versatility than is available in previous reflectometers. This versatility includes the ability to accurately measure directional-hemispherical, specular, nonspecular, and directional-annular cone reflectance. An analysis of the accuracy of the instrument indicates that an accuracy of better than one percent is possible for all engineering materials. The use of the sulfur averaging sphere also allowed the construction of a simple accurate specular reflectometer for calibration of the mirror reference standards used in these measurements.
\end{abstract}

Key Words: Averaging spheres, bidirectional reflectance, diffuse reflectance, ellipsoidal reflectometer, infrared, infrared detectors, reflectance, reflectometer, spatial sensitivity, spectral reflectance, specular reflectance.

\section{Introduction}

This paper describes the development and analysis of an ellipsoidal mirror reflectometer, which has proved to be highly versatile and capable of providing reflectance data of high accuracy. Although the instrument was developed specifically for the infrared portion of the spectrum, it is equally applicable to the visible and near infrared portions.

\section{Definition of Terms}

The terminology used in this paper is that used in the field of radiant heat transfer. Because some of the terms have different meanings than are normally used in the field of optics, they will be defined here.

Reflectance is the fraction of incident flux that is reflected by a specimen. It will vary with the wavelength of incident flux and with the direction of propagation, relative to the surface, of the incident and reflected flux. It is thus necessary to modify the term reflectance to indicate the geometric and wavelength conditions of measurement.

*The work described in this paper was done under NASA Contract No. R-09-022-032.

1 Present address: Dunn Assoc. Inc.. 910 1.aredo Rosd. Silver Spring. Md. 20901
Spectral, in a narrow wavelength bond centered at a specified wavelength. Spectral reflectance may be plotted as a function of wavelength to produce a spectral reflectance curve.

Directional, in a small solid angle about a given direction.

Hemispherical, in all possible directions from a surface.

Diffuse, incident on a surface or reflected from a surface with equal radiance in all possible directions.

Directional hemispherical reflectance is the fraction of the flux incident in a small solid angle $\Delta \omega$ about the direction $\varphi, \theta$, that is reflected into the hemisphere above the surface. Mathematically

$$
\rho(\varphi, \theta)=\frac{\int_{0}^{2 \pi} L^{\prime}\left(\varphi^{\prime}, \theta^{\prime}\right) \cos \varphi^{\prime} d \omega^{\prime}}{L \varphi, \theta \cos \varphi(\Delta \omega)}
$$

in which $L_{\varphi}, \theta$ is the incident radiance in the solid angle $\Delta \omega$ about the direction $\varphi, \theta: \varphi$ is the angle from the normal to the surface and $\theta$ is the azimuth from some fixed point on the specimen. $L^{\prime}\left(\varphi^{\prime}, \theta^{\prime}\right)$ is the functional description of the reflected radiance in the direction $\varphi^{\prime} \theta^{\prime}$. 
Specular reflectance is the fraction of the flux incident in a small solid angle $\Delta \omega$ centered about the direction $\varphi, \theta$ that is reflected into a small solid angle $\omega^{\prime}$ centered about the direction $\varphi^{\prime}, \theta^{\prime}$, where $\varphi^{\prime}=\varphi$ and $\theta^{\prime}=\theta+\pi$. For mirror surfaces, $\omega^{\prime}=\Delta \omega$, but for engineering surfaces in general, $\omega^{\prime}>\Delta \omega$. The size of $\Delta \omega$ and $\omega^{\prime}$ should be specified in each case. Mathematically

$$
\rho_{\text {specular }}=\frac{\int_{\omega^{\prime}} L^{\prime}\left(\varphi^{\prime}, \theta^{\prime}\right) \cos \varphi^{\prime} d \omega^{\prime}}{L_{\varphi, \theta} \cos \varphi \Delta \omega} .
$$

For diffusely reflecting specimens, the defined specular reflectance includes the flux that is diffusely reflected in the specular direction.

Nonspecular reflectance is the directional hemispherical reflectance (eq (l)) minus the specular reflectance (eq (2)).

Directional annular cone reflectance is the fraction of the flux incident in a small solid angle $\Delta \omega$ centered about the direction $\varphi^{\prime}, \theta^{\prime}$ that is reflected into the annular cone defined by the angles $\varphi_{1}$ and $\varphi_{2}$. Mathematically

$$
\rho(\text { d.a.c. })=\frac{\int_{\varphi_{1}}^{\varphi_{2}} \int_{0}^{2 \pi} L^{\prime}\left(\varphi^{\prime}, \theta^{\prime}\right) \cos \varphi^{\prime} \sin \varphi^{\prime} d \varphi^{\prime} d \theta^{\prime}}{L \varphi, \theta \cos \varphi \Delta \omega} .
$$

TABLE 1. Terminology: Flux Terminology

$F_{m}=$ The flux reffected by the reference mirror.

$F_{i}=$ The flux incident on the sample at the first focal point.

$F_{r}=$ The total flux reflected by the sample (not including interreflections).

$F_{\alpha}=$ The flux effectively absorbed by the ellipsoidal mirror.

$F_{u}=$ The flux absorbed by the wire divided by $\left(\bar{\rho}_{\epsilon}\right)_{u}$. (i.e., $F_{u}$ is the flux leaving the sample headed in the direction of the wires.)

$F_{s p}=$ The flux that is initially shaded from the detector by the sample divided by $\bar{\rho}_{\epsilon}$.

$F_{s r}=$ The flux that reaches the detector after multiple reflections with the sample divided by $\bar{\rho}_{\epsilon}$.

$F_{h}=$ The flux lost out the entrance hole.

$F_{s}=$ The total flux crossing the first focal plane (excluding detector ellipsoid interchanges) divided by $\left(\bar{\rho}_{\epsilon}\right)_{s}$.

$F_{s 1}=$ The total flux crossing the first focal plane when shield $A_{s h}$ is used divided by $\left(\bar{\rho}_{\epsilon}\right)_{s 1}$.

$F_{s 2}=$ The total flux crossing the first focal plane when shield $\mathrm{A}_{s 2}$ is used divided by $\left(\bar{\rho}_{\epsilon}\right)_{s 2}$.

$F_{a}=$ The total flux crossing the first focal plane when shield $A_{d}$ is used divided by $\left(\tilde{\rho}_{\epsilon}\right)_{d}$. "All fluxes are defined on the basis of the flux leaving the sample. The subscript $D$
added to the subscript of any of the above fiuxes implies the flux actually viewed by the detector when the defined fiux is measured by the detector.

\section{Reflectance Terminology}

$\rho_{\epsilon}^{\prime}=$ Effective reflectance of a point on the ellipsoidal mirror.

$\bar{\rho}_{\epsilon}=$ Effective reflectance of the central area of the ellipsoidal mirror.

$\left(\bar{\rho}_{\epsilon}\right)_{s}=$ Effective reflectance of the ellipsoidal mirror to the flux $F_{s}$.

$\left(\bar{\rho}_{\epsilon}\right)_{s 1}=$ Effective reflectance of the ellipsoidal mirror to the flux $F_{s 1}$.

$\left(\bar{\rho}_{\epsilon}\right)_{s 2}=$ Effective reflectance of the ellipsoidal mirror to the flux $F_{s 2}$.

$\left(\bar{\rho}_{\epsilon}\right)_{d}=$ Effective reflectance of the ellipsoidal mirror to the flux $F_{d}$.

$\rho_{h s}=$ The hemispherical reflectance of the sample.

$\rho_{h m}=$ The hemispherical reflectance of the reference mirror.

$\rho_{m}=$ The reflectance of the specular reference standard.

$\rho_{s}=$ The normal hemispherical reflectance, of a specimen and is approximately equal to $\rho\left(7^{\circ}, \theta\right)$.

$\left(\bar{\rho}_{e}\right)_{w}=$ The effective reflectance of the ellipsoidal mirror to the flux $F_{w}$.

$\rho_{\epsilon d}=$ Effective reflectance of the ellipsoidal mirror to diffuse flux from the second focal point.

\section{Area Terminology}

$A_{h}=$ Area of the entrance hole.

$A_{\epsilon}=$ Area of the opening of the ellipsoidal mirror in the first focal plane minus the area of the shield $A_{d}$.

$A_{s h}^{\prime}=$ Area of the shield $A_{s h}$ in the first focal plane.

$A_{s}^{\prime}=$ Area of the sample in the first focal plane.

$A_{s 1}^{\prime}=$ First focal plane area of the image of the sphere entrance port at the second focal point.

$A_{d}=$ Area of the shield used to block the specular component.

$A_{s 2}=$ Represents the shield used to establish the flux distribution for mirror loss corrections.

$A_{s h}=$ Projection of $A_{s h}^{\prime}$ from the second focal point onto the ellipsoidal mirror.

$A_{s}=$ Projection of $A_{s}^{\prime}$ from the second focal point onto the ellipsoidal mirror.

$A_{s 1}=$ Projection of $A_{s 1}^{\prime}$ from the second focal point onto the ellipsoidal mirror.

\section{Angle Terminology}

$\varphi=$ Angle of incidence, measured from the normal to the surface.

$\theta=$ Azimuth of incidence, measured from a fixed point on the specimen.

$\omega=$ Solid angle of incidence.

$\varphi^{\prime}=$ Angle of reflection, measured from the normal to a surface. 
$\Phi^{\prime}=$ Azimuth of reflection, measured from a fixed point on the specimen.

$\omega^{\prime}=$ Solid angle of reflection.

\section{Radiance Terminology}

$L=$ Radiance of incident flux, watts per steradian per square centimeter (projected normal to the direction of incidence).

$L \varphi, \theta=$ unidirectional radiance.

$L^{\prime}=$ Reflected radiance.

$L^{\prime}\left(\varphi^{\prime}, \theta^{\prime}\right)=$ Functional description of the reflected radiance in the direction $\varphi^{\prime}, \theta^{\prime}$.

\section{Miscellaneous Terminology}

$\eta=$ Efficiency of the averaging sphere (i.e., the ratio of the flux viewed by the detector to that entering the sphere).

$\eta^{\prime}=$ The ratio of flux leaving the entrance port of the sphere to that incident on the entrance port.

$f_{s-\epsilon}=$ Diffuse configuration factor from the sphere entrance port to the ellipsoidal mirror (corrected for shading effects of the sample and sample support and for the effect of the entrance hole).

\section{Methods of Measuring Reflectance}

A reflectometer was desired that would measure absolute reflectance (fraction of incident flux reflected) under conditions approximating normal irradiation and hemispherical viewing, with an accuracy of at least 1 percent, of specimens at temperatures in the range 100 to $800{ }^{\circ} \mathrm{K}$, and over the wavelength range of 1 to $15 \mu$.

A number of different methods have been used to measure reflectance. Specular reflectance of mirrors $[1]^{2}$ is of minor interest here, and will not be discussed further. Three different types of reflectometers have been used to measure directional hemispherical reflectance: (1) integrating sphere, (2) hemispherical source, and (3) integrating hemisphere instruments.

(a) Integrating sphere reflectometers [2, 3, 4] are widely used at wavelengths below about $2.5 \mu$, but are generally not suitable for use at longer wavelengths, and will not be discussed further.

(b) Hemispherical source instruments measure the reflectance factor ${ }^{3}$ under conditions of diffuse irradiation and directional viewing, which is equivalent to directional-hemispherical reflectance. The principal instrument of this type for use in the infrared is the Hohlraum reflectometer $[5]$, in which a cooled specimen is inserted into a heated blackbody cavity, where it is irradiated diffusely. The specimen and a spot on the cavity wall are viewed alternately through an

\footnotetext{
${ }^{2}$ Figures in brackets indicate the literature references at the end of this paper.

3 The ratio of the flux reflected directionally by a diffusely illuminated sample to that reflected by the diffuse complete reflector under the same conditions of irradiation and viewing.
}

opening in the cavity, and the ratio of the two fluxes is reported as the reflectance. The method is useful in the 1 to $25 \mu$ range. Major errors, that are difficult to eliminate or correct for, arise as a result of heating the specimen and thermal gradients in the cavity walls.

(c) Integrating hemisphere reflectometers [6] make use of a hemisphere to focus the flux reflected by a specimen, located at one conjugate focus of the hemisphere, onto a detector located at a second conjugate focus. Major errors, which are difficult to correct for, arise due to spherical aberrations [7], and to variations in areal and angular sensitivity of the detector [8]. Several modifications $[9,10,11]$ have been made to the basic Coblentz instrument.

None of the above instruments meets all of the requirements stated at the beginning of this section. Integrating sphere instruments in general are not useful beyond about $2.5 \mu$. Hemispherical source instruments have no provision for chopping of incident flux, and hence are not suitable for measuring reflectance of hot specimens, and integrating hemisphere instruments have errors because the mirror does not satisfactorily focus the reflected flux and available detectors do not satisfactorily measure the reflected flux. Other disadvantages which limit the usefulness of available reflectometers include (1) errors due to flux losses such as those out entrance and exit ports which are difficult to correct for accurately, (2) lack of versatility; several are restricted by design to measurements under a single set of conditions; and (3) several of them can only be used to measure relative reflectance, and hence require a reference standard calibrated in absolute reflectance, which is not available, in order to give absolute reflectance.

It was decided to build a new reflectometer that would meet all of the requirements previously outlined. The Coblentz hemisphere reflectometer was modified by replacing the hemispherical mirror by an ellipsoidal mirror which permitted the specimen and detector to be separated by about $17 \mathrm{in}$., and thus permitted heating and cooling of the specimen without affecting the detector. Use of the ellipsoidal mirror, with specimen and detector at true foci, also greatly reduced errors due to spherical aberrations.

\section{Design of the Ellipsoidal Mirror Reflectometer ${ }^{4}$}

The ellipsoidal mirror reflectometer (EMR) was initially designed in 1959 , and construction was completed in 1960 [12]. However, because of serious detector problems [13] and general secondary priority of the work, progress was slow, and the desired results were not achieved until late in 1964.

\footnotetext{
- Certain commerical instruments and equipment are identified in this paper in order to adequately specify the experimental procedure involved. In no case does such identification imply recommendation or endorsement by the National Bureau of Standards, nor does it imply that the instrument or equipment identified is necessarily the best available for the purpose.
} 
(a) Basic design. The basic design of the instru* ment is shown in figure 1 . An ellipsoidal mirror, ${ }^{5}$ $12 \frac{1}{4} \mathrm{in}$. in diameter and $3 \frac{5}{8} \mathrm{in}$. high, is the principal feature of the design. Its focal points are located about $3 \frac{5}{8}$ and $203 / 8$ in. from the apex, and it has a linear magnification factor of about 5.7.

The flux from a Globar source is chopped at $11.3 \mathrm{c} / \mathrm{s}$ and focused on the entrance slit of a Perkin-Elmer Model 83 monochromator. The exit beam from the monochromator is refocused, through a small hole in the ellipsoidal mirror, onto the first focal plane of the ellipsoid, which is in the plane of the edge of the mirror, and centered on the first focal point. A 10 -junction thermopile is used as the detector, and its output is amplified by a synchronous amplifier. The angle of incidence of the incident beam on the sample at the first focal point is $7^{\circ}$.

In the absolute mode the incident flux is measured with the detector at the first focal point, and the reflected flux is measured with the specimen at the first focal point and the detector at the second focal point. Thus, after correcting for system losses, the absolute directional hemispherical reflectance is measured for $\varphi=7^{\circ}$ (i.e., $\rho\left(7^{\circ}, \theta\right)$ ). In the relative mode the detector remains at the second focal point, and the fluxes reflected by a standard and the specimen are measured.

${ }^{3}$ Purchased from Strong Electric Corporation, City Park Avenue, Toledo, Ohio.

$36^{\prime \prime}$ RAOIUS SPHERICAL MIRROR

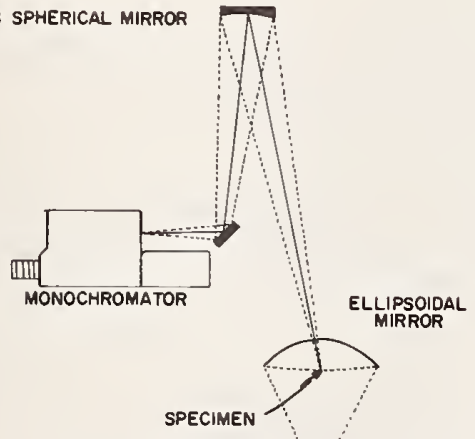

DETECTOR AT SECONO FOCAL POINT

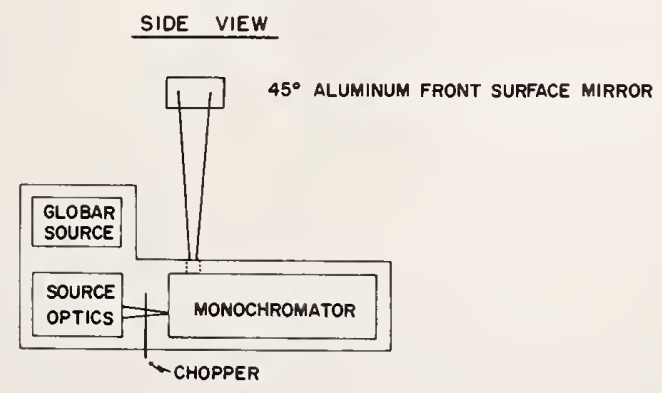

PARTIAL TOP VIEW

Flgure 1. Basic design of the ellipsoidal mirror reflectometer.
The relative measurement tends to eliminate the errors due to atmospheric absorption and to reduce errors from other sources.

It is more convenient and accurate to use the EMR in the relative mode. first because it is inconvenient and time consuming to move the detector back and forth between the focal points for an absolute measurement, and second because the use of a specular reflectance standard does not introduce a significant error into the measurement, because its reflectance can be measured to about 0.001 [1].

(b) Advantages of the EMR. The EMR has the same inherent errors as Coblentz hemisphere systems, with the following exceptions:

(1) Aberrations are reduced [7].

(2) The reflected flux is concentrated in a cone of $24^{\circ}$ half angle, instead of a whole hemisphere; hence detector angular sensitivity problems are greatly reduced.

(3) The detector and sample are separated by 17 in., hence the specimen can be heated or cooled without affecting the detector.

(4) The unique optical system permits accurate calibration of mirror and hole losses for practically all engineering surfaces (except diffraction gratings and extremely good retroreflectors).

The optics of the ellipsoidal mirror allow accurate description of the distribution of the reflected flux, because the areal distribution of the reflected energy crossing the first focal plane is related precisely to the geometric distribution of the reflected flux for small areas of irradiation at the first focal point. That is, every direction $\varphi, \theta$ in the hemisphere above the surface is represented by a point $P$ in the first focal plane, and every solid angle centered in the direction $\varphi, \theta$ is represented by an area about $P$. Thus, it is possible to select the flux that the detector views, by blocking out the unwanted flux with a shield in the first focal plane. Hence, a specular component which has a solid angle determined by the area of the opening in a shield placed in the first focal plane, can be measured. Similarly, the bidirectional reflectance for $7^{\circ}$ incidence can be measured by varying the position of a small hole in the shield. The directional annular cone reflectance can be measured by use of a set of circular disks centered on the first focal point. Further, the ability to measure the geometric distribution of the reflected flux enables precise corrections to be made for the system losses, as will be described later.

(c) Detector problems. Because of the large magnification factor of the ellipsoidal mirror, about 5.7 linear, a large-area detector is required to view the entire image of the irradiated area. The irradiated area of the specimen is about $2 \times 2 \mathrm{~mm}$ in size, and is enlarged to more than $1 \times 1 \mathrm{~cm}$ at the detector. When the instrument is used in the absolute mode it must measure equally well the flux in a $2 \times 2 \mathrm{~mm}$ image incident in a cone of $4^{\circ}$ half angle centered about a direction $7^{\circ}$ from the normal, and that in a $1.2 \times 1.2$ $\mathrm{cm}$ image incident in a cone of $24^{\circ}$ half angle centered about the normal. 


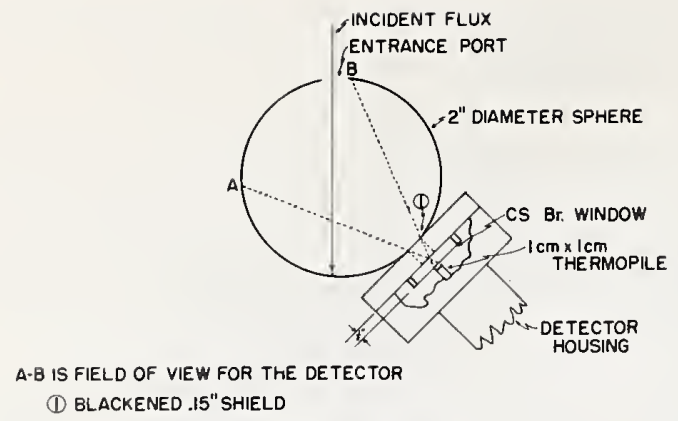

a) EXTERNAL SHIELD

Figure 2. Basic design of averaging sphere. The sphere is lined with $m \mu$ sulfur.

Preliminary tests, in which a Golay-cell detector and a 10-junction thermopile detector were used, gave reflectances that were in error by 40 to 50 percent. These large errors were later found to be due primarily to the variation in areal sensitivity of the detector [13] and overfilling of the detector sensing area. Detector response to the flux in a $1 / 16 \mathrm{in}$. diam beam was found to vary by as much as 50 percent as the beam was scanned across the sensitive area of the detector. There was also some variation of angular sensitivity, particularly at angles from the normal greater than $27^{\circ}$. Detector response to the flux in a 3 by $3 \mathrm{~mm}$ beam centered on the sensitive area of the thermopile detector varied only slightly as the angle of incidence was changed from $0^{\circ}$ to $27^{\circ}$, but fell off rapidly at angles greater than $27^{\circ}[14,15]$.

(d) Flux averaging devices. Several flux averaging devices were investigated $[14,15]$ and a 2 -in. diam sphere lined with mu sulfur, as shown in figure 2 , was selected for use with the instrument. Tests using the averaging sphere and the thermopile detector showed that the variation in areal and angular sensitivity of the combination had been reduced to a point where errors from this source could be almost completely eliminated, at wavelengths from the visible out to about $10 \mu$.

\section{Analysis of the EMR}

In the relative mode the EMR is used to measure the ratio of the flux $\left(F_{r}\right)$ reflected by the sample and the flux $\left(F_{m}\right)$ reflected by the reference standard (e.g.. an accurately calibrated mirror); the reflectance $\rho\left(7^{\circ}, \theta\right)$ of the sample is obtained by multiplying this ratio by the reflectance $\left(\rho_{m}\right)$ of the reference mirror, thus:

$$
\rho\left(7^{\circ}, \theta\right)=\left(F_{r} / F_{m}\right) \rho_{m}
$$

The relation between the flux $F_{m}$ reflected by the reference mirror and the portion of the flux $F_{m i t}$ en- tering the averaging device is simple. ${ }^{6}$ The flux $F_{m d}$ is the product of the average value of reflectance $\bar{\rho}_{e}$ of that portion of the ellipsoidal mirror receiving the reflected flux and the reflected flux $F_{m}$ itself, thus:

$$
F_{m d}=\bar{\rho}_{e} F_{m}
$$

When a diffusing sample is substituted for the reference mirror, however, the relation between the reflected flux $F_{r}$ and the detected flux may be more complex. There are four major sources of flux loss, and these must all be accounted for precisely: (1) A part, $F_{\alpha}{ }^{7}$ is absorbed or misdirected by the ellipsoidal mirror, (2) a part $F_{h}$ is lost out the hole admitting the incident beam, (3) the flux scattered and absorbed by the wire sample supports is $\left(\rho_{\epsilon}\right)_{w} F_{u},{ }^{8}$ and (4) the flux lost by sample shading is $\bar{\rho}_{\epsilon}\left[F_{s t}-\left.F_{s r}\right|^{9}\right.$ (see fig. 3 ).

The flux crossing the first focal plane is $\left(\bar{\rho}_{\epsilon}\right)_{s} F_{s}$. Thus, the total flux reflected by the sample is:

$$
F_{r}=F_{s}+F_{w}+\left[F_{s p}-F_{s r}\right]+F_{h} .
$$

All the fluxes in eq (6) are defined on the basis of the flux leaving the sample. before it is reflected by the ellipsoidal mirror. Since each one of these losses depends on the distribution of flux reflected by the sample, it is necessary to know something about this distribution.

- Neglecting until later the interchange of flux between the sample and the averaging device.

T Note that the actual mirror loss will be taken into account by considering the reflecance of directly appear in eq (6). Further, the fux terms used in this section are referenced to the flux leaving the sample and not the actual fux loss occurring in each case.

${ }^{B}\left(\bar{\rho}_{\epsilon}\right)_{, r}$ is the average effective reflectance of the ellipsoidal mirror for the given distribu. tion of $F_{s}$ on the mirror, where $x$ is a variable.

${ }^{9} \bar{\rho}_{\varepsilon}$ is the average effective reflectance of the central part of the ellipsoidal mirror, which varied by less than 0.2 percent. $F_{s p}$ is the flux leaving the sample on the first reflection that is shaded (after refection from the ellipsoidal mirror) from the sphere entrance. $F_{s r}$ is that part of $F_{s p}$ that eventually reaches the sphere entrance by any path.
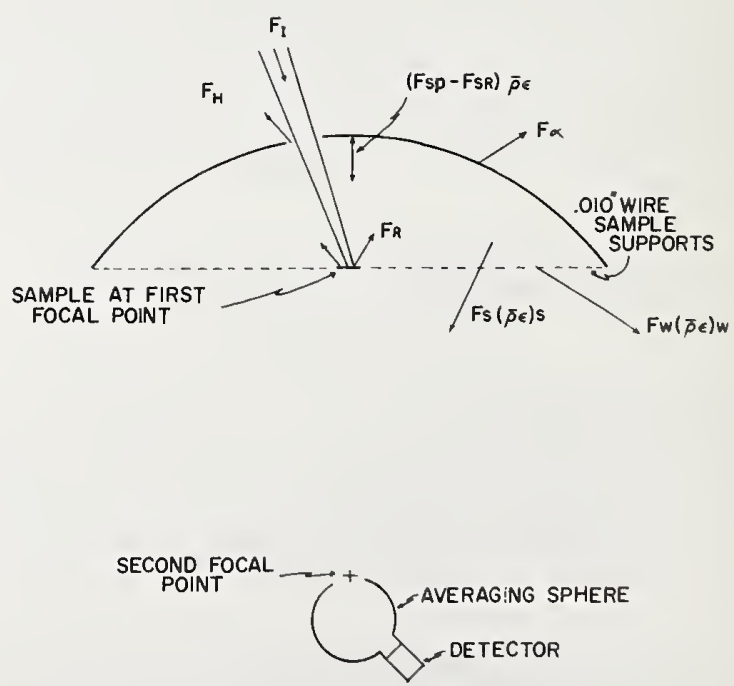

Figure 3. Flux balance of a sample in the ellipsoidal mirror reflectometer. 

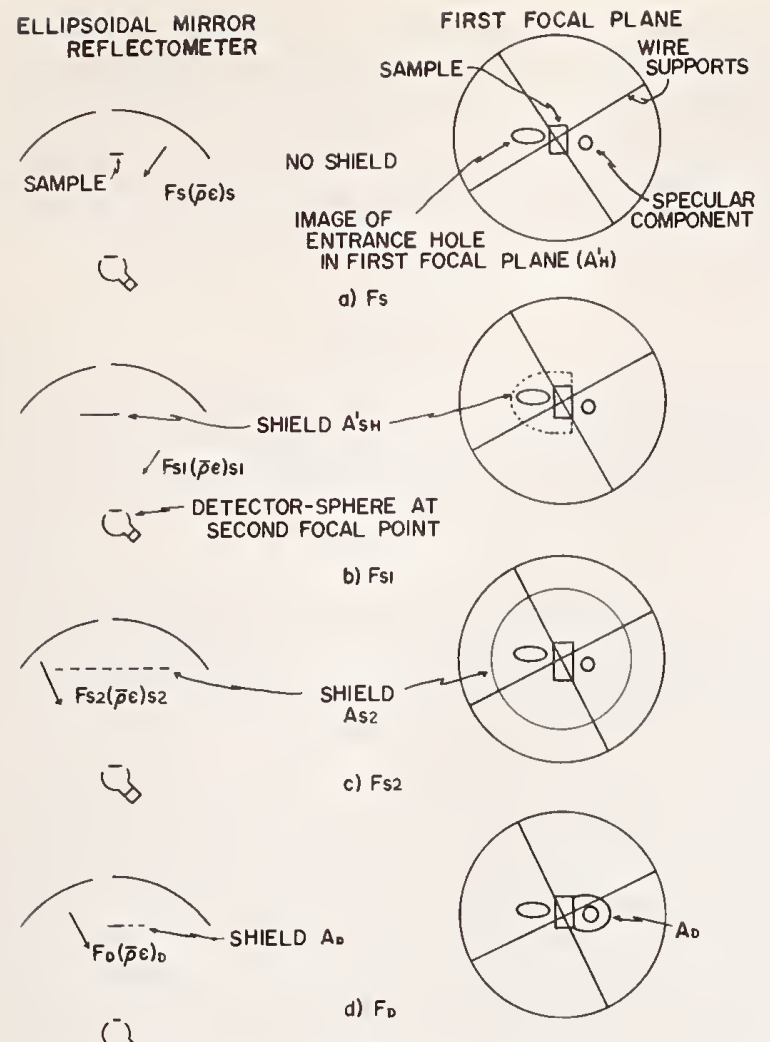

Figure 4. System configuration for definition of the fluxes $\mathrm{F}_{\mathrm{s}}, \mathrm{F}_{\mathrm{s} 1}$. $\mathrm{F}_{\mathrm{s}^{2}}$, and $\mathrm{F}_{\mathrm{d}}$.

The flux distribution throughout the system can be evaluated by means of shields placed in the first focal plane, as previously described. Thus, to aid in evaluating the fluxes in eq (6), the following fluxes are defined (see fig. 4):

$F_{s}$-the flux crossing the first focal plane, divided by the average effective reflectance of the mirror. for the particular distribution of $F_{s}$ on the mirror.

$F_{s 1}$ - the flux crossing the first focal plane, divided by $\left(\bar{\rho}_{\epsilon}\right)_{s 1}$, when shield $A_{s i t}$ is in the first focal plane.

$F_{s: 2}$ - the fiux crossing the first focal plane, divided by $\left(\bar{\rho}_{\epsilon}\right)_{s^{\prime}}$, when shield $\mathrm{S} 2$ is in the first focal plane.

$F_{t}-$ the flux crossing the first focal plane, divided by $\left(\bar{\rho}_{\epsilon}\right)_{d}$. when shield $\mathrm{D}$ is in the first focal plane.

The fluxes defined above can now be used to compute the losses in the system. For brevity, only the equation describing each loss is given. The complete derivation of each loss is given in [15].

Mirror loss, $F_{\alpha}$ : The ellipsoidal mirror will absorb some of the flux incident upon it. Further, since the mirror is not perfect, it may transmit or scatter some of the incident flux. Thus, it is necessary to know the effective reflectance $\rho_{\epsilon}^{\prime}$ of the mirror. This is defined as the ratio of the flux that reaches a predefined area at the second focal plane (the entrance port to the averaging sphere) to that incident on the ellipsoidal mirror from a defined area in the first focal plane (the irradiated area of the specimen). The "absorbed" flux $\left(\alpha=1-\rho_{\epsilon}^{\prime}\right)$ includes flux lost by absorption, transmission, scattering. and optical aberrations. This $\rho_{\epsilon}^{\prime}$ was measured for the ellipsoidal mirror, and is reported as a function of position on the mirror in tables 2 and 3 . Figure 5 shows the optical paths used in making the measurements and locations of the areas measured. Table 3 indicates that $\rho_{\epsilon n} / \rho_{\epsilon 1}$ does not vary with wavelength, but increases as the measured area moves away from the apex of the mirror. The reflectance of the outer edge of the mirror is about 1.5 percent higher than that of the apex.

TABLE 2. Absolute reflectance of ellipsoidal mirror (Point 2 in fig. 5)

\begin{tabular}{l|r|r|r|r|r}
\hline \hline & Set \#1 & Set \#2 & Set \#3 & Set \#4 & Average \\
\hline & & & & & \\
$1.5 \mu$ & 0.951 & 0.949 & 0.951 & 0.948 & 0.950 \\
$2.0 \mu$ & .964 & .963 & .959 & .961 & .962 \\
$2.5 \mu$ & .965 & .969 & .967 & .963 & .966 \\
$3.5 \mu$ & .969 & .971 & .969 & .971 & .970 \\
$4.5 \mu$ & .969 & .971 & .970 & .973 & .971 \\
$5.5 \mu$ & .970 & .971 & .973 & .970 & .971 \\
$6.5 \mu$ & .971 & .973 & .973 & .974 & .972 \\
$7.0 \mu$ & .972 & .972 & .974 & .973 & .972 \\
\hline
\end{tabular}

TABLE 3. Relative reflectance of the ellipsoidal mirror as a function of position and wavelength ${ }^{\text {a }}$

\begin{tabular}{c|c|c|c|c|c|c|c|c}
\hline Wavelengths $\rightarrow$ & $1.5 \mu$ & $2.0 \mu$ & $2.5 \mu$ & $3.5 \mu$ & $4.5 \mu$ & $5.5 \mu$ & $6.5 \mu$ & $7.0 \mu$ \\
\hline & & & & & & & & \\
Areas $\downarrow$ & & & & & & & & \\
1 & 1.000 & 1.000 & 1.000 & 1.000 & 1.000 & 1.000 & 1.000 & 1.000 \\
2 & 1.001 & 1.001 & 1.000 & 1.000 & 1.000 & 1.000 & 1.001 & 1.000 \\
3 & 1.002 & 1.002 & 1.001 & 1.000 & 1.001 & 1.001 & 1.001 & 1.002 \\
4 & 1.013 & 1.010 & 1.012 & 1.016 & 1.016 & 1.015 & 1.015 & 1.013 \\
5 & 1.000 & 1.001 & 1.002 & 1.001 & 1.000 & 1.001 & 1.000 & 1.000 \\
6 & 1.002 & 1.002 & 1.003 & 1.001 & 1.002 & 1.002 & 1.002 & 1.001 \\
7 & 1.015 & 1.013 & 1.015 & 1.015 & 1.014 & 1.014 & 1.015 & 1.013 \\
8 & 1.001 & 1.001 & 1.001 & 1.000 & 1.002 & 1.001 & 1.001 & 1.001 \\
9 & 1.002 & 1.002 & 1.003 & 1.002 & 1.003 & 1.002 & 1.003 & 1.002 \\
10 & 1.014 & 1.015 & 1.014 & 1.016 & 1.015 & 1.015 & 1.015 & 1.014 \\
11 & 1.001 & 1.002 & 1.001 & 1.000 & 1.000 & 1.001 & 1.001 & 1.002 \\
12 & 1.002 & 1.002 & 1.001 & 1.002 & 1.001 & 1.002 & 1.002 & 1.002 \\
13 & 1.013 & 1.014 & 1.014 & 1.016 & 1.014 & 1.014 & 1.015 & 1.014 \\
\hline
\end{tabular}

Average values for areas equally distant from the apex of the ellipsoid

\begin{tabular}{l|l|l|l|l|l|l|l|l}
\hline Set A & \\
Set B & 1.001 & 1.001 & 1.001 & 1.000 & 1.001 & 1.001 & 1.001 & 1.001 \\
Set C & 1.002 & 1.002 & 1.002 & 1.001 & 1.002 & 1.002 & 1.002 & 1.002 \\
& 1.014 & 1.013 & 1.014 & 1.016 & 1.015 & 1.015 & 1.015 & 1.014 \\
\hline
\end{tabular}

a Values are all referred to position No. 1, see figure 3 .

bet $A$ is composed of areas $2,5,8$, and 11 ; set $B$ is composed of areas $3,6,9$, and 12 ; and set $C$ is composed of areas $4,7,10$, and 13 .

If the reflectance of the mirror were uniform, $\rho_{\epsilon}$ would cancel out in the computation of absolute reflectance from relative measurements. Tables 2 and 3 indicate that the flux reflected by a diffusing sample as measured with the EMR should be corrected for the reflectance of the mirror on the basis of its geometric distribution. The defined flux $F_{s 2}$ was used to compute the effective reflectance of the mirror for each sample measured. This leads to the following equations for the various effective reflectances of the ellipsoidal mirror. 


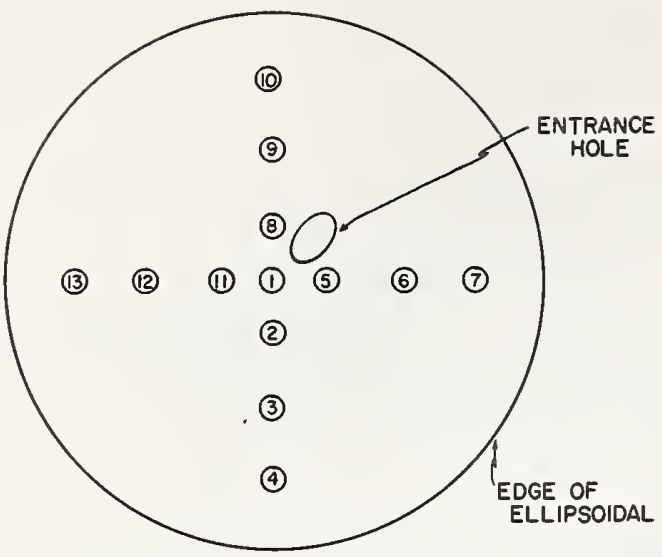

TOP VIEW OF ELLIPSOIDAL MIRROR

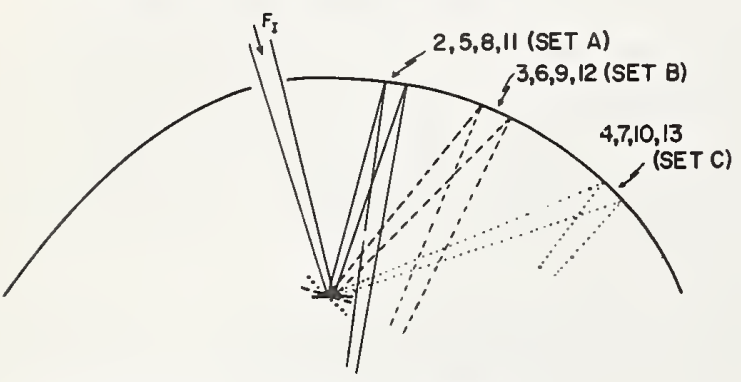

SIDE SECTION OF ELLISOIDAL MIRROR

FIGURE 5. Areas used in measurement of mirror reflectance.

$$
\begin{aligned}
& \left(\bar{\rho}_{\epsilon}\right)_{s}=\bar{\rho}_{\epsilon}\left[1+\frac{F_{s 2}}{F_{s}}(0.015)\right] \\
& \left(\bar{\rho}_{\epsilon}\right)_{s 1}=\bar{\rho}_{\epsilon}\left[1+\frac{F_{s 2}}{F_{s 1}}(0.015)\right] \\
& \left(\bar{\rho}_{\epsilon}\right)_{d}=\bar{\rho}_{\epsilon}\left[1+\frac{F_{s 2}}{F_{d}}(0.015)\right] \\
& \left(\bar{\rho}_{\epsilon}\right)_{s 2}=1.015 \bar{\rho}_{\epsilon} .
\end{aligned}
$$

Hole loss, $F_{h}$ : Some of the reflected flux will escape through the hole in the mirror which admits the incident beam. This loss is determined by the amount of flux reflected by the sample in the direction of the hole. Previous investigators have not established the magnitude of this loss, which does not necessarily lie between the condition of no loss for a specular reflector and a loss based on the diffuse configuration factor from the sample to the hole. The flux density around the entrance hole can be computed from fluxes $F_{s}$ and $F_{s 1}$, as shown in figure 4 , and the geometric relationships involved.

$$
F_{s h}=F_{s}-F_{s 1}
$$

where $F_{s h}$ is the flux incident on the area $A_{s h}$, the area on the ellipsoidal mirror of the projection of $A_{s h}^{\prime}$ (in the first focal plane) from the second focal point. Thus, the average flux density around the hole is $F_{s h} /\left(A_{s h}\right.$ $-A_{h}$ ). It is logical to assume that the flux density over the area of the hole, $A_{h}$, is the same as that over the area $A_{s h}$ surrounding it. Thus, the hole loss is

$$
F_{h}=\frac{A_{h} F_{s h}}{A_{s h}-A_{h}}
$$

or, in terms of the measured fluxes,

$$
F_{h}=\frac{A_{h}\left(F_{s}-F_{s 1}\right)}{A_{s h}-A_{h}}
$$

The assumption of uniform radiance over the small solid angle subtended by $A_{s h}$ is more reasonable and accurate than the assumption of uniform radiance over a hemisphere made by previous investigators.

Wire loss, $\left(F_{w}\right)$ : A fraction $F_{w}\left(\bar{\rho}_{\mathrm{\epsilon}}\right)_{w}$ of the flux reflected by the sample and focused toward the detector will be absorbed or scattered out of the optical path by the wire sample supports. It should be noted that the wires are oriented out of the path of the specularly reflected beam. Hence, if a shield blocks the flux in the area surrounding the direction of specular reflection, the remainder would be the nonspecularly reflected flux. If this flux is assumed to be uniformly distributed over the area $\left(A_{\epsilon}\right)$ of the first focal plane of the ellipsoid, then

$$
\left(\bar{\rho}_{\epsilon}\right)_{w} F_{w}=F_{d} \frac{A_{w}}{A_{\epsilon}}\left(\bar{\rho}_{\epsilon}\right)_{d}
$$

where $A_{\epsilon}=\pi / 4 D_{\epsilon}^{2}-A_{d}$.

( $D_{\epsilon}$ is the diameter of the ellipsoidal mirror, and $A_{d}$ is the area of shield D.)

Equation (14) reduces to

$$
F_{w}=F_{d}\left(A_{w} / A_{\epsilon}\right)
$$

since $\left(\bar{\rho}_{\epsilon}\right)_{w} \equiv\left(\bar{\rho}_{\epsilon}\right)_{d}$ when $F_{d}$ is evenly distributed over $A_{\epsilon}$.

Since $F_{w}$ is a secondary correction, it is apparent that the assumption, that the average nonspecular flux density over the first focal plane is intercepted by the wire supports, is sufficiently accurate, especially since the wire supports comprise 2 diam of the first focal plane.

Sample shielding loss, $\left(F_{s p}-F_{s r}\right)$ : Flux leaving the sample normal to its surface will be reflected back to the sample, and hence be blocked from the detector. However, any of the reflected flux incident on the specimen in the area $A_{s 1}^{\prime}$ (the image in the first focal plane formed by the ellipsoidal mirror of the sphere entrance port in the second focal plane) may be multiply reflected by the sample and mirror and focused on the detector. To correct for the sample shielding loss, the three fluxes $F_{d}, F_{s}$, and $F_{s 1}$ will be required. The flux involved in this loss is that which 
strikes the ellipsoidal mirror on the projected area of the sample, $A_{s}$ (projected from the second focal point). From figure 4 , it is seen that $A_{s}$ is partially surrounded by the shield $A_{s h}$, and will have approximately the same flux density as that on $A_{s h}$. Hence, the total flux initially shaded is

$$
F_{s p}=\frac{A_{s} F_{s h}}{A_{s h}-A_{h}} .
$$

However, a portion $A_{s 1} / A_{s}\left(A_{s 1}\right.$ is the area $A_{s 1}^{\prime}$ projected on the ellipsoid) of the flux $F_{s p}$ is reflected from area $A_{s 1}^{\prime}$ and could reach the detector. Reference 15 shows that the fraction $F_{s r}$ that eventually reaches the detector is

$$
F_{s r}=\left[\frac{F_{s h}\left(A_{s 1}\right)}{A_{s h}-A_{h}}\right]\left[\frac{F_{d}}{F_{s}} \rho_{s} \bar{\rho}_{\epsilon}\left\{\frac{A_{\epsilon}-A_{w}}{A_{\epsilon}}\right\}\right] \frac{1}{-\rho_{s} \bar{\rho}_{\epsilon}\left[\frac{F_{s}-F_{d}}{F_{s}}\right]} .
$$

The flux multiply reflected by the sample or reference and ellipsoidal mirror that eventually reaches the detector is defined by eq (18), in terms of the four measured fluxes and the area relationships involved.

One further problem arises because the detector is not black; that is, the sphere entrance port back-reflects flux into the optical path, some of which eventually gets back into the sphere and increases the flux sensed by the detector. For the $F_{s}$ measurement, flux $\left(\bar{\rho}_{\epsilon}\right)_{s} F_{s}$ enters the sphere port initially. However, some fraction $\eta^{\prime}$ of this flux is reflected back out the sphere entrance port. This flux is reflected nearly diffusely, so that a fraction $f_{s-\epsilon} \eta^{\prime} F_{s}\left(\bar{\rho}_{e}\right)_{s}$ is intercepted by the mirror and focused on the sample in the first focal plane $\left(f_{s-\epsilon}\right.$ is the standard diffuse configuration factor as defined in reference 16). The sample then reflects the flux back to the mirror, which focuses it on the sphere entrance. Thus, an amount $F_{s}^{\prime}$ is added to the flux $F_{s}\left(\bar{\rho}_{\epsilon}\right)_{s}$ that was originally incident on the sphere entrance.

$$
F_{s}^{\prime}=\rho_{h s}\left(\bar{\rho}_{\epsilon}\right)_{d}^{2} \eta^{\prime} f_{s-\epsilon}\left[F_{s}\left(\bar{\rho}_{\epsilon}\right)_{s}\right]
$$

where $\left(\bar{\rho}_{\epsilon}\right)_{d}$ is the average effective reflectance of the mirror for flux coming diffusely from the sphere entrance and $\rho_{h s}$ is the hemispherical reflectance of the sample. Further, a fraction $F_{s}^{\prime \prime}$ of the flux that reaches the sphere on the second pass will be multiply reflected back to the sphere.

$$
F_{s}^{\prime \prime}=\rho_{h s}\left(\bar{\rho}_{\epsilon}\right)_{d}^{2} \eta^{\prime} f_{s-\epsilon} F_{s}^{\prime} .
$$

This process will continue until the total flux in the sphere is

$$
F_{s s}=\left(\bar{\rho}_{\epsilon}\right)_{s} F_{s}\left[\frac{1}{1-\rho_{h s}\left(\bar{\rho}_{\epsilon}\right)_{d}^{2} \eta^{\prime} f_{s-\epsilon}}\right]
$$

The flux viewed by the detector, $F_{s d}$, will be

$$
F_{s d}=\eta F_{s s}
$$

where $\eta$ is the sphere efficiency.

Similarly, the detector views the other measured fluxes as

$F_{s 1 d}=\eta F_{s 1}\left(\bar{\rho}_{\epsilon}\right)_{s 1}\left[\frac{1}{1-\rho_{l s}\left(\bar{\rho}_{\epsilon}\right)_{d}^{2} \eta^{\prime}\left(f_{s-\epsilon}\right)_{s 1}}\right]$

$F_{s 2 d}=\eta F_{s 2}\left(\bar{\rho}_{\epsilon}\right)_{s 2}\left[\frac{1}{1-\left(\frac{F_{s}-F_{s 2}}{F_{s}}\right) \rho_{h s}\left(\bar{\rho}_{\epsilon}\right)_{d}^{2} \eta^{\prime}\left(f_{s-\epsilon}\right)_{s: 2}}\right]$

$F_{d d}=\eta F_{d}\left(\bar{\rho}_{\epsilon}\right)_{d}\left[\frac{1}{1-\rho_{h s}\left(\bar{\rho}_{\epsilon}\right)_{d}^{2} \eta^{\prime}\left(f_{s-\epsilon}\right)_{d}}\right]$.

The total flux reflected by the sample, $F_{r}$, is thus

$$
\begin{aligned}
& F_{r}=\frac{F_{s d}\left[1-\rho_{h s}\left(\bar{\rho}_{\epsilon}\right)_{d}^{2} \eta^{\prime}\left(f_{s-\epsilon}\right)_{s}\right]}{\eta\left(\bar{\rho}_{\epsilon}\right)\left\{1+\frac{F_{s-d}}{F_{s d}}(0.015)\right\}}\left[1+\frac{A_{h}+A_{s}}{A_{s h}-A_{h}}-\frac{A_{s 1}}{A_{s h}-A_{h}}\right. \\
& \left.\times\left\{\frac{F_{d d}}{F_{s d}} \rho_{s}\left(\bar{\rho}_{\epsilon}\right)_{d}^{2}\left(\frac{A_{\epsilon}-A_{w}}{A_{\epsilon}}\right)\left(\frac{1}{1-\rho_{s} \bar{\rho}_{\epsilon} \frac{F_{s d}-F_{d d}}{F_{s d}}}\right)\right\}\right] \\
& +\frac{F_{s 1 d}\left[1-\rho_{h s}\left(\bar{\rho}_{\epsilon}\right)_{d}^{2} \eta^{\prime}\left(f_{s-\epsilon}\right)_{s 1}\right]}{\eta \bar{\rho}\left\{1+\frac{F_{s ! 2 d}}{F_{s 1 d}}(0.015)\right\}}\left[-\frac{A_{h}+A_{s}}{A_{s h}-A_{h}}+\frac{A_{s 1}}{A_{s h}-A_{h}}\right. \\
& \left.\times\left\{\frac{F_{d d}}{F_{s d}} \rho_{s} \bar{\rho}_{\epsilon}^{2}\left(\frac{A_{\epsilon}-A_{w}}{A_{\epsilon}}\right) \frac{1}{1-\rho_{s} \bar{\rho}_{\epsilon} \frac{F_{s d}-F_{d d}}{F_{s t l}}}\right\}\right] \\
& +\frac{F_{d d l}\left[1-\rho_{s_{s}}\left(\bar{\rho}_{\epsilon}\right)_{d}^{2} \eta^{\prime}\left(f_{s-\epsilon}\right)_{d l}\right] A_{u}}{\eta \bar{\rho}_{\epsilon}\left\{1+\frac{F_{s:-d l}}{F_{d d}}(0.015)\right\} A_{\epsilon}} .
\end{aligned}
$$

If we now account for the interchange of flux between the reference mirror and the averaging device, the incident flux is computed as

$$
F_{1}=\frac{F_{1 d}\left[1-\rho_{h m}\left(\bar{\rho}_{\epsilon}\right)_{d}^{2} \eta^{\prime}\left(f_{s-\epsilon}\right)_{1}\right]}{\rho_{m} \bar{\rho}_{\epsilon} \eta} .
$$


The reflectance of the sample, $\rho\left(7^{\circ}, \theta\right)$, is equal to the reflected flux, $F_{r}$, eq (26), divided by the incident flux, eq (27). The simplifying assumption is made that the terms $\left(l-\rho_{t s} \bar{\rho}_{\epsilon}^{2} \eta^{\prime} f_{s-\epsilon}\right)$ for $F_{1 d} . F_{s d}, F_{s 1 d}$, and $F_{\text {sdd }}$ are equal, and that $\eta$ and $\eta^{\prime}$ are respectively identical in eqs (26) and (27). Then

$$
\begin{aligned}
& \rho\left(7^{\circ}, \theta\right)=\frac{\rho_{m}}{F_{1 d}}\left[\left\{\frac { F _ { s d } } { \{ 1 + \frac { F _ { s \geq } } { F _ { s d } } ( 0 . 0 1 5 ) \} } \left(1+\frac{A_{h}+A_{s}}{A_{s h}-A_{h}}\right.\right.\right. \\
& \left.\left.-\frac{A_{s 1}}{A_{s h}+A_{h}} \times\left[\frac{F_{d d}}{F_{s d}} \rho_{s}\left(\bar{\rho}_{\epsilon}\right)_{d}^{2}\left(\frac{A_{\epsilon}-A_{w}}{A_{\epsilon}}\right) \frac{1}{1-\rho_{s} \bar{\rho}_{\epsilon} \frac{F_{s d}-F_{d d}}{F_{s t l}}}\right]\right)\right\} \\
& +\frac{F_{\text {ddd }}}{1+\frac{F_{s: 2 d}}{F_{\text {sdd }}}(0.015)}\left[\frac{A_{u}}{A_{\epsilon}}\right]+\frac{F_{s 1 d}}{\left\{1+\frac{F_{s: 2 d}}{F_{s d d}}(0.015\}\right.}\left\{-\frac{A_{h}+A_{s}}{A_{s h}-A_{h}}\right. \\
& \left.\left.+\frac{A_{s 1}}{A_{s h}-A_{h}} \times\left[\frac{F_{d d}}{F_{s d}} \rho_{s} \bar{\rho}_{\epsilon} \frac{A_{\epsilon}-A_{w}}{A_{\epsilon}} \frac{1}{1-\rho_{s} \bar{\rho}_{\epsilon} \frac{F_{s d}-F_{d d}}{F_{s d}}}\right]\right\}\right]
\end{aligned}
$$

in which the only remaining unknown is $\rho_{s}$, which is identical to $\rho\left(7^{\circ}, \theta\right)$. A very good approximation is

$$
\rho_{s}=F_{s d} / F_{1 d}
$$

An error of up to 50 percent in $\rho_{s}$, as evaluated by eq (29), would cause only a very small error in $\rho\left(7^{\circ}, \theta\right)$, since $\rho_{s}$ occurs only in secondary flux terms. If justified, an iteration process can be used to obtain successively better values of $\rho_{s}$.

The derivation of eq (28) is, for the most part, applicable to any ellipsoidal mirror. A few of the simplifying assumptions are based on measurement with the particular mirror used in this work.

There are two sources of known errors that are not compensated for in eq (28): they are the edge loss, and detector-sample interchange.

Edge loss. If the sample is not accurately positioned in the first focal plane, some of the reflected flux will miss the ellipsoidal mirror and be lost. The amount depends upon the geometric distribution of reflected flux. For a perfect diffuser, the fraction of the total flux lost in this way is (reference 15)

$$
\frac{F_{r}-F_{e}}{F_{r}}=\frac{h^{2}}{h^{2}+r_{\epsilon}^{2}}
$$

where $F_{r}$ is the total flux reflected by the sample and $F_{e}$ is the flux hitting the mirror, $h$ is the distance between the sample and the first focal plane, and $r_{\epsilon}$ is the diameter of the ellipsoidal mirror. The magnitude of this loss is shown in figure 6.

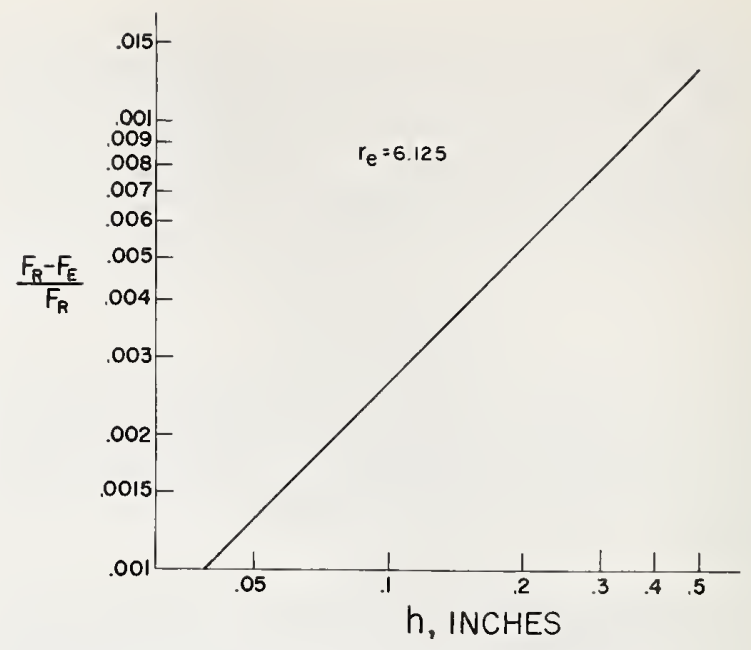

Figure 6. Edge loss for a perfect diffuser.

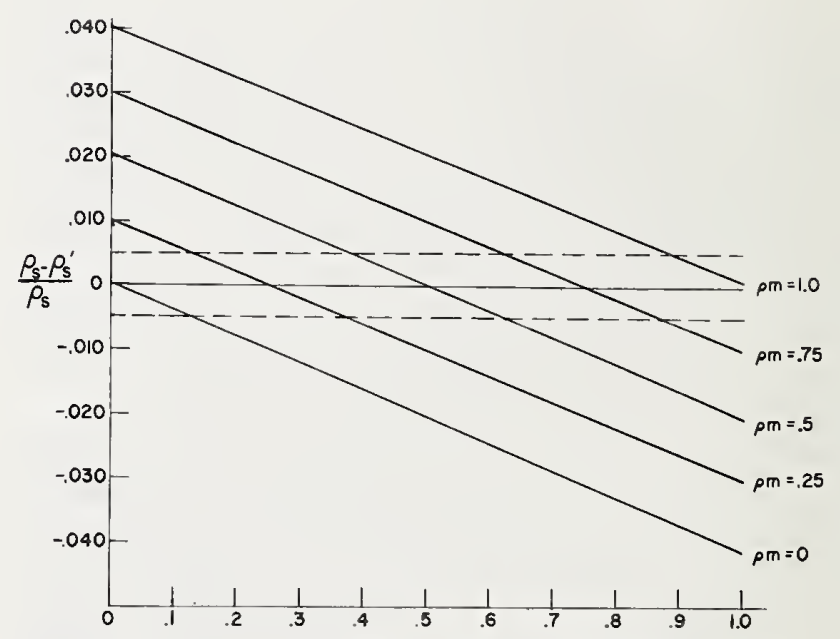

FIGURE 7. Error due to detector-ellipsoid interchange, for different values of $\rho_{\mathrm{s}}$ and $\rho_{\mathrm{m}}$.

Detector-ellipsoid (sample) interchange. In the final steps of the derivation of eq (28), the detectorellipsoid interchange was ignored. This is legitimate if $\rho\left(7^{\circ}, \theta\right) \cong \rho_{m} ;$ however. for $\rho_{m} \ll \rho\left(7^{\circ}, \theta\right)$ or $\rho_{m} \gg \rho\left(7^{\circ}, \theta\right)$, a significant error can be introduced from this source. Figure 7 shows the magnitude of this error for different values of $\rho\left(7^{\circ}, \theta\right)$ and $\rho_{m}$. The maximum value is 4 percent. The error can be avoided by using a comparison standard having a reflectance $\rho_{m}$ near that of the sample. If necessary, a calibration can be made for this factor (reference 15).

Summary. The analysis of the EMR presented here is based on the ability to measure four different fluxes. These fluxes, plus a knowledge of the system parameters, permit corrections to be made for system losses, based on reasonable assumptions about the 
geometric distribution of flux reflected from surfaces to be measured. The important features of the reflected flux distribution are measured, in order to make accurate corrections. The assumptions on which these corrections are based are believed to be more accurate than those used previously with other reflectometers.

\section{Experimental Data}

A. Reference standards. The use of a calibrated mirror as the reference standard is highly desirable, since suitable mirrors are readily available and can be calibrated by any investigator. The specular reflectometer used in this work to measure the reflectance of the reference mirrors utilized the previously mentioned sulfur-coated diffusing sphere and thermopile detector to measure the incident monochromatic flux and that reflected once each by two sample mirrors. The use of the diffuser reduces the required precision of optical alinement. The ratio of the twice reflected flux to the incident flux is the product of the reflectances of the two mirrors." If the reflectances are equal, then the ratio is the square of the reflectance. This procedure reduces the error of the measurement, since the expected error is the same whether one or more reflections are involved.

Three sets of four mirrors, each coated with vacuum deposited aluminum, gold, and rhodium, respectively, were used. Six reflectance measurements were made, two each on three different pairs from each set. This did not exhaust the six unique pairs in each set, but allowed intercomparison of all the mirrors to establish that their reflectances are indeed equal. The data are shown in table 4 , together with comparable literature values $[17,18,19,20]$.

B. Optical quality of ellipsoid. To ascertain that all of the beam of reflected flux was focused onto the entrance port of the averaging sphere at the second focal point, a Polaroid Land camera back was placed at the second focal point so that the plane of the film ${ }^{10}$ was at the position of the sphere entrance port. Two different samples were used at the first focal point: (1) an aluminum mirror, and (2) a diffuse porcelain enamel reflectance standard. Figure 8 displays the images formed in the second focal plane for the two different samples and for different exposure times. The black area around each image is the approximate size and shape of the entrance port of the averaging sphere.

The image formed with the aluminum mirror is quite clear and well-defined. The image formed when the mirror was inclined $25^{\circ}$ with respect to the first focal plane shows light gray areas surrounding the white image, which indicate that the scatter and aberration of the ellipsoidal mirror increase with distance from the apex. The image formed in the second focal plane when the porcelain enamel (a fairly good diffuser) was placed at the first focal point is enlarged, which indicated that careful location of the image on the sphere entrance port is required if one expects to collect all of the flux represented by these images. The increased image size for the diffuser is indicative of the total scatter and aberrations for this particular ellipsoidal mirror. In all cases, increased time of exposure yielded slightly enlarged images, indicating that a small amount of flux surrounds the visual image. The conclusion drawn from the results displayed in figure 8 is that essentially all of the flux does enter the sphere when care is taken to center the visual image on the entrance.

C. Directional hemispherical reflectance. Several samples were chosen for reflectance measurement with the EMR: (1) platinum -13 percent rhodium alloy. (2) gold mesh, (3) a porcelain enamel, and (4) oxidized Kanthal. ${ }^{11}$ Samples 1 and 4 are high-temperature

\footnotetext{
10 The film was Polaroid Type 47 , a 3000 speed film

"Trade name of a heat-resistant alloy.
}

TABLE 4. Measured reflectance of reference mirrors

\begin{tabular}{|c|c|c|c|c|c|c|c|c|c|}
\hline \multirow[b]{2}{*}{$\lambda$} & \multicolumn{3}{|c|}{ Reflectance of aluminum } & \multicolumn{4}{|c|}{ Reflectance of gold } & \multicolumn{2}{|c|}{$\begin{array}{l}\text { Reflectance } \\
\text { of rhodium }\end{array}$} \\
\hline & Average & $\begin{array}{l}\text { Standard } \\
\text { deviation }\end{array}$ & \begin{tabular}{|l|}
\multicolumn{1}{c|}{ Best } \\
literature \\
values [34]
\end{tabular} & Average & $\begin{array}{l}\text { Standard } \\
\text { deviation }\end{array}$ & $\begin{array}{c}\text { Literature } \\
\text { values [36] }\end{array}$ & $\begin{array}{c}\text { Best } \\
\text { literature } \\
\text { values [35] }\end{array}$ & Average & $\begin{array}{r}\text { Literature } \\
\text { values [37] }\end{array}$ \\
\hline 1.5 & 0.9608 & 0.0012 & 0.9742 & 0.9809 & 0.0014 & 0.982 & 0.9906 & 0.8383 & 0.882 \\
\hline 2.0 & .9742 & .0017 & .9779 & .9833 & .0010 & .983 & .9914 & .8850 & .905 \\
\hline 2.5 & .9757 & .0010 & .9794 & .9843 & .0014 & .983 & .9922 & .9104 & .915 \\
\hline 3.5 & .9828 & .0005 & .9816 & .9870 & .0005 & .983 & .9934 & .9339 & .932 \\
\hline 4.5 & .9840 & .0011 & .9835 & .9874 & .0008 & .983 & .9938 & .9428 & .942 \\
\hline 5.5 & .9852 & .0012 & .9850 & .9870 & .0014 & .983 & .9938 & .9470 & .946 \\
\hline 6.5 & .9852 & .0013 & .9861 & .9878 & .0005 & .983 & .9939 & .9474 & .950 \\
\hline 7.0 & .9863 & .0017 & .9866 & .9890 & .0017 & .984 & .9939 & 9510 & .953 \\
\hline
\end{tabular}

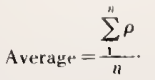

Slandard deviatim $=\sqrt{\frac{\sum_{1}^{n}(\rho-\bar{\rho})^{2}}{(n-1)} .}$. 


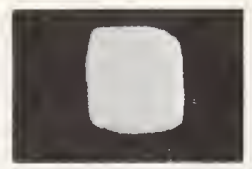

Aluminum Mirror $0^{\circ}$ Sample Holder 2-Second Exposure

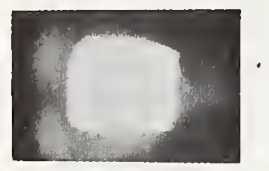

Aluminum Mirror $0^{\circ}$ Sample Holder 1-Minute Exposure

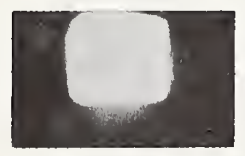

Aluminum Mirror $25^{\circ}$ Sample Holder 5-Second Exposure

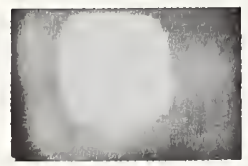

\section{Aluminum Mirror} $25^{\circ}$ Sample Holder 1.5-Minute Exposure

\section{Ename 1 \\ $0^{\circ}$ Sample Holder \\ 45-Second Exposure}

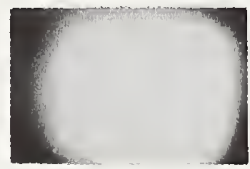

Ename 1

$0^{\circ}$ Sample Holder 2-Minute Exposure
Figure 8. Photographs of images formed in second focal plane of ellipsoidal mirror.

The black area surrounding each image is the approximate size of the sphere entrance port.

emittance standards provided by the National Bureau of Standards and described by Richmond et al., [21]. Sample 2 was provided by Bernd Linder of the Missiles and Space Division, General Electric Company, Philadelphia, $\mathrm{Pa}$. It is a 0.002-in. diam stainless steel wire screen, 135 mesh, backed by 1.5 mil Mylar, coated with vapor-deposited gold. Sample 3 is a standard of luminous daylight reflectance.

(1) Platinum-13 percent rhodium. The average values obtained in six determinations of the reflectance of each of two samples are shown in figure 9. Table 5

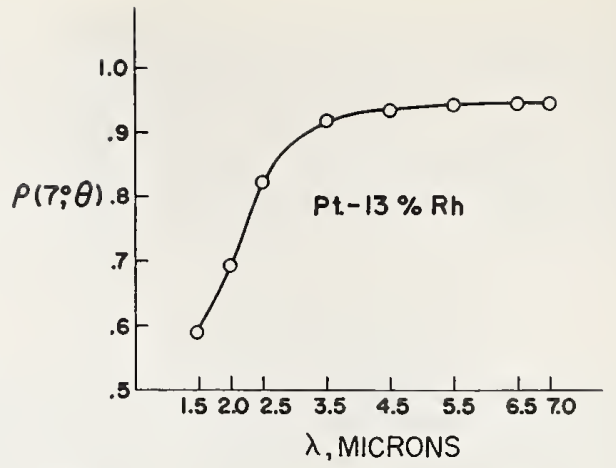

Figure 9. Spectral directional hemispherical reflectance of platinum-13 percent rhodium alloy.

Each point is the average of six determinations, three each on two specimens.

gives the individual measurements, and values from reference 21 . The six values reported in table 5 were obtained by two different operators over a period of one week. Determinations $1 \mathrm{la}$ and $2 \mathrm{a}$ were made on samples tilted $10^{\circ}$ to the first focal plane, in order to eliminate the hole and sample shading corrections. This is possible because, as the data on the specular component in table 5 indicate, the reflected flux is concentrated around the specular direction, and tilting the sample results in no edge loss. The data for the tilted samples are not significantly different from those for samples that were not tilted: hence, the corrections must have been accurate (assuming no change of reflectance for small change in angle of incidence).

(2) Gold mesh. The data for the gold mesh samples are presented in figure 10 . Each data point is the average of three determinations.

(3) Porcelain enamel. The data for the porcelain enamel reflectance standard are presented in figure 11 . No attempt was made to correct for the sphere-ellipsoid interchange.

(4) Oxidized Kanthal. The data for the oxidized Kanthal are shown in figure 12. The incident flux was attenuated by 50 percent with a sector disk for the reference measurement, but not for the sample measurement. The data were corrected for the sphereellipsoid interchange [15].

The data in figures $7,8,9$, and 10 include a value for the specular component of reflected flux. No effort was made to study the size of the shield that would give the most useful specular component: instead, the $A_{\text {sh }}$ shield was used. The specular component was computed as:

$$
\% \text { specular component }=\frac{F_{s d}-F_{d d}}{F_{s d}} \times 100 .
$$

The experimental specular component for a near perfect diffuser (mu sulfur) is 9 percent. The "true" specular component is that computed by eq (31) minus 9 percent, and is shown in table 6 . 
TABLE 5. Reflectance of platinum-13 percent rhodium alloy ${ }^{\mathrm{a}}$

\begin{tabular}{|c|c|c|c|c|c|c|c|c|c|}
\hline Wavelength & $\# 1$ a & \# $1 \mathrm{~b}$ & $\# 1 c$ & $\# 2 \mathrm{a}$ & $\# 2 \mathrm{~b}$ & $\# 2 \mathrm{c}$ & Average & $\begin{array}{l}\text { Standard } \\
\text { deviation }\end{array}$ & $\begin{array}{c}\text { Specular } \\
\text { component }\end{array}$ \\
\hline $\begin{array}{l}1.5 \\
2.0 \\
2.5 \\
3.5 \\
4.5 \\
5.5 \\
6.5 \\
7.0\end{array}$ & $\begin{array}{r}0.610 \\
.701 \\
.823 \\
.919 \\
.933 \\
.942 \\
.945 \\
.947\end{array}$ & $\begin{array}{r}0.597 \\
.691 \\
.813 \\
.905 \\
.926 \\
.936 \\
.938 \\
.940\end{array}$ & $\begin{array}{r}0.591 \\
.692 \\
.821 \\
.921 \\
.937 \\
.940 \\
.940 \\
.942\end{array}$ & $\begin{array}{r}0.566 \\
.694 \\
.826 \\
.924 \\
.935 \\
.946 \\
.947 \\
.946\end{array}$ & $\begin{array}{r}0.574 \\
.686 \\
.827 \\
.930 \\
.940 \\
.947 \\
.949 \\
.953\end{array}$ & $\begin{array}{r}0.603 \\
.696 \\
.820 \\
.913 \\
.929 \\
.932 \\
.942 \\
.943\end{array}$ & $\begin{array}{r}0.596 \\
.693 \\
.822 \\
.919 \\
.933 \\
.941 \\
.944 \\
.945\end{array}$ & $\begin{array}{r} \pm 0.005 \\
.002 \\
.002 \\
.005 \\
.004 \\
.005 \\
.005 \\
.005\end{array}$ & $\begin{array}{l}\% \\
69 \\
82 \\
83 \\
86 \\
87 \\
88 \\
90 \\
92\end{array}$ \\
\hline
\end{tabular}

a The specimens were annealed at $1825{ }^{\circ} \mathrm{K}$ prior to measurement. Two samples are represented in this table. and 2. Measurements $l a$ and $2 a$ were made with the sample tilted $10^{\circ}$ to the first focal plane.

-1) This is an approximation of the specular component by using $\left[\left(F_{* d f}-F_{\text {ndd }}\right) / F_{\text {std }}\right] \times 100 \%$, because it includes the diffuse component of flux in the solid angle about the specular direction, and does not account for obvious system corrections.

NBS Reflectance for $\mathrm{Pt} 13 \% \mathrm{Rh}$ reference 38 (various sample temperatures) ${ }^{\mathrm{C}}$

\begin{tabular}{c|c|c|c}
\hline$\wedge$ & $800^{\circ} \mathrm{K}$ & $1100^{\circ} \mathrm{K}$ & $1300^{\circ} \mathrm{K}$ \\
\hline & $\%$ & $\%$ & $\%$ \\
$1.5 \mu$ & 74.8 & 78.7 & 77.4 \\
2.0 & 80.8 & 81.5 & 80.3 \\
2.5 & 83.5 & 83.2 & 82.0 \\
3.5 & 87.4 & 85.7 & 84.5 \\
4.5 & 89.1 & 87.4 & 86.5 \\
5.5 & 90.4 & 88.9 & 87.3 \\
6.5 & 91.4 & 89.9 & 88.7 \\
7.0 & 91.6 & 90.4 & 89.2 \\
\hline
\end{tabular}

"Data are $1-\epsilon$. where $\epsilon$ is normal spectral emittance

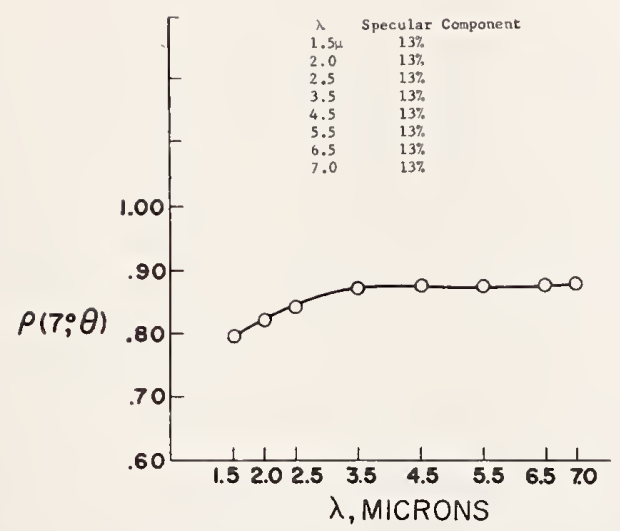

FigurE 10. Spectral directional hemispherical reflectance of gold mesh.

Each point is the average of three determinations.

D. Directional annular cone reflectance. The directional annular cone reflectance of samples of Crystex brand sulfur, $\mathrm{BaSO}_{4}$, and gold mesh was measured at $2.5 \mu$ and compared to the values computed for the perfect diffuser. The directional annular cone reflectance $\rho(d-a-c)$ is defined as follows

$$
\rho(\text { d-a-c })=\frac{\int_{0}^{2 \pi} \int_{\varphi_{1}}^{\varphi_{2}} L^{\prime}\left(\varphi^{\prime}, \theta^{\prime}\right) \cos \varphi^{\prime} \sin \varphi^{\prime} d \varphi^{\prime} d \theta^{\prime}}{L\left(7^{\circ}, \theta\right) \Delta \omega} .
$$

PORCELAIN ENAMEL

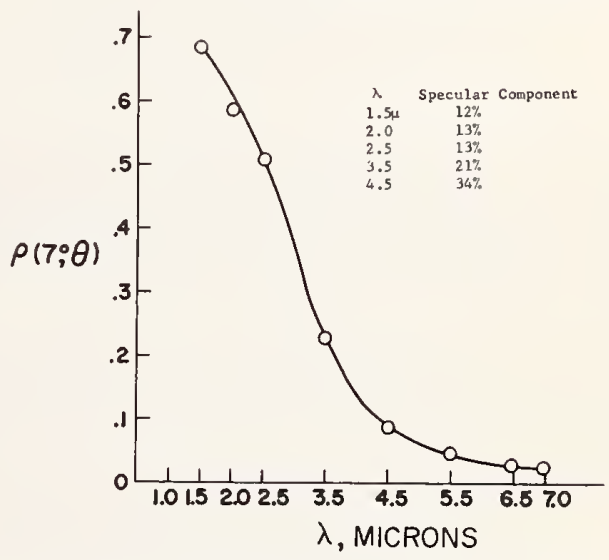

Figure 11. Spectral directional hemispherical reflectance of a porcelain enameled specimen.

In the direct measurements, $\varphi_{2}$ was always $\pi / 2$, and the flux reaching the detector was restricted to the annular solid angle between $\varphi_{1}$ and $\pi / 2$ by means of a circular disk centered on the sample and placed just below the first focal plane. Five shields were used to obtain five different values of $\varphi_{1}$. In each case, the reading with a shield in place was divided by $F_{r}$ to obtain the ratio of the directional annular cone reflectance to the directional hemispherical reflectance. By subtracting the values obtained with successively larger shields, it was possible to separate the hemispherical reflectance into five annular cone 


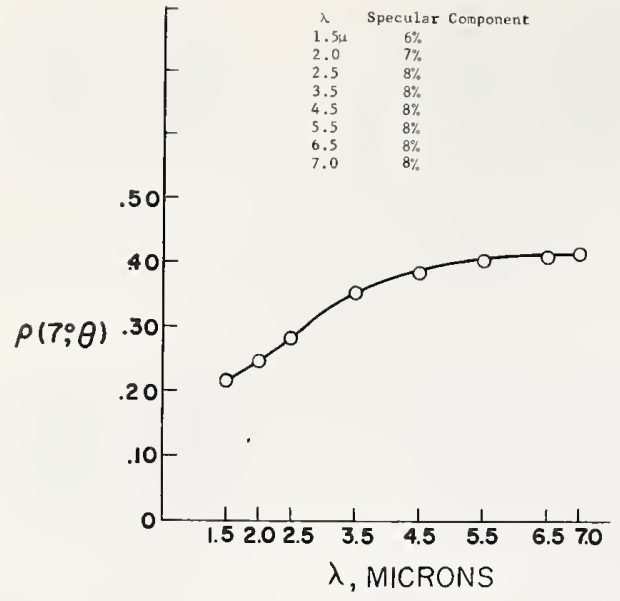

Figure 12. Spectral directional hemispherical reflectance of oxidized Kanthal.

TABLE 6. "True" specular components

\begin{tabular}{c|c|c|c|c}
\hline \hline$\lambda$ & Pt-13\% Rh & Gold mesh & $\begin{array}{c}\text { Porcelain } \\
\text { enamel }\end{array}$ & $\begin{array}{c}\text { Oxidized } \\
\text { Kanthal }\end{array}$ \\
\hline & & & & \\
\hline Micruns & $\%$ & $\%$ & $\%$ & $\%$ \\
1.5 & 60.0 & 4.0 & 3.0 & -3.0 \\
2.0 & 73.0 & 4.0 & 4.0 & -2.0 \\
2.5 & 74.0 & 4.0 & 4.0 & -1.0 \\
3.5 & 77.0 & 4.0 & 12.0 & -1.0 \\
4.5 & 78.0 & 4.0 & 25.0 & -1.0 \\
5.5 & 79.0 & 4.0 & $\ldots \ldots \ldots \ldots . .$. & -1.0 \\
6.5 & 81.0 & 4.0 & $\ldots \ldots \ldots \ldots .$. & -1.0 \\
\hline
\end{tabular}

reflectances. These are compared to the computed ratio for a perfect diffuser in table 7 . It should be noted that the specific measurement technique used will not yield the most accurate data since these data were not corrected for various system losses.

TABLE 7. Ratio of directional annular cone reflectance at $2.5 \mu$ to the directional hemispherical reflectance for diffusely reflecting samples

\begin{tabular}{c|c|c|c|c|c}
\hline$\varphi_{1}^{*}$ & $\varphi_{2}$ & $\begin{array}{c}\text { Perfect } \\
\text { diffuser }\end{array}$ & $\begin{array}{c}\text { Crystex } \\
\text { sulfur }\end{array}$ & BaSo & Gold mesh \\
\hline 0 & & & & & \\
0 & 14.5 & 0.06 & 0.07 & 0.07 & 0.08 \\
14.5 & 34.3 & .25 & .24 & .25 & .28 \\
34.3 & 43.7 & .16 & .17 & .16 & .16 \\
43.7 & 62.8 & .31 & .32 & .31 & .29 \\
62.8 & 79.5 & .18 & .17 & .17 & .16 \\
79.5 & 90.0 & .03 & .04 & .04 & .03 \\
\hline
\end{tabular}

It can be seen from the tables that the barium sulfate comes closest to being a perfect diffuser, and that the sulfur is also a very good diffuser. While the gold mesh reflects an appreciable percentage into each of the annular cones, it reflects more than a perfect diffuser at angles less than $34.3^{\circ}$, and less than a perfect diffuser at angles from $43.7^{\circ}$ io $79.5^{\circ}$. This can be interpreted to indicate appreciable increase in reflection in specular and near specular directions.

\section{Summary and Conclusions}

An ellipsoidal mirror reflectometer was designed and built. It consists essentially of (1) a source of monochromatic flux, (2) an ellipsoidal mirror reflector, and (3) a detector. A beam of chopped monochromatic flux from a monochromator equipped with a Globar source is focused through a small hole in the ellipsoidal mirror onto the specimen, which is centered on the first focal point of the ellipsoid. The reflected flux is collected by the ellipsoidal mirror, and focused onto the detector, which is centered on the second focal point. The incident flux is measured (1) (in the absolute mode) by moving the detector to the first focal point, and measuring it directly, or (2) (in the comparison mode) by substituting a mirror of known reflectance for the specimen. It should be emphasized that the reflectometer measures reflectance in absolute units by both techniques.

Serious problems were encountered, that led to errors on the order of 50 percent of the measured reflectance. These large errors were found to be due to the variation in spatial and angular sensitivity of the detector and over-filling of the detector sensitive area. These errors were eliminated by use of a sulfurcoated averaging sphere on the detector.

Because of the unique optical system, each direction from the first focal point of the ellipsoid toward the mirror corresponds to a point in the first focal plane, and each solid angle from the first focal point corresponds to an area in the first focal plane. Hence, it is possible, by the use of shields in the first focal plane, to measure the flux reflected in any desired solid angle about any desired direction. In this way, accurate estimates can be made of all known losses, and corrections applied. This also permits measurement of (1) directional-hemispherical reflectance. (2) specular reflectance, (3) nonspecular reflectance, and (4) directional annular cone reflectance.

An analysis of all known errors leads to the conclusion that the instrument is capable of measuring directional-hemispherical reflectance to an accuracy of at least 0.01 [15].

Data are presented on the directional-hemispherical reflectance at wavelengths from 1.5 to $7.0 \mu$ of annealed platinum-13 percent rhodium alloy, gold mesh, a porcelain enamel, and oxidized Kanthal; in addition, the specular component of reflected flux is separately evaluated for these materials. Data are also presented on the directional annular cone reflectance of the gold mesh, barium sulfate, and Crystex brand sulfur.

The assistance of Warren D. Hayes, Jr, and David P. DeWitt in construction of the equipment and early testing; and of John T. Perone, Jr., in making many of the measurements, is gratefully acknowledged. 


\section{References}

[1] Bennett, H. E. and Koehler, W. F., Precision measurement of absolute specular reflectance with minimized systematic errors. J. Opt. Soc. Am. 50, 1-6 (1960).

[2] Parmer, J. F., The thermal radiation characteristics of specular walled grooves in the solar space environment, Ph.D. Thesis, Oklahoma State University, May 1965.

[3] Edwards, D. K., Gier, J. T., Nelson, K. E., and Raddick, R. D., Integrating sphere for imperfectly diffuse samples, Appl. Opt. 5 1, 1279-1288 (1961).

[4] Jacques, J. A. and Kuppenheim. H. F., Theory of the integrating sphere, J. Opt. Soc. Am. 45, 460-470 (1955).

[5] Gier, J. T., Dunkle, R. V., and Bevans, J. T., Measurement of absolute spectral reflectivity from 1.0 to 15 microns. J. Opt. Soc. Am. 44, 558-562 (1954).

[6] Coblentz, W. W., The diffuse reflecting power of various substances, Bull. BS 9, 283-325 (1913).

[7] Brandenberg, W. M., Focusing properties of hemispherical and ellipsoidal mirror reflectometers, J. Opt. Soc. Am. 54, 1235-1237 (1964).

[8] Stair, R. and Schneider, W. E., Standards, sources, and detectors in radiation measurements, Symposium on Thermal radiation of solids, ed. Dr. Samuel Katzoff, NASA SP-55. To be published in 1965 .

[9] Janssen, J. E. and Torborg, R. H., Measurement of spectral reflectance using an int egrating hemisphere. Measurement of Thermal Radiation Properties of Solids, ed. J. C. Richmond. NASA SP-31, 1963, pp. 169-182.

[10] White, J. U., New method for measuring diffuse reflectance in the infrared, J. Opt. Soc. Am. 54, 1332-1337 (1964).

[11] Kozyrev, B. P. and Vershinin, O. E., Determination of spectral coefficients of diffuse reflection of infrared radiation from blackened surfaces, Optics and Spectroscopy 6, 345-350 (1959).
[12] Harrison, W. N.. Richmond, J. C., Plyler, E. K., Stair, R., and Skromstad, H. K., Standardization of Thermal Emittance Measurements. WADC Technical Report 59-510 Pt. II (Nov. 1960).

[13] Richmond, J. C., DeWitt, D. P., Dunn, S. T., and Hayes, W. D., Jr., Procedures for the Precise Determination of Thermal Radiation Properties, November 1963 to October 1964. Technical Documentary Report ML-TDR-64-257 Pt. II, Materials Laboratory, U.S. Air Force (1965).

[14] Dunn, S. T., Flux averaging devices for the infrared, NBS Tech. Note 279, 1965.

[15] Dunn, S. T., Design and analysis of an ellipsoidal mirror reflectometer, Ph.D. Thesis, Oklahoma State University, 1965.

[16] Wiebelt, J. A., Engineering Radiation Heat Transfer, (Hott, Rinehart and Winston, 1965).

[17] Bennett, H. E., Silver, M., and Ashley, E. J., Infrared reflectance of aluminum evaporated in ultra-high vacuum, J. Opt. Soc. Am. 53, 1089-1095 (1963).

[18] Bennett, J. M. and Ashley, E. J., Infrared reflectance and emittance of silver and gold evaporated in ultrahigh vacuum, Appl. Opt. 4, 221-224 (1965).

[19] Hass, G., Optucs Section, American Institute of Physies Handbook, pp. 6-19, Coordinating Editor: D. E. Gray, 1957, (McGraw-Hill Book Company, Inc., New York, N.Y.)

[20] Hass, G., Filmed surfaces for reflecting optics, J. Opt. Soc. Am. 45, 945-952 (1955).

[21] Richmond, J. C., DeWitt, D. P., and Hayes, W. D., Jr., Procedures for precise determination of thermal radiation properties, November 1962 to October 1963, NBS Tech. Note 252, 1964.

(Paper 70C2-221) 


\section{High Purity Powdered Csl as a High Reflectance Infrared Diffuser}

\section{Jon Geist, Gerhart J. Kneissl, and V. R. Weidner}

National Bureau of Standards, Washington, D.C. 20234.

Received 3 April 1967.

The purpose of this note is to suggest the use of high purity, powdered CsI as a body scattering material for use as a diffuser in the ir. The reflectance of an optically thick sample of such a material is approximately diffuse ${ }^{1}$ and dependent only upon the ratio of its absorption coefficient to its scattering coefficient. When this ratio is much less than one, the directional hemispherical reflectance of the sample is very close to $100 \%{ }^{2}$ The absorption coefficient of a powder is greater than the absorption coefficicnt of the single crystal which was ground to make the powder. However, both the scattering and absorption coefficients of the powder can be calculated from the index of refraction and absorption coefficient of the single crystal. ${ }^{3}$

The properties of CsI make it one of the most promising, readily available ionic substances for use as an ir diffuser. A perusal of the transmission curves in Ref. 4 shows that CsI has its long wavelength transmission cutoff farther into the ir than most other optical materials. The contribution of the absorption coefficient from the lattice of a pure, perfect single crystal of CsI should be negligible below at least $18 \mathrm{~mm}$ and possibly below $40 \mu \mathrm{m}$. Thèrefore powdered CsI should be a high reflectance diffuser out to wavelengths beyond $18 \mu$.

Flowers of sulfur, a well known high reflectance ir diffuser, ${ }^{5}$ 99.5\% CsI, and ultra pure CsI (assayed impurities less than 80 ppm) were obtained in powdered form from commercial sources. Aluminum flats with a cylindrical cavity, $4 \mathrm{~mm}$ deep and $50 \mathrm{~mm}$ in diameter, were used as sample holders. Some of the cavities were lined with black electrical tape and the others with aluminum foil. Enough powder was poured into the cavity to fill it, and a steel ruler was used to break up any lumps and to level the powder with the upper surface of the flat. The hemispherical directional reflectance of these samples was measured at NBS on a Cary-White 90 spectrophotometer with reflectance attachment. ${ }^{6}$ The data were corrected for the hole loss which is inherent in any instrument of this type.

Figs. 1 and 2 compare the ir reflectance of $99.5 \%$ pure CsI and ultra pure CsI with that of flowers of sulfur. These samples were backed with aluminum foil and were slightly above room temperature when measured. The reflectance of the sample of ultra pure, powdered CsI which was backed with aluminum foil was as much as $3 \%$ higher over portions of the spectrum than the reflectance of the same material backed by black electrical tape. No such cffect was observed with the $99.5 \%$ pure, powdered CsI, or with the flowers of sulfur. These results indicate that $4 \mathrm{~mm}$ of the ultra pure, powdered CsI was not quite thick enough to be opaque, even though $4 \mathrm{~mm}$ of the $99.5 \%$ pure CsI was. This is due either to a difference in the average grain size in two powders, or to a difference in the densities with which they were packed.

The reflectance of the $99.5 \%$ pure CsI sample, which was backed with aluminum foil, was also measured at $300^{\circ} \mathrm{C}$ to see what effect higher temperatures would have on the numerous absorption bands in the room temperature spectrum. This curve is included in Fig. 1. It is interesting that the band between $2800 \mathrm{~cm}^{-1}(3.6 \mu \mathrm{m})$ and $3800 \mathrm{~cm}^{-1}(2.6 \mu \mathrm{m})$ disappeared at the higher tcmperature, while the other bands showed much

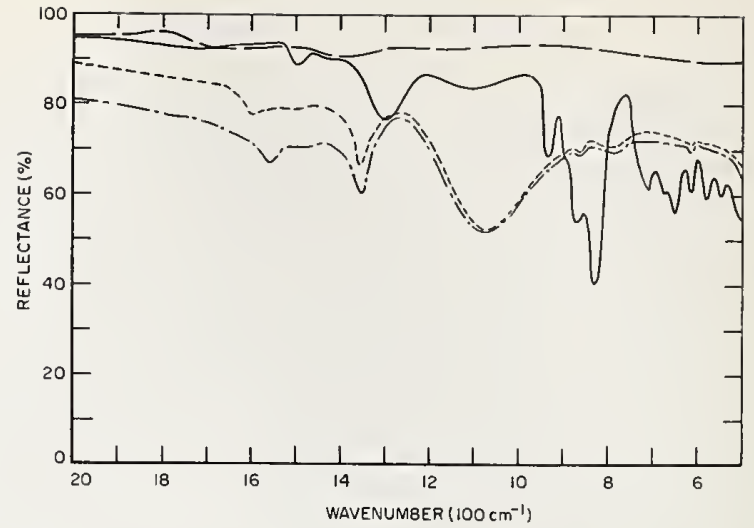

Fig. 1. Reflectance of four powdered samples: - - ultra pure CsI at room temperature; - flowers of sulfur at room temperature; $-99.5 \%$ pure $\mathrm{CsI}$ at $300^{\circ} \mathrm{C}$; and $---99.5 \%$ pure CsI at room temperature.

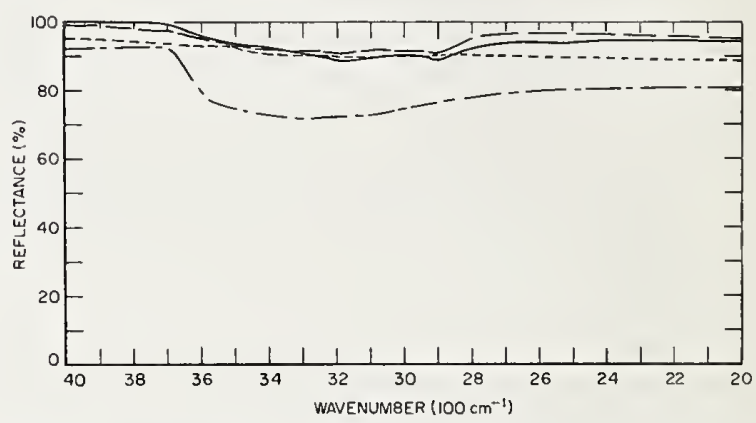

Fig. 2. Reflectance of four powdered samples: - - ultra pure CsI at room temperature; - flowers of sulfur at room temperature; $-99.5 \%$ pure $\mathrm{CsI}$ at $300^{\circ} \mathrm{C}$; and --- $99.5 \%$ pure CsI at room temperature.

less change on heating. This band is due at least partly to $\mathrm{II}_{2} \mathrm{O}$.

Figs. 1 and 2 clearly show that powdered CsI of high purity is a very good choice as an ir diffuser to beyond $20 \mu$. Comparison of the absorption bands in the reflectance spectrum of a natural sulfur crystal ${ }^{5}$ with those in the reflectance spectrum of flowers of sulfur ${ }^{5}$ suggests that the larger bands below $1400 \mathrm{~cm}^{-1}$ (beyond $7 \mu \mathrm{m})$ are intrinsic; that is, they are not due to impurities. If this is so, pure powdered CsI will have a considerably higher reflectance between $8 \mu$ and $20 \mu$ than flowers of sulfur regardless of the purity of the sulfur.

\section{References}

1. W. D. Wright, The Measurement of Colour (Adam Hilger Ltd., London, 1944), p. 16.

2. H. C. Hamaker, Philips Res. Rept. 2, 55 (1947).

3. H. C. Van Dehulst, Light Scattering by Small Particles (John Wiley \& Sons, Inc., New York, 1957).

4. S. S. Ballard, K. A. McCarthy, and W. L. Wolfe, University of Michigan, Willow Run Laboratories, Rept. No. 2389-11-S (Jan. 1959).

5. M. Kronstein, R. J. Kraushaar, and R. E. Deacle, J. Opt. Soc. Am. 53, 458 (1963).

6. J. U. White, J. Opt. Soc. Am. 54, 1332 (1964). Y 


\section{PREPARATION AND COLORIMETRIC PROPERTIES OF A MAGNFSIUM-OXIDE REFLECTANCE STANDARD}

The smoke from magnesium freely burning in air deposited on a satisfactory base forms a uniform, fine-grained, diffusing surface of high reflectance. By observing a few simple precautions one can rrepare such magnesium oxide (MgO) surfaces with reproducible properties. Hence, such surfaces serve as convenient and reliable standards.

1. The magnesium should be obtained in the form of turnings of approximately 0.5 millimeter in thickness and between 3 and 6 millimeters in width, preferably of spiral shape, containing a minimum of magnesium dust, and no cutting oil. If turnings are unavailable, ribbon may be substituted. When ribbon is employed, strips 10 to 15 centimeters long can be held by tongs so that the burning end can be moved about beneath the surface to be coated. As an alternative procedure, a mechanism can be devised to feed the ribbon, at a near constant rate, so that the burning end is maintained in the same position.

2. The oxide must be deposited on a surface not affected by the heat from the burning magnesium. A satisfactory base may be made of (a) aluminum, (b) block porcelain, (c) sheet steel coated with white vitreous enamel, or (d) a baked surface of a sprayed mixture of magnesium oxide and distilled water $(7, \mathrm{p} .21) \%$. Milk or opal glass is often unsatisfactory because it easily cracks during heating. Metallic surfaces are usually to be preferred to non-metallic surfaces. More satisfactory results are obtained with depolished or slightly roughened surfaces because of the higher adhesion of the oxide to the rougher surface. A surface having a high and spectrally non-selective reflectance, throughout the spectrum, is better than a dark surface or a spectrally selective (chromatic) surface. The former is preferred because the magnesium oxide coating is not opaque and a thinner coating can be used to achieve reproducible properties. The thinner coating is also desirable because of speed of preparation, and the coating does not chip off so readily. A suitable holder for a reference surface of magnesium oxide can be made by turning a one- to two-millimeter deep. flat-bottomed, trough in the surface of an aluminum plate. Care must be taken to remove all tool-marks from the area of the trough on which the magnesium oxide is to be deposited. Such a shallow trough provides some protection for the magnesium oxide surface and reduces the tendency of the coating to chip off near the edges.

(*) Numbers in parentheses, sometimes followed by a page number, indicate references in the bibliography. 
3. Place a small quantity (about $5 \mathrm{~g}$ ) of the chips on a refractory dish (zirconium silicate, magnesite, or graphite are suitable refractory materials) and ignite them with a hand blow torch or bunsen burner. Work the unignited chips beneath the flame until a slowly burning bal1 or clinker is formed; this gives a steady stream of smoke.

4. Place the surface to be coated about 8 to $10 \mathrm{~cm}$ above the flame and tilted about 30 degrees from the horizontal. Use of smaller distances results in a coarse-grained deposit and, further, introduces the risk of contamination by possible impurities in the magnesium (3)

5. Move either the combustion dish or the surface being coated from side to side in order to obtain a uniform deposit.

6. When the clinker has to be turned over or broken, in order to permit the magnesium to burn completely, the surface being coated should be temporarily removed, since the burst of flame is likely to carry up large dust particles.

7. Repeat the operation several times until a sufficient deposit is obtained. The layer should be so thick that further increase produces no sensible change in reflectance; the critical thickness is about half a millimeter (4, p. 17). A one- to two-millimeter thickness is preferred. Do not attempt to prepare a thick layer by exposure to a single charge of magnesium. Rather, build up the required thickness by a large number of exposures to small charges. In cases where it is inconvenient to measure the thickness of the coating, place a small dot of india ink on the original surface near the edge, then deposit oxide until the spot cannot be seen in good illumination. If the original surface is dark, put on one coat of MgO first; a deposit of black sinoke (from a candle or smoky gas flame), in a small spot near the edge, then provides a similar test.

8. The operation should be conducted under a well-ventilated hood in order to dispose of the excess oxide.

9. The operator's eye should be protected from the high intensities of visible and ultraviolet radiant energy by suitable goggles (4, p. 30).

10. Magnesium ribbon may be used for small surfaces instead of turnings. But, for large surfaces, careful manipulation is required to produce a uniform coating because of the irregular burning of the ribbon.

11. For many purposes, pressed surfaces of reagent-grade MgO powder are suitable (8).

The properties of a surface prepared by burning magnesium are as follows : 
1. It is a good diffusor (1, P. 59; 2).

2. Its reflectance to the visible portion of the spectrum is very high, 0.97 to $0.98,(1,4,5)$.

3. The reflectance varies with wavelength in the visible spectrum by less than one percent $(2,4)$ when the oxide is first prepared, but. there is some variation with time (see 5 below)

4. The apparent reflectance for 45-degree incidence and normal viewing (standard conditions adopted by the International Commission on Illumination, Cambridge, 1931) is defined to be 1.00 (4, p. 29).

5. Its reflectance varies slightly with time. Although the reflectance is apparently constant with time between 550 and $750 \mathrm{~nm}$, it decreases at wavelengths less than $550 \mathrm{~nm}$; this may anount to as much as. 3 percent in the violet (6, p. 378), and causes the oxide to become slightly yellower with time. In such a case the changes in the chromaticity coordinates, $x, y, z$, and the luminous reflectance, $Y$, computed on the 1931 CIE basis and for CIE Illuminants A and C are as follows:

$\begin{array}{lllll} & -\frac{\Delta x}{+0.001_{1}} & \frac{\Delta y}{+0.000_{9}} & -0.001_{9} & \frac{\Delta Y}{-0.001_{3}} \\ \text { CIE Illuminant A } & +0.001_{4} & +.002_{0} & -.003_{5} & -.002_{3}\end{array}$

6. It is extremely fragile. However, the tendency of the coating to chip off can be reduced if the edges are protected by deposition of the MgO into a flat trough.

The first four properties listed make this reproducible surface a convenient reference standard of reflectance; its usefulness is limited by the fifth and sixth properties (lack of constancy, fragility). which make it often desirable to use working standards of reflectance carefully calibrated in terms of the freshly prepared MgO.

\section{Bibliography}

1. McNicholas, H.J., Absolute methods in reflectometry, J. Research NBS 1,29 (1928) RP3.

2. Priest, I.G., and Riley, J.0., The selective reflectance of magnesium oxide, J. Opt. Soc. Am. 20, 156 (1930).

3. Priest, I. G., Note on the yellowness of commercial magnesium carbonate and the alleged yellowness of magnesium oxide. J. Opt. Soc. Am. 20, 157 (1930). 
4. Preston, J.S., The reflection factor of magnesium oxide; Trans. Opt. Soc. (London), 31, 15 (2929-30).

5. Tynda11, E.P.T., Reflectivity: Non-Metals, Internationa1 Critical Tables $\underline{5}, 262$ (1929).

6. Gibson, K.S., and Keegan, H.J., The calibration and operation of the General Electric recording spectrophotometer of the National Bureau of Standards, J. Opt. Soc. Am. 28, 378 (1938).

7. Moon, Parry and Severance, D.P., Some tests on radiationmixing enclosures, J. Opt. Soc. Am. 29, 21 (1939).

8. ASTM Recommended Practice for Preparation of Reference White Reflectance Standards, ASTM Designation: E 259-66, ASTM Book of Standards Parts 21, 30 (1967).

Supercedes IC 547

Spectrophotometry Section

Optical Physics Division

Institute for Basic Standards 


\title{
A Laser-Source Integrating Sphere Reflectometer
}

\author{
Gerhart J. Kneissl and Joseph C. Richmond
}

Abstract

A reflectometer was developed for measuring the absolute spectral reflectance of materials at temperatures up to $2500^{\circ} \mathrm{K}$. The equipment included (1) a helium-neon laser as the source, capable of operation at $0.6328,1.15$ or $3.39 \mu \mathrm{m}$, (2) a $35 \mathrm{~cm}$ diameter integrating sphere coated with sodium chloride, (3) a lead sulfide detector, and (4) a radio frequency generator for heating the specimen by induction. A spike filter transmitting at the laser wavelength in front of the detector absorbed most of the background radiation from the hot specimen, and a chopped incident beam together with synchronous amplification of the signal from the detector was used to eliminate the effect of the remaining background radiation. The integrating sphere could be evacuated, or operated under a slight positive pressure of purified helium. An error analysis showed that the measured absolute reflectances are in error by less than one percent. Preliminary data are presented for thoria, tungsten and graphite. Graphite was found to be stable in reflectance on heating to $2150^{\circ} \mathrm{K}$ in vacuum. Both thoria and tungsten were somewhat unstable in reflectance on heating in vacuum.

Key Words: diffuse reflectance, graphite, high temperature reflectance, infrared reflectance, integrating sphere reflectometer, laser, reflectance, sodium chloride coating, synchronous amplification, thoria, tungsten. 



\section{Laser Radiometry}

Papers

10.1. Calorimetric Measurement of Pulsed Laser Output Energy.

D. A. Jennings, IEEE Trans. Instr. \& Meas. IM-15, No. 4, 161164 (Dec. 1966).

Key words: Absorption cell; calorimeter; energy measurement; pulsed ruby laser; substitution calibration.

10.2. Radiometric Methods for Measuring Laser Output.

D. A. McSparron, C. A. Douglas, and H. L. Badger, Nat. Bu. Stand. (U.S.) Tech. Note 418, 12 pp. (Nov. 1967).

Key words: Laser; laser energy measurement; optical attenuator; photoelectric photometry; radiometric calibration; radiometry

10.3. A Laser Power Meter for Large Beams.

D. A. Jennings and E. D. West, Rev. Sci. Instrum. 41, No. 4, 565-567 (April 1970).

Key words: C. IW. laser power; diverging laser beam; large diameter laser beam; pulsed laser power

Abstract

10.4. Laser Power and Energy Measurements.

D. A. Jennings, E. D. West, K. M. Evenson, A. L. Rasmussen, and W. R. Simmons, Nat. Bur. Stand. (U.S.) Tech. Note 382, 64 pp. (Oct. 1969).

Key words: Absorption cell calorimeters ; laser energy measurement; laser power measurement; metal plate calorimeters; pulsed ruby laser 



\title{
Calorimetric Measurement of Pulsed Laser Output Energy
}

\author{
D. A. JENNINGS
}

\begin{abstract}
There are several methods by which one may measure the energy output of the pulsed laser. However, the technique which seems to be most promising as far as accuracy and precision are concerned is the calorimetric method. We have designed, built, and calibrated calorimeters for measuring the output energy of the pulsed ruby laser $(6943 \AA)$. The heart of the calorimeter is a small absorption cell containing an aqueous solution of $\mathrm{CuSO}_{4}$. The temperature of the absorption cell, as measured by a thermocouple, indicates the energy absorbed by the calorimeter. The calorimeter was calibrated in two different ways: 1) the known heat capacity of the absorption cell and the thermocouple sensitivity calibration gives a calorimeter calibration, which agrees within 0.3 percent of 2) an electrical energy substitution calibration which is obtained via a heater wire contained in the absorption cell solution. A method has been devised by which two calorimeters may be intercompared. Calorimeters which we have built and calibrated agree with each

Manuscript received June 23,1966 . This paper was presented at the 1966 Conference of Precision Electromagnetic Measurements, Boulder, Colo. Colo.

The author is with the National Bureau of Standards, Boulder,
\end{abstract}

other to about 0.7 percent. This specific calorimeter has been designed to measure energies up to $30 \mathrm{~J}$ and will take peak powers 0 up to $200 \mathrm{MW} / \mathrm{cm}^{2}$.

\section{INTRODUCTION}

(1) NE OF THE IMPORTANT parameters of pulsed laser systems is their output energy. The application of pulsedl asers requires knowing this energy with varying degrees of accuracy, depending on the application. There are several methods by which one may measure the energy output of the laser [1] [7]. In this paper we would like to describe an optical calorimeter for measuring the output energy of a pulsed laser. The calorimetric technique seems very promising as far as accuracy and precision are concerned. The calorimeter which is described in this paper was designed to measure 0.1 - to $30-J$ laser pulses in the 5000 to 10 000- $\AA$ range. 


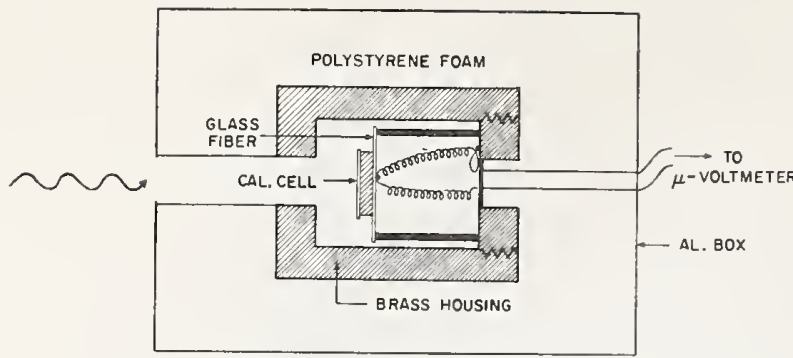

Fig. 1. Cross-sectional diagram of the calorimeter, showing layout of components, method of supporting calorimeter absorption cell, and position of the thermocouple.

\section{General Description}

The basis of the calorimetric system is the calorimeter proper, which is shown in Fig. 1. The calorimeter absorption cell, filled with an absorbing solution, was supported in a massive brass housing (heat sink) by means of small glass fibers. The brass housing was placed in polystyrene foam insulation and the entire system enclosed in an aluminum box. One junction of a $\mathrm{Cu}$-constantan thermocouple was attached to the absorption cell, and the other junction was attached to the brass housing. When a pulsed laser was fired in to the absorption cell, the cell rose in temperature and the thermocouple generated a voltage proportional to the temperature difference between the absorption cell and the brass housing. The output voltage of the thermocouple was then a measure of the energy in the laser beam. The voltage generated by the thermocouple was measured with a microvoltmeter whose output was fed in to a stripchart recorder.

\section{The Absorption Cell}

Since the heart of the system is the absorption cell, we shall describe it in detail. Figure 2 shows a crosssectional view of the absorption cell. The absorption cell was made of silver, so as to give a fast thermal equalization to the cell. The silver cell was then electroplated with $2.5 \times 10^{-4} \mathrm{~cm}$ of gold in order that the tarnish problem be kept to a minimum. The cell diameter was $3.17 \mathrm{~cm}$ and the depth was $3.0 \mathrm{~mm}$. The wall thickness was $0.50 \mathrm{~mm}$. The silver cell had three small holes near the edge. One hole was utilized for filling, and the other two were for a heater wire. The heater wire we shall describe later. The entrance window was made of quartz. Eopoxy was used to bond the quartz to the silver cell and also to seal the fill and heater-wire holes. The cell was filled with a one molar solution of $\mathrm{CuSO}_{4} \cdot 5 \mathrm{H}_{2} \mathrm{O}$. The addition of 2 drops of Bendix purple ink per $10 \mathrm{cc}$ of solution extends the useful range to $5000 \AA$, as opposed to only $6500 \AA$ with $\mathrm{CuSO}_{4}$ solution alone. An absorption curve of this solution is shown in Fig. 3. The absorption coefficient $\alpha$ is defined by the equation

$$
I=I_{0} C^{-\alpha x}
$$

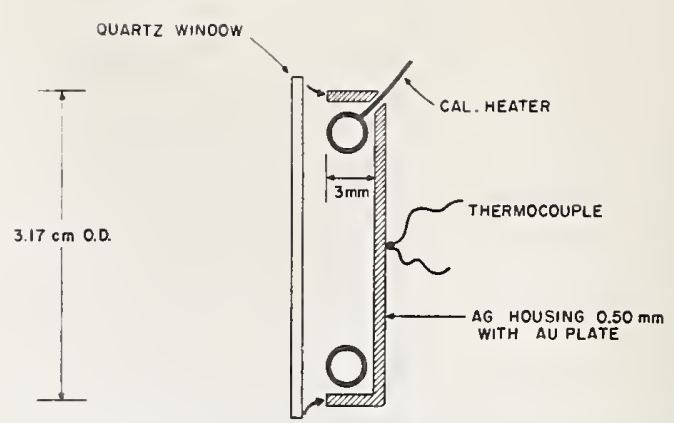

Fig. 2. Cross-sectional diagram of the calorimeter absorption cell, showing the approximate size of components and the position of the heater wire.

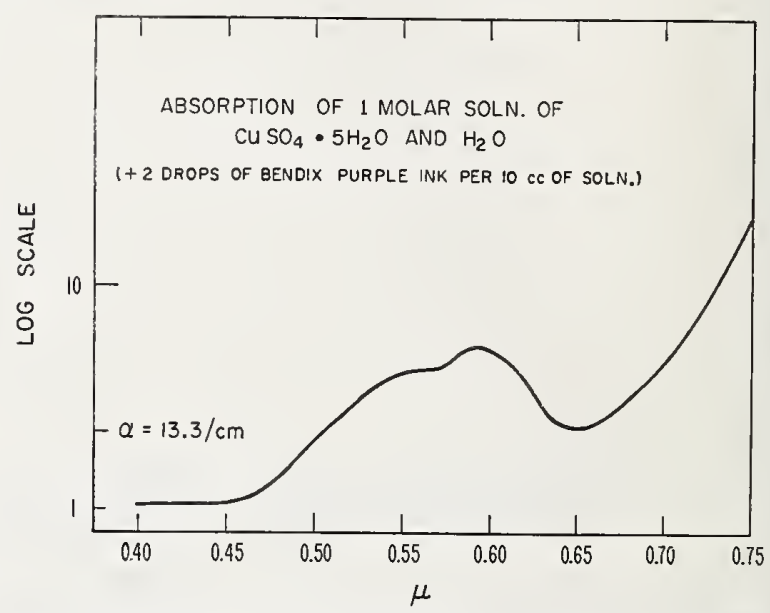

Fig. 3. Absorption spectrum of the solution used in the calorimeter absorption cell. The calibration of the arbitrary log scale is shown and was measured at $6943 \AA$.

where $I$ is the laser beam intensity at a distance $x$ in the absorbing liquid, $I_{0}$ is the initial laser beam intensity, and $x$ is the distance into the liquid as measured from the surface. The absorption coefficient $\alpha$ was measured using a low intensity incoherent light source at $6943 \AA$, and checked using the high in tensity $Q$-switched ruby laser. The cell filled with this solution will absorb 99.9 percent of the laser beam, not counting the Fresnel losses of the window.

A cell of this description gave about $0.1^{\circ} \mathrm{C}$ temperature rise for a $1-\mathrm{J}$ input.

\section{Calibration of the Calorimeter System}

Great care must be taken in the calibration of the calorimeter since the accuracy is no better than the calibration. Two independent methods of calibration were used. One calibration was based on the heat capacity of the absorption cell and the thermocouple calibration, and the second calibration was based on an electrical energy substitution via the heater wire.

The heat capacity calibration consisted of weighing all the component parts of the absorption cell and then 
using the best obtainable values for the specific heats to calculate the heat capacity of the absorption cell. All components of the absorption were accurately weighed. The thermocouple sensitivity was calibrated using standard techniques. The heat capacity calibration factor for this particular calorimeter was calculated to be $3.102 \pm .024 \mu \mathrm{V} / \mathrm{J}$. The uncertainty was obtained by taking into account the errors in the specific heats, weighing, and thermocouple sensitivity. This calibration factor has also been adjusted to correct for Fresnel reflection losses of the entrance window. The error here was very small, since the correction was only on the order of 4 percent. The true calibration was obtained by use of the following equation

$$
\mathrm{SHC}_{\imath}=\mathrm{SHC}(1-R)
$$

where $\mathrm{SHC}$ is the specific heat calibration, $\mathrm{SHC}_{\mathrm{t}}$ is the adjusted specific heat calibration, and $R$ is the reflection as calculated from the Fresnel equations.

The electrical energy substitution calibration was accomplished by passing a known dc current through the heater wire for a known length of time. The heater wire was Teflon coated and had a resistance of $16 \mathrm{ohms}$ per foot and a total resistance of $110 \mathrm{ohms}$.

The dissipation of heat outside the cell from the leads to the heater was small and corrected for in the calibration.

Prior to making the electrical energy substitution calibration, all instruments were calibrated to an accuracy of 0.2 percent.

The electrical energy calibration proceeded in the following manner. The gate output from a preset scaler, counting the line frequency ( 60 hertz), was used to turn on a transistor switch. The transistor was powered by a constant current source (constant to 0.01 percent), and the absorption cell heater wire was in the collector circuit of the transistor. The voltage drop $V$ across the heater wire was measured via the calibrated strip-chart recorder. Since the voltage $V$ and the heater-wire resistance $R$ and the time $t$ are known, the energy $J$, put in to the absorption cell, can be calculated from

$$
J=\frac{V^{2}}{R} t
$$

A typical calibration trace obtained from the stripchart recorder is shown in Fig. 4. The overshoot at the beginning of the trace was due to the close proximity of the heater wire to the silver housing of the absorption cell. The error, introduced because the silver housing was warmer during the overshoot than when in an isothermal condition, was small enough to be neglected. An estimation of this error from both convection and radiation cooling shows it to be less than 0.25 percent. Other losses, such as conduction of heat along the wire leads and quartz mounting fiber, were less than 0.1 per-

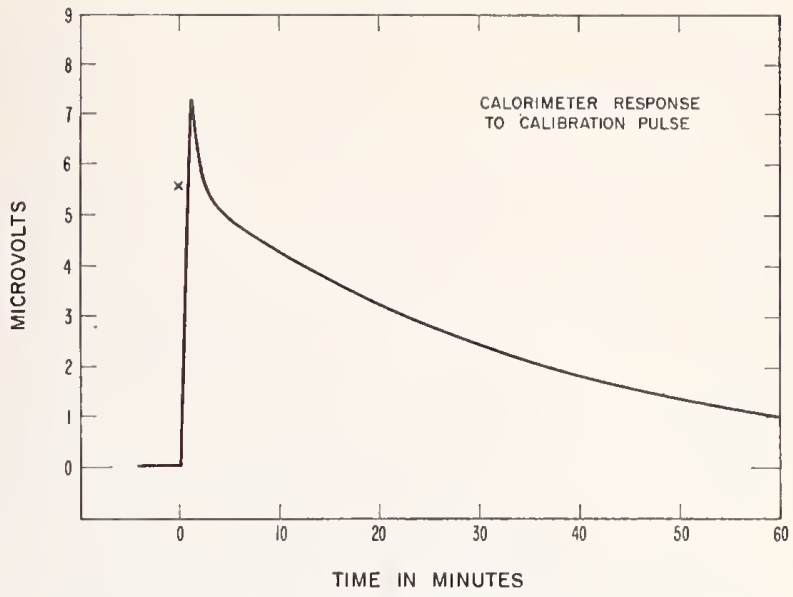

Fig. 4. Typical chart trace of the calorimeter system response to a calibration. Note the overshoot due to the close proximity of heater wire to the silver housing. The total energy input to the calorimeter here was $1.55 \mathrm{~J}$.

cent over the entire measurement (20-30 minutes).

The electrical energy substitution calibration was finally obtained by making an exponential extrapolation of the thermal decay back to time $=0$, as shown by the $x$ on the chart (Fig. 4).

The precision of the calorimeter system was determined by the reproducibility of the electrical energy substitution calibration. The electrical energy substitution calibration factor for this calorimeter system was $3.092 \mu \mathrm{V} / \mathrm{J}$. This factor was the average of 13 calibration runs. The data had a standard deviation of 0.009 . The two calibrations, specific heat and electrical energy substitution, compare very favorably with each other. From this we assume that the accuracy and precision is good to at least \pm 1 percent.

\section{RESULTS AND INTERCOMPARISONS of Calorimeters}

A typical output of the calorimeter system for a normal ruby laser is shown in Fig. 5. Notice here that there was no overshoot as compared with the electrical calibration. The output energy of the laser was found by making the exponential extrapolation to time $=0$, indicated by the $x$ on the chart, and then dividing the deflection at time $=0$ by the calibration factor.

Two calorimeter systems of the same basic design were intercompared with a setup as shown in Fig. 6. The technique was to measure the reflectivity $R$ of the beam splitter. This position of calorimeter, as shown in Fig. 6, was the $A$ position and the reflectivity of the beam splitter was $R_{A}$. Then the calorimeters were interchanged to position $B$, and $R_{B}$ measured. Now if calorimeter No. 1 was assumed to be correct, and calorimeter No. 2 was in error by a fractional amount $\Delta$ 


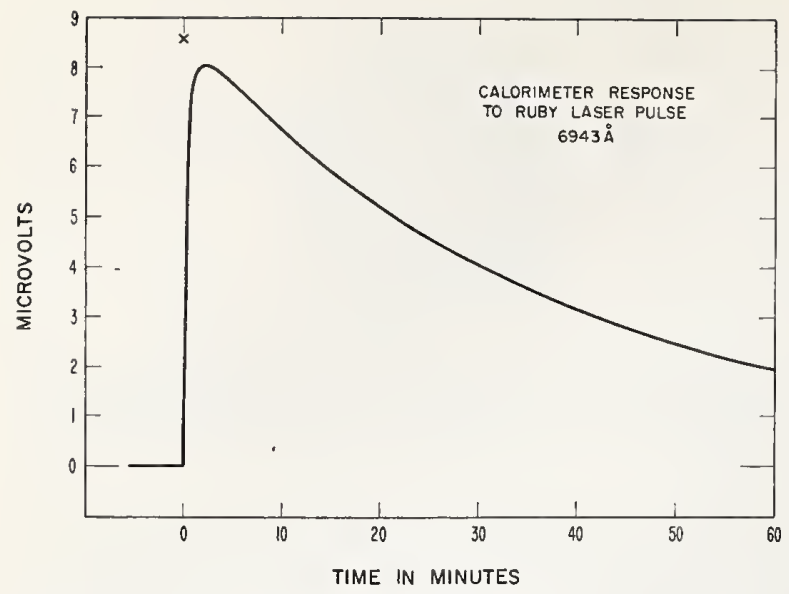

Fig. 5. Typical chart trace of the calorimeter system response to normal ruby laser pulse. Note here that there is no overshoot at the beginning of the trace. The energy in the laser pulse for this shot was $2.77 \mathrm{~J}$.

$50 \%$ BEAM SPLITTER

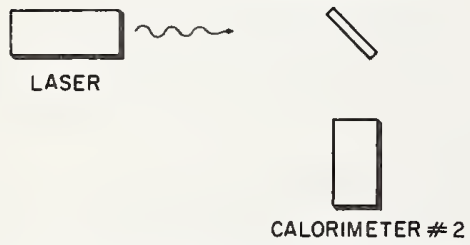

Fig. 6. Block diagram of apparatus arrangement used in the intercomparison of two calorimeters.

where

$$
\Delta=\frac{E_{2}{ }^{1}-E_{2}}{E_{2}{ }^{1}}
$$

where $E_{2}{ }^{1}$ is the true incident on calorimeter No. 2, and $E_{2}$ is the apparent energy, then $\Delta$ can be shown to be equal to

$$
\Delta=\frac{R_{A}}{R_{B}}-1
$$

For lasers which have a polarized output, i.e. $90^{\circ}$ or $60^{\circ}$-oriented ruby laser rods, the laser rod must be orien ted properly, relative to the beam splitter. This was easily done to $\pm 1^{\circ}$, and represented an error of no more than \pm 0.1 percent.

Using this technique, we find a $\Delta$ for our calorimeter systems of 0.007 . This result was from an average of 10 comparison runs. The data had a standard deviation of 0.002 .

\section{Discussion}

We have made several calorimeters modeled after the same basic design with modifications to include cone shaped absorption cells, a different absorbing liquid $\left(\mathrm{CuCl}_{2}+\mathrm{H}_{2} \mathrm{O}\right)$, and Brewster's angle input. We have tested the calorimeters with laser energies up to $15 \mathrm{~J}$ (normal laser) and powers up to 150-200 megawatts ( $Q$-switched), and found no anomalies in the calorimeters. It would seem possible to scale the calorimeter components to make calorimeter systems that would measure very high energies, say $10^{4} \mathrm{~J}$ with one percent accuracy and precision, by using a large aperature, large volume calorimeter so as not to exceed a certain energy density, in order to avoid a change in state of the absorbing fluid.

\section{ACKNOWLEDGMENT}

The author would like to thank Dr. J. L. Hall, of the Joint Institute of Laboratory Astrophysics, for the many stimulating discussions concerning the design of the calorimeters, and A. L. Rasmussen, of the National Bureau of Standards, for many of the measurements.

\section{REFERENCES}

[1] T. H. Maiman, R. H. Hoskins, I. J. D'Haenens, C. K. Asawa, and V. Evtuhov, "Stimulated optical emission in fluorescent solids. II. Spectroscopy and stimulated emission in ruby," Phys. Rev., vol. 123, p. 1151,-1157, August 15, 1961.

[2] S. Koozekanani, P. P. Debye, A. Krutchkoff and M. Ciftan, "Measurements of the laser output," Proc. IRE (Correspondence), vol. 50, p. 207 , February 1962.

[3] T. Li and S. D. Sems, "A calorimeter for energy measurement of optical masers," Appl. Oplics, vol. 1, pp. 325-328; May 1962.

[4] A. L. Glick, "A method for calibration of laser energy output," Proc. IRE (Correspondence), vol. 50, p. 1835, August 1962.

[5] R. C. C. Leite and S. P. S. Porto, "A simple method for calibration of ruby laser output," Proc. IRE (Correspondence), vol. 51, pp. 606-607, April 1963.

[6] J. A. Calviello, "An optical calorimeter for laser energy measurements," Proc. IRE (Correspondence), vol. 51, pp. 611-612, April 1963.

[7] M. Stimler, "Ballistic torsional pendulum," U. S. Gov. Res. Rept. AD-420, p. 469, October 1963. 


\title{
Radiometric Methods for Measuring* Laser Output
}

\author{
D. A. McSparron, C. A. Douglas, and H. L. Badger
}

\section{NBS Technical Note 418}

\begin{abstract}
The output of pulsed lasers may be measured with reasonable accuracy by modifications of established radiometric methods. The receiver, thermopile or phototube, is used ballistically. It is calibrated by an incandescent-lamp standard of spectral irradiance. The laser beam is attenuated to make its irradiation on the receiver comparable to that from the calibrating source. Attenuation is accomplished by intercepting the entire laser beam on a diffusely reflecting surface. The at tenuation factor is determined from direct measurement of the reflectance of this diffusing surface and the geometric constants of the system. The accuracy of the measurement of laser output is dependent upon the accuracy of calibration of the standard of spectral irradiance and the accuracy with which the spectral sensitivity of the receiver, the spectral transmittance of the band-pass filters, and the reflectance of the diffuser can be determined. Measurements made with different photoelectric receivers agree to about $1.5 \%$ using a $1 / 4-$ joule pulsed ruby laser as a source. Total uncertainty is estimated to be about $5 \%$. Measurements made using these radiometric methods have been compared with calorimetric measurements and a discrepancy of $9 \%$ was observed.
\end{abstract}

Key Words: Laser, radiometry, laser energy measurement, radiometric calibration, photoelectric photometry, optical attenuator.

\section{Introduction}

The rapid development of laser technology has left workers in the field without recognized methods of measuring the parameters of interest. The situation is particularly bad in the measurement of the energy emitted from pulsed lasers. Discrepancies of $25 \%$ or more have been common. Two widely differing approaches to the problem have been popular, namely, radiometric and calorimetric. A common calorimetric approach entails the absorption of the entire laser beam in a liquid. The temperature rise of the liquid is then a measu re of the total energy contained in the pulse. Calorimetric measurements are difficult to perform because of the low signal levels obtained from low energy pulsed lasers, usually a few microvolts, and because of the long recycling times, thirty minutes or more. In addition, no information can be obtained from calorimetric measurements on the peak power levels of the pulse. The work described in this paper employs a radiometric approach. In addition to the advantages of relatively high signal levels and fast recycling times, a radiometric method can provide information about the peak power levels in the pulse through the display of the receiver output on an oscilloscope. Although the work to date has involved only a relatively low powered, approximately $1 / 4$-joule, conventional ruby laser operated at 694.3 $\mathrm{nm}$, the measurement techniques and procedures should be readily applicable to a wide range of energies and wavelengths.

A pulsed laser beam differs from conventional sources in that it is highly monochromatic, extremely intense, of short time duration, and in some cases polarized. These properties require modification of conventional radiometric measurement techniques if existing standards are to be used for the calibration. Such receivers as vacuum phototubes, photomultipliers and thermopiles can tolerate maximum incident power levels only of the order of milliwatts $/ \mathrm{cm}^{2}$ for conventional tubes or at most watts $/ \mathrm{cm}^{2}$ for present biplanar photodiodes. Beyond these levels physical damage occurs. Since instantaneous power levels in laser beams run to megawat $\mathrm{s} / \mathrm{cm}^{2}$ and higher, some attenuation device is necessary. An ideal......

*

The complete Technical Note 418 is given in Section 6 of this Volume 7; see pages 336 to 350 . 


\title{
A Laser Power Meter for Large Beams*
}

\author{
D. A. JPRNANG, AND E. D. WEST \\ Radio Standards Physics Division, National Burean of Standards, Bualder, Colorado 80302
}

(Recuived 6 October 1969; and in final form, 29 December 1969)

\begin{abstract}
A power meter is described in detail for large or divergent laser beams, either cw or repetitively pulsed. 'The meter measures the thow of heat generated by the beam and is calibrated with an electrical heater wound just behind the alsorbing surface. The meter is capable of power measurements of 1 to $30 \mathrm{~W}$ accurate to $\pm 2.5 \%$.
\end{abstract}

\section{INTRODUCTION}

in Ili meaturement of average cw litser power is often a rovitine operation, especially if the laser beam is well collinitted and less than $1 \mathrm{~cm}$ in cross section. The measurement is not so simple when the bean is large in diameter, saly 5 to $10 \mathrm{~cm}$, or not well collimated, as in diode lasers or laser beams which have been made to diverge.

IIe would like to describe a simple device, which allows the measurement, on an absolute basis, of the average power of lasers of large diameter and/or divergent beams. The device is also useful for the measurement of average power of repetitively pulsed lasers.

The instrument is designed 10 measure average power from 1.0 to $30.0 \mathrm{~W}$; however, there seems to be no reason why the derice cannot be scaled to higher or lower powers. In what follows we shall describe in detail, the construction of the instrument, its calibration, and operating characteristics.

\section{THE INSTRUMENT}

The entire instrument, which we will call a disk calorimeter (DC), is constructed of aluminum alloy 6061 with a vapor blasted, black anodized finish. In Fig. 1 we show a cross section of the DC, which has cylindrical symmetry about a horizontal axis. The main parts of the DC are the disk, which absorbs the radiation and converts it to heat; the conducting tube, which carries the heat to the sink; the thermopile, which measures the temperature gradient in the tube; and a calibrating electric heater. The absorbing disk is $0.5 \mathrm{~mm}$ thick and the conducting tube has a wail thickness of $0.5 \mathrm{~mm}$ and is $1.2 \mathrm{~cm}$ long. In order to avoid the thermal resistances of mechanical connections, the disk, the conducting tube, and heat sink witl its vertical cooling firs are machined from a solid block of aluminum. The front surface of the disk is painted with a special flat black paint. ${ }^{2}$ The characteristics of this surface are discussed later.

The principle of operation is as follows: 'The laser beam of interest is made to fall on the disk where it is converted into heat. This heat raises the temperature of the disk and causes a temperature difference $\Delta T$ to be established along the tube connecting the disk with the heat sink. The heat is then dissipated into the air via the cooling fins. The $\Delta T$, which is generated along the tube, is measured by a fourjunction series connected copper-constantan thermopile of $0.13 \mathrm{~mm}$ wire (No. 36 gauge). Since the anodized finish forms an insulating layer, the thermocouple junctions are placed in contact with the cylinder and epoxyed in place, the hot junction near the disk and the cold junction near the heat sink. The junctions are distributed symmetrically about the cylinder.

On the back side of the disk, at a $3 \mathrm{~mm}$ distance from the cylinder, is a ledge on which is wound a heater wire of 0.13 mm manganese wire ( $\mathrm{No} .36$ gauge). It has a resistance of approximately $90 \Omega$ and is connected to feedthroughs by 18 gauge copper wire. This heater allows one to electrically calibrate the DC by Joule heating.

\section{CALIBRATION AND TEST}

The calibration is eff ected by determining the sensitivity to electrical heating $S_{e l}$ of the meter in the following manner: The output voltage of the thermocouples is amplified by a suitable microvoltmeter and then recorded on a strip chart. A known amount of power is supplied to the DC by applying a known voltage to the heater and the output in microvolts is read from the chart recorder. The $S_{l l}$ is then

$$
S_{e l}=\mu \mathrm{V} / \mathrm{W} .
$$

The data for a typical calibration run are shown in Table I.

lig. 1. Cross section of the disk cillorimeter. A laser beam incident on the disk is converted to heat which sets up a temperature gradient in the conducting tube. The gradient is sensed by the thermopile.

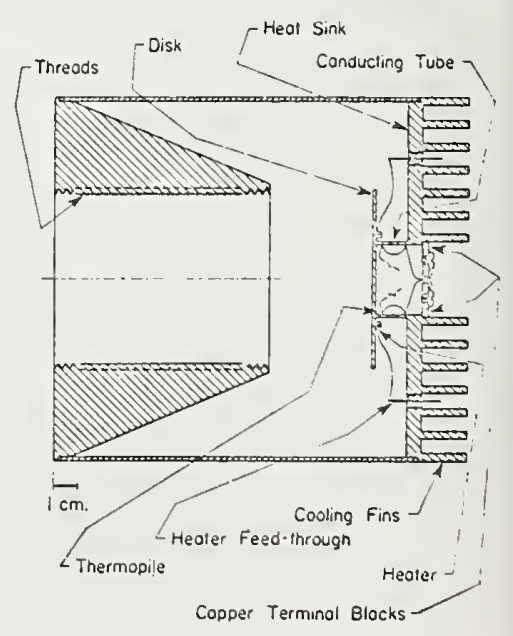




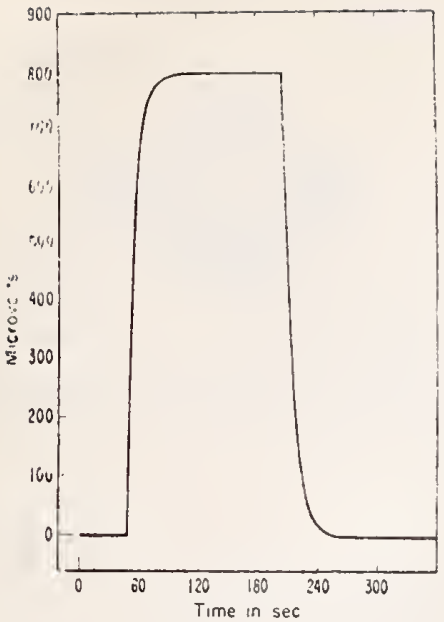

The calibration is over the power range 1-20 W. The zero clrift was meatsured over several hours and wits less than $0.5 \mu \mathrm{V}$. \%ero stablitity is required for atcumale calibration and power metsurement, If the flat black paint absorbed all of the incident laser beams then the calibration would be complete, but as one might expect, it does not. We have measured this particular paint and found it to be flat from 400 to $1060 \mathrm{~nm}$ and at $10.6 \mu$. The total reflection $r$ from the surface was measured to be $4.0 \%$. The damage threshold for the black paint, as measured by a $\mathrm{CO}_{2}$ laser, is $500 \mathrm{~W} / \mathrm{cm}^{2}$. The sensitivity to laser input $S_{l}$ is obtained from $S_{e l}$ by correcting for this reflection

$$
S_{l}=S_{e l}(1-r)^{-1} \text {. }
$$

This calibration has been compared with other methods developed at NBS and we find agreement to $3 \%{ }^{2}$

The instrument requires $50 \mathrm{sec}$ to reach $99 \%$ of its ultimate reading. A calibration trace is shown in Fig. 2. We have compared the time development of the response of the DC to both laser power and Joule heating and find no difference; however, this point is strictly relevant only to the time required to make a measurement, since the DC is used in the steady state mode.

\section{ACCURACY}

Since one unay know the voltage and resistance better than $0.1 \%$ and the absorption of the surface better than $1.0 \%$, it would scem that the accuracy is limited by other quantities not so easily measurable, such as thermal emf, a constant rate of change in ambient temperature, linearity of the readout electronics, and changes in ambient lighting conditions during a power measurement. In some power measurements one may need to be concerned with pump
TAl: $x$ T. Electrical calibration data for the disk catorinuter.

\begin{tabular}{|c|c|c|}
\hline Power $v^{2}, R$ & Response & Sensilivity $f_{,}$ \\
\hline$i(x)=w$ & $7.3 .20 \mu \mathrm{V}$ & $\times 2.76, \mu l, 16$ \\
\hline 4.914 & 40.5 .2 & $\times 2.46$ \\
\hline 9.9 .30 & 811.2 & 81.69 \\
\hline 14.94 & 1212. & 81.11 \\
\hline 19.71 & $150 \times$. & 81.08 \\
\hline
\end{tabular}

lamp light and electrical noise from the laser electronics. In various versions of the apparatus we have fabricaled the DC of copper and have used different themocouple material and different calibrated microvolt meters and chart recorders. The various versions behave in essentially the same way. The DC fabricated of copper behaves essentially as the aluminum DC, with the exception of response time which is twice as fast.

The largest source of error is geometrical variation of response over the surface of the disk. In order to ascertain the magnitude of this error, we used a laser beam of $5 \mathrm{II}$ to probe the response. The laser power level was held to $\pm 1 \%$ during the measurements. Beams of three different diameters were used, $5.5,4.0$, and $2.0 \mathrm{~cm}$. Table II shows the results of this test. We feel that the $4 \mathrm{~cm}$ beam reads very close to the ture power. The $2 \mathrm{~cm}$ beam, which exaggerates the nonuniformity, read different by only $3 \%$.

For accurate measurements the small variation of $S_{e t}$ with power (Table I) should be taken into account. We feel that this nonlinearity is due to convection cooling of the disk; however, this can be calibrated out by performing the calibration at the power level for which the DC is to be used.

With the foregoing in mind, we can now see how the errors propagate through the calibration. The sensitivity to a laser beam in terms of its factors is

$$
S_{l}=\left(R / v^{2}\right) D(1-r)^{-1} G,
$$

where $R$ is the resistance of the heater, $v$ is the voltatge applied to the heater, $D$ is the deflection in microvolts as read from the chart recorder, $r$ is the total reflection from the disk, and $G$ is the geometrical response factor. The

TABLE Ir. Power indicated by the disk calorimeter as a function of beam size with a $5 \mathrm{~W}$ input beam.

\begin{tabular}{ll}
\hline Beam size & Power \\
\hline $5.5 \mathrm{~cm}$ & $4.96 \mathrm{Wl}$ \\
4.0 & 5.0 \\
2.0 & 5.16
\end{tabular}


fractional error in $S i$ is then given by the expression

$$
\frac{d S_{l}}{S_{l}}=\frac{d R}{R}+\frac{2 d v}{i}+\frac{d D}{D}+\frac{d r}{(1-r)}+\frac{d G}{G} .
$$

In our work we estimate $d R / R-0.1 \%, 2 d v / v<0.2 \%$, $d D: D=0.1 \%, d r /(1-r)<1 \%$, and $d G / G=1 \%$ for beams 4 to $5 \mathrm{~cm}$ in diameter, ranging up to $3 \%$ for beams $2 \mathrm{~cm}$ in diameter. The corresponding error in $S_{l}$ ranges from \pm 2.4 to $\pm 4.4 \%$. 110 fecl then that if the disk is completely filled, the $D C$ gives the true power to $\pm 2.4 \%$ and in the worst case of small be:ms $\pm 4.4 \%$.

\section{ACKNOWLEDGMENT}

We are grateful to John C. Schleter of the Spectrophotometry Section for measurements of the spectral reflectance of the black painted surface.

* 'This work was supported by the Night Vision Laboratory, U. S. Army lilectronics Command, l't. Balvoir, Va., and by the Advanced Resiarch Projects Arency under order No. 891.

'l'he particular paint seal was Nextel velvet coating 101-C10 black made by 3.11 Co.

${ }^{2}$ D. A. Jennings, E. D. West, K. M. Evenson, A. L. Rasmussen, ind W. R. Simmons, Technical Note No. 382, Nationa\} l'sureau of Stimdlards, Boulder, Colo., Oct. 196\%. 


\section{Laser Power and Energy Measurements}

\section{NBS TECHNICAL NOTE 382}

\section{ISSUED OCTOBER 1969}

Nat. Bur. Stand. (U.S.), Tech. Note 382, 64 pages (Oct. 1969)

D. A. Jennings, E. D. West, K. M. Evenson

A. L. Rasmussen, and W. R. Simmons

Most laser calorimeters operate in a constant temperature environment. The calorimeters can be used as deflection devices or the data can be analyzed by extrapolation or integration. Consideration of the heat flow problem common to all of these methods points up the underlying assumptions and the possible errors involved.

Calorimeters for measuring the output energy of pulsed ruby and neodymium glass lasers have been built and calibrated. The absorbing medium is an aqueous solution of $\mathrm{CuSO}_{4}$. Calibration of laser energy detectors has been estimated uncertainty of $\pm 2 \%$ for input energies of $0.1 \mathrm{~J}$ to lo0J. Comparisons of absorption cell calorimeters with metal plate calorimeters agree within $1 \%$.

Instrumentation is described that is used for the calibration of $\mathrm{CW}$ laser power meters. The calibration unit employs an absorption cell calorimeter to calibrate the output of a photovoltaic cell transfer detector. The power meter to be calibrated is then compared to the calibrated output of the transfer detector. The calibrations are within an accuracy of $4 \%$.

A discussion and description is also given of several types of calorimeters that have been used to measure the output of a 100 watt $\mathrm{CO}_{2}$ laser. The most recent design provides for measurement from 1 watt to 5 kilowatts with a measured accuracy of better than $3 \%$.

Key words: Calorimetry; laser; laser calorimetry; laser energy; laser power. 



\section{General Photometry}

Papers

11.1. The Waidner-Burgess Standard of Light.

H. T. Wensel, Wm. F. Roeser, L. E. Barbrow, and F. R. Caldwell, J. Res. Nat. Bur. Stand. (U.S.) 6, 1103-1117 (RP325, June 1931).

Key words : Platinum blackbody; primary standard of light;

SI unit of luminous intensity

11.2. Announcement of Change in Electrical and Photometric Units.

Sect. III Photometric Units, p. 5-7 (May 1947).

Key words: International photometric units of 1948 ; platinum blackbody radiator

11.3. Vertical Distribution of Light from Gas Filled Candlepower Standards.

L. E. Barbrow, S. W. Wilson, Illum. Eng. 53, No. 12, 645-648, (Dec. 1958).

Key words: Lamp intensity ; standard lamps ; variation with angle; vertical distribution

11.4. Concepts, Terminology, and Notation for Optical Modulation.

C. S. McCamy, Photog. Sci. \& Engrg. 10, No. 6, 314-325 \&

Note (Nov.-Dec. 1966).

Key words: Absorptance; contrast; modulation reflectance ; notation; propagance; optical density; reflectance factor; symbols; transmittance factor.

11.5. The Metric System in Illuminating Engineering.

L. E. Barbrow, Illum. Eng. 62, No. 11, 638-640, (Nov. 1967). Key words: Illuminating engineering; metric system; nomenclature; photometric units; International System of Units

11.6. Optical Resource Letter on Radiometry.

Fred E. Nicodemus, J. Opt. Soc. Amer. 59, No. 3, 243-248, (Mar, 1969).

Key words: Bibliography; illumination; infrared radiometry ; light; ultraviolet radiometry

11.7. Introduction of the International Practical Temperature Scale 1968: some effects in relation to light sources, colour temperature, and colorimetry.

J. S. Preston, Lighting Res. \& Tech., 1, No.4, 248-250 (1969). Key words: Color temperature; nomenclature; SI unit; spectral distribution; luminous efficacy; tungsten-filament lamps; platinum blackbody

11.8. Photometric Unit Chart.

Francis Clark and L. E. Barbrow, Illum. Engr. 64, No. 9, 603 (Sept. 1969).

Key words: Illumination; luminance; luminous energy ; luminous exitance; luminous flux; luminous intensity; units 


\section{General Photometry-Continued}

\section{Abstracts}

11.9. Filters for the Reproduction of Sunlight and Daylight and the

Determination of Color Temperature.

Raymond Davis and K. S. Gibson, Misc. Pub. 114, Nat. Bur. Stand. (U.S.), 165 pp. (Jan. 21, 1931) ; reprinted 1970 as (PB192894).

Key words: Sunlight; color temperature, 1,600 to 20,000 K ; color filters; colorimetry; spectrophotometry

11.10. A Physical Photometer.

Ray P. Teele, J. Res. Nat. Bur. Stand. 27, RP1415, 217-228 (Sept. 1941).

Key words: CIE luminous efficiency function; luminous efficiency filter; luminous intensity; standard observer...-

11.11. New Principle of Absolute Photometery.

Calvin S. McCamy, Abstract MC16, 1963 Spring Meeting, Jacksonville, Florida. J. Opt. Soc. Am. 53, No. 4, p. 511 (Apr. 1963).

Key words: Adjustable light source; photometric comparator; quantitative attenuation; Riemann integration; sliding shutter; optical modulation

11.12. Changes in the U.S. Unit of Luminous Flux.

Tech. News Bull. 54, No. 3, p. 55 (March 1970).

Key words: Color temperature $2800 \mathrm{~K}$; gas-filled tungstenfilament lamps; total luminous flux; U.S. standard 


\title{
THE WAIDNER-BURGESS STANDARD OF LIGHT
}

\author{
By H. T. Wensel, Wm. F. Roeser, L. E. Barbrow, and F. R. Caldwell
}

AESTRACT

A source of light sufficiently reproducible to serve as a fundamental photometric reference standard has been obtained by carrying out the criginal suggestion of Waidner and Burgess to immerse a hollow inclesure in a bath of molten platinum and to make observations during the pcriod of freezing.

The platinum, of exeeptionally high purity, was contained in thorium oxide crucibles and was heated by means of a high-frequeney induction furnace. The brightness of the source, reproducible to 0.1 per cent, was 58.84 international candles per, square centimeter.

The platinum used was not appreciably contaminated by being melted and frozen over 100 times in crucibles of fused thorium oxidc. Various tests indicated that the platinum was at all times purer than 99.997 per cent.

\section{CONTENTS}

I. Existing units and standards

II. Black body radiation standards

III. Apparatus and methods

1. The furnace and crucible

2. The platinum 1108

3. 'The photometrie arrangement_............. 1109

4. Mcthod of making observations .

IV. Results_...

V. Discussion of errors

VI. Comparison of results with those of other obscrvers ... . . .

VII. Black body standards of highcr color temperatures................ 116

VIII. Summary

IX. Aeknowledgments........... 1117

\section{EXISTING UNITS AND STANDARDS}

Since visible light, by its very definition, involves physiological and psychological reactions, it is not possible to derive a unit of light from the units of a purely physical system. To measure light, an arbitrary unit must be estrblished, in addition to the arbitrary units upon which a physical system may be based. For example, a unit of luminous intensity might be defined as the light corresponding to radiation of a specified spectral distribution or quality and of a specified energy flux per unit solid angle. Such a unit would have the appearance of being derived from those of the physical system, but light is of such a nature that the derivation has no special significance, the practical value of the unit depending solely upon its reproducibility.

At present there are no means available for determining radiant energy quantitatively with an accuracy approaching that desired in modern photometry. Consequently, instead of describing the radiant energy, we are obliged to fall back upon a specification of the source which produces it. Any light source, therefore, which is reproducible from specifications, and which consequently emits visible radiation 
the quantity and quality of which are fured by such specifications, may be used as a fundamental referenco standard. As another consequence of the nature of light, any such standard defmes a unit of only limited applicability, in that it can be used to evaluate light of any other spectral distribution only by introducing an additional specification regarding the relative weights to be assigned to radiations of varions wave lengths. Fortunately, such a specification, in the form of a table of visibility factors, has already been adopted by the International Coinmission on Illumination. ${ }^{1}$

Beginning with the sperm candle defined in the British metropolitan gas act of 1860 , a large number of standards, more or less reproducible from specifications, have been proposed and some of them adopted. The most widely used have been the Pentrme lamp, described by H. E. Vernon Harcourt ${ }^{2}$ in 1877 and the Hefner lamp devised by F. von Hefner Alteneck ${ }^{3}$ in 18S4. These flame standards are not, however, satisfactory, first because the lamps themselves are not accuratcly reproducible, and sccond because their light output is dependent to a great extent on atmospheric conditions which can not be controlled with sufficiont accuracy.

A standard entirely different in principle was proposed by Violle. ${ }^{4}$ This standard is a luminous surface of platinum at its freezing point, the light from 1 square centimeter of which is called the Violle. It seemed to give promise of ending the search for a satisfactory reproducible standard and was adopted by the International Electrical Congress in 1S99, the one-twentieth part of the Violle being given the name "Bougie décimale." However, this standard proved to be even less reproducible than the flame standards. The presence of a slight amount of forcign material on the surface greatly affects the amount of light emitted. Moreover, it was found by 3 urgess ${ }^{5}$ that the emission from incandescent platinum has a well-marked discontinuity at the freczing point, a decrease of some 15 per cont for red light occurring upon freezing without change in temperature. Whatever the cause, published values ${ }^{6}$ for the light emitted by the Violle standard differ by over 20 per cent from the originally assigned value.

No fundanental reference standard reproducible accurately enough for the purposes of modern photometry has been available aud no one primary standard has been universally accepted. In 1909 the then existing units of candlepower of Great Britain, France, and the United States were brought into agreement ${ }^{7}$ and the resultant unit was termed the international candle. Germany and the other countries which had previously adopted the Hefner standard continued to use the mnit derived therefrom, but its value was accepted as being 0.9 of the international candle. The international unit hus since then been maintained by merns of carbon-filament lamps deposited in the various nationnl haboratories. While these hamps have for the present the status of primary standaris, it was recognized at the time that their adoption was only a temporary expedicnt and that they would in time be superseded by some reproducible standard.

1 Recueil des Travaux, I. C. T. Gth session, p. 67, Ceneva; 1924. See uso B. S. Sei. Papers, 19, n. 131; 19:3-

24. 'Trans. Illum. Jing. Soc., 18, p. 176; 1924; and 20, 1. 629; 1925.

i 13. A. Report, p. $845 ; 1898$.

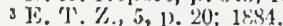

C. R., 84, 1). 171; 1879; Ann. do Chin. ot Phys., 3, 1). 373; 1884.

313. s. Bull., 2, 1). 501; 1914.

o \%.s. f. Instric., 11, 1).161; 1891; 14, 1. 207; 1894.

7 B. S. Circular No. 15; 1900. 


\section{BLACK-BODY RADIATION STANDARDS}

In 1908 Waidner and Burgess ${ }^{8}$ suggested as a standard of light a black body immersed in a bath of freezing platinum. 'This suggestion retained the one desirable feature possessed by the Violle standard, namely, a reproducible temperature at which to operate the radintor, and at the same time avoided the variations arising from the character of the radiating surface. This suggestion received much favorable comment, but it was many years before any serious attempt was made to realize the standard in practice.

In 1924, Ives ${ }^{9}$ set up a light source somewhat along the lines of the Waidner-Burgess suggestion. He fashioned cylinders of platinum foil, the ends of which were clamped in heavy terminals. An electric current passing through the platinum was regulated so as to bring the temperature up slowly to the point where the cylinders melted. A longitudinal slit in the platinum foil permitted the light from the interior "black body" to emerge and to be compared with other light sources. Although Ives did not realize the standard of Waidner and Burgess, he perfected a source which was reproducible to a higher degree than any previously devised. For the brighitness of this source Ives obtained a value of 55.40 candles per square centimeter.

In 1926, Brodhun and Hoffmann ${ }^{10}$ set up a black-body furnnce, the temperature of which was determined by the fusion of a smiall bit of pure platinum welded between the hot junctions of a $90 \mathrm{Pt}-10 \mathrm{Rh}$ to $60 \mathrm{Pt}-40 \mathrm{Rh}$ thernocouple. The melting of the pure platinum manifested itself in a slight halt in the emf.-time curve of the thermocouple, so that observations, made slightly before and slightly after the thermocouple indicated the melting temperature, could be corrected by means of the thermocouple readings. The result obtained by these workers wns 65.24 Hefners per square centimeter, which, on the basis of the conversion factor 0.9 , is equivalent to 58.72 international candles per square centimeter. Comparisons of candlepower of carbon-filament standard lamps made between the PhysiknlischTechnische Reichsnnstalt and the National Bureau of Standards during the last decade have been somewhat discordant, but on the wholo have indicated that the ratio of the Hefner unit to the candle as maintained at the bureau is somewhat less than 0.9. Measurements made on a group of lamps in 1926 gave a ratio of 0.893 . On this basis the result obtained by Brodhun and Hoffmann is equivalent to 58.26 candles per square centimeter.

Since Brodhun and Hoffmann used resistance furnaces wound with iridium and with a platinum-iridium alloy, the platinum melt sample was subject to contamination by iridium. A platinum wire which was allowed to remain in the furnace at a temperature near the melting point of platinum was found after some time to have undergone a marked change in thermoelectric properties in a direction to indicate the addition of iridium. Another source of error lies in the fact that it is practically impossible to secure temperature uniformity in a resistance-wound furnace. No estimate is given of the temperature differences existing within the furnaces used by Brodhun and Hoff-

8 Elec. World, 52, p. $625 ; 1908$.

J. Frank. Inst., 197, p. 147; 1924. Also Rec. des Travaux I. O. I. Geneva, p. 77; 1924.

10 Zeits 1. Phys., 37, p. 137; 1920. 
mann. They merely state that the reading of the thermocouple depended on its location in the furnace.

The contamination of the platinum spccimen by iridium is perhaps of no great consequence, since each specimen is in the furnace only for the length of time required to make one run. The temperature differences within the furnace present a more serious difficulty. Brodhun and Hoffmann observed the light coming from a smail auxiliary hollow inclosure within the furnace, the thermoelement and bit of platinum being behind the back wall of this small cavity. The remarkably consistent results obtained in this work do not neeessarily indicate that the specimen of platinum and the inner black body were at the same temperature, since a constant difference in temperature would likewise yield consistent results. In any case the question remains whether the conditions existing in the furnaces used in this work could be reproduced in furnaces constructed in other laboratorics.

Tleury ${ }^{11}$ and Chappius ${ }^{12}$ have been working with a black body, the temperature of which is defined by the ratio $(N)$ of its radiation (at wave length $0.622 \mu$ ) to that of a black body heid at the gold point. The ratio is measured by means of a spectrophotometer and a sector disk. For a radiator matching in color the carbon filament standard lamps $N$ lies between 450 and 470 . The precision is estimated at better than 1.4 per cent.

Since in the source used by Ives only a comparatively narrow ring is at the melting point, much of the light emitted comes initially from colder portions of the tube. It was to be expected, thercfore, that the value obtained by Brodhun and Hofimann for a black body within a furnace would be somewhat higher than the value cotained for the brightness of the platinum cylinder source, but the large diflerence actually found was somewhat disturbing. This difference showed the necessity for further experimental work, and when such was undertaken at the National Bureau of Standards in July, 1928, it was decided to set up a source in exactly the manner suggested by Waidner and Burgess, namely, to immerse a black body in a bath of molten platinum and to take observations during the time the platinum is solidifying.

\section{APPARATUS AND METHODS}

\section{THE FURNACE AND CRUCIBLE}

In the present work especial attention was given to the elimination of the two possible sources of error which may have been present in the work of Brodium and Hollmann, contammation of the platinum by metallic vapors and lack of temperature uniformity. 'The use of an induction furnace to generate the heating curents in the platinum itself eliminated all the usual sources of contamination with the caception of the refractories used to hold the platinum and to provide thermal insulation.

The uniformity of temperature, which was sought by immerine the black body in molten platinum, was further pronoted by the violent stirring in the metal which results from the action of the

$" 1$ Ann, do lhys, ot de Chinic, 5, p. 205; 1920 .

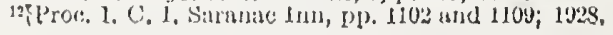


mannetic ficld of the coil of the induction furnace on the currents indured in the metal. 'Thus when the netal is allowerl to cool slowly without cntirely cutting off the power supplied to the furnace, a practically uniform temperature within the metal is assured at the instant solidification begins.

The arrangement of crucible, inmersed black body or sight tube, insulation, and platinum is shown in Figure 1 and a photograph of a
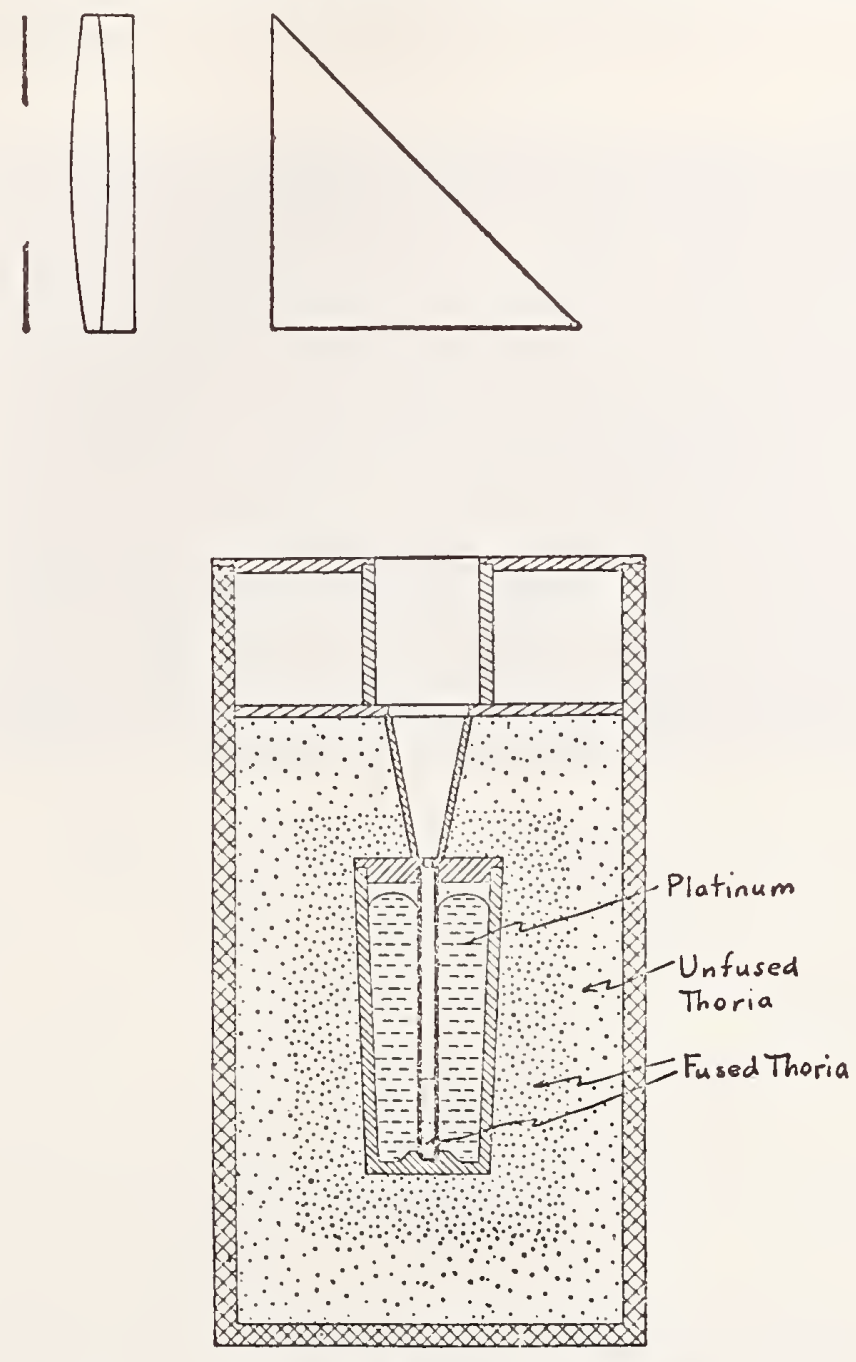

Figure 1.-Cross section showing the platinum crucible and insulation

cut-away view of the assembly in Figure 2. The erueible and sight tube, as described elsewhere in this journal, ${ }^{13}$ were made of fused thorium oxide. They were surrounded by two layers of the same material, the inner layer being fused and ground and the outer unfused. The unfused material provides the greater part of the thermal insulation, but it is subject to a large shrinkage upon heating to high

13 B. S. J. of Rescarch, 6, p. 1131; 1031. 
temperatures. The purpose of the fused material next to the crucible is to prevent leakage of the platinum through cracks which devclop in the crucible. No trouble was experienced due to cracking of the sight tubes because of the slight temperature gradicnt through them.

The inside dimensions of the crucibles were substantially as follows: Diameter at the top $22 \mathrm{~mm}$, diameter at the bottom $17 \mathrm{~mm}$, height $45 \mathrm{~mm}$. The sight tube had an inside diameter of $2.5 \mathrm{~mm}$, a wall thickness between 0.2 and $0.3 \mathrm{~mm}$, and was filled to a depth of about $15 \mathrm{~mm}$, with finely ground fused thorium oxide. The opening in the cover of the crucible was $1.5 \mathrm{~mm}$ in diameter, and was conxial with the sight tube.

\section{THE PLATINUM}

As a result of studies on the purification of the platinum mctals by Wichers, Gilchrist, and Swanger, ${ }^{14}$ the production of platinum of exceptionally high purity has bcen for some years a mattcr of routine at the National Bureau of Standards. Two lots of platinum so prepared were used. The first lot, designated as ingot No. 1, was melted and frozen some 150 times in four different crucibles of thorium oxide, and the sccond lot, designated as ingot No. 2, was melted and frozen about 100 times in two crucibles. These two ingots of platiuum were tested, both before and after use, for temperature coefficient of resistance, for thermoelectric properties, for impurities spectrographically and for iron by chemical analysis. The results of these tests, summarized in Table 1 bolow, show that the purity of the platinum was not decreased appreciably by repeated melting and freezing in the thorium oxide crucibles.

TABLE 1.-Results of tests indicative of the purity of the platinum used

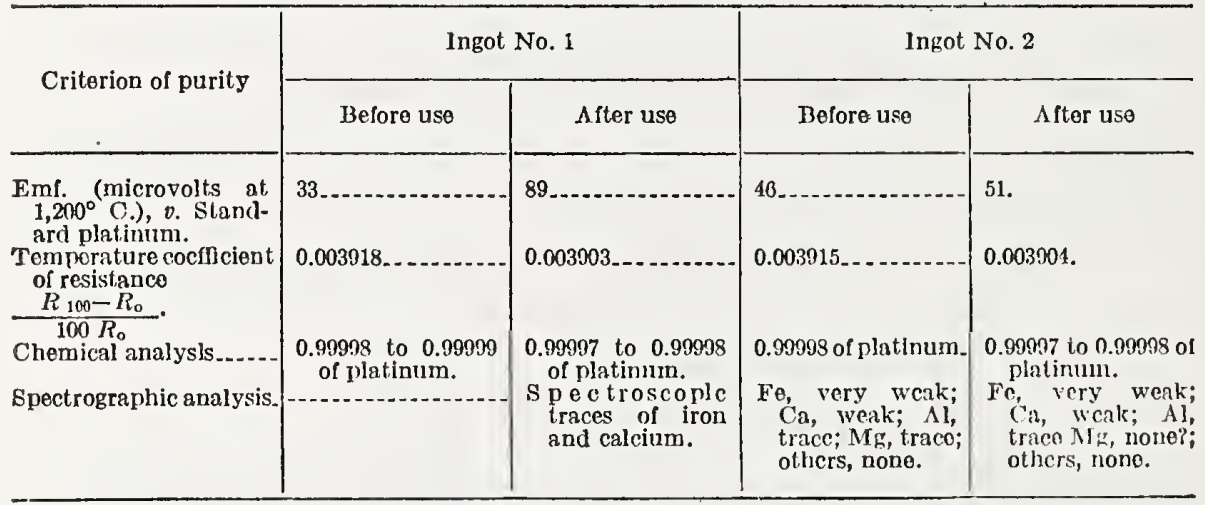

While the thermoclectric test is, perhaps, the most scusitive that can be applied in the detection of impuritics, the tempcratuie cocfficient is generally recognized as the surest guide since small amounts of any impurity will lower the temperature coefficient. The highest value of which we have record for platinum is 0.003925 , which may be taken as the value for annealed platinum free from all impurities. Nlthough there are few data available for estimating the effect of small amounts of impurities on the freezing point of platinum, the indications are that the amount of impurity present in the platinum used in this work was not great enough to change the freezing point

11 Trans. A. I. M. M. E., 76, p. 602; 1928. 


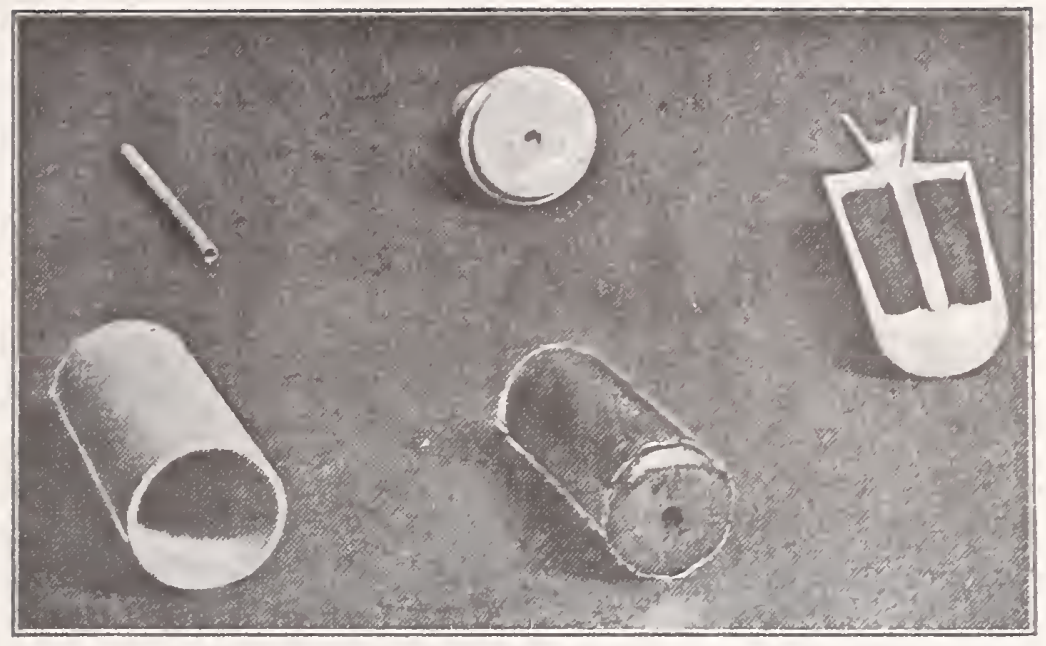

Flave 2.-Photograph of platinum ingot and refractory parts 
by more than $0.1^{\circ}$ or $0.2^{\circ} \mathrm{C}$, corresponding to a ehange in brightness of the stanclard of less than 0.2 per cent. Any platinum having a tempernture coeflicient of resistance of 0.00390 or more should serve to reproduce the standard within the limits that can be detected photometrically.

\section{THE PHOTOMETRIC ARRANGEMENT}

The substitution method of photometry was employed, the general arrangement of the apparatus being shown schematically in Figure 3. This is a horizontal plan view excepting that the black body furnaec, $F$, and the $45^{\circ}$ total 1 eflecting prism above it are shown rotated from their true position through an angle of $90^{\circ}$ about the dotted line. Light from the opening in the black body at $F$ passes vertically upward, is turned through an angle of $90^{\circ}$ by the prism and passes horizontally through the lens $L$ and the diaphragm $K$. The diaphragm was chromium plated and then blackened. The lens $J$ forms an image of the opening in the black body upon the reflecting test plate $H$ of magnesium carbonato, $N$ is a transmitting test plate

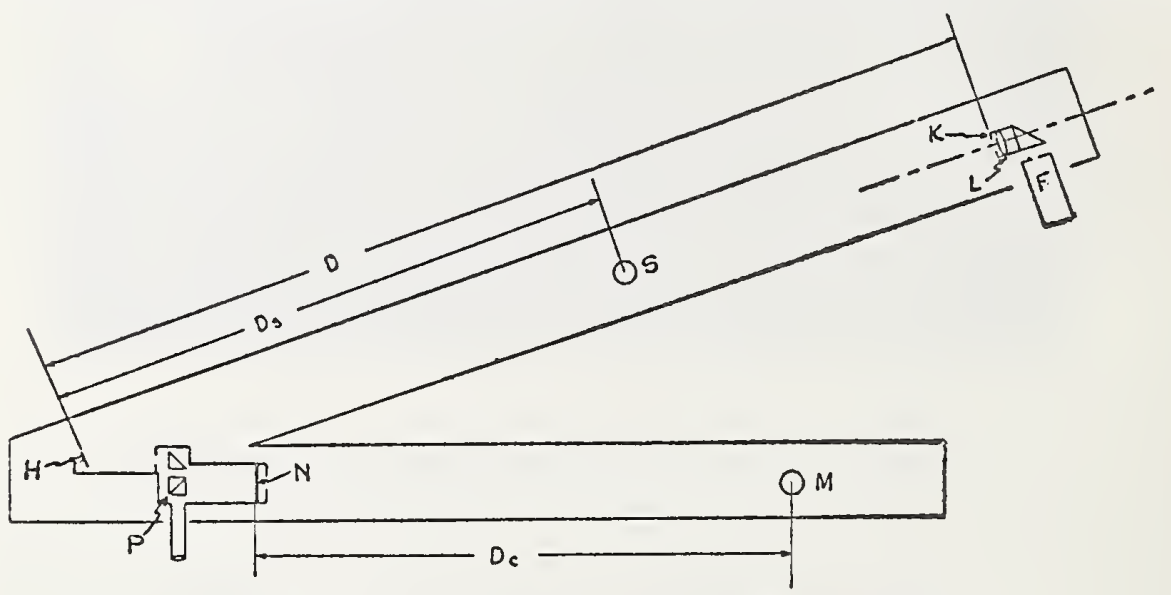

Figure 3.-Pian diagram of the photometer and track

illuminated by light from a comparison lamp $M$. The apparent brightnesses of these two test plates as seen in the Lummer-Brodhun photometer head at $P$ are adjusted to equality by moving the comparison source.

The optical principle employed is that the illumination of an image produced by an opticnl system is equal to the product of the brightness of the object and the solid angle of the cone of rays illuminating the image multiplied by the transmission of the optical system.

The illumination $E_{B}$ on the test plate $F$ due to the image of the black-body opening is given by the formula,

where

$$
E_{B}=\frac{B A T}{D^{2}}
$$

$B=$ the brightness of the black body in candles per square centimeters.

$\Lambda=$ the area in square centimeters of the opening in diaphragm $K$.

$T=$ the transmission of the lens and prism. 
and

$D=$ the distance in centimeters between the djaphragm $K$ and tho test plate $H$.

The illumination $E_{S}$ on the test plate $H$ due to the light incident on it from the standard lamp $S$ is given by the formula

$$
E_{S}=\frac{I}{D_{S}^{2}}
$$

where and

$I=$ the candle power of the standard lamp $S$.

$D_{S}=$ the distance between the standard lamp $S$ and the test plate $H$.

If the distance between the comparison lamp $M$ and the transmitting test plate $N$ is $D_{C}$ when a photometric balance is obtained with illumination $E_{B}$ on the test plate $H$, and $D_{C /}$, when a photometric balance is obtained with illumination $E_{S}$ on test plate $H$, then

$$
\frac{E_{B}}{E_{S}}=\frac{D_{C \prime}^{2}}{D_{C}^{2}}
$$

Substituting tho values above for $E_{B}$ and $E_{S}$ in equation (3)

$$
B=\left(\frac{D_{C I}}{D_{C}}\right)^{2} \cdot\left(\frac{D}{D_{S}}\right)^{2} \cdot \frac{I}{\Lambda T}
$$

In this work six standard lamps comprising one of the groups of "primary" carbon lamps by which the international candle is maintained at the National Bureau of Standards were used. The color temperature of these lamps is approximately $2,080^{\circ} \mathrm{K}$., which is only $35^{\circ}$ higher than that of the black body at the freezing point of platinum. Thus, practically no difficulties due to color difference were encountered in the photometric work.

The horizontal candlepower of the primary carbon standards used was about 17 and the distance $D_{S}$ between the standard lamps $S$ and test plate $H$ was about $125 \mathrm{~cm}$, resulting in an illumination on the test plate of approximately $11 \mathrm{~m}$ candles. By suitably choosing the distance $D$ and the size of the opening in the diaphragm $K$, approximately the same illumination was obtained when light from the black body during the freezing of the platinum was incident on the test plate. Thus, all photometric measurements that are used to calculate the black body brightness were made with the same photometer field brightness. Also, the distances $D_{C}$ and $D_{C}$, were nearly equal, so that only their difference need be known accurately. The photometric settings from which the ratio $\frac{D_{C \prime}}{D_{C}}$ was determined were automatically recorded on a drum chart, thus producing freezing and cooling curves such as the one shown in Figure 4.

\section{METHOD OF MAKING OBSERVATIONS}

As explained before, the metal in the liquid state undergoes a violent stirring action so that the entire mass cools to the freezing point before any part of it begins to solidify. For this reason only the observations taken during freezing were used, although the melts were 
usually obscrved. The mclts did not differ matcrially from the freezes, but were usually not as sharply defincd at beginning and end. $A$ s shown in figure 4 undercooling before frcezing almost invariably occurred.

The procedure in making a run was as follows: The platinum was heated, and all necessary adjustinents made to center the image of the black-body opening upon the test plate. To measure the distances from the tcst plate to the standard lamps and to the diaphragm a stecl bar was next clamped in position along the optical axis of the lens. The bar had threc calibratcd refcrence marks, onc near cach end and onc at the location of the socket for the standard lamp $S$. It was over $3 \mathrm{~m}$ in length, being some 12 to $15 \mathrm{~cm}$ shorter than the distance bctween the test plate and diaphragm. The bar was adjusted until

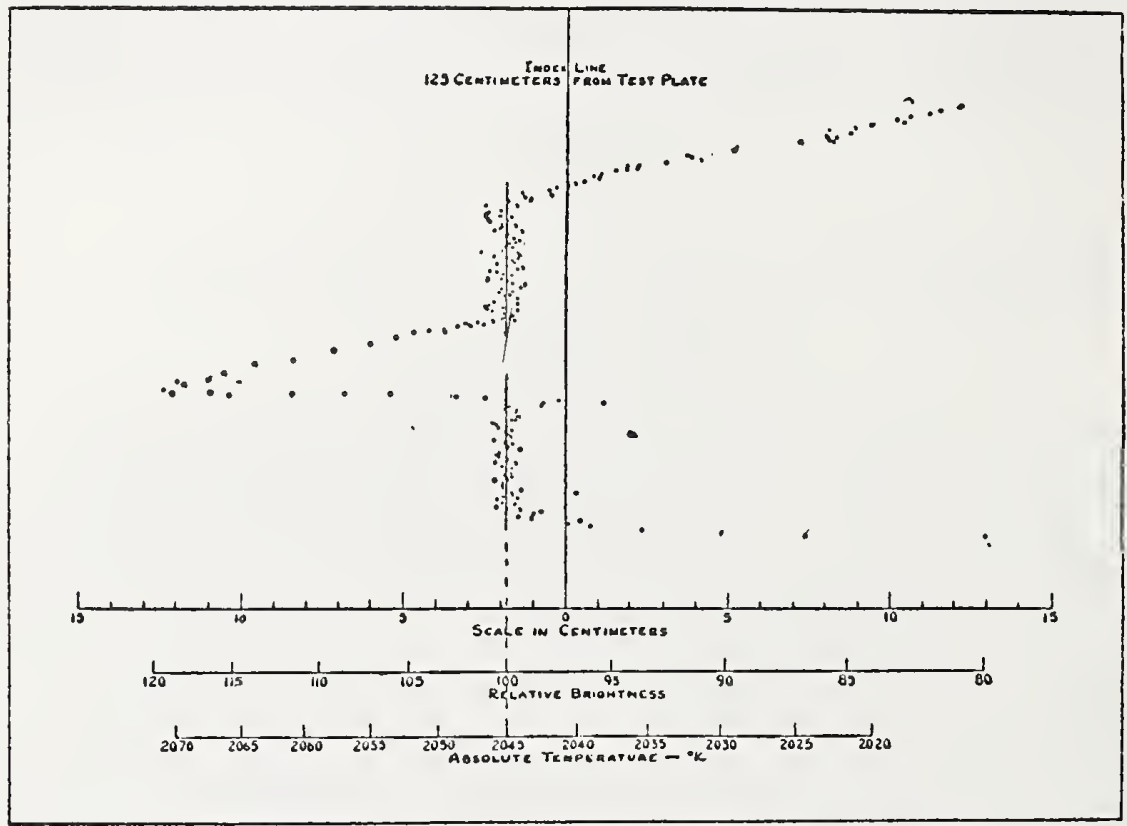

Figure 4.-Aulomatic record of a typical mell and frecze

the middle scratch coincided with the center of the lamp socket at $S$ and the distances from the diaphragm $K$ to one cnd scratch and from the test plate $\mathrm{H}$ to the othcr end scratch were measured with a short steel scale to within $0.2 \mathrm{~mm}$.

Throughout each run the carbon comparison lamp was maintained at a constant voltage such that its color tempciature was approximately midway betwcen that of the black-body standard and that of the standard carbon lamps. The observer made photometric measurements first on thrce standard lamps, then on three successive freezes of the platinum standard, and finally on three additional standard lamps. Electrical measurcments on both the standard and the comparison lamps wcre made by means of a 5-dial Dicselhorst potentiometcr. Following these photometric measurements, the stcel bar was again clamped in position on the optical axis and tho distanco measurements rcpeated.

The photometric settings made during a run were usually all recorded on a single drum chart. The distances between the average 
settings of the comparison lamp for the black-body standard and for the standard lamps were measured by a steel scale to an accuracy of about $0.2 \mathrm{~mm}$.

A sample data sheet is given below. The symbols used have the following meanings:

$D$, the distanee between the diaphragm and the test plate.

$D_{s}$, the distance between the center of the standard lamp socket and the test plate. $D_{c}$, the distanee between the eomparison lamp and the transmitting test plate when a photometrie balanee is obtained with illumination from the blaek body in freezing platinum (obtained from drum eharts).

$D_{c}^{\prime}$, the distanee between the comparison lamp and the transmitting test plate when a photometric balance is obtained with illumination from the standard lamp (obtained from drum eharts).

Date: Oetober 10, 1929.

Drum eharts: 46 and 47.

Observer: W. F. R.

Distanee measurements:

D, before photometrie measurements _... $c$ cm

D, after photometrie measurements . . . .

Menn value of $D$

$D_{R}$, before photometrie measurementis___.

$D_{s}$, after photometrie measurements_... 125.77

Mean value of $D_{0} \ldots \ldots$

Standard lamp data

\begin{tabular}{|c|c|c|c|c|c|c|}
\hline \multirow[b]{2}{*}{ Lamp No. } & \multicolumn{2}{|c|}{ Voltage } & \multicolumn{2}{|c|}{ Current } & \multirow[b]{2}{*}{$I$} & \multirow[b]{2}{*}{$D_{0}^{\prime}$} \\
\hline & Rated & $\begin{array}{c}\text { During } \\
\text { measure- } \\
\text { ments }\end{array}$ & Rated & $\begin{array}{c}\text { During } \\
\text { measure- } \\
\text { ments }\end{array}$ & & \\
\hline $\begin{array}{l}44 \\
45 \\
49 \\
50 \\
64\end{array}$ & $\begin{array}{l}\text { Volls } \\
48.960 \\
48.960 \\
49.460 \\
49.460 \\
49.460 \\
50.459\end{array}$ & $\begin{array}{l}\text { Volts } \\
48.960 \\
18.960 \\
49.460 \\
49.460 \\
49.460 \\
50.459\end{array}$ & $\begin{array}{c}\text { Amps. } \\
1.3314 \\
1.3291 \\
1.3203 \\
1.3292 \\
1.3269 \\
1.3320\end{array}$ & $\begin{array}{r}\text { Amps. } \\
1.3310 \\
1.3392 \\
1.3218 \\
1.3292 \\
1.3269 \\
1.3322\end{array}$ & $\begin{array}{r}\text { Candles } \\
17.74 \\
17.49 \\
17.58 \\
17.76 \\
17.64 \\
18.13\end{array}$ & $\begin{array}{l}c m \\
126.35 \\
126.69 \\
125.55 \\
126.20 \\
126.20 \\
125.16\end{array}$ \\
\hline Mean...... & .... & $\ldots$ & & ..... & 17.723 & 126.19 \\
\hline
\end{tabular}

Data on platinum freezes

\begin{tabular}{|c|c|}
\hline Freeze No. & $D_{0}$ \\
\hline $\begin{array}{l}1 \\
1 \\
3 \\
3\end{array}$ & $\begin{array}{c}c m \\
126.69 \\
126.99 \\
126.88\end{array}$ \\
\hline Mean.-. & 126.85 \\
\hline
\end{tabular}

$A$, area of opening in diaphragm

$T$, transmission of lens and prism_._._.

The brightness of the blaek body immersed in freezing platinum, computed from the equation (see equation (4), p. 1110)

is $B=\mathbf{5 8 . 8 2}$ eandles/em².

$$
B=\left(\frac{D c^{\prime}}{D c}\right)^{2}\left(\frac{D}{D_{s}}\right)^{2} \frac{I}{A T}
$$


The dimeter of the opening in the diaphragm $K$ was measured both with a microseope eomparator by the length measurements seetion of the National Burenu of Standards and with rages by the gnge section. The opening was found to be very slightly out of round, and this fact was taken into account in calculating the area.

The measurement of the combined trunsmission of the lens and prism, which enters in the result to the first order, and which proved to be somewhat of a problem, was made by two independent methods. In the first, termed the box method, a rectangular light box was construeted, the front of which contained a circular opening through which the rear wall could be viewed. The inside walls were coated with the fumes from burning mignesium ribbon, and several incandescent lamps were placed inside the box and sereened so ns to illuminate the rear wall as uniformly as possible. The voltage of the lamps was adjusted so that the color temperature of the light from the box and that of the comparison lamp were made equal to that of the black body. Observations were then made by matching the comparison test plate with the rear wall of the box, first directly and next with the prism and lens interposed between the light box and the photometer head. The identical area of the rear wall of the box could not be viewed in the two observations so that irregularities in the brightmess of this wall affected the consistency of the results obtained. By making a large number of determinations the efiect of nonuniformity of the brightness of the rear wall should be eliminated. However, it was decmed advisable to check the results thus obtained by an entirely different method, using an optical pyrometer.

In the optical-pyrometer method, the black-body standard itself was used as a source of uniform brightness and proper spectral distribution. An optical pyrometer was sighted through the lens and prism into the black body during freezing of the platimm and the current through the pyroineter lamp necessary to secure a match was determined. The red screen was, of course, removed from the pyrometer for this purpose. Next a sector disk having the same transmission as that which the box method lad yiclded for the lens and prism was substituted for these optical parts, and the eurrent through the pyrometer lamp necessary to secure a match during a freeze of the platinum was again determined. Two other sectors were also used, one having a slightly greater and one a slightly smaller transmission than the first in order to determine the relation between the current through the lamp and the transmission in this region. As it happened, the last two sectors were scarcely necded because the current through the lamp in the two eases, when the first sector and when the lens and prism were used, differed by only 1 part in 10,000, corresponding to a difference of 1 part in 2,000 in the transmission.

Each of three observers made 4 sets of observations, representing in all 18 freczes using the first sector and 18 freczes using the lens and prism. The values obtained are listed in Tablo 2. 
TABLE 2.-Data on transmission of lens and prism

\begin{tabular}{|c|c|c|}
\hline Observer & Box method & Optical-pyrometor method \\
\hline $\begin{array}{l}\text { H. T. W W. } \\
\text { W. F. R R. R. } \\
\text { E. Q. A } \\
\text { C. }\end{array}$ & $\begin{array}{l}\text { Per ccnt } \\
76.03 \text { (Average of } 12 \text { sets) } \\
76.93 \text {................. } \\
76.89 \\
76.97 \text { (Average of } 8 \text { sets) }\end{array}$ & $\begin{array}{l}\text { Per cent } \\
77.03 \text { (Average of } 4 \text { sets). } \\
76.93 \quad \text { Do. } \\
76.85 \quad \text { Do. } \\
\end{array}$ \\
\hline Mean...... & 76.92 & 76.94 \\
\hline
\end{tabular}

\section{RESULTS}

During the first few freezes taken on ingot No. 1, a water-cooled diaphragm was used in contact with the prism. The cooling proved too effective, the prism being cooled sufficiently below roour temperature to collect a slight film of moisture from the atmosphere. While these results did not differ appreciably from those obtained later, it was decided to discard all results from runs at the conclusion of which any film could be detected. With air cooling instead of water cooling, no further trouble from films was experienced and no results obtained after the change to air cooling have been discarded.

The photometric settings were made by four observers. Observers (H. T. W. and E. G. A.) had considerable previous experience in photometry, and observers (H. T. W., W. F. R., and F. R. C.) had considerable experience in precision optical pyrometry. The results of individual muns obtained are listed in Table 3, each run being the average of observations on three freezes and six primary standard lamps.

TABLE 3.-Data on black-body brighiness

\begin{tabular}{|c|c|c|c|c|c|c|c|}
\hline \multicolumn{4}{|c|}{ Ingot No. 1} & \multicolumn{4}{|c|}{ Ingot No. 2} \\
\hline H. T. W. & W. F.R. & F. R. O. & E. G. A. & В.T. & W. F.R. & F.R.C. & E. G.A. \\
\hline $\begin{array}{l}\mathrm{c/cm} 2 \\
58.69 \\
58.87 \\
59.15 \\
59.09\end{array}$ & $\begin{array}{l}\mathrm{c/cm} 2 \\
58.48 \\
58.54 \\
58.78 \\
58.82\end{array}$ & $\begin{array}{l}\mathrm{c} / \mathrm{cm}^{2} \\
58.92 \\
58.56 \\
59.23 \\
59.15\end{array}$ & $\begin{array}{l}c / \mathrm{cm}^{2} \\
58.46 \\
59.10 \\
58.67 \\
59.07\end{array}$ & $\begin{array}{l}\mathrm{c/cm} 2 \\
58.61 \\
58.88 \\
58.65 \\
59.14\end{array}$ & $\begin{array}{l}c / \mathrm{cm}^{2} \\
58.81 \\
58.86 \\
58.59 \\
59.01\end{array}$ & $\begin{array}{l}c / c m^{2} \\
59.13 \\
58.97 \\
58.46 \\
59.09\end{array}$ & $\begin{array}{l}\mathrm{c/cm} \\
58.53 \\
58.95 \\
58.73 \\
59.02\end{array}$ \\
\hline${ }^{1} 58.95$ & 58.66 & 58.96 & 58.83 & 58.82 & 58.82 & 58.01 & 58.81 \\
\hline \multicolumn{4}{|c|}{$\begin{array}{l}\text { Mean of ingot No. 1, } 58.85 \text { candles } \\
\text { per } \mathrm{cm}^{2} \text {. }\end{array}$} & \multicolumn{4}{|c|}{$\begin{array}{l}\text { Mean of ingot No. 2, } 58.84 \text { candles } \\
\text { per } \mathrm{cm}^{3} \text {. }\end{array}$} \\
\hline
\end{tabular}

1 Mean values.

\section{DISCUSSION OF ERRORS}

The precision attainable in visual photometry is generally considered to be no better than 1 part in 1,000, and it would be very gratifying if one could be assured that the value for the brightness of the Waidner-Burgess standard has been determined to this order of accuracy. The agreement of the results obtained with ingot No. 1 and ingot No. 2, to 1 part in 6,000 may be looked upon as an unusual coincidence. It is believed, however, that the standard as used in this work is reproducible in any suitably equipped laboratory to within 1 part in 1,000 . 
The uncertainty in the value obtained for the brightness, due to errors in the measurement of the opening in the limiting diaphragm used is too small to affect the result by more than 0.03 per cent. The distance measurements were all made with sufficient accuracy to make the resultant error negligible, about 0.02 per cent. The measurement of the transmission of the lens and prism, made by two independent measurements each of which is probably good to nearly 0.1 per cent, is considered good to 0.1 per cent. The value for the candlepower of the carbon lamps used is taken to be exact, our result being expressed relative to the candlepower of this group of six lamps. The various errors may be summarized as shown in Table 5.

TABLE 5.-Summary of possible errors due to various causes

Percent

Area of diaphragm

Distance measurements

Transmission of lens and prism...

Photometric matching _.................................. 1

Lack of black-body conditions and temperature drop in wall of sight tube- $\quad$-. 2

Sum

+0.25 to -0.45

The errors listed in Table 5 are all independent of each other and are not likely to be all of the same sign. The numerical value for thie brightness in terms of the group of six lamps used in the measurements is, therefore, probably not high by more than 0.15 per cent nor low by more than 0.35 per cent.

\section{COMPARISON OF RESULTS WITH THOSE OF OTHER OBSERVERS}

The values for the brightness of a black body at the platinum point obtained by Ives, by Brodhun and Hoffmann, and in the present work are given below in terms of the international candle as maintained at the National Bureau of Standards.

$$
\begin{aligned}
& B_{1} \text { (Ives) } \\
& B_{2} \text { (B. \& H.) } \\
& B_{3} \text { (W., R., B. \& C.) } 25.40 \pm 0.11 \\
& \text { (W. }
\end{aligned}
$$

It is obvious that the differences in the values given above can not be due to errors in the photometric comparisons, and are likewiso somewhat too large to be entirely accounted for by differences in photometric units. It would seem more logical to conclude that the sources actually differed in in trinsic brightness in the manner indicated by the measurements. It so happens that positive evidence on this point is available.

The freezing point of platinum has been determined, as described elsewhere in this journal ${ }^{15}$ by making observations with an optical pyrometer on the same black bodies for which the brightness was determined in this investigation as having the value $B_{3}$ above. The temperature obtained was $1,773.5^{\circ} \mathrm{C}$. Int. Likewise the melting point of platinum was determined by Hoffmann ${ }^{16}$ using the experimental arrangement with which the value of brightness cited as $B_{2}$ was found. The value obtained, $1,771^{\circ} \mathrm{C}$, , on the basis of $C_{2}=1.430 \mathrm{~cm} \mathrm{deg.,}$ 
becomes $1,769.5^{\circ} \mathrm{C}$. when reduced to the International Temperature Scnle.

Ives made no temperature measurements, but shortly after the publication of this work measurements ${ }^{17}$ were made at the National Bureal of Standards with an optical pyrometer on the radiation from small holes in platinum tubes $1 \mathrm{~mm}$ in diameter and having walls $0.1 \mathrm{~mm}$ thick. The temperature readings at the instant of melting ranged from $1,760^{\circ}$ to $1,766^{\circ} \mathrm{C}$. More recently Ribaud and Mohr ${ }^{18}$ have made similar measurements and report $\Omega$ value of $1,762^{\circ} \mathrm{C}$.

The correlation of the temperature mensurements made on tubes with the value found by Ives on tubes of radically different dimensions may be open to question. However, these measurements are the only available ones made under even approximately similar conditions. Neverthcless, there can be no doubt that the radiation from such tubes at the time of failure is equivalent to that from a black body at a temperature considernbly below the platinum point.

The conditions in the work of Brodhun and Hoffmanu were such that the temperature of the black body used may have been either above or below the temperature of the melting platinum. The evidence, both in the case of the brightness measurements and in the case of the tempcrature measurements, is that the black body used by Brodhun and Hoffmann emitted less radiation than the immersed black body used in the present work, which in turn could not possibly be at a temperature above that of the freezing platinum in which it was immersed.

On the basis that the equivalent temperature and brightness of the three sources used corresponded closely to the obscrved values, we may calculate the value that each would have obtained with a source at $1,773.5^{\circ} \mathrm{C}$.

\begin{tabular}{|c|c|c|c|}
\hline Source used & $\begin{array}{c}\text { Temperatute } \\
\text { of source } \\
\text { (observed) }\end{array}$ & $\begin{array}{l}\text { Brightness } \\
\text { of source } \\
\text { (observed) }\end{array}$ & $\begin{array}{c}\text { Brightncss } \\
\text { calculated } \\
\text { for a temper- } \\
\text { ature of } \\
1,773.5^{\circ} \mathrm{O} \text {. }\end{array}$ \\
\hline $\begin{array}{l}\text { Platinum tubes- } \\
\text { Resistance-wound furnace }\end{array}$ & $\begin{array}{c}{ }^{\circ} \mathrm{C} . \\
1,762.5 \pm 2 \\
1,760.5 \pm 2\end{array}$ & $\begin{array}{c}c / \mathrm{cm}^{2} \\
55.40 \pm 0.11 \\
58.20 \pm 0.15\end{array}$ & $\begin{array}{c}\mathrm{c} / \mathrm{cm}^{2} \\
59.10 \pm 0.66 \\
59.64 \pm 0.66\end{array}$ \\
\hline Black body immersed in platinum....... & $1,773.5 \pm 1$ & $58.81 \pm 0.09$ & $58.81 \pm 0.09$ \\
\hline
\end{tabular}

Since a change of $1^{\circ} \mathrm{C}$. in the temperature of a black body at the platinum point corresponds to a change of almost 0.6 per cent in brightness, the accuracy of the temperature measurements described is not as great correspondingly as the accuracy of the photometric measurements.

\section{BLACK-BODY STANDARDS OF HIGHER COLOR TEMPERATURES}

The choice of the type of lamp for use in maintaining the value of the international candle was made before the advent of incandescent lamps of higher luminous efficiencies than those with carbon filaments. To-day the carbon lamp has passed almost completcly from general

${ }^{17}$ Unpublished work by C. O. Fairchild and H. T. Wensel; Scptember-October, 1924.

18 C. R., 192, p. 37; 1931 . 
use. The question naturnlly nrises whether a black body at the color tomporature of tungsten lnmps, either vacuum or gns-filled, would bo a moro logical standard than the platinm black body to replaco tho present carbon lamps as the photometric standard.

In practice, the soveral nationnl lnborntories maintnin as working standards vacuum tungston and gas-filled tungsten lamps, which havo been calibrated in terms of the primnry carbon standards by various methods. Unfortunntely, tho mational Inborntories aro not in ngreement on the results obtained in such calibrations, so that although they agree npproximatoly on the unit of light as maintained by carbon standnrds, they do not nereo on the unit as represcuted by derived tungsten standards. Intercomparisons indicato that the discrepancies may amount to as inuch as 5 per cent. Such a condition may bo remedied in one of two ways. 'The several laboratories may agreo on a procedure which will give consistant results in comparing lamps differing in color tempornture or a black body at a hirher color temperature may bo nrbitrarily set up as a stand ard and tho unit so defined maintained by tungsten lamps. The objections ngainst defining a new unit are the same ns thoso ngainst any dual stand ard. Moreover', since the tungsten-filament incondescent lamps in use to-dny range in color tempernture from about $2,400^{\circ}$ to $3,300^{\circ} \mathrm{K}$., no ono standard will serve for all such lamps until a solution is found for the problen. of comparing two lamps diflering in color temperature by hundreds of degrees. A cooperative investigation is now being corricel on among the 11ntional laboratories in which it is hoped tho spectral transmission values of several bluo filters will be agreed upon. With tho use of these filters, the step from ono color temperature to nnother can be made without the difficulties inherent in heterochromntic photometry. 'The solution of this problem will make the position in the color-temperature scale of tho sourco chosen ns tho primmy standard a matter of secondary importance.

\section{SUMMARY}

The proposal of Waidner and Burgess for a reproducible standnirl of light has been experimentally realized. The light ernitted by a hollow inclosuro of fused thorium oxide immersed in freezing pintinum has been found to be reproducible within the limits that can bo detected by visual photometry. The brightness of this sourco was determined as $58.84 \pm 0.09$ candles per square centimeter.

The brightness of a black body at tho platinum point probably lies betweon 58.75 and 59.04 candles por squaro centimetor.

\section{ACKNOWLEDGMENTS}

Grateful acknowledgment is made to tho many ssections of tho bureau who contributed assistance in this work. In particular tho authors wish to oxpress their appreciation to L. R. Kleinschmidt and $H$. A. Buchheit for preparation of tho platinum spongo; to W. $H$. Swanger and J. S. Acken for melting the spongo into ingots; to E. Wichers for the chemical analysis; to W. F. Meggers and B. F. Scribner for the spectrographic analyses; and to $\mathbb{E}$. G. Anderson who served as one of the four obscrvors.

Washington, March 5, 1931. 


\section{*ANNOUNCEMENT OF CHANGES IN ELECTRICAL AND PHOTOMETRIC UNITS}

\section{PHOTOMETRIC UNITS}

\section{HISTORY}

Units of light have never been established by law in the United States. In 1909 the National Bureau of Standards by agreement with laboratories in France and Great Britain introduced the "international candle" [7]; the agreement, however, did not provide for any primary reference standard. Pending the development of a satisfactory basic standard the "candle" was represented by groups of carbon-filament lamps. As other types of lamps were developed, working standards of the new types were set up independently in various countries. This required visual comparison of lights of different colors, which is very difficult, and led to some diversity of units. Moreover, Germany and Austria never accepted the international units of light.

As a result of years of discussion and experimental work on standards of candlepower and on photometry of lights of different colors, general agreement was reached on two points: first, the best type of primary standard now available is a radiator of the type known as a black body at a temperature controlled by molten platinum at its freezing point; second, lights of different colors should be evaluated by using certain standard luminosity factors representing the spectral sensitivity of the average eye under specified conditions.

These two elements give a basis for a complete system of photometric units when a numerical value is assigned for the brightness of the platinum-black-body standard. Its brightness expressed in international units was experimentally determined to be 58.9 candles per square centimeter [8]. In 1936 the National Bureau of Standards proposed that the round value 60 candles per square centimeter be adopted as defining a new basic unit to be extended to lights of other colors by means of the accepted luminosity factors [9]. This proposal was approved by the United States National Committee of the International Commission on Illumination after favorable recommendation by a committee including representatives of the American Institute of Electrical Engineers, Illuminating Engineering Society, Optical Society of America, National Electrical Manufacturers' Association, Edison Electric Institute, Association of Edison Illuminating Companies, Electrical Testing Laboratories, and lamp manufacturing companies.

\footnotetext{
*Reprinted from NBS Circular C459, issued May 15, 1947.
} 
The new system of units was adopted by the International Committee on Weights and Measures in 1937 and approved by the International Commission on Illumination in 1939 with the expectation that it would be introduced in practical use as of January 1, 1940. As in the case of the electrical units, the execution of this plan was delayed hy the war, and the date of January 1, 1948, has now been chosen so as to make the change in both sets of units at the same time.

\section{MAGNITUDE OF UNITS}

The light from the platinum-black-body radiator is not greatly different in color from that given by carbon-filament standard lamps as the color temperature of the lamp filaments is about $2,100^{\circ} \mathrm{K}$, whereas the freezing point of platinum is $2,046^{\circ}$. In this range of color the new unit of intensity is about 1.9 percent smaller than the old international candle, and sources of light are correspondingly given higher numerical ratings. However, when light sources of higher color temperature are compared with these basic standards, the accepted spectral luminosity factors give slightly lower values for the "whiter" sources than were obtained by visual measurements when the present international units were established. The difference between the two scales therefore grows less as the color temperature of the sources measured is increased, and for sources in the range of ordinary vacuum tungsten-filament lamps, around $2,500^{\circ} \mathrm{K}$, the new scale crosses the international scale as used in the United States. Furthermore, when the range of standards was extended to gas-filled tungsten-filament lamps and other new types, the measurements were made by methods nearly in accord with the luminosity factors. Consequently, the present ratings of tungsten-filament lamps in this country will be practically unaffected by the change, no type being changed by more than 1 percent.

In European countries somewhat higher values have been assigned for light from the high-temperature sources. Introduction of the new scale will bring about reductions of several percent in their ratings, but these changes will not be so great as would have resulted if the standard luminosity factors had been used to step up from the international candle as represented by carbon-filament lamps.

\section{LEGAL STATUS OF UNITS}

When the electrical units were defined by law (Public Law No. 105, 53d Cong.) in 1894 it was supposed that the international units were practically identical with the corresponding multiples of the centimetergram-second 3lectromagnetic system. Alternative definitions were given for most of the units, and those definitions which appear to be legally controlling were taken partly from one system and partly from the other. The joule and the watt, for example, are clearly defined as multiples of the cgs units. In brief, the absolute units have as good a legal basis under the terms of that act as do the present international units. New legislation is being proposed to remove the ambiguities of the old act, but there should be no objection on legal grounds to the general adoption of the absolute units even in advance of Congressional action. 
The photometric units now in use were adopted by common consent of those interested, and as previously stated have no statutory basis. The new system might therefore be adopted in the same way, but authorization for it is included in the proposed legislation.

\section{NAMES OF UNITS}

During the period of transition to the new units, in order to avoid any doubt as to the units used in giving precise data, the International Committee on Weights and Measures has recommended that the adjectives "international" (abbreviation "int") and "absolute" (abbreviation "abs") be used with the names of the electrical units. Similarly, with names of photometric units the use of the adjectives "international" and "new" is recommended. The use of these distinguishing terms will become unnecessary, except in referring to old data, within a few years.

\section{SELECTED REFERENCES}

[1] Procès-verbaux, Comité International des Poids et Mesures, 1929, p. 58 and 63

[2] Comptes rendus, $8^{\text {me }}$ Conférence Générale des Poids et Mesures, 1933, p. 53.

[3] Electric units and standards, Circular of the Bureau of Standards C60 (1920). (This is out of print, but may be found in reference libraries.)

[4] Systems of electrical and magnetic units, National Research Council Bulletin No. 93 (1933)

[5] Announcement of a change in the value of the international volt, Circular of the Bureau of Standards C29 (1910). (Out of print.)

[6] Harvey L. Curtis, Review of recent absolute determinations of the ohm and the ampere, J. Research NBS 33, 235 (October 1944) RP1606. See also, A. Pérard, Les unités principales, etc., Rev. métrologie 22, 3 and 25 (Jan. and Feb. 1944).

[7] The international unit of light, Circular of the Bureau of Standards C15, (1909). (Out of print.)

18] H. T. Wensel, Wm. F. Roeser, L. E. Barbrow, and F. R. Caldwell, The Waidner-Burgess standard of light, BS J. Research 6, 1103 (1931) RP325. G. Ribaud, Realisation de l'étalon primaire d'intensité lumineuse, Rev. optique 12, 289 (1933). National Physical Laboratory (Great Britain) Report, 1935, p. 79.

[9] K. S. Gibson and E. P. T. Tyndall, Visibility of radiant energy, Sci. Pap. BS 19, 131 (1923) S475; Trans. Illum. Eng. Soc. 19, 176 (1924); For adoption of factors, see Recueil des Travaux, Comm. Int, de l'Eclairage, 1924, p. 67 and p. 232.

Washington, March 5, 1947. 


\title{
Vertical Distribution of Light From Gas-Filled Candlepower Standards
}

\author{
BY L. E. BARBROW \\ S. W. WILSON
}

\section{Introduction}

In recent years, gas-filled tungsten-filament lamps have been used in increasing numbers as standards of luminous intensity. These lamps are operated base down and have coiled monoplane filaments and clear bulbs. When used as standards, they are marked with fiducial lines so that they can be accurately aligned about a vertical axis.

Middleton and $\mathrm{Mayo}^{1}$ have reported on the variation of candlepower of such lamps about the vertical axis. Reported herein are the variations of candlepower of several sizes of such lamps about a horizontal axis through the filament center perpendicular to the photometric axis. Table I lists the types of lamps studied and the number of lamps of each type.

\section{Method}

The lamps were operated at a distance of approximately 2 meters ( 80 inches) from a barrier-layer photocell, the current output of which was measured in a "zero-resistance" circuit. ${ }^{2}$ The photocell was diaphragmed to 1.2 centimeters diameter, so that the receiver diameter subtended an angle of approximately 0.36 degree at the lamp. Each lamp was first oriented about a vertical axis so that the vertical lines etched on the bulb were in a plane through the center of the photocell diaphragm. By visual inspection, the plane of the filament was set to be vertical by tilting the lamp about the horizontal axis perpendicular to the photometric axis. This vertical angle is designated as 0 degrees. The candlepower of each lamp was measured at this angle and at one-degree intervals through \pm 5 degrees.

In accord with a practice in common use, a diaphragm $21 / 2$ centimeters high and $91 / 2$ centimeters wide was placed $4 \frac{1}{2}$ centimeters from the center of the socket, toward the receiver. Ample baffles were used so that stray light was excluded.

A paper presented at the National Technical Conference of the Illuminating Engineering Socjety, August 17.22, 1958, Toronto, Ont. AUTHORS: Nationa! Bureau of Standards, Washington, D. C. This paper accepted by the Papers Committee of I,E.S. as a Transsction of the Mluminsting Engineering Society.

\section{Results}

The data obtained on the individual lamps are given in Figs. 1-4. It will be noted that the variation of candlepower through the range of tilt of \pm 5 degrees from vertical may be almost 10 per cent for the 500 -watt lamps and somewhat less for the 50- and 100-watt lamps (excluding lamp 4398 which gave atypical results). These results point up (1) the need for specifying a procedure for orienting coiled-filament, clear bulb lamps about a horizontal axis as well as about a vertical axis if their use as eandlepower standards is to be continued, or (2) the need for a change in the type of lamps used as gas-filled standards of luminous intensity, if no special precautions are to be taken for oricntation of the lamp and for controlling the vertical angle subtended by the photocell.

Since the lamp and diaphragm were tilted as a unit, the obtaining of data through a wider range of angles was precluded. To obtain such data, measurements of the lamps in Group 4 were made without the diaphragm. These measurements were made at.2-degree intervals. The data obtained are shown in Fig. 5. In this figure, 0 degrees is the vertical angle of maximum candlepower instead of the angle at which the filament plane is approximately vertical as in Figs. 1-4. Plotted on this figure also is a cosine curve with an amplitude of 5 per cent of maximum candlepower and a period 18 degrees. This curve can be represented by the equation $100\left(I_{o}-I\right) / I_{o}=5(1-\cos 20 \Phi)$ where $I_{o}$ is the maximum luminous intensity and $I$ is the luminous intensity at vertical angle $\Phi$.

It will be noted that this cosine curve represents fairly well the average variation of candlepower of the lamps with angle of tilt. An apparent explana-

TABLE I - Types of Lamps Studied.

\begin{tabular}{ccccc}
\hline $\begin{array}{c}\text { Group } \\
\text { Number }\end{array}$ & Watts & $\begin{array}{c}\text { Number } \\
\text { of Lamps }\end{array}$ & $\begin{array}{c}\text { Filament } \\
\text { Form }\end{array}$ & Bulb \\
\hline 1 & 50 & 5 & C-13 & T-10 \\
2 & 100 & 6 & C-13 & T-10 \\
3 & 500 & 6 & C-13 & T-20 \\
1 & 500 & 5 & C-13B & T-20 \\
\hline
\end{tabular}




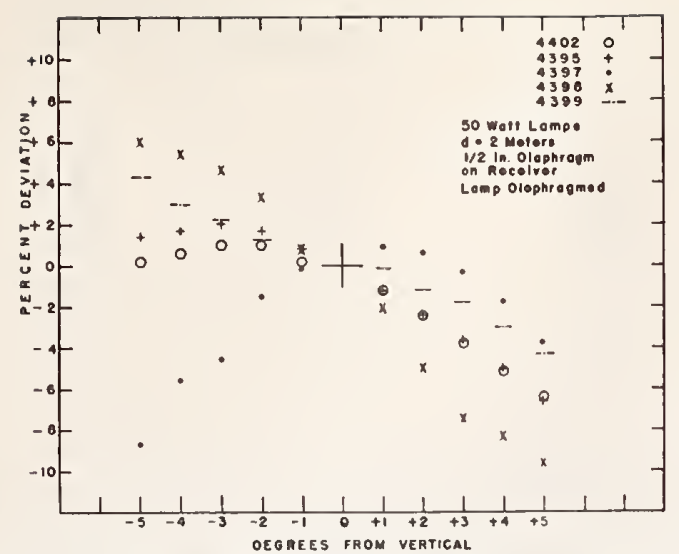

Figure 1. Per cent deviation of candlepower $v$ s. angle of tilt of 50-watt, C-13 filament lamps, Group No. 1.

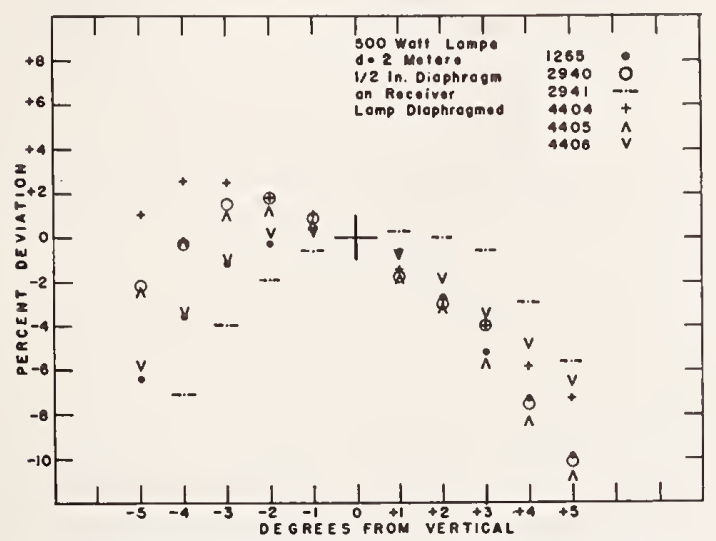

Figure 3. Per cent deviation of candlepower vs. angle of tilt of 500-watt, C-13 filament lamps, Group No. 3.

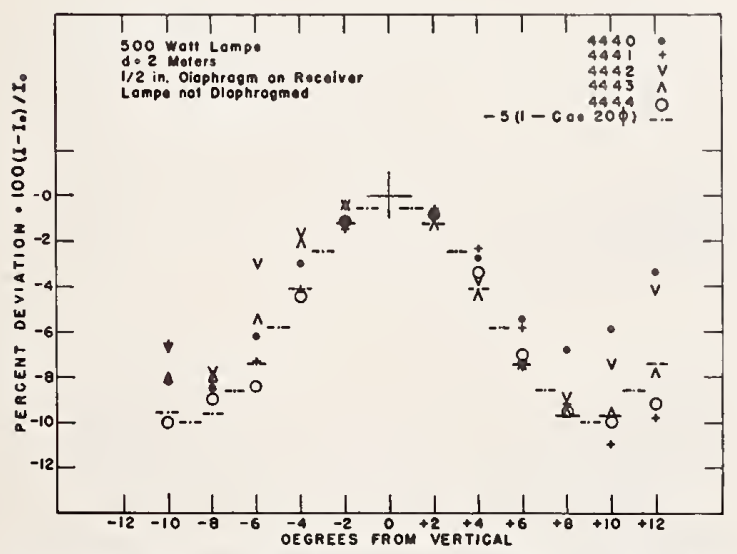

Figure 5. Per cent deviation of candlepower v8. angle of tilt of 500-watt C-13B filament lamps, Group No. 4. Also, a cosine curve with an amplitude of five per cent of maximum candlepower and a period of 18 degrees.

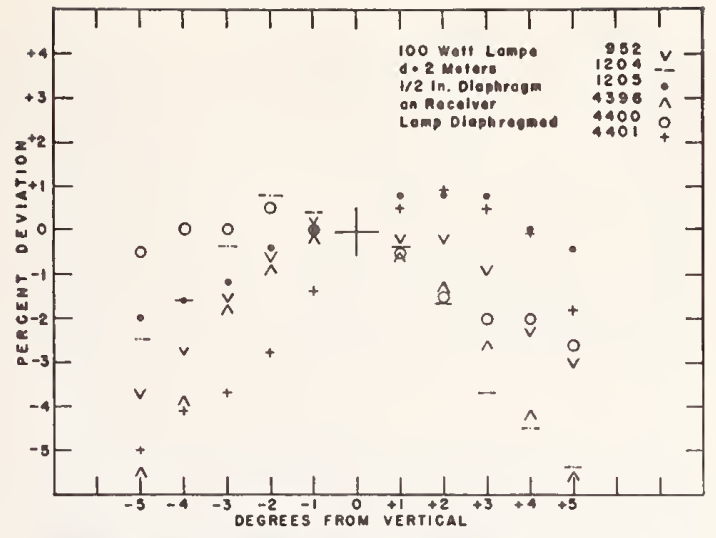

Figure 2. Per cent deviation of candlepower vs. angle of tilt of 100-watt, C-13 filament lamps, Group No. 2.

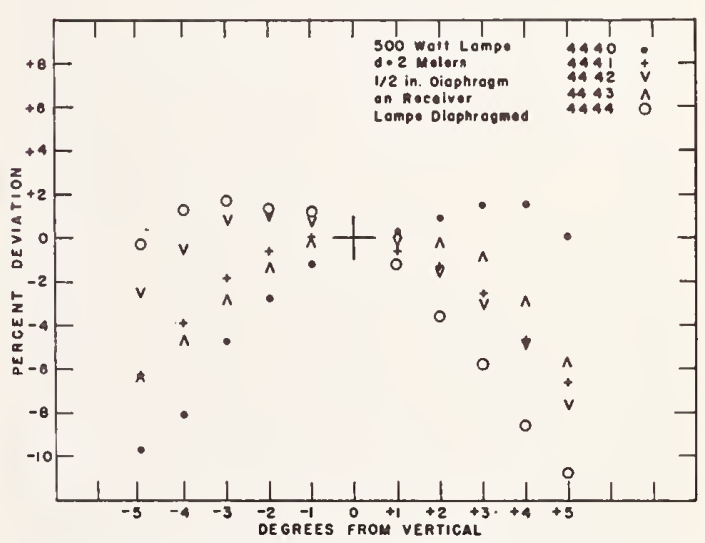

Figure 4. Per cent deviation of candlepower $v s$. angle of tilt of 500-watt, C-13B filament lamps, Group No. 4.

tion of this cyclic variation of candlepower with angle of tilt is that maximum candlepower is obtained when the orientation of the filament is such that the filament section at the rear of the coil is maximally viewed between the front turns of the coil, whereas minimum candlepower is obtained when this rear section is maximally eclipsed by one of the front turns. A measurement of the pitch of coil of several of the lamps verified this explanation.

\section{Discussion}

The procedure which has been followed at the National Bureau of Standards in calibrating gasfilled candlepower standards has included orienting each lamp so that the plane of the filament was vertical and perpendicular to the photometric axis. In view of the data herein reported, it is apparent 
that such a means of orientation is not adequate since frequently the filament plane is not accurately definable. In addition, and quite unfortunately, the need for properly orienting coiled-filament lamps about a horizontal axis has not been stressed and it can be assumed that various laboratories use rarious methods of orienting about a horizontal axis, some laboratories merely screwing the lamps into existing sockets which affords means for orientation about a vertical axis but not about a horizontal axis.

To remedy this situation, two actions have been taken at the National Bureau of Standards. First, regarding the use of elear-bulb, gas-filled lamps as candlepower standards, the practice of specifying that each such standard must be oriented so that its axis is perpendicular to the photometric axis has been adopted. In addition, these standards are selected so that when they are oriented as specified above, the variation of candlepower of each standard with change of tilt about a horizontal axis will not cxceed one per cent per degree of tilt. Users of the standards should have no difficulty in orienting lamps so that they are well within one degree from vertical.

In those cases in which greater reproducibility of results is required, such as in interlaboratory intercomparisons, in addition to vertical fiducial lines on each standard as in the past, horizontal fiducial lines are placed on each standard. These lines are $1 \frac{1}{4}$ inches above the filament center and instructions are given to orient the lamp so that these lines are in a plane which is $1 \frac{1 / 4}{4}$ inches above the photometric axis. The lines are etched on each lamp in a position such that when the lamp is properly oriented, the candlepower is a maximum. Accordingly, small deviations from proper orientation have a minimum effect. This procedure, however, as will be explained presently, is not deemed to give an adequate solution to the problem.

Second, steps have been taken that will probably lead to the use of a different type of lamp as gasfilled standards of candlepower. Several series of measurements of 300- and 500-watt lamps with inside frosted T-20 bulbs and medium-bipost bases (trade designations 300T20/2 and 500T20/4) indicate that such lamps show only small variation of candlepower $v s$. angle of either tilt or rotation about a vertical axis. This variation was less than 1 per cent for changes of angle of 10 degrees about either axis $( \pm 5$ degrees from the perpendicular to the filament plane, which was taken to be in the plane of the base pins).

Inside frosted lamps have not previously been nsed as candlepower standards, presumably because of the large size of source and the uncertainty

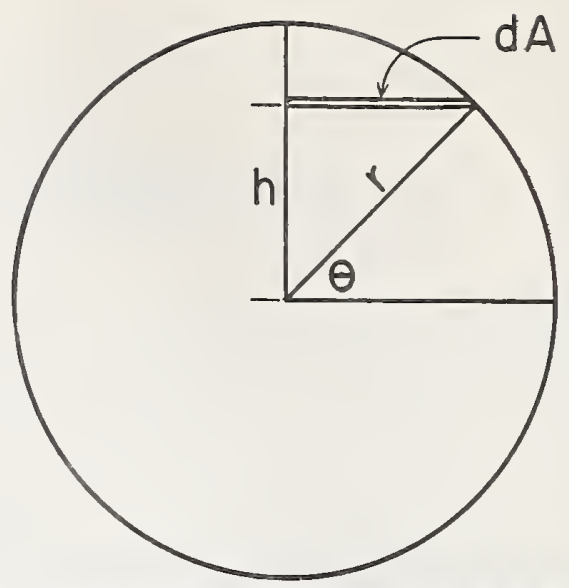

Figure 6. Element of area displaced at an angle $\Phi$ from the horizontal as viewed from the lamp filament.

of the point in the lamp from which the photometric distances should be measured. Preliminary measurements on the change of apparent candlepower of the lamps with change of distance from the receiver indicate that the inverse square law may be used for distances of 50 centimeters and greater without introducing errors of as much as 1 per cent, the distances being measured from the plane of the base pins to the receiver. The errors found in these preliminary measurements were, in general, of the opposite sign from those predicted by assuming that the lamps are cylindrical sources.

Steps have already been taken to have 100 -watt lamps of the same type made up as photometric standards. It is possible that by the time this paper is presented, 100-, 300-, and 500-watt lamps of this type, specially made as photometric standards, will be issued as standards of eandlepower in lieu of the clear-bulb lamps previously issued.

In connection with the use of clear-bulb coiledfilament lamps as standards of candlepower, it should be noted that the measured candlepower will not be independent of the vertical angle subtended by the receiver at the filament since the luminous intensity of such lamps varies so markedly with vertical angle. Assuming a circular receiver of radius, $r$, we see by referring to Fig. 6 that the luminous flux, $F$, received is :

where:

$$
F=4 \int_{h=0}^{h=r} E d A=4 \int_{0}^{r} E r \cos \theta d h
$$

$E$ is the illumination on the element of area $d A$,

$h$ is the distance of $d A$ from the center of the receiver.

Since :

$$
\begin{aligned}
h & =r \sin \theta \\
d h & =r \cos \theta d \theta, \text { and } \\
F & =4 \int_{0}^{\pi / 2} E r^{2} \cos ^{2} \theta d \theta .
\end{aligned}
$$




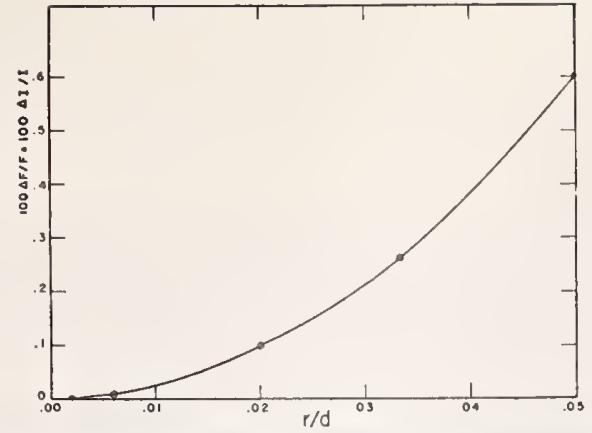

Figure 7. Per cent error of intensity measurements vs. ratio of radius of receiver to distance betwcen lamp and receiver.

Since $E=K I$, where $K$ is a constant and $I$ the luminous intensity of the lamp in the direction $d A$, and since from previous discussion of the properties of these lamps it is evident that $I$ can be expressed as :

$$
I=I_{o}[1-0.05(1-\cos 20 \Phi)],
$$

where $I_{o}$ is the maximum luminous intensity and $\Phi$ is the angular displacement of $d A$ from the horizontal as viewed from the lamp filament, it follows that:

$$
\begin{aligned}
F & =4 K I_{o} \int_{0}^{\pi / 2}[1-0.05(1-\cos 20 \Phi)] r^{2} \cos ^{2} \theta d \theta \\
& =4 K I_{o} r^{2}\left[\pi / 4-0.05 \int_{0}^{\pi / 2}(1-\cos 20 \Phi) \cos ^{2} \theta d \theta\right] .
\end{aligned}
$$

The per cent difference between the flux incident on the receiver when $I$ does not vary with $\Phi$, and the flux when $I$ does vary with $\Phi$ in accordance with the above relationship is given by:

$$
\begin{aligned}
& 100 \Delta F / F=\frac{20}{\pi} \int_{0}^{\pi / 2}(1-\cos 20 \Phi) \cos ^{2} \theta d \theta \\
& \text { Since } \tan \Phi=\frac{h}{d}=\frac{r \sin \theta}{d}, \text { where } d \text { is the distance }
\end{aligned}
$$

from lamp to receiver, the indicated integration can be made by a summation process for any assumed value of $r / d$. This was done for five values of $r / d$, from .002 to .05 , and the results obtained are plotted in Fig. 7. It will be noted that for the maximum plotted value of $r / d$, where the distance from lamp to receiver is 20 times the receiver radius or 10 times the receiver diameter, the error introduced is 0.6 per cent which is a minor error compared to that introduced by tilting the lamp.

In view of the deficiencies of clear-bulb, coiledfilament lamps as standards of luminous intensity, their use, except possibly in special applications, should be avoided wherever possible. Recommended as replacements, if maintenance measurements prove them suitably stable, are inside-frosted lamps similar to the 300- and 500-watt lamps referred to above. These lamps, in addition to the advantage of relative uniformity of candlepower with change of angle cited above, will have a medium-bipost base instead of the serew base now used on nearly all candlepower standards. The medium-bipost base will enable the user of such standards to orient his socket properly at the start of a series of measurements and relieve him of the necessity of orienting each standard individually. In addition, the use of a diaphragm in front of the standard, which has been a great inconvenience, will be eliminated. The frosted-bulb lamps will have two fiducial marks: one to show which side of the bipin plane faces the photometer, the second to show the vertical position of the filament center so that this position can be placed in the horizontal plane through the photometric axis.

\section{References}

1. Middleton, W. E. Knowles and Mayo, Eleanor G.: Journal of the Optical Society of America, 41, 513 (1951).

2. Barbrow, L. E.: Journal of Research, National Bureau of Stand. ards, 25. 703 (1940) RP1348. 


\title{
Concepts, Terminology, and Notation for Optical Modulation*
}

\author{
C. S. McCamy, Refractometry and Photography Section, National Bureau of Standards, Washington, \\ D. C.
}

\begin{abstract}
Reflectance, transmittance, and optical density are regarded as kinds of flux modulation factors. Transmittance is defined as the ratio of transmitted flux to the incident flux, while transmittance factor is here defined for an optical system as the ratio of the emergent flux with the sample in the system to the emergent flux with the sample removed. Transmission density is defined as the negative logarithm of transmittance factor. This is essentially a new definition to accord with an old practice. Reflection, transmission, and fluorescence are considered generically as propagation and generalized modulation terms are proposed. A coordinate system and functional notation are adopted to systematize the description of optical systems which use or measure modulation. Simplified notation of the form $D\left(g ; S: g^{\prime} ; S^{\prime}\right)$ describes most cases. Terms, symbols, and notation are proposed for standardization.
\end{abstract}

Key Words: Modulation reflectance, transmittance, reflectance factor, transmittance factor, propagance, absorptance, optical density, contrast, symbols, and notation.

The photographic effect is usually measured and expressed in terms of transmission density or reflection density. Optical density is therefore one of the fundamental quantities in photographic science. ${ }^{1-3}$

American standards define various types of density and provide symbols to designate the types. ${ }^{4-7}$ The types are differentiated on the basis of the geometrical arrangement of the optical components of the measuring system, the spectral quality of the flux incident on the sample, and the spectral sensitivity of the receiver used to evaluate the emergent flux. Certain arrangements which may be regarded as geometrically extreme cases are specified in simple terms. These include diffuse density (also called totally diffuse density), specular density, and doubly diffuse density. Two spectral types of density are defined: visual density and printing density. These spectral types correspond to the two principal uses of photographic materials, direct or projection viewing and photographic printing.

In mid-1963, the American Standards Association requested the author to form a committee to revise the existing standard on transmission density, in accordance with the customary schedule of revision. The charge to the committee was later extended to include all American standards on optical density. At the first meeting on November 18, 1963 , I offered to propose a system of terms and symbols, including the functional notation which

Received August 10, 1966.

* The proposed functional notation presented at the Annual Conference, Cleveland, Ohio, May 18, 1965, is given here with some revisions. The other concepts were presented at the Annual Conference, San Francisco, Calif., May 12, 1966.

1. W. de W. Abney, Phil. Mag., 48: 161 (1874).

2. W. de W. Abney, Phil. Mag., N. S., I1: 38 (1887).

3. F. Hurter and V. C. Driffield, J. Soc. Chem. Ind., 9: 445 (1890).

4. American Standard Spectral Diffuse Densities of Three-Component Subtractive Color Films, PH2.1-1952.

5. American Standard Diffuse Reflection Density, PH2.17-1958.

6. American Standard Diffuse Transmission Density, PH2.19-1959.

7. American Standard Photographic Printing Density (Carbon Step Tablet Method), PH2.25-1965.
I had developed for solving problems in precise density calibration at the National Bureau of Standards.

In optical literature one often finds optical density defined as the common logarithm of the ratio of the incident flux to the reflected or transmitted flux. Although this definition is usually cited in photographic literature, in actual practice and in photographic standards, the term "density" has taken on a somewhat different connotation. Density has come to be regarded as a measure of the modulation of flux by a sheet, often bearing a graphic image, when the sheet is employed in a given type of optical system. The optical system may be as simple as the eye directly viewing a print or as complex as an optical motion-picture printer. Various methods of measuring density have been developed, using devices which simulate the important features of the optical systems in which materials are used. Thus in photographic science, and indeed in most other fields involving optical modulators, the optical density of an area on a given sample may have various values depending on the intended use and the corresponding method of measurement adopted to simulate it.

When this revision was undertaken it was not generally recognized that the usually expressed definition of density was not the one needed or used in most practical cases and, for this reason, there was no systematic terminology for the quantities involved. For years there have been differences between some of the terms and symbols used in photography and those generally accepted in photometry and colorimetry. Since there was much to be gained by bringing the standard terminologies and symbols of these disciplines into harmony, I had several conferences in June of 1965 with L. E. Barbrow and Deane B. Judd of the Photometry and Colorimetry Section of NBS. Judd had just returned from a meeting of the Committee on 
Nomenclature of The International Commission on Illumination to revise the International Lighting Vocabulary, and was therefore familiar not only with the official actions but with current attitudes of the representatives of various nations toward alternate terms. The concepts, terms, symbols, and notation are proposed in the hope that they will be acceptable in photometry as well as photography. The terms "transmission density" and "reflection density" and most of the symbols in current ASA photographic standards are retained.

\section{Optical Modulation}

When radiant energy interacts with matter, some of it is absorbed, and is converted into another form of energy, while some may be propagated by reflection, refraction, transmission, diffraction, or scattering. Some of the photons may be absorbed and then reradiated almost immediately, in less than $10^{-8} \mathrm{sec}$, with a reduction in frequency. This process is called fluorescence. For most purposes, that part of the energy which is reradiated in this way is effectively propagated. The term "propagation" is a useful general term for several of the processes with which we must deal. It is particularly useful in discussions involving both transmission and reflection.

We of ten need a general term for a measure of the effect of an object on the propagation of radiant energy from a source to a receiver. The object may change the direction, amplitude, frequency, phase, or polarization of the electromagnetic wave. The technical term for the process of varying the char. acteristics of a carrier wave to convey information is modulation. The graphic images produced by photography are mainly used as devices which modulate the flow of radiant energy and thereby convey information. Modulation has been used in about this sense for many years in sound-on-film technology. ${ }^{8}$ This usage is standard in sensitometry..$^{9-14}$ The spatial variation of modulation of the light falling on objects around us permits us to receive visual information about these objects and to photograph them.

The modulation factor of communication theory is defined with respect to the amplitude of the signal that modulates the carrier wave. ${ }^{15}$ Optical modulation factors, on the other hand, are usually measured in terms of ratios of fluxes and the flux (power)

8. J. G. Frayne and H. Wolfe, Sound Recording, Wiley and Sons, N. Y. (1949), Chapters 15-19.

9. American Standard Sensitometry and Grading of Photographic Papers, PH2.2-1953.

10. American Standard Method for Determining Speed of Photographic Negative Materials (Monochrome, Continuous-tone), PH2.5-1960.

11. American Standard Method for the Sensitometry of Industrial X-ray Films for Energies Up to 3 Million Electron Volts, PH2.81964.

12. American Standard Method for the Sensitometry of Medical X-ray Films, PH2.9-1964.

13. The Theory of the Photographic Process, C. E. K. Mees and T. H. James, eds., Macmillan Co., N. Y., 3rd ed. p. 412.

14. T. H. James and G. C. Higgins, Fundamentals of Photographic Theory, Morgan \& Morgan, Inc., N. Y. (1960).

15. W. D. Hershberger, Principles of Communications Systems, Prentice-Hall, Inglewood Cliffs, N. J. (1955), p. 77 et seq. propagated is proportional to the square of the amplitude of the electric displacement associated with the electromagnetic wave. I suggest the term "flux modulation" to distinguish the modulation measured in terms of ratios of fluxes from amplitude modulation, phase modulation, polarization modulation, and frequency modulation; all of which have more relevance to photography and related fields than is generally recognized. We may note a few examples. Pictures printed on paper containing fluorescent dyes are effectively frequency modulators as well as flux modulators. Various effects observed when objects are placed between polarizers are utilized in high-speed shutters, stress analysis, thickness measurement, and chemical polarimetry. These applications depend on polarization modulation. The phase contrast microscope is used to observe small objects which vary only in thickness or refractive index by utilizing the phase modulation resulting from these variations. A similar optical system is used to project photographic images produced by photoplastic recording. Thus "modulation" is a fitting generic term for a number of useful optical processes including the effects of objects such as pictures on the propagation of radiant energy. Transmittance and optical density are two of many measures of optical modulation.

Although phase modulation and polarization modulation are of interest in themselves, they are often converted by optical devices to flux modulation so that the effects may be observed visually or detected by flux-sensing devices. Since optical frequency detection is the basis of color vision and instrumental receiver sensitivities depend on frequency, spectral aspects of modulation are always considered along with the flux modulation. The remainder of this paper deals with flux modulation only, with due regard for fluorescence phenomena.

Although one could regard a whole optical, photographic, or television system, including the display, as an "object" which is irradiated on one "side" and viewed on the other, the usual conceptions of optical modulators are typified by sheet materials and the surfaces of bodies.

It should be noted that modulation does not necessarily mean attenuation. Oftentimes objects cause a gain in the flow of radiant energy from a source to a receiver.

These apparently far-ranging observations are made in the hope that optical modulation measurement can be viewed in a perspective sufficiently broad that the concepts, terms, and notation adopted will have the generality to cope with current problems and many yet to come.

\section{Terms and Symbols for Fluxes}

The measures of flux modulation most often used are ratios of fluxes or logarithms of these ratios. Therefore, the fundamental quantities to be identified and be given symbols are the fluxes. We deal in radiant flux, using the symbol $\Phi$. Luminous flux 
TABLE I. Fluxes Involved in Optical Modulation

\begin{tabular}{|c|c|c|}
\hline Term & Symbol & Definition \\
\hline Incident flux & $\Phi_{0}$ & $\begin{array}{l}\text { Flux incident on scan- } \\
\text { ning aperture }\end{array}$ \\
\hline Absorbed flux & $\Phi_{\mathrm{a}}$ & $\begin{array}{l}\text { Flux absorbed by } \\
\text { sample }\end{array}$ \\
\hline Propagated flux & $\Phi_{\mathrm{p}}$ & $\begin{array}{l}\text { Flux propagated by } \\
\text { sample and utilized }\end{array}$ \\
\hline Extra flux & $\Phi_{\mathrm{q}}$ & $\begin{array}{l}\text { Flux propagated out- } \\
\text { side spatial and spec- } \\
\text { tral range of receiver }\end{array}$ \\
\hline Lost flux & $\Phi_{\mathrm{L}}$ & Flux not utilized \\
\hline Reflected flux & $\Phi_{\mathrm{r}}$ & $\begin{array}{l}\text { Flux reflected by sam- } \\
\text { ple and utilized }\end{array}$ \\
\hline Transmitted flux & $\Phi_{\mathrm{t}}$ & $\begin{array}{l}\text { Flux transmitted by } \\
\text { sample and utilized }\end{array}$ \\
\hline $\begin{array}{l}\text { Reference propagated } \\
\text { flux }\end{array}$ & $\Phi_{\mathrm{ps}}$ & $\begin{array}{l}\text { Flux propagated by } \\
\text { reference standard } \\
\text { and utilized }\end{array}$ \\
\hline $\begin{array}{l}\text { Reference reflected } \\
\text { flux }\end{array}$ & $\Phi_{\mathrm{r} 8}$ & $\begin{array}{l}\text { Flux reflected by ref- } \\
\text { erence standard and } \\
\text { utilized }\end{array}$ \\
\hline $\begin{array}{l}\text { Reference transmitted } \\
\text { flux }\end{array}$ & $\Phi_{t s}$ & $\begin{array}{l}\text { Flux transmitted by } \\
\text { reference standard } \\
\text { and utilized }\end{array}$ \\
\hline Aperture flux & $\Phi_{\mathrm{j}}$ & $\begin{array}{l}\text { Flux utilized with no } \\
\text { sample in scanning } \\
\text { aperture }\end{array}$ \\
\hline
\end{tabular}

is regarded as a specific case not fundamentally different from any other specific spectral distribution of flux. The symbol $\Phi$ is given an appropriate lower case subscript to identify the particular flux. The terms, symbols, and definitions of fluxes involved in optical modulation are given in Table $\mathbf{I}$.

In the past, confusion and misunderstanding have stemmed from the failure of authors to state clearly whether the phrase "transmitted flux" necessarily meant all of the transmitted flux or might mean some specified part of it. To economize on symbols and terminology and at the same time make the notation completely explicit in all cases, I prefer to consider each transmitted, reflected, or propagated flux to be a specified part of the whole. In each case, one of the many possible specified parts would be all of the flux. Notation for specifying what part of the flux is utilized for measurement or practical purposes is given in a later section of this paper. There is usually no doubt about whether all or part of the incident flux is under consideration but since the value of modulation depends on the geometrical and spectral nature of the incident flux, the part of space and the spectrum from which the incident flux comes is denoted in a manner exactly analogous to that used to denote the part of space and the spectrum in which the emergent flux is utilized. Once the utilized part of the emergent flux is specified, the terms "transmitted," "reflected," and "propagated" mean transmitted, reflected, and propagated in the specified part of space and the spectrum. To provide a term and symbol for all of the flux, the prefix "total" may be used and the subscript may be capitalized.
The flux $\Phi_{0}$ which is incident on a specified area (defined by a scanning aperture) on a body is either absorbed by the body, propagated to a specified receiver, or propagated in directions or parts of the spectrum other than that specified. The flux which is absorbed is called absorbed flux $\Phi_{\mathrm{a}}$, that propagated to the specified receiver is called propagated flux $\Phi_{\mathrm{p}}$, and that propagated outside the specified spatial or spectral range of the receiver is called extra flux $\Phi_{\mathrm{q}}$. That which is not propagated in the specified direction is called lost flux $\Phi_{\mathrm{L}}$ and is the sum of the absorbed and extra fluxes. That which is not absorbed is called total propagated flux $\Phi_{\mathrm{P}}$.

When the propagated flux emerges from the surface on which the incident flux falls, it is called reflected flux $\Phi_{r}$. When the propagated flux passes through the body and emerges from another surface, it is called transmitted flux $\Phi_{t}$. The general term "propagation" is useful when the mode of propagation (transmission, reflection, scattering, fluorescence) is not known or it is known to be some combination.

The aperture flux $\Phi_{\mathrm{j}}$ is the flux propagated from the source to the receiver with the sample removed from the scanning aperture but the remainder of the optical system undisturbed, as when a slide is removed from a projector.

In discussions of the propagation of flux within a medium it is convenient to have a symbol for the flux transmitted by the first interface and a symbol for the flux incident on the second interface. Sometimes several media are involved, in which case there are several interfaces. The interfaces can be numbered in the order in which they would be encountered by energy passing through the system and the terms and symbols for incident flux, reflected flux, and transmitted flux can be applied to the interactions at each interface, using the number designating the interface as a subscript. For example, in the simple case of a glass window we would have:

$$
\Phi_{01}, \Phi_{\mathrm{r} 1}, \Phi_{\mathrm{t} 1} \text { and } \Phi_{02}, \Phi_{\mathrm{r} 2}, \Phi_{\mathrm{t} 2}
$$

Flux may be reflected back and forth between interfaces, in which case the subscripts for the interfaces may be appended in series. The notation " $\Phi_{\text {r21435" }}$ means that the flux was reflected by the

TABLE II. Measures of Flux Modulation

$\begin{array}{cc}\text { Arithmetic (modulation } & \text { Logarithmic (optical } \\ \text { factor, } m \text { ) } & \text { density, } D \text { ) }\end{array}$

Transmittance, $\tau=\Phi_{\mathrm{t}} / \Phi_{0} \quad$ Transmittance density, $D_{\tau}=-\log _{10} \tau$

Reflectance, $\rho=\Phi_{r} / \Phi_{0} \quad$ Reflectance density, $D_{\rho}=-\log _{10} \rho$

Propagance, $\psi=\Phi_{\mathrm{p}} / \Phi_{0} \quad$ Propagance density, $D_{\psi}=-\log _{10 \psi}$

Transmittance factor, $T=\Phi_{\mathrm{t}} / \Phi_{\mathrm{j}}$

Transmission density, $D_{\mathrm{T}}=-\log _{10} T$

Reflectance factor, $R=\Phi_{\mathrm{r}} / \Phi_{\mathrm{r}:}$

Propagance factor, $P=\Phi_{\mathrm{p}} / \Phi_{\mathrm{j}}$
Reflection density, $D_{\mathrm{R}}=-\log _{10} R$ Propagation density, $D_{\mathrm{r}}=-\log _{10} P$ 
second, first, fourth, third and fifth interfaces, in that order. If the number of interfaces exceeds nine, the subscript numbers would be separated by commas. To avoid confusion, it should be explicitly noted that the subscript for incident flux is a zero, not the fifteenth letter of the alphabet. This is generally accepted usage.

In discussions of instruments utilizing or measuring optical modulators, it is convenient to define two kinds of stray flux: internal stray flux $\Phi_{\mathrm{xi}}$, any flux affecting the sensor, from the source intentionally used to irradiate the sample but arriving at the receiver by any path other than the one specified for operation, and external stray flux $\Phi_{\text {xe }}$, any flux affecting the sensor and coming from any source other than that used to irradiate the sample.

\section{Terms and Symbols for Measures of Modulation}

The terms, symbols, and defining equations for the various measures of flux modulation are given in Table II.

As they are defined here, transmittance, propagance, and reflectance are "absolute" measures in that they are independent of arbitrary reference standards. Reflectance factor is clearly a relative measure because a reference surface is used in the measurement.

Propagance factor and transmittanve factor may be considered relative if one considers the air which normally replaces the sample as a "reference standard," but they are essentially absolute for most practical purposes. In photography we sometimes measure the modulation produced by a processed film relative to the modulation produced by the same kind of processed material with no exposure. Chemists often measure the modulation produced by a cuvette containing a solution relative to the modulation of the same or an identical cuvette containing pure solvent. One sometimes hears these measurements referred to as "relative transmittance" even though transmittance is defined, as it is here, as the ratio of transmitted to incident flux. Clearly, the concept employed in such instances is what we would call transmittance factor relative to processed unexposed film or relative to solvent. In the cases where a reference standard is substituted for the sample, the terms "relative transmittance factor, $T_{\mathrm{r}}$ " and "relative propagance factor, $P_{\mathrm{r}}$ " may be used and the defining equations become $T_{\mathrm{r}}=\Phi_{\mathrm{t}} / \Phi_{\mathrm{ts}}$ and $P_{\mathrm{r}}=\Phi_{\mathrm{p}} / \Phi_{\mathrm{ps}}$. The corresponding logarithmic terms would be "relative transmission density, $D_{T_{\mathrm{r}}}$ " and "relative propagation density, $\mathrm{D}_{P \mathrm{r}}$ " and the defining equations would be

$$
D_{T \mathrm{r}}=-\log _{10} T_{\mathrm{r}}
$$

and

$$
D_{P \mathrm{r}}=-\log _{10} P_{\mathrm{r}}
$$

Although it would be logically consistent to call $-\log _{10} R$ "relative reflection density," such usage would probably be much more confusing than helpful. The only "less relative" type that comes to mind would be a measurement with respect to the flux reflected by the scanning aperture with no sample or reference standard in place. Such a procedure is little used in reflectometry but if the occasion arises we have the general term "propagation density" defined in exactly this way. It is much more common, because it is useful, to use the term "relative reflection density" for measurements of reflection density using unexposed but processed photographic paper or blank printing paper as a reference standard instead of magnesium oxide or a standard plaque. When such a distinction is useful, the symbol " $D_{R \mathrm{r}}$ " can be used for such relative reflection density.

Hurter and Driffield defined opacity as the reciprocal of transmittance and the term has been used this way in photography ever since. ${ }^{3}$ To be consistent, we may define opacity factor as the reciprocal of transmittance factor, however, the use of these opacity terms is not encouraged because of frequent confusion with the concept of hiding power, the ability of a sheet or film to obscure a black-and-white pattern on which it is placed, as when a blank sheet of paper is laid on a printed page. In general, hiding power is not determined by transmittance alone, because the reflectance of the sheet must also be taken into account.

If we adopt the symbol $m$ as the general symbol for any of the arithmetic forms of optical flux modulation $\tau, o, \psi, T, R$, or $P$, and the symbol $D$ as the general symbol for any of the logarithmic forms which we call optical density, we may give the general definition of optical density by the equation $D=-\log _{10} m$.

Theoretical computations can often be simplified by the use of natural density* $D_{\mathrm{e}}$ defined by the equation $D_{\mathrm{e}}=-\log _{\mathrm{e}} m$. The symbols for the various types would be $D_{\mathrm{e} \tau}, D_{\mathrm{e} \rho}, D_{\mathrm{e} \psi}, D_{\mathrm{e} T}, D_{\mathrm{e} R}$, and $D_{\mathrm{e} P}$. The terms would be formed as in "natural transmission density." When necessary, the prefix "common" may be used to specify densities defined by logarithms to base ten. The two types of density are related by the equation $D=N D_{\mathrm{e}}$ where, $N=\log _{10} \mathrm{e}=0.43429448$. Hurter and Driffield defined density in terms of $\log _{e}$ but then said, "For general convenience we use vulgar and not hyperbolic logarithms." 3 I have heard objection to the term "common" but it appears that we have made satisfactory progress in this regard in the past 76 years.

\section{Terms and Symbols for Measures of Optical Attenuation}

Most optical modulation measurements are made to find what part of the incident flux is propagated, but at times interest centers on the flux that is

\footnotetext{
* This name derives from the name of natural logarithms, often called Napierian logarithms. Strictly speaking, Napier's $\log x$ was not $\log \mathrm{e}^{x}$, see, e.g., Websters New International Dictionary, second edition, under "logarithm."
} 
absorbed. The nature of the absorption of flux within an optical medium is an important characteristic of the medium, intimately related to its other chemical and physical characteristics. In studies of photochemical reactions, the absorbed flux is of primary importance because it provides the energy to initiate reactions. The absorbed flux is the difference between the incident flux and the total propagated flux; $\Phi_{\mathrm{n}}=\Phi_{0}-\Phi_{\mathrm{P}}$.

In the process of modulation, flux may be lost not only by absorption but by reflection, refraction, diffraction, scattering, fluorescence, or transmission, depending on what part of the propagated flux is evaluated or utilized. The lost flux is the difference between the incident flux and the specified part of the total propagated flux; $\Phi_{\mathrm{L}}=\Phi_{0}-\Phi_{\mathrm{p}}$. If absorbed flux is to be measured indirectly, all other losses must be taken into account.

If the nature of the medium is such that in a collimated beam the differential loss of flux in each differential layer of thickness is a constant fraction of the flux incident on that layer, that is if $d \Phi / d z=$ $-a_{b} \Phi$, where $z$ is the distance in the direction of the beam and $a_{b}$ is the thickness attenuation coefficient, then we find by integration that the internal transmittance of a layer of thickness $b$ is $\tau_{i}=\Phi_{02} / \Phi_{t 1}=$ $\exp \left(-a_{b} b\right)$. This equation, which holds for optical media in which there is negligible scattering and fluorescence, is known as Bouguer's law.

If the thickness attenuation coefficient is proportional to the concentration $c$ of the substance which causes the attenuation, that is, if $a_{b}=a c$, where $a$ is the attenuation coefficient, then the internal transmittance of a layer of thickness $b$ and concentration $c$ is $\tau_{\mathrm{i}}=\exp (-a b c)$.

The chemist, interested in the concentration variation for a constant thickness, uses a concentration attenuation coefficient $a_{\mathrm{c}}=\alpha b$ and the expression for internal transmittance becomes $\tau_{i}=\exp \left(-a_{c} c\right)$. This equation which is a good approximation for many materials, is called Beer's law. When $a_{c}$ holds for a thickness $b$ of one unit and $c$ is expressed in molar concentrations, $a_{c}$ is the molar attenuation coefficient.

The conditions imposed on these relationships should not be neglected. For some substances, Beer's law fails. If a material scatters a great deal, multiple scattering of flux invalidates the assumption of constant fractional attenuation in each differential of thickness. Nonetheless, Beer's law is useful in chemistry and Bouguer's law is useful in atmospheric optics.

In studies of the visibility of signal lights, it has been found that the atmosphere scatters very little flux in the backward direction. The flux reflected in the second, third, and other successive reflections is negligible for a receiver, such as one or a few retinal receptors of the eye, which is sensitive over a small angle. Practically all scattered light is lost, as though it were absorbed. In such cases, scattering may be treated mathematically in the same manner as absorption, the conditions for the derivation of the law of exponential attenuation hold, and we may write $\tau_{\mathrm{i}}=\exp -\left(\alpha_{\mathrm{a}}+a_{\mathrm{s}}\right) b c$, where $\boldsymbol{\alpha}_{\mathrm{a}}$ is the absorption coefficient and $a_{\mathrm{s}}$ is the scattering coefficient. In general, the relationship between modulation and the absorption and scattering coefficients is by no means this simple.

Since all materials have molecular density variations, all exhibit some scattering. For this reason the term absorption coefficient should not be used in place of the more general term attenuation coefficient, but should be reserved for its appropriate use.

Any of the exponential expressions for internal transmittance can be written in terms of powers of ten rather than e. I suggest that such coefficients be called "coefficients to base 10 " to differentiate them from "coefficients to base $e$ " and that such coefficients to base 10 be identified by the prime symbol, for example $a^{\prime}$. The corresponding coefficients are related as follows: $a^{\prime}=N a$, where $N=$ $\log _{10} \mathrm{e}=0.43429448 \ldots$

Absorption and scattering characteristics are, of course, dependent on wavelength. Thus the various attenuation coefficients are functions of wavelength.

The various measures of attenuation are defined in Table III.

Absorptivity is usually defined as the absorptance of a layer of unit thickness. However, in some studies of radiation and modulation, absorptivity is defined as the limiting absorptance as the thickness increases infinitely, in a manner analogous to that shown for reflectivity in Table III. To differentiate these kinds of absorptivity, I suggest the following terms and symbols: "limiting absorptivity" $\alpha_{\infty}$ and "unit absorptivity" $\alpha_{L_{L} \text { * }}$

Since the reflectance of a body depends on the condition of its surface, the measurement of the characteristic reflectivity of a material requires careful attention to the condition of the surface.

\section{TABLE III. Measures of Optical Attenuation and Related Quantities}

Properties of a body
Internal propagance

Internal propagance density

Internal transmittance

Internal transmittance density

Absorptance

Internal absorptance

$$
\begin{aligned}
\psi_{\mathrm{i}} & =\Phi_{02} / \Phi_{\mathrm{t} 1} \\
D_{\psi_{\mathrm{i}}} & =-\log _{10} \psi_{\mathrm{i}} \\
\tau_{\mathrm{i}} & =\Phi_{02} / \Phi_{\mathrm{t} 1} \\
D_{\tau \mathrm{i}} & =-\log _{10} \tau_{\mathrm{i}} \\
\alpha & =\Phi_{\mathrm{a}} / \Phi_{0} \\
\alpha_{\mathrm{i}} & =\Phi_{\mathrm{a}} / \Phi_{\mathrm{t} 1}
\end{aligned}
$$

Properties of a material
* The value of transmissivity or absorptivitiy depends on the unit of thickness, which must be stated. 


\section{Contrast}

Optical contrast is an evaluation of the relationship between two magnitudes of some attribute of appearance, compared simultaneously or successively. Luminance contrast and the modulations that produce it are of interest here.

Several objective measures have been used, some of which were chosen to correlate with subjective visual evaluations. According to Weber's Law, as it applies to luminance, the least preceptible difference between the luminances of two adjacent fields is proportional to the luminance at which the comparison is made. Thus the ratio of the difference of two luminances to the mean value is a measure of contrast proportional to the least perceptible difference at any mean value of luminance, over a large part of the useful range. The difference is sometimes divided by the lower of the two values instead of the mean of the two. The current edition of the International Lighting Vocabulary ${ }^{16}$ gives three expressions for objective measures of contrast, referring to them as expressions (a), (b), and (c), without assigning symbols. I propose the use of the symbol $C$, always with a subscript, for contrast and the use of $a, b$, and $c$, as subscripts to correspond with the types given in the Vocabulary, thus:

$$
\begin{aligned}
& C_{\mathrm{a}}=\frac{L_{2}-L_{1}}{L_{1}} \\
& C_{\mathrm{h}}=\frac{L_{2}-L_{1}}{\left(L_{2}+L_{1}\right) / 2}
\end{aligned}
$$

and

$$
C_{\mathrm{c}}=L_{2} / L_{1}
$$

In photographic tone reproduction theory the term "contrast" usually refers to a contrast ratio $C_{c}$, which may be the ratio of scene luminances or it may be the ratio of two transmittance factors or reflectance factors on a photograph. This property of the photograph may also be indicated by a difference of optical densities. This measure of contrast is particularly suitable for describing a scene to be photographed or a negative to be printed. The ratio of scene luminances determines the ratio of exposures for any shutter and aperture settings so that, with appropriate settings and choice of film, the exposure scale of the film can be matched, within practical limits, to the ratio of exposures. The density difference on a negative determines the log exposure difference in the printing operation, regardless of the amount of flux in the beam modulated by the negative. The print material may be selected on the basis of the log exposure difference and the flux and exposure time are adjusted to the speed of the print material. Weber's Law has no

16. International Lighting Vocabulary, 2nd Ed., Vol, 1, International Commission on Illumination Bureau Central, 57 Rue Cuvier, Paris 5, France. bearing, since the negative is not being viewed directly.

One should clearly distinguish between contrast, which is a characteristic of the scene or the photograph, and gamma, the slope of the straight-line portion of the characteristic curve, which is a characteristic of the photographic process. The contrast between two parts of a picture depends on the scene as well as gamma. Incidentally, the contrast between the lightest and darkest areas may not be the contrast of interest. For example, when a person's face is photographed by artificial light outdoors at night, the contrast between parts of the face may be low while the contrast of the face to background may be extremely high.

In the case of a photograph viewed directly or by projection the mean luminance depends not only on the photograph but on the illumination. (Only with constant illumination, as in a standardized motion-picture review room, is the mean luminance determined by the photograph.) Nevertheless, the subjective contrast is determined by the photograph. If we define contrast $C_{\mathrm{m}}$ as the luminance difference $\left(L_{2}-L_{1}\right)$ divided by the least perceptible difference $\Delta L$ at luminance $L$ and assume Weber's Law

$$
\Delta L / L_{\text {mean }}=k
$$

then

$$
\begin{aligned}
& C_{\mathrm{m}}=\left(L_{2}-L_{1}\right) / \Delta L=\left(L_{2}-L_{1}\right) / k L_{\text {mean }} \\
& C_{\mathrm{m}}=2\left(L_{2}-L_{1}\right) / k\left(L_{2}+L_{1}\right)
\end{aligned}
$$

Given a transparency with areas having transmittance factors $T_{1}$ and $T_{2}$ and uniform illuminance $E$, then

$$
C_{\mathrm{m}}=2\left(E T_{2}-E T_{1}\right) / k\left(E T_{2}+E T_{1}\right)
$$

and

$$
C_{\mathrm{m}}=2\left(T_{2}-T_{1}\right) / k\left(T_{2}+T_{1}\right)
$$

Let $C_{\mathrm{M}}=(k / 2) C_{\mathrm{m}}$, then

$$
C_{\mathrm{MI}}=\left(T_{2}-T_{1}\right) /\left(T_{2}+T_{1}\right)
$$

a quantity which I propose to call modulation contrast because the above derivation demonstrates that it is clearly a measure of contrast, it is computed from measures of modulation, and it has of ten been referred to as "modulation" in a special sense.

According to Stevens ${ }^{17}$ the value of $k$ is about 0.016 . Therefore

$$
C_{\mathrm{M}}=C_{\mathrm{m}} / 125=\left(L_{2}-L_{1}\right) / 125 \Delta L
$$

that is, $C_{\mathrm{M}}$ is a measure of contrast, the "units" of which are about 125 times the least perceptible difference. Since the constant $k$ varies with luminance on the high and low ends of the luminance

17. S. S. Stevens, "Sensation and Psychological Measurement," in Edwin G. Boring, Herbert S. Langfeld, and Harry P. Weld, eds., Foundations of Psychology. Wiley, 1948, p. 268. 


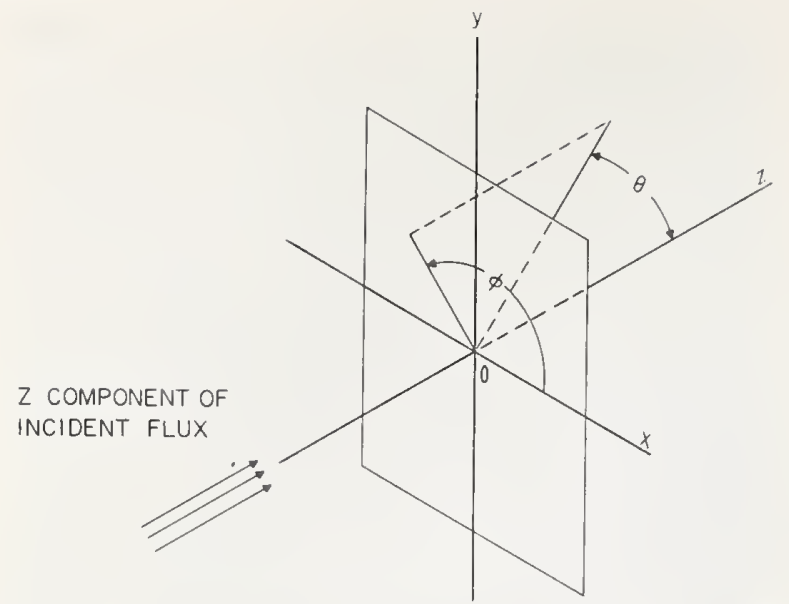

Fig. 1. Coordinate system for describing geametrical factors affecting aptical modulation.

scale and with the nature of the field of view, the relationship given is approximate.

The ratio of the difference to the sum $\left(C_{M}\right)$ is equal to the ratio of half the difference to the mean. This expression corresponds to one of the fundamental quantities in communication theory, the ratio of the amplitude to the mean value of a sinusoidally varying amplitude modulation of a carrier wave. It is called the "modulation factor" or simply "modulation." This specific application of the term "modulation" should be distinguished from the generic term which is used on this paper and is well established in "frequency modulation" and "phase modulation." (The "modulation index" in frequency modulation theory is quite different from the "modulation factor" in amplitude modulation.)

\section{Coordinate System}

Figure 1 provides a coordinate system for describing the geometrical factors affecting optical modulation. The coordinates relate to a reference plane, the $x y$ plane, which is the plane in which the first surface of the sample is placed for measurement or use. The point on the sample placed at the origin 0 is the center of the area said to have its density measured. The $z$ axis is directed with the component of incident flux normal to the reference plane.

The colatitude of a ray is the angle $\theta$ between the ray and the $z$ axis. Unless otherwise stated, the colatitude $\theta$ of incident rays and reflected rays shall be measured from the negative $z$ axis while the colatitude of transmitted and propagated rays shall be measured from the positive $z$ axis. This special convention should be the most convenient for most cases but the description of some measurements and applications would be simplified by measuring all colatitudes from the positive $z$ axis, according to the usual mathematical convention. The longitude of a ray is the angle $\phi$ measured in the $x y$ plane, from the positive $x$ axis in the direction of the positive $y$ axis to the projection of the ray on the $x y$ plane. The direction of a ray is given by the coordinates $\theta, \phi$, in that order. Angle $\phi$ is less than $360^{\circ}$ and $\theta$ is $180^{\circ}$ or less.

If the sample is thick, it may be desirable to refer the description of the efflux to a secondary coordinate system $x^{\prime}, y^{\prime}, z^{\prime}, \theta^{\prime}, \phi^{\prime}$, having its origin $0^{\prime}$ displaced a specified distance $b$, corresponding to the thickness, along the positive $z$ axis, so that $x^{\prime}=x$, $y^{\prime}=y, z^{\prime}=z-b$ and the angles are defined in a corresponding manner.

Subscripts are used for the direction angles of rays to correspond to the subscripts for fluxes, that is, " 0 " for incident, " $r$ " for reflected, " $t$ " for transmitted, and " $p$ " for propagated.

\section{Functional Notation}

Finctional notation provides a convenient and generally understood means of denoting a dependent variable and the independent variables, known as "parameters," on which its value depends. The symbol for the dependent variable is followed by the symbols for the various parameters, or their values in a given case, in parentheses. Using this notation, we may write the fact that optical density $D$ depends on the conditions of measurement or use, as follows:

$$
D\left(C: C^{\prime}\right)
$$

where $C$ is the influx function, the complete description of the flux incident on the sample and $C^{\prime}$ is the efflux function, the complete description of the sensitivity of the system receiving flux from the sample and using or evaluating it. Let $C$ and $C^{\prime}$ be given the generic name "condition functions." The influx function is, in the general case, the radiance $L_{\mathrm{e}}$ at the sample as a function of angles $\theta$ and $\phi$, wavelength in vacuum $\lambda$, and the spatial coordinates $x$ and $y$,

$$
C=L_{\mathrm{e}}(\theta, \phi, \lambda, x, y)
$$

The efflux function is, in the general case, the sensitivity $S_{\mathrm{e}}$ of the receiver system as a function of angles, wavelength, and spatial coordinates,

$$
C^{\prime}=S_{\mathrm{e}}(\theta, \phi, \lambda, x, y)
$$

If the samples to be considered are uniform over the entire area of interest, the dependence of the condition functions on $x$ and $y$ can usually be disregarded, since the ratios of fluxes are not influenced. Then

$$
C=L_{r}(\theta, \phi, \lambda)
$$

and

$$
C^{\prime}=S_{\mathrm{e}}(\theta, \phi, \lambda)
$$

Furthermore, in most applications, the measured value of modulation does not depend on the absolute magnitudes of $L_{e}$ and $S_{e}$, but only on their relative 
spectral and spatial distributions. In the remainder of this paper it should be understood that relative distributions are implied, with the understanding that in all questionable cases, the constancy or variation of modulation with the absolute magnitude of $L_{\mathrm{e}}$ should be determined by measuring modulation with various values of incident radiance. The absolute sensitivity is not involved because modulations are defined in terms of ratios of fluxes.

If samples are nonuniform with respect to $x$ or $y$ or both, the measured modulation may depend on the product $L_{\mathrm{e}}(x, y) S_{\mathrm{e}}(x, y)$ known as the aperture function. Photographic films are usually nonuniform for any aperture function having an area less than one square millimeter. For this reason, the aperture function is of particular interest in microdensitometry, image evaluation, and applications such as sound-on-film technology involving film scanning. In such instances the aperture function should be specified. It is often regarded as constant over a rectangle or circle. In some applications, as in granularity measurement, the scanning aperture function is symmetrical with respect to the $z$ axis and can be most easily described as a function of a polar coordinate $r$, the distance from the origin in the reference plane.

The condition functions $C$ and $C^{\prime}$ describe both geometrical and spectral conditions. In very many practical cases either geometrical or spectral factors are of interest, but not both. For this reason and because it leads to a more simplified notation in most practical cases, let us separate the geometrical and spectral factors as follows:

$$
\begin{gathered}
C=g ; S \\
C^{\prime}=g^{\prime} ; S^{\prime}
\end{gathered}
$$

where

$g=$ the symbol for the influx geometry, the spatial distribution of radiance at the irradiated surface of the sample;

$S=$ the symbol for the influx spectrum, the spectral distribution of the flux incident on the sample;

$g^{\prime}=$ the symbol for the efflux geometry, the spatial distribution of the effectiveness of the optical system in utilizing radiant flux received from the sample;

$S^{\prime}=$ the symbol for the efflux spectral sensitivity, the spectral sensitivity of the receiver which utilizes the radiant flux from the sample, including the effect of any filters, lenses, integrating sphere, or other optical components.

The dependence of optical density on these parameters may be denoted as follows:

$$
D\left(g ; S: g^{\prime} ; S^{\prime}\right)
$$

In the general case, $g$ is the radiance as a function of $\theta, \phi, x$, and $y$,

$$
g=L_{\mathrm{e}}(\theta, \phi, x, y)
$$

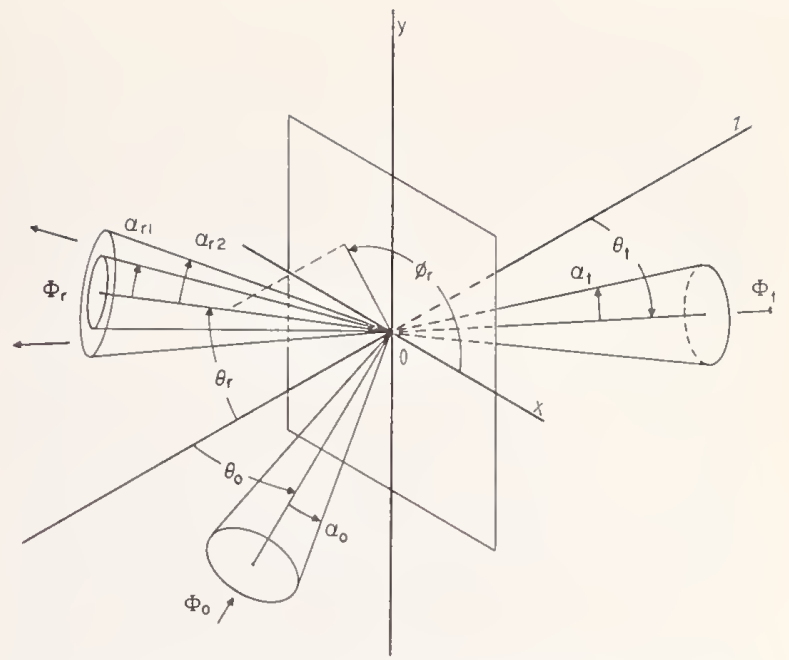

Fig. 2. Coordinate system for simplifled description of geametrical factors affecting optical modulation. $\alpha_{0}, \alpha_{t}, \alpha_{\mathrm{r}}=$ half cone angle of incident, transmitted and reflected pencils. $\theta_{0}, \theta_{\mathrm{t}}, \theta_{\mathrm{\tau}}=$ calotitude, also called ongle of incidence, transmission, or reflection (of pencil axis) with respect to the normal $\left(\theta_{0}\right.$ and $\theta_{\mathrm{r}}$ ore measured from the negative $z$ axis). $\phi_{0}, \phi_{\mathrm{t}}, \phi_{\mathrm{r}}=$ longitude of oxis of incident, transmitted, and reflected pencils.

$S$ is the radiance as a function of $\lambda$,

$$
S=L_{\mathrm{e}}(\lambda)
$$

$g^{\prime}$ is the sensitivity of the receiver as a function of $\theta, \phi, x$, and $y$,

$$
g^{\prime}=S_{\mathrm{e}}(\theta, \phi, x, y)
$$

and $S^{\prime}$ is the sensitivity of the receiver as a function of $\lambda$,

$$
S^{\prime}=S_{\mathrm{e}}(\lambda)
$$

In general, the description is rather complex. The description in general form is complex even for the simple conditions most often used in photographic instruments and adopted for measurements. Fortunately, by the adoption of certain conventions the description can be very greatly simplified in most cases.

A very large percentage of practical geometrical arrangements used in photography can be described adequately in terms of uniform pencils bounded by right circular cones. In projectors and enlargers (ideally considered), the projection lens utilizes flux uniformly within the conical solid angle it subtends at the point on the film on the optical axis. In enlargers with diffuse illumination, the point is regarded as being uniformly illuminated from all angles throughout the hemisphere. When condensers are used, they are designed to image the source on the projection lens so that all rays from the condenser pass through the projection lens. It follows that the rays within the cone subtended by the projection lens on axis at the film plane would come from within an influx cone subtending the same angle. The same conical conditions are found 
in many densitometers. For the description of these cases a special simplified form of notation is adopted.

The simplified notation is applicable if, for every point on the area of the sample under consideration, ${ }^{1}$ the angular distribution of incident radiance is adequately described as uniform at all angles within a right circular cone with its apex at the point but zero at all other angles and ${ }^{2}$ the angular distribution of the sensitivity of the receiver (including the effect of any filters, integrating sphere, or other optical components) is adequately described as uniform at all angles within a right circular cone with its apex at the point (or between two co-axial cones in the case of "annular collection") but zero at all other angles.

Then it is no longer necessary to treat the radiance $L_{\mathrm{e}}$ as a general function and the influx geometry may be described by three parameters, shown in Fig. $2 ; \alpha_{0}$, the half-angle of the incident cone, and $\theta_{0}$ and $\phi_{0}$, the direction angles of the axis of the cone:

$$
g=\alpha_{0}, \theta_{0}, \phi_{0}
$$

The efflux geometry may be described in a similar manner:

$$
\begin{aligned}
& g^{\prime}=\alpha_{\mathrm{r}}, \theta_{\mathrm{r}}, \phi_{\mathrm{r}} \quad \text { in the reflection case } \\
& g^{\prime}=\alpha_{\mathrm{t}}, \theta_{\mathrm{t}}, \phi_{\mathrm{t}} \quad \text { in the transmission case }
\end{aligned}
$$

The geometry of the simple cases may be specified by giving these angles in degrees. The angle $\alpha$ is less than $180^{\circ}$.

As it happens, in the great majority of applications in photography, the sample, is irradiated and "viewed" normally so $\theta_{0}=\theta_{\mathrm{r}}=\theta_{\mathrm{t}}=0$. If $\theta=0$, $\phi$ is indeterminate and, by convention, both can be omitted. Thus in most cases, the geometrical conditions may be specified adequately by one angle for the influx geometry and one for the efflux geometry. For transmission, the form would be $D_{T}\left(\alpha_{0} ; S: \alpha_{\mathrm{t}} ; S^{\prime}\right)$.

Two alternatives to the specification of $\alpha$ in degrees are useful, particularly in applications involving projectors or microdensitometers. If the sample is irradiated by an optical system focused on the sample and the efflux is received by an optical system focused on the sample, the influx and efflux geometries may be specified by the influx numerical aperture $A_{n}$ and the efflux numerical aperture $A^{\prime}{ }_{n}$, where

$$
\begin{aligned}
& A_{n}=n \sin \alpha_{0} \\
& A^{\prime}{ }_{n}=n^{\prime} \sin \alpha_{t}
\end{aligned}
$$

$n$ is the refractive index of the medium between the irradiating optics and the sample and $n^{\prime}$ is the refractive index of the medium between the sample and the receiver optics. For systems operating in air, $n=n^{\prime}=1$. To indicate that geometries are specified by numerical apertures rather than angles, the subscript $n$ is placed after the parenthetic statement of parameters, for example, $D_{T}(0.25$; $\left.2850^{\circ} \mathrm{K}: 0.40 ; \mathrm{S}-4\right)_{n}$. As another alternative, the influx and efflix geometries may be specified by the influx $f$-number $A_{f}$ and the efflux $f$-number $A^{\prime}$, where the $f$-numbers are those of the irradiating and receiving optics, but only when both optical systems are focused on the sample, the distance from the exit pupil of the irradiating optical system to the sample is nearly equal to the equivalent focal length of that system, and the distance from the sample to the entrance pupil of the receiver optical system is nearly equal to the equivalent focal length of that system. In the case of condenser optics, as used in projectors, where the condenser is designed to just fully irradiate the projection lens, $A_{f}=A^{\prime}{ }_{s}$. The requisite focal conditions are usually fulfilled for a system simulating a projector projecting a picture on a screen more than 25 focal lengths away from the lens. To indicate that geometries are specified by $f$-numbers rather than angles, the subscript $f$ is placed after the parenthetic statement of parameters, for example $D_{T}\left(3.5 ; 2850^{\circ} \mathrm{K}: 3.5, V\right)_{f}$.

Having simplified the description of geometric conditions in most practical cases, we turn to the spectral conditions and find that the same degree of simplification can be effected. The spectral distributions of many of the sources of interest in photography are satisfactorily represented by color temperature in degrees Kelvin. Others can be

TABLE IV. Notation for Parameters of Modulation

Influx function $C \quad$ : $\quad$ Efflux function $C^{\prime}$

Reference/ standard $s$

Influx geometry $g$; Influx spectrum $S$ : Efflux geometry $g^{\prime}$; Efflux spectral sensitivity $S^{\prime} /$ standard $s^{\prime}$

$$
L_{\mathrm{e}}(\theta, \phi) \quad ; \quad \mathrm{L}_{\mathrm{e}}(\lambda) \quad: \quad S_{\mathrm{e}}(\theta, \phi) \quad S_{\mathrm{e}}(\lambda) \quad / \quad s
$$

Simplified notation when the angular distribution of irradiation and collection are uniform within right circular cones, zero elsewhere, and uniform over the sample (shown for the case of reflection):

$$
\alpha_{0}, \theta_{0}, \phi_{0} \quad ; \quad S \quad \alpha_{\mathrm{r}}, \theta_{\mathrm{r}}, \phi_{\mathrm{r}} \quad ; \quad S^{\prime} \quad s
$$

Simplified notation when above conditions are met and $\theta_{0}=\theta_{\mathrm{r}}=0$.

$$
\begin{array}{llllllll}
\alpha_{0} & ; & S & \alpha_{\mathrm{r}} & ; & S^{\prime} & s
\end{array}
$$

For annular irradiation, when above conditions are met: 
specified by symbols adopted by standardizing organizations for certain standardized sources, such as CIE-C, or phosphors, such as P-11. If the influx spectrum is a very narrow band of frequencies, it may be specified by the wavelength, in a vacuum, preferably in nanometers (abbreviated $\mathrm{nm}$ and formerly called millimicrons).

Similarly, the efflux spectral sensitivity can be denoted by the symbols for standardized spectral sensitivity functions such as $V$ for visual, $P$ for the standard sensitivity representing commonly used photographic printing materials, S-4 (for example) for a particular photocell response, or when appropriate, a wavelength in nanometers.

To indicate that a measurement was made relative to a reference standard, it is proposed that a symbol for the reference standard, $s$, be included at the end of the list of parameters and be separated from the efflux parameters by a diagonal, as follows:

$$
D\left(C: C^{\prime} / s\right)
$$

The most common reference standards used in photography are magnesium oxide, base, and base plus fog. These may be given the symbols $\mathrm{MgO}$, $b$, and $b f$, respectively. Other references may be assigned symbols or the general symbol $s$ may be used when appropriate. The functional notation in the general and simplified forms can be used to specify the parameters of any of the arithmetic measures of modulation, as well as the various kinds of optical density, as follows:

$$
\begin{array}{r}
\tau\left(C: C^{\prime}\right), \rho\left(C: C^{\prime}\right), \psi\left(C: C^{\prime}\right), T\left(C: C^{\prime}\right), R\left(C: C^{\prime}\right), \\
\text { and } P\left(C: C^{\prime}\right)
\end{array}
$$

The general features of the system of notation and the various simplifications are shown in Table IV.

In some standard systems of measurements, flux is incident uniformly in the annular region between two co-axial cones or the efflux is uniformly evaluated between two such cones. In such cases the notation would be as shown in these examples for the reflection case:

$$
D_{R}\left(\alpha_{01} \text { to } \alpha_{02} ; S: \alpha_{\mathrm{r}} ; S^{\prime}\right)
$$

or

$$
D_{R}\left(\alpha_{0} ; S: \quad \alpha_{\mathrm{r} 1} \text { to } \alpha_{\mathrm{r} 2} ; S^{\prime}\right)
$$

When an angular subtense is broken into two or more discrete regions it may be described by the use of the "plus" sign, for example,

$$
\alpha_{01} \text { to } \alpha_{02}+\alpha_{03} \text { to } \alpha_{04}
$$

Similarly one may indicate a region deleted by the use of the "minus" sign, for example,

$$
0 \text { to } 360^{\circ}-170^{\circ} \text { to } 190^{\circ}
$$

It should be noted that the punctuation used in the notation has been formalized in the interest of clarity. The influx function and efflux function are separated by a colon. The geometrical and spectral functions are separated by semicolons. The angular paraneters of the geometrical functions are separated by commas.

If a second coordinate system for efflux description is established at a distance $b$ along the $z$ axis, as discussed in the section dealing with the coordinate system, the distance $b$, with proper unit of length, may be indicated between two colons and this group of symbols may replace the usual single colon, as follows:

$$
D_{T}\left(\alpha_{0} ; S: \quad b: \alpha_{\mathrm{t}} ; S^{\prime}\right) .
$$

\section{Examples of Functional Notation}

The current American standard for diffuse transmission density defines two types. American standard diffuse visual density, Type Vl-b would be written in the proposed notation as follows:

$$
D_{T}\left(90^{\circ} ; 3000^{\circ} \mathrm{K}: \leq 10^{\circ} ; V\right)
$$

This notation means that the incident flux is uniformly distributed at all angles over the hemisphere and has the spectral distribution of a full radiator at $3000^{\circ} \mathrm{K}$ and that the flux is evaluated uniformly in a cone of half-angle less than or equal to $10^{\circ}$ with its axis on the normal, by a receiver having a response proportional to the standard photopic spectral luminous efficiency function. The second type, American Standard Diffuse Printing Density, Type P2-b would be written in the proposed notation as follows:

$$
D_{T}\left(90^{\circ} ; 3000^{\circ} \mathrm{K}: \leq 10^{\circ} ; P\right)
$$

One can see at a glance that the only difference from the first type is that the spectral sensitivity of the receiver is that of a standard photographic print paper. The current American Standard Visual Diffuse Reflection Density, Type $3000 \mathrm{~K}$, would be written:

$$
D_{R}\left(40^{\circ} \text { to } 50^{\circ} ; 3000^{\circ} \mathrm{K}: 5^{\circ} ; \mathrm{V} / \mathrm{MgO}\right)
$$

which means that the incident flux is uniformly distributed at all longitudes at colatitudes from $40^{\circ}$ to $50^{\circ}$ and has the spectral distribution of a full radiator at $3000^{\circ} \mathrm{K}$; that the flux is uniformly evaluated at all longitudes at colatitudes from 0 to $5^{\circ}$ by a receiver having a response proportional to the standard photopic spectral luminous efficiency function; and that the measurement is made relative to a magnesium oxide reference standard.

A microdensitometer might measure $D_{T}(0.25$; $\left.2850^{\circ} \mathrm{K}: 0.40 ; \mathrm{S}-4\right)_{n}$. The illuminating optical system has a numerical aperture of 0.25 , the source is characterized by a color temperature of $2850^{\circ} \mathrm{K}$, the receiver optical system has a numerical aperture of 0.40 , and the receiver has the spectral sensitivity of a type S-4 photodetector.

A projector with a projection lens having an $f$ number of 3.5 might utilize $D_{T}\left(3.5 ; 2850^{\circ} \mathrm{K}: 3.5\right.$; $V)_{s}$.

In some studies in photography the primary interest is in the effect of geometry on modulation measurements while the spectral conditions are of 
little interest. In such cases, the influx spectrum and efflux spectral sensitivity can be omitted from the notation. The general form of the notation then becomes simply $D\left(g: g^{\prime}\right)$.

For example, the three geometrically extreme cases in transmission densitometry mentioned in American Standards can be written as shown in Table V. It should be noted that the notation provides a means of differentiating the two types of diffuse density measurement generally regarded as equivalent.

In the proposed notation, the Callier " $Q$ " factor is defined by the equation

$$
Q=D_{T}(0: 0) / D_{T}(1.00: 0)_{n}
$$

The $q$ introduced by Altman ${ }^{18}$ may be written as follows:

$$
q=D_{T}\left(g: g^{\prime}\right) / D_{T}(1.00: 0)_{n}
$$

He used the symmetrical case, $g=g^{\prime}$, that is, $\alpha_{0}=$ $\alpha_{\mathrm{t}}$ and $\theta_{0}=\theta_{\mathrm{t}}=0$. I have found this ratio useful, permitting $g$ and $g^{\prime}$ to take on different values.

\section{Discussion}

Present day concepts in densitometry are largely based on the excellent analysis of experimental determination of photographic density by Koerner and Tuttle, thirty years ago. ${ }^{19}$ There is, however, a basic difference between their point of view and that adopted here. They said:

"From the definition it is apparent that, in order to measure density, it is necessary only to determine the flux incident on, and the flux transmitted by, the silver deposit. Since there are no qualifying statements attached to the above definition, it would seem quite beside the point to inquire what portion of the fluxes should be measured; one naturally supposes that all the incident and all the transmitted flux is meant. Any other interpretation would add an equivocal quality to the term "photographic density" as defining a basic physical quantity, and although it is true that Hurter and Driffield were not aware of certain properties of the photographic image, particularly the light-scattering properties, at the time the definition was formulated, it is also probably true that, had they appreciated these factors, their definition would still have remained as it now is."

No one kind of optical density is any more a "basic physical quantity" than any other. Indeed, it is the very "equivocal quality" of "photographic density" which underlies most of the problems of measurement and application. The first step in standardization was a natural one, the adoption of

18. J. H. Altman, Appl. Opt., 3: 35 (1964).

19. A. M. Koerner and C. Tuttle, J. Opt. Soc. Am., 27: 241 (1937), the proposal of Koerner and Tuttle, "that the integrating sphere used with an incident light, no ray of which departs from normal incidence by more than $10^{\circ}$, shall be considered as the standard optical set-up for determination of photographic density according to both letter and spirit of the Hurter and Driffield definition." There was, of course, a very great advantage to photographic science in having one standard density well defined rather than none. However, the most important justification for continuing diffuse geometry in existing standards is not that it satisfies the definition of Hurter and Driffield and is essentially unaffected by small variations in sample thickness but that it simulates the conditions of use in contact printing and in viewing transparencies against a diffuse source and that these are important applications. If the quantities to be measured are the quantities to be used, the generalized concept of density which has come into use and is here systematized, must be recognized and adopted. There is, however, some danger of "undoing standardization," in this point of view. If every kind of density were "standard" we would have no standardization other than, perhaps, a standard notation for describing the density. The reasons adduced by Koerner and Tuttle and the need for continuity are sufficient to warrant the continued use of diffuse density as the kind to be used when there is no reason to use another. Then, it seems imperative in the interest of standardization to choose and standardize representative parameters for other types of density for which there is a real need. Experience in science and industry has demonstrated a need for a projection density, a diffusesource contact-printing density, and a reflection density which can be used to characterize photographic images on surfaces such as brushed metals, which have a considerable variation in reflection density, $D_{R}\left(5^{\circ}, 45^{\circ}, 0^{\circ} ; S: 5^{\circ}, 45^{\circ}, 180^{\circ} ; S^{\prime}\right)$, as the sample is rotated about the $z$ axis.

When the task of revising existing density standards was undertaken, it was clear that several new kinds of density would be needed but the prospect of adding to the plethora of specialized terminology, which for years has been the curse of photometry, was not inviting. The functional notation is a convenient form of expression for the values of the parameters affecting the measurement of optical modulation. It is to be hoped that the infinite flexibility of this mathematical notation will obviate the need for more words to denote the various types

TABLE V. Notation for Transmission Density with Extreme Geometrical Conditions

\begin{tabular}{lc}
\hline Name of type & Simplified notation \\
\hline Regular $^{a}$ & $D_{T}(0: 0)$ \\
Diffuse & $D_{T}\left(0: 90^{\circ}\right)$ or $D_{T}\left(90^{\circ}: 0\right)$ \\
Doubly diffuse & $D_{T}\left(90^{\circ}: 90^{\circ}\right)$ \\
\hline
\end{tabular}

"Sometimes called specular although many regard this term as a direct reference to a mirror and therefore inappropriate to any case other than reflection. 
other than such terms as for example, "projection density" for a type of density defined in such a way that the measurement conditions simulate the conditions in a typical or "average" projector. The adoption of the present proposals should more than satisfy the requirement stated in $N_{c}$ ees and James, "It is desirable that standard terms be adopted which would serve to differentiate the limiting true specular density from densities lying between this and totally diffuse density."

New terms are proposed only because calibration and standardization activities have demonstrated a real need for some such terms. Prescriptive definitions and arbitrary systems of notation cannot be wrong, of course, but they can be good or bad. I hope these will be found useful.

20. The Theory of the Photographic Process, C. E. K. Mees and T. H. James, eds., Macmillan Co., N. Y. 3rd ed. (1966) p. 426.
The concepts of propagance factor, transmittance factor, and reflectance factor should appeal to the systems engineer. This "systems approach" provides solutions to a number of modulation problems which would baffle a densitometrist clinging faithfully to the definitions of transmittance and reflectance. The author did not invent these concepts but recognized them in use, though they were ill defined. Mr. L. Barbrow suggested the use of the suffix term "factor."

\section{Acknowledgment}

I am grateful to Mr. L. Barbrow and Dr. Deane B. Judd for the definitions of terms adopted or under consideration by international committees and to the members of the ASA subcommittee on densitometry, who encouraged the development of these proposals and authorized the publication of the fact that they are being considered. 


\section{The Metric System in Illuminating Engineering}

Reprinted from ILLUMINATING ENGINEERING

Vol. LXII, No. 11, November 1967

\author{
By L. E. Barbrow
}

AT SOME TIME, possibly not far in the future, the British system of units will be abandoned in the United States, in favor of the metric system which should more properly be called the International System of Units (SI) as designated in 1960 by the Eleventh General Conference on Weights and Measures (see Appendix). The Illuminating Engineering Society has requested its Nomenclature Committee to consider this matter and to present, for study and discussion, recommendations on two questions: (1) adoption by IES of the policy that primary emphasis in the publications of the Society be given to SI units to supersede the footcandle, footlambert, and candela per square inch, the units most commonly used by illuminating engineers in the United States and (2) "preferred" prefixes for SI units when the SI units are not of appropriate size.

As to question (1), because the SI units are already used exclusively in nearly all of the countries of the world and will now be receiving increased use in the English-speaking countries, and because of the almost exclusive use of the SI units in the scientific field and the need for uniformity of units used in science and engineering, the IES Nomenclature Committee unanimously recommends that the IES approve the policy of having the SI units given primary use in its publications, with equivalent values in the British, system given in parentheses if desired. The Nomenclature Committee suggests early approval of this policy.

In a discussion of question (2) the relationships with which we are concerned are:

$1 \mathrm{fc}=10.76 \mathrm{lux}=1.076$ dekalux $=0.1076$

hectolux $=1 \mathrm{~lm} / \mathrm{ft}^{2}$.

$1 \mathrm{~lx}=1 \mathrm{~lm} / \mathrm{m}^{2}$.

$1 \mathrm{fL}=10.76$ apostilb $=1.076$ dekapostilb $=$

0.1076 hectoapostilb $=1 \mathrm{~cd} / \pi \mathrm{ft}^{2}$.

I $\mathrm{asb}=1 \mathrm{~cd} / \pi \mathrm{m}^{2}$.

$1 \mathrm{~cd} / \mathrm{in}^{2}=1550$ nit $=1.55$ kilonit.

$1 \mathrm{nt}=1 \mathrm{~cd} / \mathrm{m}^{2}$.

$1 \mathrm{fL}=3.426$ nit.

Because one footcandle is equal to 1.076 dekalux, the use of dekalux would be advantageous in terms of

AUTHOR: Chairman. JES Nomenclature Committee, National Bureau of Standards, Washington. D. C. Note: Only those recommendations so marked and indicated by italics, received unanimous approval of the JES Nomenclature Committee and are submitted at this time for approval of the IES Council. continuity with the past insofar as literature and recommended practices are concerned. Also the scales of the multiude of footcandle meters now in use could continue to be used by adding a 7.6 per cent correction to the reading to take into account the difference in size of these two units. On the other hand it must be noted that in the course of the extensive use abroad of the SI units over a period of many years, the lux without any prefix has been used almost without exception as the unit of illumination. The Nomenclature Committee is almost equally divided on the question of giving preference to lux or dekalux as the unit of illumination. Perhaps no firm choice should or even needs to be made now between lux and any of its multiples; experience gained by use of the SI units may reveal whether a choice in the future will be advisable.

As for the two currently used units of luminance ( $\mathrm{fL}$ and $\mathrm{cd} / \mathrm{in}^{2}$ ), because luminance is a directional quantity, the Nomenclature Committee is unanimous in recommending that the IES should promote the deprecation of "lumens per unit area" as a unit of luminance, and sponsor its use solely as a unit of luminous exitance (formerly emittance).

The kilonit (knt) which equals about $0.65 \mathrm{~cd} / \mathrm{in}^{2}$ is a conveniently sized unit that might well be used to describe or specify the luminance of sources, while the nit (about $0.3 \mathrm{fL}$ ) might well be used to report the luminance of surrounds.

The metric units lux and nit have been adopted by the CIE but only one of these two units, lux, has been adopted by the General Conference on Weights and Measures while the other term, nit, has never received much use. On this account, and also because of the connotation of the word in English, there has developed some sentiment for avoiding the use of the name "nit" and for using instead the self-explanatory name "candela per square meter" which is the SI unit. This is too long a name for a commonly used unit, so the majority of the Nomenclature Committee recommends the use of "nit" and decimal multiples thereof for expressing values of luminance. There is, however, a minority view that "candela per square meter," the SI unit, be preferred. A suggested interesting solution to this dilemma is the use of "SI unit," which would readily be recognized as being equivalent to "candela per square meter." Here, as in the choice of a preferred unit for illumination, perhaps experience 
gained by use of the SI units may reveal whether the choice of a preferred luminance unit is necessary.

A matter of concern to some illuminating engineers in deprecating "lumens per unit area" as a unit of luminance is the loss of the easily remembered (but often erroneously applied) relationship: $L=\rho E$, in which $L$ is luminance in footlamberts, $E$ is illumination in footcandles and $\rho$ is hemispherical reflectance for the particular incidence geometry. Of course this relationship applies only to a Lambertian (perfectly diffusing) surface. For such a surface the relationship when the recommended metric units are used is: $L=\rho E / \pi$, in which $L$ is luminance in nits, $E$ is illumination in lux, and $\rho$ is hemispherical reflectance. It can be at least hoped that the presence of $\pi$ in this equation will remind illuminating engineers that this relationship is applicable only to the extent that the surface illuminated approximates a Lambertian sur. face. For a non-Lambertian surface the relationship that should be used is: $L=\beta E / \pi$, in which $L$ is luminance in nits, $E$ is illumination in lux and $\beta$ is the luminance factor of the surface. Luminance factor, $\beta$, is not defined in Z7.1-1942 but will be defined as follows in 27.1-1967 which has been approved as a USA Standard but is not yet published: "Luminance Factor, $\beta$ : Luminance factor is the ratio of the luminance (photometric brightness) of a surface or medium under specified conditions of incidence, observation, and light source, to the luminance (photometric brightness) of a perfectly reflecting or trans- mitting, perfectly diffusing surface or medium under the same conditions.

"Note: Reflectance or transmittance cannot exceed unity, but luminance factor may have any value from zero to values approaching infinity."

For your further information, the accompanying material of particular interest to illuminating engineers is being considered for incorporation in a forthcoming USA Standard Letter Symbols for General Use.

\begin{tabular}{rll}
\multicolumn{3}{c}{ Prefixes } \\
Factor by which the unit \\
is multiplied $\quad$ Prefix & Symbol \\
$1,000,000,000,000$ & $=10^{12}$ tera & $\mathrm{T}$ \\
$1,000,000,000$ & $=10^{9}$ giga & $\mathrm{G}$ \\
$1,000,000$ & $=10^{6}$ mega & $\mathrm{M}$ \\
1,000 & $=10^{3}$ kilo & $\mathrm{k}$ \\
100 & $=10^{2}$ hecto & $\mathrm{h}$ \\
10 & $=10^{1}$ deka & $\mathrm{da}$ \\
0.1 & $=10^{-1}$ deci & $\mathrm{d}$ \\
0.01 & $=10^{-2}$ centi & $\mathrm{c}$ \\
0.001 & $=10^{-3}$ milli & $\mathrm{m}$ \\
$0.000,001$ & $=10^{-6}$ micro & $\mu$ \\
$0.000,000,001$ & $=10^{-9}$ nano & $\mathrm{n}$ \\
$0.000,000,000,001$ & $=10^{-12}$ pico & $\mathrm{p}$ \\
$(0.000,000,000,000,001$ & $=10^{-18}$ atto & $\mathrm{a},)^{* *}$ \\
$(0.000,000,000,000,000,001$ & $=10^{-18}$ femto & $\mathrm{f},)^{* *}$
\end{tabular}

"Translator's note: This prefix is spelled "déca" in French. **Added in 1964 .

\begin{tabular}{|c|c|c|}
\hline Unit & Symbol & Notes \\
\hline lux & Ix & The SI unit of illuminance. One $1 \mathrm{x}=$ one $\mathrm{Im} / \mathrm{m}^{2}$. \\
\hline phot & $\mathrm{ph}$ & $\begin{array}{l}\text { The CGS unit of illuminance. One ph }=\text { one } 1 \mathrm{~m} / \mathrm{cm}^{2} \text {. Use of the SI unit, } \\
\text { the lux (lumen per square meter), is preferred. }\end{array}$ \\
\hline footcandle & fc & $\begin{array}{l}\text { A British unit of illuminance. One } \mathrm{fc}=\text { one } \mathrm{Im} / \mathrm{ft}^{2} \text {. The name lumen per } \\
\text { square foot is preferred for this unit. }\end{array}$ \\
\hline nit & nt & $\begin{array}{l}\text { The name sometimes given to the SI unit of luminance (candela per square } \\
\text { meter). One } \mathrm{nt}=\text { one } \mathrm{cd} / \mathrm{m}^{2} \text {. }\end{array}$ \\
\hline apostilb & asb & $\begin{array}{l}\text { A unit of luminance in the metric system. One asb }=\text { one } \mathrm{cd} / \pi \mathrm{m}^{2} \text {. One } \\
\mathrm{Im} / \mathrm{m}^{2} \text { leaves a surface whose luminance is one apostilb in all directions } \\
\text { within a hemisphere. If luminance is measured in metric units, the can- } \\
\text { dela per square meter is preferred. }\end{array}$ \\
\hline stilb & sb & $\begin{array}{l}\text { A CGS unit of luminance. One } s b=\text { one } \mathrm{cd} / \mathrm{cm}^{2} \text {. Use of the SI unit, the } \\
\text { candela per square meter, is preferred. }\end{array}$ \\
\hline lambert & $\mathbf{L}$ & $\begin{array}{l}\text { A unit of luminance in the CGS system. One } L=\text { one } \mathrm{cd} / \pi \mathrm{cm}^{2} \text {. One } \\
\mathrm{Im} / \mathrm{cm}^{2} \text { leaves a surface whose luminance is one lambert in all directions } \\
\text { within a hemisphere. If luminance is measured in CGS units, the stilb } \\
(\mathrm{sb}) \text { is preferred. }\end{array}$ \\
\hline footlambert & $\mathrm{fL}$ & $\begin{array}{l}\text { A British unit of luminance. One } \mathrm{fL}=\text { one } \mathrm{cd} / \pi \mathrm{ft}^{2} \text {. One } 1 \mathrm{~m} / \mathrm{ft}^{2} \text { leaves a } \\
\text { surface whose luminance is one footlambert in all directions within a } \\
\text { hemisphere. If luminance is measured in British units, the candela per } \\
\text { square foot }\left(\mathrm{cd} / \mathrm{ft}^{2}\right) \text { is preferred. }\end{array}$ \\
\hline
\end{tabular}




\section{APPENDIX}

Translation from French of Records of Meetings of the Eleventh General Conference on Weights and Measures, Paris, October 11-20, 1960.

\section{International System of Units Resolution No. 12}

The Eleventh General Conference on Weights and Measures, Considering:

Resolution No. 6 of the Tenth General Conference on Weights and Measures by which six units have been adopted which are intended to serve as a practical base for a system of measures for international purposes:

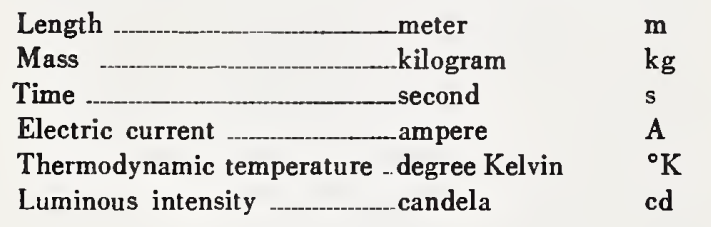

Resolution No. 3 adopted by the International Com. mittee on Weights and Measures in 1956.

The recommendations adopted by the International Committee on Weights and Measures in 1958 concern. ing the symbols and prefixes to be used in this system for the formation of multiples and submultiples of the units, Decides:

1. The system based on the six basic units above mentioned is referred to as the International System of Units.

2. The international abbreviation of the name of this system is: SI.

3. The names of multiples and submultiples of the units are formed with the prefixes shown in the accompanying table.

4. The units shown below will be used in this system, without prejudice to other units which could be added in the future:

\section{SUPPLEMENTARY Units}

$\begin{array}{ll}\text { Plane angle } & \text { rad } \\ \text { Solid angle } & \text { sradian }\end{array}$

\section{DeRIVEd UnITS}

Area

Volume

Frequency

Density

Velocity

Angular velocity

Acceleration

Angular acceleration

Force

Pressure

Kinematic viscosity

Dynamic viscosity

Work, energy, quantity of heat

Power

Electric charge

Voltage, potential difference,

electromotive force

Electric field strength

Electric resistance

Electric capacitance

Magnetic flux

Inductance

Magnetic flux density

Magnetic field strength

Magnetomotive force

Flux of light.

Luminance

Illumination square meter

cubic meter

hertz

kilogram per cubic meter

meter per second

radian per second

meter per second squared

radian per second squared

newton

newton per square meter

square meter per second

newton-second per square meter

joule

watt

coulomb

volt

volt per meter

ohm

farad

weber

henry

tesla

ampere per meter

ampere

lumen

candela per square meter

lux $\mathrm{m}^{2}$

$\mathrm{m}^{3}$

$\mathrm{Hz}$

$\mathrm{kg} / \mathrm{m}^{3}$

$\mathrm{m} / \mathrm{s}$

$\mathrm{rad} / \mathrm{s}$

$\mathrm{m} / \mathrm{s}^{2}$

$\mathrm{rad} / \mathrm{s}^{2}$

$\mathrm{N}$

$\mathrm{N} / \mathrm{m}^{2}$

$\mathrm{m}^{2} / \mathrm{s}$

$\mathrm{N} \cdot \mathrm{s} / \mathrm{m}^{2}$

$\mathrm{J} \quad \mathrm{N} \cdot \mathrm{m}$

W

C

$\mathrm{J} / \mathrm{s}$

A.s

V

$\mathrm{V} / \mathrm{m}$

$\Omega \quad$ V/A

F $\quad A \cdot S / V$

Wb V.s

$\mathrm{H} \quad$ V.s/A

$\mathrm{T} \quad \mathrm{Wb} / \mathrm{m}^{2}$

$\mathrm{A} / \mathrm{m}$

A

Im

$\mathrm{cd} / \mathrm{m}^{2}$

lx

$\operatorname{lm} / \mathrm{m}^{2}$ 


\title{
Optical Resource Letter on Radiometry
}

\author{
FRED E. NICODEMUS \\ Sylvania Electronic Systems-Western Division, Mountain View, California 94040
}

\begin{abstract}
These Optical Resource Letters are patterned after the well-established Resource Letters published in the American Journal of Physics and distributed by the American Association of Physics Teachers. The purpose is to provide, in an important field of optics, briefly annotated references which should be of value to teachers, researchers, engineers and technicians interested in the subject area. No claim is made for completeness or exhaustiveness of coverage; rather, it is hoped that the references quoted will be helpful in themselves, and will be representative, or at least suggestive, of the broad literature on this subject.

The present Optical Resource Letter, OR-2, and that published last month, OC-1 on Colorimetry, were prepared under the direction of the OSA Ad Hoc Committee on Resource Letters: K. M. Baird, H. E. Breed, M. A. Jeppesen, J. M. Stone, and S. S. Ballard (Chairman).

Notation: The letter $\mathrm{E}$ following an item number indicates that the reference is on a more-or-less elementary level; the letter A indicates an advanced level.

Additional copies are available from the Executive Office of the Optical Society of America, 2100 Pennsylvania Avenue, N. W., Washington, D. C. 20037. Request Optical Resource Letter OR-2, and please enclose a stamped, return-addressed envelope.
\end{abstract}

Radiometry is seldom taught or pursued by and for itself; usually this is done in connection with some other subject (e.g., astrophysics or meteorology) or attention is confined to just one of its major subdivisions (e.g., photometry, illumination engineering, or optical pyrometry). Those who conduct radiometric studies and publish their results tend to group themselves according to these categories, usually with poor communication between different groups. Each group tends to publish in its own journals and to develop its own scheme of concepts, terminology, symbols, sacred cows, and jargon, and thus further discourages intergroup communication.

In these circumstances, not only is the disclaimer of completeness for this resource letter particularly apt, but it is also extremely difficult, if not impossible, even to achieve representative coverage of the field or, in fact, to determine what is truly representative of radiometry as a whole. Accordingly, the first person is used occasionally, as a reminder that it is radiometry as seen through the author's eyes that is being presented.

Radiometry is that branch of optics concerned with the measurement of the radiant energy or power flowing in beams of optical electromagnetic radiation including, primarily, incoherent ultraviolet, visible, and infrared radiation, as well as longer or shorter wavelengths in circumstances wherein the laws of geometrical optics apply. With the rapidly increasing interest in coherent radiation, resulting from the explosive development of laser technology, there is growing need for more adequate treatment of the problems of measuring radiant power in coherent beams as well, in which its distribution is strongly affected by the phase relations that give rise to interference. Radiometric relations based on the classical geometrical optics of the literature cited here should not be applied blindly to situations involving coherent radiation. ${ }^{1}$

The largest subdivision of radiometry includes photometry and illumination engineering, wherein radiant energy is measured in terms of its effect on the average or "standard" human eye (spectral luminous efficacy of radiant flux $K(\lambda)$, formerly called "luminosity factor"). In optical pyrometry, radiant energy is measured to determine temperatures. Radiometry plays a major role in the radiant-heat-transfer area of mechanical and chemical engineering. Meteorologists and astrophysicists are also interested in radiative heat transfer, but from a somewhat different standpoint. Radiant-heat-

${ }^{1}$ A. Walther, "Radiometry and Coherence," J. Opt. Soc. Am. $58,1256(1968)$. 
transfer problems are increasing in importance and are encountering new conditions in connection with spacecraft temperature control. Ceramic engineers are greatly concerned with radiometric considerations, not only in developing materials for space applications but also in mundane areas such as the cooling of glass sheets during their manufacture. Ophthalmologists and optometrists, as well as optical-instrument designers and manufacturers, are also concerned with radiometric relations and measurements. Radiometry occupies a large place in the growing military applications of electro-optical devices, especially in the infrared. Meteorologists and climatologists, foresters, and other biologists concerned with the effects of radiant energy, particularly solar radiant energy, on plants and animals make extensive use of radiometric relations and measurements. Radiometric instruments are used in medicine, for example, to detect and map anomalous patterns of skin temperature associated with diseased conditions, particularly circulatory difficulties. They are also used in industrial inspection and control, e.g., to detect defective circuits and components, and poor layout and design of electronic assemblies, by the infrared radiation resulting from undue heating. This listing is far from exhaustive.

I have collected a working list of over 1000 references, primarily in connection with a continuing effort to improve and expand a preliminary general treatment of radiometric calibration. ${ }^{2}$ These references have been gathered partly through subject searches in Science Abstracts, Engineering Index, etc., but mainly on the basis of citations encountered as particular problems have been explored from time to time; these, in turn, have led to others, and so on. I am convinced that a really systematic and exhaustive collection effort would turn up many more pertinent items. For this resource letter, only a few of the most important basic references - those having the widest application-have been selected.

\section{GENERAL TREATMENTS}

There seems to be no completely general treatment of radiometry, as such. There are good optics texts that cover many fundamentals, but most of them do not devote much attention to radiometric considerations. Then there are treatments of radiometry which manage to achieve enough generality to be useful as basic references even though they are primarily concerned with certain aspects, such as infrared physics, rather than with radiometry as a whole.

1E. Measurement of Optical Radiations-The Detection of Electromagnetic Radiation from Ultraviolet to Infrared. Georg Bauer (The Focal Press, London and New York, 1965). Translation by K. S. Ankersmit of Strahlungs-

2 "Methods of Radiometric Calibration," Fred E. Nicodemus and George J. Zissis. (The University of Michigan, Contract No. SD-91 with the Advanced Research Projects Agency, Report 4613-20-R, October 1962) (Defense Documentation Center No. AD-289,375). messung im optischen Spektralbereich (Friedr. Vieweg und Sohn Verlag, Braunschweig, 1962). This probably comes as close as any to being a good introductory treatment of radiometry in general. In a good review E. J. Gilham deplores Bauer's bias toward principles rather than practice in such an introductory volume. However, this is probably unavoidable in any general treatment and I welcome it, particularly since it can easily be supplemented by good accounts of actual measurement practices in reports of specific experiments and studies. It is unfortunate that there are some shortcomings in the translation, but some of the same difficulties of inadequate or ambiguous terminology are also frequently encountered in texts written directly in English, so the reader may as well learn from the outset to cope with them (see discussion of nomenclature, below). There are also citations of sources for more extensive and intensive treatments of particular topics, but many of them are in German. [Reviewed by: Gilham, Brit. J. Appl. Phys. 16, 1407 (1965); MacAdam, J. Opt. Soc. Am. 55, 898 (1965).]

2E. Measurement of Radiant Energy. W. E. Forsythe, Ed. (McGraw-Hill Book Co., New York, 1937). This, too, is a general treatment of radiometry, as its title indicates. Unfortunately, it is somewhat out of date. However, it is still a good reference on many topics and certainly is an excellent summary as of 1937 , as well as being almost the only reference in which all of the topics treated can be found together in a single volume.

3E. Handbook of Military Infrared Technology. William L. Wolfe, Ed. (Office of Naval Research, Washington, D. C., 1965, Supt. of Doc., U. S. G. P. O. Washington, D. C. 20402-\$3.75). 906 pp. While its emphasis is on the infrared region of the spectrum and on military applications, this reference volume contains much useful information on radiometric fundamentals; also, the coverage is complete enough in many places so that, to some extent, it can serve as a text as well as a reference volume. At the price, it is a real bargain. [Reviewed by: Howard, Appl. Opt. 6, 2200 (1967); MacAdam, J. Opt. Soc. Am. 57, 988 (1967).]

4E. Applied Optics and Optical Engineering. Vol.IV, R. Kingslake, Ed. (Academic Press Inc., New York, 1967). (Vols. I and II, 1965; Vol. III, 1966; Vol. V is in preparation). Chapter 8 , p. 263 , in Vol. IV presents a general treatment of radiometry in very limited space. Fortunately, some topics such as instrumentation and atmospheric phenomena are covered in other chapters of the same treatise. The chapter is organized around an examination of reasons why the precision and accuracy achievable in radiometry are so poor, in contrast to those attained in most other types of physical measurements. I have since become increasingly aware of the existence of many more significant publications in addition to those I consulted in writing that chapter. A good example is Gershun [5]. [Reviewed by: Ballard, Appl. Opt. 7, 252 (1968); Hills, J. Opt. Soc. An. 58, 591 (1968). 7 
5A. The Light Field. A. Gershun. English translation by Parry Moon and Gregory Timoshenko. J. Math. Phys. 18, 51 (1939). (MIT, Elect. Eng. Dept. Contribution No. 164, 1939). This is a vector treatment of the geometrical relations of radiometry. A study of portions of it reveals it to be elegant and complete. It treats radiometric quantities as field quantities, as I have long felt they should be treated, and clearly states the invariance properties of radiance, pointing out their power and usefulness. ${ }^{3}$ Although photometric terminology is used throughout, it is clearly stated at the outset that the geometrical relations apply equally to all radiometry, regardless of the units in which radiant power is measured, so that radiometric quantities may be substituted everywhere for the corresponding photometric quantities. It seems surprising that this publication is not more widely known and recognized.

6A. "Temperature Radiation of Solids." G. A. W. Rutgers, in Handbuch der Physik, Vol. 26, S. Flügge, Ed. (Springer-Verlag, Berlin, 1958), p. 129. As indicated by the title, this treatment does not cover directly certain important phases of radiometry, such as radiation from gases. However, it is an authoritative presentation of much material that is basic to all radiometry.

7E. Light. R. W. Ditchburn (Interscience Publishers, John Wiley and Sons, Inc., New York, 1963), 2nd ed. This comprehensive treatise emphasizes physical optics. It includes a good, though brief, discussion of much basic material on radiometry, particularly in $\mathrm{Ch}$. X, "Detection and Measurement of Radiation." [Reviewed by: Davis, Appl. Opt. 3, 14 (1964); MacAdam, J. Opt. Soc. Am. 55, 898 (1965).]

8A. The Theory of Optics. Paul Drude. Translated from the German by C. Riborg Mann and Robert A. Millikan (1902). (Dover Publications, Inc., New York, 1959). (Preface to German edition is dated January 1900). Although not modern, this classic stands up well. It contains much useful material on basic principles of radiometry, especially in Part III, "Radiation." In particular, Kirchhoff's law relating emissivity and absorptance is correctly stated in terms of the directional quantities, and the implication which follows concerning polarization of oblique emitted rays is clearly pointed out (something which, strangely, has been overlooked in recent texts). ${ }^{4}$

9A. The Detection and Measurement of Infrared Radiation. R. A. Smith, F. E. Jones, and R. P. Chasmar. (The Clarendon Press, Oxford, 1968), 2nd ed. Fundamentals are well presented with primary attention to the infrared, especially the properties of detectors for that spectral region. [1st ed. (1957) reviewed by: Gates, J. Opt. Soc. Am. 48, 198 (1958).]

10E. Proceedings IRE, Vol. 47, No. 9, September 1959-Infrared Issue. This special issue was prepared

${ }^{3}$ This is my message in "Radiance." Fred E. Nicodemus, Am. J. Phys. 31, 368 (1963).

"Oscar Sandus, "A Review of Emission Polarization," Appl. Opt. 4, 1634 (1965). primarily by workers in military applications of infrared, under the general supervision of Stanley S. Ballard as guest editor, to summarize the growing body of unclassified knowledge in this field. Dr. Ballard has also summarized the evolution of subsequent publications in this field, including a number of excellent book reviews, in his Applied Optics feature "Optical Activities in the Universities," especially in 1, 585 (1962); 2, 323 (1963); 3, 219 (1964); 5, 1043 (1966); and 5, 1256 (1966).

11E. "Infrared Target and Background Radiometric Measurements-Concepts, Units and Techniques." G. Kelton, G. F. Levy, E. Bell, L. M. Biberman, G. E. Brown, J. N. Hamilton, R. C. Jones, D. S. Lowe, R. Paulson, G. J. Zissis, M. R. Nagel, L. Nichols, N. E. Beardsley, E. Wormser, E. M. Sevadjian, and J. A. Sanderson. Infrared Phys. 3, 139 (1963). Also report of the Working Group on Infrared Backgrounds (WGIRB). Report No. 2389-64-T, IRIA, Inst. of Sci. and Tech., The University of Michigan, Ann Arbor (AD-275810) (Jan. 1962). A tremendous essay at standardization and simplification was worked out very thoroughly by a rather large committee, the Working Group on Infrared Backgrounds (WGIRB), in the area of military infrared applications. This is an excellent source of radiometric fundamentals.

12A. Fundamentals of Infrared Technology. M. R. Holter, S. Nudelman, G. H. Suits, W. L. Wolfe, and G. J. Zissis. (Macmillan Co., New York, 1962). This text grew out of the lecture notes for a summer course of the same title at the University of Michigan. The authors are concerned primarily, though not exclusively, with military applications. [Reviewed by: Langton, Appl. Opt. 2, 364 (1963).]

13E. Photometry. J. W. T. Walsh. (Constable and Company, Ltd., London, 2nd ed., rev., 1953). [Also 3rd ed., 1958; reprinted by Dover Publications, Inc., New York, 1965.] An authoritative classic with a wealth of useful information on basic radiometry as well as on the more specialized considerations of photometry. [Reviewed by: Middleton, Appl. Opt. 5, 147 (1966).] 14A. The Scientific Basis of Illuminating Engineering. Parry Moon. (McGraw-Hill Book Co., New York, 1936). (Dover Publications reprint, with additional conversion tables and bibliography, New York, 1961). This volume seems harder to read than [13], in part, perhaps, because I have had less occasion or opportunity to refer to it. It is frequently cited as a classic.

15A. "Radiant-Heat Transmission." Hoyt C. Hottel. In Heat Transmission, W. H. McAdams, (McGraw-Hill Book Co., New York, 1954), 3rd ed., Ch. 4. Radiometry is presented here as developed by engineers concerned with radiative heat transfer. While the underlying principles are the same, the approaches and terminology are quite different in many respects from those in the previously cited sources.

16A. "Atmospheric Transmission: Concepts, Symbols, Units and Nomenclature," I. J. Spiro, R. Clark 
Jones, and D. Q. Wark. Infrared Phys. 5, 11 (1965). Although the title indicates that this paper is concerned primarily with nomenclature, there is an appendix on radiometry by Jones which outlines fundamentals from a fresh and stimulating point of view (see [25] below) in an attempt to deal more adequately with situations involving propagation through the atmosphere, and emission and reflection by natural objects. The paper was written at the instigation of the Specialty Group on Infrared Backgrounds and Atmospheric Physics of the Infrared Information Symposia (IRIS), sponsored by the Office of Naval Research.

17A. Vision'Through the Atmosphere. W. E. K. Middleton. (Univ. of Toronto Press, Toronto, Canada, 1952). Many workers, even when not directly concerned with atmospheric visibility, have found this a very useful text and reference on radiometric and photometric fundamentals, including nomenclature.

There are three new publications that, unfortunately, I have not had the opportunity to study sufficiently to be able confidently to assess their value in relation to the others listed above. However, from skimming them or from information about them, it appears that they should not be omitted from this list.

18A. Infrared Radiation: A Handbook for Applications. M. A. Bramson. (Plenum Press, New York, 1968). The treatment of thermal radiation and associated radiance contrasts seems to be particularly complete, with many useful tables. The implications and interrelationships between spectral distributions in terms of wavelength, wave number, or frequency and in terms of radiant energy or quanta, and their graphs or measures on logarithmic or linear scales, also appear to be covered quite thoroughly.

19A. Radiation Heat Transfer. E. M. Sparrow and R. D. Cess. (Brood/Cole Publishing Co., Belmont, Cal.,' Div. of Wadsworth Publ. Co., Inc., 1966). This book might replace [15] as a more up-to-date treatment from the standpoint of heat-transfer engineering. Incidentally, there appears to be a good discussion and a convenient catalogue of angle factors (projected solid angles in steradians divided by $\boldsymbol{\pi}$, the projected solid angle of a hemisphere).

20A. Infrared System Engineering. Richard D. Hudson, Jr. (John Wiley \& Sons, Inc., New York, 1969). Although I have not yet seen this book, I am informed ${ }^{5}$ that it is "strictly up-to-date on the physics and technology of the infrared, written from the systems point-of-view. See especially Ch. 2, Infrared Radiation, and Appendix 2, Symbols and Nomenclature for Radiometry and Photometry."

\section{NOMENCLATURE}

Nomenclature (concepts, terms, and symbols) is important in three ways. First, well-defined self-con-

- Private communication from S. S. Ballard, editor of the series in which this book appears. sistent nomenclature is essential for clear thinking, for the formulation of problems and propositions about radiometric quantities and their interrelationships, and for their successful manipulation to derive new relationships and to solve problems. Second, a somewhat broader appreciation of the variations and subtleties in the nomenclature in current use is needed for effective communication with contemporaries. Finally, a much more comprehensive grasp of the wide variations that occur in nomenclature, past as well as present, is needed for full access to and use of the large body of heterogeneous literature on radiometry that is only sampled here.

From the present introduction to the diversity in radiometric literature, it is clear that nomenclature differences lie at the core of the problem of achieving maximum availability and use of existing resources in that literature. Even if, by some miracle, complete standardization could eliminate the plague of nomenclature differences overnight, there would still remain the very substantial body of existing literature wherein the diversity is an established fact that cannot be ignored if we are to make use of it. However, before considering how to cope with this problem, in the absence of adequate keys and tables of corresponding items in the different nomenclature systems and practices, we note the recent rapid and substantia! progress toward standardization. Probably the best example is:

21E. USA Standard Nomenclature and Definitions for Illuminating Engineering, RP-16 (USAS Z7.1-1967). Approved August 16, 1967, by USA Standards Institute. Sponsored, published by, and available from the Illuminating Engineering Society, 345 E. 47th St., New York, N. Y. 10017. This monumental document is clear, complete, and well indexed. As indicated in its Foreword, it is the culmination of an extensive effort, including wide coordination on an international scale. ${ }^{6}$

Adoption of the radiometric nomenclature of [21] is rapidly gaining momentum and it clearly seems destined for wide acceptance. In particular, I understand that the editors of the Journal of the Optical Society of America and Applied Optics, and of the forthcoming OSA Handbook of Optics as well, have indicated that they will adopt the nomenclature of [21], strongly recommending its use in contributions to their publications. However, I hope that they continue to permit variations and departures when an author has strong reasons for them, lest the standards become a straitjacket that stifles desirable innovation and progress. Also, in view of this accelerating crystallization of a long-needed standard nomenclature, it is important to recognize aspects that make some of us accept it with less than complete enthusiasm, even while we recognize that the advantages far outweigh our objections.

\footnotetext{
"See also D. B. Judd, "Symbols, Nomenclature and Units," J. Opt. Soc. Am. 52, 954 (1962), and several related articles in the June 1960 issue (Vol. 15, No. 6) of Physics Today. See also OSA Nomenclature Committee Report, J. Opt. Soc. Am. 57, 854 (1967), and review of [21] in J. Opt. Soc. Am. 58, 864 (1968).
} 
Although the definitions and interrelationships are clearly stated in [21], the adoption of symbols that come from photometry, with subscripts added where needed to distinguish the radiometric from the corresponding photometric quantities, may unintentionally encourage illogically anthropomorphic tendencies to treat the standard human eye as a primary detector. It is important to point this danger out, particularly when introducing the nomenclature to students. This is not just a quibble over prestige between radiometry and its overshadowing branches of photometry and illumination engineering: important practical considerations are involved, as has been eloquently pointed out by Biberman [22]:

22E. "Apples, Oranges and Unlumens." Lucien M. Biberman. Appl. Opt. 6, 1127 (1967). “...Luminosity is related to the human eye, not to an S-20 cathode or photographic emulsion. The absurdity of applying photometric units to non-eye-related functions is illustrated and a plea made for proper use of radiometric terminology." [From author's abstract.] This letter to the editor touched off a series of letters in which the topic is further explored: F. H. Barr and E. H. Eberhardt, "On the Elimination of the Lumen from the Responsivity Calibration of Photodetectors," Appl. Opt. 6, 1575 (1967); H. K. Hammond III, "Phototube Response Evaluation," Appl. Opt. 7, 985 (1967); F. E. Nicodemus, "Radiometry with Spectrally Selective Sensors," Appl. Opt. 7, 1649 (1968).

To summarize briefly, radiometry is, logically, the broad field covering all forms of radiant-power measurements, including those with selective instruments having responsivities which vary as functions of one or more of the radiation parameters: wavelength or frequency, position, direction, fluctuation or modulation frequency, and polarization. The output of a radiometer is then just the weighted integral of the distribution function, describing the incident radiation in terms of these parameters, weighted by the responsivity function of the instrument. Photometric units are a particular case, albeit a very important one, of such a weighted response, wherein the weighting function is the luminosity function representing the spectral responsivity of the standard photopic human eye. But any intercomparison of photometric results with those of other selective sensors (e.g., photocells or photographic films) requires computations that are equivalent to transforming all results to absolute radiometric units.

As suggested in the comments on [1], the reader of the radiometric literature is constantly plagued by inadequate and ambiguous nomenclature. Until there is time for standardization to take full effect, the problem remains; I have found two techniques effective for coping with it : to prepare a glossary or translation table as I read, and to make frequent use of dimensional analysis. When I want to be meticulous, I set down in a list each of the author's radiometric terms and symbols, as they are introduced, together with their definitions, if given. The corresponding terms or sym. bols in my own familiar nomenclature are listed as soon as I am able to identify them, rechecking as I go along to see if the author's use of them is consistent with my identification. In addition, by applying dimensional analysis to each relationship and equation, the consistency can be even more thoroughly checked through particular attention to the units given for each quantity Not infrequently, inconsistencies appear, possibly even because the author is in error; then it becomes a problem to discover where the error occurred and to determine what is really meant by the statements made. Admittedly this takes time, but it is essential for understanding. Often, however, such elaborate precautions are not needed, especially after one has formed the habit of making these critical examinations and can do much of it mentally as he reads. But the confusion resulting from failure to understand and interpret a particular term or symbol correctly can frequently cost far more in lost time and effort than is required for this procedure; a few such instances will usually convince one that the time spent in systematically translating any difficult analysis or derivation into more familiar terms and symbols will prove to be a time-saving rather than a time-consuming exercise.

The general references already cited above are, of course, also sources for radiometric nomenclature. The possible shortcomings of Bauer [1], arising out of translation, have been noted. There is a good summary of much of the radiometric nomenclature in Wolfe [3], particularly in Ch. 2, Radiation Theory. In [4], I made some mention of variations in nomenclature but, for the most part, I concentrated on trying to present a clear, consistent scheme of nomenclature based on my own preferences at that time (now I would follow [21]). Gershun [5], Walsh [13], Moon [14], and Middleton [17] are good sources for the nomenclature used in photometry and illumination engineering, and Moon [14] also tabulates, im the latest Dover edition, the "improved system" of quite different nomenclature which is employed primarily by Domina Eberle Spencer and himself in numerous publications on illumination engineering (see [30], below). Hottel [15] and Sparrow and Cess [19], as indicated, employ the numenclature of radiative-heat transfer engineering. Spiro, Jones, and Wark [16] are primarily concerned with standardization of nomenclature for situations involving atmospheric propagation.

23E. The Science of Color. Optical Society of America, Committee on Colorimetry. (Thomas Y. Crowell Co., New York, 1953, reprinted 1966 by Optical Society of America, available from address given in preface of this Resource Letter). The Glossary-Index of this excellent volume is one of the best available sources for authoritative information about optical (including radiometric) nomenclature, particularly the nomenclature used by a large group in the Optical Society of America (OSA). A greatly expanded glossary of this type, with references 
to all publications containing significant material on radiometric nomenclature, preferably supplemented by extensive tables of equivalents, would be tremendously valuable as a dictionary to make much more of the existing radiometric literature available and useful to a much larger community of scientists and engineers. 24E. "Radiometric Quantities, Symbols and Units." E. E. Bell. Proc. IRE 47, 1432 (1959). A presentation of essentially the nomenclature recommended by Kelton et al. (the WGIRB) [11] that has been rather widely adopted in the community of scientists and engineers working on military infrared applications in this country.

25E. "Terminology in Photometry and Radiometry." R. Clark Jones. J. Opt. Soc. Am. 53, 1314 (1963). This is a proposal for an ingenious system of concepts and terminology that clearly recognizes the underlying geometrical relations common to radiometry and photometry and to the propagation of any quantity that obeys the laws of geometrical optics-one that is propagated in straight lines in a homogeneous isotropic medium, at a constant speed characteristic of the medium and without interference. Jones has coined the term phluometry for this underlying system of common geometrical relationships. His ideas, developed further in the appendix to [16], are important even if his proposed nomenclature is never widely used.

There follow some sources for the nomenclature currently in wide use in various areas, some for that used in earlier times (which will still be encountered when cited references are consulted), and some that intercompare the nomenclature used in different areas.

26E. "How to Understand and Use Photometric Quantities." Kodak Tech Bits No. 1, pp. 2-5, 15. (Eastman Kodak Co., Rochester, New York, 1965). This is a good, simple, practical summary of the most generally used photometric nomenclature, with some illustrative applications.

27A. "Bridging the Language Gap in Radiation Heat Transfer." Thaddeus C. Grimm. Chem. Eng. 72, 117 (1965). A direct intercomparison is made between the nomenclature used in photometry and illumination engineering and that used in heat-transfer engineering.

28E. Journal of Heat Transfer (ASME Trans., Ser. C). This journal follows the commendable practice of tabulating the nomenclature used in each paper on the first page of that paper. Unfortunately, the definitions of the symbols and terms are not always complete or rigorous, thus leaving room for ambiguity and misunderstanding, particularly since the units or physical dimensions for each quantity are not always given. Nevertheless, a quick scan through these tabulations in a few issues of the journal can give a very good idea of the nomenclature generally used by radiative-heat-transfer engineers.

29A. "The Units and Nomenclature of Radiation and Illumination." H. E. Ives. Astrophys. J. 45, 39 (1917). This is primarily of historical interest. It is perhaps encouraging to note that, confused as the present situation seems, it has been even more confused in times past.

30A. "Study of Photometric Nomenclature." Parry Moon and Domina Eberle Spencer. J. Opt. Soc. Am. 36, 666 (1946). The proposals and the arguments in support of them, as presented here, have not gained wide acceptance. However, much interesting historical and factual material is also presented.

31A. "Survey of Radiometric Quantities and Units." W. Viezee. The RAND Corp., Santa Monica, California, Research Memorandum No. RM-2492 (AD-241, 636) (July 12, 1960). Even though this report is not available in a recognized journal, it is cited here because, for those who wish to obtain a copy, ${ }^{7}$ it is a very good discussion of a wider scope than most. The radiativeheat-transfer nomenclature of the meteorologist or astrophysicist, rather than that of the engineer, is related to the historical development of photometric nomenclature.

\section{ACKNOWLEDGMENTS}

I am grateful to Prof. Stanley S. Ballard and the OSA Committee on Resource Letters for the opportunity to submit this letter and for encouragement and guidance in its preparation, and to Nelson M. Blachman and Harold Chatland of Sylvania for helpful discussions and criticism.

${ }^{7}$ Available directly from the RAND Corp., 1700 Main Street, Santa Monica, California 90406 (or, to qualified requestors, from the Defense Documentation Center-DDC). 


\title{
Introduction of the International Practical Temperature Scale 1968: some effects in relation to light sources, colour temperature, and colorimetry
}

\author{
J.S.PRESTON, M.A., C.Eng., F.I.E.E., F.Inst.P., F.Illum.E.S.
}

(1) Introductory and general

Since 1927 an internationally agreed temperature scale has been in use. In 1948 the International Temperature Scale, later named the International Practical Temperature Scale 1948 (IPTS-48), came into force. This scale has now been replaced by the IPTS $-68,{ }^{1}$ adopted in this country as from January I, I969. A change of scale does not, very obviously, mean any change in the actual temperatures associated with critical physical phenomena or states, for example the freezing point of pure gold under standard conditions. Chiefly it means an adjustment in the numerical values assigned to such temperatures in the light of increased accuracy of measurement, ${ }^{2}$ or more accurate knowledge of certain physical constants, or both. Any change in the unit of the scale, or in agreed conventions about methods of measurement may also play a part. One may imagine some ideally reliable thermometer continuing to give a certain unvarying reading while, from time to time, its calibration is adjusted, in the light of new research, to yield a corresponding numerical value nearer to the truth, according to the agreed conventions.

In the range above the gold point-the range of direct interest to lighting engineers and colorimetrists - the conventional procedure for determining a temperature is to compare it with that of the gold point with an optical pyrometer. In this, the value of the gold point fixes what we may call the 'datum level' of the scale of the pyrometer, while the value taken for the radiation constant $c_{2}$ (in the Planck formula) determines the 'scale expansion factor' of the instrument. In the change from IPTS-48 to IPTS-68, not only has the accepted value of $c_{2}$ been changed from 0.0I4 380 to 0.014388 $\mathrm{m} \mathrm{K}$, but the gold point value also has been changed from $1336 \cdot 15$ to $1337 \cdot 58 \mathrm{~K}$. Because this 'datum level' has been changed independently of the change in $c_{2}$, one cannot derive a temperature value on IPTS-68 simply by multiplying the 1948 value by the ratio new $c_{2} /$ old $c_{2}$, although only the ratio $T / c_{2}$ appears in the Planck formula. It is as well to hold this point clearly in mind. It is quite distinct from the case where one can use this ratio procedure in order to be able to use radiation tables which have been computed from an out-of-date value of $c_{2}$.

The intention of this note is not to go into great detail for, indeed, the effects of the change of scale in lighting technology will generally be less than that of other experimental uncertainties. Moreover, the error-tolerance on colour temperatures, as at present assigned by the standards laboratories, is of the same order as the change in temperature values. Nevertheless, the present description should interest workers who wish to be knowledgeable on background, or whose requirements are exacting.

\section{(2) The unit, nomenclature and symbols}

First, nomenclature and the unit. A temperature is a physical quantity of which the unit is the kelvin, symbol $\mathrm{K}$, now included in the SI system. The use of degree Kelvin and of the symbol ${ }^{\circ} \mathrm{K}$ has been discontinued. The size of this unit is defined by assigning the value $273 . \mathrm{I} 6 \mathrm{~K}$ to the temperature of the triple point of water, i.e. $273 \cdot 16$ kelvins above absolute zero. The ice point is $0.01 \mathrm{~K}$ below the triple point and so has the value $273.15 \mathrm{~K}$. It also corresponds to $0^{\circ} \mathrm{C}$ on the Celsius scale which is still permissible and widely used. So if $T$ is the value of a temperature in kelvins, and $t$ its value in degrees Celsius, $T=t+273 \cdot 15 \mathrm{~K}$, over the whole range of temperatures. The symbol ${ }^{\circ} \mathrm{C}$ is retained for the Celsius scale, but the use of Centigrade for Celsius is deprecated.

\section{(3) Colour temperature and spectral}

\section{distributions}

Next, colour temperatures of lamps. Consider, first, the use of a tungsten filament lamp to simulate the (visible) spectrum from a black
This paper is a communication from the National Physical Laboratory. Teddington. The paper was received on February 13, 1969. 
body radiator at, say, $2703 \mathrm{~K}$. Suppose that last year the lamp had been set at colour temperature $2703^{\circ} \mathrm{K}$ (IPTS-48). It is now required to be re-set; and suppose that the standards laboratory sets it at $2703 \mathrm{~K}$ (IPTS-68)-a slightly different temperature. What has happened? Simply, that the closeness of simulation has been improved. It is as though the standards laboratory had last year set and measured the temperature of an actual black body, but this year had decided that a slight adjustment of its actual temperature would bring its spectrum into even closer agreement with the theoretical one (for the stated temperature value), and had then, after the adjustment, re-set the lamp to match it.

However, it may also be necessary to know what this theoretical spectrum is. This can be obtained from tables of black body radiation at the page headed 2703. Last year this page was computed from the Planck formula using temperature 2703 and $c_{2}=0.014380$. Now new tables should be used ${ }^{3}$, based on 2703 and 0 - or 4388 . Alternatively, old tables can be used, but for a different temperature $T$, given by $T / 14380=2703 / 14388$, or $T=2701 \cdot 5 \mathrm{~K}$, since only the ratio $T / c_{2}$ affects values given by the formula. Indeed, this must be done to achieve consistency, for the new value of $c_{2}$ is one of the things involved also in the slightly changed setting of the lamp.

On the other hand, the level of the colour temperature of the lamp may not be as important as knowing exactly what it is. We will suppose, too, that the lamp has been used so little that a re-check for deterioration alone would be a pointless extravagance. It is then only necessary to enquire what temperature on IPTS-68 corresponds to $2703^{\circ} \mathrm{K}$ (IPTS-48), and to use the former as the revised, better, colour temperature of the lamp and as the indication of what page to consult in new tables for the matching black body spectrum. If careful interpolation is carried out between values given in the Table at the end of this paper, it will be found that this IPTS-68 value is $2707.3 \mathrm{~K}$. If old tables are used it will be necessary to look up temperature $T^{\prime}$ such that $T^{\prime} / 14380=$ $2707 \cdot 3 / 14388$, or $T^{\prime}=2705 \cdot 8 \mathrm{~K}$.

\section{(4) Colorimetric illuminant $A$}

The case of the colorimetrist, using a tungsten filament lamp to simulate standard illuminant $\mathrm{A}$, is different. The (relative) spectrum of this source was defined (CIE I93I) as that given by the Planck formula if one puts into it $T=2848 \mathrm{~K}$ and $c_{2}=0.014350 \mathrm{~m} \mathrm{~K}$. As we have seen, insertion of any other pair of such values, having the same ratio, produces the same computed spectrum. Thus, on the IPTS -48 with $c_{2}=$ $0.014380, T$ became $2854^{\circ} \mathrm{K}$, while on the IPTS-68 with $c_{2}=0.014388, T$ becomes
$2855 \cdot 5 \mathrm{~K}$. The colorimetrist will therefore now get a lamp set at $2855.5 \mathrm{~K}$ (IPTS-68) for best simulation. At first sight it may seem strange that, against this, the IPTS- 68 value of $2854^{\circ} \mathrm{K}$ is not $2855 \cdot 5 \mathrm{~K}$ but $2858 \cdot{ }_{7} \mathrm{~K}$; but this is because the physicist has been concerned not only with a specification anchored to theory alone, but with progressively improving the aceuraey of all factors involved in emulating theory in terms of practical sources. Although a particular lamp now set at $2855.5 \mathrm{~K}$ will emit a somewhat different spectrum from that which the same lamp emitted when previously set at $2854^{\circ} \mathrm{K}$ (IPTS-48) (the spectrum would be unchanged if it were now set at $2858 \cdot 7 \mathrm{~K}$ (IPTS-68)), the new spectrum is a better simulation of standard illuminant $A$ as a result of work in revising the temperature scale.

Again, however, the colorimetrist whose lamp has not deteriorated and who, anyway, probably works on the basis of known spectral values rather than exact simulation, may decide not to have a lamp re-set. He will then assign to it the new colour temperature $2858 \cdot 7 \mathrm{~K}$, and look up this temperature in new tables, or $2858.7 \times 14380$ / $14388=2857 \cdot 1^{\circ} \mathrm{K}$ in old tables.

\section{(5) The platinum point and the luminous} efficacy of radiation

Lastly, the freezing point of platinum. Historically, this point was of importance as a fixed point in setting up the 'scale' of colour temperature as preserved by means of tungsten standard lamps; but here we consider it in the different context of the radiation properties of the primary standard of light (but see note below Table $\mathrm{I}$, relevant in both respects).

\section{Table 1 Equivalent temperature values}

For convenient reference, some pairs of equivalent temperature values are given in this table. A temperature which was designated by the numeral in the first column is, as from January 1, 1969, designated by the numeral in the second column.

\begin{tabular}{ll}
\hline IPTS-48 & IPTS-68 \\
\hline $2042 \cdot 15^{\circ} \mathrm{K}$ & $2044 \cdot 9 \mathrm{~K}^{\circ}$ \\
2200 & $2203 \cdot 1$ \\
2353 & $2356 \cdot 5$ \\
2400 & $2403 \cdot 6$ \\
2600 & $2604 \cdot 1$ \\
2788 & $2792 \cdot 5$ \\
2800 & $2804 \cdot 6$ \\
2854 & $2858 \cdot 7$ (colorimetrists see text) \\
3000 & $3005 \cdot 1$ \\
3200 & $3205 \cdot 7$ \\
3400 & $3406 \cdot 2$ \\
\hline
\end{tabular}

-The decimal places, here, arise from the machinery of conversion of Scales 1927 to 1948 to 1968 , chiefly the interpolation used. They have usually been included when the values have been used in the computations referred to. However, as a fixed point, the platinum point is not now regarded as known to this degree of precision. In the IPTS-68 it is given s mply es $2045 \mathrm{~K}$-and even this figure may need revision followil $a$ any future practical re-determination. Any such revision may well heve e significant effect on the computed value of $K_{\mathrm{m}}$. 
Obviously, changing a 'book' value of the freezing point of platinum, or of $c_{2}$, has no effect on the light or radiation emitted by the primary standard; but changing cither will affect the radiation which the Planck formula says it emits. Therefore, since this calculated radiation spectrum enters into interesting computations of the absolute luminous efficacy of radiation, it is as well to use the most recent data available. The freezing point of platinum, which was taken as $2042 \cdot 15^{\circ} \mathrm{K}$ (IPTS-48), now becomes $2044.9 \mathrm{~K}$. Use of this latter value, together with the I 968 value of $c_{2}$, leads to a value of $673 \mathrm{~lm} / \mathrm{W}$ for $K_{\mathrm{m}}$, the luminous efficacy of radiation at wavelength $0.555 \mu \mathrm{m}$.

\section{Acknowledgment}

This article has been prepared as part of the work of the National Physical Laboratory in this field. The author is indebted to several colleagues, particularly $\mathrm{Mr} \mathrm{C}$. R. Barber of the Division of Quantum Metrology, for assistance and critical discussion.

\section{References}

1 The International Practical Temperature Scale of I 968 , HMSO.

${ }^{2}$ Changes in the numerical values of temperature resulting from the adoption of the International Practical Temperature Scale I968 (on request from Director, National Physical Laboratory, Teddington, Middx.)

3 Tables of spectral power distribution of tungsten and black body radiators, with explanatory introduction (enquiries to Superintendent, Division of Quantum Metrology, National Physical Laboratory, Teddington, Middx.) 


\section{PHOTOMETRIC UNIT CHART}

This chart, developed by Francis Clark, Lighting Services, Waterbury, Conn., and L. E. Iarbrow, National Bureau of Standards, Washington, D. C., ilustrates the interrelatiunship among U: six most commonly used photometric units.

UnITS OF LUMINOUS INTENSITY, LUMINOUS FLUX, LUMINOUS ENEFIGY, ILLUMINATION, LUMINANCE, and LUMINOUS EXITANCE

The *CANDELA is the luminous infensity of $1 / 60$ of $1 \mathrm{~cm}^{2}$ of projected ore of a blackbidy rodiotor operoting of the femperoture of solidification of plotinum, $2045 \mathrm{k}$.

The *UMEN is the luminous flux from I condelo in all directions thru o unit solid angle of one steradion

The * LUMEN-SECOND (which is 1 TALBOT) is the luminous energy delivered cy $\mid$ lumen $i$. one second

Point $A$ io ony $B$ is $1 \mathrm{~cm}$ $B B 8 B$ is $1 \mathrm{~cm}^{2}$
Point A to ony $C$ is $19 t$ $\operatorname{CCCC}$ is $1 \mathrm{ft}^{2}$
Point A to any $D$ is 1 met: DODD is $1 \mathrm{~m}^{2}$

\section{UNITS OF ILLUMINATION $\longrightarrow$}

The PHOT is the unit of illuminotion resulting from the flux of I lumen folling on $B B B B$ from $A=1$ conde 0
The FOOTCANDLE is the unit of illumination resulting from the flux of I lumen folling on CCCC from $A=1$ condelo
The * LUX is the ur.." $c^{*}$ illumination resulting fium inc. flux of I lumen folling ir DDDD from $A=1$ condela

\section{— UNITS OF LUMINANCE}

Assume $100 \%$ of the luminous flux from A (I condelo in oll directions) is reflected in o perfectiy diffuse monner by $8 B 8 B-C C C C-$ or- DDDD-then

$B B B B$ will have o directionally uniform luminance of 1 lumen $/ \mathrm{cm}^{2}$ which is 1 LAMBERT or $1 / \pi$ condelo/ $\mathrm{cm}^{2}$ which is $1 / \pi$ STILB CCCC will have o direcionally uniform luminance of I lumen $/ \mathrm{ft}^{2}$ which is 1 FOOTLAMBERT or $1 / \pi$ condelo $/ \mathrm{ft}^{2}$ DODD will have o directionally uniform luminonce of 1 lumen $/ \mathrm{m}^{2}$ which is 1 APOSTILB or $1 / \pi$ * condelo $/ \mathrm{m}^{2}$ which is $1 / \pi$ NIT

- UNITS OF LUMINOUS EXITANCE Assume $100 \%$ of the luminous flux from A (I condelo in all directions) is reflected by $8 B 8 B$ - $\mathrm{CCCC}-$ or - DDDD- then

$B B B 8$ will hove on exitance of 1 lumen $/ \mathrm{cm}^{2}$

CCCC will hove on exitance of 1 lumen $/ f:^{2}$

DDDD will have on exitonce of "Iumen/: $r^{2}$ 


\title{
FILTERS FOR THE REPRODUCTION OF SUNLIGHT AND DAYLIGHT AND THE DETERIIINATION OF COLOR TEMPERATURE ${ }^{1}$
}

\author{
By Raymond Davis and K. S. Gibson
}

\section{ABSTRACT}

A liure number of filters, as listed in the introduction of the paper, liave been developed for use in photographic sensitometry, colorinetry, and photometry. 'They are reproducible from speeification and may be prepared in the laboratory. They consist of a 2-compartment eell with three borosilicate crown glass windows, the two compartunents being filled, respectively, with solutiona $\mathrm{A}$ and $\mathrm{B}$, having compositions as follows:

\section{Solution $A$}

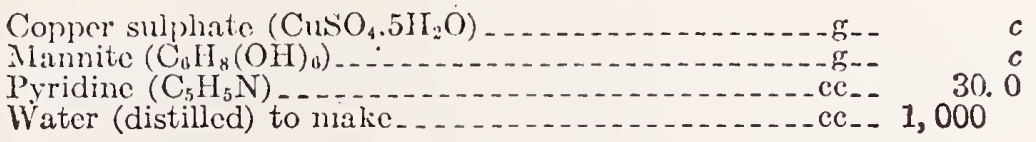

Solution $B$

Copper sulphate $\left(\mathrm{CuSO}_{4} .5 \mathrm{H}_{2} \mathrm{O}\right)$ Cobalt ammonim sulphate $\left(\mathrm{CoSO}_{4} \cdot\left(\mathrm{NH}_{4}\right)_{2} \mathrm{SO}_{4} \cdot 6 \mathrm{H}_{2} \mathrm{O}\right)_{-\mathrm{g}--}$ Sulphuric acid (specific gravity 1.835) ...................

Water (distilled) to makic 10.0

The values of $c, c_{1}$, and $c_{2}$ vary from one fitter to another.

The design of the filters has been based upon extensive spectrophotometrie measurements of the component solutions and cell, including a study of Beer's law over the ranges of concentrations used, and upon methods of computation giving accurate color matches and relatively good spectral cnergy matches between the sourec-and-filter combination and the energy distribution which it was sought to duplicate. Detailed studies have been made of the reliability of the data, the spectrophotometric reproducilsility of the chenicals, the permanence of the solutions, their temperature coefleients, and various other factors of importance in the preparation and use of the filters.

The paper contains 33 figures and 26 tables, giving among other things the chergy distributions and fundamental spectrophotometric and colorimetric data used in the computations, auxiliary spectrophotometric and colorimetric information obtained during the investigation, and the trilinear coordinates of the various source-and-filter combinations and of the Planckian radiator from $1,600^{\circ}$ to $20,000^{\circ} \mathrm{K}$. computed on the basis of the adopted mean sun as the "ncutral" stimulus. The filters are further described in 38 charts, each chart giving the chemical formula, the spectral transmission, and the light transmission of the filter, as well as the spectral energy distribution of the source, that of the source and filter combined, and the energy distribution which serves as the ideal in that particular casc.

1 Partial reports of this investigation have been published as follows:

1. Davis and Gibson, Reprodueible Liquid Fitters for the Produetion of "White Light" (abstract), J. Opt. Soc. Am. and Kev, sei. Inst., 14, p. 135; Februnry, 1927.

2. Davis and Gibson, Reproducible Ijicuid Filters for the Determination of the Color Temperatures of Incandescont lamps (abstract), Pliys. KRev. (2), 27, p. 916; June, 1027.

3. Davis and Gibson, Filters for the Teproductiou of Sualight and the Determination of Color Temperature (abstract), J. Opt. Soe. $\Lambda \mathrm{m}$. and Rev. Sici. Inst., 16, p. 332; May, 1925.

4. Gibson and Davis, Methods for Determining the Color of Sunlight and Daylight (abstract), J. Opt. Soc. Am. and Rev. Sei. Inst., 1S, p. 442; June, 1929.

5. Divis and Gibson, Artifieial Sunlight for Photographie Sensitometry, Trans. Soc. Motion-Picture Engineers, 12, No. 33, pp. 225-236; 192s; Proc., Seventh International Congress of Photography, July, 1925, pp. 161-173.

6. $\Lambda$ Filter for Changing the Color Tempernture of $2,848^{\circ} \mathrm{K}$. to $\Lambda$ verage Daylight, B. S. Teeh. News Bull., No. 135, pp: 143-144; No. 139, p. 156; 1828.

7. Filters for the Reproduetion of Sunlight and the Determination of Color Temperaturo. (Proposed Working Standards for Sonsitornetry, Colorimetry, and Photometry.) Standards Ycarbook, pp. 18s-101; 1928; also Standards Yearbook, pp. 138-141; 1030. 
Fart of Journal of Research of the National Bureau of Standards, Volume 27. September 1941

\title{
A PHYSICAL PHOTOMETER
}

\author{
By Ray P. Teele
}

\section{ABSTRACT}

The unreliahle and differing charncteristics of observers' cyes have prevented photometry from being an exact science. A physical plotometer has been cunstructed, consisting of a thermopile, a potentiometer, and a filter which has nt anch wavelength a transmission proportional to the ICI luminosity factor for that ravelength. 'This photometer gives results which are consistent with the ICI luminosity factors and which are more accurate than those obtained by visual nhacrvere when the pliotometric ficlds are not colnr-matched.

\section{CONTENTS}

Page

I. Introduction

II. The physical photometer

1. Inminosity filter

2. Jeceiver

3. Potentiometer

4. Precantions to be observed.

III. Metlind of taking observations

IV. Fxperimental tests...

1. Measurement of spectrally selective filters _............... 225

2. Measurement of rotating sectored disks

3. Stability

V. Application to measurement of lamps

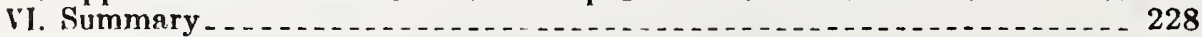

\section{INTRODUCTION}

Photometry has been carricd on for many years by means of visual nhservations, not because the eye is an accurnte measuring instrument hut because there has been no physical apparatus which would respond in radiant energy in the same manner as the human eye with an nccurncy comparable with that of visual obscrvations made under the bret condilions.

$V$ ision is a combined physiological and psychological proress. In the visual photometry of colored lights, otherwise normal observers may obtain markedily differing positions of photometric balnnce hecause of their differing brightness evalunations of different parts of the spectrum.

Experience shows not only that the results obtained in heterochromatic photometry are influenced by the characteristics of the nye of the observer, but also that nearly all obscrvers using the equality-of-brightness method will change their dccision as to a photometric balance over a period of a month or more. Some observers even exhibit a variation in this decision during a single day. 
Wyen in lomochromstic photometry the results ohtrined nre influeneed by the at.titude of the oleservere nond by his ocular norl bodile

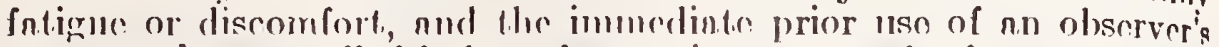
eye must, be controlled before observations nre made; for exnmple, nn oherver enteringe a photometric lnborntory from n, brightly lighted room requires a periond of time to become properly ndepted to thr light conditions of the photometer room. All of these varintions makr it desirnble to devise n physienl instrmment to ovaluate light.

Various cxperimenters linve worked on the problem. It is suffi. cient bere to noto the experiments of Ives and Kingshury, nnd of Ives, in 1915, in which a lmminosity filter and $\Omega$ thermopile werr cmployed. Tho luminosity "filter" in ono caso was a templnto which wes placed in the path of the radinnt energy dispersed by a prism. The optical system required and the mechanical difficulties encomstered in constructing, mounting, nnd alining a template has madr this methor imprenctical. In the other case the luminosity filter consisted of a liquid solution. In both cases a Thomson galvanometer was used. The susceptibility of this galvanometer to mechanical disturbances and drift of zero, and the leck of exact proportionnlity between current and deflection in the circuit, imposed serious limitntions on their mensurements. In order to nvoid the difficultirs which Jves and Kingshury encountered, which are present even with the best of modern galvanometers, it was decided in the presentwork to measure the cmf developed, instead of drawing and measuring current from the thermopile. Since this emf is small, it was necessnry to develop a specinl potentiometer and procedure in order to make thin mensurements to the required accuracy, and these have been described in a previous paper. ${ }^{2}$ The prisent paper gives a more complet description of the physical photometer which was constructed and describes the method of operation and the results of tests which wern made to determine its performsnce. 
11. 12 .

\section{CHANGES IN THE U.S. UNIT OF LUMINOUS FLUX}

The Bureau announces a change of 1.9 percent in the U.S. unit of total luminous flux for gas-filled tungsten-filament lamps. This change is necessary to bring the U.S. unit into agreement with the world mean. Owners of gas-filled tungsten-filament lamp standards of luminous flux supplied on the old basis should multiply the presently assigned values by the factor 1.019 to obtain values on the new basis.

Several international intercomparisons ${ }^{1-4}$ of luminous flux measurements have been conducted since the establishment of the platinum-point black body ${ }^{5}$ as the primary standard of luminous intensity. These intercomparisons indicated that the U.S. unit of total luminous flux at the color temperature of gas-filled tungsten-filament lamps (approx. $2800 \mathrm{~K}$ ), maintained at NBS, is discrepant with respect to the world mean. Recent work at NBS has confirmed that this discrepancy represents a misassignment of the U.S. unit.

In the past 30 years NBS has set up standards of total luminous flux for several categories of gasdischarge lamps. These standards have not been referenced to the gas-filled tungsten lamp standards of luminous flux, but rather have been calibrated by the more fundamental method of distribution photom. etry based on the NBS standards of horizontal luminous intensity. Since no change is indicated for the other U.S. photometric units, flux standards derived directly from standards of luminous intensity will not change. Lamps falling specifically in this category for which no change in rated values is necessary include mercury vapor ${ }^{6}$ and fluorescent lamps.

\footnotetext{
${ }^{1}$ Comité Consultatif de Photométrie, Session de 1952, ProcésVerbaux des SÉances, Annexe P3, 46-75.

${ }^{2}$ Ibid, Annexe P4, 76-100.

${ }^{3}$ Comité Consultatif de Photomét;ie, $4^{e}$ Session (1957), ProcésVerbaux des Séances, Annexe P8, 74-103.

"Comité Consultatif de Photométrie, $5^{\text {e }}$ Session (1962), Annexe 9, 63-87.

5 Announcement of changes in electrical and photometric units, Nat. Bur. Stand. (U.S.), Circ. C459, 12 pages, (May 1947).

${ }^{\circ}$ Nat. Bur. Stand. (U.S.), Tech. News Bull. 35 (1) 3-5 (1951).
}

MC16. New Principle of Absolute Photometry. CaLvin S. MícCAMY, National Burcan of Stuataids, Washington 25, D. C.-For absolute photometry, a standard source, photometric comparator, and quantitative attemuator are required. Heretofore, quantitative attenuation has heen based upon the inverse-square law or Malns' law of polari\%ation. The principle of attenuation presented here is an optical analog of Riemann integration. A rectangular aperture in a light path is fitted with a sliding straight-edged shutter. The light flux througin the aperture is then approximately proportional to the open-aperture area. A thin slit, instead of the shutier, is moved across the aperture while the light passing though the slit to a detector is compared to a constant source. If the response of the detector is constant as the slit moves, the shutter is replaced, and the flux through the aperture to the detector is known to be proportional to shutter displacement, which can be measured preciscly. If this conclition is not found, the edges of the aperture may be shaped or an optical compensator may be placed in the aperture to produce the required condition. Two slits may be used to establish an exponential or inverse-square relationship to shutter displacement. The nicthod requires less space than the inversesquare method and is less subject to certain errors.

11.11. 


\section{Applied Photometry}

Papers

12.1. The Use of Zonal Constants in the Calculation of Beam Flux. Page

Theodore H. Projector, Illum. Eng. 48, No. 4, 189-191 (April (1953).

Key words: Luminous flux; searchlights ; floodlights ; zonal

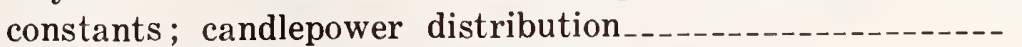

12.2. Versatile Goniometer for Projection Photometry.

Theodore H. Projector, Illum. Eng. 48, No. 4, 192-196 (April 1953).

Key words: Goniometry; projection photometry; 100-foot photometric range; searchlights

12.3. Photometry of Projectors at the National Bureau of Standards.

L. Chernoff, Nat. Bur. Stand. (U.S.) Tech. Note 198, 28 pages, (Dec. 1963).

Key words: Projectors; searchlights

12.4. Review of Elementary Theory of the Photometry of Projection Apparatus.

C. A. Douglas, Illum. Eng. 62, No. 4, 246-253. (Apr. 1967).

Key words : Critical distance ; illumination ; light projectors ; photometric distance; signal lighting

12.5. Photometer for Measurement of Effective Intensity of Condenser-Discharge Lights.

C. A. Douglas, Illum. Eng. 53, No. 4, 205-208, (Apr. 1958).

Key words: Flashed light intensity; photometer

12.6. Effective Intensity of Flashing Lights.

T. H. Projector, Illum. Eng. 52, No. 12, 630-640, (Dec. 1957). Key words: Flashing light; effective intensity

12.7 Computation of the Effective Intensity of Flashing Lights.

C. A. Douglas, Illum. Eng. 52, No. 12, 641-646 (Dec. 1957).

Key words: Flashing light; effective intensity; computation

12.8. Photometer for Luminescent Materials.

Ray P. Teele, J. Opt. Soc. Amer. 35, No. 6, 373-378 (June 1945).

Key words: Dark adapted eye; low luminance photometry ; luminescent materials

12.9. On the Standard Source for Low Level Photometry.

J. Terrien, J. Opt. Soc. Amer. 39, No. 10, Letter to Editor

p. 888 (Oct. 1949).

Key words: Color temperature $2042 \mathrm{~K}$; dark adapted observer; low Iuminance; platinum blackbody; Purkinje effect

12.10. The Photometry of Colored Light.

A. C. Wall, Illum. Eng. 62, No. 4, 239-242 (Apr. 1967).

Key words: Filters; photocells; photometers; phototubes ; spectral correction 


\section{Applied Photometry-Continued}

Abstracts

12.11. Absolute Light-Scattering Photometer: I, Design and Operation.

Page

Donald McIntyre and G. C. Doderer, J. Res. Nat. Bur. Stand. (U.S.) 62, No. 4, 153-159 (April 1959).

Key words: Absolute photometry; angular scattering; photometer design; polymer solutions; ratio scattered to incident light; research photometer.

12.12. Absolute Light-Scattering Photometer: II, Direct Determination of Scattered Light from Solutions.

Donald McIntyre, J. Res. Nat. Bur. Stand. (U. S.) 68A, No. 1, 87-96 (Jan.-Feb. 1964).

Key words: Absolute photometry ; polymer solutions ; transmission by solutions; transverse measurements of light scattering 


\section{The Use of Zonal Constants in the Calculation of Beam Flux}

\section{From Candlepower Distribution Data}

BY THEODORE H. PROJECTOR

$\mathrm{T}$ HE LUMINOUS FLUX in the beams from searchlights, floodlights, and other projection lighting equipment is frequently calculated from candlepower distribution data with the aid of tables of zonal constants. The beams are divided into convenient zones and the flux in each zone is obtained by multiplying the average candlepower in the zone by the solid angle subtended by the zone at the light source (the zonal constant). For axially symmetrical beams (where a cross-section of the beam is radially symmetrical), annular zones are convenient, and the constants may be computed readily from the formula:

$$
\text { Zonal Constant }=2 \pi\left(\cos \alpha_{1}-\cos \alpha_{2}\right)
$$

where $\alpha_{1}$ and $\alpha_{2}$ are the plane angles defining the inner and outer limits of the zone, respectively. As early as 1908, Weinbeer published a table of constants for annular zones at 10-degree intervals, ${ }^{1}$ and many similar tables have been published since then, giving constants for zones at 1-, 2-, 5-, and 10-degree intervals., ${ }^{2,3}$

In the event that the beam from the projector is not axially symmetrical, more finely subdivided zones must be selected. Zones may be defined in a number of ways and the method selected in any given case is determined principally by the type of goniometer used and the availability of tables of constants. If the goniometer used measures cone angles (the angles that define annular zones) and polar angles (angles around the optical axis of the test equipment) then subdivision of annular zones into segments is practical. For a zonal segment defined by two cone angles, $\alpha_{1}$ and $\alpha_{2}$, and by two polar angles, $\beta_{1}$ and $\beta_{2}$, the zonal constant is given by :

$$
\text { Zonal Constant }=\frac{\beta_{2}-\beta_{1}}{360} 2 \pi\left(\cos \alpha_{1}-\cos \alpha_{2}\right)
$$

( $\beta_{1}$ and $\beta_{2}$ are expressed in degrees.)

If zonal constants for annular zones $\left(\alpha_{1}, \alpha_{2}\right)$ are available, they may be used to compute segmental constants. It is evident by comparison of equations

AUThor: National Burean of Standards, Photometry and Colorimetry Section, Washington, D. O
(1) and (2) that zonal constants for segments defined by $\alpha_{1}, \alpha_{2}, \beta_{1}$, and $\beta_{2}$ are given by:

$$
\left(\begin{array}{c}
\text { Zonal } \\
\text { Constant }
\end{array}\right)_{\alpha_{1} \alpha_{2} \beta_{1} \beta_{2}}=\frac{\beta_{2}-\beta_{1}}{360}\left(\begin{array}{c}
\text { Zonal } \\
\text { Constant }
\end{array}\right)_{\alpha_{1} \alpha_{2}}
$$

Goniometers suitable for cone angle measurements are occasionally used but the majority of goniometers employed in projection photometry are of the types illustrated in Fig. 1.4,12* In these, a direction of measurement in the beam is given by a "horizontal" and a "vertical" angle. In a type A goniometer, "vertical" angles are measured around a fixed horizontal axis of rotation while "horizontal" angles are measured around an axis which is vertical only when the "vertical" angle is zero (as shown) or $180^{\circ}$. In a type B goniometer, "horizontal" angles are measured about a fixed vertical axis and "vertical" angles are measured about a movable horizontal axis. It may be emphasized at this point

*See following article: "Versatile Goniometer for Projection Photometry."
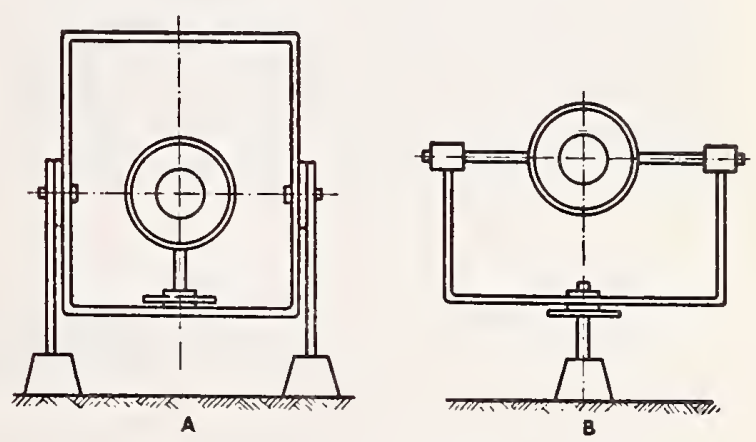

Figure 1. (After Bergmans and Keitz, 1947) The type A goniometer measures "vertical" angles about a fixed horizontal axis and and "horizontal" angles about an axis which is vertical at zero "vertical" angle (beam horizontal) but is inclined from the vertical as the "vertical" angle is moved from the zero position. With the type $B$ goniometer, "horizontal" angles are measured about a fixed vertical axis of rotation and "vertical" angles are measured about a horizontal axis which rotates as the "horizontal" angle is changed. 


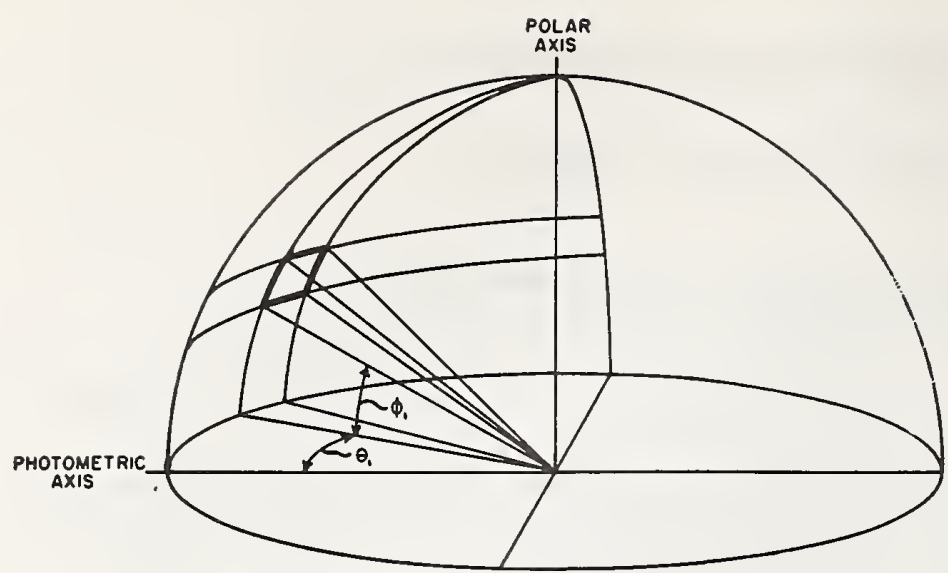

Figure 2. Geometrical construction of spherical quadrilateral zones for type A goniometers. $\theta_{1}$ is the "horizontal" angle and $\phi_{1}$ is the "vertical" angle to the corner of the zone nearest the photometric axis. When the goniometer is swung through the angles $\theta_{1}$ and $\phi_{1}$ this corner of the zone is coincident with the photometric axis.

that this is the most obvious distinction between the two types of goniometers: in a type A goniometer, the horizontal axis is fixed, and in a type $\mathrm{B}$ goniometer, the vertical axis is fixed.

For computation of flux from candlepower measurements made with a type A or a type B goniometer, "square" or "rectangular" zones are selected and are defined as spherical quadrilaterals, each bounded by ares corresponding to two horizontal and two vertical angles set on the goniometer. AIthough two ares on opposite sides of the quadrilateral represent equal angular intervals as measured on the goniometer, they are not in general equal in length. The solid angle subtended by any given quadrilateral zone depends on which type of goniometer is in use. ${ }^{4}$ The manner in which spherical zones are defined is illustrated in Figs. 2 and 3, corresponding respectively to the goniometers shown in Figs. 1A and 1B. In these figures, the "photometric axis" is the direction from which horizontal and vertical angles are measured. To present the nearest corners of the outlined zones to the photometer, the goniometer is swung through hori- zontal angle, $\theta_{1}$, and vertical angle, $\phi_{1}$. In type A goniometers (Fig. 2), horizontal angles are meridional and vertical angles are latitudinal, while the reverse is true in type $\mathrm{B}$ goniometers (Fig. 3 ). The polar axes indicated in the figures are the axes through the intersections of the meridians. The polar axes correspond to the movable axes of rotation on the goniometers of Fig. 1 rather than to the axes that remain physically fixed.

Zonal constants for a Type A goniometer (geometry of Fig. 2) may be computed from the formula :

$$
\text { Zonal Constant }=\pi \frac{\theta_{2}-\theta_{1}}{180}\left(\sin \phi_{2}-\sin \phi_{1}\right)
$$

where $\phi_{1}$ and $\phi_{2}$ are the vertical angles $\theta_{1}$ and $\theta_{2}$ are the horizontal angles (measured in degrees) bounding the spherical quadrilateral zone. If the goniometer is of type B then the horizontal angles, $\theta$, and the vertical angles, $\phi$, must be interchanged in the formula.

Thus the zonal constants for zones defined by "horizontal" and "vertical" angle boundaries must
Figure 3. Geometrical construction of spherical quadrilateral zones for type B goniometers. As in Fig. 2, $\theta_{1}$ is the "horizontal" angle and $\phi_{1}$ the "vertical" angle to the corner of the zone nearest the photometric axis.

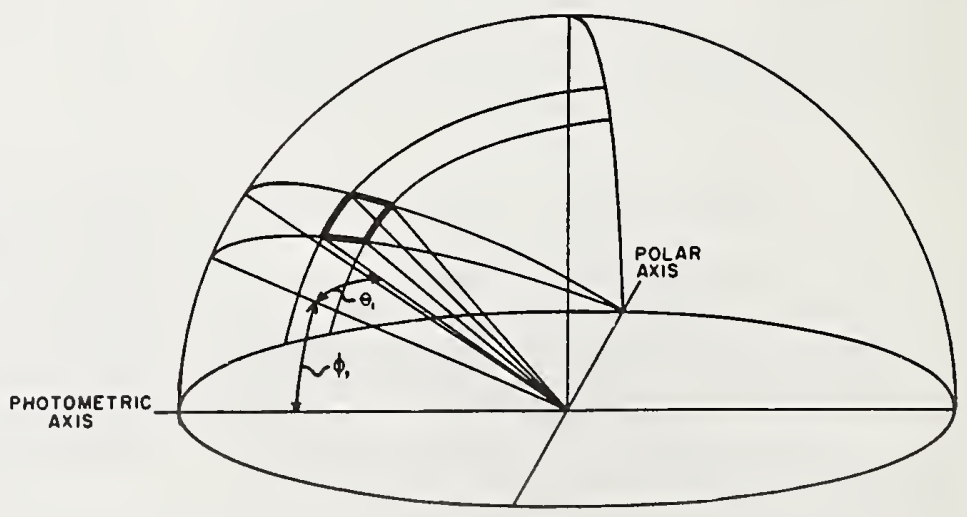

$580-190$ 
be specified for angles measured with one of the two types of goniometers. If the goniometer used is not of the type for which the constants in a table are computed, correct constants can be obtained by interchanging the horizontal and vertical angle designations in the table. To assist in determining the type of goniometer for which zonal constants are given, it is especially helpful to note that the angular arguments in a table are always the settings made around the fixed axis of rotation of the goniometer.

The distinction between the two types of goniometers has not been made very clear in any of the published tables of zonal constants for "square" or "rectangular" zones, and recently, in fact, tables have been published in which the goniometer type has not been mentioned at all. In 1927, the Association of Railway and Electrical Engineers published a report on "A General Procedure for Photometry of Incandescent Floodlights," ${ }^{5}$ later incorporated into an I.E.S. Testing Specification, "Testing Procedures for Narrow Beam Inclosed Projectors,"2 which included zonal constants for "square" zones. A description of a recommended goniometer of type B and a brief discussion of its geometry was included in the accompanying text but without any warning that goniometers may be of type A and that in such a case, the tables would have to be used with the precautions described in this paper. In a subsequent revision of the I.E.S. Testing Specification ${ }^{6}$ the textual material pertaining to the design of the recommended goniometer and the discussion of the geometry of the zones was repeated but the column heading in the tables which had previously been designated "horizontal angle" was now labeled merely "angles," thus adding further to the possibility of misuse.

Tables of zonal constants for the type B goniometer have been republished and extended since the publication of the revised I.E.S. Testing Specification in 1936, but in all of the published sources that have come to my attention, including the I.E.S. Lighting Handbook, no mention at all is made of the fact that the tables are computed for type B goniometers. ${ }^{3,7,8,9}$ Two tables of constants for type A goniometers have appeared. ${ }^{1011}$ Bóth were published for use in the photometry of street lighting luminaires and the texts clearly described distribution photometers with type A goniometers.

It is recommended therefore that notations be made on tables of zonal constants that may be used for reference, describing the type of goniometer for which the tables are computed, and noting that if a goniometer of the other type is to be used, the tables are valid provided that the angular arguments are taken as the angles about the fixed axis of rotation.

References

1. E. W. Weinbeer: "A Simple Method of Determining the Mean Spherical Intensity of a Source of Light," Illuminating Engineer, London, Vol. 1 (1908).

2. "Testing Procedures for Narrow Beam Inclosed Projectora," Trans. I.E.S., p. 494 (June 1933).

3. I.E.S. Lighting Handbook, Second Edition, p. A-20 and p. A-22 (1952).

4. J. Bergmans and H. A. E. Keitz, "Determining the Light Digtribution and Luminous Flux of Projectors," Philips Technical Review, p. 114 (1947).

5. "General Procedure for Photometry of Incandescent Floodlights," Proc. of Assoc. of Railway Electrical Engineers, p. 133, Vol. 18 (1927).

6. "Testing Procedure for Narrow Beam Enclosed Projectors," Trans. I.E.S., p. 508 (May 1936).

7. "Specifications for Testing Lighting Equipment. Section II. Incandescent Filament Floodlights," Illuminating ENgINEERING, p. 671 (June 1941).

8. "Photometric Testing Procedure for Searchlights," NEMA Stand. ard FL8-10 (May 1945)

9. "I.E.S. Guide for the Photometric Testing of Floodlights of 10-160 Degrees Total Beam Spread," ILuUminating Enginfierina, p. 163 (March 1951).

10. G. A. Horton: Modern Photometry of Street Lighting Luminaires," Illuminating Engineering, p. 989 (November 1948).

11. "I.E.S. Guide for Photometric Testing of Street Lighting Luminaires - 1948," ILLUMINATING ENGINERRING, p. 416 (July 1949).

12. H. J. Turner: "The Use of Sinusoidal Webs in Plotting Light Distributions from Fluorescent Lamp Fittings," Light and Lighting, p. 218 (September 1949) and Corrigenda, ibid., p. 322 (November 1949).

Made in U.S.A.

Reprinted from ILLUMinating Engineering

Vol. XLVIII, No. 4, April 1953 


\section{Versatile Goniometer For Projection Photometry}

BY THEODORE H. PROJECTOR

A GONIOMETER of unusual versatility and precision has been designed and constructed for use on the 100 -foot photometric range at the National Bureau of Standards. The new gonioineter incorporates a table with $\mathrm{T}$-slots for mounting test equipment, a choice of goniometric geometries, provision for minimizing errors due to backlash and torsion, and is generally constructed for optimum rigidity and convenience.

Goniometers for general purpose photometric ranges must meet severe requirements. Test equipmnt may vary in weight over a very wide range and the distribution of weight and bulk may be quite variable. For longer searchlight ranges, accuracies of the order of $\pm 0.05^{\circ}$ or better are required and goniometers must be massive to accommodate heavy bulky searehlights without severe angular distortion.

Searchlights and other large, narrow-beam projectors are measured at the National Bureau of Standards on a 900 -foot range using a goniometer constructed from a 60 -inch searchlight, ${ }^{1}$ but smaller equipment is measured on the 100 -foot range. Because of the great variety of equipment measured on the 100 -foot range and the many types of photometric measurements made, it was necessary to design a goniometer having considerable flexibility.

As noted in the previous paper, ${ }^{*}$ conventional goniometers have two axes of rotation, one for measuring "horizontal" angle and the other for measuring "vertical" angle, although occasionally. goniometers are designed in which cone angles are measured around one axis and polar angles around the other axis. ${ }^{2}$ In the discussion of the geometry of goniometers, it was shown that goniometers measuring "horizontal" and "vertical" angles may" be divided into two types, $A$ and $B$, depending on which of the axes of rotation is fixed. The new NBS goniometer has been designed to permit use a a type A or B goniometer or, if desired, the goniometer may be used for cone and polar angle measurements.

*The Use of Zonal Constants in the Calculation of Beam Flux from Candlepower Distribution Data. (This issue, p. 189.) AUTHOR: National Bureau of Standarls, Photometry and Colorimetry Section, Washington. D. C.

\section{Construction of New NBS Goniometer}

Fig. 1 is a photograph of the new goniometer. Three axes of rotation are provided: two for measuring "horizontal" angles and one for "vertical" angles. In any given set of measurements, only one of the two "horizontal" angle rotations is used, the choice being dependent on the desired goniometric geometry or on the mounting requirements of the test equipment. The "horizontal" angle rotations are obtained with rotary tables of the type used on machine tools. The lower rotary table, with a table surface 25 inches in diameter, is attached to a heavy welded base frame. The frame is leveled and supported on eight adjustable pads which may be raised if necessary to permit moving the goniometer on its casters. An outer U-frame made of welded iron channels is mounted on the lower rotary table.

A second, inner, U-frame, construeted from lengths of iron pipe welded together, is mounted

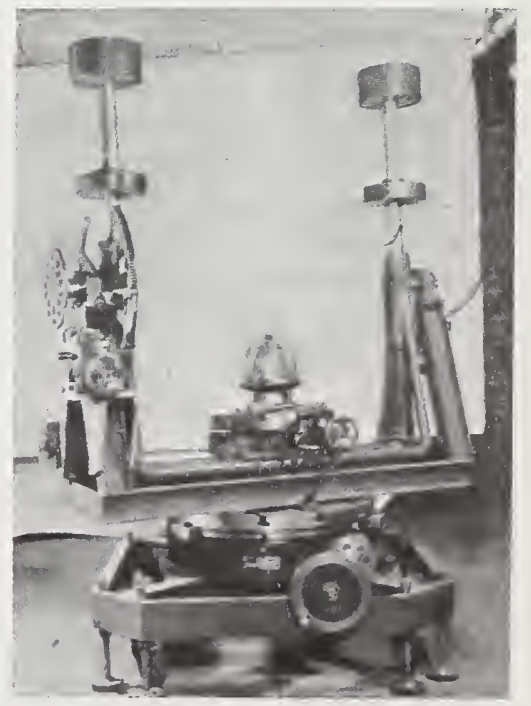

Figure 1. New NBS goniometer for $100^{\circ}$-foot pnotometric range. Three axes of rotation are provided, instead of the conventional two. By selection of the proper rotations and suitable orientation, measurements of type $A$ or type B "horizontal" and "vertical" candlepower distributions, or of distributions with respect to cone and polar angles may be made. 


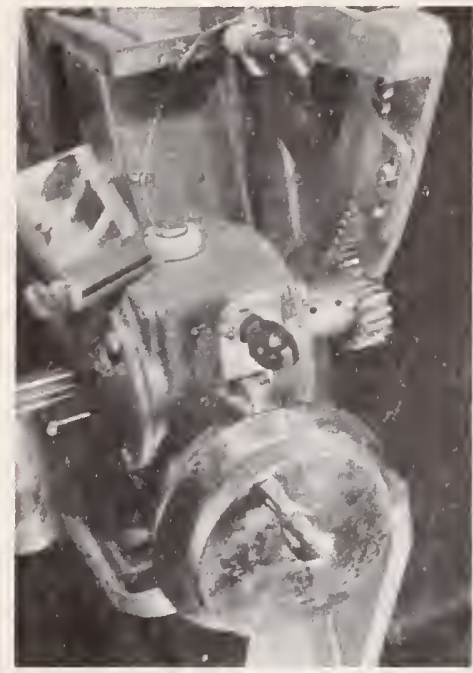

Figure 2. Close-up of "vertical" angle drive. To balance the inner $\mathrm{U}$-frame of the goniometer, the pinion gear at the right in photos, shown engaged with the sector gear in Fig. 2a (left), is disengaged by sliding it along its shaft to the position shown in Fig. 2b (right).

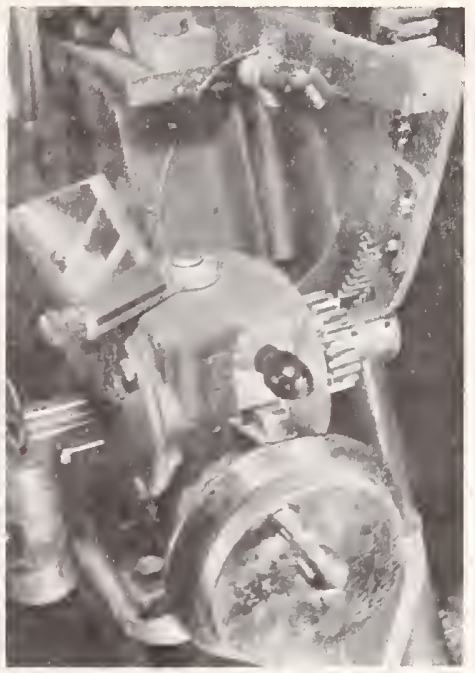

within the outer U-frame. The inner frame may be rotated around a horizontal axis extending through ball bearings set on pedestals at the tops of the arms of the outer frame. The handwheel drive for this "vertical" angle rotation is linked to the inner U-frame by a gear reducer, a pinion, and a large sector gear, all at the left in the picture. A pair of fixed counterweights is attached to the ends of tubular shafts on each arm of the inner U-frame to balance the frame about its axis of rotation. In addition a pair of adjustable weights is provided to permit balancing the frame with test equipment mounted in place.

Test equipment is usually mounted on the upper rotary table, set at the bottom of the inner U-frame, utilizing the T-slots in the 15 -inch surface plate. A collection of T-head bolts, spacers, clamping bars, etc., is provided, to facilitate rigid mounting and alignment of a variety of test equipment without special setup work.

To balance the inner frame after the test equipment is mounted on the rotary table, the pinion gear is disengaged from the large sector gear by depressing the small spring-loaded locking pin visible in Fig. 2a and sliding the pinion along its shaft until it has cleared the sector as shown in Fig. 2b. The inner frame is then freely rotated until the arms are horizontal, in which position the balaneing weights may be adjusted easily and balance determined accurately. During balancing, the small weight at the far left in Fig. 1 is disconnected from the pulley as shown in Fig. 3a. When balancing is completed and the pinion gear is reengaged with the sector, the weight is replaced on

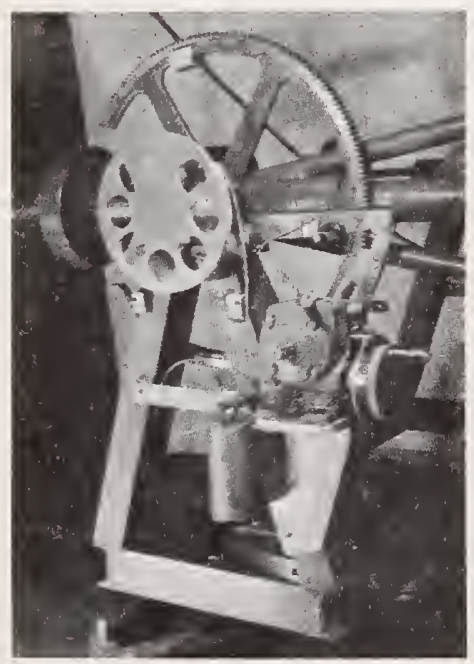

Figure 3. During balancing of the inner U-frame, the small weight used to apply a constant torque to the frame is removed from its pulley as shown in photo at left. When balancing is completed and the pinion gear re-engaged with the sector as in Fig. 2, the weight is replaced on the pulley as shown at right.

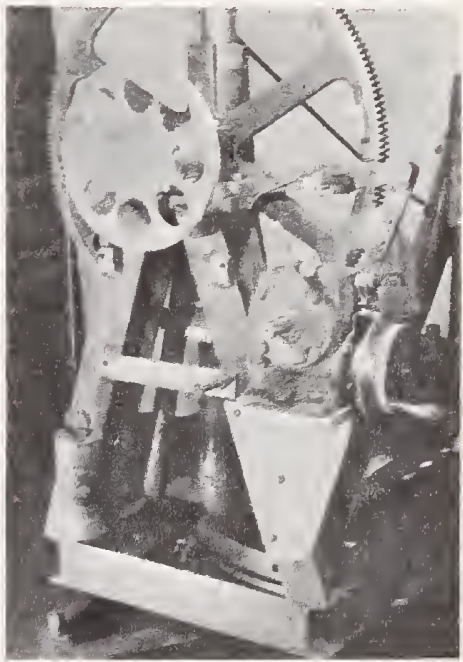


the pulley as in Fig. 3b. With the inner frame balanced and the small weight in place, a constant torque of about four pound-feet is applied to the frame at all settings of the "vertical" angle.

The two rotary tables have scale markings at the edges of their surface plates graduated in degrees. The lower table has a graduated dial, attached to its drive shaft, with scale graduations at intervals of $0.05^{\circ}$. The drive shaft dial of the upper rotary table is graduated at intervals of $0.1^{\circ}$. Fractions of the smaliest intervals on both dials may be estimated when necessary. The large sector gear is graduated on its outer face at the periphery in degrees (see Fig. 3) and a vernier index may be used to read the "vertical" angle to $0.1^{\circ}$. A small adjustable mirror is mounted on the top of the gear reducer (see Fig. 2) so that the graduations on the outside of the sector gear may be read by an operator behind the goniometer on the other side of the sector. The dial on the handwheel for the "vertical" angle drive is graduated in hundredths of a degree Small lighting fixtures provide illumination for all the angle scales.

The worm drives on both rotary tables may ke disengaged to permit free rotation of the tables as required to facilitate rotation through large angles.

\section{Goniometric Geometry}

For most photometric work, the upper rotary table is used for "horizontal" angle measuremer together with the drive for the inner frame for "vertical" angles. This combination constitutes a type A goniometer, as described in the previous paper, in which the axis of "vertical" angle measurements (the horizontal axis) remains fixed and the axis of "horizontal" angle measurements rotates as the "vertical" angle setting is changed. If the geometry of a type B goniometer is desired, then the lower rotary table is used instead of the upper one and the axis of "horizontal" angle measurements is the fixed one. Since, for most work, the choice between A or B geometry is not important, the choice of the type A geometry is usually made with the new goniometer because of the greater ease of operation of the upper table. However, apart from the choice of geometries, the lower table has been found useful occasionally, when it was necessary to mount test equipment in such a way that the upper table could not be rotated either because the test equipment was too bulky to clear the arms of the inner frame or because the inner frame was used in the mounting of the test equipment.

For cone angle measurements, the inner U-frame is rotated into the position where its arms are horizontal, and the beam from the test equipment projects horizontally through the open end of the Uframe toward the photometer. From this position of the inner frame, cone angles are set by rotation of the inner frame (the "vertical" angle drive when the goniometer is of type A or B) and polar angles are set by rotation of the upper rotary table.

\section{Accuracy}

Backlash and variable torque errors in "vertical" candlepower distributions have been greatly reduced in the new NBS goniometer by the provision of the adjustable balancing arrangement and the constant torque device. Backlash errors are minimized in conventional goniometers by rotating the goniometer in one direction only during a traverse. This is generally satisfactory for horizontal traverses when the axis of rotation is vertical and the only torque involved is that of friction in the drive system and bearings. However, in a vertical traverse, there may be considerable variation in the torque loading on a goniometer lacking a balancing arrangement, as the goniometer is rotated. In many cases, the goniometer will pass through a balance point during the traverse, with torques of opposite sense applied on either side of the balance point. Under these conditions, backlash errors may be large and may vary considerably from test to test. (It should be noted that backlash errors due to variable torque, including occasionally a reversal of the sense of the torque, may occur under certain conditions with horizontal traverses taken on a type A goniometer. In this type, the axis of rotation for the horizontal traverse may depart appreciably from the vertical. If the test equipment is asymmetrical in weight distribution about the axis, then the torque applied may vary during the horizontal traverse, depending on the amount of asymmetry, the angles traversed and the inclination of the axis from the vertical. With the new NBS goniometer. this can be avoided, if other considerations permit. by using the type $\mathrm{B}$ combination, in which the axis of horizontal traverses remains fixed vertically.)

Since the adjustable balancing weights on the new NBS goniometer are confined to positions along the arm extensions of the inner U-frame, it is not possible to balance the frame with the weights for all "vertical" angle settings if the center of gravity of test equipment cannot be made to lie in the plane of symmetry of the frame containing the axis of rotation. Balance for all angular settings may be achieved in such a case by a two-step process. First the balancing weights are moved along the arm extensions to the innermost position nearest the axis of rotation. The inner frame, with the pinion disengaged, will now hang like a pendulum with the rotary table near its lowest position and the arms nearly vertical. The departure of the arms from the vertical is a measure of the asymmetry of the center of gravity of the test unit. 
Weights are then fastened to the inner U-frame, as required, to balance the test unit about the plane of symmetry. Balance is indicated by the arms being vertical. The inner frame is then rotated $90^{\circ}$ and balancing with the adjustable weights is done as previously described. After this double balaneing operation, the goniometer has been fully balanced and backlash error throughout any vertical traverse is avoided.

Although backlash error is the most usual source of error found in goniometry, an error due to variable torsion may sometimes occur. In any goniometer similar to the new NBS goniometer the driving torque delivered to the inner U-frame is delivered to one side of the frame only. If the weight distribution of the equipment under test is asymmetric with respect to the vertical plane in which the axis of the upper rotary table rotates during a vertical traverse. then under certain conditions, a torsional stress, varying with "vertical" angle, may be applied to the inner frame, resulting in a distortion of the frame and a consequent error in the "vertical" angle measurement. Torsional errors of this type have been kept to a minimum by the rigid welded construction of the inner frame. In case of seriously asymmetrical loading, some compensation may be obtained by unequal setting of the two adjustable counterweights, but no provision has beer made for accurate compensation of this type, and the extent of the required inequality of the settings must be estimated.

Ideally in projection photometry, the equipment under test should be mounted on the goniometer in such a way that the effective center of the aperture of the unit is coincident with the center of rotation of the goniometer. (It is frequently difficult to ascertain with precision the effective center of the aperture, but the uncertainty usually involves negligible photometric error.) It is conventional on photometric ranges to set the photometer at the same height as the center of rotation of the goniometer. When the center of aperture of the test unit is located at the center of rotation of the goniometer the photometric distance is equal to the range length (the distance between the center of rotation of the goniometer and the photometer) throughout any traverse.

The first step in obtaining this coincidence is to mount the test unit on the horizontal surface of the mounting plate (with the goniometer at zero "vertical" angle) so that a vertical line through the center of aperture passes through the center of rotation. The next step is to adjust the height of the test unit so that complete coincidence is achieved.* The first step is usually easily made

*The airport boundary light shown in Fig. 1, is mounted below the center of rotation. It will be shown later that errors due to offcenter mounting are of ten negligible. simply by proper location of the test unit on the mounting surface. The second step is often more difficult. In some goniometers, the second adjustment is obtained by a built-in vertical adjustment of the mounting surface plate. Such a built-in adjustment has not been incorporated into the new NBS goniometer, since it was felt that the added convenience would be more than offset by the increased mechanical complexity of the goniometer and the difficulty of maintaining adequate rigidity in the goniometer structure to insure high angular accuracy. The permanent location of the upper rotary table is such that for most work the vertical height of the test unit with a simple mounting will fall very near the center of rotation or a few inches below it. In the latter case, the unit may be raised the necessary distance by the insertion of simple spacers between it and the mounting plate.

In order to determine the accuracy required in mounting a test unit, or conversely, to determine the errors resulting from any degree of inaccuracy in the mounting, it is customary in each case to make simple calculations based on the required photometric measurements and the geometry of the range. In almost all cases, the total error can be made negligible with relative ease. The total error consists of two parts: (1) the error in the measurement of distribution angles due to any angular difference between the range axis, or the line connecting the center of rotation of the goniometer with the photometer, and the actual photometric axis, or the line connecting the center of the aperture of the test unit with the photometer, and (2) the error due to any difference between the nominal photometric distance and the actual distance between the center of aperture of the test unit and the photometer.

The error in angle measurement may be of two types. The first type of error arises when angular measurements in a distribution are made with re spect to an external reference direction such as the range axis. The second type arises when angle measurements are referred to an axis determined in the course of the measurements, such as the direction of peak candlepower in the traverse.

Suppose for example, that a test unit is mounted on the upper rotary table in such a way that its center of aperture is four inches below the center of rotation of the goniometer. Suppose further that a mechanical axis is indicated on the test unit and that when the unit is mounted on the horizontal surface plate, this axis is horizontal. The photometer is then four inches above this axis. For a range distance of 100 feet, the candlepower measurement at what is indicated as zero "vertical" angle will actually be made at an angle above zero equal in radians to the ratio 4 inches to 100 feet $=$ $1 / 300$ radian $=0.2$ degree. If this error is not 
negligible, the height adjustment must be improved, or the angle measurement corrected.

In the event that the error in the measurement of angular interval within a traverse (the second type of error) is of primary concern in the above case, then the length and direction of the traverse and the actual angles traversed must be considered in determining the error. Suppose that a 60 -degree vertical traverse is to be taken with 30 degrees lying on either side of zero "vertical" angle. The error in the measurement of the angular interval between the center and either end of the traverse, arising from the mounting height being four inches too low will be less than 0.03 degree.

The maximum error in the photometric measurement in this example due to the difference (2 inches) between the nominal photometric distance of 100 feet and the actual photometric distance will occur at the ends of the traverse and is equal to 0.33 per cent.

Measurements of the angular accuracy obtainable with the new NBS goniometer have been made br comparing angular intervals measured with a tran- sit mounted on the goniometer with those indicated by the goniometer. The transit was mounted on the upper rotary table in place of a test unit. The results are summarized in the following table:

\begin{tabular}{|c|c|c|c|c|c|}
\hline & \multicolumn{5}{|c|}{$\begin{array}{c}\text { Maximum error, in degrees, of the } \\
\text { measurement of any angular interval } \\
\text { in a traverse when the total } \\
\text { traverse length is: }\end{array}$} \\
\hline & 10 & $3^{\circ}$ & $10^{\circ}$ & $30^{\circ}$ & $90^{\circ}$ \\
\hline Upper rotary table .............. & 0.02 & 0.02 & 0.02 & 0.03 & 0.03 \\
\hline Lower rotary table ................ & 0.02 & 0.02 & 0.02 & 0.03 & 0.03 \\
\hline "Vertical" angle drive ........ & 0.02 & 0.03 & 0.04 & 0.05 & 0.10 \\
\hline
\end{tabular}

The assistance of various members of the Photometry and Colorimetry Section at the National Bureau of Standards who contributed suggestions and advice, and of the Shops Division where the basic goniometer was constructed and many design details worked out, is gratefully acknowledged.

\section{References}

1. F. C. Breckenridge and T. H. Projector: "Construction of a Goniometer for Use in Determining the Candlepower Characteristics of Beacons," CAA Technical Development Report No. 39 (February 1944).

2. J. Bergmans aud H. A. E. Keitz: "Determining the Light Dis tribution and Luminous Flux of Projectors," Philips Technical Review, p. $114(1947)$.

\author{
Reprinted from \\ ILLUMINATING ENGINEERING \\ Vol. XLVIII, No. 4, Apr. 1953
}




\title{
PHOTOMETRY OF PROJECTORS AT THE NATIONAL BUREAU OF STANDARDS
}

\author{
L. Chernoff
}

NBS Technical Notes are designed to supplement the Bureau's regular publications program. They provide a means for making available scientific data that are of transient or limited interest. Technical Notes may be listed or referred to in the open literature.

For sale by the Superintendent of Documents, U.S. Government Printing Office Washington, D.C., 20402 - Price 25 cents 
1. INTRODUCTION -- 1

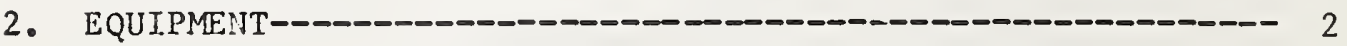

2.1 Ranges-ー- - - - - - - - -

a. 30-lleter Range----C-n- 3

b. 279-Meter Range--- 8

2.2 PAR Lampholder-- 12

2.3 Photosensors-- 12

a. Barrier-Layer photocel1--

b. Vacuum Phototube-- 15

3. CALIBPATION OF TIIE PIIOTOMETRIC SYSTEM--

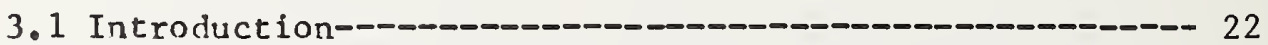

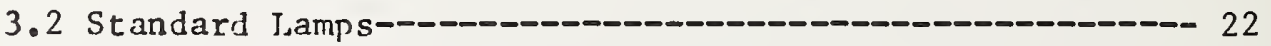

3.3 Attenuators-C--

3.4 Calibration Procedure-- -

a. External Shunt Circuit--

b. Phototube with Electrometer Amplifier Circuit---- 24

c. Zero-Resistance Circuit---

d. Special Procedures-- 26

4. TESTING PRDCEDURE--

5. ACKNONLEDGIENT--

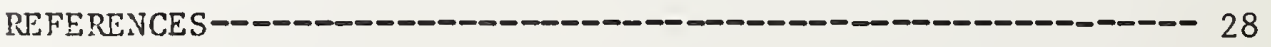


Photometry of Projectors at the National Bureau of Standards

\section{Chernoff}

A projector consists of a light source and optical system which produces a beam of light. In the photometry of projectors, the intensity of the projector as a function of angle of viewing is measured. For many years the National Bureau of Standards has conducted photometric tests on various types of projectors, and a variety of techniques and equipment has correspondingly been developed.

Photometric testing is carried out on a photometric range of which there are two at NBS: a short range on which the test distance may be varied up to a maximum of 30 meters and a longer range with a fixed test distance of 279 meters. Photometric measurements are made by comparison with a standard lamp of known luminous intensity in a specified direction. These comparisons are made with photosensors which are color-corrected by filters so that the spectral response is similar to the CIE luminous efficiency function. While precision requirements are not severe, considerable care is required to keep the experimental errors within the desired limits.

\section{INTRODUCTION}

One of the most complicated problems encountered in practical photometry is the measurement of the luminous intensity of a light projector at various angles of view. A projector consists of a light source and an optical system which produces a beam of light. A common example of a projector is the PAR-type lamp which is a sealed-reflector lamp consisting of a filament placed at the focus of a parabolic reflector with a glass cover. Another example is a light source placed at the focus of a Fresnel lens.

For measuring the luminous intensity of a projector, a photometer employing an electrical photosensor is used. The response of the photosensor is read or recorded on an electrical measuring device such as a self-balancing recording potentiometer. This response is a function of the illuminance on the face of the photosensor; the 111 uninance is expressed by the inverse-square law as follows:

$$
E=\frac{I}{d^{2}}
$$

where $E$ is the illuminance at the photosensor,

$\underline{I}$ is the luminous intensity of the 11 ght source, and

d is the distance between the light source and the photosensor. 
The photometer is calibrated by using a standard 1 amp of known luminous intensity in a specified direction oriented in that direction at a known distance from the photosensor.

Projectors are mounted at one end of a photometric range. There are two ranges for the photometric measurement of projectors at the National Bureau of Standards. On the shorter range, the distance between the projector and the photosensor can be varied to a maximum of 30 meters. On the longer range, this distance is a fixed 279 meters.

For many years the National Bureau of Standards has made photometric measurements of various kinds of projectors, particularly those units used in airfield and aircraft lighting. Since these projectors have been of many sizes, shapes, beam characteristics, and intensities, a variety of procedures, techniques, and equipment have been correspondingly developed for their measurement.

When photometric measurements are to be made, each projector must be considered individually, and it is not possible to put forth any one general method for testing them. The organization of this paper, therefore, is first tc describe the photometric equipment most commonly used, with emphasis on its application to particular types of projectors. Next, the theory and practical considerations of the various calibration procedures are discussed. Finally, there is a general description of the procedures used in these measurements.

The information and data contained herein have been obtained from many photometric tests of projectors by the Photometry and Colorimetry Section of the Metrology Division, NBS, and have specific reference to the work of this section*. Some of the material has been presented in papers 1 isted in References but has been reviewed briefly in this paper in the interest of completeness. While this paper deals specifically with photometry of projectors, the techniques and equipment mentioned are adaptable to the photometry of other kinds of light units.

\section{EQUIPMENT \\ 2.1. Ranges}

There are two ranges for the photometry of projectors at the National Bureau of Standards. The choice of range and the actual photometric setup are determined by the intensity, size, shape, and type of the unit to be tested.

*There are many publications which treat projection photometry in general. Among these are:

IES Lighting Handbook, 3d ed., Sec. 4 (Illuminating Engineering Society, New York, N.Y., 1959).

Waldram, J.M., The Photometry of Projected Light, I11. Eng. 47, 397 (1952).

Walsh, J.W.T., Photometry, Chap. XIV (Constable and Company Ltd., London, Engl and, 1953). 
Smaller units are usually tested on the 30-meter range, which is located in the attic of the East Building of the National Bureau of Standards. The photosensor and standard lamp for calibrating it are mounted on a movable "photometer bar", shown in figures $1 \mathrm{~A}$ and 1B. By moving the photometer bar, a maximum distance of 30 meters can be obtained between the unit under test and the photosensor. The standard lamp can be moved in and out of the calibration position by remote control.

Test units are mounted on a goniometer, a device which can rotate the unit through known angles about a horizontal and a vertical axis. The test unit can then be set at a given angle with respect to one axis, and when photometric measurements are made, a traverse can be taken at this angle by rotating the goniometer about the other axis, thus obtaining an intensity distribution.

The goniometer which is located at one end of the 30-meter range is shown in figure 2. Units mounted on it can be rotated about a horizontal axis through two pivot points on the U-shaped inner frame. There are two rotary tables for horizontal traverses which permit the goniometer to be ${ }_{1}$ used as either a class A or class B goniometer as described by Projector. The inner table on which the test unit is mounted is used almost exclusively, however. This table provides rotations about a secondary axis corresponding to the vertical, perpendicular to the horizontal axis which in this case is fixed. When the goniometer is operated in this manner, it is a class A goniometer.

Horizontal traverses obtained by rotating the larger table on which the outer frame is mounted result in rotations about a fixed vertical axis. When the goniometer is operated in this manner, it is a class $B$ goniometer.

The goniometer is gear-driven in the horizontal and vertical directions and is usually turned by means of synchronous motors. When a self-balancing recording potentiometer is used to record the output of the photosensor, the recorder chart of the potentiometer is driven by another synchronous motor which is powered from the same source as is the goniometer motor. Gear ratios for the recorder and the goniometer can be varied to make available several choices of speed of rotation and chart speed, and hence provide a range of angular scales (degrees per division) on the chart.

One source of error in goniometry is the backlash of the driving gears. For horizontal traverses, the errors caused by backlash are minimized by running traverses in only one direction. In the vertical direction, backlash can result from inconstant torque and from the goniometer cradle passing through a balance position in the course of the traverse. To minimize these effects, with the test unit mounted on the goniometer the pinion gear on the vertical drive is disengaged, and the inner frame 


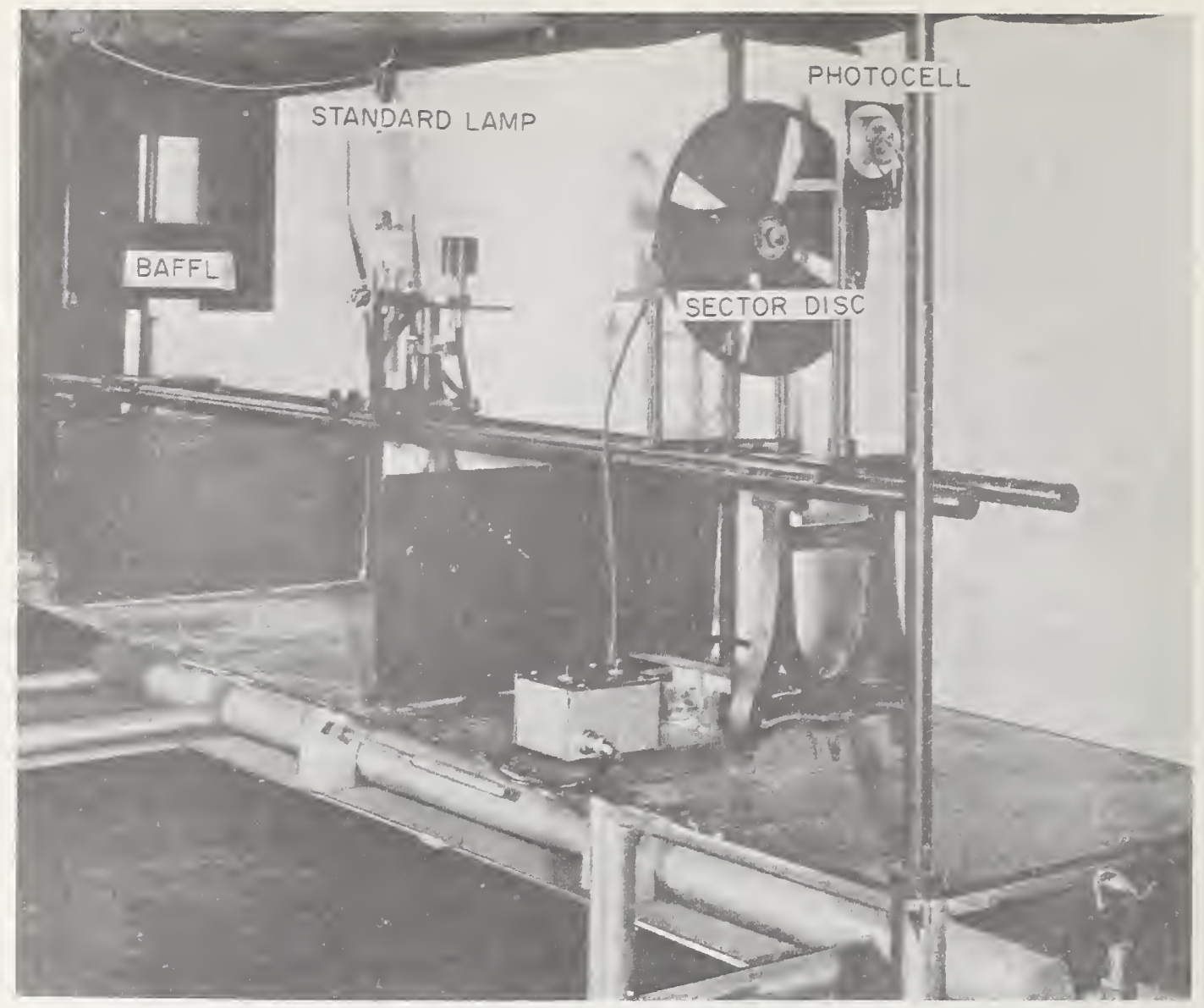

Figure 1A THE PHOTOMETER BAR OF THE 30-METER RANGE- Mounted on the bar is the equipment used in calibrating, however the shielding for stray light between the standard $1 \mathrm{amp}$ and the photocell has been removed, and a white background has been substituted for the normally black one.
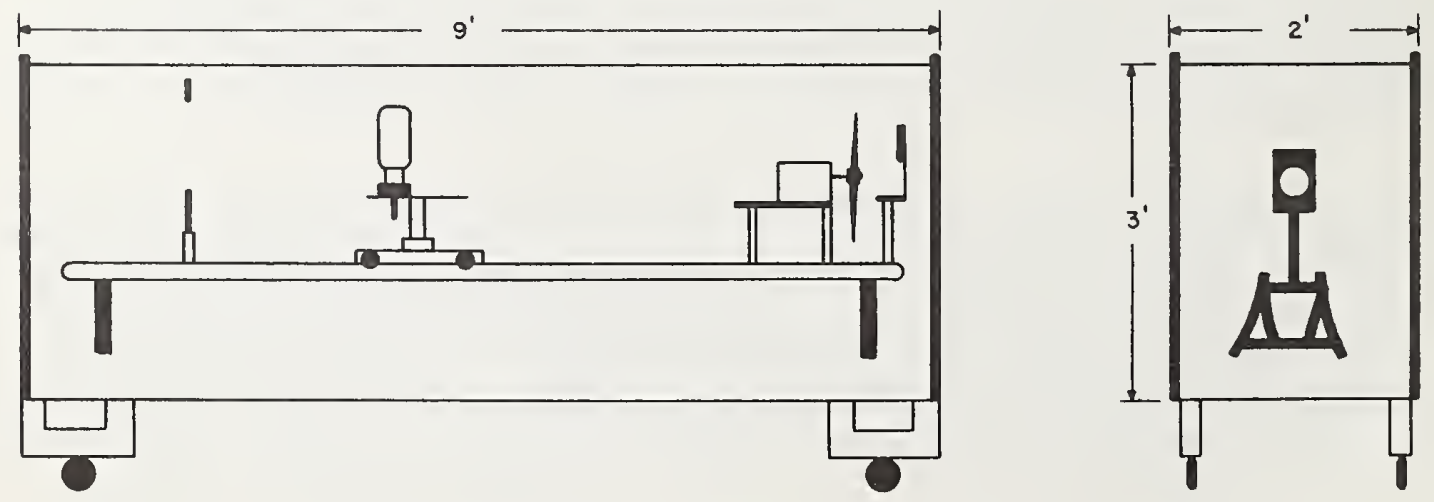

Figure 1B. DIMENSIONAL DIAGRAM OF THE PHOTOMETER BAR 


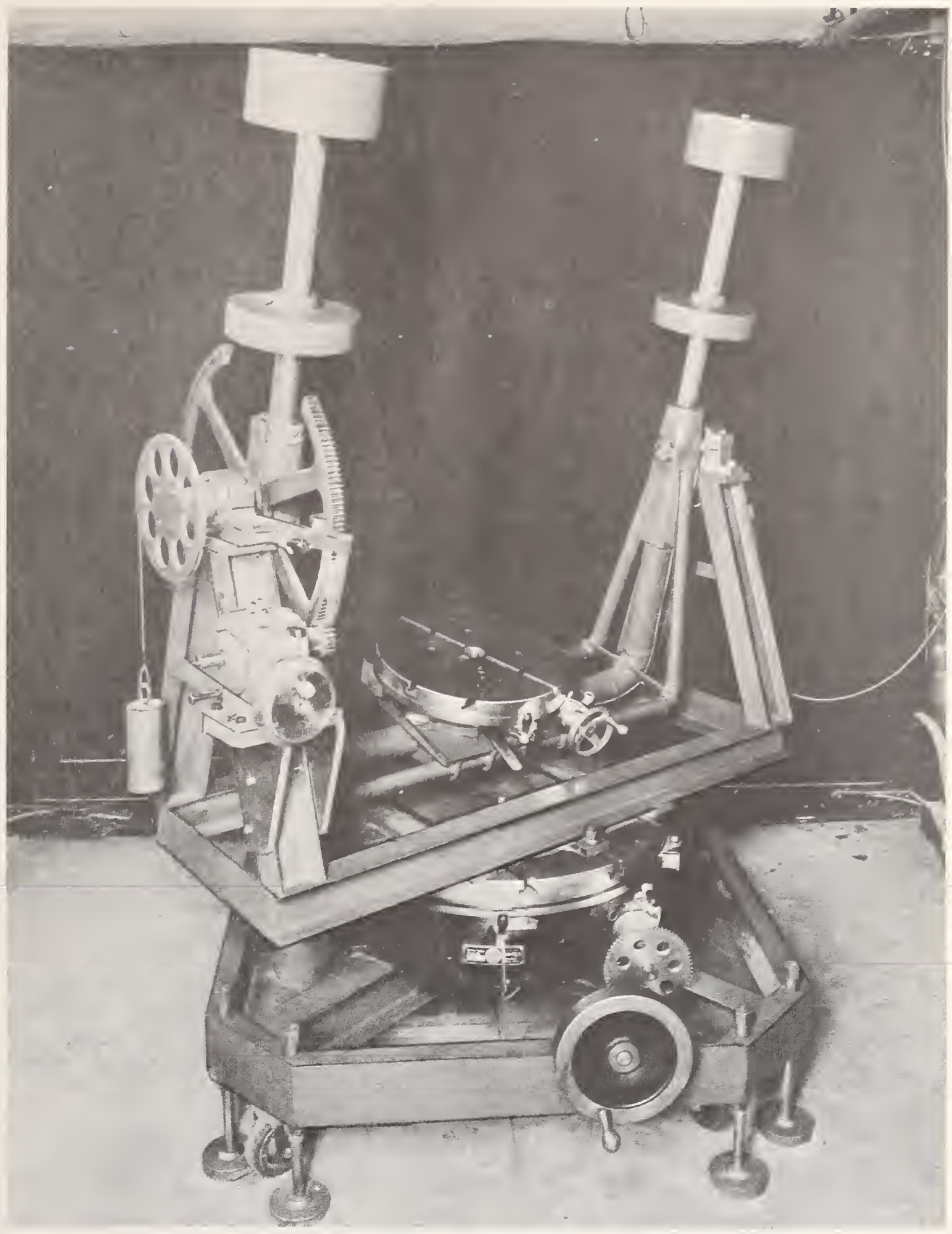

Figure 2 THE GONIOMETER OF THE 30-METER RANGE 
is balanced by means of the counterwelghts at the top of the goniometer. After this balancing, a constant torque is applied by means of a small weight at the end of a cable, which passes over a pulley connected to the vertical drive shaft. The pulley and welght are seen to the left of the goniometer (figure 2).

In order to minimize the errors caused by stray light from spurious reflections, the photometer bar is provided with a series of baffles. One of these baffles is seen in figure 1. In addition, there are adjustable baffles situated along the range between the goniometer and the photometer bar. The walls behind and around the goniometer and the background of the photometer bar are black.

The minimum test distance used in photometry of projectors is called the "minimum inverse-square distance". "The 1llumination from the light unit, measured at distances greater than this minimum, obeys the inversesquare law which is a necessary criterion for the determination of luminous intensity. The photometric distance is made greater than this minimum distance. The minimum inverse-square distance is determined by the type and size of the light source, lens, reflector, etc., and must be considered individually for each unit. If this distance is more than 30 meters (100 feet), the 30-meter range can not be used.

The case of the photometry of a searchlight with a finite sized light source and emitting a collimated beam is shown in figure 3 . For this case, the angle subtended by the optic (reflector) of the searchlight at the photosensor must be less than the angle subtended at the point on the reflector farthest from the light source by the smallest projected dimension of the light source. If the reflector is viewed through a telescope at the position of the photosensor, the reflector will then appear bright over all the aperture.

From these considerations, the minimum inverse-square distance, $\mathrm{L}_{0}$ is given by

$$
\mathrm{L}_{0}=\frac{\mathrm{ad}}{6 \mathrm{~s}} *
$$

where

Lo is the minimum inverse-square distance (in feet),

a is the distance from the point on the reflector that is farthest from the light source to the axis of the searchlight(in inches),

d is the distance from the light source to point "a" (in inches), and $s$ is the smallest projected dimension of the light source as viewed from point "a" (in inches).

To illustrate, these considerations may be applied to the photometry of two different sealed-reflector lamps of the PAR-64 type, one with a 300-watt, 6.6-ampere f1lament, and the other with a 120-watt, 6-volt(20ampere) filament. These lamps have the same overall dimensions and differ

* The number 6 appears in this equation as a result of the mixture of length units. 


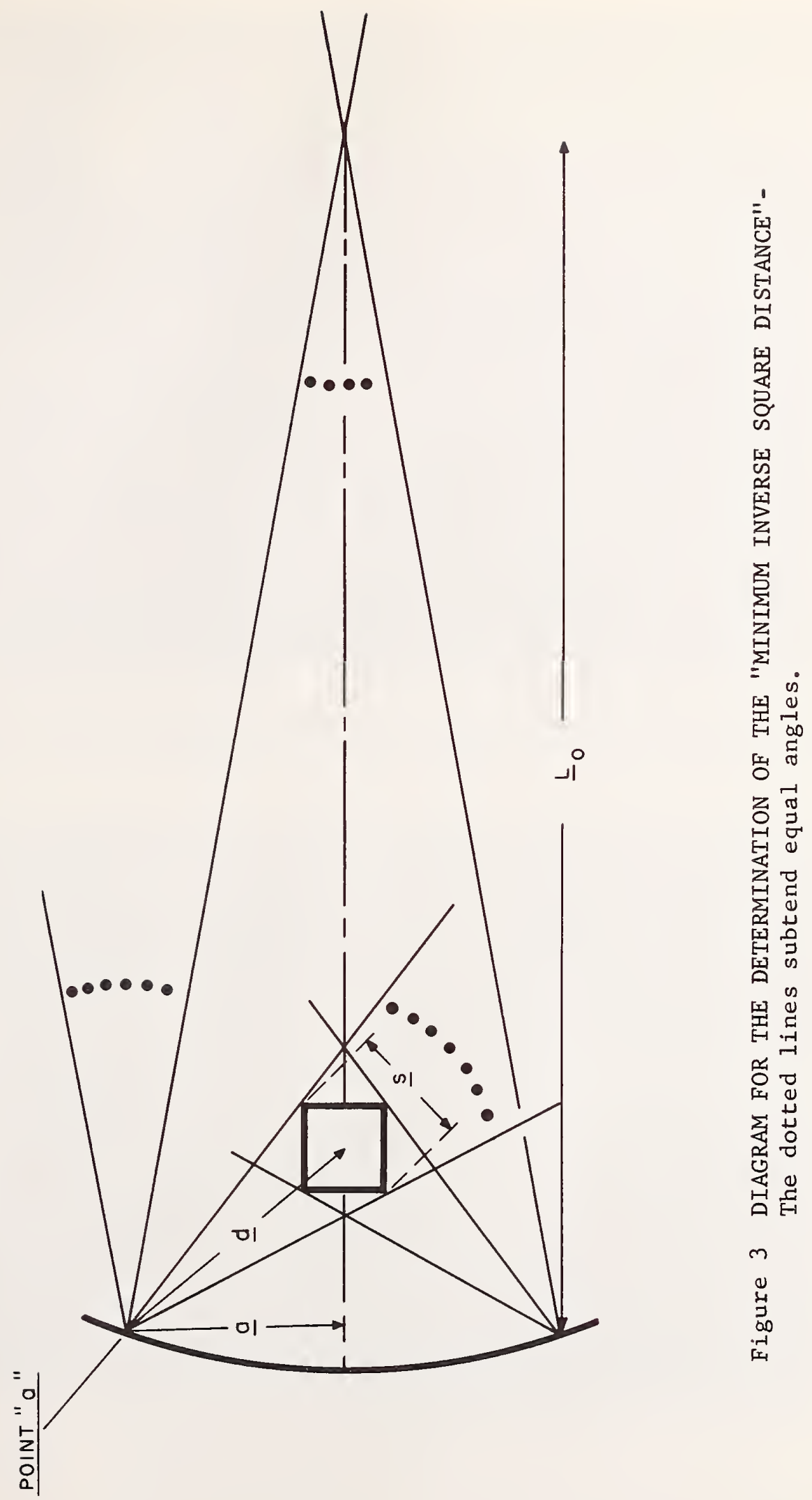


only in the size and construction of the filaments. Both lamps have clear covers and parabolic reflectors, and both emit collimated beams of 1 ight. For both lamps, the dimension $a$ is 3.7 inches and the dimension d is 3.8 inches.

The filament of the 300-watt lamp is of the CC-6 type; that is, the filament wire is wound in a helix, and the helix is again wound into a larger helix. The axis of the larger helix is perpendicular to the axis of the reflector. The smaller helix is wound so tightly that its diameter can be considered the smallest dimension of the light source, and its projected dimension, dimension $s$, is this diameter, 0.033 inch. $L_{0}$ is therefore 70 feet, which permits the lamp to be photometrically measured on the 30 -meter range.

The filament of the 120-watt lamp is of the C-6 type. The filament wire is wound into a single helix. This helix is wound so loosely that the single turns of the coil can be discerned. Therefore, the diameter of the filament wire itself, 0.020 inch, is considered its smallest projected dimension. $L$ is 120 feet, and on this basis this lamp should be photometered on the 279-meter range described below. However, the separate turns of the helix cannot be discerned from the extreme regions of the reflector. For the region of the reflector where the separate turns of the helix can just be discerned, both a and $\underline{d}$ are about 2.2 inches, and $L_{0}$ computed for this region is 40 feet. At the extreme regions of the reflector, the diameter of the helix, $0.083 \mathrm{inch}$, is the smallest projected dimension of the light source, and $J$ is 28 feet. This lamp could also, therefore, be photometrically measured on the 30-meter range.

The above discussion for the determination of the minimum inversesquare distance is exact only for axial measurements. For measurements off the axis, test distances of two or three times these computed minimum inverse-square distances are sometimes required but are often impractical. Measurements near the axis are usually the most important in the testing of projectors; therefore, test distances only slightly greater than the computed minimum inverse-square distances are necessary for most practical purposes.

\section{b. 279-Meter Range}

Larger lights are usually tested on the 279-meter range. The photosensor and the standard lamp are located in an enclosure on the roof of the Industrial Building at the National Bureau of Standards. The range has been designed with the photosensor facing south so that during the day the photosensor views the shaded side of the building in which the goniometer is located and the shaded sides of the intervening stray-light baffles. A baffling tube and two additional stray-light baffles are situated on the same roof. This arrangement can be seen in figures $4 \mathrm{~A}$ and $4 \mathrm{~B}$. 


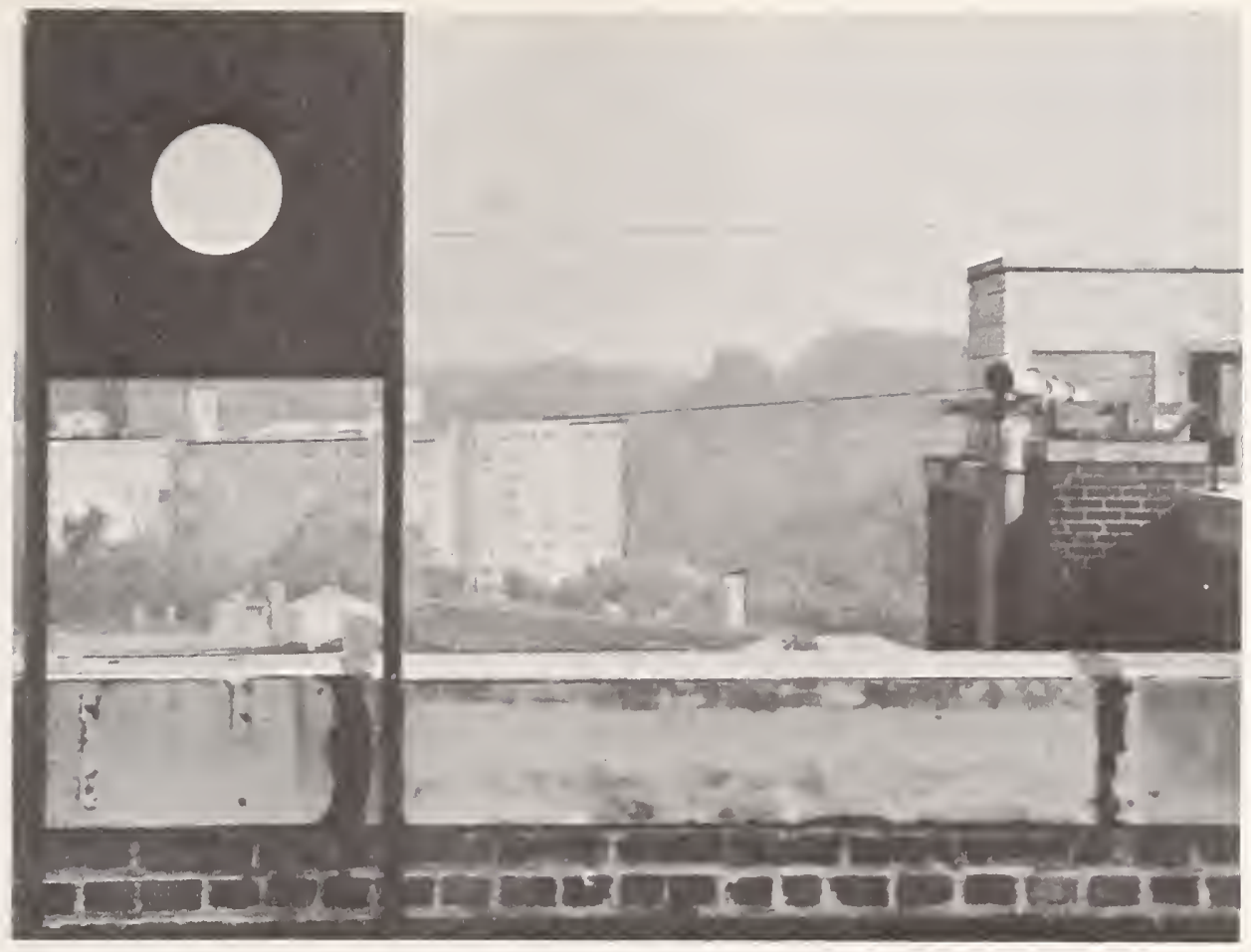

Figure 4A THE BAFFLING TUBE OF THE 279-METER RANGE- Seen are the baffling tube and one of the two other baffles.

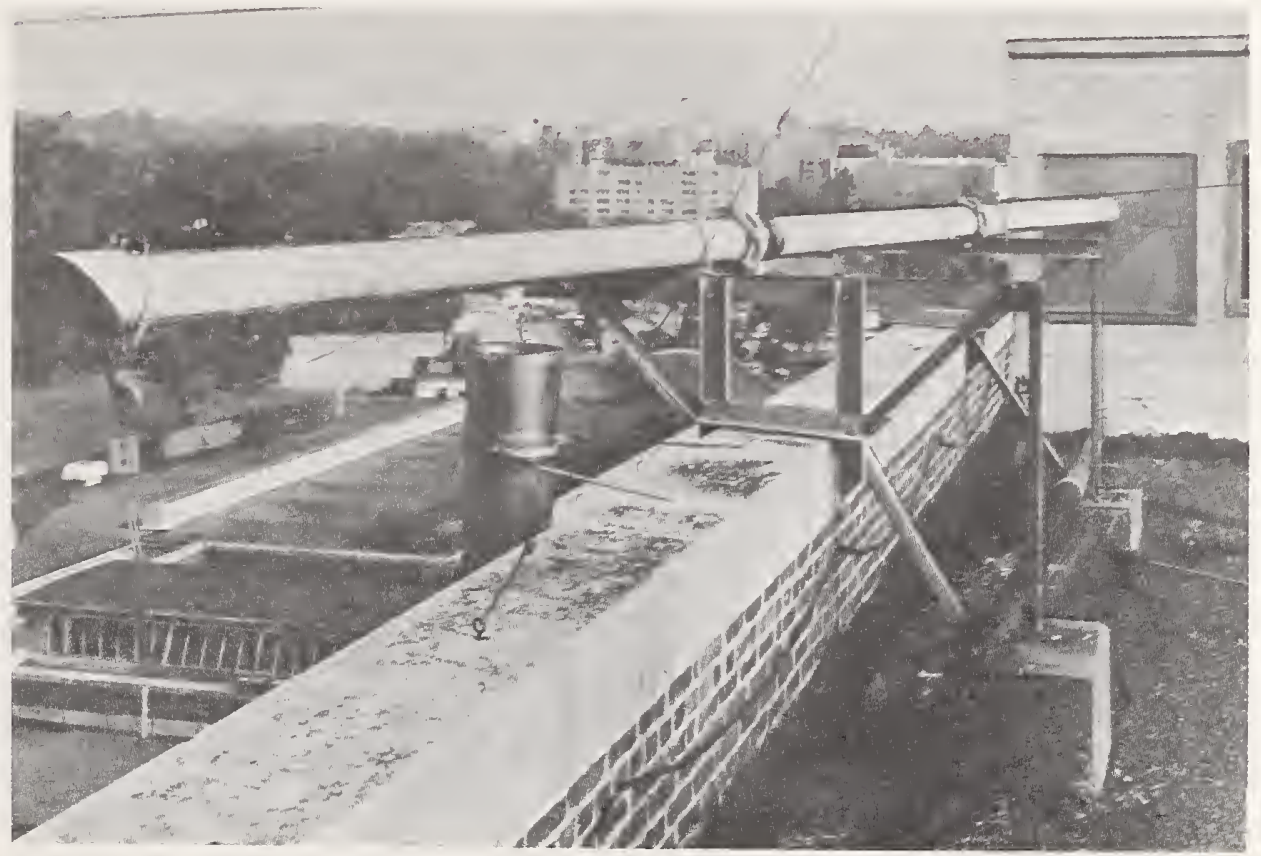

Figure 4B A CLOSE-UP VIEW OF THE BAFFLING TUBE- The front of the tube is covered with a removable protective shield. 
The gonlometer, recording potentiometer, and all electrical controis are located in a room on the top floor of the Computer Laboratory. The distance between the photocell and the goniometer is fixed at. 279 meters.

This goniometer was constructed from an available 60-inch coastdefense searchlight and is shown in figure 5. To obtain greater precision in the angle settings, the old gearing, which had a great deal of play and friction, was removed, and more precise gearing was installed. Angles are measured through the gears because they can thereby be more conveniently read than if they were measured directly on the goniometer table. The accuracy achieved by this procedure is sufficient for the purpose. Tests have shown that the angles for small traverses can be set with an error of less than $0.03^{\circ}$ in both horizontal and vertical settings.

Units mounted on this goniometer can be rotated about a fixed vertical axis and about a secondary axis perpendicular to it. Errors resulting from inaccurate vertical centerings of the test unit are generally negligible; e.g., if a unit is mounted 2 feet below or above the center of rotation of the goniometer, for a vertical rotation of $30^{\circ}$ there will be an error of 0.2 percent in the candlepower reading due to the change in photometric distance, and a corresponding error in the vertical angle readings of $0.015^{\circ}$. The goniometer is so sturdy that there is very little flexure resulting from inconstant torque. It was found that under an extremely asymmetrical loading of $a_{4}$ test unit the angular error resulting from flexure was within $0.04^{\circ}$.

This goniometer is usually driven by hand al though motor drives are installed. An " $x-y$ " recorder is used to trace the goniophotometric distribution. The chart drive of the recorder, a servo-motor, is controlled by a self-balancing potentiometer. A voltage signal from a potential divider attached to the drive mechanism of the goniometer is connected to the input of the y-axis amplifier, thus making the chart position a function of the goniometer angle.

The baffling tube and some of the other stray-light baffling seen in figures $4 \mathrm{~A}$ and $4 \mathrm{~B}$ block out all but a 6 -foot diameter area of view at the goniometer. This baffling is effective enough to permit much of the photometry on the 279-meter range to be conducted during the day. The illumination from the background is measured at the time of each test and has been found to be equivalent to or less than that of a light with an intensity of about 1 kilocandle. Correction is made for background illumination in making photometric measurements. During the course of a traverse, the background illumination will usually not vary by more than 10 percent. Therefore, as a general rule, any light having a peak intensity over 100 kilocandles can be photometrically measured during daylight.

A barrier-layer photocell is usually used as the photosensor on the 279-meter range. A barrier-layer photocell is mounted in a fixed position in the enclosure at the receiving end of the range. 


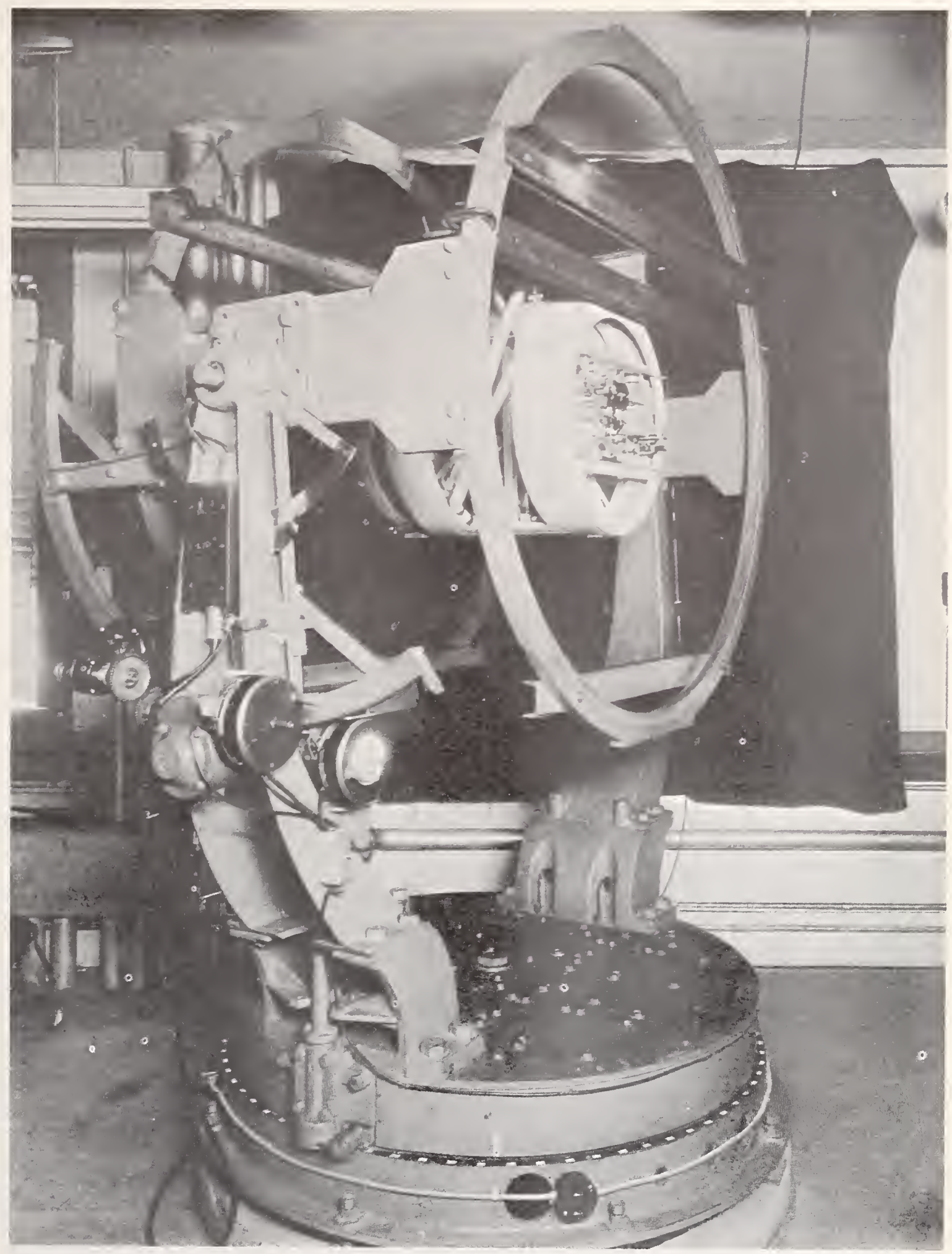

Figure 5 THE GONIOMETER OF THE 279-METER RANGE- An aircraft searchlight is mounted on the goniometer 
As shown in figure 6 the standard 1 amp and photocell are so arranged that they do not have to be moved for calibration or test measurements. The photometric axes from the standard lamp and from the test unit are respectively $5^{\circ}$ left and right of an axis perpendicular to the face of the photocel1. The response of the photocell used in the 279-meter range was tested for symmetry and was found to be symmetric with respect to these angles of incidence of the light illuminating it. Therefore, such an arrangement introduces negligible experimental errors.

A correction for the transmittance of the 279-meter air path must be made. The transmittance at the time of photometry is measured by means of the transmissometer described by Douglas and is used as a correction in the calibration of the photometer.

\subsection{PAR Lampholder}

A special holder for PAR-type lamps is used to facilitate the mounting of lamps of this type. Since PAR-type lamps are usually tested on the 30-meter range, the holder for PAR-type lamps has been designed to be mounted easily on the goniometer of this range. It is shown in figure 7. A set of removable mounting rings makes it possible to mount any of the several sizes of PAR lamps on the holder.

The holder contains a telescope which is used to align the holder with a mark at the other end of the range so that the axis of the PAR lamp reflector will coincide with the photometric axis. It is therefore possible to remove the holder from the goniometer and to replace it at some future time, aligned as before.

\subsection{Photosensors}

The photosensors used in the photometric measurements are constantcurrent devices which produce currents proportional to the illuminance on their faces. All photosensors used are color-corrected by means of optical filters in order to make their spectral response similar to the CIE spectral luminous efficiency function. Two different types of photosensors are in general use, the barrier-layer photocell and the vacuum phototube. Although photomultiplier tubes are available, there has been no occasion to use them for the photometry of projectors at the National Bureau of Standards laboratories in the last few years.

\section{a. Barrier-Layer Photocel1}

The barrier-1ayer photocell, a solid-state photoelectric device, is used in most of the photometric measurements. In the selection of a photocell for photometric testing, several cells which have been colorcorrected by means of color-correcting filters are checked for linearity and similarity of spectral response to the CIE luminous efficiecy function. In order to check the adequacy of the color-correction, the luminous transmittance of several colored filters for light of a specified 


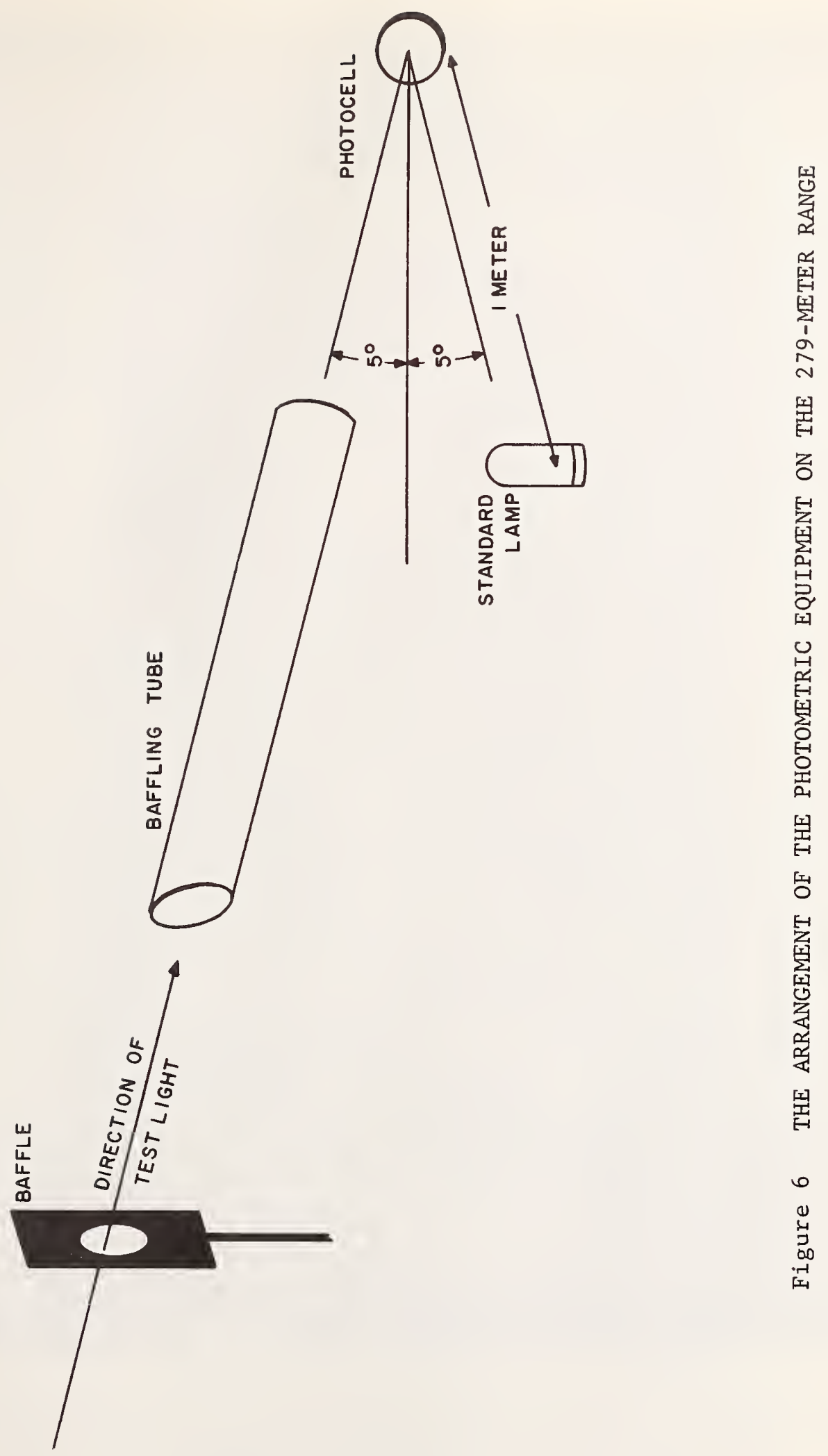

$601-13$ 


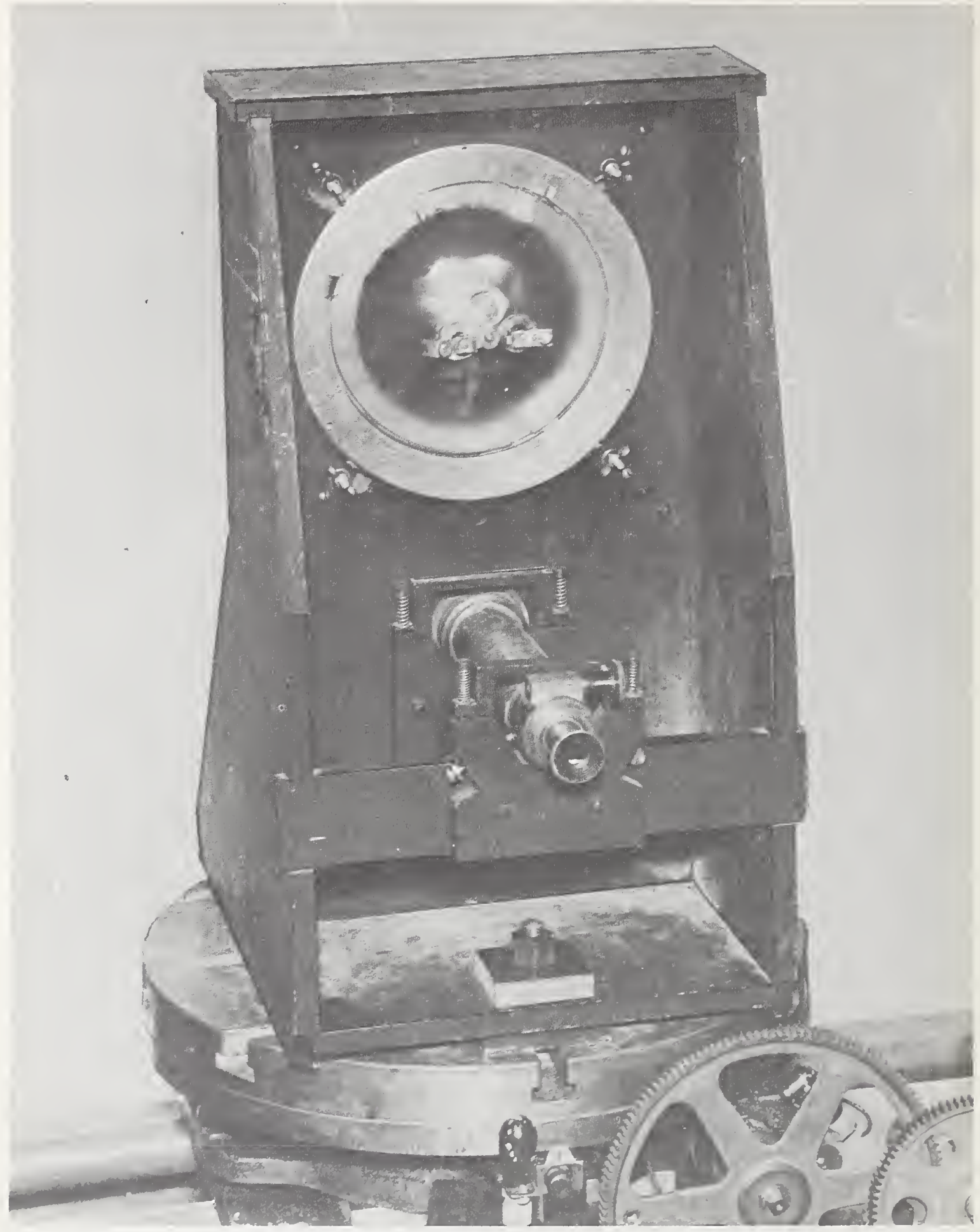

Figure 7 A REAR VIEW OF THE PAR LAMP HOLDER- Seen are the telescope for aligning, the mounting ring, and the back of a PAR-56 lamp which has been mounted on the holder. 
color temperature is measured with these photocells. These measurements are compared with transmittances determined from spectrophotometric measurements. The results of one such series of measurements, using a lamp operating at Source A (color temperature $2854^{\circ} \mathrm{K}$ ) are given in table $\mathrm{I}$.

The photocells with good color response are then tested for linearity; the one most nearly linear in its response is selected for use. The response of the photocell being used at present, cell number 3 of table $I$, was found to be linear to better than $0.1 \%$ in the most useful range, which is sufficiently linear for photometric testing. Here, linearity was tested using several standard lamps of known horizontal intensity in turn at distances from 1 to 30 meters from the photocell. The results of the linearity measurements of this photocell are shown in figure 8.

The barrier-1ayer photocell is used with two different circuits. For most photometric work with this type photocel1, an external shunt is used. The voltage drop across the shunt is amplified by means of a linear amplifier, and the output of the amplifier is recorded on the recorder chart of a self-balancing potentiometer. This circuit is shown in figure 9. However, when greater precision is required in the measurements, or when the illumination of the face of the photocell is either very large or very small, a "zero-resistance" circuit is employed, and jntensity measurements are made by using a Kohlrausch potentiometer.

\section{b. Vacuum Phototube}

For flashing 1 ights of short flash duration such as condenser-discharge lights, and for lights of very low intensity, a G.E. type PJ-14B vacuum phototube is used. The phototube with its power supply is housed in a metal box as shown in figure 10. The phototube is color-corrected by means of a specially designed filter; the accuracy of this color correction is indicated in figures $11 \mathrm{~A}$ and $11 \mathrm{~B}$.

A d-c electrometer-amplifier is used to amplify the output of the phototube. The electrometer has been modified so that it may also be used as a null-detector for a self-contained potentiometer when greater accuracy is necessary.

In the photometry of flashing lights, it is desirable to compute the effective intensity* of the flash, which is determined from a measurement of the average intensity of the flash. In order to read the average intensity, a resistance-capacitance network is placed across the phototube so that the photometer circuit will have a suitable time constant. A diagram of the photometer circuit employing the PJ-14B phototube, together with such a resistance-capacitance network, is shown in figure 12. The combination of $d-c$ amplifier and phototube is useful in measuring steady illuminations as low as 0.00001 footcandle.

* The effective intensity of $\bar{a} \bar{f} \bar{l} \overline{a s h} \overline{i n g} \bar{l}$ ight is equal to the intensity of a steady-burning light of the same color which will produce the same visual effect under identical conditions of observation. 


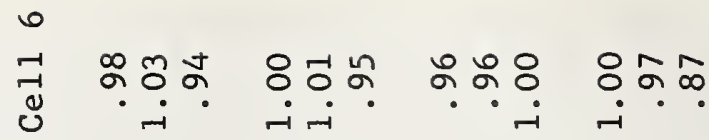

in

નૅ

离

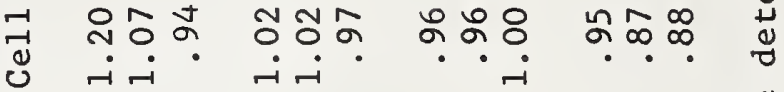

䒕

N

$m$

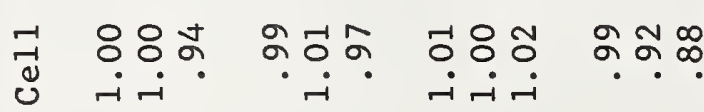

-

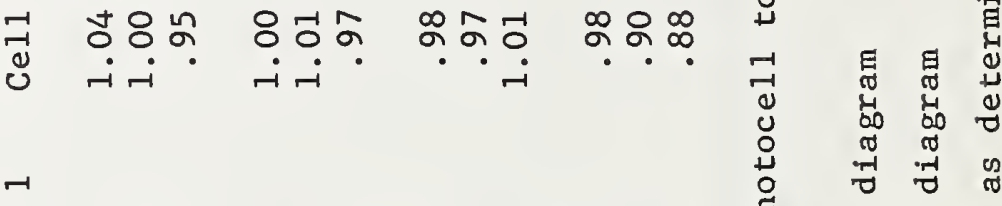

נj

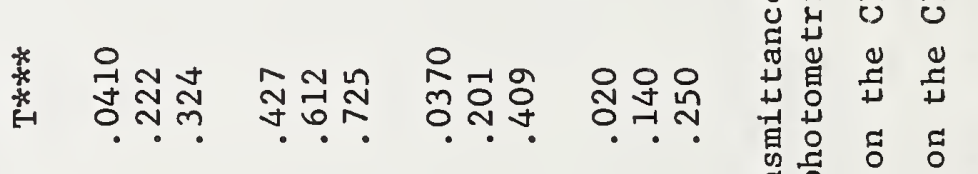

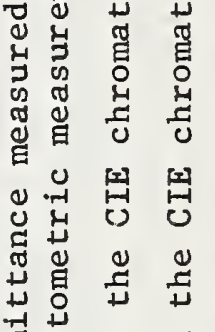

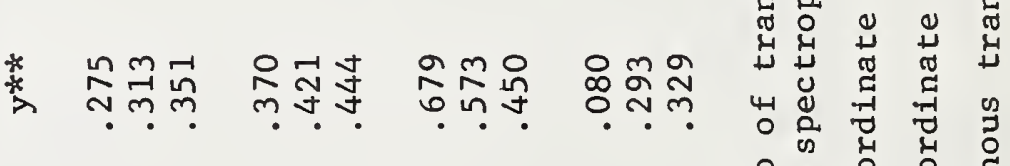

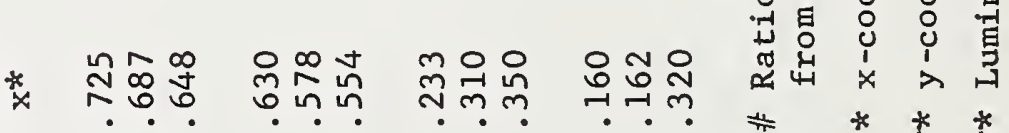

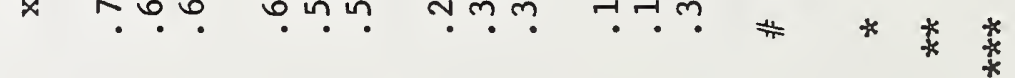

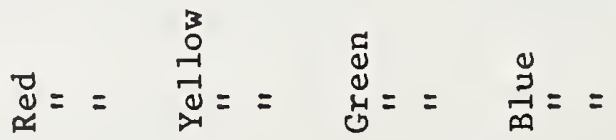




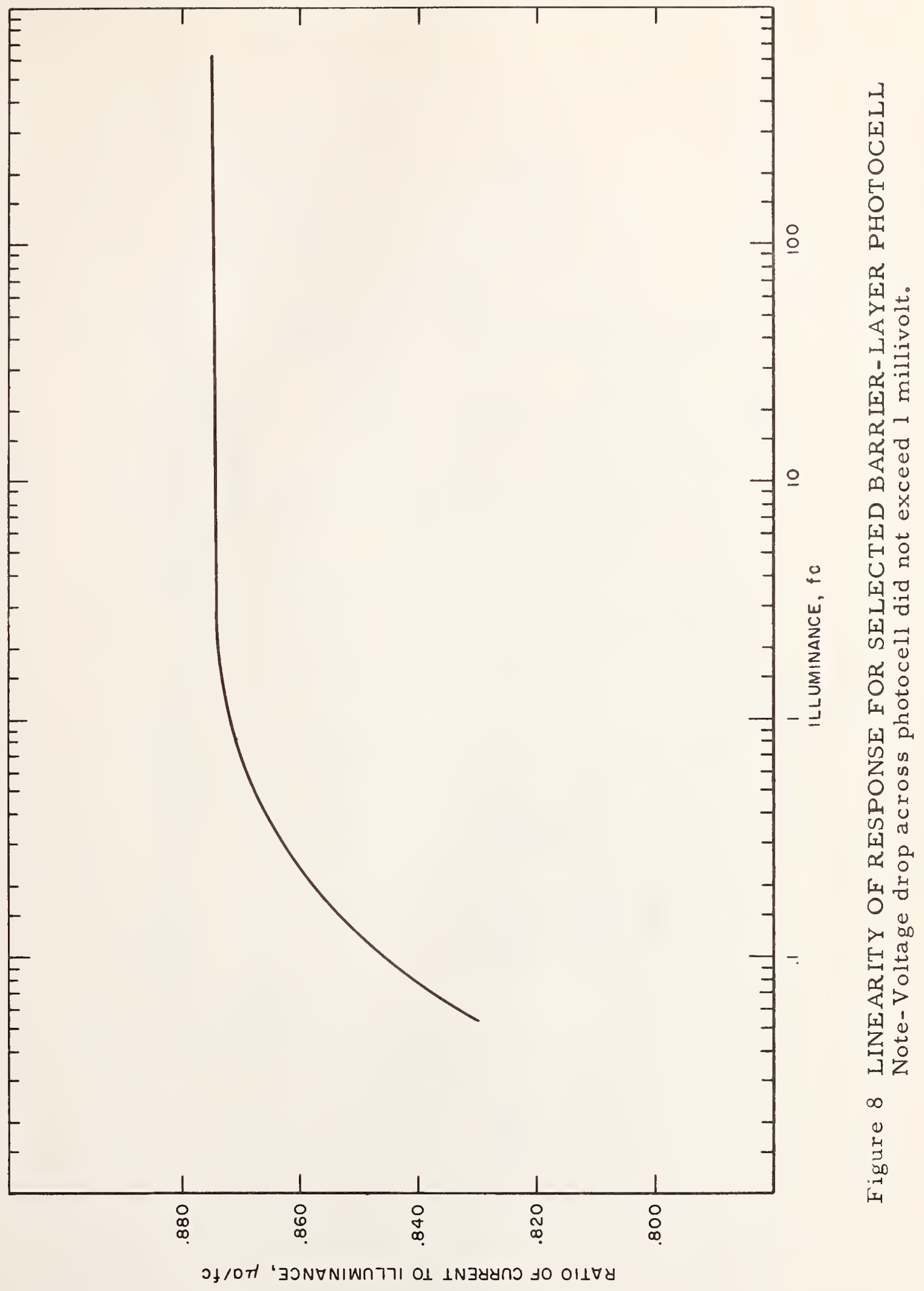

$605-17$ 


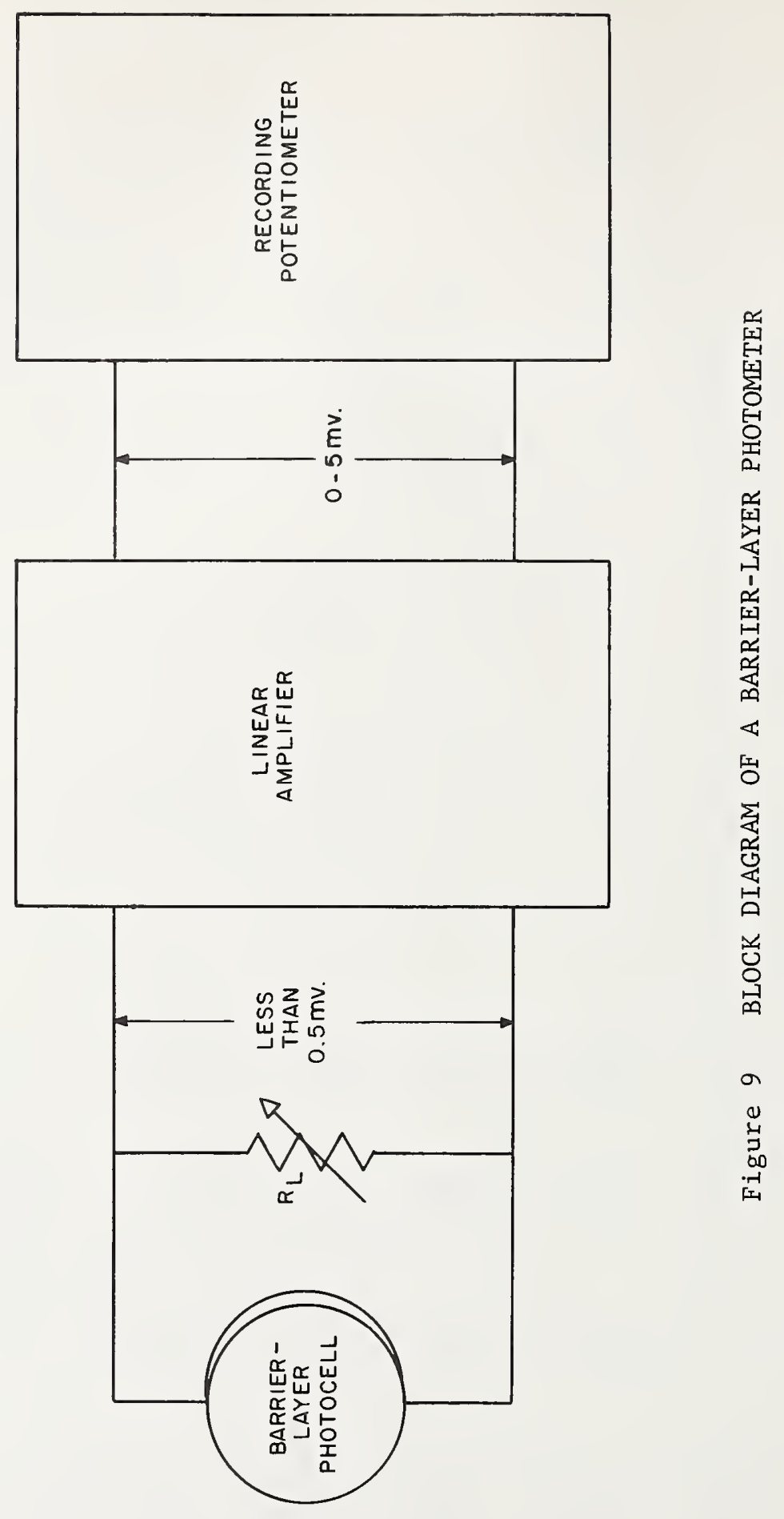

$606-18$ 


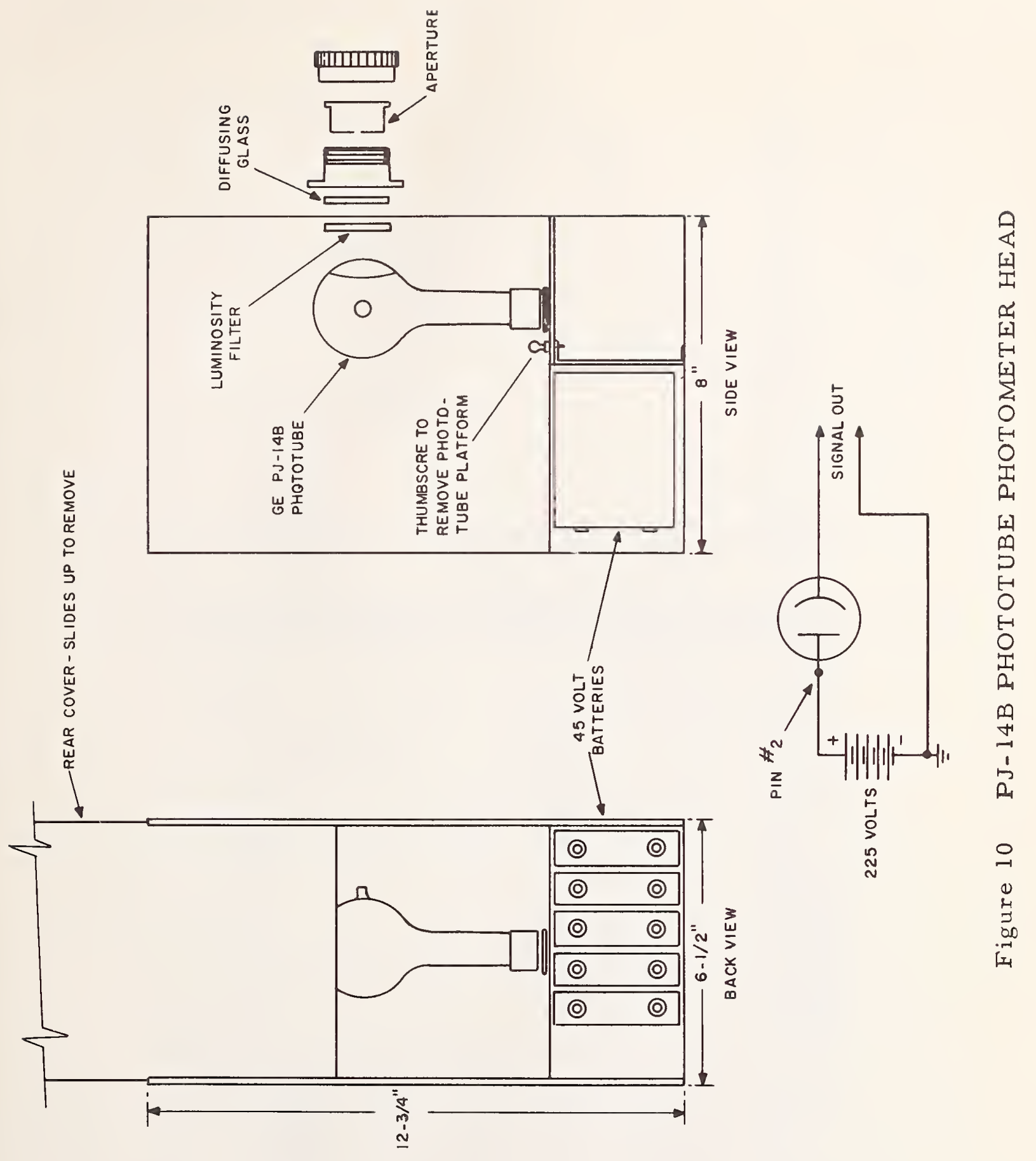




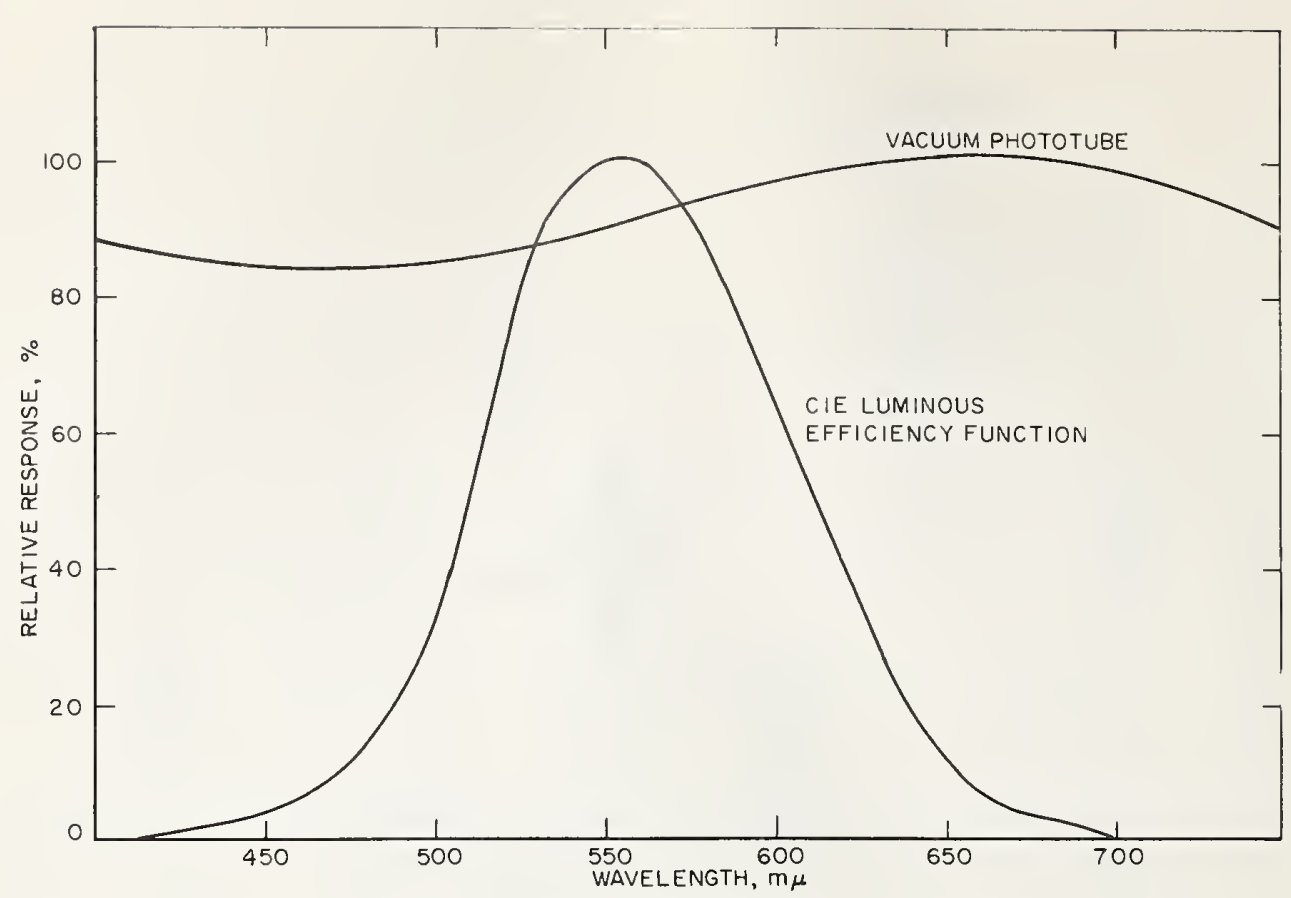

Figure 11A RELATIVE SPECTRAL RESPONSE OF UNCORRECTED PJ-14B VACUUM PHOTOTUBE AND CIE LUMINOUS EFFICIENCY FUNCTION

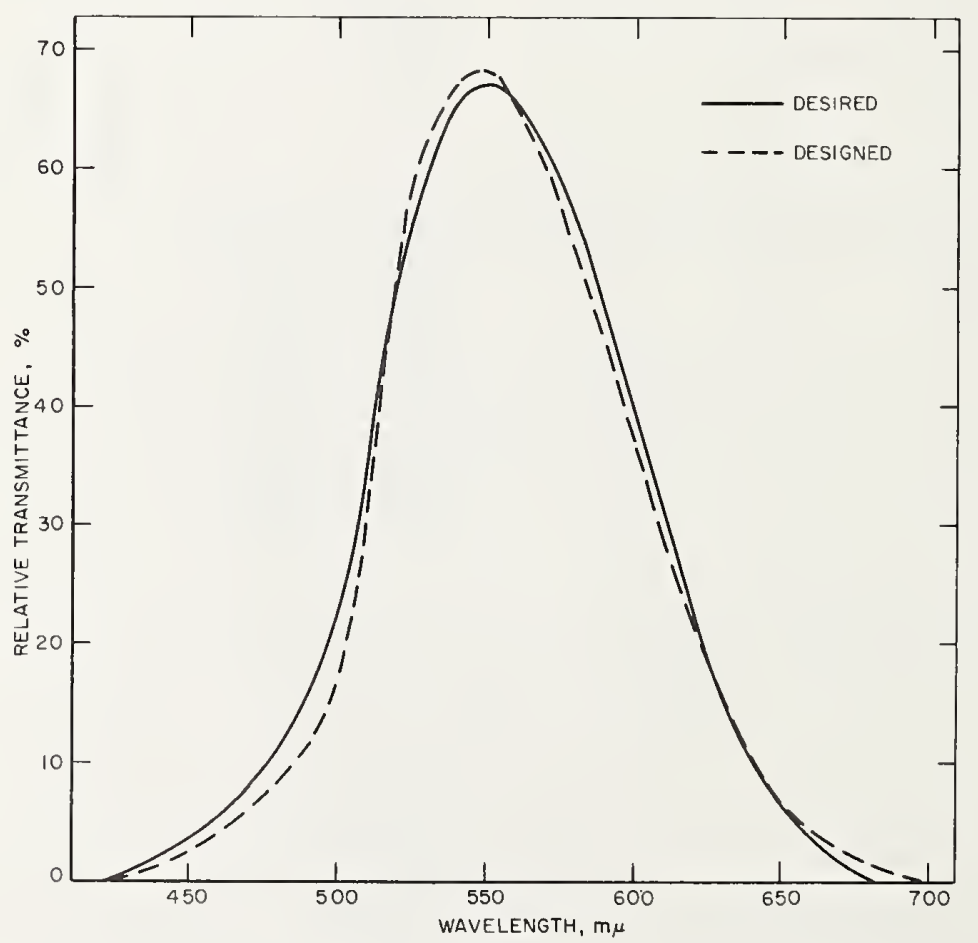

Figure 11B SPECTRAL TRANSMITTANCE OF DESIRED AND DESIGNED COLOR-CORRECTING FILTER 


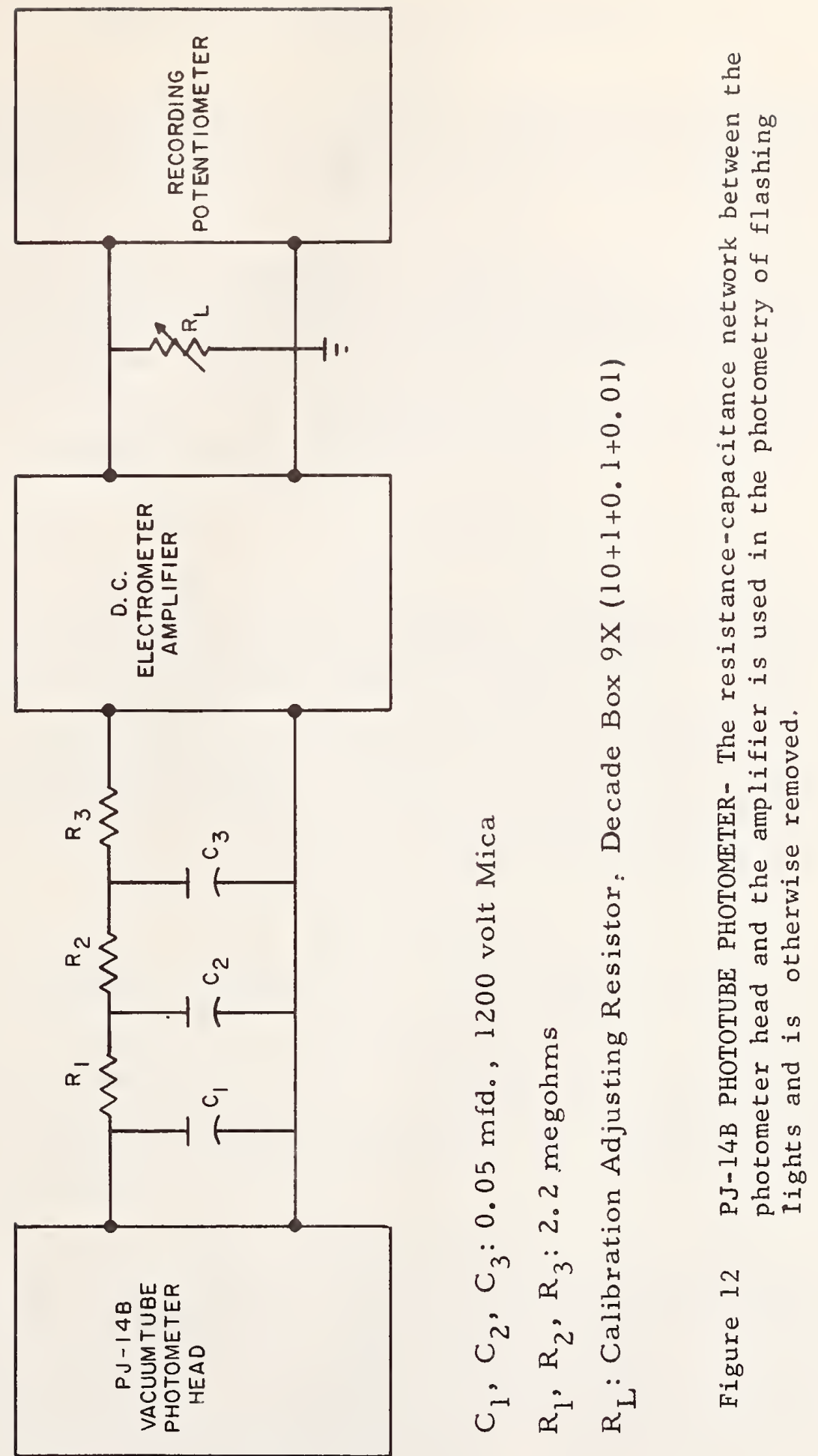


Lamp standards of luminous intensity are used for calibrating the photometric testing equipment; a separate calibration is made before each test, and a record is kept of the photometer sensitivity in order to detect any irregularities. The illumination on the photosensor produced by the standard lamp is adjusted to some typical value of the illumination produced by the test light, usually in the range of $75 \%$ to $100 \%$ of the peak illumination produced by the test light. This procedure minimizes errors resulting from nonlinearity of the response of the photosensor. The adjustment of the illumination of the standard lamp on the photosensor is accomplished by varying the distance of the standard lamp from the photocell and by using optical attenuators. The photometer is usually calibrated so that it is direct reading in luminous intensity.

\subsection{Standard Lamps}

The standard lamps used are "working standards" whose luminous iftensity in a given direction has been determined at a given voltage.
Standard lamps are available ranging in intensity from about 8 to 900 candles. When a colored light is being tested, a filter is placed between the standard lamp and the photosensor, which results in a standard lamp-filter combination having approximately the same spectral characteristics as those of the light to be tested. This procedure minimizes errors resulting from inadequate spectral correction of the photosensor. In this procedure, a standard lamp of known color temperature as well as of known luminous intensity is needed.

\subsection{Attenuators}

Sector disks are almost always used for light attenuation, although neutral filters are also available. A sector disk is usually placed between the standard lamp and the photosensor to calibrate the photometer for the proper range of illumination. However, when the intensity of the light being photometrically measured is unusually high, the sector disk may be used to attenuate the illumination from the test 1 ight. The range of sector disks available is from $1 \%$ to $80 \%$ transmittance.

When a sector disk is used, it is placed within a few inches of the photocell in order to reduce error from stray light. The disk is rotated at a few hundred revolutions per minute, which is fast enough to minimize error from apparent flicker. When a high illumination is attenuated by a sector disk of low transmittance, there is an error which results from only one part of the photocell being illuminated at a time; this error is successfully eliminated by placing a condenser of about $4 \mathrm{mfd}$. across the output of the photocell. (In utilizing this technique, one must be careful to obtain a capacitor which does not itself generate an emf.) 


\subsection{Calibration Procedure}

The calibration involves illuminating the photosensor with light from a standard lamp placed at a given distance from the photosensor, and then adjusting the sensitivity of the photometric system to some desired value.

If 1 is the photosensor current,

$\bar{I}$ is the intensity of the light illuminating the photosensor, and $\underline{D}$ is the distance from the test unit to the photosensor, then, since the photosensor produces a current proportional to the illuminance on its face,

$$
i=k I / D^{2}
$$

where $k$ is the sensitivity of the photosensor.

It is usually convenient to calibrate the photometer to be direct reading, so that

$$
I=N \delta
$$

where $\delta$ is the reading of the potentiometer of the measuring circuit, and where $\mathrm{N}$ is an integral power of 10 or the product of an integer. usually 2 or 5 , and an integral power of 10 .

The photometer is then calibrated by using a standard 1 amp of known horizontal luminous intensity. If

I is the luminous intensity of the standard 1 amp,

$\mathrm{D}^{\mathrm{S}}$ is the distance of the standard lamp from the photosensor,

$\delta_{s}^{s}$ is the potentiometer reading and $i$ is the photosensor

current when the photosensor is 111 uminated by 11 ght from the standard lamp placed at the distance $\underline{D}_{s}$ from the photosensor, then

$$
1_{s}=k I_{s} / D_{s}^{2}
$$

and since the potentiometer reading is proportional to the photosensor current,

$$
i_{s} / \delta_{s}=1 / \delta
$$

Combining (1), (2), (3), and (4),

$$
\delta_{s}=I_{s} D^{2} / N_{s}^{2}
$$

Calibration is accomplished by the following procedure: $I_{\text {s }}$ and $\mathrm{D}_{\mathrm{S}}$ are chosen so that $I^{\prime} / \mathrm{D}$ will be approximately equal to $\mathrm{I} / \mathrm{D}^{2}$, where
$\mathrm{I}$ is some typical value of the intensity of the light to be tested. A suitable value of $\mathbb{N}$ is then selected. Calibration to make the photometer direct reading is completed by one of the three following procedures, depending on the photoineter circuit used.

\section{a. External Shunt Circuit}

A diagram of this circuit is shown in figure 9. In this circuit 


$$
\delta_{s}=\frac{R_{L} S k I}{D_{s}{ }^{2}}
$$

where $S$ is the sensitivity of the photometer circuit and $R_{L}$ is the resistance of the shunt.

Calibration, therefore, requires that, with the photocell illuminated by light from the standard lamp, the external shunt resistance is set so that the potentiometer indicates the value $\delta$ given in equation (5). The other parameters of the calibration are usually chosen so that the shunt resistance will be of the order of a few ohms. This order of resistance is used as it is large enough to be set accurately, and small enough so that the voltage developed across the photocell will not cause the photocell to respond nonlinearly. The practice is to maintain the sensitivity of the recorder at a fixed value of 5 millivolts for full-scale deflection. The sensitivity of the preamplifier is therefore set so that this recorder sensitivity and desired range of resistance may be used.

\section{b. Phototube with Electrometer Amplifier Circuit}

The procedure for calibration is the same as that for procedure a. (See figure 12.) The load resistor on the phototube and the controls of the amplifier are adjusted for the optimum performance range of the amplifier. Also, the output of the amplifier should not exceed 5 milliamperes. Hence, other parameters are adjusted so that $R_{L}$ is greater than $1 \mathrm{ohm}$ and is less than 5 ohms.

\section{c. Zero-Resistance Circuit}

A diagram of this circuit is shown in figure 13. In this circuit, if the photometer is balanced so that no current flows through the galvanometer, then

$$
i=i_{a} a / r_{x}
$$

where

$i$ is the photocell current,

$\underline{i}_{a}$ is the current through the slidewire between 0 and A(figure 13), $\mathrm{a}^{\mathrm{a}}$ is the resistance of the slidewire between 0 and A (figure 13), and $\bar{r}$ is the resistance of the resistor, $r_{x}$.

Assuming the slidewire is graduated from 0 to 100 , the reading of the indicator of the slidewire is

$$
\delta=\mathrm{a} / \mathrm{a}_{\mathrm{o}}
$$

*This equation is an approximation which depends on $i$ being much greater than $\underline{i}$. In practice, $\underline{i}$ is kept at about $10 \mathrm{milliamperes,} \mathrm{and} \mathrm{the} \mathrm{range}$ of $\underline{i}$ is from 1 to 20 microamperes. If $\underline{i}$ is 20 microamperes, the error resulting from the use of this approximation will be $0.3 \%$. For larger values of $\underline{i}$, a correction in the calibration can be made. ${ }^{6}$ 


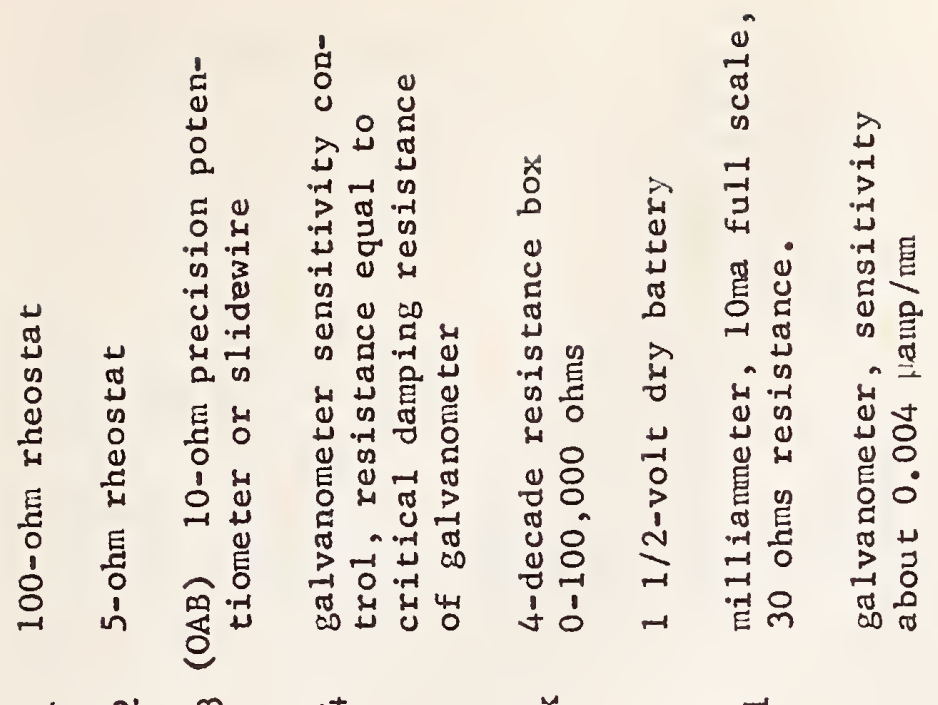

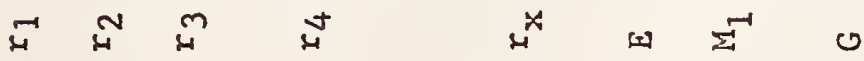

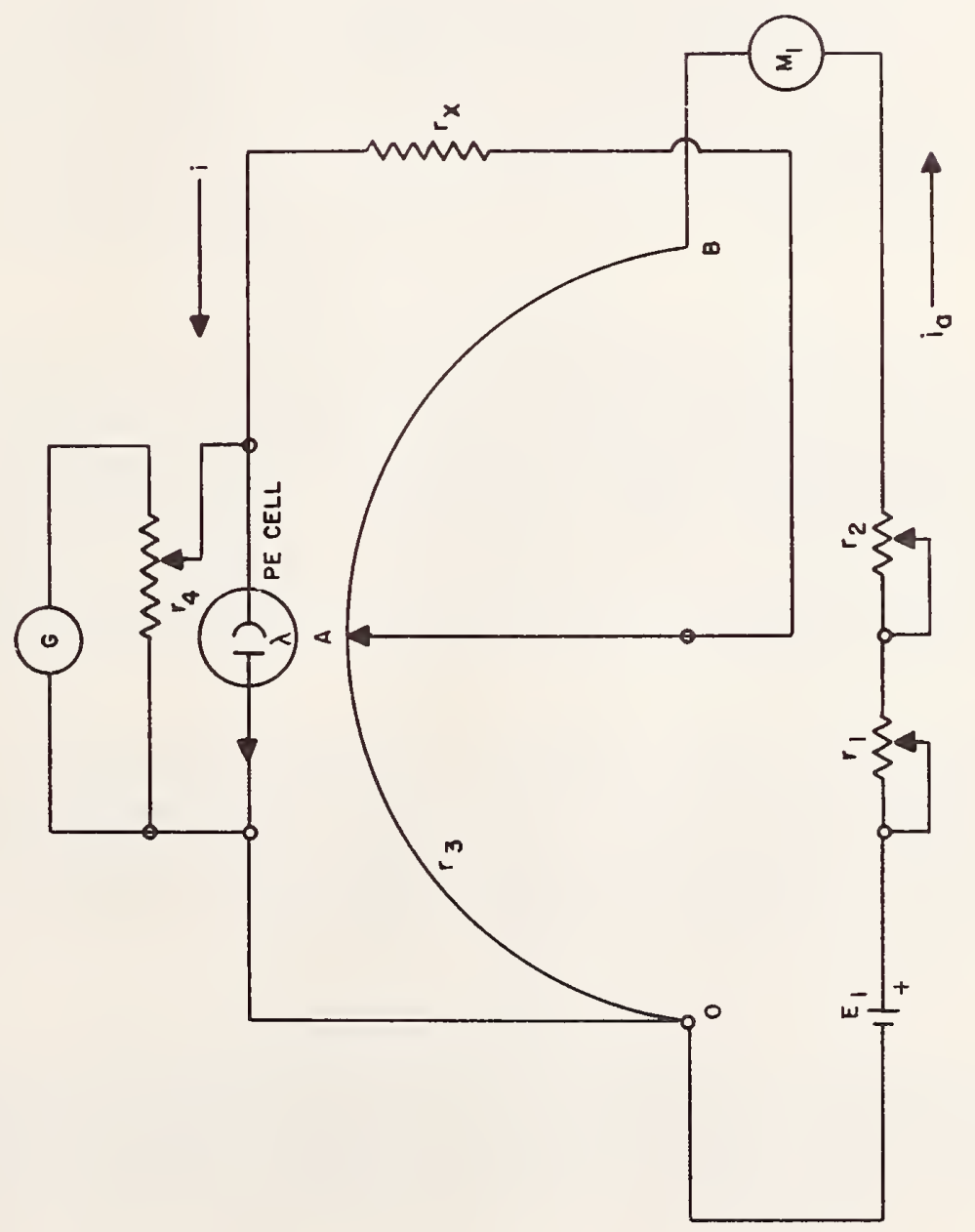

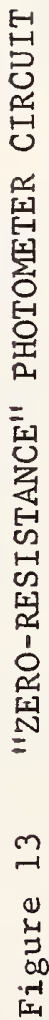


where $a_{0}$ is the total resistance of the slidewire (the resistance between 0 and $\vec{B}$ in figure 13 ).

$$
\begin{aligned}
& \text { Then, combining (1), (7), and (8), } \\
& \qquad \delta=\frac{r_{x} k I}{i_{a} a_{0} D^{2}}
\end{aligned}
$$

In the calibration of the zero-resistance circuit, $i_{a}$ is usually kept constant and $r_{x}$ is varied.

When the photocell is illuminated by light from the standard lamp, $I$ is adjusted to obtain a zero reading of the galvanometer when the sildewire is set at the value $\delta$ of equation (5) for a given test distance, D. With the photometer thus calibrated, the intensity of the test i ight is given by equation (2).

\section{d. Special Procedures}

While photometric data are usually presented for a test light operating under the design condition, photometry of the test light under operating conditions other than the design condition is often desirable. Equation (5) can be generalized, taking into account this condition as well as the transmittance of any filters or sector disks used in caiibrating, so that

$$
\delta_{s}=\gamma_{c} \gamma_{s} F I_{s} \frac{D^{2}}{N_{s}^{2}}
$$

where $Y_{C}$ is the transmittance of the color filter at the color temperature of the standard lamp, $\underline{Y}_{s}$ is the transmittance of the sector disk, and $\underline{F}$ is the ratio of the output of the light under test when it is operated under the design conditions to the output of the light when it is operated under test conditions. This ratio may be, for example, the ratio of the rated lumen output of the test lamp to the output of the lamp at the test voltage. It also may be the ratio of the intensity in a given direction at the operating voltage to the intensity in this direction at the test voltage.

In the case of lights which are flashed in service but on which photometric measurements are made with the light burning steadily at a selected voltage, the factor $F$ is the ratio of the effective intensity of the flash in a given direction to the steady intensity at the selected voltage in this direction of view.

\section{TESTING PROCEDURE}

The photometer is calibrated as described under "Calibration Procedure" (Section 3.4) to an illuminance range determined by the intensity of the light being tested, the test distance, and the information desired. 
The test unit is mounted on the goniometer and is aligned. The angular settings of the goniometer are adjusted so that the origin of the goniometer settings will correspond to the desired axis. This axis usually is chosen with respect to either the seating plane of the unit or some characteristic of the beam such as its peak.

The baffling for stray light is put into place. The eye is placed in the position normally occupied by the photosensor. Examination can then be made to insure that the baffling is properly placed so that no obstructions exist between the light and the photosensor and so that reflections from the walls, floor, and ceiling of the range are intercepted before they reach the photosensor. When the 279-meter range is used, the baffling tube is periodically checked to insure that it is not being obstructed by birds.

If a sealed-reflector lamp is being photometrically measured, the lamp is usually operated at either rated voltage or rated current. Other lamps, such as those used in combination with an optical system, are usually operated at or corrected to rated lumen output. Power for the test and standard lamps is usually obtained from storage batteries, which are periodically recharged. Voltage and current are measured on a potentiometer, and photometric measurements are not made until the lamp has reached stability.

If the goniometer is to be motor driven, the gear ratios are chosen so that the traverse will be slow enough to insure the accurate recording of the characteristics of the light.

\section{ACKNOWLEDGMENT}

The contributions of the many members of the Photometry and Colorimetry Section of the Metrology Division, NBS, who through the years have developed procedures and instrumentation described here is gratefully acknowledged. 
1. T. H. Projector, Versatile Goniometer for Projection Photometry, I11. Eng. 48, 192 (1953)

2. IES Lighting Handbook, 3d ed., p. 4-35 (I1luminating Engineering Society, New York, N.Y., 1959)

3. J. W. T. Walsh, Photometry, Chap. XIV (Constable and Company Ltd., London, England, 1953)

4. F. C. Breckenridge and T. H. Projector, Construction of a Goniometer for Use in Determining the Candlepower Characteristics of Beacons, CAA Tech. Dev. Rep. No. 39 (Feb. 1944)

5. C. A. Douglas, Visibility Measurements by Transmissometer, Electronics 20, $106(1947)$

6. Louis E. Barbrow, A Photometric Procedure Using Barrier Layer Photocells, NBS J. Res. 25, 703 (1940)

7. T. H. Projector, M. K. Laurer, and C. A. Douglas, An Improved "Zero-Resistance" Circuit for Photo-Cell Photometry, Rev. Sci. Instr. 15, 107 (1944)

8. C. A. Douglas, Photometer for Measurement of Effective Intensity of Condenser-Discharge Lights, I11. Eng. 53, 205 (1958)

9. C. A. Douglas and M. R. Freund, Potentiometer for Measurement of Small Photoelectric Currents, NBS Rep. 6422

10. IES Lighting Handbook, 3d ed., p. 4-5 (I1luminating Engineering Society, New York, N.Y., 1959) 


\title{
Review of Elementary Theory
}

\section{Of the Photometry of Projection Apparatus}

\author{
By C. A. Douglas
}

\begin{abstract}
Equations based upon simple geometric relations arc developed for the illuminance* produced by a projector such as a scarchlight, beacon, or floodlight at a distance from the projector. When the beam is rotationally symmetrical but not collimated and the image, virtual or real, subtends a smaller angle at the point of observation than does the objective of the projector, illuminance varies inversely as the square of the dis. tance to the image. If the angle subtended by the image is larger than that subtended by the objective, the illuminance varies inversely as the square of the distance to the objective. The distance at which the two angles are equal is defined as the critical distance. Equations relating critical distance to the radius of the sourcc, the radius of the objective, and the magnification of the system are devcloped. Approximations for use when the beam of the projector is asymmetric are developed. Very good agreement was found between the computed variation of illuminance with distance and the measured variation of illuminance with distance for a projector forming a virtual image 150 feet behind the objective.
\end{abstract}

\section{Introduction}

$\mathrm{T}$ HE luminous intensity, $I$, of a projector is obtained by measuring the illuminance, $E$, on a surface at a distance, $d$, from the projector and computing $I$ from the relation

$$
I=E d^{2}
$$

The use of this relation without qualification implies that the value of $I$ is independent of the distance at which the illuminance, $E$, is measured.

If measurements could be made at increasing distances, in a perfectly transmitting atmosphere (or in any atmosphere if corrections are made for atmospheric losses), of the illuminance produced by a projector emitting a collimated beam, the value of the

A paper presented at the National Technical Conference of the Illuminating Engineering Society, August 21 to 26, 1966, Min. nespolis, Minn. Author: National Bureau of Standards, Wash. ington, D. C. product $E_{1} f^{2}$ increases and approaches a limiting value. The relation

$$
I=\lim _{d \rightarrow \infty} E d^{2}
$$

may be considered as the definition of $I$.

In practice it is usually found for these projectors that beyond a certain distance, the critical distance. there is no measurable change in the intensity com. puted by means of Equation (1).**

The concept of the intensity of a projector producing a collimated beam is valid, and useful, only when this critical distance is exceeded, for then and only then can illuminance at one distance be computed from measurements of illuminance at another distance.

\footnotetext{
* Illumination.

* Such terms as "minimum inverse-square distance" and "limiting distance for the application of the inverse-square law" aro frequently used for this distance. The term "critical distance" is used throughout this report as it is a brief, convenient term.
} 
In the photometry of projectors, the photometric distance is made greater than this critical distance whenever it is feasible to do so. Hence, determination of the critical distance is important. The relation between critical distance for a collimated-beam projector and the dimensions of the projector and the source has been extensively treated. ${ }^{1-5}$ For a parabolic reflector with a spherical source at the focus, the critical distance along the axis of the reflector is

$$
d_{c}=[R F / r]\left[1+(R / 2 F)^{2}\right]
$$

and for a disk source

$$
d_{c}=[R F / r]\left[1+(R / 2 F)^{2}\right] /\left[1-(R / 2 F)^{2}\right]
$$

where

$d_{c}$ is the critical distance,

$R$ is the distance from the axis to the edge of the reflector,

$r$ is the radius of the source, and

$F$ is the focal length of the reflector.

Similar equations may be written for a spherical lens. ${ }^{4}$

The relation given in the IES Lighting Handbook, Third Edition, ${ }^{5}$ may be written as

$$
d_{c}=R d_{e} / r
$$

where $d_{c}$ and $R$ are as before, and $d_{e}$ is the distance from the focal point to the edge of the reflector.

For each of these conditions, $d_{c}$ is given approximately by

$$
d_{c}=R F / r \text {. }
$$

The error in computing $d_{c}$ from this relation is insignificant in most practical applications, particularly when $r$ is small in comparison to $R$, and $F$ is two or more times $R$. Although many projectors are intended for use as collimated-beam projectors, with the source at the focus, there are many types of projectors where this is not the case. For example, in the lens cells of the Fresnel-lens optical landing system, the source is between the focus and the lens so that the lens forms a virtual image of the source 150 feet behind the lens. Spread lenses used in conjunction with collimated beams form either real images in front of the spread lens or virtual images behind the spread lens. The lens cells of the Fresnel-lens optical landing system include a 50-degree horizontal spread lens.

The purpose of this paper is to present an analysis of the factors affecting critical distances and the relation between illuminance and distance of noncollimated beam projectors. Throughout this paper rigor and detailed accuracy of analysis have been subordinated in order to obtain simple, easily interpreted relations free from the complicating and often confusing effects of correction terms. The relations developed are therefore approximations only. They are, however, sufficiently accurate to be used in a qualitative analysis of the performance of a projector system. The performance of the usual types of projector systems is significantly affected by such factors as errors in the shape of the optics and by the variations in illuminance across the surface of the source. Such factors vary from unit to unit and cannot be readily treated in a theoretical analysis. Quantitative analyses of projector systems can, and should, be made by direct measurement on a photometric range.

\section{Case 1. Projectors Having the Source Between the Focus and the Objective}

\section{I Illuminance Produced by a Positive Lens}

Consider the optical system shown in Fig. 1. The system is symmetric about the line $X X^{\prime}$ and consists of a positive lens of focal length $F$ and radius $R$. (Throughout this analysis $F$ is assumed to be large in comparison to $R$.)

A source $S$ of radius $r$ and of uniform luminance $L$ is placed at a distance $u$, less than $F$, from the lens.

\section{SYMBOLS}

$L \quad$ Luminance of source $S$

$d$ Distance between the projector and the point of observation or measurement

$d_{r}$. Critical distance

$E$ Illuminance at the point of observation or measurement

F Focal length of the optic of the projector

$I \quad$ Intensity of the projector

$I_{s} \quad$ Intensity of the source $S$

$I_{i} \quad$ Intensity of the image $S^{\prime}$ of the source

$m$ Magnification of optical system

$R$ Radius of the objective (lens or reflector) when the objective is the field stop of the projector

$r \quad$ Radius of the source $S$

$r^{\prime} \quad$ Radius of image $S^{\prime}$ of source $S$

$S$ Light source (assumed to be of uniform luminance)

$S^{\prime} \quad$ Source image formed by the projector

$\tau$ Transmittance of the optical system, if lens type; reflectance of the optic, if reflector type

$u$ Distance between the source and the objective

$v$ Distance between the image and the objective

$y \quad$ Radius of flashed zone on the objective

$\theta \quad$ The beam spread produced by a spread lens

$\vec{y} \quad$ Dimension of asymmetric source in $x-y$ plane

$\bar{Y} \quad$ Dimension of asymmetric optic in $x-y$ plane

$\bar{z}$ Dimension of asymmetric source in $x-z$ plane

$\vec{Z} \quad$ Dimension of asymmetric optic in $x-z$ plane 
Figure 1. Optical system with the source between the focus and a positive objective (lens). (Not to scale.)

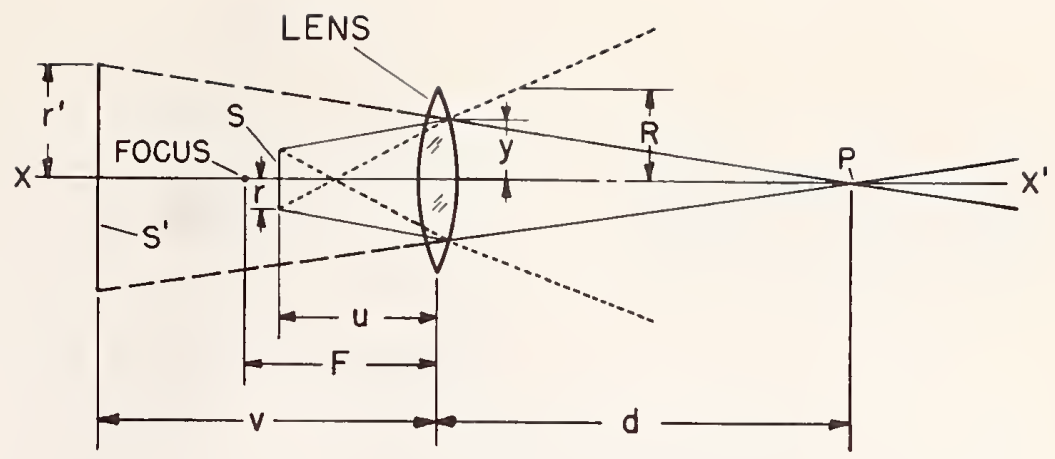

The system will form a virtual image $S^{\prime}$ of the source at a distance $v$ from the lens. Assume the observer's eye, or the photometer, is at the point $P$, a distance $d$ from the lens. With a lossless optical system the luminance of the virtual image, $L^{\prime}$, would be equal to the luminance of the source, $L$. With a real optical system the luminance

$$
L^{\prime}=L_{\tau}
$$

where $\tau$ is defined as the transmittance of the optical system. The intensity of the source is given by

$$
I_{8}=\pi r^{2} L,
$$

and the intensity of the image

$$
\begin{array}{ll} 
& I_{i}=\pi\left(r^{\prime}\right)^{2} L \tau \\
\text { or } & I_{i}=I_{s} m^{2} \tau
\end{array}
$$

When the virtual image, $S^{\prime}$, is viewed through the lens from the point $P$ and $d$ is less than a critical distance, $d_{c}$, to be determined later, the entire virtual image will be seen. The illuminance at $P$ will then be

$$
E=I_{i} /(v+d)^{2} . \quad\left(d \leqslant d_{c}\right)
$$

Since Equation (9) is of the form Illuminance $=$ Constant $/$ Distance $^{2}$,

$I_{i}$ can be considered to be, by definition, the intensity of the projector applicable to this condition of view with distance to be measured from the position of the virtual image, not from the lens.

From Fig. $l$ it is evident that from the point $P$ a zone on the lens of radius $y$ will be flashed, that is, appear to be the source of light, and that the illuminance at $P$ is also given by

$$
E=\pi y^{2} L \tau / d^{2} . \quad\left(d \leqslant d_{o}\right)
$$

If the radius of the virtual image, $r^{\prime}$, is less than the radius of the lens, the flashed zone will never fill the lens however large $d$ is made. For this condition, Equations (9) and (10) are valid for all distances. However, if $r^{\prime}$ is greater than $R$ and the distance, $d$, is equal to or greater than a critical distance, $d_{c}$, the entire lens will be filled and the illuminance at a point on the axis will then be given by

$$
\begin{array}{lll} 
& E=\pi R^{2} L \tau / d^{2} & \left(d \supseteq d_{c}\right) \\
\text { or } & E=I / d^{2} & \left(d \supseteq d_{c}\right)
\end{array}
$$

where $\quad l=\pi R^{2} L \tau$.

$I$ is a constant and hence is by definition the intensity of the projector, applicable to all distances equal to or greater than the critical distance.

Note that when $d$ is equal to $d_{c}$, Equations (8) and (11) are both valid. Hence at this distance

$$
I=I_{i} d_{c}^{2} /\left(v+d_{c}\right)^{2} \text {. }
$$

This leads to the somewhat surprising conclusion that at one distance it is sometimes possible for a projector to have two intensities depending upon whether the source is considered to be located at the objective or at the virtual image position.

Note that in practice it is seldom feasible to determine $I_{i}$ or $I$ to the desired degree of accuracy by Equations (6), (7), (8) or (13). Instead, the illuminance is measured at a known distance from the projector and Equation (9) or Equation (12) is used as applicable.

\subsection{Determination of the Critical Distance}

Note from Equation (11) that for distances greater than the critical distance the axial illuminance varies inversely as the square of the distance to the lens and is independent of the position of the source with respect to the focus. However, the critical distance, $d_{c}$, is not independent of the position of the source with respect to the focus. It may be determined as follows.

If there is a critical distance, at this distance

Therefore, since

$$
y=R . \quad\left(d=d_{c}\right)
$$

$$
\begin{array}{ll} 
& y / d=r^{\prime} /(v+d), \\
\text { and } & r^{\prime} / r=v / u=m, \\
& R=r v d_{c} / u\left(v+d_{c}\right) \\
\text { or } & R=r m d_{c} /\left(v+d_{c}\right) . \\
\text { Hence } & d_{c}=R u /(r-u R / v), \\
\text { and } & d_{c}=R v /(r m-R) .
\end{array}
$$

Note that as $u$ approaches $F, v$ becomes infinite, and Equation (14a) becomes the familiar relation used for collimated-beam projectors,

$$
\lim _{u \rightarrow F} d_{c}=R F / r .
$$

From Equations (14a) and (14b), note that $d_{c}$ becomes infinite when 
Figure 2. Optical system with the focus between the source and a positive objective (lens).

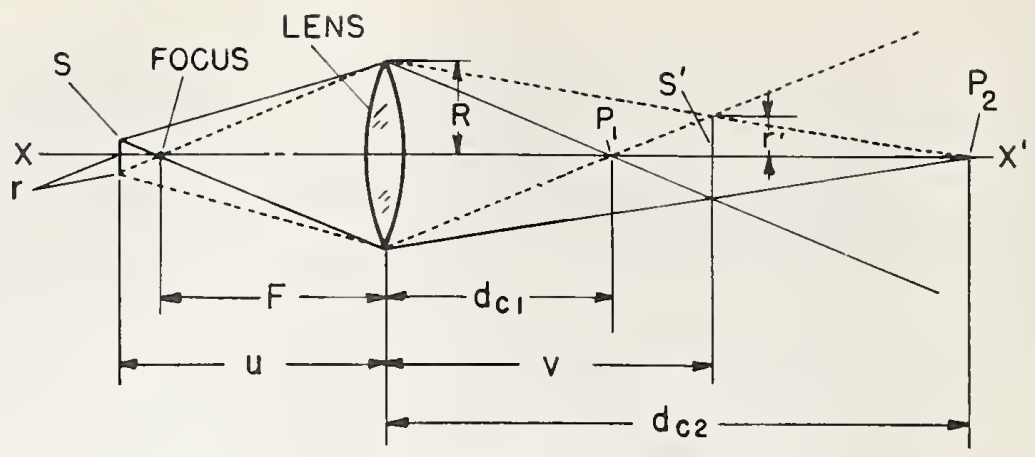

$$
r=R u / v=R / m,
$$

for then the radius, $r^{\prime}$, of the virtual source is just equal to the radius of the lens, $R$, and the lens will appear flashed only when viewed from an infinite distance. If $r$ is less than $R / m$, the value obtained for $d_{c}$ is negative indicating that $r^{\prime}$ is less than $R$ and that the virtual source will not completely fill the lens at any viewing distance. Hence, under this condition Equation (9) is valid for all distances.

\section{Case 2. Projectors Having the Focus Between the Source and the Lens}

\section{I Illuminance Produced by a Positive Lens}

If the distance from the source to the lens, $u$, is greater than the focal length of the lens, $F$, the system will form a real image of the source a distance $v$ from the lens, as shown in Fig. 2.

Consider first the case in which the radius of the image, $r^{\prime}$, is less than the radius of the lens. It is evident from Fig. 2, on which the limiting rays from the edge of the lens are shown, that the entire lens will appear to be flashed only between the points $P_{1}$ and $P_{2}$. The illuminance at a point on the axis is, as for the flashed lens of Case 1 ,

$$
E=\pi R^{2} L \tau / d^{2} . \quad\left(d_{c 1} \leq d \leq d_{c 2}\right)
$$

The inverse square law holds with distance measured from the lens to the point of observation, or measure- ment, and as before

$$
I=\pi R^{2} L_{\tau} . \quad\left(d_{c 1} \leq d \leq d_{c 2}\right)
$$

When the lens is observed from $P_{4}$ (see Fig. 3 ) where $d$ is equal to or greater than $d_{c 2}$, the entire image will be visible and the illuminance at $P_{4}$ is given by

$$
E=I_{i} /(d-v)^{2} . \quad\left(d \geqslant d_{c 2}\right)
$$

If the lens is viewed from a point $P_{3}$ on the axis between the point $P_{1}$ and the lens, the entire lens will not be flashed, for rays from the outer part of the lens to the image cross the axis at or beyond the point $P_{1}$. Instead, a zone of radius $y$ will appear flashed, as is evident from the limiting rays shown in Fig. 4. The illuminance at $P_{3}$ will be

$$
\begin{array}{ccc} 
& E=\pi y^{2} L \tau / d^{2} . \quad\left(d \leq d_{c 1}\right) \\
\text { Since } & y=r^{\prime} d /(v-d), & \\
& E=\pi\left(r^{\prime}\right)^{2} L \tau /(v-d)^{2} & \left(d \leq d_{c 1}\right) \\
\text { or } \quad & E=I_{i} /(d-v)^{2} . & \left(d \leq d_{c 1}\right)
\end{array}
$$

Note that $(v-d)$ and $(d-v)$ are both the distance between the point of observation and the image formed by the lens.

\subsection{Determination of the Critical Distances For Lenses Forming Real Images}

If $r^{\prime}$ is less than $R$, the critical distances, $d_{c 1}$ and $d_{c 2}$, may be found by noting that for observations from $P_{3}$ (Fig. 4),

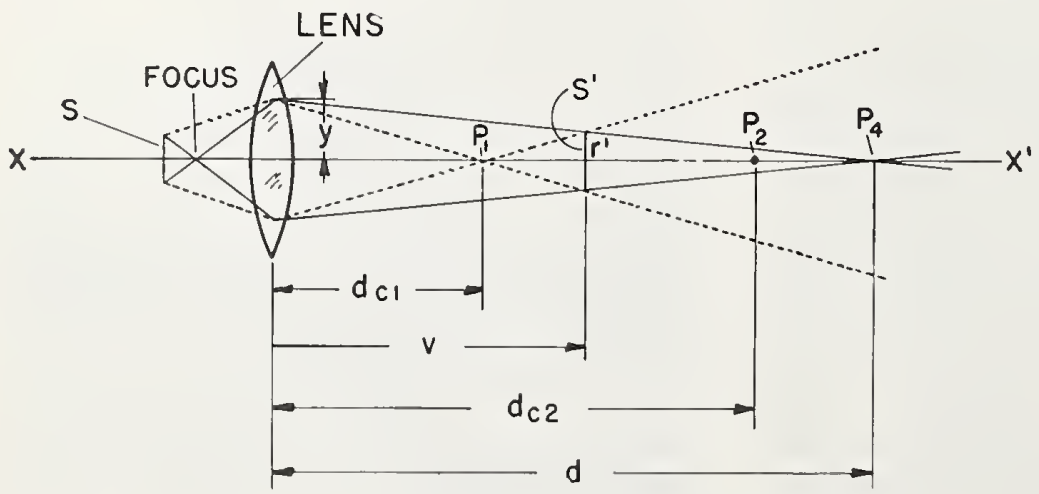

Figure 3. Flashed zone of an op. tical system forming a real image with the image between the point of observation and the objective. 


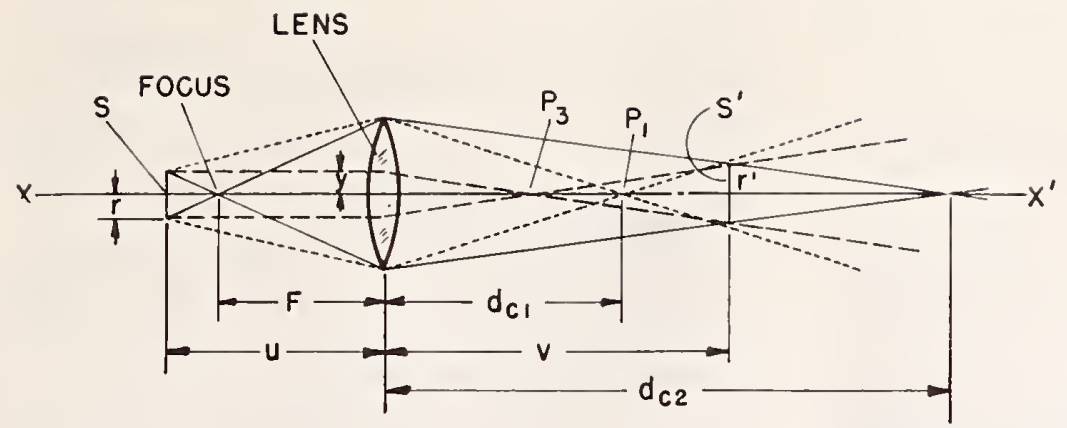

Figure 4. Flashed zone of an oplical system forming a real image with the point of observation between the objective and the image.

$$
y=r m d /(v-d)=r d / u(1-d / v)
$$

and substituting $R$ for $y$, and $d_{c 1}$ for $d$ obtaining

$$
\begin{array}{cc} 
& d_{c 1}=R v /(R+m r) \\
\text { or } & d_{c 1}=R u v /(R u+r v) .
\end{array}
$$

Similarly, for observations from $P_{4}$ (Fig. 3 )

$$
\begin{array}{cc} 
& d_{c 2}=R v /(R-m r) \\
\text { or } & d_{c 2}=R u v /(R u-r v) . \\
\text { Since } & r^{\prime}=m r,
\end{array}
$$

when $r^{\prime}$ is equal to or greater than $R, d_{c 2}$ becomes infinite, and the lens is flashed for all distances greater than $d_{c 1}$. For this condition, Equation (11) is applicable for all distances greater than $d_{c 1}$.

When the source is placed at the focus,

$$
u=F \text {, }
$$

$m$ and $v$ become infinite, and Equation (22) reduces to Equation (4). That is,

$$
d_{c 1}=R F / r \text {, }
$$

and $d_{c 2}$ becomes infinite.

\subsection{Measurement of Beam Angles}

When the optical system forms an image, virtual or real, of the source instead of producing a collimated beam, and the point of observation or measurement is at a distance such that the objective of the system is not completely flashed, angles of observa. tion as well as the illuminance are determined, using the distance between the image and the point of observation as a base, not the distance between the $o b$ - jective and the point of observation. That is, the vertex of the angle between the line of sight and the axis of the light is located at the image of the source, not at the objective of the projector. However, when a light is mounted on a goniometer, it is usually more convenient to measure angles whose vertices are at the projector. The relation between the two angles is easily found by the use of Fig. 5 .

Since

and

$$
\tan \psi=a / d
$$

for small angles

$$
\tan \phi=a /(d+v),
$$

and

$$
\psi \doteq a / d,
$$

$$
\phi \doteq a /(d+v)
$$

where $a$ is the distance from the point of observation to the axis $X X^{\prime}$.

Hence,

or

$$
\phi \doteq[d /(d+v)] \psi,
$$

$$
\phi=[d /(d+m u)] \psi \text {. }
$$

\subsection{Asymmetric Beams}

Throughout the preceding discussion it has been assumed that the optical system is rotationally sym. metric about the optical axis of the system. Thus, a disk or spherical source and an optical system com.

Figure 5. Correction of angles of an optical system forming a virtual image.

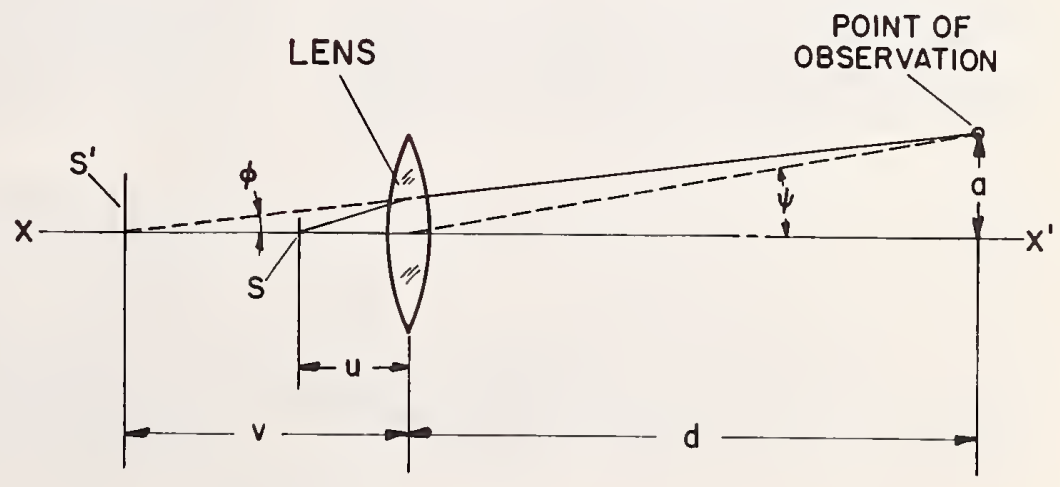




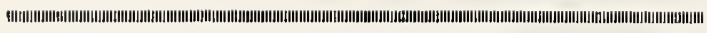

Table 1-Photometric Measurements of an Asymmetric Projector

PHOTOMETRIC DISTANCE (d) (FEET)

50
100
920

PEAK INTENSITY (I) (KILOCANDELAS)

32.0

31.3

34.8

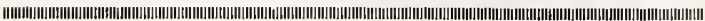

posed of spherical elements have been assumed. In such systems the beam pattern is the same in every plane containing the axis of the optical system.

However, lights having unequal vertical and hori. zontal beam spreads are often required. These unequal beam spreads may be obtained by using sources in which the width (the dimension in the $z$ direction) and the height (the dimension in the $y$ direction) are not equal and/or by adding cylindrical elements, with the axis of the cylinders parallel to the $y$ or $z$ axis. When cylindrical elements are used, the image of the source formed by a narrow section in the $y$ direction through the center of the lens will not be the same distance from the lens as the image formed by a narrow section in the $z$ direction. That is, the projection of the image of the source on the $x-y$ plane will not be in the same position as the projection of the image on the $x-z$ plane. Under these conditions the equations of the type given below will give approximate values. The applicable equations of Section 2.2 are applied to determine if the viewing, or test, distance exceeds the critical distance using the dimensions of the projection on the $x-y$ and the $x-z$ planes, in turn, to determine $d_{c y}$ and $d_{c z}$, respectively.

Thus, from Equations (14a) and (14b), if the projection system forms a virtual image,

$$
d_{c y}=\bar{Y} u_{y} /\left(\bar{y}-u_{y} \bar{Y} / v_{y}\right),
$$

or

$$
d_{c y}=\bar{Y} v_{y}\left(\bar{y} m_{y}-\bar{Y}\right)
$$

where

$\bar{Y}$ is the dimension of the lens in the $x-y$ plane,

$\bar{y}$ is the dimension of the source in this plane, and the subscript $y$ refers to quantities measured in the $x \cdot y$ plane.

Similar equations can be written for $d_{c z}$ where $\bar{Z}, \bar{z}$, and the subscript $z$ replace $\bar{Y}, \bar{y}$, and the subscript $y$.

If the observation distance is less than the critical distance in both the $x-y$ and the $x-z$ planes, that is,

$$
d \leq d_{c \nu}
$$

and

$$
d \leq d_{c z},
$$

Equation (8) may be written as

$$
I_{i}=I_{8} m_{\eta} m_{z} \tau
$$

and Equation (9) may be written as

$$
I_{i}=E\left(v_{y}+d\right)\left(v_{z}+d\right) .
$$

Equation (27) may be considered as the definition of intensity under these conditions.

$$
\begin{aligned}
& \text { Similarly, if } \\
& d \leq d_{c y} \\
& d \supseteq d_{c z}
\end{aligned}
$$

and

Similar equations can, of course, be written interchanging the $y$ 's and the $z$ 's.

Similar expressions can, of course, be developed for systems which form real images in either or both planes by using the terms $(v-d)$ or $(d-v)$ in place of $(v+d)$, as applicable.

\subsection{Experimental Confirmation}

The principles developed in Section 3.4 were applied to a cell of the Fresnel-lens optical landing system. The cell has a lens with vertical cylindrical elements forming a real image of the source ahead of the unit to produce a wide horizontal spread, with the following dimensions (approximate) :

$\bar{Z}=0.16$ inch $(2 \bar{Z}$ is the width of one cylindrical element)

$\bar{z}=0.38$ inch

$v_{z}=0.5$ inch (computed from design beam spread of 40 degrees)

$u_{z} \doteq 24$ inches.

From these dimensions it is apparent that the first critical distance in the $x-z$ (horizontal) plane, $d_{c z 1}$, is very small. (This is usually the case in the plane in which there is an appreciable spread.)

From Equation (22b), $d_{c z 1}=0.48$ inch

The second critical distance in the horizontal plane is also very small. From Equation (23b),

$$
d_{c z 2}=0.52 \text { inch }
$$

In the $x-y$ (vertical) plane, the optical system forms a virtual image of the source 150 feet behind the lens. Pertinent dimensions are as follows:

$$
\begin{aligned}
& \bar{Y}=0.4 \text { foot approximately } \\
& \bar{y}=0.003 \text { foot approximately } \\
& v_{y}=150 \text { feet } \\
& u_{y}=2 \text { feet } \\
& m_{y}=75 \\
& r^{\prime}{ }_{y}=m_{y} \bar{y}=0.2 \text { foot }
\end{aligned}
$$

Since $r^{\prime}{ }_{y}$ is less than $Y$, the lens will never be completely flashed in the $y$ direction.

The relation between illuminance produced by the system and the luminous "intensity" is

$$
l=E\left(v_{y}+d\right)\left(d-v_{z}\right)
$$

or

where $d$ is in feet.

$$
I=E(150+d)(d-0.04)
$$

Or, since $v_{z}$ is very small compared to typical values of $d$,

$$
I=E(150+d)(d)
$$

Measurements were made at three distances of the illuminance produced by a Fresnel-lens optical landing system cell and the luminous intensity was com. 
puted by Equation (27). Results are given in Table I. The consistency of the values of $l$ is surprisingly good.

\subsection{Practical Considerations}

\subsubsection{Simplified Equations}

Although the equations which have been developed describe the performance of a projector, they are in forms which often are too complex to be used to best advantage by the photometrist, who, given the task of determining the intensity distribution of a projector, desires only sufficient information to permit him to choose a photometric distance greater than the critical distance. Often he is given very little information concerning the design parameters of the projector. The relations given below contain only parameters which are easily measured.

Consider first a projector which forms a virtual image of the source. From Equation (14a) it is evident that

$$
d_{c} \supseteq R u / r
$$

Hence, if the photometric or observation distance is less than $R u / r$, that is, if

$$
d<R u / r
$$

the entire lens is not flashed and illuminance varies inversely as the square of the distance to the image.

The objective of a projector forming a real image of the source will appear flashed at all distances equal to or greater than the image distance if (and only if) the radius of the image is equal to or greater than the radius of the objective. Hence,

$$
r^{\prime}=r v / u \supseteq R
$$

or

$$
v \supseteq R u / r
$$

(The distance $v$ can usually be determined readily from direct observation of the position of the image.)

Hence, when a real image is formed at a distance equal to or greater than $R u / r$, illuminance varies inversely as the square of the distance from the objective for all distances greater than the image distance, $v$. It is apparent from simple geometric relations (see Fig. 2) that, if the condition of relation (33) is met, the objective will be flashed for distances in the range $v / 2$ to $v$ inclusive also.

\subsubsection{Application to Spread Lenses}

Projcctors often contain lens elements to increase the beam spread of the light from the projector. These lens elements form images, real or virtual, which are too small to flash the element. Hence, dis. tance should be measured from the position of the image. (Note these elements usually are asymmetric and the image distances in the $y$ and $z$ planes are different. For simplicity, only one plane is treated here.) The geometric relations of these spread elements are illustrated in Fig. 6.

The angle $\theta$, in degrees, is commonly called the beam spread of the lens. The relation between image distance and beam spread is

$$
v=(b / 2) \operatorname{ctn} \theta / 2
$$

where $b$ is the width of the spreader elements. The following approximation is sufficiently accurate for most purposes.

when $\theta$ is in degrees.

$$
v \approx 60 b / \theta
$$

Note that when a spread lens is used, each spreader element acts as a separate projector, and that usually these elements will not be completely flashed at practical observation distances. Under these conditions, distance should properly be measured from the images produced by these spreader elements. With the usual projectors, $v$ is so small in comparison to $d$ that no significant error results when the term $(v+d)$ is replaced by $d$. For example, consider a five-degree spread lens with cylindrical elements one inch wide. From Equation (35), $v$ is found to be 12 inches. As this is a rather extreme example, $v$ will usually be considerably less than this. The criteria determining the photometric distance are then:

1. The photometric distance, $d$, should be large in comparison to the image distance $v$.

2. The angular subtense of the light unit from the point of measurement should be small in comparison to the beam spread of the light.

\subsubsection{Incandescent Lamps as Sources}

The filament of an incandescent lamp is not a source of uniform luminance. Hence, the overall dimensions of the filament should not be considered as the size of source in determining the critical distance.

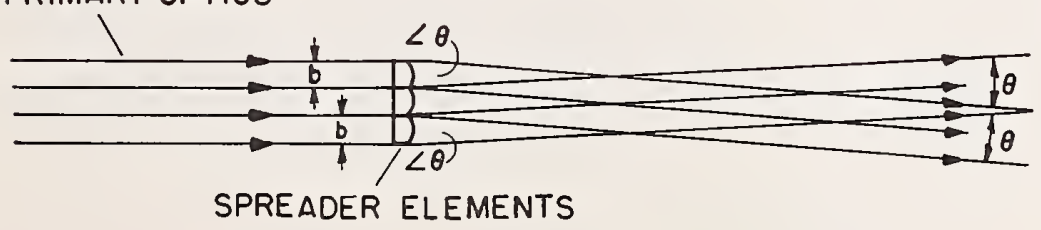

Figure 6. Geometric relations of a spreader element. 
Instead, the size of the smallest separable element of the filament should be used. 5 The smallest separable element will usually be the filament wire itself but at times it will be the helix. The dimensions of both the filament wire and the helix must be considered, as the separate turns of the filament wire may be dis. cernible from the central part of a reflector while only the helix may be discernible from the outer part of the reflector. ${ }^{8}$

\subsubsection{Application to Objectives Of Small f-Number}

The development of the equations of this report is based on the assumption that the radius of the objective is small compared to its focal length. In practice, the radius of the objective is often as large as or larger than the focal length. Frequently, when applied to projection systems of the latter type, these equations yield results sufficiently accurate for engineering purposes if $u$ and $v$ are measured to the most listant part of the objective instead of along the axis of the projection system. ${ }^{5,6}$

\section{Conclusion}

A review of the elementary theory of the photometry of projectors has been made. Use was made of simple geometric relations to develop equations relating distance with the illuminance produced by projectors. Particular attention has been given to projectors producing noncollimated beams and to the effect of spreader elements in the optical system. Equations have been developed relating critical distance to the radius of the source, the radius of the objective and the magnification of the system. Equations applicable to asymmetric beams have been stated. The agreement between values computed from these relations and direct measurements is very good.

Acknowledgment

The photometry of the Fresnel-lens optical landing system was performed by my colleague A. C. Wall.

\section{References}

1. Waldram, J. M.: "The Plintometry of Projectel Light," Transactions of the Illuminating Engineering Societll (London), Vol. 46. p. 187 (1951); also lli.uminativg Ensinkering, Vol. XLVII, p. 397 (July 1952).

2. Walsh, J. W. T.: Photnmetry, Chapter XIV, Constable and Co., Ltd., London, England (1953).

3. Benford, Frank: "Studies in the Projection of Light," General Electric Review, Vol. 26, pp. 75, 160, 230, 280. 575, 624, 780, 818 (1923).

4. Fampton, W. M.: "The Photometry of Projectors," Transactions of the Illuminating Engineering Society (London), Vol. 3, p. 52 (1938).

5. IES Lighting Handbook. Third Erlition, p. 4.35, Illuminating Engineering Society, New York. N. Y. (1959).

6. Chernoff, L.: "Photometry of Projectors at the National Buresu of Standards," NBS Technicul .Note 198 (December 17. 1963). 


\section{Photometer for Measurement of Effective Intensity of Condenser-Discharge Lights}

By CHARLES A. DOUGLAS

\section{Introduction}

In the past, measurements of the effective intensity of condenser-discharge lights have generally been made by two methods : (1) coupling the output of a phototube to a cathode-ray oscilloscope, photographing the trace of the instantaneous intensity against time, integrating the area under the curve and computing the effective intensity; and (2) charging a capacitor by the photoelectric current generated by one or more flashes and measuring the voltage developed with a vacuum-tube voltmeter. Both of these methods are time consuming when intensity distribution measurements of a projector with a flashing source are being made. In addition, when the source is a condenser-discharge lamp, there has often been uncertainty about the accuracy of the correction of the spectral response of the photometric system to the CIE standard observer luminosity function; the sensitivity may be so low that short photometric distances are required; although the average photoelectric current is low, peak currents are high, and, unless suitable precautions are taken, the phototube may be saturated during part of the flash, destroying the linearity of the system. A method that will allow measurements of the effective intensity distribution of condenserdischarge units producing repetitive flashes to be made and recorded automatically is very desirable. The development of such a method, designed to avoid difficulties of the types described above and making use of commercially available equipment, wherever possible, is described below.

\section{Effective Intensity Photometer}

The effective-intensity photometric system is shown schematically in Fig. 1. The test and standard distances, the intensity of the standard lamp, and the transmittance of the sector disc are chosen so that the average illumination on the photocell during calibration is of the same order as the illu-

This paper was prepared as a part of the work in the development of a viation ground lighting conducted at the National Bureau of Standards under the sponsorship of the Visual Landing Aids Branch of the Bureau of Aeronautics and the Aeronautical Accessories

Lahoratory, Wright Air Development Center. AUTHor: National

Bureau of Standarảs, Washington, D. C. mination from the projectors under test. The sector dise was used to obtain flashes during calibration which had roughly the same intensity-time relation as the flashes from the lights under test. (As will be shown below, this latter condition is not necessary.) With this system it is possible to obtain in approximately three minutes an intensity distribution curve of a projector using a condenser-discharge lamp which produces repetitive flashes.

Light from either the test unit or the standard lamp falls on a diffusing glass so that the distribution of illumination on the photosensitive surface of the phototube is independent of the distance of the light source. A small aperture in front of this glass is used to control the illumination on the phototube. The light then passes through the luminosity filter to the phototube. This filter is so designed* that the spectral response of the phototube-filter-diffusing glass combination is essentially that of the luminosity function of the CIE standard observer. ${ }^{1}$

A type PJ-14B phototube is used because it is stable, it has a low dark current, and its relatively flat spectral response simplifies the design of the luminosity filter. The correction for the spectral sensitivity of the phototube by the luminosity filter is sufficiently accurate for the photometry of any non-chromatic ("white") light using an incandescent lamp or a conventional flashtube. The luminous sensitivity of the phototube-filter combination is sufficiently high so that the photometric distance may be made as large as necessary.

The output current of the phototube is smoothed by the resistance-capacitance network so that the phototube will not be saturated and the d-c electrometer-amplifier will not be overloaded during the flashes and so that output current will be sufficiently stable to produce a smooth curve on the recording potentiometer. The output of the system is, of course, proportional to the average photoelectric current and hence to the average illumination at the phototube.

The General Radio Type 1230A d-c amplifier and electrometer is operated with its "Ground" switch

*The luminosity filter was designed by Mr. I. Nimeroff of the National Bureau of Standards. 


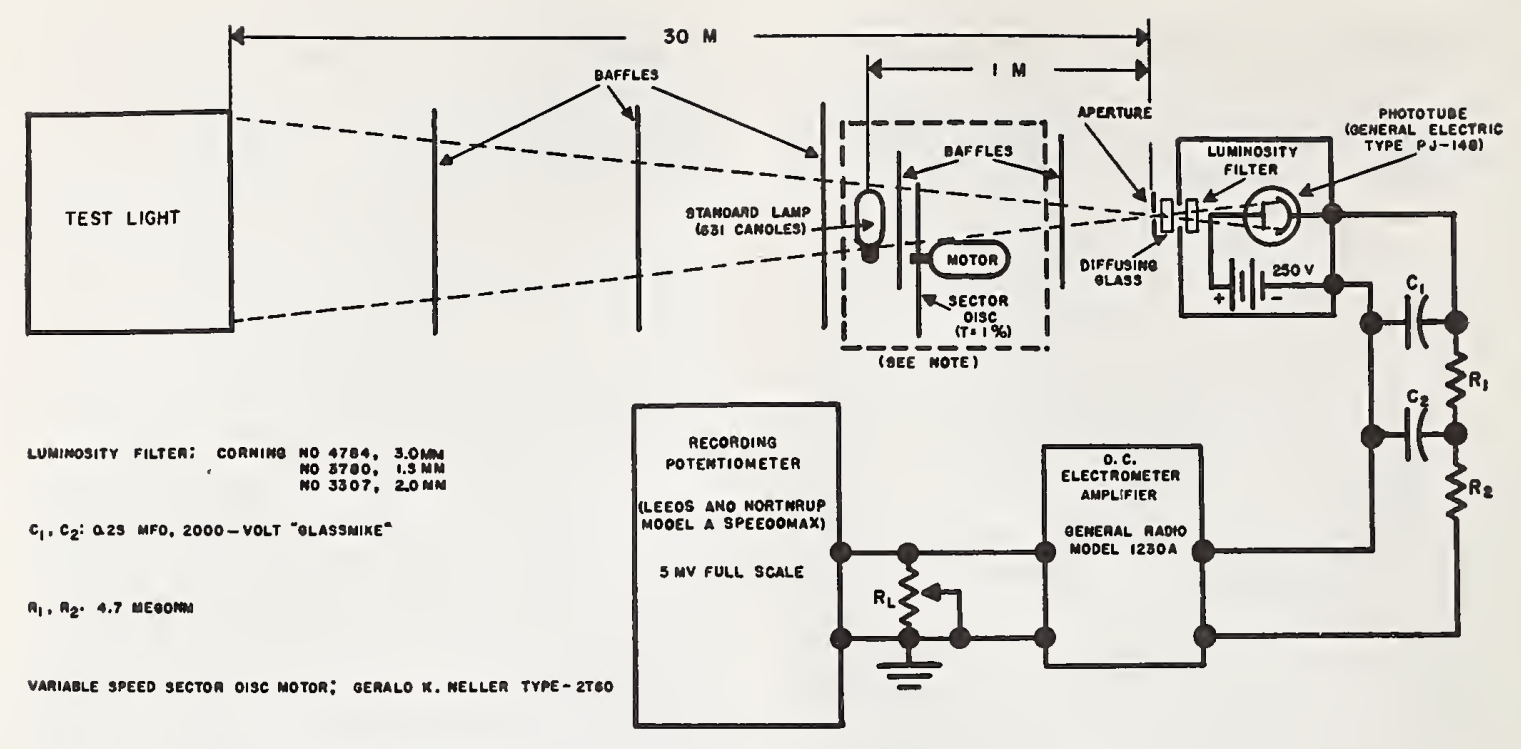

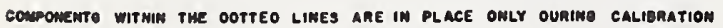

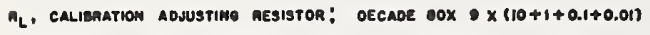

Figure 1. Block diagram of effective-intensity photometric system. Components with dotted lines are in place only during calibration. $\boldsymbol{R}_{L}$, calibration-adjusting resistor, is a decade box adjustable by 0.01-ohm steps to 99.99 ohms.

in the "I" position. With this arrangement, use of a single capacitor across the input of the instrument is unsatisfactory because the feedback in the instrument produces low-frequency oscillations (of the order of 0.1 to $0.5 \mathrm{cps}$ ) in the output. A network of the type shown eliminates these oscillations. With the "Ground" switch in the "E" position, a single capacitor across the input may be used. The time constant of the circuit is then a function of the capacitance across the input and the input resistance selected by means of the "Input Resistance" switch of the electrometer-amplifier. The arrangement shown is considered preferable unless very long time constants are required, for the time constant is relatively independent of the input resistance selected and may be readily adjusted by changing the values of $R_{1}$ and $R_{2}$. Ordinary carbon resistors are satisfactory, but capacitors with very high leakage-resistance are required to obtain stability of calibration.

The electrometer-amplifier is designed to operate an external five-milliampere full-scale meter or recorder. However, the recording potentiometer available had a five-millivolt full-scale range. Load resistor $R_{L}$ is used to obtain a suitable input signal for the recording potentiometer from the output current of the electrometer-amplifier. It also provides a convenient means of making small adjust- ments of the over-all sensitivity of the photometric system. The minimum value used for $R_{L}$ is one ohm in order that a full-scale reading of the potentiometer may be obtained before the electrometer is overloaded.

\section{Theory of Operation}

The method of obtaining the effective intensity of a condenser-discharge light flashed at a known rate from measurements of the average intensity is developed below.

\subsection{Definitions of Terms and Symbols.}

The terms and symbols used in this paper are defined as follows:

$I$ the instantaneous intensity of the test light during a cycle

$\bar{I}$ the average intensity of the test light during a complete flash cycle

$I_{e}$ the effective intensity of the test light

$I_{s}$ the intensity of the standard lamp

$T$ the transmittance of the sector dise

$D$ the distance between the test light and the diffusing glass of the photometer

$d$ the distance between the standard lamp and the diffusing glass of the plotometer

$\tau$ the time in seconds required for a complete flash cycle 
$R$ the recorder reading for the test source

$R_{s}$ the recorder reading during calibration with the standard lamp and rotating sector dise.

\subsection{Basic Relations.}

The effective intensity is defined as

$$
I_{e}=\frac{\int_{t_{1}}^{t_{2}} I d t}{0.2+t_{2}-t_{1}},
$$

where $t_{1}$ is the time in seconds of the beginning of the flash and $t_{2}$ is the time of the end of the flash. An equation of this form was first suggested by Blondel and Rey but has rarely been used. ${ }^{2}$

The times $t_{1}$ and $t_{2}$ are chosen so that $I_{e}$ is equal to $I$ at these times. ${ }^{3}$ However, if the effective intensity is sufficiently small in comparison to the instantaneous intensity over most of the flash then $\int_{t_{1}}^{t_{2}} I d t$ may be replaced by $\int_{0}^{\tau} I d t$ without introducing significant errors. In addition, the flash of a condenser-discharge light is so short (generally less than 0.001 second) that $t_{2}-t_{1}$ is negligibly small in comparison to 0.2 .

Therefore equation (1) may be rewritten as

$$
\begin{aligned}
& I_{e}=5 \int_{0}^{\tau} I d t . \\
& \int_{0}^{\tau} I d t \\
& \text { But } \\
& \bar{I}=\frac{\int_{0}}{\tau} \text {. } \\
& \text { Therefore } \quad I_{e}=5 \bar{I} \tau \text {. }
\end{aligned}
$$

Note that $\tau$, the period of the flash cycle, must be known, and must be stable, to the same degree of accuracy as is desired for the effective intensity.

The time constant of the input circuit is sufficiently long so that the recorder indication is nearly steady and proportional to the average current of the phototube. During a flash the photoelectric current charges capacitor $C_{1}$ and the voltage across the phototube is not decreased appreciably. Hence the phototube does not saturate and the photoelectric current is at all times proportional to the illumination on the phototube.

Therefore, the recorder reading is given by

$$
R=\frac{k \vec{I}}{D^{2}},
$$

where $k$ is a constant of proportionality which is a function of the exposed area of the diffusing glass, the input resistance of the d-c amplifier, the range on which the amplifier is set, the load resistance across the output of the amplifier, and the sensitivity of the recorder.
Combining equations (4) and (5),

$$
\frac{I_{e}}{R}=\frac{5 \tau D^{2}}{k} \text {. }
$$

For ease in interpretation of the recorder charts, the ratio $I_{e} / R$ is made the product of either an integer, or the reciprocal of an integer, and an appropriate power of ten by adjusting the parameters of the circuit to obtain the proper value of $k$. The recorder chart can then be graduated in a convenient number of effective candles per chart division.

\subsection{Calibration.}

The calibration of the photometric system to obtain the proper value of $k$ is accomplished by means of a standard lamp.

$$
\begin{gathered}
\quad R_{s}=\frac{k I_{s} T}{d^{2}}, \\
\text { then } \quad R_{s}=5 \tau D^{2} \frac{R}{I_{e}} \cdot \frac{I_{s} T}{d^{2}} .
\end{gathered}
$$

A value of $I_{e} / R$ is chosen and the parameters of the photometric system are adjusted to obtain the desired $R_{s}$. The aperture of the photometer is kept sufficiently small so that the phototube will not be saturated during a flash. The electrometer-amplifier is generally operated on the 100- or 300-millivolt range using an input resistance of $10^{7}$ or $10^{8} \mathrm{ohms}$. The maximum output of the instrument is kept near, but below, the design maximum. Final adjustment of the calibration is obtained by adjusting the value of the Resistor, $R_{L}$, across the amplifier output until the voltage drop across this resistor drives the recorder to the desired reading, $R_{s}$.

\subsection{Performance Tests.}

The photometric system was calibrated by means of a standard lamp one meter from the photometer and two 2-aperture sector dises having transmittances of 0.0098 and 0.0252 . These sector discs were used as designed and also with one aperture blocked, resulting in transmittances of 0.0049 and 0.0126 . The dises were driven at speeds of $80 \mathrm{rpm}$ and greater. Under these conditions the variation of the illumination on the photometer during the time of exposure approximates that obtained with condenser-discharge lamps. No measurable change of response with variation in speed of rotation of the sector dises from 30 to $1000 \mathrm{rpm}$ was found. The recorder readings were proportional to the four transmittances of the sector dises within the accuracy with which the recorder chart could be read.

Measurements have been made automatically of 


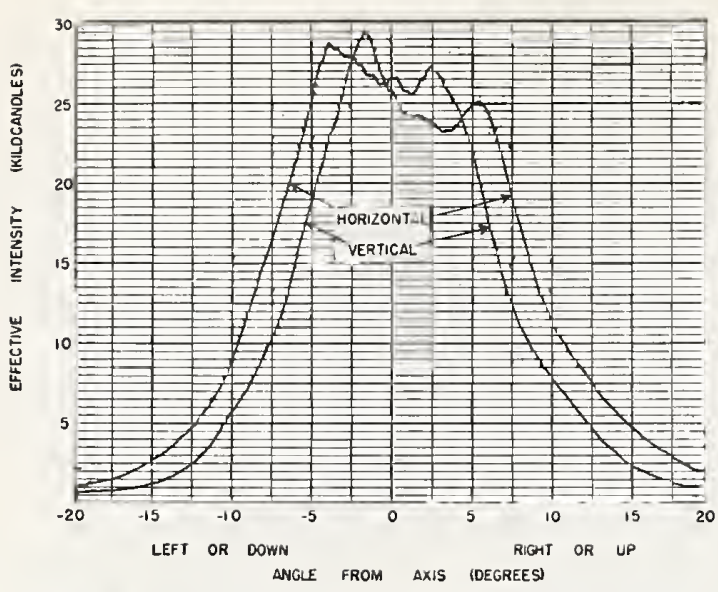

Figure 2. Typical horizontal and vertical intensity distributions made automatically with the effective-intensity photometer of a projector with a flash rate of two per second.

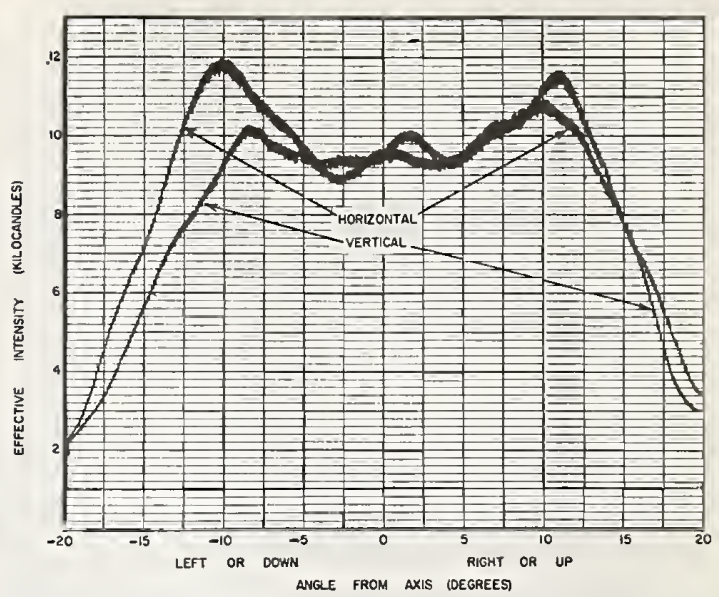

Figure 3. Typical horizontal and vertical intensity dis. tributions made automatically with the effective-intensity photometer of a projector with a flash rate of one per second.

have been so long that the sean rate of the goniometer would have had to be considerably slower than the 7.5 degrees per minute rate used when these figures were recorded.

\section{Discussion}

The photometer system described here is, of course, not limited to the photometry of condenserdischarge lights only, but is applicable to any light having a flash duration of less than about one millisecond (between the times when the intensity is about 5 per cent of peak intensity). The effective intensity distributions of lights with flash durations somewhat longer than one millisecond (up to about 0.01 second) can often be recorded automatically with this system if a suitable correction factor is included in the calibration. This factor is the ratio of the effective intensity computed by equation (1) to that computed by equation (2).

The components used in the system described here can, of course, be replaced with components having similar characteristics. Those listed in Fig. 1 were used because they were readily available.

\section{References}

1. IES Lighting Handbook, Second Edition (1952) Section 1. 2. Blondel, A. and Rey, J.: Journal de Physique, 1 (5th Series), 643 (1911).

3. Douglas, C. A.: "Computation of the Effective Intensity of Flashing Lights," Illuminating ExgINeERING, Vol. LII, No. 12 (December 1957).

Reminted from ILLuminating EngineERing 
Flasming lights are in widespread use as signals, markers and warnings because of their distinctiveness and their superiority over steady burning lights in attracting attention. Complete evaluation of flashing lights or lighting systems employing flashing lights usually requires the weighing of many factors suel as configuration, background, information to be presented, etc., and may involve complex, elusive, psychological elements. In most applications of flashing lights to land, sea, air navigation or traffic control, one of the fundamental considerations is the apparent or "effective" intensity of the lights. This has been the subject of considerable researel for over a century, especially since 1911, when the classical work of Blondel and Rey was published. It is the purpose of this paper to review existing psychophysical knowledge in the field essential to the design and evaluation of flashing lights.

The evaluation of the effective intensity of a flashing light is relatively easy under some eonditions and very difficult nnder others. If the flashing light is viewed under conditions such that the illuminance at the observer's eye is at or very near lis visual thresloold, then the problem is relatively simple. For threshold viewing, Blondel and Rey defined the effective intensity of a flashing light as the intensity of a steady light which is seen at threshold under the same conditions. The experimental techniques for aseertaining the threshold for a steady light may be used to determine the equivalent threshold for a flashing light. If, however, the flashing light is viewed under conditions in which the illuminance at the eye is above threshold, and especially if it is far above threshold, it is difficult if not impossible to exclude from any judgment as to effective intensity psychological elements which are not present at low levels. Observers find it difficult to distinguish between the judgments "equally intense" and "equally irritating." In spite of this difficulty, the concept of effective intensity has heen extended to abovethreshold viewing, and has been found useful at

A paper presented at the National Technical Conference of the Illuminating Engineerinf Society, September 9-13, 1957, Atlanta, Ga. AUTHor: Photometry and Colorimetry Section, National Bureau of Standards, Washington, D. C. This raper was prepared as a part of the work in the development of aircraft lighting conducted at the National Bureau of Standards under sponsorslip of the Bureau of Aeronautics. Accepted by the Papers Committee of I.E.S. as a Transaction of the Illuminating Engineering Society. least at relatively low levels, that is, up to about twenty times threshold. The experimental technique for above-threshold measurements of effective intensity involves the comparison of a flasling and a steady-burning light, one of which is varied in intensity until the observer reports apparently equal intensity. Such judgments are difficult to make, requiring as they do the equating of profomully dissimilar appearances. The resuts of above-threshold measurements indicate that the effective intensity of a flashing light varies with the level of illuminance at the eye, and is thus fundamentally different from the intensity of a steadyburning source, which is a fixed property of a light source, essentially independent of the viewing condition. The concept of "effective intensity" may be useful only at the lower levels of illuminance at the eye where the fundamental ability to see is of great importance, so that the difficulty of making intensity judgments at higher levels may not have practical implications. In any event any measure of effective intensity of flashing lights must be related to the viewing conditions, and the use of the eoncept should te avoided at high levels of illuminance.

\section{Classical Work of Blondel and Rey}

The experimental work reported by Blondel and Rey in $1911^{1}$ consisted of a series of determinations of threshold illuminance with the olselvers viewing a centrally fixated point source of incandescentlamp light which was flashed with a shutter at controlled durations from 0.001 to 3 seconds. The wave form of the flash was approximately square; that is, the light appeared at full intensity at the onset of the flash, remained at full intensity for the duration of the flash and then fell abruptly to zero at the end of the flash. For each flash duration, the intensity of the light was varied to establish the threshold. The results of the experiments are shown in Fig. 1.

The ordinate of a point is the ratio of the energy of a threshold test flash to the energy of a threesecond threshold flash, expressed as $E t / 3 E_{3}$. where $E$ is the instantaneons illuminance at the eye during the test flash and $E_{33}$ is the instantaneons illuminance at the eye during the three-second flash. A three-second flash was chosen for comparison 


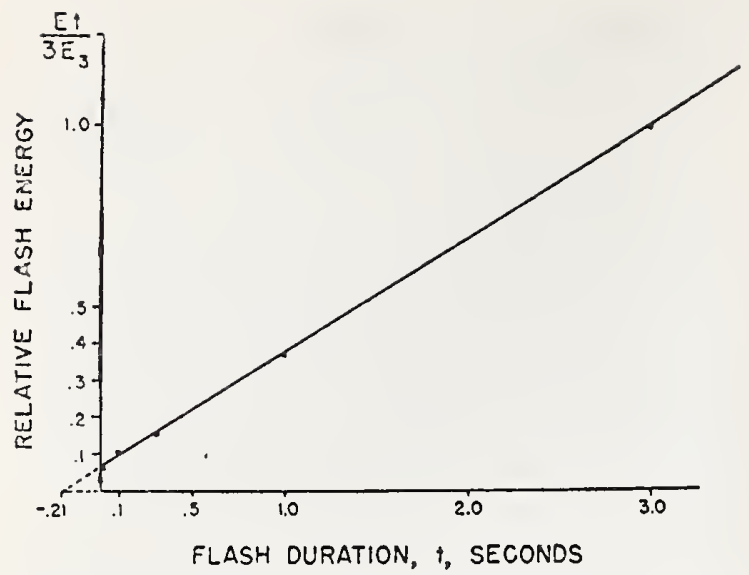

Figure 1. Ratio of test flash cnergy required for threshold excitation to encrgy of a threshold flash of 3 seconds duration as a function of test flash duration. (Blondel and Rey).

with the test flash as representing a duration sufficiently long that the effective intensity would be closely equal to the intensity of the same lamp burning steadily. The abseissas are the durations of the flashes. Blondel and Rey found that a straight line eould be fitted very well to the experimental points plotted on the graph, and that the equation of this line could be expressed in the forns:

$$
\frac{E_{o}}{E}=\frac{t}{.21+t}
$$

where $E_{0}$ is the threshold illuminanee for a steady source and $E$ is the illuminance during the time $t$ of a flashing light at threshold. If it is kept in mind that the equation applies to threshold illuminanee under a given set of viewing conditions, then intensity may be used interchangeably with illuminanee :

$$
\frac{E_{0}}{E}=\frac{I_{0}}{I}=\frac{t}{.21+t}
$$

and one may refer to the effeetive intensity as a property of the flashing light.

The Blondel-Rey equation has been investigated extensively sinee 1911 by Blondel and Rey themselves, ${ }^{2}$ and by many others. ${ }^{3,4.5}$ (The references cited above and in what follows are not intended to be complete but are seleeted as illustrative, or as of special importance. Work done prior to 1911 was summarized by Blondel and Rey, ${ }^{1}$ and many additional references will be found in the other referenees given in this paper.) The validity of the equation has been generally well established, not only under the speeial conditions studied by Blondel and Rey, but also with sourees of different sizes, ${ }^{B}$ with observers in different states of dark adaptation, ${ }^{7}$ with observations made in peripheral areas of the retina, ${ }^{8,6,10}$ and with lifferent colors. ${ }^{8}$ Researeh work on the relation between effective intensity and the wave form of the finsh, extremcly short durations, the level of illuminance at the observer's eye, and flash repetition rate will be discussed in detail later. In aldition to the investigations which have been more or less directly eon. neeted with effective intensity, there have been a number of studies of more eomplex visual problems connected with flashing light signals in whieh effeetive intensity played an important part. ${ }^{11.12}$ Such studies have yielded resnlts eonsonant with the Blondel-Rey equation.

\section{Analysis of the Blondel-Rey Equation}

The Blondel-Rey equation has been found to have sueh widespread applicability that it might be well to analyze its implieations before discussing its speeialized aspeets. Its simplest general expression (for flashes of square wave form) is

$$
\frac{I_{e}}{I}=\frac{t}{a+t}
$$

where $I_{e}$ is the effeetive intensity, $I$ is the instantaneous intensity during the flasl, $t$ is the duration of the flash in seconds, and $a$ is a eonstant. The value of $a$ found by Blondel and Rey, 0.21, has been confirmed by subsequent work, although the inherent impreeision of experimental work in this ficld makes it desirable to round the constant to 0.2 . As will be shown later, the constant $a$ appears to decrease as the level of illuminanee at the cye inereases, with the value of 0.2 representing its maximum value at threshold illuminance.

For purposes of analysis, methods of flashing lights may be divided into two eategories. In the first, the light is burned steadily, and flashing is obtained by oeeulting the light with a shutter or similar deviec so as to eoneeal the light from the observer exeept during the flash interval. In this method, the light from the source is not visible during the dark intervals and the luminous energy emitted during the dark interval, as well as the eor. responding eleetrieal or other form of energy whieh produees it, may be eonsidered "wasted." In the seeond eategory, the light source is energized only during the "on" interval, as when flashes are produced with an cleetrical switeh, or by discharging the electrical enerry stored in a condenser into an electronie flash lamp. Flashes produeed by these methods do not involve "wasted" energy" although the efficieney of utilization of energy may vary with the type of light souree and the method of producing the flash. Flashes produeed by oscillat- 
ing or rotating steady burning light are considered to belong in this second category, since the light energy is fully utilized when, as is usually the ease, all the directions swept by the beam are of importance

Fig. 2 gives curves of the Blondel-Rey equation for flashing lights of the first category, where a steady burning light of intensity $I$ is exposed for a time $t$, yielding an effective intensity $I_{e}$. The effective intensity for extremely short flash durations approaches zero, increases rapidly as duration increases, and finally approaches the steady intensity I asymptotically as durations become relatively long. According to the mathematics of the equation, it would take an infinitely long duration to achieve full equality of $I_{e}$ and $I$, but for practical purposes the two are equal when the duration is of the order of two or more seconds, depending somewhat on illuminance level. The relation between illuminance level, as represented by a corresponding value of $a$, and effective intensity, is shown in the figure. For example, for a duration of 0.2 seconds, $I_{e}$ is about 50 per cent of $I$ at threshold when $a=0.2$, but about 90 per cent of $I$ when the illuminance is such that $a=0.02$. Thus, if a given flash of light appears equal in intensity to a given steady burning light when both appear to be at threshold, then the flashing light will appear more intense than the steady light when the conditions of observation are such that they both appear to be well above threshold. This effect may explain a number of apparently anomalous field observations

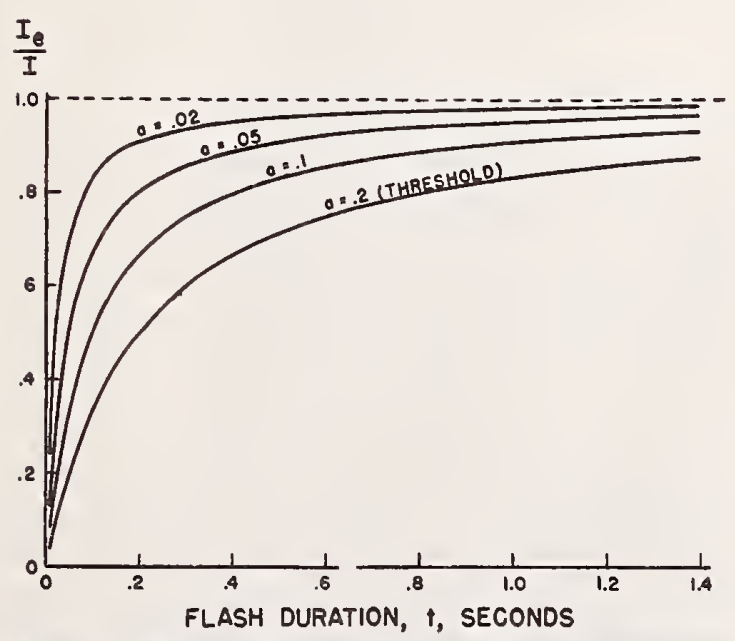

Figure 2. Effective intensity ratio, $I_{e} / I$, as a function of flash duration, according to the Blondel-Rey equation (equation 1), for the case where a steady burning light of intensity $I$ is exposed for a time, $t$. Curves are shown for threshold illuminance when $a=0.2$, and for abovethreshold illuminances when $a$ has the values $0.1,0.05$, and 0.02 .

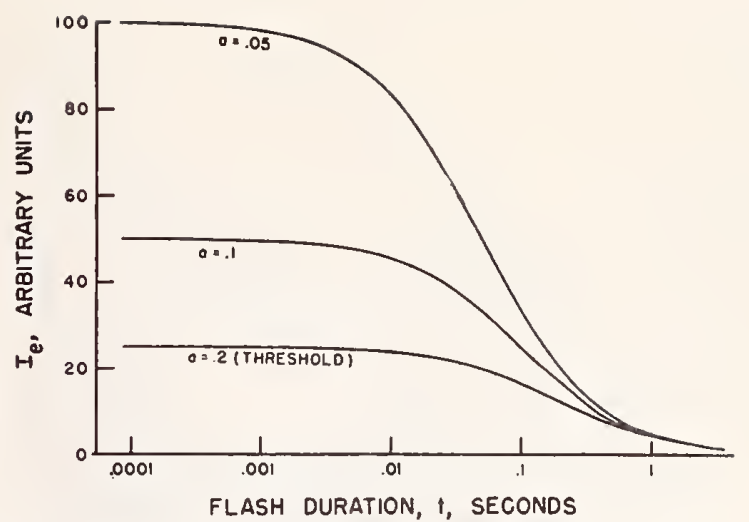

Figure 3. Effective intensity, $\boldsymbol{I}_{e}$, as a function of flash duration, $t$, for flashes of equal energy, at $a=0.2$ (threshold illuminance), 0.1 , and 0.05 , plotted from equation 3 , with $k$ arbitrarily chosen as equal to 5 .

wherein comparisons between flashing and steady lights or between flashing lights of very different flash durations do not give the same results when the comparisons are made close to the lights and illuminances are high as they do at considerable distances where the illuminances are very low.

For flashing lights of the second eategory, where there is no "waste" energy, a more meaningful presentation of the Blondel-Rey equation is shown in Fig. 3. In these curves the effective intensity for flashes of equal luminous energy is plotted against flash duration.

To obtain the curves of Fig. 3, the numerator of the right side of the Blondel-Rey equation, written in the form:

$$
I_{e}=\frac{I t}{a+t}
$$

is kept constant by varying $I$ and $t$ inversely with respect to each other so that

$$
I_{e}=\frac{k}{a+t},
$$

and plotting $I_{e}$ against $t$. $I_{e}$ becomes zero if $t$ is increased sufficiently. As $t$ is decreased, $I_{e}$ increases, and finally, when $t$ becomes very small compared to $a, I_{e}$ levels off to a value given by:

$$
I_{e}=\frac{I t}{a} \text {. }
$$

At threshold, when $a=0.2$,

$$
I_{e}=5(I t) \text {. }
$$

As in the case of shutter flashing in the first category, the relative effectiveness of an abovethreshold flash is greater than it is at threshold. For example when $a=0.05$,

$$
I_{e}=20(I t) \text {. }
$$




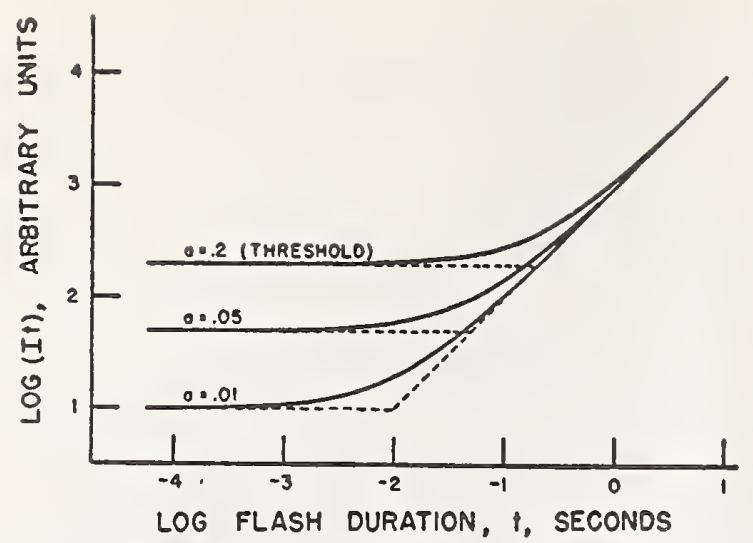

Figure 4. Flash energy (plotted as $I t$ ) required to pro. duce a given effective intensity, as a function of flash duration, at $a=0.2$ (threshold illuminance), 0.05 and 0.01 .

It will be noted that as $a$ is decreased, the duration time $t$ at which the curves level off is decreased, but in every case, leveling off occurs, and further reduction of the time of the flash produces no further increase in $I_{0}$ for flashes of constant energy. Thus for extremely short flashes, such as those obtained with electronic flash tubes ("strobe" lights), $I$ is reciprocally related to $t$ for a given $I_{0}$ according to the Blondel-Rey equation in a manner analogous to the equations used for determining photographic exposure.

The curves of Fig. 3 show the relationship between luminous energy in the flash and flash duration. A variation of this form of presentation, used by many research workers, ${ }^{10,11}$ is shown in Fig. 4. In this figure, the luminous energy (plotted as $I t$ ) necessary to produce a given effective intensity is plotted against flash duration. When the BlondelRey equation is plotted in this way, two distinct parts of the curves are clearly indicated. The portion at the left, for very short flash durations, shows the reciprocal relation betwcen $I$ and $t$, as does Fig. 3, where

$$
I t=\text { constant. }
$$

The part at the right indicates that for long durations

$$
I=\text { constant. }
$$

Between these two regions the curve blends smoothly from one limiting relation to the other. The two limiting relations are plotted as straight lines and their intersections, shown in the figurc by dashed lines, define the "eritical duration" or the duration marking the boundary time between the two rclations. It may be noted that the critical duration at threshold is about 0.1 second. Fig. 4 may be comparcd with Fig. 1, from Blondel and Rey, which is similarly plotted execpt that the scales of Fig. 1 are linear rather than logarithmic. The logarithmic form of Fig. 4 shows clearly the two parts of the eurve.

For a clear understanding of the significance of the curves of Fig. 4, and particularly to clarify the relationship of the curves for different values of $a$, it may be helpful to describe an experimental procedure for obtaining them. With observers stationed at some arbitrary distance from the light source, the intensity of the flash at very long duration is adjusted until the flashes appear to be at threshold. The curve for $a=0.2$ is then obtained by reducing the flash duration by set amounts and determining the relative energy required to maintain the light at threshold. When that is done the light is readjusted to the intensity and duration prevailing at the beginning of the experiment and the observers are stationed at a point closer to the light by an amount sufficient to raise the illuminance to a level above threshold corresponding in this case to $a=0.05$. A curve for $a=0.05$ is then obtained as before (except that a comparison technique must be used instead of threshold observations) and this procedure is repeated for any additional values of $a$ desired.

\section{Integral Form of the Blondel-Rey Equation}

Shortly after the publication of their classic paper on abrupt flashes, Blondel and Rey took up the question of flashes having time distributions other than square. On purely intuitive grounds they proposed the following modification of their original equation for effective intensity

$$
I_{0}=\frac{\int_{t_{1}}^{l_{8}} I d t}{.21+\left(t_{2}-t_{1}\right)} .
$$

In this equation the numerator of the right side represents the light energy contained in the flash between the time limits of integration, $t_{1}$ and $t_{2}$. For the case where the flash is abrupt (square in shape) this reduces to the original equation

$$
I_{0}=\frac{I\left(t_{2}-t_{1}\right)}{.21+\left(t_{2}-t_{1}\right)}=\frac{I t}{.21+t} .
$$

The difficulty with the integral equation proposed by Blondel and Rey is that there is an ambiguity about the choice of the time limits, $t_{1}$ and $t_{2}$. Blondel and Rey recognized this difficulty and could ofler 110 rigorous solution fur it. They did, however, suggest that $t_{1}$ and $t_{2}$ should be chosen as the times when $I$ was equal to the threshold value of $I$ for the case of steady illumination.

Recently Douglas" has proposed that the am-

- Seo Part 1I, japer by C. A. Douglas. 
biguity in the selection of time limits in the integral form of the Blondel-Rey equation be resolved by selection of the limits so as to maximize $I_{\rho}$. When the limits of integration are so chosen, it turus ont that the mistataneous value of intensity at these time limits is the steady light threshold intensity, exactly as proposed by Blondel and Rey in 1911. Douglas further shows that in general, for any value of $a$, the maximum value of $I_{c}$ is obtained when the limits of integration are those ralues of $t$ at which the instantaneous value of $I$ is equal to $I_{c}$.

Oddly, although most practical flashing light sources do not produce abrupt flashes, Blondel and Rey's proposal of the integral form of their equation has been generally ignored since 1911. Stiles, Bennett, and Green ${ }^{4}$ took note of the proposal in 1937, but commented that there had been little or no experimental verification of it. Blondel and Rey ${ }^{2}$ themselves, in 1916, made some measurements of effective intensity on rotating beacons with abrupt square form flashes, and with non-abrupt flashes of both long and short duration. The conditions of their experimental work were such as to give imprecise results, but within the error of their measurements, they confirmed the original equation and the integral form of it. As Douglas has shown, the uncertainty in the computation of $I_{e}$ resulting from the uncertainty in the choice of the limits of integration is not very large, especially for flashes of relatively short duration, so that experimental verification of the validity of the method of maximization of $I_{e}$ is very difficult to obtain. The uncertainty of Blondel and Rey's measurements was so great that the choice of limits of integration had no significant effect on their results.

Neeland, Laufer, and Schaub, ${ }^{5}$ in 1938, reported the results of a series of measurements of effective intensity of rotating beacons, with both square and non-square flash characteristics. They used the results of their measurements to compute the value of $a$ in the Blondel-Rey equation, but fitted both the square and non-square flashes to equation (2). For the non-square flashes they took $I$ as the peak of the candlepower-time distribution and $t$ as the interval between those points on the distribution where the instantaneous intensity was 10 per cent of the peak intensity. They thus attributed much more energy to the flash than was actually there. The values of " they obtained fo" souarefom flashes eonformed fairly well with those obtanned by others, but the values of $a$ for the non-square flashes were siguficantly higher. If, however, their results are computed from the integral form of the equation, then the values of $a$ are in approximate agreement with those obtained by others.

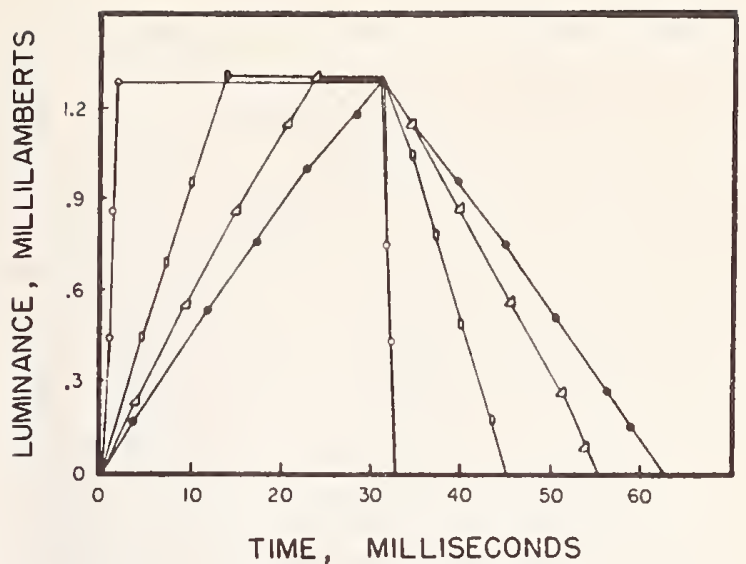

Figure 5. Curves showing four of the seven wave forms used by Long in determining the relation between wave form of a flash and effective intensity. The wave forms are so designed as to provide equal total flash energies.

In 1951, Long ${ }^{10}$ reported an investigation of the relation between the wave form of the flash and the effective intensity. He used seven wave forms ranging from a square wave to a triangular wave. Fig. 5 shows four of the seven wave forms. The other three were intermediate in shape. The wave forms were so designed as to contain the same light energy per flash. The viewing condition was 15 degrees peripheral and the light source subtended a visual angle of 2 minutes. Long did not use the integral form of the Blondel-Rey equation nor did he concern himself with the choice of the limits of integration. He computed the total energy under the curve for each wave shape. Long felt that he was well within the range of reciprocity since his flash durations were quite short, and therefore looked only for the relation between the total

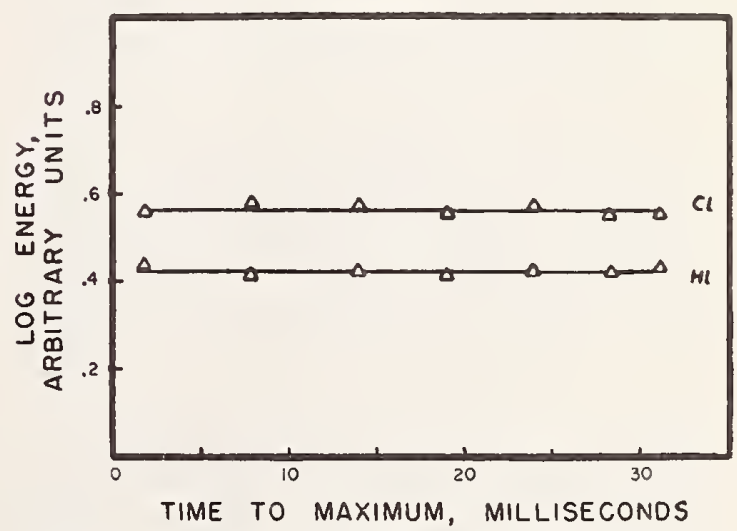

Figure 6. Flash energy required for threshold excitation for flashes of seven wave forms (four of them shown in Fig. 5) by two observers. The seven wave forms are distinguished by the time required for the intensity 10 rise to a maximum. (Long). 
chergy in the flash and the offective intensily. II results are shown in Wig. 6 (Long identified the lifferent wave shapes by the time it took for the liminance to rise to a maximum.) It will be noted that the eonstancy of the energy required for thresluold exeitation with the differcnt wave shapes was rather remarkible, in view of the experimental crrors usually found in work of this kind.

It is of interest to eonsider Long's work from the point of view of Douglas' proposed method for computing $I_{c}$. For the ease of the square form fasil or the nearly square trapezoidal flashes, there is a negligibly small differenee between $I_{e}$ computed from the total energy and $I_{e}$ naximized by Donglas' method. IIowever, as the wave form becomes more nearly triangular, a larger differeñee may be expected. The eomputed difference for the triangular wave turns out to be about 10 per cent; that is, althongh the square form flash and the triangular form flash have equal total energy, $I_{e}$ for the triangular flash is 10 per eent less than $I_{e}$ for the square fiash when both are eomputed from the integral form of the Blondel-Rey equation by Douglas' method of maximization. However, this differenee amounts to only about $0.05 \mathrm{log}$ unit on the ordinate scale of Fig. 6 and it is evident therefore that Long's results are not greatly affected by the ehange in the method of eomputing $I_{e}$.

\section{Extremely Short Flashes}

As shown above, the relation between intensity and duration is reciprocal, aeeording to the Blondel-Rey equation, for flashes with durations appre(iably shorter than the "eritical duration." It has been suggested, especially since the introduetion of clectronic flash lamps with flash durations of the order of fractions of a milliseeond, that the reciprocity relationship does not hold for extremely short flashes, and that such flashes have higher effective intensity than that which would be predicted from the Blondel-Rey equation. On the other hand, some early researeh workers obtained results that suggested a failure of reciprocity in the other direetion for short flashes, that is, the effeetive intensity was less than $I_{e}$ eomputed from the Blondel-Rey equation. Reeves, ${ }^{6}$ for example, in 1918 , foum that it took about half again more enery in a flash at a duration of two milliseconds than in a flash of 10 milliseconds duration to obtain threshotel illuminance. Piéron, ${ }^{7,8}$ in 1920 , found a similar reciprocity failure, althongh his results were less consistent. Stiles, Bennett, and Green, in 3937, expressed doubt as to the validity of the inference of reciprocity drawn ly l'ierón and Roveres from their datal. It appears likely, in view of the absence of any reninrority lisilure in the results obtained in other work, most of it carried out with eonsiderably mo: "are, that the experimental error in the work of Piéron and Recves was sufficiently great that an inference of reciprocity could heve been clrawn as valiclly as one of tialure of reeiprocity. 'Their papers furuished only sparse information as to experimental techniques, but it is likely for example that there may have been substantial errors in their measurements of the dnrations or energies of short flashes. Jaumgardt, ${ }^{9}$ in 1949, measured effeetive intensity in the duration range from four microseconds to one millisecond and found reciprocity throughout this range. Iis results are shown in Fig. 7 .

The weight of the evidenee for reeiproeity is sufficiently great as to offer no support for the existence of a failure of reciproeity in either direc. tion with any light sonrces now in use.

\section{Above Threshold Illuminances}

Blondel and Rey observed during the course of their work on threshold measurements that a short duration flash that appeared equal in intensity to a long duration flash at threshold, appeared noticeably more intense than the long flash when the illuminance at the eye was wcll above threshold. They did not, however, pursue the question raised by this observation. Toulnin-Smitl and Green, ${ }^{13}$ in 1933, reported the results of a series of experiments with dark-adapted observers in which ere illuminances were varied from 0.2 mile-eandle (slightly above threshold) to 4.0 mile-eandles. In connection with signal lights used in navigation, the eoncept of "useful" threshold had been developed, as a measure of the lower limit of illuminance at whieh, under practical conditions, reliable recognition of the existence of light signals might be expeeted. It is generally agreed that this is in the neighborhood of 0.5 mile-candle, although under the variable eonditions so often found in practice, wide departures from this valne may be found. (Toulmin-Smitl and Green used the ratler overpreeise value of 0.425 mile-candle as the "useful" threshold.) Their range of illuminances was therefore from about $1 / 2$ to abont 10 times the "usefnl" threshold, and bracketed fairly well the ranne of illuminanees in which signal siglitings usually oceur. The flash durations were from 0.05 to 0.5 seconds. Fir. 8 shows the data obtained at an illuminance of 0.5 mile-eandle and indieates the spread of the individual observations. Fig. 9 shows the family of enrves obtained at the five ilhmi-

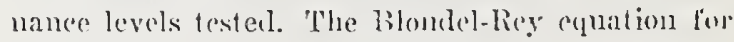
threshold illuminanee is plotfed in the firmre and 


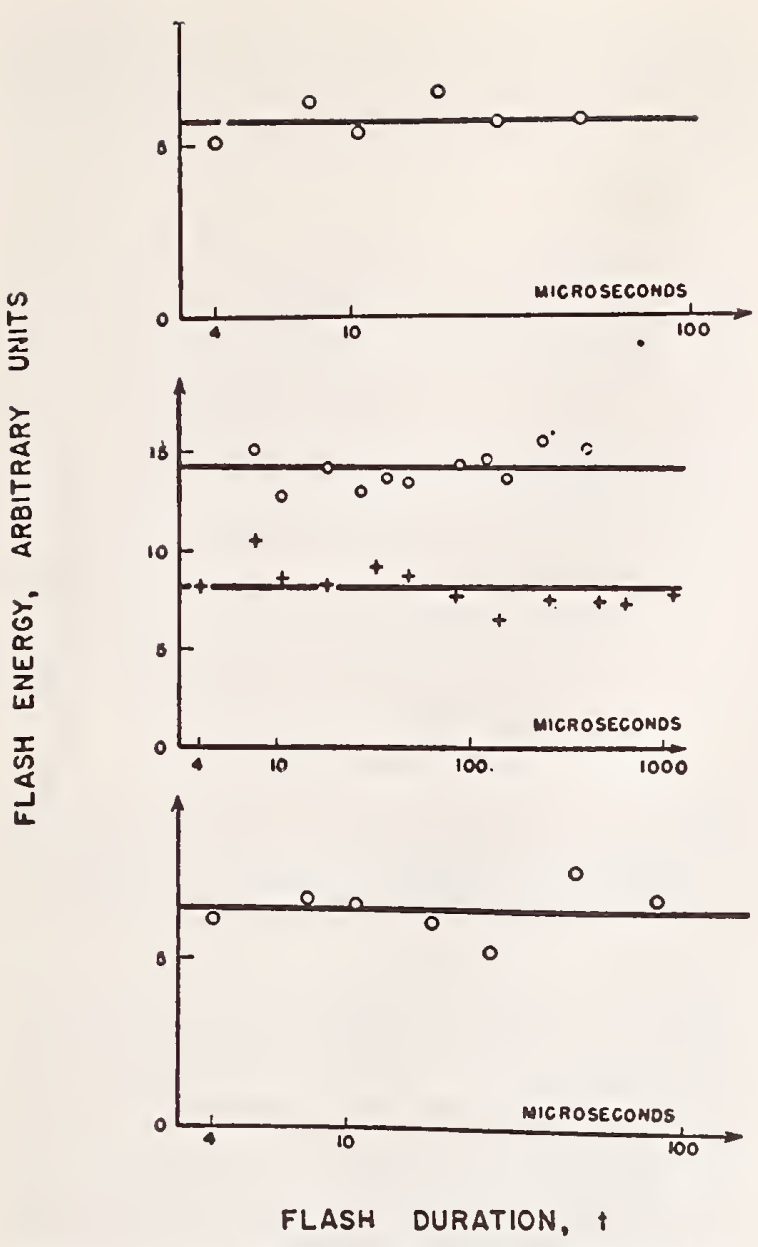

Figure 7. Flash energy required for threshold excitation $12^{\circ}$ from the fovea as a function of flash duration, $t$, for values of $t$ between 4 microseconds and 1 millisecond. Data for four observers. (Baumgardt)

shows very close eorrespondence to the experimental curve at 0.2 mile-candle. Toulmin-Smith and Green tried to determine an equation of close fit to their data for the "uscful" threshold at 0.425 mileeandle (obtained by interpolation from their experimental results) as shown in Fig. 10, in which are plotted the Blondel-Rey equation, interpolated test data for 0.425 mile-candle, and the fitted curve for the equation

$$
\frac{I_{e}}{I}=1.1 \frac{t}{.15+t} \text {. }
$$

Hampton ${ }^{14}$ in 1934 objected to the ToulminSmith and Green equation because of the coefficient 1.1, asserting that an adequate fit to all of their data could be obtained by an equation in the Blondel-Rey form with the constant, $a$, treated as a function of the illuminance level. On this assumption, he plotted Toulmin-Smith and Green's data as

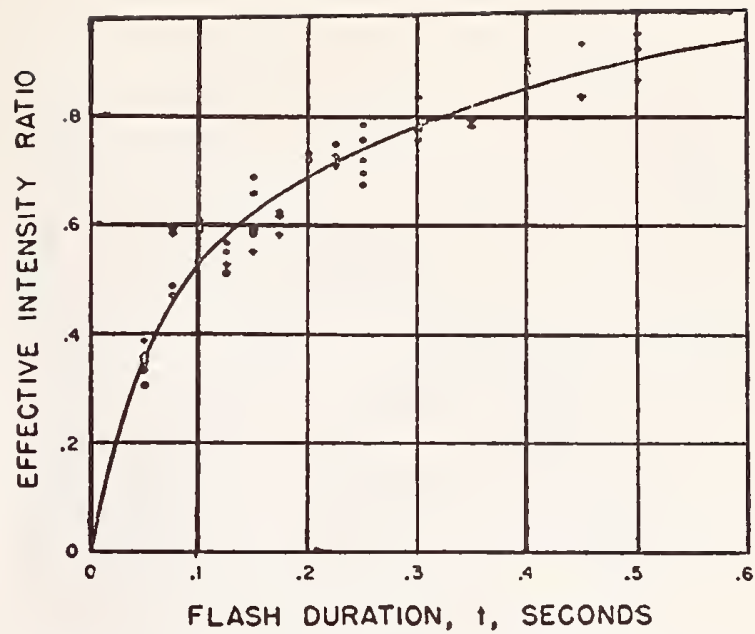

Figure 8. Effective flash intensity relative to fixed intensity, as a function of flash duration, $t$, for each of two observers, at an illuminance of 0.5 mile-candle. (Toulmin-Smith and Green).

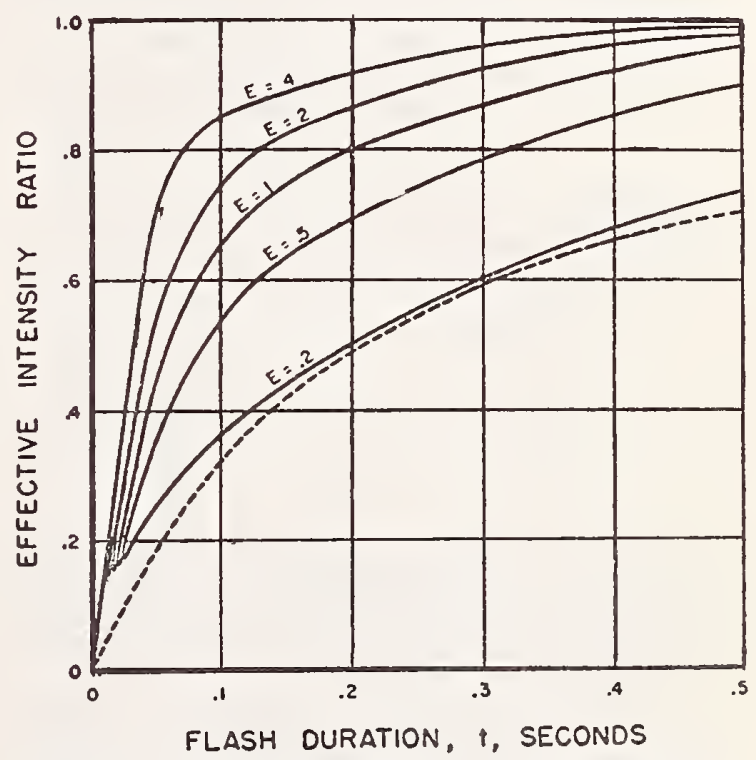

Figure 9. Effective flash intensity relative to fixed intensity, as a function of flash duration, $\ell$, at illuminances $E=0.2,0.5$ (data shown in Fig. 8), 1, 2, and 4 mile: candles. The dashed curve is the Blondel-Rey equation (equation 1) for threshold illuminance (Toulmin-Smith and Green'.

shown in Fig. 11, and from this obtained the equation,

$$
\frac{I_{c}}{I}=\frac{t}{(.0255 / E) \cdot 81+t}
$$

where $E$, the illuminance at the observer, is given in mile-candles. In this equation, $a$ has the follow- 


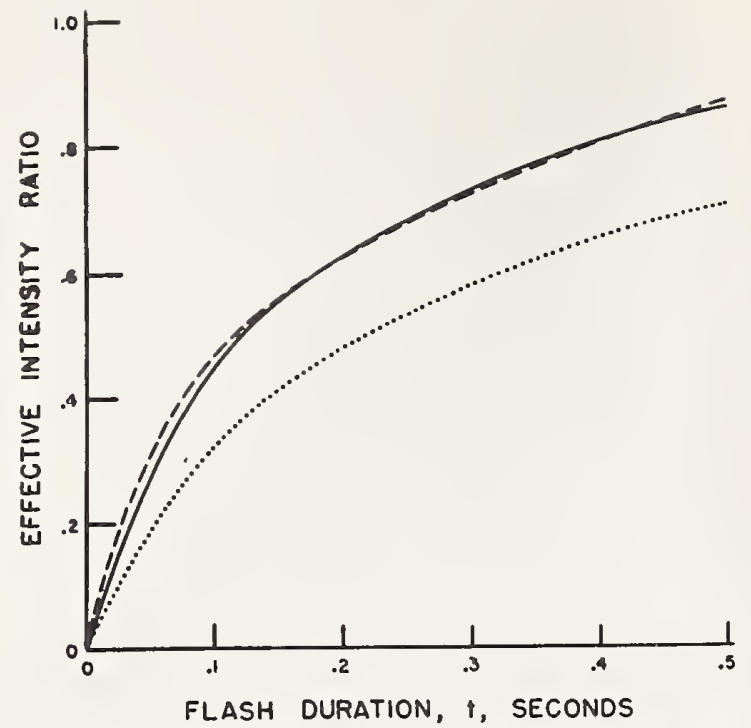

Figure 10. Curve fitted to test data at 0.425 mile-candle ("useful" threshold) by Toulmin-Smith and Green. The dashed curve is the test data, obtained by interpolation from the data represented by the curves of Fig. 9. The solid curve is the fitted equation (equation 5 ). The lower dotted curve is the Blondel-Rey equation (equation 1) for threshold illuminance.

ing values at the illuminance levels used in the original experiments :

$\begin{array}{cc}\text { Illuminance, } \\ \text { Mile-Candles } \\ .2 & a \\ .5 & .19 \\ 1.0 & .09 \\ 2.0 & .05 \\ 4.0 & .03 \\ & .017\end{array}$

Hampton then replotted the original curves, using his equation, and obtained the curves of Fig. 12, not significantly different from those of ToulminSmith and Green (Fig. 9).

In Fig. 13, Hampton compared his equation (equation 6), Toulmin-Smith and Green's formula (equation 5), and the experimental data for 0.425 mile-candle. None of the differences is significant, in view of the experimental errors inherent in this type of measurement.

Apart from the work of Toulmin-Smith and Green, there appears to have been no systematic effort to ascertain the relationship between effective intensity and illuminances above threshold. Some qualitative observations and occasional rough measurements ${ }^{1,2,12}$ have shown that effective intensity is relatively greater above threshold than at threshold, but have not confirmed with any precision the values of $a$ ealculated by Hampton from the data of Toulmin-Smith and Green. On the

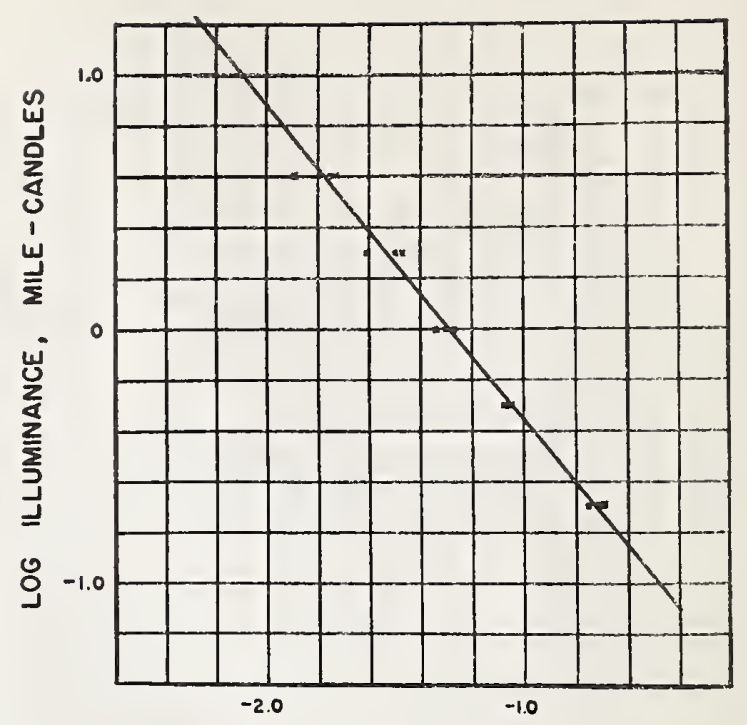

LOG (0)

Figure 11. Relation between the value of $a$ and the illuminance, plotted by Hampton on the assumption that the Toulmin-Smith and Green data could be represented by the Blondel-Rey equation (equation 1 ).

other hand, Schuil, ${ }^{15}$ in the course of some work in which the value of $a$ was incidental, noted that he did not confirm the Toulmin-Smith and Green value of $a$ at an illuminance of 2.0 mile-candles although he did confirm it at 0.5 mile-candle. He did not give any details nor did he mention the extent of the disagreement. In the absence of any definitive work calling for a modification, it appears that

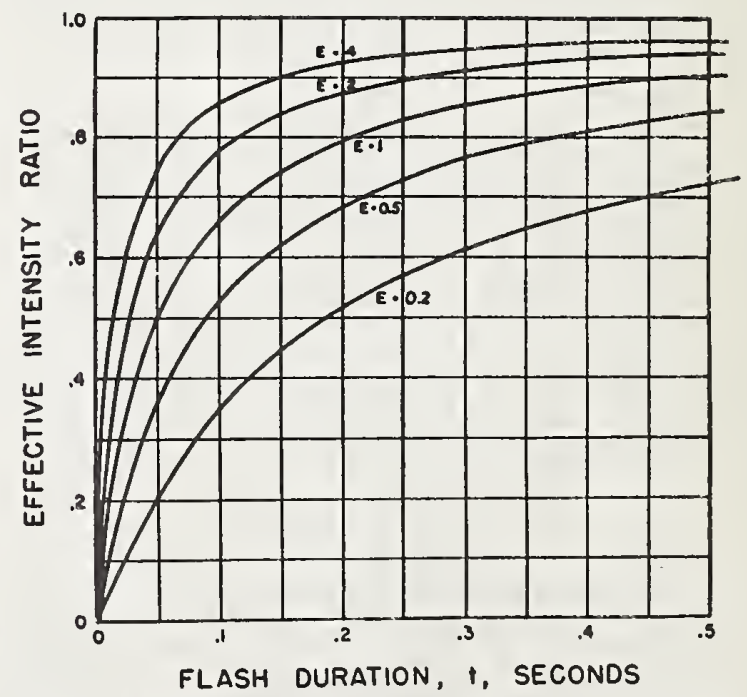

Figure 12. Representation of Toulmin-Smith and Green data (Fig. 9) as recast by Hampton in the form of the Blondel-Rey equation, with the value of a empirically determined from the data (equation 6). 


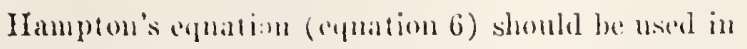
ealeulating ethective intensity for above-thresholl illuminances in the ramere covered by the observations of Toulnin-Smith and Green.

\section{Repeated Flashes}

The foregroing discussion of light flashes has considered a light flash as an isolated event in time, and the effective intensity of sueh isolated fiashes is well deseribed by the Bhondol-hey law. If flashes oceur in more or less rapil suceession, interaetion effeets may oeeur and it is of interest to know what these effects are. Many flashing sirnals used in praetiee do involve repetitive flashing simnals, for example, aireraft "anti-eollision" lights, traffie obstruetion markers, liglithouse beaeons, ete., so that the question is of some importanee. For flashes above the eritieal flicker frequeney, that is, at a repetition rate great enough so that the observer is unaware of the discontinuous eharaeter of the signal, Talbot's law holds, and the apparent intensity is equal to the average intensity. Under sueh eonditions, it is immaterial low the luminous energy is distributed in time: two light sourees with distributions whose time averages of intensity are equal will appear equally intense. Sehuil ${ }^{15}$ investigated this problem for flashes of $1 / 10$ and $1 / 40$ seeond duration, and with repetition rates from one per second up to frequeneies well above the limit of noticeable flieker. In his experimental procedure he kept the flash duration constant (at eitler $1 / 10$ or $1 / 40$ seeond) and varied the duration of the dark interval between flashes. Thus the energy per flash was eonstant but the time average of the intensity inereased as the dark interval deereased. Sehuil's results, for an instantaneous illuminanee during the flashes of 0.5 mile-eandle, are shown in Fig. 14. Three regions, indieated in the figure for eurve 2, the experimental results for a flash of $1 / 40$ seeond duration, may be distinguished. In the first, $A$, at the left, the light appears steady, and Talbot's law (eurve 4 for the $1 / 40$ second flash) holds. This region begins at the point where the dark interval is zero and the total period of the flash and its duration are equal, the light therefore being physieally eoutinuous. The region ends at about .075 seeond when the repetition rate is about 13 flashes per second and the light-dark ratio is about $1: 3$. The effeetive intensity is then about one-third of the instantaneous intensity during the flash in aeeordanee with Talbot's law. As the dark interval is inereased further and the repetition rate eorrespondingly deereased (B), flieker is observed until the period is about 0.2 seeond long, eorresponding to a repetition rate of five flashes per seeond. Be-

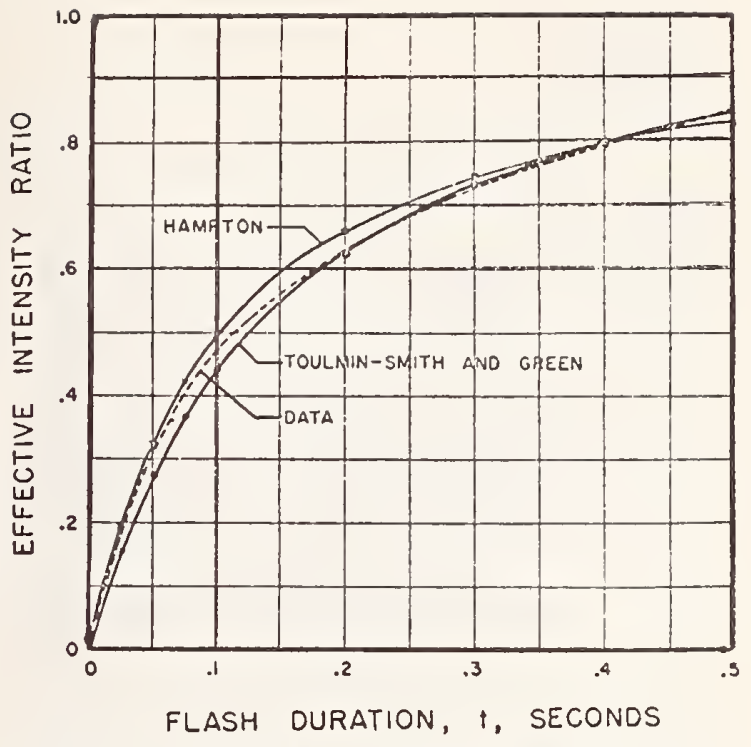

Figure 13. Comparison of Toulmin-Smith and Green equation, Hampton equation, and data, at illuminance of 0.425 mile-candle. (Hampton)

yond this point (C) the flashes are observed as more or less distinctly separate, and the effective intensity levels off to a value equal to that called for by the Blondel-Rey equation, with the value for a eomputed by Hampton from the data of Tou!min-Smith and Green. The data show a smooth transition from the region at the left where Talbot's law holds to the region at the right where the effeetive intensity of the flashes is equal to that of isolated flashes in aeeordanee with the Blondel-Rey law. Sehuil observed, however, as noted previously, that while the general relationship between effective intensity and repetition rate which he obtained at 2.0 mile-eandles was similar to that obtained at 0.5 mile-candle, the effeetive intensities for the limiting frequeneies at the right differed from those of Toulmin-Smitls and Green.

Although Sehuil's work shows a smooth transition from the region of Talbot's law to the region of the Blondel-Rey law, some other work sugrests that at eertain middle frequeneies in the flickering range, the effeetive intensity is aetually greater if a steady burning light is oeeulted than if it is viewed eonstantly. Bartley ${ }^{16}$ found for example that at a repetition rate of about 8 or 9 flashes per seeond, a flashing field of view was mateled in apparent brightness with a similar strady field when the luminanee of the flashing field was only about 60 per eent of the luminanee of the steady field. This result was obtained with a steady field luminance of about 300 footlamberts and a light-dark ratio for the flashing field of $1: 1$. Bartley did not give 


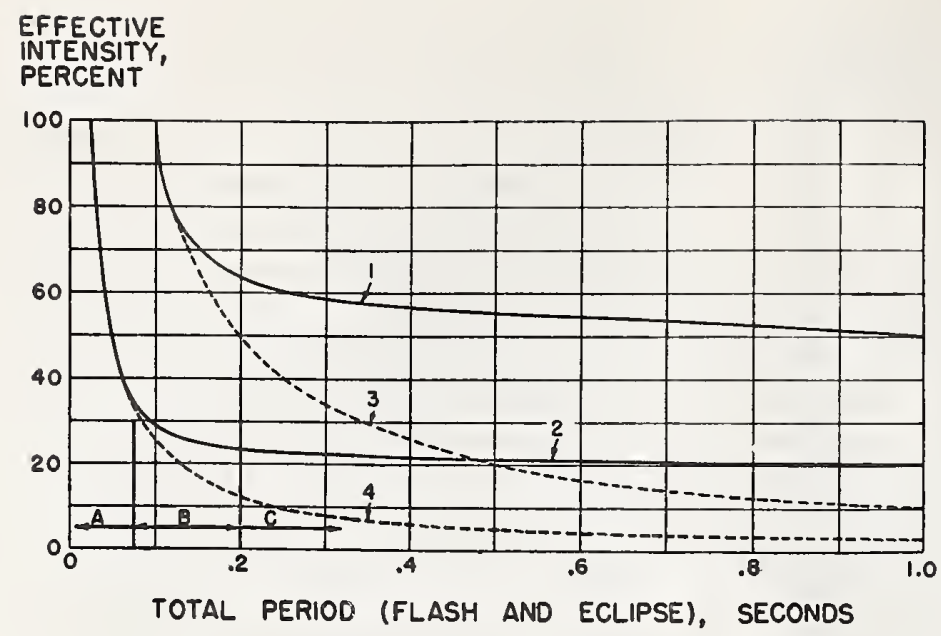

Figure 14. Ffective intensity of Mash relative to fixed intesnity, for repeated flashes, as a function of the period of the flash cycle, with the flash duration kept constant and the eclipse time varied to obtain different repettion rates. (Schuil).

Curve 1, experimental curve for $1 / 10$ second flash.

Curve 2, experimental curve for $1 / 40$ second flash.

Curve 3, Talbot's law for $1 / 10$ sccond flash.

Curve 4, Talbot's law for $1 / 40$ second flash.

(Note: Three regions for curve 2 are distinguished: $A$, in which the lighs appears steady, B, in which the light appears to flicker, and $C$, in which the flashes are seen as separate and distinct.) any data as to the size of the fields viewed, but it is presumed that the fields had some extent since he dealt with them in terms of luminance rather than intensity. Bartley gave data only for the results otbained with the 300 -footlambert steady field, but noted that he obtained essentially similar results with steady field luminances from about 14 to about 1200 footlamberts. It will be noted that Bartley's minimum luminance was very far above threshold: 500 times threshold even for a lightadapted observer. Schuil, on the other hand, worked close to threshold. The author has carried out rough measurements which confirm the smooth transition found by Schuil in the low illuminance region. It appears likely that at illuminance levels where the concept of effective intensity is useful repetitive flashes that appear separate and distinct from each other may be treated in accordance with the Blondel-Rey law.

\section{Conclusions}

Since it was first proposed in 1911, the Blondel and Rey equation

$$
I_{e}=\frac{\int_{t_{1}}^{t_{2}} I d t}{a+\left(t_{2}-t_{1}\right)}
$$

defining the effective intensity of a flashing light in terms of the duration of the flash and the instantancous intensity during the flash has become well established. A considerable body of research has verified the general validity of the equation over a large range of observing conditions. There are nevertheless many areas where the precise application of the equation is in some doubt and where considerably more work is called for in order to establish valid relationships or to confirm relationships at present only poorly supported.

For point sources of non-chromatic light, foveally viewed at threshold with the dark-adapted eye, Blondel and Rey's value of about 0.2 for the constant, $a$, seems fairly well established, although somewhat higher and lower values have been reported, ranging from about 0.15 to 0.25 . The inherent lack of precision of measurements in this field suggests that the value of 0.2 be used in all computations as representative of the values most often found. Observations of flashing lights not falling within the narrow confines set forth above, for example, lights that are chromatic, or with some extent, or viewed peripherally, seem to be subject to more or less the same relationship as long as effective intensity is-defined in terms of the same source burned steadily and viewed under the same conditions.

The general effect of raising the illuminance at the eye above threshold or of providing a light background against which the lights are viewed is to lower the value of $a$ in the Blondel-Rey equation. For the case of above-threshold viewing of flashing lights against a dark background, valucs of $a$ related to illuminance at the eye have becn established, but considerably more work conld be done in this area to put these values on a firmer foundation. Because of the critical dependence of the threshold illuminance on the conditions of observation, it may be profitable in researeh work to relate the results to the steady light threshold for the same conditions of observation.

Finally it seems clear that the concept of cflective intensity should be limited in application to 
low levels of illuminance, with the illuminance here considered as a multiple of the marginal amount of illuminance necessary to distinguish a flashing light from its background.

\section{References}

1. Blondel. A., and Rey, J.: "The Perception of Lights of Sbort Duration at Their Rs nge Limits," Transactions, IES, London, Vol. VII, No. 8, p. 625, (November 1912). This paper is a complete account in English of the material contained in two papers published in 1911 in the Journal do Physigus, Vol, I, Series 5, page 530 and psge 643 .

2. Blondel, A., and Rey, J.: "Comparaison, au point de vue de le portée, des signaux lumineux brefs produit, au moyen d'un appareil rotatif, par des sources de luinière donnant des durées d'impression différent. Conditions d'efficacité maxima du flux lumineux utilise," Comptes Rendus, Vol, 162, page 861, (1916).

3. Langmuir, I., and Westendorp, W. F.: "Study of Light Signals in Aviation and Navigation," Physics, Vol, 1, No. 5, p. 273, (November 1931).

4. Stiles, W. S., Bennett, M. G., snd Green, H. N.; "Visibility of Light Signals witb Special Reference to Aviation Lights. (Review of Existing Knowledge)." Aeronautical Research Committee Reports and Memoranda No. 1793, May 11, 1937.

5. Neeland, G. K., Laufer, M. K., and Schaub, W. R.: "Measure. ment of tbe Equivalent Luminous Intensity of Rotating Beacons," Journal of Optical Sociely of America, Vol. 28 , No. 8, p. 280 , (August 1938).

6. Reeves, P.: "The Effect of Size of Stimulus and Exposure Time on Retinal Thresbold," Astrophysical Journal, Vol. XLVIII, p. 141, (April 1918).
7. Piéron, H.: "De la Variation de l'Energie Liminaire en Fonction de la Durée d'Excitation nour la Vision Fovenle," Comptes Rendus. Vol. 170, p. 525, (1920).

8. Piéron, H.: "De la Varistion de l'energie Liminsire en Fonction de la Durée d'Excitation pour la Vision Póriphérique, (Loi des Cônes et Loi des Battonets)," Comptes Rendus, Vol. 170, p. 1203. (1920).

9. Baumgardt, E.: "Les Théories Photochimique Classiques et Quantiques de la Vision et l'Inhibition Nerveuse on Vision Liminaire," Revus d'Optique, Vol. 28, No. 12, p. 661, (December 1949).

10. Long, G. E.: "The Effect of Duration of Onset and Cessation of Light Flash on the Intensity-Time Relation in the Peripheral Retina," Journal of the Optical Society of America, Vol. 41, No. 11, p. 743, (November 1951).

11. Brown, R. H.: "Velocity Discrimination snd the IntensityTime Relation," Journal of the Optical Society of America, Vol. 45 No. 3, p. 189, (March 1955).

12. Leibowitz, H. W., Meyers, N. A., and Grant, D. A.: "Radial Localization of a Single Stimulus as a Function of Luminsnce and Duration of Exposure," Journal of ths Optical Society of America, Vol. 45, No. 2, p. 76, (February 1955).

13. Toulmin-Smith, A. K., and Green, H. N.: "The Fized Light Equivalent of Flsshing Lights," Illuminating Enginesr, London, Vol. XXVI, p. 304, (December 1933).

14. Hampton, W. M.: "The Fixed Light Equivalent of Flashing Lights," Illuminating Engineer, London, Vol. XXVII, p. 46, (February 1934 ).

15. Schuil, A. E.: "The Effect of Flash Frequency on the Apparent Intensity of Flashing Lights Having Constant Flash Duration," Transaclions, IES, London, Vol. V, No. 8, p. 117, (1940).

16. Bartley, S. H.: "Subjective Brightness in Relation to Flash Rate and the Light-Dark Ratio," Journal of Experimental Psy"/,w ogy, Vol. 23, No. 3, p. 313, (September 1938).

Reprinted from:

ILLUMINAT ING ENGINEER ING

Vol. 52, No. 21,

December 1957 


\section{Computation of the Effective Intensity Of Flashing Lights}

BY CHARLES A. DOUGLAS

$I_{T}$ Is GENERALLY recognized that when a light signal consists of separate flashes, the instantaneous intensity during the flashes must be greater than the intensity of a steady light in order to obtain threshold visibility. Blondel and Rey ${ }^{1}$ found that the threshold illuminance for an abrupt flash (a flash produeing a relatively eonstant illuminanec throughout its duration) is

$$
E=E_{0}(a+t) / t \text {, }
$$

where $E_{\mathrm{o}}$ is the threshold illuminanee for a steady light, $t$ is the flash duration, and $a$ is a eonstant. They found that $a$ was equal to 0.21 when $t$ is in seconds.

It is convenient to evaluate flashing lights in terms of their effeetive intensity, $I_{e}$, that is, the intensity of a fixed light which will appear equally bright. Then

$$
I_{e}=I E_{0} / E
$$

where $I$ is the instantaneous intensity producing the illuminance, $E$,

$$
I_{\theta}=\frac{I t}{a+t} .
$$

Later Toulmin-Smith and Green ${ }^{2}$ found that somewhat different effective intensities were obtained when the illuminanee at the eye was above threshold. However, Hampton ${ }^{3}$ showed that their experimental results eould be adequately expressed by equation (2) when $a$ is a funetion of the illuminance at the eye.

The flash from most lights used in aviation service, such as airway beaeons and anti-collision lights, is not abrupt. The instantaneous intensity often rises and falls gradually and may vary appreciably during the flash. If the flash duration is very short or if the times of rise and fall of intensity are short in comparison to the flash duration, only small uncertainties would be introduced in the determination of flash duration and by the use of the product

A paper presented at the Nationai Technical Conference of the Illuminating Engineering Society, September 9.13, 1957, Atlanta, Ga. AUTHOR: Photometry and Colorimetry Section, National Bureau of Standards, Washington, D. C. This peper was prepared as a part of the work in the development of aviation ground lighting conducted at the National Bureau of Btandards under the sponsorship of the Vieual Landing Aide Branch of the Bureau of Aeronautics and the Lighting Bection, Aeronautics Accesories Laboratory, Wright Air

Development Center. Arcepted by the Papers Committee of I.E.8. as a Tranaction of the Illuminating Engineering Society. of the peak intensity during the flash and the flash duration for the quantity $I t$. However, in many cases signifieant errors would be introduced. Some modification of equation (2) is therefore required.

Some of the speeifications for flashing lights have evaluated their signals in terms of the candleseeonds in the flash, integrating over a period of not more than 0.5 seeond, that is

$$
\text { Candle-seeonds }=\int_{t_{1}}^{t_{2}} I d t
$$

where $I$ is the instantaneous intensity and $t_{2}-t_{1}$ does not exceed 0.5 second. This method of evaluation provides a measure of eomparison between lights of roughly the same intensity variation with time but is not suited to the comparison of lights of different flash eharacteristics nor to the computation of visual ranges.

Others have used the relation

$$
I_{e}=\frac{I_{\max } t}{a+t},
$$

where $I_{\max }$ is the maximum instantaneous intensity during the flash and $t$ is the flash duration. Often the value of $a$ is adjusted for the characteristics of the flash so that the eomputed value of $I_{\theta}$ is in reasonable agreement with the observed value.

When the specification for aircraft anti-collision lights was being drafted, it was suggested that a modified form of equation (2) be used for the computation of effeetive intensity, so that

$$
I_{e}=\frac{\int_{t_{1}}^{t_{2}} I d t}{.2+t_{2}-t_{1}} .
$$

An equation of this form was originally suggested by Blondel and Rey, ${ }^{4}$ but has rarely been used.

The question of choice of limits was immediately raised. Rather than use an arbitrary set of limits, such as choosing for $t_{1}$ and $t_{2}$ the times when $I$ was 10 per cent of the peak of the flash, a choiee of limits which would make $I_{\theta}$ a maximum was suggested. This immediately poses the problem of developing a method, other than trial and error, of obtaining the maximum value of $I_{e}$. The development of such a method is the purpose of this paper. 


\section{Fundamental Theorems}

The method of obtaining the maximum value of Io will be developed by means of one theorem and two corollaries. The proofs follow.

Theorem. $I_{\theta}$ is a maximum when the limits $t_{1}$ and $t_{2}$ are the timcs when the instantaneous intensity is equal to $I_{0}$.

This theorem may be readily proved by applieation of the calculus of variations." 'l'he proof given below is included because of the application of the method and equations used to later sections of the paper.

Consider instantaneous intensity, $I$, in a flash as any eontinuous, non-negative, single-valued function of time such that $I$ is less than $I_{0}$ in the intervals $t^{\prime \prime}{ }_{1}$ to $t_{1}$ and $t_{2}$ to $t^{\prime \prime}{ }_{2}$, and $I$ is greater than $I_{0}$ in the intervals $t_{1}$ to $t^{\prime}{ }_{1}$ and $t^{\prime}{ }_{2}$ to $t_{2}$, where $t^{\prime \prime}{ }_{1}<t_{1}<$ $t_{1}^{\prime}<t^{\prime}{ }_{2}<t_{2}<t^{\prime \prime}{ }_{2}$, and $I_{0}$ is defined in equation (4):

$$
I_{0}=\frac{\int_{t_{1}}^{t_{2}} I d t}{a+t_{2}-t_{1}} \text {. }
$$

Fig. 1 shows $I$ \&s a function of $t$ for a simple onepeak flash meeting these requirements.

\section{Case 1.}

Consider the case where the integration is performed over the time interval $t_{1}^{\prime}$ to $t_{2}^{\prime}$ whieh lies within the interval $t_{1}$ to $t_{2}$.

Then the intensity $I^{\prime}$ at the times $t_{1}^{\prime}$ and $t^{\prime}{ }_{2}$ is greater than $I_{\text {o. }}$.

Let

$$
I^{\prime}>I_{0}
$$

$$
I_{0}^{\prime}=\frac{\int_{t_{1}^{\prime}}^{t_{\prime \prime}^{\prime}} I d t}{a+t_{2}^{\prime}-t_{1}^{\prime}} .
$$

Then

$$
\int_{t_{1}}^{t_{3}} I d t=\int_{t_{1}}^{t_{1}} I d t+\int_{t^{\prime},}^{t_{1} y_{2}} I d t+\int_{t^{\prime},}^{h_{1}} I d t,
$$

so that

$$
I_{0}\left(a+t_{2}-t_{1}\right)=\int_{t_{2}}^{t_{1}^{\prime}} I d t+I_{0}^{\prime}\left(a+t_{2}^{\prime}-t_{1}^{\prime}\right)+\int_{t^{\prime}}^{h_{2}} I d t \text {. }
$$

But

and

$$
\int_{t_{1}}^{t_{1}{ }_{1}} I d t>I_{0}\left(t^{\prime}{ }_{1}-t_{1}\right)
$$

Substituting and combining terms, we have

$$
I_{0}\left(a+t_{2}^{\prime}-t^{\prime}{ }_{1}\right)>I_{\mathrm{e}}^{\prime}\left(a+t_{2}^{\prime}-t^{\prime}{ }_{1}\right) \text {. }
$$

Therefore

$$
I_{0}>I_{0}^{\prime}
$$

*Tho anthor fo indoblod to Dr. H. H. Sollger of the National Bureiv of Standarde for an olegant proof asing the celculus of rarletione.

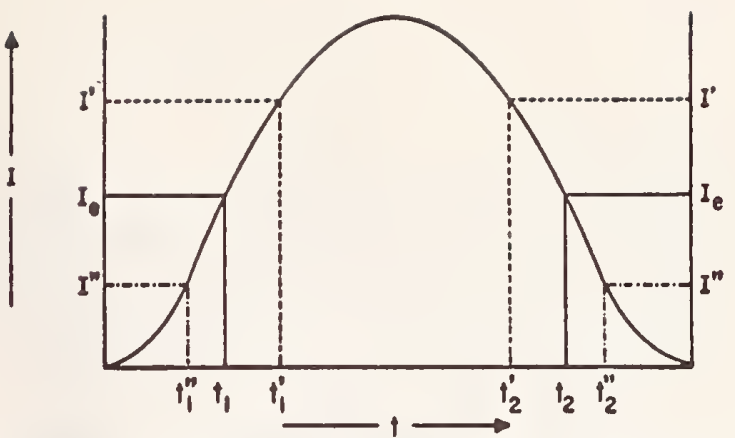

Figure 1. Intensity-time distribution of simple flash. (See text.)

Casc II.

Consider now the ease where the integration is performed over the time interval $t^{\prime \prime}{ }_{1}$ to $t^{\prime \prime}{ }_{2}$, which includes the interval $t_{1}$ to $t_{2}$.

Then the intensity $I^{\prime \prime}$ at the times $t^{\prime \prime}{ }_{1}$ and $t^{\prime \prime}$ is less than $I_{6}$.

$$
I^{\prime \prime}<I_{0} \text {. }
$$

Let

Then

$$
I^{\prime \prime}=\frac{\int_{t^{m_{1}}}^{I_{n_{2}}} I d t}{a+t^{\prime n_{2}-t^{\prime \prime}}} .
$$

and

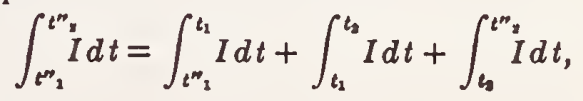

$I^{\prime \prime} \cdot\left(a+t^{m}{ }_{2}-t^{\prime \prime}{ }_{1}\right)=\int_{t^{m_{1}}}^{h_{1}} I d t+I_{0}\left(a+t_{2}-t_{1}\right)+\int_{t_{3}}^{t^{\prime \prime}} \stackrel{I}{I} d t$.

But

$$
\int_{t^{\prime \prime}{ }_{1}}^{t_{1}} I d t<I_{v}\left(t_{1}-t^{\prime \prime}{ }_{1}\right)
$$

and

$$
\int_{t_{2}}^{t^{\prime \prime}} I d t<I_{0}\left(t^{\prime \prime}{ }_{2}-t_{2}\right)
$$

Substituting and eombining terms, we have

$$
I_{0}>I^{\prime \prime} \text {. }
$$

Thus $I_{\theta}$ is greater than both $I^{\prime}$, and $I^{\prime \prime}{ }_{0}$. Therefore, the maximum value which can be obtained from the Blondel-Rey relation, equation (4), is that obtained when the intensity at the beginning and end of the interval of integration is equal to the effeetive intensity.

Corollary 1. If the instantaneous intensity is integrated over a period of time $t_{1}^{\prime}$ to $t_{2}{ }_{2}$ shorter than $t_{1}$ to $t_{2}$, and $I^{\prime}$ is the instantaneous intensity at these times, a value $I^{\prime}$ ' is obtained for the effective intensity that is always less than $I^{\prime}$.

From equation (6) we have

$$
I_{0}>I_{\text {. }} \text {. }
$$


But

Therefore

$$
I^{\prime}>I_{e}
$$

$$
I^{\prime}>I_{e}^{\prime} .
$$

Corollary 2. If the instantaneous intensity is in. tegrated over a period of time $t^{\prime \prime}{ }_{1}$ to $t^{\prime \prime}{ }_{2}$ longer than $t_{1}$ to $t_{2}$, and $I^{\prime \prime}$ is the instantaneous intensity at the times $t^{\prime \prime}{ }_{1}$ and $t^{\prime \prime}{ }_{2}$, a value $I^{\prime \prime}{ }_{e}$ is obtained for the effective intensity that is always grcater than $I^{\prime \prime}$.

From equation ( 7 ) we have

$$
I^{\prime \prime}{ }_{e}\left(a+t^{\prime \prime}{ }_{2}-t_{\cdot{ }_{1}}\right)=\int_{t_{1}{ }_{2}}^{t_{1}} I d t+I_{c}\left(a+t_{2}-t_{1}\right)+\int_{t_{2}}^{t^{\prime \prime}{ }_{2}} I d t .
$$

But

$$
\int_{t^{\prime \prime}}^{t_{1}} I d t>I^{\prime \prime}\left(t_{1}-t^{\prime \prime}{ }_{1}\right)
$$

and

$$
\int_{t_{2}}^{t^{\prime \prime}} I d t>I^{\prime \prime}\left(t^{\prime \prime}{ }_{2}-t_{2}\right)
$$

Also

$$
I_{e}\left(a+t_{2}-t_{1}\right)>I^{\prime \prime}{ }_{e}\left(a+t_{2}-t_{1}\right) .
$$

Substituting these into equation (7) and simplifying, we have

$$
I^{\prime \prime}>I^{\prime \prime}
$$

\section{Computations of Effective Intensity}

Guides for the computation of the effective intensity from an intensity-time distribution curve may be obtained from the theorem and corollaries.

\section{a. Computation of $I_{e}$}

1. Make an estimate $I^{\prime}$ of the value of the effective intensity and solve equation (3) using the values of $t$ corresponding to this intensity, obtaining $I_{e_{1}}$.

2. Repeat step 1 above, using as limits the values of $t$ corresponding to the $I_{e_{1}}$ obtained in step 1 , obtaining $I_{e_{2}}$. Repeat as often as necessary to obtain the desired accuracy.

Note that if the estimated effective intensity is too high ( $I^{\prime}$ in Fig. 1), the effective intensity, $I_{E_{1}}$, computed in step 1 will be below $I_{e}$ ( $I^{\prime \prime}$ of Fig. 1) and thus $I_{e}$ lies between $I^{\prime}$ and $I_{e_{1}}$. If the initial estimate is lower than $I_{e}$ ( $I^{\prime \prime}$ of Fig. 1), $I_{e}$ will be greater than both $I^{\prime \prime}$ and $I_{e_{1}}$ and a "straddle" is not obtained but $I_{\theta}$ is approached continuously from the low side.

\section{b. Determination of Conformance of a Flashing}

\section{Light to Specification Requirements}

1. Compute $I_{e_{1}}$ using the time limits corresponding to the specified effective intensity $I_{R}$. If $I_{\theta_{1}}$ is greater than $I_{s}$, the unit obviously complies, for the conditions are those of Fig. 2a (corollary 2).

2. If $I_{e_{1}}$ is equal to $I_{s}$, the unit just complies, for then $I_{e}=I_{s}=I_{e_{1}}$ (theorem).

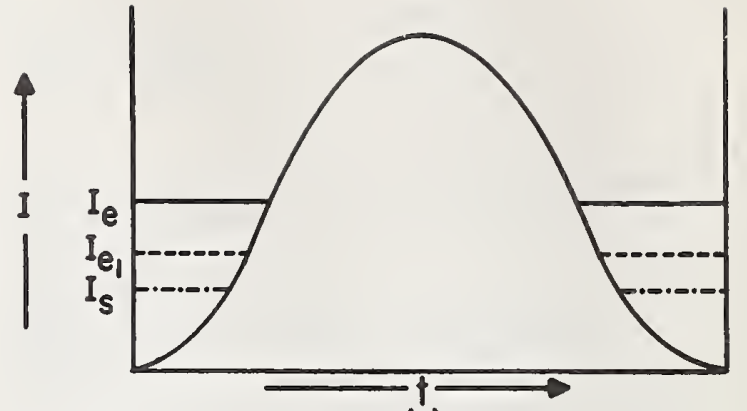

(o)

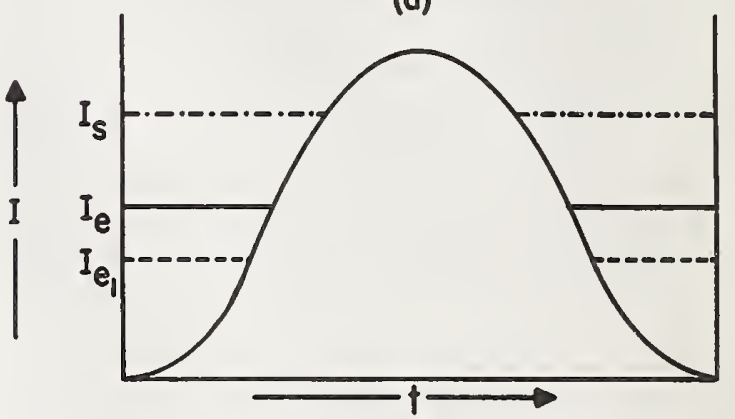

(b)

Figure 2. Illustrating the method of determining conformance to specification requirements of effective intensity. (See text.)

3. If $I_{e_{1}}$ is less than $I_{s}$, the unit fails for then the conditions are those of Fig. 2b (corollary 1 ).

Note that the degree by which the unit exceeds or fails to meet the specification requirements is not given by the single computation described here. The method outlined in section $a$ must be used for this purpose.

\section{c. Visual Range Computations}

If the visual range of the light, under specified conditions of transmittance and threshold, is desired, compute the effective intensity by using the method outlined in section $a$ and compute the visual range by using Allard's Law.

If the problem is only the determination of whether the light can be seen at a given distance under specified conditions of transmittance and threshold, use Allard's Law to compute the fixed intensity required to make the source visible at this distance. Then, by using the method outlined in section $b$, determine if the effective intensity of the unit exceeds this intensity.

\section{Application to Complex Intensity-Time Curves}

Not all units have single-peak intensity-time distribution curves similar to the curve shown in Fig. 1. Consider an intensity-time distribution curve of the type whose rise is shown in Fig. 3 where $I_{0}$ 


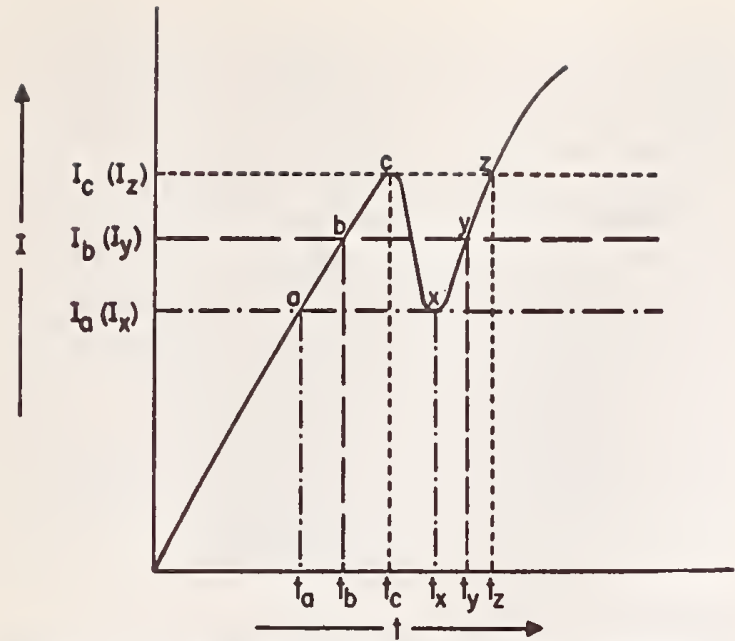

Figure 3. Illustrating application of the method to multi-peak intensity-time distributions. (See text.)

is the average intensity in the time interval $t_{0}$ to $t_{v}$. (The time interval $t_{z}-t_{o}$ is sufficiently short so that the momentary decrease in intensity is not visible.) If $I_{\theta}$ is less than $I_{a}$ or is greater than $I_{\varepsilon}$, then the restrictions on the shape of the curve stated in theorem 1 are met and there is no problem in the determination of $I_{c}$.

Consider the case where $I_{b}$ lies between $I_{a}$ and $I_{z}$. It may be easily shown by means of equations (5) and (8) that as the shape of other parts of the intensity-time distribution curve changes, the lower limits of time to be used to obtain the maximum value of equation (4) will lie between $t_{a}$ and $t_{b}$ or between $t_{y}$ and $t_{z}$ and will never lie between $t_{b}$ and $t_{y}$. If $I_{\theta}$ is equal to $I_{b}$, then either $t_{b}$ or $t_{\nu}$ can be used as the lower limit.

\section{Application to Groups of Short Flashes}

In general a signal from a flashing light consists of regularly spaced single flashes of light and the

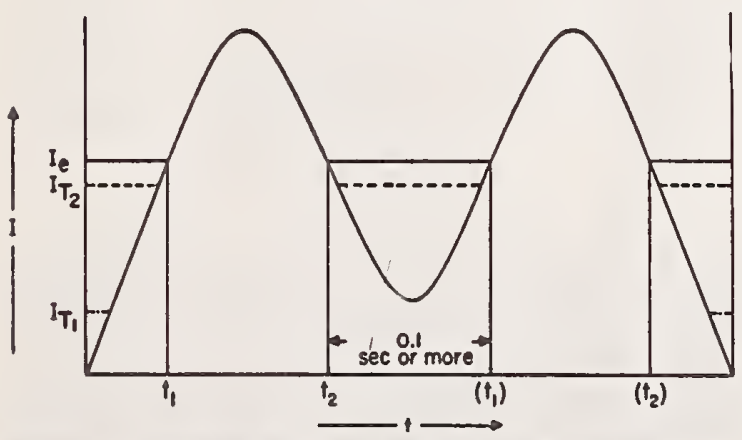

Figure 4. Illustrating application of the method to twopeak intensity-time eurves. (See text.) interval between flashes is so great that each flash has little influence on the effective intensity of the adjoining flashes.

Consider first a flash with the intensity-time distribution shown in Fig. 4. This flash is similar to that of the "split-beam" beacon used at military airfields. If the threshold intensity required to make a steady light visible is much less than $I_{0}$ $\left(I_{T_{1}}\right)$, the flash will be seen as a continuous flash with two peaks. However, if the threshold intensity is about equal to $I_{0}\left(I_{T_{2}}\right)$, two separate flashes will be seen. The maximum distance at which the light can be secn will be determined by the effective intensity of a single flash computed over the time interval $t_{1}$ to $t_{2}$.

There are lights that produce a number of very short flashes in rapid succession so that this group of flashes is seen as a single flash. An example of a light of this type is a unit using a number of condenser-discharge lamps to produce a single flash.

There appear to be no published data reporting studies of the effects of groups of flashes where the interval between flashes is short. Behavior of the eye under somewhat similar conditions suggests that if in a group of flashes the periods during which the instantaneous intensity of the light is below the effective intensity of the flash are of the order of 0.01 second or less, the eye will perceive this group as a single flash. The effective intensity of the group should then be computed by equation (12), choosing as times $t_{1}$ and $t_{2}$ the first and the last times the instantaneous intensity is $I_{e}$.

$$
I_{0}=\frac{\int_{t_{1}}^{t_{a}} I d t+\int_{t_{b}}^{t_{c}} I d t+\int_{t_{d}}^{t_{a}} I d t+\int_{t_{t}}^{t_{2}} I d t}{a+t_{2}-t_{1}} .
$$

Note that $I_{e}$ is the effective intensity of the group and not that of a single flash.

If the periods during which the effective intensity is less than $I_{e}$ are of the order of 0.1 second or more, it is believed that the individual flashes will be seen. Therefore, the effective intensity should then be computed on the basis of a single flash.

When the dark period is between 0.01 and 0.1 second, the effective intensity will lie between that of a single flash and that of the group. The behavior during the transition is not known.

\section{Numerical Examples}

Although the precise determination of the maximum value of $I_{0}$ may appear laborious, it is relatively easy. Since a change in the times chosen as the limits in equation (4) changes the denominator and the numerator in the same direction, it is not necessary to determine the correct limits, $t_{1}$ and $t_{2}$, 


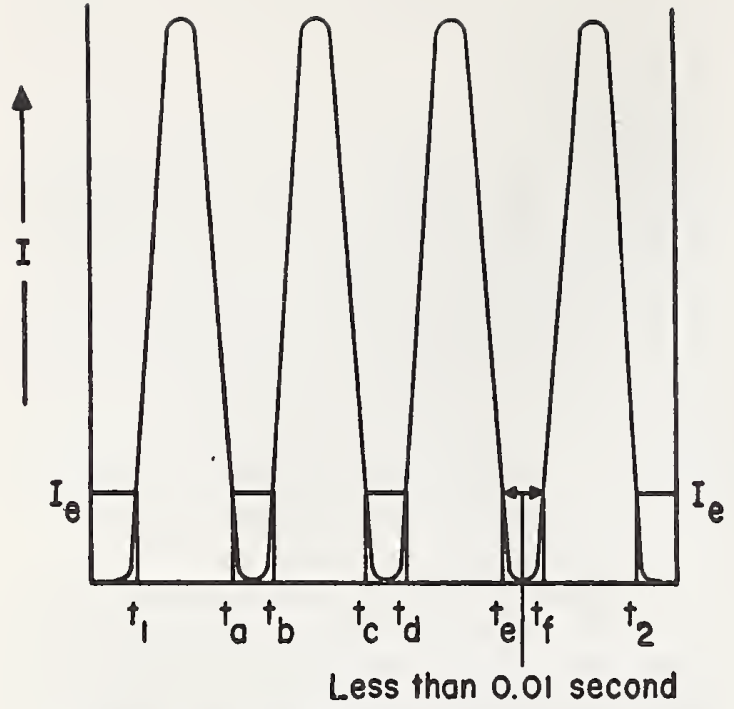

Figure 5. Illustrating application of the method to multiple flashes.

with great precision in order to obtain a satisfactorily precise determination of $I_{e}$. This is illustrated in Fig. 6 which shows two representative intensity-time distributions, one for a flashing light with a flash duration of about one-quarter second, and one with a flash duration of about one-twentieth second. The values of $I_{e}$ are computed for seven sets of time limits. The values obtained are indicated at the abscissa of the time limits. The middle value of each group is the maximum $I_{e}$ computed according to the method outlined above. Note that this value is equal to the instantaneous intensity at the corresponding time limits. Typically, the maximum effective intensity occurs lower on the curve for the short duration flash than for the long one and the variation of computed values of effective intensity with changes in time limits is smaller.

Experience indicates that if the times chosen for the initial integration are the times when the instantaneous intensity is about 20 per cent of the peak intensity, only one additional step is required to obtain a value for the effective intensity which is within one or two per cent of the maximum value. This is within the limits of accuracy with which the integral is evaluated by means of a planimeter. Often a single computation is sufficient if, instead of using as limits for the initial integration the times when $I_{\theta}$ is 20 per cent of the peak intensity, the times used are the times when the instantaneous intensity is equal to the product of the peak intensity and the number of seconds between the times when the instantaneous intensity is roughly five per cent of the peak intensity.
The maximum value of $I_{e}$ for the curves of Fig. 6 were computed by using as limits for the first integration the times corresponding to an intensity equal to about 20 per cent of the peak intensity and as limits for each succeeding integration the times corresponding to the instantaneous intensity obtained from the preceding step. (The curves have the shape of probability curves so the values of integrals may be computed with the desired accuracy. The accuracy is not limited by the accuracy of planimetric measurements.) Successive values for $I_{\theta}$ of $1.66,1.75$, and 1.75 kilocandles were obtained for the longer flash and of $3.41,3.42$, and 3.42 kilocandles for the shorter flash.

\section{Discussion}

As noted above, concern has frequently been expressed about the choice of the limits for the integral of the Blondel-Rey relation for computing the visual range of a flashing light. It seems illogical to extend the limits of the integral beyond the

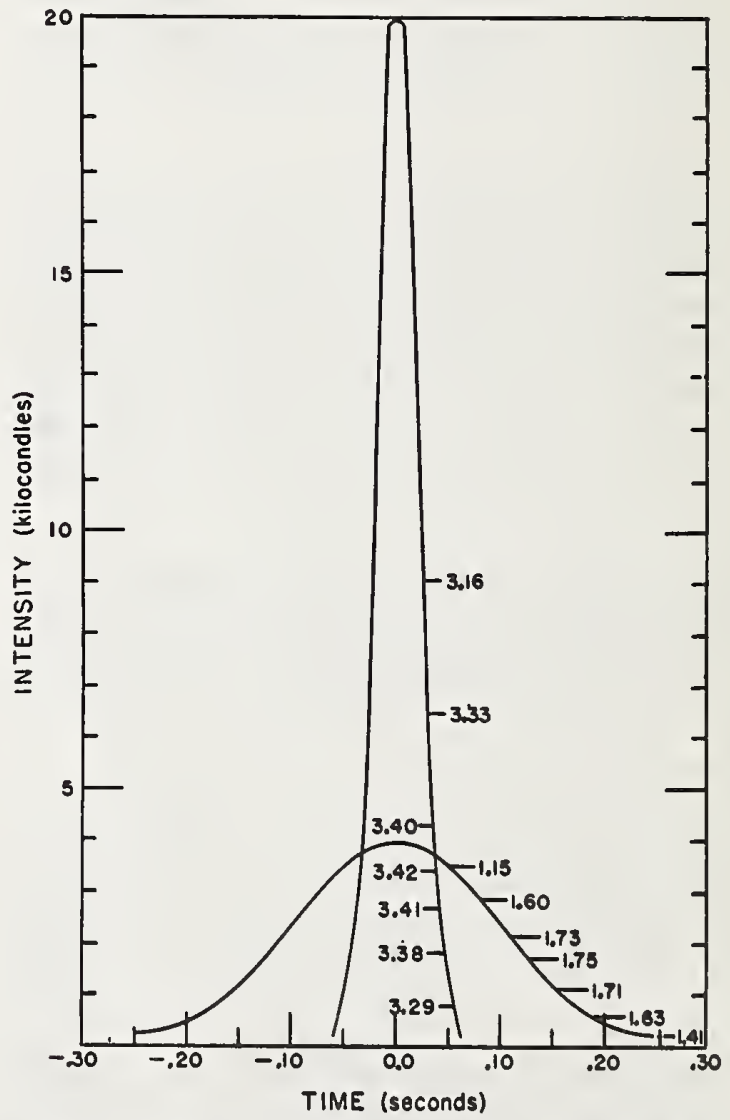

Figure 6. Examples of the effects of time limits on the computed value of effective intensity. The effective intensities are indicated at the abscissa of the time limits used in the computations. 
times when the instantaneous intensity is below the threshold intensity for steady burning lights so that intensities which are below threshold, even for a steady burning light, are included, or to exclude intensities which are above threshold for steady burning lights. Using this reasoning, Blondel and Rey ${ }^{4}$ suggested that the limits of the integral of equation (4) be the times when the instantaneous intensity is equal to the threshold intensity. $\Lambda$ s shown above, these are also the limits which make the computed visual range of the light a maximum. Therefore, the use of these limits in evaluating the performance of a lighting unit appears to be a logical choice.

The use of the maximum value of $I_{c}$ as the effective intensity of a flashing light is probably not valid except when the light is at or near threshold. When the light is well above threshold, not only will the value of $a$ in equation (4) be decreased, thus tending to increase the value of $I_{e}$, but also the limits of the integral should probably be extended to include the entire portion of the flash which is above threshold, thereby tending to decrease the value of $I_{e}$. In many cases this latter effect will be predominant. This is consistent with the decrease in effective intensity of airway beacons with increase in illuminance at the eye found by Neeland, Laufer, and Schaub. ${ }^{6}$

This analysis should be considered only as a mathematical treatment of equation (4). The analysis neither proves nor disproves the validity of this equation in determining the effective intensity of flashing lights nor the validity of the principle of choosing the limits of integration so that the effective intensity is a maximum.

\section{References}

1. Blondel, A., and Rey, J.: "Sur la Perception des Lumieres Breves a lo Limito de Lenr Port6e," Journal do Physigue, 1 (5th Series), 530 (1911).

2. Toulmin-Smith, A. K., and Green, H. N.: "The Fixed Equivalent of Flashing Lights," Illuminating Engineer, Iondon, XXVI, 305 (1933).

8. Hampton, W. M.: "The Fixed Equivalent of Flashing Lights," Illuminating Engineer, London, XXVII, 46 (1934).

4. Blondel, A., and Rey, J.: "Application anx Bignaux de la Loi de Perception des Lumières Brèves a ls Limite de Leur Porté," Journal de Phyoique, 1 (5th Series), 643 (1911).

b. Neeland, G. K., Lauler, M. K., and Schanb, W. R.: "Measureinent of the Equivalent Luminous Intensity of Rotating Beacons," J. Optical socisty of 4 mertea, 28,280 (1938).

\section{DISCUSSION}

F. E. Carlson: * These interesting papers should prove very helpful in evaluating the many lighting problems involving flashing lights. It is assumed that the ealculations deseribed hold for viewing conditions approximating the ideal. I wonder if the authors could tell us something about the influence of such factors as atmospheric transmission, other sources of light in the fleld of viow, otc.
G. A. Horton: * Mesars. Projector and Douglas are to be lighly eommended for presenting two papers on a subject which has been long neglected. The papers are particularly timely because of recent application of condenser discharge lights as aviation runway approach lights.

In tho eondenser light, tho longth of tho flash may be of the order of 100 microseconds, while the peak of the flash may reach several million candlepower. Since the time required for the eye to reach its maximum response is of the order of one-tenth of a second, it is clear that the effeetive candlepower as seen by the eye is not the same as the meas. urable average candlepowcr developed by the flash.

However, by application of the formulas developed by the authors of these two papers to the measured data can the effectivo candlepower of the condenser lights be expressed.

I should like to ask the authors if visual field cheeks of the eondenser lights have been made to substantiate the theory developed in the papers and, if so, what degree of agreement was obtained.

T. H. Projector and C. A. Douglas: " It is a pleasure to acknowledge the comments of Mcssrs. Carlson and Horton and to reply to their questions.

Mr. Carlson raises the question of the relation between effective intensity and the conditions of observation. The term "effective intensity" is used for convenience, and implies narrowly restricted conditions, usually threshold illuminance, dark adaptation, ete. Strictly, tho term should be "effective illuminance" for greater generality, thus automatically taking into account the effect of atmospheric attenuation on illuminance. The effect of other sources of light in the field of view and, in general, of the background eondition, state of adaptation, etc., has not been sufficiently explored and is a fertile field for further investigation.

In reply to $\mathrm{Mr}$. Horton, there have been a number of visual field checks of the applicability of the Blondel-Rey law to condenser discharge lights data in the United States and in Europe. All of the results of thesc ehecks show no significant deviations from the Blondel-Rey law. To our knowledge nono of these results has as yet been published formally. Publication of the results of work at the National Bureau of Standards is expeeted in the future. It should be noted that these remarks apply only to the direct light from the source when the illuminance from the light is near threshold. Frequently, in a foggy or hazy. atmosphere, the visual range of the glow of a flashing light will be considerably greater than the visual range of the direct light.

\#estinghouse Electric Corp., Clevelsnd, Ohio.
*Authors.

\section{Reprinted from}

\section{ILLUMINAT ING ENGINEER ING}

Vo1. 52, No. 12, December 1957 


\title{
Photometer for Luminescent Materials
}

\author{
Ray P. TEele \\ National Bureau of Standards, Washington, D. C.
}

(Received March 12, 1945)

\begin{abstract}
In evaluating the usefulness of luminescent materials it is necessary to take into account the behavior of the human eye at low values of luminance. A photometer that provides for the determination of low luminances, with due regard for the characteristic behavior of the eye at such values, is described. It is interesting to note that both the luminescent materials and some of the phenomena of vision for the nearly dark-adapted eye have been known for many years, although the use of modern lamps to produce higher and higher illuminations has made it generally unnecessary to consider these phenomena. However, the use of the airplane for bombing with the counter measure of blacking out as a means of passive defense and the need for markers in the interiors of blacked-out ships have shown many of the luminescent materials to be practical instead of merely novel, and has led to development of methods for measuring the luminances which they yield.
\end{abstract}

\section{INTRODUCTION}

GOME of the luminescent materials have been $S$ known for hundreds of years but were considered more or less as novelties until recently, when their practical usefulness became apparent. Luminescence not outlasting the excitation (fluorescence) was, in all probability, not observed until after the invention of irradiating sources and devices especiatly designed for the purpose of detecting such luminescence. Luminescence such as that of glowworms and rotting wood and that which outlasts the excitation (phosphorescence) must have been noticed by man in his carly existence. Aristotle's pupil, Theophrastus, says that a carbuncle exposed to sunlight glows like a live coal; ${ }^{1}$ while Aristotle, himself (about 350 B.C.) mentions the luminescence of rotting wood.

${ }^{1}$ P'. I'ringshein and M. Vorel, Luminescence of Liquids and Solids and its P'ractical Applicalions (Interscience P'ublishers, Inc., New York, 1943).
Benvenuto Cellini tells of seeing a white sapphire which illuminated a perfectly dark room. ${ }^{2}$ As early as 1652 Peter Poterius made little toy animals from phosphorescent material.' An alchemist, Vicenzo Cascariolo, in Bologna, Italy, about 1600 found a stone which seemed heavier than one of its size should be; upon heating it, in the hope of finding gold, he discovered that it would glow in the dark, "sometimes for as long as an hour" and also found how to make it glow at will. ${ }^{3}$

The luminance ${ }^{4}$ of the luminescent light from phosphorescent materials ranges downward from that of a white surface viewed in full moonlight. In measuring such luminances it is necessary to

${ }^{2}$ S. HI. Ball, Sci. Monthly 47, 497 (1938).

${ }^{3} \mathrm{G}$. T. Schmidling, Prolective and Decorative Coatings (John Wiley and Sons, Inc., New York), Vol. III, p. 657. Also reference 1.

1OSA Committee on Colorinetry, "Psychophysics of color," J. Opt. Soc. Anı. 34, 245 (1944). 
consider the behavior of the weakly illuminated eye. In $1825 \mathrm{~J}$. Purkinje ${ }^{6}$ showed that after a red surface and a blue surface have been illuminated so as to have the same brightness, a reduction of the illumination of both surfaces in the same proportion will cause the red surface to appear darker than the blue after a certain limit of reduction has been passed. The dependence of the observed values also upon the size of the field of view for the nearly dark-adapted eye was determined by $\mathrm{P}$. Reeves ${ }^{6}$ in 1917 . The trend to higher and higher illumination for lighting purposes had made it unnecessary for the photometrist to remember these effects in the measurements customarily made in the laboratory. However, as the use of luminescent materials was shown to be practical it became necessary to take account of both of these phenomena in constructing a photometer for the measurement of their luminance.

\section{PHOTOPIC, MESOPIC, AND SCOTOPIC VISION}

The human cye has the ability to adapt for light conditions over a wide range. The approximate upper limit is represented by the condition of viewing fresh snow in full sunlight, which is uncomfortable and, if long continued, results in temporary blindness, "snow blindness," or if

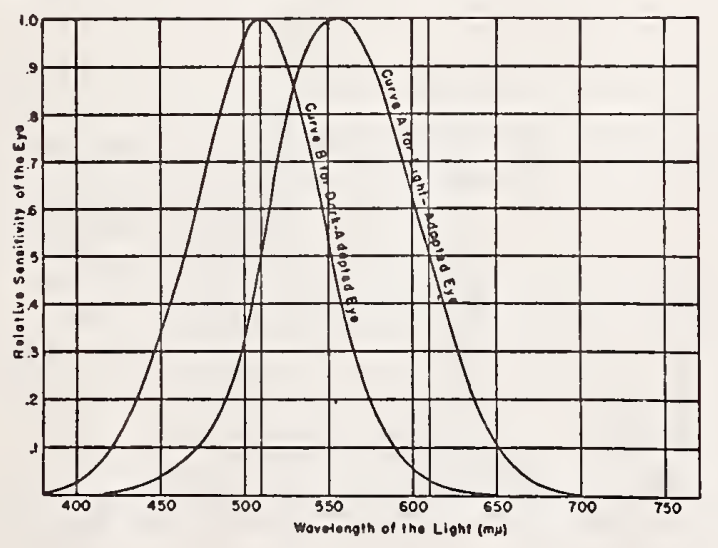

Fig. 1.

viewed for prolonged periods, may result in permanent injury to the eye. The lower limit is considerably below the condition of viewing a

5 J. Purkinje, Mag. f.d. gesammte Heilkunde 20, 199 (1826).

'P. Reeves, J. Opt. Soc. Am. 1, 148 (1917); P. G. Nutting, Trans. I. E. S. 11, No. 9939 (1916). white surface on a clear, moonless night. The change in size of the pupil of the eye is easily observed; and, as everyone knows, the pupil is small if strongly illuminated, and as the illumination weakens, the pupil becomes larger. There are other less obvious changes, such as the change in the luminosity curve, which explains the Purkinje effect and the loss of the ability to detect detail (acuity) and of the ability to detect chromaticity differences, all associated with the transition from cone to rod vision.

When we view a surface of high brightness with photopic vision, that is, when the eye may be said to be light-adapted, we find that the eye has a nearly constant luminosity curve, independent of the luminance range under consideration. All definitions of units of luminance (photometric brightness) imply that comparisons between differently-colored surfaces be made at values of luminance sufficiently high to insure that the olserver's eye is in a state of light adaptation. This is to insure that luminance values obey the additive law, by which a luminance of $y$ units superimposed upon one of $x$ units will provide a luminance of $(x+y)$ units. If this law is not obeyed, the ordinary inverse square law, the ratio of areas of openings in diaphragms, and the ratio of the areas of the open and opaque sectors of a rotating disk (Talbot's law) maty not be applied to the luminance values under consideration. If differently-colored surfaces are viewed, obedience to the above-mentioned laws occurs only if the luminosity curve of the eye is constant and independent of the adaptive state of the eye throughout the range of luminane under consideration. Fortunately the eye in observing luminances greater than 1000 microlamberts does possess a nearly constant luminosity curve, and for this condition (when the eyc is said to be light-adapted) we have photopic vision and the values of luminance (photometric brightness) for differently-colored surfaces not only obey the additive law but also correlate well with brightness, subjectively evaluated. IVe may speak of such values as photopic luminance.

Unfortunately the luminosity curve of the eye does not remain constant when the luminance of an extended surface is reduced below 1000 nicrolamberts; in fact, the eye lecomes progressively more sensitive to short wave (1)lue) 

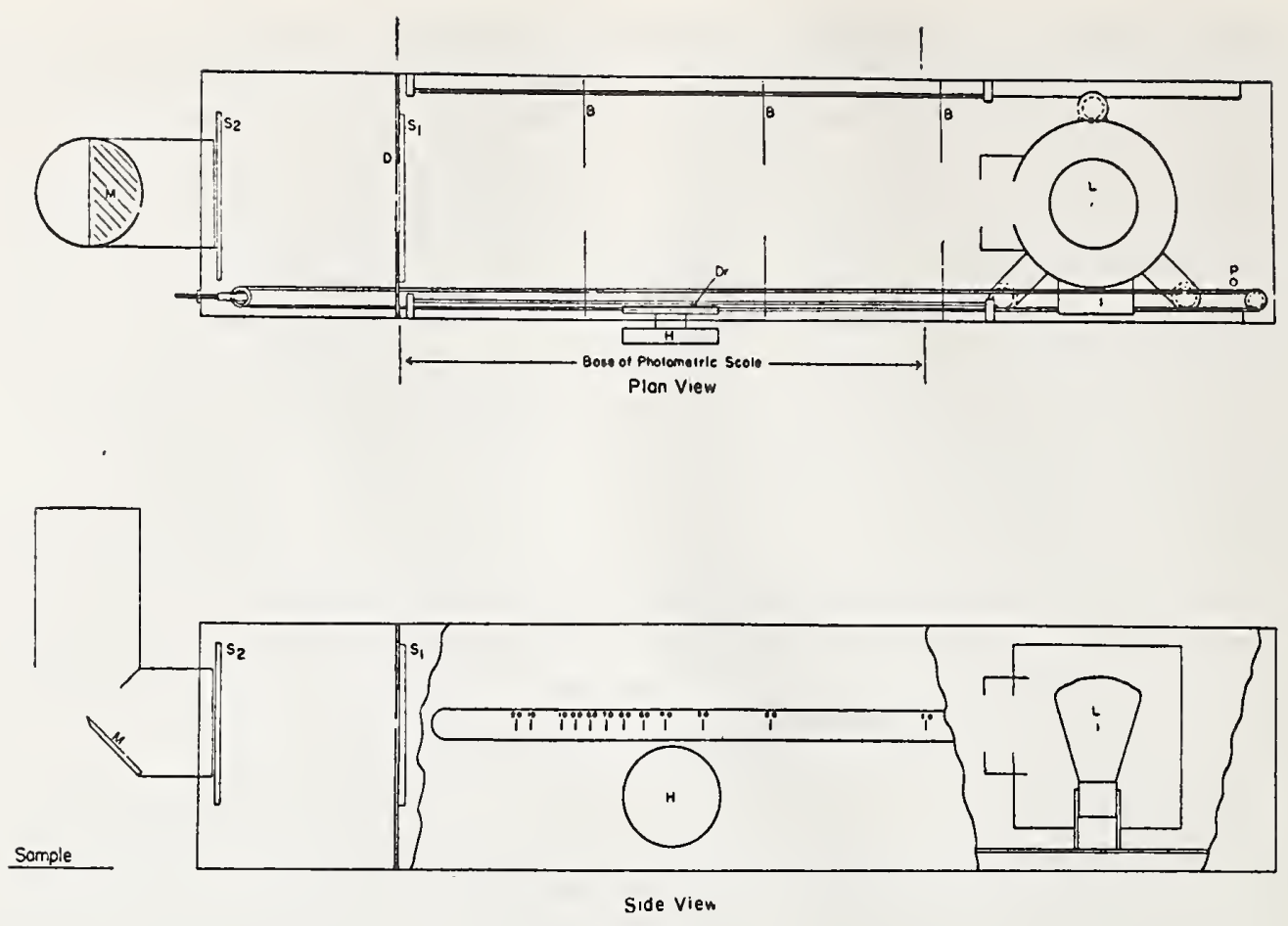

FIG. 2.

and less sensitive to long wave (red) light as the luminance to which it is adapted is reduced from 1000 to about 0.5 microlambert, the rate of change being most pronounced between 200 and 1 microlambert. This shift of the luminosity curve towards shorter wave-lengths explains the Purkinje effect. For adlaptation to luminances below 0.5 microlambert, the eye again reaches a steady state, with a constant luminosity curve characteristic of scotopic vision and the cye may be regarded as dark-adapted. We may speak of these luminances as scotopic luminañces. In Fig. 1 the adopted luminosity curve of the lightackapted ${ }^{7}$ eye and an average curve for the darkarlapted ${ }^{8}$ eye are shown. As the luminance of the observed surface is diminished we pass through the region of mesopic vision and the luminosity curve moves progressively from that of the lightadapted eye towards that of the dark-adapted.

\footnotetext{
${ }^{7} \Lambda$ résunee of the data on which the standard ICI luminosity factors are based and of the present status of these factors is given in a paper by Kasson S. Gibson, J. Opt. Soc. $\Lambda \mathrm{m} .30,51(1940)$.

"Simmatry of American Opinion on BS/ARI" 18. Brilish Siandard Specification for Fluoresent and Phosphorescent ['aint" prepared for the $\Lambda$ merican Standards Asseciation by L. $\Lambda$. Jones under date of June 15, 1942, gives an average of the luminosity data for low luminances ileternined by Hecht and Williams and by Weaver.
}

There are several ways to assign numerical values to a scale of luminance below the photopic region to include mesopic and scotopic luminance.

$A$. Arbitrary application of the photopic luminosity function to all luminances by making visual comparison of fluorescent and phosphorescent materials only with a standard of similar spectral composition, the standard to be evaluated by way of the photopic luminosity function.

$B$. Adoption of an arbitrary photoelectric procedure for evaluating the radiant energy from fluorescent and phosphorescent materials.

$C$. Definition of the unit and scale of mesopic and scotopic luminance in terms of the hypothetical equal-energy source.

$D$. Definition of the unit and scale of mesopic and scotopic luminance in terms of an incandescent lamp operating at $2360^{\circ} \mathrm{K}$ color temperature.

There have been attempts to make the standard photopic luminosity function $(A)$ do. Each material characterized by racliant energy of a new spectral composition, $x$, requires the setting up and evaluation of a new stanclarcl. For various samples of the same material, the method gives useful comparisons; but because of the Purkinje 
effect, photopic luminance of one material compared to another does not necessarily correlate with the observable brightness in the mesopic and scotopic regions of the materials.

A better correlation is obtainable by arbitrary adoption of a photoelectric procedure in whics. the source-filter photo-cell combination is approximately equivalent to the luminosity function somewhere within the mesopic range $(B)$. It is obvious, however, that no one luminosity function can be generally valid.

Procedures $C$ and $D$ are similar and give values of mesopic and scotopic luminance that correlate well with brightness. The use $(C)$ of the hypothetical equal-energy source would bring a unique logical simplicity to the general concept of luminance, but the predominant opinion ${ }^{8}$ is that an incandescent-lamp source at $2360^{\circ} \mathrm{K}$ is more convenient in practice and, on that account, preferable for tentative standardization and use at the present time. By choice the comparison source is assigned mesopic and scotopic luminances by the same methods (inverse-square law, sector-disk relation, aperture relations) used for photopic luminance.

\section{DESCRIPTION OF THE PHOTOMETER}

\section{(a) Photometric Details}

The photometer is shown in Fig. 2. The views depart from conventional drawing practice by showing the openings in the baffles and diaphragms as in a section taken through the axis of the beam. Otherwise Fig. 2 shows conventional plan and elevation views.

The lamp illum inates the flashed opal diffusing glass screen, $S_{1}$, which in turn illuminates a similar screen, $S_{2}$, the outer surface of which viewed in the front-surface mirror, $M$, forms the comparison field of the photometer. The other or test field is the surface of the sample viewed directly. The luminance of the surface of $S_{2}$ facing the mirror depends upon the light reaching the surface toward $S_{1}$, which is closely proportional to the product of the area of the opening in the cliatphragm $D$ and the luminance of the surface of $S_{1}$ exposed to $S_{2}$, since the distance between $S_{1}$ and $S_{2}$ is constant. The luminance of the surface of $S_{1}$ away from the lamp is proportional to the illuminance of the surface toward the lamp which, of course, depends upon the distance between $S_{1}$ and the lamp.

Let the luminance of $M$ as viewed from the sight tube be $B_{M}$, the reflectance of the mirror be $r$ and the luminance of the surface of $S_{2}$ facing the mirror be $B_{2}$. We then have that

$$
B_{M}=r B_{2} \text {. }
$$

The luminance $B_{2}$ is proportional to the illuminance $E_{2}$. on the surface of $S_{2}$ away from the mirror, or

$$
B_{2}=k_{2} E_{2},
$$

the constant of proportionality, $k_{2}$, being characteristic of the transmissive properties of $S_{2}$. Hence

$$
B_{M}=r k_{2} E_{2} \text {. }
$$

$E_{2}$, however, is equal to the product of the area, $A$, of the opening in the diaphragm $D$ and the luminance, $B_{1}$, of the surface of $S_{1}$ facing $S_{2}$, divided by the square of the distance $d_{1}$, between $S_{1}$ and $S_{2}$,

$$
E_{2}=A B_{1} / d_{1}^{2},
$$

and we acrordingly have the expression

$$
B_{M}=r k_{2} A B_{1} / d_{1}{ }^{2},
$$

for the luminance of the mirror. The luminance $B_{1}$ is proportional to the illuminance $E_{1}$ on the surface of $S_{1}$ toward the lamp, or

$$
B_{1}=k_{1} E_{1} \text {, }
$$

where $k_{1}$ is characteristic of the transmissive propertics of $S_{1}$, which gives us

$$
B_{M}=r k_{2} A k_{1} E_{1} / d_{1}{ }^{2} .
$$

Finally $E_{1}=I / d^{2}$ where $I$ is the luminous intensity of the lamp, $I_{2}$, in the direction of $S_{1}$ and $d$ is the distance of $L$ from $S_{1}$, so that

$$
B_{M}=r k_{2} A k_{1} I / d_{1}^{2} d^{2} \text {. }
$$

In this we see that $r, k_{2}, k_{1}, I$, and $d_{1}$ are fixed characteristics of any particular photometer and can be grouped under a single characteristic, $P$, and hence we can write that

$$
B_{i}=P A / d^{2} \text {. }
$$

The photometric scale is graduated from 20 to 0.5 , and a set of diaphragms having holes with areas differing by factors of about 10 has been 
made. These were made with ordinary drills and then calibrated. The photometric scale and the ratios of the areas of the openings in the diaphragms overlap, and values near the encls of the scale can be measured by means of either of two diaphragms. The use of these mechanical means to control the luimnance of the comparison field gives a long range with no change in spectral composition. When the photometer is used without any of the removable diaphragms the maximum reading is more than 150,000 times the minimum reading with the smallest-aperture diaphragm. The opening in the metal plate holding $S_{1}$ is a limiting diaphragm when none of the removable diaphragms are used.

The field is a plain elliptical field (diametrically divided circle viewed at $45^{\circ}$ ), the major axis being $1 \frac{1}{2}$ inches long. The major axis coincides with the dividing line of the field, which usually is viewed so that the two halves are seen side by side. Since the end of the sight tube is 4 inches from the mirror, the angles subtended at the eye by the field are 15 and 20 degrees for the minor and major axes respectively. The mirror when placed as shown in Fig. 2 serves as a baffle to prevent any light from the screen $S_{2}$ falling on the test surface.

\section{(b) Mechanical Details}

The mechanical details may, of course, be varied to suit the maker's materials and choice. The photometer used at the Bureau employs the loox, track, lamp housing, and scale of a Sharpllillar ${ }^{9}$ photometer. The photometric cube and eyepiece were removed, and the diaphragmliffusing-screen arrangement described in the previous section installed. Since this type of photometer is no longer conmercially available a description of the mechanical details will serve as a guide for anyone wishing to construct one.

The box (Fig. 2) is about $4 \times 4 \times 22$ inches. The lamp, $L$, is moved by means of an endless cord which passes over a drum, $D r$, which is turned by

\footnotetext{
* Metric drills of $0.5,1.6,5$, and $16 \mathrm{~mm}$ will give areas proportional to $0.25,2.56,25$, and 256 provided new accurately ground drills are available. For some purposes thesc area ratios may be sufficiently close to the desired factors of 10 .

${ }^{2}$ C. H. Sharp and P. S. Millar, Elec. World 51, 181 (1908); Elec. Rev. 52, 141 (1908); and Electrician 60, 562 $(1907-08)$.
}

the handwheel, $I I$. The sight tube may be turned in its collar to view the test surface at various angles. In order to avoid crrors caused by light reflected from the interior of the box a series of baffles, $B$, macle of fiber is placed between the lamp and the screen $S_{1}$, and the interior is painted with a flat (mat) black paint. These baffes are carried on two light rods and are attached to each other and the lamp housing by cords. When the lamp moves toward $S_{1}$, the housing pushes the baffles successively in front of it; and when it moves away from $S_{1}$, the cords pull the baffles one after another into their original position. The lamp housing carries an index, $I$, the shadow of which falls upon the translucent scale in the side of the box, and thus there is no parallax. The scale is covered by red plastic to preserve the dark adaptation of the photometric observer. This arrangement also makes it unnecessary to provide a light for reading the scale, which is a great convenience, and avoids the usual scale marked on a spacewasting rod protrucling from the box. Since readings are taken in a dark room, no stray light will enter the photometer through the translucent scale. It would be necessary to provide a shutter. to cover the translucent scale if readings were taken in a lighted room, the shutter being opened only to read the scale after a setting had been taken.

The lamp housing has a second index on the side opposite the scale index to facilitate the accurate positioning of the filament of the lamp at the unity mark of the photometric scale. The housing runs on a track made of angle brass fastened to the sides of the box. The single wheel has a spring forcing it against the track so that sidewise motion is prevented.

\section{SUMMARY}

A photometer, such as described in this paper makes possible the determination of scotopic and mesopic luminance such as that of fluorescent and phosphorescent materials. The use of a comparison field of color temperature $2360^{\circ} \mathrm{K}$ and the mechanical control of the luminance of the comparison field is in accord with current American opinion on the datum and method of evaluating luminances in the mesopic and scotopic 
regions. Luminance so evaluated takes the Purkinje effect into account and correlates perfectly with brightness, subjectively evaluated.

The photometer has been used for nearly 3 years in routine measurements of the luminancetime (brightness decay) curve of phosphorescent materials as well as to determine both the luminance and chromaticity of the fluorescent light from papers impregnated or coated with fluorescent chemicals. In measuring the luminance of phosphorescent materials, different observers agree within about 5 percent in the region near 10 effective microlamberts, while the spread between observers increases to about 25 percent when the luminance is in the region of 0.005 effective microlambert. The measurement of fluorescent materials has not been extensive.
However, some rieasure of the effect of color is given by tests of blue fluorescence at about 100 effective microlamberts where four observers macle observations within a little less than 25 percent and tests of yellow fluorescence where (with the same observers) the results did not spread by as much as 10 percent at about 200 effective microlamberts. The use for determining chromaticity (where the spectral composition of the comparison source must remain constant while the luminance is varied) has been so satisfactory at low luminances that a photometer has been designed with a much wider range of luminance than the present photometer possesses. 'This increased range will be adequate for measuring the chromaticity of non-luminescent materials in the photopic range of luminance.

\section{Reprinted from: \\ Journal of the Optical Society of America \\ June, 1945 - Vol. 35 , No. 6}

12.9 .

On tise Standard Source for Low Level Photometry

J. TERRIEN

Bureau International des Poids et Wesures, Sères, Seine-et-Oise. France

July 25,1949

THE definitions and agreenents of photometry are now well-

L scttled and they received the sanction of the Comité International and the Conférence Gúnérale des Poids ct Mesures. ${ }^{1}$ Although these definitions, the sing? official ones today, have been adopted without any iimitation of the scope of their validity, they are likely to be modified in the theld of low iuminance levels, in order to take into account the Purkinje ellect, and new international agrecments, less sinple that the present ones, are likely to be adopted in the fuitur. An almost unanimons agrecment has already been achicved at the eleventh session of the C.I.E. (Puris $19.18)$; it settles the choice of the primary standiurd source for ali future photometric system and lic magnitude of the unit of what wili be, in these systems, the anivgy of luminance (er piotometric brightness). The text of the resolution aclopted unanimously by a!l the nations (cxecpt Austratia) which attenceci the C.I.E., and in particular by the $U$. S. A., reads as follows:

"Light and Vision. Resolution 2. It is reconmencied that, when for special purposes the luminous effects of raliant encreits of various spectrai compositions are evaluated by methods that do not rest on the stindard uminosity function adopted by the C.I.E. in 1924, the unit of the cuantity corresponding to luminance (piotometric brightness) should alwatys be chosen so that this quantity has the numerial value 60 (c.g.s. system) for a black boly at the iemperature of freezing latinum." 


\section{The Photometry of Colored Light}

Reprinted from Illuminativg ENGINEERING Vol. IdXII, No. 4, April 1967
A. C. Wall
DRES RESENT day photometers usually incorporate a photosensitor for evaluating the illumination from an unknown source by comparing it with the illumination from a standard source. The photosensitor must be spectrally corrected to provide a sensitivity curve as close as possible to that of the photopic luminous efficiency function of the CIE standard observer.* This function is the design goal of a photosensitor-filter combination. An example of the goal and the realized spectral response of a particular phototube-filter combination is shown in Fig. 1. (More details will be given below.) The overall match is good and the photosensitor gives excellent results in the photometric measurements of light with a spectral distribution close to that of the light with which the photosensitor is calibrated.

Consider, however, red light transmitted by a filter with a shortwave cutoff at about 630 nanometers and with a transmittance curve as shown in Fig. 2. The output $R$ of the photosensitor is given approximately by

A paper presented at the National Technical Conference of the Illuminating Engineering Society, August 21 to 26, 1966, Minneapolis. Minn. AUthOR: National Bureau of Standards, Washington, D. 0 .

* For a two-degree field of view.

$$
R=K \Sigma_{\lambda} E_{\lambda} \tau(\lambda) S(\lambda) \Delta \lambda
$$

where $K$ is the proportionality factor, $E_{\lambda}$ is the spectral irradiance, $S(\lambda)$ is the relative spectral response of the photosensitor, and $\tau(\lambda)$ is the spectral transmittance of the correction filter.

It can be seen from Fig. 2 that the $S(\lambda) \tau(\lambda)$-real. ized curve is much different from the $S(\lambda) \tau(\lambda)$-goal curve at wavelengths above 630 nanometers. Hence the outputs of the phototube will not have the same constant of proportionality for the illuminations from the red light and the "white" standardizing light.

The investigation reported herein is an evaluation of the adequacy of several photosensitor-filter combinations when used in the measurement of "colored light," which in this paper designates light that has a spectral distribution different from that of the light with which the photometer was calibrated.

\section{Photosensitors and Correction Filters}

The type PJ-14B vacuum phototube, with an S-7 cathode surface, though not available today, is ideally suited to general low-illumination photometry as well as high-illumination flash photometry. ${ }^{1}$ It has low

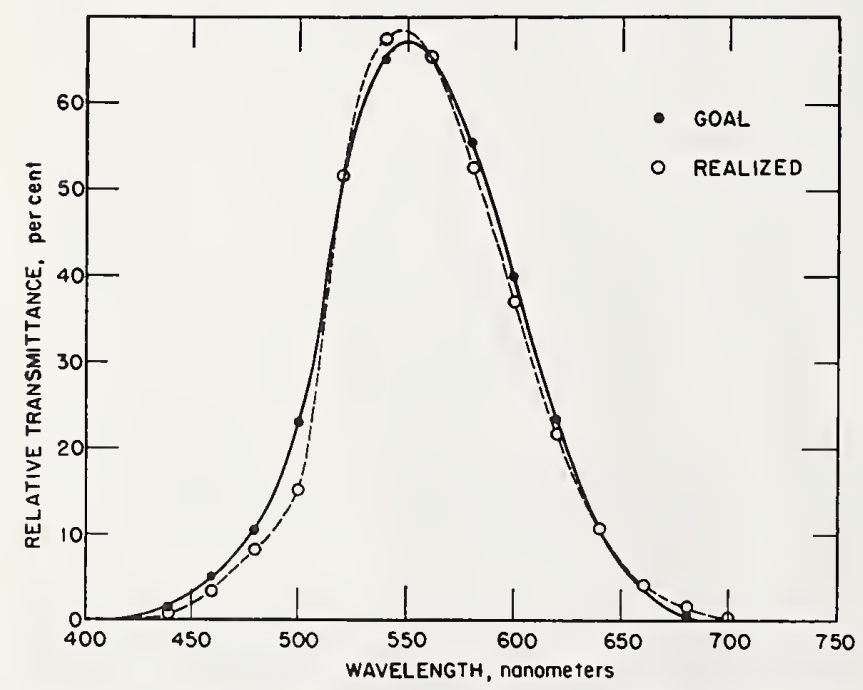

Figure 1. Spectral transmittance goal of a filter and the transmittance as realized.

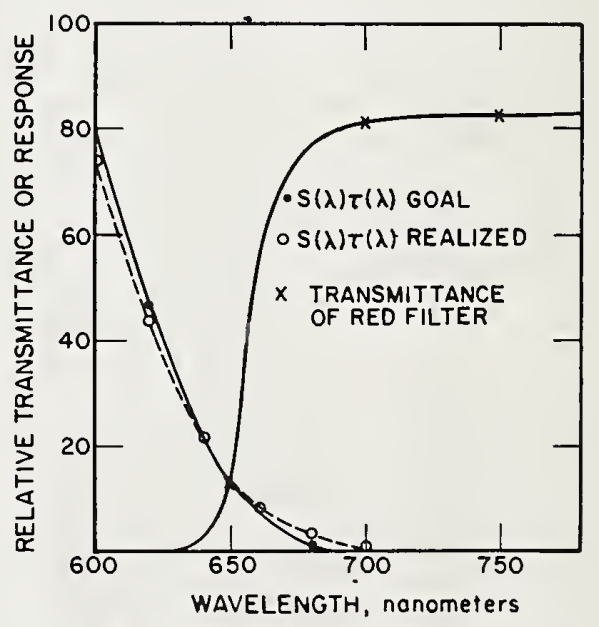

Figure 2. Relative spectral response goal of a phototube-filter combination, the response as realized, and the spectral transmittance of a filter transmitting red light. 
Table I-Correction Factors for Four Color-Corrected Photosensitors with S.4 Surfaces

\begin{tabular}{cl}
$\begin{array}{c}\text { TEST } \\
\text { FILTER }\end{array}$ & \multicolumn{1}{c}{$\begin{array}{c}\text { COLOR } \\
\text { NUMBER }\end{array}$} \\
& \multicolumn{1}{c}{ DESIGNATION* } \\
1 & Purplish blue \\
2 & Blue \\
3 & Blue \\
4 & Blue (lunar white) \\
5 & Blue (lunar white) \\
6 & Bluish green \\
7 & Green \\
8 & Green \\
9 & Green \\
10 & Green \\
11 & Green \\
12 & Yellow-green \\
13 & Yellowlsh green \\
14 & Yellowish orange \\
15 & Yellowish orange \\
16 & Orange \\
17 & Reddish orange \\
18 & Reddish orange \\
19 & Red \\
20 & Red \\
21 & Red \\
29 & Red \\
&
\end{tabular}

\begin{tabular}{|c|}
\hline $\begin{array}{l}\text { LUMINOUS } \\
\text { TRANSMITTANCE }\end{array}$ \\
\hline .020 \\
\hline .039 \\
\hline .102 \\
\hline .166 \\
\hline .246 \\
\hline .130 \\
\hline .198 \\
\hline .249 \\
\hline .110 \\
\hline .047 \\
\hline .049 \\
\hline .405 \\
\hline .138 \\
\hline .740 \\
\hline .760 \\
\hline .659 \\
\hline .519 \\
\hline .304 \\
\hline .154 \\
\hline .106 \\
\hline .050 \\
\hline .0254 \\
\hline
\end{tabular}

1
1.08
1.10
.86
.91
.94
1.15
1.14
1.15
1.16
1.17
1.90
1.16
1.10
1.01
.98
.94
.90
.81
.79
.65
.46
.33

2
.97
1.03
.93
.95
.96
1.14
1.10
1.10
1.19
1.15
1.17
1.14
1.09
1.00
.90
.94
.92
.87
.80
.74
.55
.48

* The chromaticity coordinates are shown in Fig. 3.

dark current and an essentially flat spectral response in the visible Pegion, which makes the design of a correction filter relatively easy.

Optical filters were designed at the National Bureau of Standards for selected tubes of this type to provide phototube-filter combinations with spectral responses which essentially match the CIE standard observer spectral luminous efficiency function $V(\lambda)$. Thus the goal is to design a filter so that

$$
K_{n} \tau(\lambda) S(\lambda)=V(\lambda)
$$

where $\tau(\lambda)$ is the spectral transmittance of the filter, $S(\lambda)$ is the relative spectral response of the phototube at wavelength $\lambda$, and $K_{n}$ is the normalizing factor.

The spectral transmittance of a desired filter was determined by solving Equation (2) for $K_{n} \tau(\lambda)$ in terms of $V(\lambda)$ and $S(\lambda)$. Having determined the desired function $K_{n} \tau(\lambda)$, a filter consisting of a suitable combination of four glasses in series was chosen and the spectral transmittance of samples of each glass was determined. From these data the desired thickness of each component was computed. The components were then ground and polished to the desired thicknesses and the spectral transmittances were measured. In those instances in which the measured spectral transmittance did not agree sufficiently well with the desired spectral transmittance, the components were reground to a thickness which gave the desired spectral response.

Fig. 1 shows the spectral response of the desired and the designed phototube-filter, indicating a reason. ably good approximation.

The Gillod-Boutry-type phototube is an end-on vacuum phototube with a $\mathrm{CsBi}$ cathode surface. The phototube saturates at less than two volts, has a spectral sensitivity that peaks near $500 \mathrm{~nm}$ and extends beyond $700 \mathrm{~nm}$, and has low dark current, about $10^{-13}$ ampere. The phototubes were offered to mem. bers of the International Bureau of Weights and Measures in 1957 and were subsequently made by a French manufacturer on the basis of orders received. Fifty of the phototubes were purchased by the $\mathrm{Na}$ tional Bureau of Standards for precision photometric measurements. J. S. Laufer of NBS designed a filter to correct a spectrally matched group of the tubes so as to have a spectral response which is essentially the CIE spectral luminous efficiency function. The filter design is similar to that described by Nimeroff and Wilson $^{2}$ for a sector division filter: the filter is composed of six sectors of varying angular extent, each segment being made of several layers of glass.

The type 929 and the type A29 are vacuum phototubes having an S-4 cathode surface that peaks in response at about $4.00 \mathrm{~nm}$. The Wratten No. 106 filter is designed to correct a typical phototube with an S-4. surface spectrally to match the CIE luminous effciency function. The S-4 cathode is found in many of the commercially available photometers, usually with the Wratten No. 106 filter.

The 856 barrier-layer photocell has a selenium pho. tosensitive surface. The cells used incorporate a twolayer spectral correcting filter. Although the manufacturer indicates more than a ten per cent deviation from linearity between 20 to 200 footcandles with a 200-ohm external resistance, Barbrow ${ }^{3}$ shows a maximum of 1.4 per cent error between 0.6 and 180 footcandles relative to the reading at 0.6 footcandle with a "zero resistance" circuit for cells of this type. 


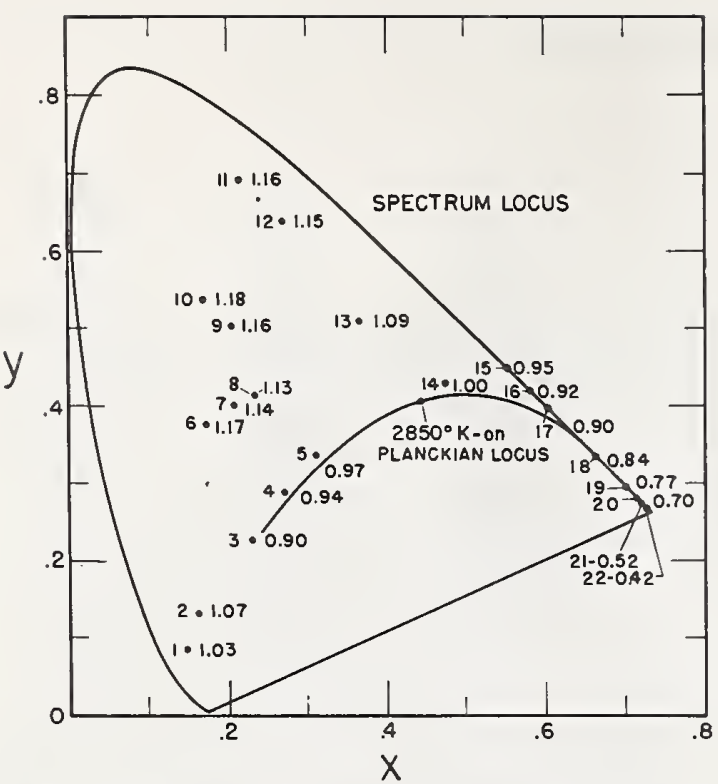

Figure 3. The 22 filters used plotted on the CIE chromaticity diagram. Shown is the set of correction factors $k$ for one color-corrected S-4 phototube.

To show that uncorrected photosensitors are useful only for measuring illumination having exactly the same spectral distribution as that of the light with which they are calibrated, measurements were made with the following uncorrected phoiosensitors: four phototubes with S-4 sensitive surfaces; three phototubes with S-1 sensitive surfaces (peak response at
$800 \mathrm{~nm})$; two type PJ-14B tubes; a Gillod-Boutry phototube; and a selenium barrier-layer cell.

\section{Procedure}

The photosensitors were calibrated by exposing them to illumination from a lamp operating at $2854^{\circ} \mathrm{K}$; 22 two- by two-inch squares of colored glass, whose spectral transmittance was known from several sets of independent spectrophotometric measurements, were placed in turn in the light beam and readings of the photosensitors were taken. The color designations (see Table I) are those suggested by Kelly. ${ }^{4}$

For each of the 22 colors a correction factor $k$ was calculated, such that

$$
k=\tau_{s} / \tau_{m}
$$

where $\tau_{g}$ is the transmittance of the filter as determined by a set of spectrophotometric measurements and $\tau_{m}$ is the transmittance as indicated by two pho. tosensitor readings.

$$
\text { Since } \begin{gathered}
E_{s}=\tau_{g} E_{o} \text {, and } \\
\tau_{m}=E_{m} / E_{o}
\end{gathered}
$$

where $E_{o}$ is the measured illuminance on the phototube without the filter, $E_{s}$ is the "correct" illuminance with the filter, and $E_{m}$ is the measured illuminance with the filter,

$$
E_{s}=k E_{m}
$$

\begin{tabular}{|c|c|c|c|c|c|c|c|}
\hline \multirow{3}{*}{$\begin{array}{l}\text { TEST } \\
\text { FILTER } \\
\text { NUMBER }\end{array}$} & \multirow[b]{3}{*}{$\begin{array}{l}\text { COLOR } \\
\text { DESIGNATION* }\end{array}$} & \multirow[b]{3}{*}{$\begin{array}{l}\text { LUMINOUS } \\
\text { TRANSMITTANCE }\end{array}$} & & & & & \\
\hline & & & & \multirow[b]{2}{*}{$\begin{array}{c}\text { PJ-1 4B } \\
2\end{array}$} & \multirow[b]{2}{*}{$\begin{array}{l}\text { GILLOD- } \\
\text { BOUTRY }\end{array}$} & \multicolumn{2}{|c|}{ BARRIER LAYER } \\
\hline & & & $\begin{array}{c}\mathrm{PJ}-1 \text { 4B } \\
1\end{array}$ & & & 2 & 3 \\
\hline 1 & Purplish blue & .020 & 1.08 & 1.11 & .85 & .93 & .93 \\
\hline 2 & Blue & .039 & 1.05 & 1.05 & .87 & 1.00 & 1.00 \\
\hline 3 & Blue & .102 & .96 & .95 & & 1.01 & 1.03 \\
\hline 4 & Blue (lunar white) & .166 & .97 & .94 & $1.0 i$ & .99 & .99 \\
\hline 5 & Blue (lunar white) & .246 & .97 & .95 & 1.01 & .99 & .99 \\
\hline 6 & Bluish green & .130 & .99 & .92 & .96 & .97 & .99 \\
\hline 7 & Green & .198 & .99 & .92 & .99 & .97 & 1.00 \\
\hline 8 & Green & .249 & .99 & .93 & .99 & .98 & 1.00 \\
\hline 9 & Green & .110 & .98 & .89 & .99 & .97 & .98 \\
\hline 10 & Green & .047 & .99 & .87 & .98 & .94 & .98 \\
\hline 11 & Green & .049 & .98 & .86 & .98 & & .98 \\
\hline 12 & Yellow-green & .405 & 1.00 & .95 & & 1.03 & 1.06 \\
\hline 13 & Yellowish green & .138 & .99 & .90 & 1.00 & .98 & 1.00 \\
\hline 14 & Yellowish orange & .740 & .99 & .99 & & 1.02 & 1.04 \\
\hline 15 & Yellowish orange & .760 & .98 & .98 & 1.02 & .98 & .99 \\
\hline 16 & Orange & .652 & .98 & 1.01 & 1.04 & .99 & .99 \\
\hline 17 & Reddish orange & .519 & 1.00 & 1.06 & 1.05 & 1.00 & 1.00 \\
\hline 18 & Reddish orange & .304 & 1.00 & 1.10 & 1.02 & .99 & .96 \\
\hline 19 & Red & .154 & .97 & 1.12 & 1.01 & .99 & .93 \\
\hline 20 & Red & .106 & .94 & 1.12 & .98 & .99 & .92 \\
\hline 21 & Red & .050 & .79 & .98 & & .89 & .83 \\
\hline 22 & Red & .0254 & .67 & .88 & & .88 & .79 \\
\hline
\end{tabular}

or, $k$ times the measured illuminance gives the "correct" illuminance which is incident on the phototube.

Table II-Correction Factors for Color-Corrected Photosensitors 
Table III-Correction Factors for Uncorrected Photosensitors

\begin{aligned} & \multicolumn{1}{c}{ COLOR } \\ & DESIGNATION* \\ 1 & Purplish blue \\ 2 & Blue \\ 3 & Blue \\ 4 & Blue (lunar white) \\ 5 & Blue (lunar white) \\ 6 & Bluish green \\ 7 & Green \\ 8 & Green \\ 9 & Green \\ 10 & Green \\ 11 & Green \\ 12 & Yellow-green \\ 13 & Yellowish green \\ 14 & Yellowish orange \\ 15 & Yellowish orange \\ 16 & Orange \\ 17 & Reddishorange \\ 18 & Reddish orange \\ 19 & Red \\ 20 & Red \\ 21 & Red \\ 22 & Red \end{aligned}

LUMINOUS
TRANSMITTANCE
.020
.039
.102
.166
.246
.130
.198
.249
.110
.047
.049
.405
.138
.740
.760
.659
.519
.304
.154
.106
.050
.0254

\begin{tabular}{|c|c|c|c|}
\hline \multicolumn{4}{|c|}{ S.4 SURFAC :S } \\
\hline 1 & 2 & 3 & 4 \\
\hline .08 & .10 & .08 & .09 \\
\hline .14 & .16 & .14 & .15 \\
\hline .26 & .28 & .25 & .27 \\
\hline .37 & .41 & .37 & .39 \\
\hline .48 & .52 & .48 & .50 \\
\hline .45 & .46 & .43 & .45 \\
\hline .52 & .53 & .50 & .52 \\
\hline .56 & .58 & .54 & .56 \\
\hline .53 & .55 & .52 & .54 \\
\hline .46 & .49 & .46 & .47 \\
\hline .96 & .93 & .96 & .93 \\
\hline 1.08 & 1.03 & 1.06 & 1.04 \\
\hline .99 & .98 & 1.01 & .98 \\
\hline 1.23 & 1.21 & 1.24 & 1.22 \\
\hline 2.08 & 1.89 & 2.18 & 2.03 \\
\hline 2.43 & 2.20 & 2.66 & 2.35 \\
\hline 2.75 & 2.48 & 3.09 & 2.71 \\
\hline 3.21 & 3.02 & 4.16 & 3.52 \\
\hline 3.28 & 3.21 & 4.91 & 3.98 \\
\hline 2.94 & 3.03 & 4.49 & 3.41 \\
\hline 2.17 & 2.27 & 3.60 & 2.60 \\
\hline 1.69 & 2.03 & 3.30 & 2.44 \\
\hline
\end{tabular}

BARRIER
LAYER
.29
.34
.55
.66
.76
.81
.88
.92
.96
.90
1.29
1.25
1.30
1.08
1.13
1.07
1.00
.81
.64
.56
.43
.36

\begin{tabular}{rrr}
\multicolumn{3}{c}{ S.1 SURFACES } \\
\hline \multicolumn{1}{r}{1} & \multicolumn{1}{c}{2} \\
.59 & .51 & .53 \\
.58 & .52 & .55 \\
.13 & .14 & .13 \\
.29 & .22 & .29 \\
.31 & .32 & .31 \\
6.67 & 7.43 & 8.13 \\
8.61 & 6.39 & 7.62 \\
7.66 & 5.47 & 6.92 \\
8.80 & 8.80 & 8.46 \\
7.84 & 7.84 & 7.52 \\
19.60 & 12.30 & 16.33 \\
4.74 & 4.09 & 4.63 \\
15.33 & 10.23 & 15.33 \\
1.44 & 1.47 & 1.45 \\
.93 & .94 & .94 \\
.77 & .77 & .77 \\
.62 & .63 & .63 \\
.37 & .37 & .37 \\
.19 & .20 & .20 \\
.13 & .14 & .13 \\
.07 & .07 & .07 \\
.04 & .04 & .04 \\
& &
\end{tabular}

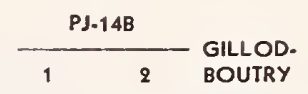

* The chromaticity coordinates are shown In Flg. 3.

\section{Results}

The $k$ values for one photosensitor with a colorcorrected S-4 surface are given in Fig. $3 ; k$ values for all four color-corrected S-4 surfaces are given in Table I. Correction factors between 0.81 and 1.20 are applicable to all colors except the reds. Factors for phototube No. 3 are within \pm 0.05 of 1.00 for 11 colors, while the other three phototubes are within \pm 0.05 for only three or four colors.

The PJ-14B phototube No. 1 is within \pm 0.08 of 1.00 for all colors except the two deepest reds and within \pm 0.05 for all but the two reds and the purplish blue, as shown in Table II. PJ.14B phototube No. 2 shows better correction for the reds than No. 1; correction in the blues and greens is less adequate, however. (The average correction factor for both phototubes is 0.97). The Gillod-Boutry is within \pm 0.05 for all but two of the 17 filters for which measurements were made.

The $k$ values for the noncolor-corrected photosensitors are given in Table III.

\section{Discussion}

The data for the barrier-layer type of photocells show that, when they are calibrated with light at a color temperature of $2854^{\circ} \mathrm{K}$, many of the colors shown in the tables can be measured with small errors. Also, more tellingly in the reds, if the photocell is calibrated with a filter close on the chromaticity diagram to the color being measured, the error is reduced. For example, a photocell calibrated with light passing through filter No. 19 could be used for measuring light through filter No. 20 within one per cent (on the basis of the data of barrier-layer cell No. 3 ).

Since many of the correction factors of Table I for adjacent filters are within a few per cent of each other, photosensitors with S-4 surfaces and Wratten No. 106 filters can also be calibrated with light spectrally close to the light being measured for increasing accuracy.

Each set of correction factors of Tables I through III applies only to a specific photosensitor and not to the type in general. The factors do, however, suggest the magnitude of the errors that might be expected when measuring the intensity of light of the color indicated.

Although the PJ-14B phototube is no longer available commercially, there is a comparable replacement, the type Z-1454, which is, however, too expensive for general photometric use. The Gillod-Boutry phototube is included as an example of a phototube for precision measurements, which has been made, and could, in the future, be made available to a group of interested purchasers at a reasonable price.

References

1. Douglas, C. A.: "Photometer for Measurement of Effective Intensity of Condenser-Discharge Lights," ILluminatiNa ENGINEerING, Vol. LIII, p. 205 (April 1958).

2. Nimeroff, I. and Wilson, S.: "A Colorimeter for Pyrotechnic Smokes," Journal of Research of the National Bureau of Stand. ards, Vol. 52, p. 195 (April 1954).

3. Barbrow, L. E.: "A Photometric Procedure Osing BarrierLayer Photocells," Journal of Research of the National Bureau of Standards, Vol. 25, p. 703 (December 1940).

4. Kelly, K. L.: "Color Designations for Lights," Journal of the Optical Society of America, Vol. 33, p. 627 (November 1943). 


\section{Absolute Light-Scattering Photometer: I. Design and Operation}

12.11. Donald McIntyre and G. C. Doderer ${ }^{1}$

A new light-seatering photometer lats been designed and builh for retrermining the absolute sentlering from polymer solutions. The instrument is also capable of performing as a rescareh instrument for making moasurements at very low and very high angles, and at very low and very high intensitics of seatered light. The instrument seans the angular seithering cither mammily or automatically while measuring continuously the ratio of the scattrerel light to the inciclent light.

p. 153

JOURNAL OF RESEARCH of the National Bureau of Standards-A. Physics and Chemistry

Vol, 68A, No. 1, January February 1964

\section{An Absolute Light Scattering Photometer: II. Direct Determination of Scattered Light From Solutions}

12.12.

\section{Donald McIntyre}

(August 9, 19633)

The light scattering photometox recently reseribed in this journal by MeTutyre: and 1)olerer has been eximined to defermine its ability to measure the absolinte scattering of liculds. The alsolute seattering of polymer solutions was determined from transmission mousurements and from two different transverse measurements. Ploe ciperimental results

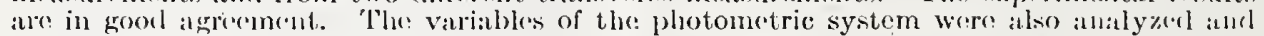
experimentally studied to dotermine its ability to measure absolute scattering of liquids

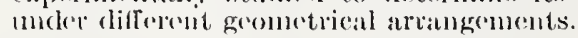


B

Badger, H. L., MeSparron, D. A., and Douglas, C. A., Radiometric methods for measuring laser output, Nat. Bur. Stand. (U.S.) Tech. Note 418, 12 pp. (Nov. 1967)

$7-336$

Barbrow, L. E., Memorandum on a procedure for obtaining spectral radiant intensities of tungsten-filament lamps, 400-700 $\mathrm{m} \mu$, J. Opt. Soc. Am. 49, No. 11, 1122 (Nov. 1959)

Barbrow, L. E., The metric system in illuminating engineering, Illum. Eng. 62, No. 11, 638604 (Nov. 1967)

Barbrow, L. E., and Wilson, S. W., Vertical distribution of light from gas filled candlepower standards, Illum. Eng. 53, No. 12, 645-648 (Dec. 1958) $7-544$

C

Caldwell, B. P., Goebel, D. G., and Hammond, H. K., III, Use of an auxiliary sphere with a spectrorefiectometer to obtain absolute reflectance, J. Opt. Soc. Am. 56, No. 6, 783-788 (June 1966)

Canfield, L. R., Johnston, R. G., Codling, K., and Madden, R. P., Comparison of an ionization chamber and a thermopile as absolute detectors in the extreme ultraviolet, Appl. Opt. 6, No. 11, 1886-1888 (Nov. 1967) _..-_-

Chamberlain, J. L., Spangenberg, D. B., and Strang A. G., Effect of surface texture on diffuse spectral reflectance: B. Surface texture measurement of metal surfaces, Symp. Thermal Radiation of Solids, San Francisco, Calif., Mar. 4-6, 1964, pp. 165-177 (NASA Sp-55, Nat. Aeron. and Space Admin., Washington, D.C. 1965) -

Chernoff, L., Photometry of projectors at the National Bureau of Standards, Nat. Bur. Stand. (U.S.) Tech. Note 198, 28 pp. (Dec. 1963) ---

Clark, H. E., Dunn, S. T., Geist, J. C., Moore, D. G., and Richmond, J. C., Thermal radiation property measurement techniques (abstract only), Nat. Bur. Stand. (U.S.) Tech. Note 415, 77 pp. (Apr. 27, 1967)

Clark, H. E., and Moore, D. G., A rotating cylinder method for measuring normal spectral emittance of ceramic oxide specimens from $1200^{\circ}$ to $1600^{\circ} \mathrm{K}, \mathrm{J}$. Res. Nat. Bur. Stand. (U.S.) 70A, No. 5, 393-415 (Sept.-Oct. 1966) -- 7-151

Codling, K., Canfield, L. R., Johnston, R. G., and Madden, R. P., Comparison of an ionization chamber and a thermopile as absolute detectors
DeWitt, D. P., Comments on the Surface characterization of real metals. Symp. Thermal Radiation of Solids, San Francisco, Calif., Mar. 4-6, 1964, pp. 141-144 (NASA SP-55, Nat. Aeron, and Space Admin., Washington, D.C. 1965)

DeWitt, D. P., and Richmond, J. C., Theory and measurement of the thermal radiative properties of metals (abstract only), submitted for book Techniques of Metals Research, Vol. VI (John Wiley \& Sons, New York, N.Y. (1970) _- 7-262

DeWitt, D. P., Richmond, J. C., Dunn, S. T., and Hayes, W. D., Jr., Procedures for precise determination of thermal radiation properties (abstract only), November 1963 to October 1964, Nat. Bur. Stand. (U.S.) Tech. Note 267, 62 pages (Dec. 17, 1965)

DeWitt, D. P., Richmond, J. C., and Hayes, W. D., Jr., Procedures for precise determination of thermal radiation properties (abstract only), November 1962 to October 1963, Nat. Bur. Stand. (U.S.) Tech. Note 252, 55 pages (Nov. 20, 1964)

Douglas, C. A., Review of elementary theory of the photometry of projection apparatus, Illum. Eng. 62, No. 4, 246-253 (Apr. 1967)

Douglas, C. A., Computation of the effective intensity of flashing lights, Illum. Eng. 52, No. 12, 641-646 (Dec. 1957) $7-640$

Douglas, C. A., Photometer for measurement of effective intensity of condenser-discharge lights, Illum. Eng. 53, No. 4, 205-208 (Apr. 1958

Douglas, C. A., MeSparron, D. A., and Badger, H. L., Radiometric methods for measuring laser output, Nat. Bur. Stand. (U.S.) Tech. Note 418, 12 pages (Nov. 1967) _._.

Dunn, S. T., Flux averaging devices for the infrared (abstract only), Nat. Bur. Stand. (U.S.) Tech. Note 279, 14 pages (Dec. 1965) _-_-_-- 7-456

Dunn, S. T., Geist, J. C., Moore, D. G., Clark, H. E., and Richmond, J. C., Thermal radiation property measurement techniques (abstract only), Nat. Bur. Stand. (U.S.) Tech. Note 415, 77 pages (Apr. 27, 1967) _-_._- 7-210

Dunn, S. T., Richmond, J. C., DeWitt, D. P., Hayes, W. D., Jr., Procedures for precise determination of thermal radiation properties ( $a b-$ stract only), November 1963 to October 1964, Nat. Bur. Stand. (U.S.) Tech. Note 267, 62 pages (Dec. 17, 1965)

Dunn, S. T., Richmond, J. C., Parmer, J. F., Survey of infrared measurement techniques and computational methods of radiant heat transfer, J. Spacecraft and Rockets, 961-965 (July 1966) 


\section{Author Index}

Dunn, S. T., Richmond, J. C., and Wiebelt, J. A., Ellipsoidal mirror reflectometer, J. Res. Nat. Bur. Stand. (U.S.), 70C, No. 2, 75-88 (Apr. June 1966)

Dunn, S. T., Application of sulfur coatings to integrating spheres, Appl. Opt. 4, No. 7, 877 (July 1965) $7-455$

\section{E}

Erminy, D. E., Kostkowski, H. J., Hattenburg, A. T., High accuracy spectral radiance calibration of tungsten strip lamps, Ch. 4, Advances in Geophysics, Vol. 14, Academic Press, New York, N.Y. (1970)

Eubanks, A. G., and Peavy, B. A., Periodic heat flow in a hollow cylinder rotating in a furnace with a viewing port, Symp. on Measurement of Thermal Radiation Properties of Solids, Dayton, Ohio, Sept. 5-7, 1962, pp. 553-563 (NASA SP-31, Nat. Aeron. and Space Admin., Washington, D.C., 1963)

\section{F}

Fussell, W., and Geist, J., Approximate normal emissivity in the infrared at elevated temperatures of single-crystal and polycrystalline calcium fluoride, Appl. Opt. 6, No. 1, 119-124 (Jan. 1967)

Fussell, W. B., Stair, R., and Schneider, W. A., A standard for extremely low values of spectral irradiance, Appl. Opt. 4, No. 1, 85-89 (Jan. 1965)

Fussell, W. B., Stair, R., and Schneider, W. E., The new tungsten-filament lamp standards of total irradiance, Appl. Opt. 6, No. 1, 101-106 (Jan. 1967)

Geist, J. C., Dunn, S. T., Moore, D. G., Clark, H. E., and Richmond, J. C., Thermal radiation property measurement techniques (abstract only), Nat. Bur. Stand. (U.S.) Tech. Note 415, 77 pages (Apr. 27, 1967)

Geist, J., and Fussell, W., Approximate normal emissivity in the infrared at elevated temperatures of single-crystal and polycrystalline calcium fluoride, Appl. Opt. 6, No. 1, 119-124 (Jan. 1967)

Geist, J., High purity powdered $\mathrm{CcI}$ as a high reflectance infrared diffuser, Appl. Opt. 6, No. 7, 1280 (July 1967)

Goebel, D. G., Generalized integrating-sphere theory, Appl. Opt. 6, No. 1, 125-128 (Jan. 1967)
Volume and Page

Goebel, D. G., Caldwell, B. P., and Hammond, H. K., III, Use of an auxiliary sphere with a spectroreflectometer to obtain absolute reflectance, J. Opt. Soc. Am. 56, No. 6, 783-788 (June 1966)

\section{H}

Halback, E. W., Stair, R., and Johnston, R. G., Standard of spectral radiance for the region of 0.25 to 2.6 microns, J. Res. Nat. Bur. Stand. (U.S.) 64A, No. 4, 291-296 (July-Aug. 1960) -- 7-361

Hammond, H. K., III, Goebel, D. G., and Caldwell, B. P., Use of an auxiliary sphere with a spectroreflectometer to obtain absolute reflectance, J. Opt. Soc. Am. 56, No. 6, 783-788 (June 1966)

Harrison, W. N., and Richmond, J. C., Equipment and procedures for evaluation of total hemispherical emittance, Am. Ceram. Soc. Bull. 39, No. 11, 668-673 (Nov. 15, 1960)

Harrison, W. N., and Richmond, J. C., Total hemispherical emittance of coated and uncoated Inconel and types 321 and 430 stainless steel, J. Res. Nat. Bur. Stand. (U.S.) 66C, No. 3, 261269 (Sept. 1962)

Harrison, W. N., Richmond, J. C., and Shorten, F. J., An approach to thermal emittance standards Symp. Measurement of Radiation Properties, Dayton, Ohio, Sept. 5-7, 1962, pp. 403423 (NASA SP-31, Nat. Aeron. and Space Admin., Washington, D.C. 1963)

Harrison, W. N., Richmond, J. C., Shorten, F. J., and Joseph, H. M., Standardization of thermal emittance measurements Part IV: Normal spectral emittance $800-1400^{\circ} \mathrm{K}$, abstracted from Tech. Rept. WADC-TR-59-510, Part IV, Wright Patterson Air Force Base, 90 pages (1963)

Hattenburg, A. T., Spectral radiance of a low current graphite arc, Appl. Opt. 6, No. 1, 95100 (Jan. 1967)

Hattenburg, A. T., Kostkowski, H. J., and Erminy, D. E., High accuracy spectral radiance calibration of tungsten strip lamps, Ch. 4, Advances in Geophysics, Vol. 14, Academic Press, New York, N.Y. (1970) -7-373

Hayes, W. D., Jr., Richmond, J. C., and DeWitt, D. P., Procedures for precise determination of thermal radiation properties, November 1962 to October 1963 (abstract only), Nat. Bur. Stand. (U.S.) Tech. Note 252,55 pages (Nov. $20,1964)$

Hayes, W. D., Jr., Richmond, J. C., Dunn, S. T., and DeWitt, D. P., Procedures for precise determination of thermal radiation properties, November 1963 to October 1964 (abstract only), Nat. Bur. Stand. (U.S.) Tech. Note 267, 62 pages (Dec. 17,1965$)$ 


\section{Author Index}

Jackson, J. K., Schneider, W. E., and Stair, R., Spectral irradiance as determined through the use of prism and filter spectroradiometric techniques, Appl. Opt. 6, No. 9, 1479-1486 (Sept.

$1967)$
Jackson, J. K., Stair, R., and Schneider, W. E., A new standard of spectral irradiance, Appl. Opt. 2, No. 11, 1151-1154 (Nov. 1963) ------ 7-274

Jackson, J. K., Stair, R., Schneider, W. E., and Waters, W. R., Some factors affecting the sensitivity and spectral response of thermoelectric (radiometric) detectors. Appl. Opt. 4, No. 6, 703-710 (June 1965)

Jennings, D. A., Calorimetric measurement of pulsed laser output energy, IEEE Trans. Instr. \& Meas., IM-15, No. 4, 161-164 (Dec. 1966) -- 7-513

Jennings, D. A., A laser power meter for large beams, Rev. Sci. Instrum. 41, No. 4, 565-567 (April 1970)

Jennings, D. A., Laser power and energy measurements, Nat. Bur. Stand. (U.S.) Tech. Note 382, 64 pp. (Oct. 1969)

Johnston, R. G., Canfield, L. R., Codling, K., and Madden, R. P., Comparison of an ionization chamber and a thermopile as absolute detectors in the extreme ultraviolet, Appl. Opt. 6, No. 11, 1886-1888 (Nov. 1967)

Johnston, R. G., and Madden, R. P., On the use of thermopiles for absolute radiometry in the far ultraviolet, Appl. Opt. 4, No. 12, 15741580 (Dec. 1965)

Johnston, R. G., Maki, A. G., and Stair, R., Apparatus for the measurement of the normal spectral emissivity in the infrared, J. Res. Nat. Bur. Stand. (U.S.) 64C, No. 2, 99-102 (Apr.June 1960)

Johnston, R. G., Stair, R., and Halback, E. W., Standard of spectral radiance for the region of 0.25 to 2.6 microns, J. Res. Nat. Bur. Stand. (U.S.) 64A, 291-296 (July-Aug. 1960) 7-361

Joseph, H. M., Harrison, W. N., Richmond, J. C., and Shorten, F. J., Standardization of thermal emittance measurements Part IV: Normal spectral emittance $800-1400^{\circ} \mathrm{K}$, (abstract only), Tech. Rept. WADC TR-59-510, Part IV, Wright-Patterson Air Force Base, 90 pages (1963)

Judd, D. B., Terms, definitions and symbols in reflectometry, J. Opt. Soc. Am. 57, No. 4, 445452 (Apr. 1967)

Kashima, T., A 5-meter integrating sphere, Appl. Opt. 6, No. 4, 757-771 (April 1967)
Volume and Page

Keegan, H. J., Schleter, J. C., and Weidner, V. R., Effect of surface texture on diffuse spectral reflectance: A. Diffuse spectral reflectance of metal surfaces, Symp. Thermal Radiation of Solids, San Francisco, Calif., Mar. 4-6, 1964, pp. 165-177 (NASA SP-55, Nat. Aeron. and Space Admin., Washington, D.C., 1965) --_-- 7-477

Kelley, D. L., Richmond, J. C., Kneissel, G. J., and Kelly, F. J., Procedures for precise determination of thermal radiation properties, November 1964 to October 1965 (abstract only), Nat. Bur. Stand. (U.S.) Tech. Note 292, 80 pages (Feb. 10, 1967)

Kelly, F. J., An equation for the local thermal emissivity at the vertex of a diffuse conical or V-groove cavity, Appl. Opt. 5, No. 6, 925-927 (June 1966)

Kelly, F. J., On Kirchhoff's Law and its generalized application to absorption and emission by cavities, J. Res. Nat. Bur. Stand. (U.S.) 69B, No. 3, 165-171 (July-Sept. 1965)

Kelly, F. J., and Moore, D. G., A test of analytical expressions for the thermal emissivity of shallow cylindrical cavities, Appl. Opt. 4, No. 1, 31-40 (Jan. 1965) --_-_-_-_-_-_-

Kelly, F. J., Richmond, J. C., Kneissel, G. J., and Kelley, D. L., Procedures for precise determination of thermal radiation properties (abstract only) November 1964 to October 1965, Nat. Bur. Stand. (U.S.) Tech. Note 292, 80 pages (Feb. 10, 1967) -......-

Kneissel, G. J., Richmond, J. C., A laser-source integrating sphere reflectometer (abstract only), Nat. Bur. Stand. (U.S.) Tech. Note 439, 69 pages (Feb. 1968)

Kneissel, G. J., Richmond, J. C., Kelley, D. L., and Kelly, F. J., Procedures for precise determination of thermal radiation properties, November 1964 to October 1965 (abstract only), Nat. Bur. Stand. (U.S.) Tech. Note 292, 80 pages (Feb. 10, 1967) --_-_-_-_-_-_

Kneissel, G. J., High purity powdered CsI as a high reflectance infrared diffuser, Appl. Opt. 6, No. 7, 1280 (July 1967)

Knodo, Y., A 5-meter integrating sphere, Appl. Opt. 6, No. 4, 757-771 (April 1967)

Kostkowski, H. J., A new radiometric equation and its application, Appl. Opt. 5, No. 12, 1959 (Dec. 1966)

Kostkowski, H. J., Erminy, D. E., and Hattenburg, A. T., High accuracy spectral radiance calibration of tungsten strip lamps, Ch. 4, Advances in Geophysics, Vol. 14, Academic Press, New York, N.Y. (1970)

Kostkowski, H. J., and Lee, R. D., Theory and methods of optical pyrometry (abstract only), Nat. Bur. Stand. (U.S.) Monogr. 41, 28 pages (reprinted Sept. 1962) 


\section{Author Index}

$\mathbf{L}$

Lee, R. D., Construction and operation of a simple high-precision copper-point blackbody and furnace (abstract only), Nat. Bur. Stand. (U.S.) Tech. Note 483, 15 pages (May 1969) -- 7-403

Lee, R. D., The NBS photoelectric pyrometer and its use in realizing the International Practical Temperature Scale above $1063^{\circ} \mathrm{C}$ (abstract only), Metrologia 2, No. 4, 150-162 (Oct.

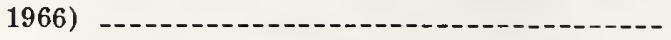

Lee, R. D., and Kostkowski, H. J., Theory and methods of optical pyrometry (abstract only), Nat. Bur. Stand. (U.S.) Monogr. 41, 28 pages (reprinted Sept. 1962)

Lee, R. D., and Lewis, E., Radiance temperature at $6550 \AA$ of the graphite are (abstract only), Appl. Opt. 5, No. 11, 1858 (Nov. 1966) $7-403$

Lewis, E., and Lee, R. D., Radiance temperature at $6550 \AA$ of the graphite arc (abstract only), Appl. Opt. 5, No. 11, 1858 (Nov. 1966)

$7-403$

\section{M}

Madden, R. P., Canfield, L. R., Johnson, R. G., and Codling, $\mathrm{K}$, Comparison of an ionization chamber and a thermopile as absolute detectors in the extreme ultraviolet, Appl. Opt. 6, No. 11, 1886-1888 (Nov. 1967) -...-

Madden, R. P., and Johnston, R. G., On the use of thermopiles for absolute radiometry in the far ultraviolet, Appl. Opt. 4, No. 12, 1574-1580 (Dec. 1965)

Maki, A. G., Plyler, E. K., Method of measuring emissivities of metals in the infrared, J. Res. Nat. Bur. Stand. (U.S.) 66C, No. 3, 283-287 (Sept. 1962)

Maki, A. G., Stair, R., and Johnston, R. G., Apparatus for the measurement of the normal spectral emissivity in the infrared, J. Res. Nat. Bur. Stand. (U.S.) 64C, No. 2, 99-102 (Apr.June 1960) -.-.--

McSparron, D. A., Douglas, C. A., and Badger, H. L., Radiometric methods for measuring laser output, Nat. Bur. Stand. (U.S.) Tech. Note 418, 12 pages (Nov. 1967)

Moore, D. G., Investigation of shallow reference cavities for high-temperature emittance measurement; Symp. Measurement of Thermal Radiation Properties of Solids, Dayton, Ohio, Sept. 5-7, 1962, pp. 515-526 (NASA SP-31, Nat. Aeron. and Space Admin., Washington, D.C., 1963)

Moore, D. G., and Kelly, F. J., A test of analytical expressions for the thermal emissivity of shallow cylindrical cavities, Appl. Opt. 4, No. 1, 31-40 (Jan. 1965)

Moore, D. G., and Clark, H. E., A rotating cylinder method for measuring normal spectral emittance of ceramic oxide specimens from $1200^{\circ}$ to $1600^{\circ} \mathrm{K}$, J. Res. Nat. Bur. Stand. (U.S.) 70 A, No. 5, 393-415 (Sept.-Oct. 1966) -
Moore, D. G., Dunn, S. T., Geist, J. C., Clark, H. E., and Richmond, J. C., Thermal radiation property measurement techniques (abstract only), Nat. Bur. Stand. (U.S.) Tech. Note 415, 77 pages (Apr. 27, 1967)

\section{$\mathbf{N}$}

Nonaka, M., A 5-meter integrating sphere, Appl. Opt. 6, No. 4, 757-771 (April 1967) $7-456$

\section{$\mathbf{0}$}

Office of Standard Reference Materials, Certificate of normal spectral emittance, standard reference materials 1402 to 1409 , Platinum-13 percent rhodium alloy, 7 pages (1966) _..._- 7-100

Office of Standard Reference Materials, Certificate of normal spectral emittance, standard reference materials 1420-1428, oxidized Kanthal, 4 pages (1965)

Office of Standard Reference Materials, Certificate of normal spectral emittance, standard reference materials 1440 to 1447 , oxidized inconel

Office of Standard Reference Materials, Instructions for handling emittance standards, 1 page (1965)

\section{$\mathbf{P}$}

Parmer, J. F., Dunn, S. T., and Richmond, J. C., Survey of infrared measurement techniques and computational methods of radiant heat transfer, J. Spacecraft and Rockets, 961-975 (July 1966)

Peavy, B. A., A note on the numerical evaluation of thermal radiation characteristics of diffuse cylindrical and conical cavities, J. Res. Nat. Bur. Stand. (U.S.) 70C, No. 2, 139-148

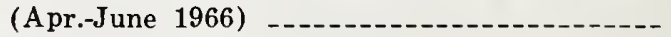

Peavy, B. A., and Eubanks, A. G., Periodic heat flow in a hollow cylinder rotating in a furnace with a viewing port, Symp. on Measurement of Thermal Radiation Properties of Solids, Dayton, Ohio, Sept. 5-7, 1962, pp. 553-563 (NASA SP-31, Nat. Aeron. and Space Admin., Washington, D.C., 1963)

Plyler, E. K., and Maki, A. G., Method of measuring emissivities of metals in the infrared, J. Res. Nat. Bur. Stand. (U.S.) $66 \mathrm{C}$, No. 3, 283-287 (Sept. 1962)

Projector, T. H., The use of zonal constants in the calculation of beam flux, Illum. Eng., 48, No. 4, 189-191 (April 1953)

Projector, T. H., Versatile goniometer for projection photometry, Illum. Eng. 48, No. 4, 192-196 (April 1953)

Projector, T. H., Effective intensity of flashing lights, Illum. Eng. 52, No. 12, 630-640 (Dec. 1957) 


\section{Author Index}

$\mathbf{R}$

Volume and Page

Richmond, J. C., Effect of surface roughness on emittance of nonmetals, J. Opt. Soc. Am. 56, No. 2, 253-254 (Feb. 1966)

Richmond, J. C., Importance of surface films, Symp. Thermal Radiation of Solids, San Francisco, Calif., Mar. 4-6, 1964, pp. 157-158 (NASA SP-55, Nat. Aeron. and Space Admin., Washington, D.C., 1965) $7-149$

Richmond, J. C., Relation of emittance to other optical properties, J. Res. Nat. Bur. Stand. (U.S.) 67C, No. 3, 217-226 (Sept. 1963) $7-236$

Richmond, J. C., Thermal radiation properties of ceramic materials, Proc. Symp. Mechanical and Thermal Properties of Ceramics, Gaithersburg, Md., Apr. 1-2, 1968, Nat. Bur. Stand. (U.S.) Spec. Publ. 303, pp. 125-137 (May 1969) ---- 7-223

Richmond, J. C., and DeWitt, D. P., Theory and measurement of the thermal radiative properties of metals (abstract only), submitted for book Techniques of Metals Research, Vol. VI (John Wiley \& Sons, New York, N.Y., 1970 _- 7-262

Richmond, J. C., DeWitt, D. P., and Hayes, W. D., Jr., Procedures for precise determination of thermal radiation properties, November 1962 to October 1963 (abstract only), Nat. Bur. Stand. (U.S.) Tech. Note 252, 55 pages (Nov. 20, 1964)

Richmond, J. C., Dunn, S. T., DeWitt, D. P., and Hayes, W. D., Jr., Procedures for precise determination of thermal radiation properties, November 1963 to October 1964 (abstract only), Nat. Bur. Stand. (U.S.) Tech. Note 267, 62 pages (Dec. 17, 1965)

7-216

Richmond, J. C., Dunn, S. T., Geist, J. C., Moore, D. G., and Clark, H. E., Thermal radiation property measurement techniques (abstract only), Nat. Bur. Stand. (U.S.) Tech. Note 415, 77 pages (Apr. 27, 1967)

Richmond, J. C., Dunn, S. T., and Parmer, J. F., Survey of infrared measurement techniques and computational methods of radiant heat transfer, J. Spacecraft and Rockets, 961-975 (July 1966)

Richmond, J. C., Dunn, S. T., and Wiebelt, J. A., Ellipsoidal mirror reflectometer, J. Res. Nat. Bur. Stand. (U.S.) 70C, No. 2, 75-88 (Apr.June 1966)

Richmond, J. C., and Harrison, W. N., Equipment and procedures for evaluation of total hemispherical emittance, Am. Ceram. Soc. Bull. 39, No. 11, 668-673 (Nov. 15, 1960)

Richmond, J. C., and Harrison, W. N., Total hemispherical emittance of coated and uncoated Inconel and types 321 and 430 stainless steel, J. Res. Nat. Bur. Stand. (U.S.) 66C, No. 3, 261269 (Sept. 1962)
Volume and Page

Richmond, J. C., Harrison, W. N., and Shorten, F. J., An approach to thermal emittance standards, Symp. Measurement of Radiation Properties, Dayton, Ohio, Sept. 5-7, 1962, pp. 403423 (NASA SP-31, Nat. Aeron. and Space Admin., Washington, D.C., 1963)

Richmond, J. C., Harrison, W. N., Shorten, F. J., and Joseph, H. M., Standardization of thermal emittance measurements Part IV: Normal spectral emittance $800-1400^{\circ} \mathrm{K}$, abstracted from Tech. Rept. WADC-TR-59-510, Part IV, Wright Patterson Air Force Base, 90 pages (1963)

Richmond, J. C., and Kneissel, G. J., A lasersource integrating sphere reflectometer (abstract only), Nat. Bur. Stand. (U.S.) Tech. Note 439, 69 pages (Feb. 1968) _-_-_-_-_ 7-509

Richmond, J. C., Kneissel, G. J., Kelley, D. L., and Kelly, F. J., Procedures for precise determination of thermal radiation properties, November 1964 to October 1965 (abstract only), Nat. Bur. Stand. (U.S.) Tech. Note 292, 80 pages (Feb. 10, 1967)

Richmond, J. C., and Stewart, J. E., Spectral emittance of ceramic-coated and uncoated specimens of Inconel and stainless steel, J. Am. Ceram. Soc. 42, No. 12, 633-640 (Dec. 1959)

Rosa, E. B., Theory, construction, and use of the photometric integrating sphere, Bul. BS 18, 281-323, (1922-23) S447

\section{$\mathbf{S}$}

Schleter, J. C., Keegan, H. J., and Weidner, V. R., Effect of surface texture on diffuse spectral reflectance: A. Diffuse spectral reflectance of metal surfaces, Symp. Thermal Radiation of Solids, San Francisco, Calif., Mar. 4-6, 1964, pp. 165-177 (NASA SP-55, Nat. Aeron. and Space Admin., Washington, D.C., 1965) _--- 7-477

Schneider, W. E., A one solar constant irradiance standard, Appl. Opt. 9, No. 6, 1410-1418 (June 1970)

Schneider, W. E., and Stair, R., Standards, sources, and detectors in radiation measurement, Symp. Thermal Radiation of Solids, San Francisco, Calif. (Mar. 4-6, 1964), pp. 217231 (NASA SP-55, Nat. Aeron. and Space Admin., Washington, D. C., 1965) _...---

Schneider, W. E., Stair, R., and Fussell, W. B., The new tungsten-filament lamp standards of total irradiance, Appl. Opt. 6, No. 1, 101-106 (Jan. 1967) 


\section{Author Index}

Volume and Page

Schneider, W. E., Stair, R., and Jackson, J. K., A new method of spectral irradiance, Appl. Opt. 2, No. 11, 1151-1154 (Nov. 1963) $7-274$

Schneider, W. E., Stair, R., and Fussell, W. B., A standard for extremely low values of spectral irradiance, Appl. Opt. 4, No. 1, 85-89 (Jan. 1965)

Schneider, W. E., Stair, R., and Jackson, J. K., Spectral irradiance as determined through the use of prism and filter spectroradiometric techniques, Appl. Opt. 6, No. 9, 1479-1486 (Sept. 1967) 7-289

Schneider, W. E., Stair, R., Waters, W. R., and Jackson, J. K., Some factors affecting the sensitivity and spectral response of thermoelectric (radiometric) detectors, Appl. Opt. 4, No. 6, 703-7-0 (June 1965)

Shorten, F. J., Harrison, W. N., Richmond, J. C., and Joseph, H. M., Standardization of thermal emittance measurements Part IV: Normal spectral emittance $800-1400^{\circ} \mathrm{K}$, abstracted from Tech. Rept. WADC-TR-59-510, Part IV, Wright Patterson Air Force Base, 90 pages (1963)

Shorten, F. J., Richmond, J. C., Harrison, W. N., An approach to thermal emittance standards, Symp. Measurement of Radiation Properties, Dayton, Ohio, Sept. 5-7, 1962, pp. 403-423 (NASA SP-31, Nat. Aeron. and Space Admin., Washington, D.C., 1963)

Spangenberg, D. B., Strang, A. G., and Chamberlain, J. L., Effect of surface texture on diffuse spectral reflectance: B. Surface texture measurement of metal surfaces, Symp. Thermal Radiation of Solids, San Francisco, Calif., Mar. 4-6, 1964, pp. 165-177 (NASA SP-55, Nat. Aeron. and Space Admin., Washington, D.C., 1965)

Stair, R., The measurement of solar radiation with principal emphasis on the ultraviolet component, Air and Wat. Pollut. Int. J. 10, 665688 (1966)

Stair, R., Fussell, W. B., and Schneider, W. E., A standard for extremely low values of spectral irradiance, Appl. Opt. 4, No. 1, 85-89 (Jan. 1965) $7-280$

Stair, R., Johnston, R. G., and Halback, E. W., Standard of spectral radiance for the region of 0.25 to 2.6 microns, J. Res. Nat. Bur. Stand. (U.S.) 64A, No. 4, 291-296 (July-Aug. 1960) -- 7-361

Stair, R., Maki, A. G., and Johnston, R. G., Apparatus for the measurement of the normal spectral emissivity in the infrared, J. Res. Nat. Bur. Stand. (U.S.) 61C, No. 2, 99-102 (Apr.-June 1960)
Stair, R., and Schneider, W. E., Standards, sources, and detectors in radiation measurement, Symp. Thermal Radiation of Solids, San Francisco, Calif. (Mar. 4-6, 1964) pp. 217-231 (NASA SP-55, Nat. Aeron. and Space Admin., Washington, D.C., 1965) -

Stair, R., Schneider, W. E., and Fussell, W. B., The new tungsten-filament lamp standards of total irradiance, Appl. Opt. 6, No. 1, 101-106 (Jan. 1967)

Stair, R., Schneider, W. E., and Jackson, J. K., A new standard of spectral irradiance, Appl. Opt. 2, No. 11, 1151-1154 (Nov. 1963)

Stair, R., Schneider, W. E., and Jackson, J. K., Spectral irradiance as determined through the use of prism and filter spectroradiometric techniques, Appl. Opt. 6, No. 9, 1479-1486 (Sept. 1967)

Stair, R., Schneider, W. E., Waters, W. R., and Jackson, J. K., Some factors affecting the sensitivity and spectral response of thermoelectric (radiometric) detectors, Appl. Opt. 4, No. 6, 703-710 (June 1965)

Stewart, J. E., and Richmond, J. C., Spectral emittance of ceramic-coated and uncoated specimens of Inconel and stainless steel, J. Am. Ceram. Soc. 42, No. 12, 633-640 (Dec. 1959) -- 7-255

Strang, A. G., Spangenberg, D. B., and Chamberlain, J. L., Effect of surface texture on diffuse spectral reflectance: B. Surface texture measurement of metal surfaces, Symp. Thermal Radiation of Solids, San Francisco, Calif., Mar. 4-6, 1964, pp. 165-177 (NASA SP-55, Nat. Aeron. and Space Admin., Washington, D.C., 1965)

\section{$\mathbf{T}$}

Taylor, A. H., Theory, construction, and use of the photometric integrating sphere, Bul. BS 18, 281-323 (1922-23), S447

\section{V}

Venable, W. H., Jr., Effects upon radiant intensity measurements due to scattering by optical elements, Appl. Opt. 9, No. 3, pp. 609-615 (March 1970). Key words: Instrument function; radiance; scattering

$7-351$

\section{W}

Wall, A. C., The photometry of colored light, Illum. Eng. 62, No. 4, 239-242 (Apr. 1967) --- 7-652

Waters, W. R., Stair, R., Schneider, W. E., and Jackson, J. K., Some factors affecting the sensitivity and spectral response of thermoelectric (radiometric) detectors, Appl. Opt. 4, No. 6, 703-710 (June 1965) 


\section{Author Index}

Weidner, V. R., Keegan, H. J., and Schleter, J. C.,

Volume and Page Effect of surface texture on diffuse spectral reflectance: A. Diffuse spectral reflectance of metal surfaces, Symp. Thermal Radiation of Solids, San Francisco, Calif., Mar. 4-6, 1964, pp. 165-177 (NASA SP-55, Nat. Aeron. and Space Admin., Washington, D.C., 1965) ------ 7-477

Weidner, V. R., High purity powdered CsI as a high reflectance infrared diffuser, Appl. Opt. 6, No. 7, 1280 (July 1967) _._._. 7-504 Wiebelt, J. A., Dunn, S. T., Richmond, J. C., Ellipsoidal mirror reflectometer, J. Res. Nat. Bur. Stand. (U.S.), 70C, No. 2, 75-88 (Apr.June 1966)
Wilson, S. W., and Barbrow, L. E., Vertical distribution of light from gas filled candlepower standards, Illum. Eng. 53, No. 12, 645-648 (Dec. 1958) $7-544$

\section{$\mathbf{Z}$}

Zipin, R. B., The apparent thermal radiation properties of an isothermal V-groove with specularly reflecting walls, J. Res. Nat. Bur. Stand. (U.S.) 70C, No. 4, 275-280 (Oct.-Dec. 1966) -- 7-50 


\section{Subject Index}

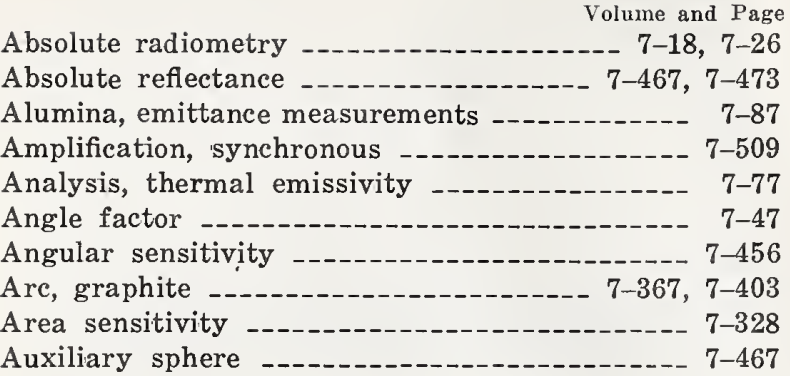

B

Bibliography, illumination

$7-563$

Blackbody, radiant cavity

$7-47$

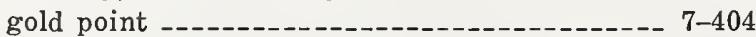

copper-point temperature --_-_-_-_-_-_-_- 7-403

platinum radiation _-_-_-_-_-- 7-525, 7-541, 7-569

radiance measurements _-_-_-_-_-_-_-_-_-_- 7-361

temperature measurements _-_-_-_-_- 7-367, 7-373

temperature scale _-_-_-_-_-_-_-_-_-- 7-390

Calibration, radiometric -

substitution measurements -_-_-_-_-_- 7-513

Calibration techniques, irradiance standard --_- 7-285

Calorimeters, absorption cell _-_-_-_-_-_-_-- 7-521

laser energy measurement _-_-_-_-_-_- 7-513

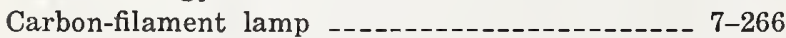

Cavities, conical-cylindrical _-_-_-_-_-38

Cavity radiation _._- 7-50, 7-87 absorption _-_-

detectors -_- $7-3$

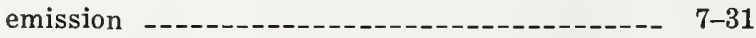

Cesium iodide, high purity _-_-_-_-_-_-_--- 7-504

Chloride, sodium coating -

Chromaticity coordinates -_-

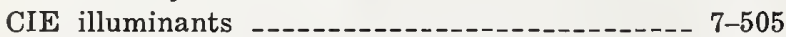

CIE luminous efficiency function -

Colorimetry, filters-daylight and sunlight --_- 7-573

Comparator, photometric _-_- 7-576

Conical cavity -_._- 7-47, 7-50

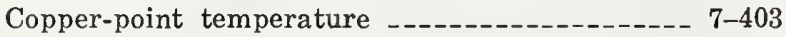

C.W. laser power

D

Density, optical -_-

Detectors, infrared _-_- 7-490

Diffuser, infrared _._- 7-504
Electromagnetic theory Volume and Page

Electron cooling

Ellipsoidal reflectometer _-_-_-_-_- 7-490

Emissivity _-_-_-_-_-_-38, 7-50

Emissivity in infrared _..-178

elevated temperatures --_-_-_-_-_-_--- 7-178

poly-crystalline calcium fluoride --_--_-_-_-- 7-178

single-crystal calcium fluoride -.--_-_-_-- 7-178

Emissivity measurements _-_-_-_-140

Emittance, change on heating -

Emittance measurements _-_-_-_-- 7-77, 7-87, 7-119

Emittance of metals, roughness effect _._._...- 7-145

surface characterization -_-_-_-_-_-_-_-145

thermal history _-_-_-_-_-_-_-_-_- 745

Emittance of nonmetals _-_-_-_-_-_-_- 7-221

effect of surface roughness -_-_-_-_-_- 7-221

surface roughness -_-_-_-_-_- 7-221

Emittance of oxidized Inconel _-_-_-111

Emittance standards _-_-_-- 7-99, 7-100, 7-107, 7-111

Energy, luminous _-_-_-_-_-_-_-_-_-_ 7-572

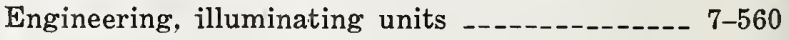

\section{$\mathbf{F}$}

Filter spectroradiometer

Filters, color -_-_-_-_-_-_-_- 7-573

daylight and sunlight _-_-_-_-_-_- 7-573

luminous efficiency -_-_-

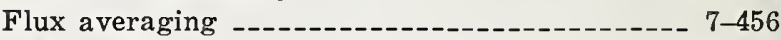

luminous -- $7-572$

total luminous -

G

Gold-black radiometry --_-_-_-_-_-_-_- 7-18

Gold point -...-...- 7-390, 7-404

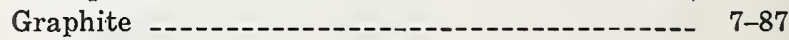

Graphite are _-_-_-_-_- 7-403

H

Handling emittance standards -_-_-_-_-_-- 7-99

Hemispherical emittance _-_-_-_-_-_-_-_-_ 7-246

ceramic coatings --.---.--

coated and uncoated Inconel _-_-_-- 7-149, 7-246

measurements -------------_-------------- 7-134

stainless steels-type 321 and 430 -.-------- 7-246

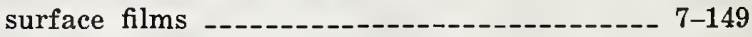

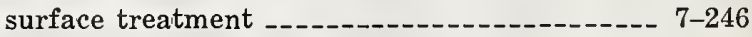

High-temperature measurement -------- 7-87, 7-373 


\section{Subject Index}

Volume and Page

Illumination $7-563$

photometric unit

$7-572$

Inconel, oxidized

$7-111$

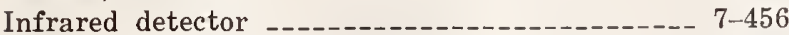

Infrared measurement techniques _-_-_-_-_- 7-195 computational methods _-_______-_-_-_ 7-195

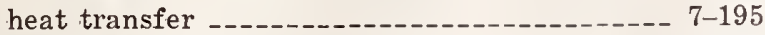

Instrument function _-_-_-_-_-_-_-_-_-_ 7-351

Irradiance, new-lamp standard _-_-___-_-_-_ 7-266 new standard _-_-_-_-_-_-_-_-_-_-_-_-- 7-274

Irradiance, quartz-iodine lamp _-_._._-_-_ 7-278 spectral _-_-_-_-_-_-_-_-_-_-_-_-_--- 7-289 standard, one solar constant standard for low values -_-_-_-_-_-_-- 7-280 tungsten-filament lamp _-_-_-_-_-_-_-_-_- 7-271

Integrating sphere _-_-_-409, 7-455, 7-456 general theory _-_-_-_- 7-473

Intensity, luminous _-_-_-_-_-_-_-_-_- 7-574

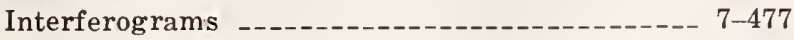

Ionization chamber -_-_-_-_-_-_-_- 7-26

Isothermal _._-

\section{$\mathbf{K}$}

Kanthal, oxidized

7-107

Kirchhoff's Law

$7-31$

L

Lamps, gas-filled standards $7-544$

Lamps, tungsten-filament $7-569,7-576$

Lamps, U.S. Standard _-_-_-_-_-_-_-576

Laser, energy measurements _-_-_ 7-336, 7-517, 7-521 measurement output energy _-_-_-_-_-_-_-_- 7-513 meter for large beams _-_-_-_-_-_-_-_- 7-518 pulsed ruby _-_-_-_-_-_-_-_-_-_-_- 7-521

Light, candlepower standards _-__-__-_-_-_ 7-544 primary standard _-_--_-_- -

\section{M}

Measurement techniques _-_-_-_-_-289

Measurements, luminous flux -_-_-_-_ 7-409, 7-456 pulsed ruby laser _-_-_-_-_-_-_-_-_- 7-513

Meter, laser power -_-_-_- 7-518

\section{$\mathbf{N}$}

NBS lamp standards

$7-271$

NBS 1000-W quartz-iodine lamp

$7-278$

NBS photoelectric pyrometer

7-390

Nomenclature, illuminating engineering _-_-_-- 7-560

reflectometry _-_-_-_-_-_-_-_-_-_-_- 7-459

temperature scale 1968 _-_-_-_-__-_- 7-569

Nonblackbody radiation

nonisothermal _._- 7-38
Volume and Page

Observer, standard _-______-_-574

Optical attenuator

Optical pyrometry _-_-4-403, 7-404

Oxide, magnesium -_-_-_-_-_-505

Oxidized nickel _-_-87

\section{P}

Paper lined cavity _._-_-_-_-87

Photometric, integrating sphere -_-_-_-_-_ 7-409

Photometry, absolute-new principle --_-_-_-- 7-576

photoelectric _-_-_-_-_-_-_-_-_-_-_-_-_- 7-517

Planck equation --_-_-_-_-_- 7-25

Platinum, blackbody radiator _-_._-_____-_ 7-541

Platinum-rhodium alloy _-_-_-_- 7-100

Power, pulsed laser _-_-_-_-_- 7-518

Pyrometer, NBS photoelectric ____-___-_-_ 7-390

$\mathbf{Q}$

Quartz-iodine lamp $7-274,7-278$

$\mathbf{R}$

Radiance, intensity measurements _-_-_-_-_-351

Radiant heat transfer _-_-_-_-_-_-_-_-_- 7-47

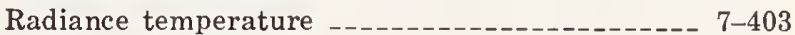

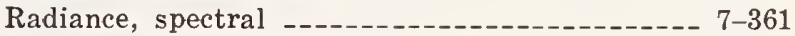

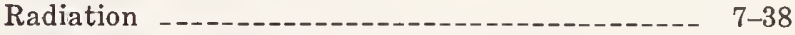

Radiation properties _-_-_-_-_-_-_-_--- 7-50

Radiation measurement techniques _-_-_-_- 7-3, 7-210 averaging spheres _-____-_-_-_10

infrared reflectance _._._._-_._-_._- 7-210

spectral reflectance

Radiation, ultraviolet _-_-_-_-_-_-_-_-_-_-_ 7-_

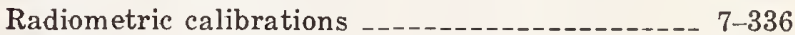

equations, radiometric _._._._._._. 7-25

standards, measurements

Radiometry -._-_-_-_- 7-25, 7-26, 7-373, 7-403 definition in reflectometry _._._._._. 7-459

Radiometry, infrared _-_-5-563

laser measurement _._-_-_-_-_- 7-517

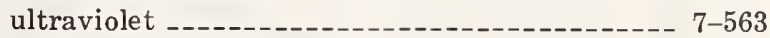

Reflectance, bidirectional _-_-_-_-490

factor _-_-_-_-_-504

high temperature _-_-_- 7-509

luminous -_-_-_-_-_-_-_- 7-505

modulation -_-_-_-_-_-_- 7-548

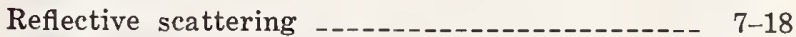

Reflectometer, ellipsoidal _-_-_-_-_-_-_-_-_-_ 7-40

laser source _-_-_- 7-509

Relation of emittance _-_-_-_-_- 7-236

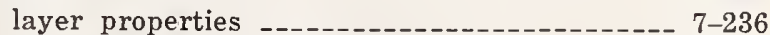

mathematical relations _-_-_-_-_-_-_- 7-236

to other optical properties _-_-_-_-_----- 7-236

Riemann integration -.--_-_-_-5 7-576

Rotating-specimen furnace 


\section{Subject Index}

Volume and Page

Scale, temperature, 1968

Scattering, optical instruments _-_-_-_-_-_ 7-351

Sensitive-surface uniformity _-_.-_-_-_-- 7-3

Shallow cavity reflectance

Shutter, sliding -_-_-_-576

Solar radiation measurement _-_-_-_-_-_-_-_-_ 7-297

Spectral emissivity measurement _-_-_-_----- 7-151

alumina _-_---_-_-_-151

ceramic oxides _-_-_-_-151

error sources _-_-_-_-_-_-_-151

oxidized Inconel -_-_-_-_-_- 7-174

platinum _-_.-- 7-174

rotating cylinder _-_-_-151

thoria -_-

zirconia --_--_--_-_-_-_-_-151

Spectral emittance _-_-_-_-_- 7-119, 7-184, 7-255

ceramic-coated materials _-_._-_._-_-_-_ 7-255

Inconel specimens _-_-_-_-_-_-_-_-_- 7-255

measurements _-_-_-_-_-_-_-_-_-_-_--- 7-56

stainless steel specimens _-_._- 7-255

standards _._-_-_- 7-56

uncoated specimens _-_-_-_-_- 7-255

Spectral irradiance, new standard _._._-_-_-_ 7-274

solar constant _-_-_-_-_-_- 7-285

standard for low values _-_-_-_-_-_-_-- 7-280

Spectral radiance _-_-_-_- 7-25, 7-361, 7-403 graphite arc _-_-_-_-_-_-_-_-_-_ 767

tungsten-strip lamp _ant

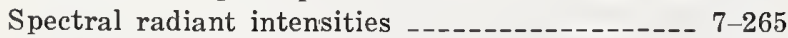

lamp standards 400-700 $\mathrm{m} \mu$-_-_-_-_-_-_-- 7-265

Spectral reflectance, metal surfaces _-_-_-_-_-_ 7-477

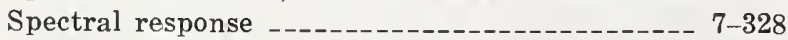

Spectrophotometry, absolute reflectance _-_-_-_- 7-467

Spectroradiometer, graphite arc

Spectroradiometry, filter _-_-_-_- 7-289

Spectroradiometry, prisms _-_-_-_-_-_-_--- 7-289

Specular component _._-___- 7-77

Spheres, averaging _-_-_-_- 7-490

Spheres, efficiency _-_-_-_-_- 7-473

Spherical candlepower _._...-_- 7-409

Standard observer _-_-_-_-_-_-_-_-_- 7-574

Standard reference materials _--_-7-100, 7-107, 7-111

Standardization, emittance measurements _-_--- 7-119

Standards, gas-filled lamps _-_-_-_-_-_-_-_ 7-544 quartz-iodine lamp -_-_-_-_-_-- 7-274, 7-278

spectral ilradiance _-_-_-_-_-_-_-_-_- 7-280

spectral radiance _-_-_-_-_-_-_-_-_-_-_ 761

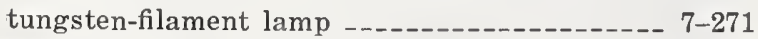

Surface texture, spectral reflectance

Sulphur coating -_-_-_-_-_-_-_-_-_-_-_ 7-455

Surface sensitivity maps _-_-_-_-_-_-_-_-_-_ 7-18

Stylus technique, measurement of reflectance _-- 7-477

Symbols for optical modulation

System, metric units _-___._-_-_-560

SI unit, luminous intensity -_-_-_-_-_--- 7-525

SI units, temperature scale 1968
Volume and Page

Temperature, color-2800 K _.

color-1600 to $20,000 \mathrm{~K}$-_._-_-_- $7-573$

Temperature scale, international practical _7-390, 7-569

Terminology, optical modulation _-_._-_-5-548

Thermal detectors -

Thermal radiation properties

$7-211,7-216,7-217,7-223,7-262$

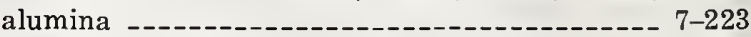

ceramic materials _-_-_-_-_-_- 7-223

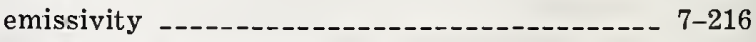

emissivity of metals _-_-_-_-_-_-_-_- 7-211

emittance -_-

emittance from shallow cavity _-_-_-_-_-_- 7-217

infrared reflectance -_-_-_-_- 7-216

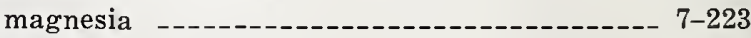

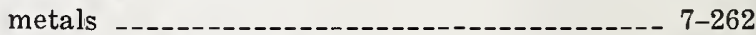

measurement techniques _-_--_-_-_-_-_-_---7-262

spectral reflectance -_-_-_-_-_- 7-216

standards calibration _-_-_-_-_-_-_ 7-211, 7-217

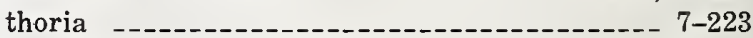

total emittance computation _._- 7-211

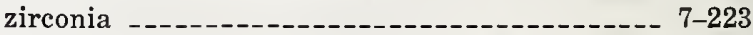

Thermopiles -

Thoria and tungsten _-_-___-_-_-_-_-_- 7-509

Total emittance -

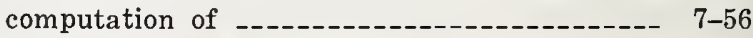

Total irradiance, lamp standards _-_-_-_-_-- 7-266

Tungsten-filament lamps _-_-_-_-_ 7-265, 7-266, 7-271

Tungsten-strip lamps _-_-_-_-_ 7-25, 7-361, 7-373

\section{$\mathrm{U}$}

Ultraviolet radiometry -__-_- 7-18, 7-26, 7-297

Units, photometric chart _-_-_-_-_-_-_-_-572

international photometric _-_-_-_-_-_--- 7-541

photometric, international system _-_-_-_- 7-560

V

V-groove cavity --_- 7-47, 7-50

W

Waidner-Burgess standard _-_-_-_-_-_-_-_-_- 7-525

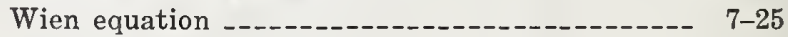




\section{Announcement of New Volumes in the NBS Special Publication 300 Series Precision Measurement and Calibration}

Superintendent of Documents, Government Printing Office, Washington, D. C. 20402

Dear Sir:

Please add my name to the announcement list of new volumes to be issued in the series: National Bureau of Standards Special Publication 300, Precision Measurement and Calibration.

Name

Company

Address

City State Zip Code

(Notification key N-353) 

FORM NBS.114A $(1.71)$

\begin{tabular}{|c|c|c|c|}
\hline $\begin{array}{l}\text { U.S. DEPT. OF COMM, } \\
\text { BIBLIOGRAPHIC DATA } \\
\text { SHEET }\end{array}$ & $\begin{array}{l}\text { 1. PUBLICATION OR REPORT NO. } \\
\text { NBS-SP-30O- V.'? }\end{array}$ & $\begin{array}{l}\text { 2. Gov't Accession } \\
\text { No. }\end{array}$ & 3. Recipient's Accession No. \\
\hline \multirow{2}{*}{\multicolumn{3}{|c|}{$\begin{array}{l}\text { Precision Measurement and Calibration } \\
\text { Radiometry and Photometry }\end{array}$}} & $\begin{array}{l}\text { 5. Publication Date } \\
\text { November } 1971\end{array}$ \\
\hline & & & 6. Performing Organization Code \\
\hline \multicolumn{3}{|c|}{$\begin{array}{l}\text { 7. AUTHOR(S) } \\
\text { H. K. Hammond III, H. L. Mason }\end{array}$} & 8. Performing Organization \\
\hline \multicolumn{3}{|c|}{ 9. PERFORMING ORGANIZATION NAME AND ADDRESS } & 10. Project/Task/Work Unit No. \\
\hline \multicolumn{3}{|c|}{$\begin{array}{l}\text { NAT IONAL BUREAU OF ST AND ARDS } \\
\text { DEPARTMENT OF COMMERCE } \\
\text { WASHINGTON, D.C. } 20234\end{array}$} & 11. Contract/Grant No. \\
\hline \multirow{2}{*}{\multicolumn{3}{|c|}{ 12. Sponsoring Organization Name and Address }} & $\begin{array}{l}\text { 13. Type of Report \& Period } \\
\text { Covered } \\
\text { Final }\end{array}$ \\
\hline & & & 14. Sponsoring Agency Code \\
\hline
\end{tabular}

15. SUPPLEMENTARY NOTES

16. ABSTRACT (A 200-word or less factual summary of most significant information. If document includes a significant bibliography or literature survey, mention it here.)

This volume is one of an extended series which brings together some of the previously published papers, monographs, abstracts and bibliographies by NBS authors dealing with the precision measurement of specific physical quantities and the calibration of the related metrology equipment. The contents have been selected as being useful to the standards laboratories of the United States in tracing to NBS standards the accuracies of measurement needed for research work, factory production, or field evaluation.

Volume 7 contains reprints of more than 60 items on radiometry and photometry publisher between 1957 and 1970. The material has been placed in groups according to the following subjects: general radiometry, emissivity standards, emissivity measurements and techniques, material properties, irradiance standards, radiometric measurement techniques, radiance standards, flux measurement, reflectometry, general photometry, projectors, flashing lights, and chronological bibliography of publications by NBS staff.

17. KEY WORDS (Alphabetical order, separated by semicolons)

emissivity; flashing light; flux measurement; irradiance; material properties; photometry; projectors; radiance; radiometry; reflectometry.

18. AVAILABILITY STATEMENT

X] UNLIMITED.

FOR OFFICI AL DISTRIBUTION. DO NOT RELEASE TO NTIS.

\begin{tabular}{|l|c|}
$\begin{array}{l}\text { 19. SECURITY CLASS } \\
\text { (THIS REPORT) }\end{array}$ & $\begin{array}{c}\text { 21. NO. OF PAGES } \\
\text { UNCL ASSIFIED }\end{array}$ \\
\hline $\begin{array}{l}\text { 20. SECURITY CLASS } \\
\text { (THIS PAGE) }\end{array}$ & 22. Price \\
UNCLASSIFIED & $\$ 7.00$ \\
\hline
\end{tabular}








\section{Official SI Unit Names and Symbols}

Fot complete statement of NBS practice, see NBS Tech. News Bull. Vê. 55, No, 1, Jan. 1971, 30ל."* Sealso NBS Special Publication 330, The International System of Units SI, Jan. 1971, 15 $\phi^{\text {* }}$.

"failable by purchase from the Supt. of Documents, Government Printing Office, Washington, D. C. 20402

\begin{tabular}{|c|c|c|c|}
\hline Name & Symbol & Name & Symbol \\
\hline meter & $\mathrm{m}$ & newton & $\mathrm{N}$ \\
\hline kilogram_-_- & $\mathrm{kg}$ & 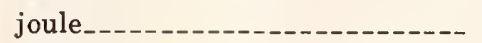 & $\mathbf{J}$ \\
\hline second & $\mathrm{s}$ & watt_- & W \\
\hline ampere & A & 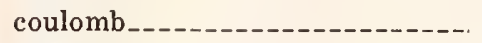 & $\mathrm{C}$ \\
\hline 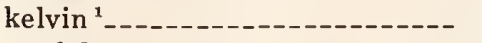 & $\mathrm{K}$ & volt_---- & V \\
\hline 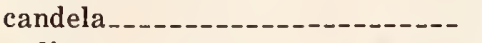 & $\mathrm{cd}$ & ohm & $\Omega$ \\
\hline radian_-_-n- & $\mathrm{rad}$ & 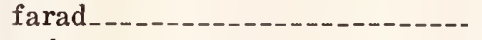 & $\mathbf{F}$ \\
\hline steradian. & sr & weber-1- & $\mathrm{Wb}$ \\
\hline 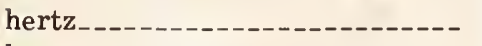 & $\mathrm{Hz}$ & henry & $\mathrm{H}$ \\
\hline 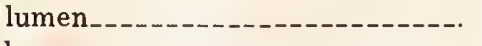 & $\operatorname{lm}$ & tesla_. & $\mathbf{T}$ \\
\hline lux & $\mathrm{lx}$ & & \\
\hline
\end{tabular}

Additional Names and Symbols approved for NBS use

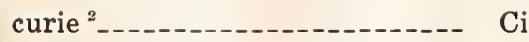

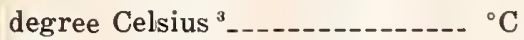

gram

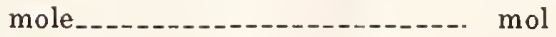

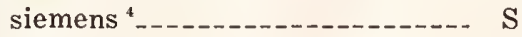

I The same name and symbol are used for thermodynamic temperature and teinperature interval. (Adopted by the 13th General Conference on Weights \& Measures, 1967).

accepted by the General Conference on Weights \& Measures for use with the SI, but to be eventually abandoned.

3 For expressing "Celsius temperature"; may also be used for a temperature interval.

4 Adopted by IEC and ISO.

\section{Table for Converting U.S. Customary Units to Those of the International System (SI) ${ }^{5}$}

To relate various units customarily used in the United States to those of the International System, the National Bureau of Standards uses the conversion factors listed in the "ASTM Metric Practice Guide", designation:E 380-70. These are based on international agreements effective July 1, 1959, between the national standards laboratories of Australia, Canada, New Zealand, South Africa, the United Kingdom, and the United States.

\section{To convert from:}

(1) inches to meters, multiply by 0.0254 exactly.

(2) feet to meters, multiply by 0.3048 exactly.

(3) feet (U.S. survey) to meters, multiply by $1200 / 3937$ exactly.

(4) yards to meters, multiply by 0.9144 exactly.

(5) miles (U.S. statute) to meters, multiply by 1609.344 exactly.

(6) miles (international nautical) to meters, multiply by 1852 exactly.

(7) grains $(1 / 7000 \mathrm{lbm}$ avoirdupois) to grams, multiply by 0.06479891 exactly.

(8) troy or apothecary ounces mass to grams, multiply by $31.10348 \ldots$

(9) pounds-force (lbf avoirdupois) to newtons, multiply by $4.448222 \ldots$.

(10) pounds-mass (lbm avoirdupois) to kilog rams, multiply by $0.453592 \ldots$

(11) fluid ounces (U.S.) to cubic centimeters, multiply by $29.57 \ldots$

(12) gallons (U.S. liquid) to cubic meters, multiply by $0.003785 \ldots$

(13) torr $\left(\mathrm{mm} \mathrm{Hg}\right.$ at $\left.\mathrm{O}^{\circ} \mathrm{C}\right)$ to newtons per square meter, multiply by 133.322 exactly.

(14) millibars to newtons per square meter, multiply by 100 exactly.

(15) psi to newtons per square meter, multiply by $6894.757 \ldots$

(16) poise to newton-seconds per square meter, multiply by 0.1 exactly.

(17) stokes to square meters per second, multiply by 0.0001 exactly.

(18) degrees Fahrenheit to kelvins, use the relation $T_{e s}=\left(t_{\mathrm{F}}+459.67\right) / 1.8$.

(19) degrees Fahrenheit to degrees Celsius, use the relation $T_{\mathrm{Bs}}=\left(t_{\mathrm{F}}-32\right) / 1.8$.

(20) curies to disintegrations per second, multiply by $3.7 \times 10^{10}$ exactly

(21) roentgens to coulombs per kilogram, multiply by $2.579760 \times 10^{-4}$ exactly.

\footnotetext{
${ }^{5}$ Systeme International d'Unites (designated SI in all languages).
} 
i.

(1)

(3)

(4)

$13(3)$

ais

1.

all

(i)
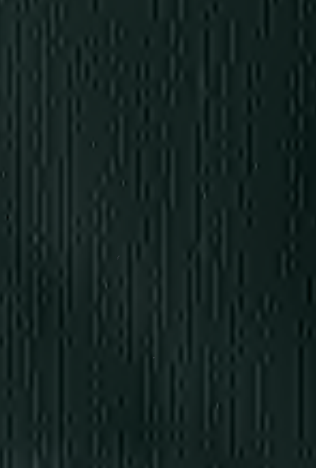

(ii) id 